\title{
ПАNЕПІ
}

\section{ТМНМА ПАНРОФОРІКНУ}

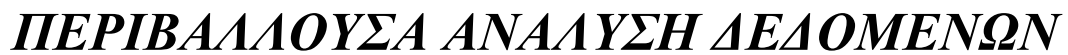

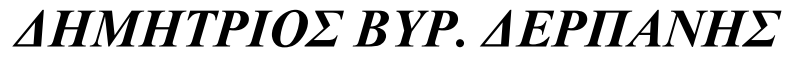

$\triangle$ I $\Delta$ AKTOPIKH $\triangle$ IATPIBH

- ПЕIPAIA 2009 - 


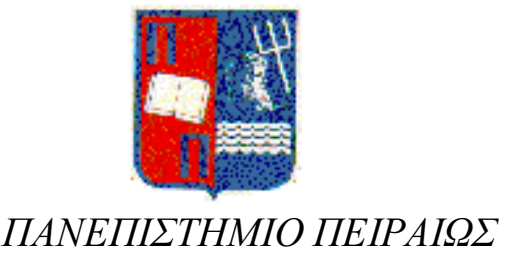

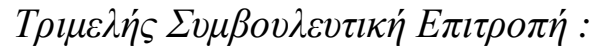

$E \pi \imath \beta \lambda \varepsilon ́ \pi \omega v$ :

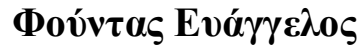

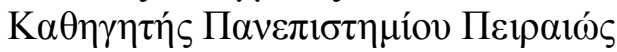

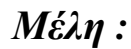

Nuкó $\lambda \alpha 0 \varsigma A \lambda \varepsilon \xi \alpha v \delta \rho \eta ́ \varsigma$

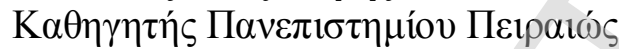

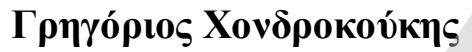

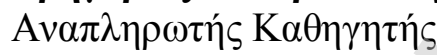

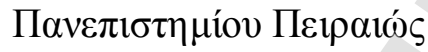

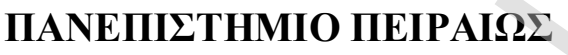 ТМНМА ПАНРОФОРІКНЕ}

\section{$\triangle I A T P I B H$}

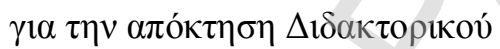

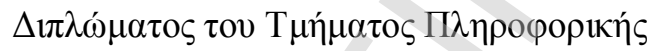

$\Delta \eta \mu \eta \tau \rho i ́ o v ~ B . ~ \Delta \varepsilon \rho \pi \alpha ́ v \eta$

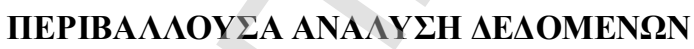

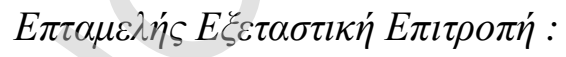

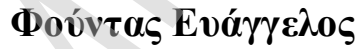

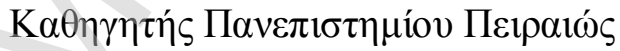

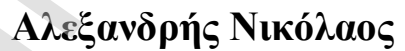

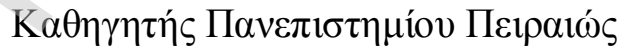

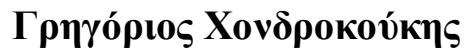

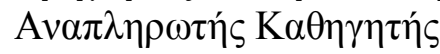

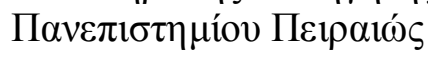

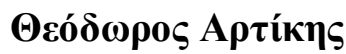

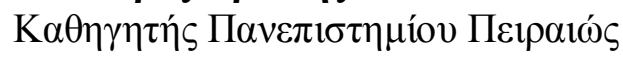

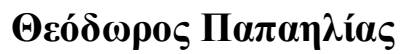

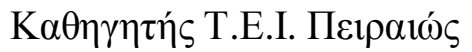

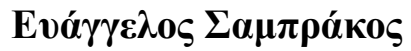

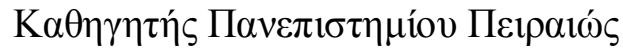

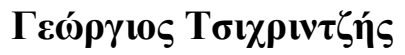

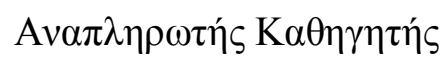

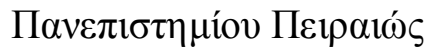


«' $\mathcal{H} \theta \varepsilon \lambda \alpha, \pi \rho \imath \nu \mu \varepsilon \varsigma \sigma \tau ' \dot{\alpha} \pi \varepsilon \imath \rho o \sigma \imath \omega \pi \dot{\eta} \sigma \omega$,

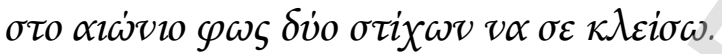

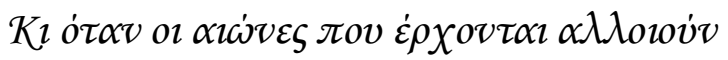

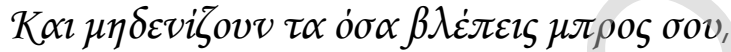

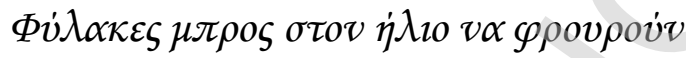

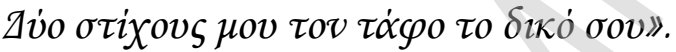

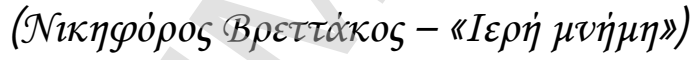

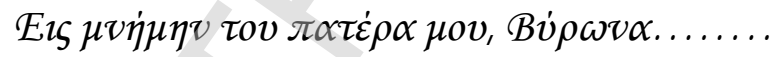




\section{ПЕРІАНЧН}

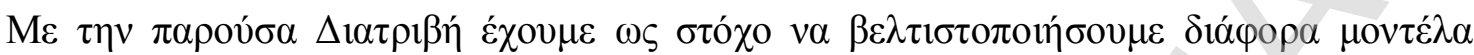

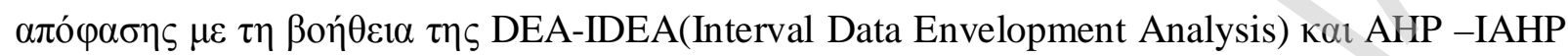

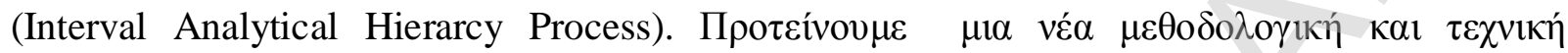

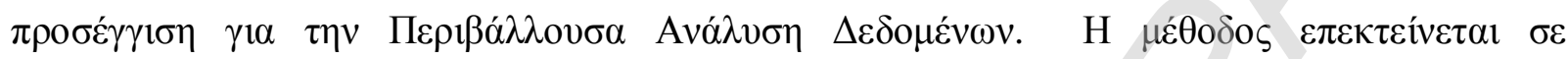

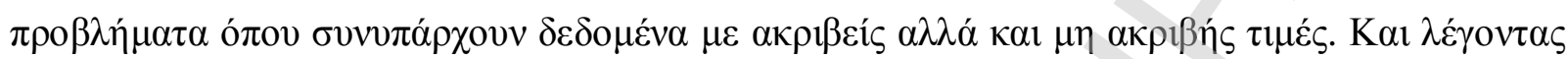

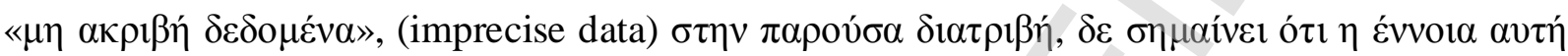

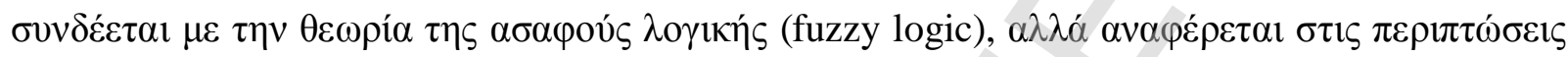

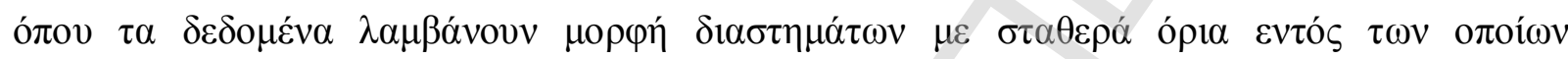

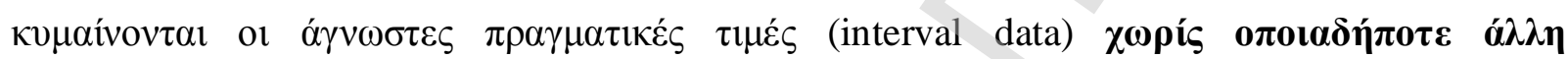

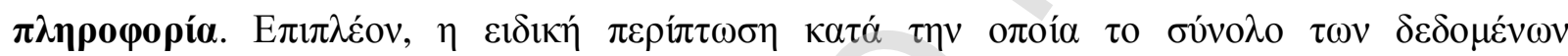

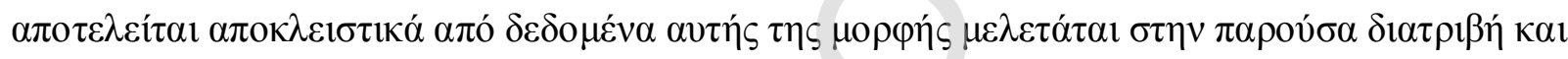

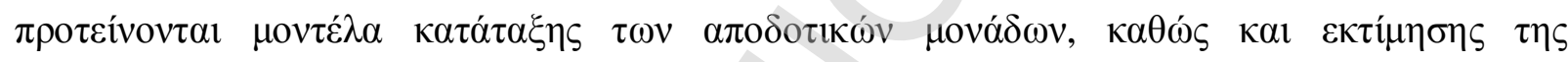

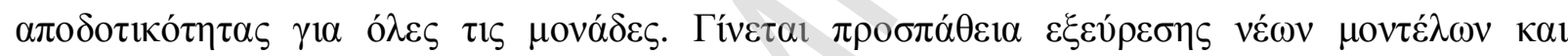

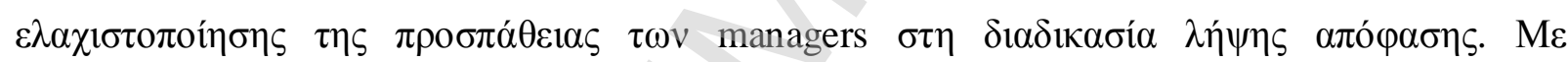

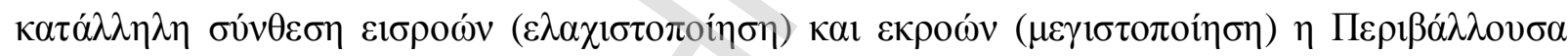

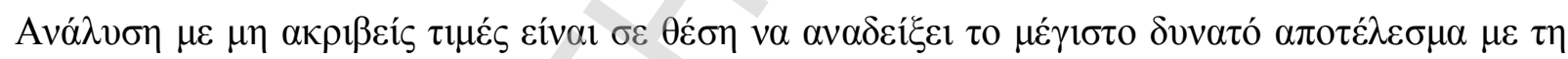

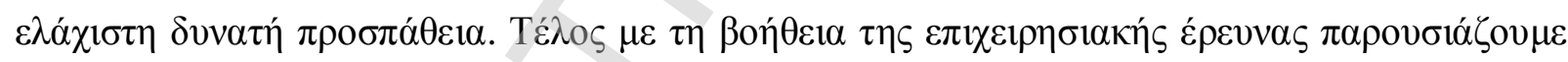

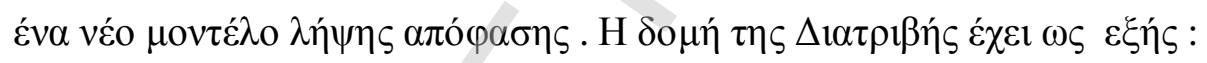

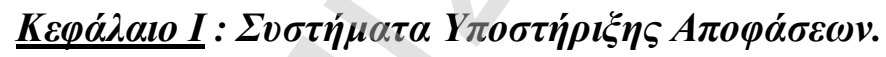

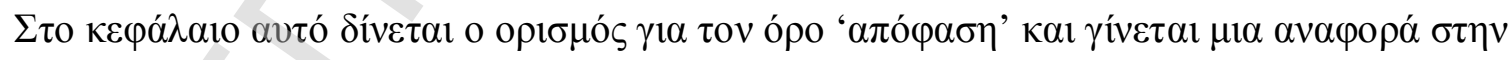

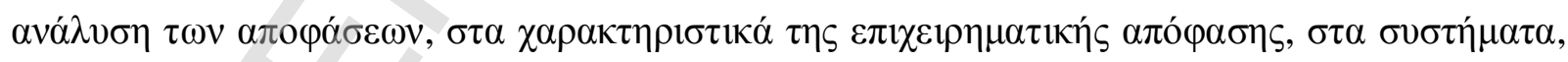
ó

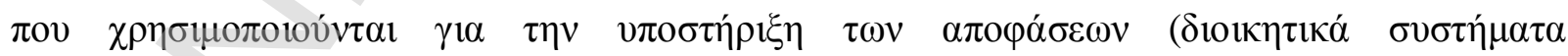

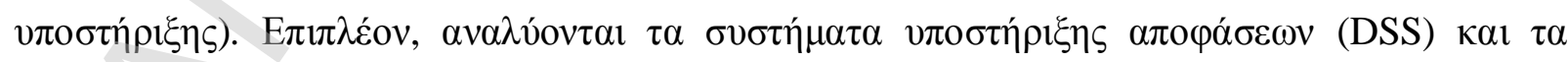

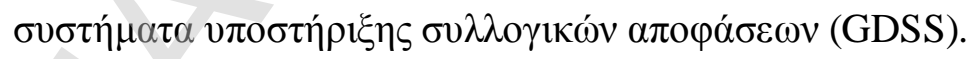




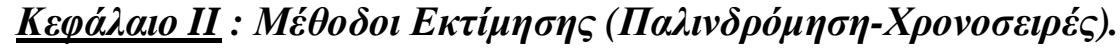

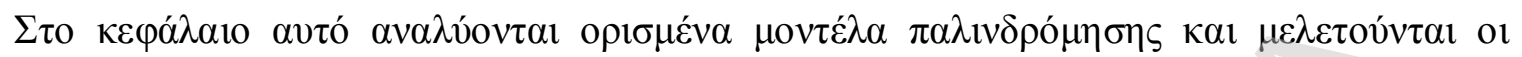

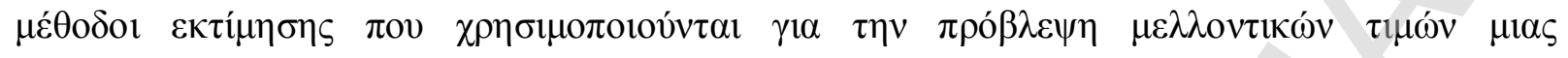

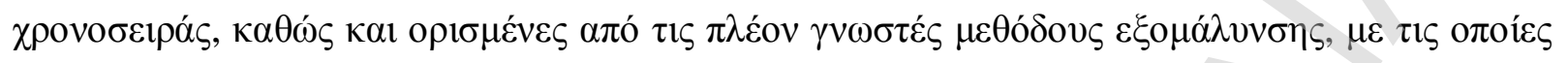

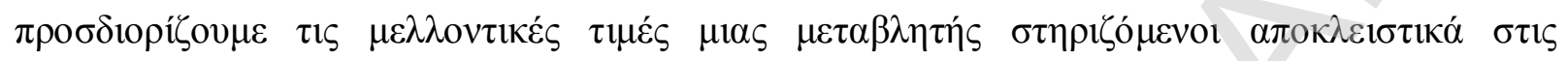

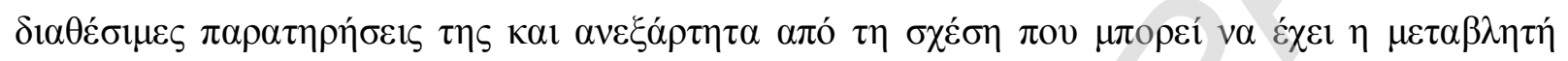
$\alpha v \tau \eta \dot{~} \mu \varepsilon \alpha \dot{\alpha} \lambda \eta \eta \dot{~} \alpha \lambda \lambda \varepsilon \varsigma \mu \varepsilon \tau \alpha \beta \lambda \eta \tau \varepsilon \dot{\varepsilon}$.

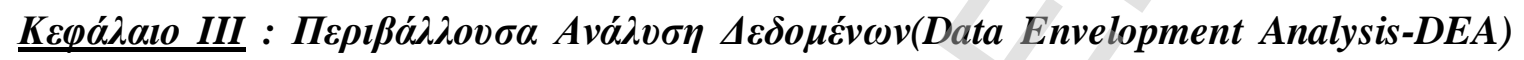

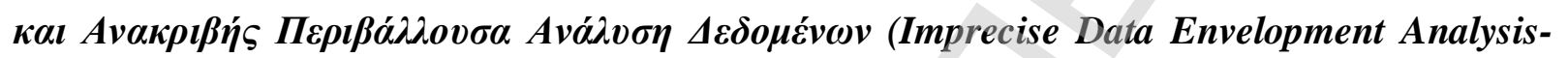
IDEA).

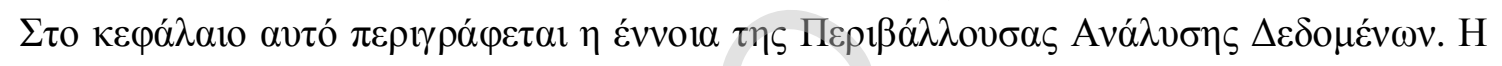

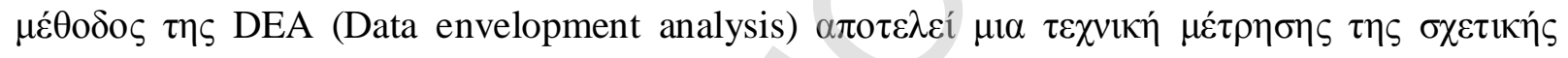

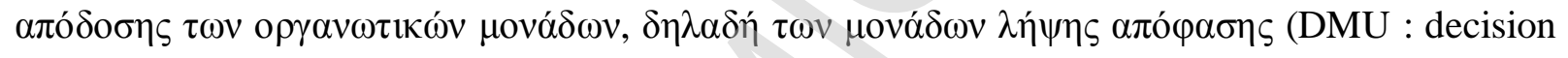

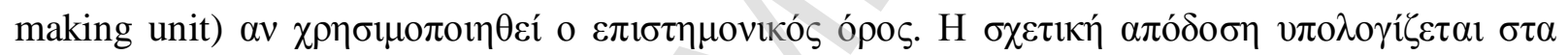

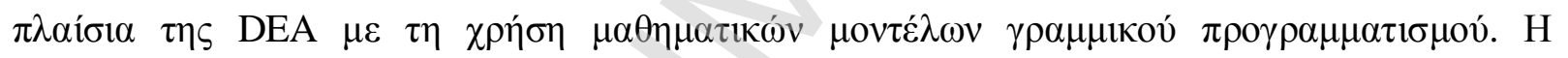

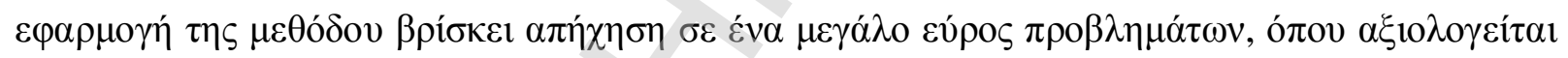

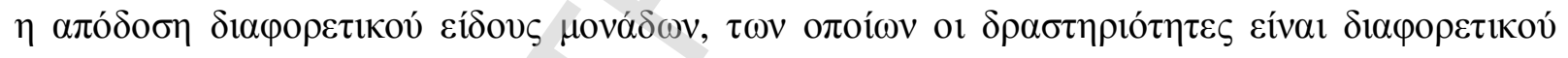

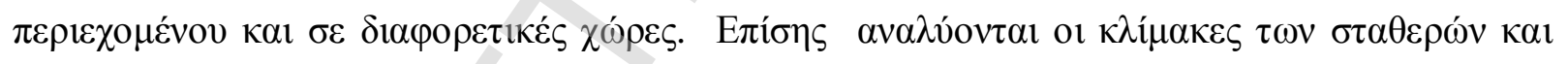

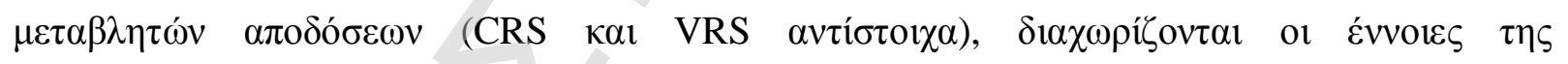

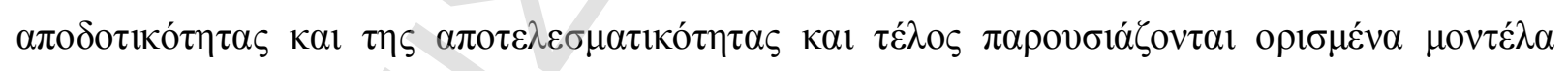

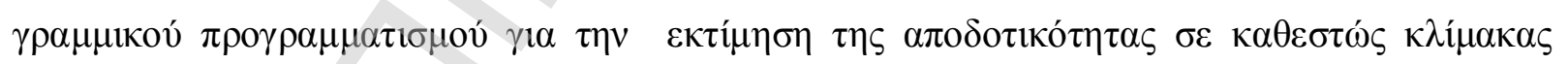

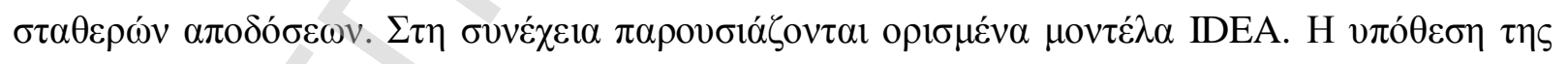

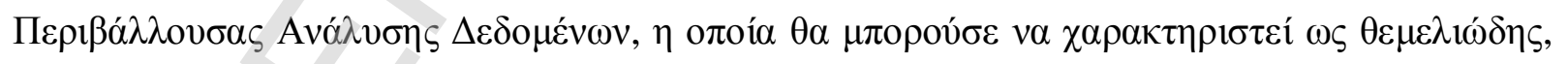

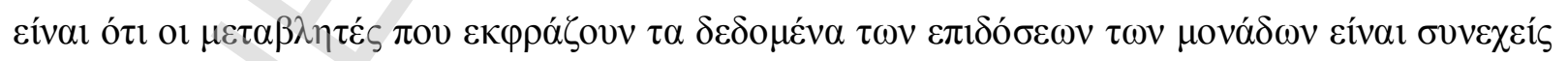

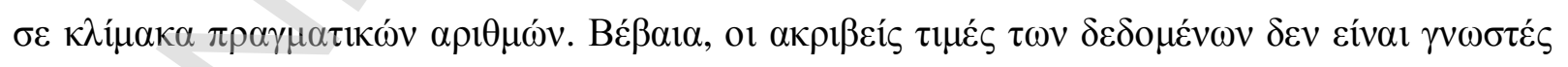

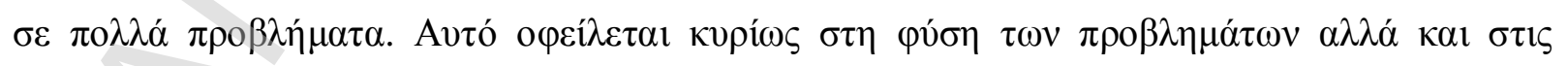

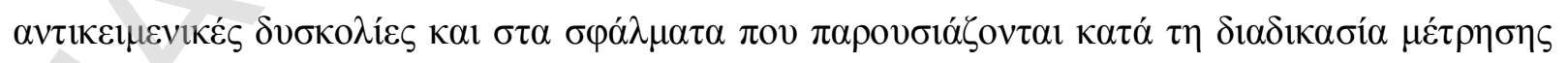

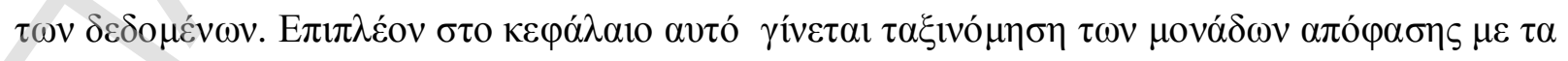

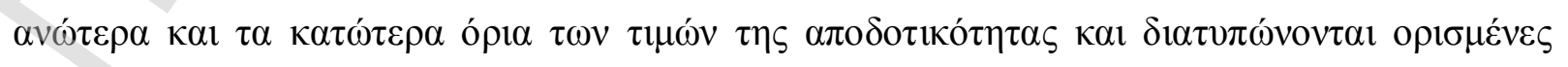

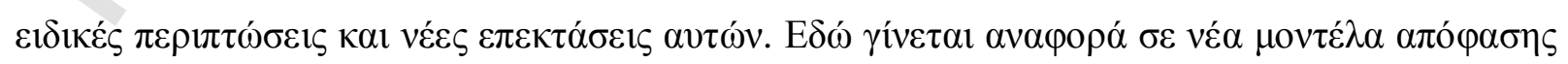

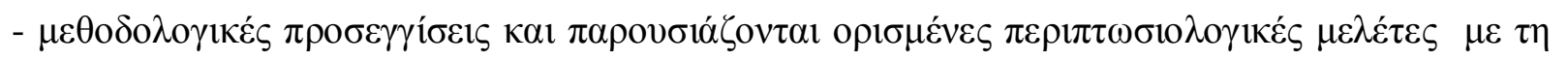




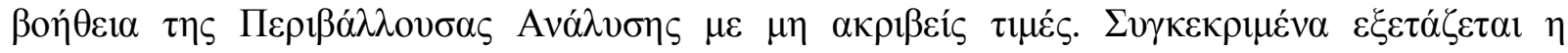

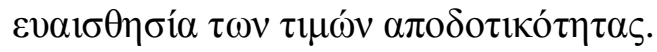

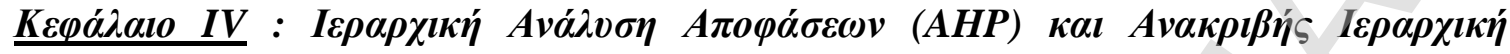

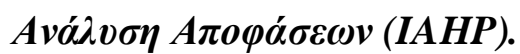

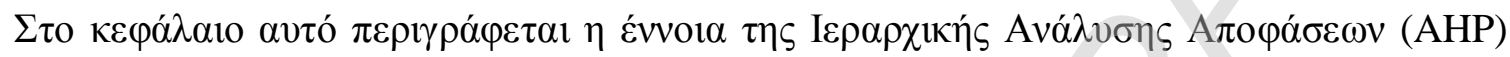

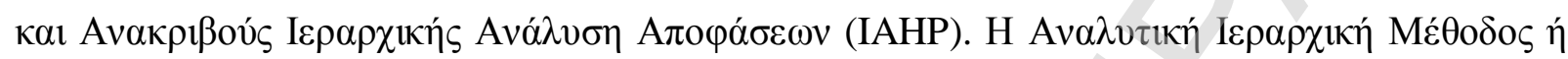

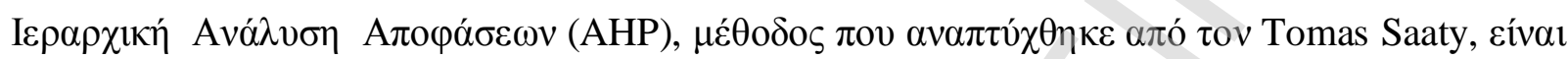

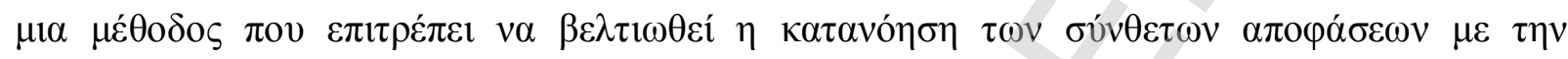

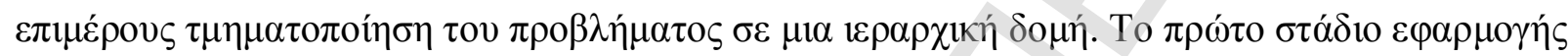

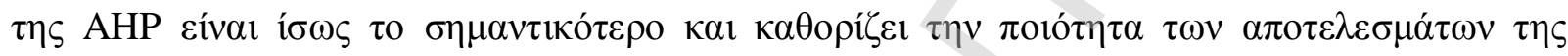

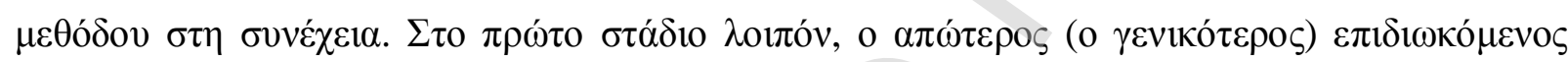

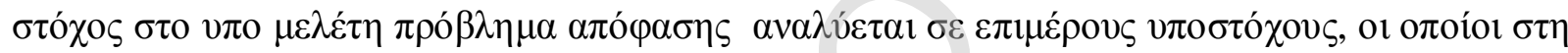

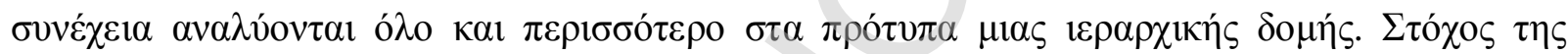

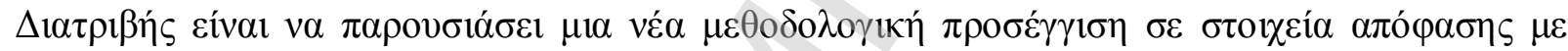

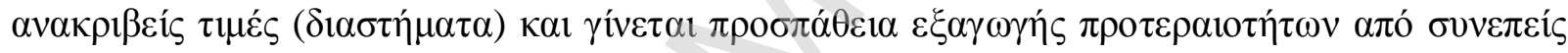

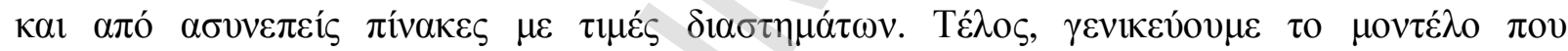

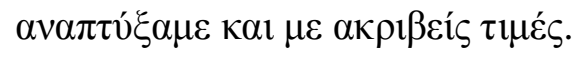

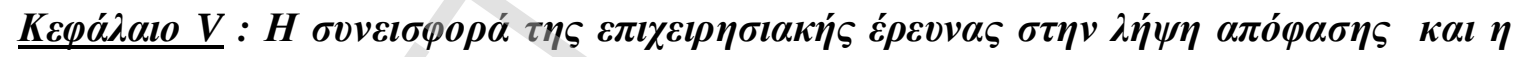

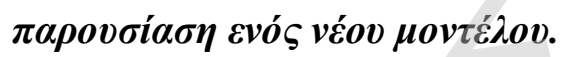

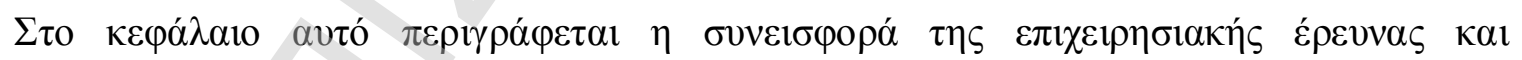

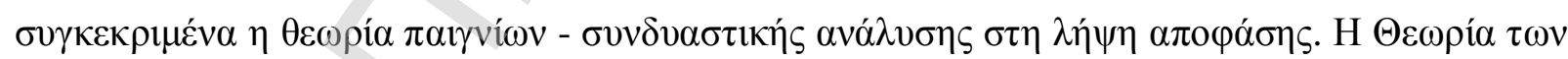

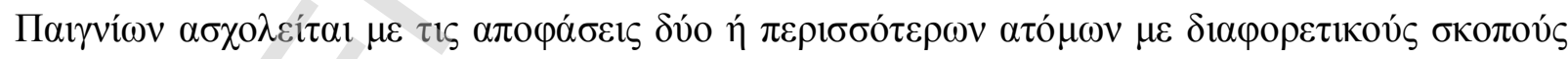

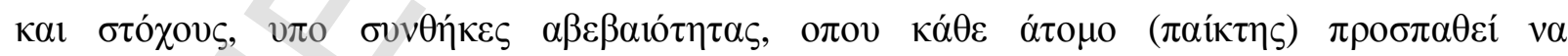

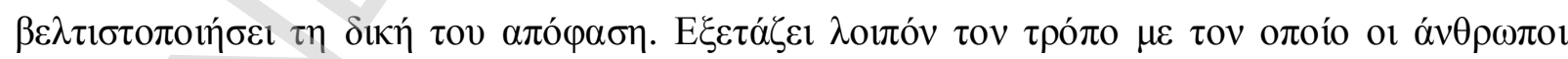

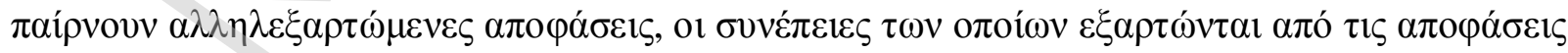

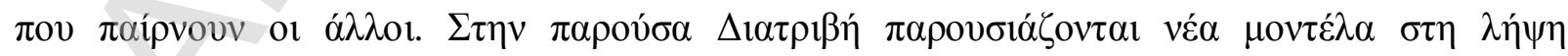

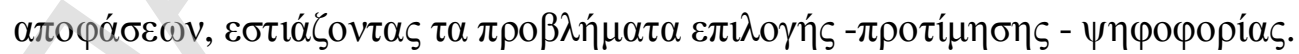




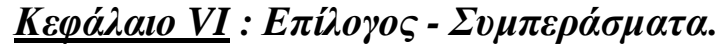

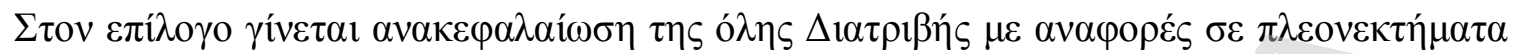

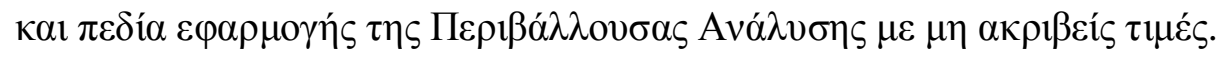

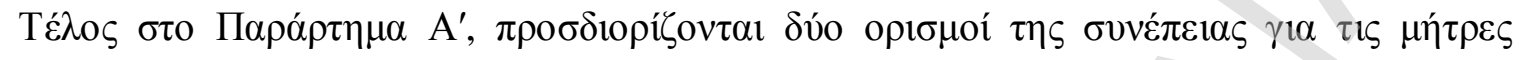

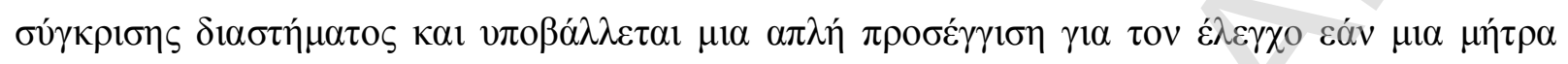

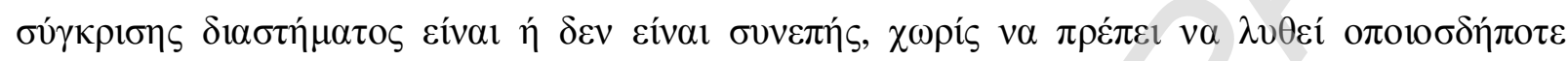

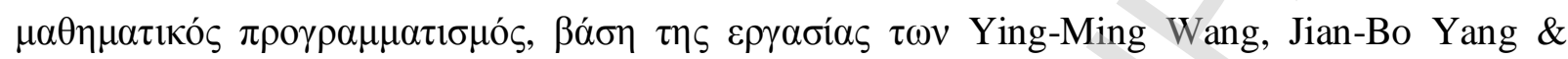

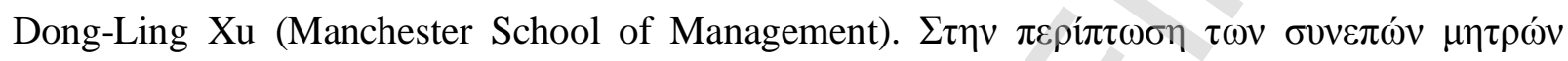

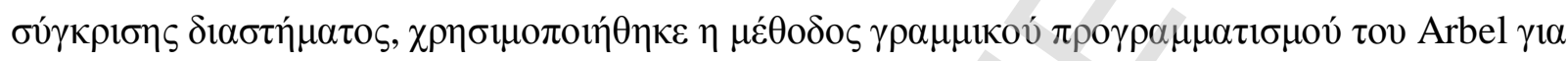

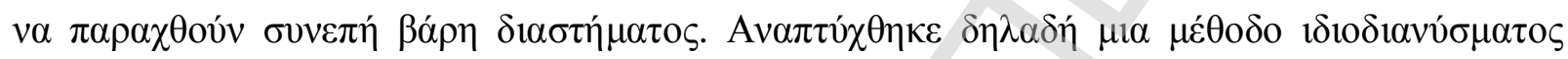

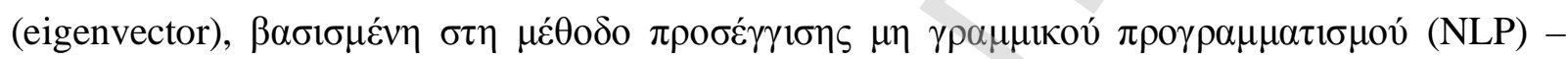

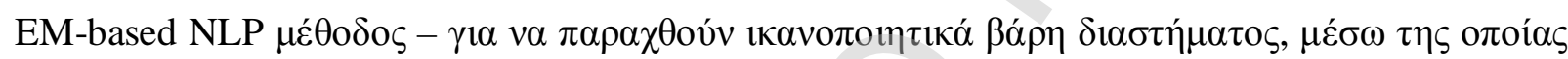

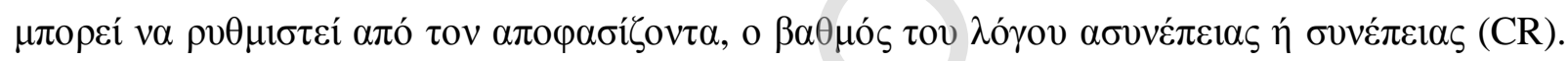

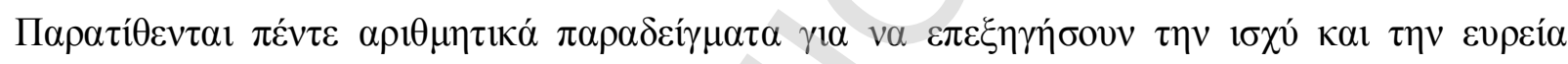

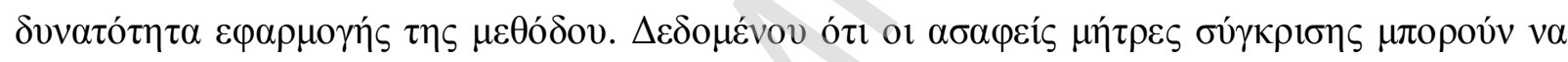

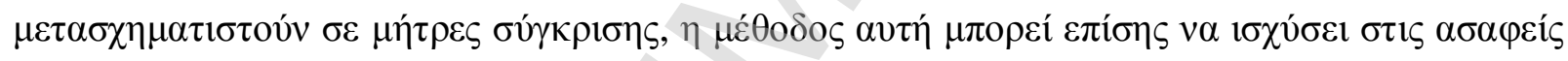

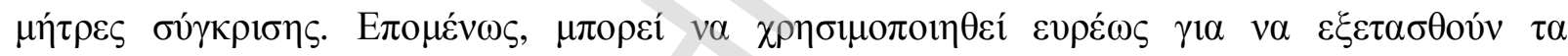

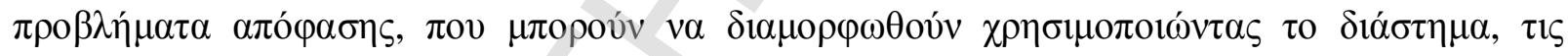

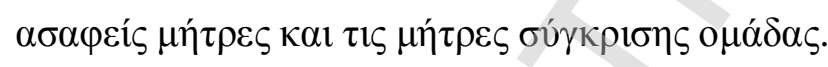




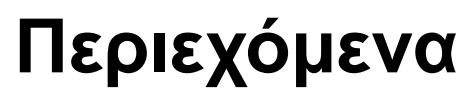

ПEPIEXOMENA

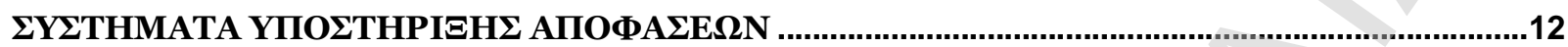

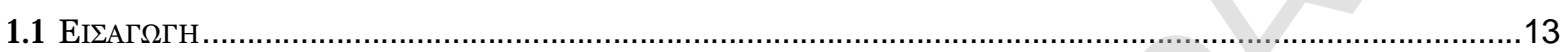

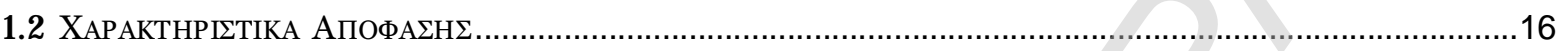

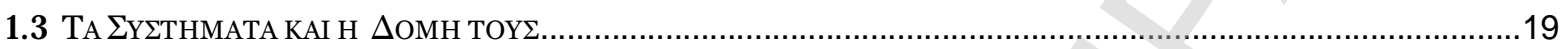

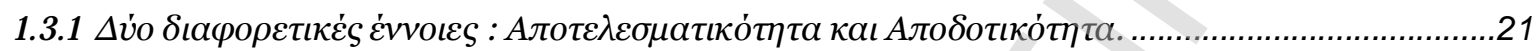

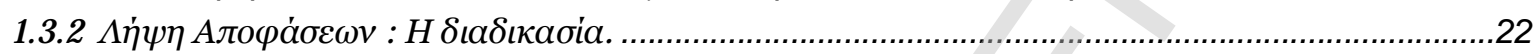

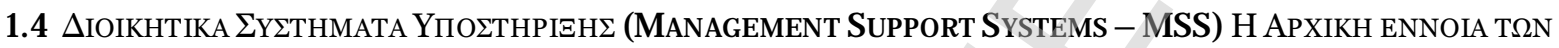

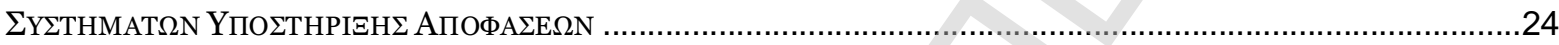

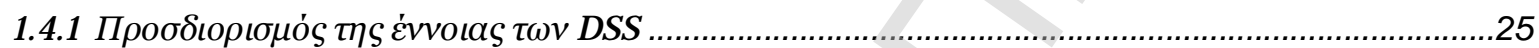

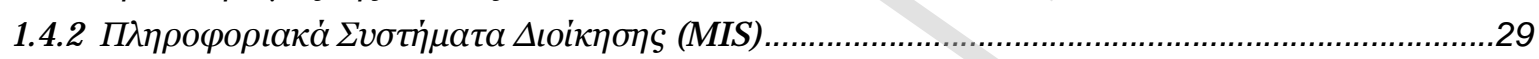

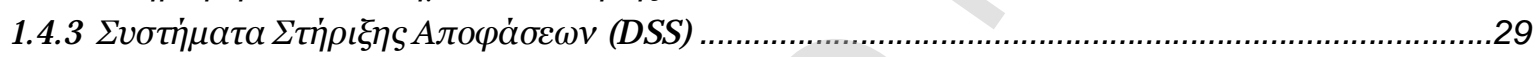

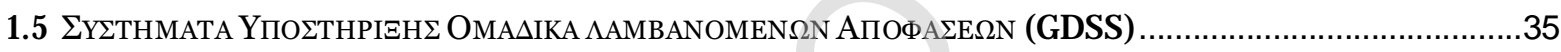

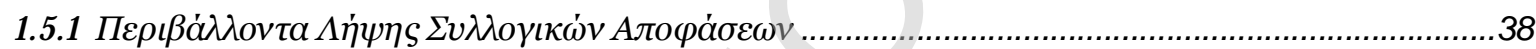

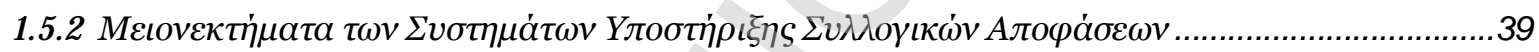

1.6 П

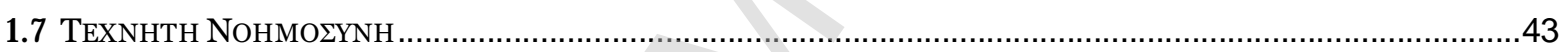

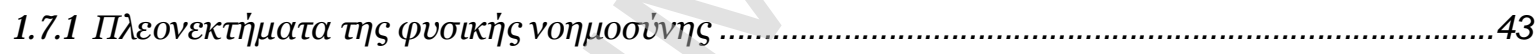

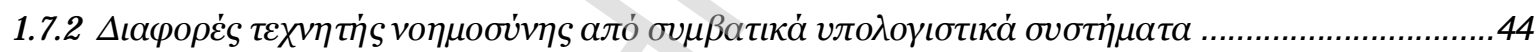

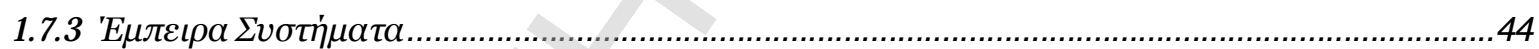

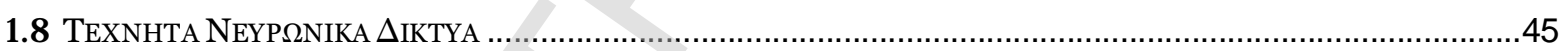

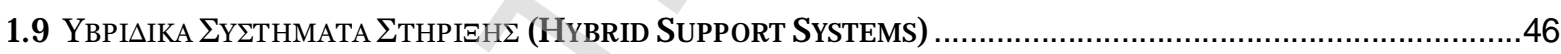

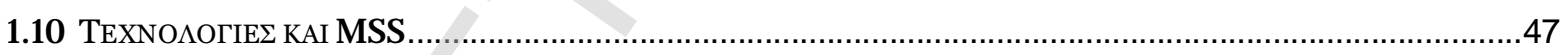

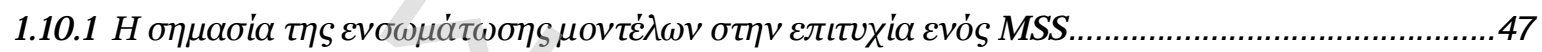

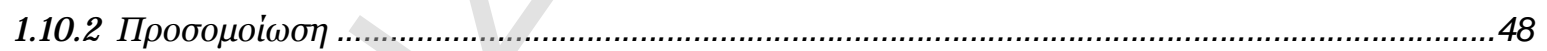

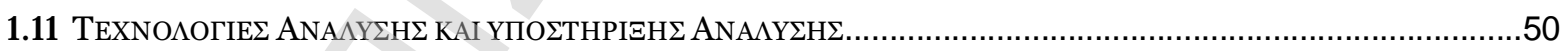

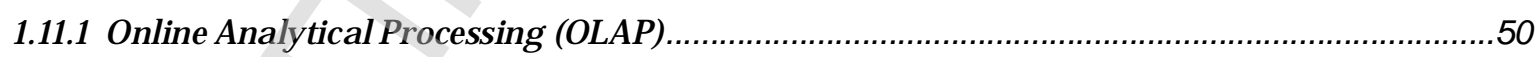

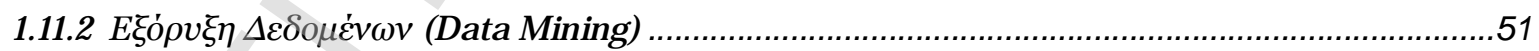

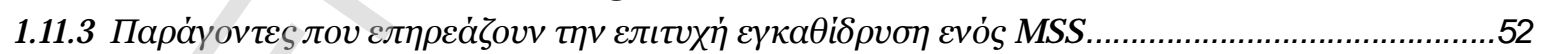

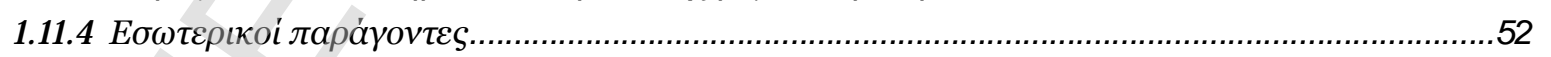

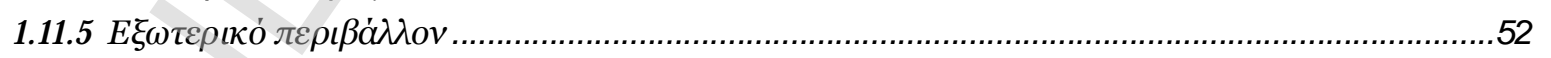

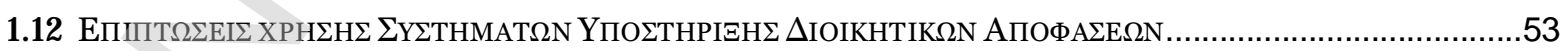

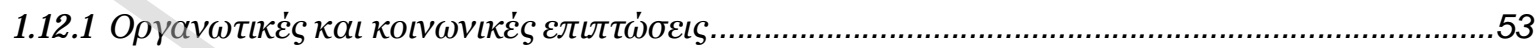

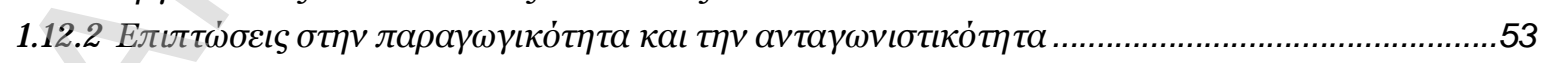

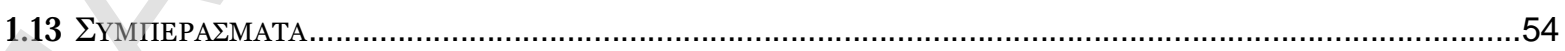

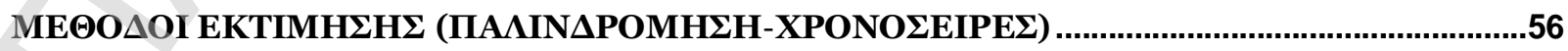

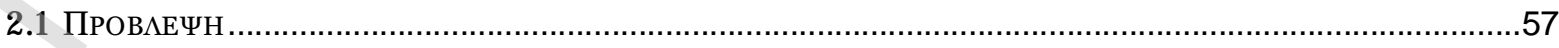

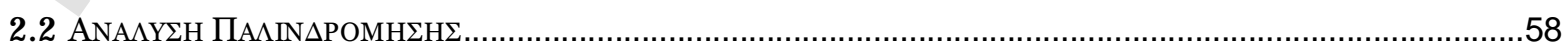

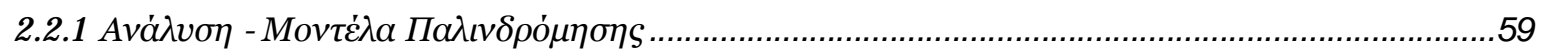




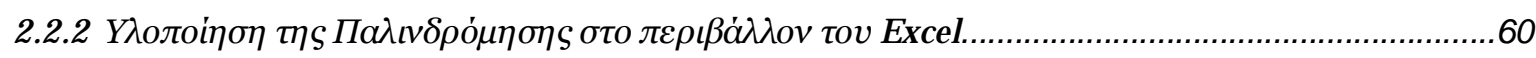

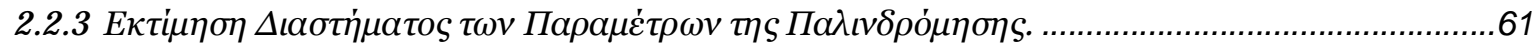

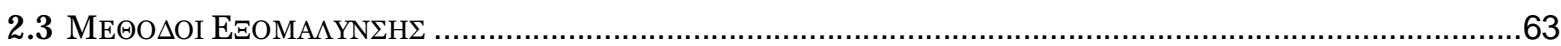

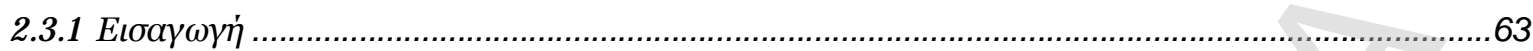

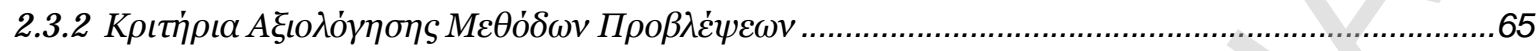

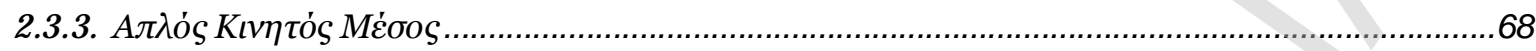

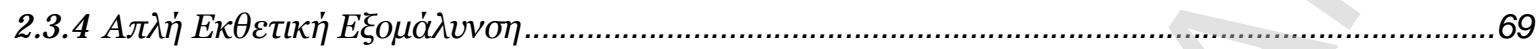

2.4 XPONOEEIPE

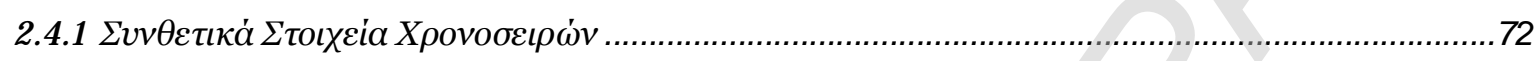

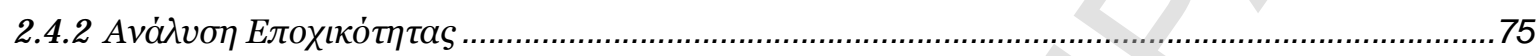

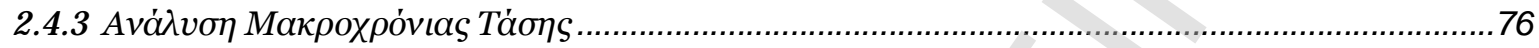

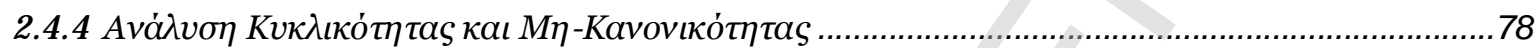

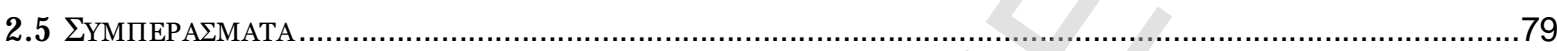

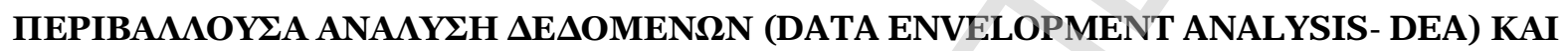

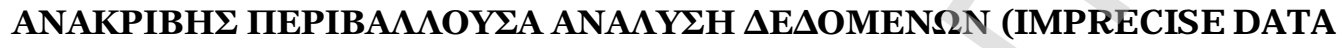

ENVELOPMENT ANALYSIS - IDEA) ........................................................................................82

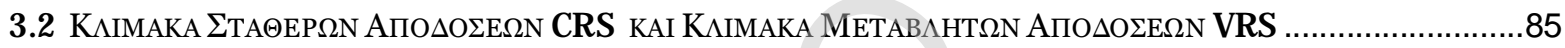

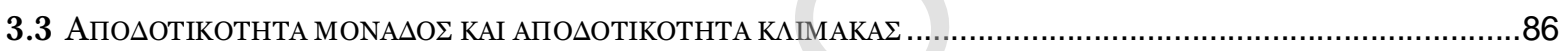

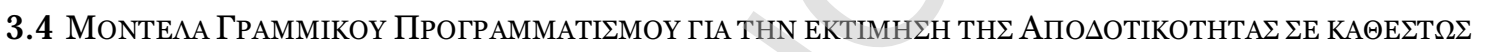

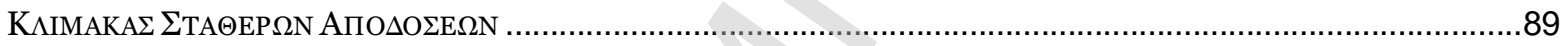

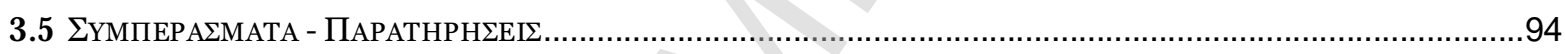

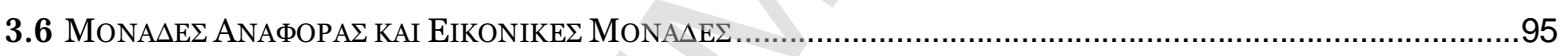

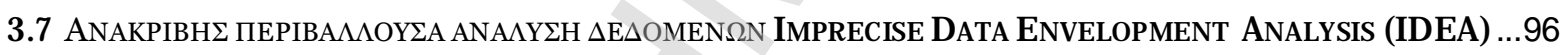

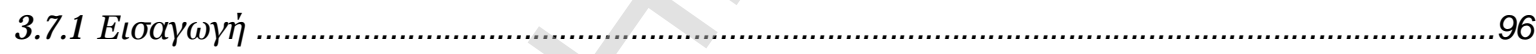

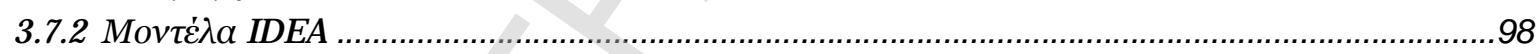

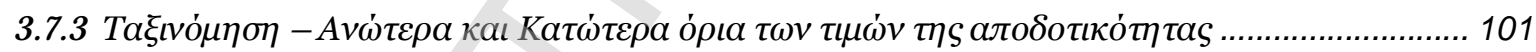

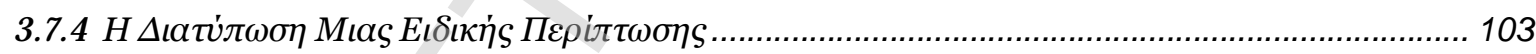

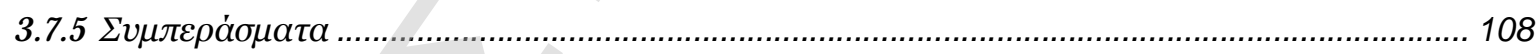

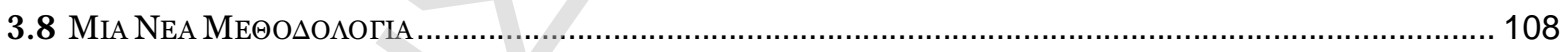

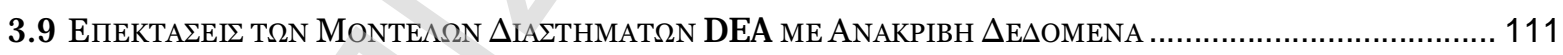

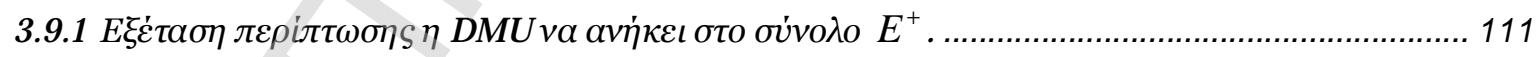

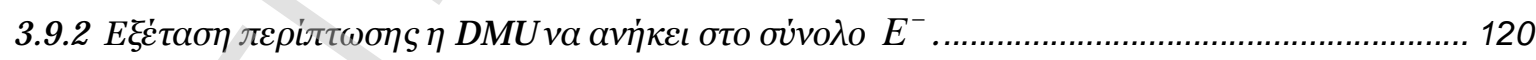

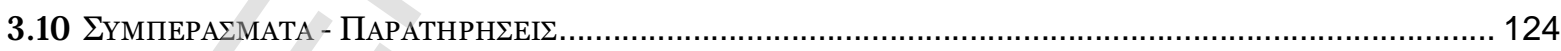

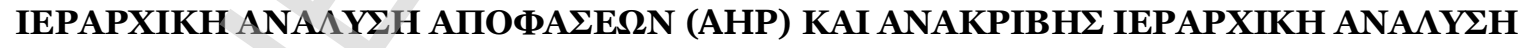

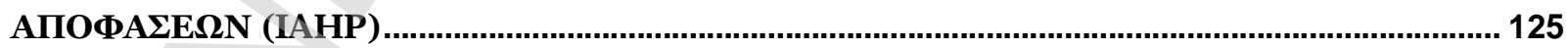

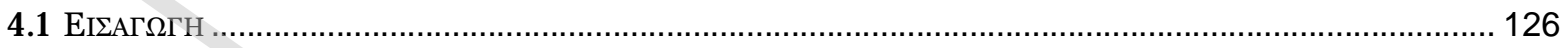

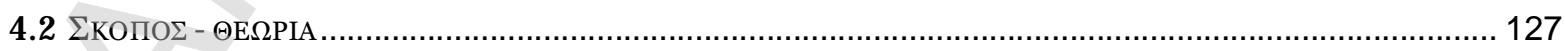

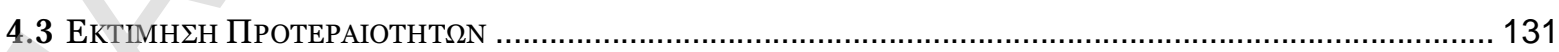

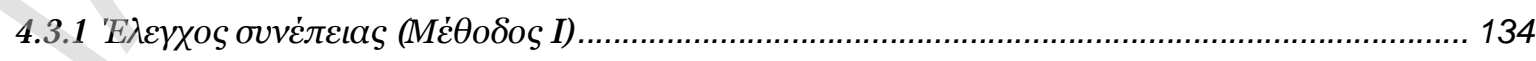

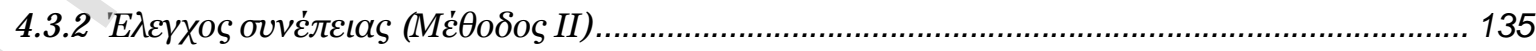

4.4 ҮПОАОГІ

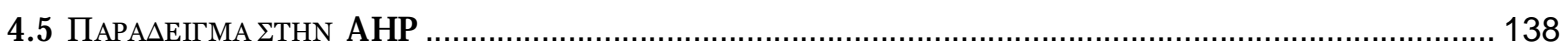




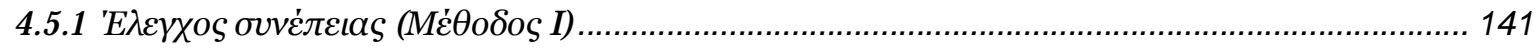

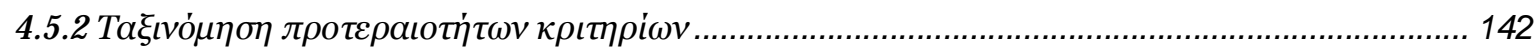

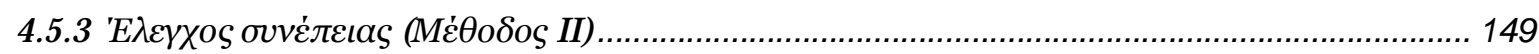

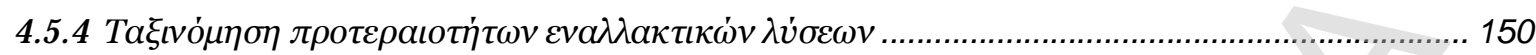

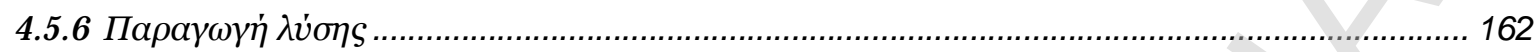

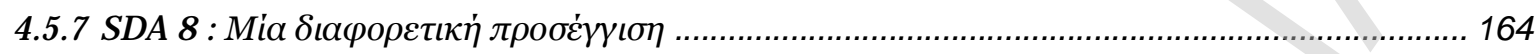

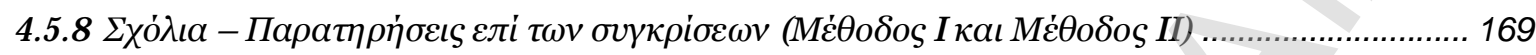

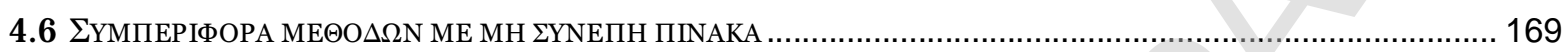

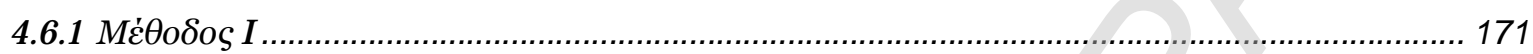

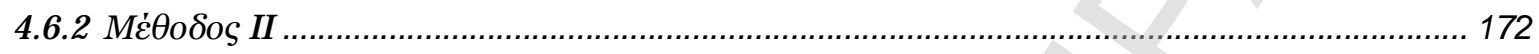

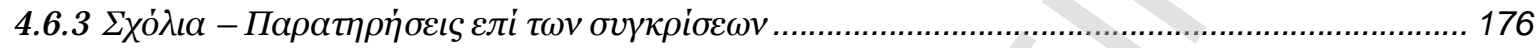

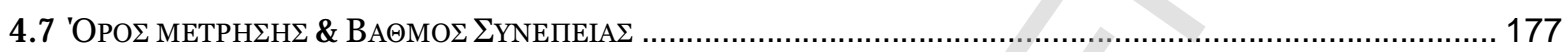

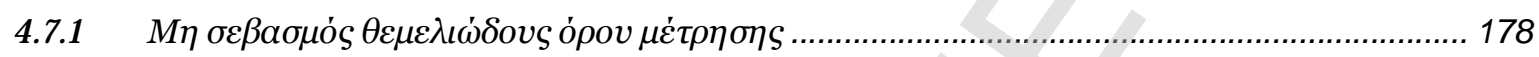

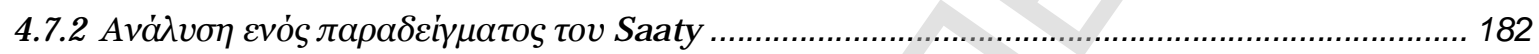

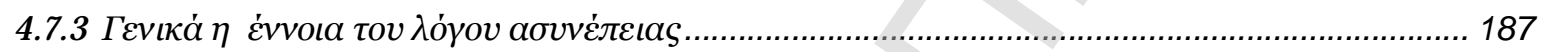

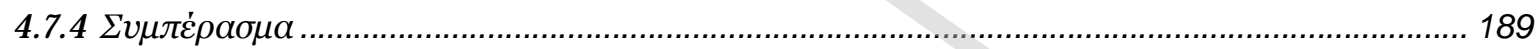

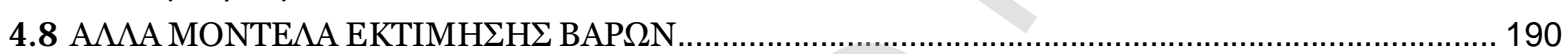

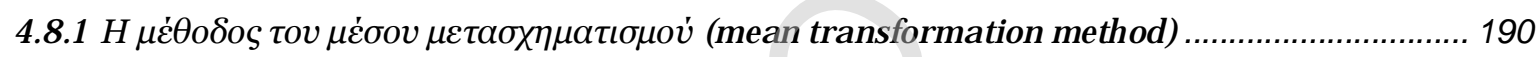

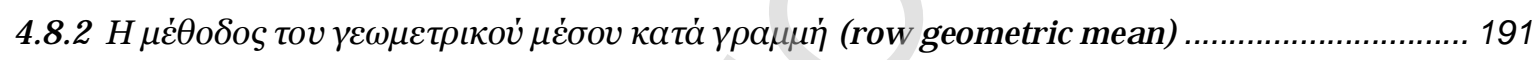

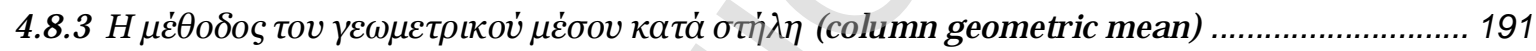

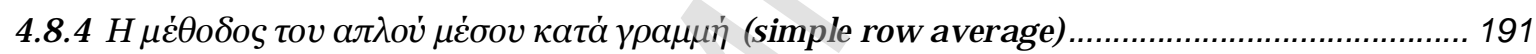

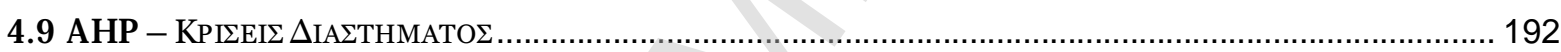

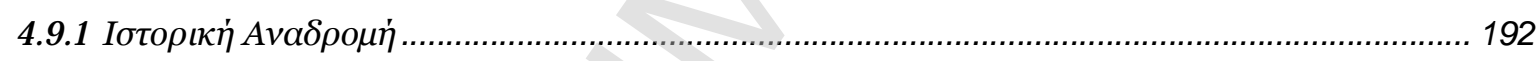

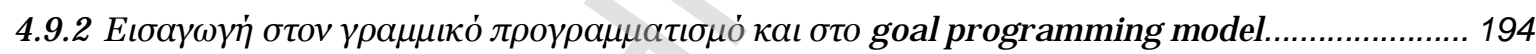

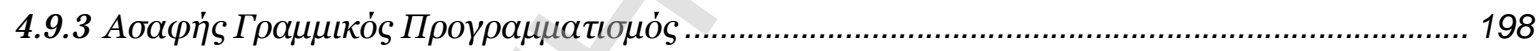

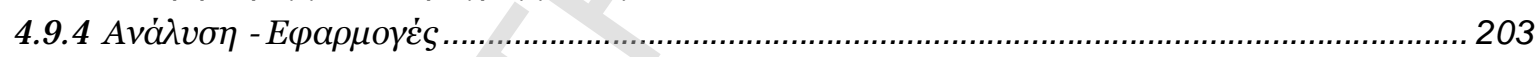

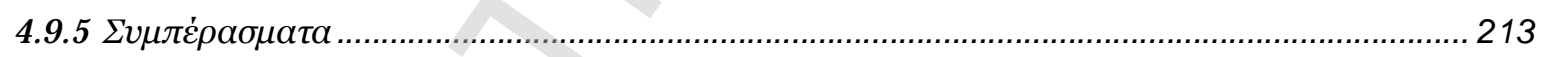

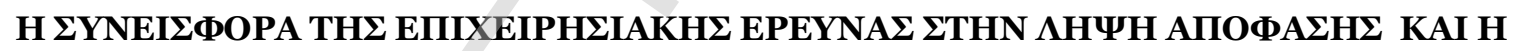

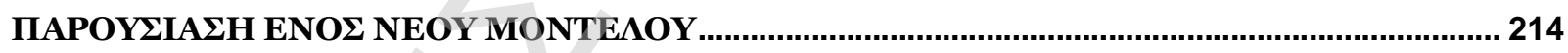

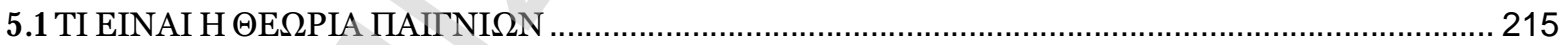

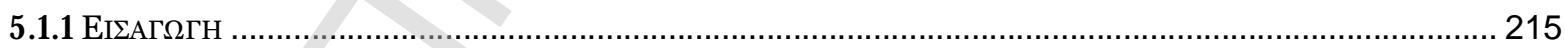

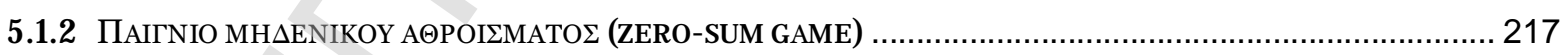

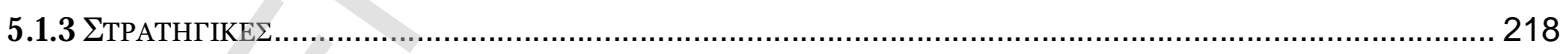

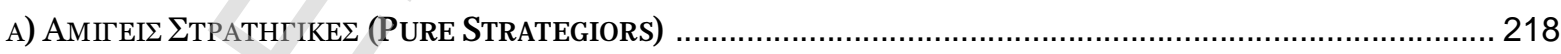

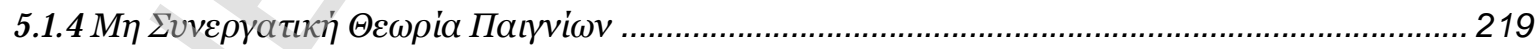

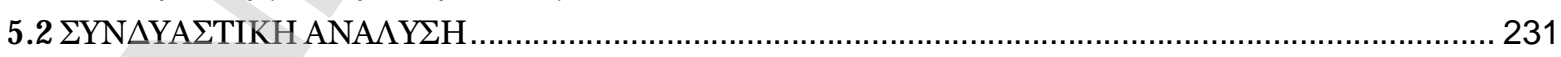

5.2 .1 A $\lambda \gamma о \rho \iota \theta \mu \iota \kappa \dot{~} \Delta \iota \dot{\tau} \tau \alpha \xi \eta \eta$

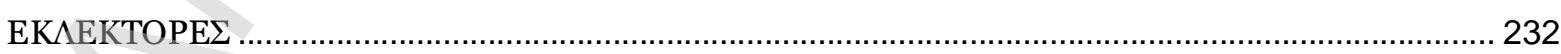

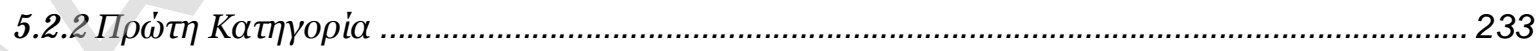

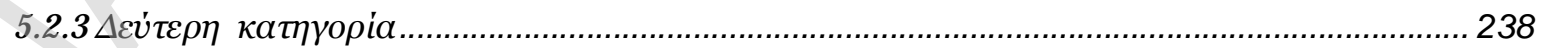

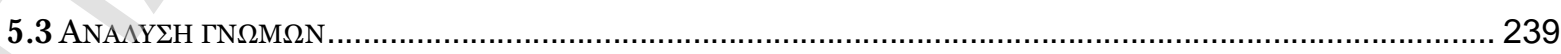

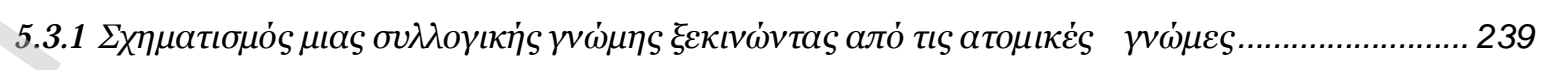

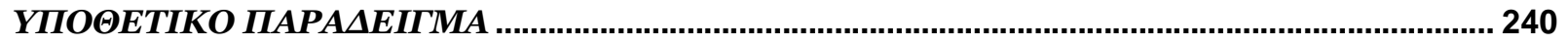




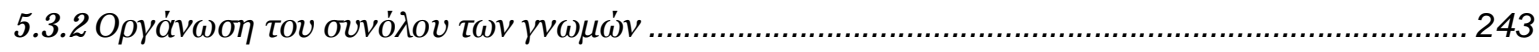

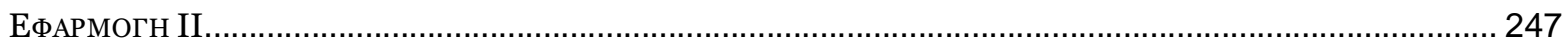

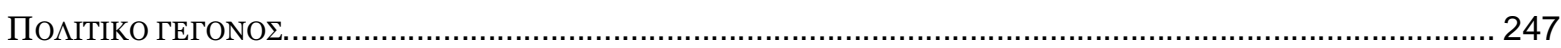

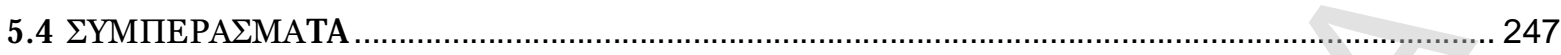

ЕПІИОГО

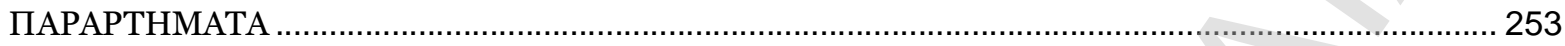

ПАРАРТНМА А'

$A P \Theta P A$.

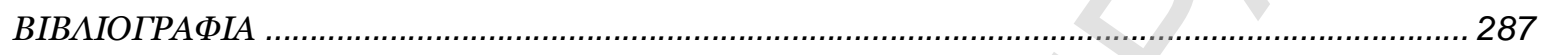




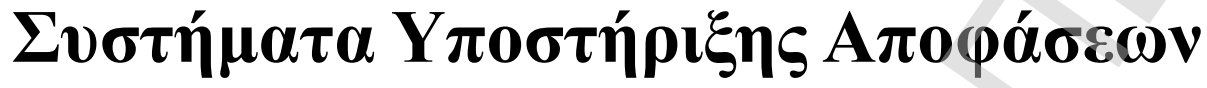

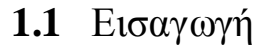

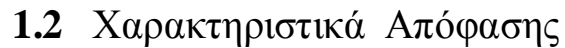

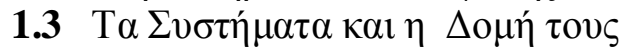

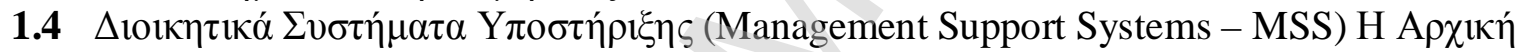

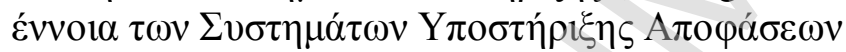

$1.5 \Sigma v \sigma \tau \eta \dot{\mu} \mu \tau \alpha$ Y

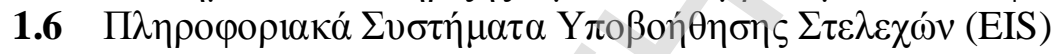

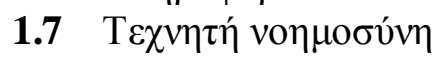

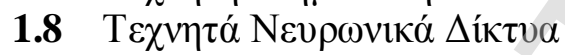

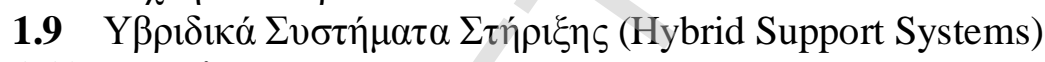

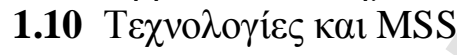

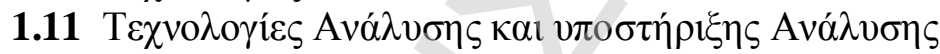

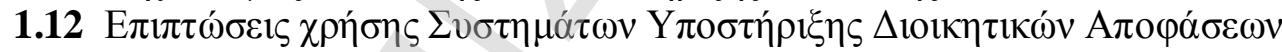

$1.13 \Sigma v \mu \pi \varepsilon \rho \alpha ́ \sigma \mu \alpha \tau \alpha$ 


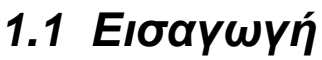

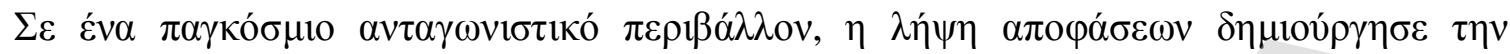

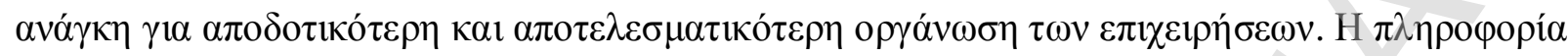

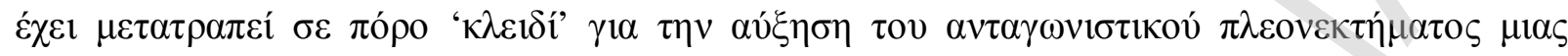

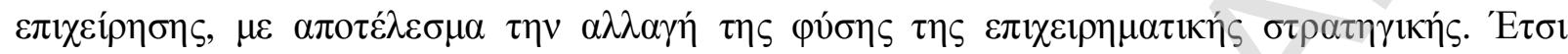

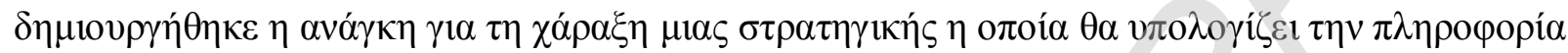

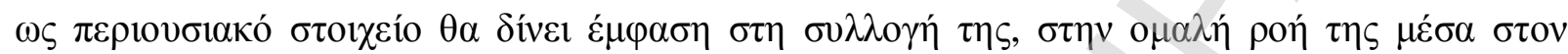

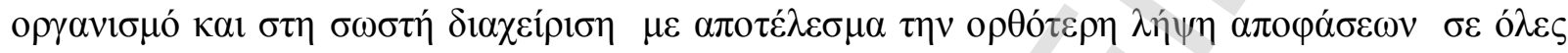

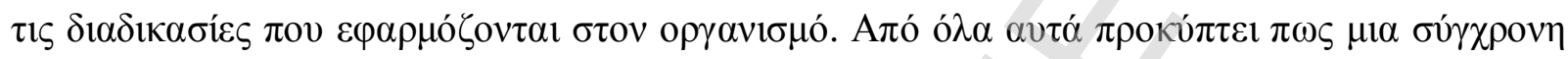

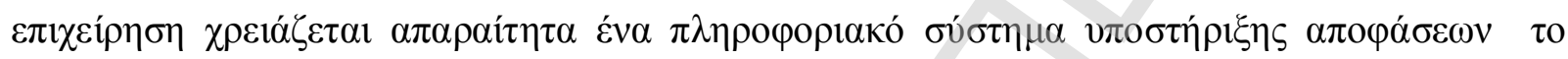

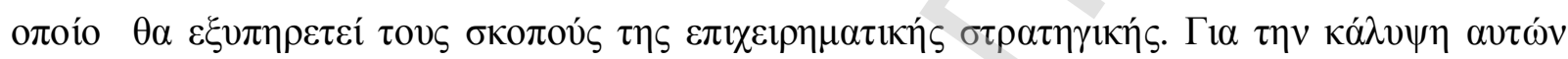

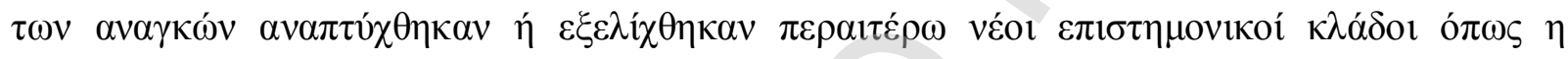

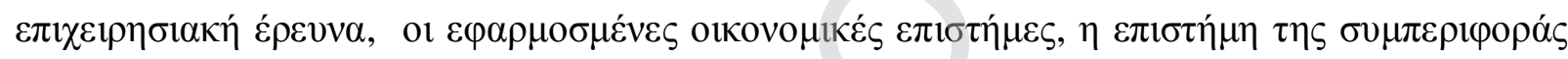

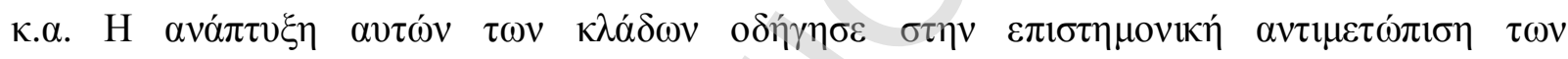

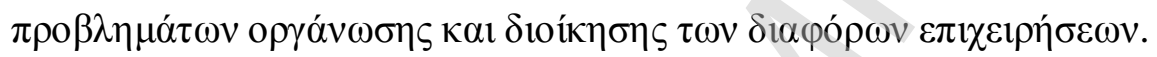

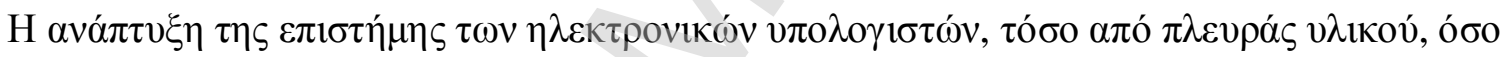

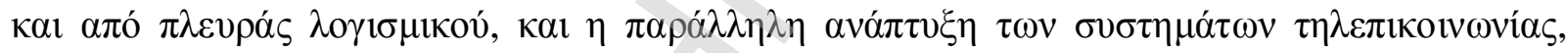

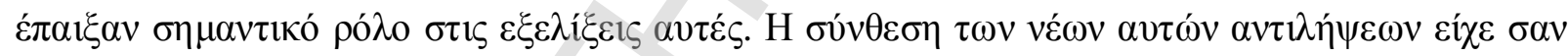

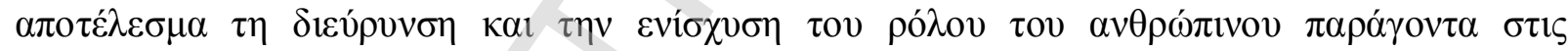

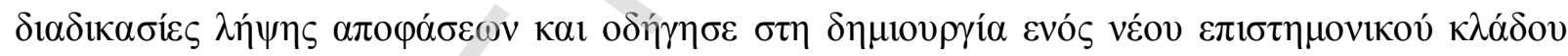

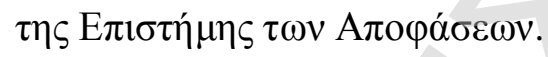

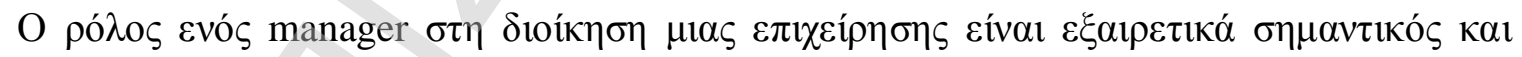

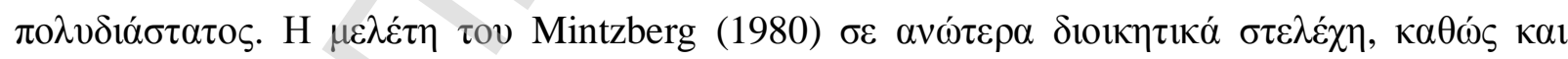

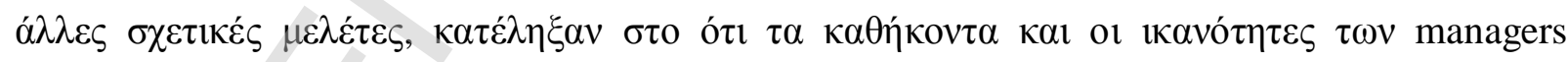

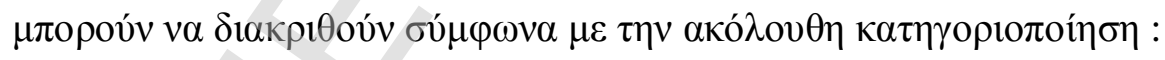

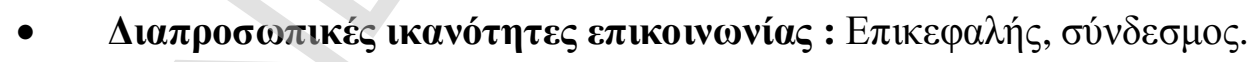

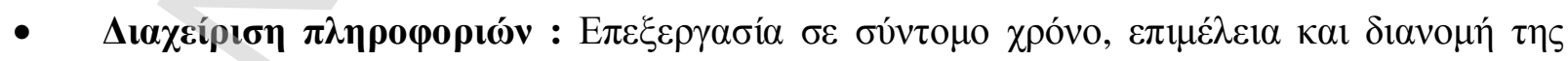

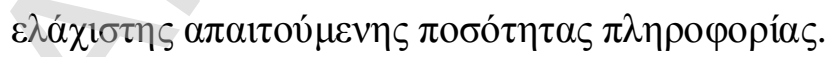

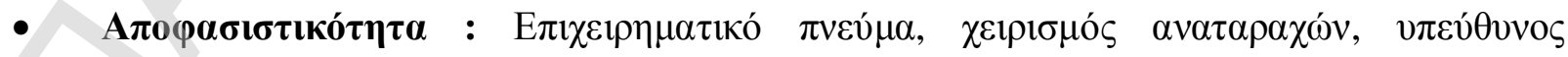

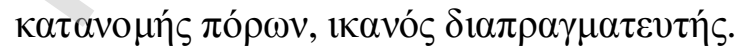

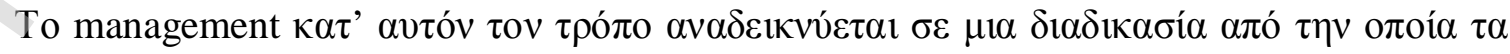

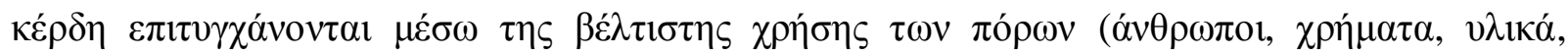




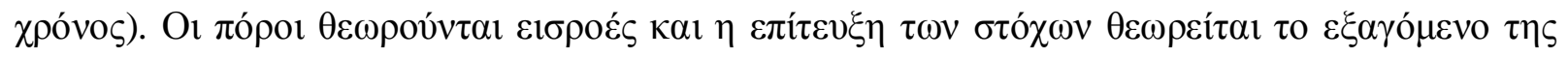

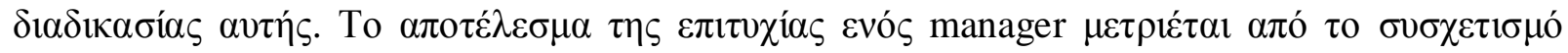

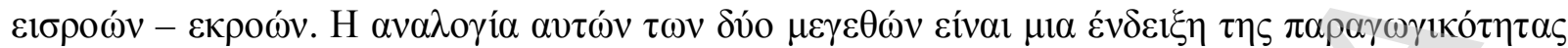

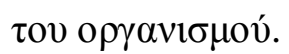

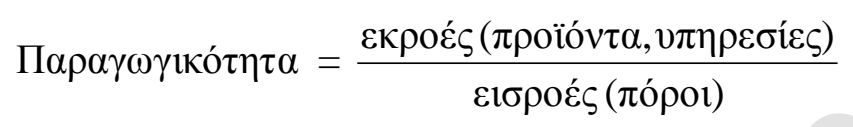

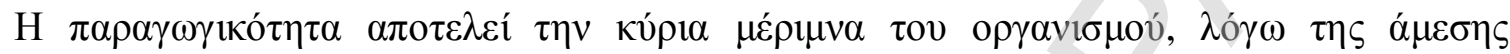

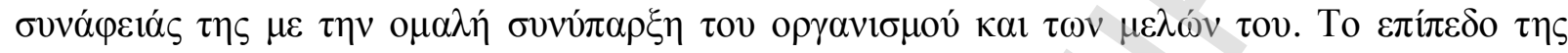

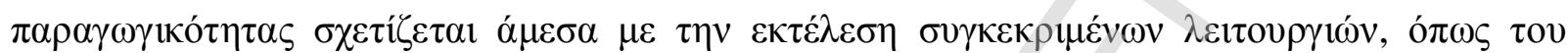

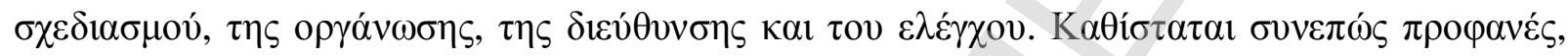

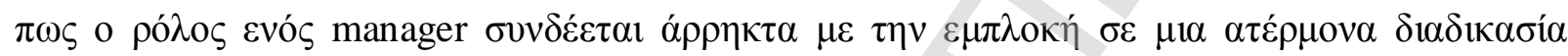

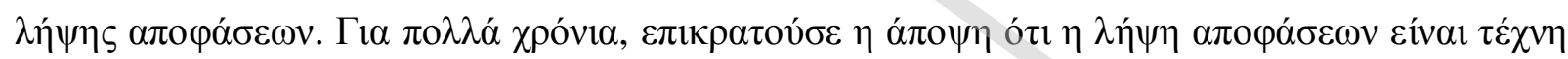

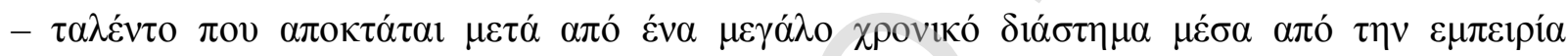

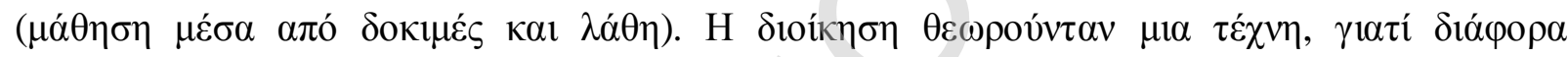

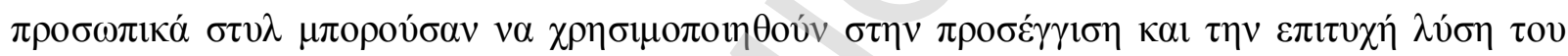

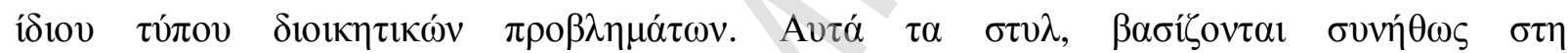

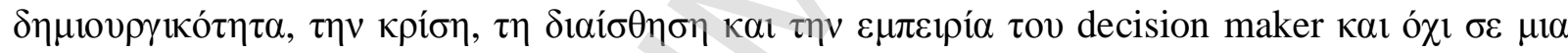

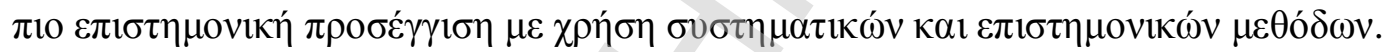

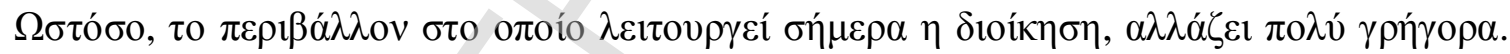

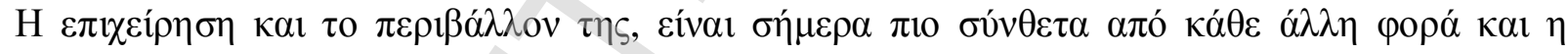

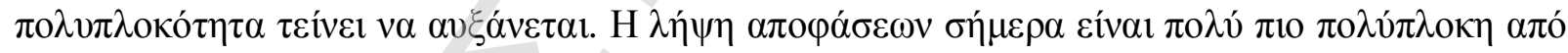

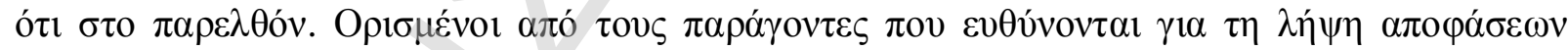
síval:

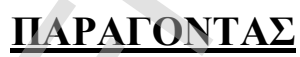

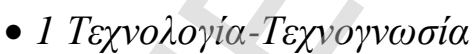

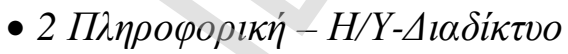

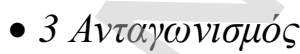

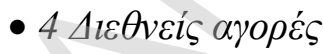

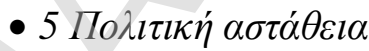

- 6 Kv $\beta \varepsilon \rho v \eta \tau \imath \kappa \varepsilon ́ \varsigma ~ \pi \alpha \rho \varepsilon \mu \beta \alpha ́ \sigma \varepsilon ı \varsigma$

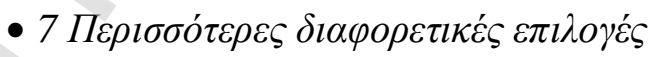

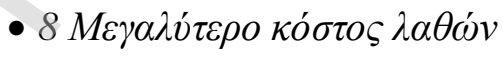

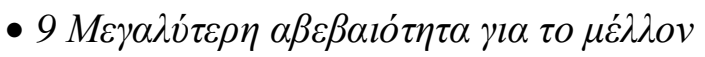




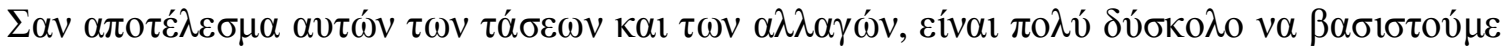

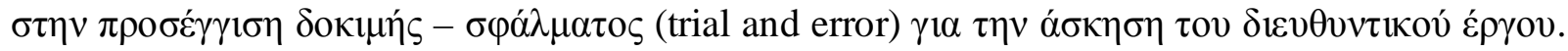

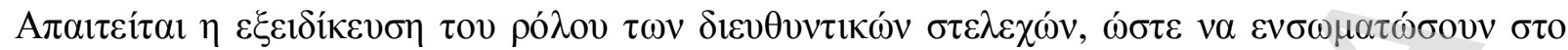

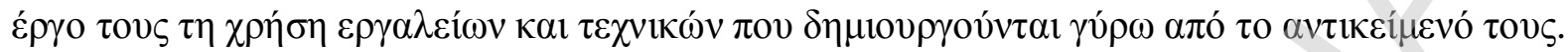

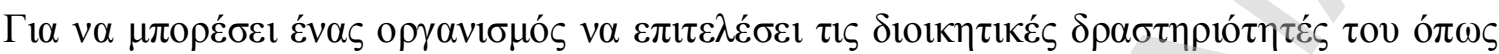

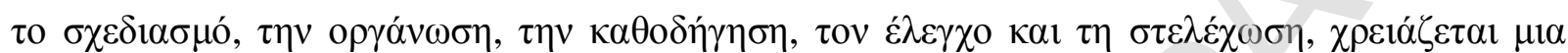

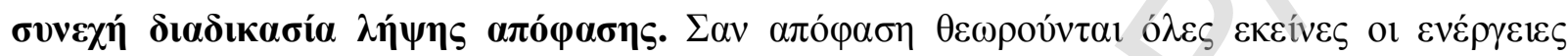

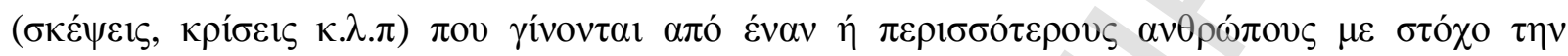

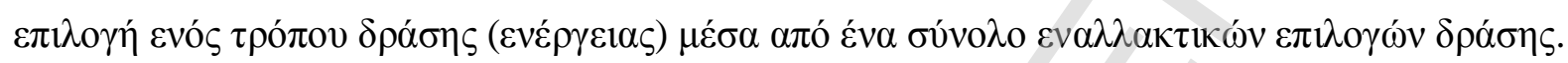

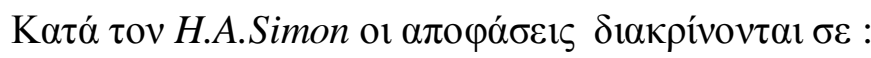

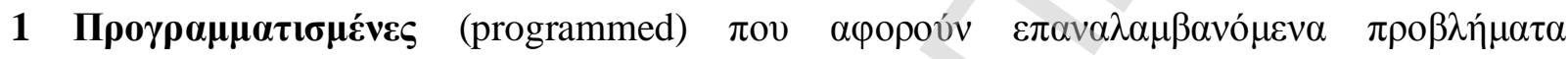

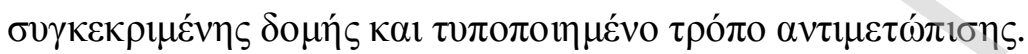

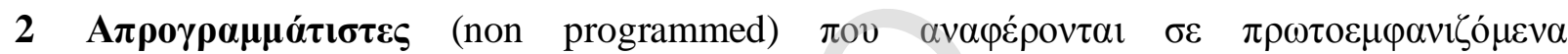

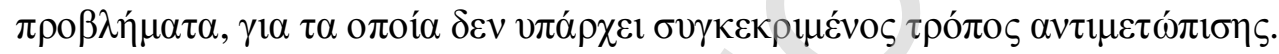

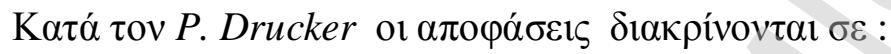

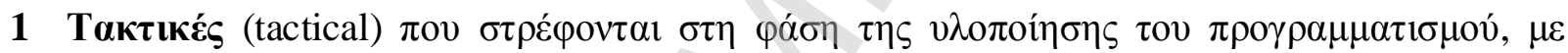

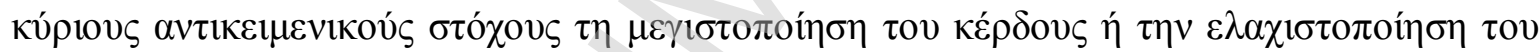

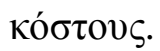

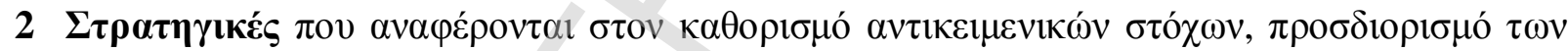
$\pi$ ó $\rho \omega v, \kappa \lambda \pi$.

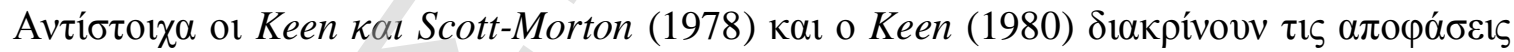

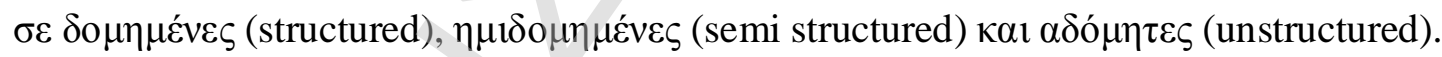

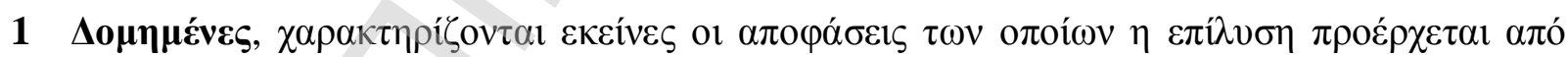

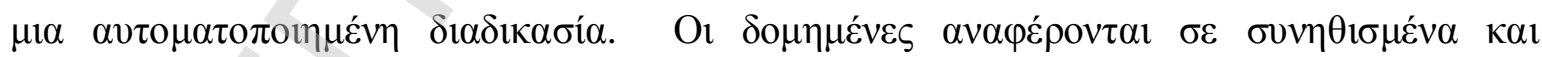

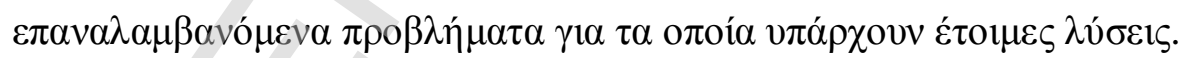

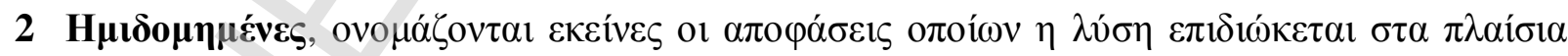

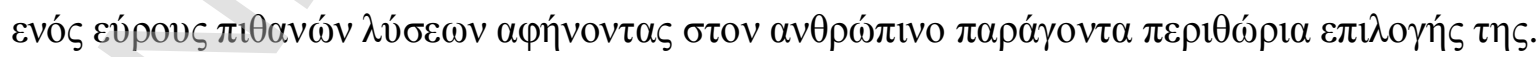

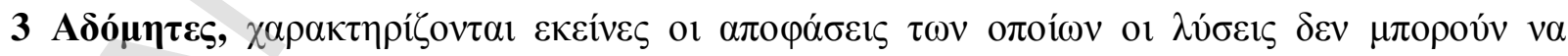

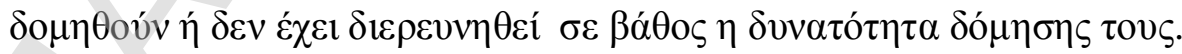

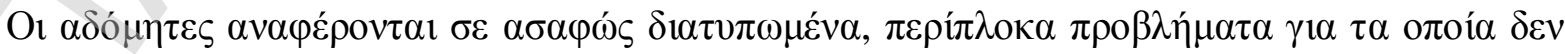

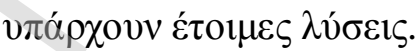




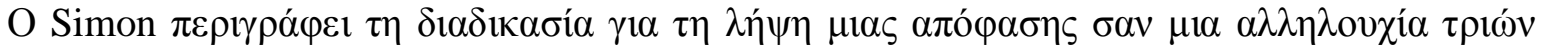

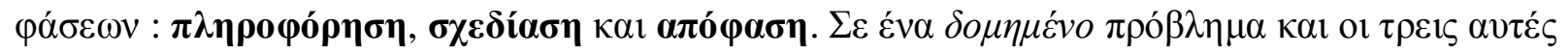

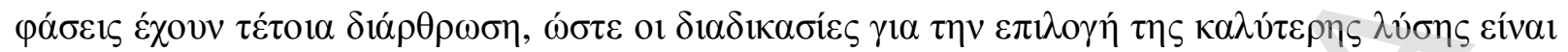

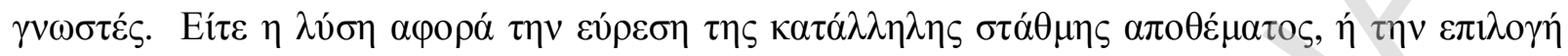

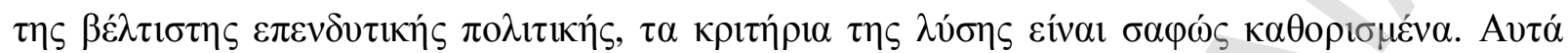

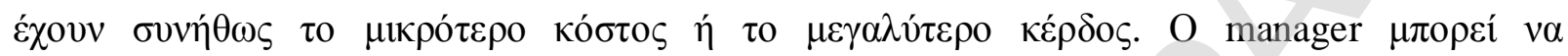

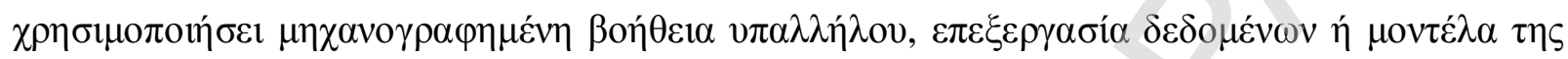

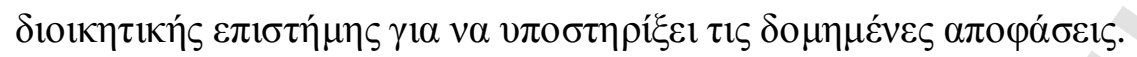

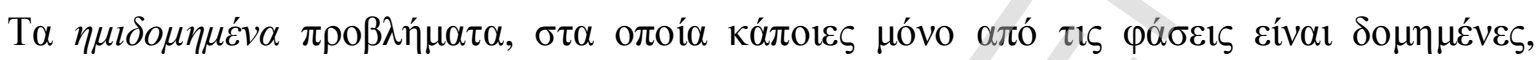

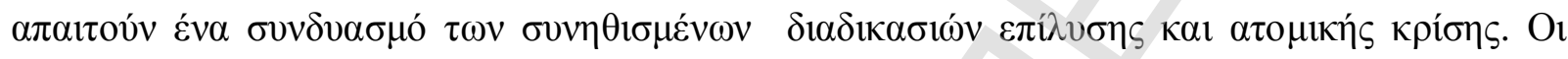

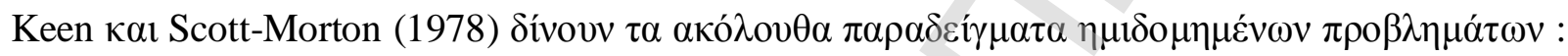

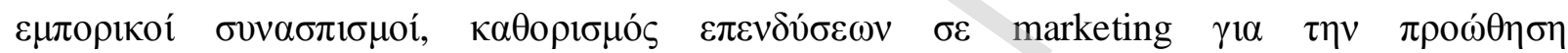

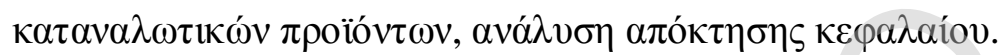

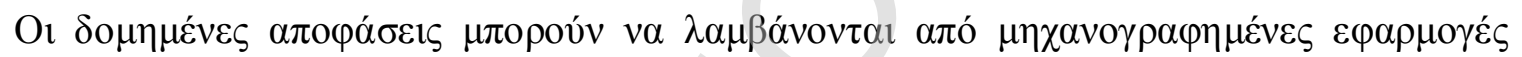

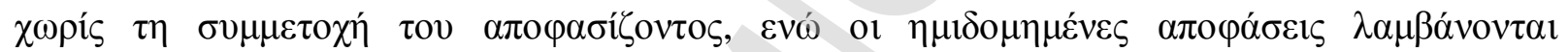

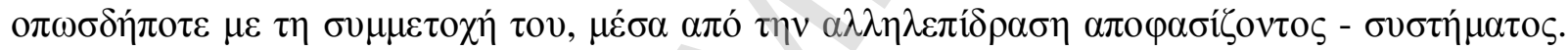

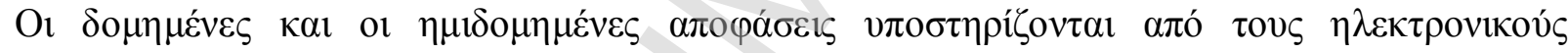

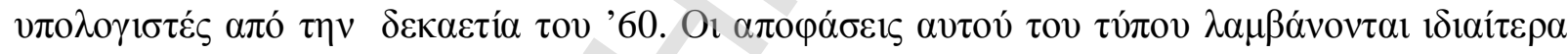

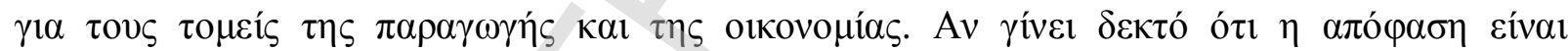
$\alpha \pi$

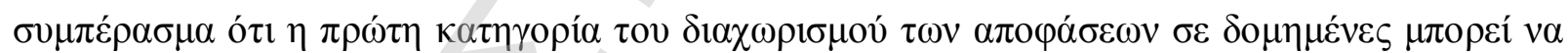

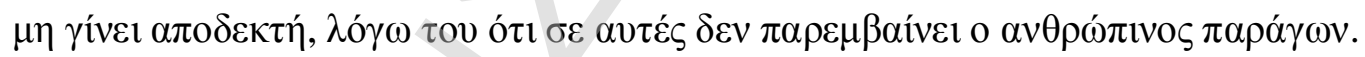

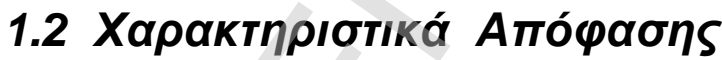

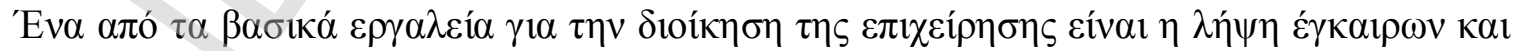

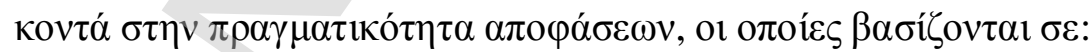

- $\quad \Pi \rho о \beta \lambda \varepsilon ́ \psi \varepsilon \varepsilon \varsigma$.

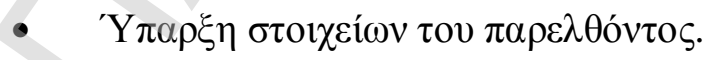

- $\Delta \varepsilon \imath \gamma \mu \alpha \tau 0 \lambda \eta \psi i ́ \varepsilon \varsigma$.

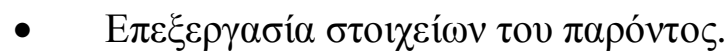




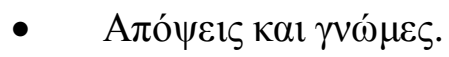

$\mathrm{H} \lambda \eta \dot{\psi \eta} \alpha \pi \circ \varphi \alpha ́ \sigma \varepsilon \omega v \alpha \pi \alpha \imath \varepsilon \dot{~: ~}$

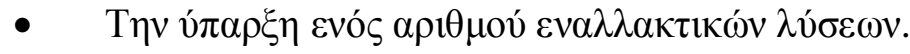

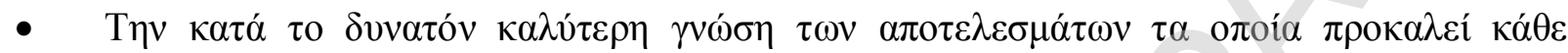
$\varepsilon v \alpha \lambda \lambda \alpha \kappa \tau \iota \kappa \eta ́ ~ \lambda v ́ \sigma \eta$.

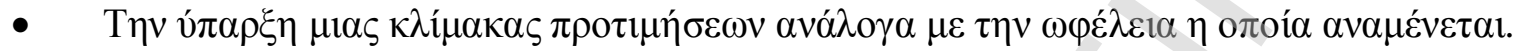

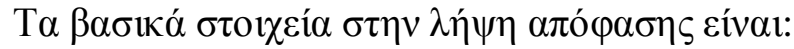

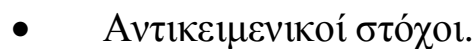

- O1 $\alpha \alpha \tau \alpha \sigma \tau \alpha ́ \sigma \varepsilon 1 \varsigma \tau \eta \varsigma \varphi v ́ \sigma \eta \varsigma$ (States of the nature).

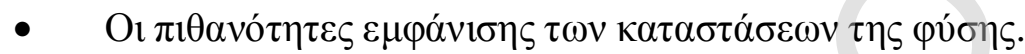

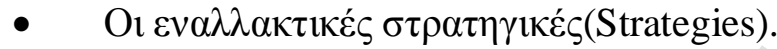

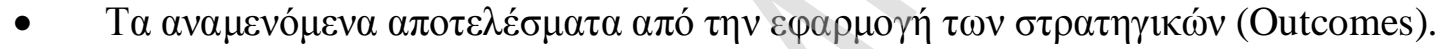

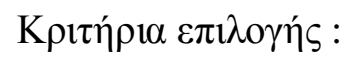

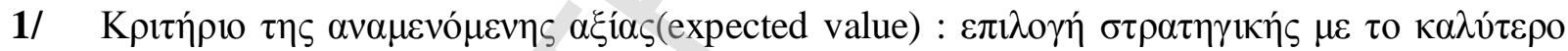
$\alpha v \alpha \mu \varepsilon v o ́ \mu \varepsilon v o$ $\theta \varepsilon \tau \imath \kappa o ́ ~ \alpha \pi \circ \tau \varepsilon ́ \lambda \varepsilon \sigma \mu \alpha$.

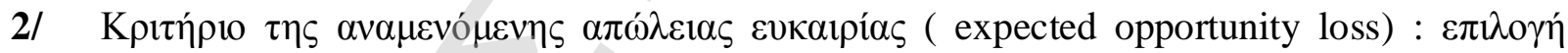

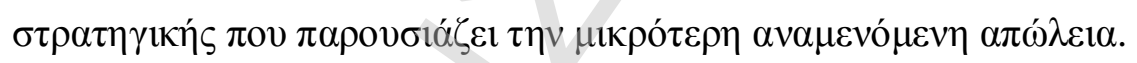

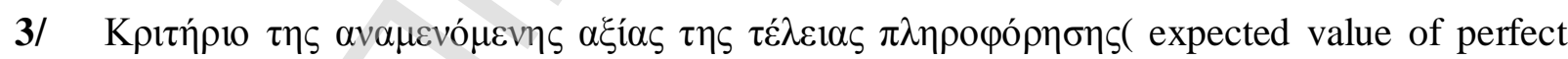
information).

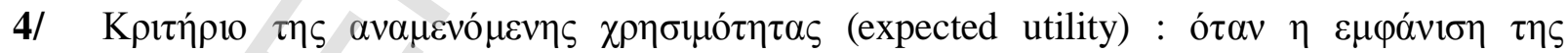

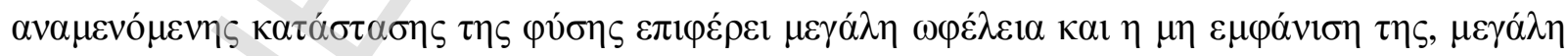

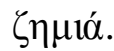

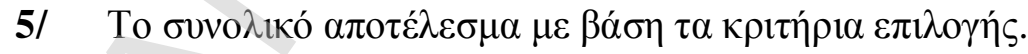

6/ Mи́

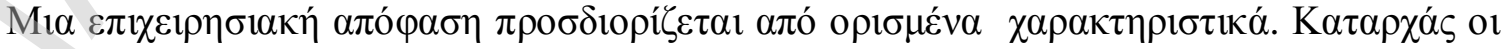

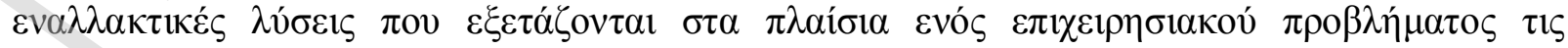

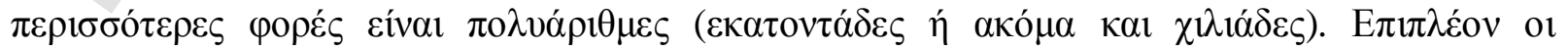




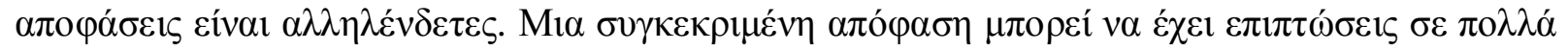

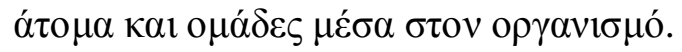

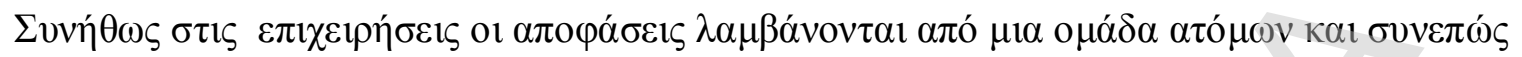

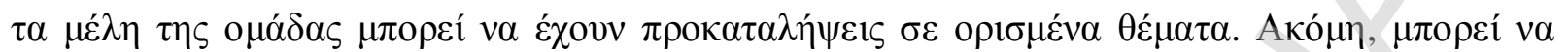

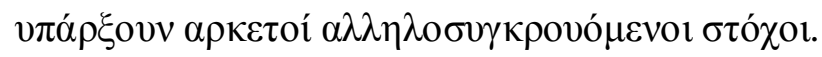

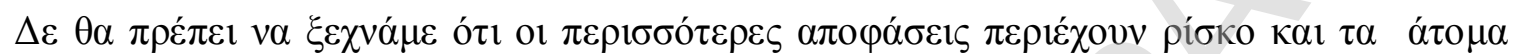

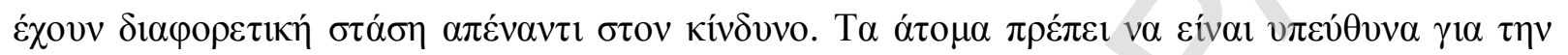

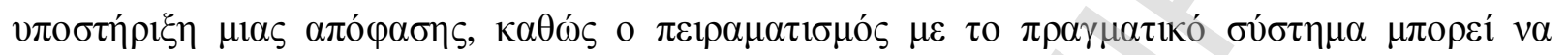

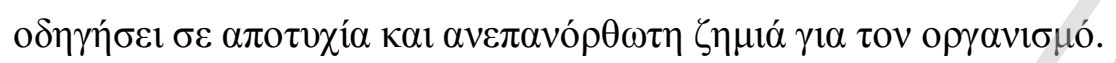

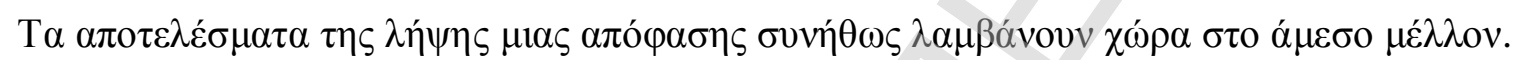

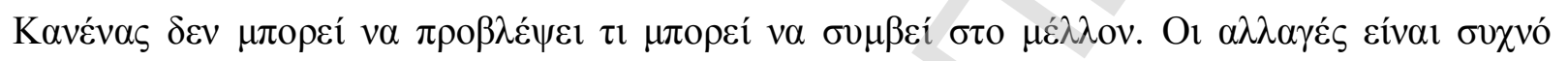

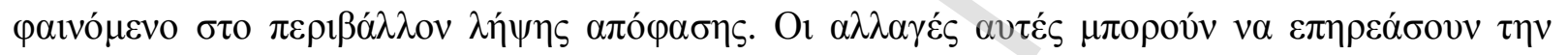

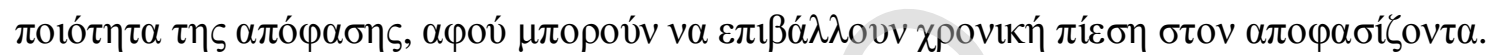

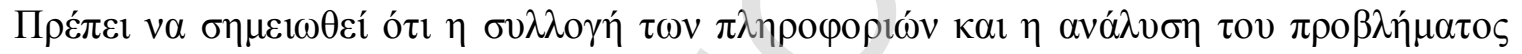

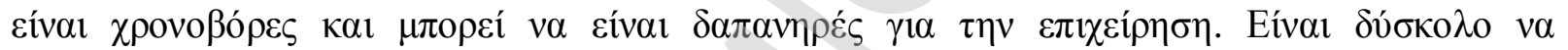

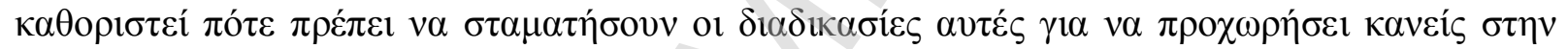

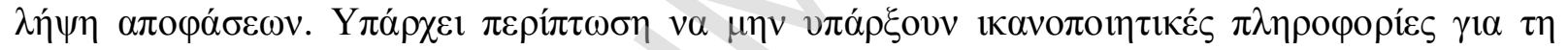

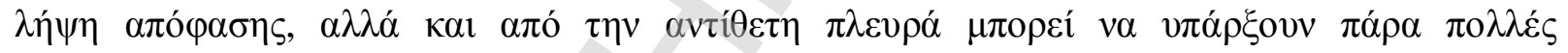

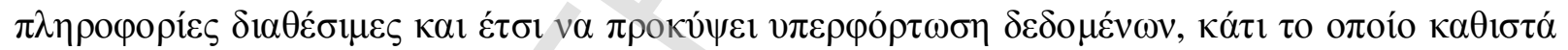
$\tau \eta v \lambda \eta ́ \psi \eta \alpha \pi o ́ \varphi \alpha \sigma \eta \varsigma \delta \delta ́ \sigma \kappa o \lambda \eta$.

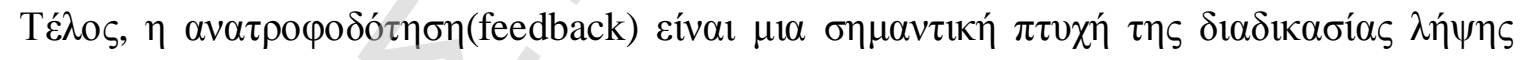

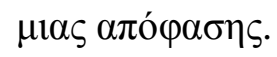

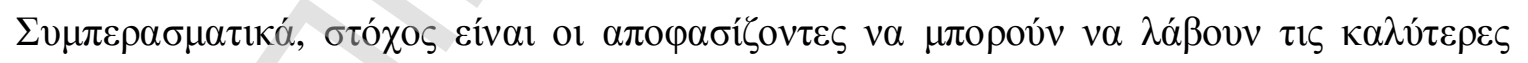

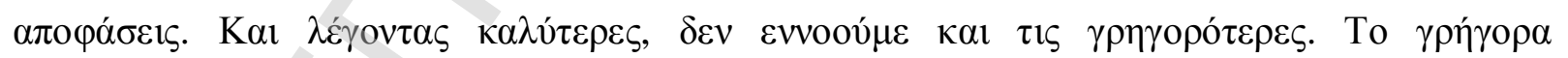

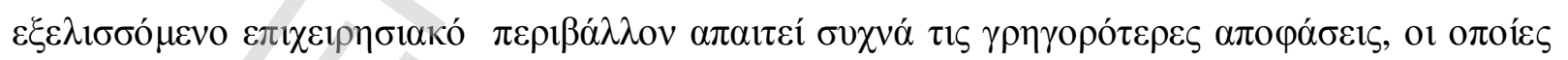

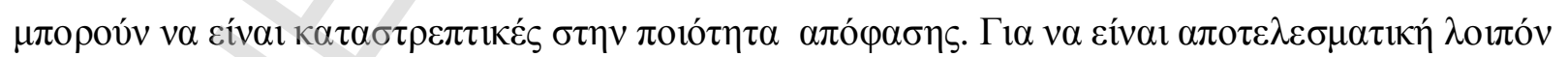
$\eta \delta$

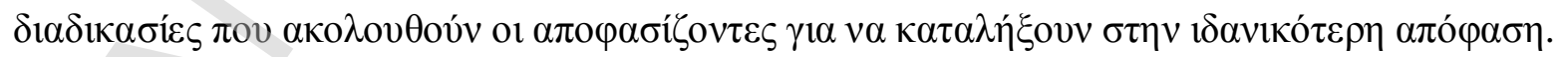

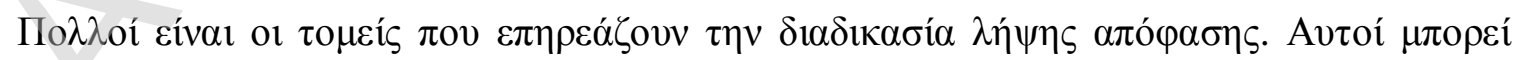

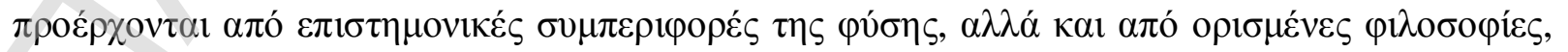

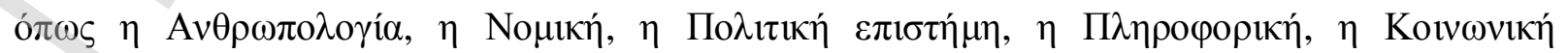

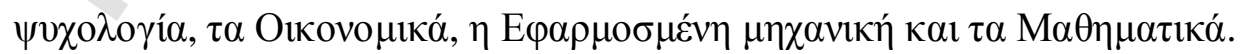




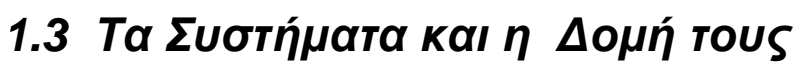

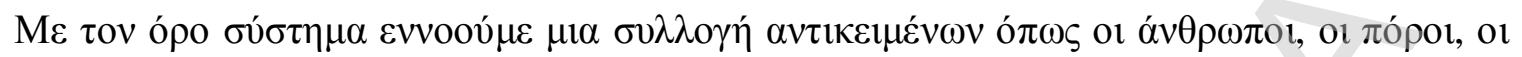

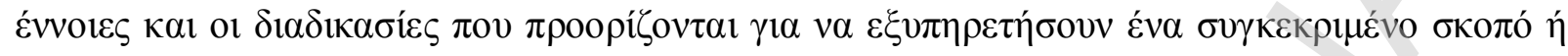

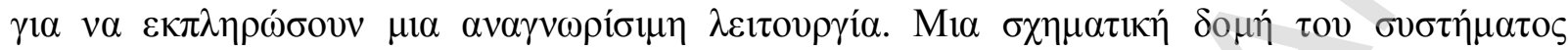

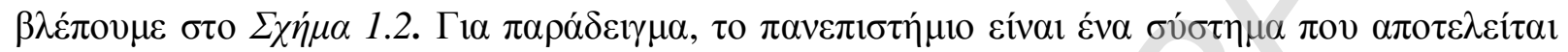

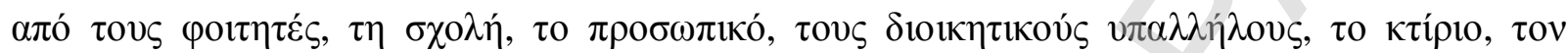

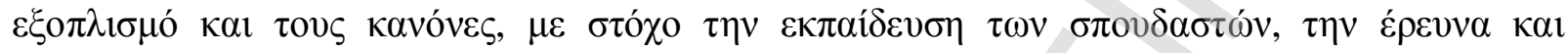

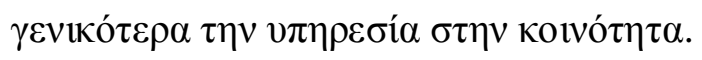

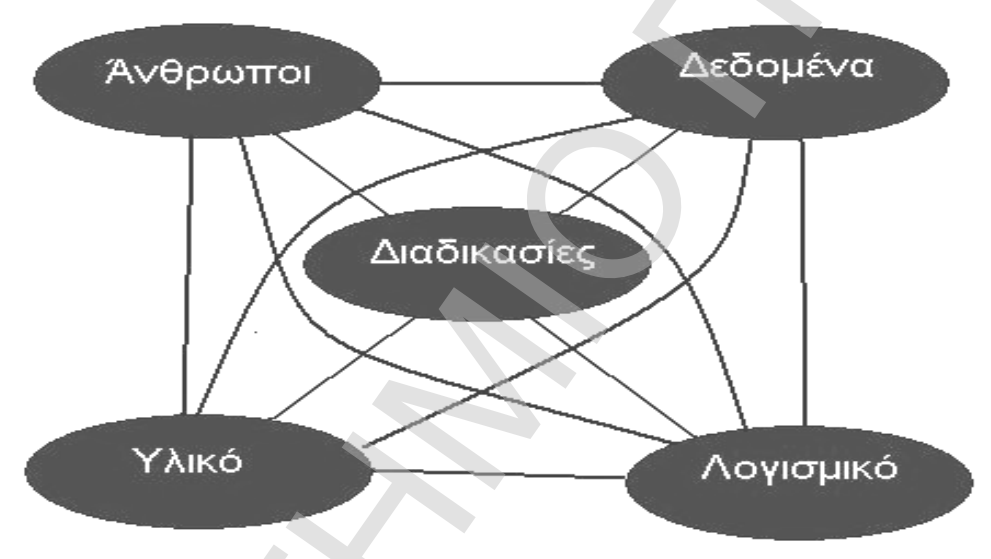

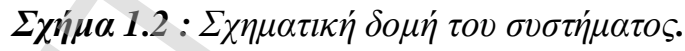

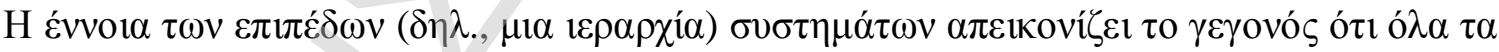

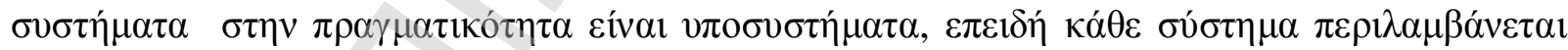

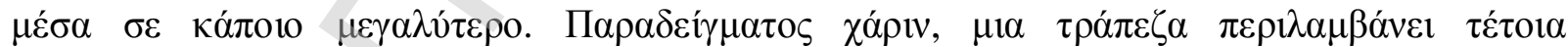

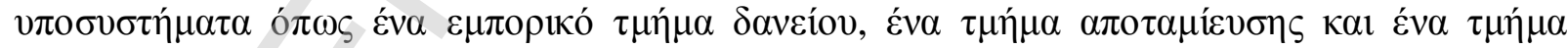

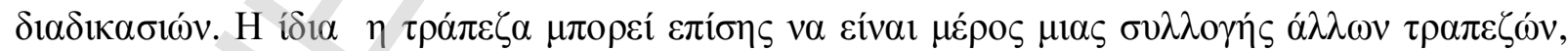

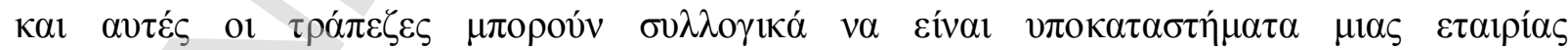

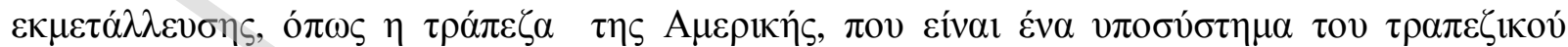

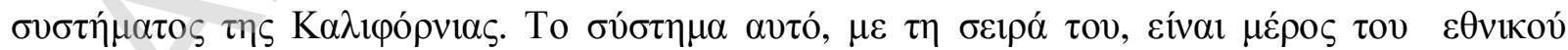

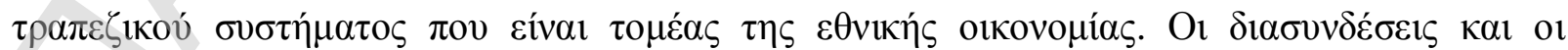

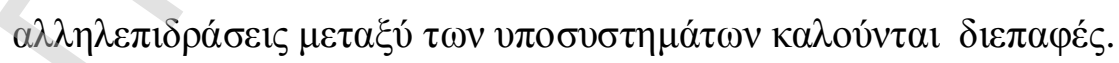

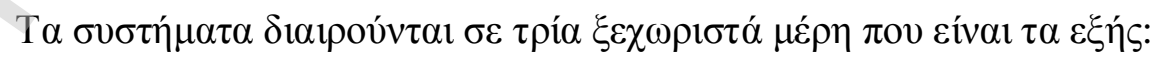




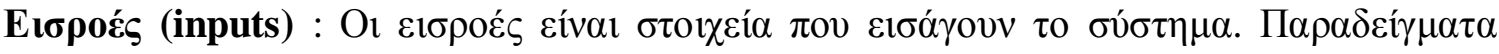

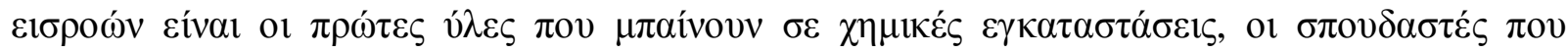

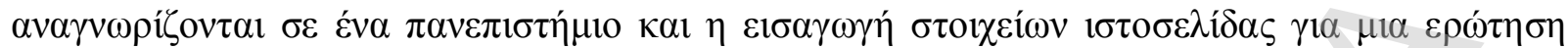
$\beta \alpha ́ \sigma \varepsilon \omega v \delta \varepsilon \delta o \mu \varepsilon ́ v \omega v$.

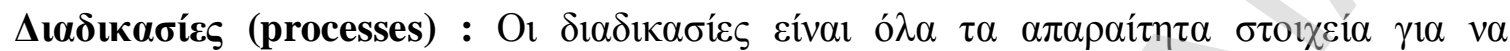

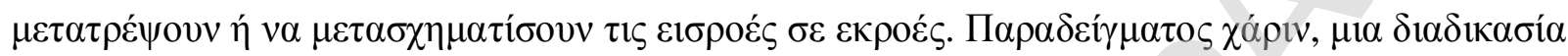

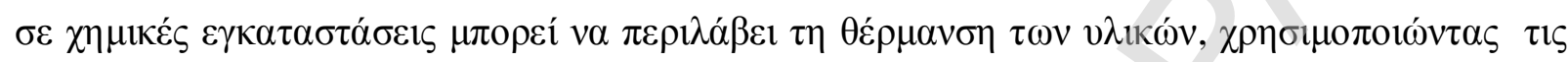

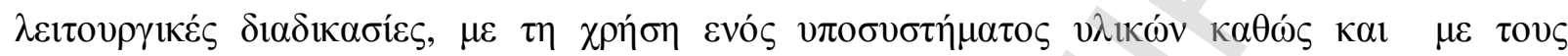

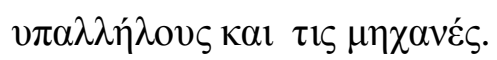

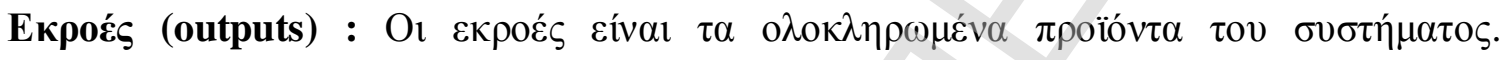

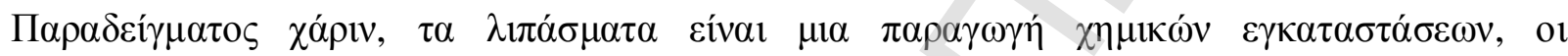

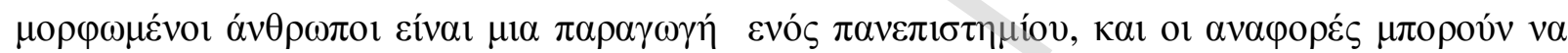

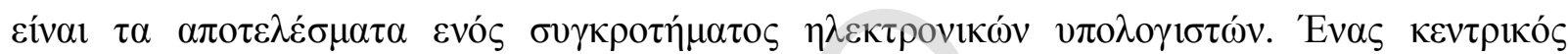

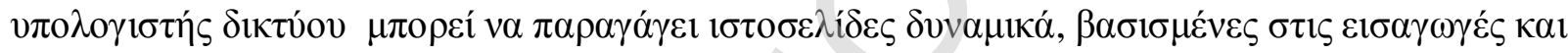
$\tau \iota \varsigma \delta 1 \alpha \delta 1 \kappa \alpha \sigma i ́ \varepsilon \varsigma \tau o v$.

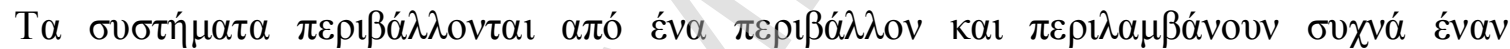

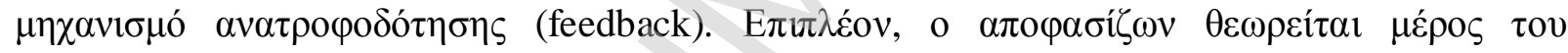

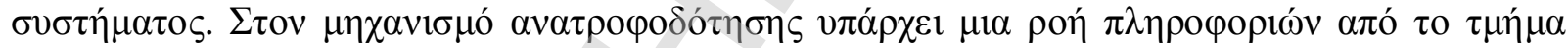

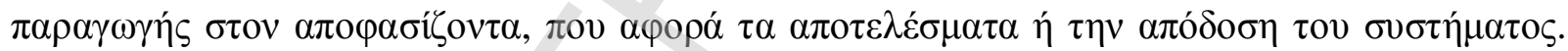

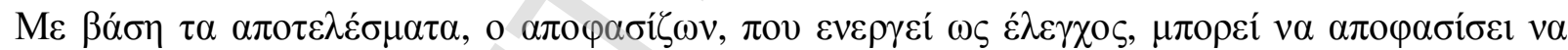

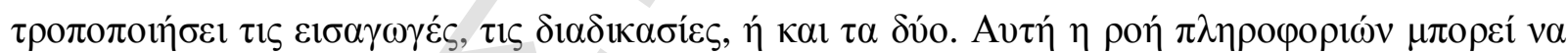

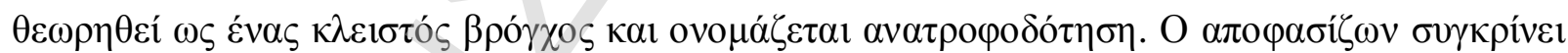

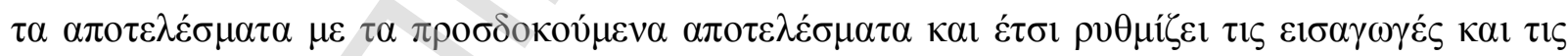

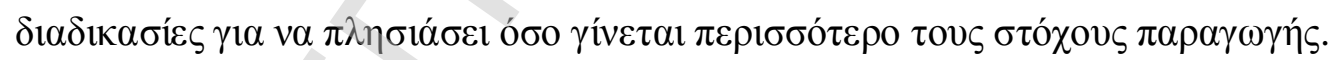

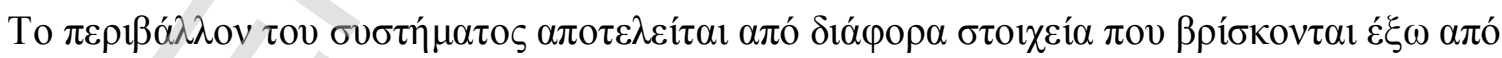

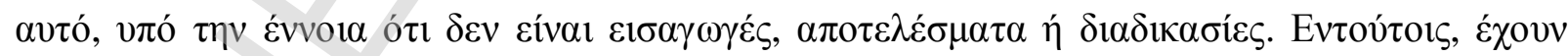

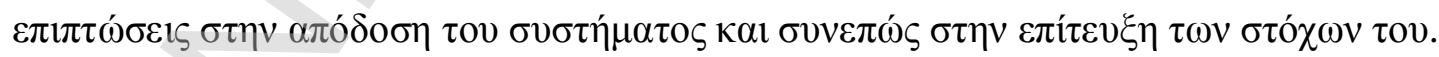

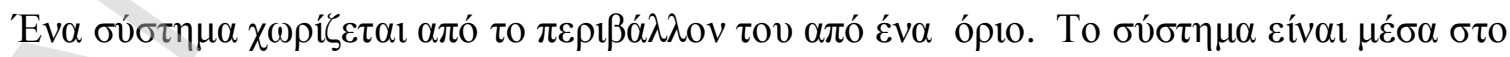

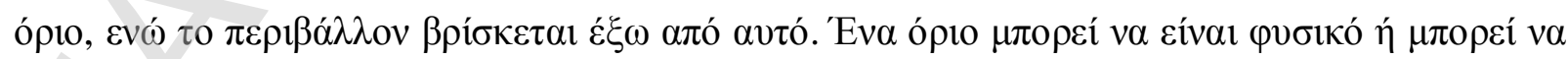

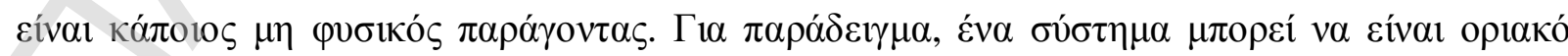

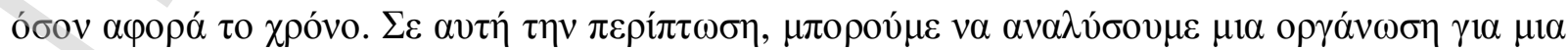

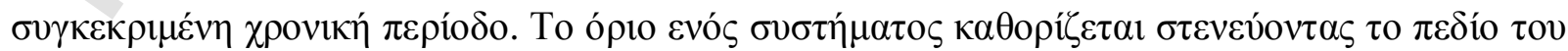

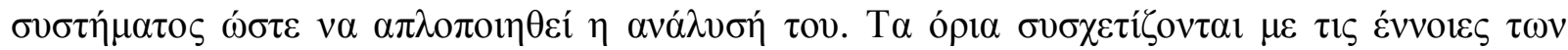




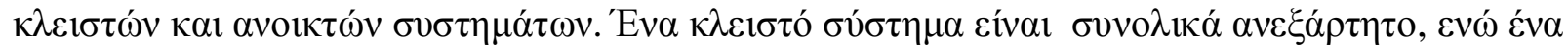

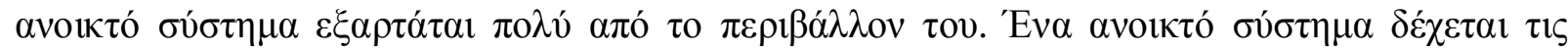

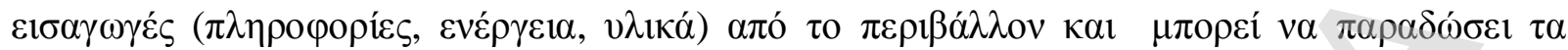

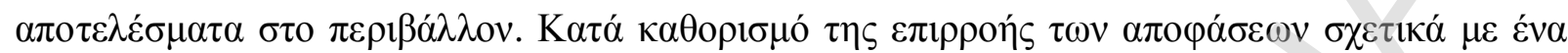

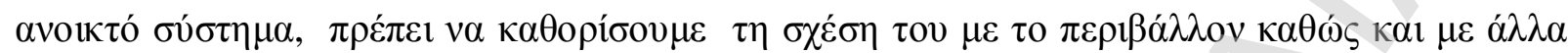

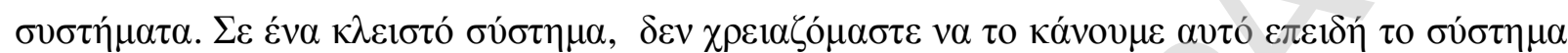

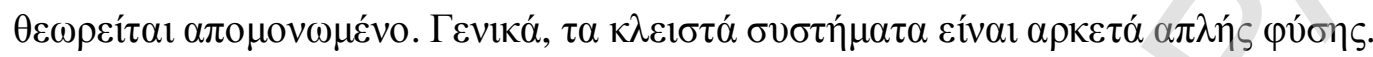

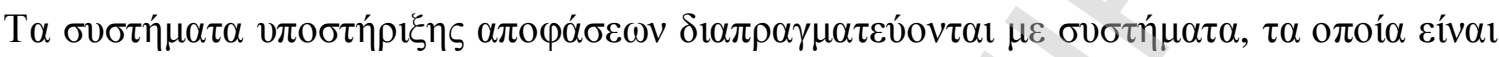

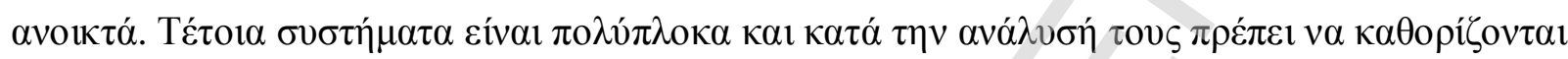

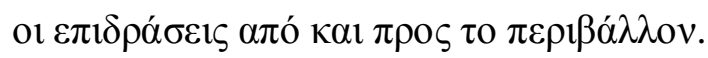

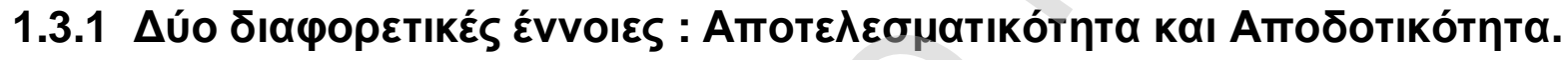

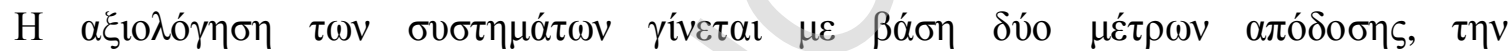

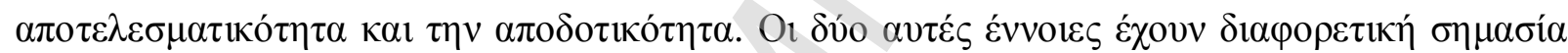
$\gamma \alpha \tau \eta \nu \alpha \xi_{10} \lambda \hat{\gamma} \eta \sigma \eta \tau \omega \nu \sigma v \sigma \tau \eta \mu \alpha ́ \tau \omega \nu$.

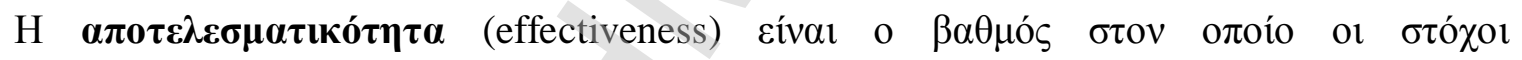

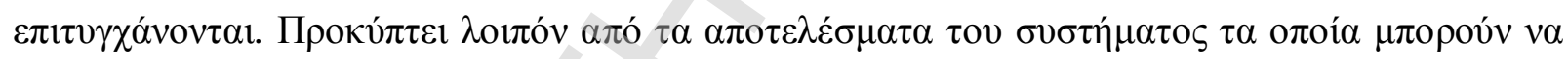

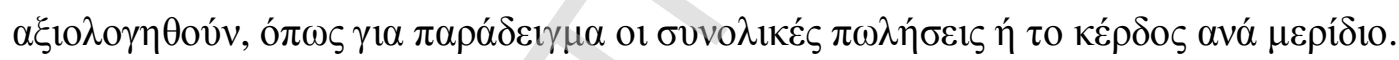

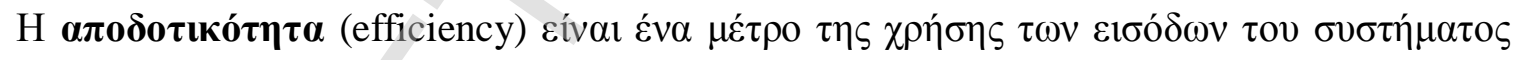

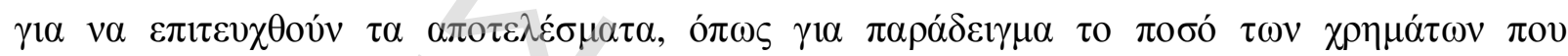

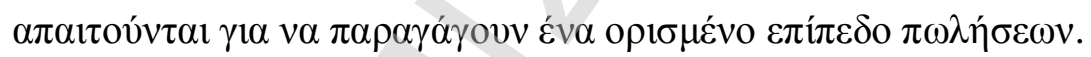

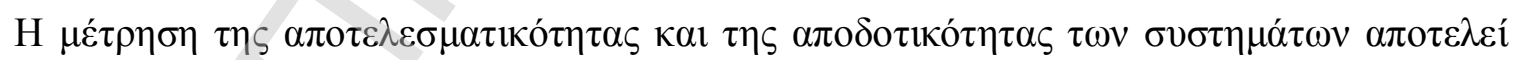

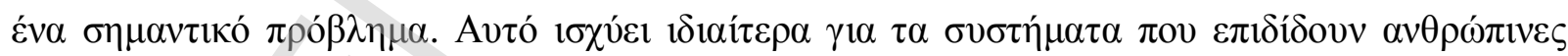

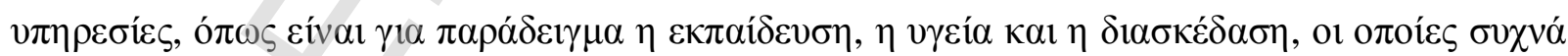

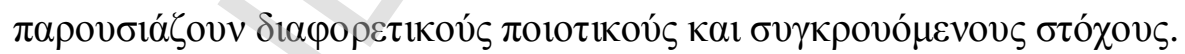

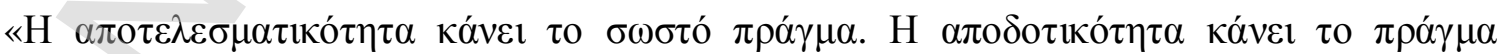
$\sigma \omega \sigma \tau$ ó». «Effectiveness is doing the right thing, efficiency is doing the thing right.»(Peter Drucker). 


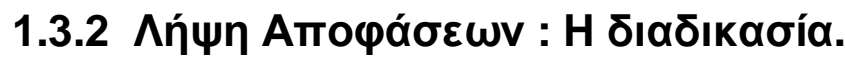

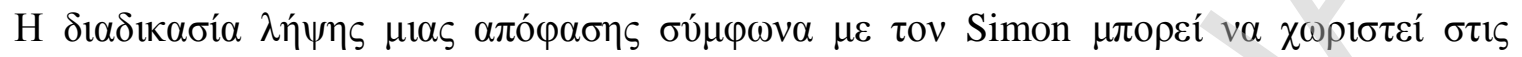

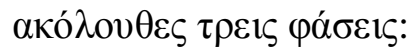

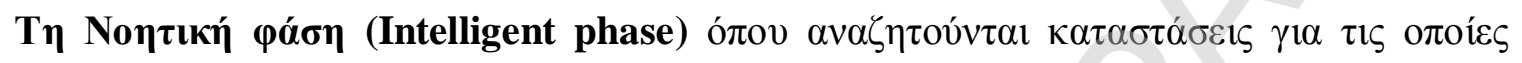

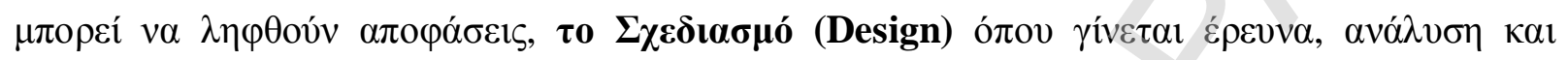

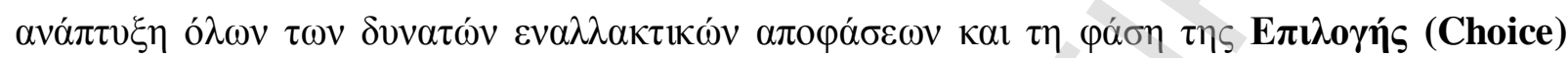

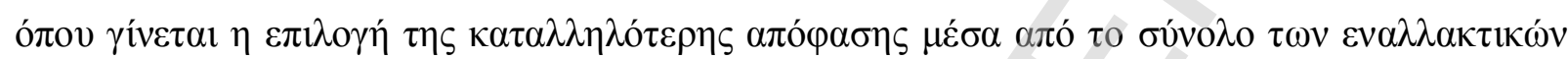
$\alpha \pi \circ \varphi \alpha ́ \sigma \varepsilon \omega v$.

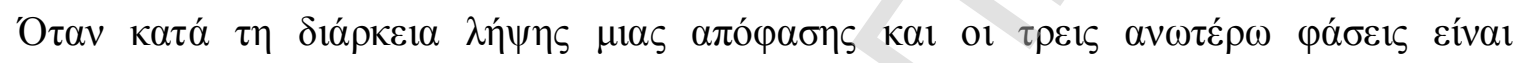

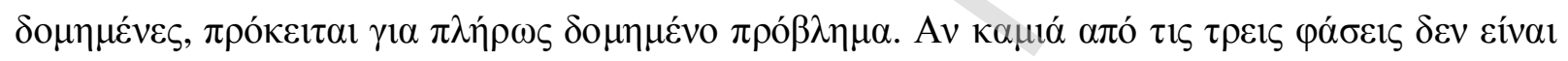

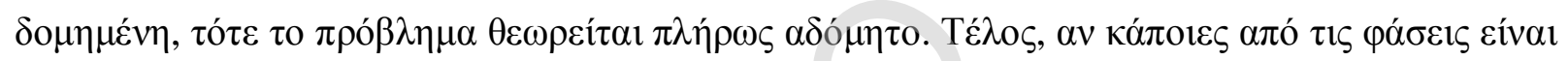

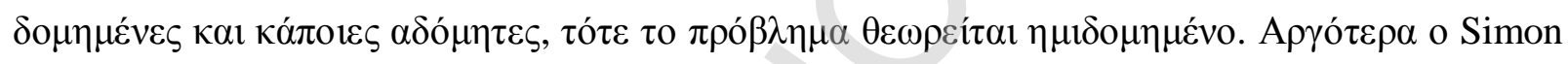

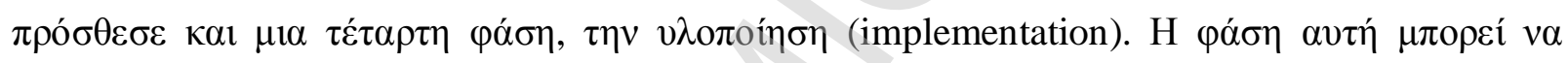

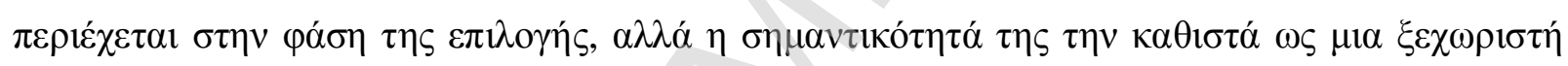

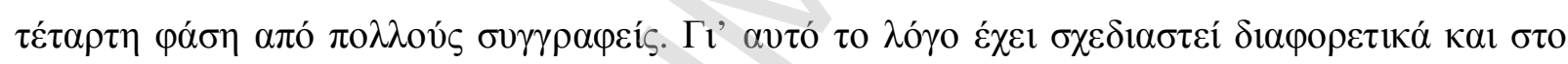

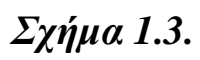




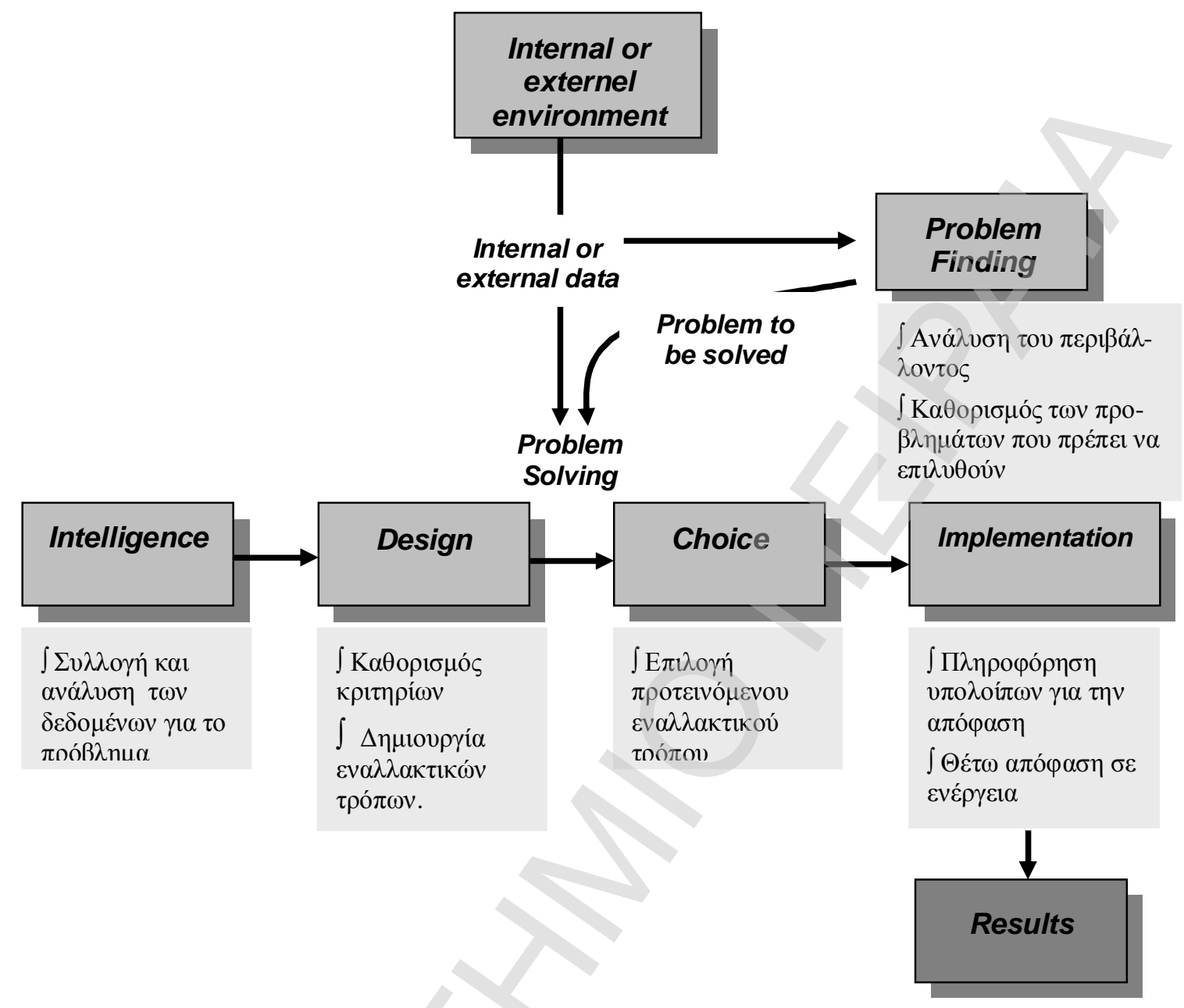

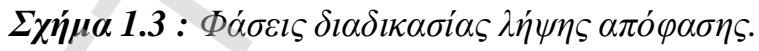

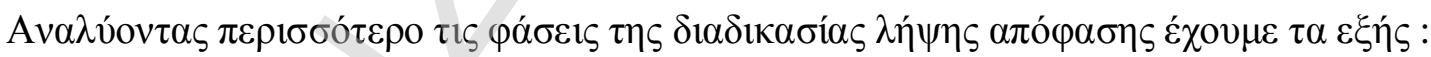

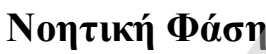

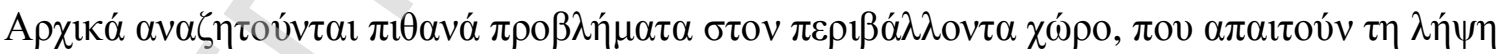

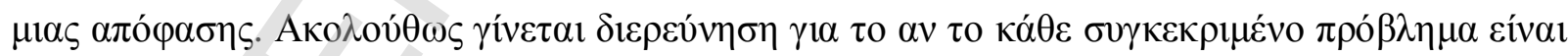

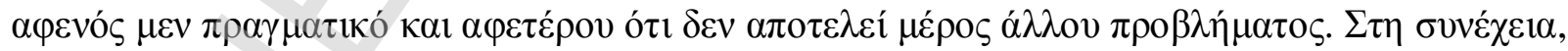

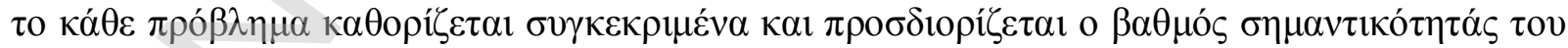

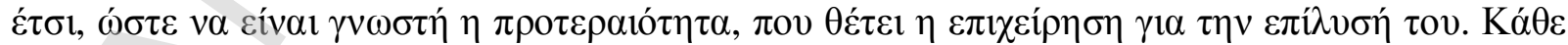

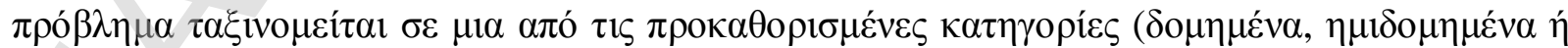

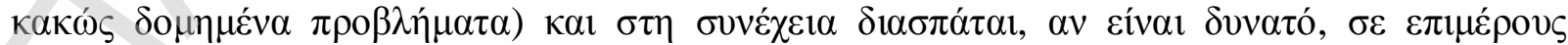

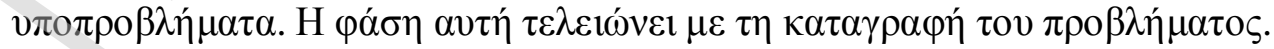




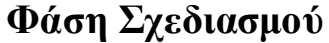

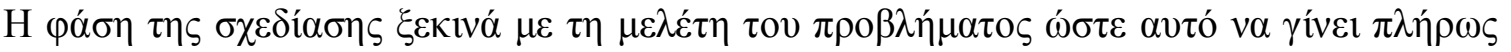

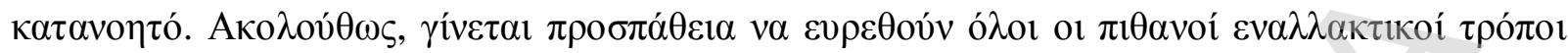

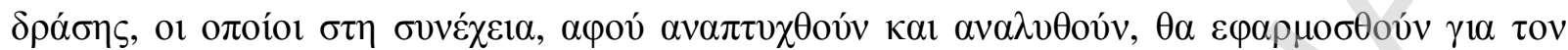

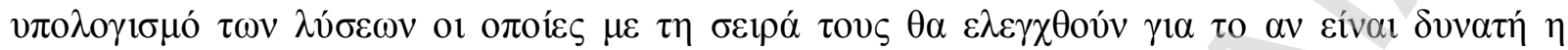

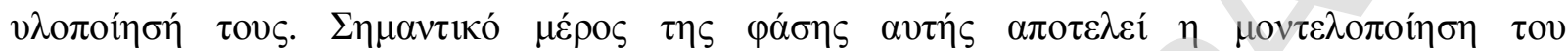

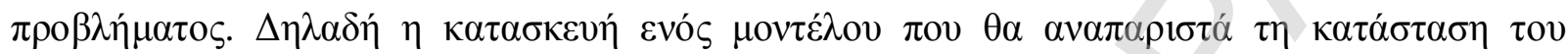

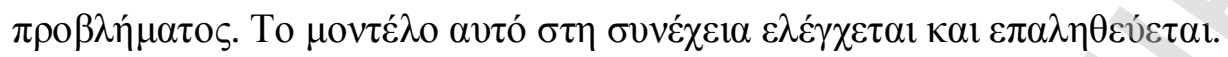

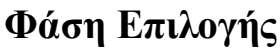

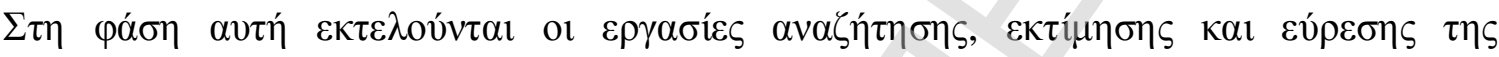

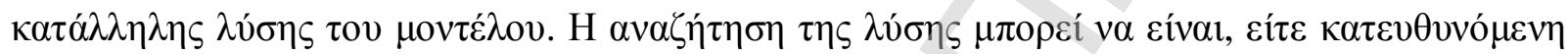

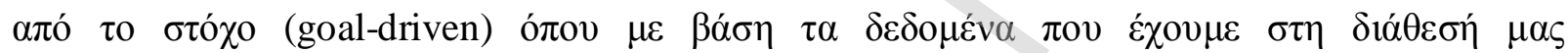

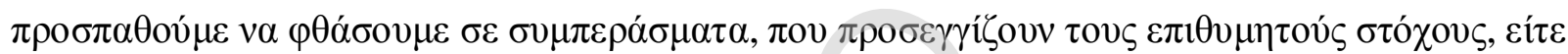

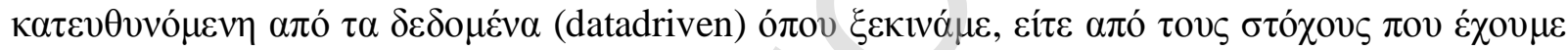

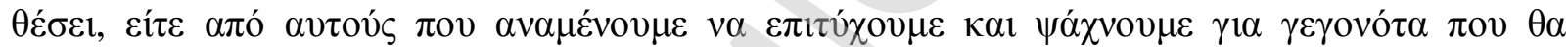

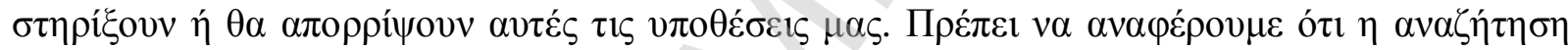

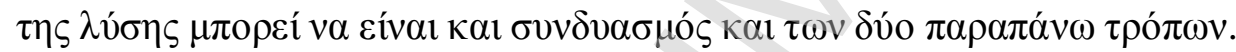

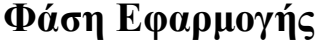

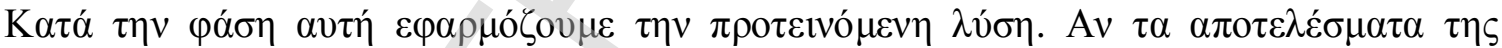

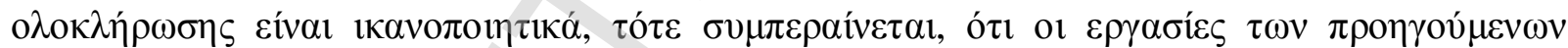

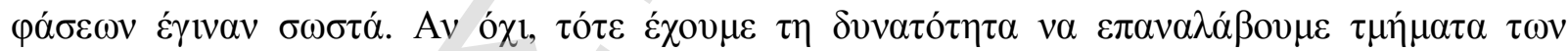

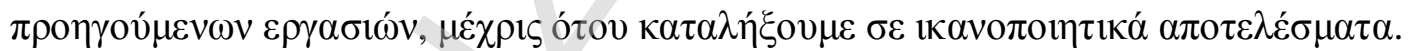

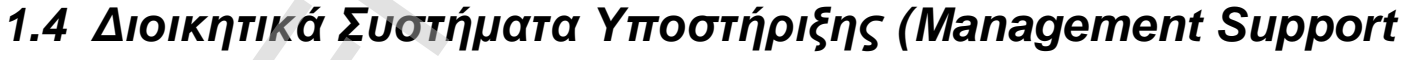

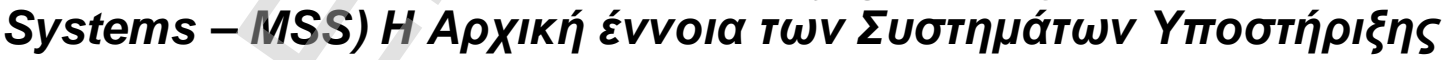

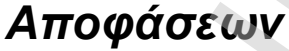

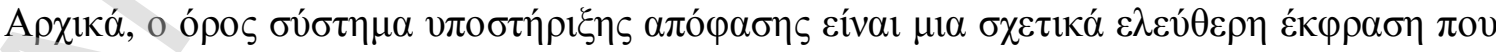

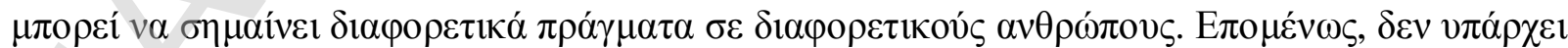

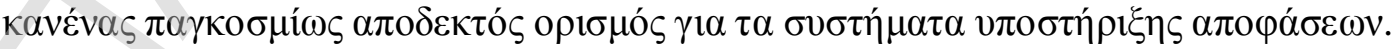




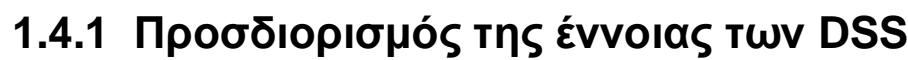

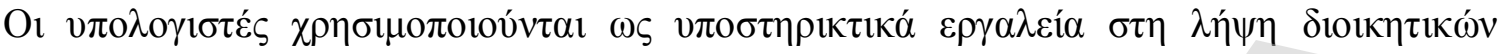

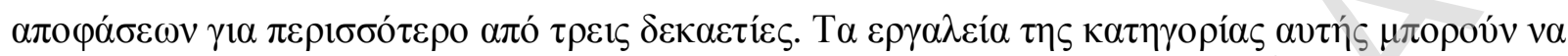

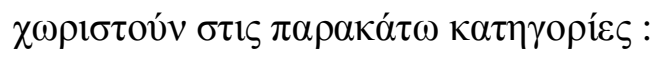

- $\quad \Sigma v \sigma \tau \eta ́ \mu \alpha \tau \alpha \delta 1 \alpha \chi \varepsilon i ́ p ı \sigma\rceil \varsigma \sigma v v \alpha \lambda \lambda \alpha \gamma \omega ́ v$ (Transaction Process Systems - TPS)

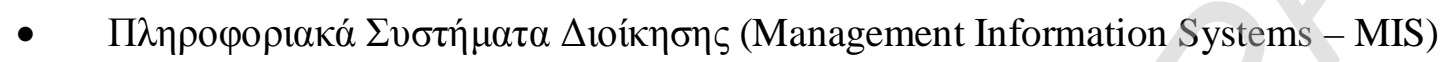

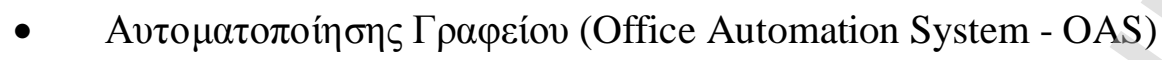

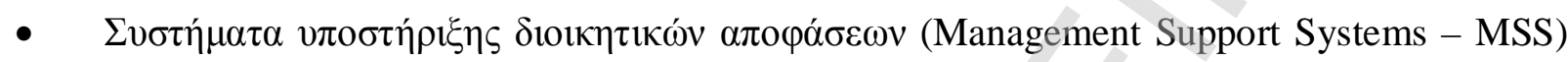

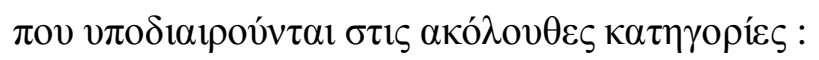

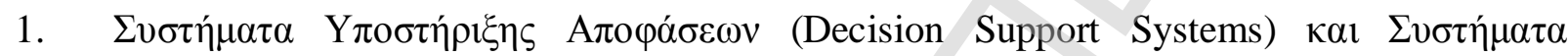

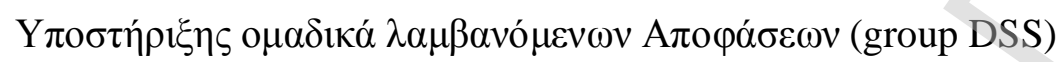

2. ${ }^{\circ} \mathrm{E} \mu \pi \varepsilon \iota \rho \alpha \sigma v \sigma \tau \eta \dot{\mu} \mu \tau \alpha$ (Expert Systems)

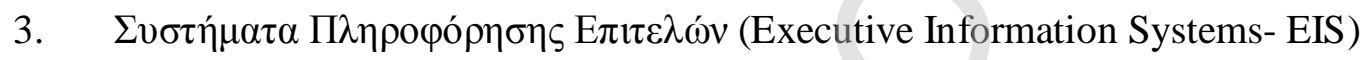

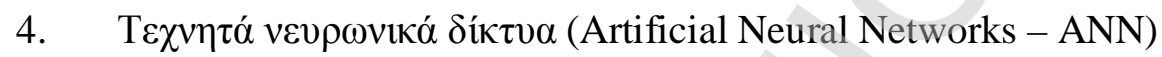

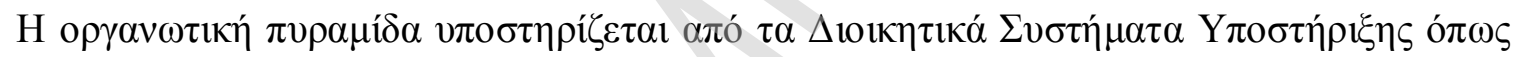

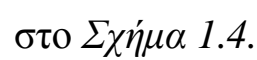

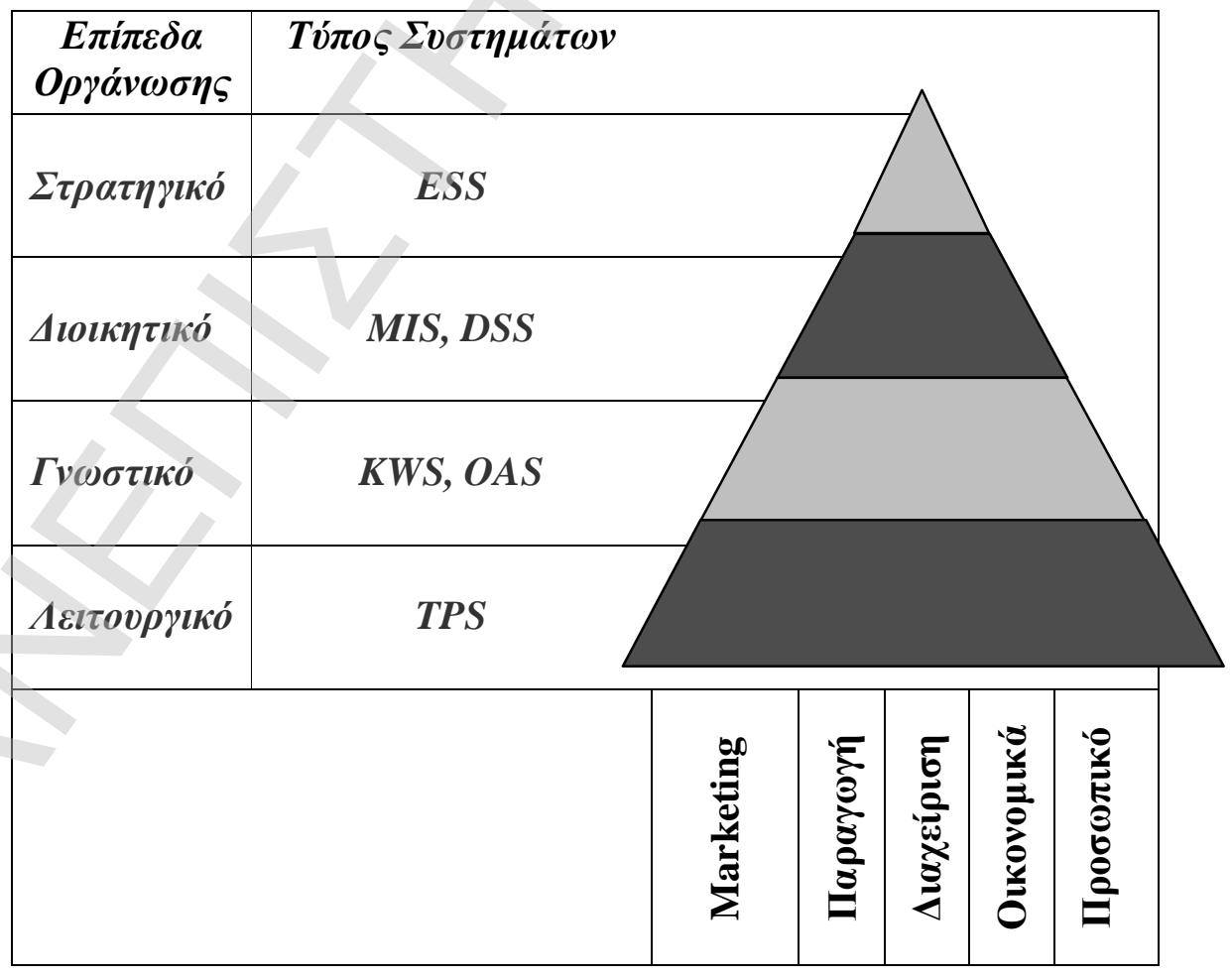

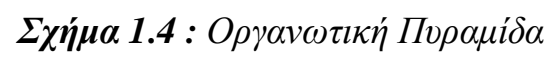




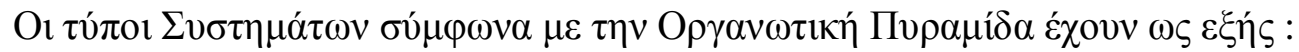

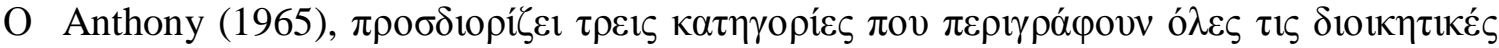

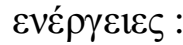

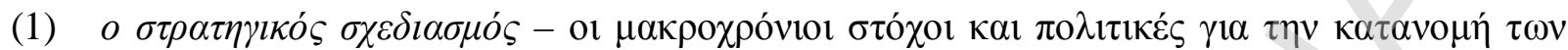
$\pi$ ó $\rho \omega v$

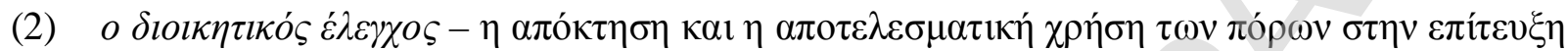

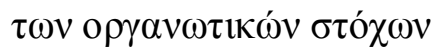

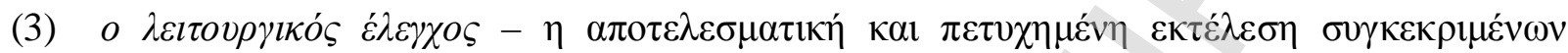

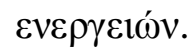

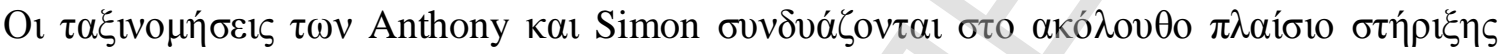

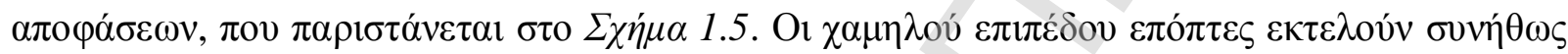

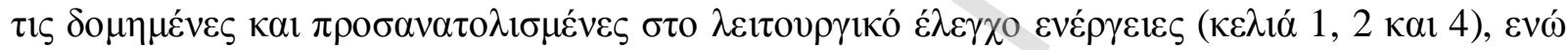

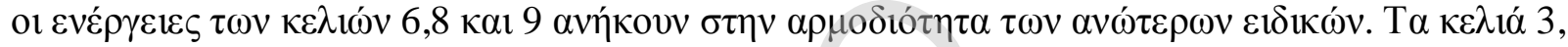

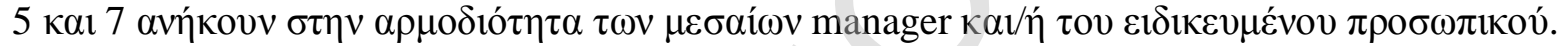

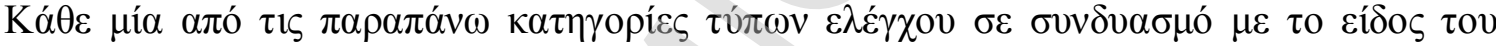

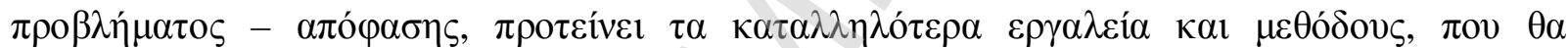

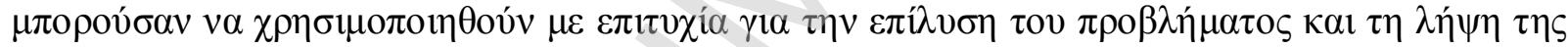

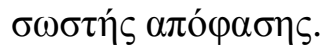




\begin{tabular}{|c|c|c|c|c|c|}
\hline & \multicolumn{4}{|c|}{ 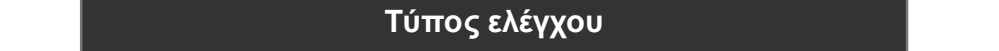 } & \\
\hline $\begin{array}{l}\text { Túmos } \\
\text { amóфaons }\end{array}$ & \multicolumn{2}{|l|}{ 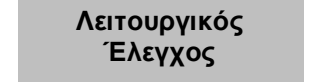 } & 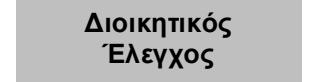 & 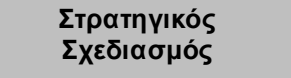 & 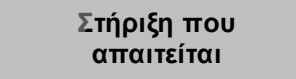 \\
\hline 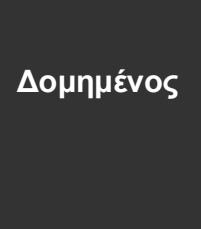 & \multicolumn{2}{|l|}{ 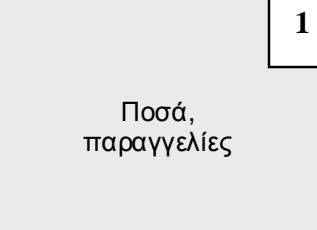 } & 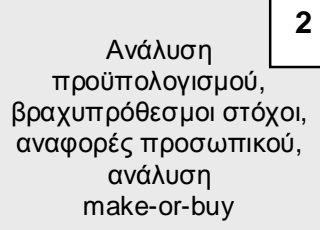 & 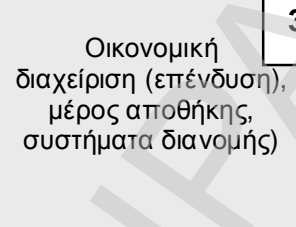 & 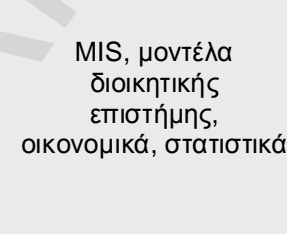 \\
\hline 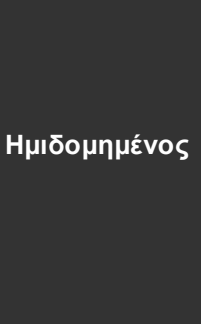 & \multicolumn{2}{|c|}{ 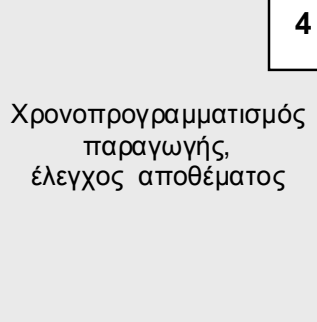 } & 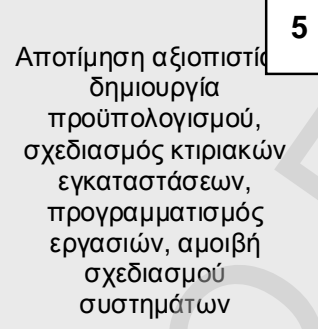 & 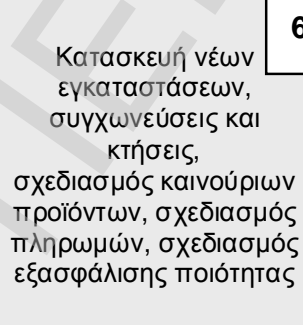 & DSS \\
\hline 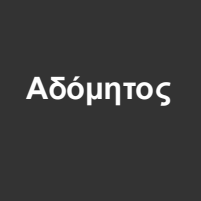 & \multicolumn{2}{|l|}{ 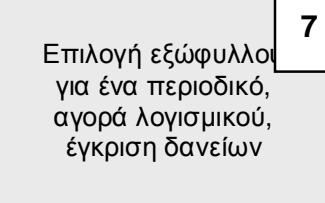 } & 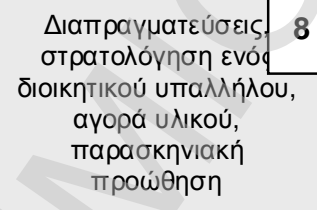 & 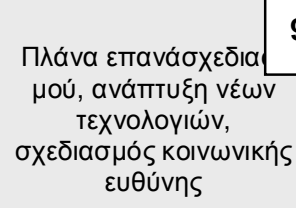 & 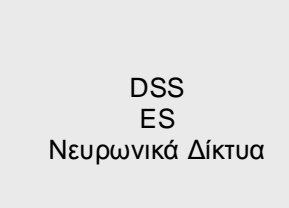 \\
\hline 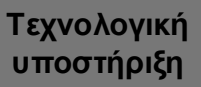 & \multicolumn{2}{|l|}{ 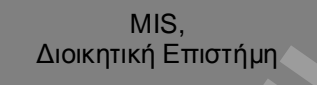 } & 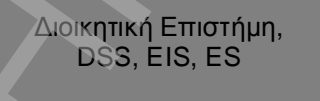 & 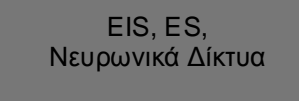 & \\
\hline
\end{tabular}

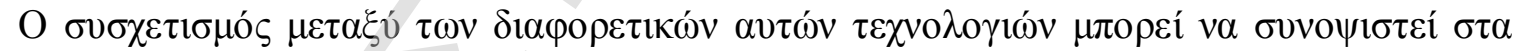
$\alpha \kappa o ́ \lambda o v \theta \alpha$ :

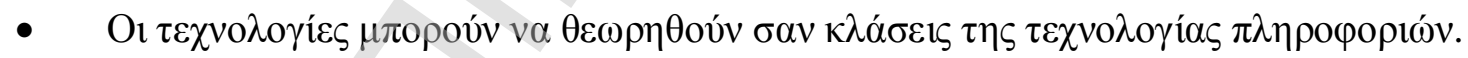

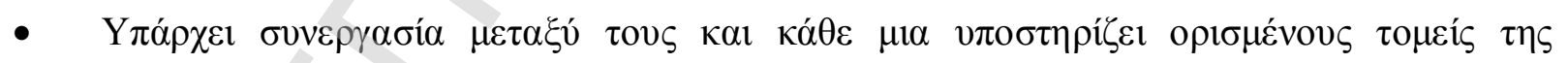
$\delta 1 \alpha \delta 1 \kappa \alpha \sigma i ́ \alpha \varsigma \alpha \pi \circ \varphi \alpha ́ \sigma \varepsilon \omega v$.

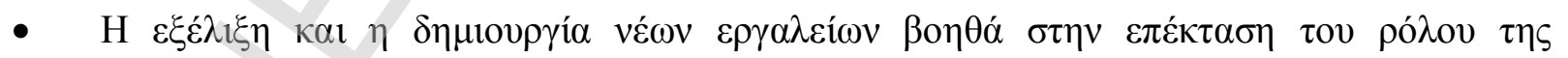

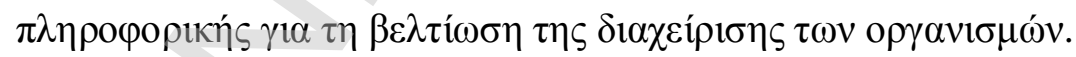




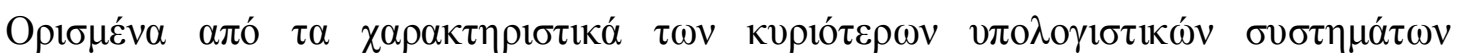

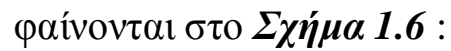

\begin{tabular}{|c|c|c|c|c|c|}
\hline & $\begin{array}{l}\text { Transaction } \\
\text { Processing } \\
\text { Systems } \\
\text { (TPS) }\end{array}$ & $\begin{array}{c}\text { Management } \\
\text { Information systems (MIS) }\end{array}$ & $\begin{array}{c}\text { Decision } \\
\text { Support } \\
\text { Systems (DSS) }\end{array}$ & $\begin{array}{l}\text { Expert Systems } \\
\text { (ES) }\end{array}$ & $\begin{array}{l}\text { Executive } \\
\text { Information } \\
\text { Systems } \\
\text { (EIS) }\end{array}$ \\
\hline Ерариоүе́s & 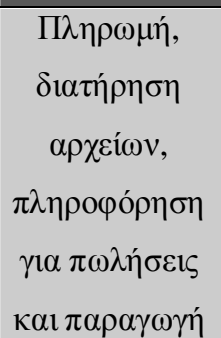 & $\begin{array}{l}\text { 'Е } \lambda \varepsilon \gamma \chi 0 \varsigma \pi \alpha \rho \alpha \gamma \omega \gamma \eta ́ \varsigma, \\
\pi \rho o ́ \beta \lambda \varepsilon \psi \eta \pi \omega \lambda \eta \dot{\sigma} \sigma \omega \nu\end{array}$ & $\begin{array}{c}\sum \tau \rho \alpha \tau \eta \gamma 1 \kappa o ́ \varsigma \\
\sigma \chi \varepsilon \delta 1 \alpha \sigma \mu o ́ \varsigma \\
\mu \alpha \kappa \rho \alpha ́ s \\
\varepsilon \mu \beta \varepsilon ́ \lambda \varepsilon 1 \alpha \varsigma\end{array}$ & 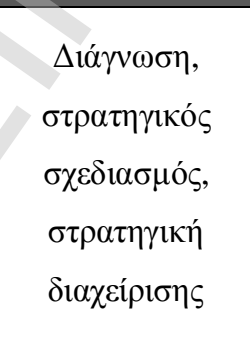 & 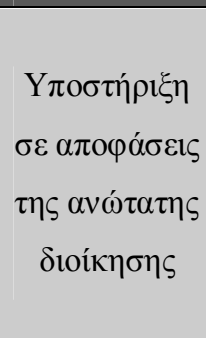 \\
\hline 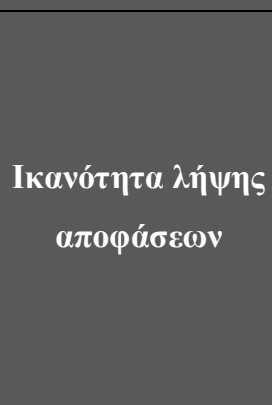 & 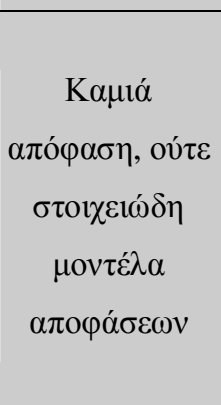 & 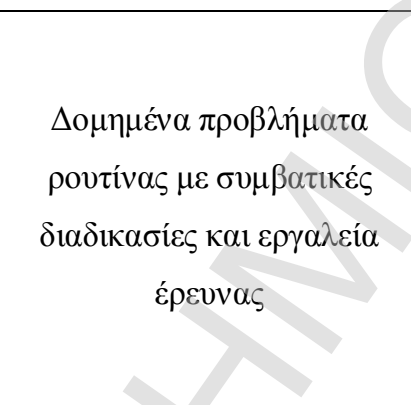 & 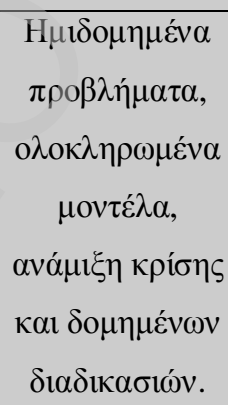 & 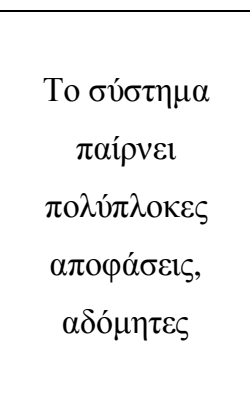 & 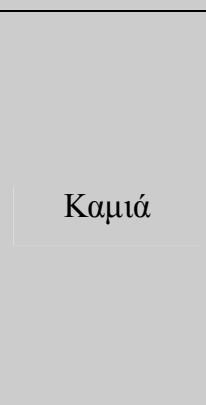 \\
\hline 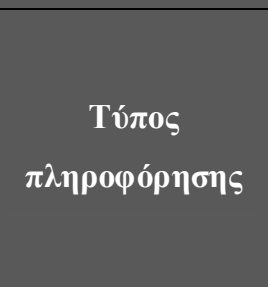 & 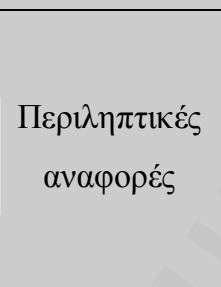 & 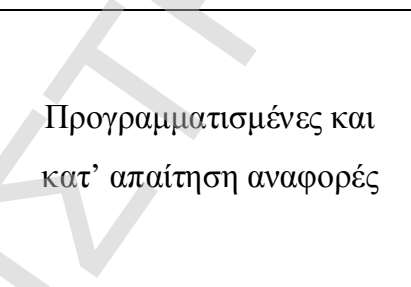 & 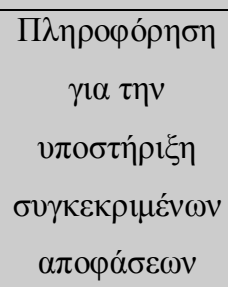 & 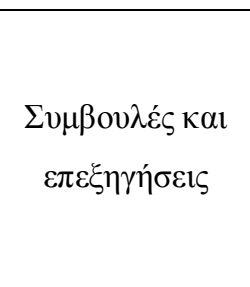 & 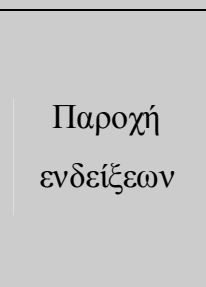 \\
\hline
\end{tabular}

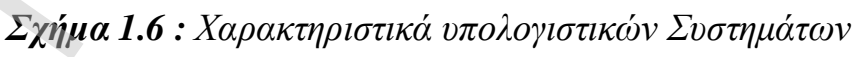

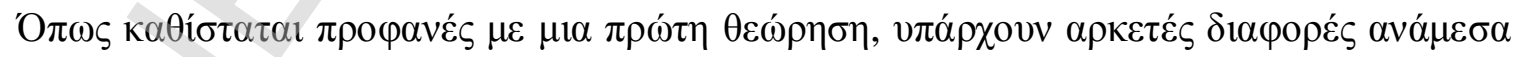

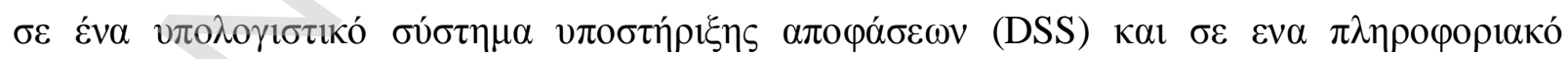

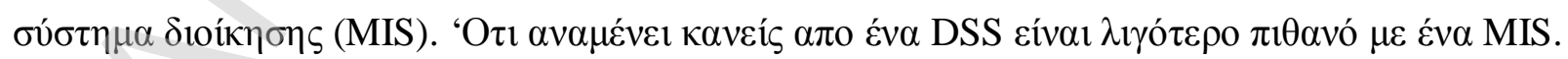

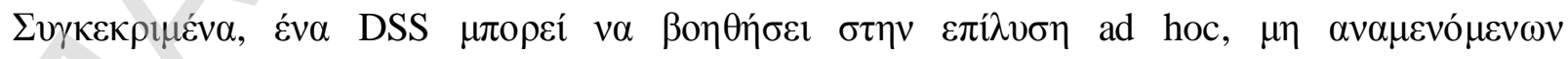

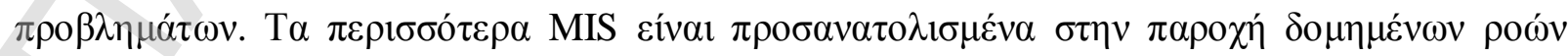

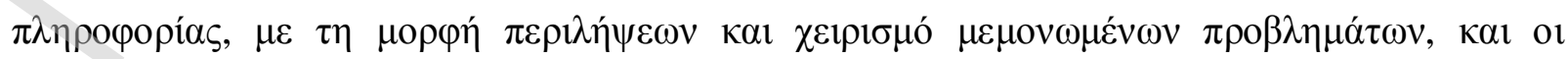

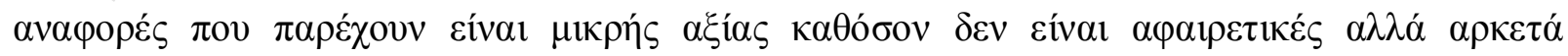




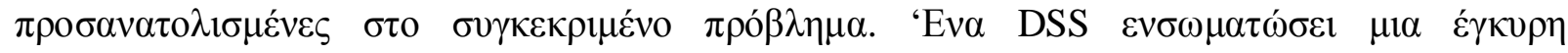

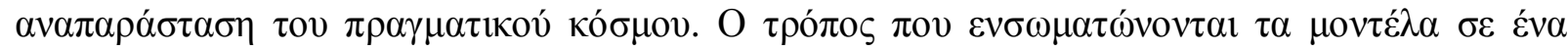

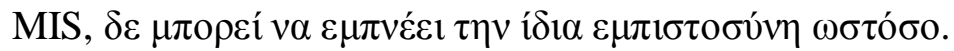

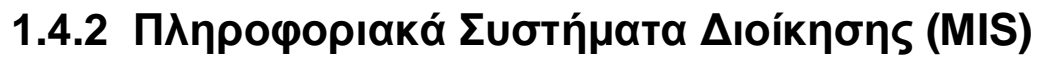

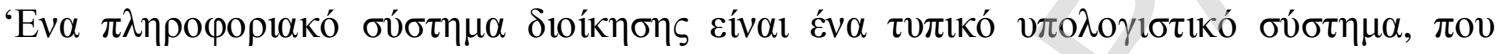

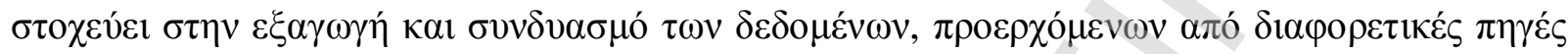

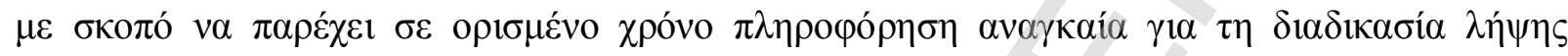

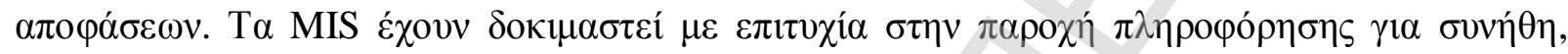

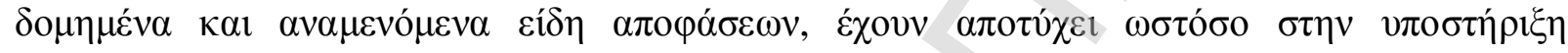

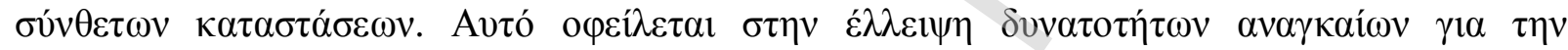

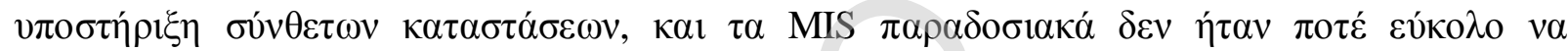

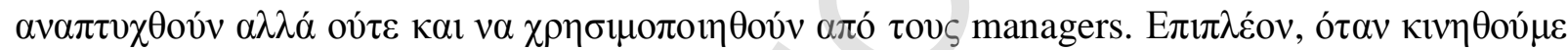

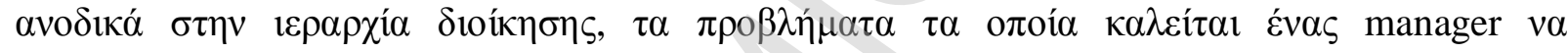

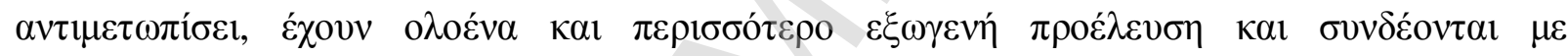

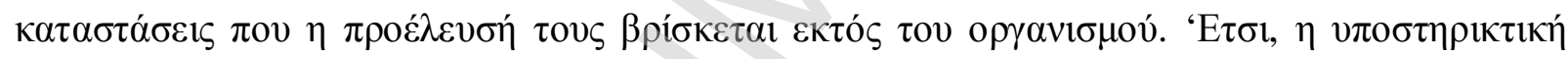

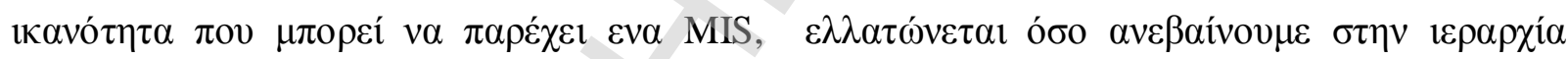

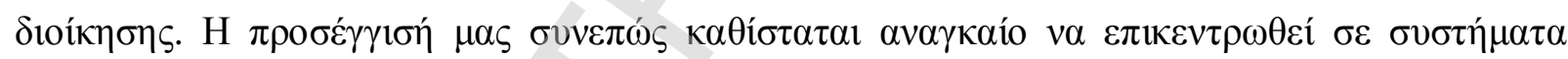

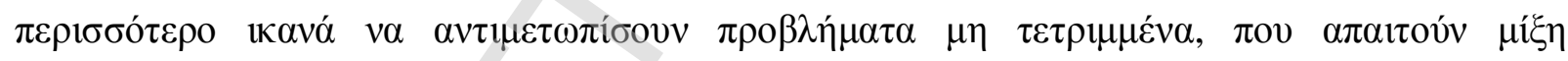

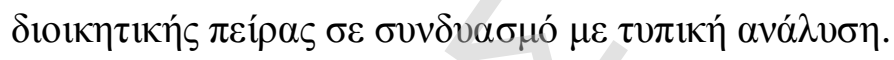

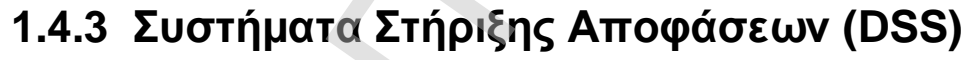

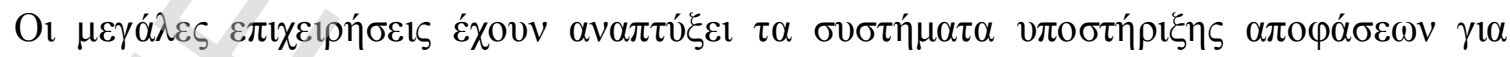

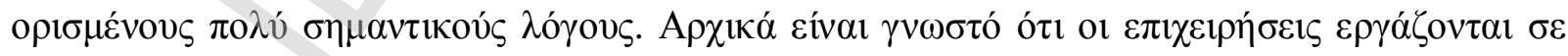

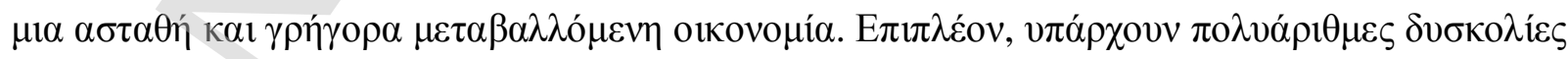

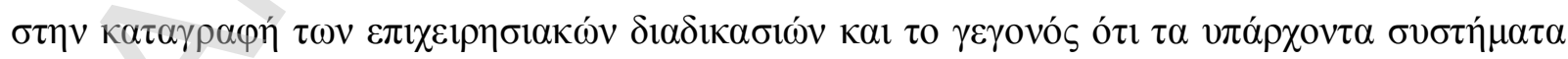

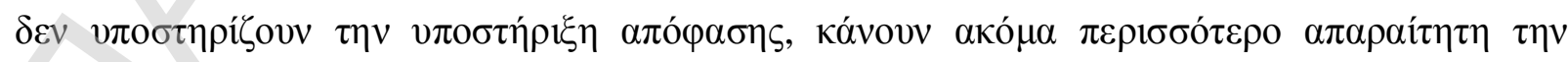

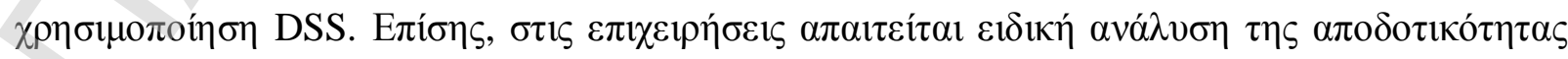

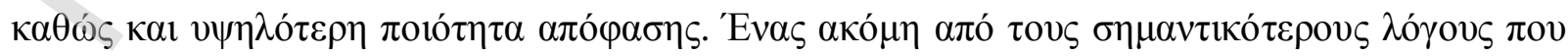

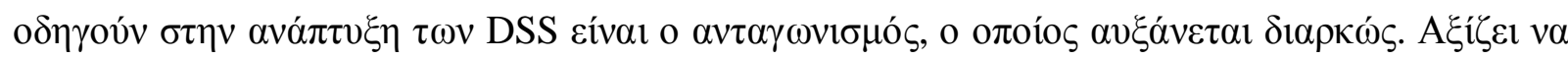




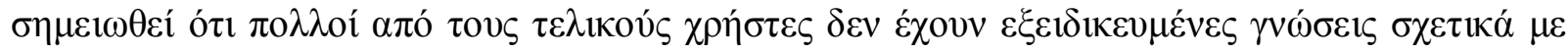

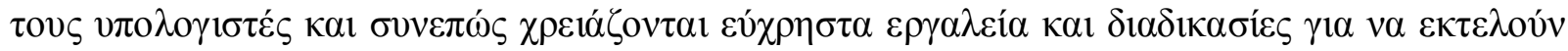

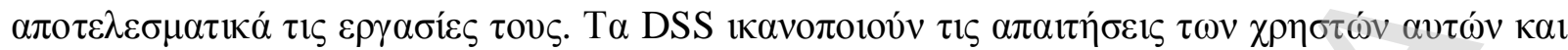

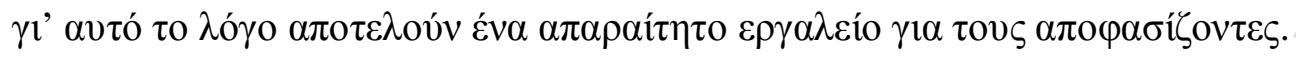

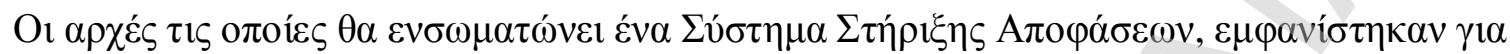

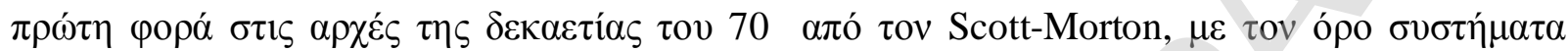

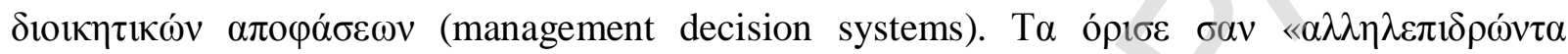

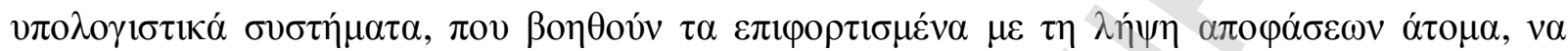

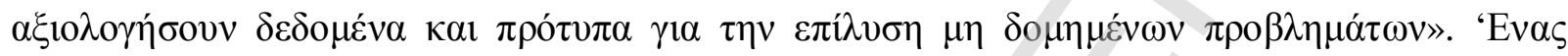

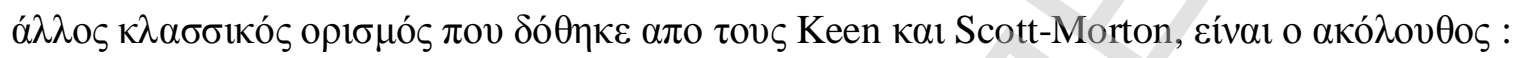

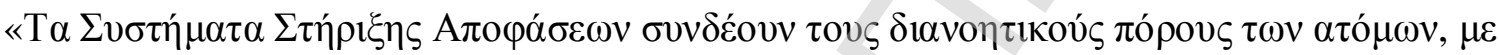

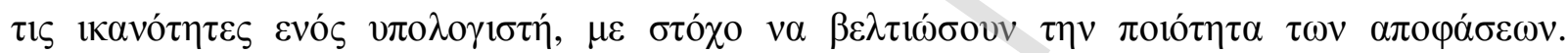

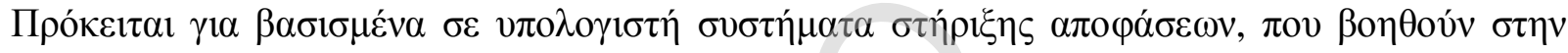
$\varepsilon \pi \hat{\imath} \lambda v \sigma \eta \eta \mu 1 \delta o \mu \eta \mu \varepsilon ́ v \omega v \pi \rho \circ \beta \lambda \eta \mu \alpha ́ \tau \omega v »$.

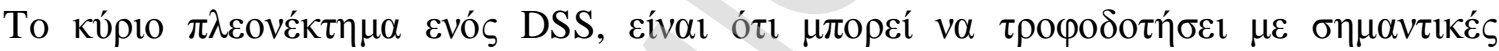

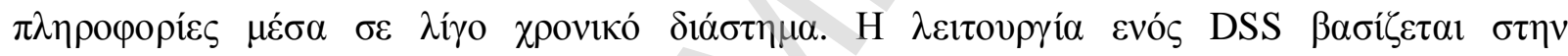

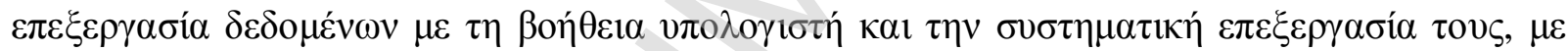

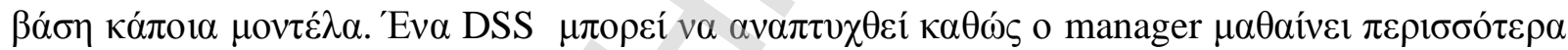

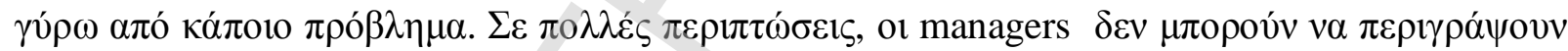

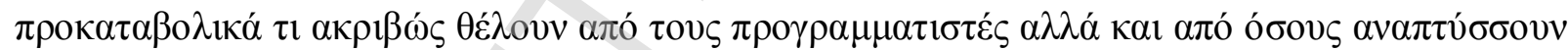

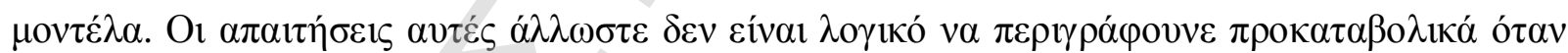

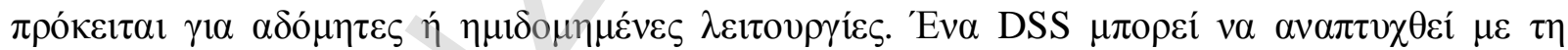

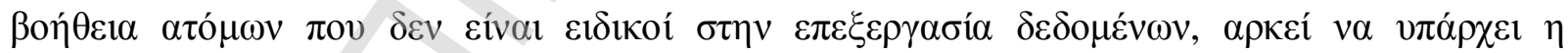

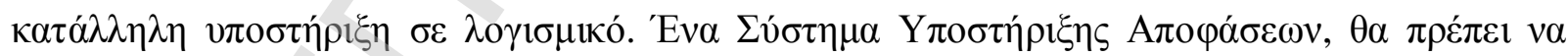

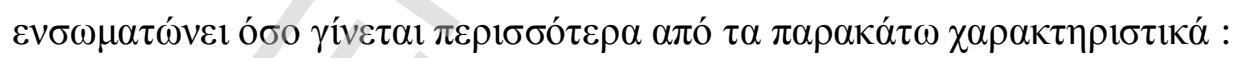

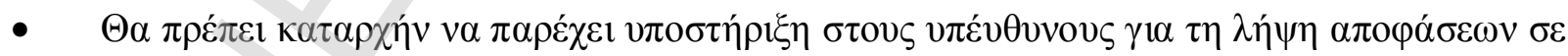

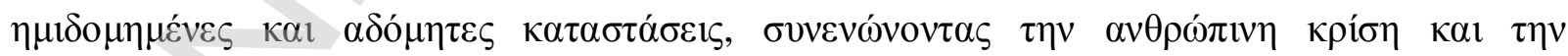

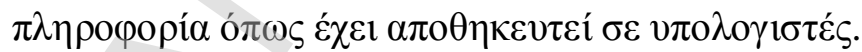

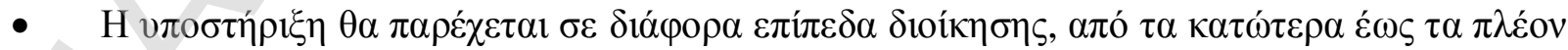

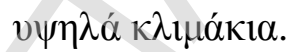

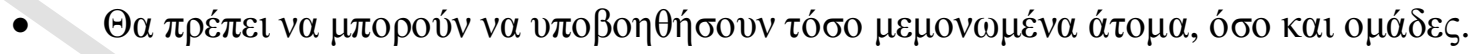




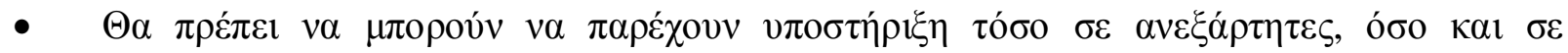
$\delta 1 \alpha \delta 0 \chi 1 \kappa \varepsilon ́ \zeta \delta 1 \alpha \delta 1 \kappa \alpha \sigma i ́ \varepsilon \varsigma$.

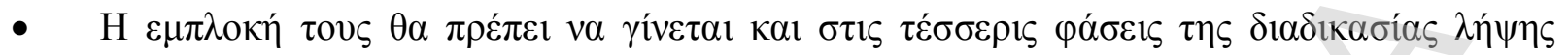

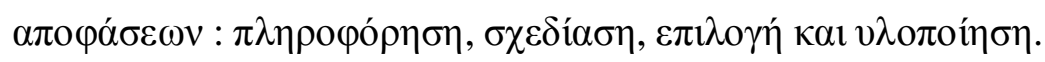

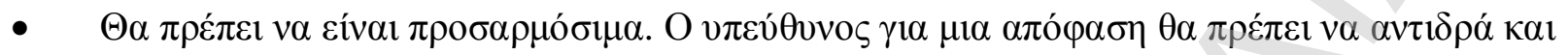

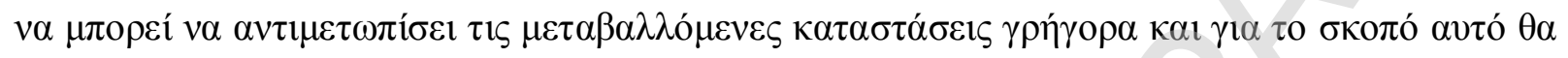

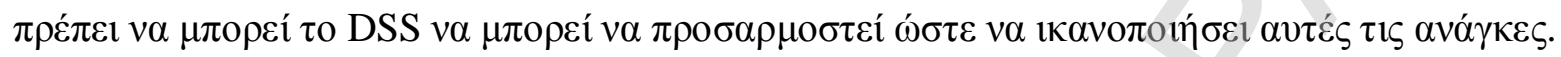

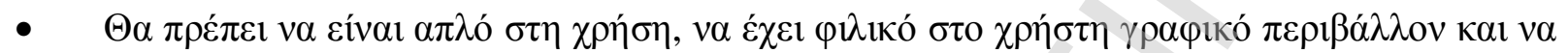

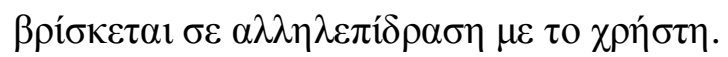

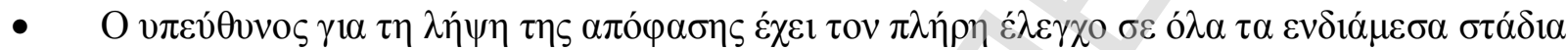

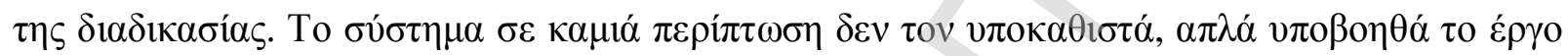

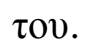

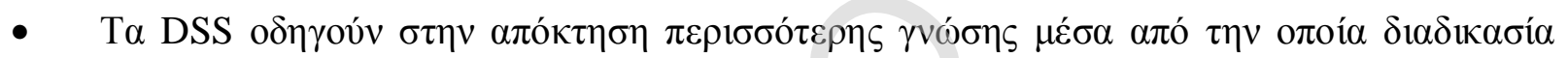

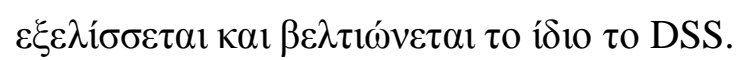

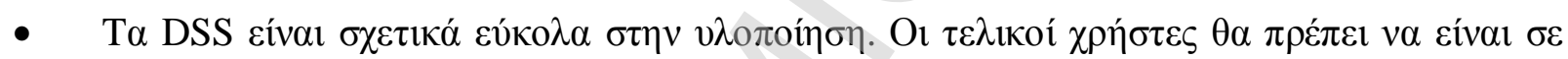

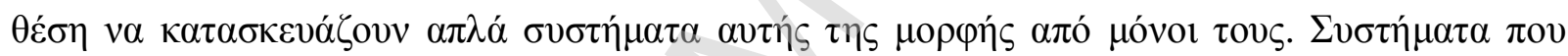

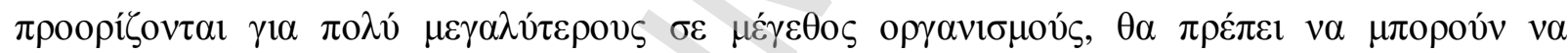

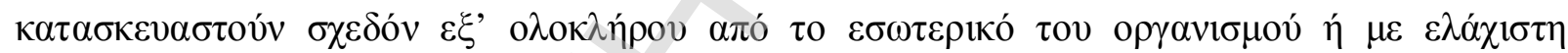

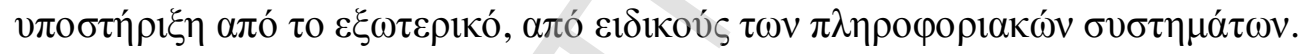

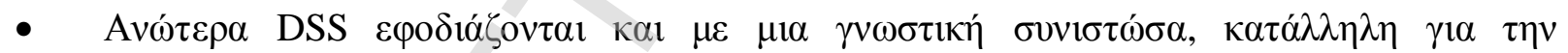

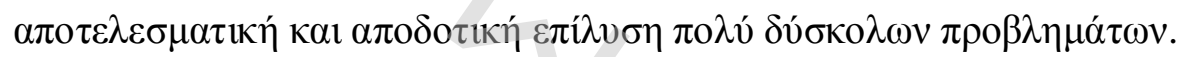




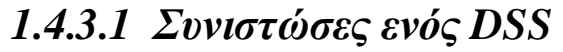

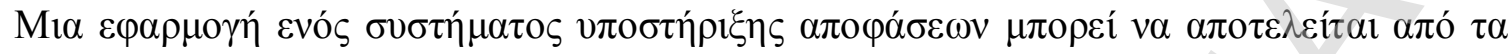

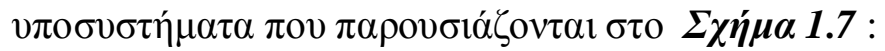

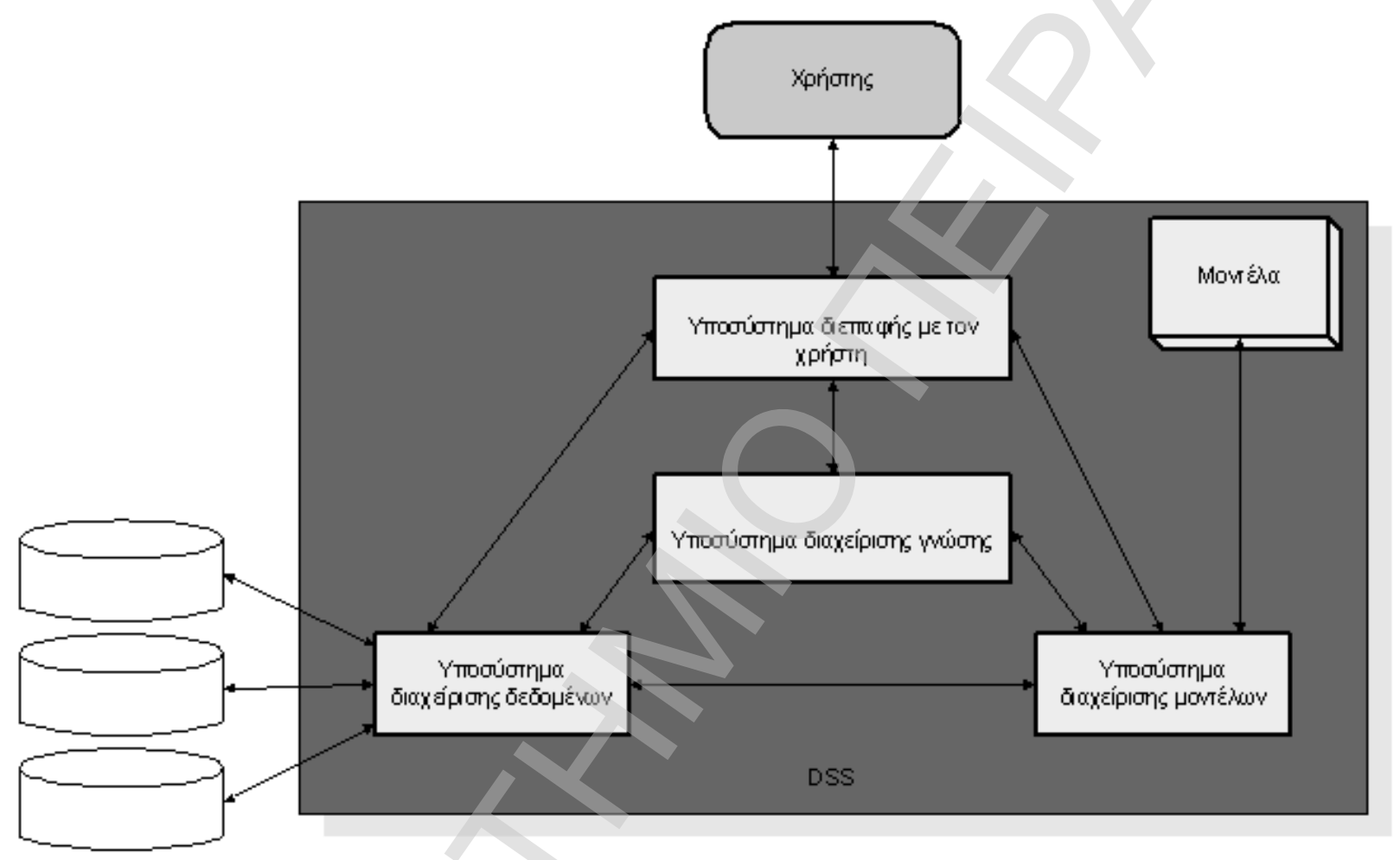

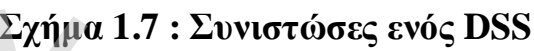

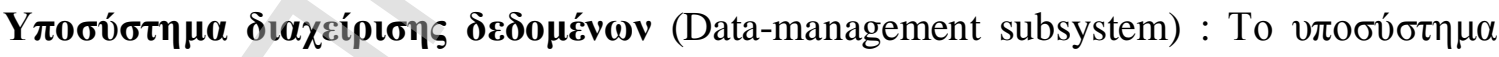

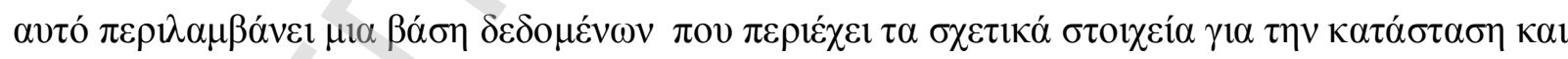

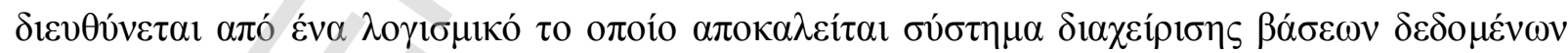

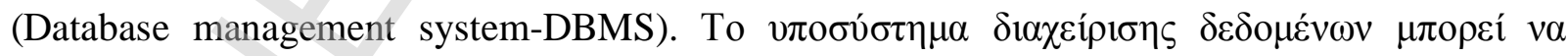

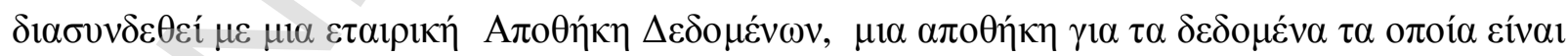

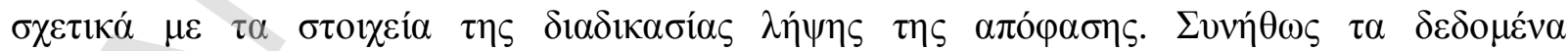

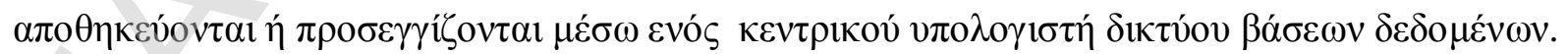

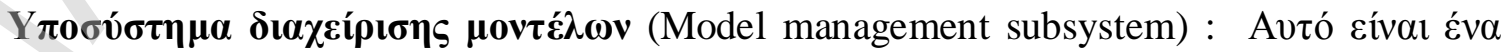

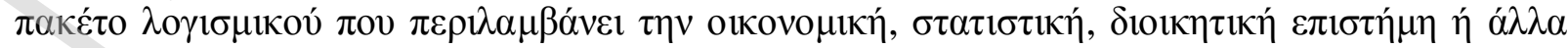

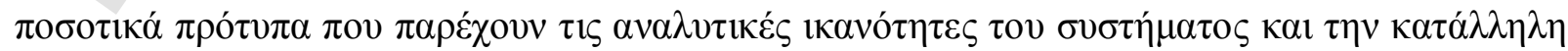

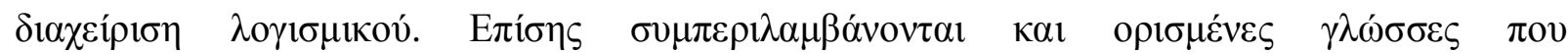




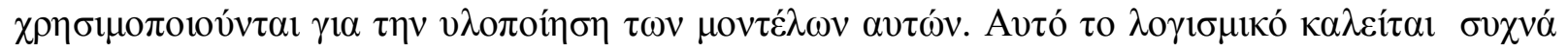

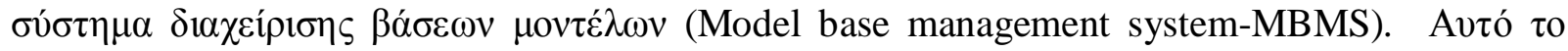

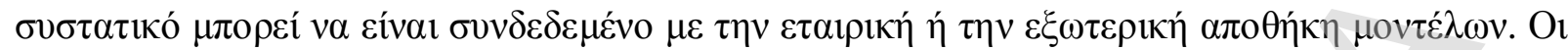

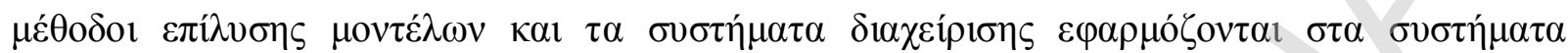

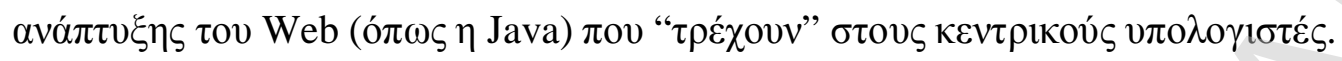

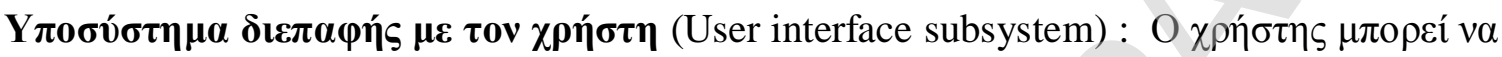

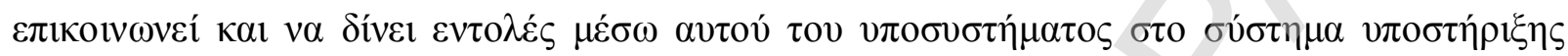

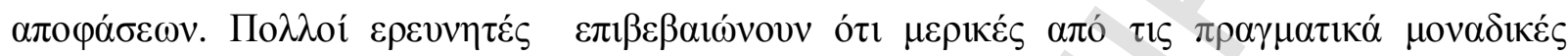

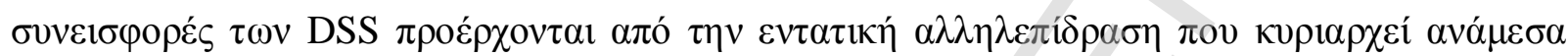

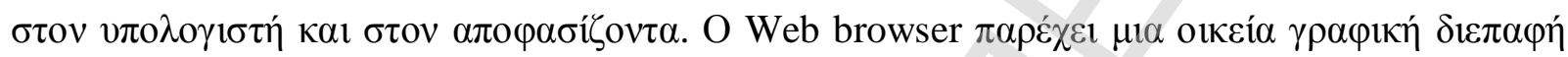

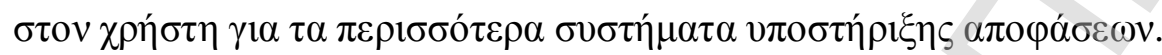

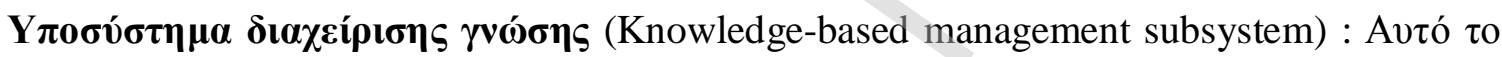

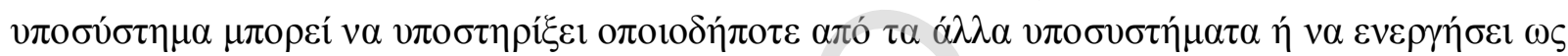

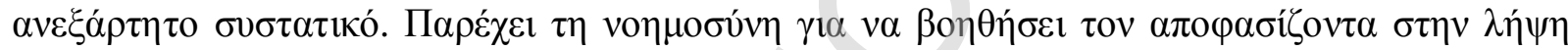

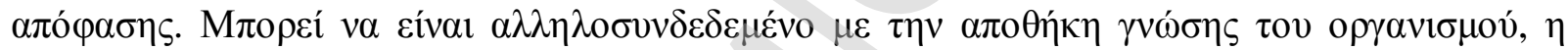

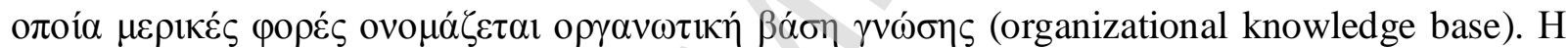

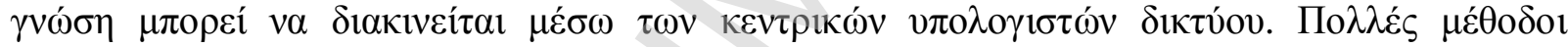

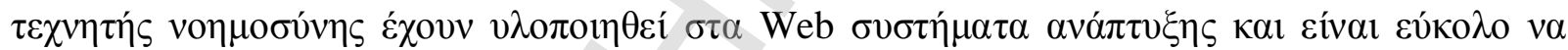

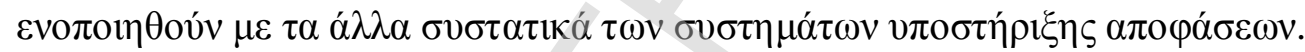

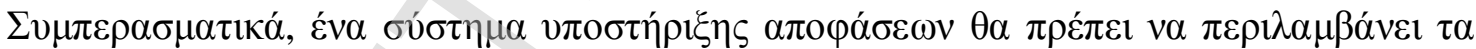

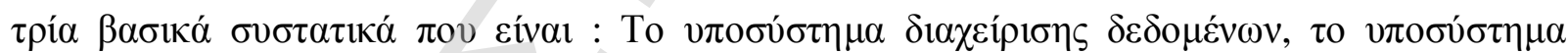

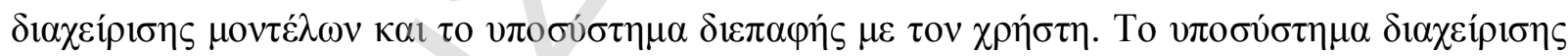

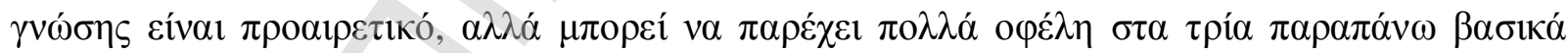

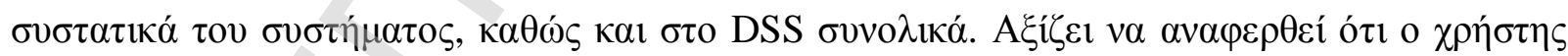

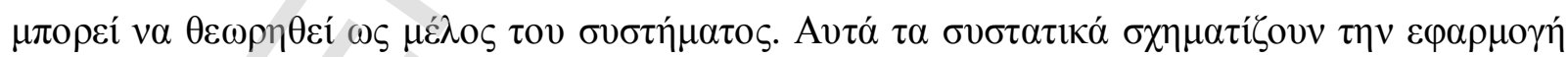

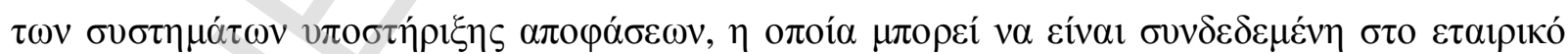

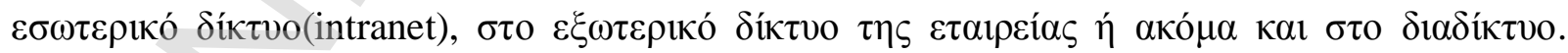

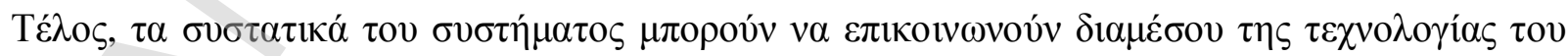
$\delta 1 \alpha \delta 1 \kappa \tau v ́ o v$. 


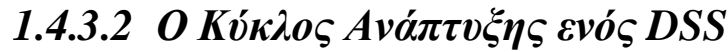

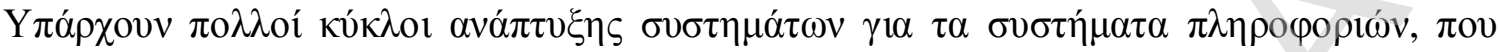

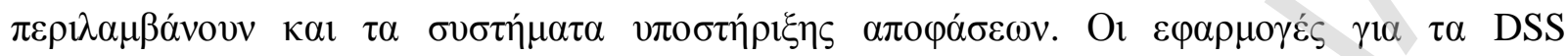

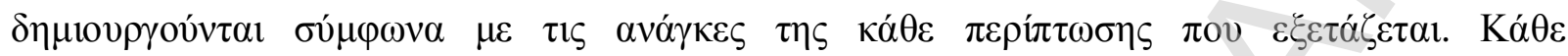

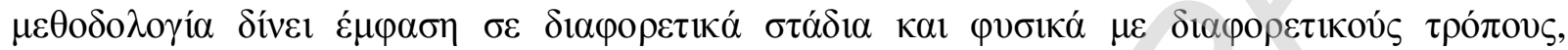

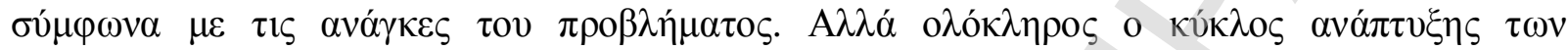

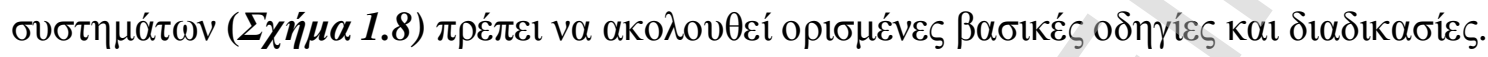

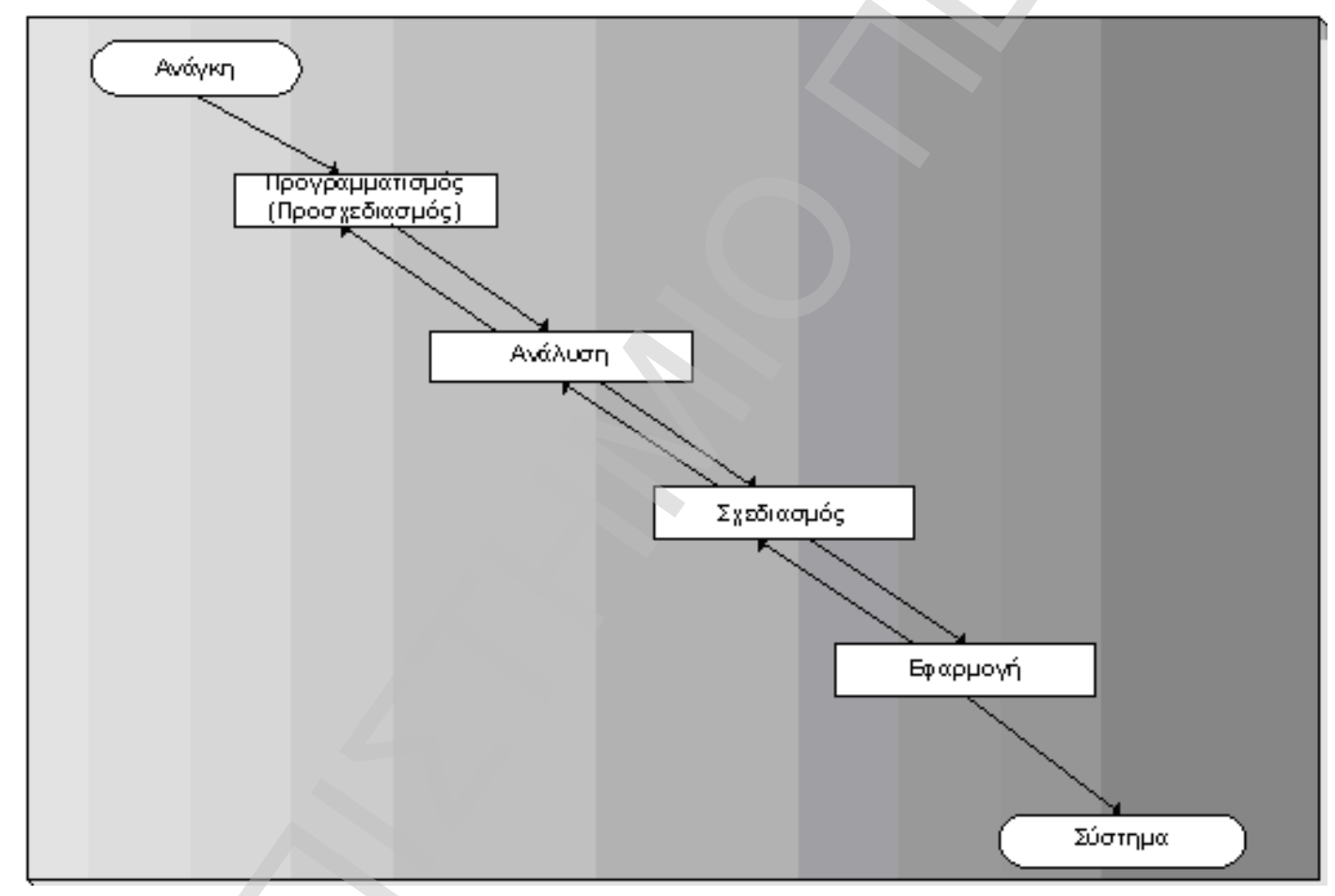

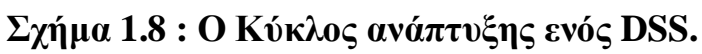

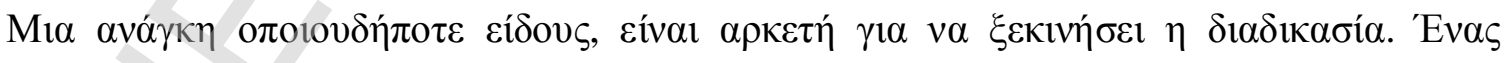

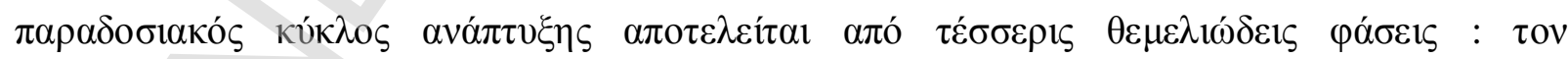

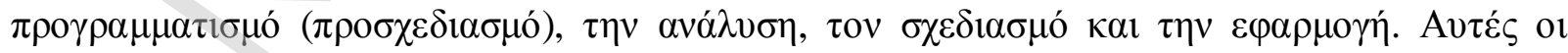

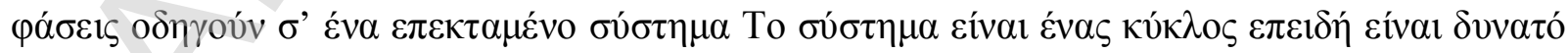

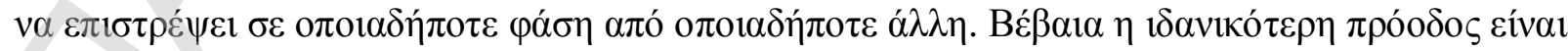

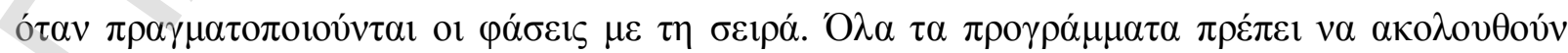

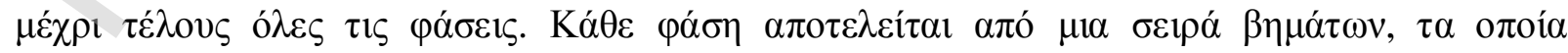

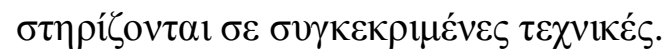




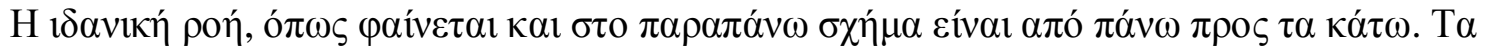

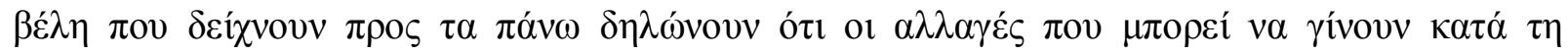

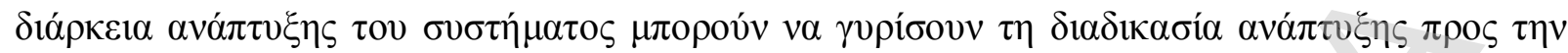

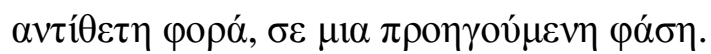

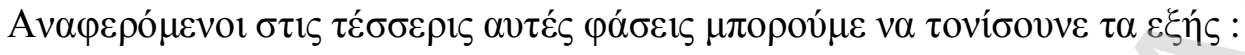

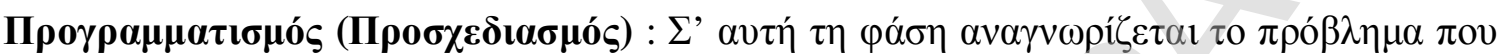

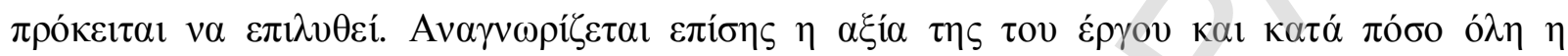

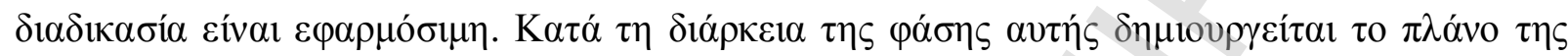

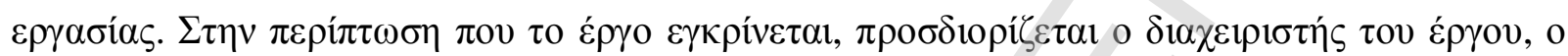

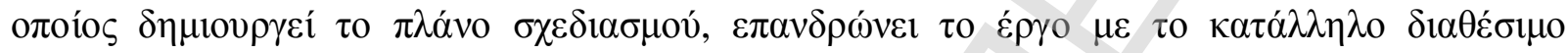

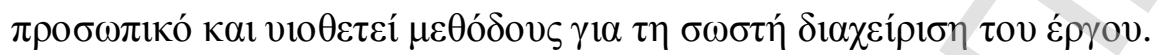

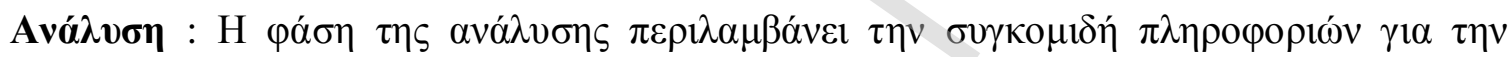

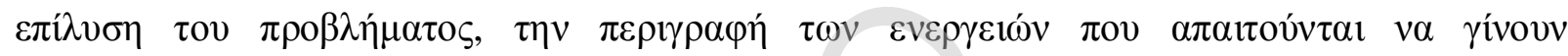

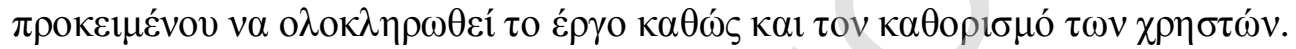

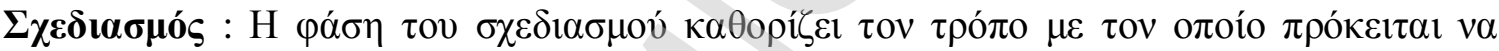

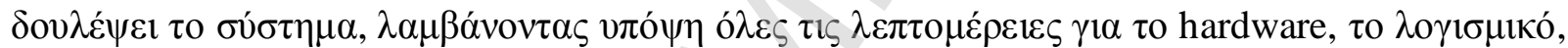

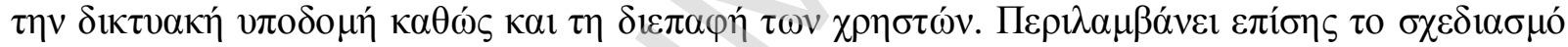

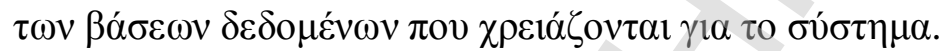

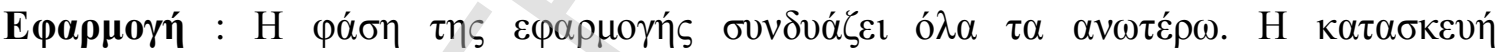

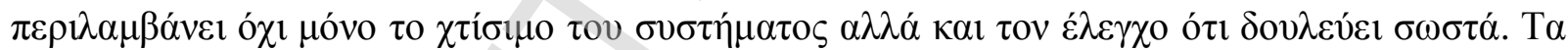

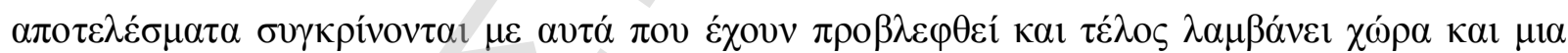

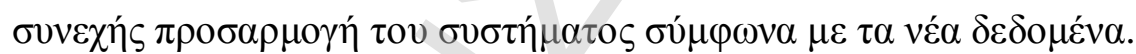

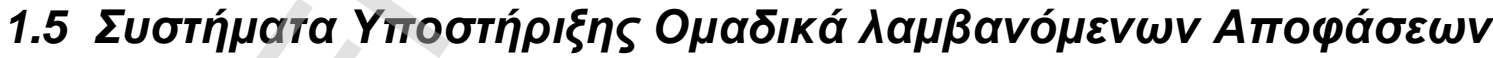 (GDSS)}

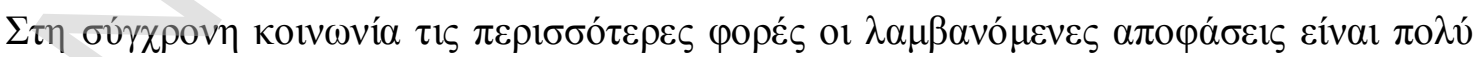

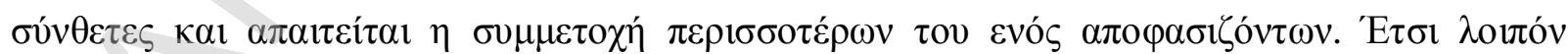

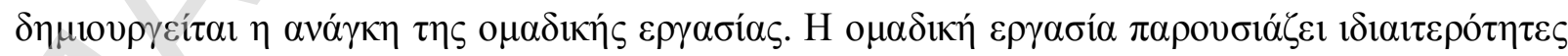

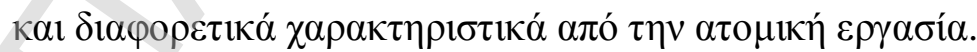

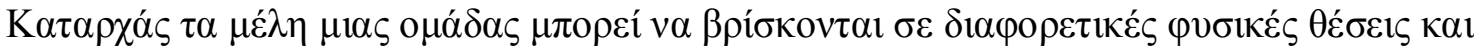

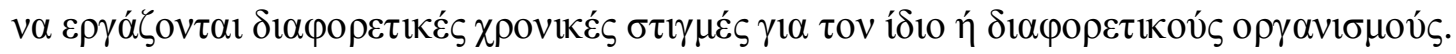




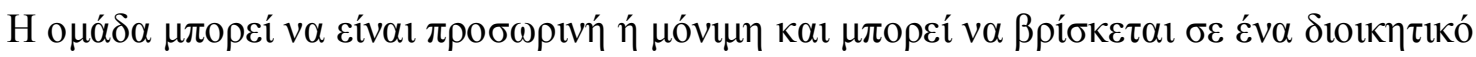

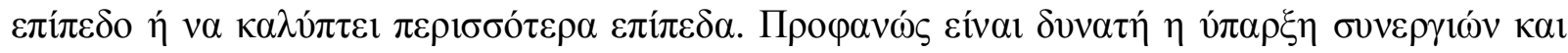

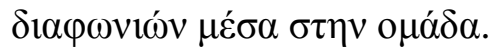

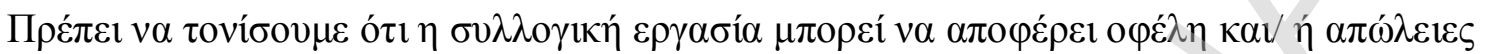

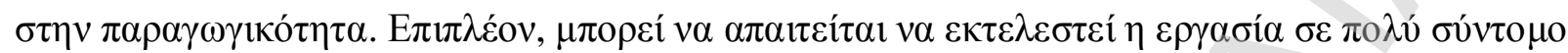

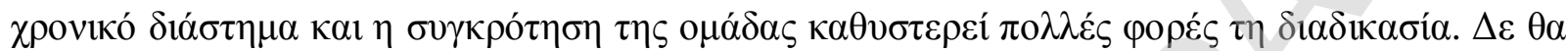

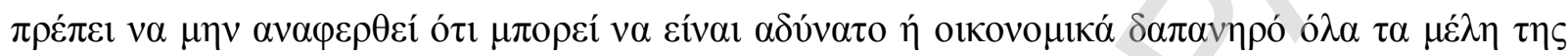

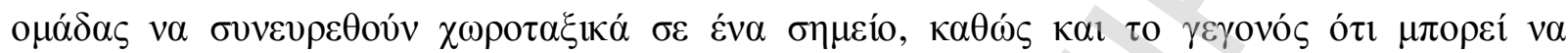

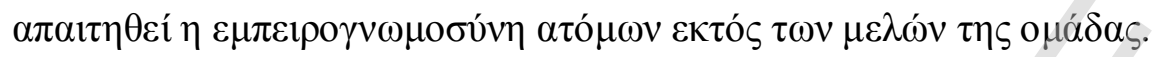

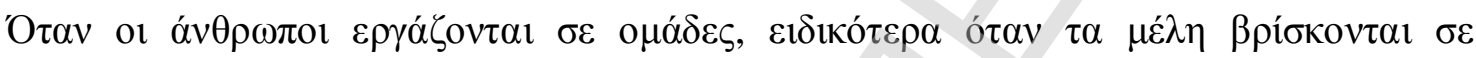

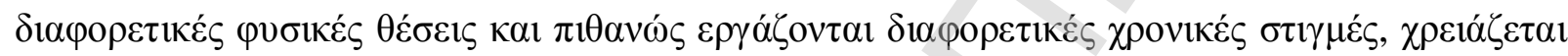

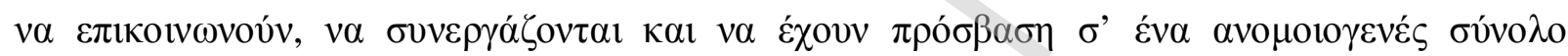

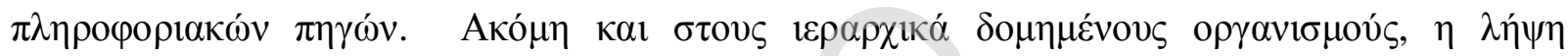

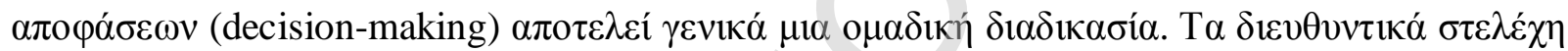

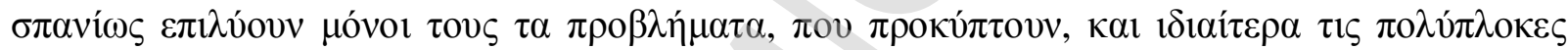

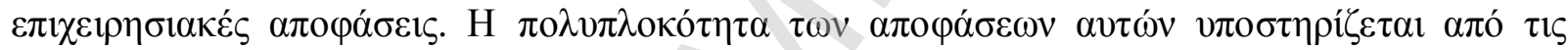

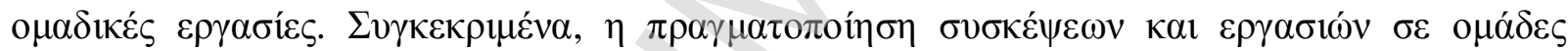

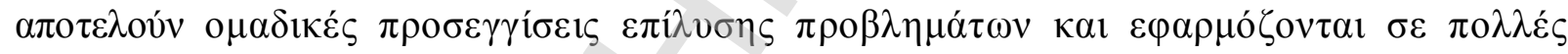

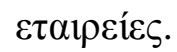

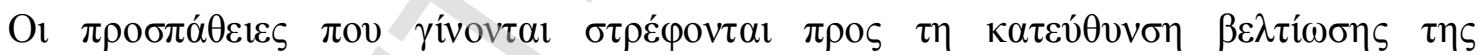

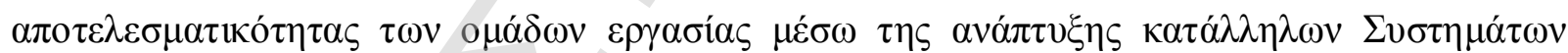

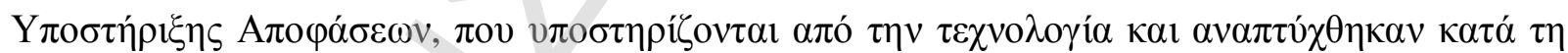

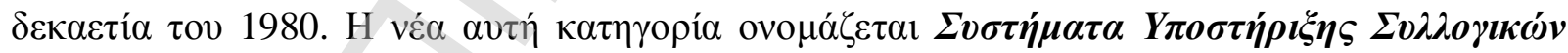

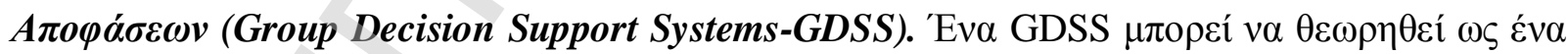

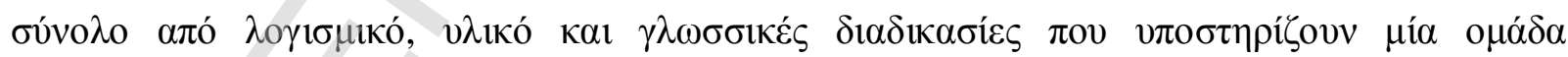

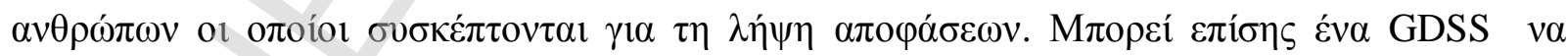
o

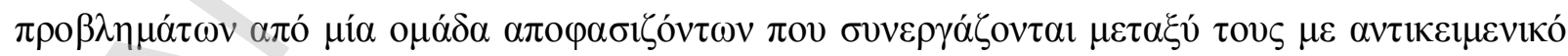

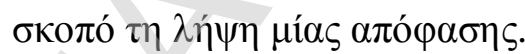

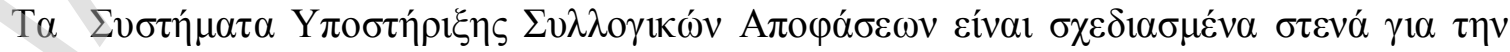

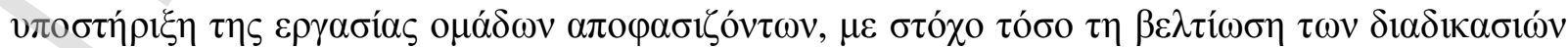

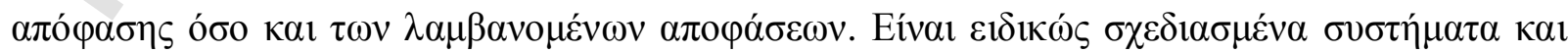

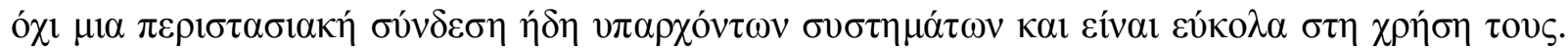




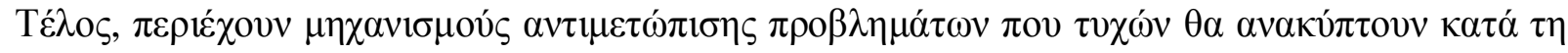

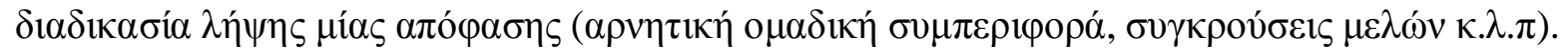

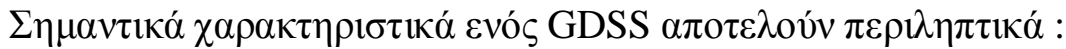

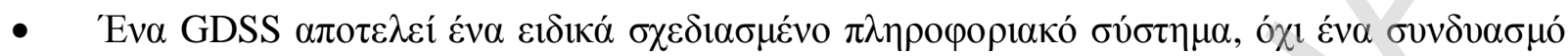
$\alpha \pi \hat{~ \eta ́ \delta \eta ~ v \pi \alpha ́ \rho \chi o v \sigma \varepsilon \varsigma ~ \sigma o v i \sigma \tau \omega ́ \sigma \varepsilon \varsigma . ~}$

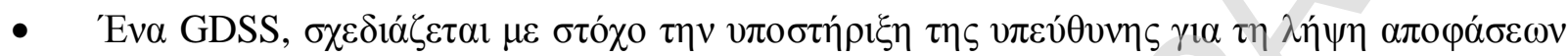

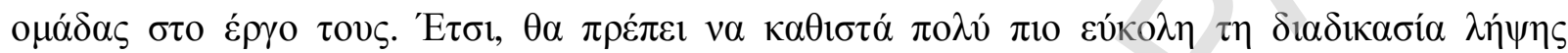

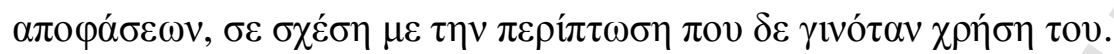

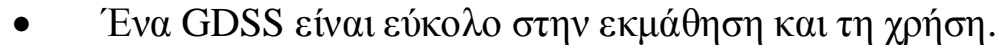

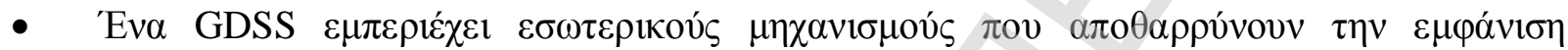

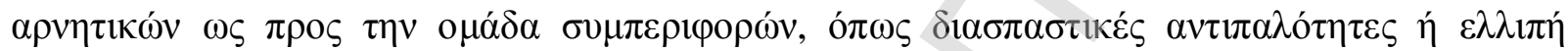

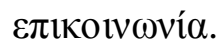

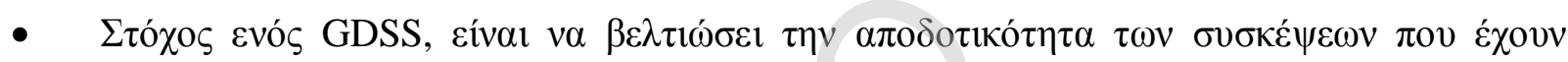

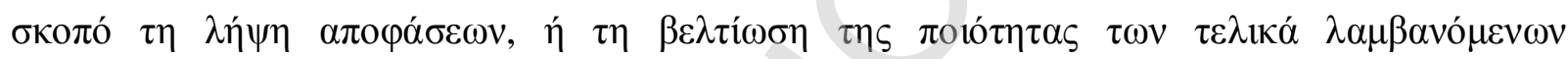

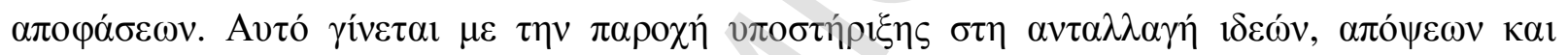

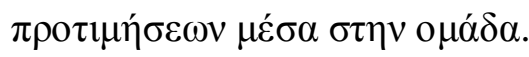

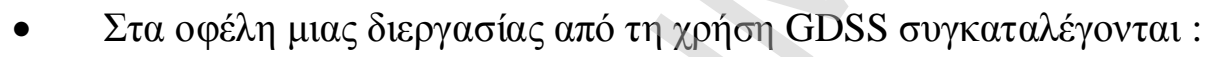

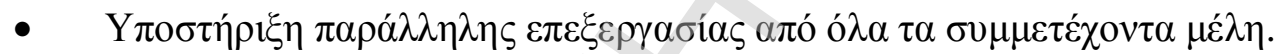

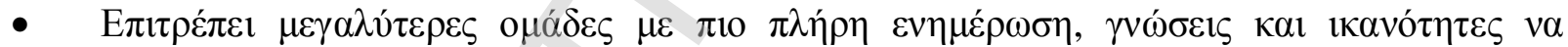

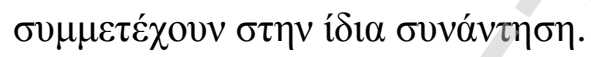

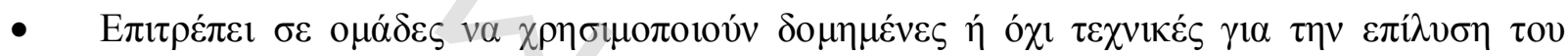
$\pi \rho \circ \beta \lambda \dot{\mu} \mu \alpha \tau$ s.

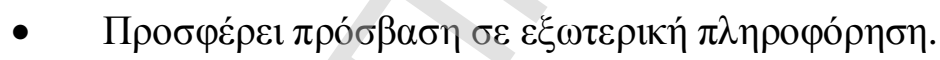

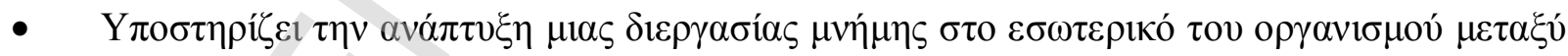
$\delta 1 \alpha \delta o \chi \iota \kappa \omega ́ v ~ \sigma v \sigma \kappa \varepsilon ́ \psi \varepsilon \omega v$.

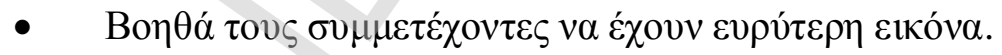

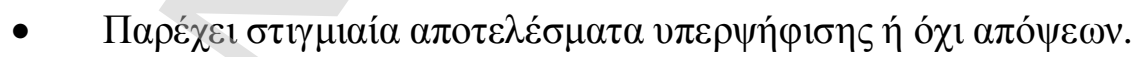

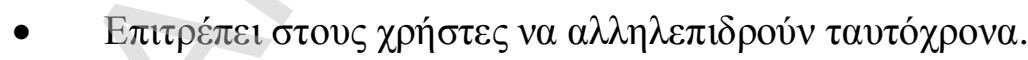

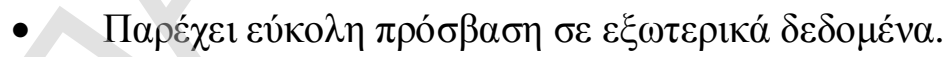

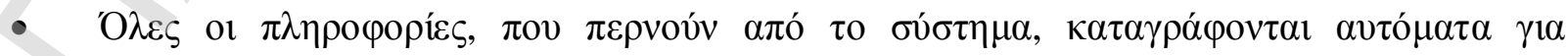

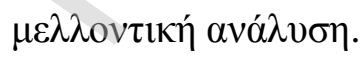




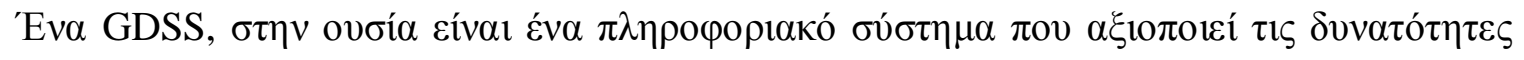

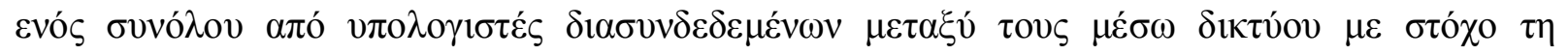

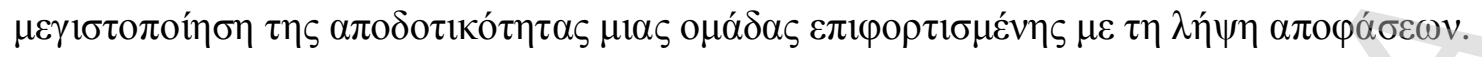

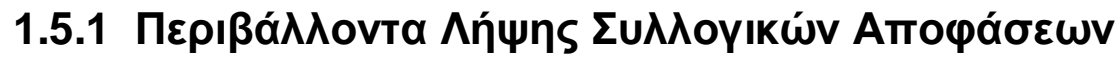

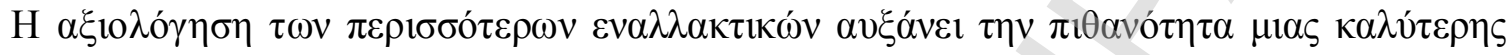

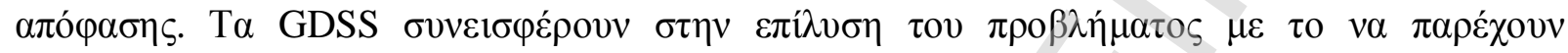

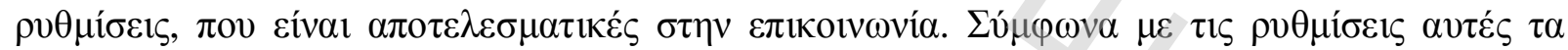

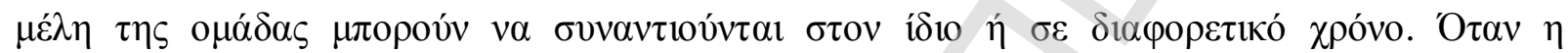

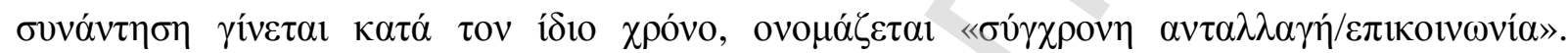

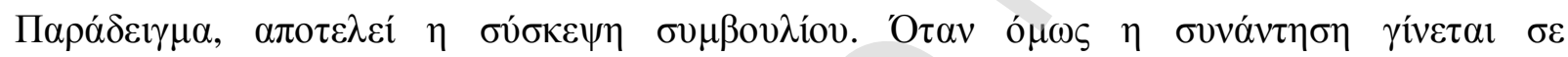

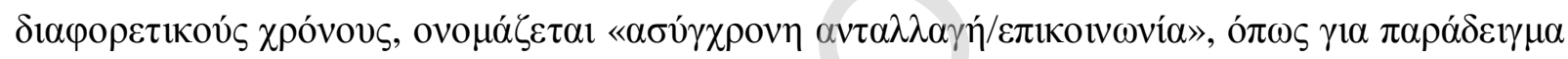

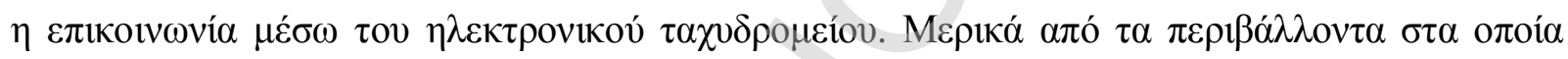

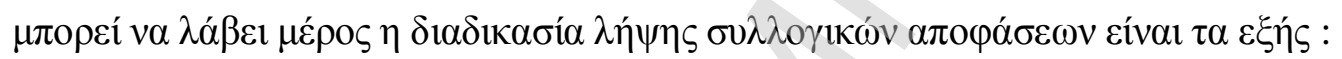

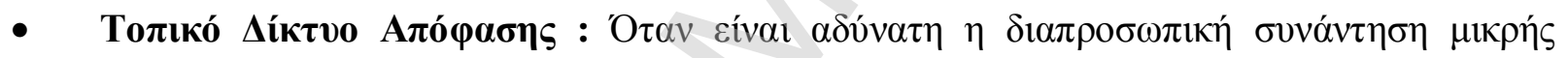

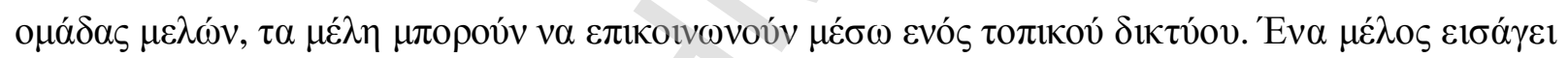

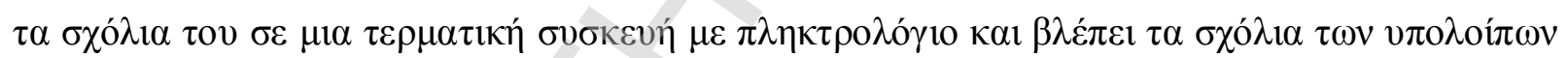

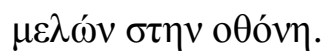

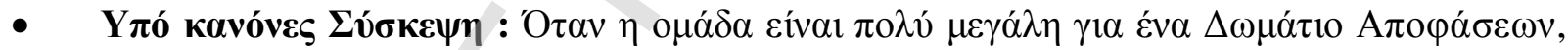

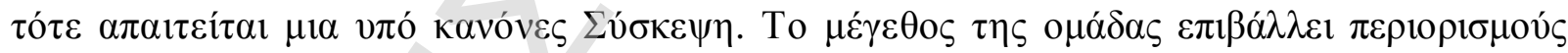

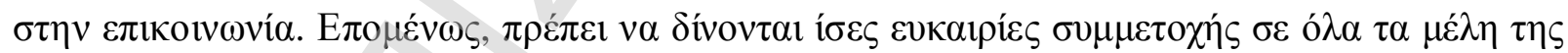

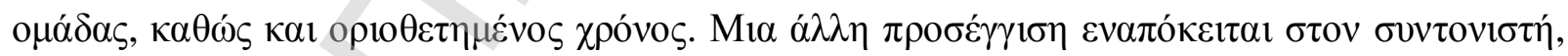

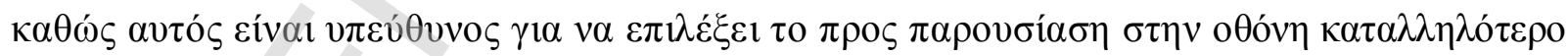
$v \lambda$ «ó $\tau \omega v \mu \varepsilon \lambda \omega ́ v$.

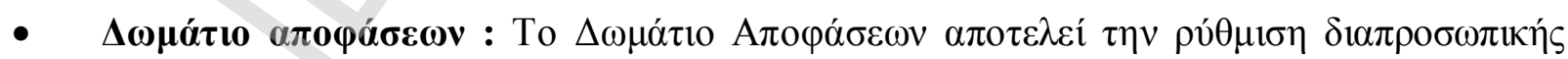

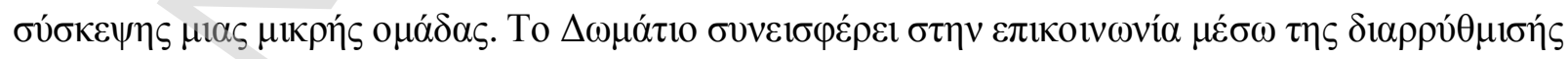

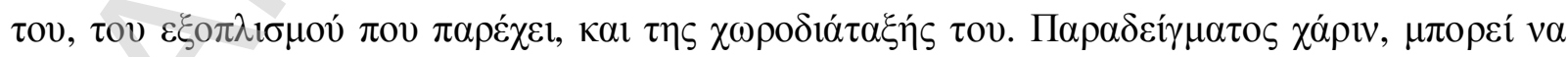

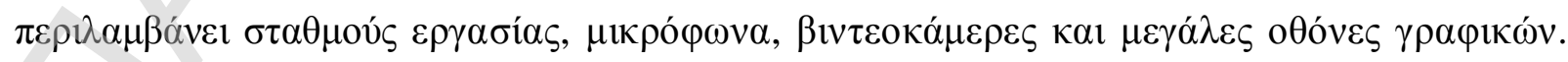

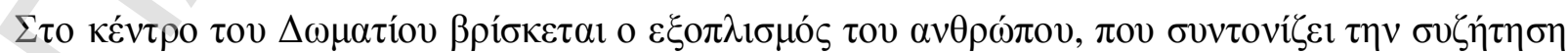

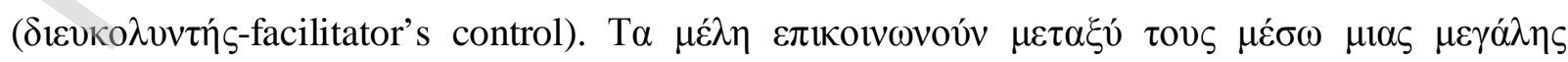




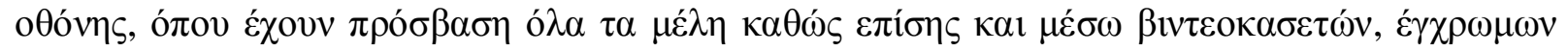
$\delta ı \alpha \varphi v \varepsilon \iota \omega ́ v \kappa \alpha \iota$ slides.

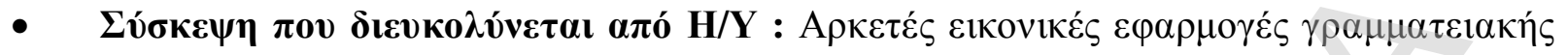

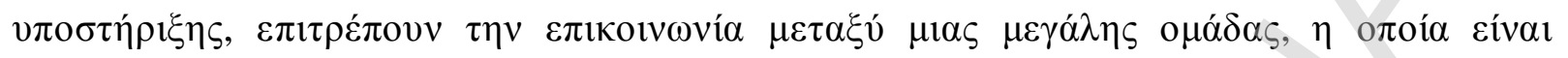

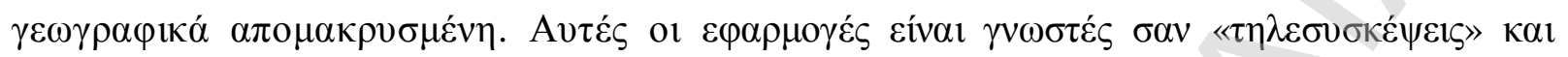

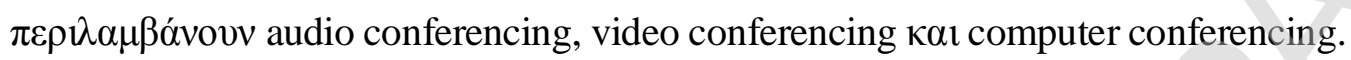

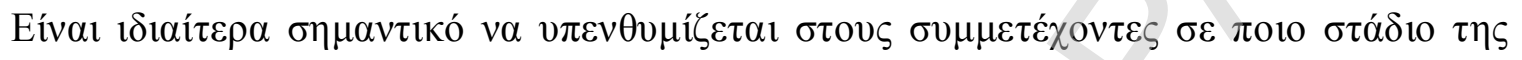

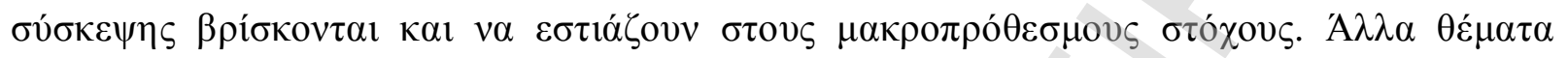

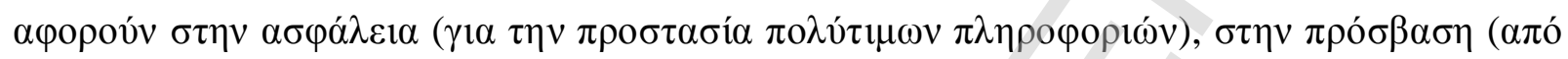

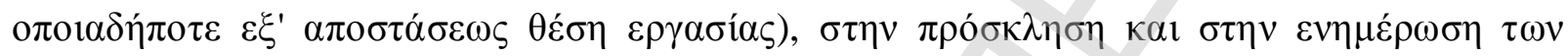

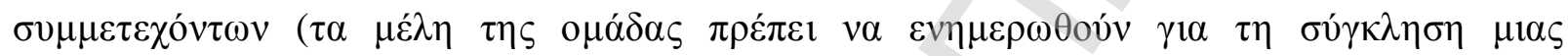

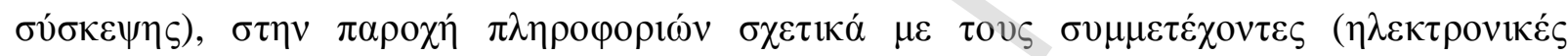

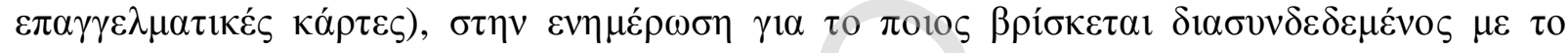

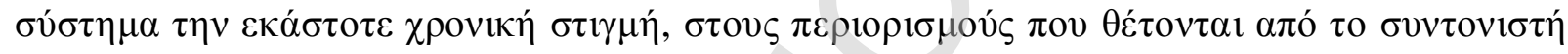

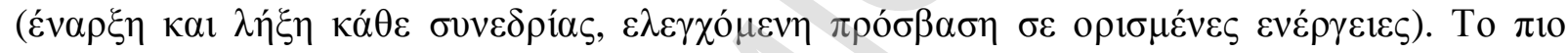

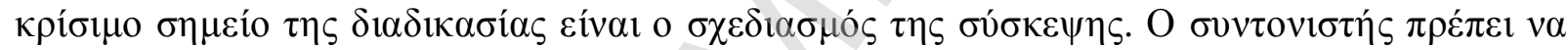

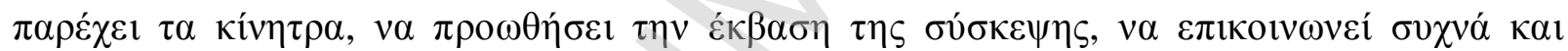

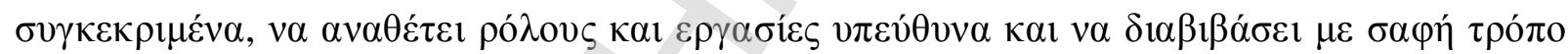

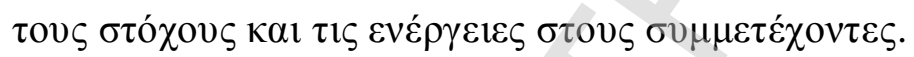

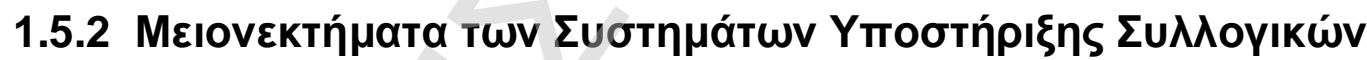

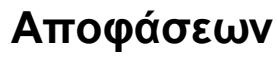

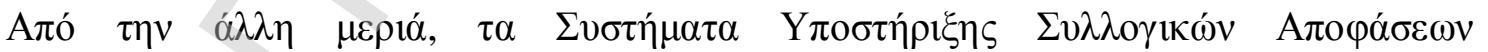

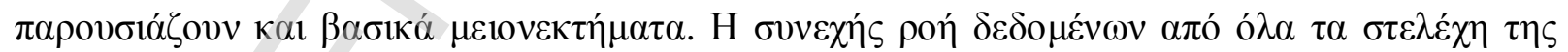

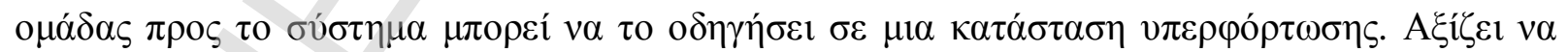

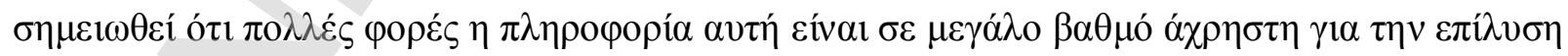

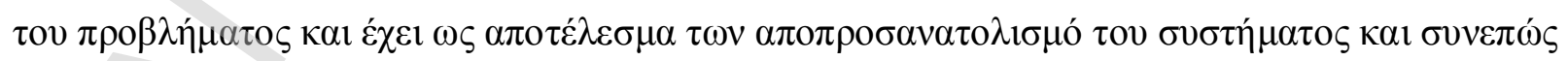

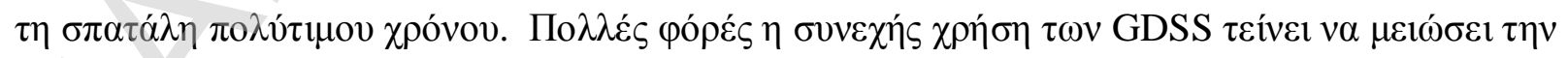

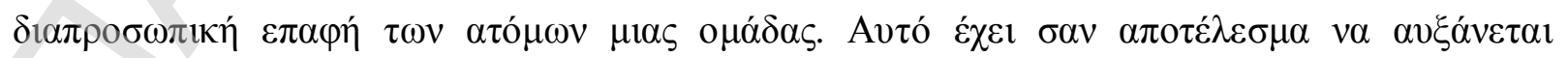

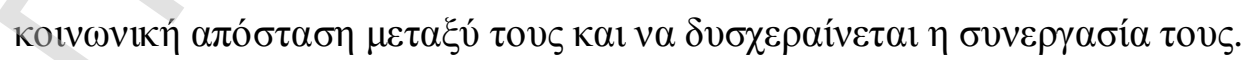

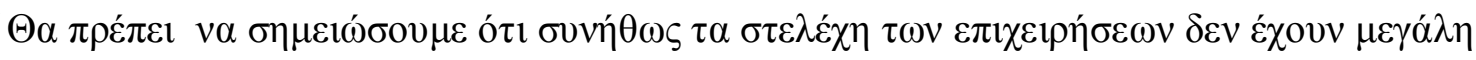

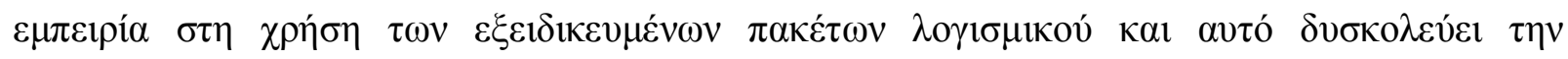




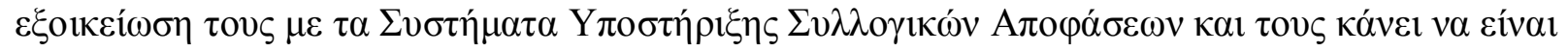

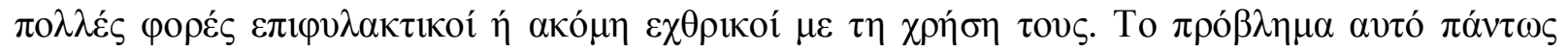

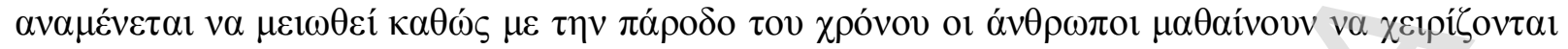

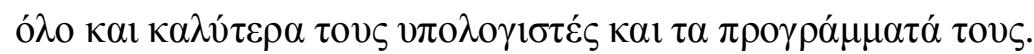

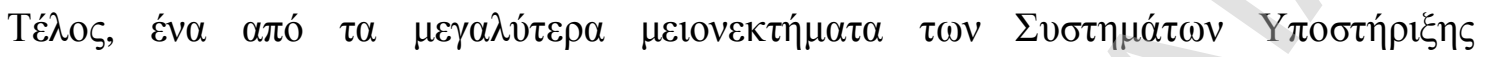

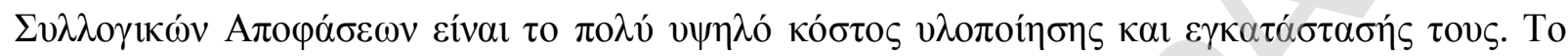

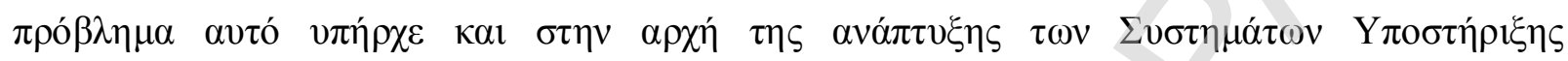

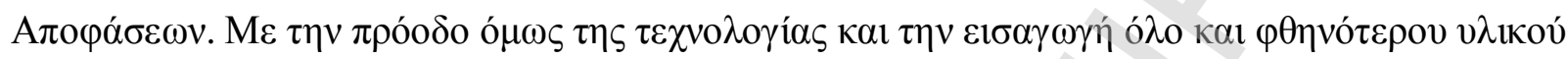

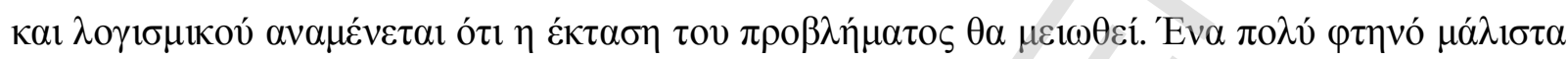

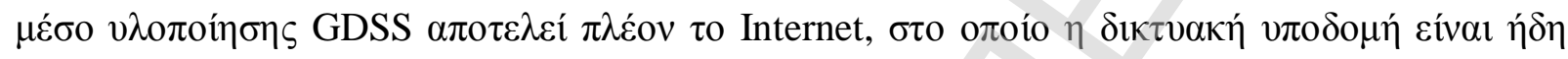

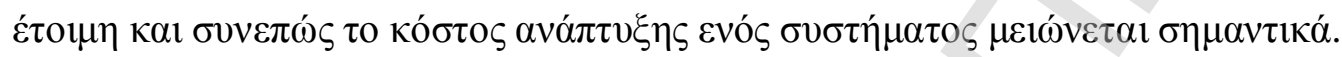

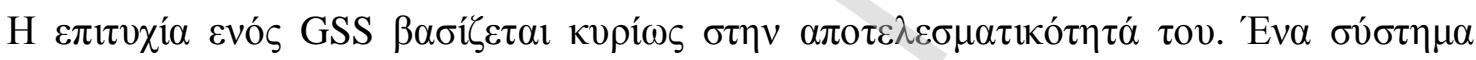

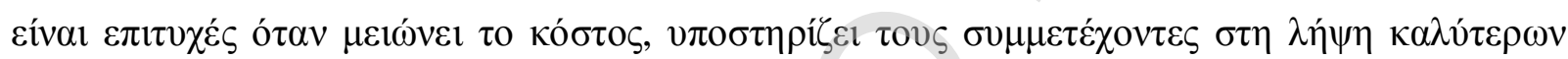

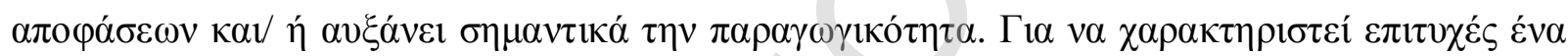

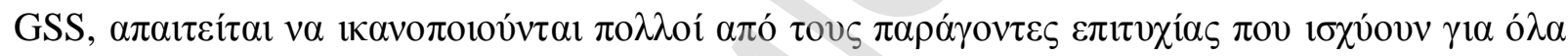

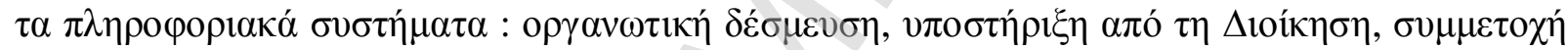

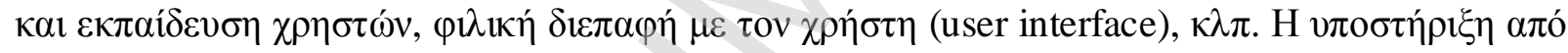

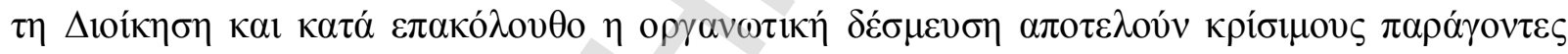

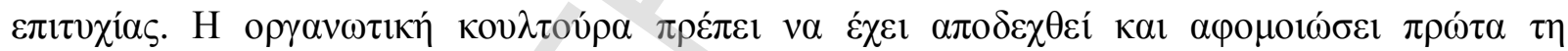

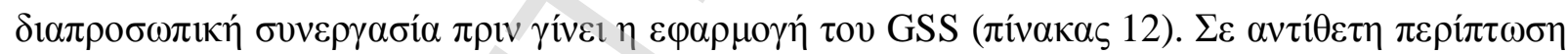

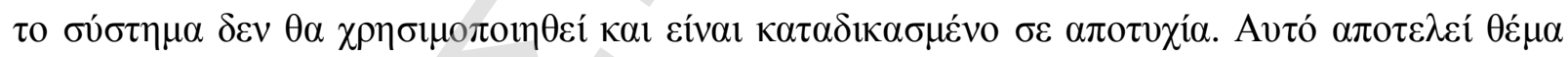

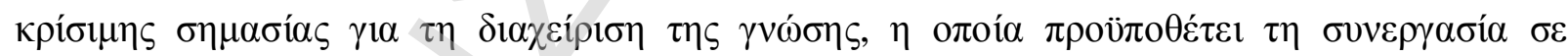

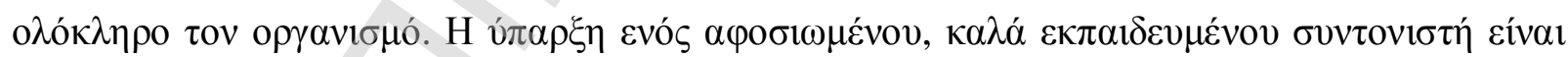

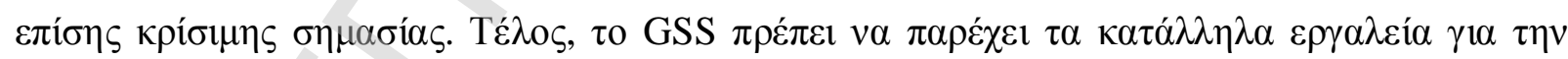

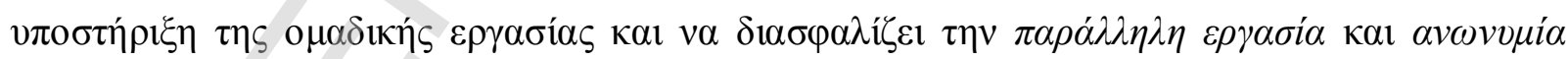

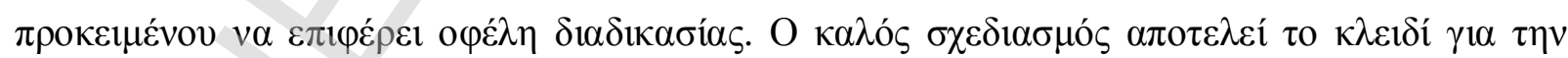

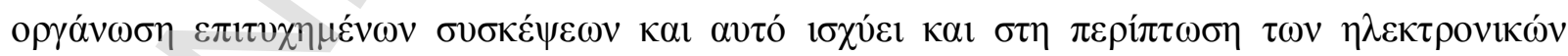

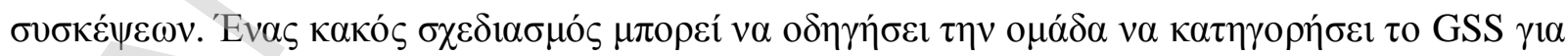

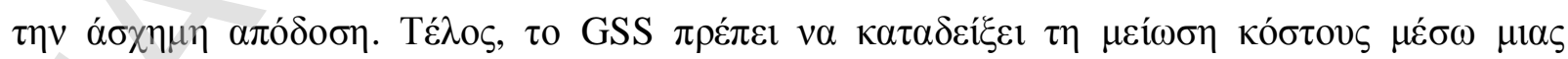

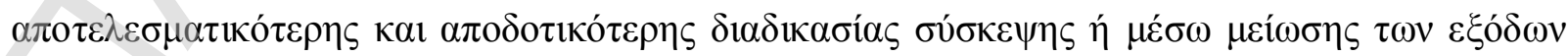
$\mu \varepsilon \tau \alpha \kappa i ́ v \eta \sigma \eta \zeta$. 


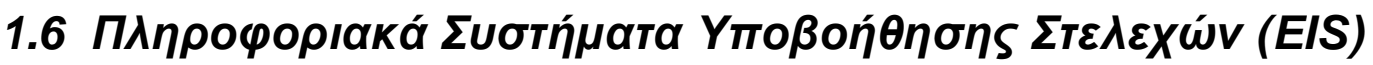

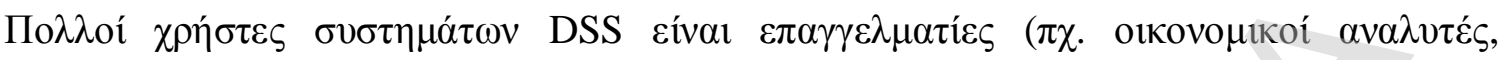

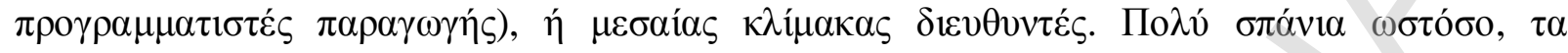

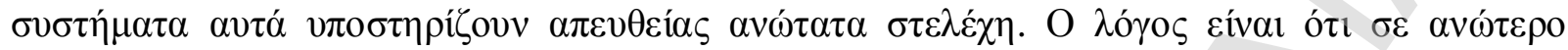

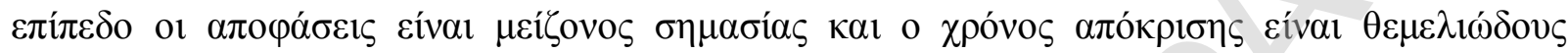

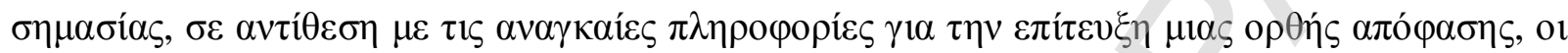

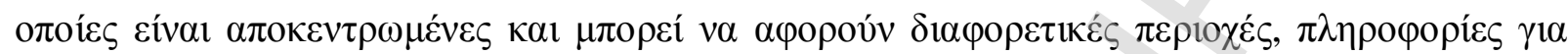

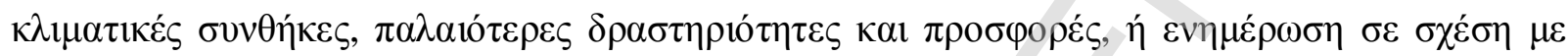

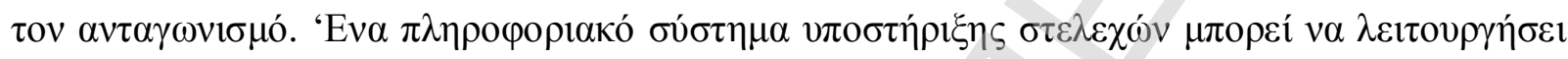

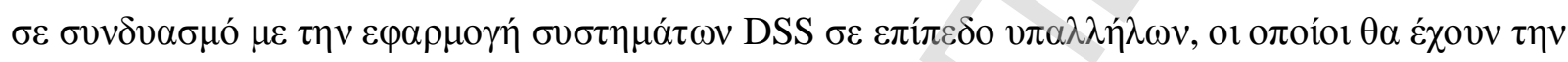

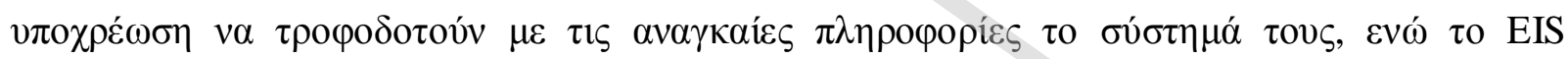

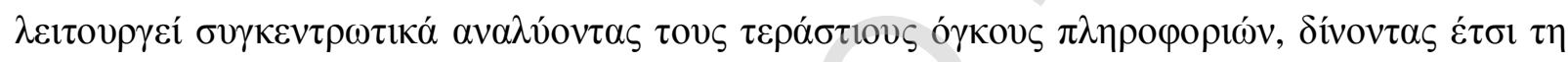

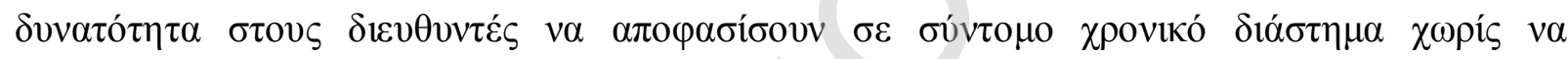

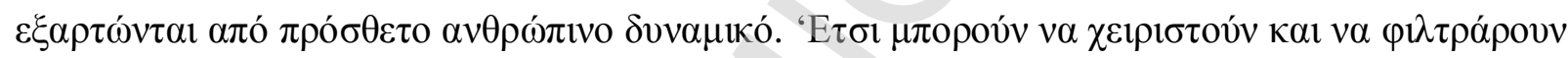

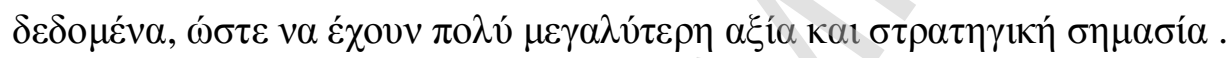

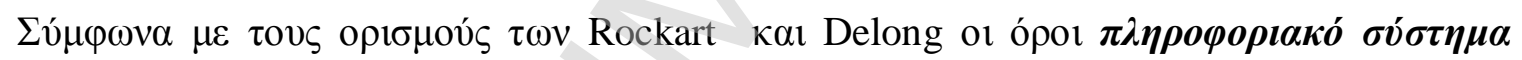

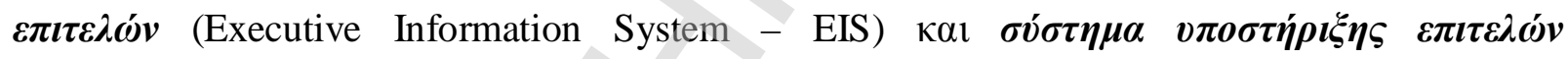

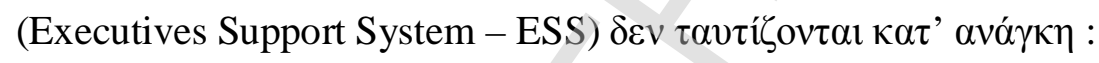

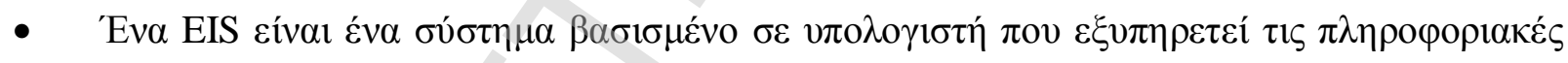

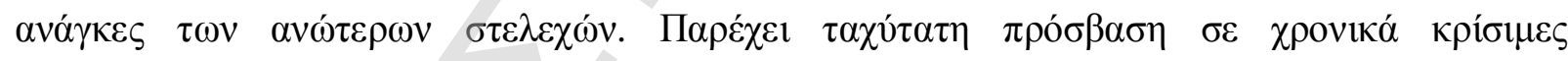

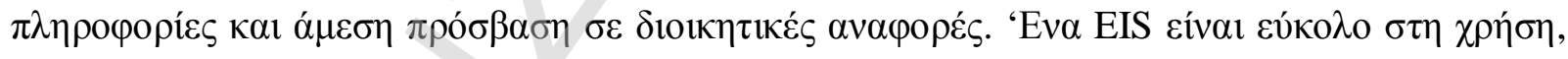

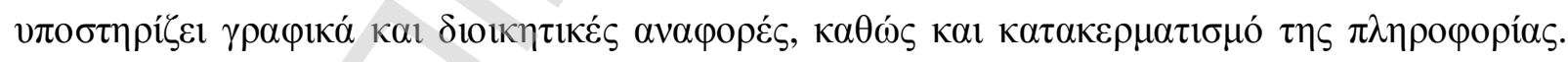

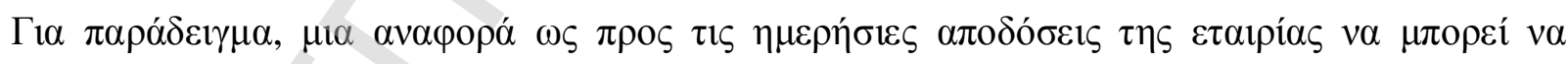

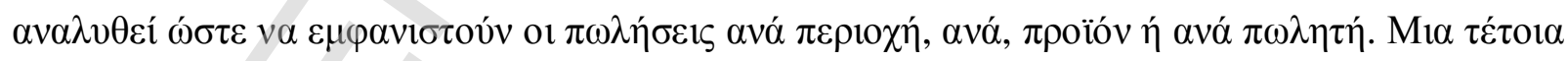

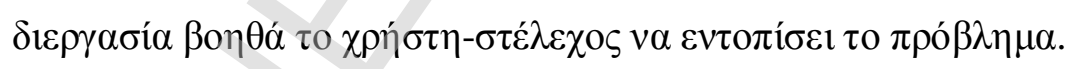

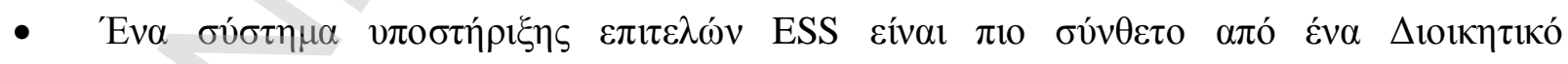

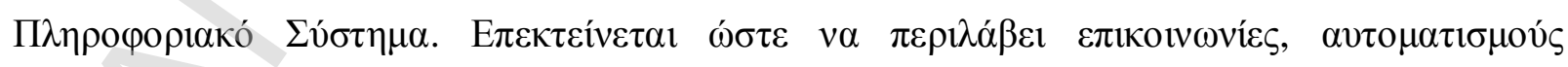

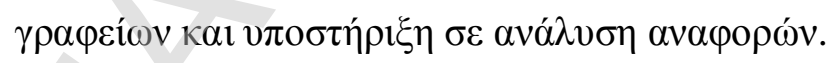

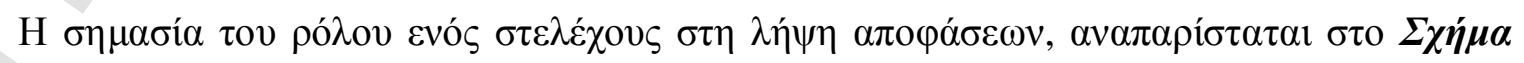
1.9 : 


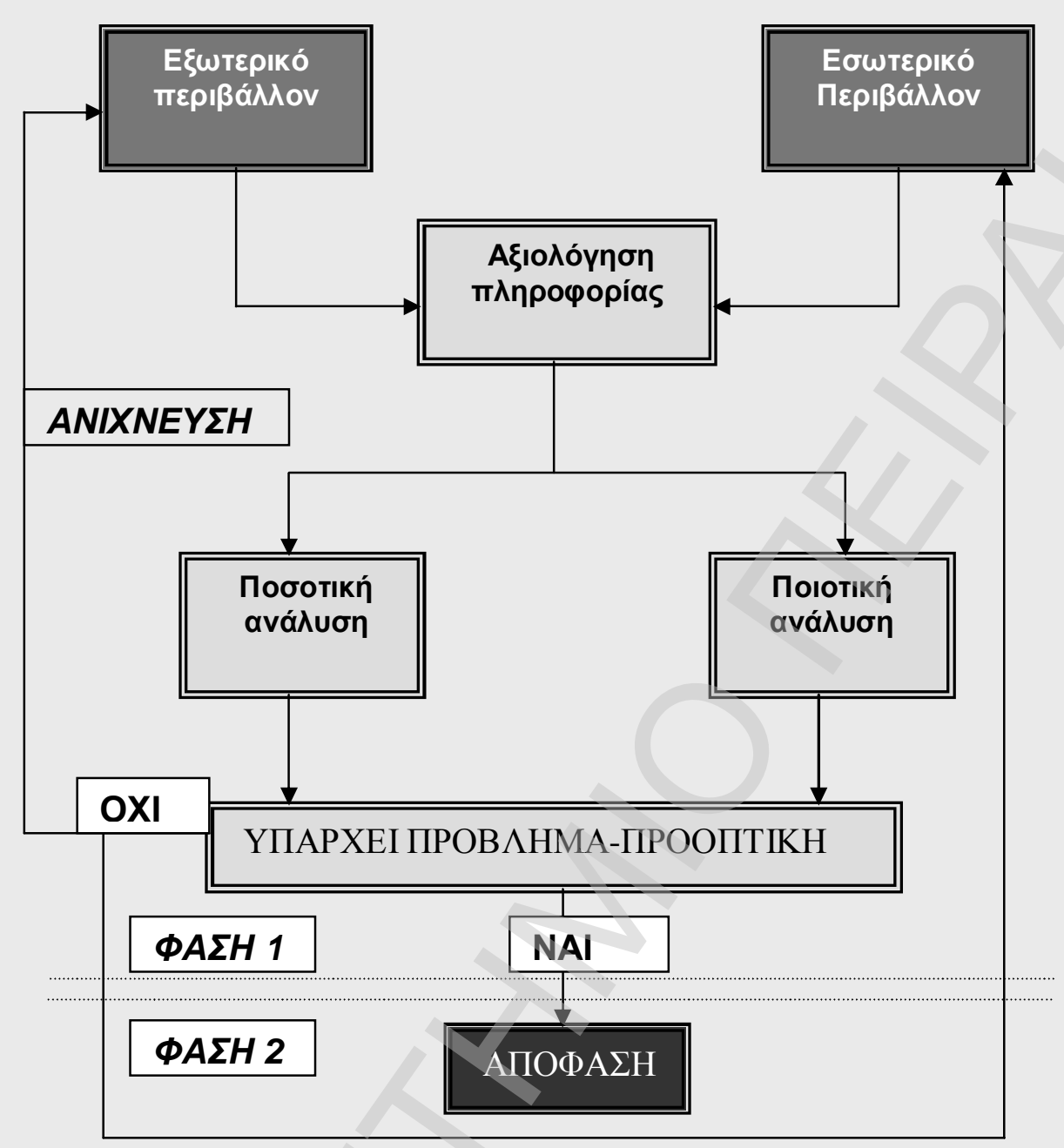

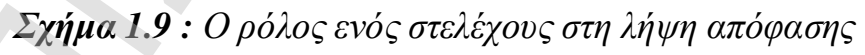

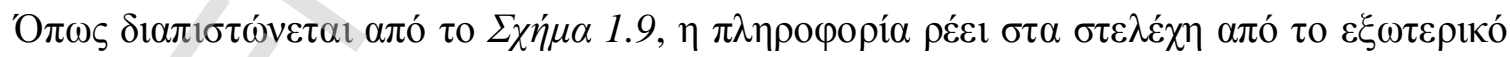

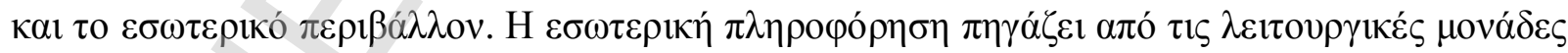

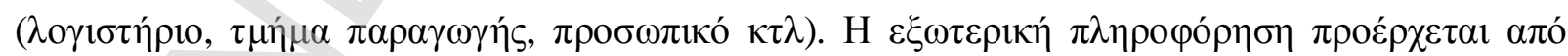

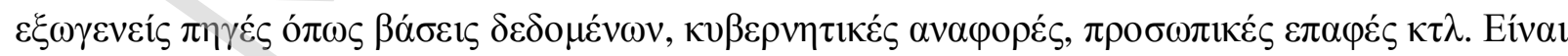

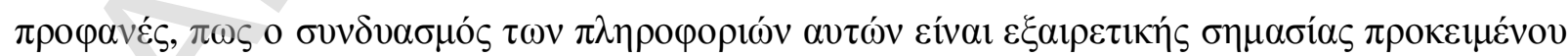

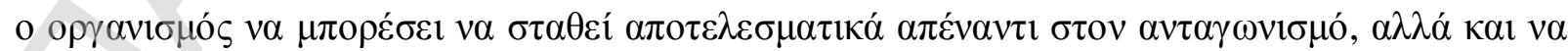

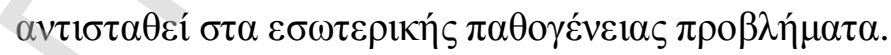




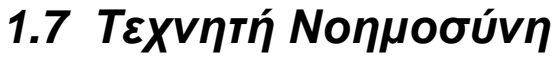

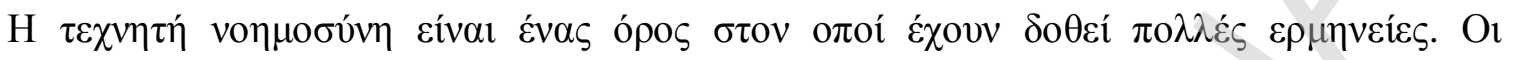

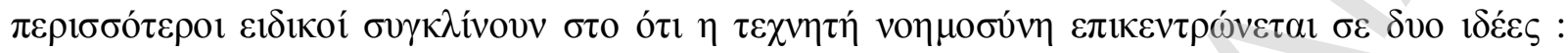

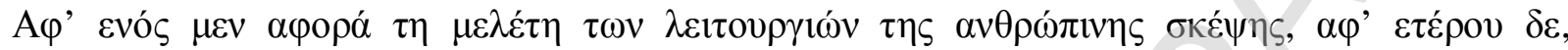

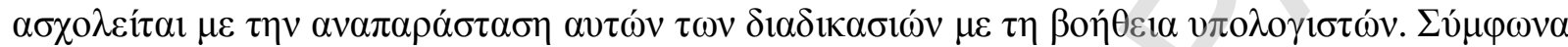

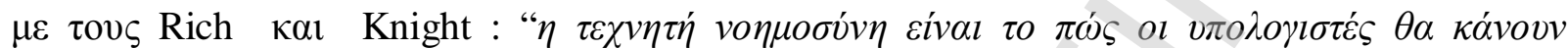

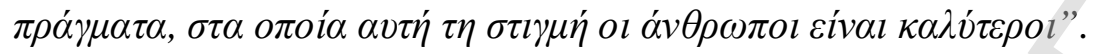

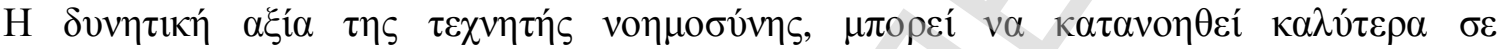

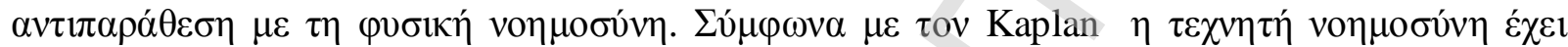

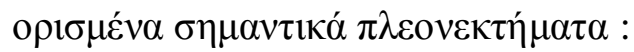

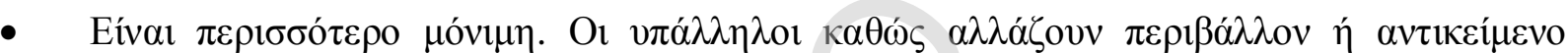

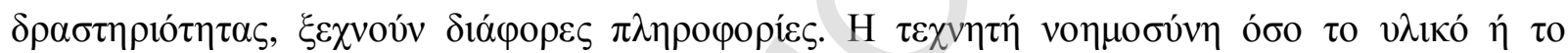

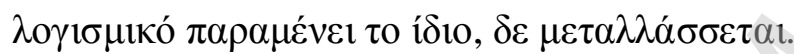

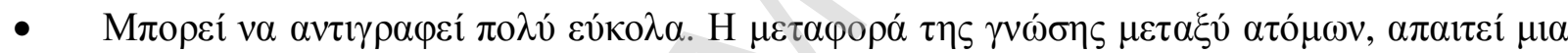

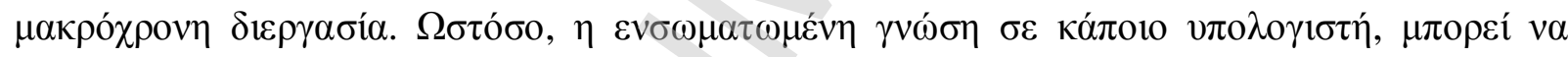

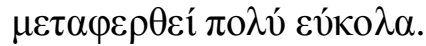

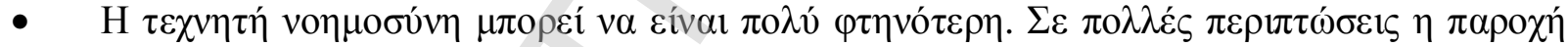

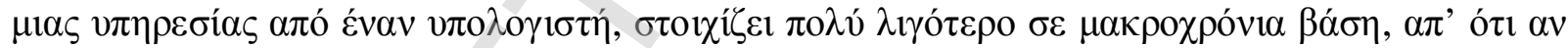

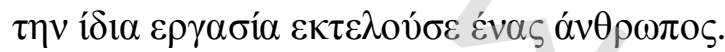

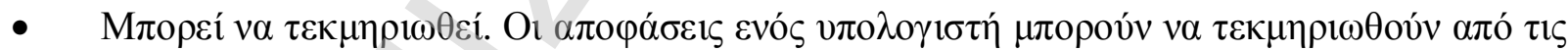

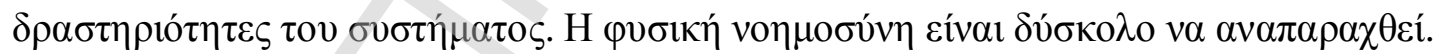

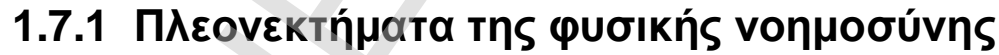

- $\quad$ H

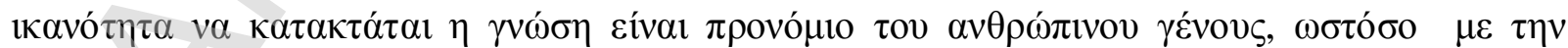

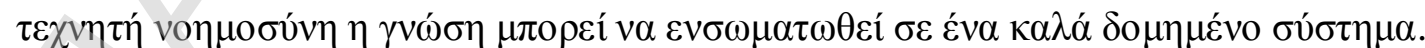

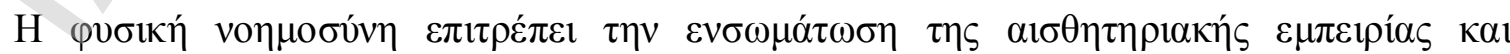

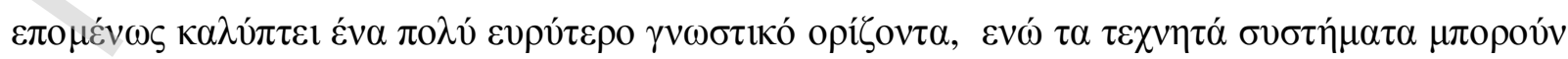

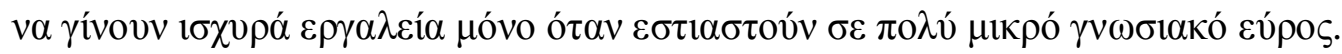




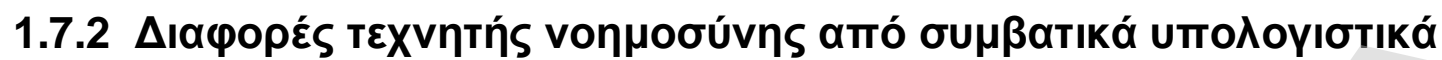

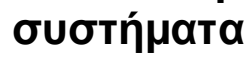

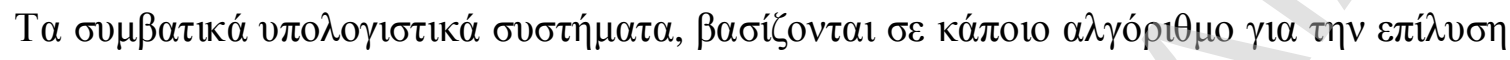

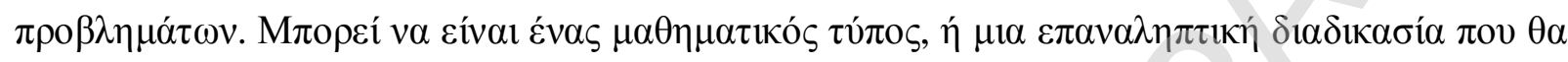

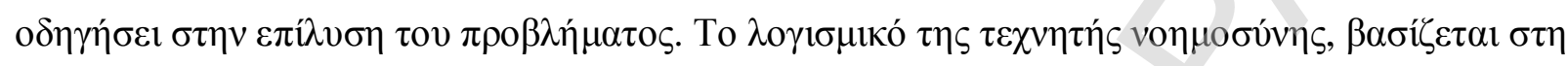

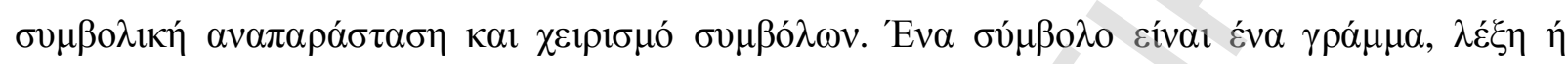

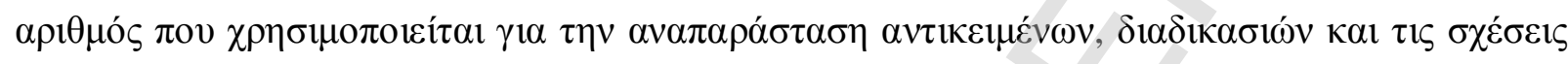

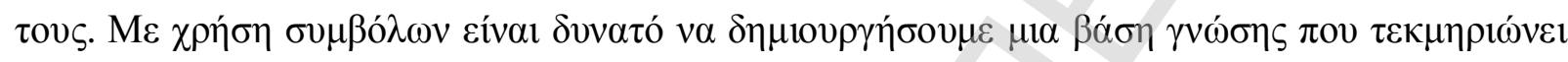

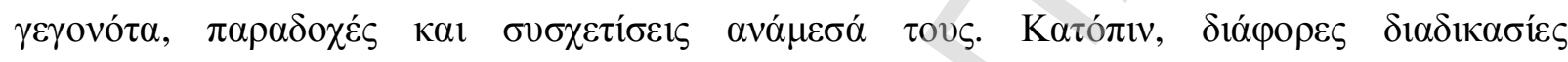

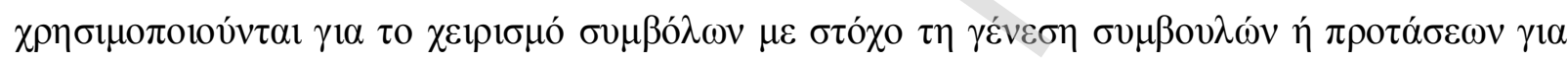
$\tau \eta v \varepsilon \pi \hat{\lambda} v \sigma \eta \pi \rho \circ \beta \lambda \eta \mu \alpha ́ \tau \omega v$.

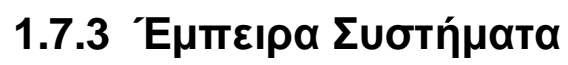

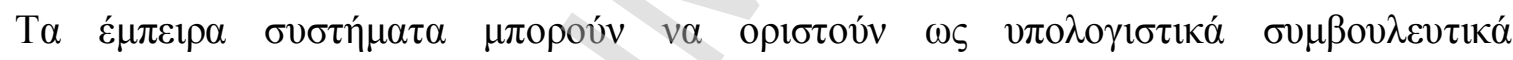

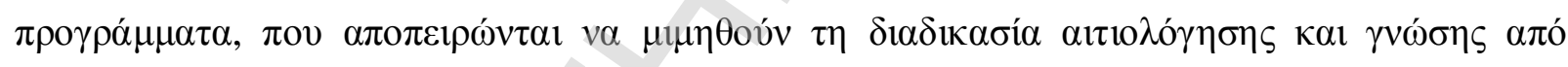

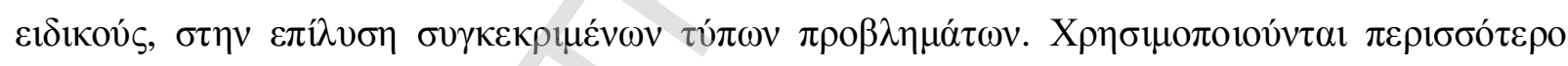

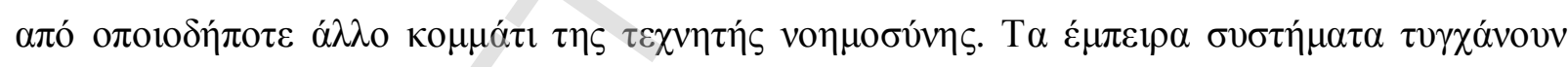

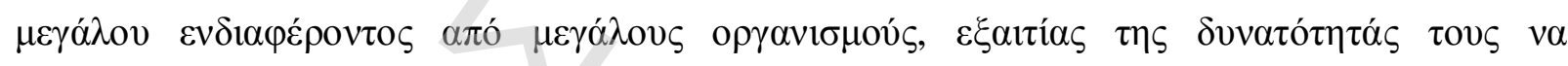

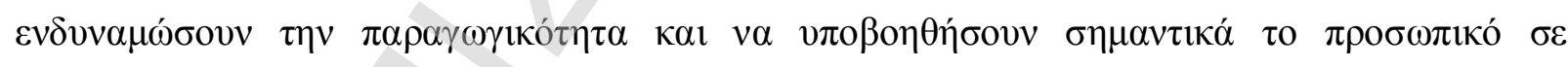

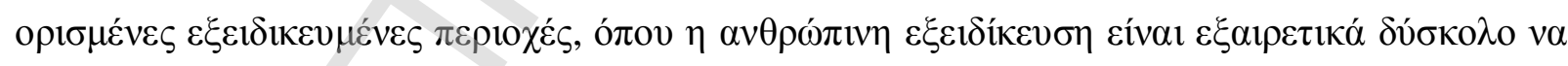

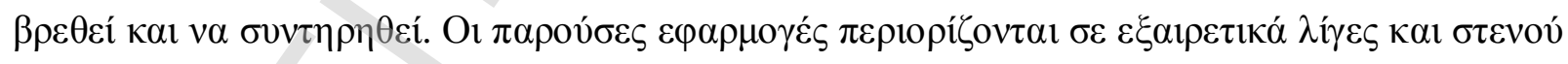

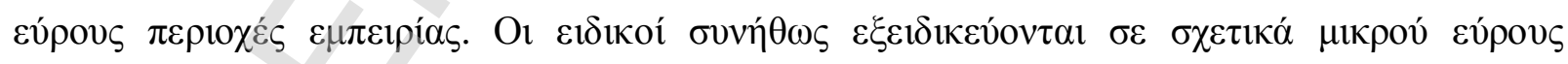

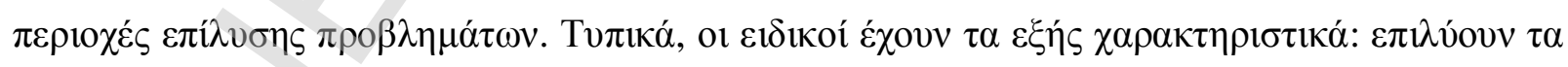

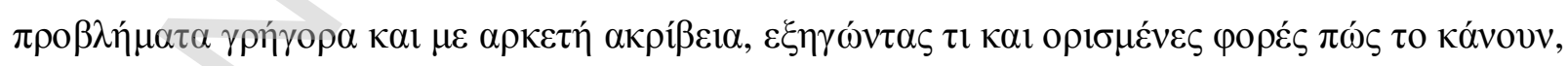

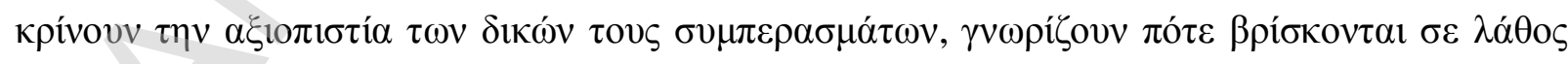

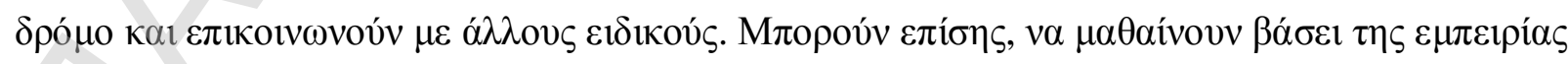

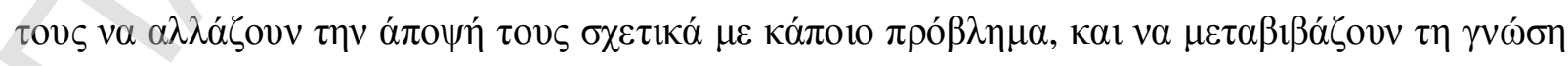

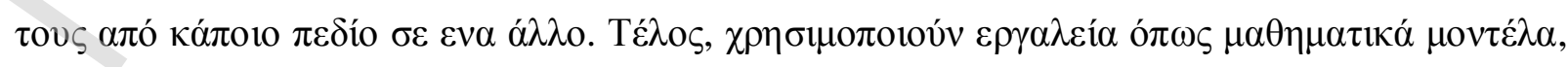

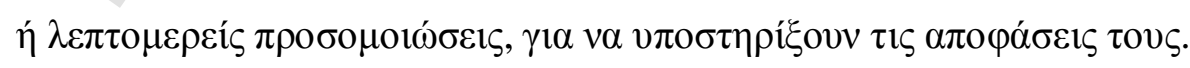




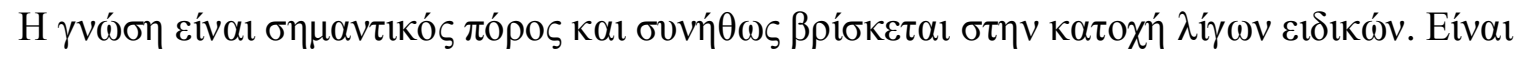

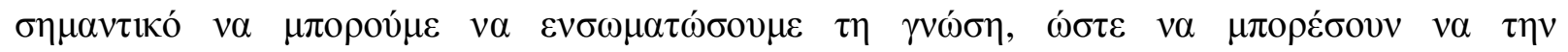

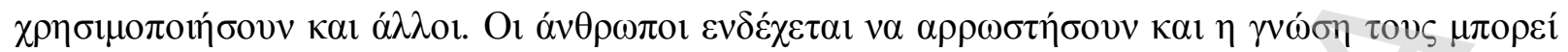

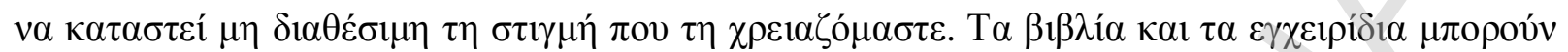

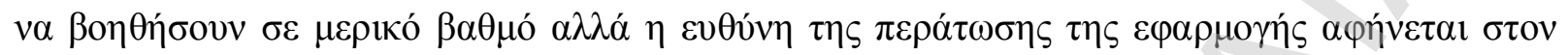

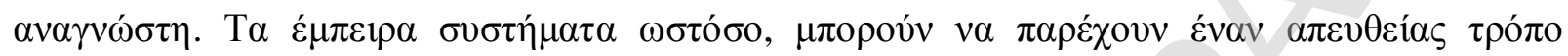

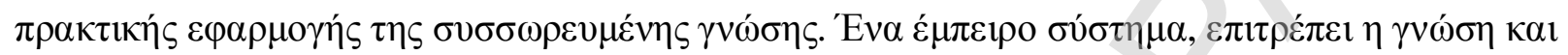

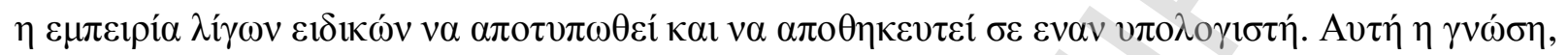

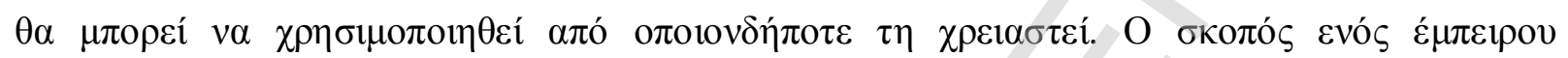

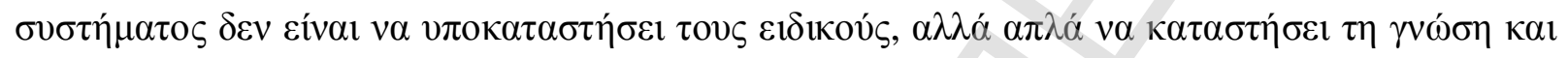

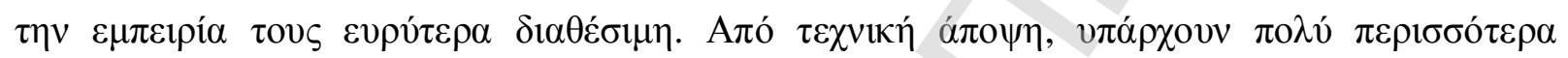

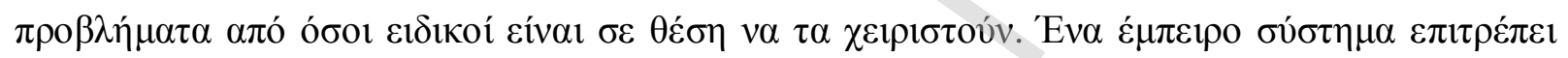

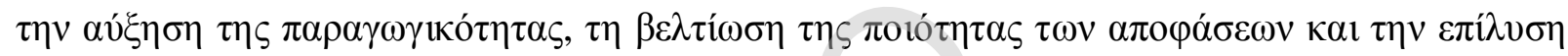

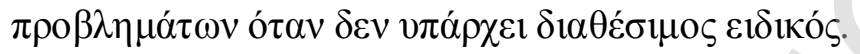

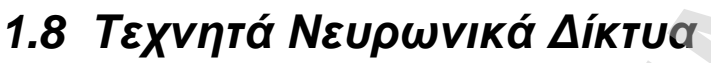

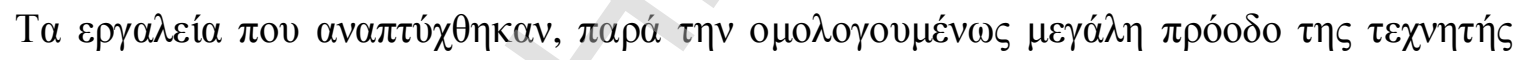

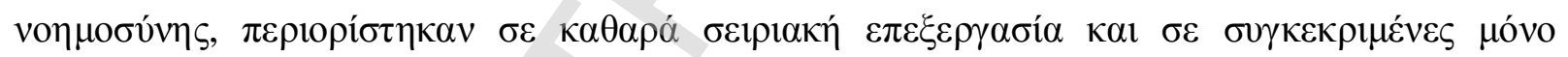

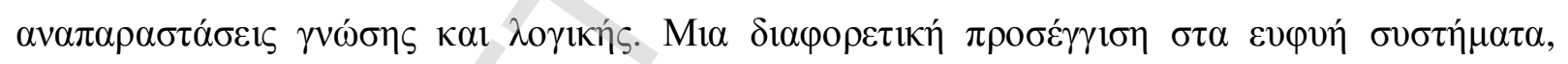

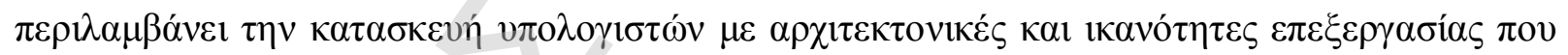

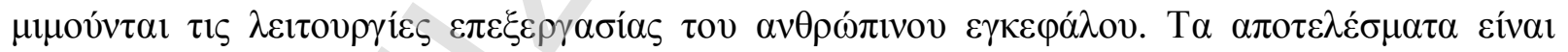

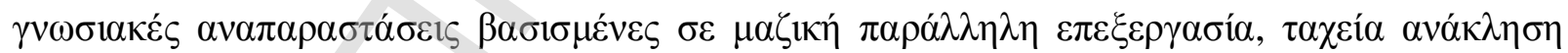

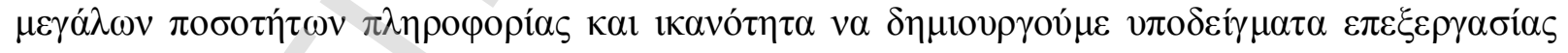

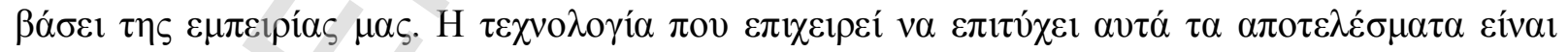

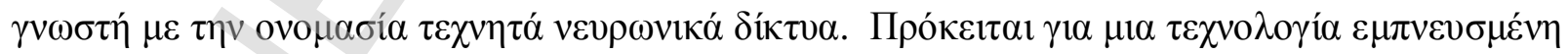

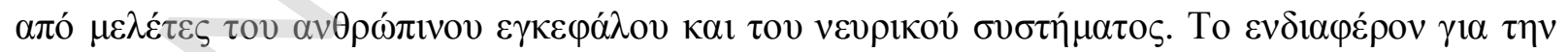

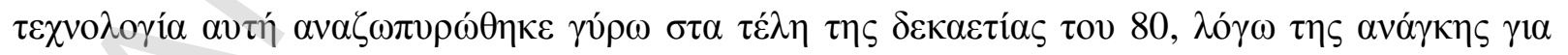

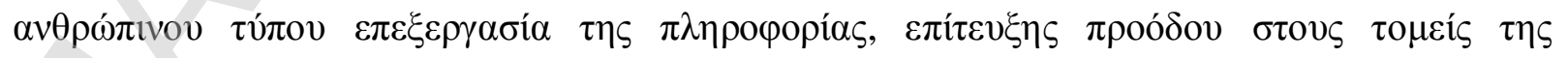

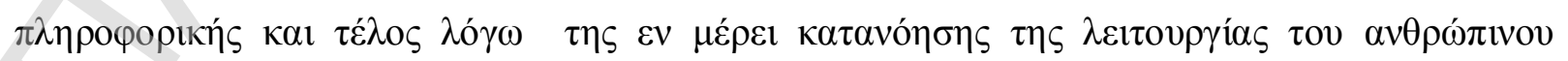

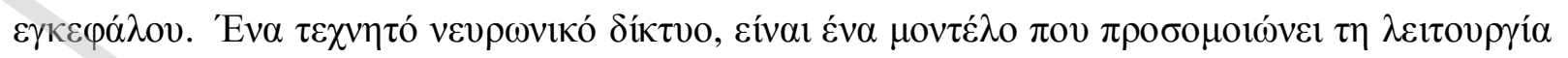

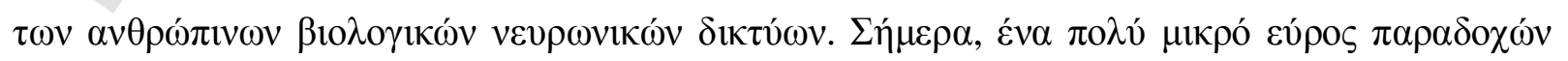




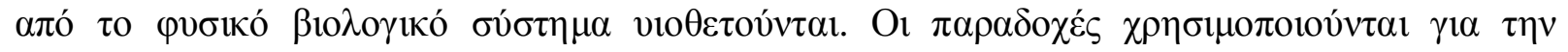

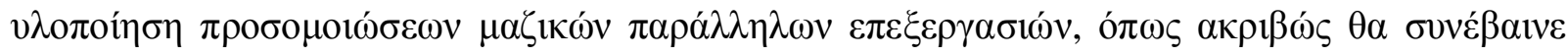

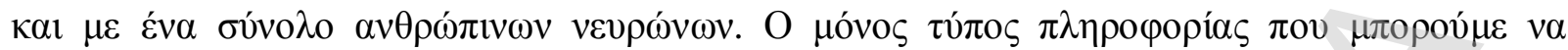

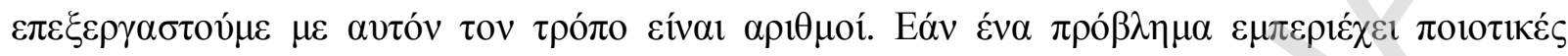

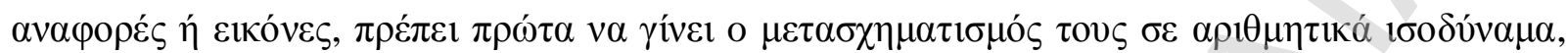

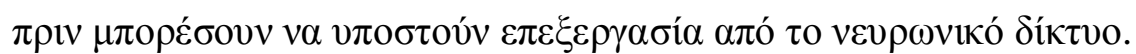

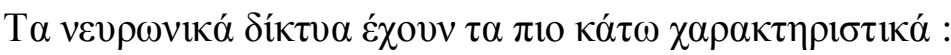

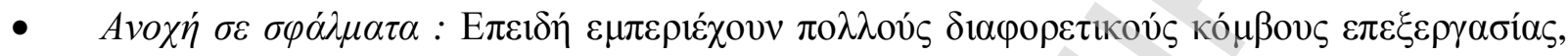

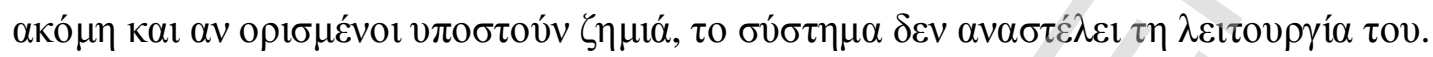

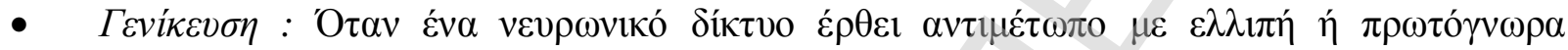

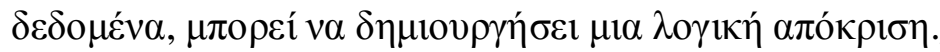

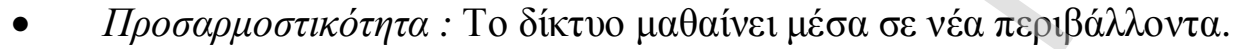

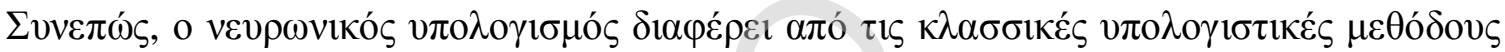

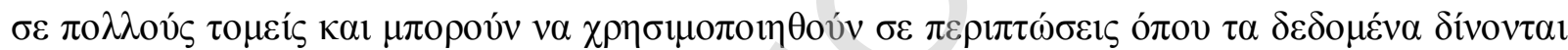

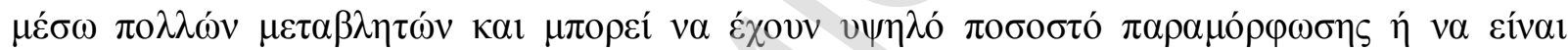

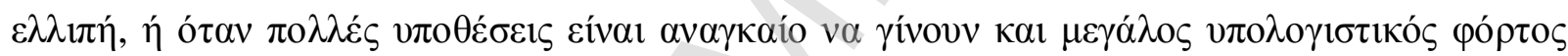
$\alpha \pi \alpha \imath \varepsilon i ́ \tau \alpha$.

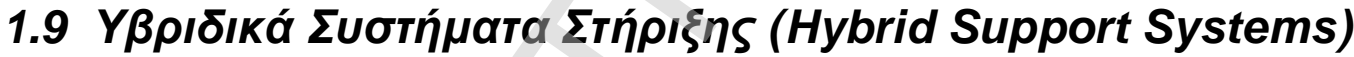

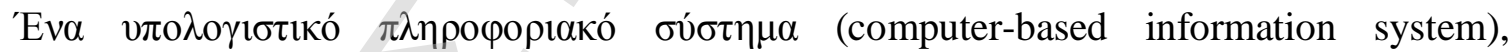

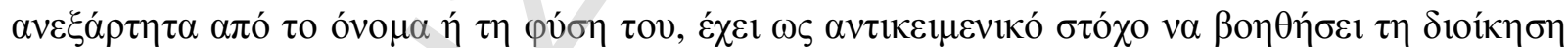

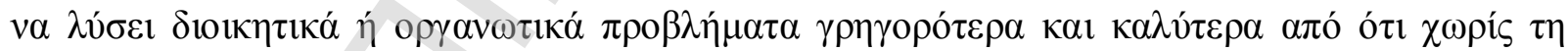

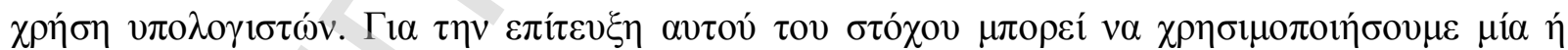

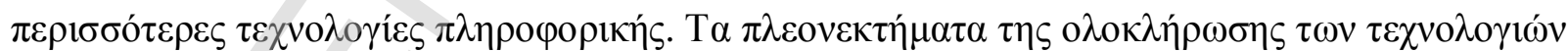
$\varepsilon \xi \varepsilon \tau \alpha ́ \sigma \tau \eta \kappa \alpha \nu$ a

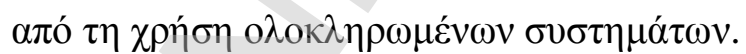

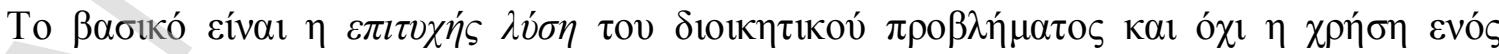

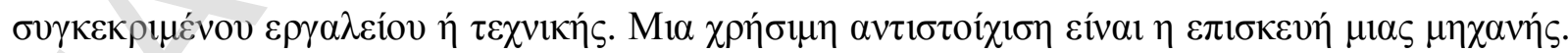

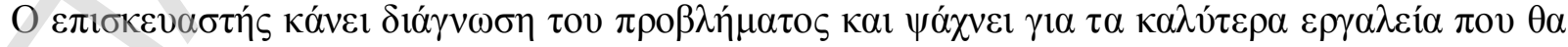

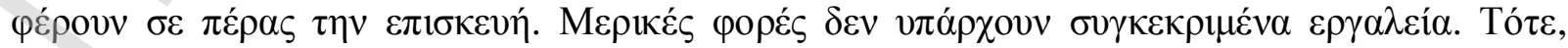

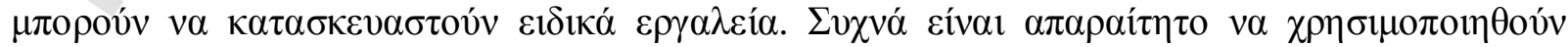

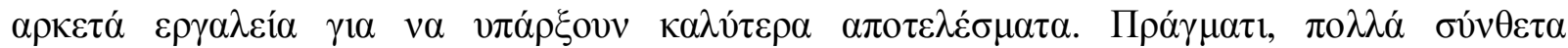




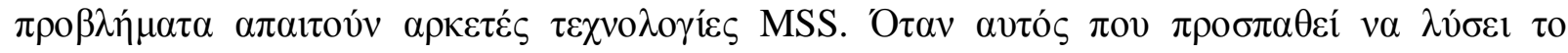

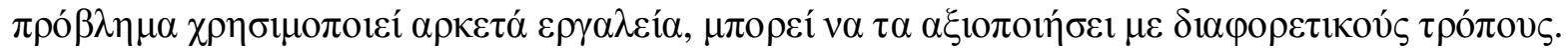

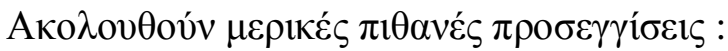

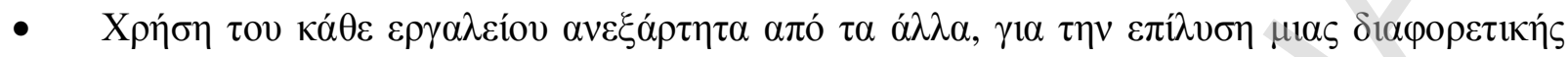
$\pi \lambda \varepsilon v \rho \alpha ́ \varsigma \tau$

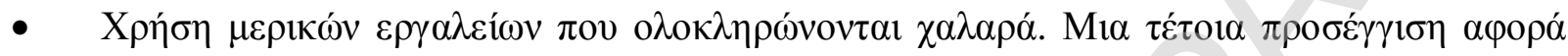

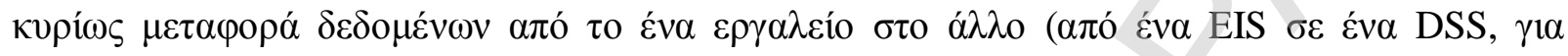
$\pi \alpha \rho \alpha ́ \delta \varepsilon \imath \gamma \mu \alpha) \gamma 1 \alpha \pi \varepsilon \rho \alpha \imath \varepsilon \dot{\varepsilon} \rho \omega \varepsilon \pi \varepsilon \xi \varepsilon \rho \gamma \alpha \sigma i ́ \alpha$.

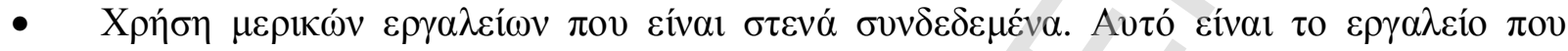

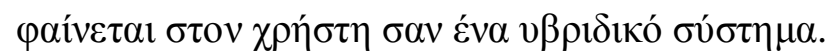

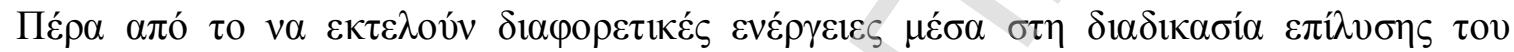

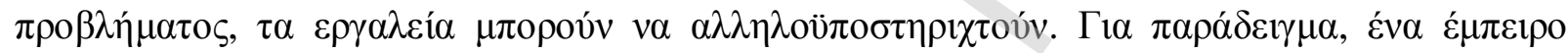

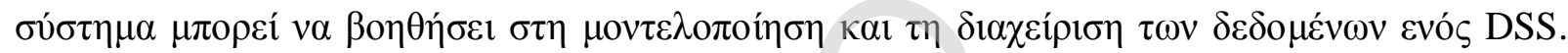

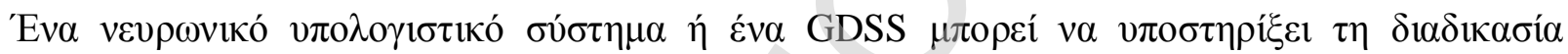

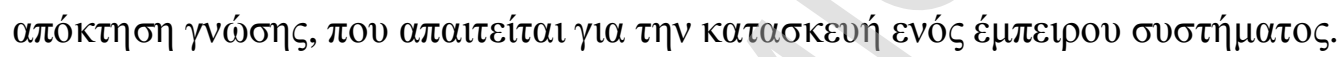

\subsection{TEXVoגoyíss kaı MSS}

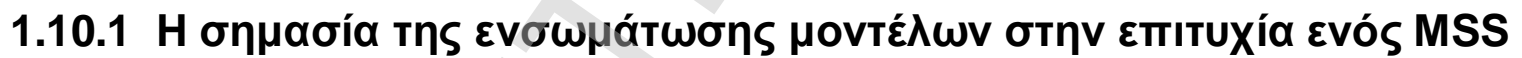

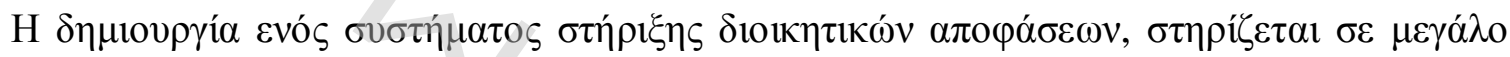

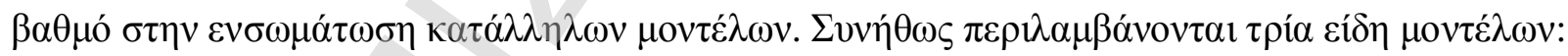

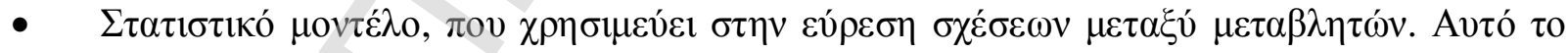

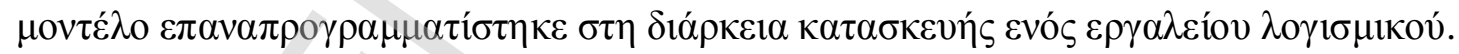

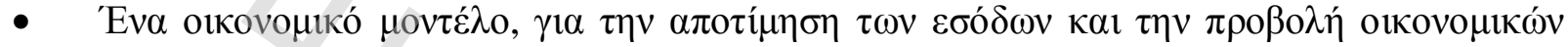

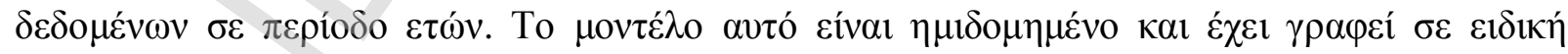
$\gamma \lambda \omega ́ \sigma \sigma \alpha$.

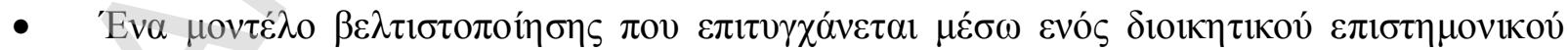

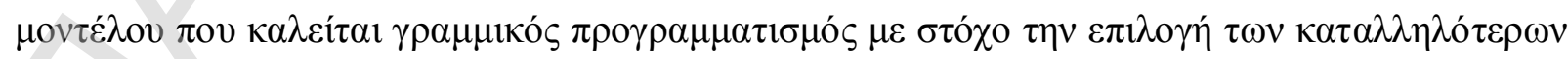
$\mu \varepsilon ́ \sigma \omega v$.

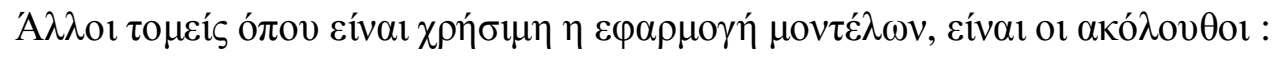




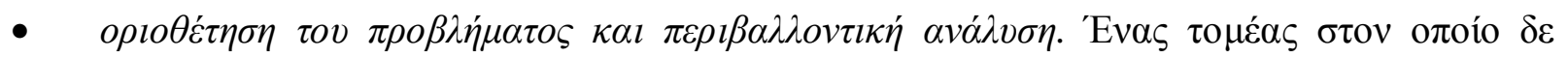

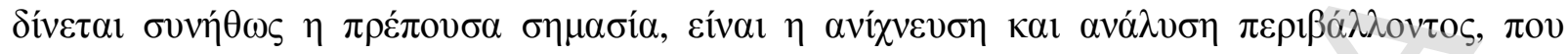

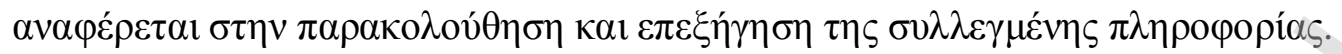

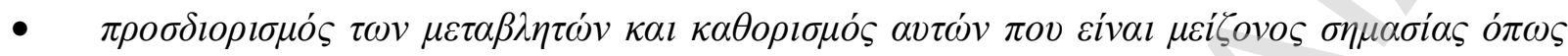
$\kappa \alpha l \tau \omega v \sigma \chi \varepsilon \dot{\sigma} \sigma \varepsilon \omega v \mu \varepsilon \tau \alpha \xi \dot{v} \tau o v \varsigma$.

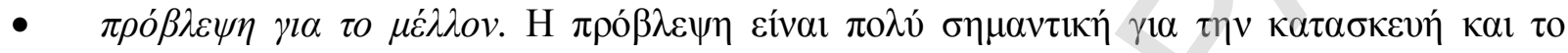

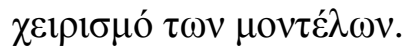

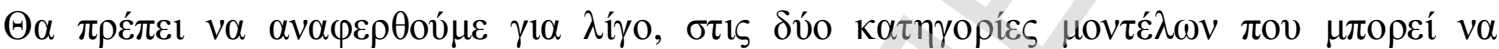

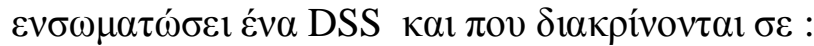

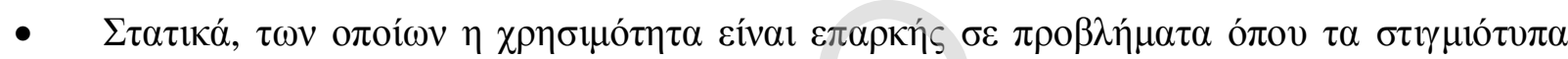

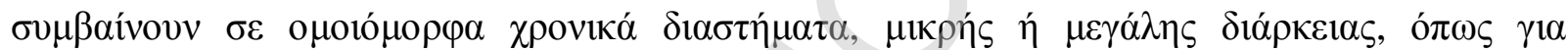

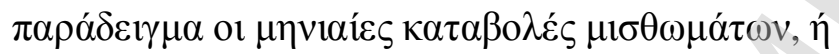

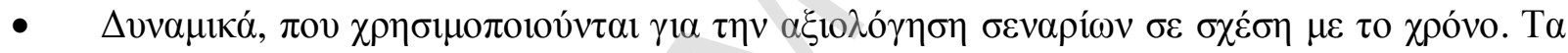

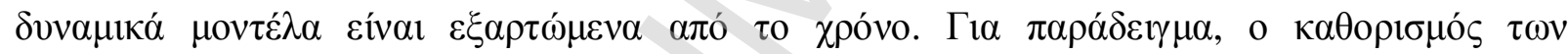

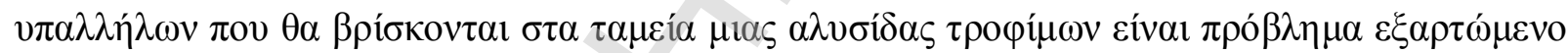

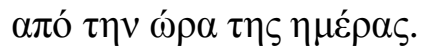

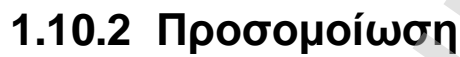

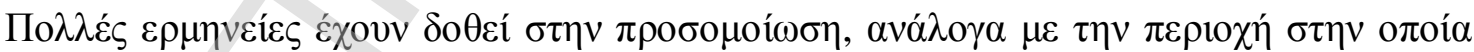

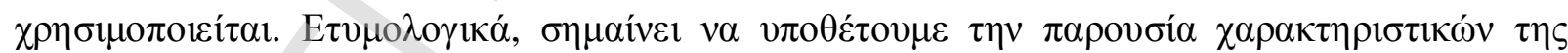

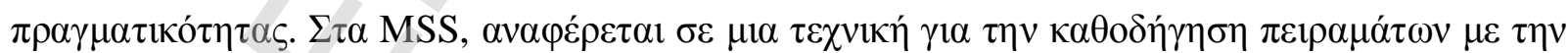

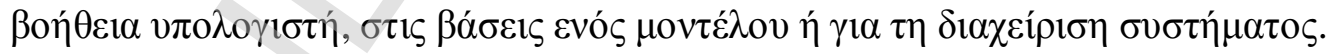

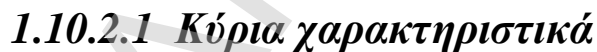

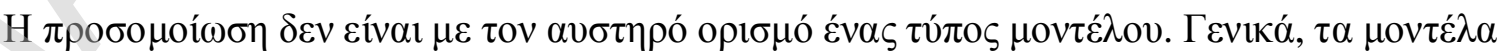

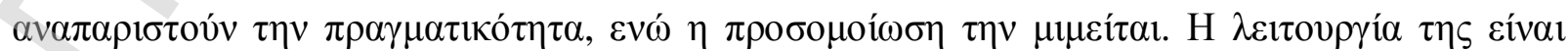

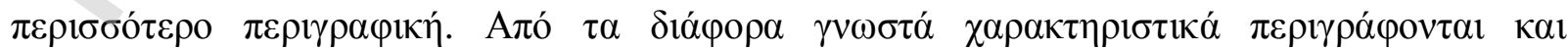

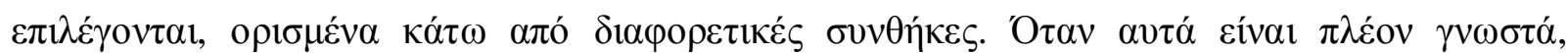




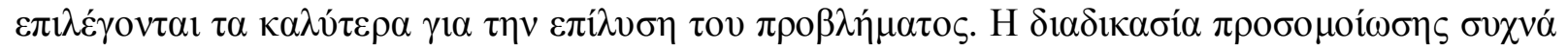

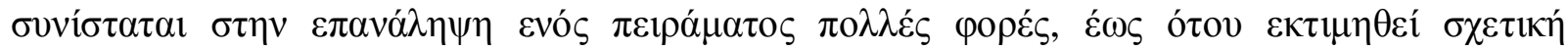

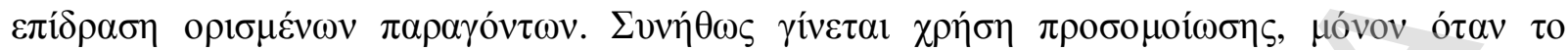

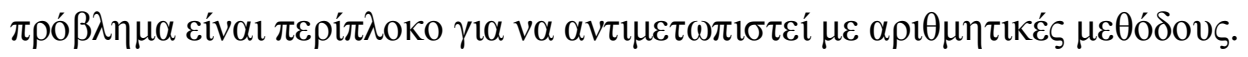

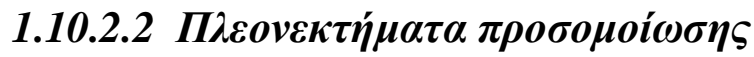

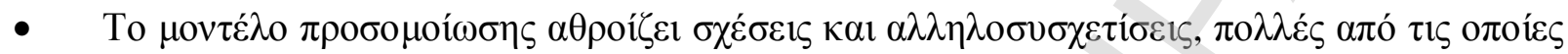

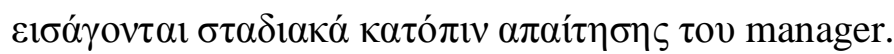

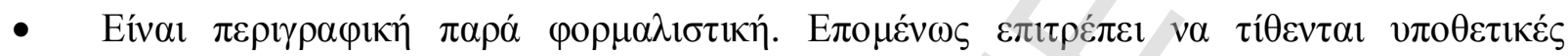

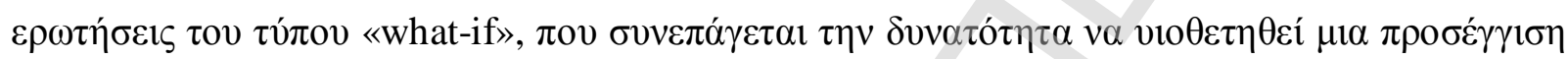

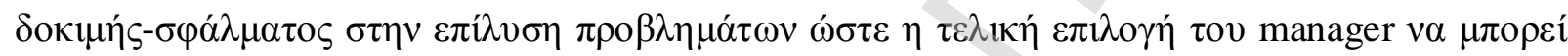

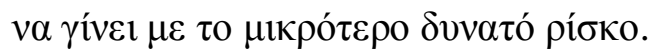

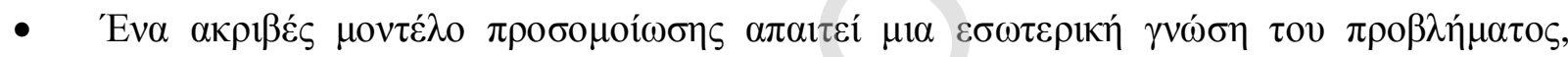

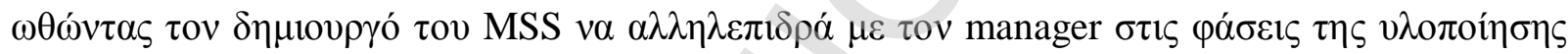

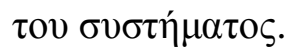

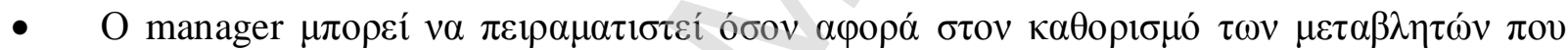

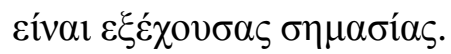

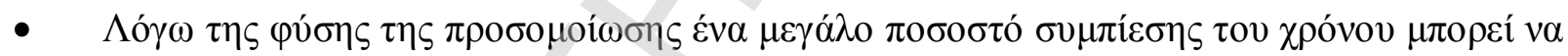

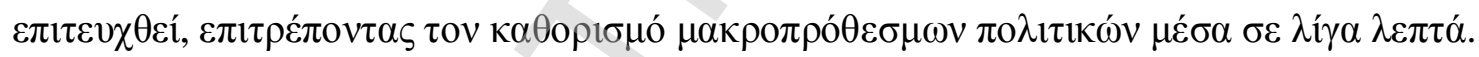

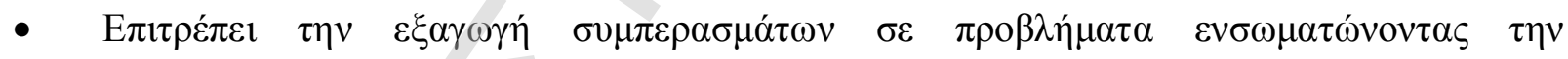

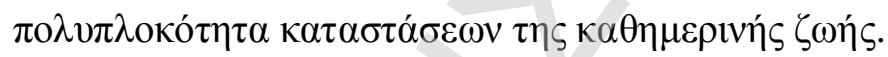

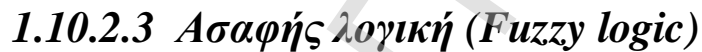

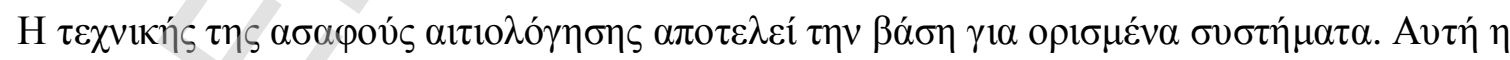

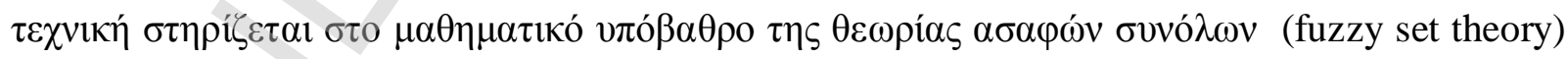

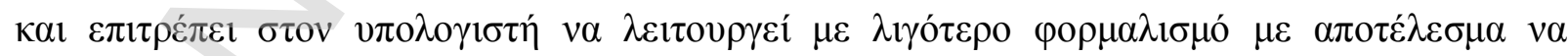

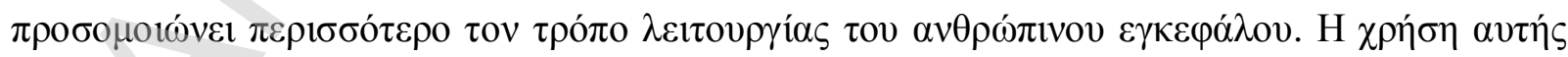

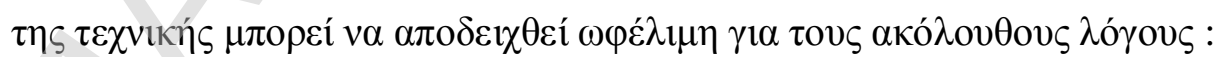

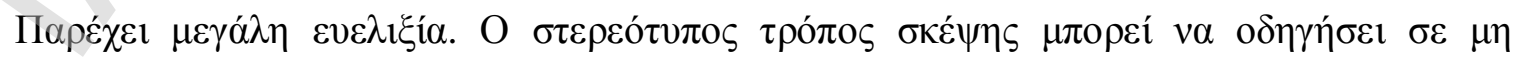

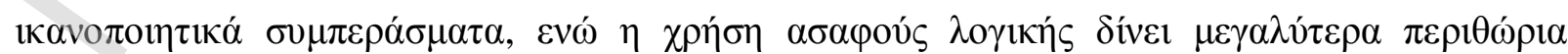

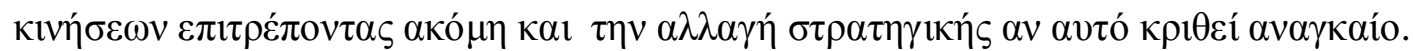




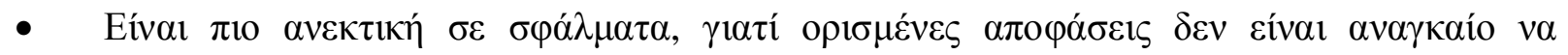

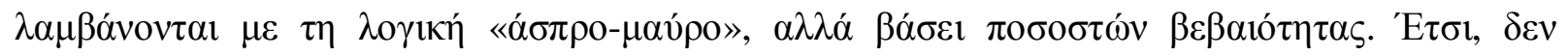

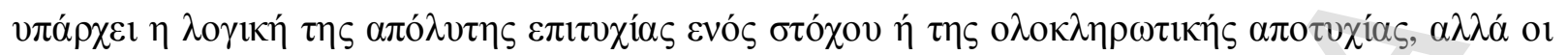

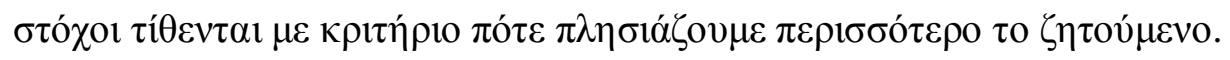

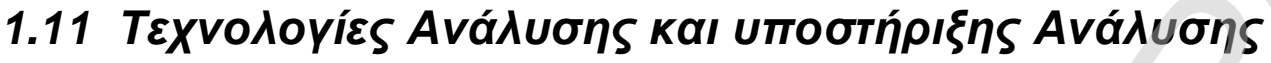

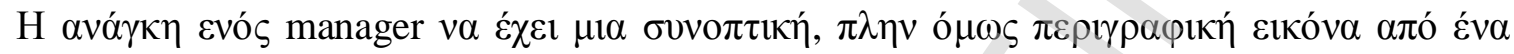

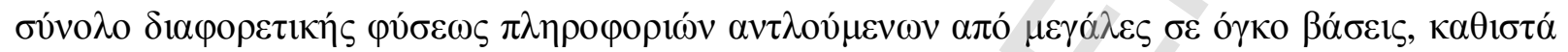

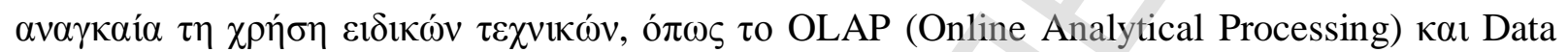
Mining.

\subsubsection{Online Analytical Processing (OLAP)}

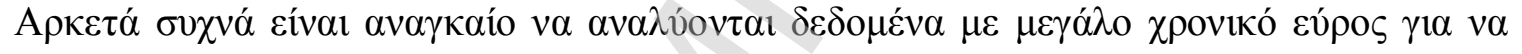

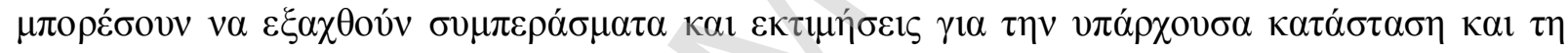

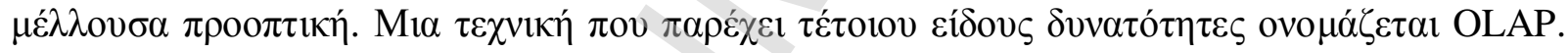

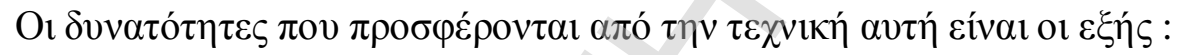

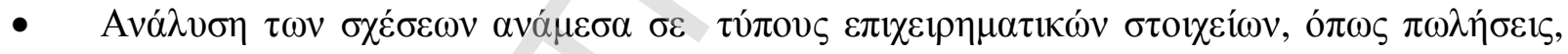

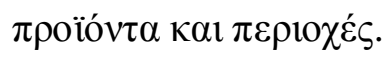

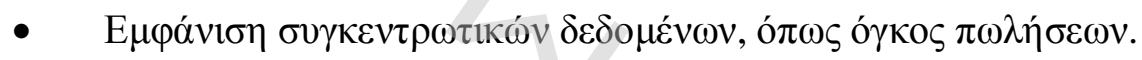

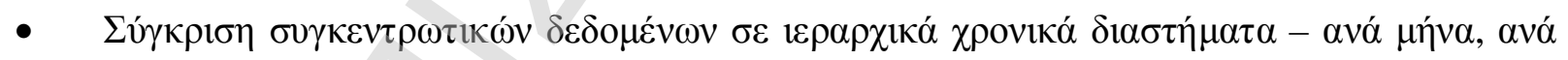

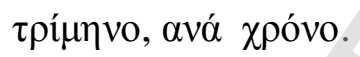

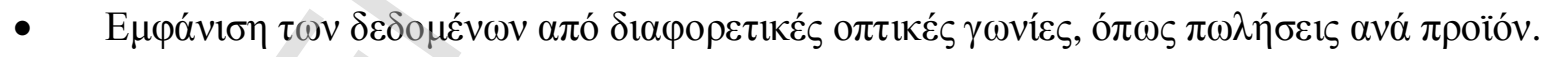

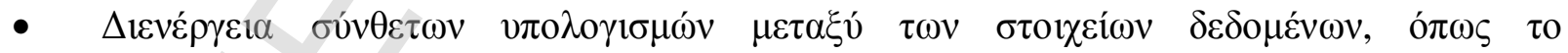

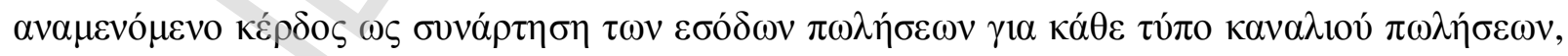
$\sigma \varepsilon \mu 1 \alpha \sigma v \gamma \kappa \varepsilon \kappa \rho \mu \varepsilon \dot{v \eta \eta} \pi \varepsilon \rho 10 \chi \eta ́$. 


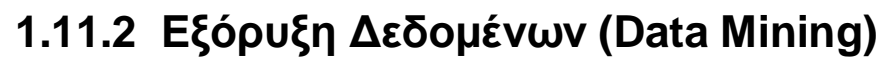

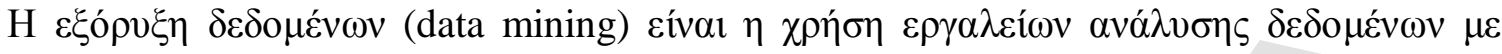

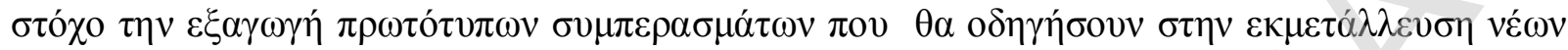

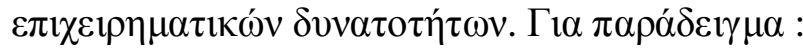

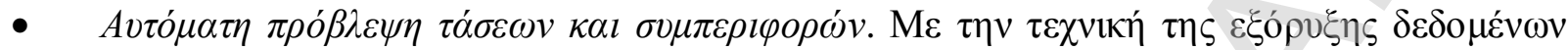

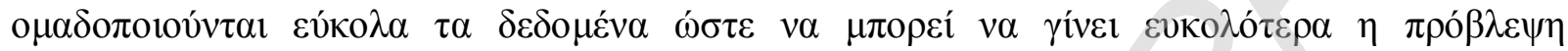

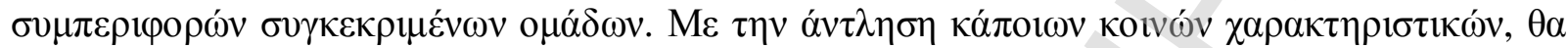

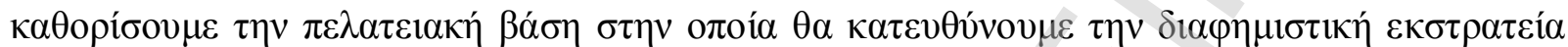

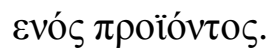

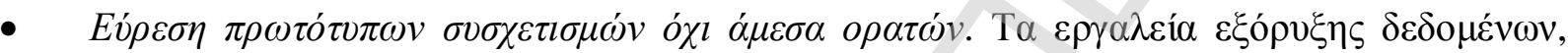

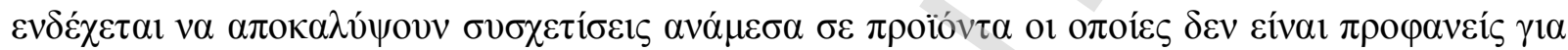

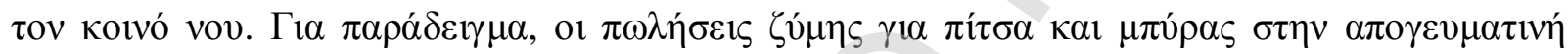

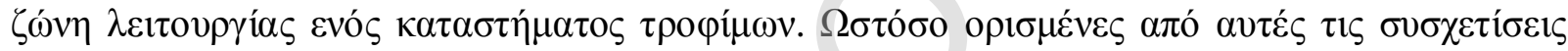

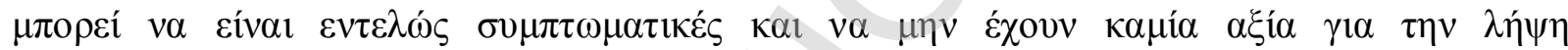

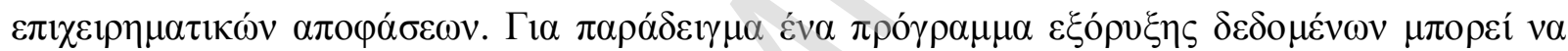

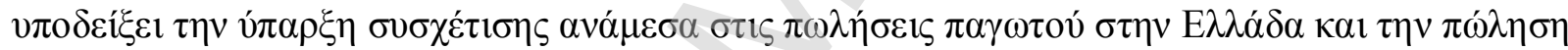


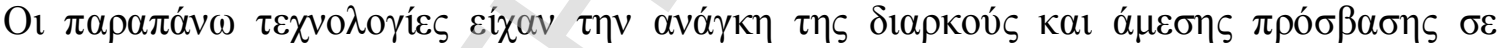

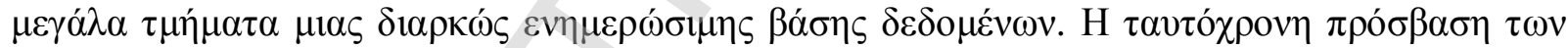

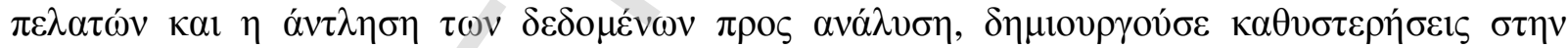

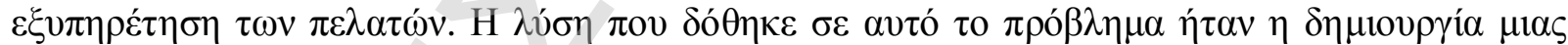

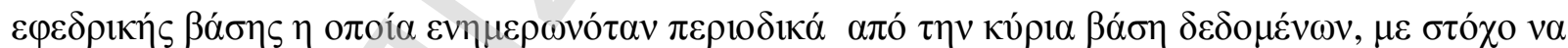

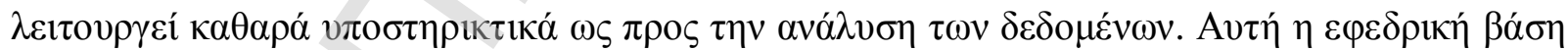

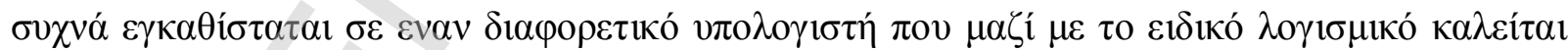
$\alpha \pi \circ \theta \dot{\eta} \kappa \eta \delta \varepsilon \delta o \mu \varepsilon ́ v \omega v$ (data warehouse). 


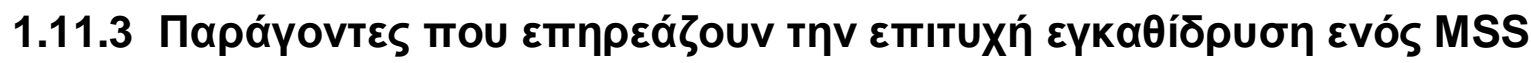

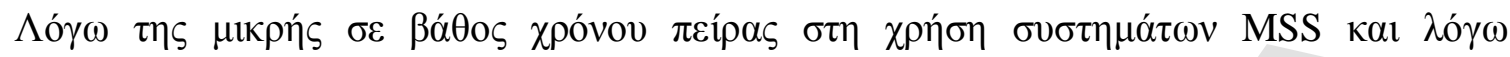

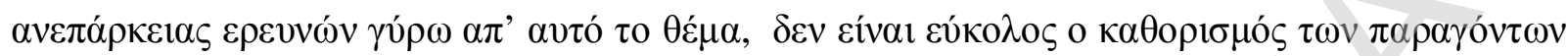

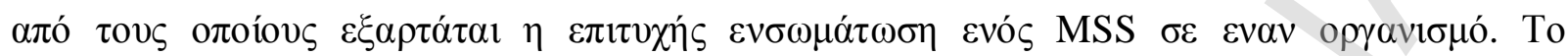

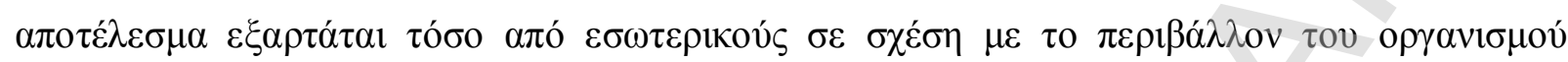

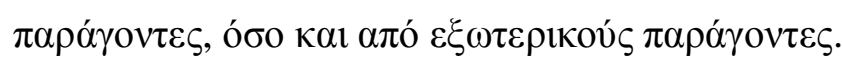

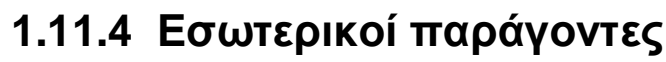

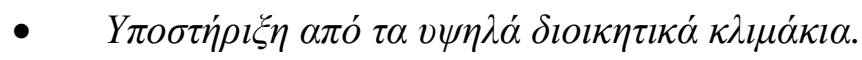

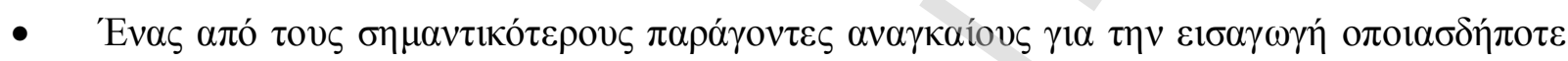

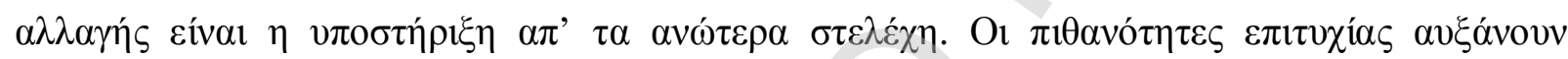

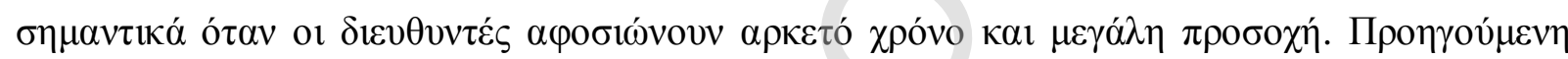

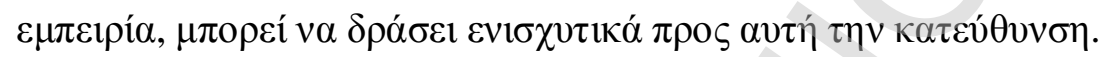

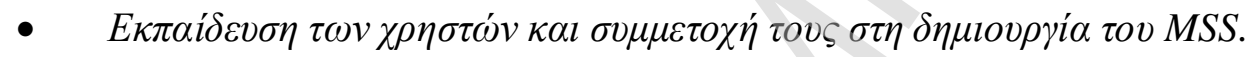

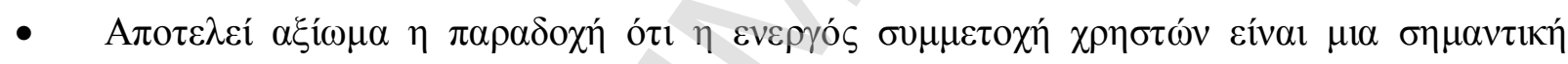

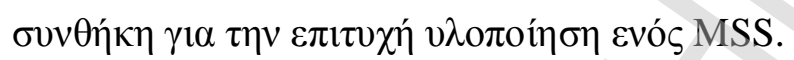

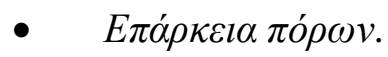

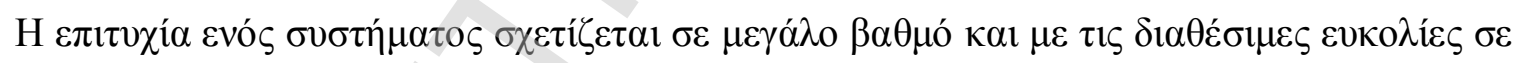

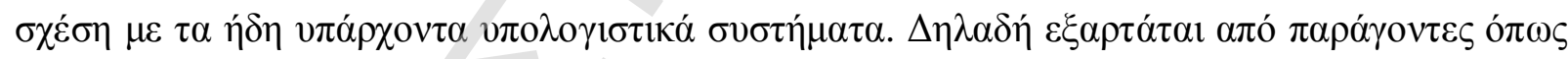

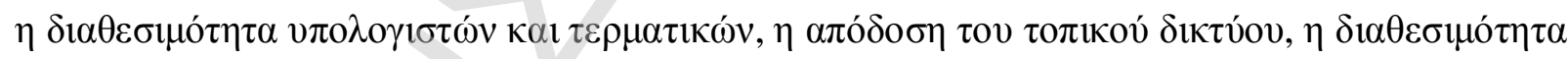

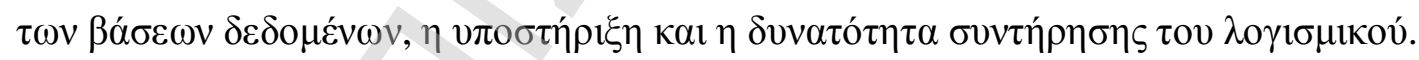

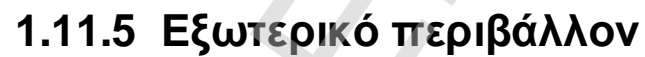

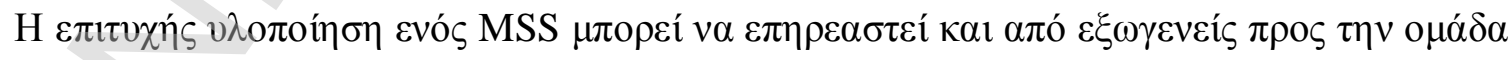

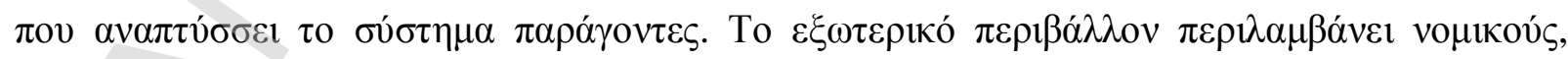

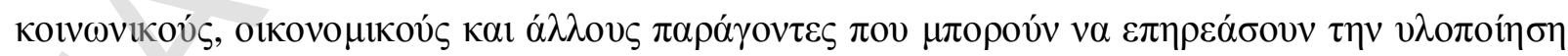

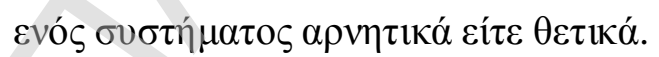

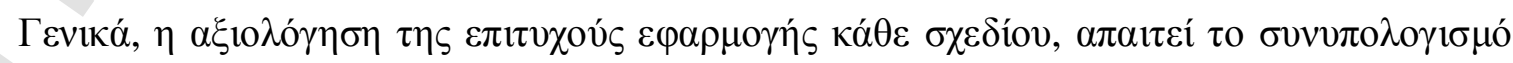

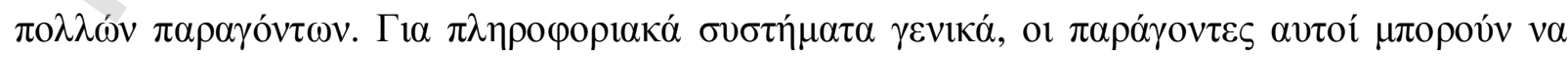

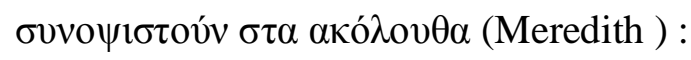




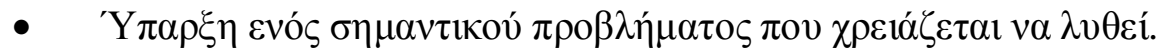

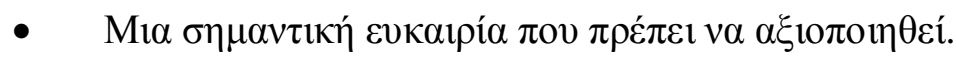

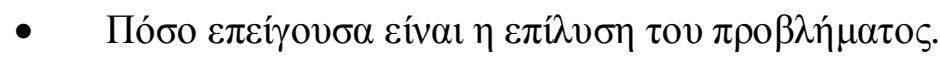

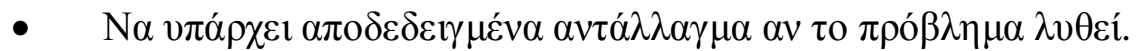

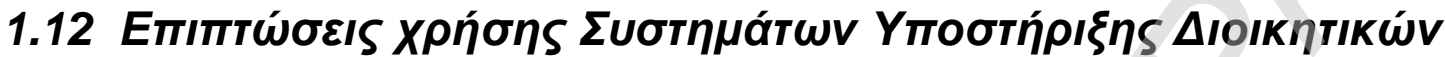

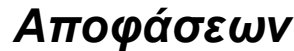

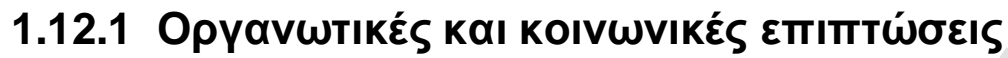

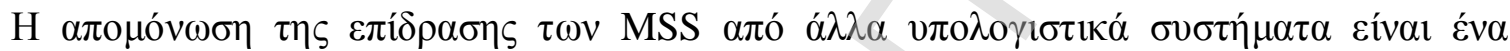

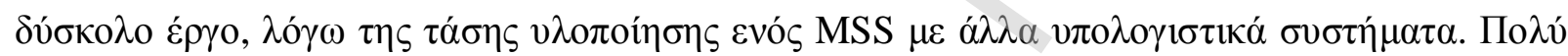

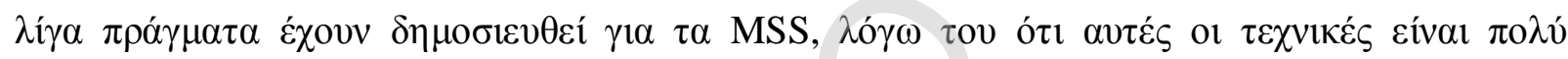

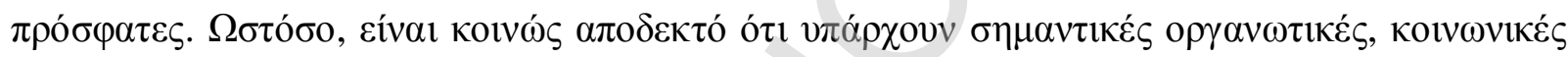

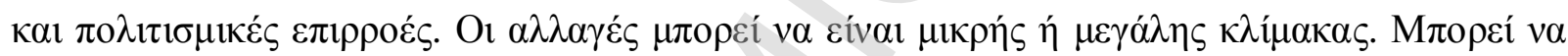

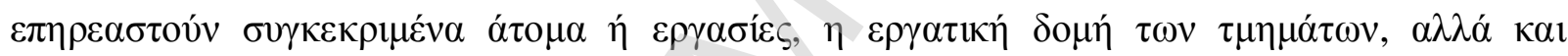
$\mu$

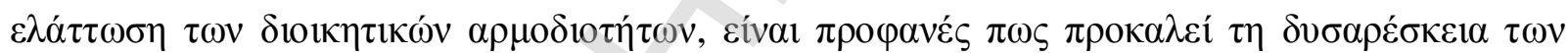

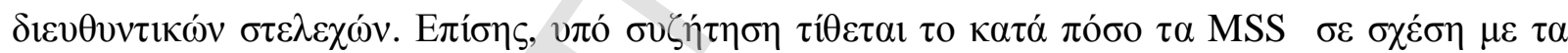

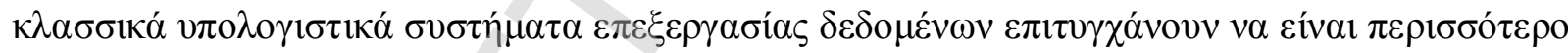

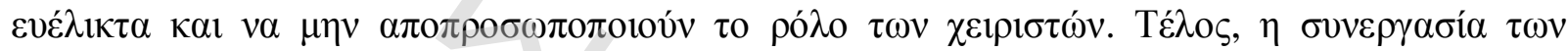

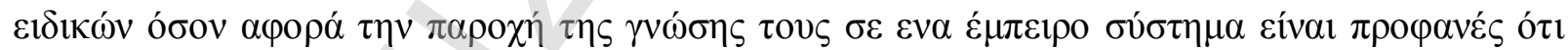

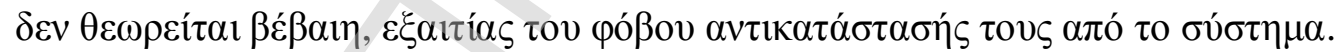

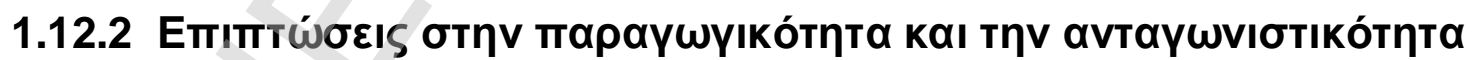

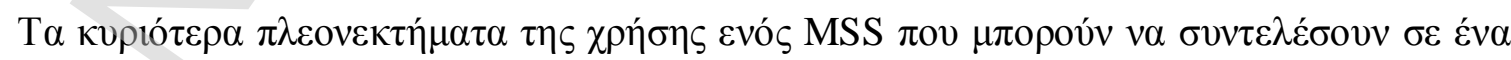

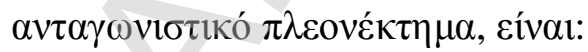

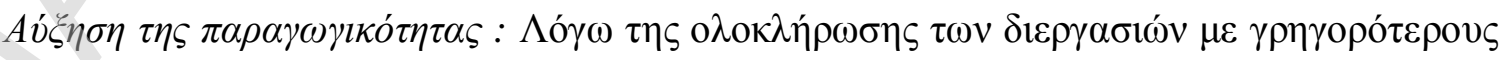

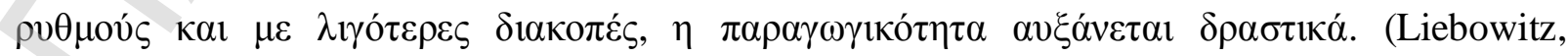
Sviolka). 


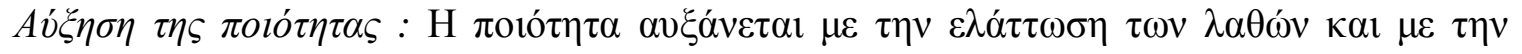

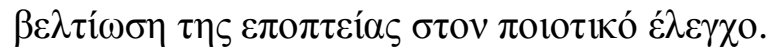

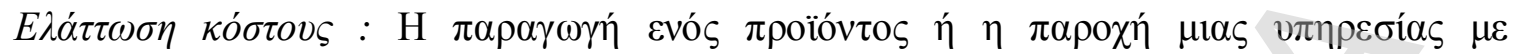

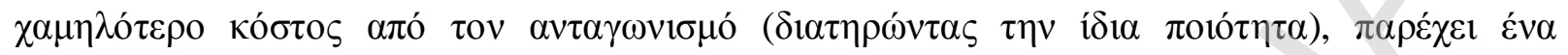
$\alpha \nu \tau \alpha \gamma \omega v i \sigma \tau 1 \kappa o ́ ~ \pi \lambda \varepsilon \sigma v \varepsilon ́ \kappa \tau \eta \mu \alpha$.

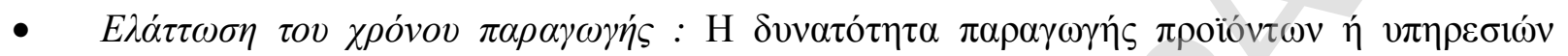

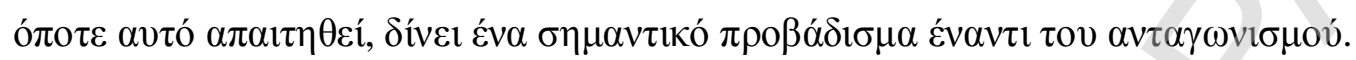

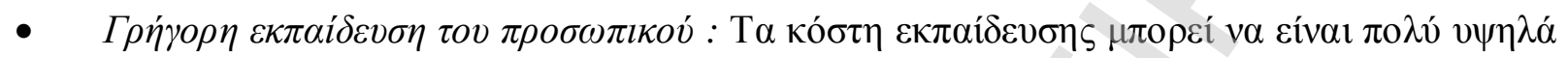

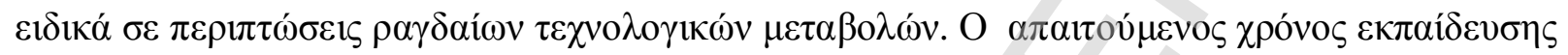

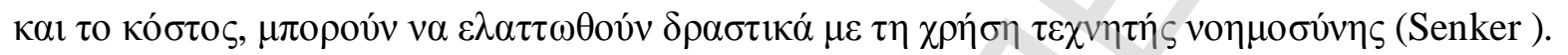

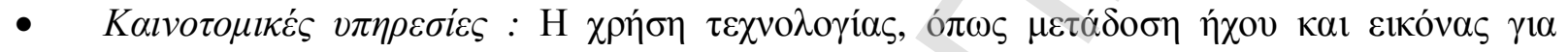

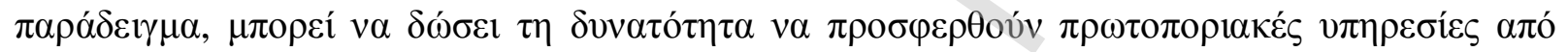

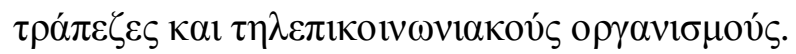

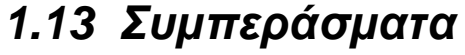

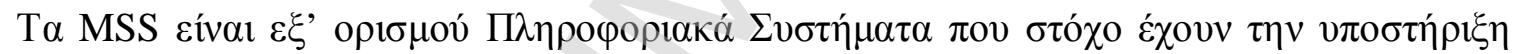

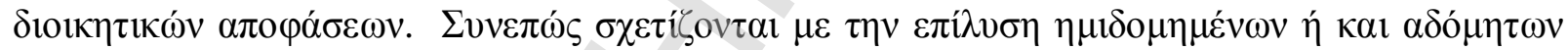

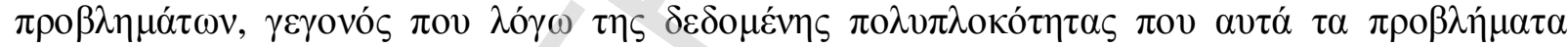

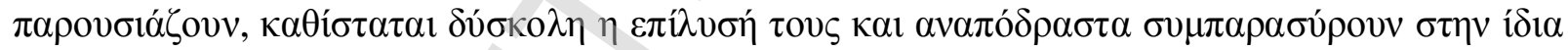

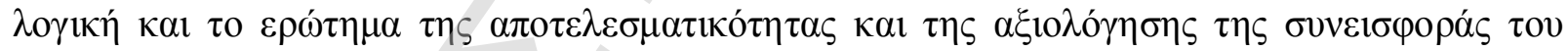

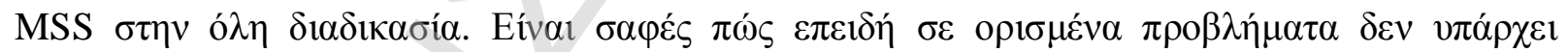

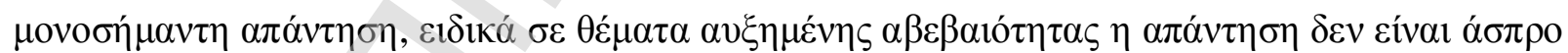

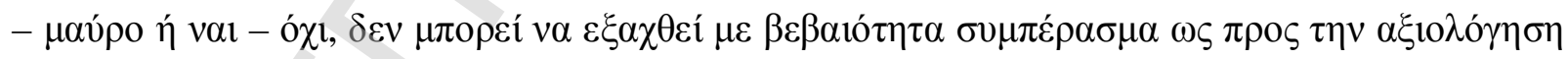

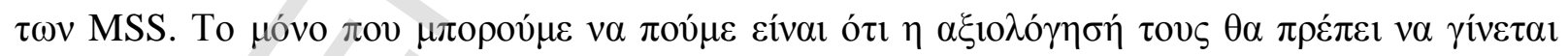

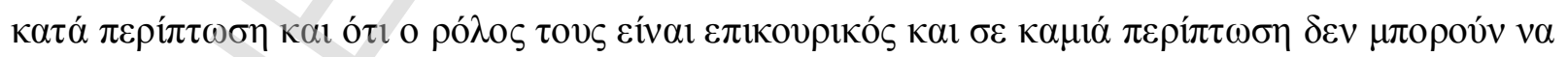

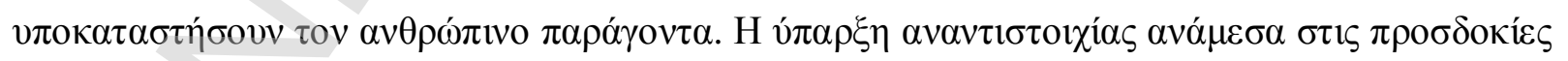

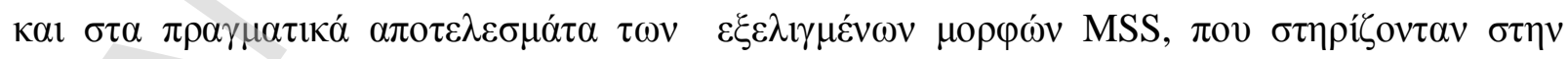

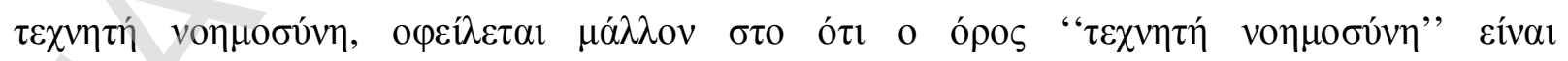

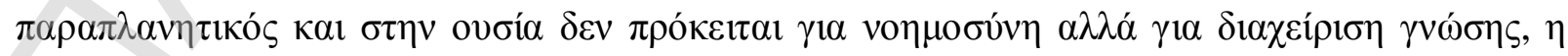

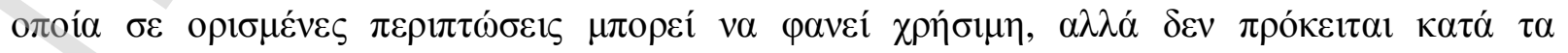

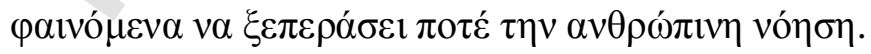




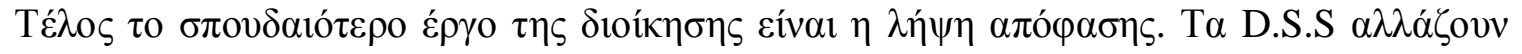

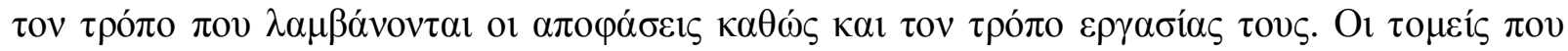
$\varepsilon \pi \eta \rho \varepsilon \alpha ́ \zeta o v \tau \alpha 1$ síval :

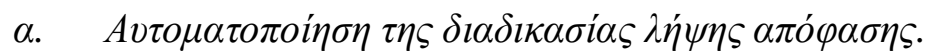

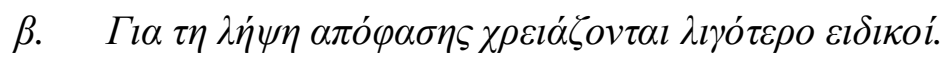

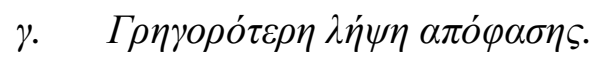

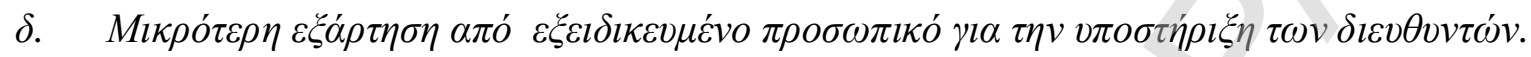

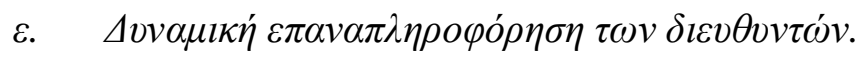

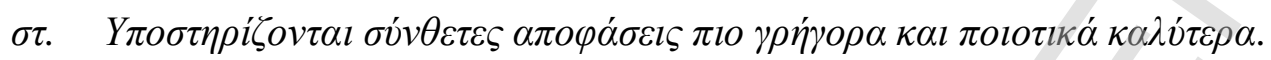

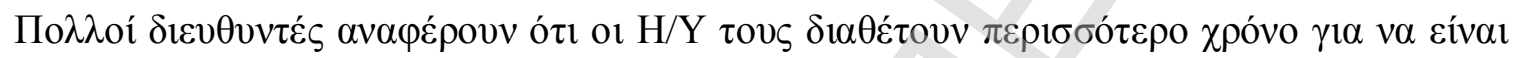

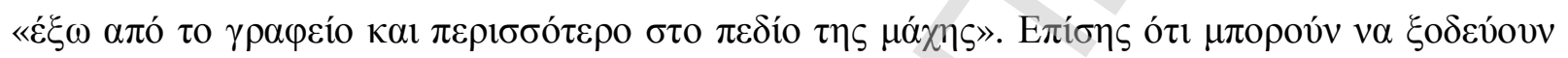

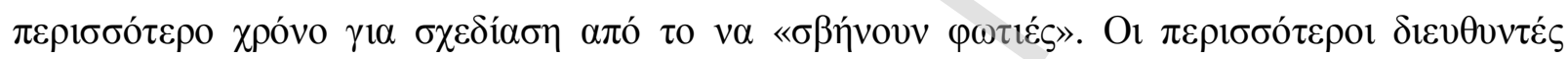

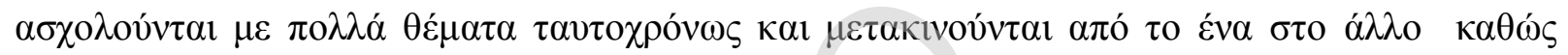

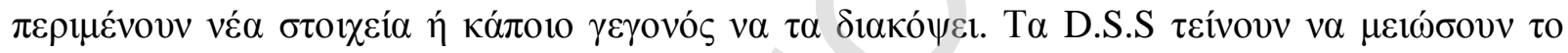

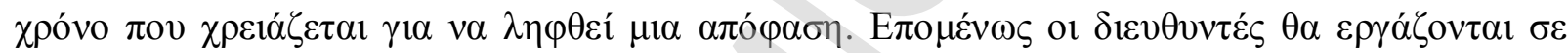

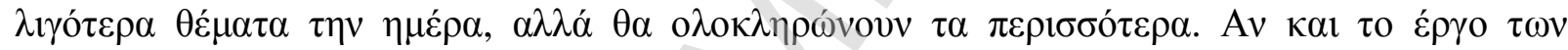

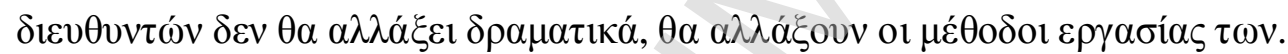




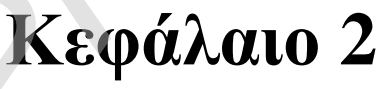

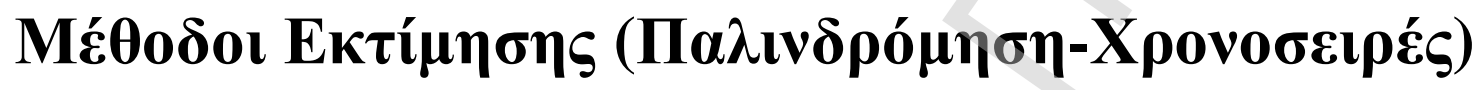

2.1 Про́ $\beta \lambda \varepsilon \psi \eta$

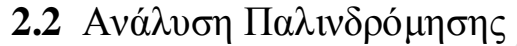

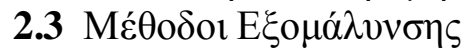

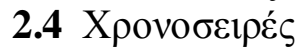

$2.5 \Sigma v \mu \pi \varepsilon \rho \alpha ́ \sigma \mu \alpha \tau \alpha$ 


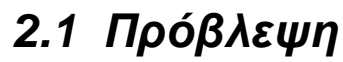

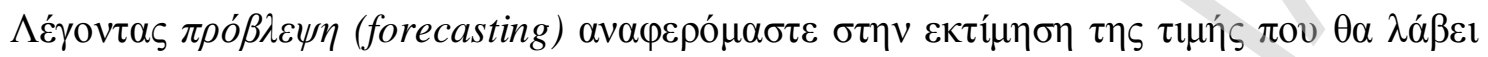

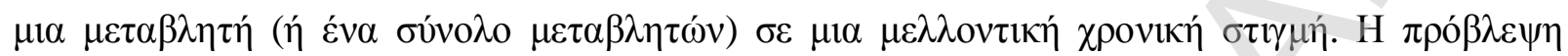

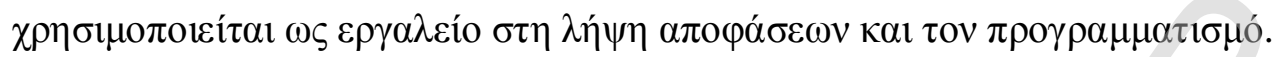

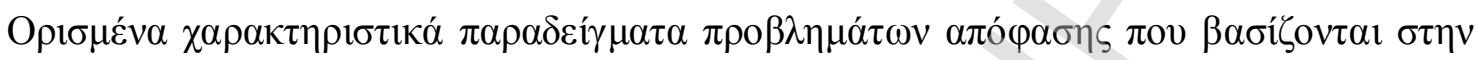

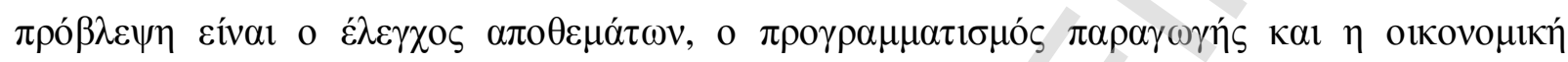

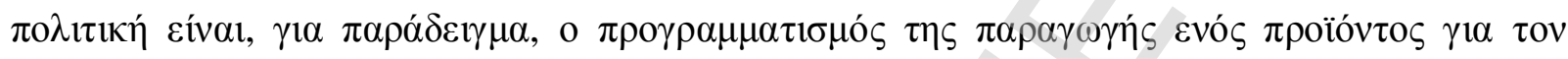

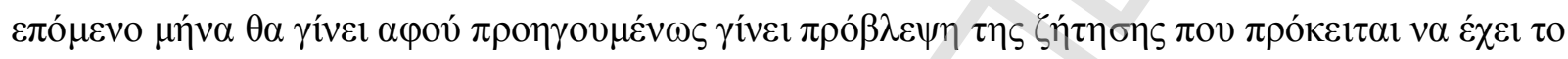
$\pi \rho 0$ ö́v.

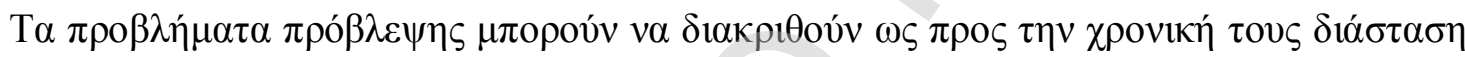

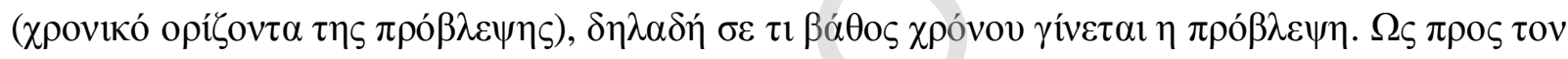

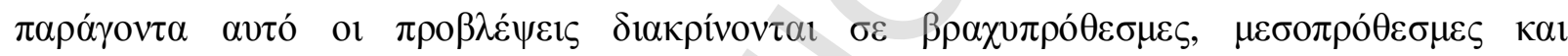

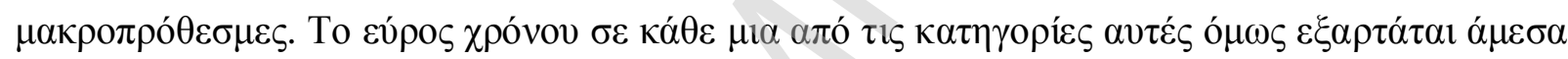

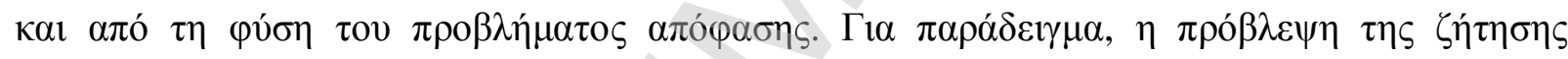

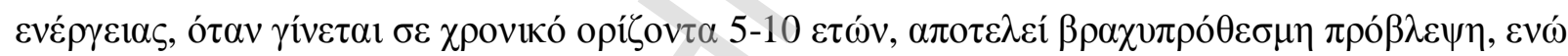

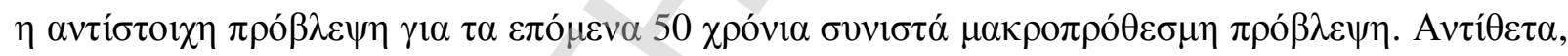

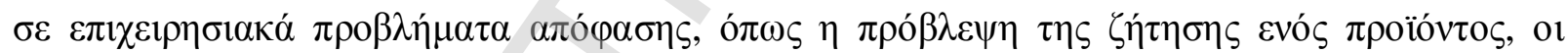

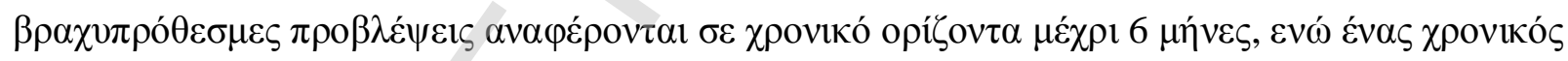

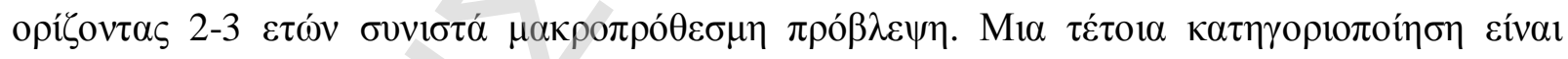

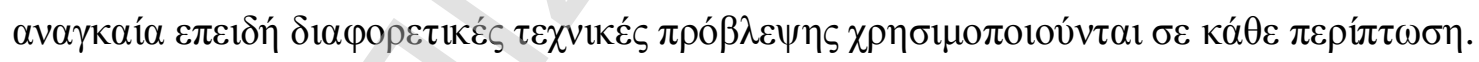

\begin{tabular}{|c|c|c|}
\hline Xpovikós opí̧ovtas & 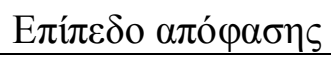 & 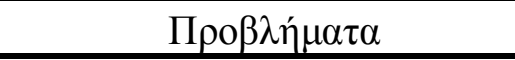 \\
\hline 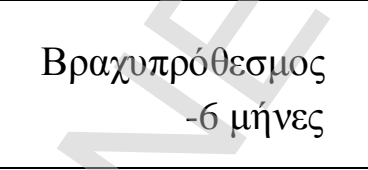 & 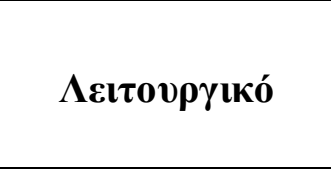 & 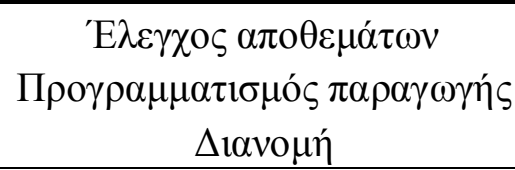 \\
\hline 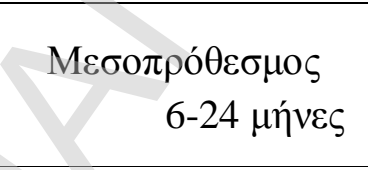 & 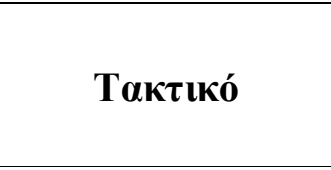 & 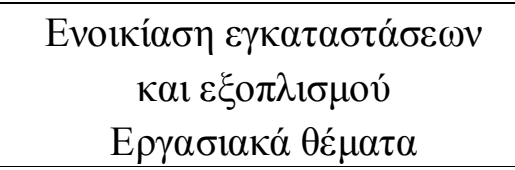 \\
\hline 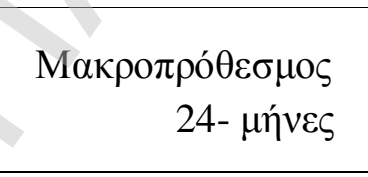 & $\Sigma \tau \rho \alpha \tau \eta \gamma\langle\kappa o ́$ & 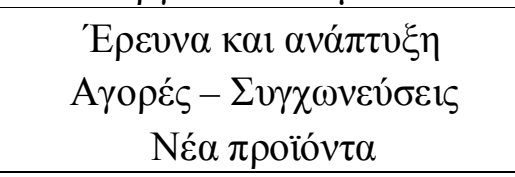 \\
\hline
\end{tabular}




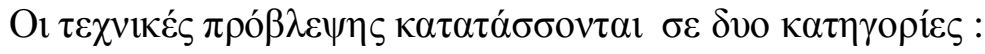

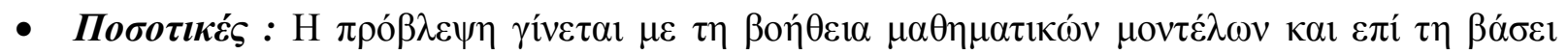

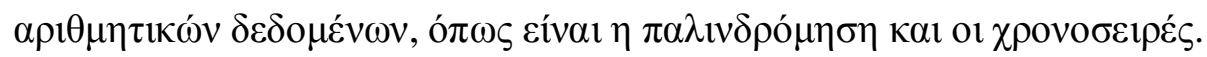

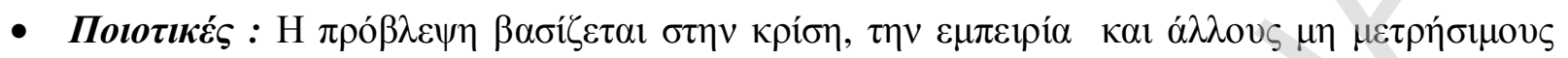
$\pi \alpha \rho \alpha ́ \gamma o v \tau \varepsilon \varsigma$.

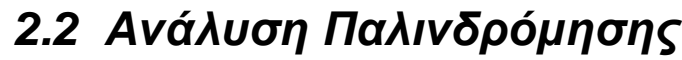

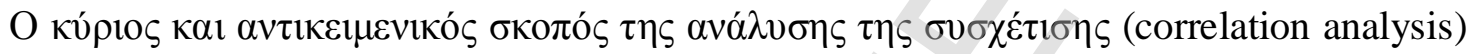

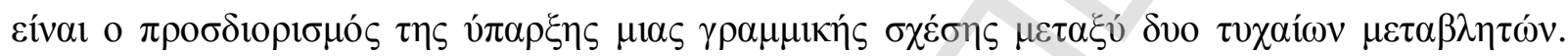

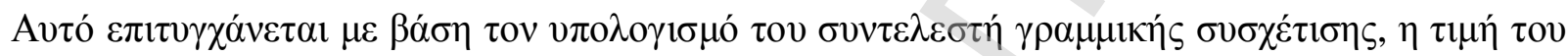

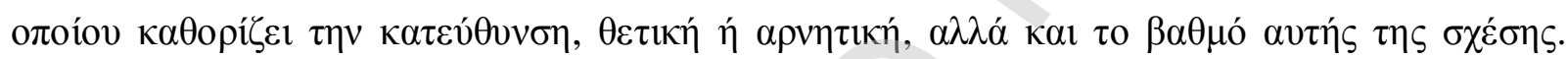

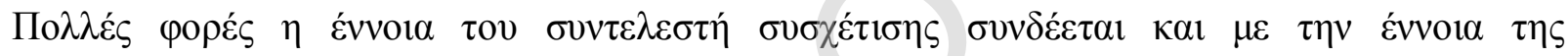

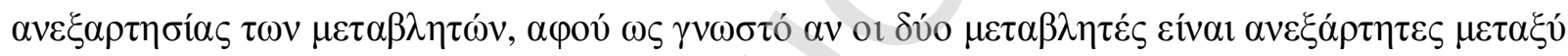

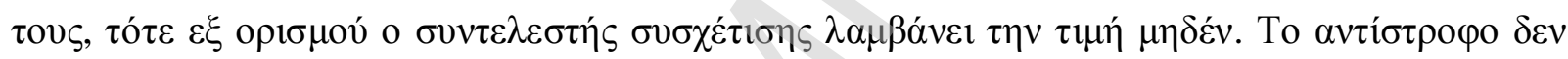

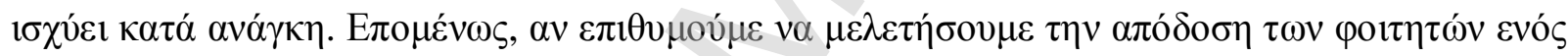

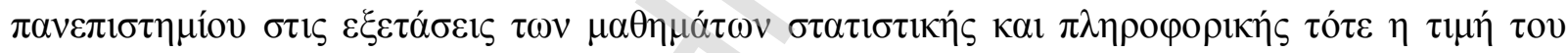

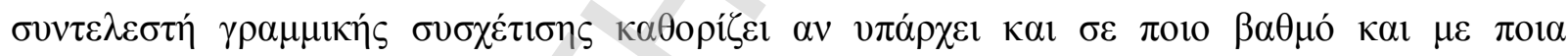

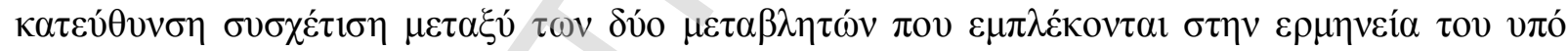

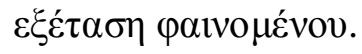

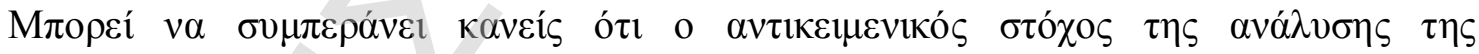

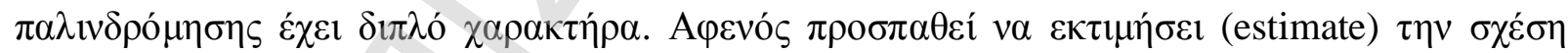

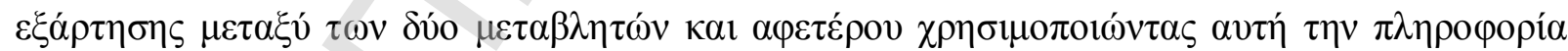

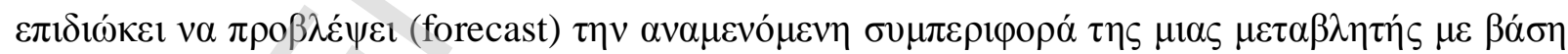

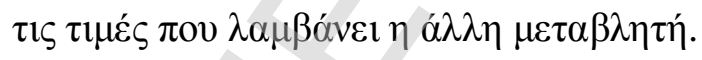

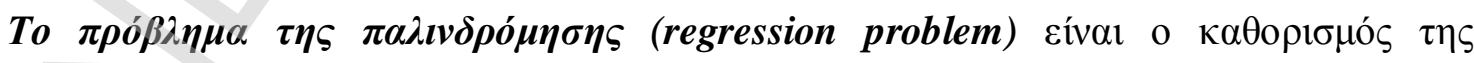

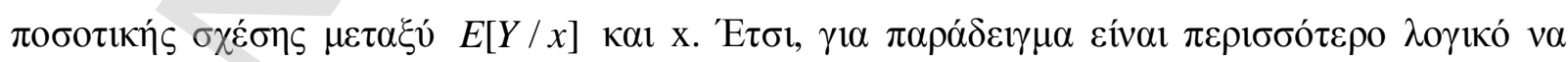

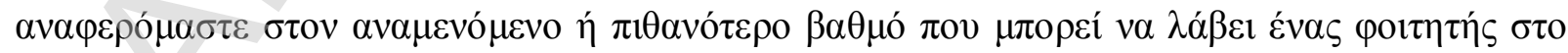

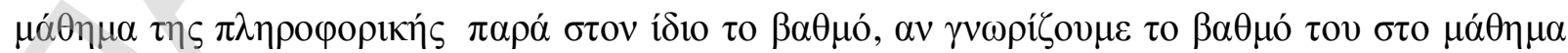

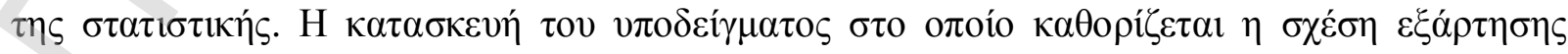

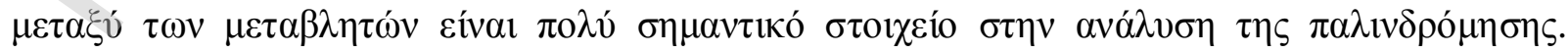




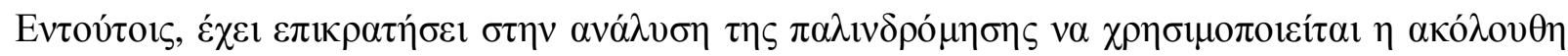

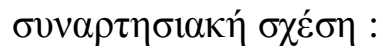

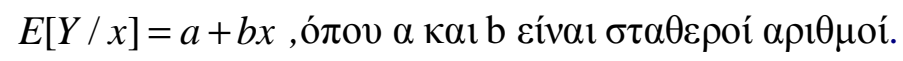

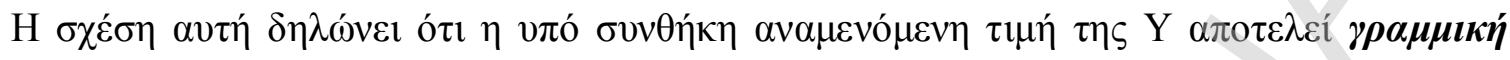

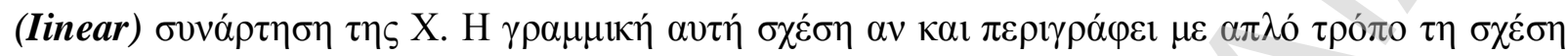

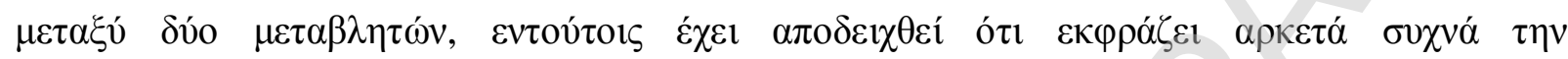
$\pi \rho \alpha \gamma \mu \alpha \tau \iota \kappa o ́ \tau \eta \tau \alpha$.

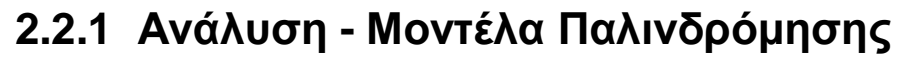

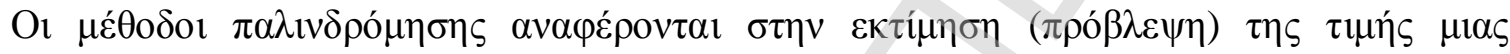

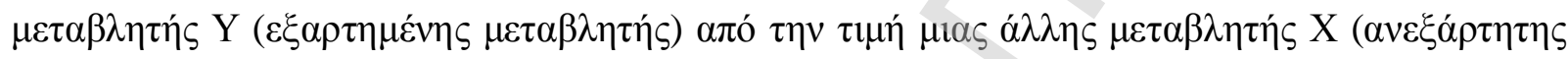

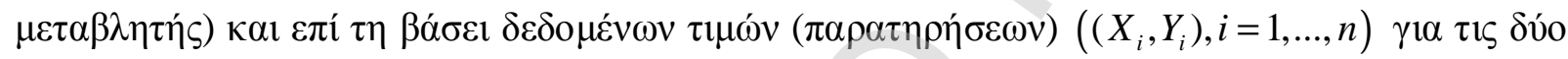

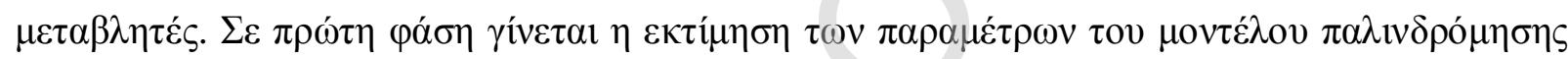

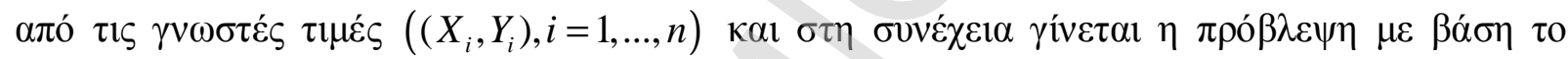

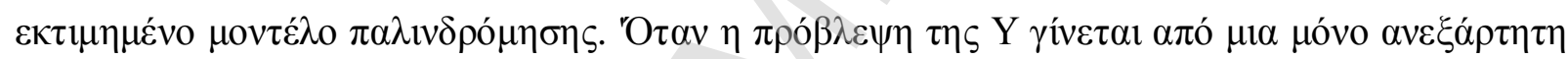

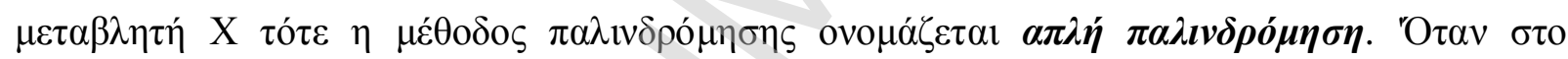

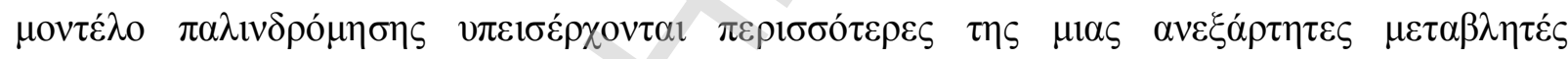

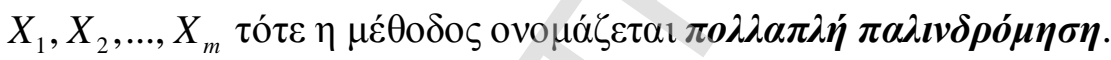

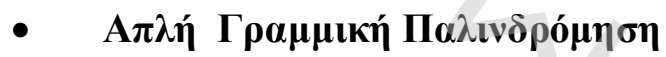

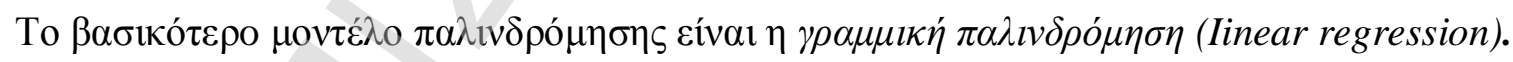

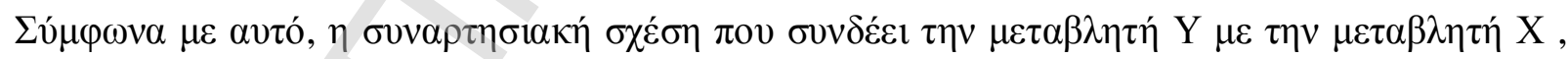

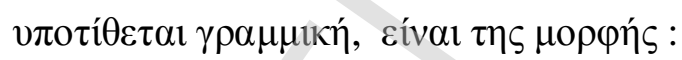

$$
Y=a x+b
$$

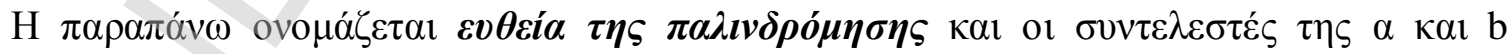

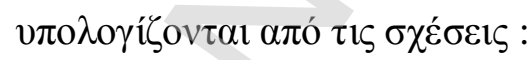

$$
\begin{gathered}
b=\left[n \sum x y-\left(\sum x\right)\left(\sum y\right)\right] / n \sum x^{2}-\left(\sum x\right)^{2} \\
a=\bar{y}-b \bar{x}
\end{gathered}
$$




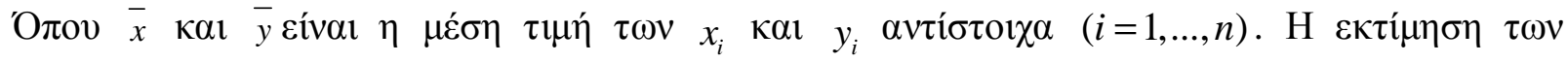

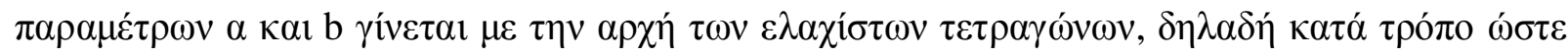

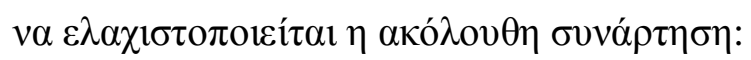

$$
e(a, b)=\sum_{i=1}^{n}\left(y_{i}-Y_{i}\right)^{2}=\sum_{i=1}^{n}\left(y_{i}-a-b x_{i}\right)^{2}
$$

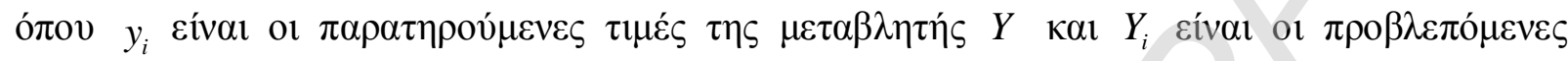

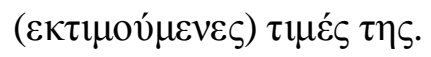

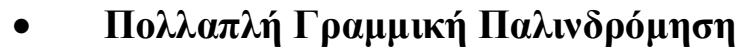

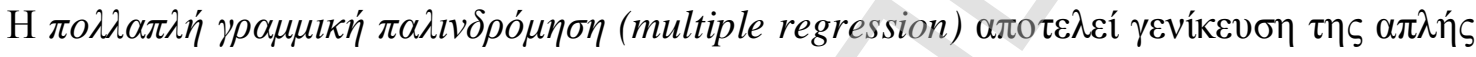

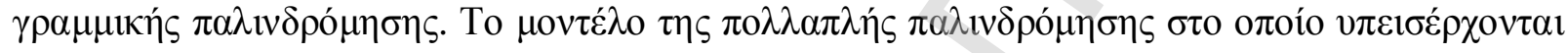

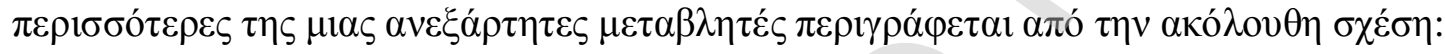

$$
\mathrm{Y}_{\mathrm{i}}=\alpha+\mathrm{b}_{1} \mathrm{X}_{1}+\mathrm{b}_{2} \mathrm{X}_{2}+\ldots+\mathrm{b}_{\mathrm{m}} \mathrm{X}_{\mathrm{m}}
$$

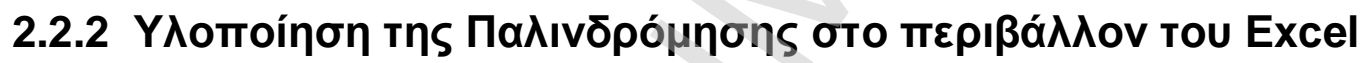

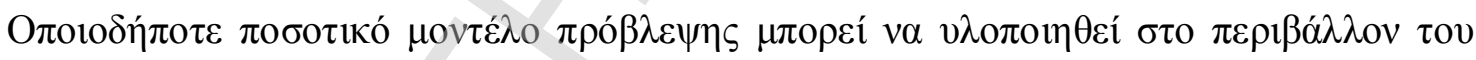

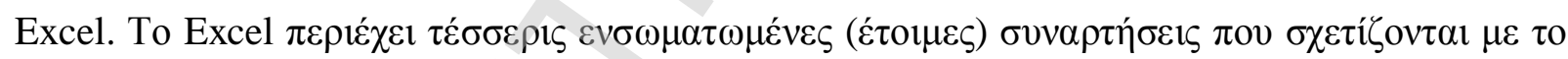

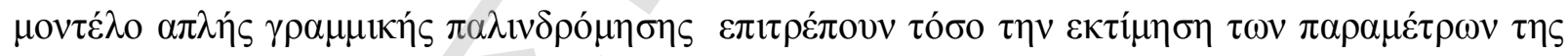

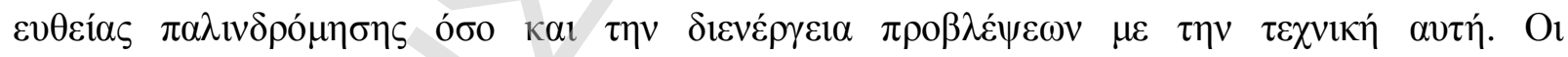

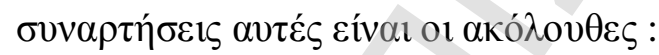

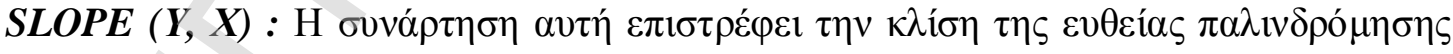

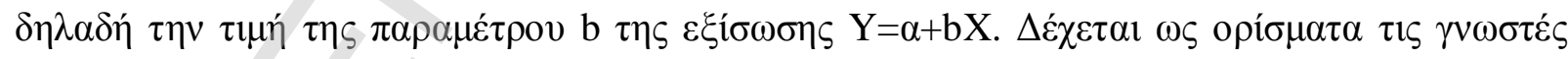

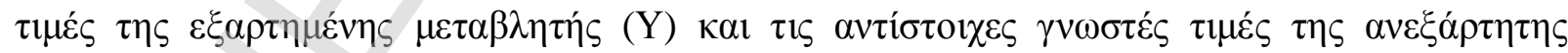
$\mu \varepsilon \tau \alpha \beta \lambda \eta \tau \dot{n} \varsigma(\mathrm{X})$.

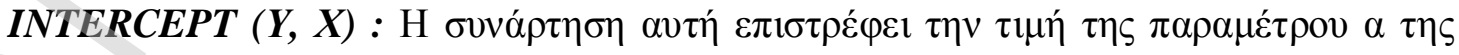

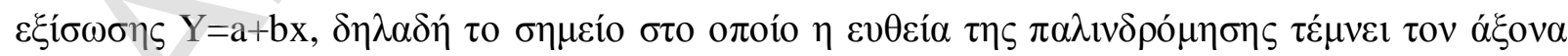

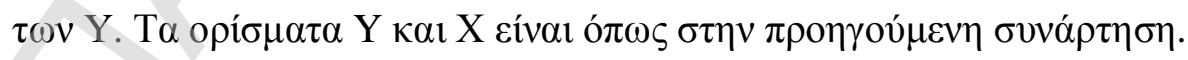

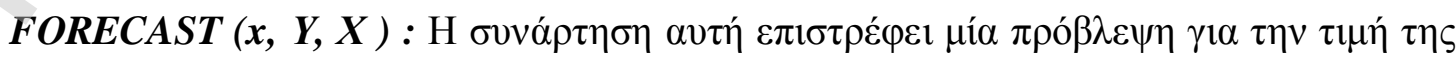

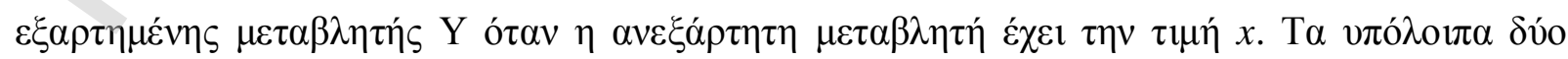

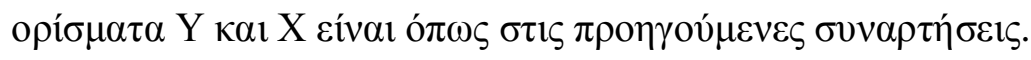




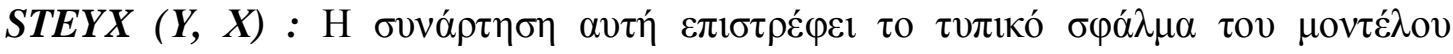

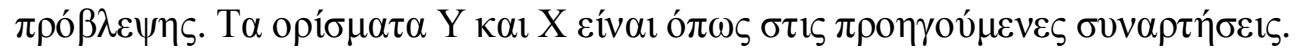

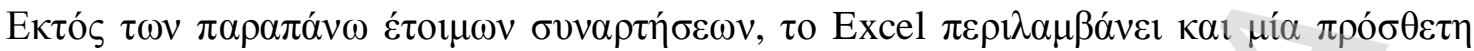

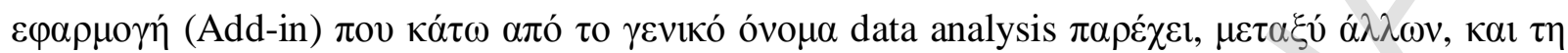

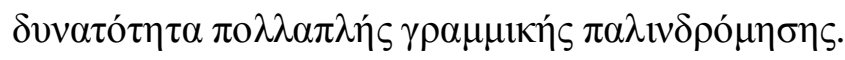

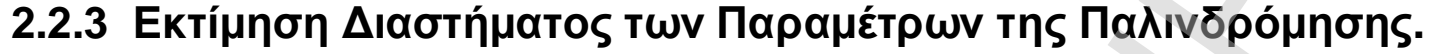

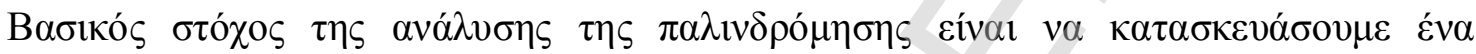

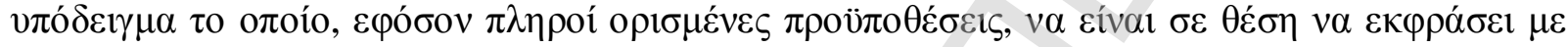

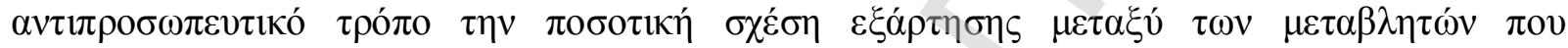

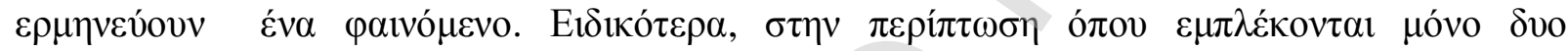

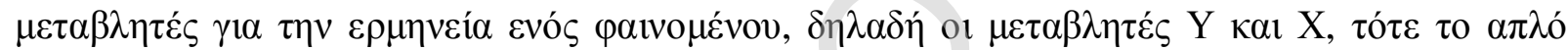

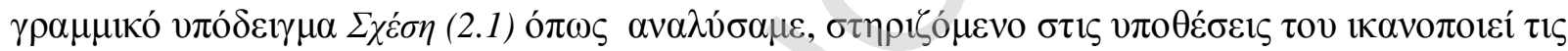

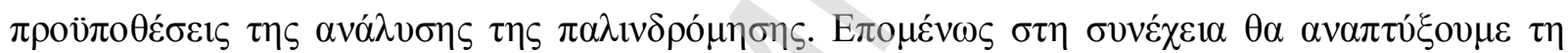

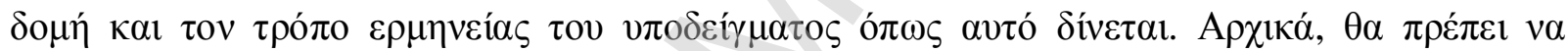

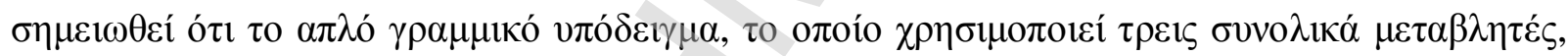

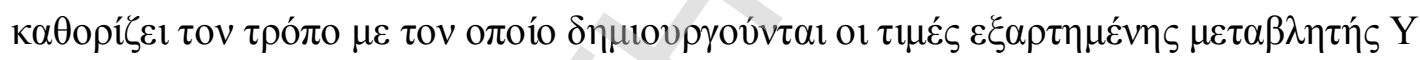

$$
\mathrm{Y}_{\mathrm{i}}=\alpha+\mathrm{b} \mathrm{X}_{\mathrm{i}}+\varepsilon_{\mathrm{i}} \quad \Sigma \chi \varepsilon \dot{\varepsilon} \sigma \eta(2.2)
$$

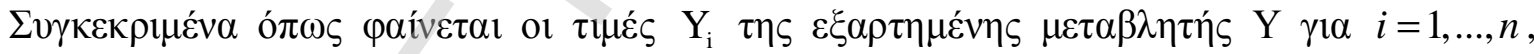
$\pi \rho 0 \sigma \delta$ เo

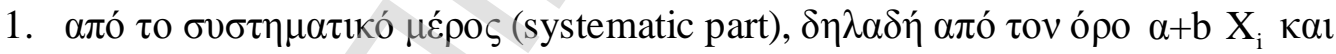

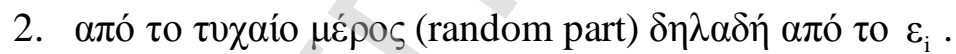

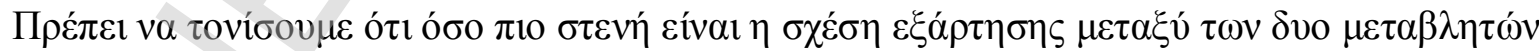

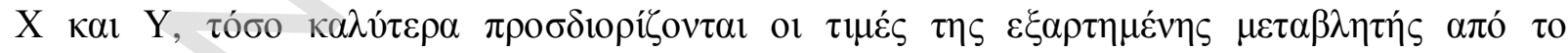

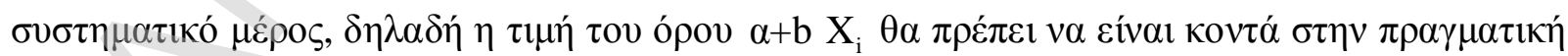

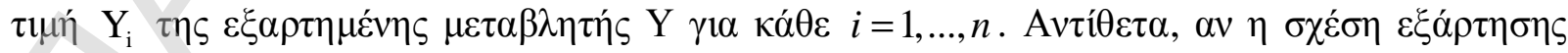

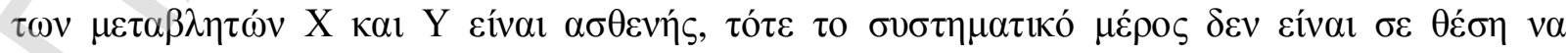

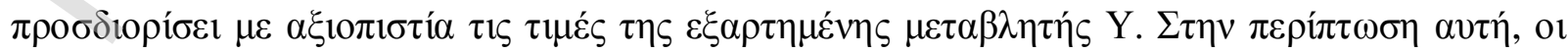

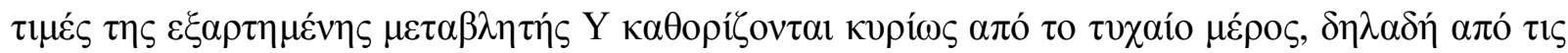




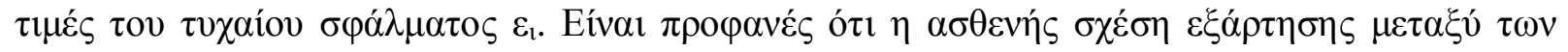

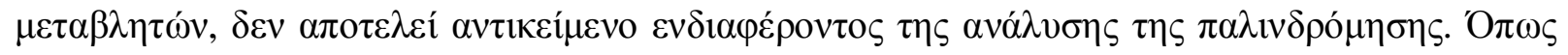

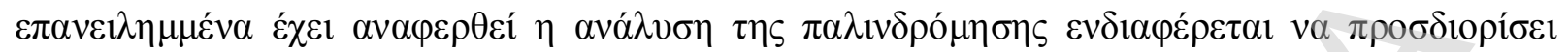

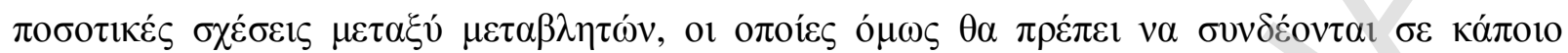

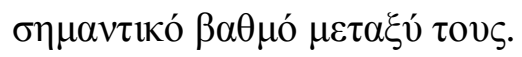

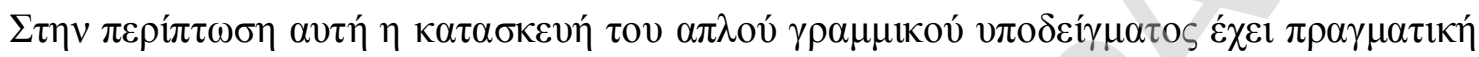

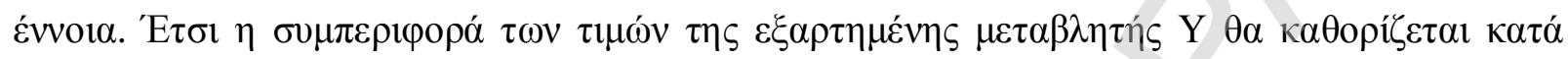

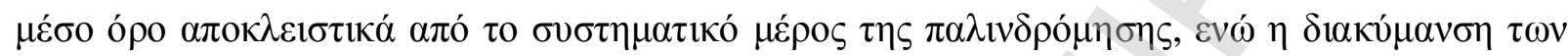

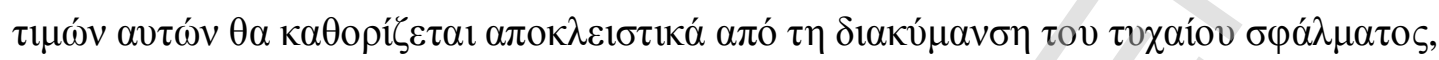

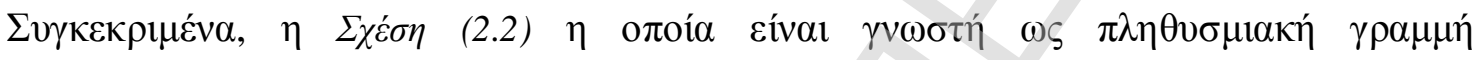

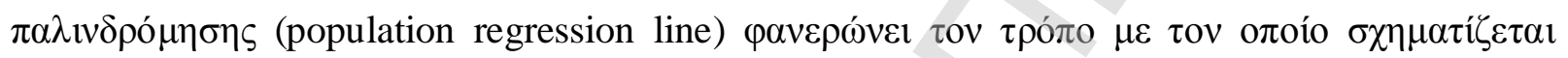

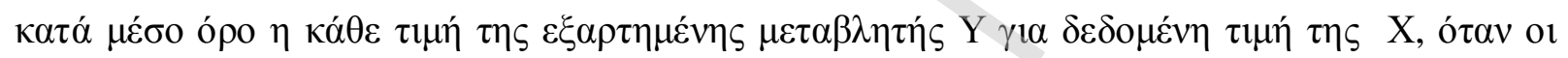

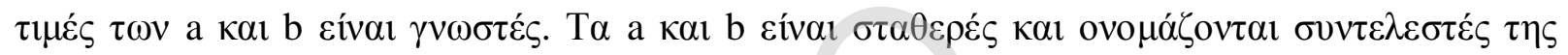

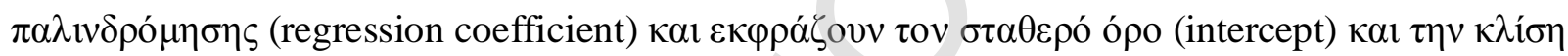
$\tau \eta \varsigma \gamma \rho \rho \mu \mu \eta ́ s ~ \pi \alpha \lambda \imath v \delta \rho o ́ \mu \eta \sigma \eta \varsigma \alpha v \tau i ́ \sigma \tau o \chi \alpha$.

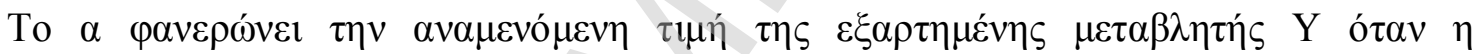
$\alpha v \varepsilon \xi \alpha \dot{\alpha} \rho \tau \tau \eta \eta ⿻ コ 一$

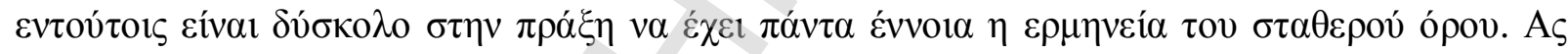

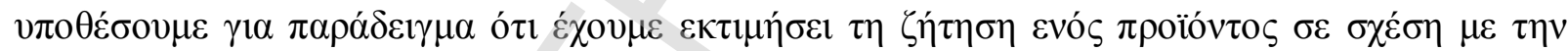

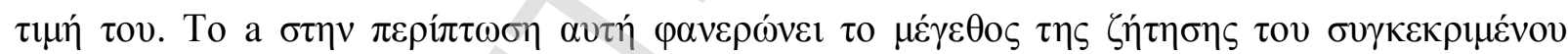

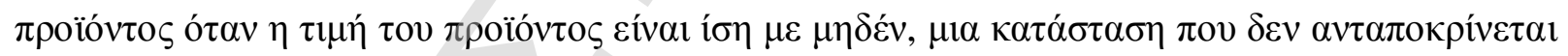

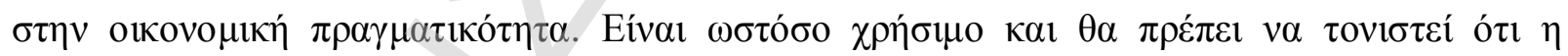

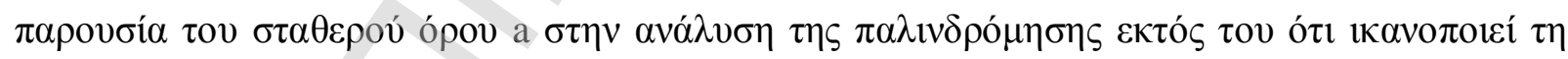

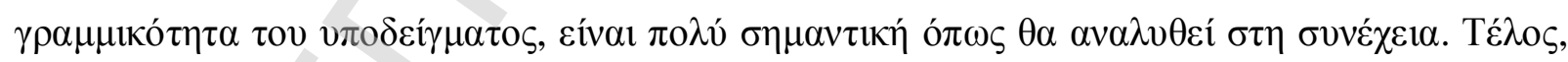

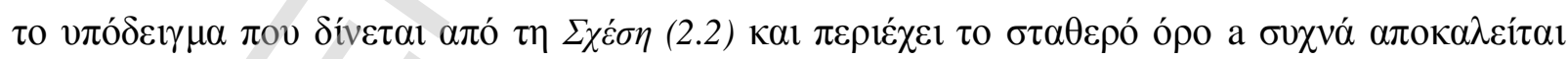

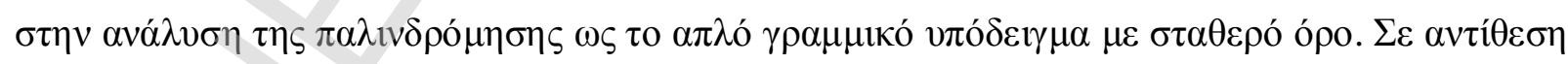

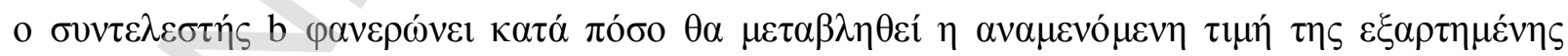

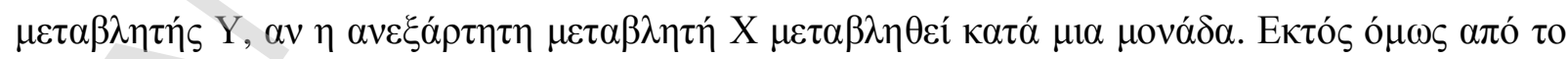

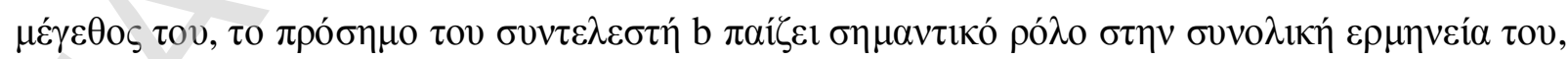

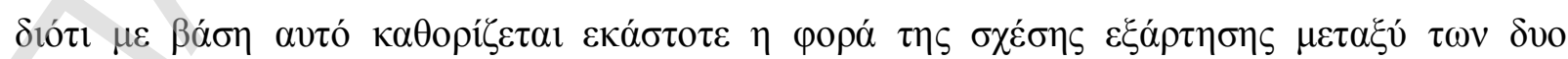

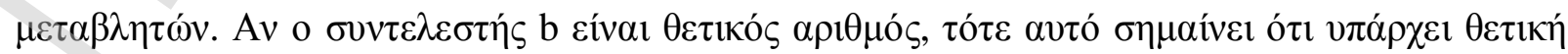

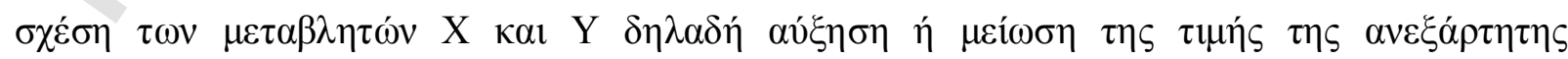

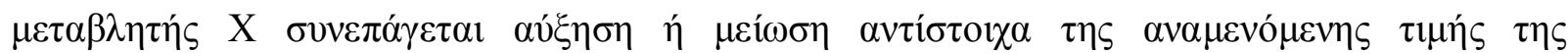




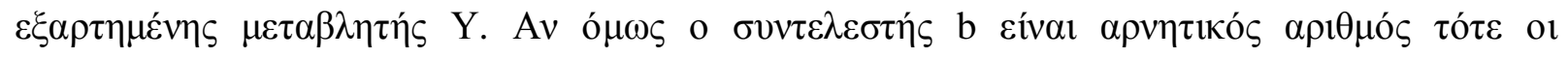

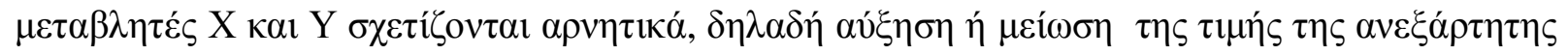

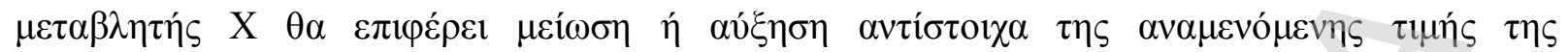
$\varepsilon \xi \alpha \rho \tau \eta \mu \varepsilon ́ v \eta \varsigma \mu \varepsilon \tau \alpha \beta \lambda \eta \tau \dot{\zeta} \mathrm{Y}$.

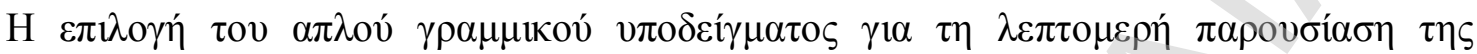

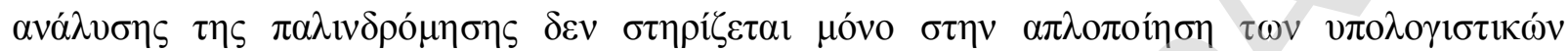

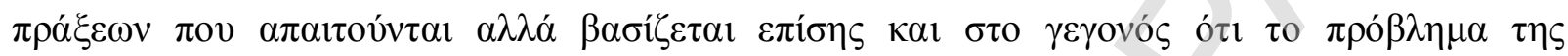

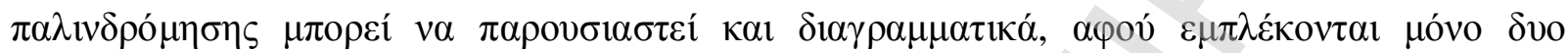

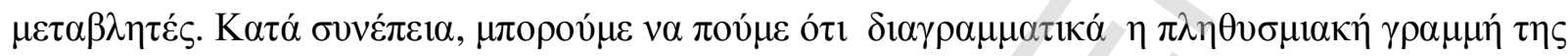

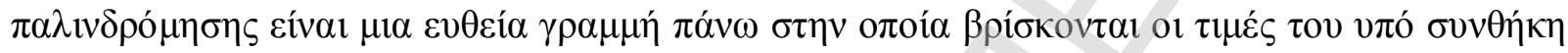

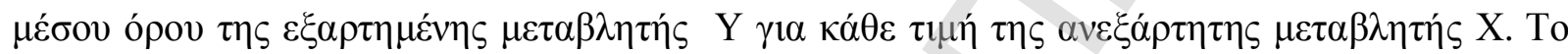

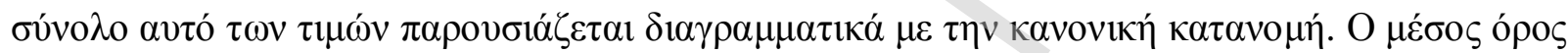

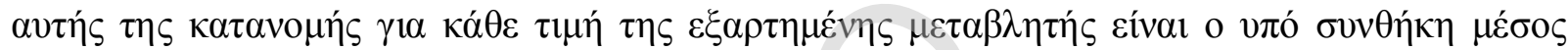

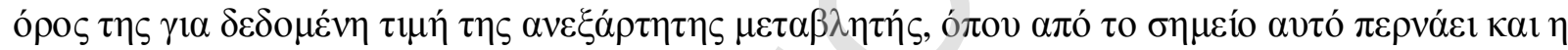

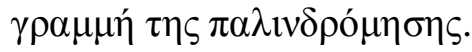

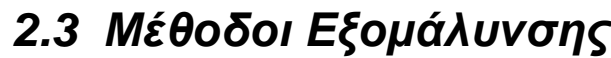

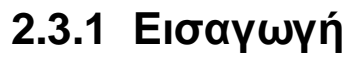

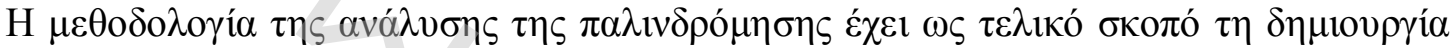

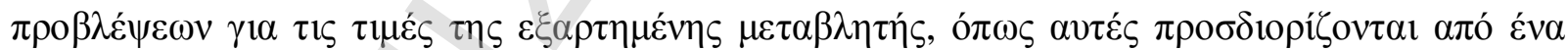

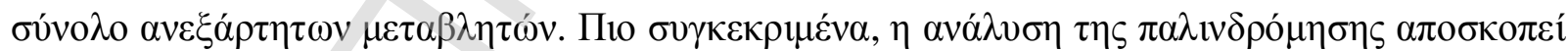

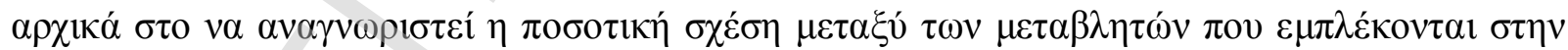

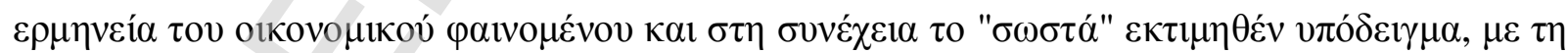

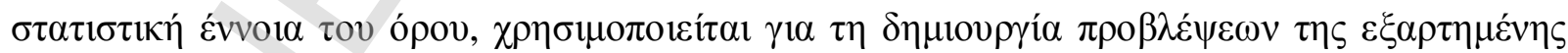

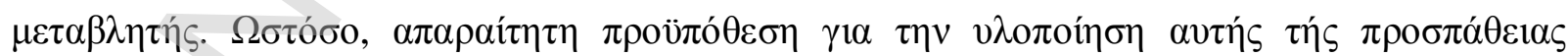

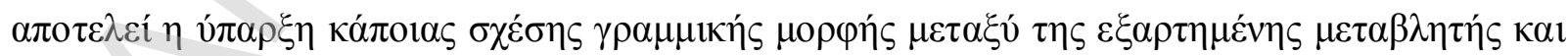

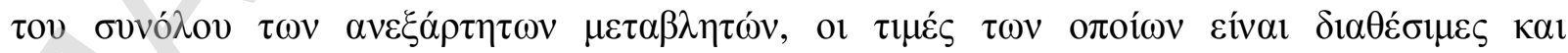

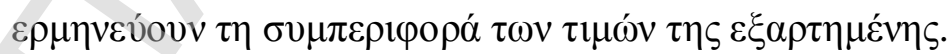

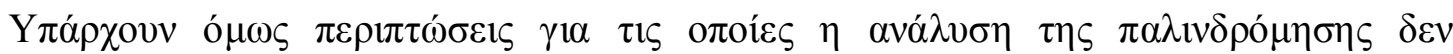

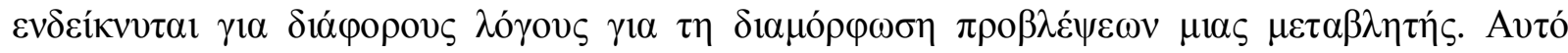




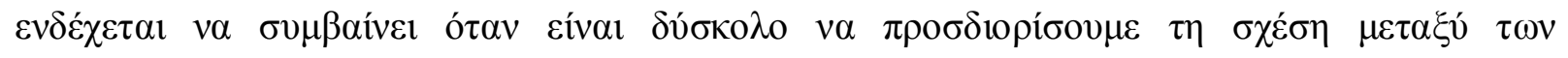

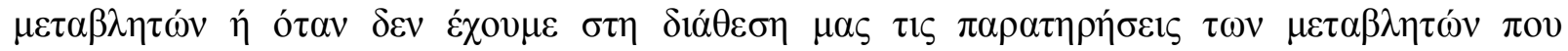

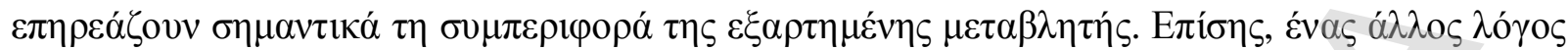

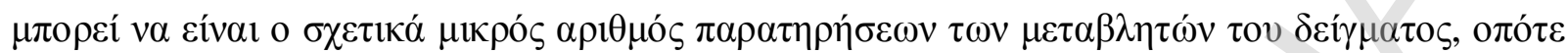

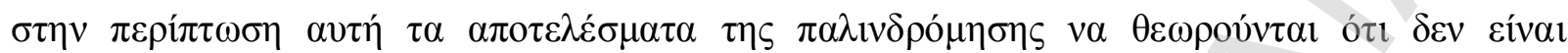

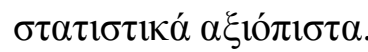

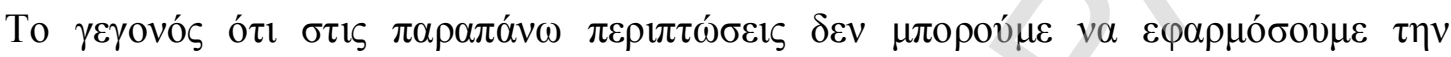

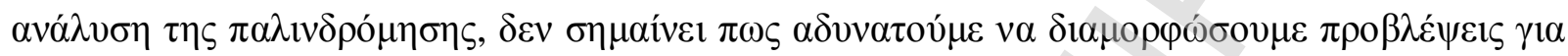

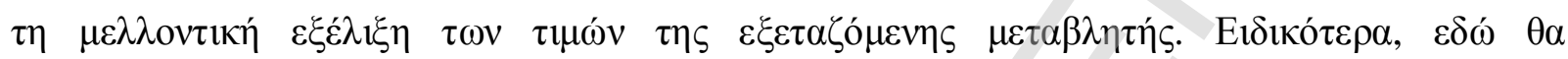

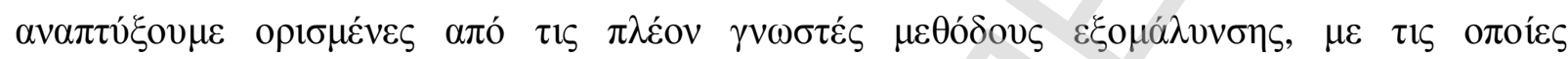

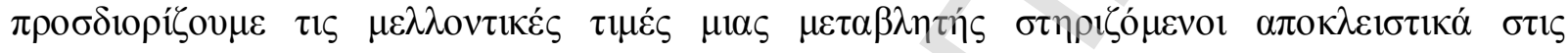

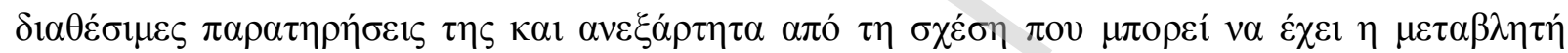

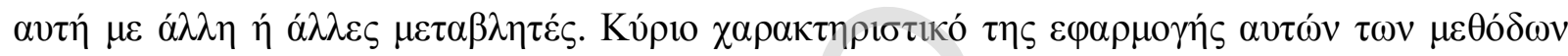

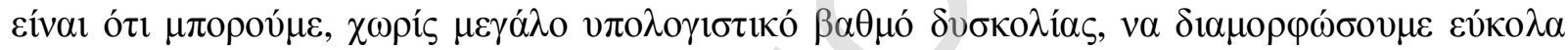

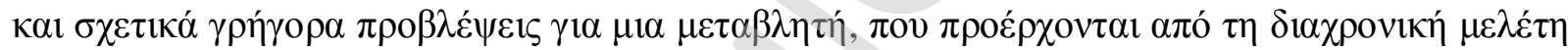

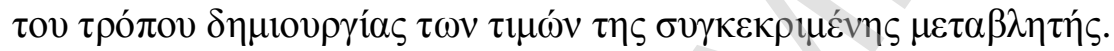

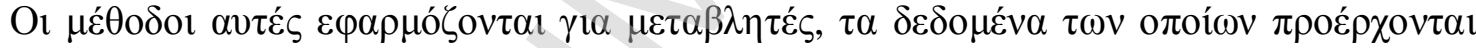

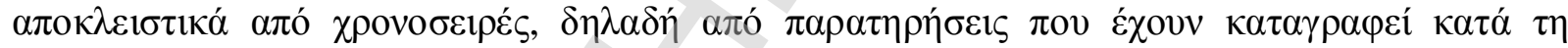

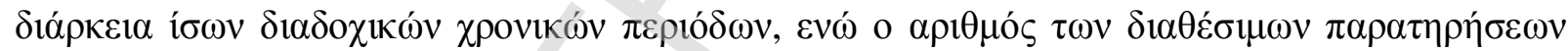

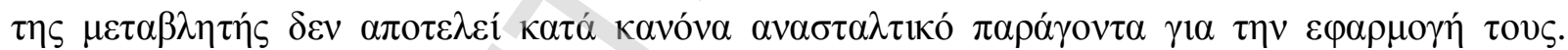

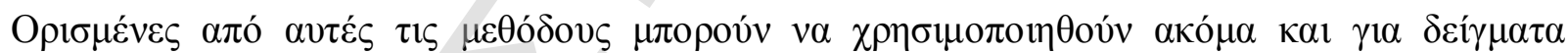

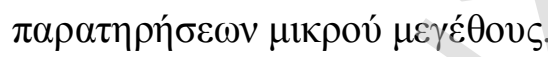

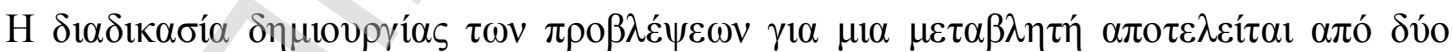

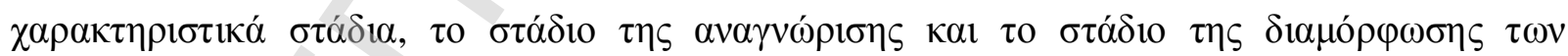

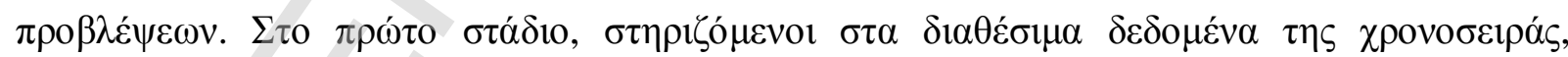

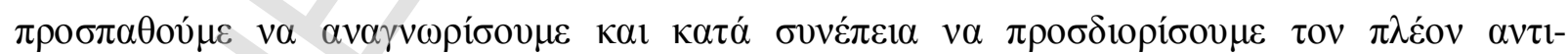

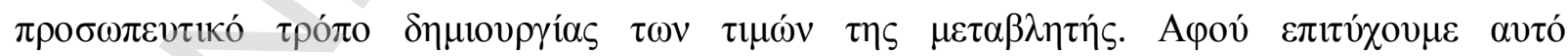

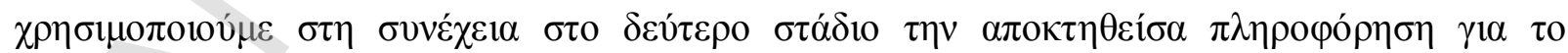

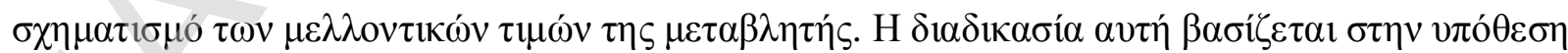

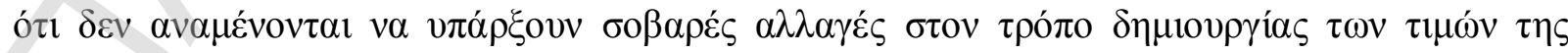

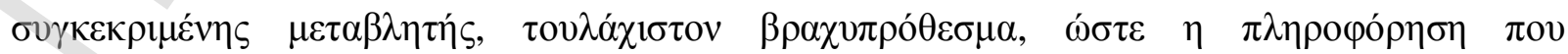

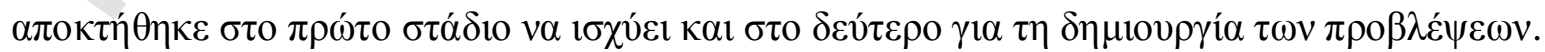




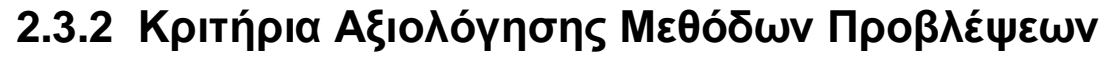

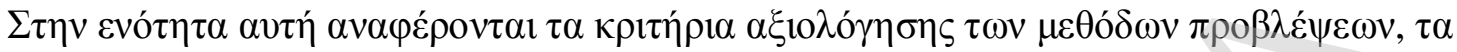

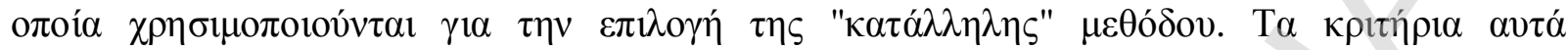

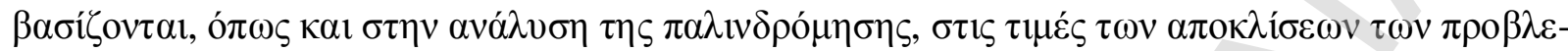

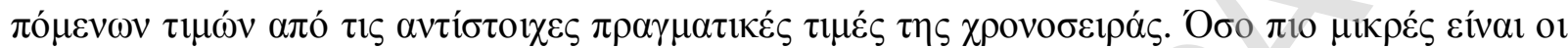

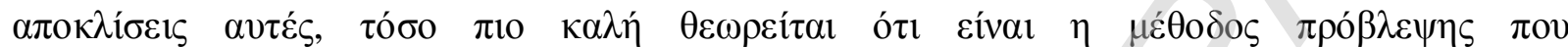

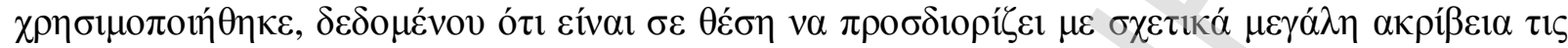

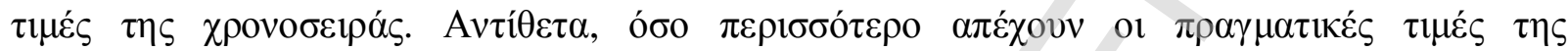

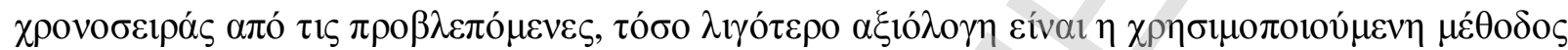

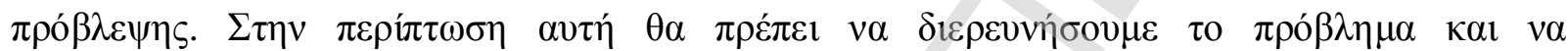

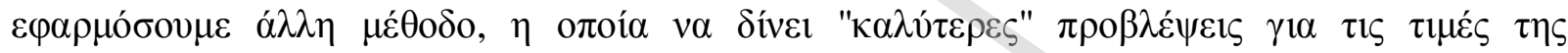

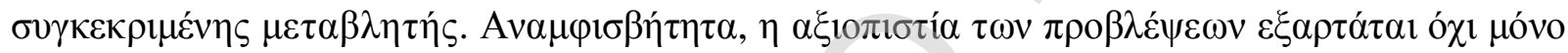

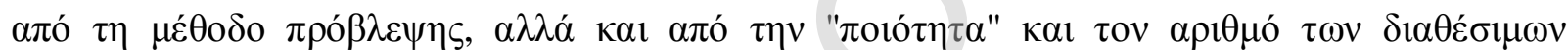

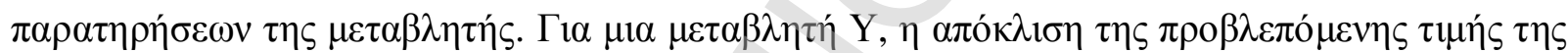

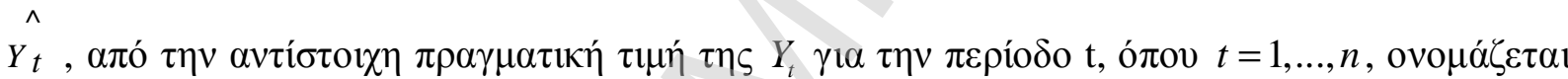

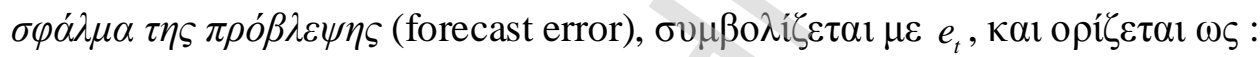

$$
e_{t}=Y_{t}-\hat{Y}_{t} \quad \Sigma \chi \bar{\varepsilon} \sigma \eta(2.3)
$$

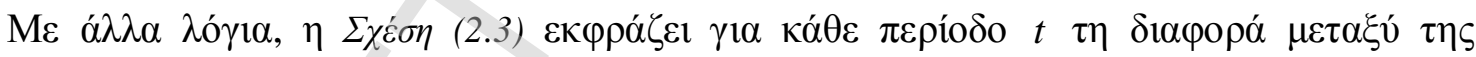

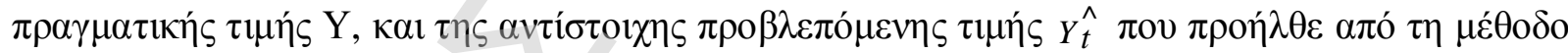

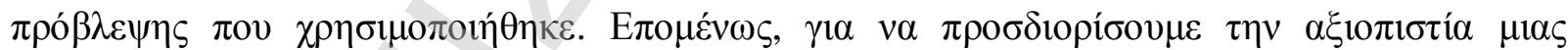

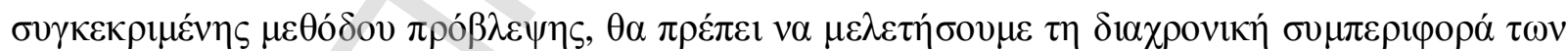

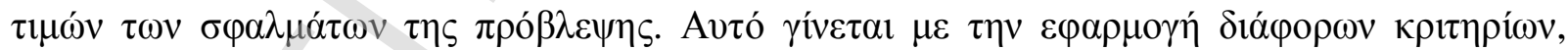

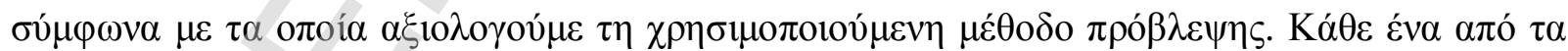

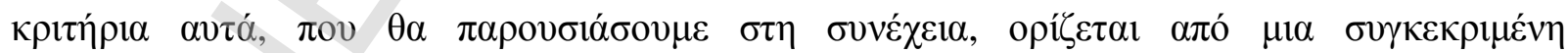

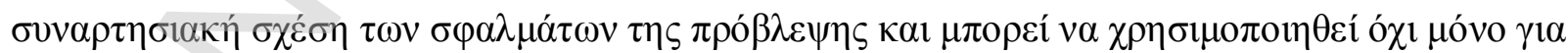

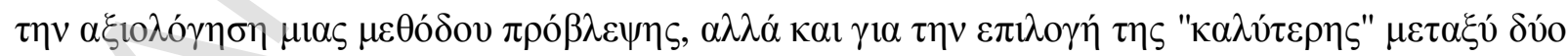

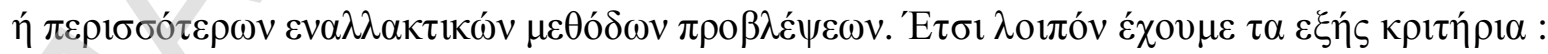




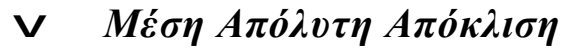

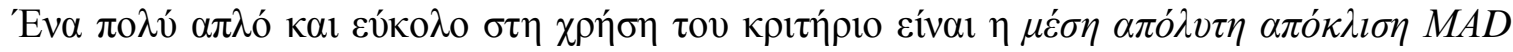

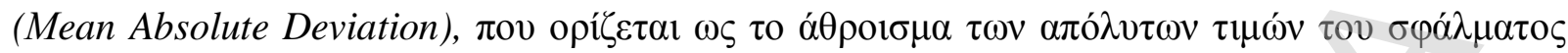

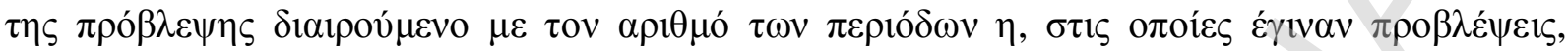
$\delta \eta \lambda \alpha \delta \eta \dot{~: ~}$

$$
M A D=1 / n \sum_{t=1}^{n}\left|Y_{t}-\hat{Y_{t}}\right|=\sum_{t=1}^{n}\left|e_{t}\right| \quad \Sigma \chi \bar{\varepsilon} \sigma \eta(2.4)
$$

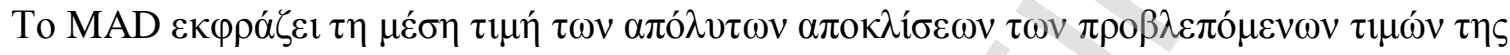

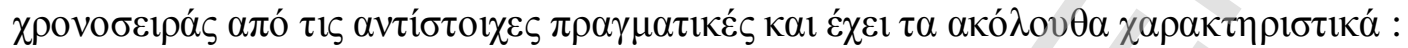

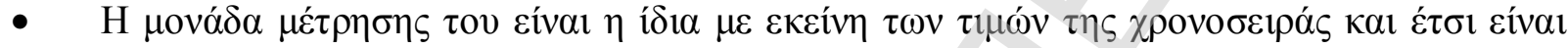

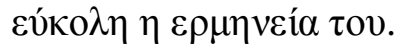

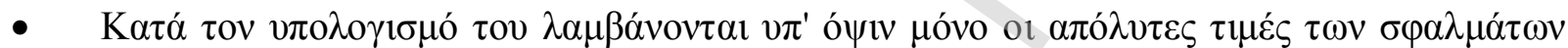

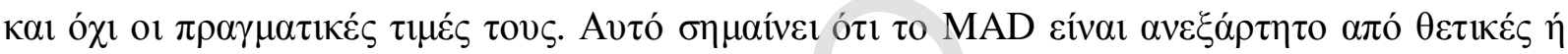

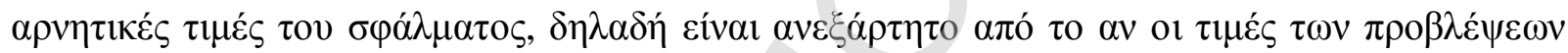

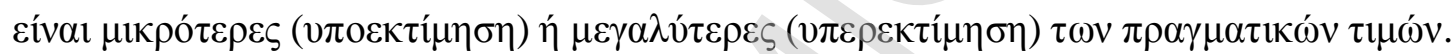

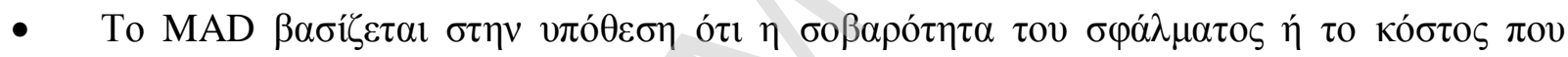

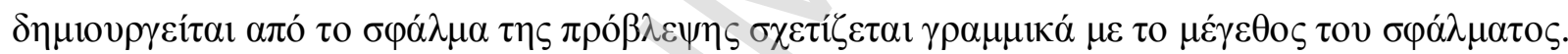

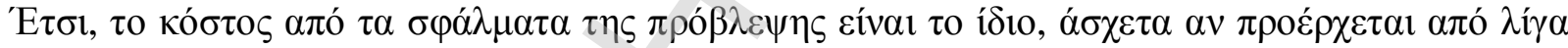

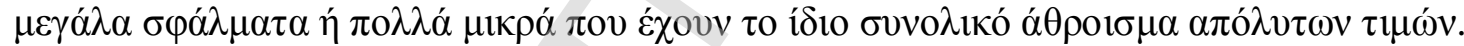

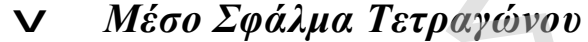

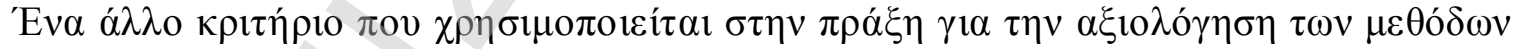

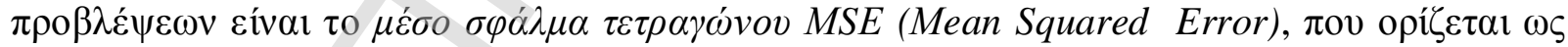

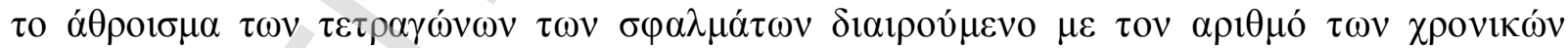

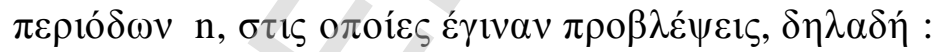

$$
M S E=1 / n \sum_{t=1}^{n}\left(Y_{t}-Y_{t}^{\wedge}\right)^{2}=\sum_{t=1}^{n} e_{t}^{2}
$$

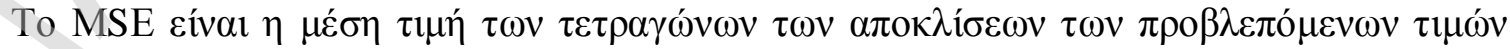

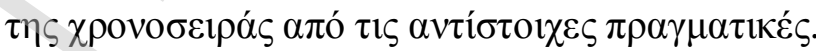

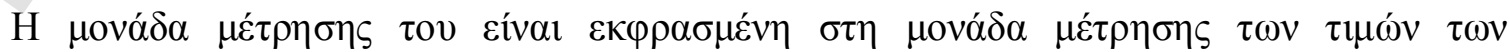

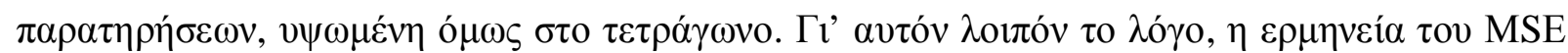




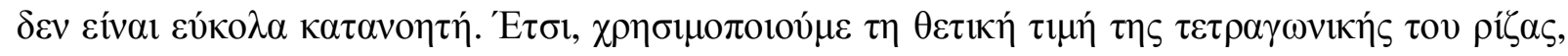

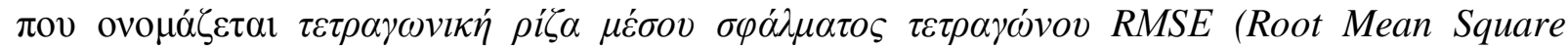
Error), $\delta \eta \lambda \alpha \delta \eta ́$ cíval :

$$
R M S E=\sqrt{M S E}=\sqrt{1 / n} \sqrt{k} \quad \Sigma \chi \varepsilon ́ \sigma \eta(2.6)
$$

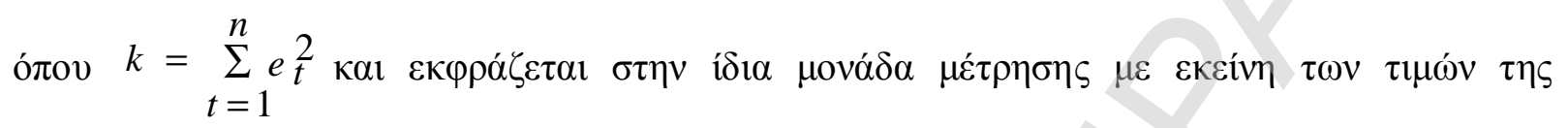

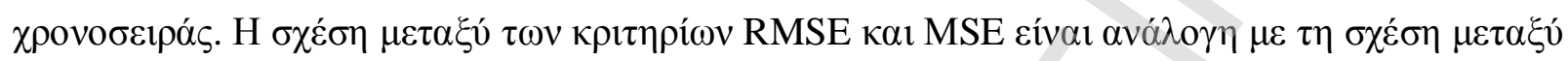

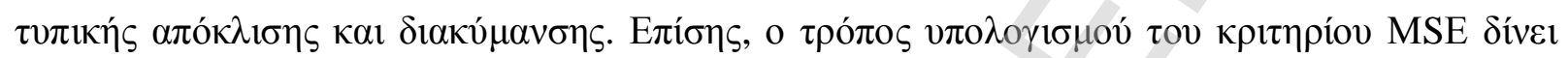

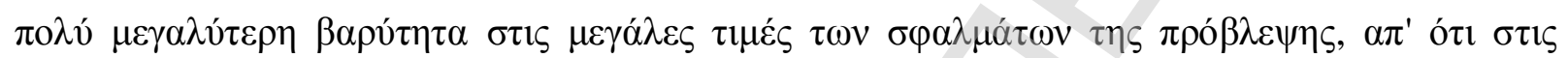

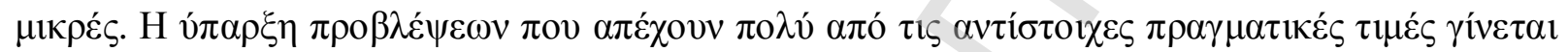

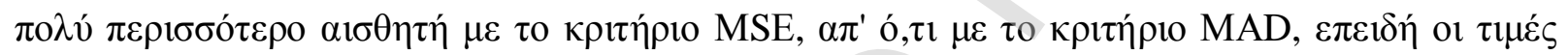

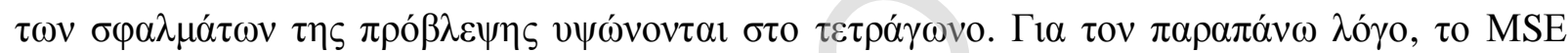

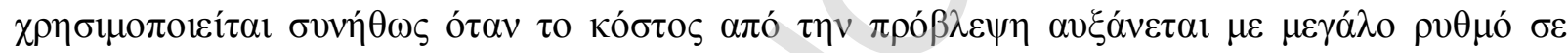

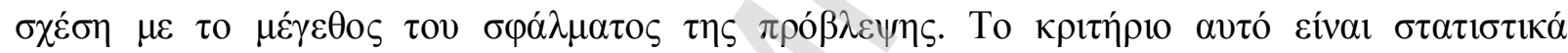

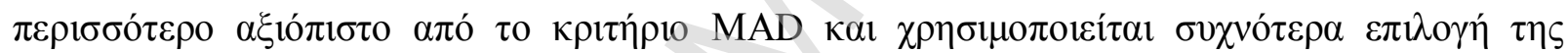

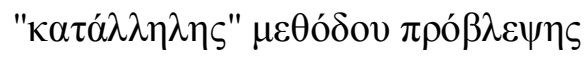

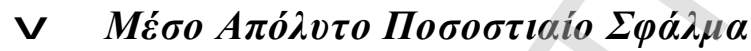

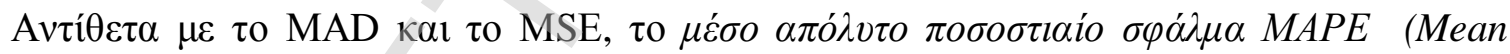

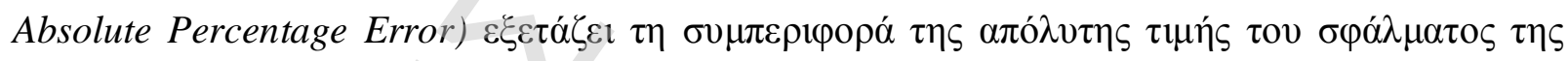

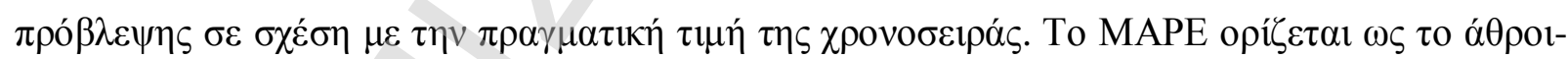

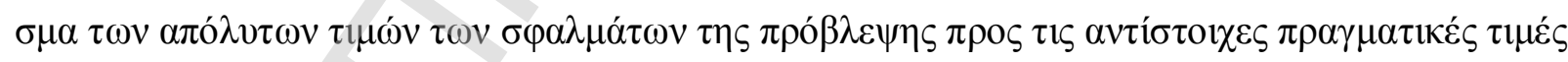

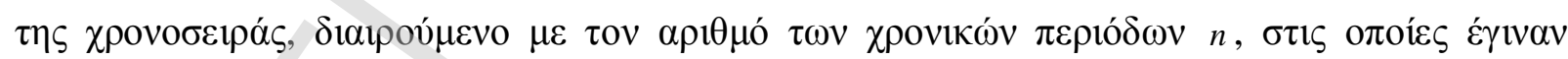
$\pi \rho \circ \beta \lambda \varepsilon \dot{\psi \varepsilon \varepsilon \varepsilon, \delta} \delta \eta \alpha \delta \eta \dot{~: ~}$

$$
\left.M A P E=1 / n \sum_{t=1}^{n}\left|\left(Y_{t}-Y_{t}^{\wedge}\right)\right| / Y_{t}\right)=1 / n \sum_{t=1}^{n}\left(\left|e_{t}\right| / Y_{t}\right)
$$

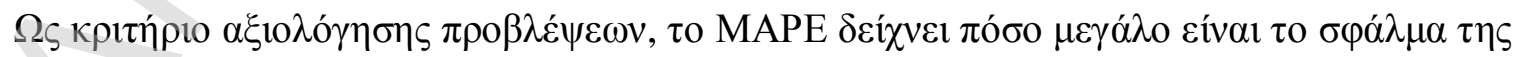

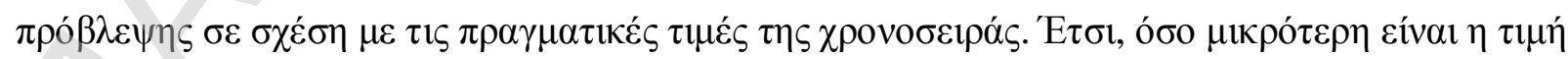

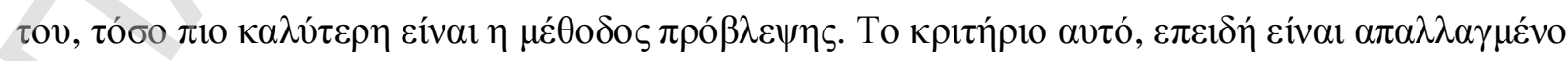

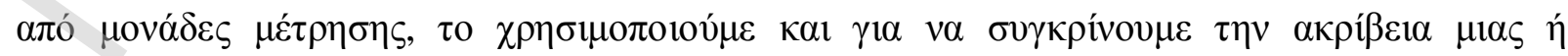

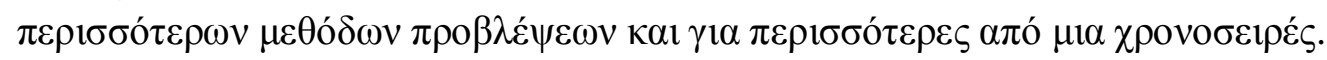




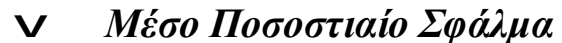

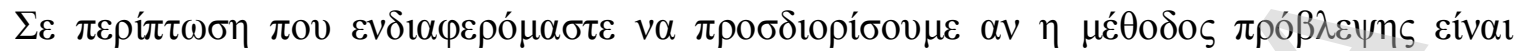

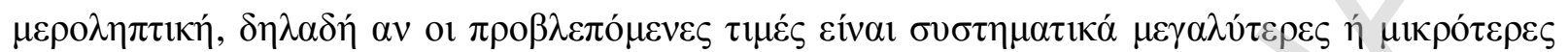

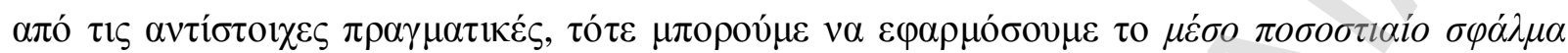

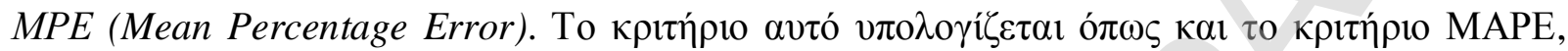

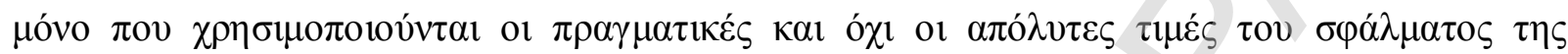
$\pi \rho o ́ \beta \lambda \varepsilon \psi \eta \varsigma, \delta \eta \lambda \alpha \delta \eta ́$ :

$$
\left.\left.M P E=1 / n \sum_{t=1}^{n}\left(Y_{t}-Y_{t}^{\wedge}\right) / Y_{t}\right)=1 / n \sum_{t=1}^{n}\left(e_{t}\right) / Y_{t}\right) \quad \Sigma \chi \dot{\varepsilon} \sigma \boldsymbol{\eta}(\mathbf{2 . 8})
$$

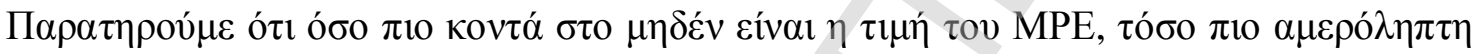

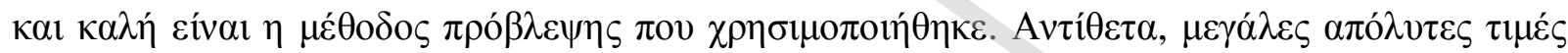

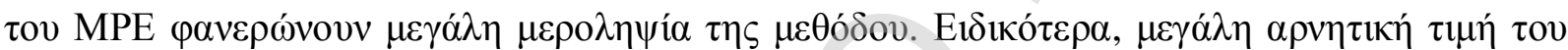

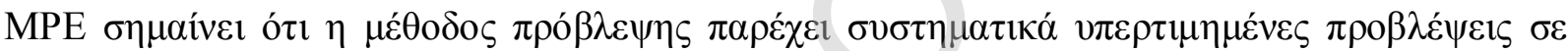

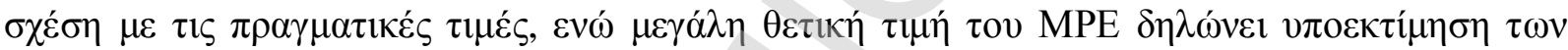

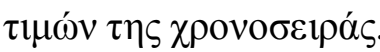

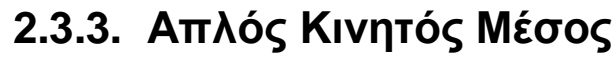

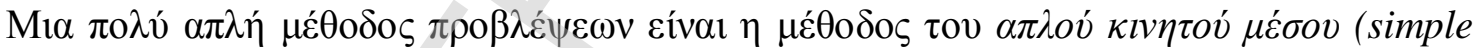

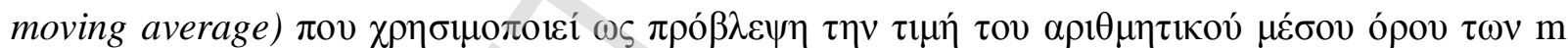

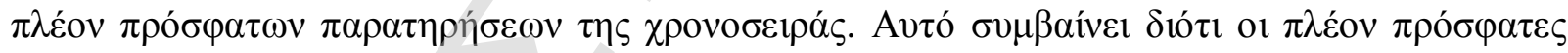

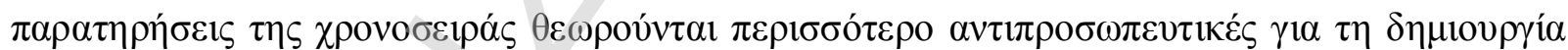

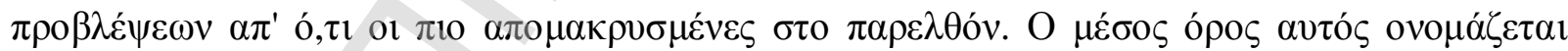

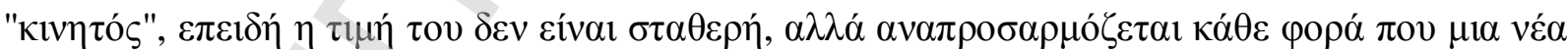

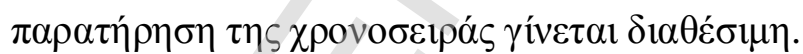

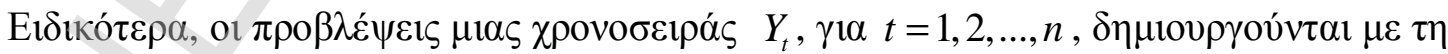

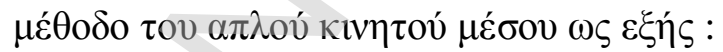

$$
Y_{t+1}^{\wedge}=M_{t+1}=1 / m \sum_{j=1}^{m} Y_{t-j+1}=1 / m\left(Y_{t}+Y_{t-1}+Y_{t-2} \cdots . . Y_{t-m+1}\right)
$$

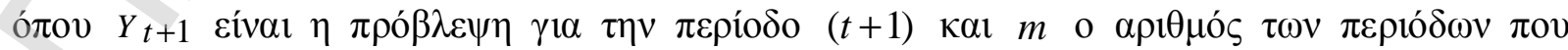

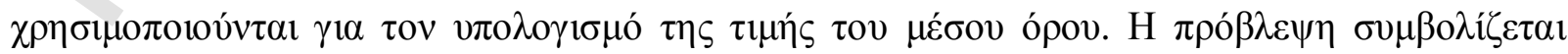

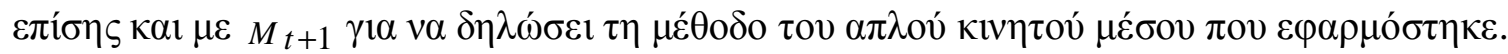




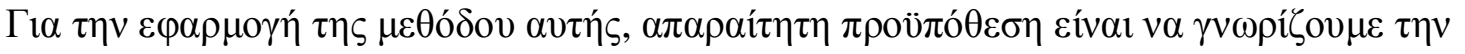

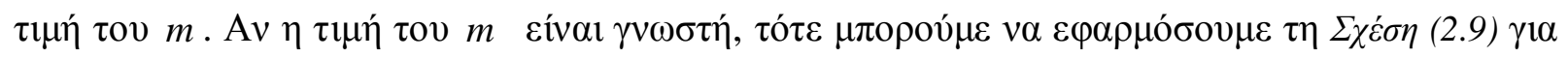

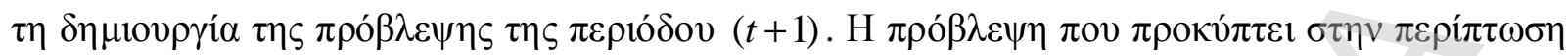

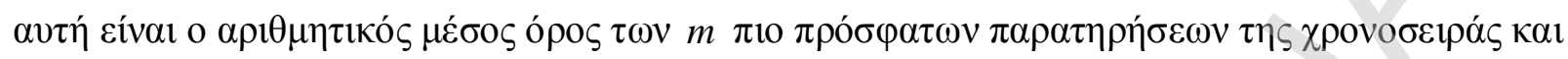

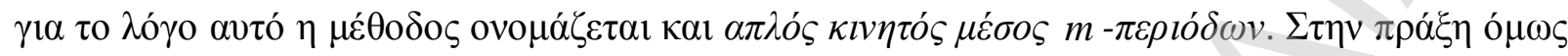

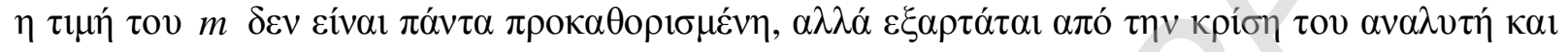

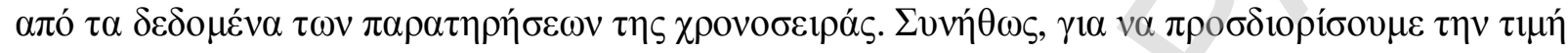

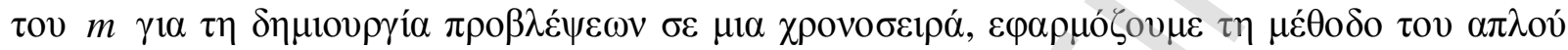

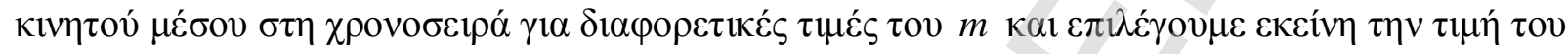

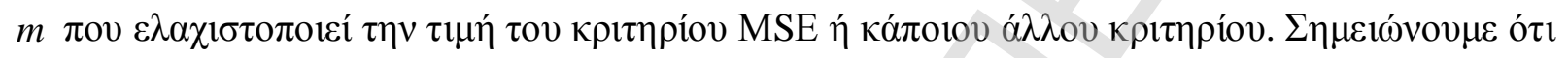

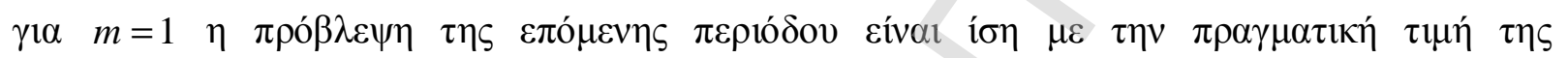

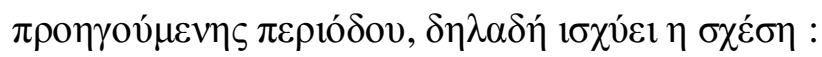

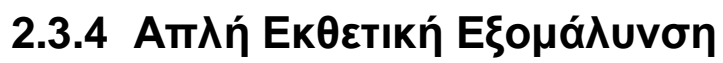

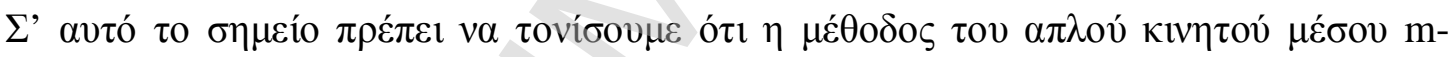

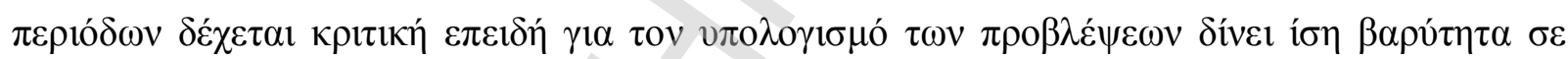

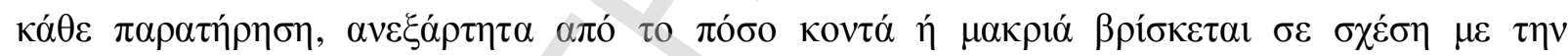

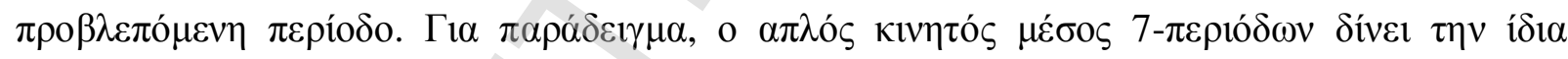

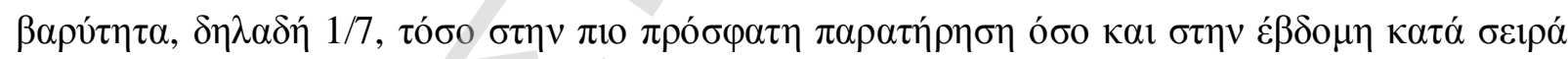
$\pi 10$ a

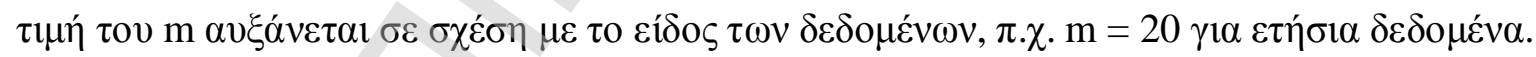

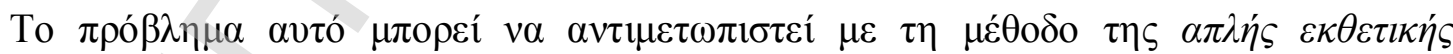

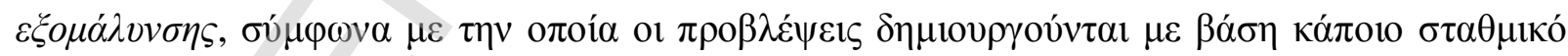

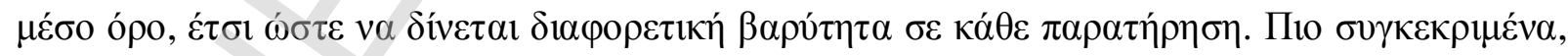

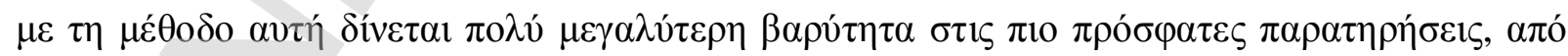

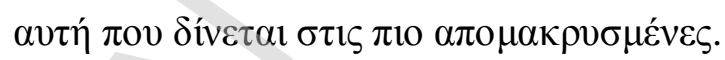

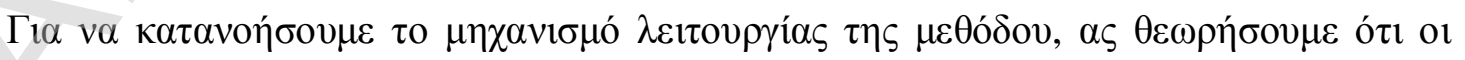

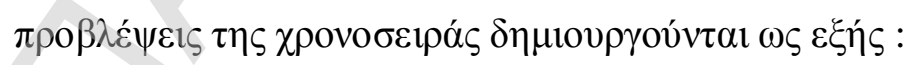

$$
\hat{Y}_{t+1}=a Y_{t}+a(1-a) Y_{t-1}+a(1-a)^{2} Y_{t-2}+\ldots \ldots .
$$




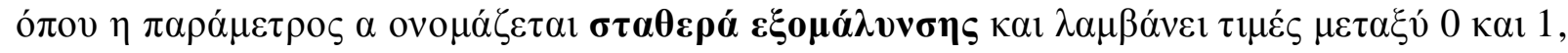
$\delta \eta \lambda \alpha \delta \eta ́$ : $\quad 0<a<1$

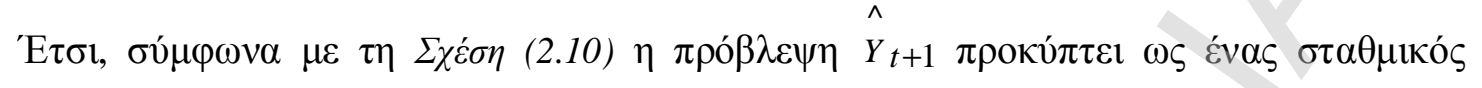

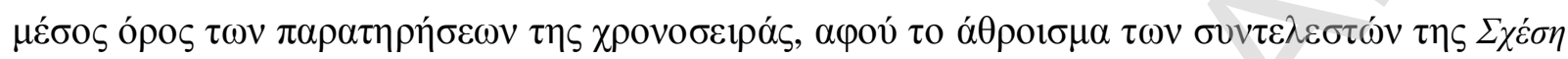

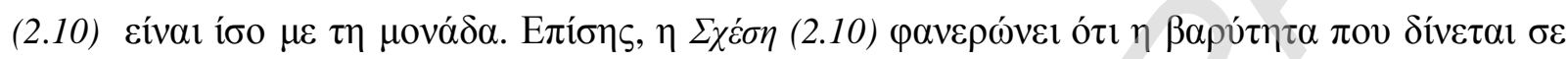

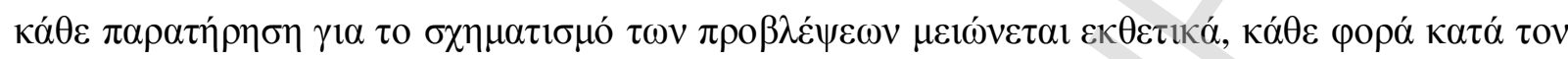

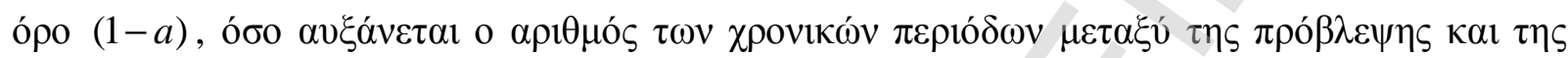

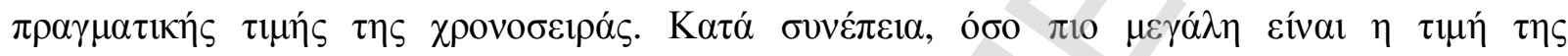

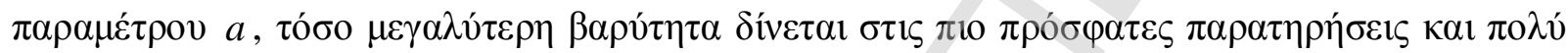

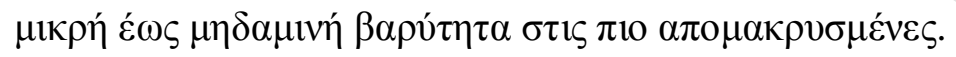

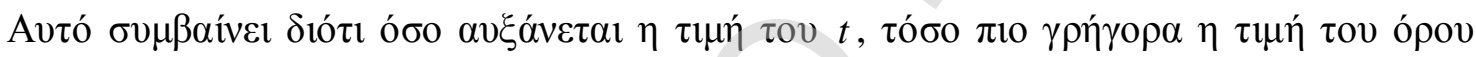

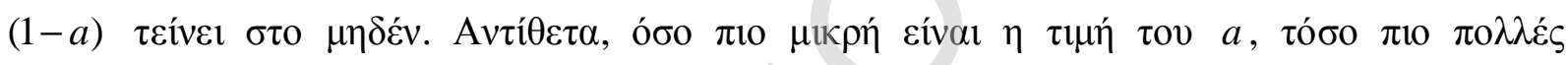

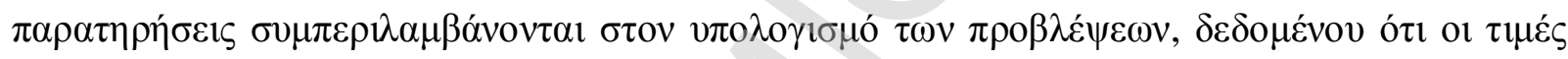

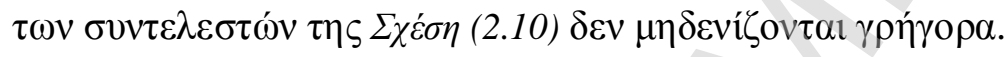

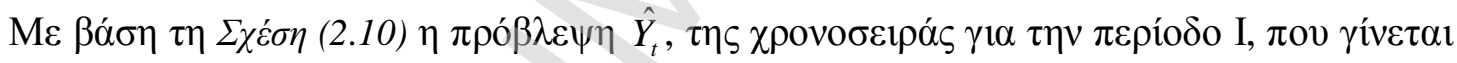

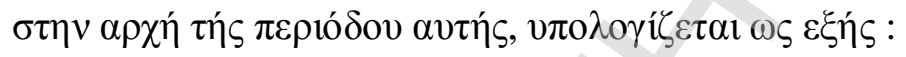

$$
\hat{Y}_{t}=a Y_{t-1}+a(1-a) Y_{t-2}+a(1-a)^{2} Y_{t-3}+\ldots \ldots
$$

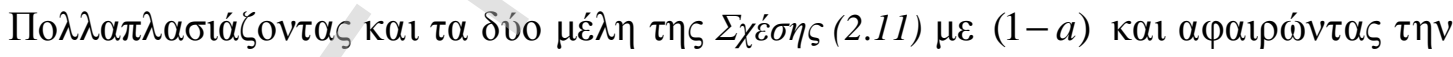

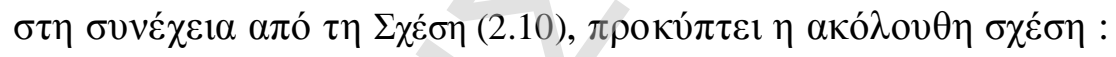

$$
\hat{Y}_{t+1}=a Y_{t}+(1-a) \hat{Y}_{t}
$$

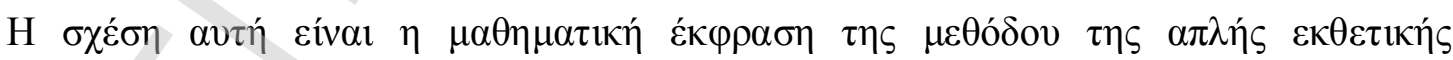

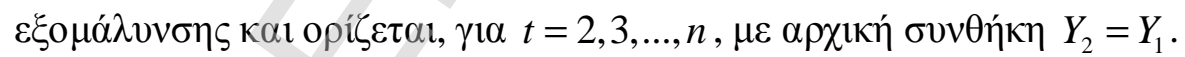

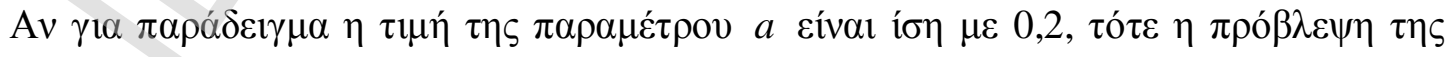

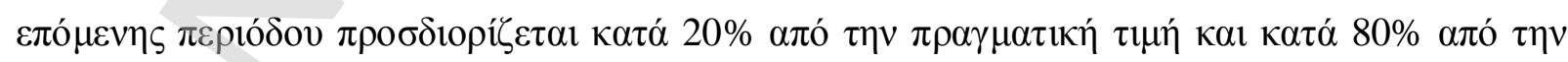

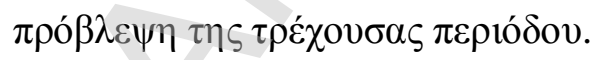

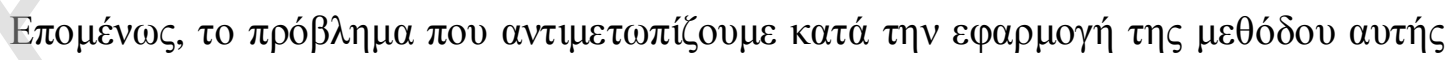

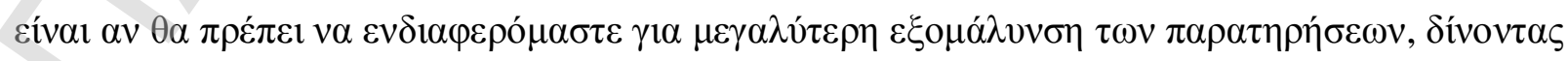

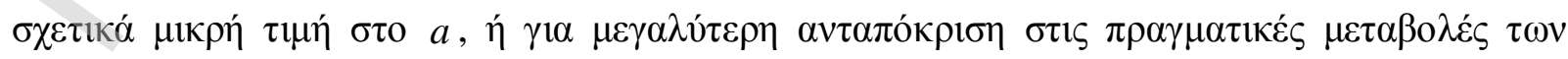

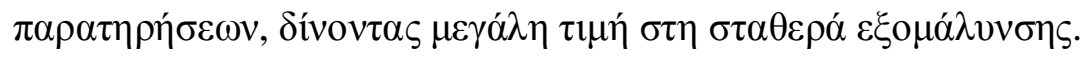




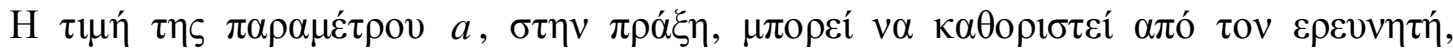

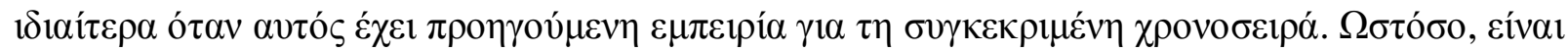

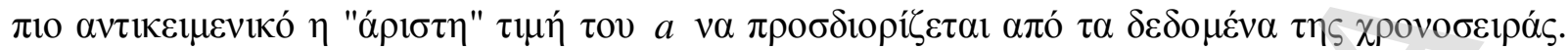

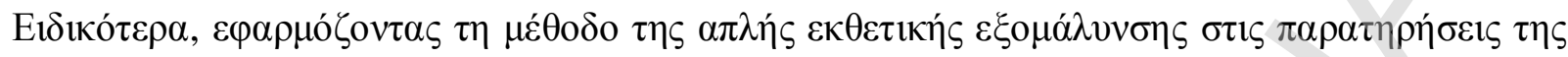

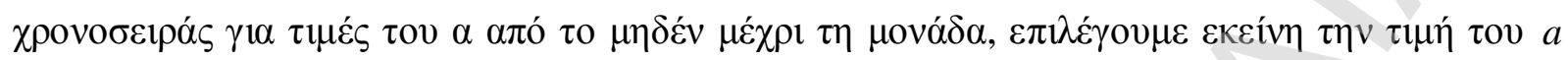

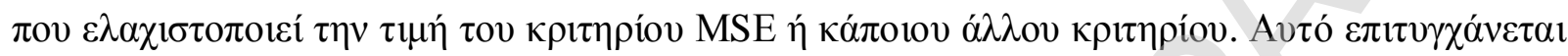

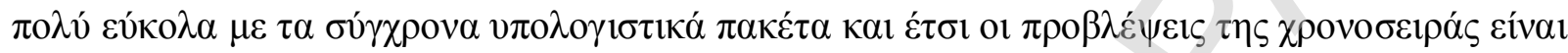

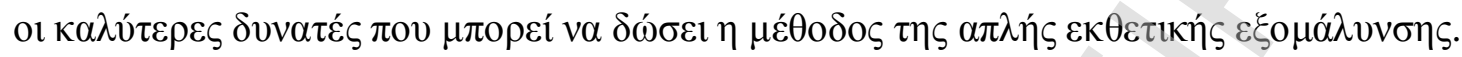

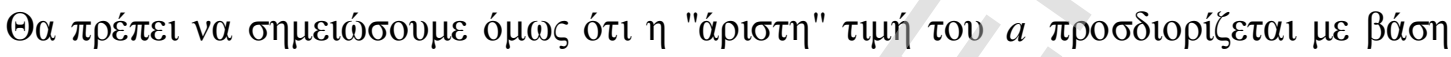

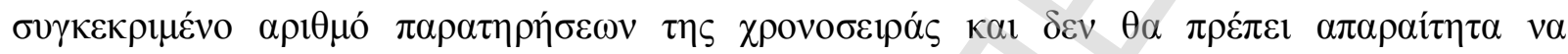

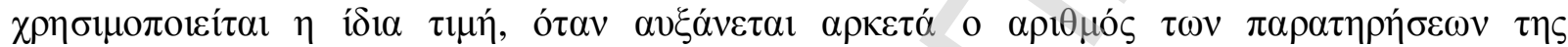

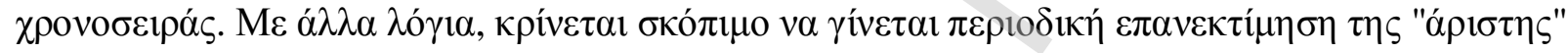

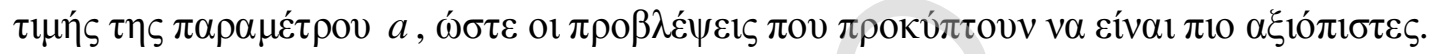

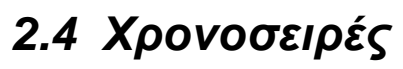

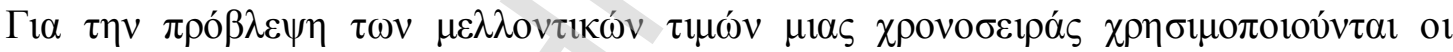

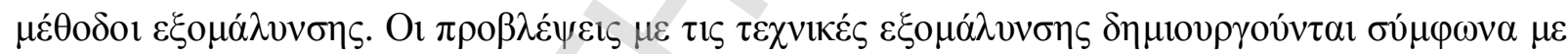

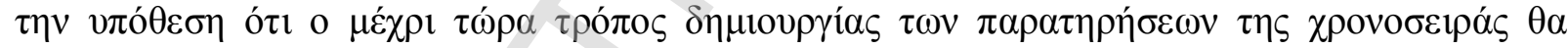

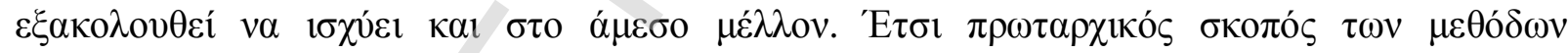

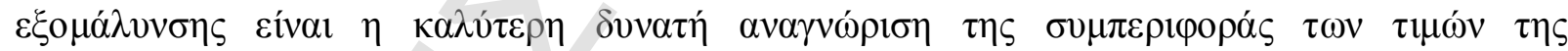

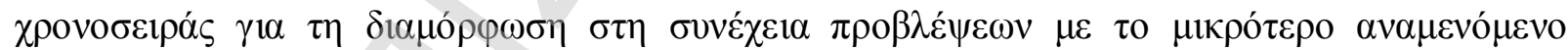

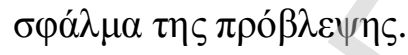

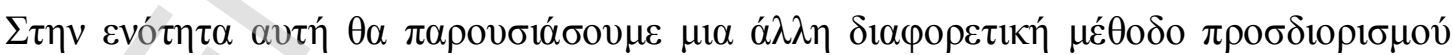

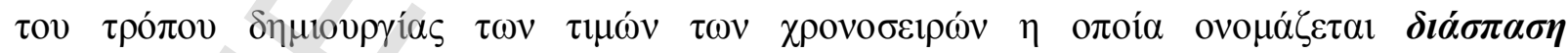

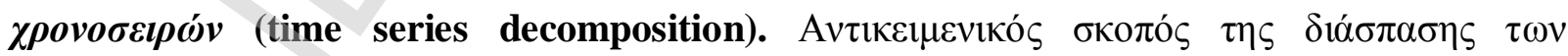

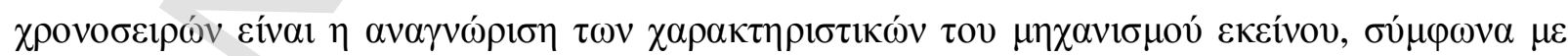

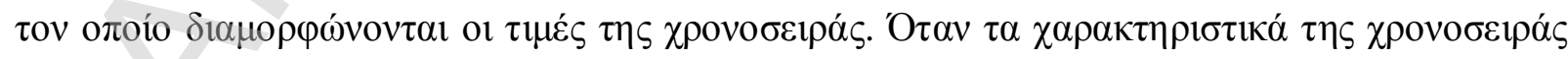

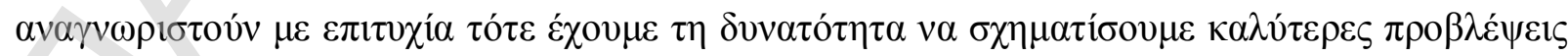

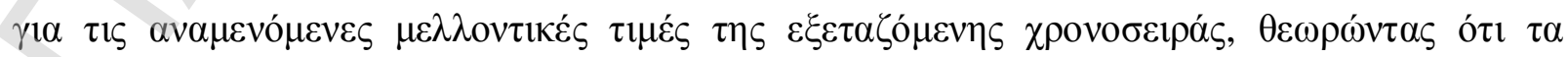

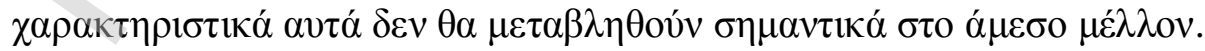




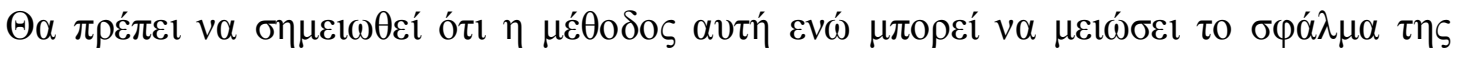

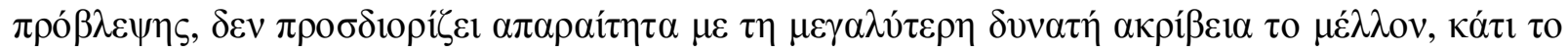

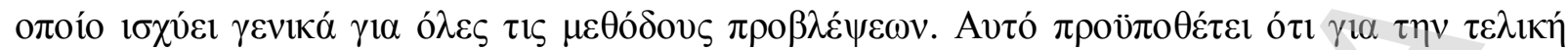

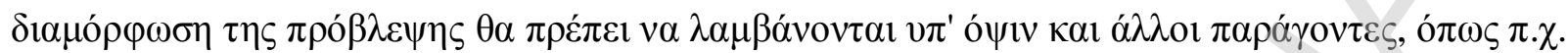

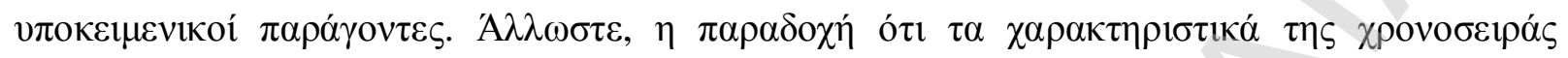

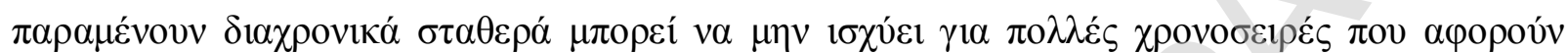

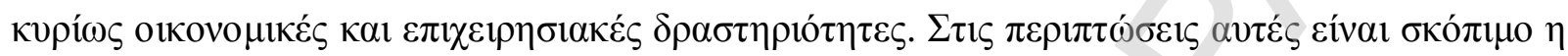

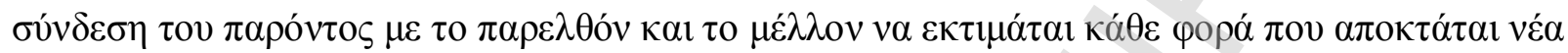

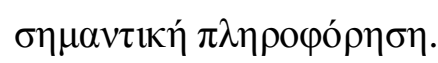

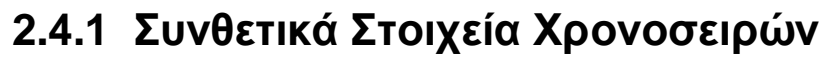

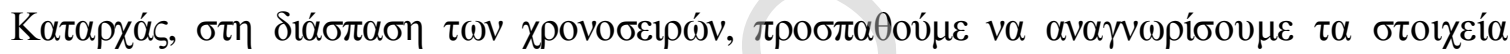

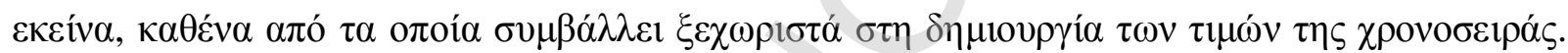

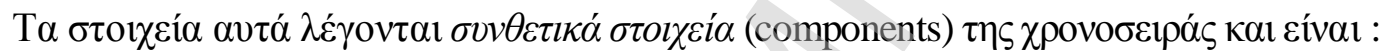

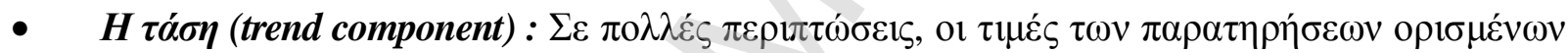

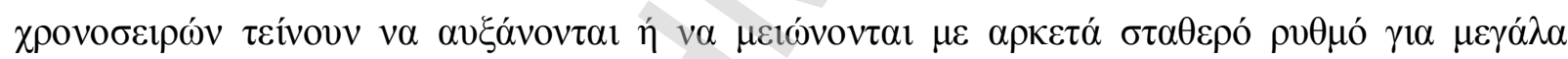

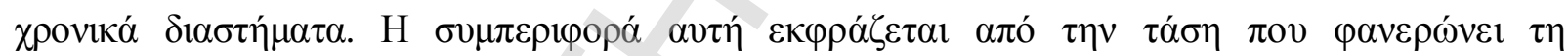

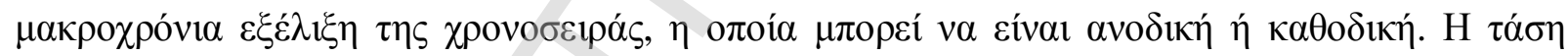

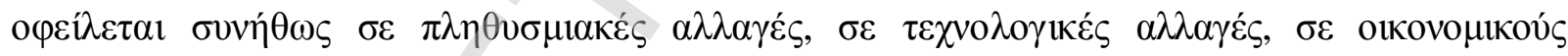

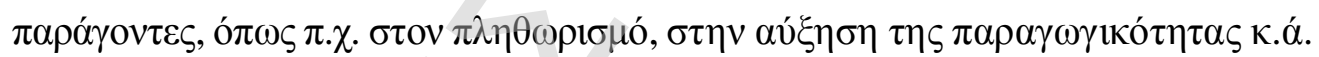

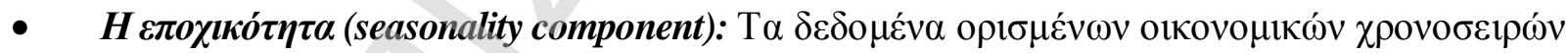

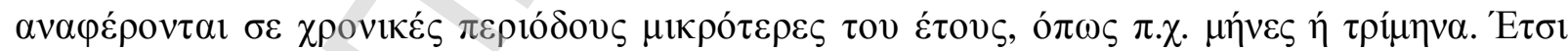

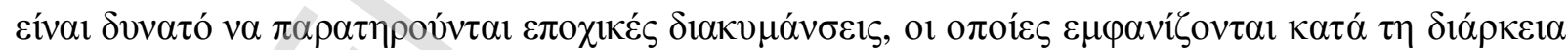

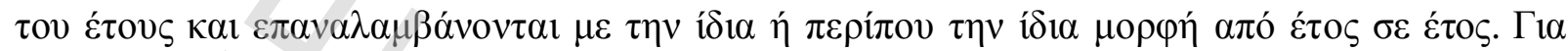

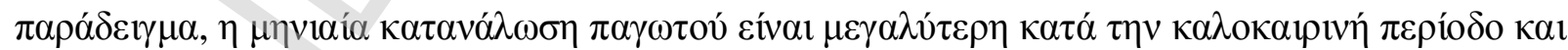

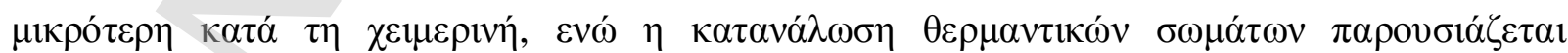

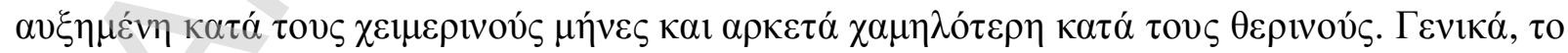

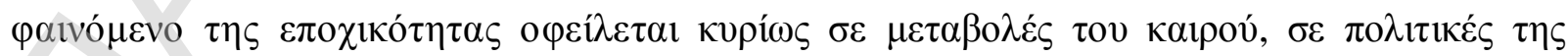

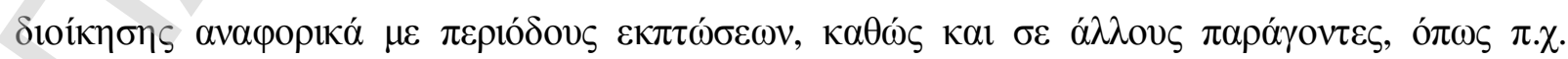

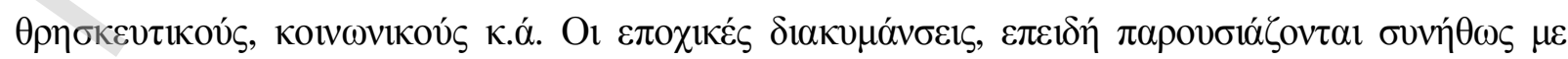

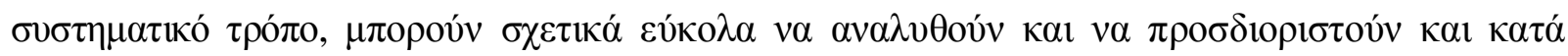




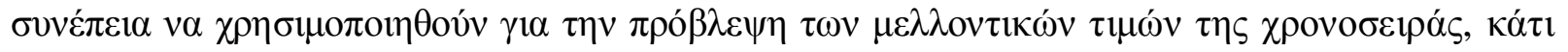

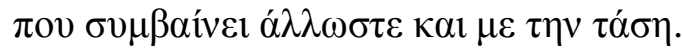

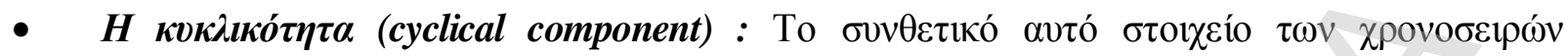

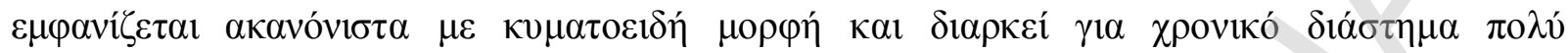

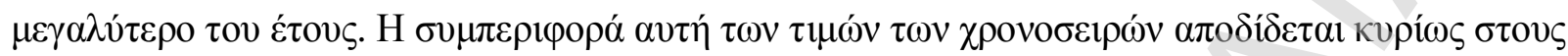

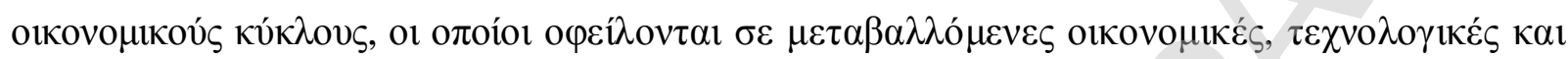

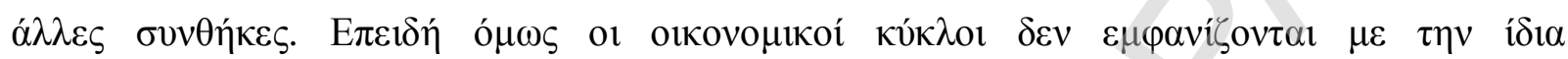

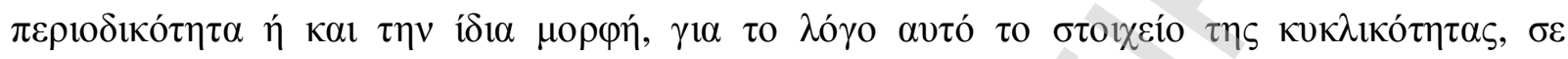

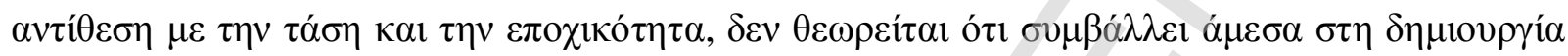

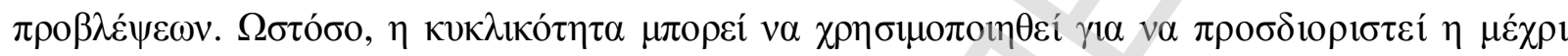

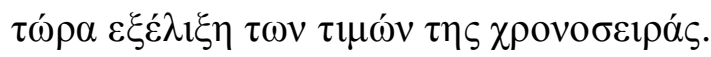

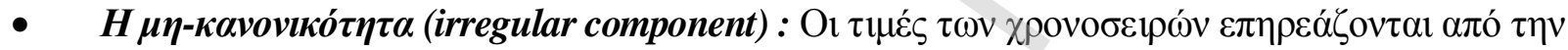

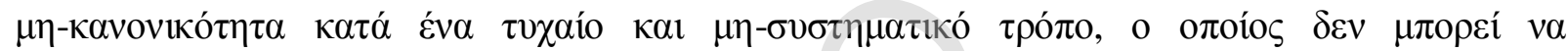

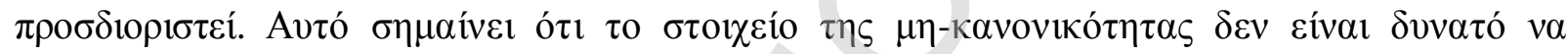

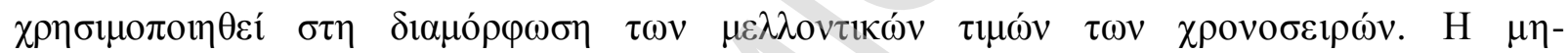

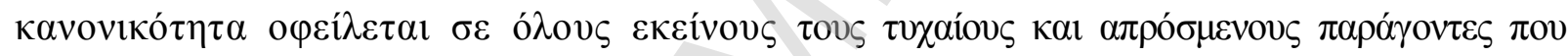

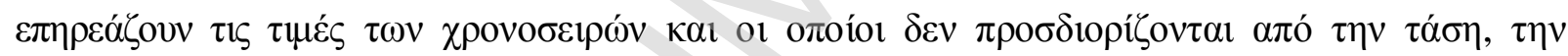

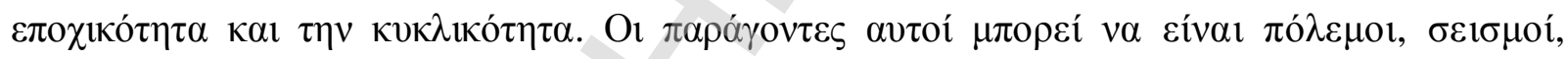

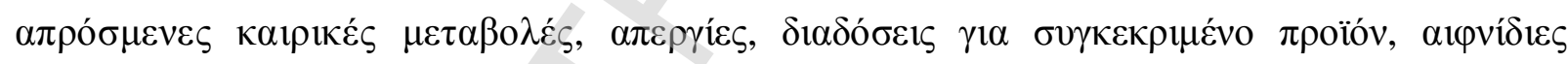

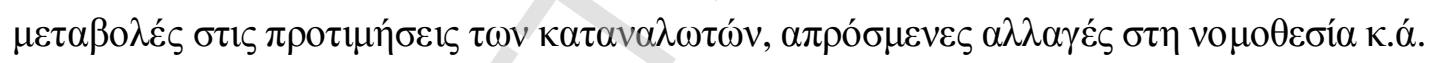

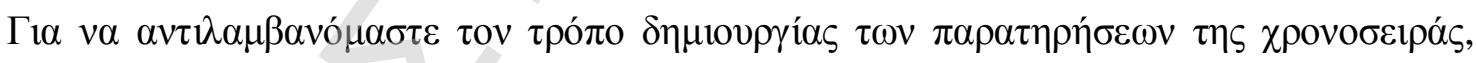

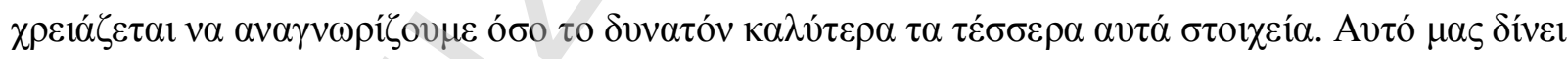

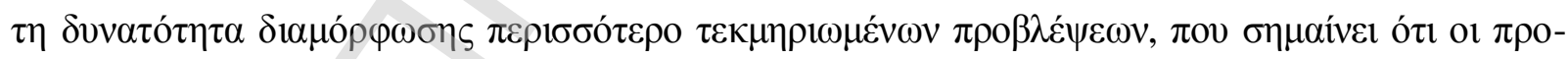

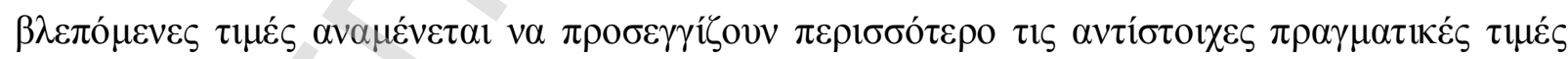

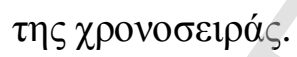

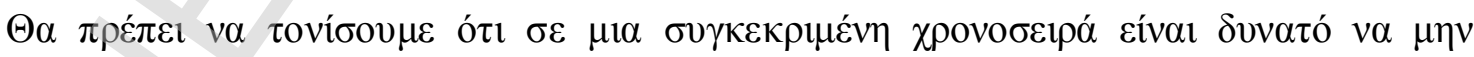

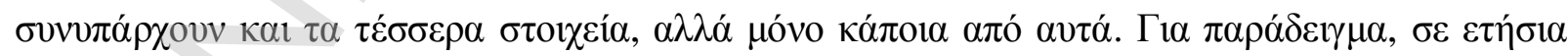

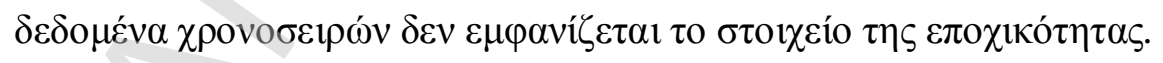

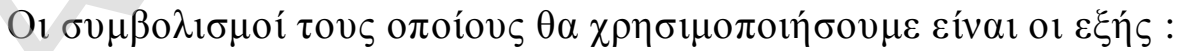

$\Gamma \imath \alpha \quad \mathrm{t}=1,2, \ldots, \eta \dot{\varepsilon} \chi 00 \mu \varepsilon:$

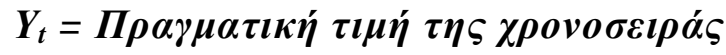

$$
\begin{aligned}
& T_{t}=T \alpha \dot{\sigma} \sigma \eta
\end{aligned}
$$




$$
\begin{aligned}
& S_{t}=E \pi \sigma \chi ๘ \kappa o ́ \tau \eta \tau \alpha \\
& C_{t}=K v \kappa \lambda \iota \kappa o ́ \tau \eta \tau \alpha \\
& I_{t}=M \eta-\kappa \alpha v o v i \kappa o ́ \tau \eta \tau \alpha
\end{aligned}
$$

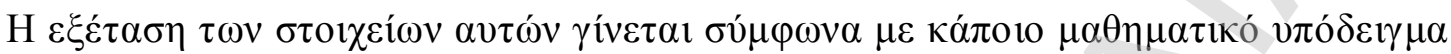

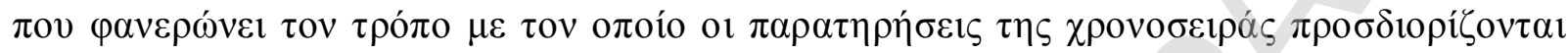

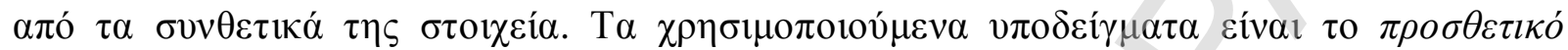

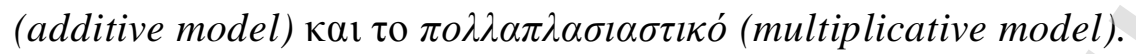

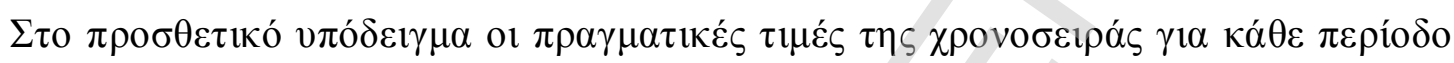

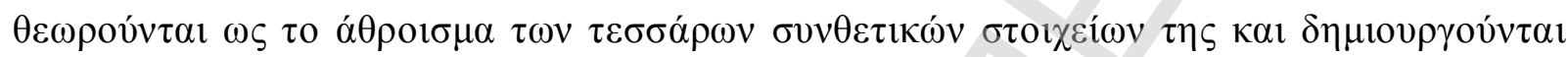

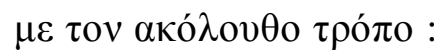

$$
m_{6}=(40+44+41) / 3=41.67
$$

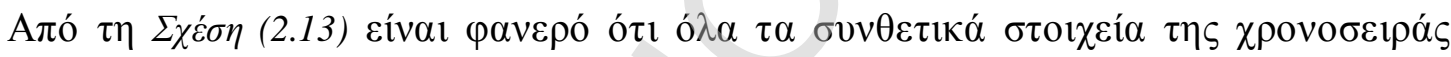

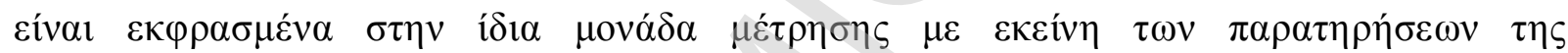

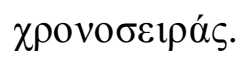

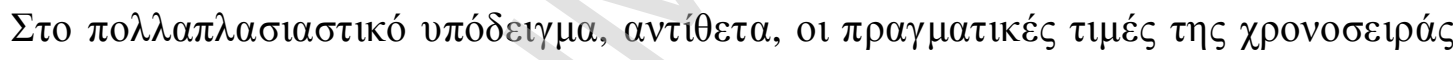

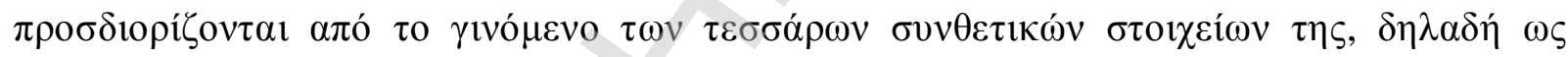
$\alpha \kappa \circ \lambda \circ \theta_{\theta \omega \varsigma}$ :

$$
Y_{t}=T_{t} \cdot S_{t} \cdot C_{t} \cdot I_{t} \quad \Sigma \chi \bar{\varepsilon} \sigma \eta(2.14)
$$

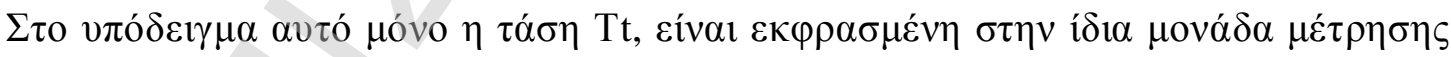

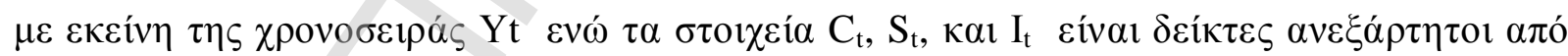
$\mu о \vee \alpha ́ \delta \varepsilon \varsigma \mu \varepsilon ́ \tau \rho \eta \sigma \eta \varsigma$.

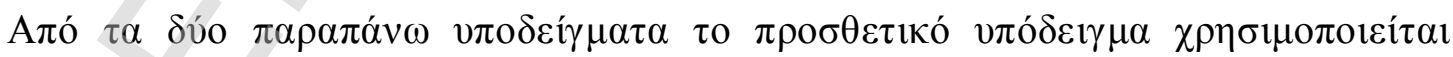

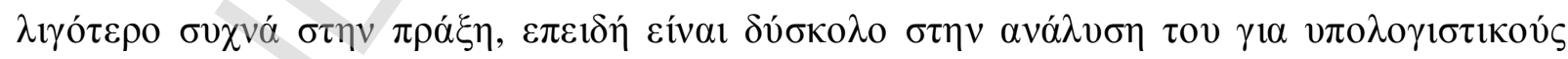

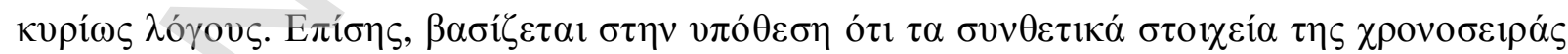

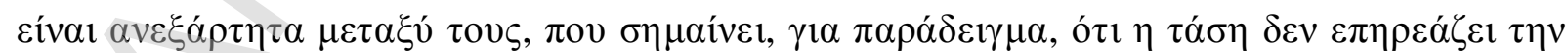

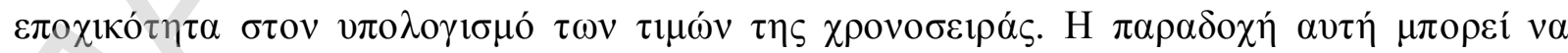

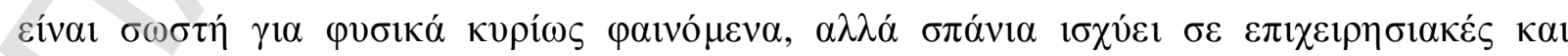

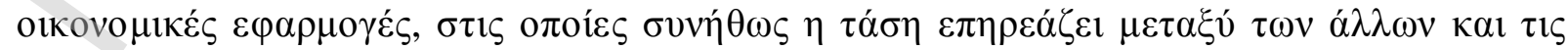

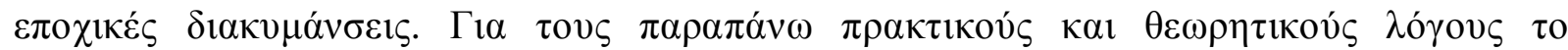




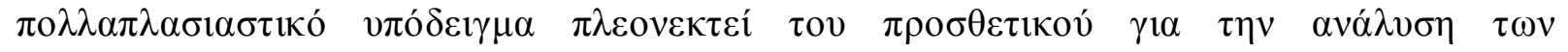

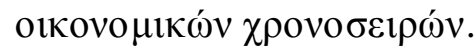

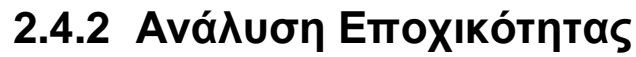

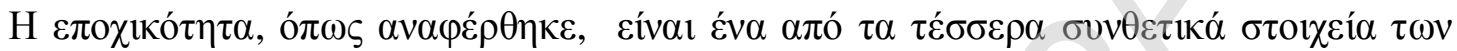

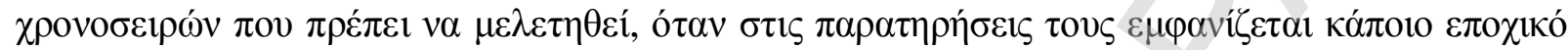

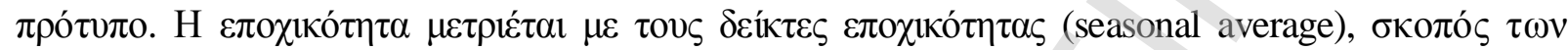

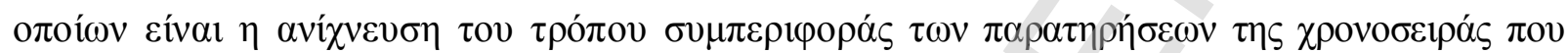

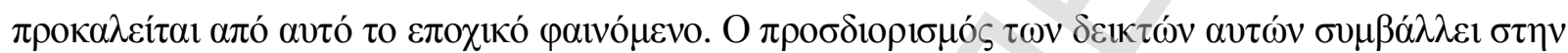

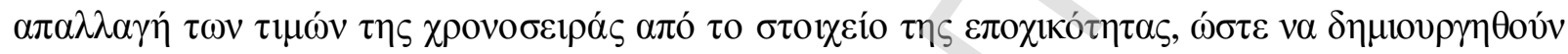

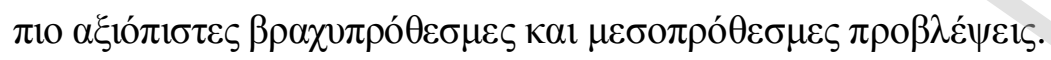

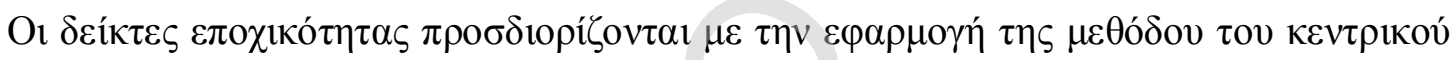

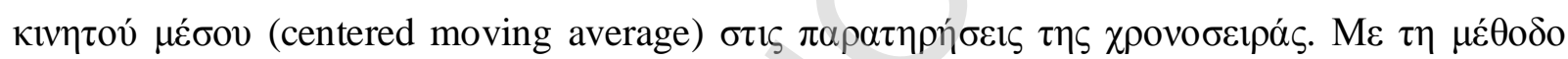

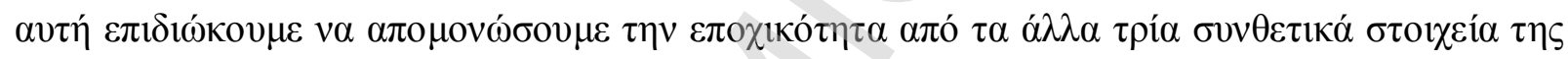

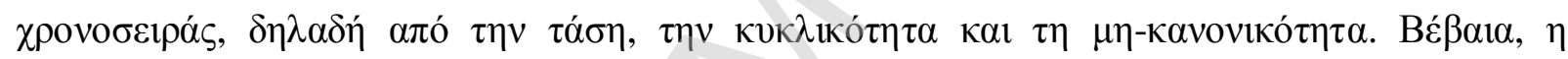

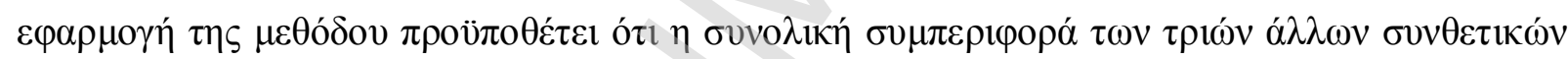

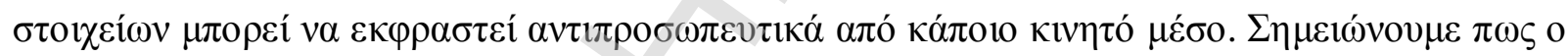

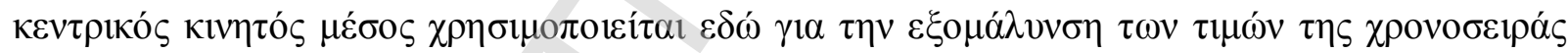

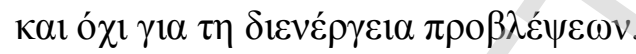

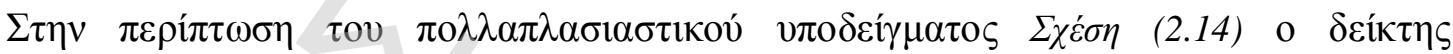

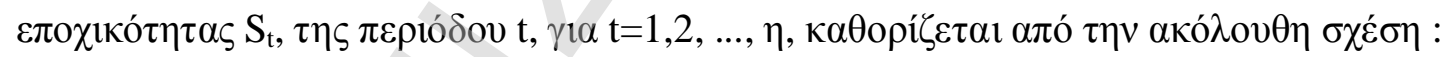

$$
S_{t}=\frac{Y_{t}}{C A_{t}}=\left(T_{t} S_{t} C_{t} I_{t}\right) / T_{t} C_{t} I_{t} \quad \Sigma \chi \dot{\varepsilon} \sigma \eta(2.15)
$$

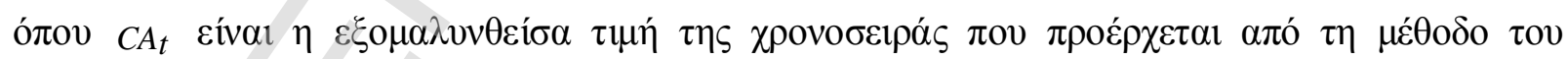

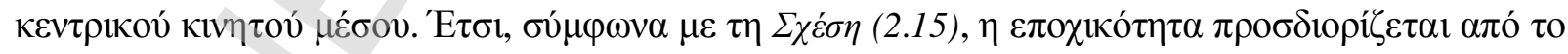

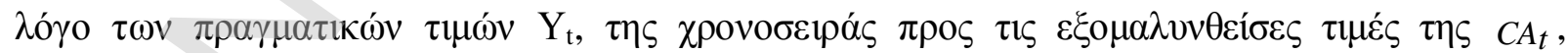

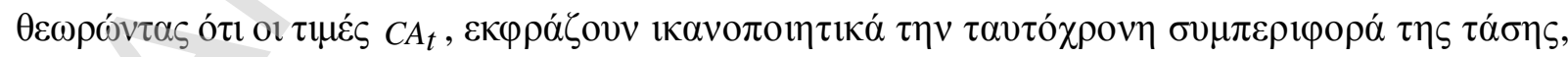

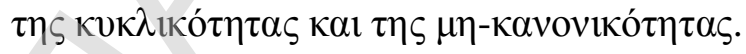




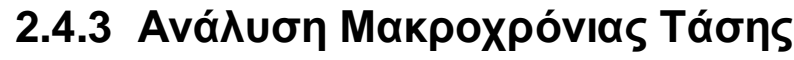

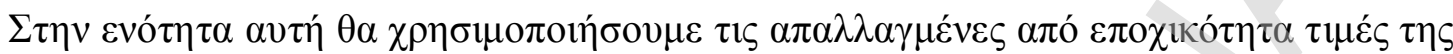

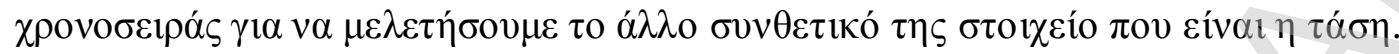

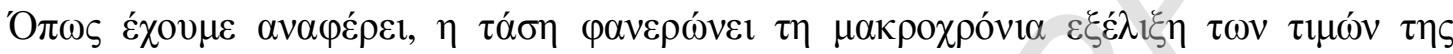

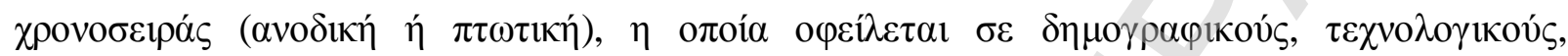

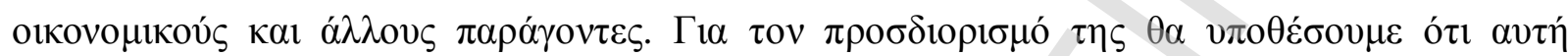

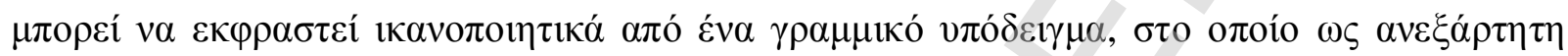

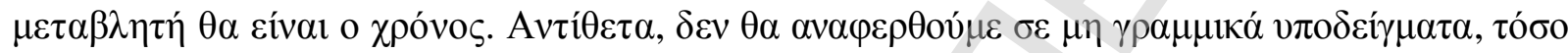

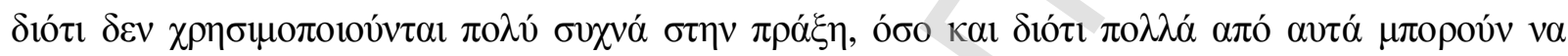

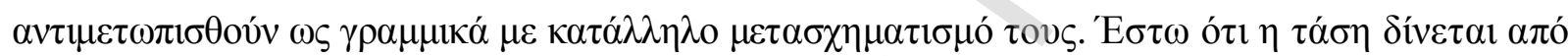

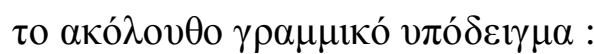

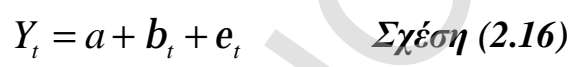

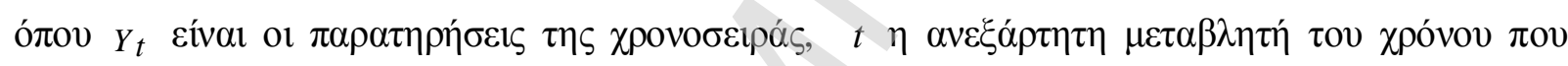

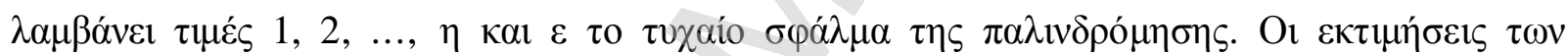

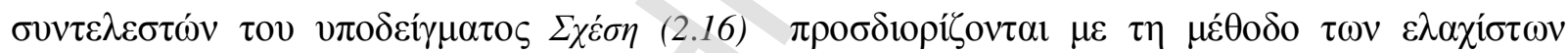
$\tau \varepsilon \tau \rho \alpha \gamma \omega ́ v \omega \nu$ OLS $\omega \varsigma \varepsilon \xi \dot{\eta} \varsigma$ :

$$
\begin{gathered}
\hat{\beta}=\frac{\left[n \sum_{t=1}^{n} t Y_{t}-\left(\sum_{t=1}^{n} t\right)\left(\sum_{t=1}^{n} Y_{t}\right)\right]}{n \sum_{t=1}^{n} t^{2}-\left(\sum_{t=1}^{n} t\right)^{2}} \\
\mathrm{Kat} \quad \hat{a}=\frac{1}{n} \sum_{t=1}^{n} Y_{t}-\hat{\beta} \frac{1}{n} \sum_{t=1}^{n} t
\end{gathered}
$$

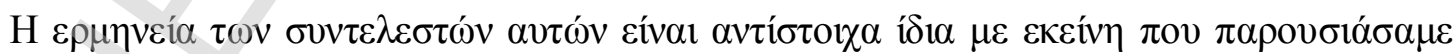

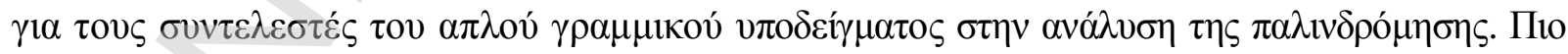

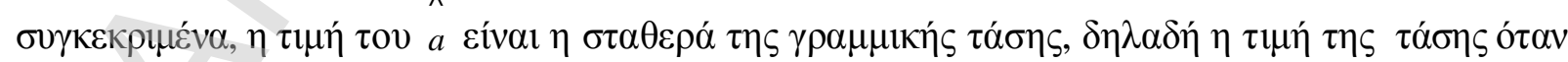

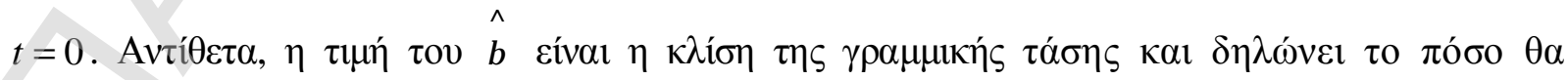

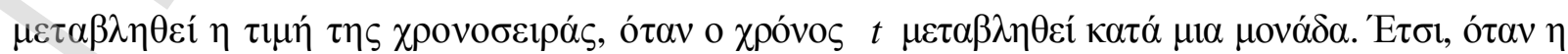




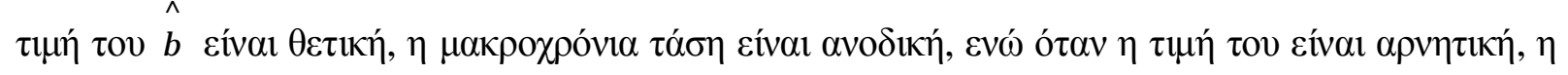

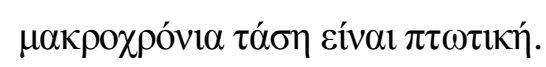

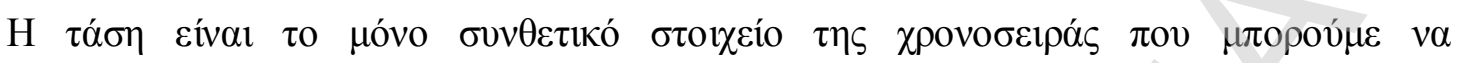

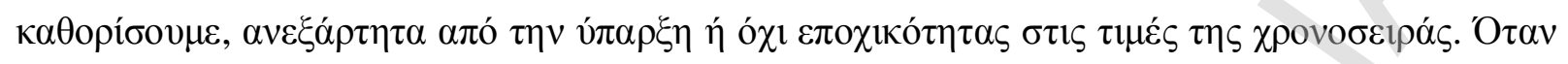

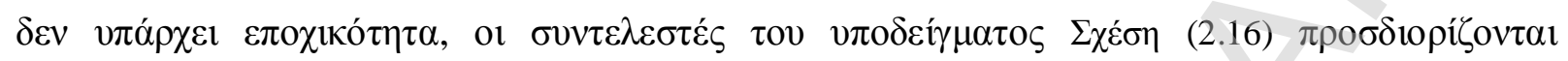

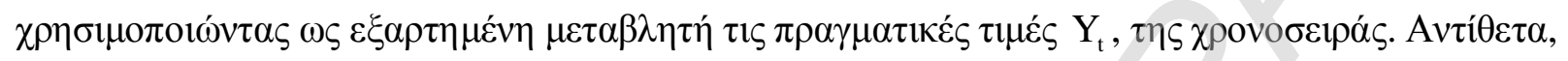

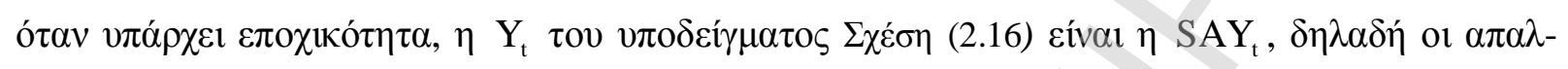

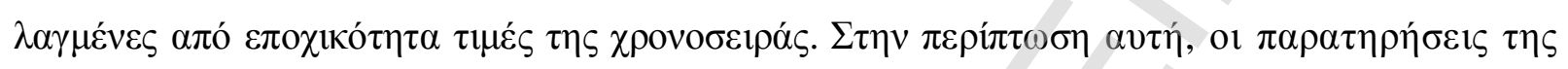

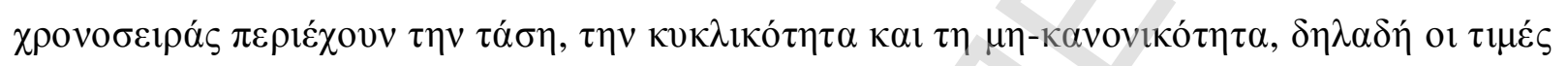
$\mathrm{SAY}_{\mathrm{t}}, \pi \rho \circ \sigma \delta 10 \rho i ́ \zeta o v \tau \alpha 1 \omega \varsigma$ :

$$
\mathrm{SAY}_{\mathrm{t}}=T_{t} \cdot C_{t} \cdot I_{t} \quad \Sigma \chi \varepsilon \dot{\sigma} \sigma \eta(2.19)
$$

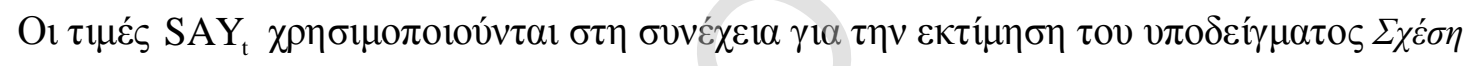

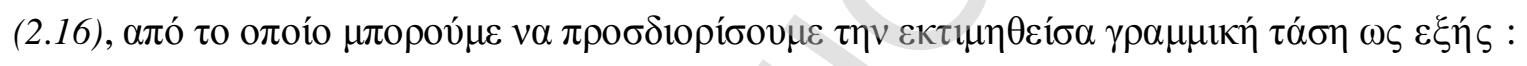

$$
T_{t}=\hat{a+\beta t} \quad \Sigma \chi \varepsilon \dot{\varepsilon} \sigma \eta(2.20)
$$

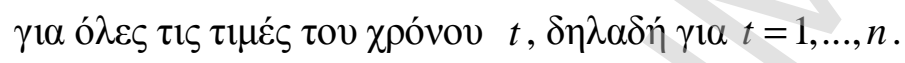




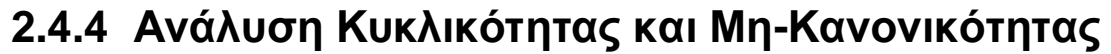

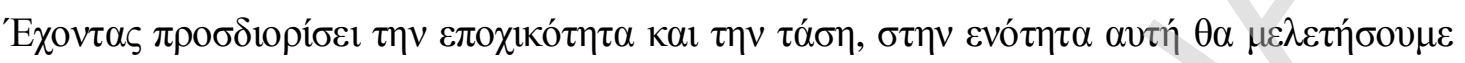

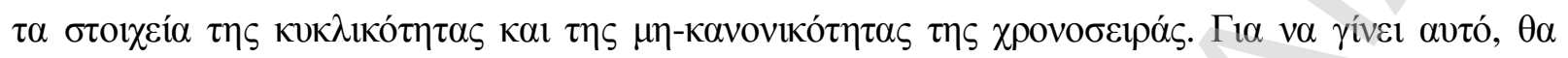

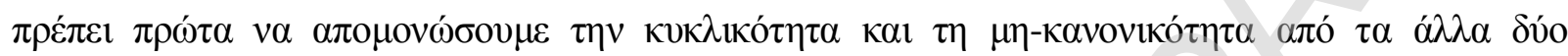

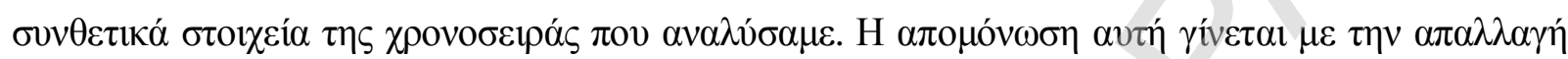

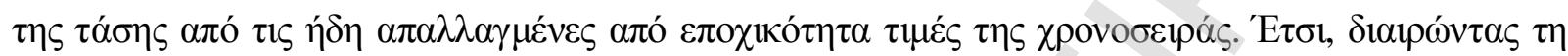

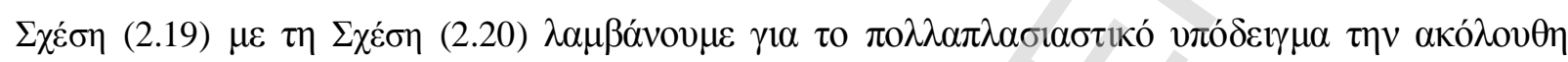
$\sigma \chi \varepsilon \dot{\varepsilon} \sigma$ :

$$
T A Y_{t}=\frac{S A Y_{t}}{T_{t}}=C_{t} I_{t},
$$

$\Sigma \chi \varepsilon ́ \sigma \eta(2.21)$

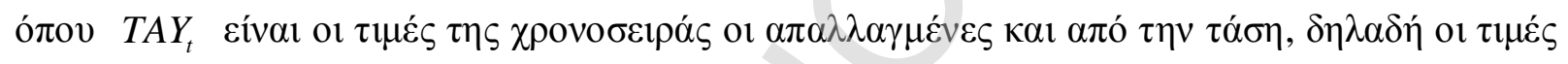

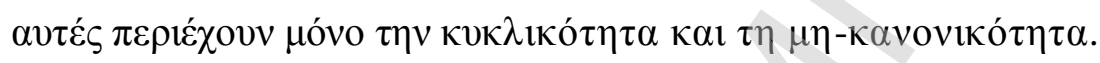

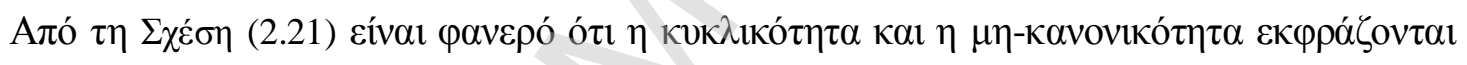

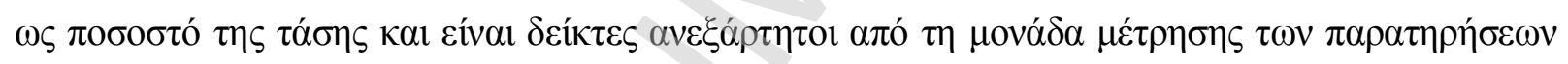

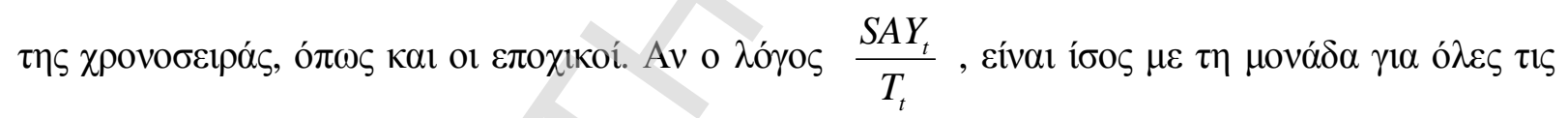

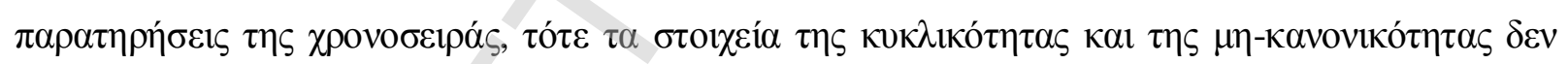

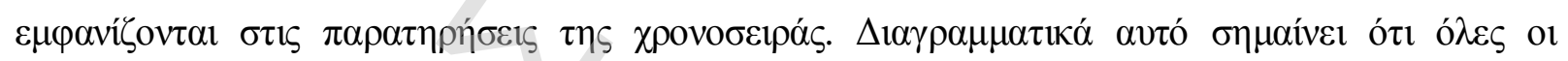

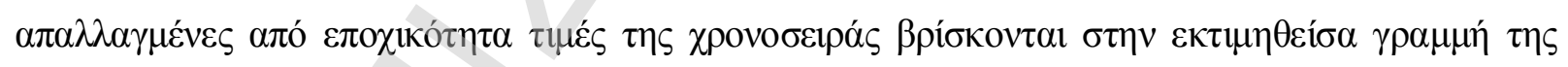

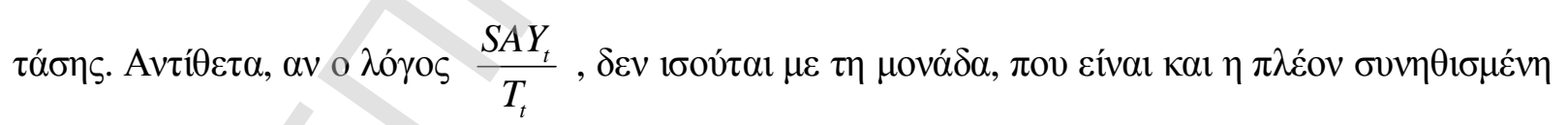

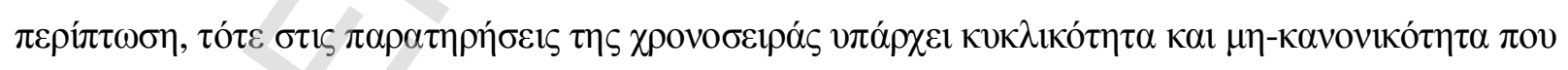

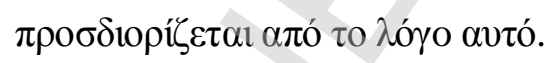

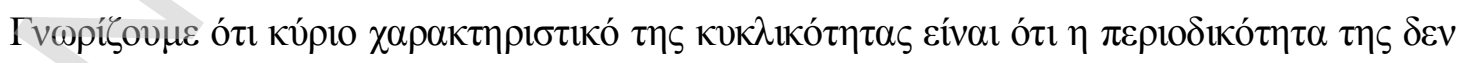

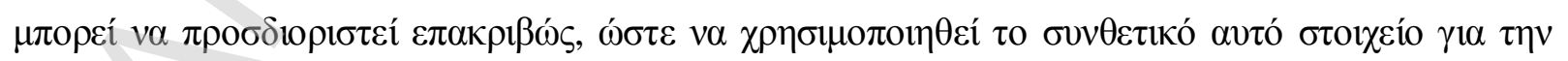

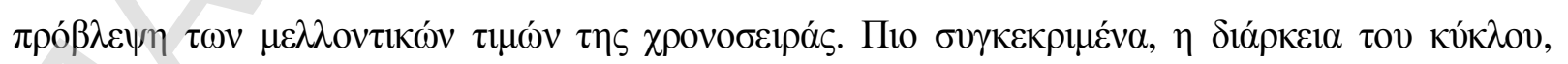

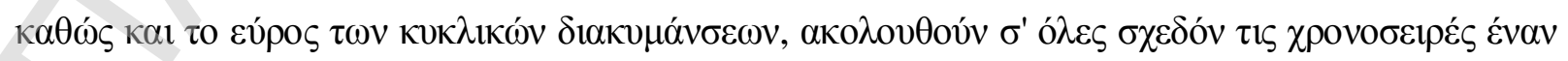

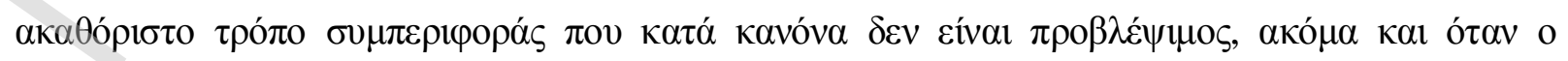

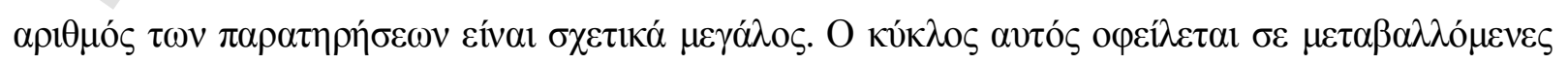




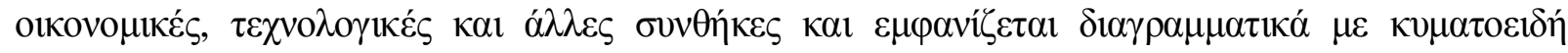

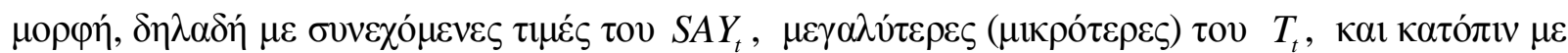

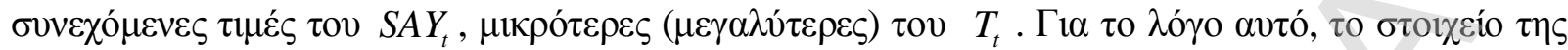

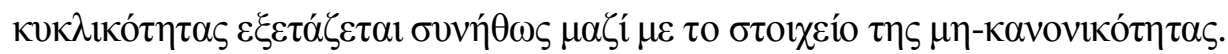

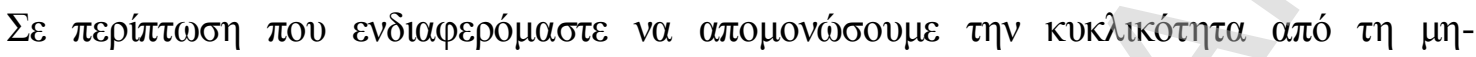

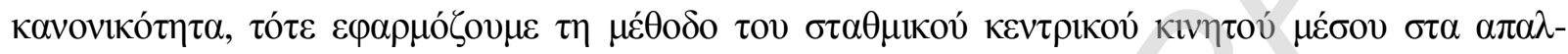

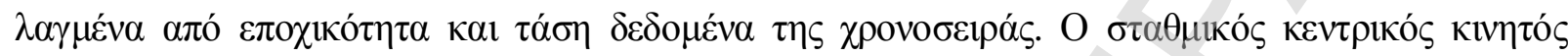

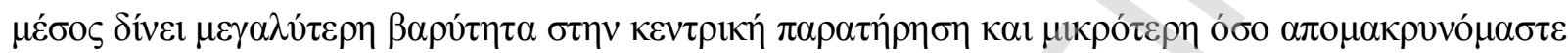

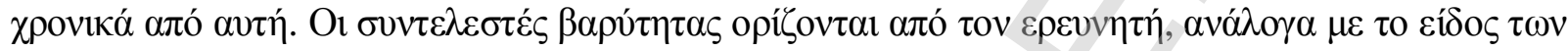

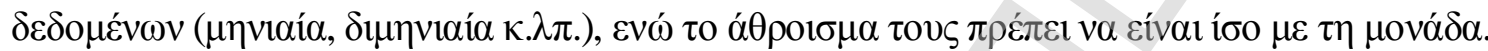

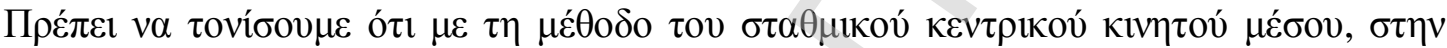

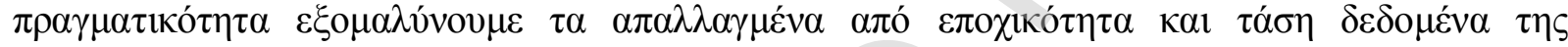

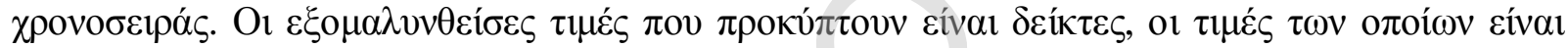

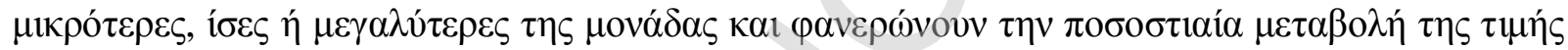

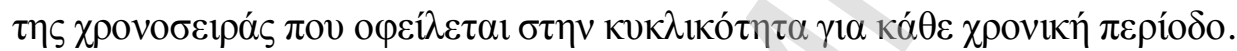

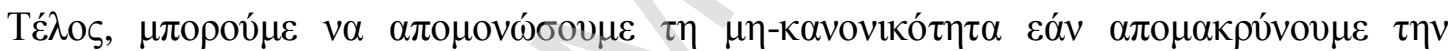

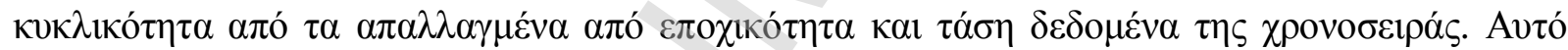

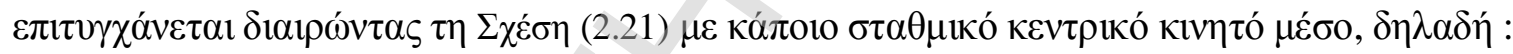

$$
C A Y_{t}=\frac{T A Y_{t}}{W A_{t}}=I_{t}
$$

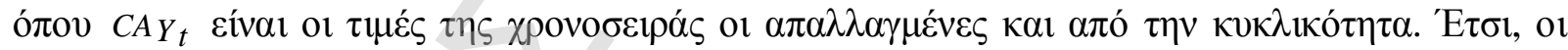

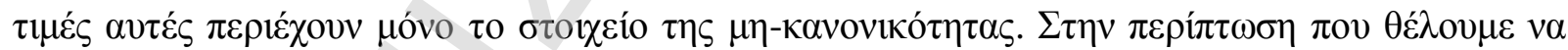

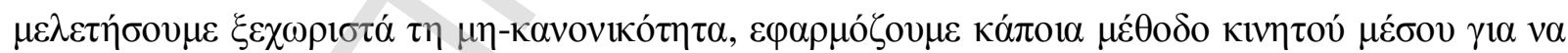

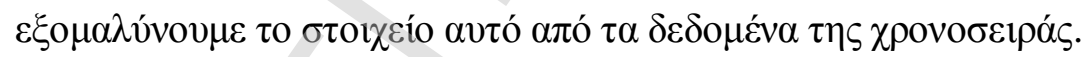

\section{$2.5 \Sigma \cup \mu \pi \varepsilon \rho \alpha ́ \sigma \mu \alpha T \alpha$}

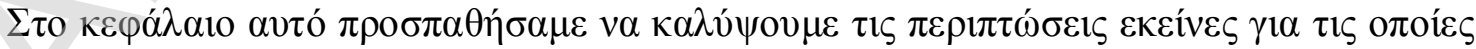

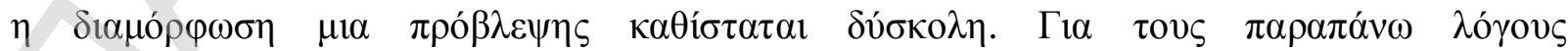

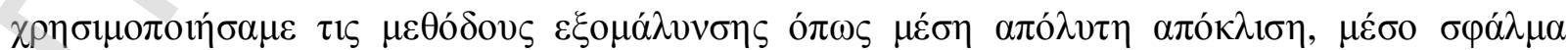

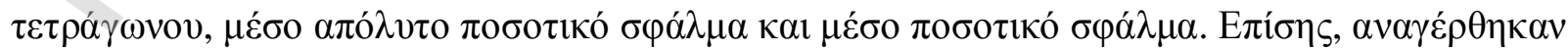

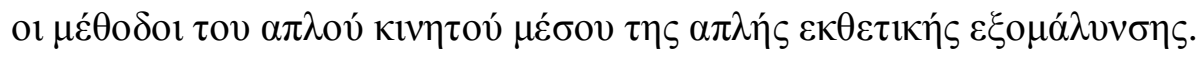




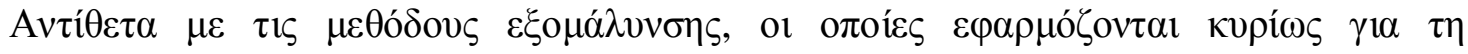

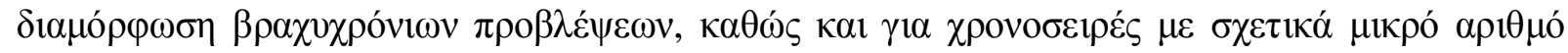

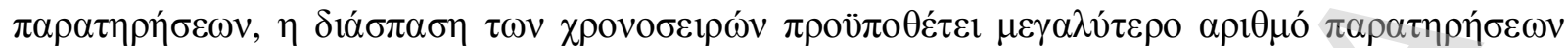

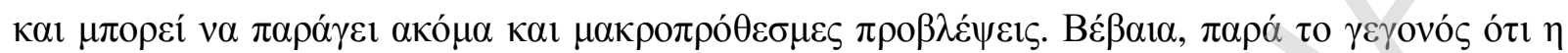

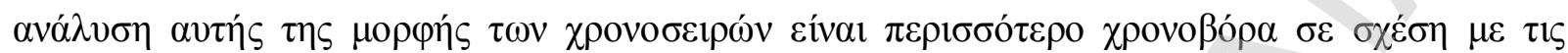

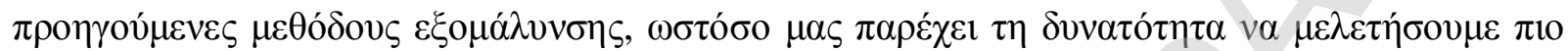

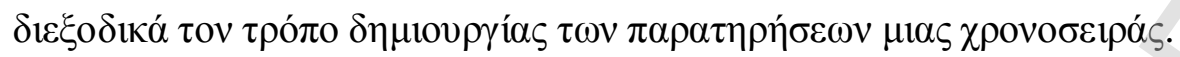

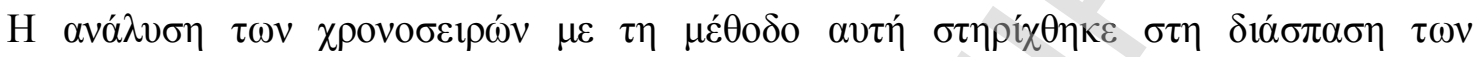

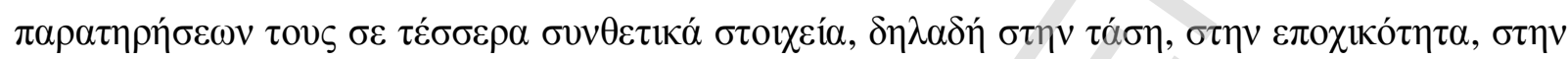

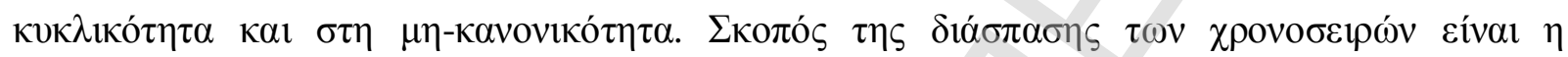

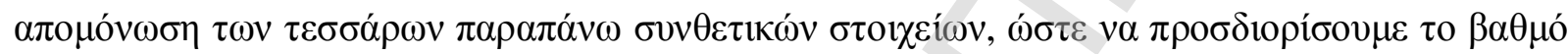

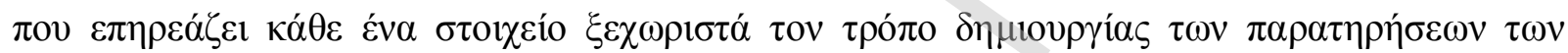

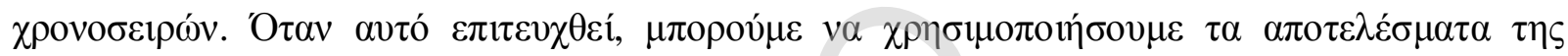
$\alpha v \alpha ́ \lambda v \sigma \eta \varsigma \gamma 1 \alpha \tau \eta \delta 1 \alpha \mu o ́ \rho \varphi \omega \sigma \eta \pi \rho \circ \beta \lambda \varepsilon ́ \psi \varepsilon \omega v$.

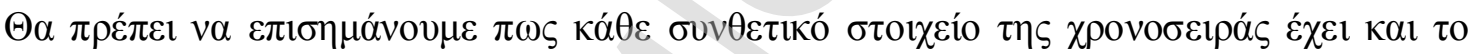

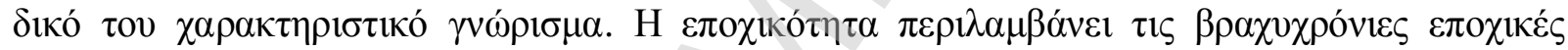

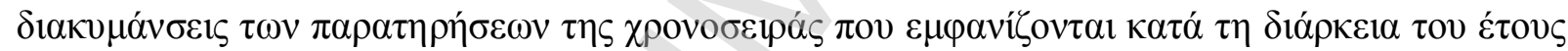

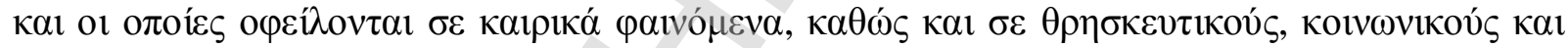

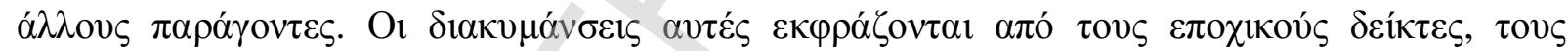

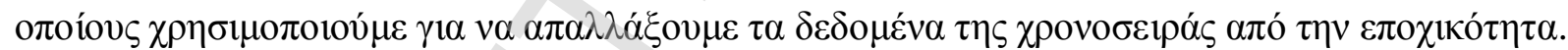

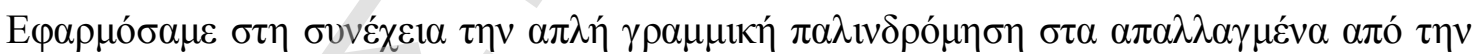

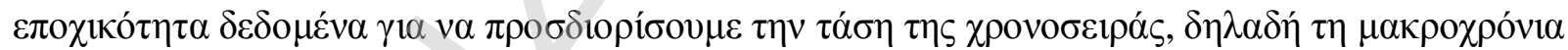

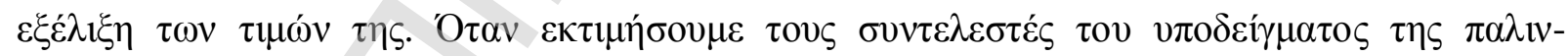

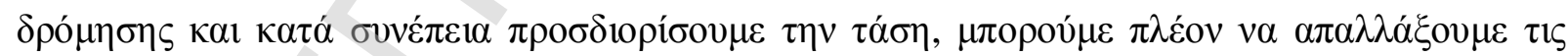

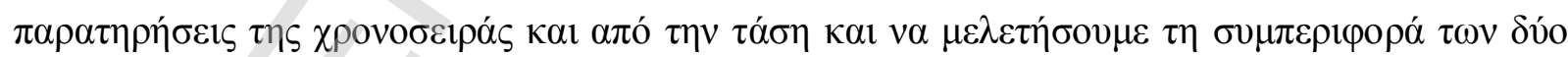

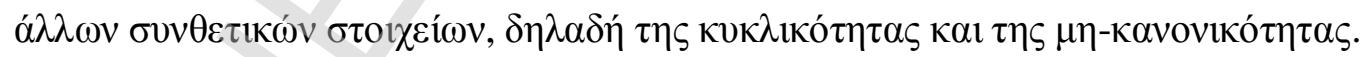

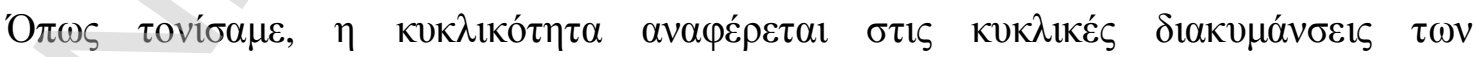

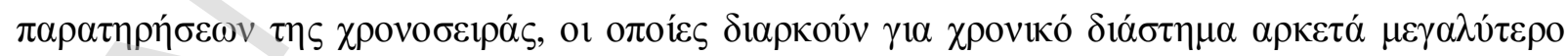

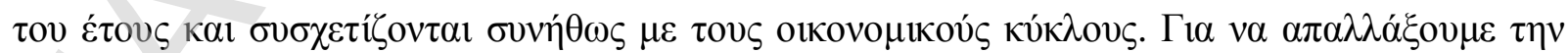

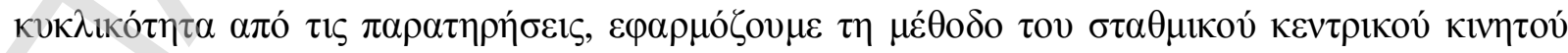

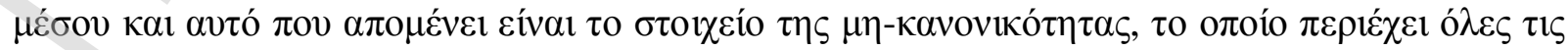

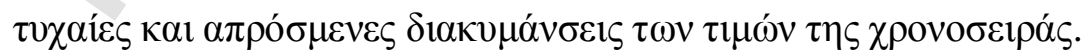




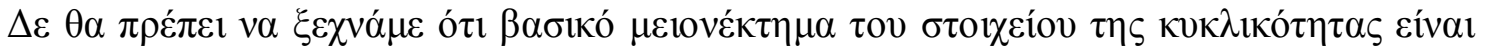

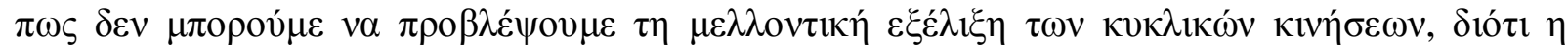

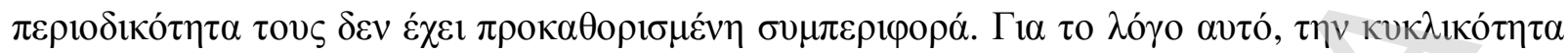

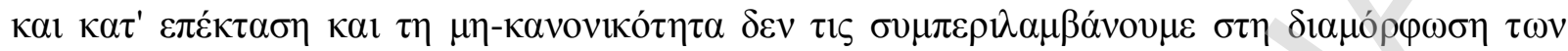

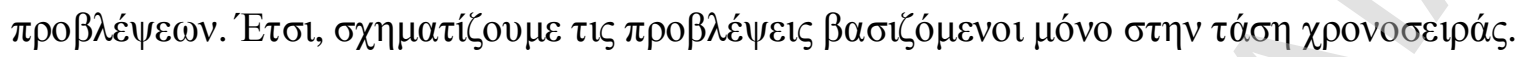




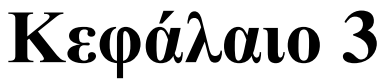

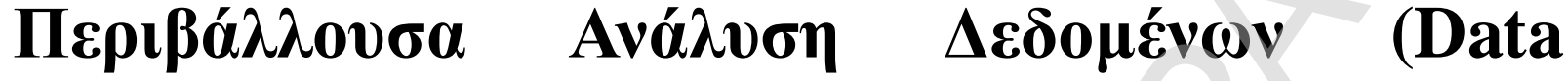

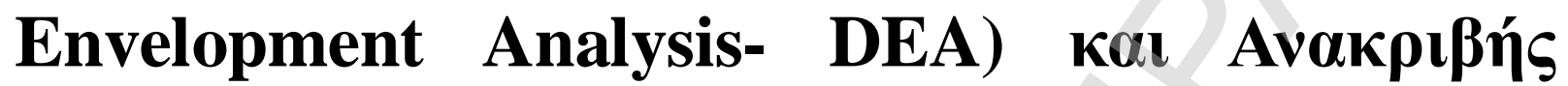

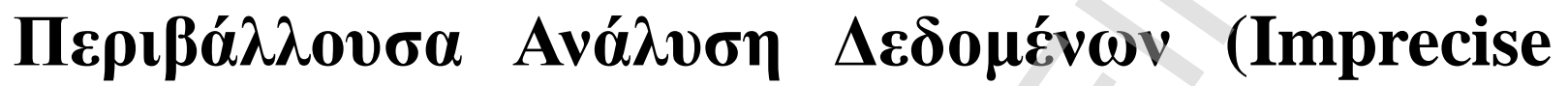 Data Envelopment Analysis - IDEA)}

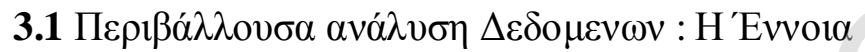

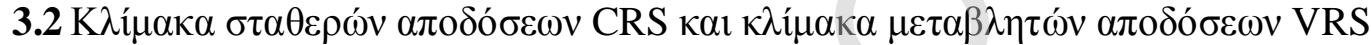

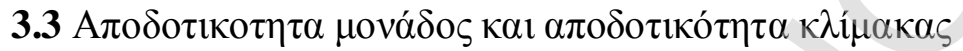

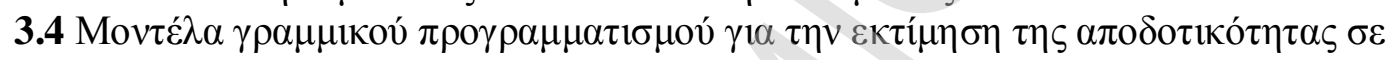
$\kappa \alpha \theta \varepsilon \sigma \tau \omega ́ \varsigma \kappa \lambda i ́ \mu \alpha \kappa \alpha \varsigma \sigma \tau \alpha \theta \varepsilon \rho \omega ́ v ~ \alpha \pi \circ \delta \delta ́ \sigma \varepsilon \omega v$

$3.5 \Sigma v \mu \pi \varepsilon \rho \alpha ́ \sigma \mu \alpha \tau \alpha-П \alpha \rho \alpha \tau \eta \rho \dot{\sigma \varepsilon} \measuredangle \varsigma$

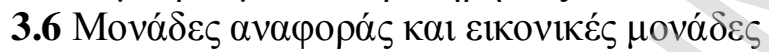

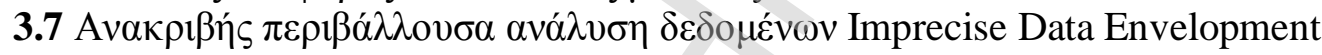
Analysis(IDEA)

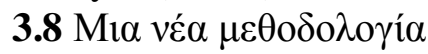

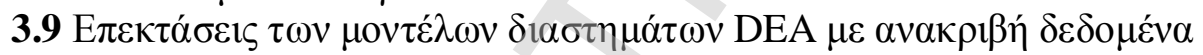

3.10 $\Sigma v \mu \pi \varepsilon \rho \alpha ́ \sigma \mu \alpha \tau \alpha-\Pi \alpha \rho \alpha \tau \eta \rho \eta ́ \sigma \varepsilon 1 \varsigma$ 


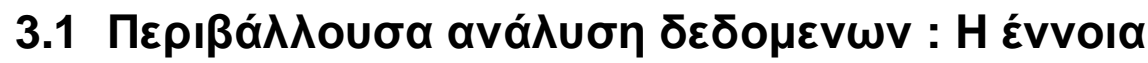

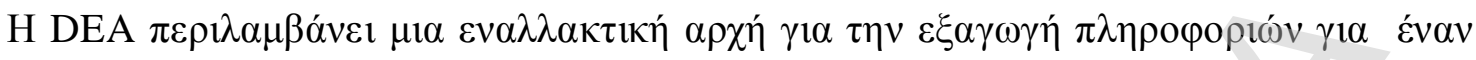

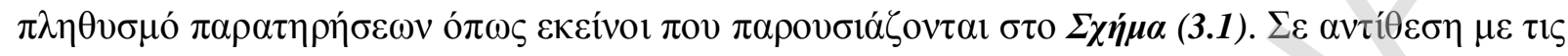

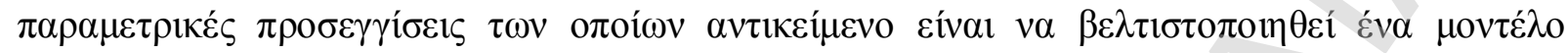

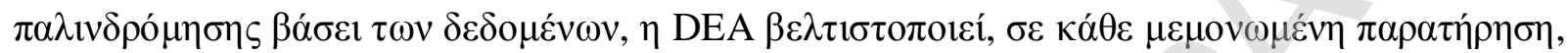

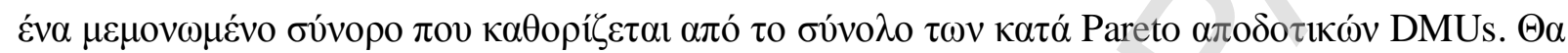

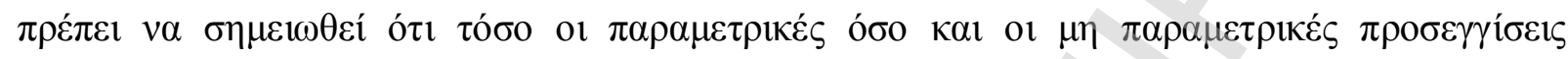

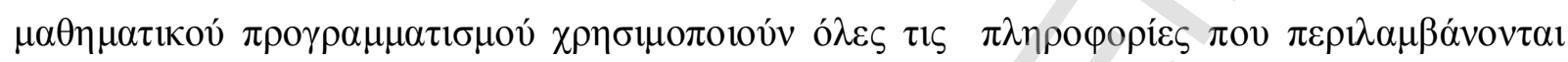

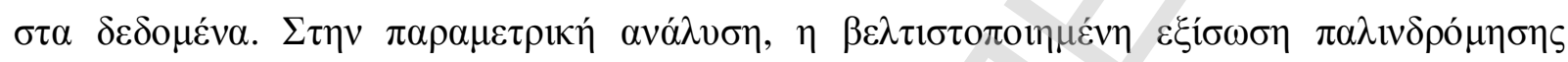

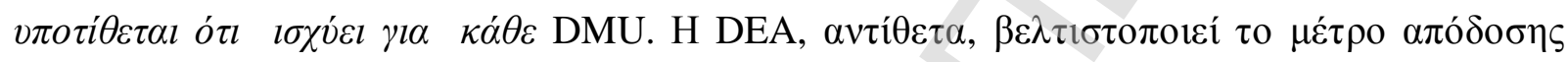

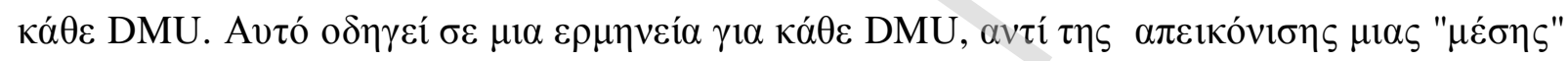

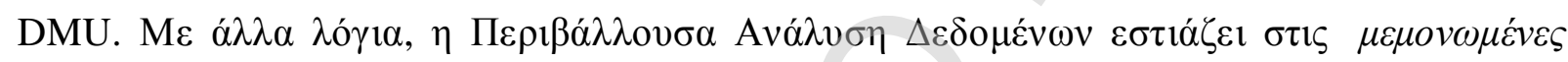

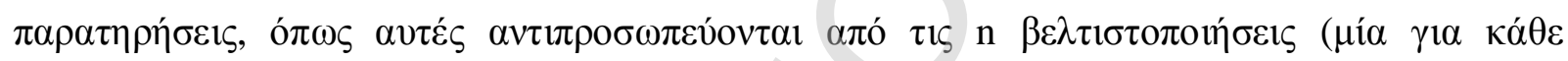

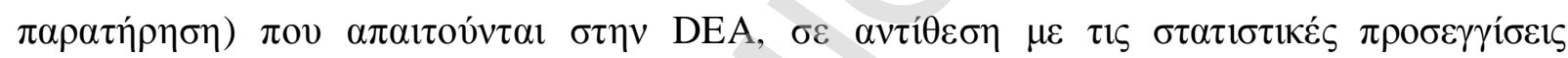

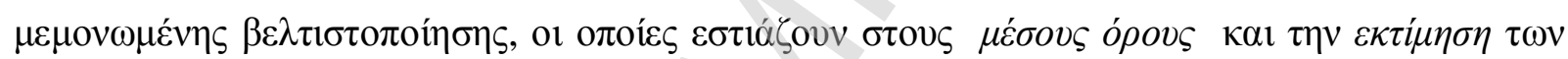
$\pi \alpha \rho \alpha \mu \varepsilon ́ \tau \rho \omega v$.

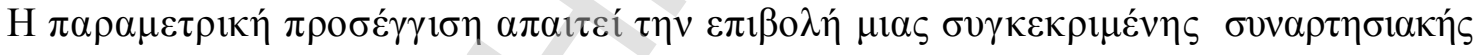

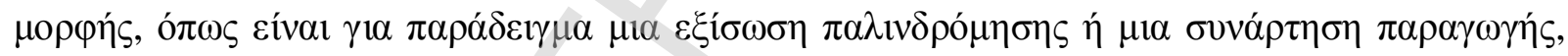

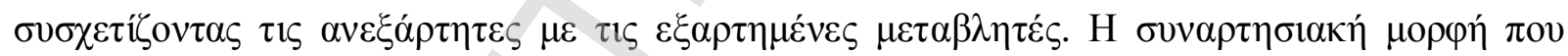

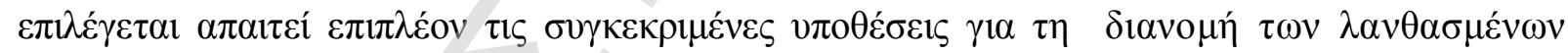

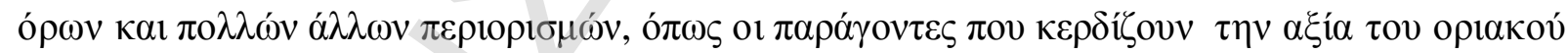

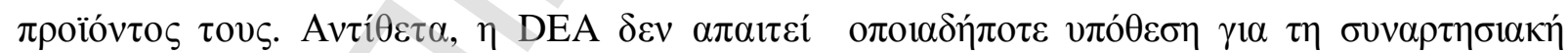

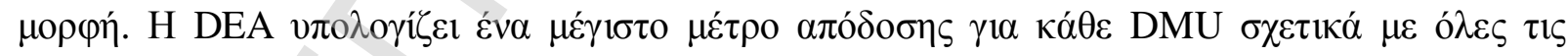

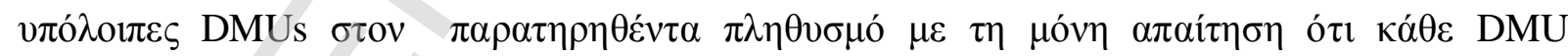

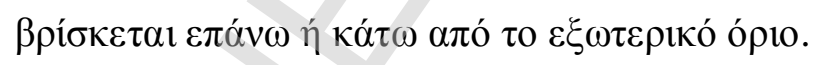

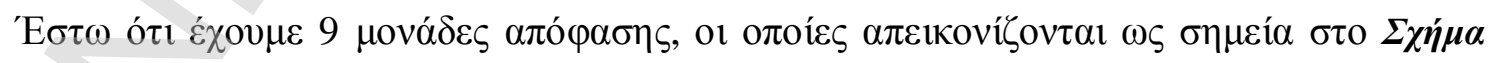
(3.1). 


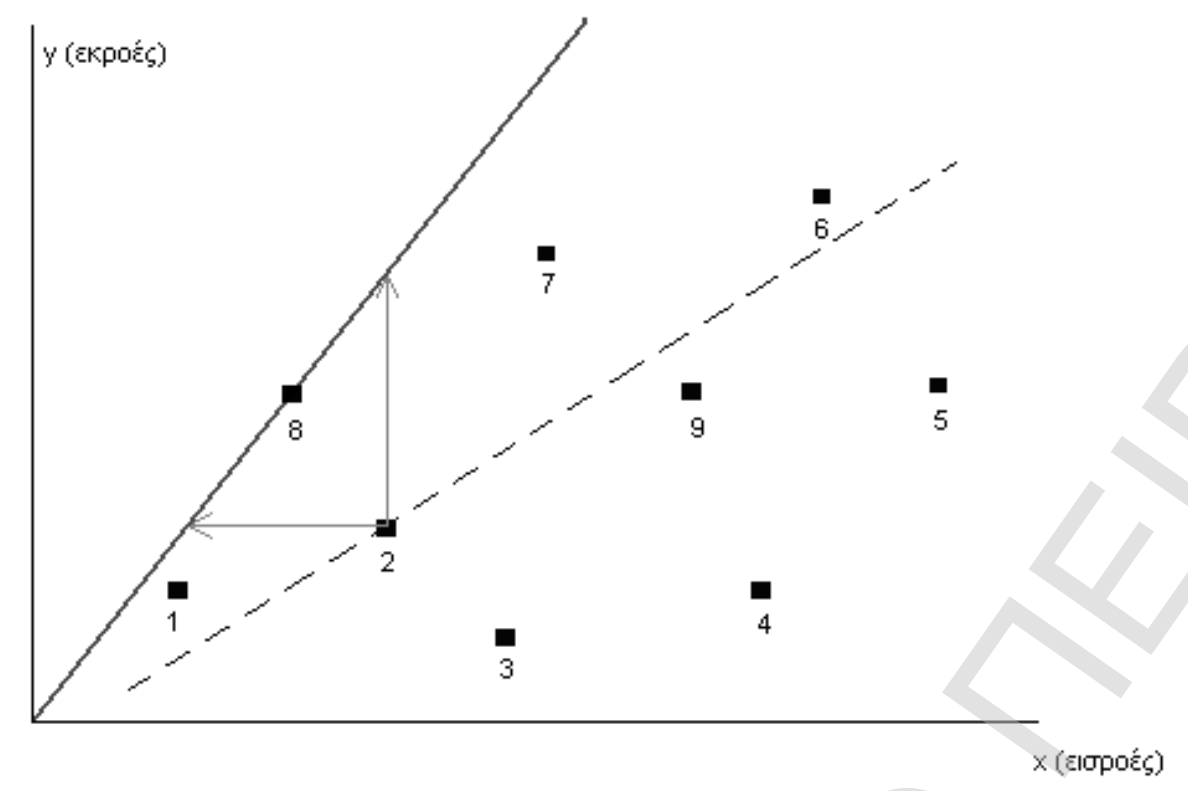

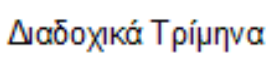

$\Sigma \chi \eta \dot{\mu} \mu(3.1)$

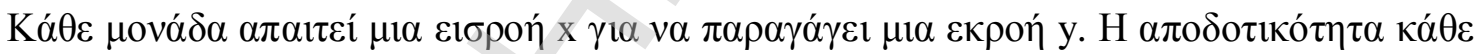

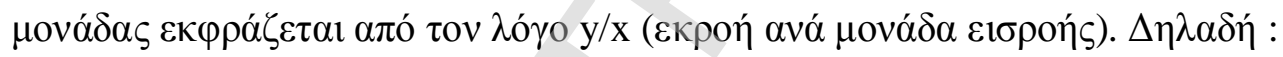

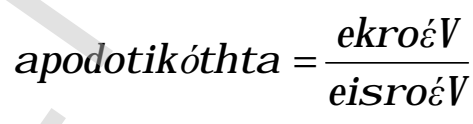

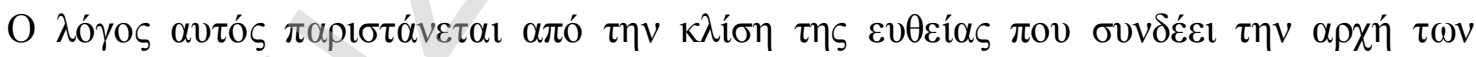

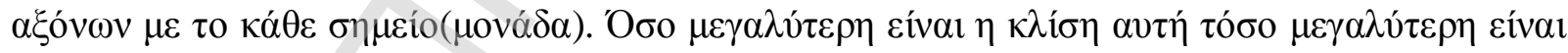

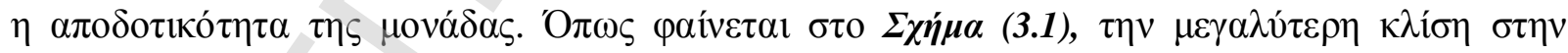

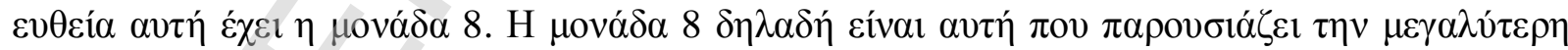

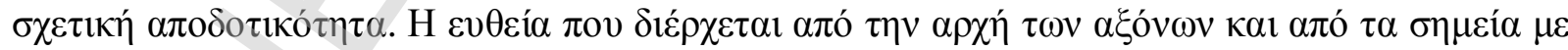

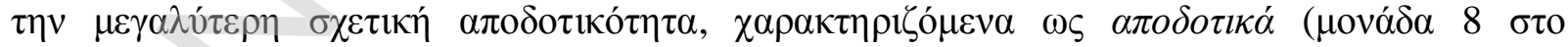

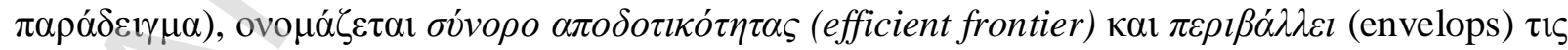

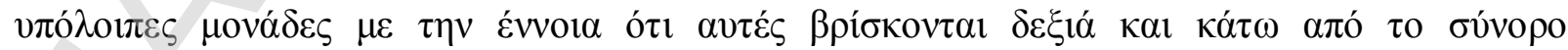

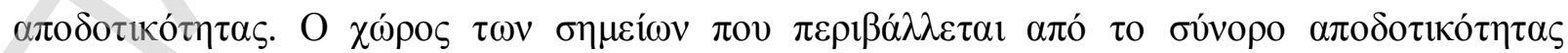

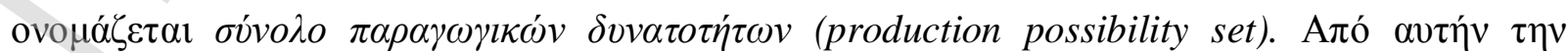

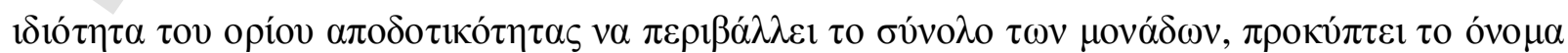

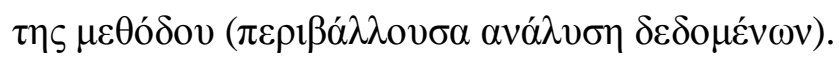




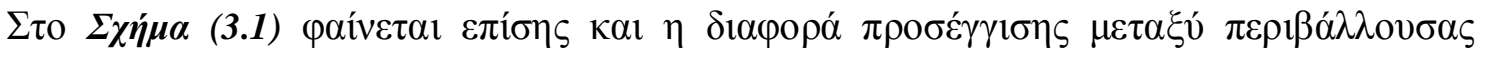

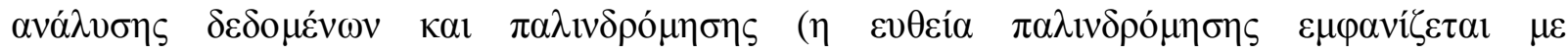

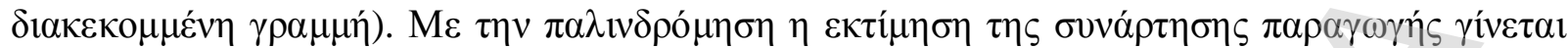

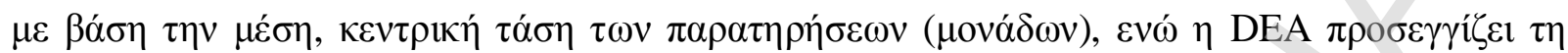

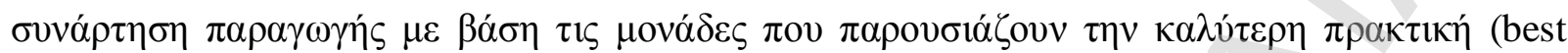
practice units), ó $\pi \omega \varsigma \pi \rho \circ \alpha v \alpha \varphi \varepsilon ́ \rho \theta \eta \kappa \varepsilon$.

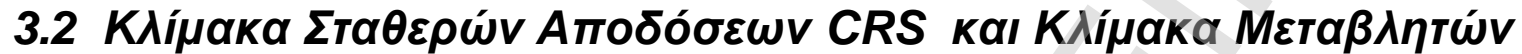

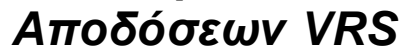

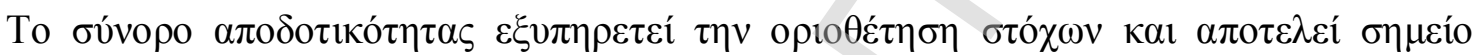

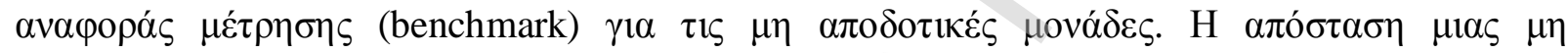

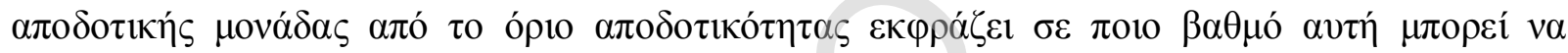

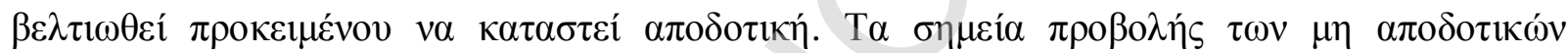

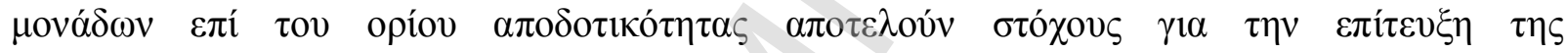

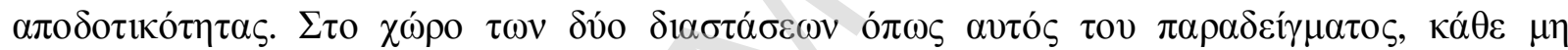

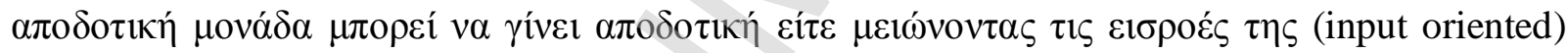

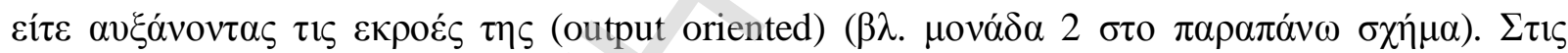

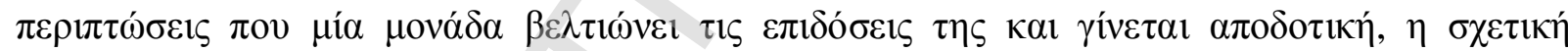

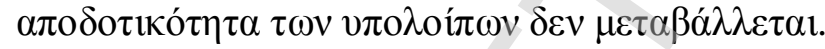

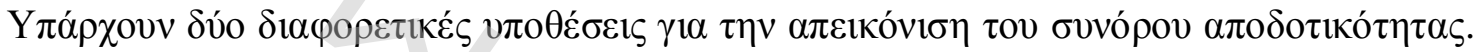

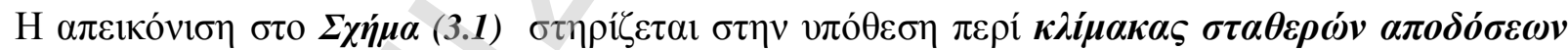

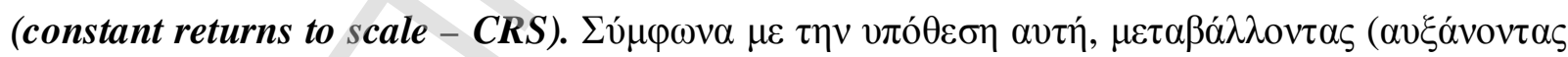

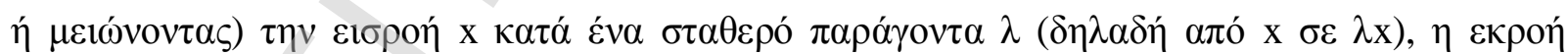

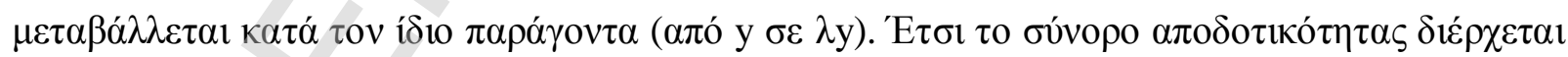

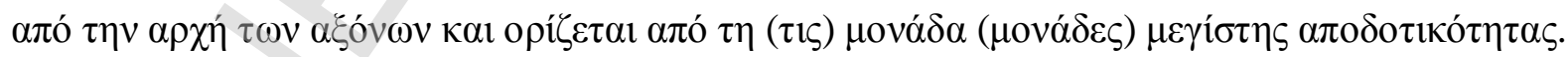

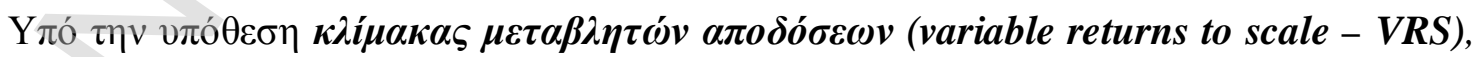

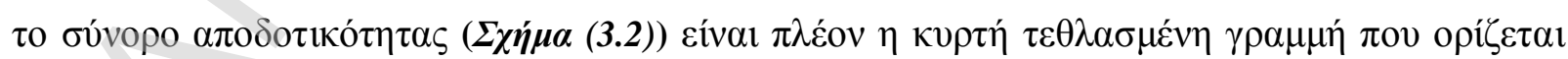

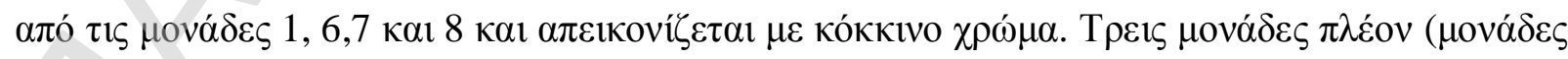

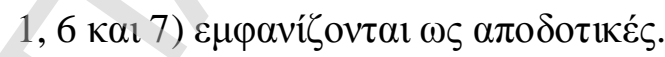




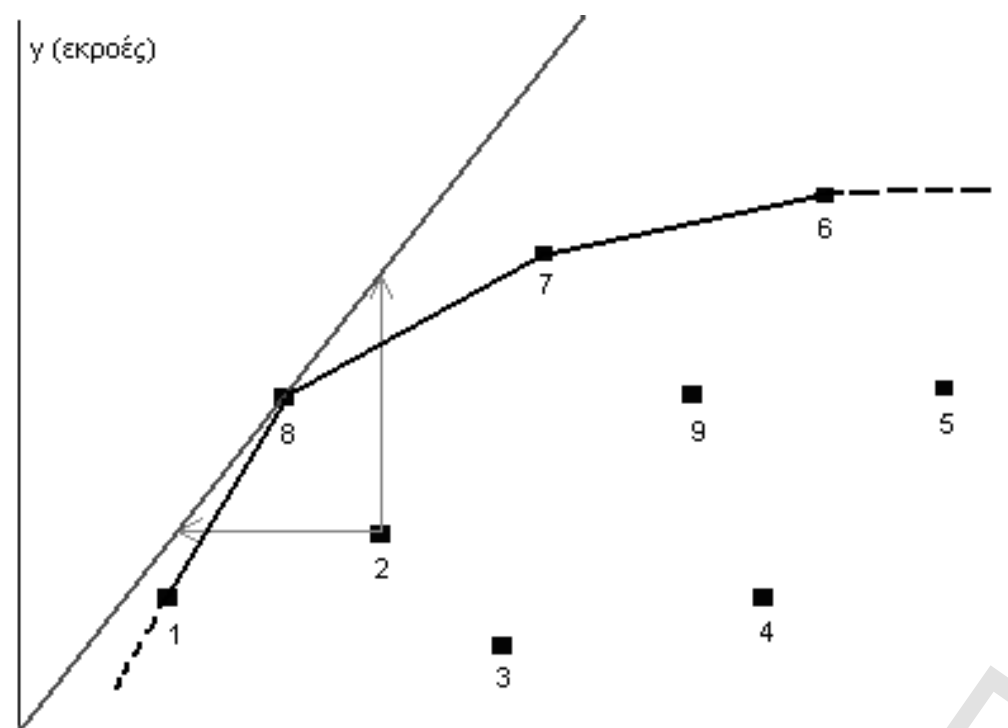

$x(\varepsilon ı \sigma p o c ́ c)$

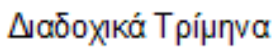

$\Sigma \chi \eta \dot{\mu \alpha}(3.2)$

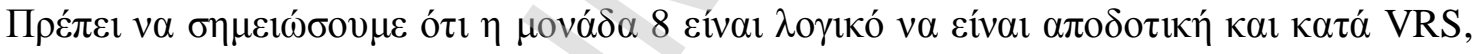

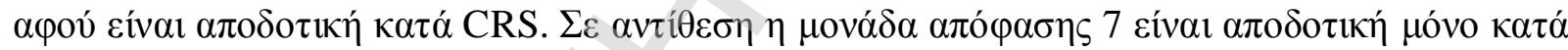
VRS.

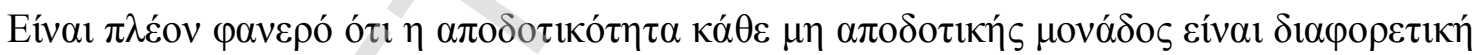

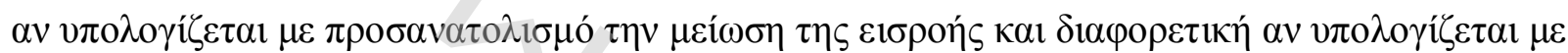

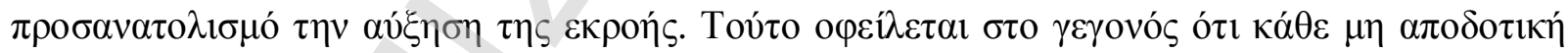

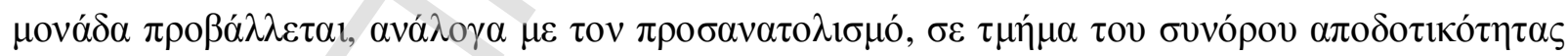

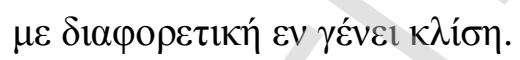

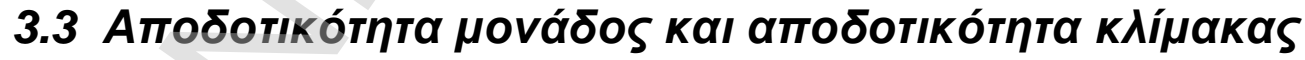

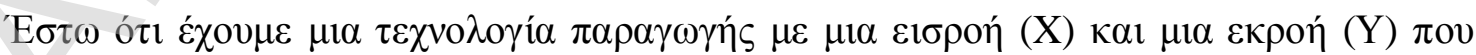

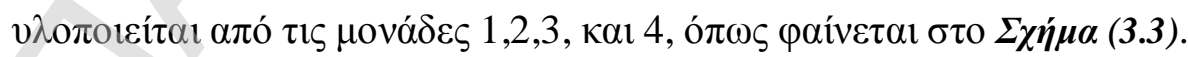




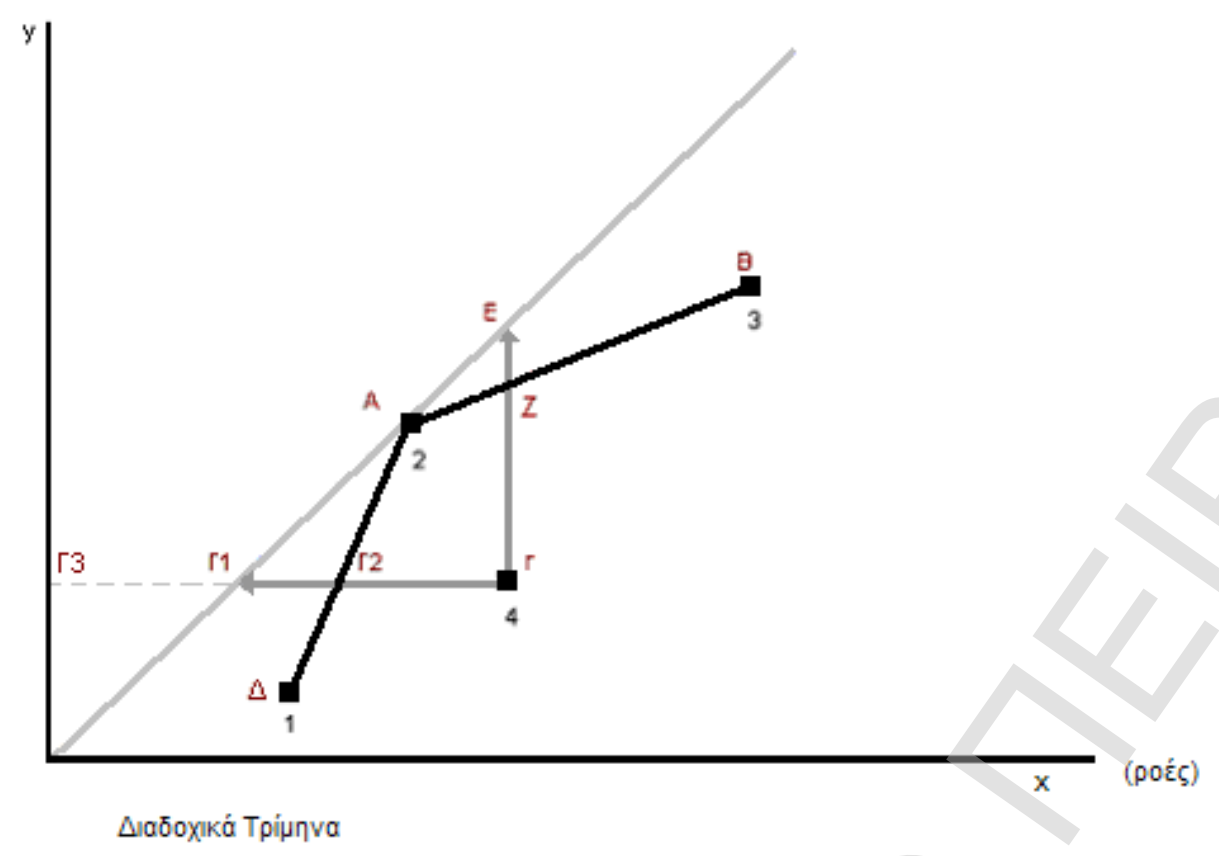

$\Sigma \chi \eta ́ r \mu \alpha(3.3)$

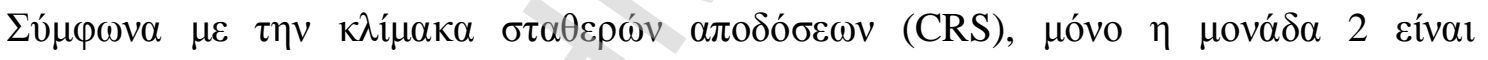

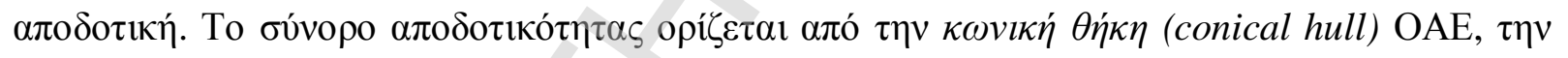

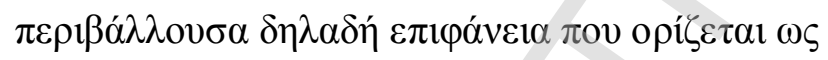

$$
\left\{\lambda_{1}\left(\mathrm{X}_{1}, \mathrm{Y}_{1}\right)+\lambda_{2}\left(\mathrm{X}_{2}, \mathrm{Y}_{2}\right) \backslash \lambda_{1}+\lambda_{2}=1\right\}
$$

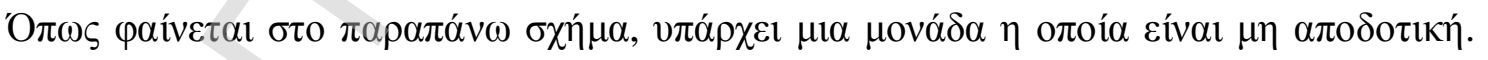

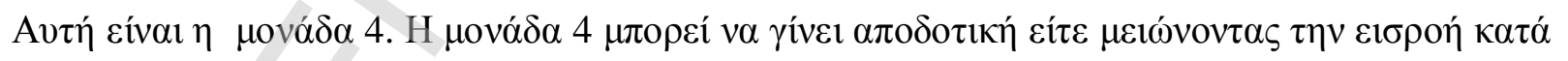

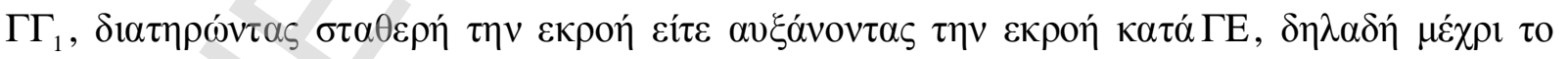

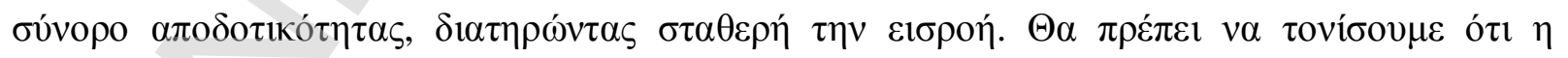

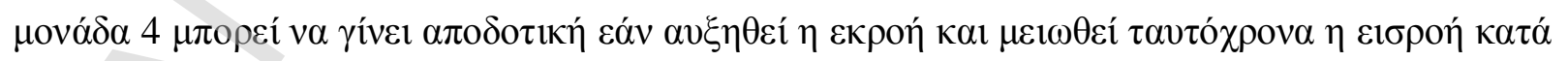

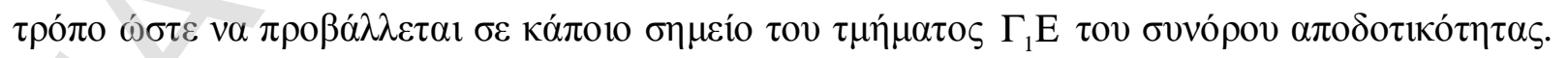

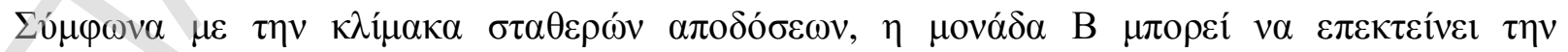

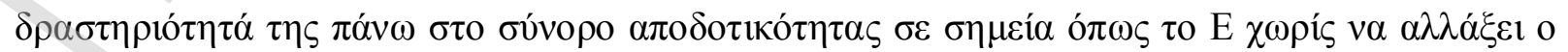

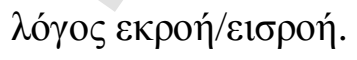




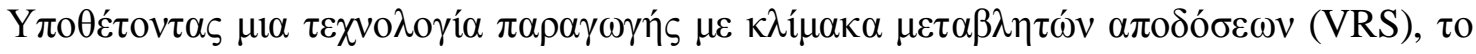

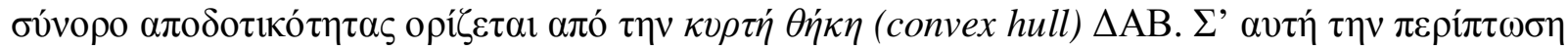

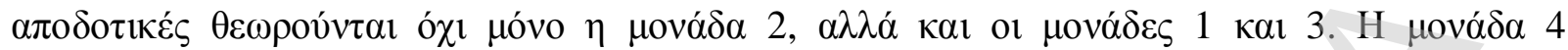

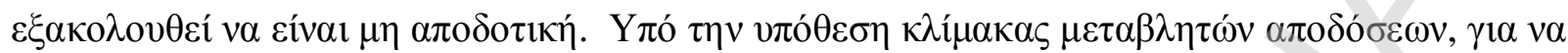

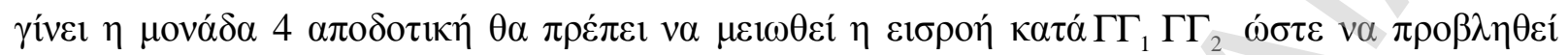

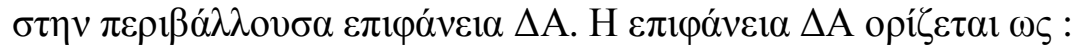

$$
\left\{\lambda_{1}\left(\mathrm{X}_{1}, \mathrm{Y}_{1}\right)+\lambda_{2}\left(\mathrm{X}_{2}, \mathrm{Y}_{2}\right) \backslash \lambda_{1}+\lambda_{2}=1\right\}
$$

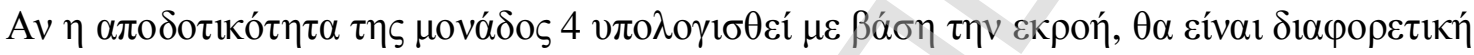

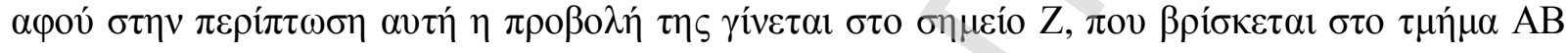

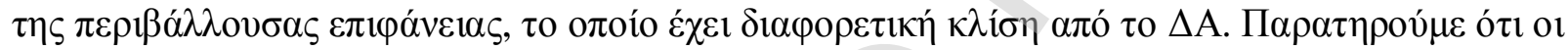

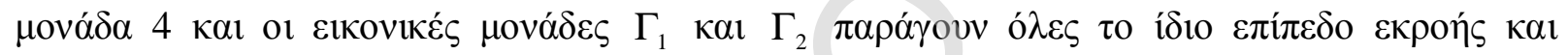

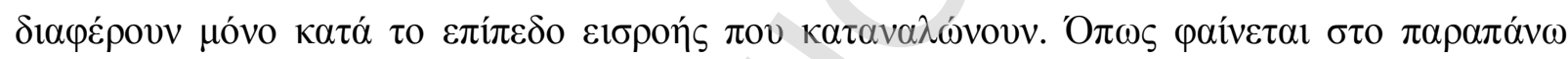

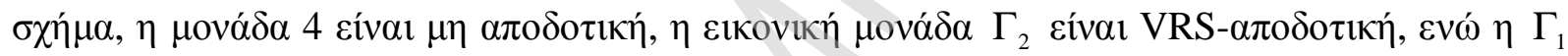

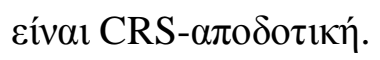

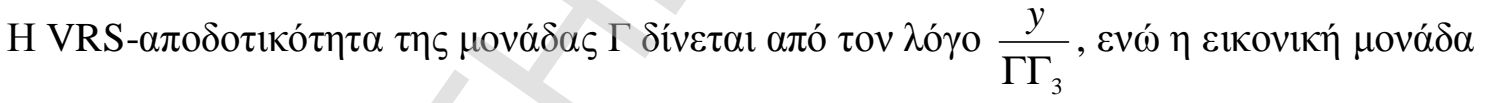

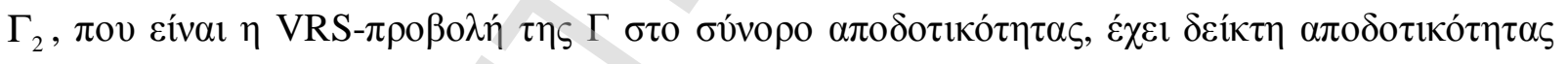

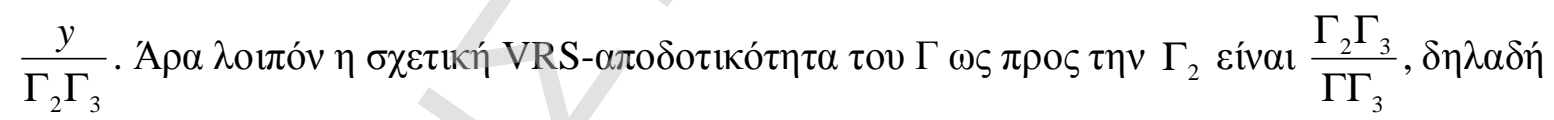

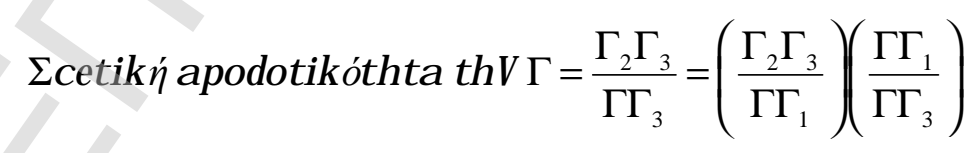

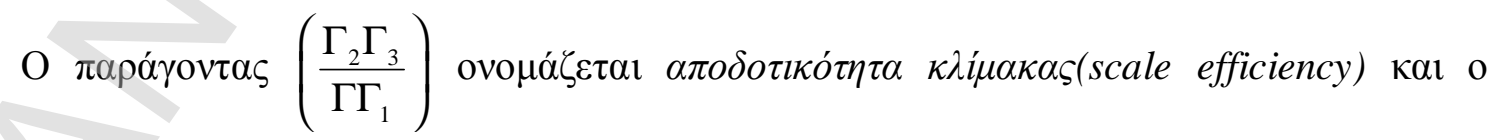

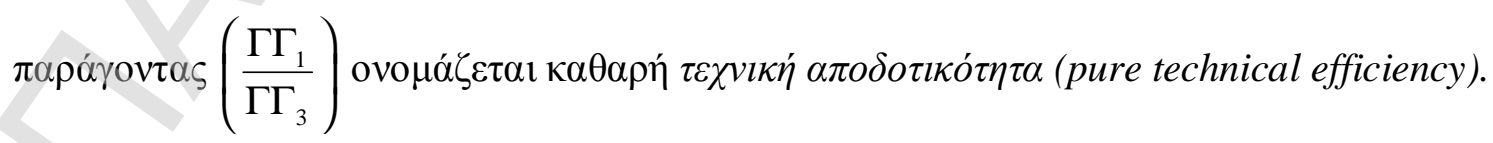




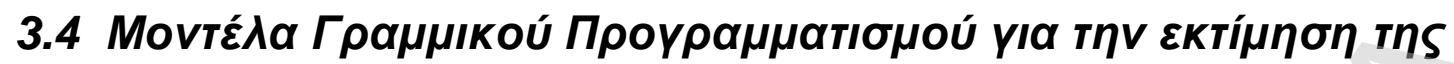

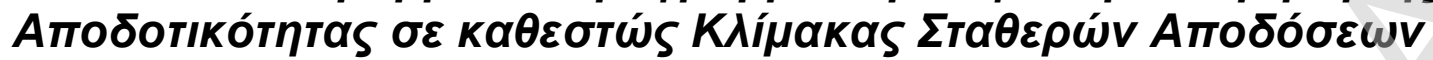

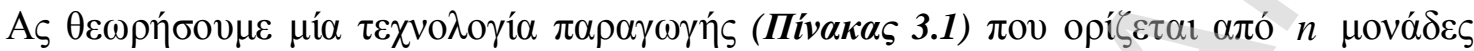
$\pi \alpha \rho \alpha \gamma \omega \gamma \eta ́ \varsigma$ (

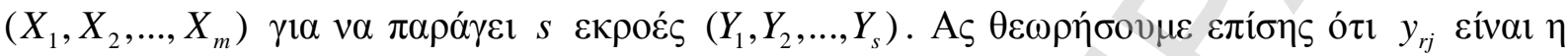

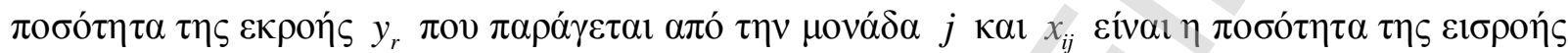
$x_{i} \pi \mathrm{ov} \kappa \alpha \tau \alpha v \alpha \lambda i ́ \sigma \kappa \varepsilon \tau \alpha \iota l \alpha \pi$ $\tau \eta v \mu o v \alpha ́ \delta \alpha j$.

\begin{tabular}{ccccccccc}
\multicolumn{3}{c}{ Екров́ $\left(Y_{1}, \ldots, Y_{r}, \ldots, Y_{s}\right)$} & \multicolumn{5}{c}{$\operatorname{Ei\sigma \rho о\varepsilon ́s~}\left(X_{1}, \ldots, X_{i}, \ldots, X_{m}\right)$} \\
\hline$D M U_{1}$ & $y_{11}$ & $y_{r 1}$ & $\ldots$ & $y_{s 1}$ & $x_{11}$ & $x_{i 1}$ & $\ldots$ & $x_{m 1}$ \\
$D M U_{2}$ & $y_{12}$ & $y_{r 2}$ & $\ldots$ & $y_{s 2}$ & $x_{12}$ & $x_{i 2}$ & $\ldots$ & $x_{m 2}$ \\
$\ldots$ & $\ldots$ & $\ldots$ & $\ldots$ & $\ldots$ & $\ldots$ & $\ldots$ & $\ldots$ & $\ldots$ \\
$D M U_{j}$ & $y_{1 j}$ & $y_{r j}$ & $\ldots$ & $y_{s j}$ & $x_{1 j}$ & $x_{i j}$ & $\ldots$ & $x_{m j}$ \\
$\ldots$ & $\ldots$ & $\ldots$ & $\ldots$ & $\ldots$ & $\ldots$ & $\ldots$ & $\ldots$ & $\ldots$ \\
$D M U_{n}$ & $y_{1 n}$ & $y_{r n}$ & $\ldots$ & $y_{s n}$ & $x_{1 n}$ & $x_{i n}$ & $\ldots$ & $x_{m n}$ \\
& $u_{1}$ & $u_{r}$ & $\ldots$ & $u_{s}$ & $v_{1}$ & $v_{i}$ & $\ldots$ & $v_{m}$
\end{tabular}

Mívakas 3.1

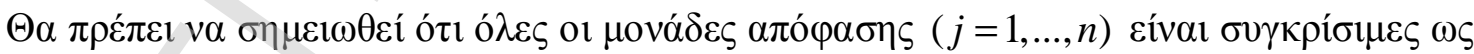

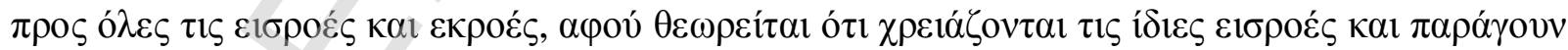

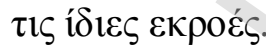

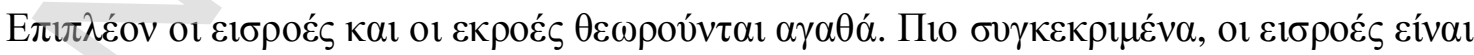

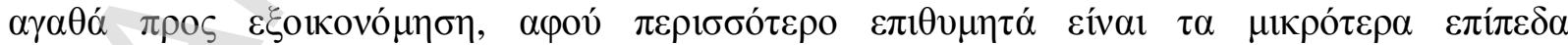

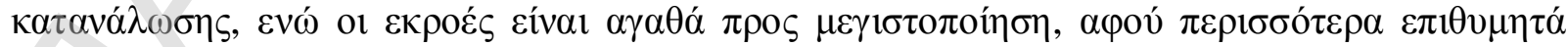

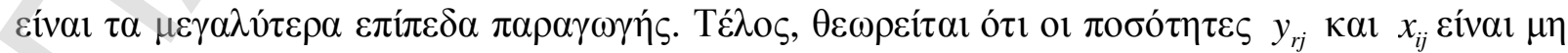
$\alpha \rho \vee \eta \tau \iota \kappa \varepsilon ́ \varsigma$. 


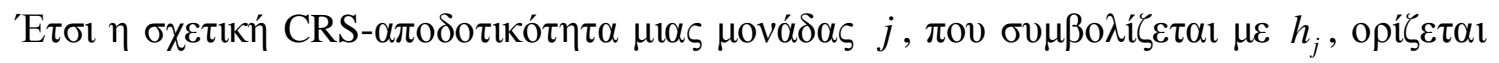

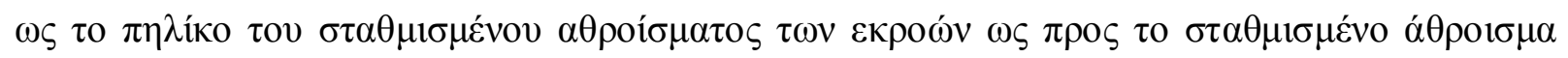

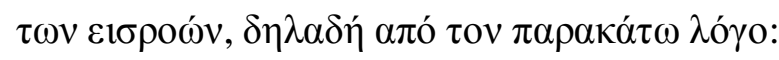

$$
h_{j}=\frac{\sum_{r=1}^{s} u_{r} y_{r j}}{\sum_{i=1}^{m} v_{i} x_{i j}}
$$

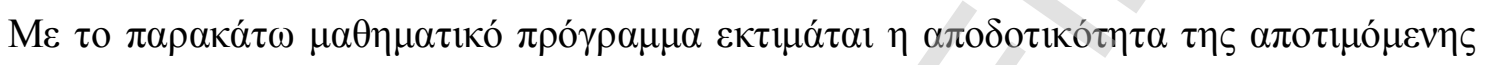

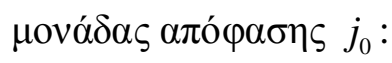

$$
\begin{aligned}
& \max h_{j_{0}}(u, v)=\frac{\sum_{r=1}^{s} u_{r} y_{r j_{0}}}{\sum_{i=1}^{m} v_{i} x_{i j_{0}}} \\
& \text { s.t } \\
& \sum_{r=1}^{s} u_{r} y_{r j}-\sum_{i=1}^{m} v_{i} x_{i j} \leq 0 \quad j=1, \ldots, n \\
& u_{r}, v_{i} \geq \varepsilon \quad \forall r, i
\end{aligned}
$$

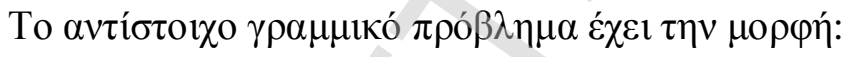

$$
\begin{aligned}
& \max _{j_{0}}(u, v)=\sum_{r=1}^{s} u_{r} y_{r j_{0}} \\
& \text { s.t. } \\
& \sum_{i=1}^{m} v_{i} x_{i j_{0}}=1 \\
& \sum_{r=1}^{s} u_{r} y_{r j}-\sum_{i=1}^{m} v_{i} x_{i j} \leq 0 \quad j=1, \ldots, n \\
& u_{r}, v_{i} \geq \varepsilon \quad \forall r, i
\end{aligned}
$$

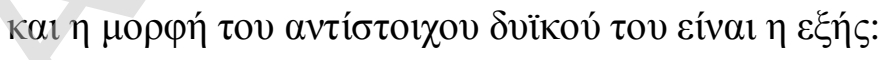


$\min E$

s.t.

$$
\begin{aligned}
& \sum_{j=1}^{n} y_{r j} \lambda_{j}-y_{r j_{0}} \geq 0 \quad r=1, \ldots, s \\
& \sum_{j=1}^{n} x_{i j} \lambda_{j}-x_{i j_{0}} E \leq 0 \quad i=1, \ldots, m \\
& \lambda_{j} \geq 0 \quad j=1, \ldots, n
\end{aligned}
$$

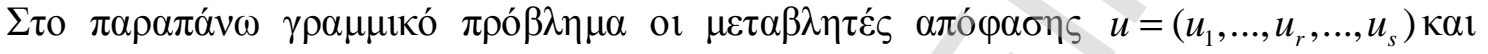

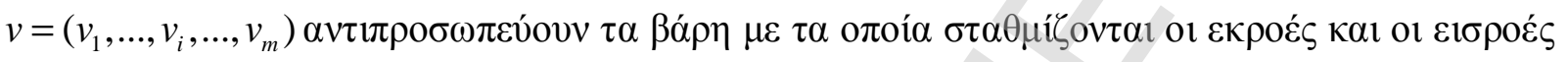

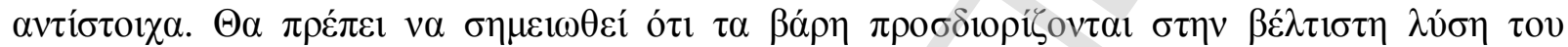

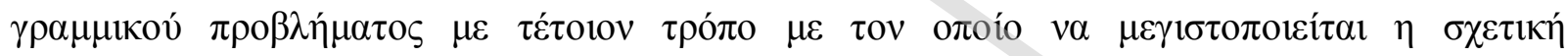

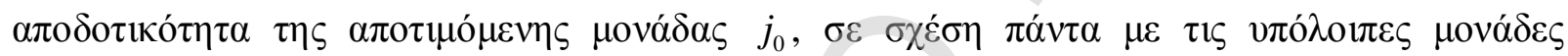

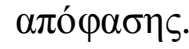

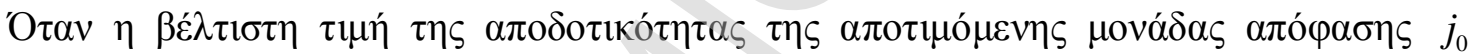

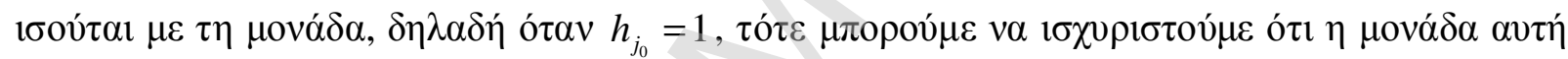

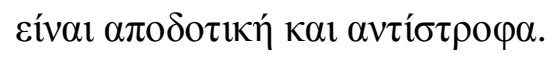

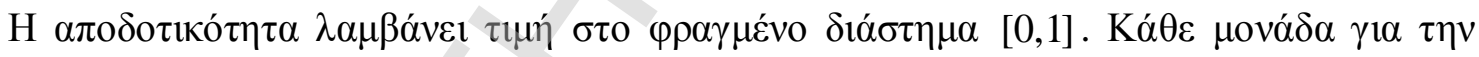

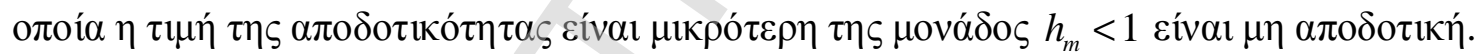

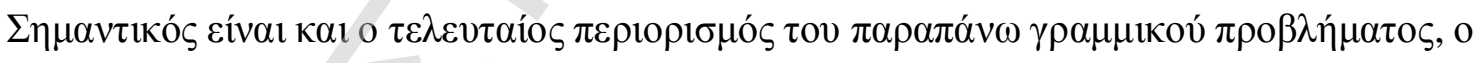

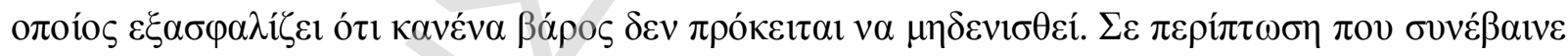

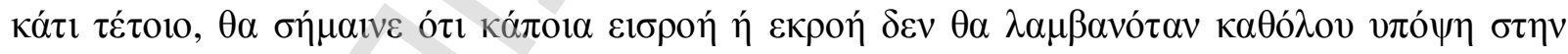

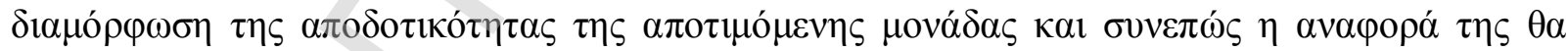

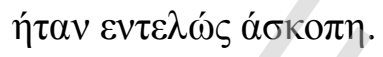




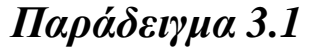

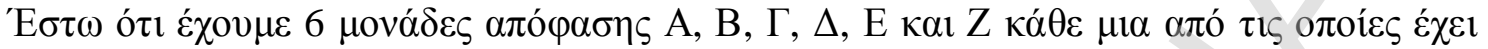

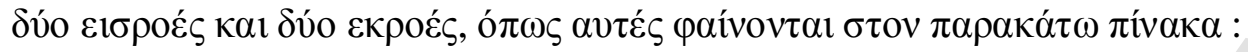

\begin{tabular}{|c|c|c|c|c|}
\hline \multirow[b]{2}{*}{ Movádes } & \multicolumn{2}{|c|}{ Еıбров́ } & \multicolumn{2}{|c|}{ Екров́ৎ } \\
\hline & $X_{1}$ & $X_{2}$ & $Y_{1}$ & $Y_{2}$ \\
\hline $\mathbf{A}$ & 1,50 & 0,20 & 1,40 & 0,35 \\
\hline B & 4,00 & 0,70 & 1,40 & 2,10 \\
\hline$\Gamma$ & 3,20 & 1,20 & 4,20 & 1,05 \\
\hline$\Delta$ & 5,20 & 2,00 & 2,80 & 4,20 \\
\hline $\mathbf{E}$ & 3,50 & 1,20 & 1,90 & 2,50 \\
\hline $\mathbf{Z}$ & 3,20 & 0,70 & 1,40 & 1,50 \\
\hline
\end{tabular}

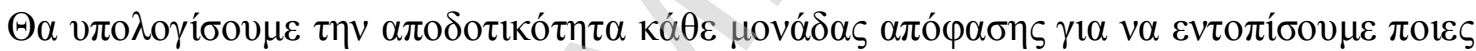

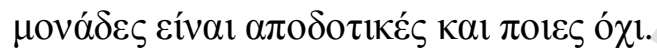

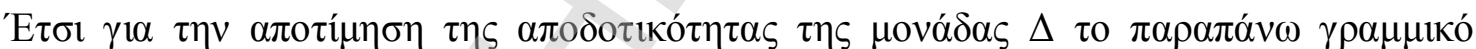

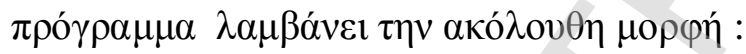

$$
\begin{aligned}
& \max h_{\Delta}=2,80 u_{1}+4,20 u_{2} \\
& \text { s.t. } \\
& 5,20 v_{1}+2,00 v_{2}=1 \\
& 1,40 u_{1}+0,35 u_{2}-1,50 v_{1}-0,20 v_{2} \leq 0 \\
& 1,40 u_{1}+2,10 u_{2}-4,00 v_{1}-0,70 v_{2} \leq 0 \\
& 4,20 u_{1}+1,05 u_{2}-3,20 v_{1}-1,20 v_{2} \leq 0 \\
& 2,80 u_{1}+4,20 u_{2}-5,20 v_{1}-2,00 v_{2} \leq 0 \\
& 1,90 u_{1}+2,50 u_{2}-3,50 v_{1}-1,20 v_{2} \leq 0 \\
& 1,40 u_{1}+1,50 u_{2}-3,20 v_{1}-0,70 v_{2} \leq 0 \\
& u_{1}, u_{2}, v_{1}, v_{2} \geq \varepsilon
\end{aligned}
$$

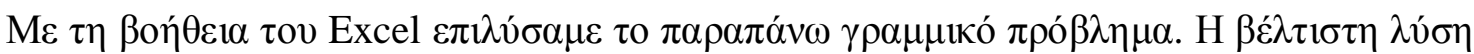

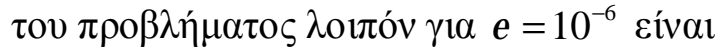




$$
\begin{aligned}
& v_{1}=0,104 \\
& v_{2}=0,227 \\
& u_{1}=0,102 \\
& u_{2}=0,169
\end{aligned}
$$

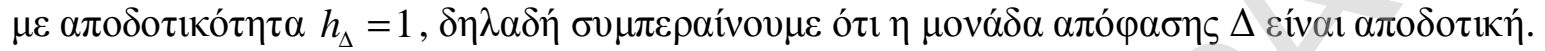

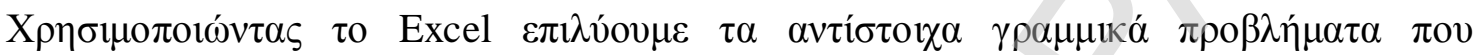

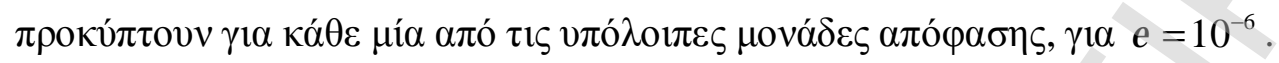

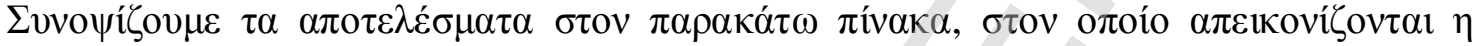

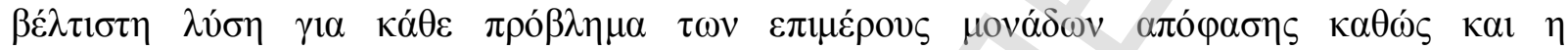

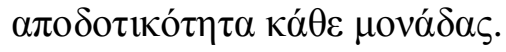

\begin{tabular}{ccccccc} 
& $\mathbf{A}$ & $\mathbf{B}$ & $\boldsymbol{\Gamma}$ & $\boldsymbol{\Delta}$ & $\mathbf{E}$ & $\mathbf{Z}$ \\
\hline$v_{1}$ & 1,346 & $10^{-6}$ & 0,312 & 0,104 & 0,109 & 0,154 \\
$v_{2}$ & 1,346 & 1,428 & $10^{-6}$ & 0,227 & 0,512 & 0,721 \\
$u_{1}$ & 0,483 & $10^{-6}$ & 0,238 & 0,102 & 0,115 & 0,162 \\
$u_{2}$ & 0,921 & 0,476 & $10^{-6}$ & 0,169 & 0,303 & 0,427 \\
$h$ & $h_{\Gamma}=1$ & $h_{B}=1$ & $h_{\Gamma}=1$ & $h_{\Delta}=1$ & $h_{\mathrm{E}}=0.997$ & $h_{\mathrm{Z}}=0.867$
\end{tabular}

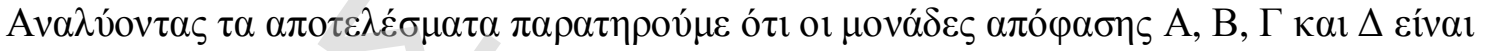

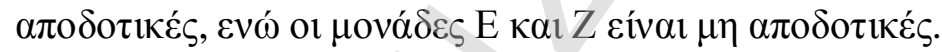




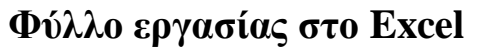

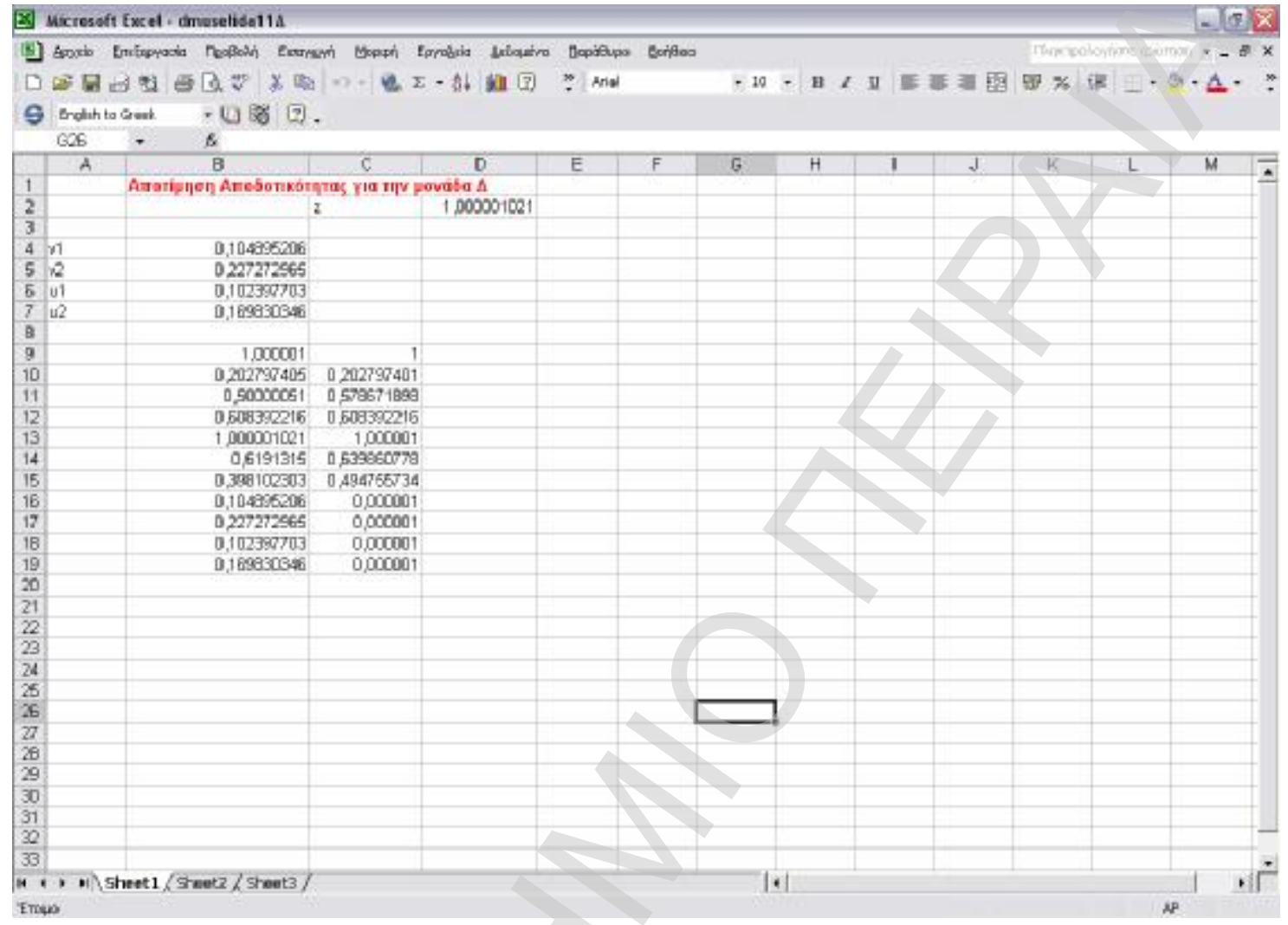

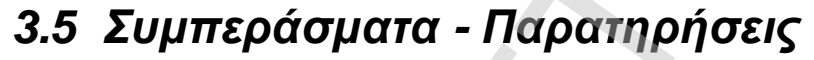

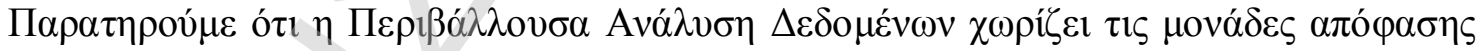

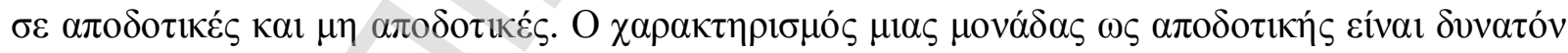

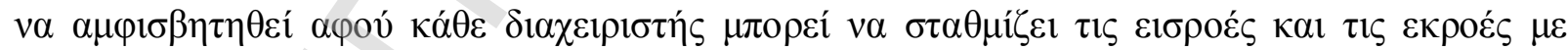

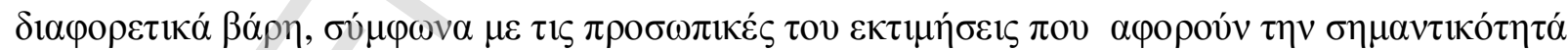

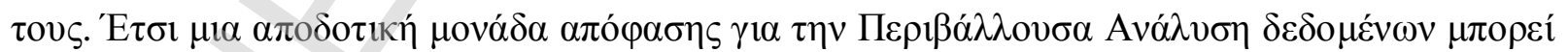

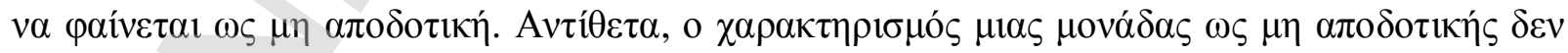

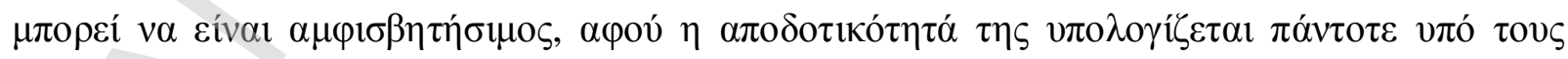

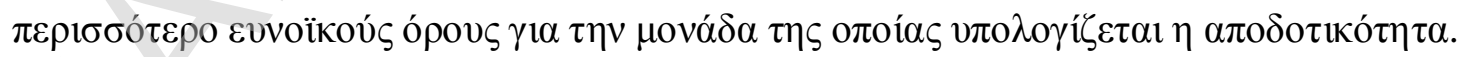

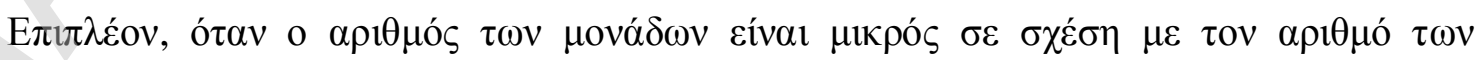

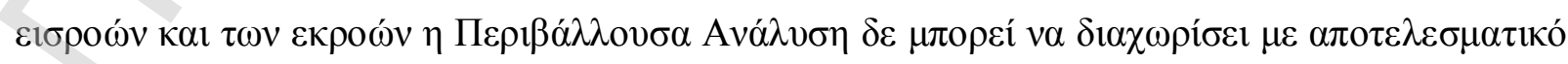

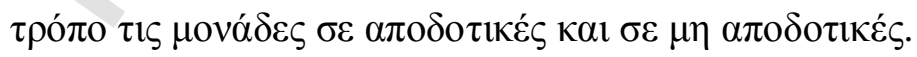




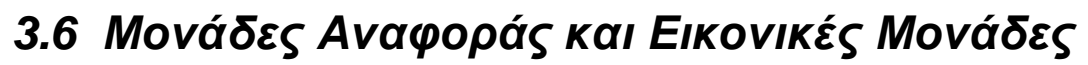

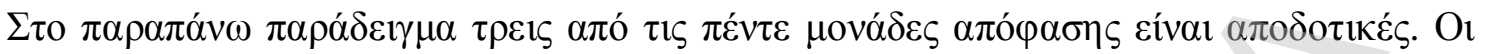

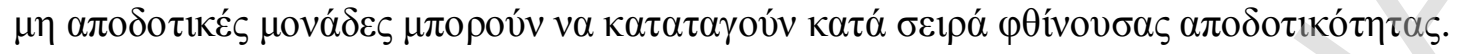

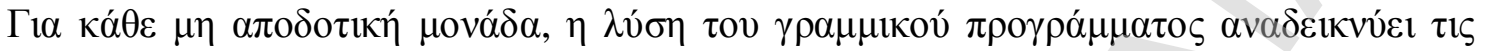

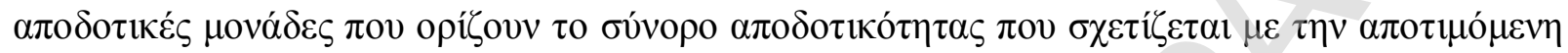

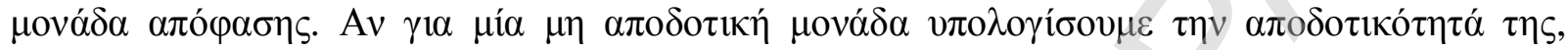

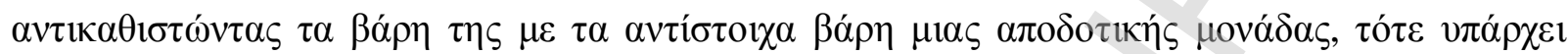

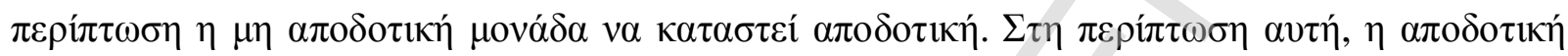

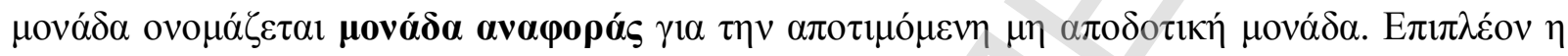

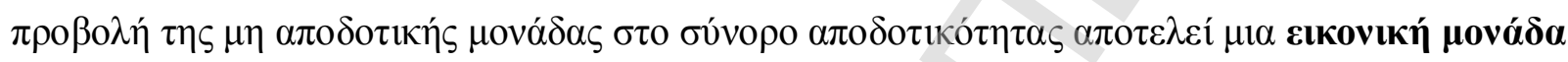

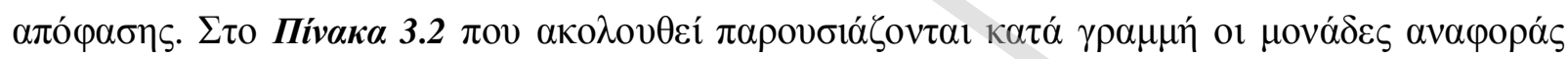

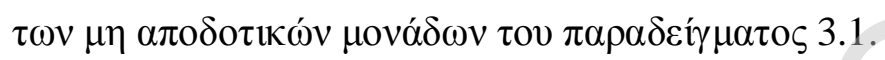

\begin{tabular}{|c|c|c|c|c|}
\hline & $\mathbf{A}$ & $\mathbf{B}$ & $\boldsymbol{\Gamma}$ & $\Delta$ \\
\hline $\mathbf{E}$ & $\mathbf{1}$ & $\mathbf{1}$ & & \\
\hline $\mathrm{Z}$ & 1 & & & \\
\hline
\end{tabular}

Пívakas 3.2

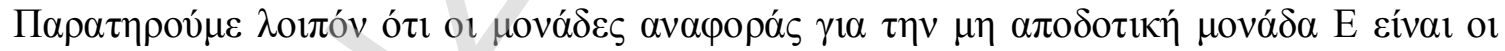

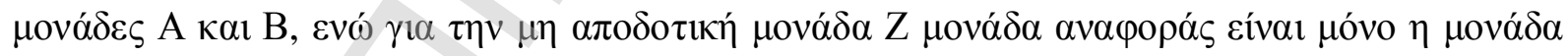
$\alpha \pi$ $\varphi \alpha \sigma \eta \varsigma$ A. 


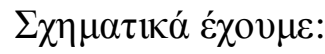

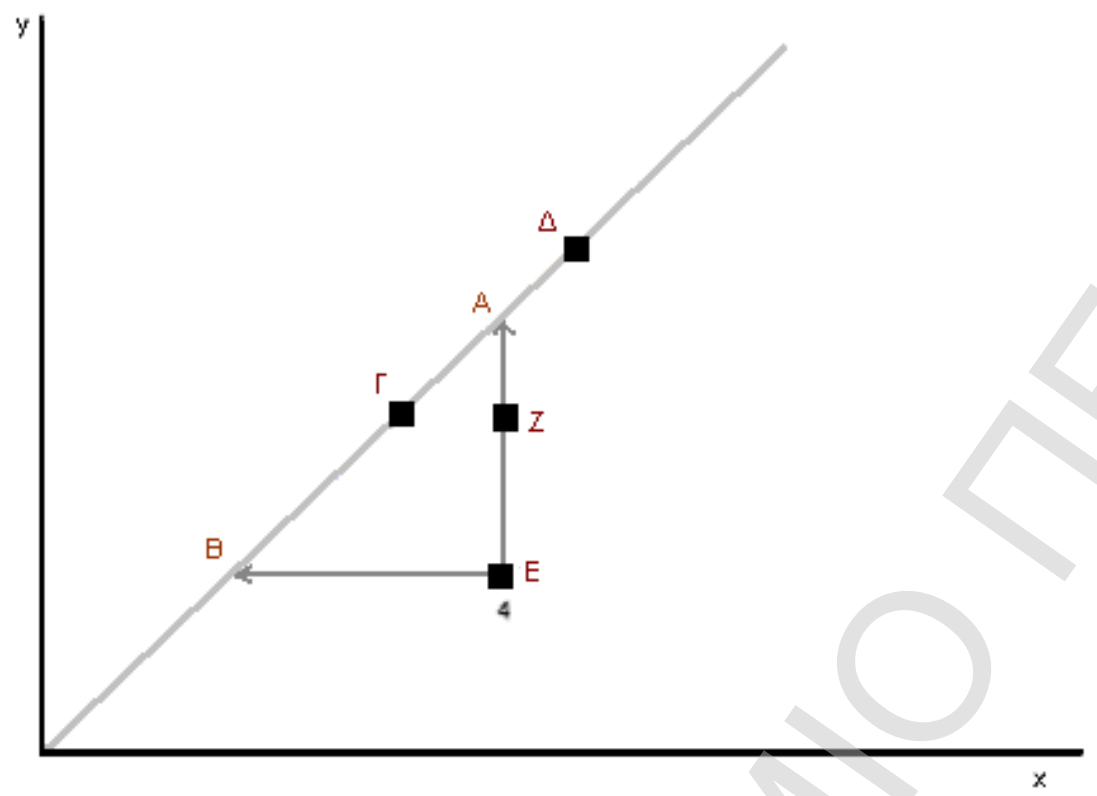

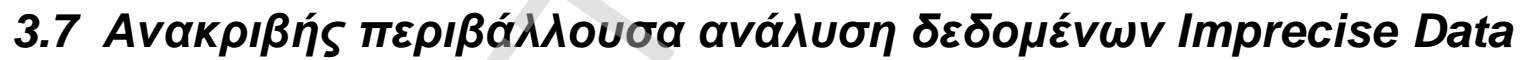 Envelopment Analysis (IDEA)}

\subsubsection{Eıøaywyń}

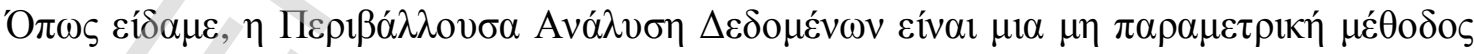

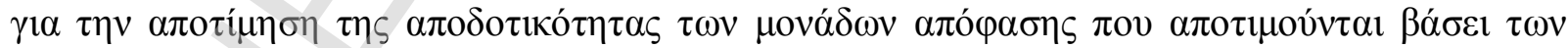

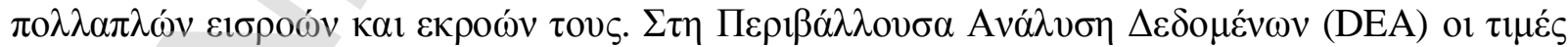

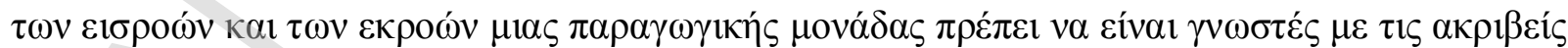

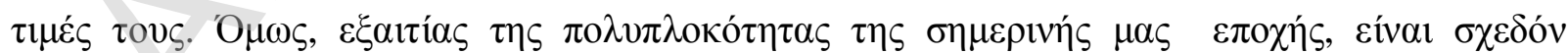

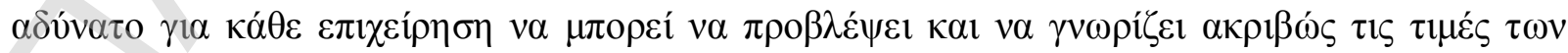

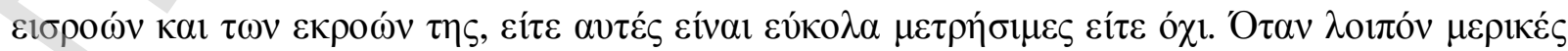

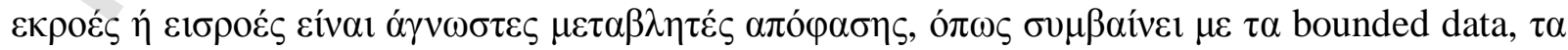

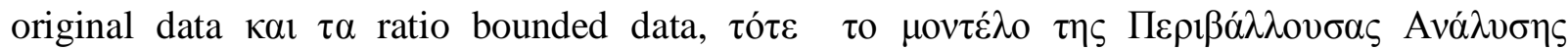




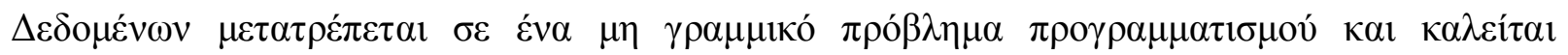

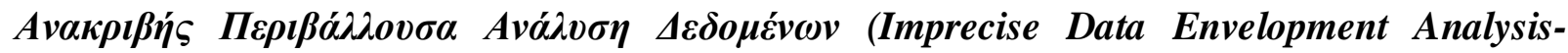

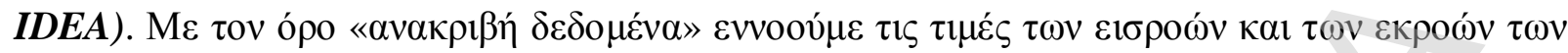

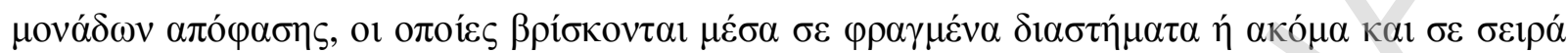

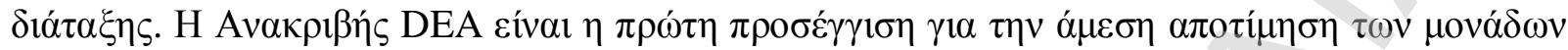
$\alpha \pi$

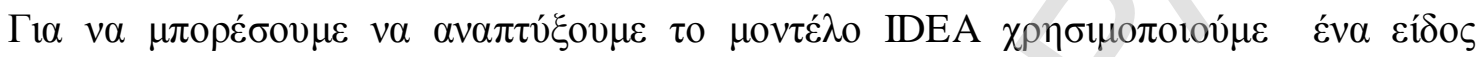

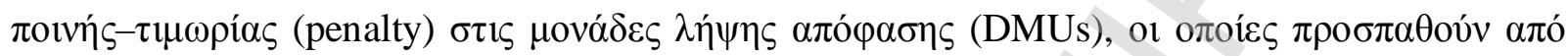

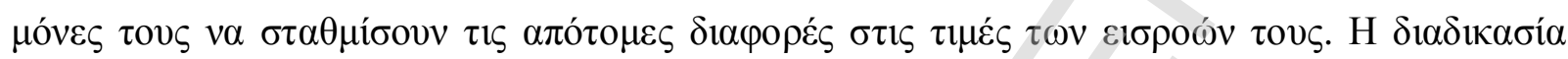

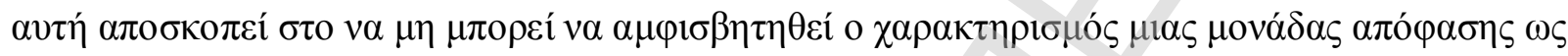

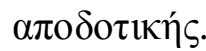

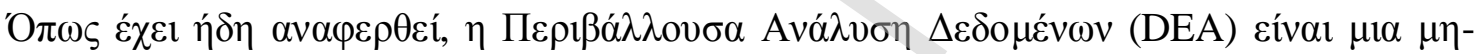

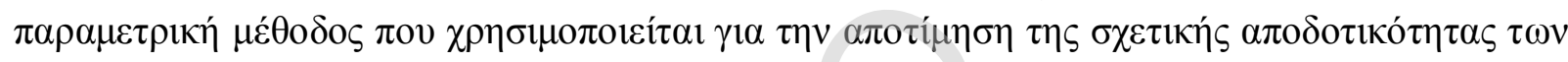

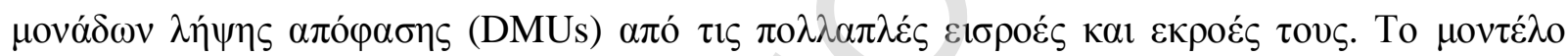

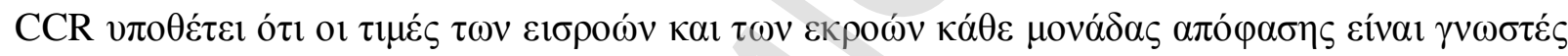

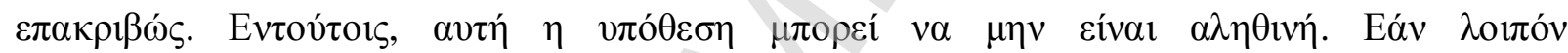

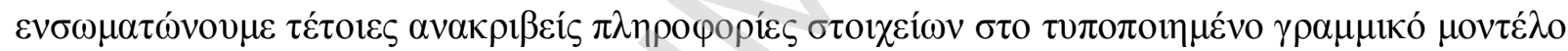

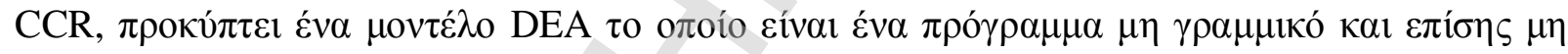

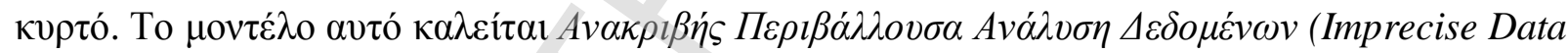

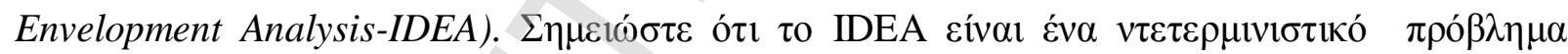

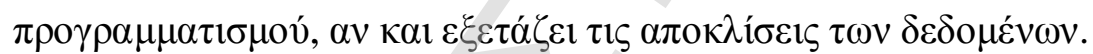

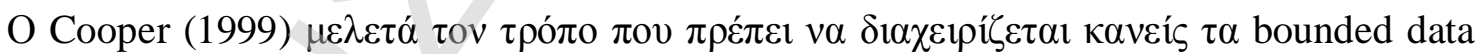

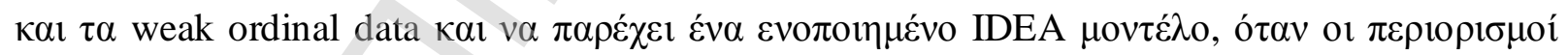

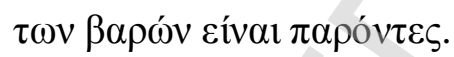

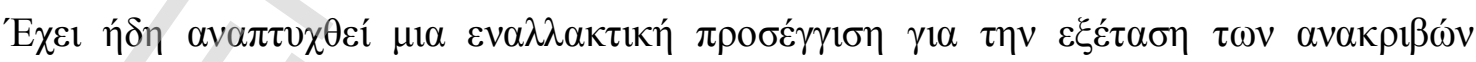

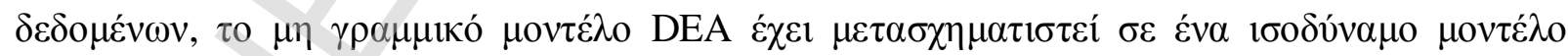

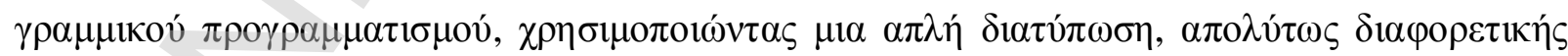

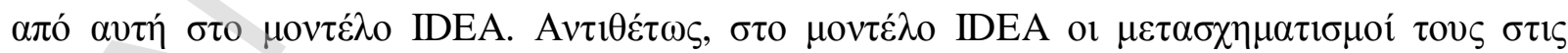

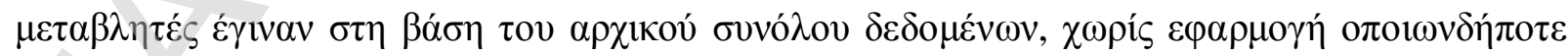

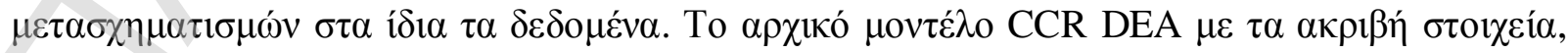

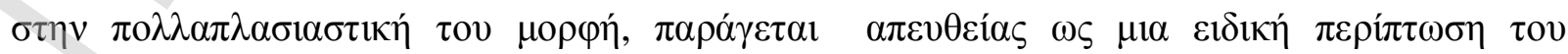

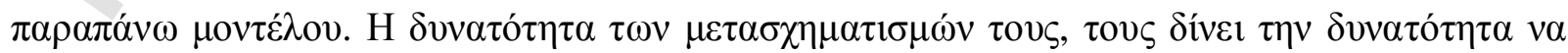

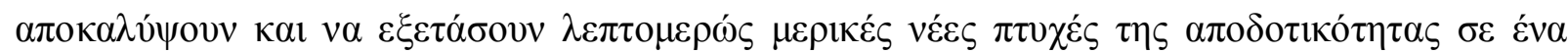




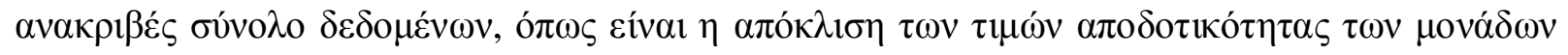

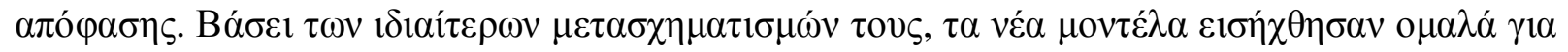

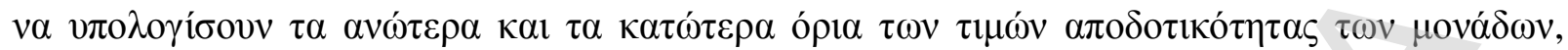

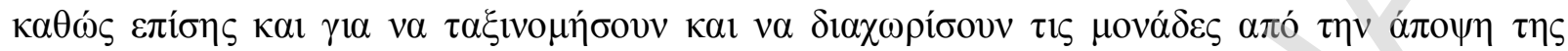

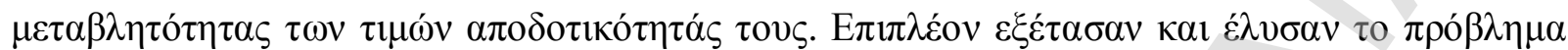

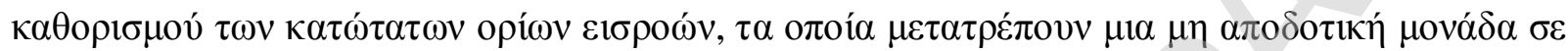

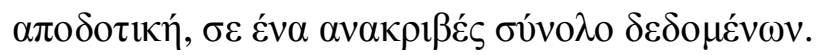

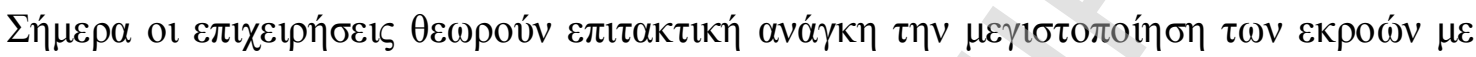

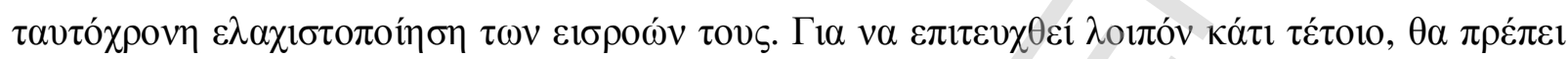

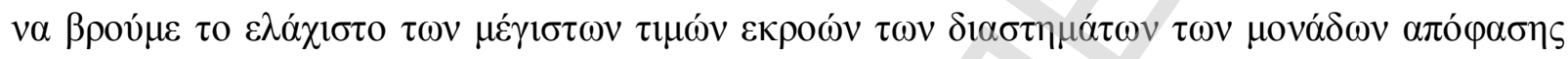

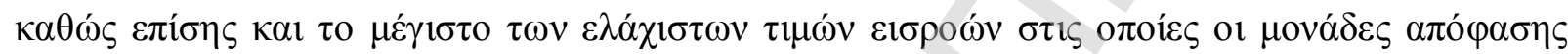

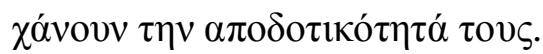

\subsubsection{Movtéরa IDEA}

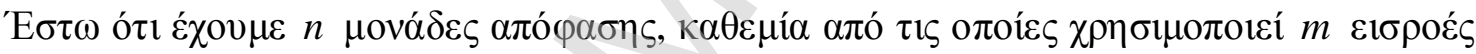

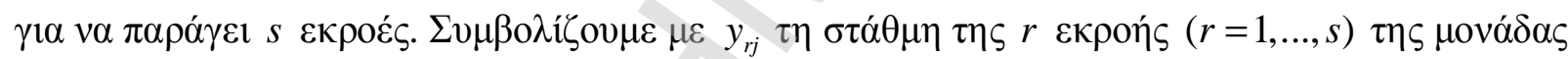

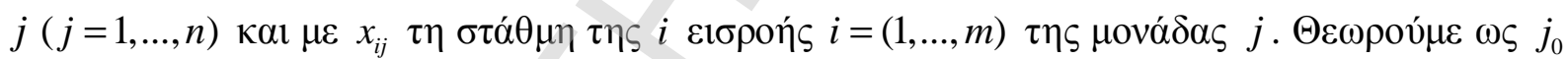

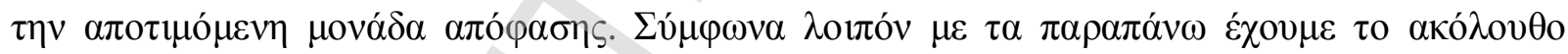

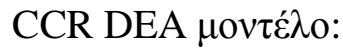

$$
\begin{aligned}
& \max _{j_{0}}=\sum_{r=1}^{S} u_{r} y_{r j_{0}} \\
& \text { s.t } \\
& \sum_{i=1}^{m} v_{i} x_{i j_{0}}=1 \\
& \sum_{r=1}^{S} u_{r} y_{r j}-\sum_{i=1}^{m} v_{i} x_{i j} \leq 0 \\
& j=1, \ldots, n \\
& u_{r}, v_{i} \geq \varepsilon, \quad \forall r, i
\end{aligned}
$$

$\operatorname{Mov\tau \varepsilon ́\lambda o~(I)~}$ 


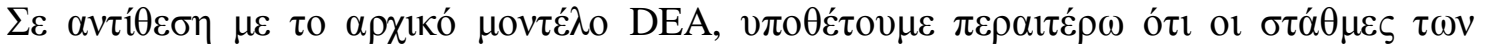

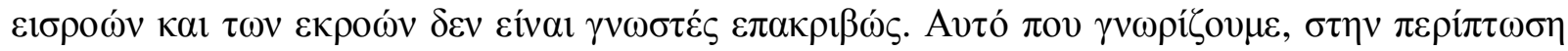

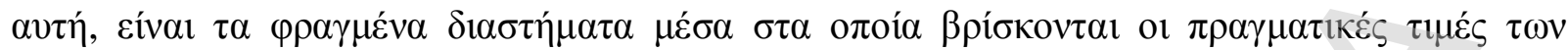

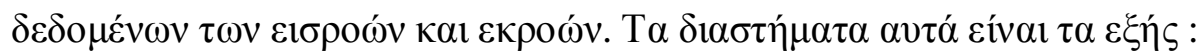

$$
x_{i j} \in\left[x_{i j}^{L}, x_{i j}^{U}\right] \quad \kappa \alpha 1 \quad y_{r j} \in\left[y_{r j}^{L}, y_{r j}^{U}\right]
$$

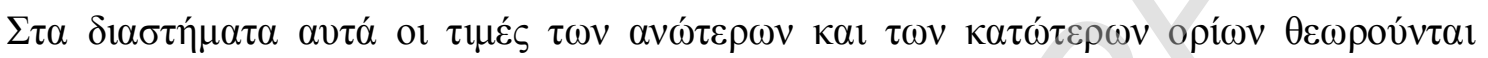

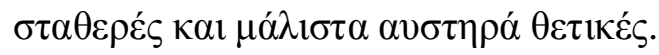

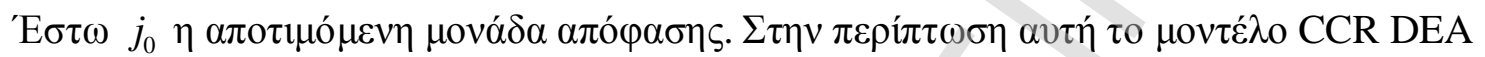

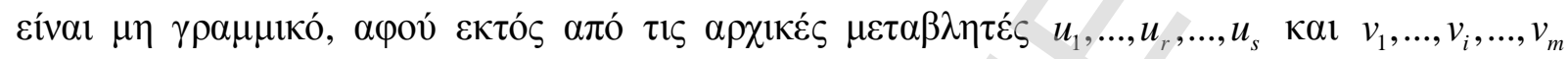

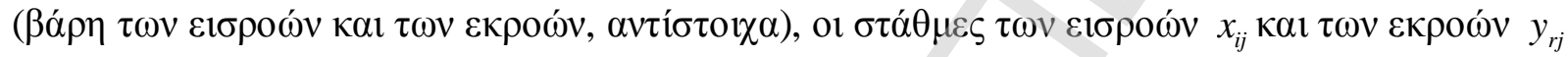

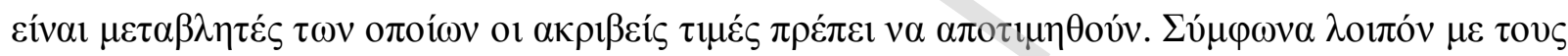

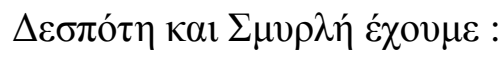

$$
\begin{gathered}
x_{i j}=x_{i j}^{L}+s_{i j}\left(x_{i j}^{U}-x_{i j}^{L}\right), i=1, \ldots, m ; j=1, \ldots, n \quad \mu \varepsilon 0 \leq s_{i j} \leq 1 \\
\kappa \alpha l \\
y_{r j}=y_{r j}^{L}+t_{r j}\left(y_{r j}^{U}-y_{r j}^{L}\right), r=1, \ldots, s ; j=1, \ldots, n \quad \mu \varepsilon 0 \leq t_{r j} \leq 1
\end{gathered}
$$

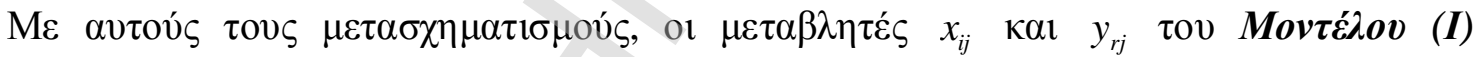

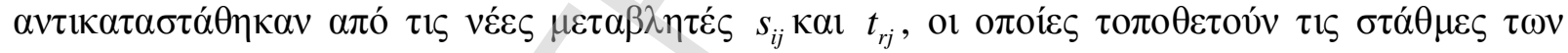

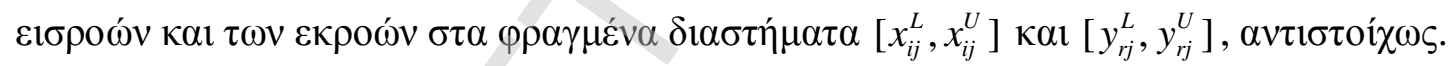

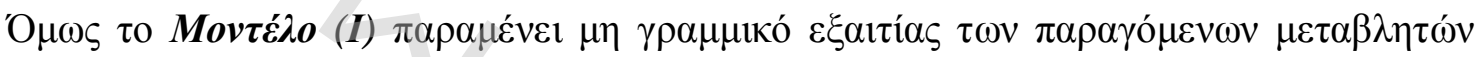

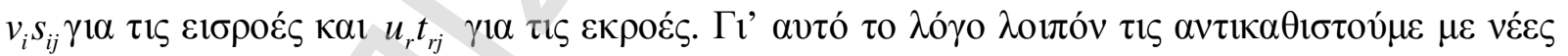
$\mu \varepsilon \tau \alpha \beta \lambda \eta \tau \dot{\varepsilon} \varsigma q_{i j}=v_{i} s_{i j} \kappa \alpha 1 p_{r j}=u_{r} t_{r j}$.

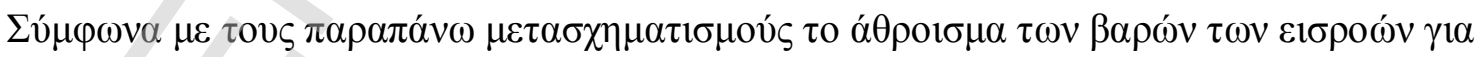

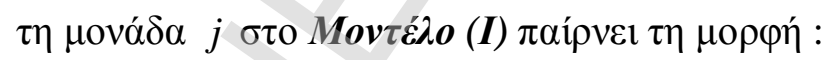

$$
\begin{aligned}
\sum_{i=1}^{m} v_{i} x_{i j} & =\sum_{i=1}^{m} v_{i}\left[x_{i j}^{L}+s_{i j}\left(x_{i j}^{U}-x_{i j}^{L}\right)\right] \\
& =\sum_{i=1}^{m} v_{i} x_{i j}^{L}+v_{i} s_{i j}\left(x_{i j}^{U}-x_{i j}^{L}\right) \\
& =\sum_{i=1}^{m} v_{i} x_{i j}^{L}+q_{i j}\left(x_{i j}^{U}-x_{i j}^{L}\right)
\end{aligned}
$$




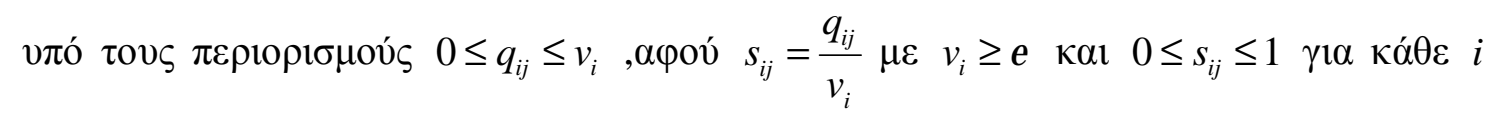
$\kappa \alpha_{1} j$.

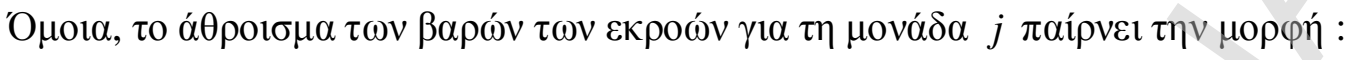

$$
\begin{aligned}
\sum_{r=1}^{S} u_{r} y_{r j} & =\sum_{r=1}^{S} u_{r}\left[y_{r j}^{L}+t_{i j}\left(y_{r j}^{U}-y_{r j}^{L}\right)\right] \\
& =\sum_{r=1}^{m} u_{r} y_{r j}^{L}+u_{r} t_{r j}\left(y_{r j}^{U}-y_{r j}^{L}\right) \\
& =\sum_{r=1}^{S} u_{r} y_{r j}^{L}+p_{r j}\left(y_{r j}^{U}-y_{r j}^{L}\right)
\end{aligned}
$$

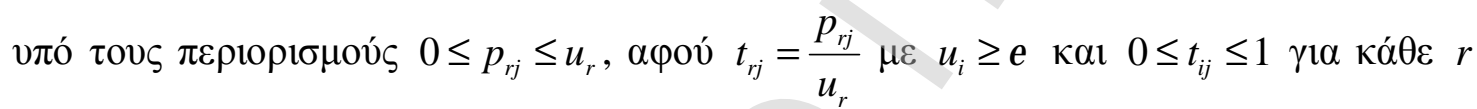
$\kappa \alpha 1 j$.

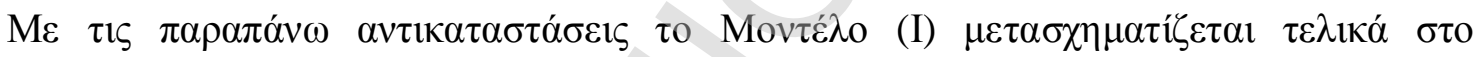
$\pi \alpha \rho \alpha \kappa \alpha ́ \tau \omega ~ \gamma \rho \alpha \mu \mu \iota \kappa o ́ ~ \pi \rho o ́ \gamma \rho \alpha \mu \mu \alpha$ :

$$
\begin{aligned}
& \max h_{j_{0}}=\sum_{r=1}^{s} u_{r} y_{r j_{0}}^{L}+p_{r j_{0}}\left(y_{r j_{0}}^{U}-y_{r j_{0}}^{L}\right) \\
& \text { s.t. } \\
& \sum_{i=1}^{m} v_{i} x_{i j_{0}}^{L}+q_{i j_{0}}\left(x_{i j_{0}}^{U}-x_{i j_{0}}^{L}\right)=1 \\
& \sum_{r=1}^{s} u_{r} y_{r j}^{L}+p_{r j}\left(y_{r j}^{U}-y_{r j}^{L}\right)-\sum_{i=1}^{m} v_{i} x_{i j}^{L}+q_{i j}\left(x_{i j}^{U}-x_{i j}^{L}\right) \leq 0 \\
& j=1, \ldots, n \quad j \neq j_{0} \\
& p_{r j}-u_{r} \leq 0 \quad r=1, \ldots, s ; j=1, \ldots, n \\
& q_{i j}-v_{i} \leq 0 \quad i=1, \ldots, m ; j=1, \ldots, n \\
& u_{r}, v_{i} \geq \varepsilon \quad \forall r, i \\
& p_{r j} \geq 0, \quad q_{i j} \geq 0 \quad \forall r, i, j
\end{aligned}
$$

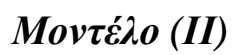




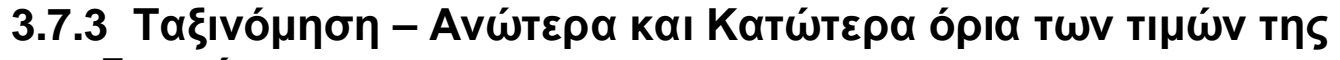 aтாоботікótnTas}

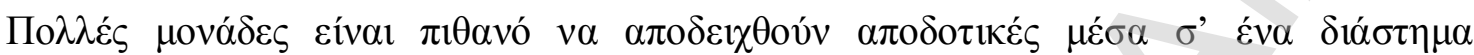

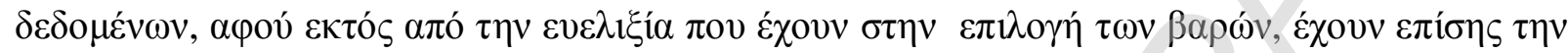

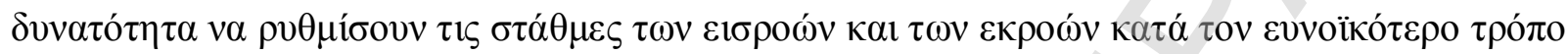

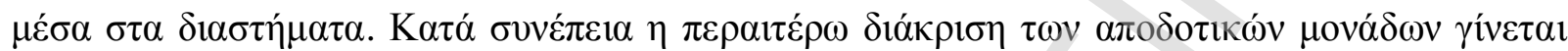

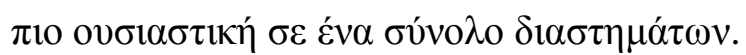

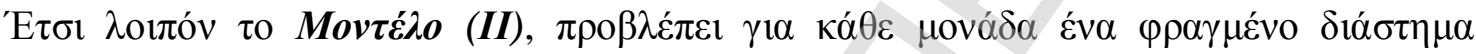

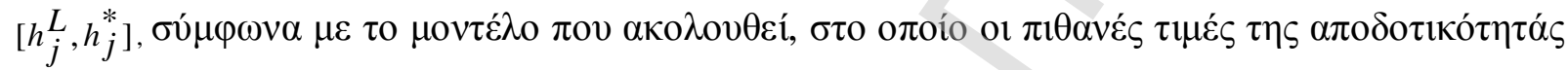

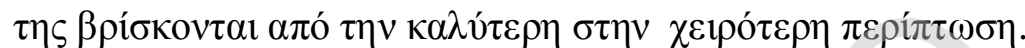

$$
\begin{aligned}
& \max h_{j}^{*}=\sum_{r=1}^{S} u_{r} y_{r j_{0}}^{U} \\
& \text { s.t } \\
& \sum_{i=1}^{m} v_{i} x_{i j_{0}}^{L}=1 \\
& \sum_{r=1}^{S} u_{r} y_{r j_{0}}^{U}-\sum_{i=1}^{m} v_{i} x_{r j_{0}}^{L} \leq 0 \\
& \sum_{r=1}^{s} u_{r} y_{r j}^{L}-\sum_{i=1}^{m} v_{i} x_{r j}^{U} \leq 0 \\
& j \neq j_{0} \quad, j=1, \ldots, n ; \\
& u_{r, v_{i}} \geq \varepsilon, \forall r, i
\end{aligned}
$$

$\operatorname{Mov\tau \varepsilon ́\lambda o~(III)~}$

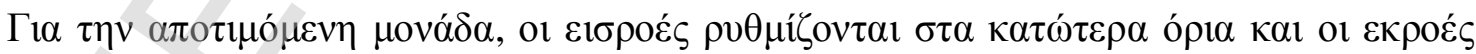

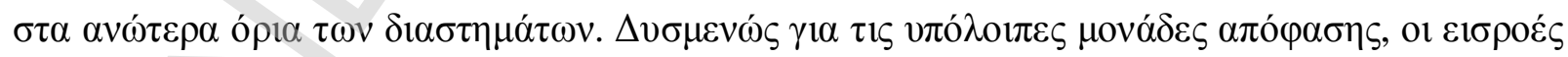

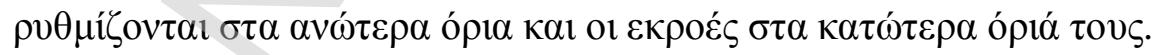




$$
\begin{aligned}
& \max h_{j}^{L}=\sum_{r=1}^{s} u_{r} y_{r_{j_{0}}}^{L} \\
& \text { s.t } \\
& \sum_{i=1}^{m} v_{i} x_{i j_{0}}^{U}=1 \\
& \sum_{r=1}^{s} u_{r} y_{r_{0}}^{L}-\sum_{i=1}^{m} v_{i} x_{i j_{0}}^{U} \leq 0 \\
& \sum_{r=1}^{s} u_{r} y_{r j}^{U}-\sum_{i=1}^{m} v_{i} x_{i j}^{L} \leq 0 \\
& j \neq j_{0} \quad j=1, \ldots, n \\
& u_{r}, v_{i} \geq \varepsilon, \forall r, i
\end{aligned}
$$

$\operatorname{Mov\tau \varepsilon ́\lambda o(IV)~}$

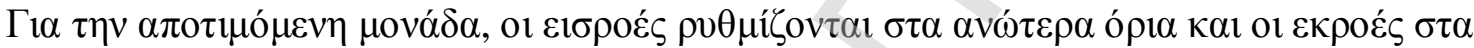

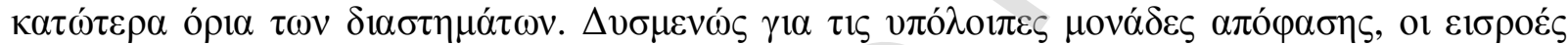

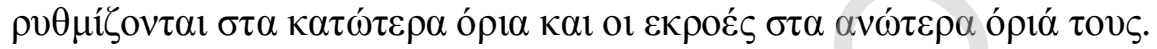

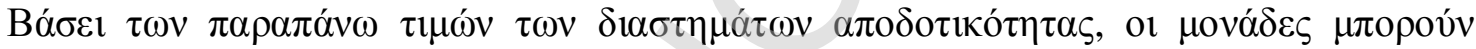

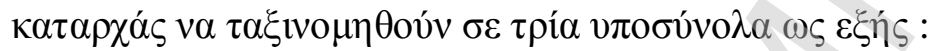

$$
\begin{aligned}
& E^{++}=\left\{j \in J / h_{j}^{L}=1\right\} \\
& E^{+}=\left\{j \in J / h_{j}^{L}<1 \kappa \alpha \iota h_{j}^{*}=1\right\} \\
& E^{-}=\left\{j \in J / h_{j}^{*}<1\right\}
\end{aligned}
$$

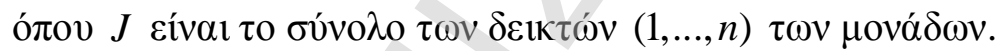

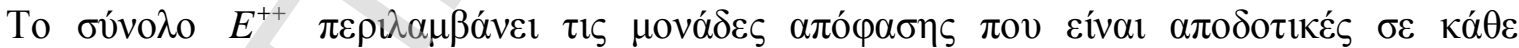

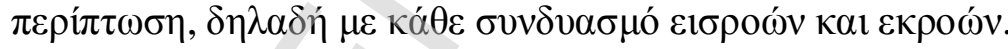

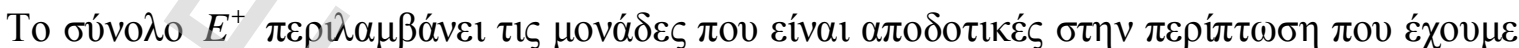

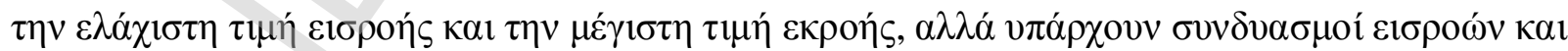

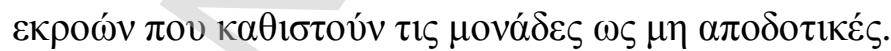

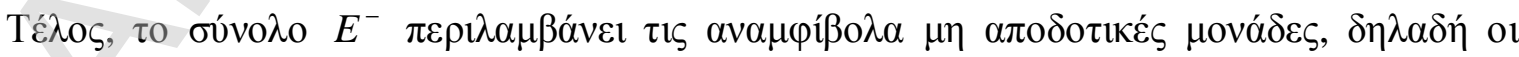

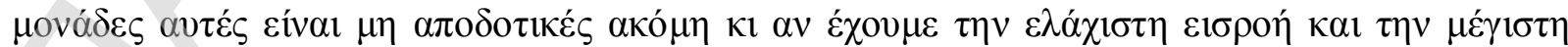

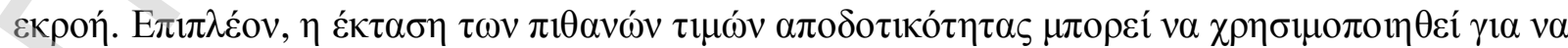

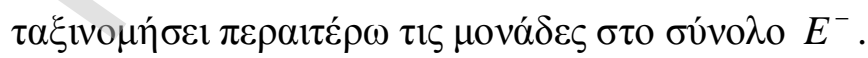




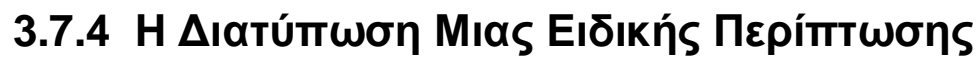

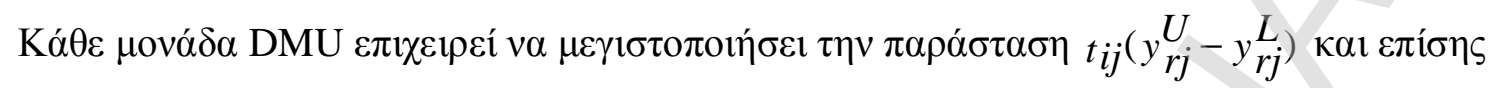

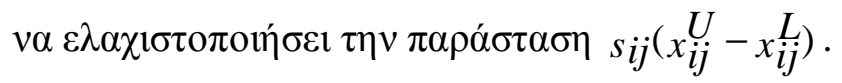

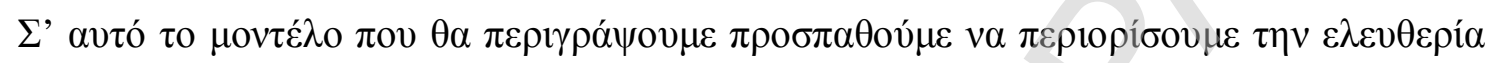

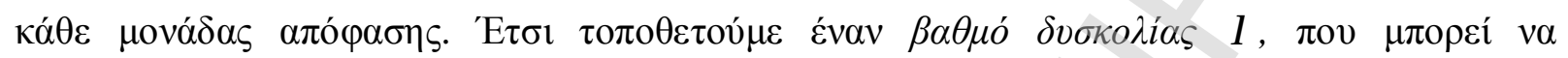

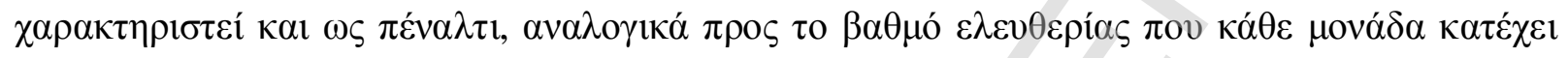

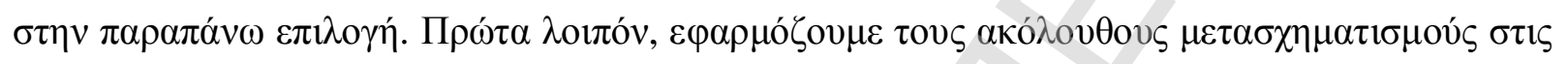
$\mu \varepsilon \tau \alpha \beta \lambda \eta \tau \dot{\varepsilon} \varsigma x_{i j} \kappa \alpha 1 \quad y_{r j}:$

$$
\begin{aligned}
& x_{i j}=x_{i j}^{L}+s_{i j}\left(x_{i j}^{U}-x_{i j}^{L}\right)+\lambda\left[x_{i j}^{U}-x_{i j}^{L}-s_{i j}\left(x_{i j}^{U}-x_{i j}^{L}\right)\right] \\
& i=1, \ldots, m ; \quad j=1, \ldots, n \quad \mu \varepsilon \quad 0 \leq s_{i j} \leq 1 \\
& y_{r j}=y_{r j}^{L}+t_{r j}\left(y_{r j}^{U}-y_{r j}^{L}\right)-\lambda t_{r j}\left(y_{r j}^{U}-y_{r j}^{L}\right) \\
& r=1, \ldots, s ; \quad j=1, \ldots, n \quad \mu \varepsilon \quad 0 \leq t_{r j} \leq 1, \\
& 0 \leq \lambda \leq 1
\end{aligned}
$$

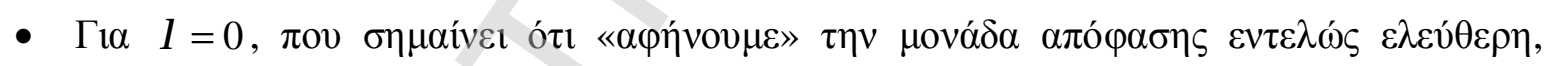
$\varepsilon \chi \chi 00 \mu \varepsilon$ :

$$
\begin{gathered}
x_{i j}=x_{i j}^{L}+s_{i j}\left(x_{i j}^{U}-x_{i j}^{L}\right) \\
\kappa \alpha \iota \\
y_{r j}=y_{r j}^{L}+t_{r j}\left(y_{r j}^{U}-y_{r j}^{L}\right)
\end{gathered}
$$

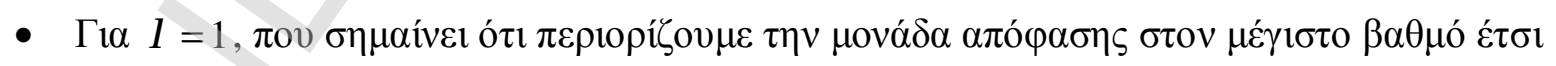

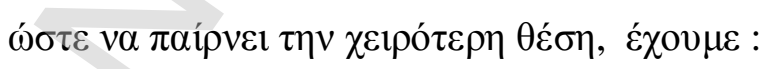

$$
\begin{gathered}
x_{i j}=x_{i j}^{U} \\
\kappa \alpha \iota \\
y_{r j}=y_{r j}^{L}
\end{gathered}
$$




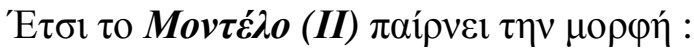

$$
\begin{aligned}
& \max h_{j_{0}}=\sum_{r=1}^{s} u_{r} y_{r j_{0}}^{L}+p_{r j_{0}}\left(y_{r j_{0}}^{U}-y_{r j_{0}}^{L}\right)-\sum_{r=1}^{s} \lambda p_{r j_{0}}\left(y_{r j_{0}}^{U}-y_{r j_{0}}^{L}\right) \\
& \text { s.t. } \\
& \sum_{i=1}^{m} v_{i} x_{i j_{0}}^{L}+q_{i j_{0}}\left(x_{i j_{0}}^{U}-x_{i j_{0}}^{L}\right)+ \\
& \quad \sum_{i=1}^{m} \lambda\left[v_{i} x_{i j_{0}}^{U}-v_{i} x_{i j_{0}}^{L}-q_{i j_{0}}\left(x_{i j_{0}}^{U}-x_{i j_{0}}^{L}\right)\right]=1 \\
& \sum_{r=1}^{s} u_{r} y_{r j_{0}}^{L}+p_{r j_{0}}\left(y_{r j_{0}}^{U}-y_{r j_{0}}^{L}\right)-\sum_{r=1}^{s} \lambda p_{r j_{0}}\left(y_{r j_{0}}^{U}-y_{r j_{0}}^{L}\right) \leq \\
& \quad \sum_{i=1}^{m} v_{i} x_{i j_{0}}^{L}+q_{i j_{0}}\left(x_{i j_{0}}^{U}-x_{i j_{0}}^{L}\right)+\sum_{i=1}^{m} \lambda\left[v_{i} x_{i j_{0}}^{U}-v_{i} x_{i j_{0}}^{L}-q_{i j_{0}}\left(x_{i j_{0}}^{U}-x_{i j_{0}}^{L}\right)\right] \\
& \sum_{r=1}^{s} u_{r} y_{r j}^{L}+p_{r j}\left(y_{r j}^{U}-y_{r j}^{L}\right)-\sum_{i=1}^{m} v_{i} x_{i j}^{L}+q_{i j}\left(x_{i j}^{U}-x_{i j}^{L}\right) \leq 0 \\
& j=1, \ldots, n \quad j \neq j_{0} \\
& p_{r j}-u_{r} \leq 0 \quad r=1, \ldots, s ; j=1, \ldots, n \\
& q_{i j}-v_{i} \leq 0 \quad i=1, \ldots, m ; j=1, \ldots, n \\
& u_{r}, v_{i} \geq \varepsilon \quad \forall r, i \\
& p_{r j} \geq 0, q_{i j} \geq 0 \quad \forall r, i, j \\
& 0 \leq \lambda \leq 1 \quad
\end{aligned}
$$

$\operatorname{Mov} \tau \varepsilon \dot{\lambda} \lambda(V)$

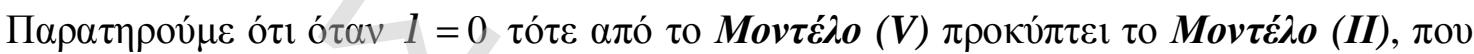

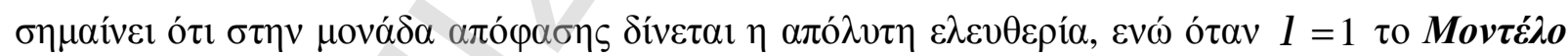

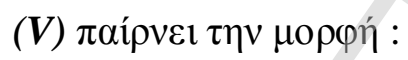




$$
\begin{aligned}
& \max h_{j_{0}}=\sum_{r=1}^{s} u_{r} y_{r j_{0}}^{L} \\
& \text { s.t. } \\
& \sum_{i=1}^{m} v_{i} x_{i j_{0}}^{U}=1 \\
& \sum_{r=1}^{s} u_{r} y_{r j}^{L}+p_{r j}\left(y_{r j}^{U}-y_{r j}^{L}\right)-\sum_{i=1}^{m} v_{i} x_{i j}^{L}+q_{i j}\left(x_{i j}^{U}-x_{i j}^{L}\right) \leq 0 \\
& j=1, \ldots, n \quad j \neq j_{0} \\
& p_{r j}-u_{r} \leq 0 \quad r=1, \ldots, s ; j=1, \ldots, n \\
& q_{i j}-v_{i} \leq 0 \quad i=1, \ldots, m ; j=1, \ldots, n \\
& u_{r}, v_{i} \geq \varepsilon \quad \forall r, i \\
& p_{r j} \geq 0, q_{i j} \geq 0 \quad \forall r, i, j \\
& 0 \leq \lambda \leq 1
\end{aligned}
$$

\section{$\operatorname{Mov\tau \varepsilon ́\lambda o~(VI)~}$}

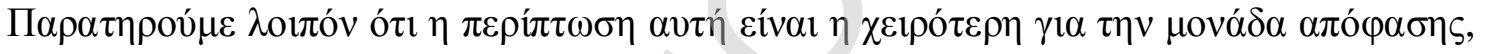

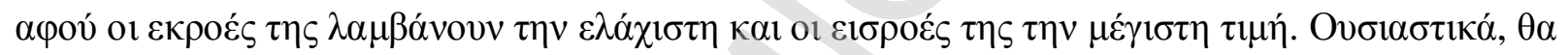

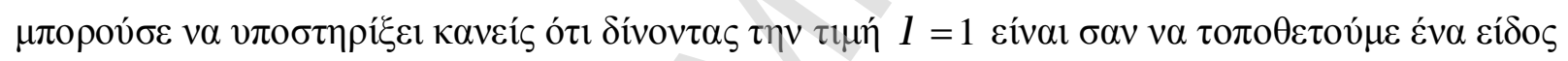

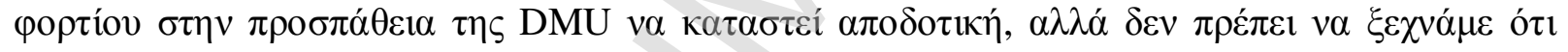

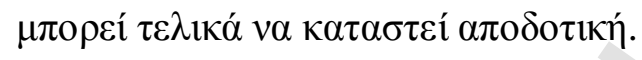

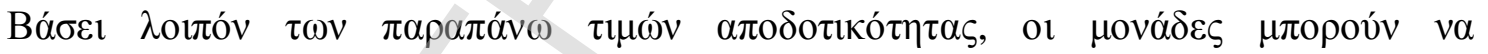

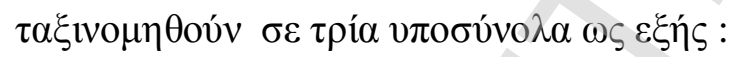

$$
\begin{aligned}
& D^{++}=\left\{j \in J / h_{j}=1\right\} \\
& D^{+}=\left\{j \in J / h_{j}=[0 . .1]\right\} \\
& D^{-}=\left\{j \in J / h_{j}<1\right\}
\end{aligned}
$$

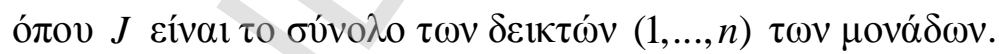

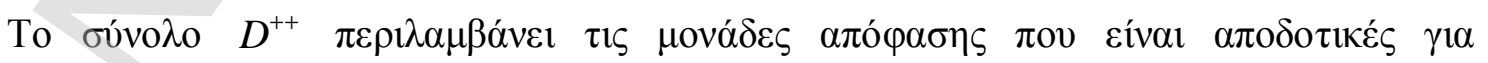
o

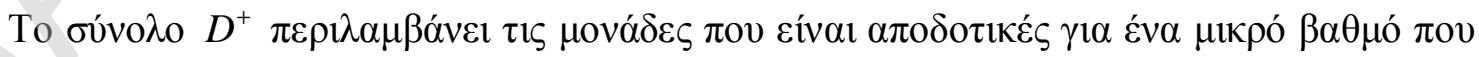

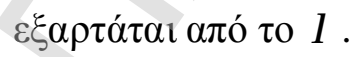

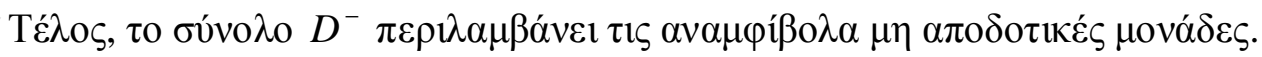




\section{$\Pi \alpha \rho \alpha ́ \delta \varepsilon \imath \gamma \mu \alpha 3.2$}

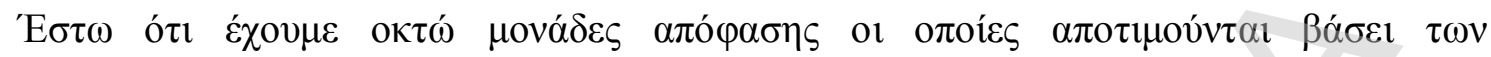

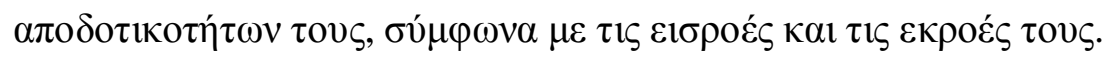

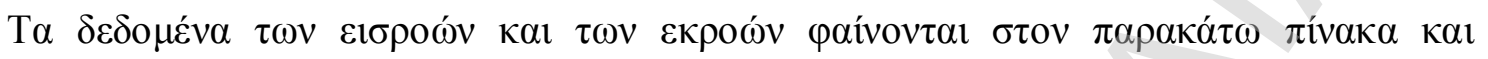

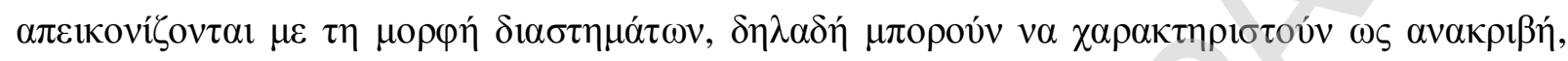

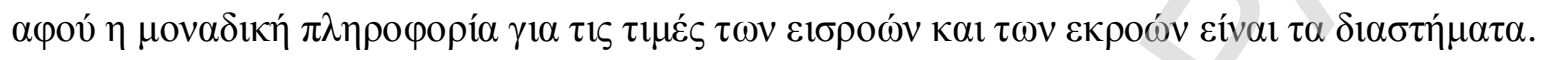

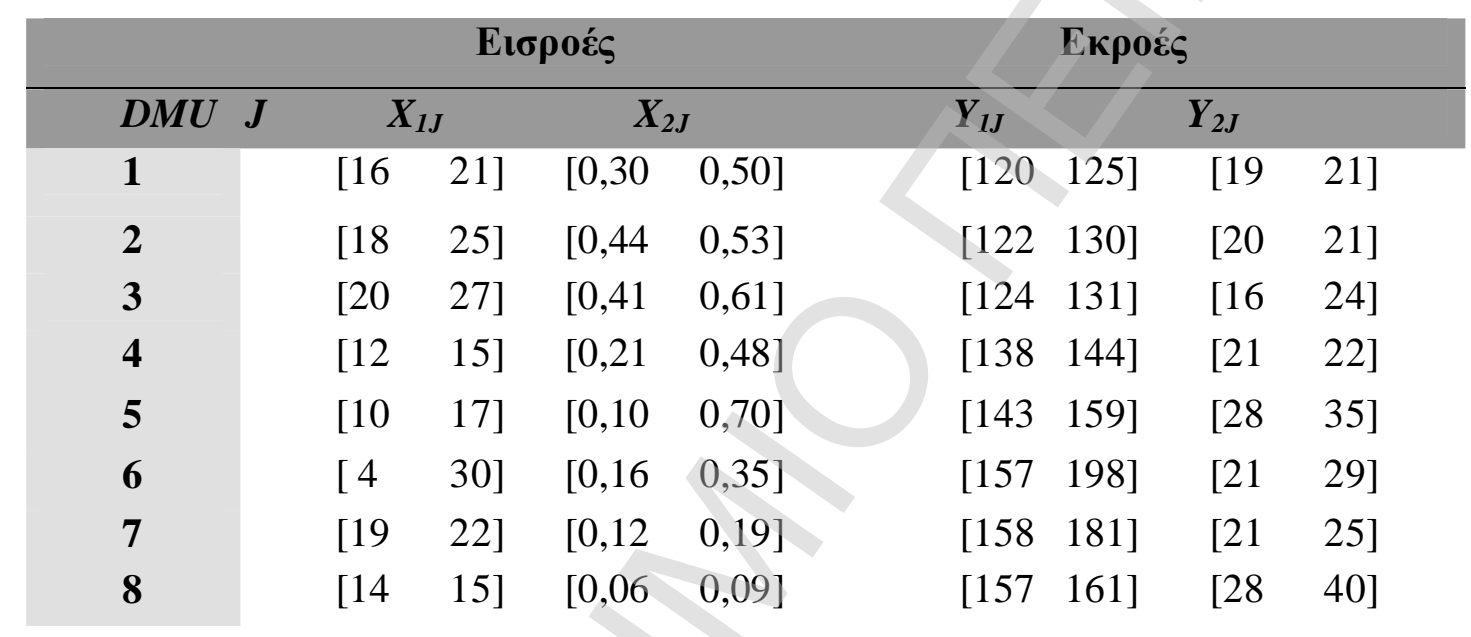

\section{Пívaкаऽ 3.2}

Е

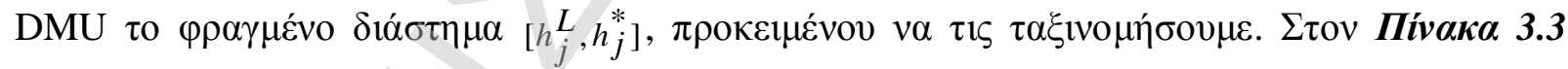

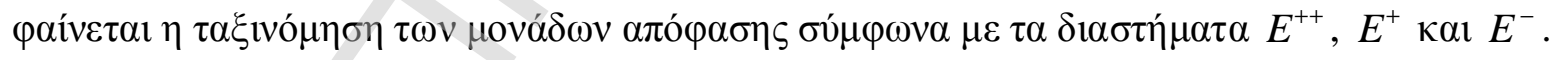




\begin{tabular}{|c|c|c|c|c|c|c|c|c|c|c|}
\hline & \multicolumn{4}{|c|}{ Eıбров́ } & \multicolumn{3}{|c|}{ Екров́ } & & & \multirow[b]{2}{*}{ 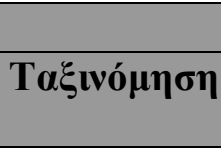 } \\
\hline DMU & & $X_{I}$ & & $Y_{2}$ & & $Y_{1}$ & $Y_{2}$ & 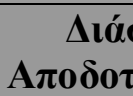 & $\begin{array}{l}\eta \mu \alpha \\
\text { ót } \tau \eta \alpha \varsigma\end{array}$ & \\
\hline 1 & {$[16$} & 21] & {$[0,30$} & $0,50]$ & {$[120$} & 125] & {$\left[\begin{array}{ll}19 & 21\end{array}\right]$} & {$[0,177$} & $0,746]$ & $\mathbf{E}^{-}$ \\
\hline 2 & {$[18$} & 25] & {$[0,40$} & $0,53]$ & {$[122$} & 130] & {$\left[\begin{array}{ll}20 & 21\end{array}\right]$} & {$[0,165$} & $0,693]$ & $\mathbf{E}^{-}$ \\
\hline 3 & {$[20$} & 27] & {$[0,41$} & $0,61]$ & {$[124$} & 131] & {$\left[\begin{array}{ll}16 & 24\end{array}\right]$} & {$[0,148$} & $0,643]$ & $\mathbf{E}^{-}$ \\
\hline 4 & {$[12$} & 15] & {$[0,21$} & $0,48]$ & {$[138$} & 144] & {$\left[\begin{array}{ll}21 & 22\end{array}\right]$} & {$[0,224$} & $1,000]$ & $\mathbf{E}^{+}$ \\
\hline 5 & {$[10$} & 17] & {$[0,10$} & $0,70]$ & {$[143$} & 159] & {$\left[\begin{array}{ll}28 & 35\end{array}\right]$} & {$[0,227$} & $1,000]$ & $\mathbf{E}^{+}$ \\
\hline 6 & {$[4$} & 30] & {$[0,16$} & $0,35]$ & {$[157$} & 198] & {$\left[\begin{array}{ll}21 & 29\end{array}\right]$} & {$[0,823$} & $1,000]$ & $\mathbf{E}^{+}$ \\
\hline 7 & {$[19$} & 22] & {$[0,12$} & $0,19]$ & {$[158$} & 181] & {$\left[\begin{array}{ll}21 & 25\end{array}\right]$} & {$[0,445$} & $0,907]$ & $\mathbf{E}^{-}$ \\
\hline 8 & {$[14$} & 15] & {$[0,06$} & $0,09]$ & {$[157$} & 161] & {$\left[\begin{array}{ll}28 & 40\end{array}\right]$} & {$[1,000$} & $1,000]$ & $\mathbf{E}^{++}$ \\
\hline
\end{tabular}

\section{Пívakas 3.3}

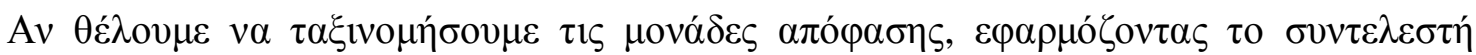

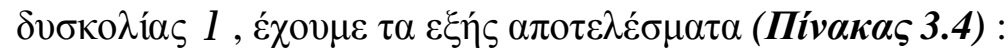

\begin{tabular}{|c|c|c|c|c|c|c|c|c|}
\hline$\Lambda$ & DMU1 & DMU2 & DMU3 & DMU4 & DMU5 & DMU6 & DMU7 & DMU8 \\
\hline 0.0 & 0,746 & 0,693 & 0,643 & 1 & 1 & 1 & 0,907 & 1 \\
\hline 0.1 & 0,720 & 0,660 & 0,600 & 1 & 1 & 1 & 0,880 & 1 \\
\hline 0.2 & 0,695 & 0,630 & 0,578 & 1 & 1 & 1 & 0,860 & 1 \\
\hline 0.3 & 0,671 & 0,600 & 0,557 & 1 & 1 & 1 & 0,835 & 1 \\
\hline 0.4 & 0,650 & 0,580 & 0,537 & 1 & 1 & 1 & 0,812 & 1 \\
\hline 0.5 & 0,630 & 0,550 & 0,518 & 1 & 1 & 1 & 0,790 & 1 \\
\hline 0.6 & 0,600 & 0,538 & 0,500 & 0,970 & 1 & 1 & 0,767 & 1 \\
\hline 0.7 & 0,590 & 0,518 & 0,480 & 0,940 & 1 & 1 & 0,746 & 1 \\
\hline 0.8 & 0,570 & 0,500 & 0,467 & 0,920 & 1 & 1 & 0,725 & 1 \\
\hline 0.9 & 0,550 & 0,480 & 0,450 & 0,900 & 0,94 & 1 & 0,705 & 1 \\
\hline 1.0 & 0,540 & 0,466 & 0,438 & 0,870 & 0,88 & 1 & 0,686 & 1 \\
\hline Class & $D^{-}$ & $D^{-}$ & $D^{-}$ & $D^{+}$ & $D^{+}$ & $D^{++}$ & $D^{-}$ & $D^{++}$ \\
\hline
\end{tabular}

Пívaкаs 3.4

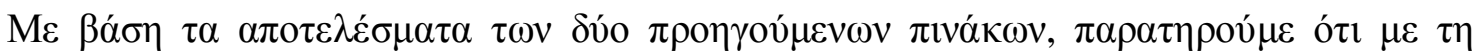

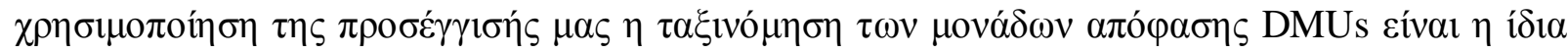

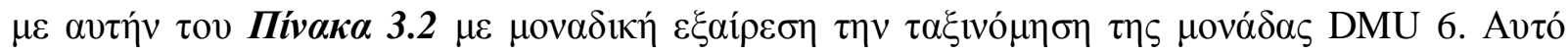

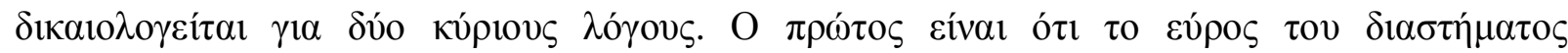

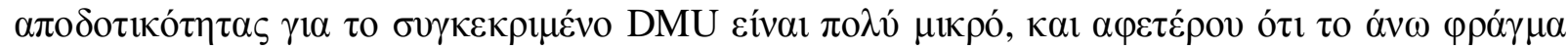

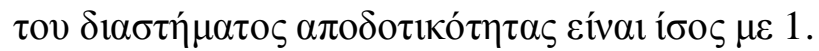




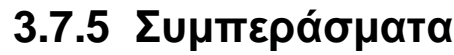

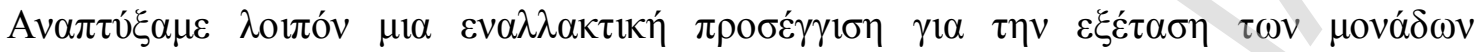

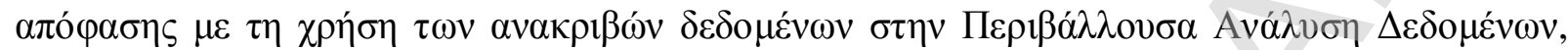

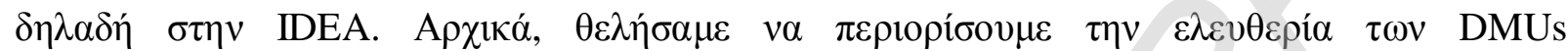

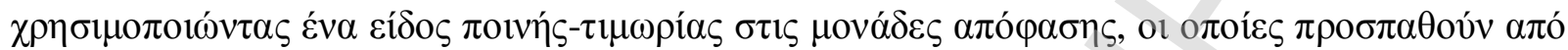

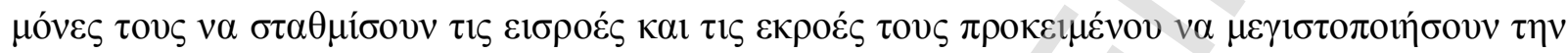

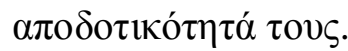

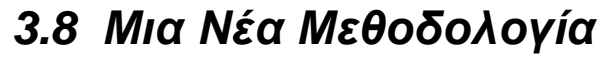

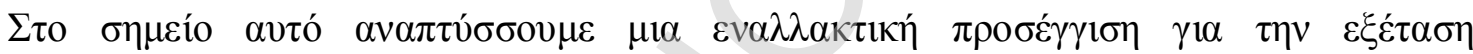

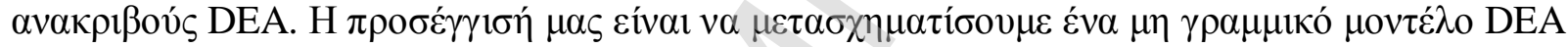

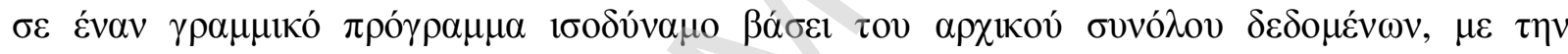

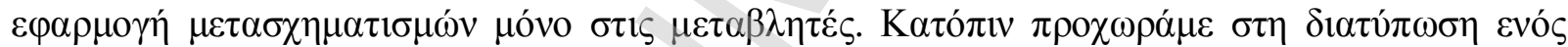

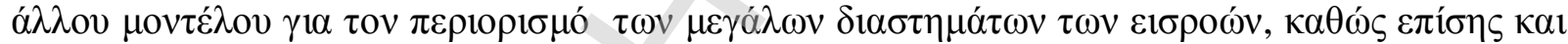

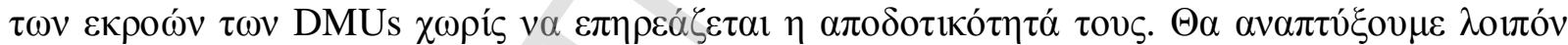

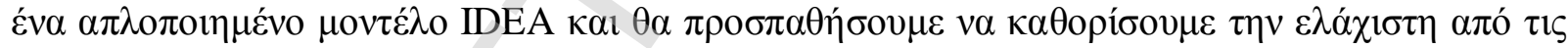

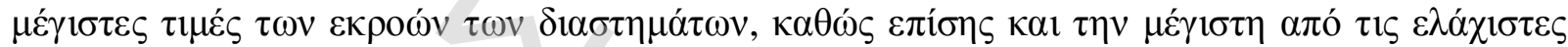

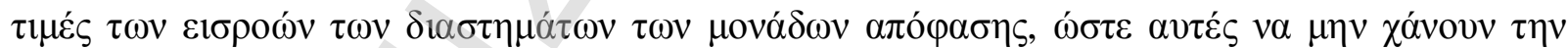

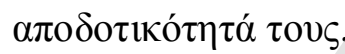

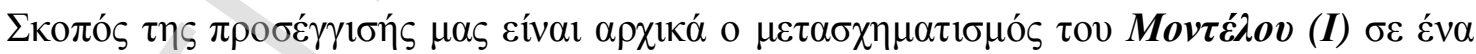

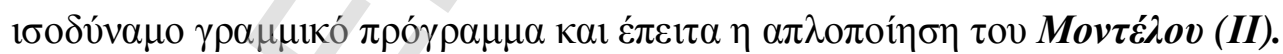

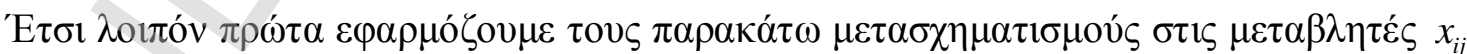
Kal $y_{r j}$ :

$$
\begin{gathered}
x_{i j}=x_{i j}^{L}+\alpha_{i j} \quad i=1, \ldots, n \quad \mu \varepsilon \quad 0 \leq a_{i j} \leq\left(x_{i j}^{U}-x_{i j}^{L}\right) \\
\kappa \alpha \iota \\
y_{r j}=y_{r j}^{L}+\beta_{r j} \quad r=1, \ldots, s \quad \mu \varepsilon \quad 0 \leq \beta_{r j} \leq\left(y_{r j}^{U}-y_{r j}^{L}\right)
\end{gathered}
$$




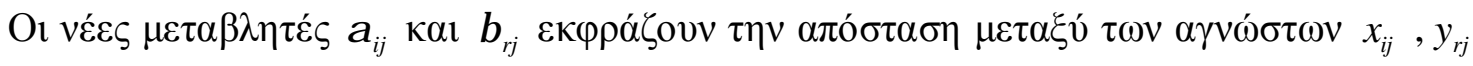

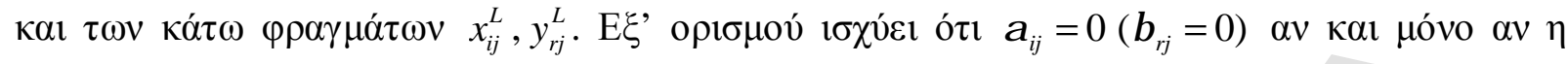

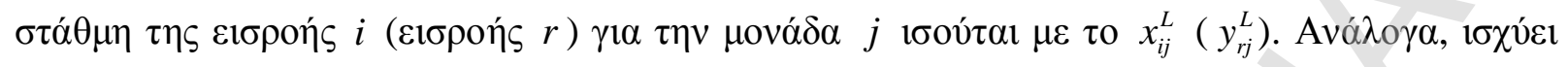

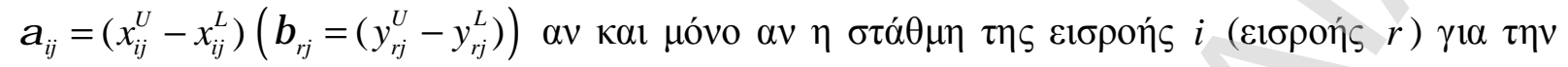

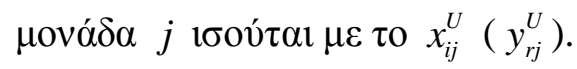

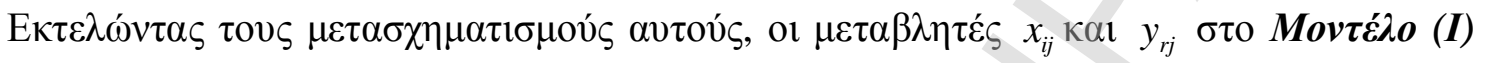

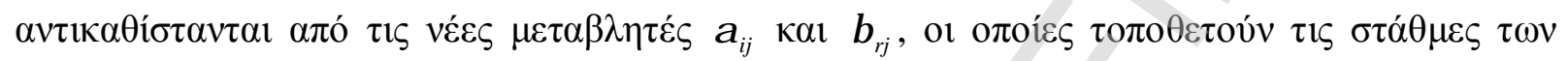

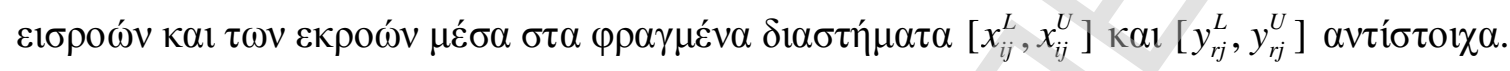

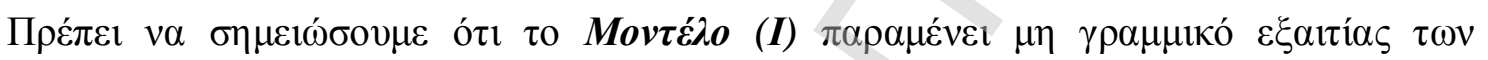

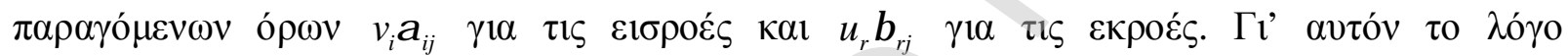

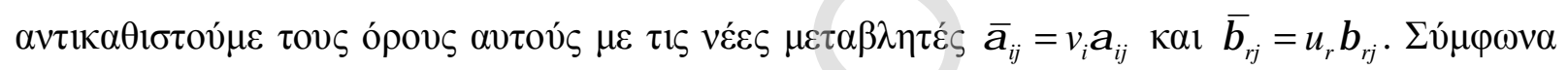

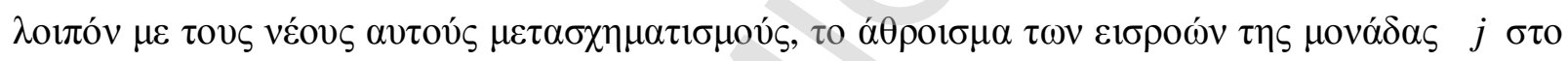

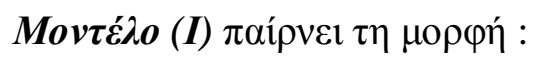

$$
\begin{aligned}
\sum_{i=1}^{m} v_{i} x_{i j} & =\sum_{i=1}^{m} v_{i}\left[x_{i j}^{L}+\alpha_{i j}\right] \\
& =\sum_{i=1}^{m} v_{i} x_{i j}^{L}+v_{i} \alpha_{i j} \\
& =\sum_{i=1}^{m} v_{i} x_{i j}^{L}+\bar{\alpha}_{i j}
\end{aligned}
$$

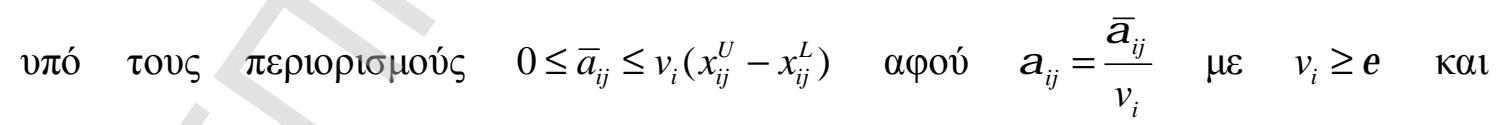
$0 \leq a_{i j} \leq\left(x_{i j}^{U}-x_{i j}^{L}\right) \gamma 1 \alpha \kappa \alpha ́ \theta \varepsilon i \kappa \alpha l j$.

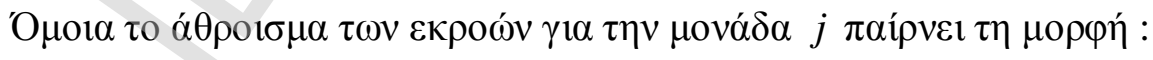

$$
\begin{aligned}
\sum_{r=1}^{S} u_{r} y_{r j} & =\sum_{r=1}^{S} u_{r}\left[y_{r j}^{L}+\beta_{r j}\right] \\
& =\sum_{r=1}^{m} u_{r} y_{r j}^{L}+u_{r} \beta_{r j} \\
& =\sum_{r=1}^{S} u_{r} y_{r j}^{L}+\bar{\beta}_{r j}
\end{aligned}
$$




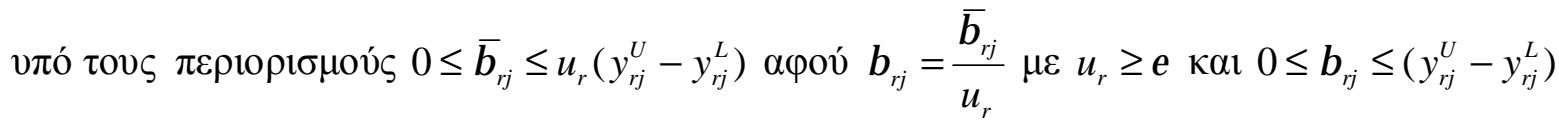
$\gamma 1 \alpha \kappa \alpha ́ \theta \varepsilon r \kappa \alpha i j$.

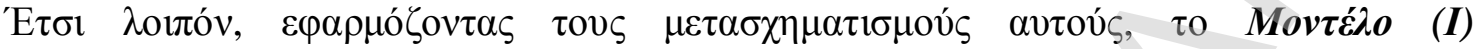

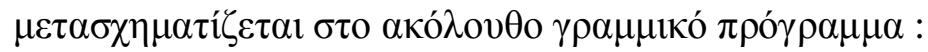

$$
\begin{aligned}
& \max _{j_{0}}=\sum_{r=1}^{S} u_{r} y_{r j_{0}}^{L}+\bar{\beta}_{r j} \\
& \text { s.t } \\
& \sum_{i=1}^{m} v_{i} x_{i j_{0}}^{L}+\bar{a}_{i j}=1 \\
& \sum_{r=1}^{S} u_{r} y_{r j}^{L}+\bar{\beta}_{r j}-\sum_{i=1}^{m} v_{i} x_{i j}^{L}+\bar{\alpha}_{i j} \leq 0 \quad j=1, \ldots, n \\
& 0 \leq \bar{\beta}_{r j} \leq u_{r}\left(y_{r j}^{U}-y_{r j}^{L}\right) \quad r=1, \ldots, s ; j=1, \ldots, n \\
& 0 \leq \bar{a}_{i j} \leq v_{i}\left(x_{i j}^{U}-x_{i j}^{L}\right) \quad i=1, \ldots, m ; j=1, \ldots, n \\
& u_{r}, v_{i} \geq \varepsilon, \quad \forall r, i
\end{aligned}
$$

\section{$\operatorname{Mov\tau \varepsilon ́\lambda o~(VII)~}$}

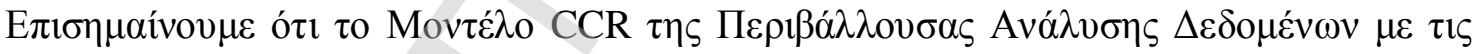

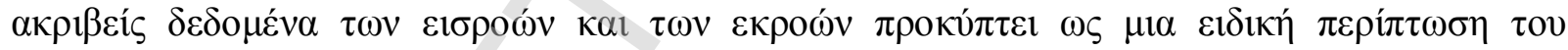

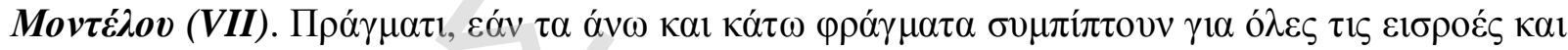

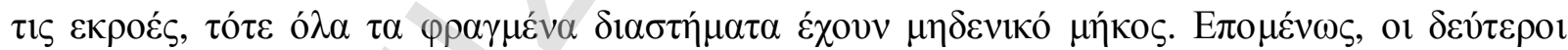

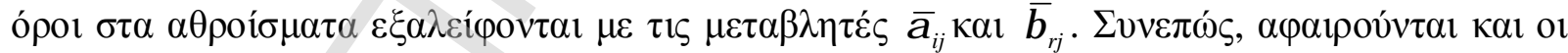

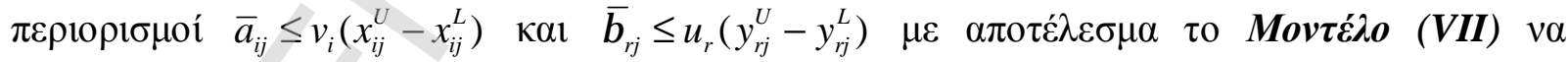

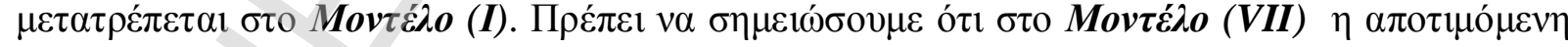

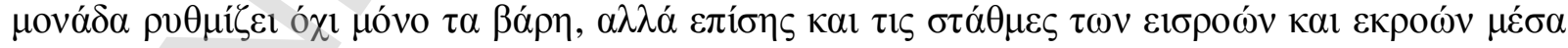

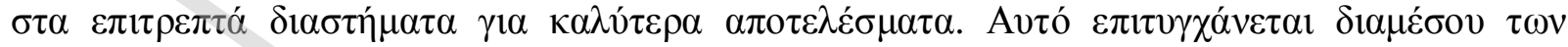

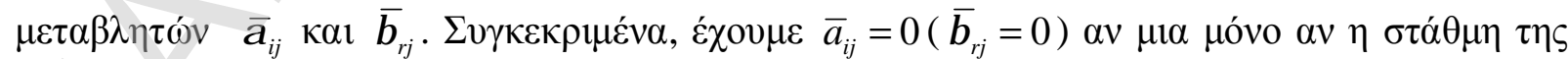

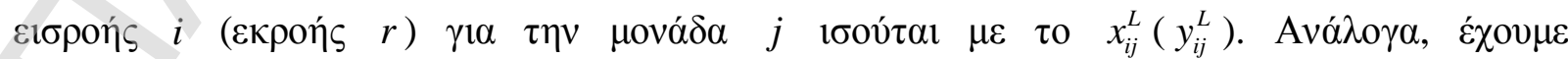

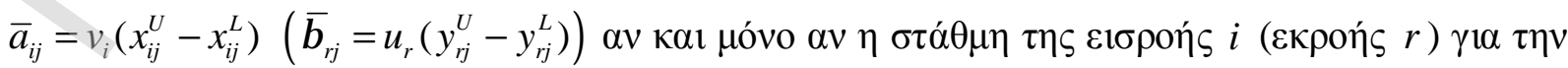

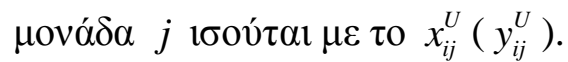




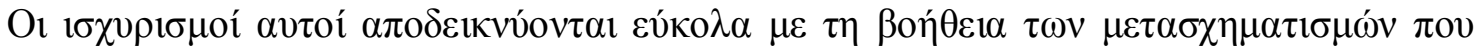

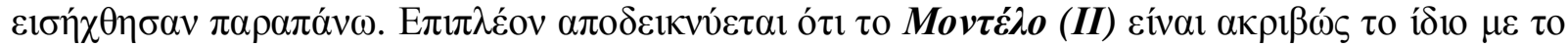

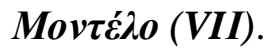

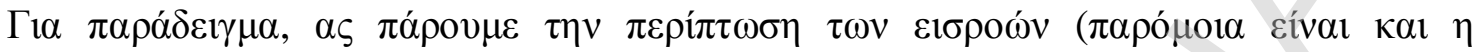
$\pi \varepsilon \rho i ́ \pi \tau \omega \sigma \eta \tau \omega v \varepsilon \kappa \rho о \omega ́ v)$.

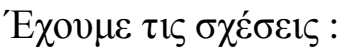

$$
\begin{aligned}
& x_{i j}=x_{i j}^{L}+s_{i j}\left(x_{i j}^{U}-x_{i j}^{L}\right), i=1, \ldots, m ; j=1, \ldots, n \quad \mu \varepsilon 0 \leq s_{i j} \leq 1 \\
& x_{i j}=x_{i j}^{L}+\alpha_{i j}, i=1, \ldots, m ; j=1, \ldots, n \quad \mu \varepsilon \quad 0 \leq \alpha_{i j} \leq\left(x_{i j}^{U}-x_{i j}^{L}\right)
\end{aligned}
$$

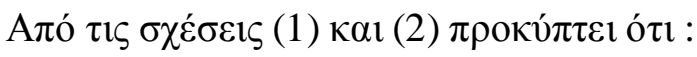

$$
\begin{aligned}
& 0 \leq s_{i j} \leq 1 \equiv 0 \leq \alpha_{i j} \leq\left(x_{i j}^{U}-x_{i j}^{L}\right) \quad \Leftrightarrow \\
& 0 \leq v_{i} s_{i j} \leq v_{i} \equiv 0 \leq v_{i} \alpha_{i j} \leq v_{i}\left(x_{i j}^{U}-x_{i j}^{L}\right) \Leftrightarrow \\
& 0 \leq q_{i j} \leq v_{i} \equiv 0 \leq \bar{a}_{i j} \leq v_{i}\left(x_{i j}^{U}-x_{i j}^{L}\right) \quad \forall i, j
\end{aligned}
$$

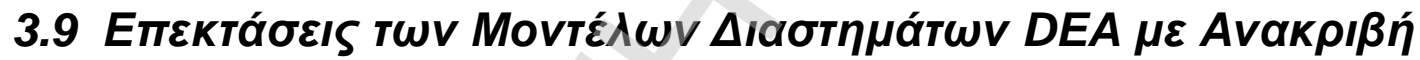 $\Delta \varepsilon \delta \circ \mu \varepsilon ́ v \alpha$}

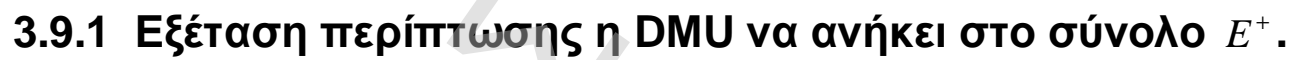

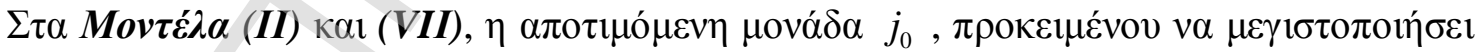

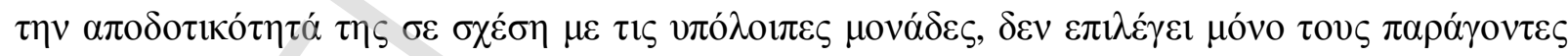

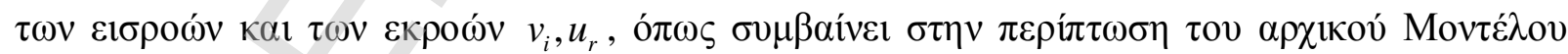

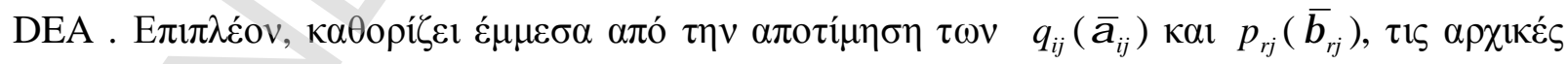

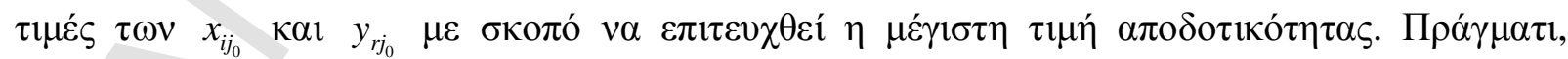

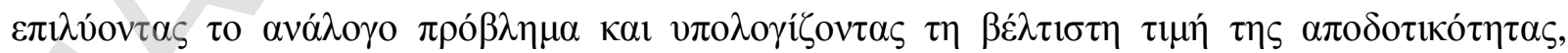




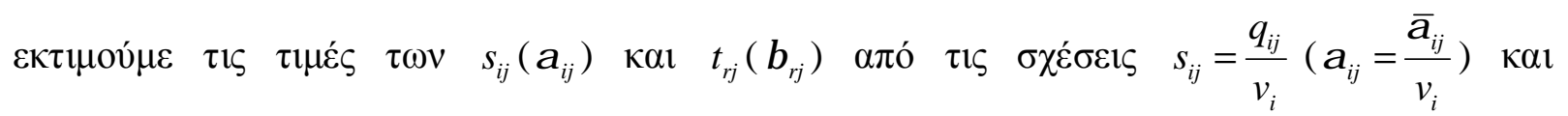
$t_{r j}=\frac{p_{r j}}{u_{r}}\left(\beta_{r j}=\frac{\overline{\beta_{r j}}}{u_{r}}\right)$

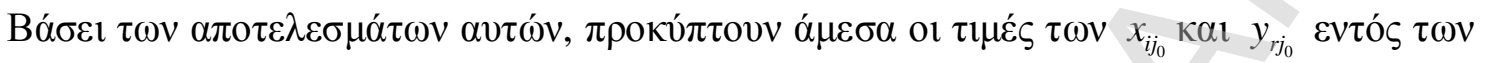

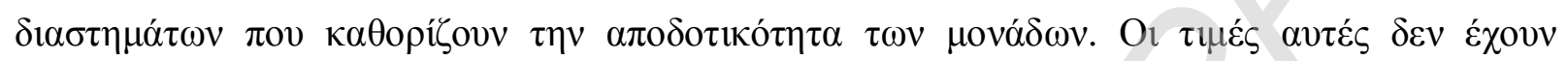

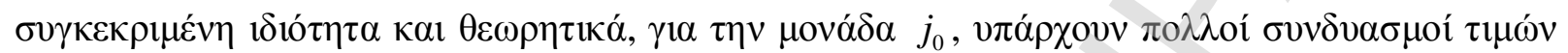

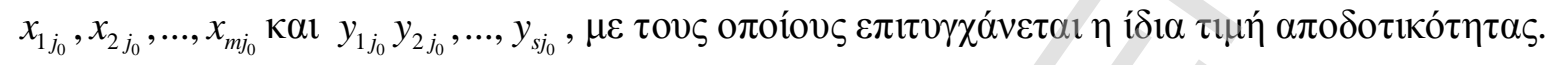

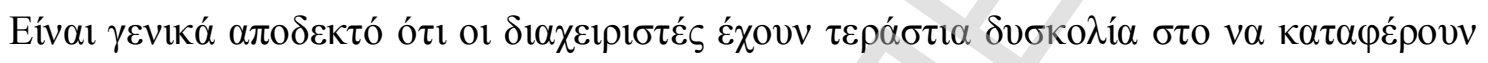

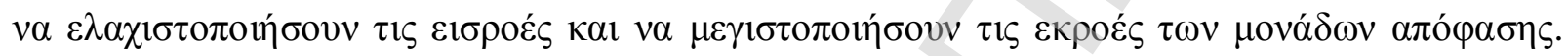

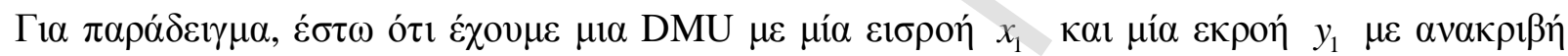

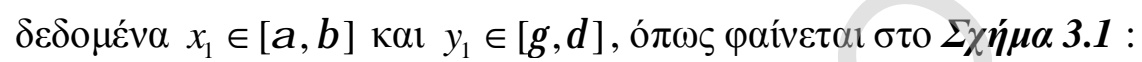

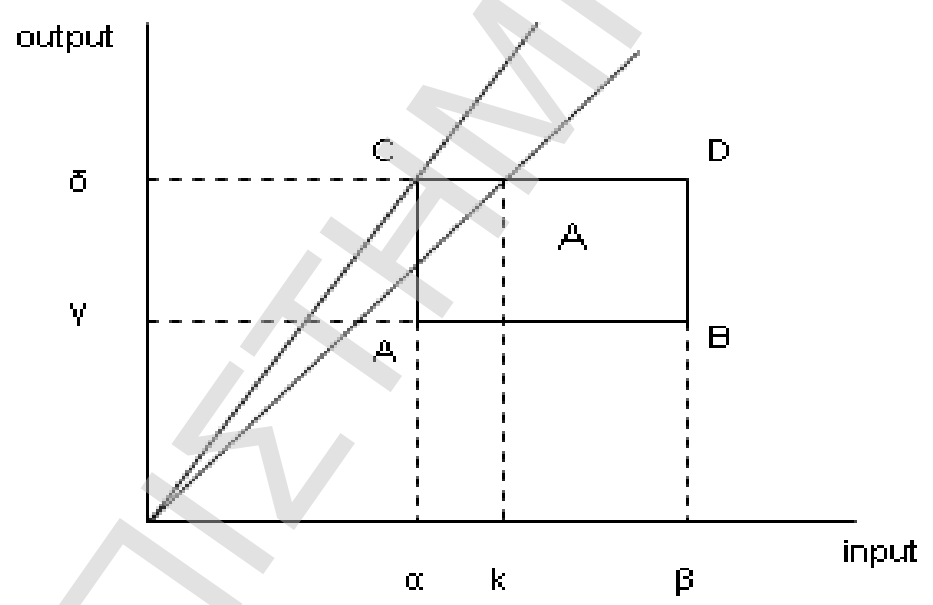

$\Sigma \chi \eta ́ \eta \alpha 3.1$

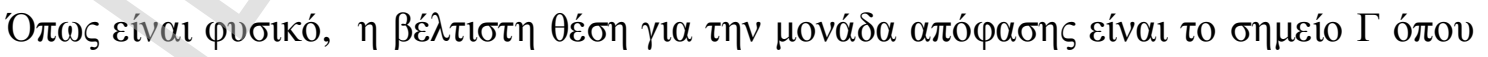

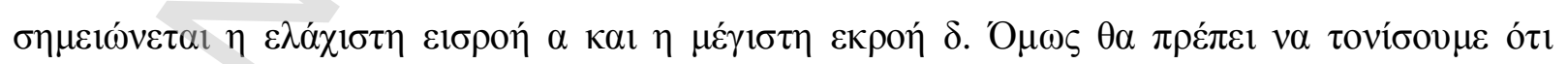

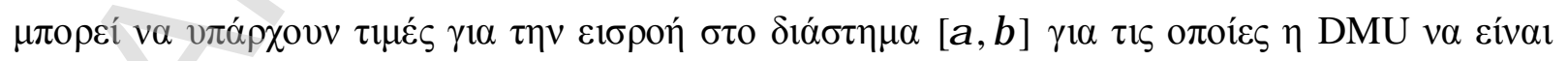

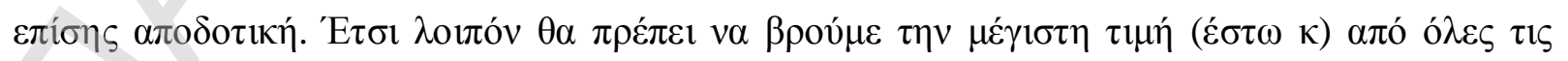

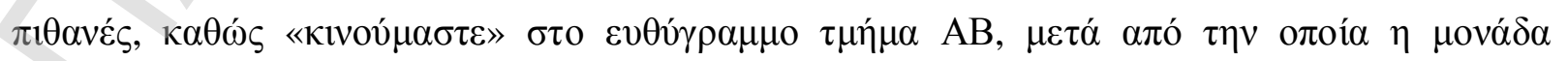

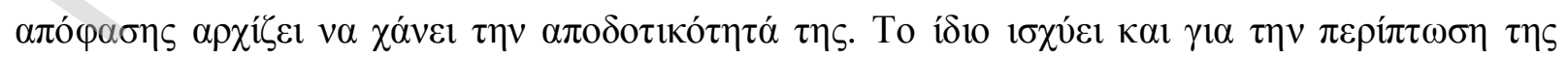

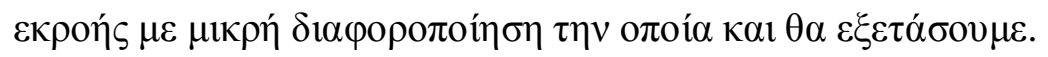




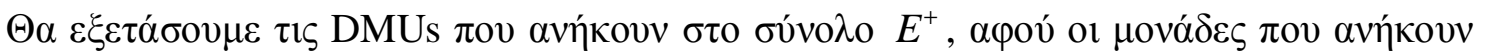

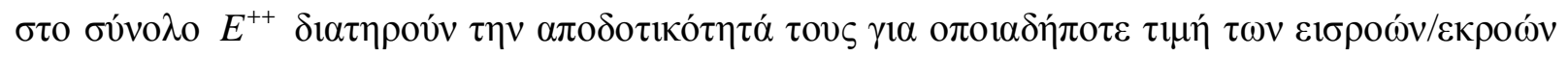

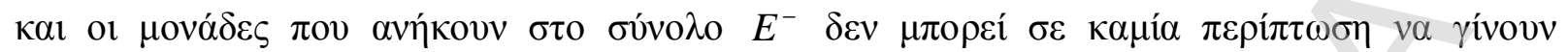
$\alpha \pi \mathrm{o \delta o \tau \imath \kappa \varepsilon ́s.}$

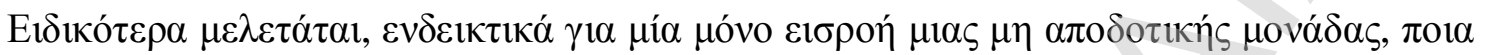

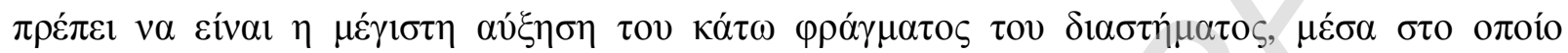

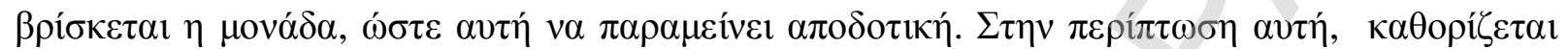

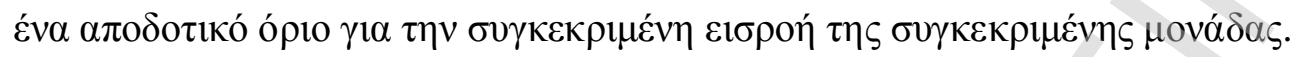

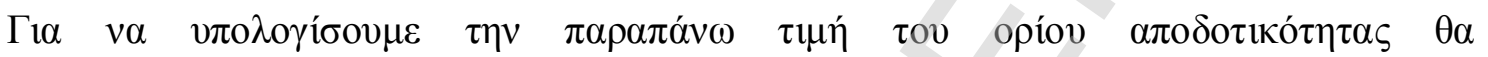

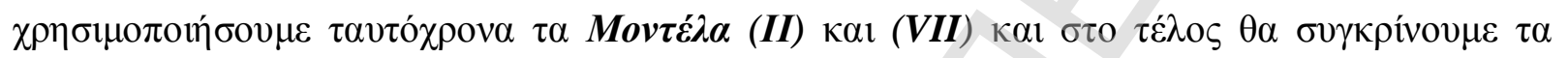

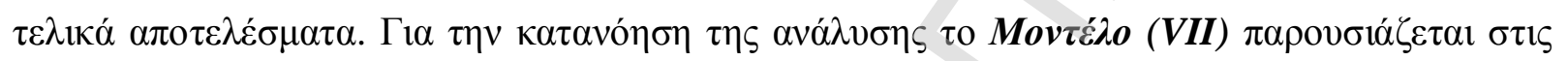
$\pi \alpha \rho \varepsilon v \theta \varepsilon \dot{\sigma \varepsilon \varepsilon 1 \varsigma \pi \circ v ~ \alpha \kappa o \lambda o v \theta o v ́ v ~ \tau o ~ M o v \tau \varepsilon ́ \lambda o ~(I I) . ~}$

$\mathrm{E} \xi \varepsilon \tau \alpha ́ \zeta o v \mu \varepsilon \tau \eta \mu$ ovó $\delta \alpha j_{0}$ :

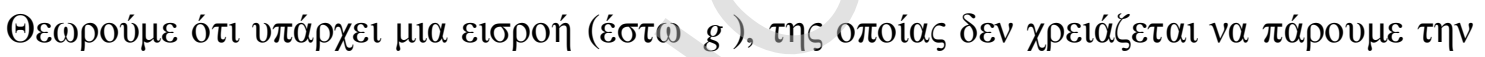

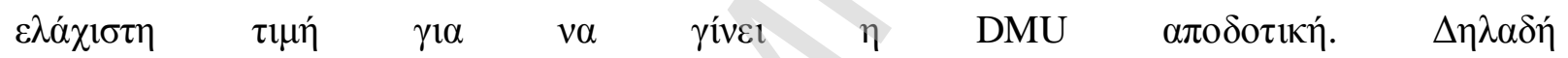

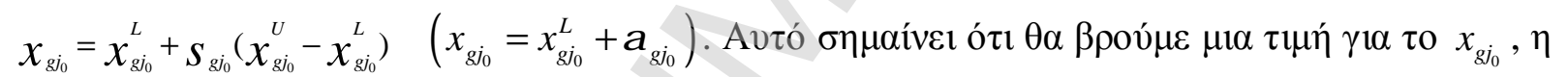

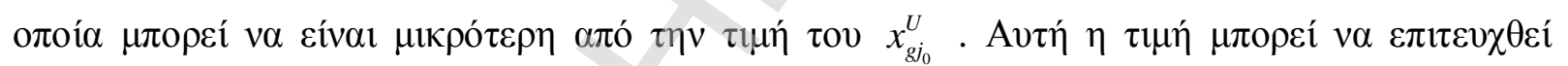

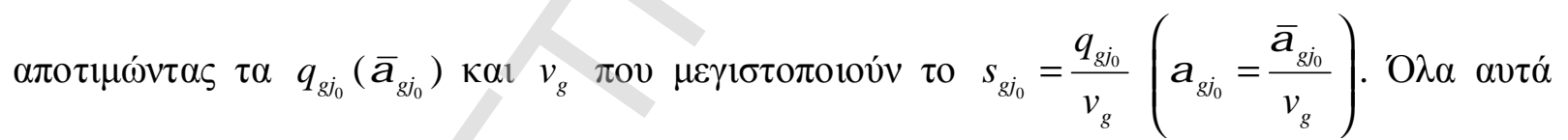

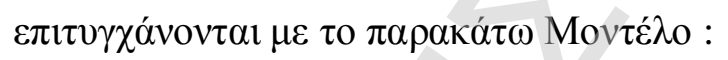

$$
\begin{aligned}
& \max z \\
& \text { s.t } \\
& (u, v, Q, P) \in S \\
& q_{g j_{0}}-z v_{g} \geq 0
\end{aligned}
$$$$
\max z
$$

s.t

$(u, v, Q, P) \in S$

$\bar{\alpha}_{g j_{0}}-z v_{g} \geq 0$

о́тоу $\quad u=\left(u_{r} \quad r=1, \ldots, s\right), \quad v=\left(v_{i} \quad i=1, \ldots, m\right), \quad Q=\left(q_{i j} \quad i=1, \ldots, m ; \quad j=1, \ldots, n\right) \quad \kappa \alpha \iota$

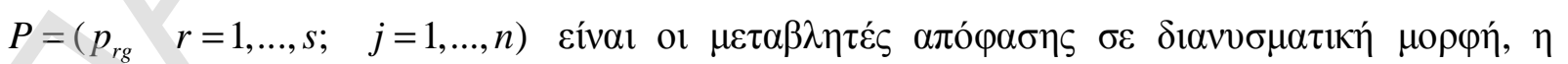




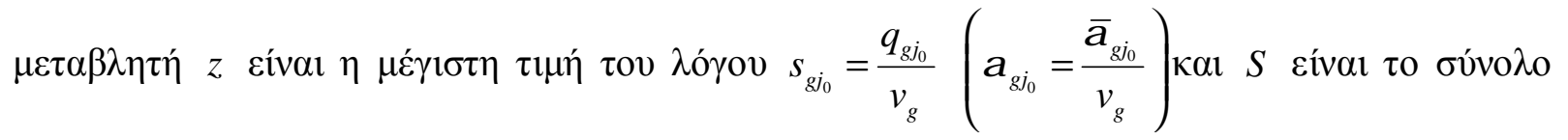

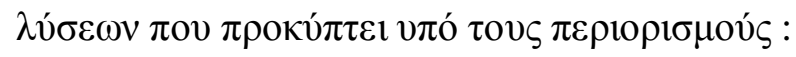

$$
\begin{array}{ll}
\sum_{i=1}^{m} v_{i} x_{i j_{0}}^{L}+q_{i j_{0}}\left(x_{i j_{0}}^{U}-x_{i j_{0}}^{L}\right)=1 & \sum_{i=1}^{S} v_{i} x_{i j_{0}}^{L}+\bar{a}_{i j_{0}}=1 \\
\sum_{r=1}^{S} u_{r} y_{r j_{0}}^{L}+p_{r j_{0}}\left(y_{i_{0}}^{U}-y_{i j_{0}}^{L}\right)- & \sum_{r=1}^{S} u_{r} y_{r j}^{L}+\bar{\beta}_{r j_{0}}-\sum_{i=1}^{m} v_{i} x_{i j_{0}}^{L}+\bar{\alpha}_{i j_{0}}=0 \\
\sum_{i=1}^{m} v_{i} x_{i j_{0}}^{L}+q_{i j_{0}}\left(x_{i j_{0}}^{U}-x_{i j_{0}}^{L}\right)=0 & \sum_{r=1}^{S} u_{r} y_{r j}^{L}+\overline{\beta_{r j}}-\sum_{i=1}^{m} v_{i} x_{i j}^{L}+\bar{\alpha}_{i j} \leq 0 \\
\sum_{r=1}^{S} u_{r} y_{r j}^{L}+p_{r j}\left(y_{i j}^{U}-y_{i j}^{L}\right)-\sum_{i=1}^{m} v_{i} x_{i j}^{L}+q_{i j}\left(x_{i j}^{U}-x_{i j}^{L}\right) \leq 0 & r=1 \\
j=1, \ldots, n \quad j \neq j_{0} & j=1, \ldots, n \quad j \neq j_{0} \\
p_{r j}-u_{r} \leq 0 \quad r=1, \ldots, s ; j=1, \ldots, n & 0 \leq \bar{\beta}_{r j} \leq u_{r}\left(y_{r j}^{U}-y_{r j}^{L}\right) \quad r=1, \ldots, s ; j=1, \ldots, n \\
q_{i j}-v_{i} \leq 0 \quad i=1, \ldots, m ; j=1, \ldots, n & 0 \leq \bar{a}_{i j} \leq v_{i}\left(x_{i j}^{U}-x_{i j}^{L}\right) \quad i=1, \ldots, m ; j=1, \ldots, n \\
p_{r j} \geq 0 \quad \forall r, j \quad q_{i j} \geq 0 \quad \forall i, j & u_{r}, v_{i} \geq \varepsilon, \quad \forall r, i \\
u_{r}, v_{i} \geq \varepsilon \quad \forall r, i &
\end{array}
$$

\section{Movtย่́̇o (VIII)}

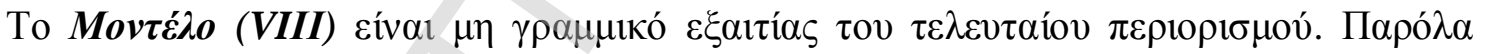

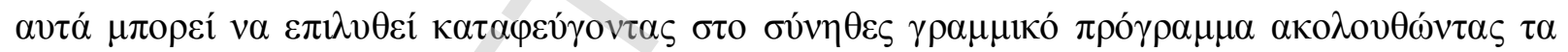

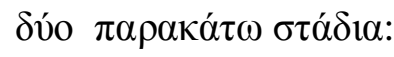

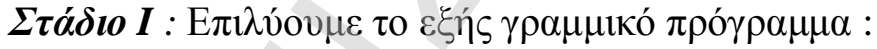

$$
\begin{array}{ll}
\max q_{g j_{0}} & \max \bar{\alpha}_{g j_{0}} \\
\text { s.t } & \text { s.t } \\
(u, v, Q, P) \in S & (u, v, Q, P) \in S
\end{array}
$$

\section{$\operatorname{Mov\tau \varepsilon ́\lambda o~(IX)~}$}

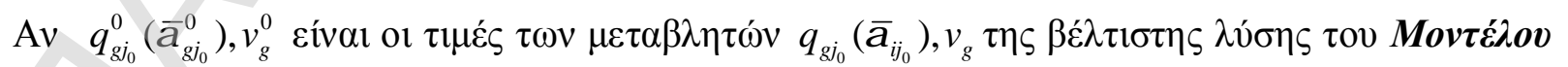

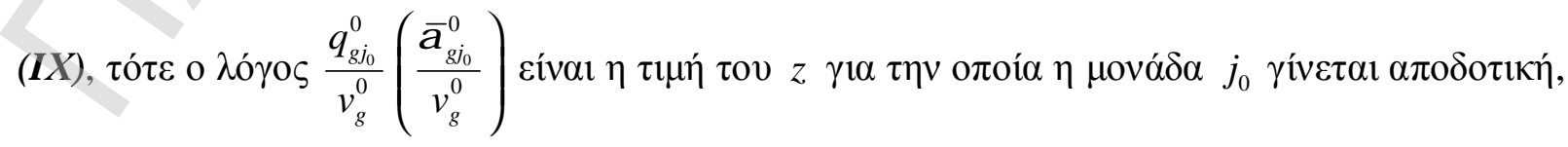

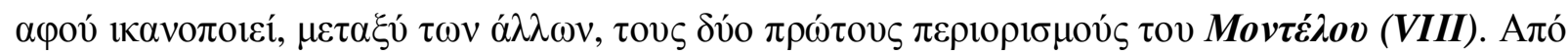




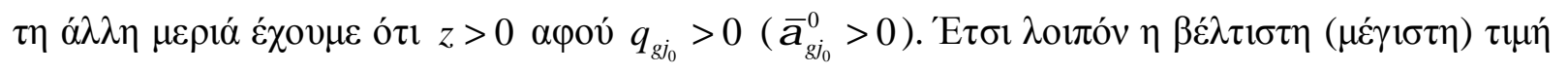

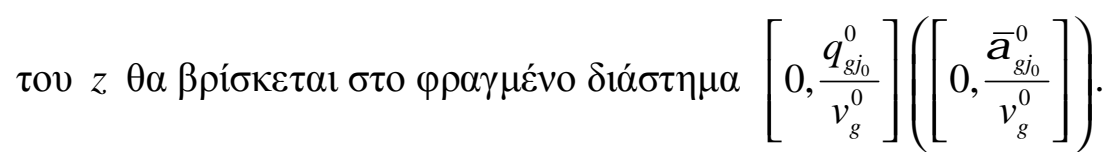

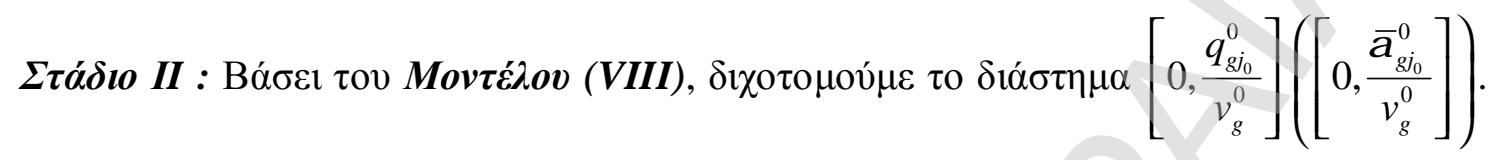

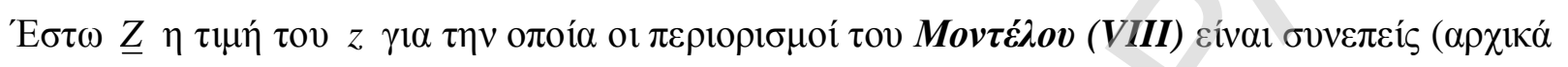

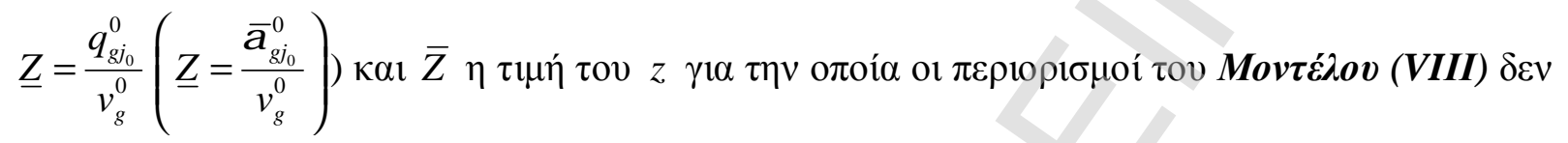

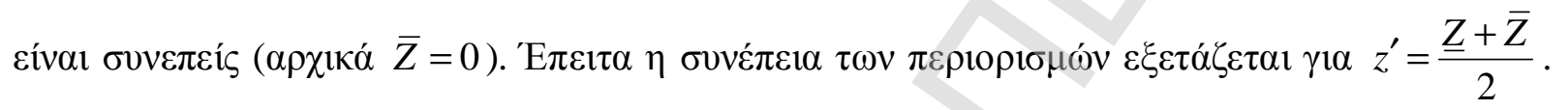

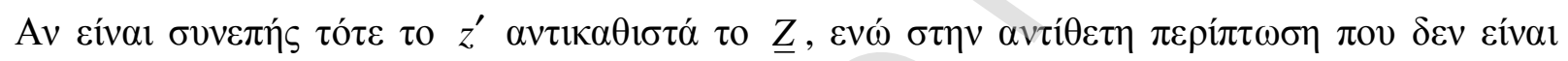

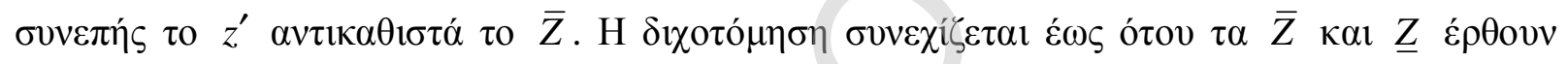

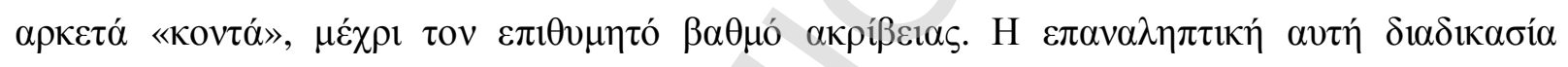

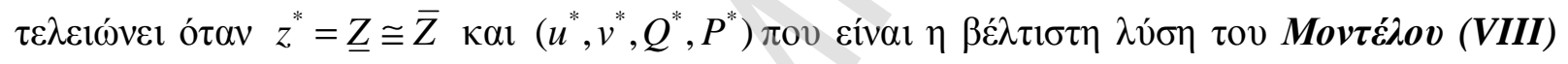

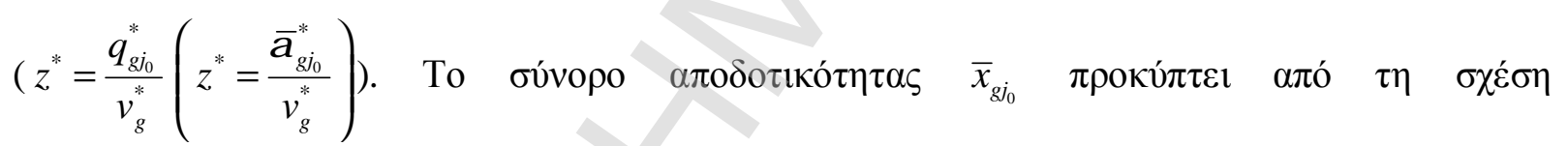

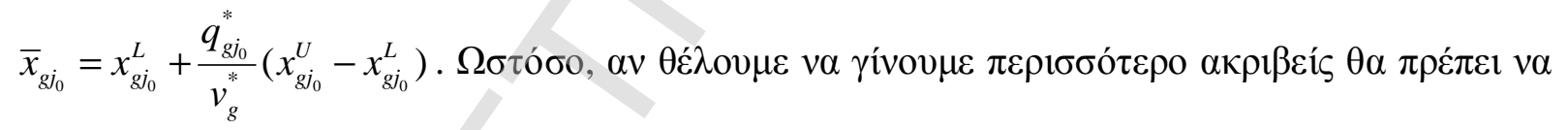

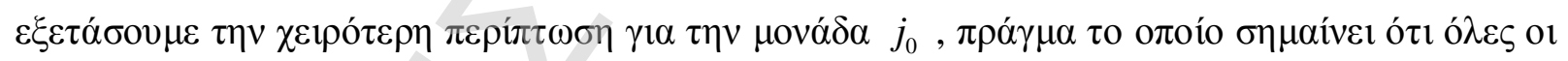

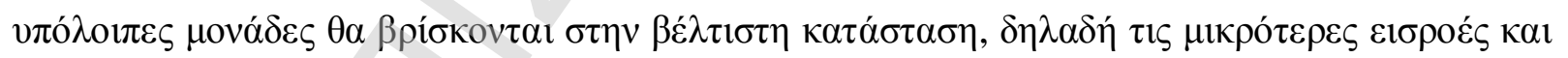
$\tau ı \varsigma \mu \varepsilon \gamma \alpha \lambda v \dot{\tau \varepsilon \rho \varepsilon \varsigma \varepsilon \kappa \rho о \varepsilon ́ \varsigma . ~}$ 


$$
\begin{array}{ll}
\max q_{g j_{0}} & \max \bar{\alpha}_{j g_{0}} \\
\sum_{i=1}^{m} v_{i} x_{i j_{0}}^{L}+q_{i j_{0}}\left(x_{i j_{0}}^{U}-x_{i j_{0}}^{L}\right)=1 & \sum_{i=1}^{m} v_{i} x_{i j_{0}}^{L}+\bar{\alpha}_{i j_{0}}=1 \\
\sum_{r=1}^{s} u_{r} y_{r j_{0}}^{L}+p_{r j_{0}}\left(y_{r j_{0}}^{U}-y_{r j_{0}}^{L}\right)- & \sum_{r=1}^{s} u_{r} y_{r_{0}}^{L}+\bar{\beta}_{i j_{0}} p_{r j_{0}}-\sum_{i=1}^{m} v_{i} x_{i j_{0}}^{L}+\bar{\alpha}_{i j_{0}}=0 \\
\quad \sum_{i=1}^{m} v_{i} x_{i j_{0}}^{L}+q_{i j_{0}}\left(x_{i j_{0}}^{U}-x_{i j_{0}}^{L}\right)=0 & \sum_{r=1}^{s} u_{r} y_{r j}^{U}-\sum_{i=1}^{m} v_{i} x_{i j}^{L} \leq 0 \quad j=1, \ldots, n \quad j \neq j_{0} \\
\sum_{r=1}^{s} u_{r} y_{r j}^{U}-\sum_{i=1}^{m} v_{i} x_{i j}^{L} \leq 0 & 0 \leq \overline{\beta_{r j}} \leq u_{r}\left(y_{r j}^{U}-y_{r j}^{L}\right) \quad r=1, \ldots, s ; j=1, \ldots, n \\
j=1, \ldots, n \quad j \neq j_{0} & 0 \leq \bar{a}_{i j} \leq v_{i}\left(x_{i j}^{U}-x_{i j}^{L}\right) \quad i=1, \ldots, m ; j=1, \ldots, n \\
p_{r j}-u_{r} \leq 0 \quad r=1, \ldots, s ; j=1, \ldots, n & u_{r}, v_{i} \geq \varepsilon \quad \forall r, i \\
q_{i j}-v_{i} \leq 0 \quad i=1, \ldots, m ; j=1, \ldots, n & \\
p_{r j} \geq 0, \quad q_{i j} \geq 0 \quad \forall r, i, j & \\
u_{r}, v_{i} \geq \varepsilon \quad \forall r, i &
\end{array}
$$

\section{$\operatorname{Mov\tau \varepsilon ́\lambda o~(X)~}$}

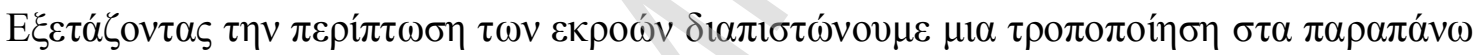

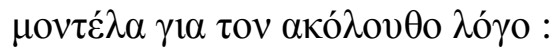

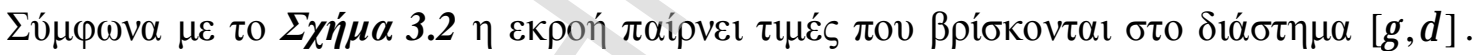

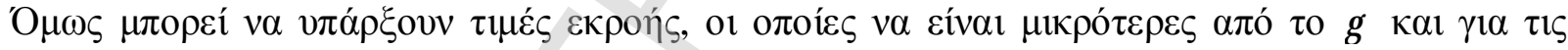

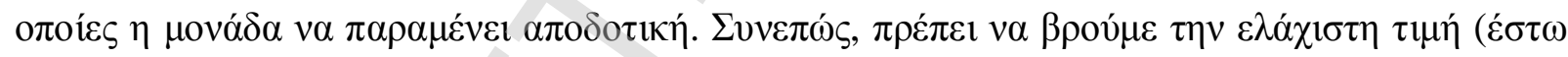

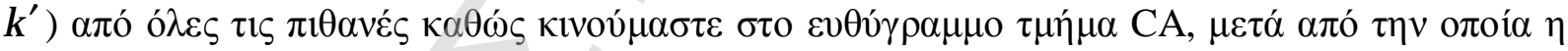
$\mu$

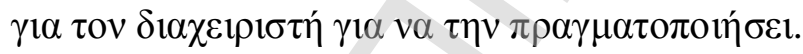




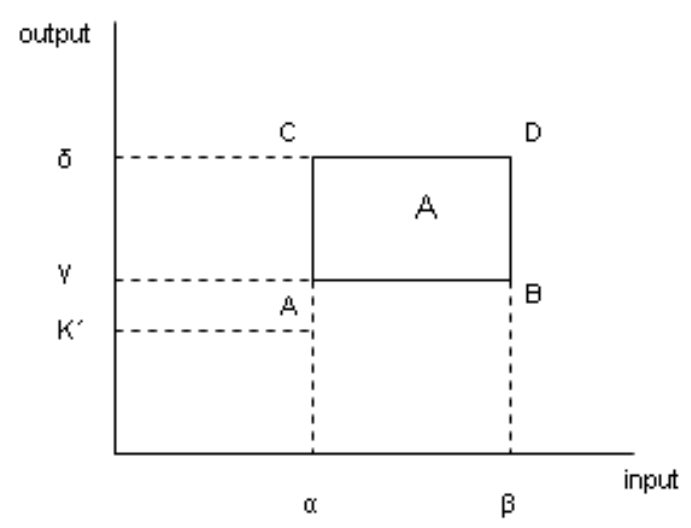

$\Sigma \chi \dot{\eta}_{\mu \alpha} 3.2$

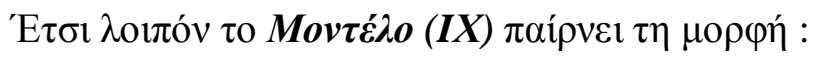

$$
\begin{aligned}
& \min p_{g j_{0}} \\
& \sum_{i=1}^{m} v_{i} x_{i j_{0}}^{L}+q_{i j_{0}}\left(x_{i j_{0}}^{U}-x_{i j_{0}}^{L}\right)=1 \\
& \sum_{r=1}^{s} u_{r} y_{r j_{0}}^{L}+p_{r j_{0}}\left(y_{r j_{0}}^{U}-y_{r j_{0}}^{L}\right)- \\
& \sum_{i=1}^{m} v_{i} x_{i j_{0}}^{L}+q_{i j_{0}}\left(x_{i j_{0}}^{U}-x_{i j_{0}}^{L}\right)=0 \\
& \min \bar{\beta}_{g j_{0}} \\
& \sum_{i=1}^{S} v_{i} x_{i j_{0}}^{L}+\bar{a}_{i j_{0}}=1 \\
& \sum_{r=1}^{s} u_{r} y_{r j_{0}}^{L}+\bar{\beta}_{i j_{0}}-\sum_{i=1}^{m} v_{i} x_{i j_{0}}^{L}+\bar{\alpha}_{i j_{0}}=0 \\
& \sum_{r=1}^{s} u_{r} y_{r j}^{L}+p_{r j}\left(y_{r j}^{U}-y_{r j}^{L}\right)-\sum_{i=1}^{m} v_{i} x_{i j}^{L}+q_{i j}\left(x_{i j}^{U}-x_{i j}^{L}\right) \leq 0 \quad \sum_{r=1}^{S} u_{r} y_{r j}^{U}-\sum_{i=1}^{m} v_{i} x_{i j}^{L} \leq 0 \\
& j=1, \ldots, n \quad j \neq j_{0} \\
& p_{r j_{0}}-u_{r} \leq 0 \quad r=1, \ldots, s ; j=1, \ldots, n \\
& j=1, \ldots, n \quad j \neq j_{0} \\
& q_{i j_{0}}-v_{i} \leq 0 \quad i=1, \ldots, m ; j=1, \ldots, n \\
& \bar{\beta}_{r j} \leq u_{r}\left(y_{r j}^{U}-y_{r j}^{L}\right) \quad r=1, \ldots, s ; j=1, \ldots, n \\
& p_{r j} \geq 0, q_{i j} \geq 0 \quad \forall r, i, j \\
& u_{r}, v_{i} \geq \varepsilon \quad \forall r, i \\
& p_{g j_{0}} \text { free } \\
& 0 \leq \bar{a}_{i j} \leq v_{i}\left(x_{i j}^{U}-x_{i j}^{L}\right) \quad i=1, \ldots, m ; j=1, \ldots, n \\
& \bar{\beta}_{g j_{0}} \text { free } \\
& u_{r}, v_{i} \geq \varepsilon, \quad \forall r, i
\end{aligned}
$$

\section{$\operatorname{Mov\tau \varepsilon ́\lambda }(X I)$}

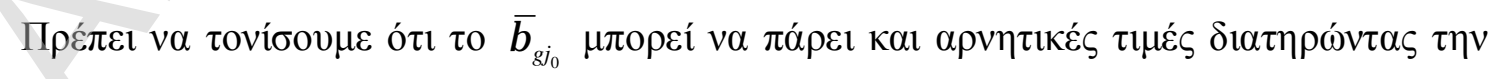

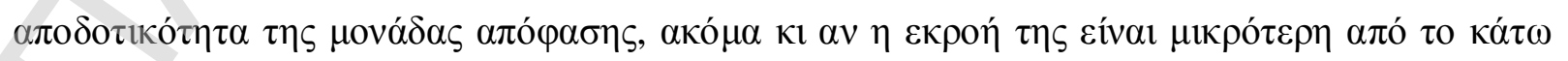

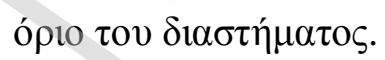




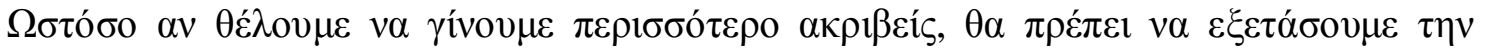

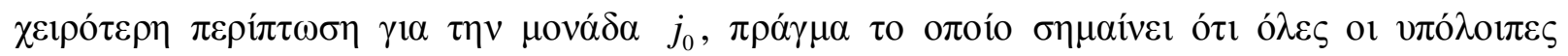

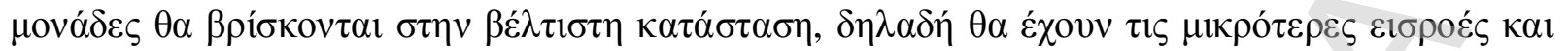

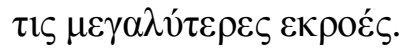

$$
\begin{array}{ll}
\min p_{g j_{0}} & \min \bar{\beta}_{g j_{0}} \\
\sum_{i=1}^{m} v_{i} x_{i j_{0}}^{L}+q_{i j_{0}}\left(x_{i j_{0}}^{U}-x_{i j_{0}}^{L}\right)=1 & \sum_{i=1}^{s} v_{i} x_{i j_{0}}^{L}+\bar{a}_{i j_{0}}=1 \\
\sum_{r=1}^{s} u_{r} y_{r j_{0}}^{L}+p_{r j_{0}}\left(y_{r j_{0}}^{U}-y_{r j_{0}}^{L}\right)- & \sum^{S} u_{r} y_{r j_{0}}^{L}+\overline{\beta_{i j}}-\sum_{i=1}^{m} v_{i} x_{i j_{0}}^{L}+\bar{\alpha}_{i j_{0}}=0 \\
\sum_{i=1}^{m} v_{i} x_{i j_{0}}^{L}+q_{i j_{0}}\left(x_{i j_{0}}^{U}-x_{i j_{0}}^{L}\right)=0 & r=1 \\
\sum_{r=1}^{s} u_{r} y_{r j}^{L}-\sum_{i=1}^{m} v_{i} x_{i j}^{L} \leq 0 & \sum_{r=1}^{s} u_{r} y_{r j}^{U}-\sum_{i=1}^{m} v_{i} x_{i j}^{L} \leq 0 \\
j=1, \ldots, n \quad j \neq j_{0} & \\
p_{r j_{0}}-u_{r} \leq 0 \quad r=1, \ldots, s ; j=1, \ldots, n & j=1, \ldots, n \quad j \neq j_{0} \\
q_{i j_{0}}-v_{i} \leq 0 \quad i=1, \ldots, m ; j=1, \ldots, n & \overline{\beta_{r j}} \leq u_{r}\left(y_{r j}^{U}-y_{r j}^{L}\right) \quad r=1, \ldots, s ; j=1, \ldots, n \\
p_{r j} \geq 0, q_{i j} \geq 0 \quad \forall r, i, j & 0 \leq \bar{a}_{i j} \leq v_{i}\left(x_{i j}^{U}-x_{i j}^{L}\right) \quad i=1, \ldots, m ; j=1, \ldots, n \\
u_{r}, v_{i} \geq \varepsilon \quad \forall r, i & \overline{\beta_{g j}} \text { free } \\
p_{g j_{0}} \quad \text { free } & u_{r}, v_{i} \geq \varepsilon, \quad \forall r, i
\end{array}
$$

$\operatorname{Mov\tau \varepsilon ́\lambda o~(XII)~}$ 


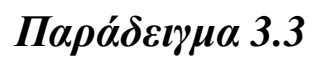

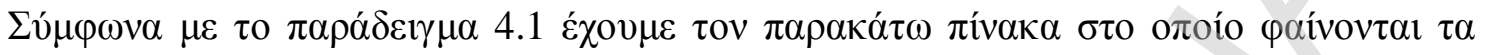

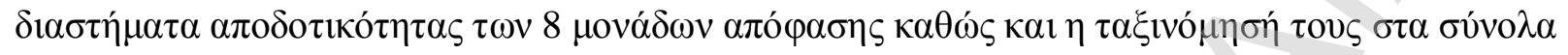
$E^{-}, E^{+} \kappa \alpha l E^{++}$.

\begin{tabular}{|c|c|c|c|c|c|c|c|c|c|c|}
\hline & \multicolumn{4}{|c|}{ Еıбров́ร } & \multicolumn{3}{|c|}{ Екров́с } & & & \multirow[b]{2}{*}{ 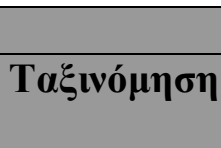 } \\
\hline DMU & & $Y_{1}$ & & $\overline{Y_{2}}$ & & $\overline{Y_{1}}$ & $Y_{2}$ & 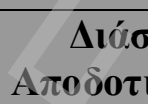 & $\begin{array}{l}\eta \mu \alpha \\
\text { об } \tau \eta \tau \alpha \varsigma\end{array}$ & \\
\hline 1 & {$[16$} & 21] & {$[0,30$} & $0,50]$ & {$[120$} & $125]$ & {$\left[\begin{array}{ll}19 & 21\end{array}\right]$} & {$[0,177$} & $0,746]$ & $\mathbf{E}^{-}$ \\
\hline 2 & {$[18$} & $25]$ & {$[0,40$} & $0,53]$ & {$[122$} & 130] & {$\left[\begin{array}{ll}20 & 21\end{array}\right]$} & {$[0,165$} & $0,693]$ & $\mathbf{E}^{-}$ \\
\hline 3 & {$[20$} & 27] & {$[0,41$} & $0,61]$ & {$[124$} & 131] & {$\left[\begin{array}{ll}16 & 24\end{array}\right]$} & {$[0,148$} & $0,643]$ & $\mathbf{E}^{-}$ \\
\hline 4 & {$[12$} & $15]$ & {$[0,21$} & $0,48]$ & {$[138$} & 144] & {$\left[\begin{array}{ll}21 & 22\end{array}\right]$} & {$[0,224$} & $1,000]$ & $\mathbf{E}^{+}$ \\
\hline 5 & {$[10$} & 17] & {$[0,10$} & $0,70]$ & {$[143$} & 159] & {$\left[\begin{array}{ll}28 & 35\end{array}\right]$} & {$[0,227$} & $1,000]$ & $\mathbf{E}^{+}$ \\
\hline 6 & {$[4$} & 30] & {$[0,16$} & $0,35]$ & {$[157$} & 198] & {$\left[\begin{array}{ll}21 & 29\end{array}\right]$} & {$[0,823$} & $1,000]$ & $\mathbf{E}^{+}$ \\
\hline 7 & {$[19$} & 22] & {$[0,12$} & $0,19]$ & {$[158$} & 181] & {$\left[\begin{array}{ll}21 & 25\end{array}\right]$} & {$[0,445$} & $0,907]$ & $\mathbf{E}^{-}$ \\
\hline 8 & {$[14$} & $15]$ & {$[0,06$} & $0,09]$ & {$[157$} & $161]$ & {$\left[\begin{array}{ll}28 & 40\end{array}\right]$} & {$[1,000$} & $1,000]$ & $\mathbf{E}^{++}$ \\
\hline
\end{tabular}

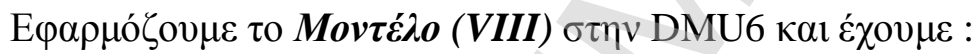

$$
q_{16}^{*}=0,030328 \kappa \alpha \iota v_{1}^{*}=0,054083 .
$$

'E $\tau \sigma \iota$

$$
\bar{x}_{g j_{0}}=x_{g j_{0}}^{L}+\frac{q_{g j_{0}}^{*}}{v_{g}^{*}}\left(x_{g j_{0}}^{U}-x_{g j_{0}}^{L}\right)=4+\frac{0,030328}{0,052864}(30-4)=18,9
$$

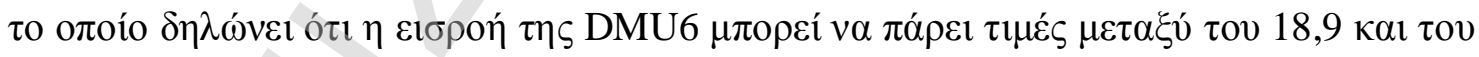

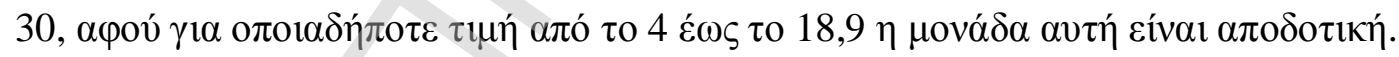

Е

$$
q_{16}^{*}=0,026105 \kappa \alpha v_{1}^{*}=0,080314 .
$$

'E $\tau \sigma \mathrm{l}$

$$
\begin{aligned}
& \bar{x}_{k j_{0}}=x_{k j_{0}}^{L}+\frac{q_{k j_{0}}^{*}}{v_{k}^{*}}\left(x_{k j_{0}}^{U}-x_{k j_{0}}^{L}\right)=4+\frac{0,026105}{0,080314}(30-4)=12,4 \\
& \bar{x}_{14}=17,105
\end{aligned}
$$

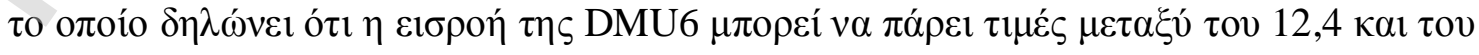

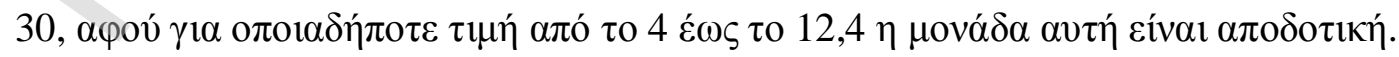

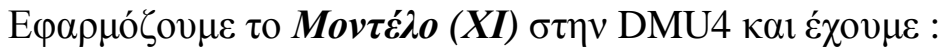




$$
p_{14}^{*}=-0,016449 \kappa \alpha 1 u_{1}^{*}=0,007959 .
$$

'E $\tau \sigma \iota$

$$
y_{g j_{0}}=y_{g j_{0}}^{L}+\frac{p_{g j_{0}}^{*}}{u_{g}^{*}}\left(y_{g j_{0}}^{U}-y_{g j_{0}}^{L}\right)=138-\frac{0,016449}{0,007959}(144-138)=125,6
$$

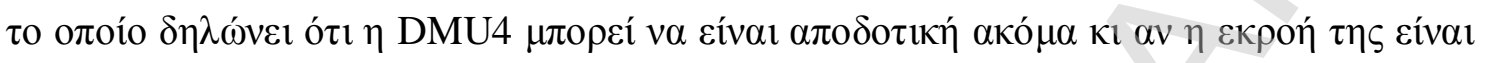

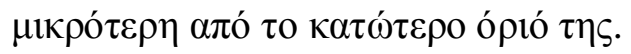

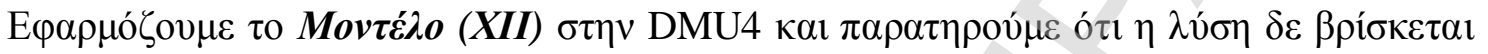
$\mu \varepsilon ́ \sigma \alpha \sigma \tau o \delta$ ió $\sigma \tau \eta \mu \alpha$.

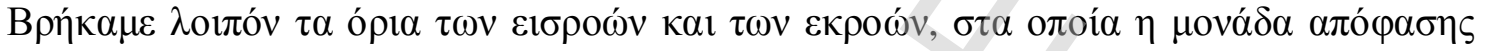

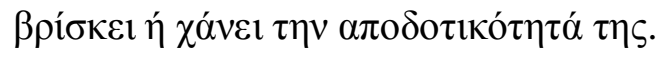

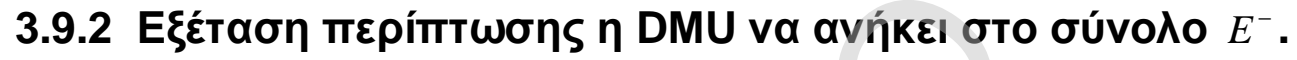

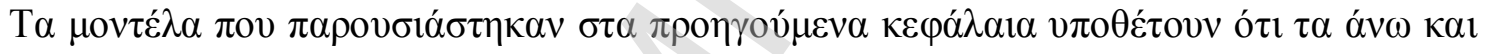

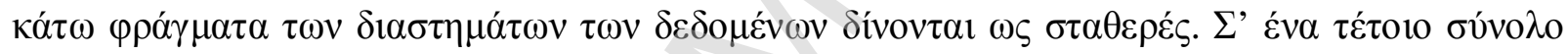

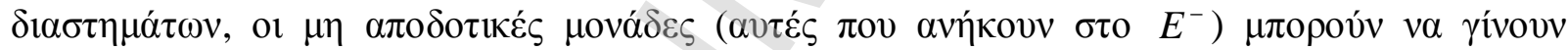

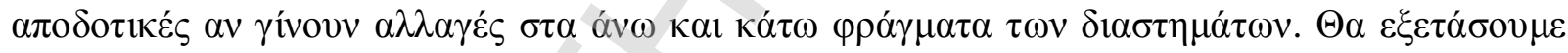

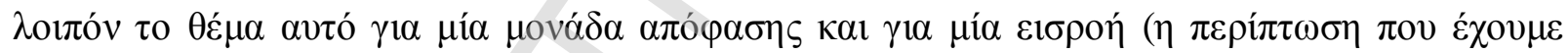

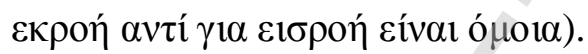

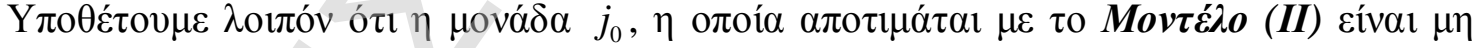

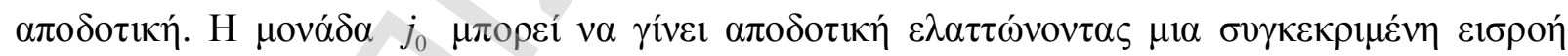

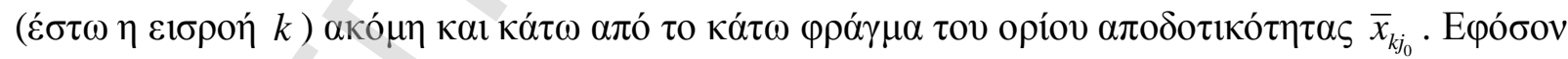

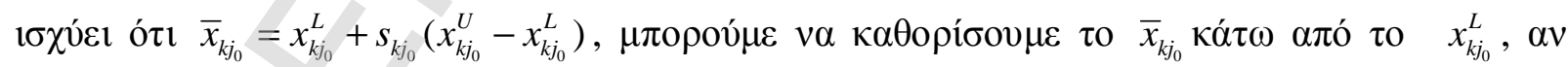

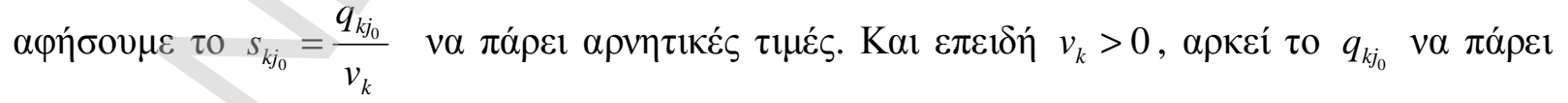

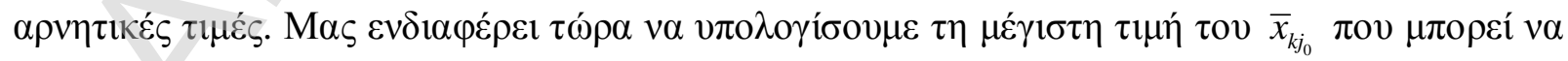

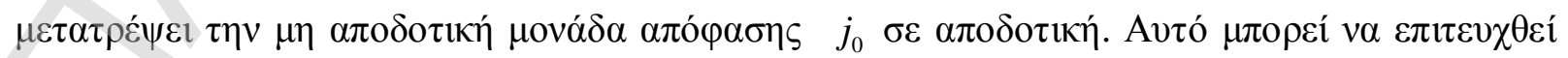




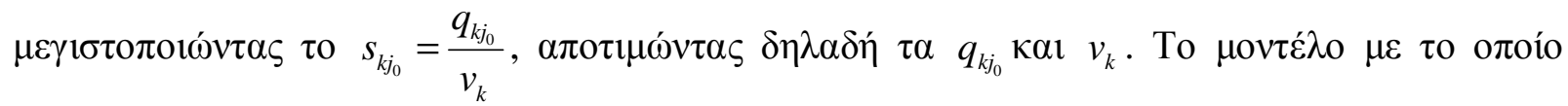

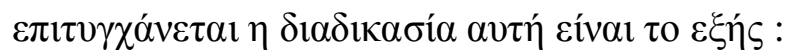

$$
\begin{aligned}
& \max z \\
& \text { s.t } \\
& (u, v, Q, P) \in S \\
& q_{k j_{0}}-z v_{k} \geq 0
\end{aligned}
$$

\section{$\operatorname{Mov\tau \varepsilon ́\lambda o~(XIII)~}$}

о́тоv $\quad u=\left(u_{r} \quad r=1, \ldots, s\right), \quad v=\left(v_{i} \quad i=1, \ldots, m\right), \quad Q=\left(q_{i j} \quad i=1, \ldots, m ; \quad j=1, \ldots, n\right) \quad \kappa \alpha 1$

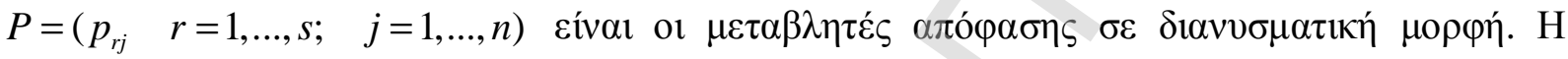

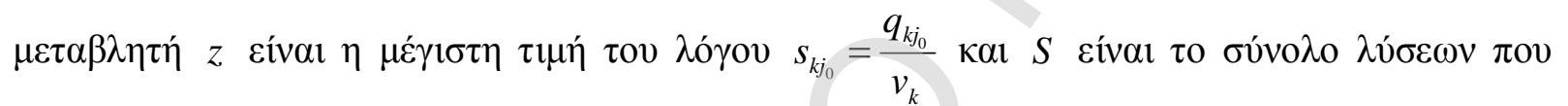

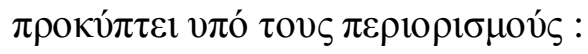

$$
\begin{aligned}
& \sum_{i=1}^{m} v_{i} x_{i j_{0}}^{L}+q_{i j_{0}}\left(x_{i j_{0}}^{U}-x_{i j_{0}}^{L}\right)=1 \\
& \sum_{r=1}^{s} u_{r} y_{r j_{0}}^{L}+p_{r j_{0}}\left(y_{r j_{0}}^{U}-y_{r j_{0}}^{L}\right)- \\
& \quad \sum_{i=1}^{m} v_{i} x_{i j_{0}}^{L}+q_{i j_{0}}\left(x_{i j_{0}}^{U}-x_{i j_{0}}^{L}\right)=0 \\
& \sum_{r=1}^{s} u_{r} y_{r j}^{L}+p_{r j}\left(y_{r j}^{U}-y_{r j}^{L}\right)-\sum_{i=1}^{m} v_{i} x_{i j}^{L}+q_{i j}\left(x_{i j}^{U}-x_{i j}^{L}\right) \leq 0 \\
& j=1, \ldots, n \quad j \neq j_{0} \\
& p_{r j}-u_{r} \leq 0 \quad r=1, \ldots, s ; j=1, \ldots, n \\
& q_{i j}-v_{i} \leq 0 \quad i=1, \ldots, m ; j=1, \ldots, n \\
& p_{r j} \geq 0 \quad \forall r, i \\
& q_{i j} \geq 0 \quad \forall i \neq k, j \neq j_{0} \\
& u_{r}, v_{i} \geq \varepsilon \quad \forall r, i \\
& q_{k j_{0}} \quad \text { free }
\end{aligned}
$$

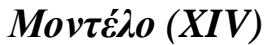

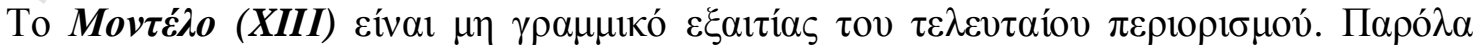

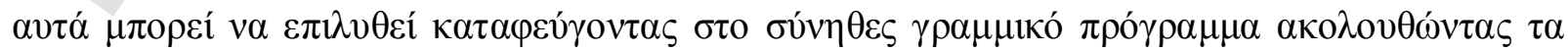

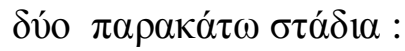




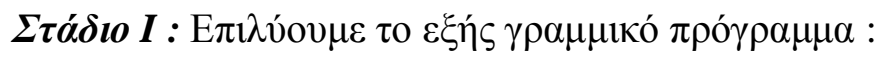

$$
\begin{aligned}
& \max q_{k j_{0}} \\
& \text { s.t } \\
& (u, v, Q, P) \in S
\end{aligned}
$$

\section{$\operatorname{Mov\tau \varepsilon ́\lambda o~(XV)~}$}

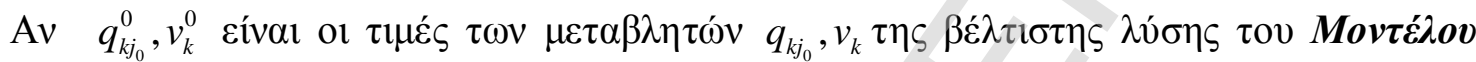

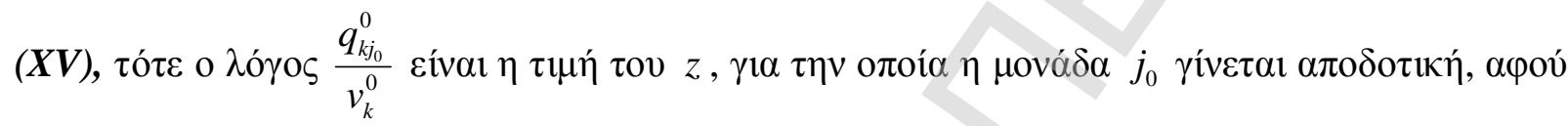

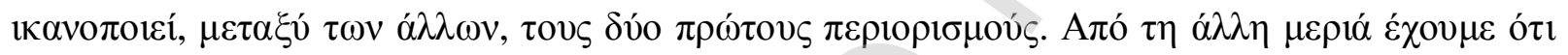

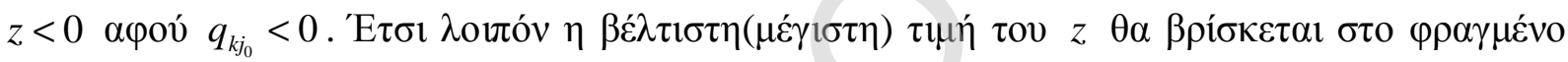
$\delta i \alpha ́ \sigma \tau \eta \mu \alpha\left[\frac{q_{k j_{0}}^{0}}{v_{k}^{0}}, 0\right]$.

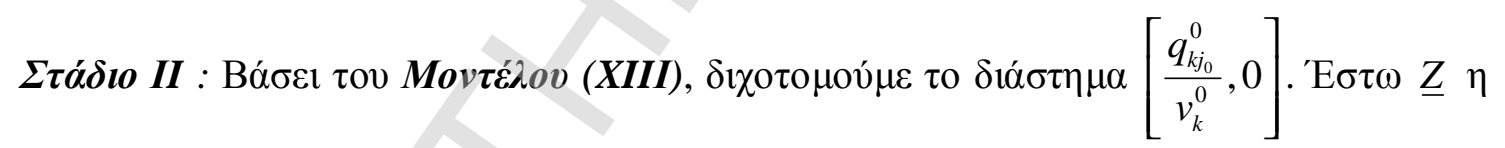

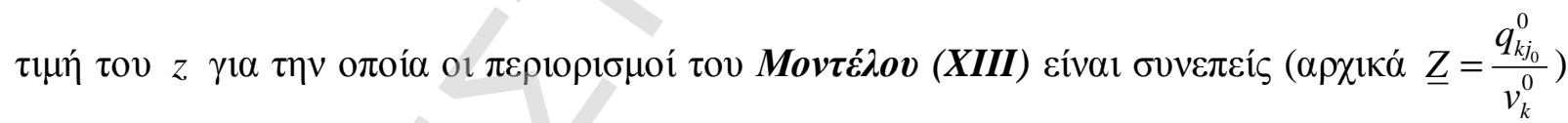

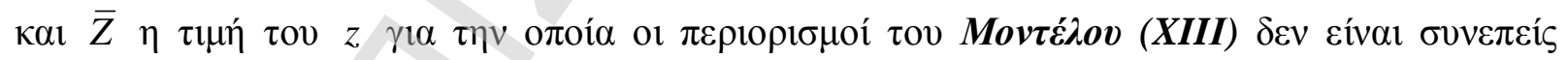

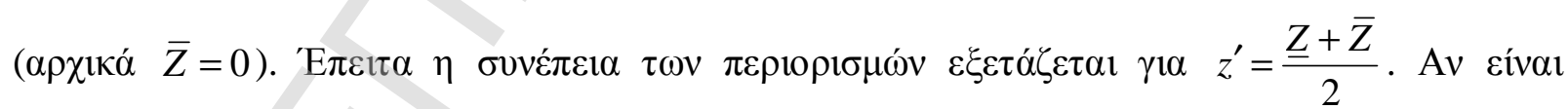

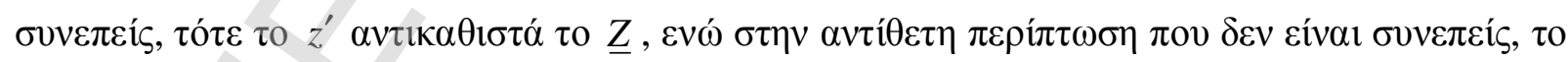

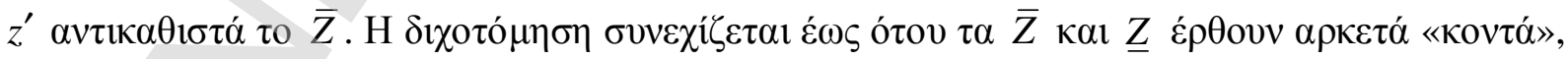

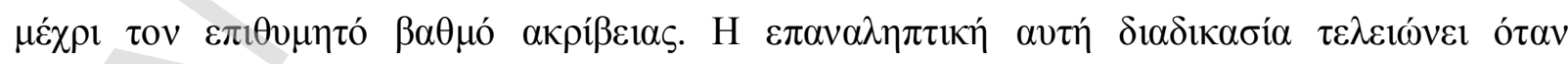

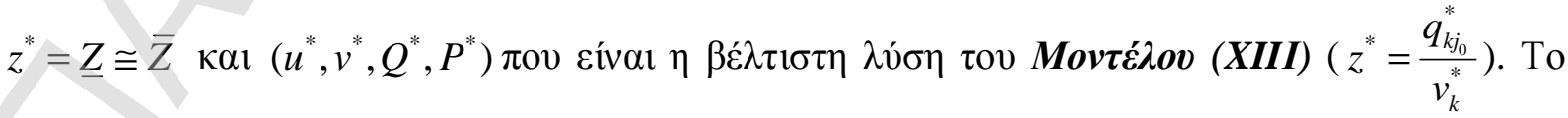

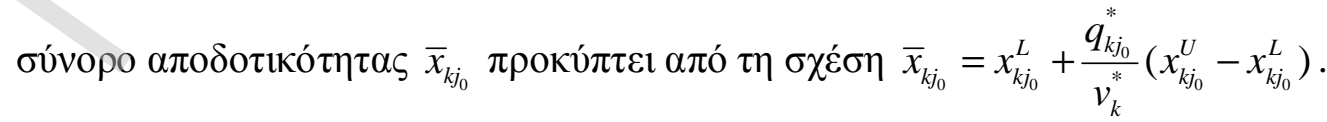




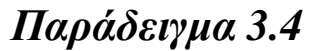

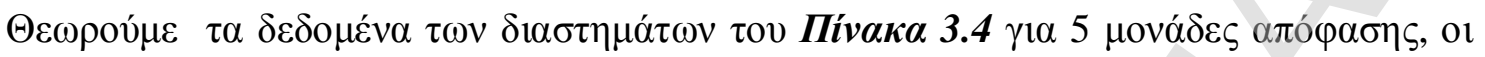

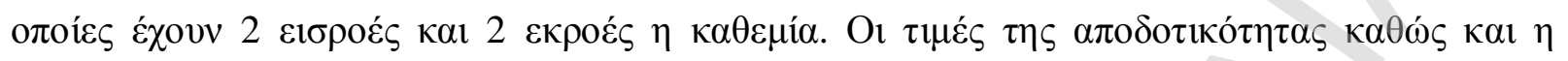

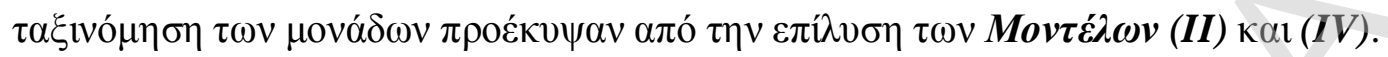

\begin{tabular}{|c|c|c|c|c|c|c|c|}
\hline & \multicolumn{2}{|c|}{ Eıбров́ } & \multicolumn{2}{|c|}{ Екров́s } & \multirow{2}{*}{\multicolumn{2}{|c|}{ 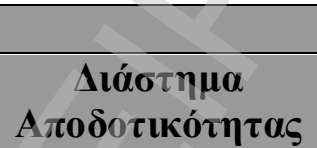 }} & \multirow[b]{2}{*}{ 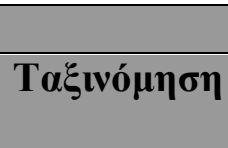 } \\
\hline DMU & $X_{1 j}$ & $X_{2 j}$ & $Y_{1 j}$ & $Y_{2 j}$ & & & \\
\hline 1 & 15] & $0,48]$ & {$[138$} & {$\left[\begin{array}{ll}21 & 22\end{array}\right]$} & {$[0,224$} & $1,000]$ & $\mathbf{E}^{+}$ \\
\hline 2 & {$[10$} & $0,70]$ & {$[143$} & {$\left[\begin{array}{ll}28 & 35\end{array}\right]$} & {$[0,227$} & $1,000]$ & $\mathbf{E}^{+}$ \\
\hline 3 & 12] & $0,35]$ & {$[157$} & {$[21$} & {$[0,823$} & $1,000]$ & $\mathbf{E}^{+}$ \\
\hline 4 & {$\left[\begin{array}{ll}19 & 22\end{array}\right]$} & $0,19]$ & {$[158$} & {$\left[\begin{array}{ll}21 & 25\end{array}\right]$} & {$[0,445$} & $0,907]$ & $\mathbf{E}^{-}$ \\
\hline 5 & {$\left[\begin{array}{ll}14 & 15\end{array}\right]$} & $0,09]$ & {$[157$} & {$\left[\begin{array}{ll}28 & 40\end{array}\right]$} & {$[1,000$} & $1,000]$ & $\mathbf{E}^{++}$ \\
\hline
\end{tabular}

Е

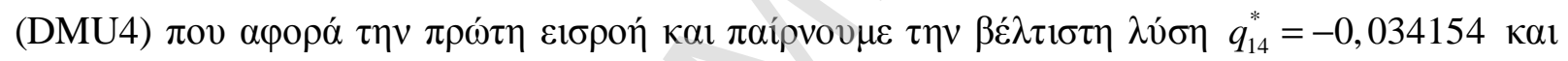

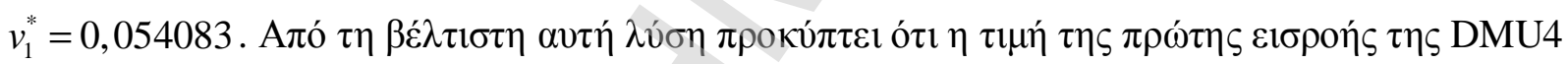

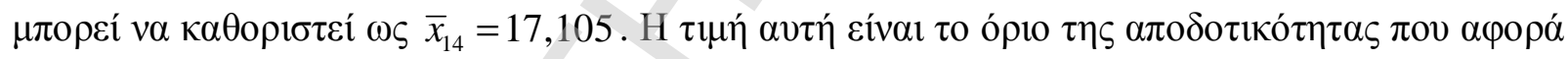

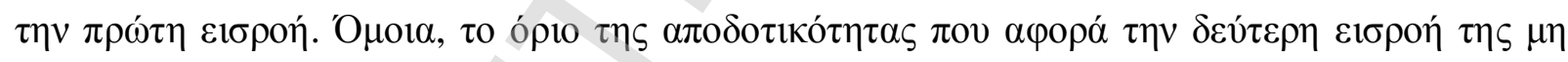

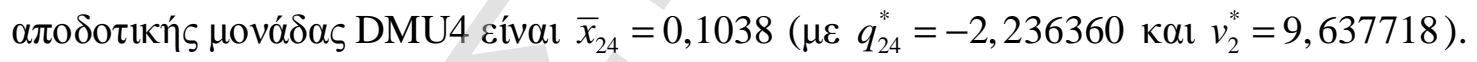




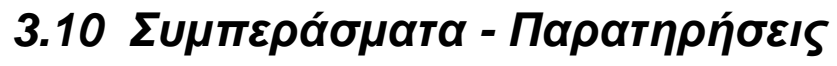

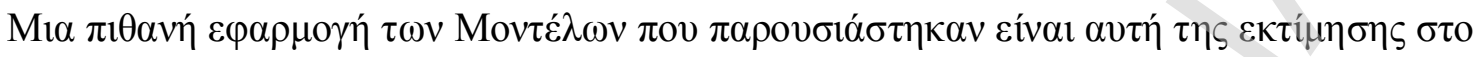

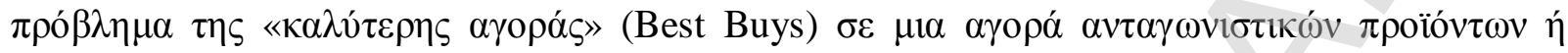

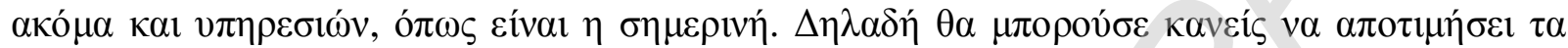

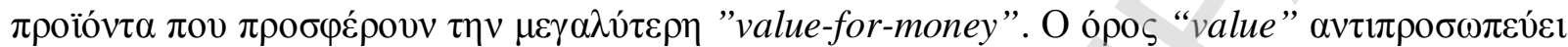

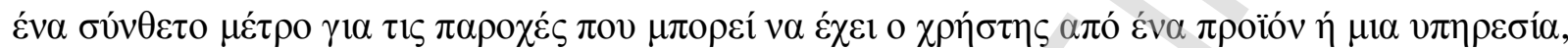

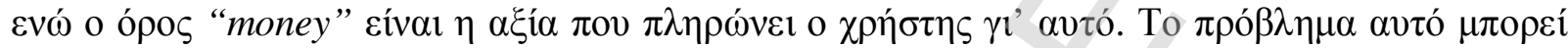

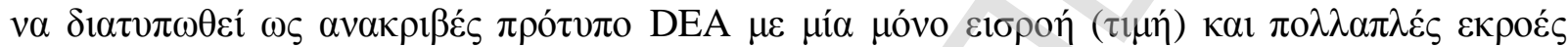
( $\delta \varepsilon i ́ \kappa \tau \varepsilon \varsigma \kappa \alpha 1 \varepsilon \kappa \tau \imath \mu \eta ́ \sigma \varepsilon 1 \varsigma \alpha \pi o ́ \delta o \sigma \eta \varsigma)$.

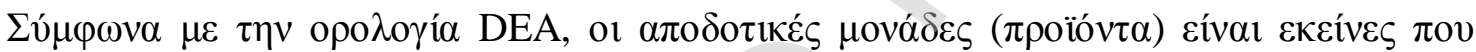

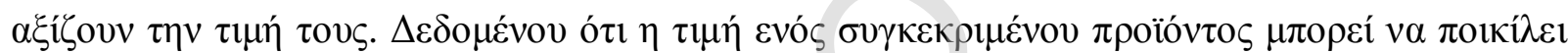

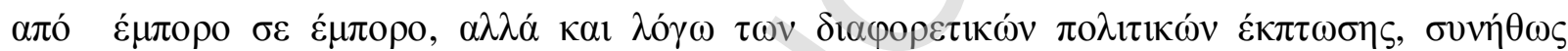

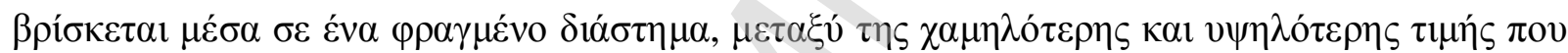

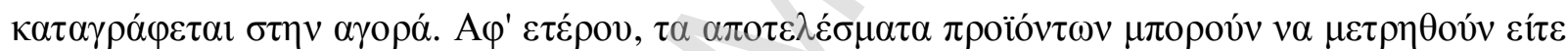

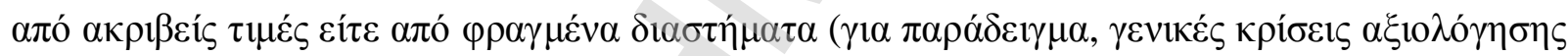

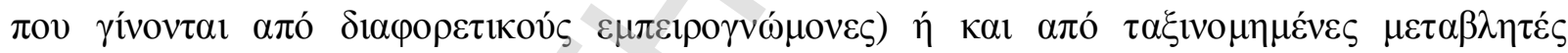

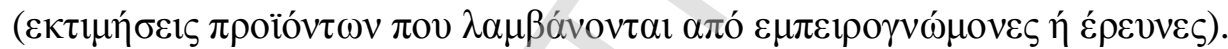

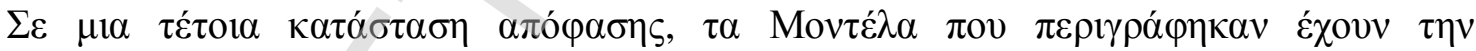

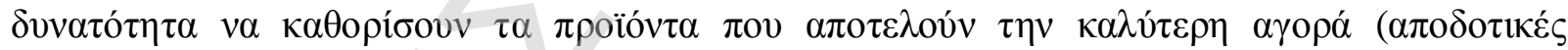

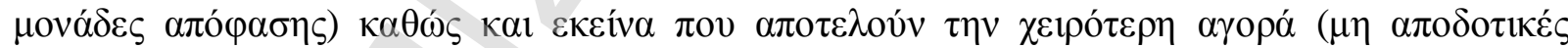

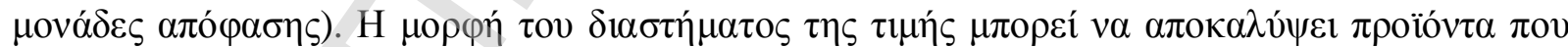

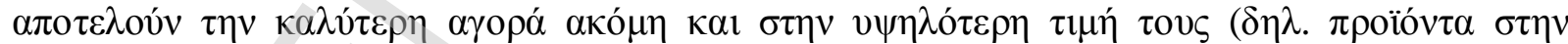

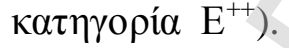




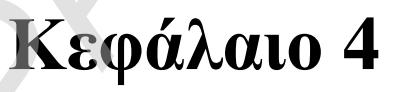

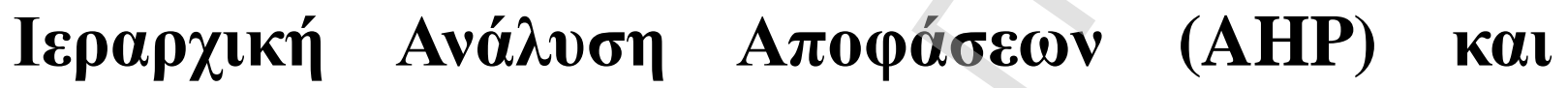

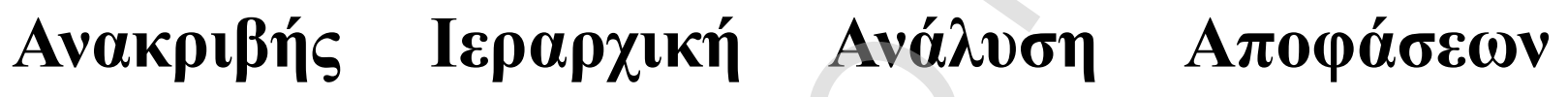 (IAHP)}

4.1 Eıб

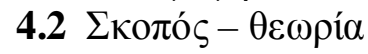

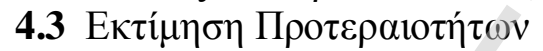

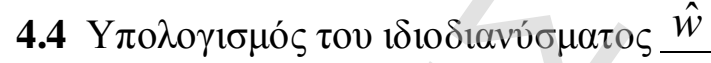

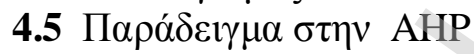

4.6 $\Sigma v \mu \pi \varepsilon \rho і \varphi о \rho \alpha ́ ~ \mu \varepsilon \theta o ́ \delta \omega v ~ \mu \varepsilon \mu \eta ~ \sigma v v \varepsilon \pi \eta ́ ~ \pi i ́ v \alpha \kappa \alpha$

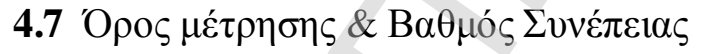

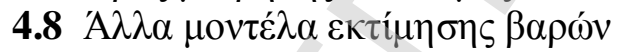

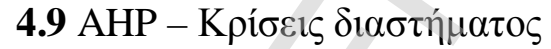




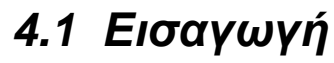

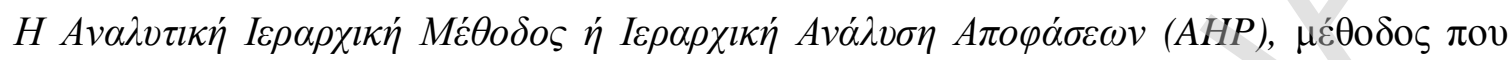

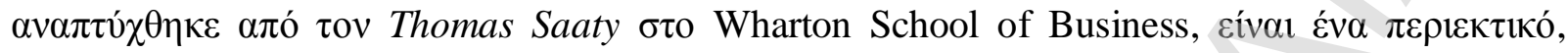

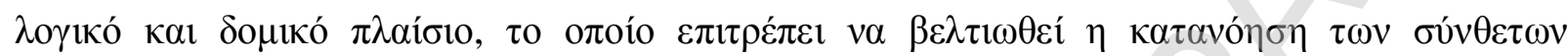

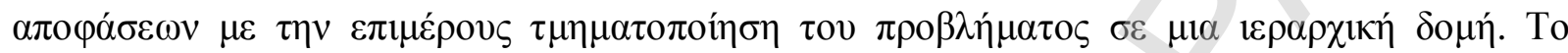

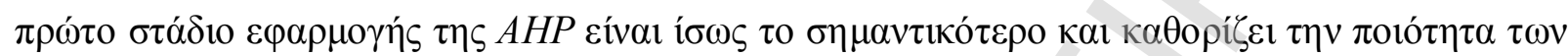

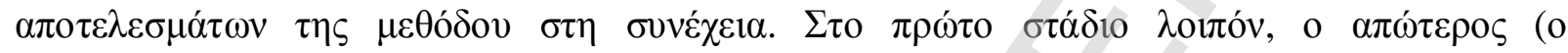

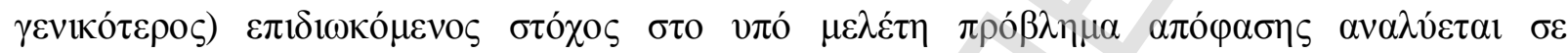

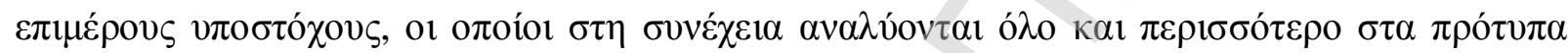

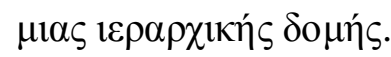

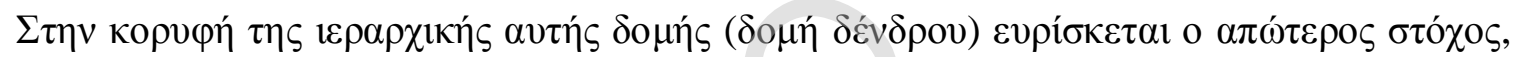

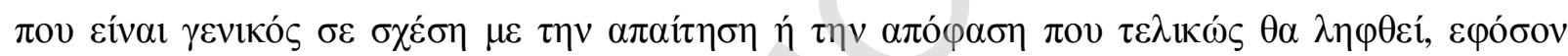

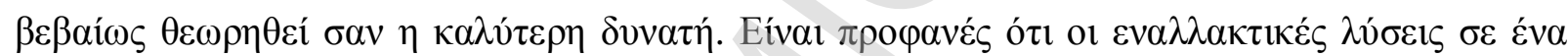

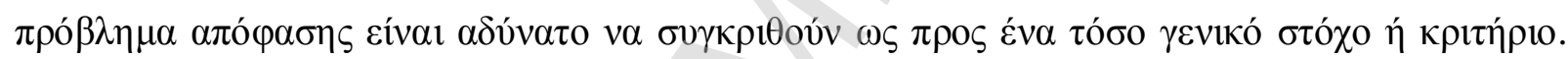

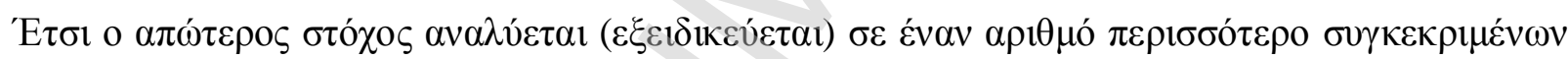

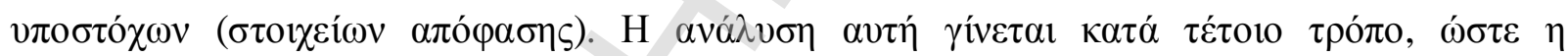

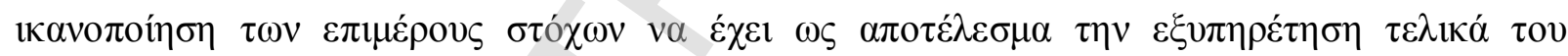

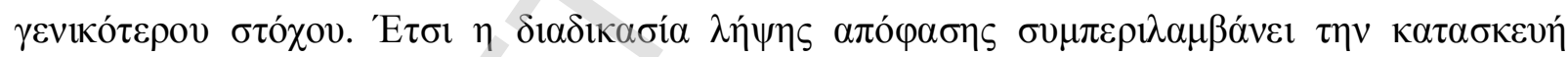

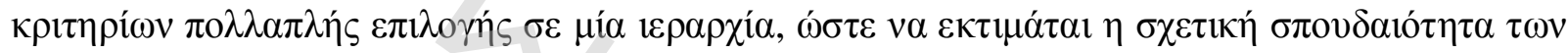

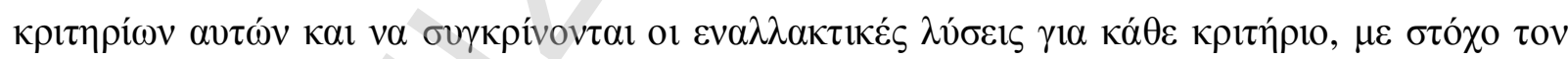

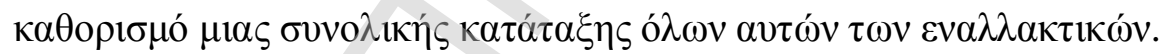

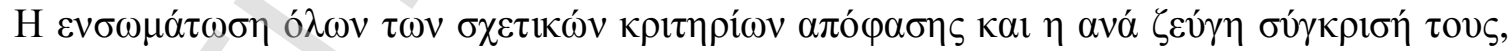

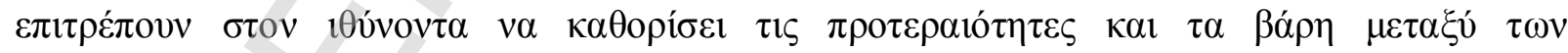

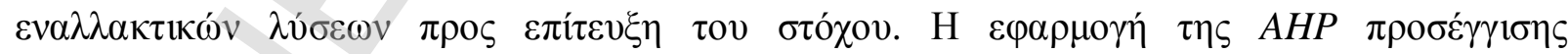

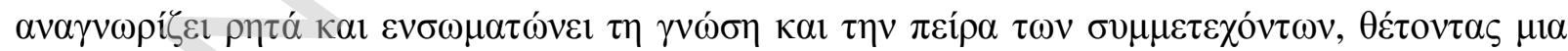

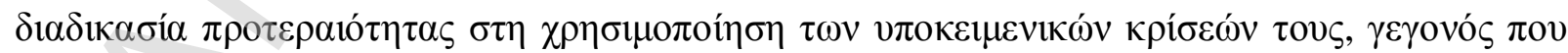

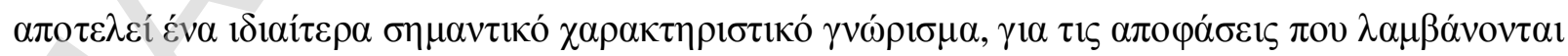

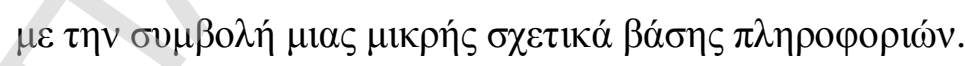




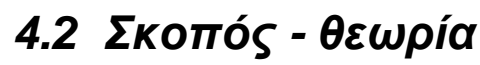

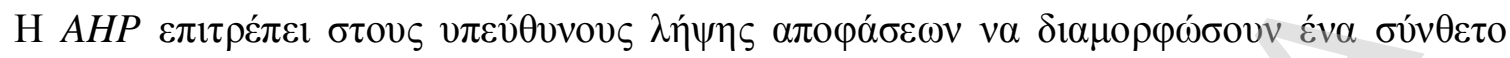

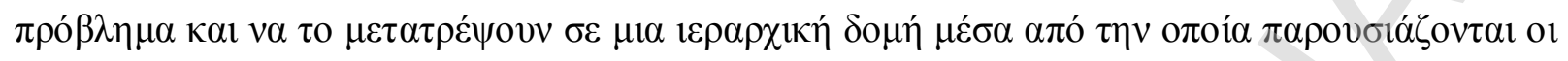

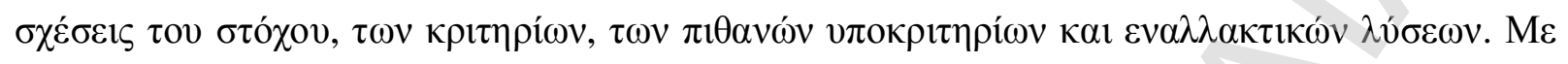

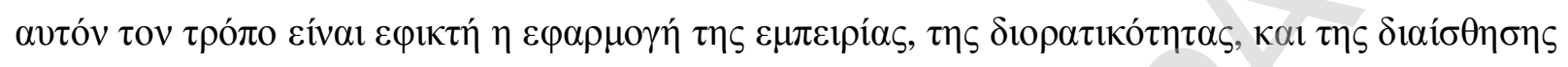

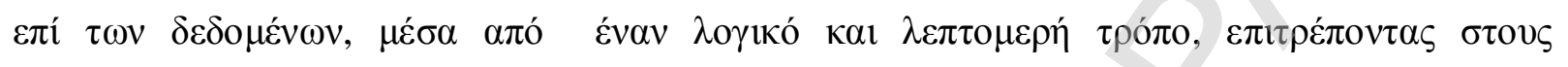

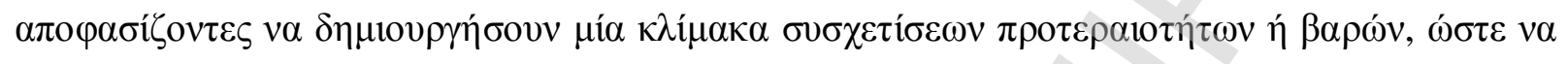

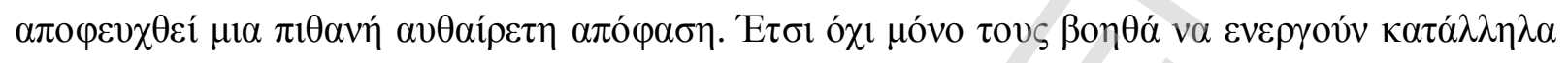

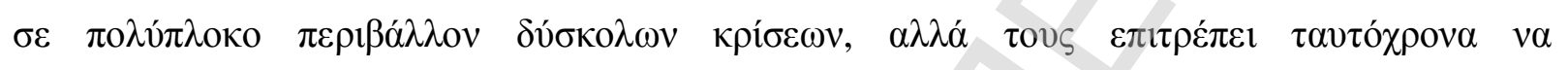

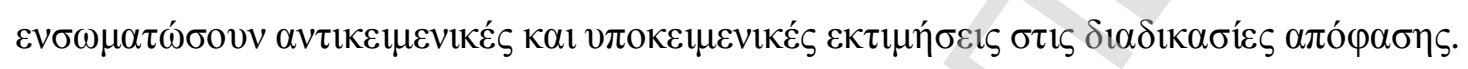

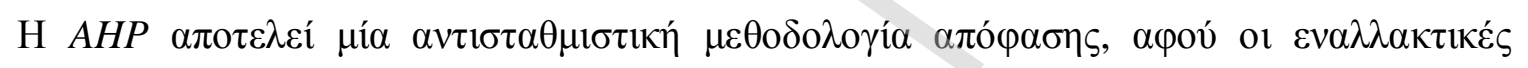

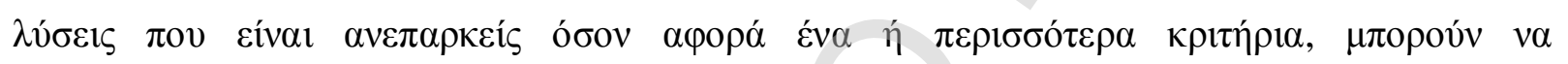

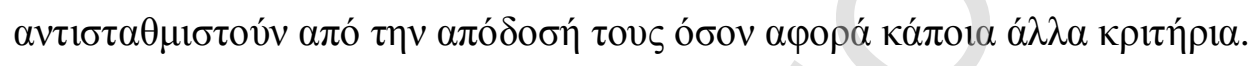

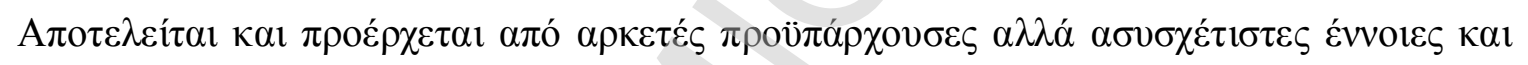

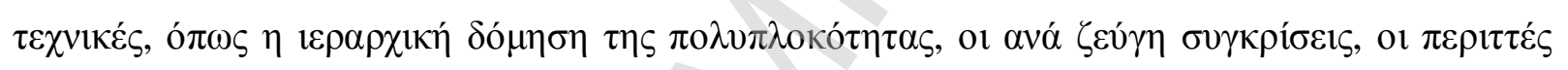

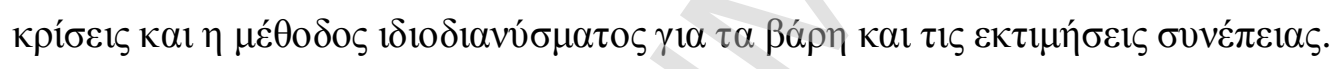

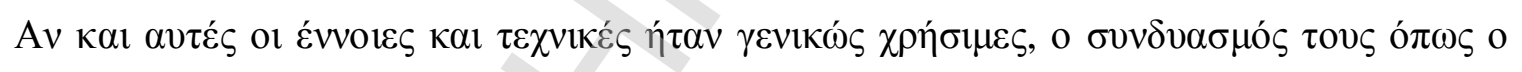

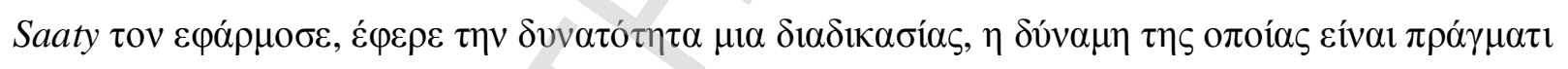

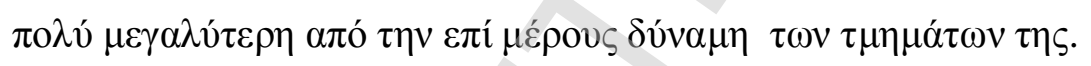

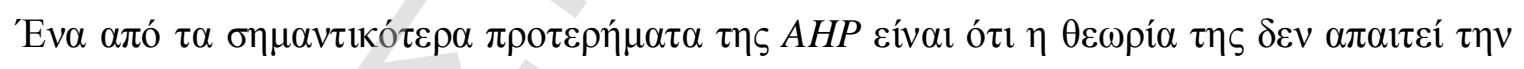

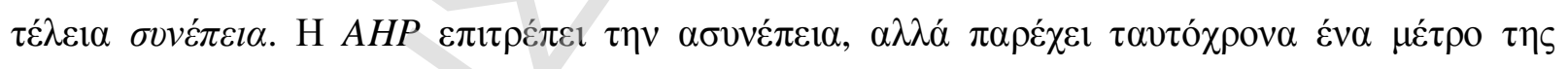

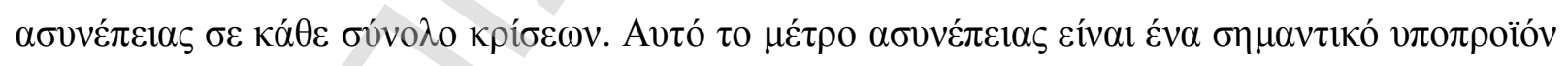

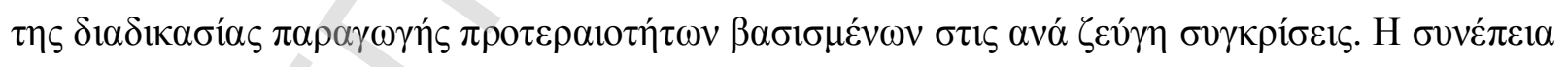

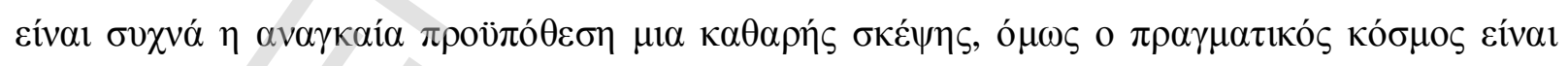

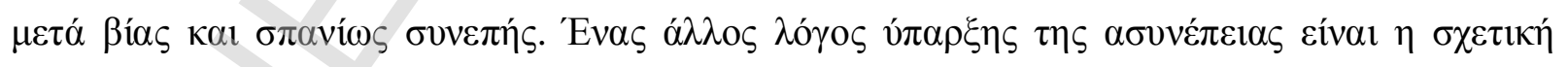

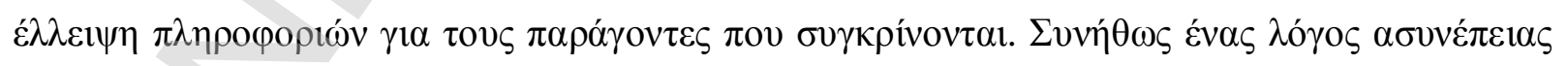

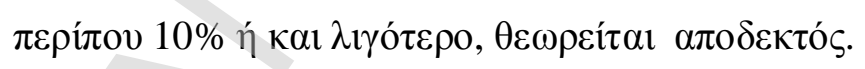

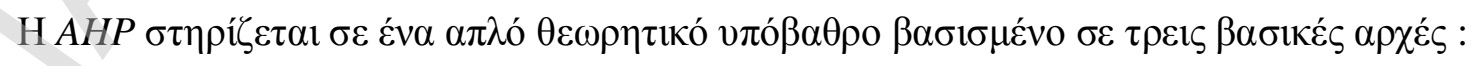

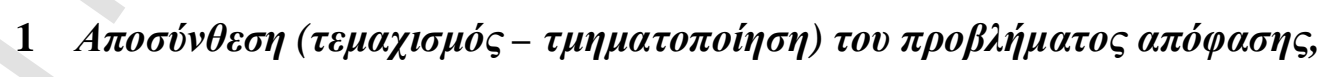

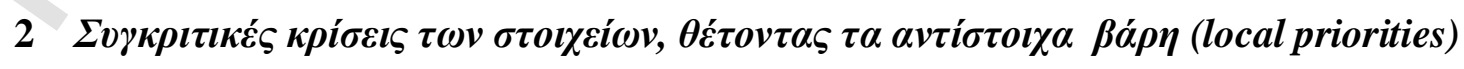

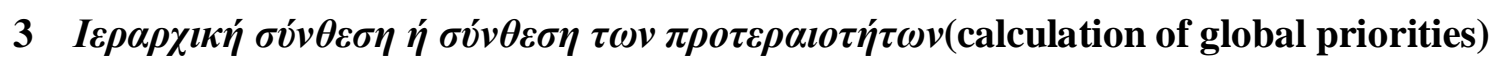




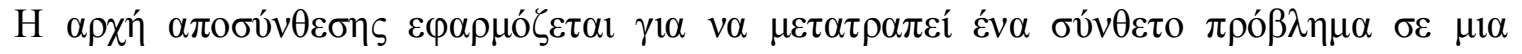

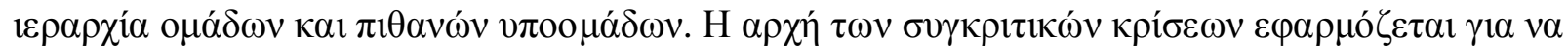

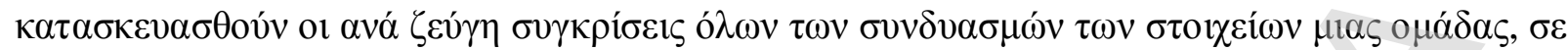

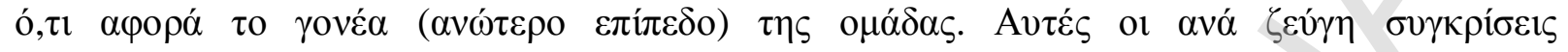

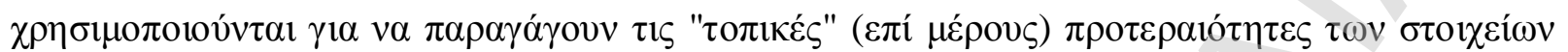

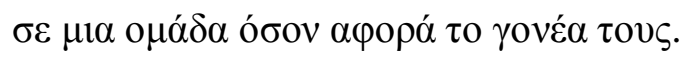

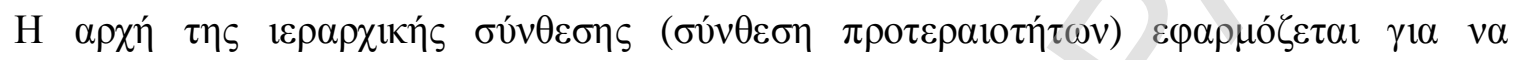

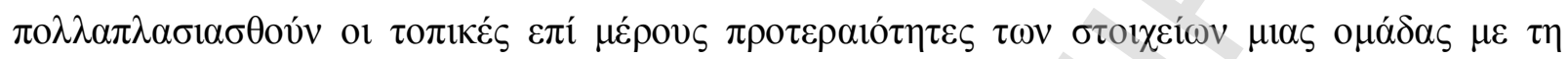

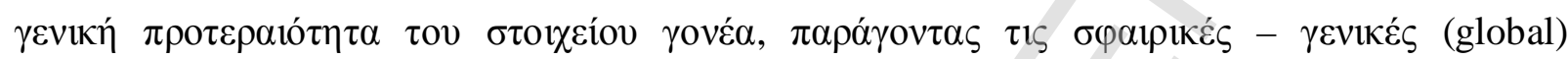

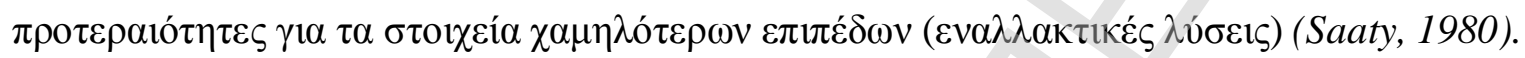

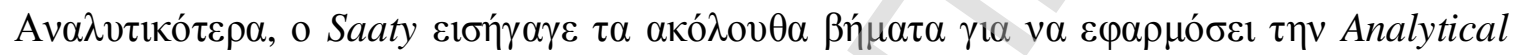
Hierarchy Process - AHP :

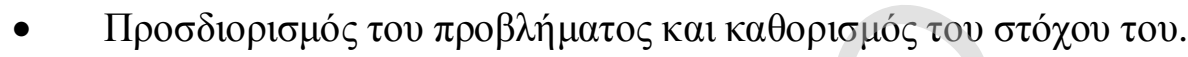

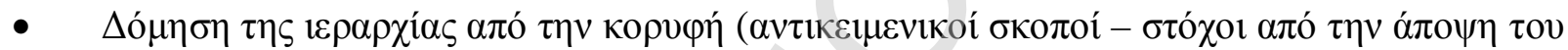

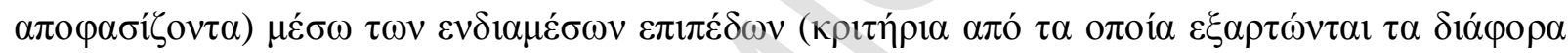

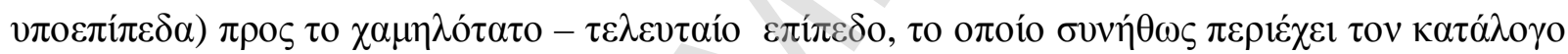
$\alpha \pi$ ó $\tau 1 \zeta \varepsilon v \alpha \lambda \lambda \alpha \kappa \tau 1 \kappa \varepsilon ́ \varsigma \lambda \hat{\sigma} \sigma \varepsilon ı$.

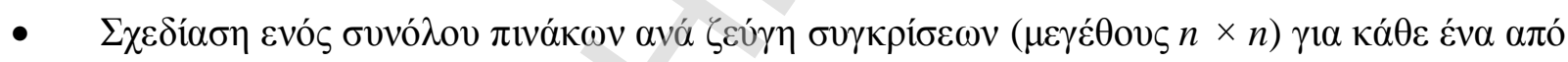

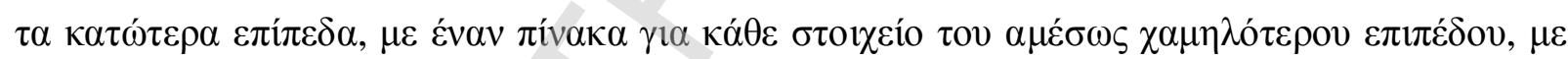

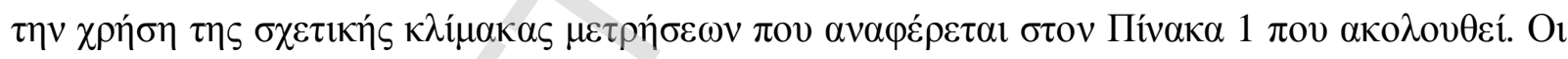

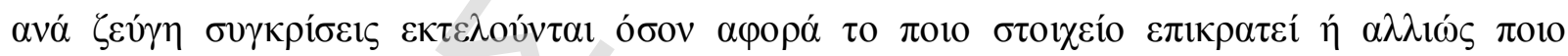

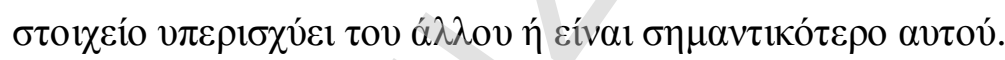

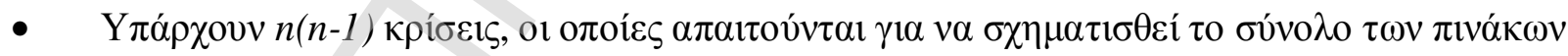

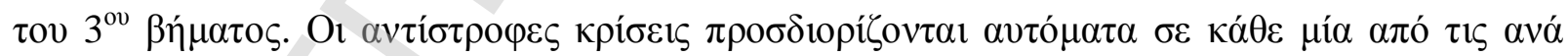

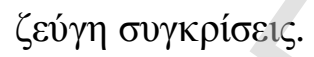

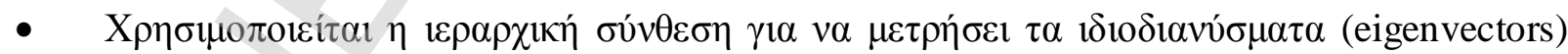

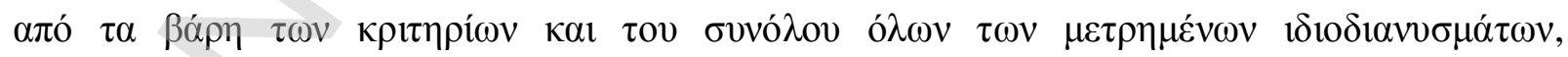

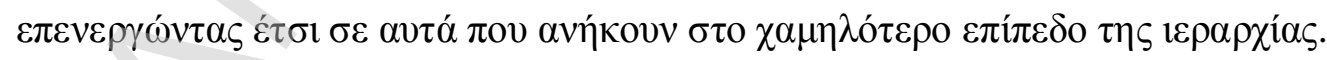

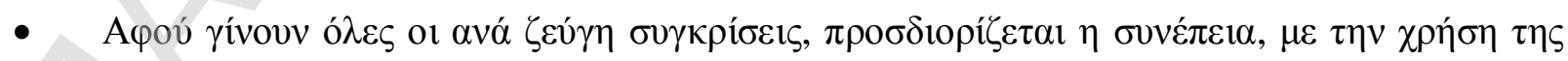

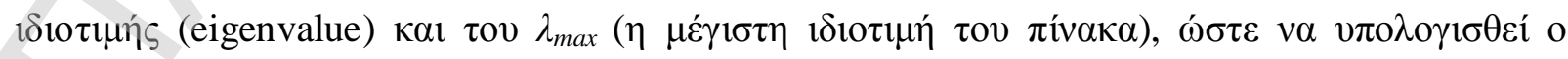

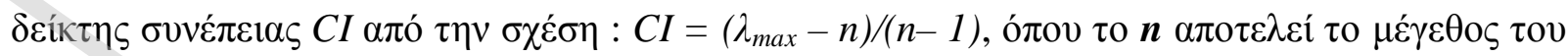

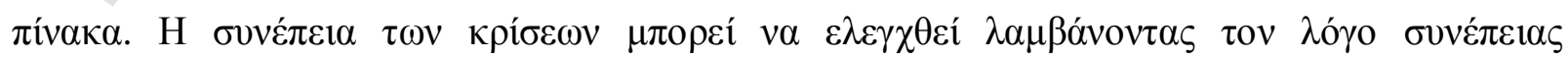




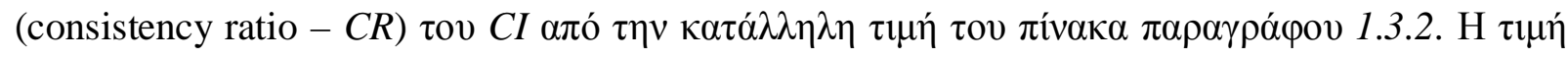

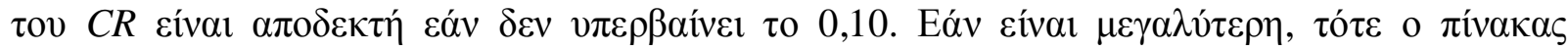

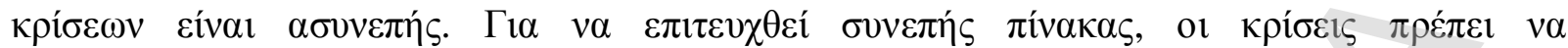
$\varepsilon \pi \alpha v \alpha \pi \rho \circ \sigma \delta 10 \rho ı \theta 0 v ́ v$.

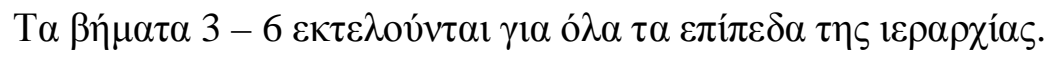

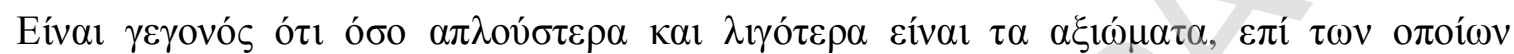

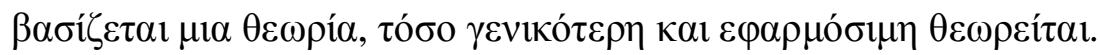

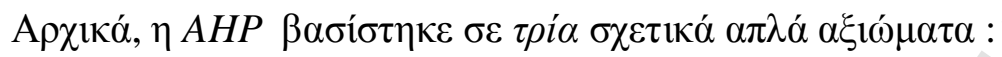

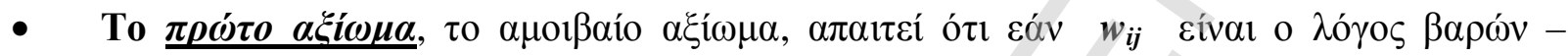

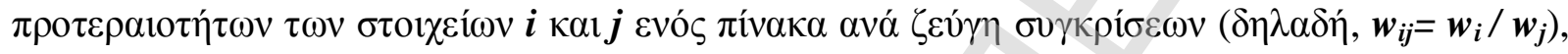

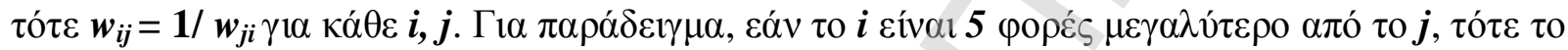

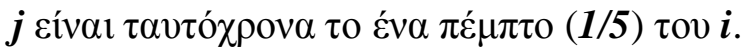

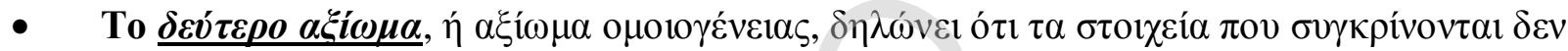

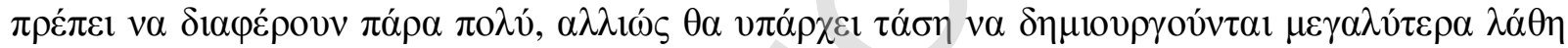

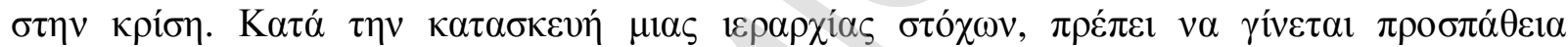

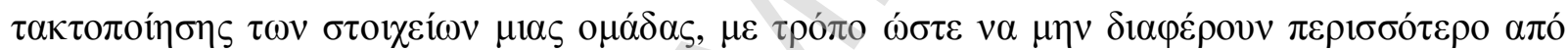

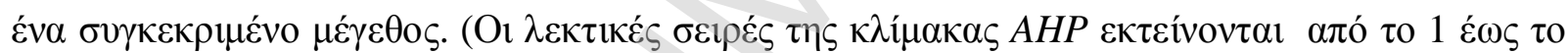

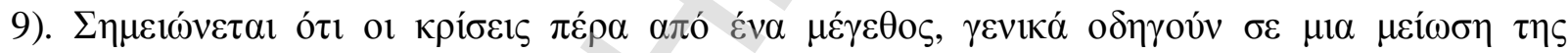

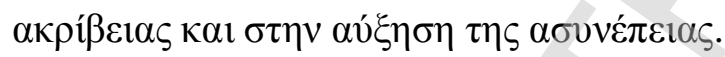

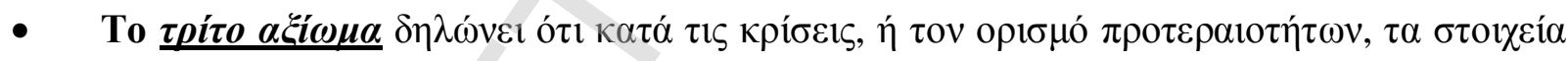

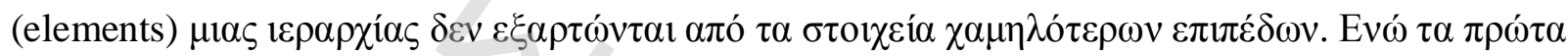

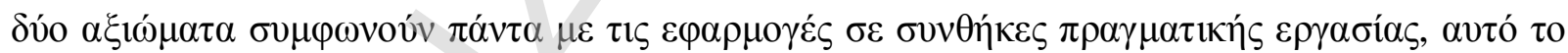

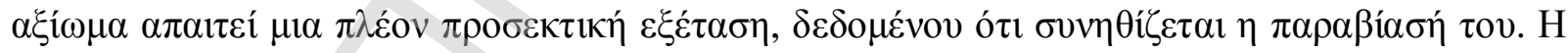

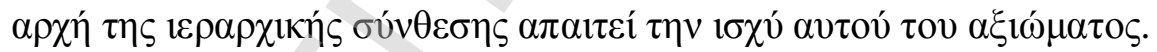

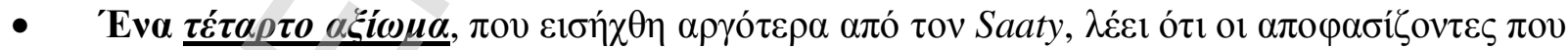

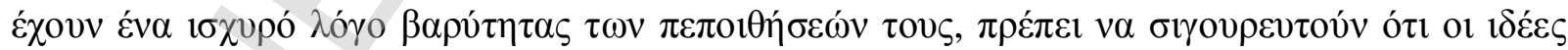

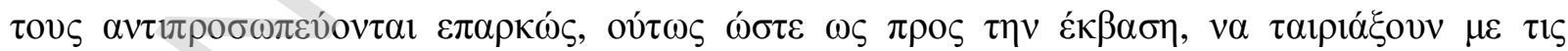

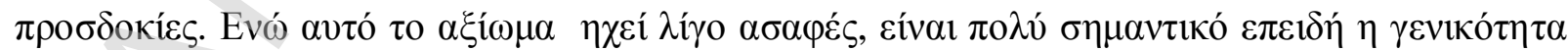

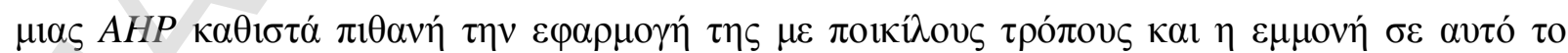

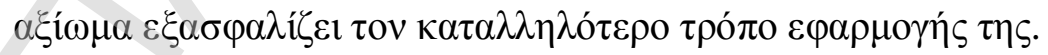

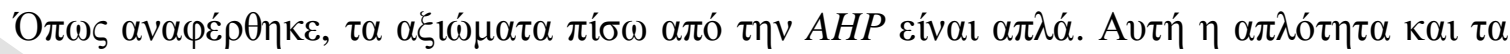

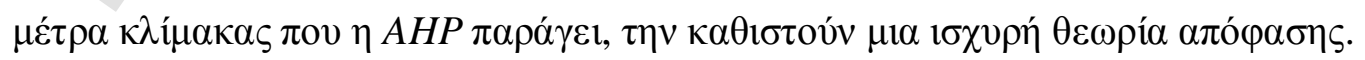




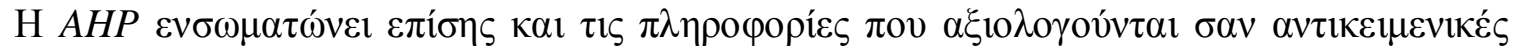

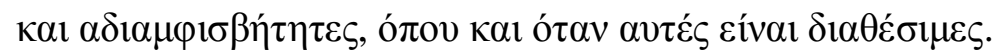

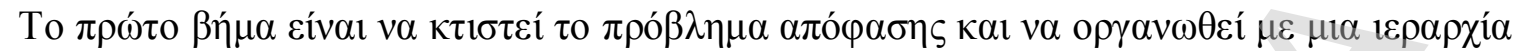

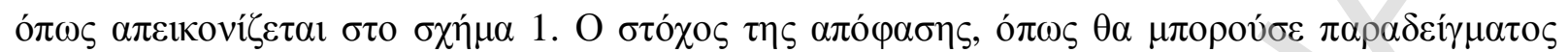

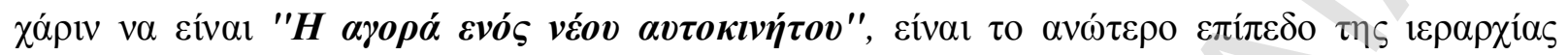

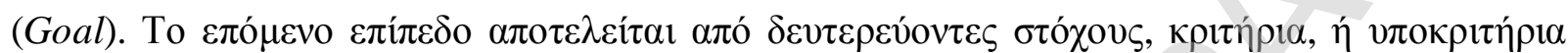

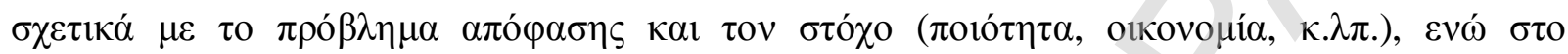

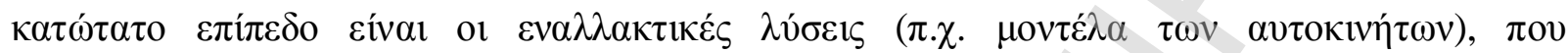

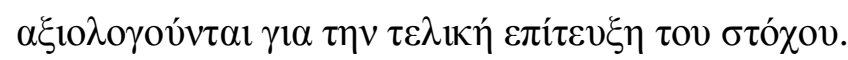

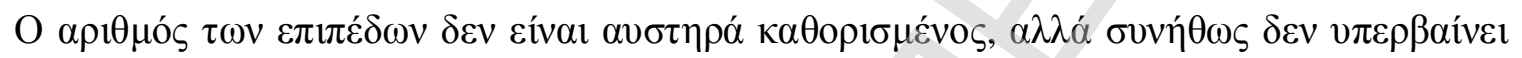

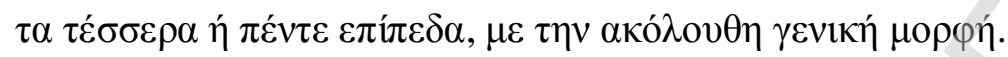

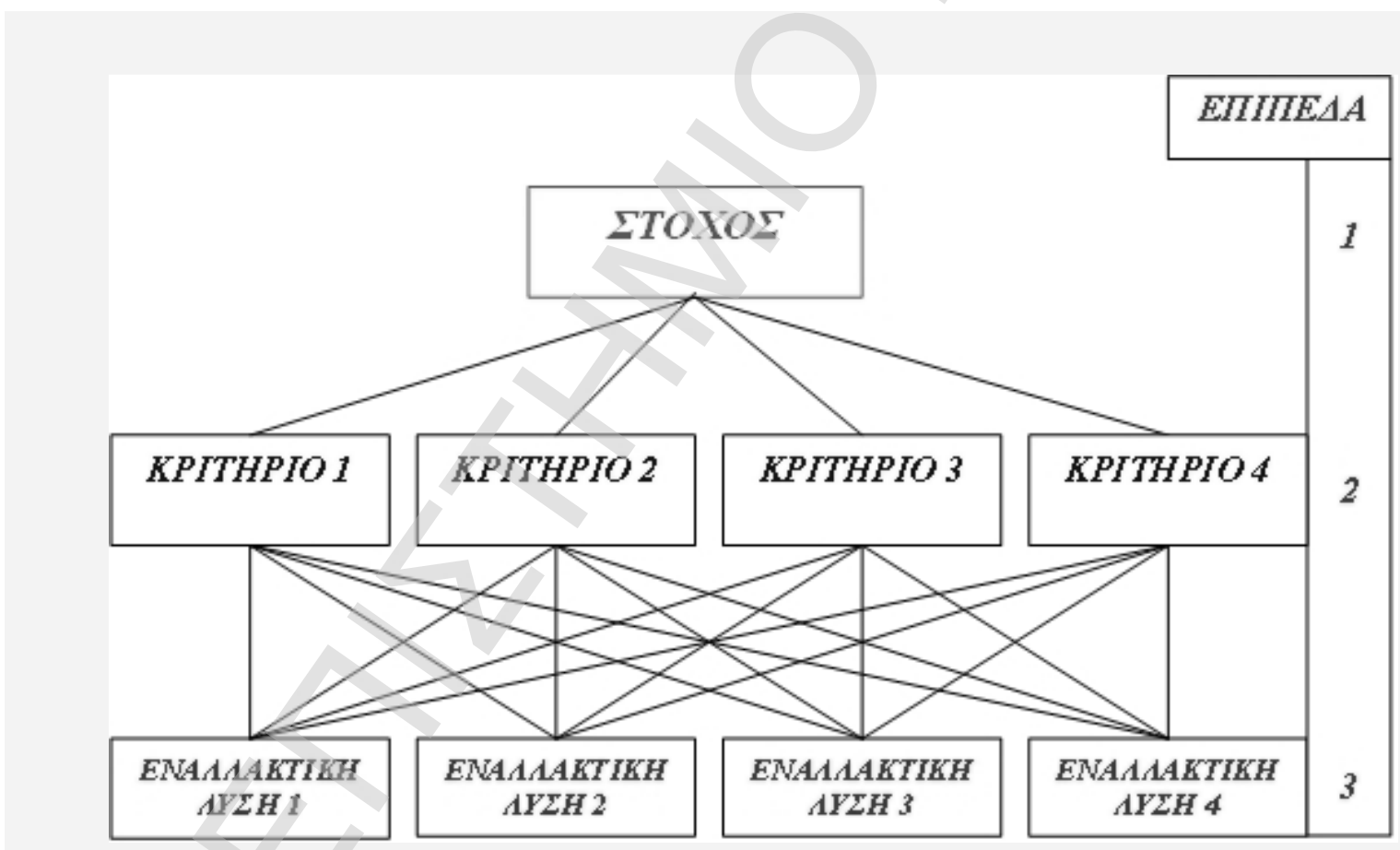

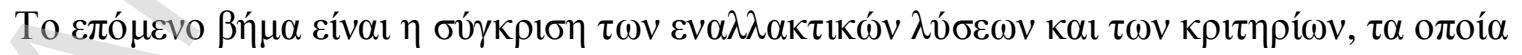

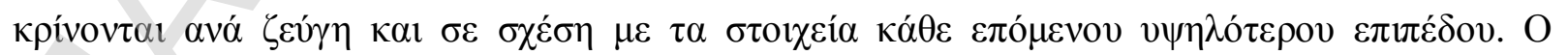

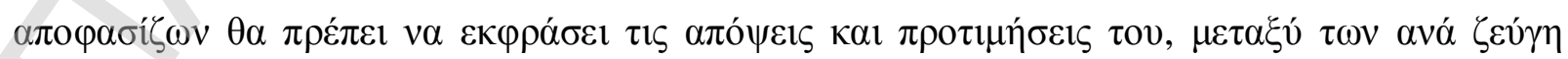

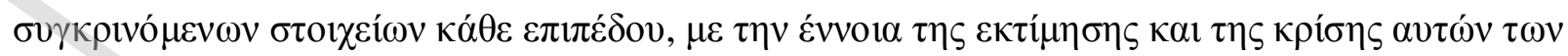

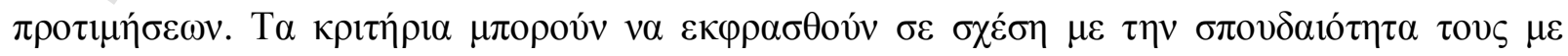




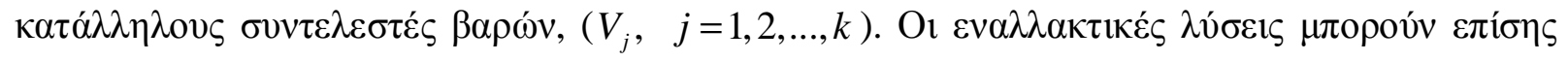

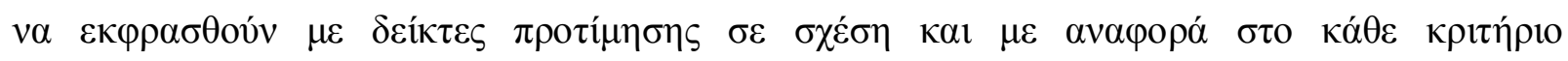

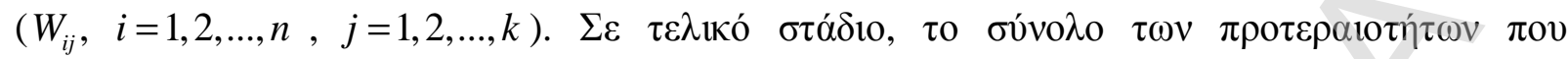

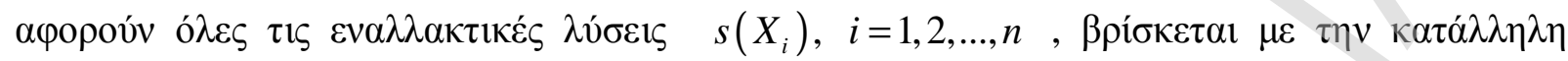

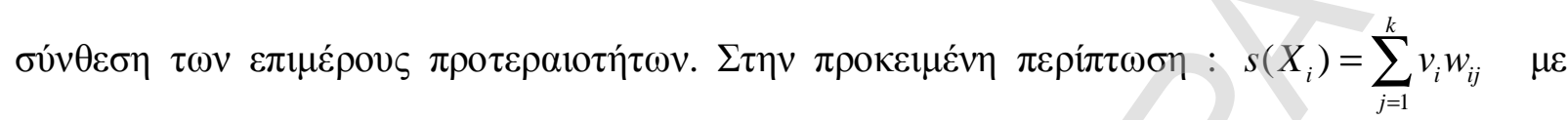
$i=1,2, \ldots, n$.

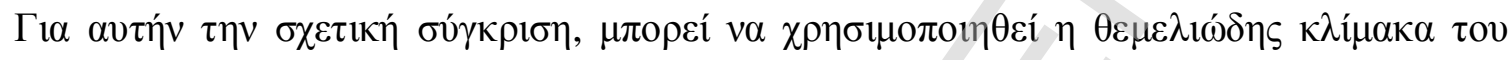

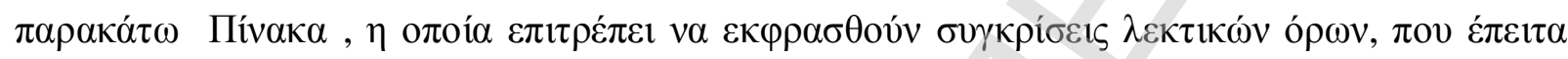

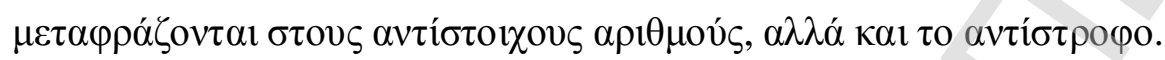

\begin{tabular}{|c|c|c|}
\hline 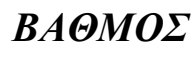 & ПЕРІГРАФН & $E \Xi H \Gamma H \Sigma H$ \\
\hline 1 & 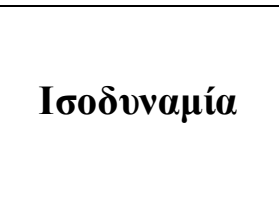 & 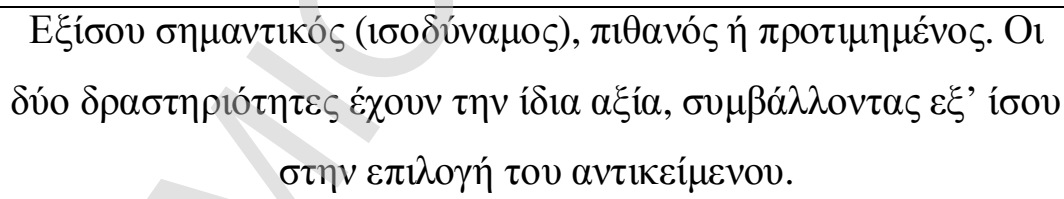 \\
\hline 3 & 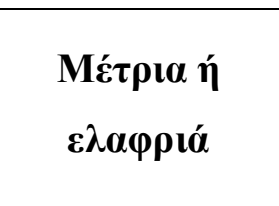 & 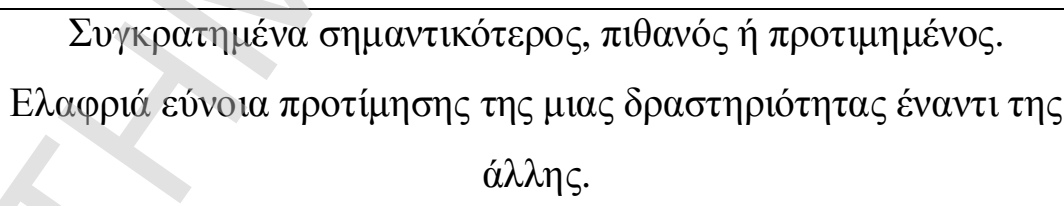 \\
\hline 5 & 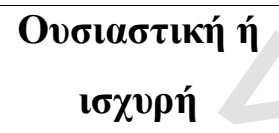 & 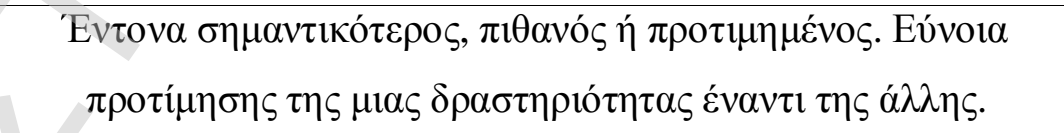 \\
\hline 7 & 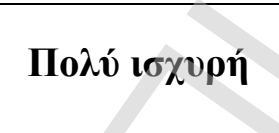 & 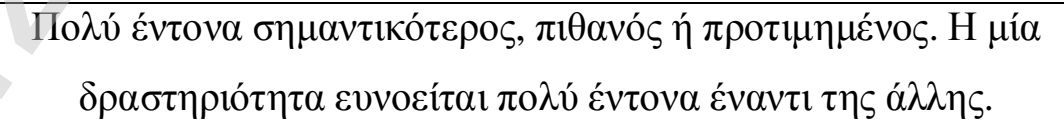 \\
\hline 9 & Aлó $\lambda v \tau \eta$ & 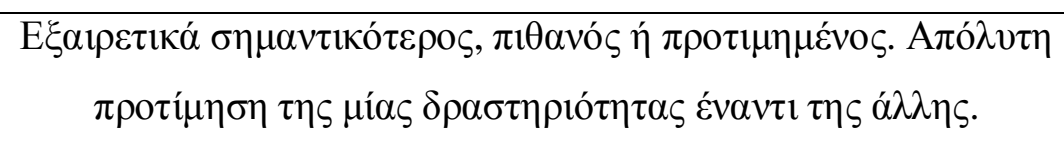 \\
\hline $2,4,6,8$ & $\begin{array}{c}\text { Evoเó } \mu \varepsilon \sigma \varepsilon \varsigma \\
\tau \imath \mu \varepsilon ́ \varsigma\end{array}$ & 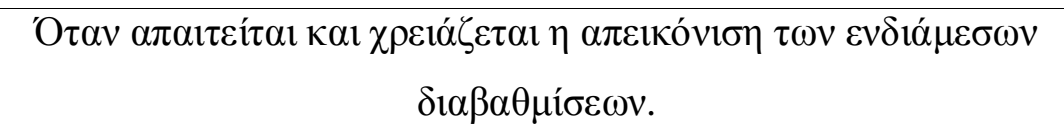 \\
\hline
\end{tabular}

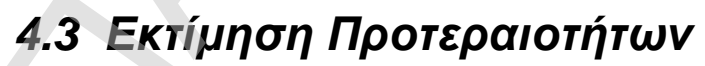

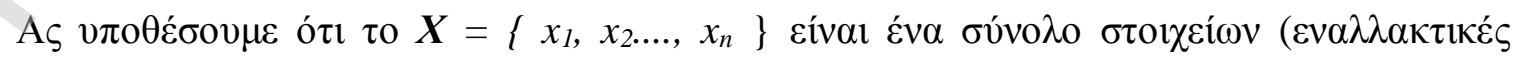

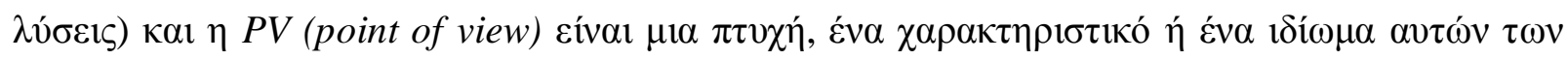




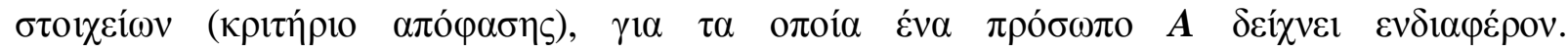

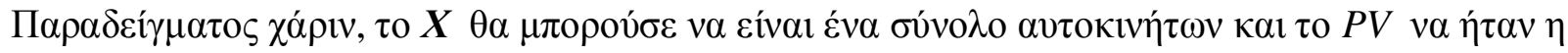

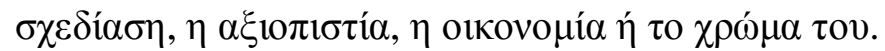

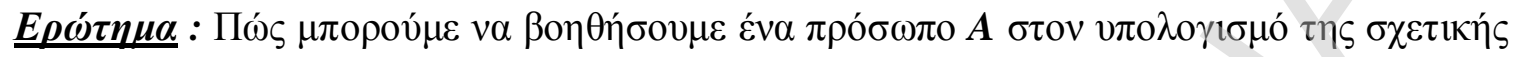

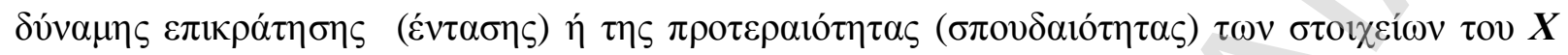
a

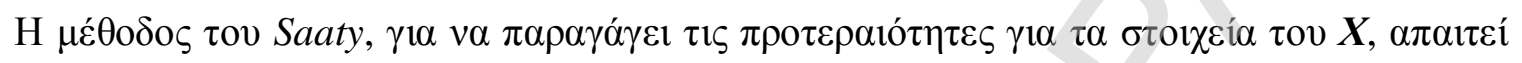

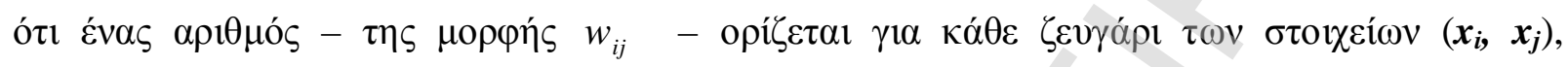

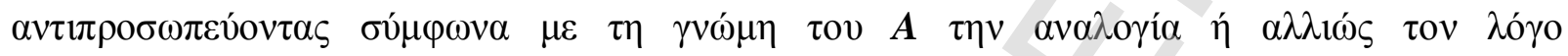

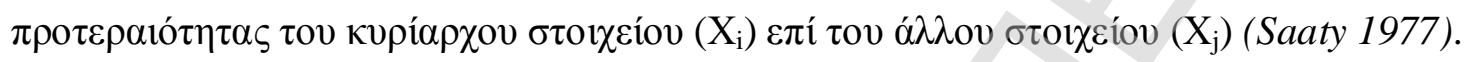

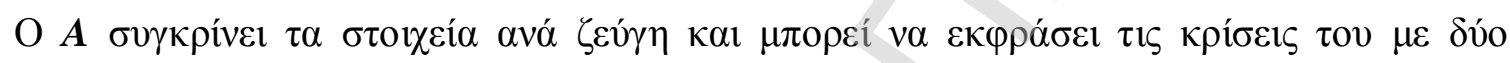

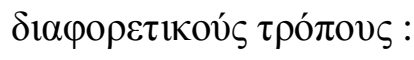

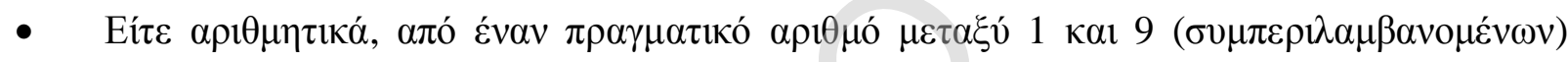

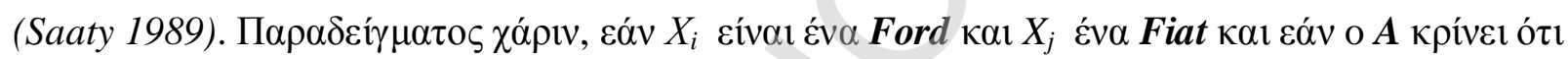

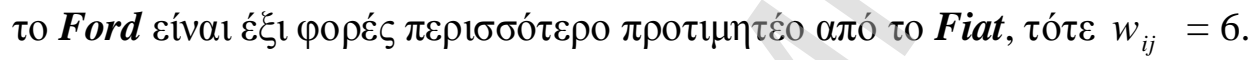

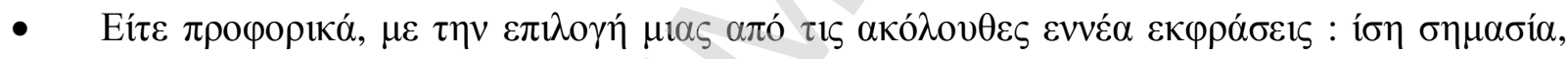

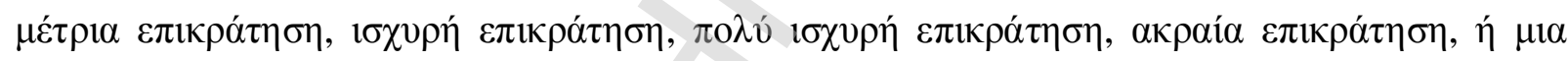

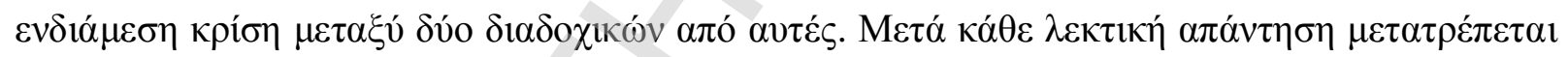

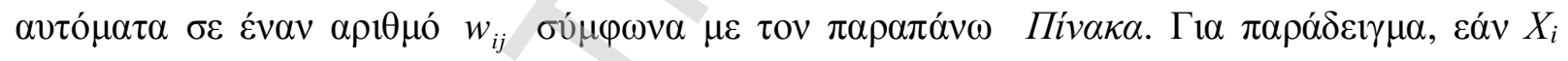

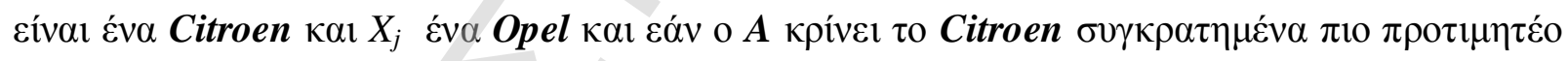

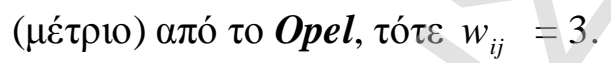

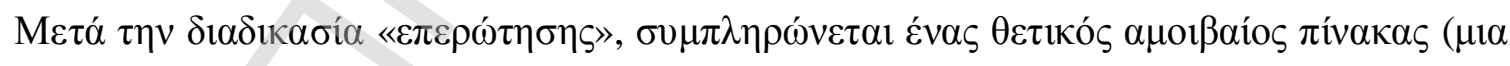

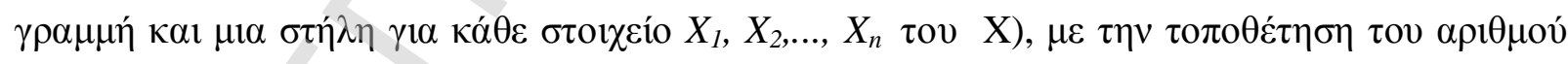

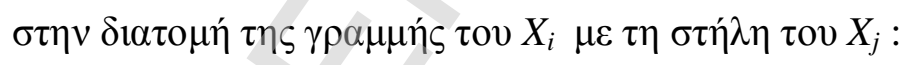

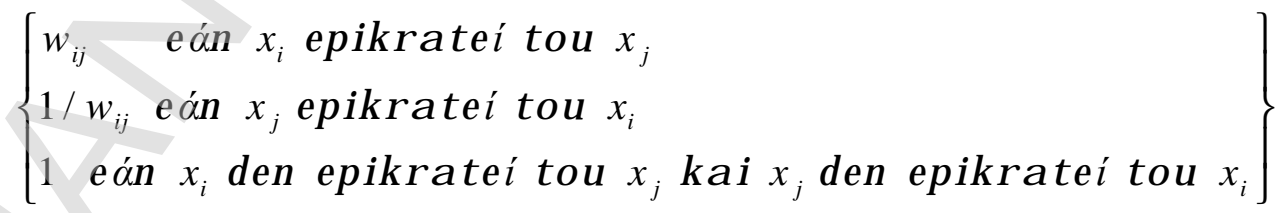

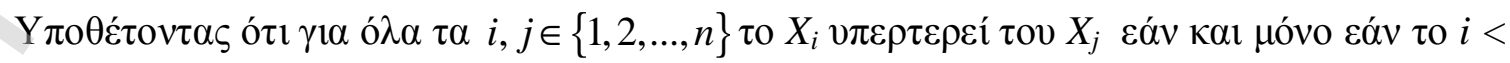

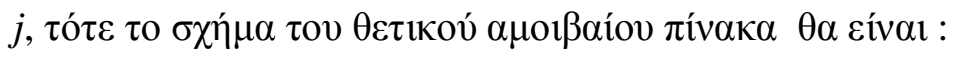




$$
w=\left[\begin{array}{lccc}
1 & w_{12} & \mathrm{~K} & w_{1 n} \\
w_{21} & 1 & \mathrm{~K} & w_{2 n} \\
\mathrm{~K} & \mathrm{~K} & \mathrm{~K} & \mathrm{~K} \\
w_{n 1} & w_{n 2} & \mathrm{~K} & 1
\end{array}\right]=\left[\begin{array}{lccc}
1 & w_{12} & \mathrm{~K} & w_{1 n} \\
1 / w_{12} & 1 & \mathrm{~K} & w_{2 n} \\
\mathrm{~K} & \mathrm{~K} & \mathrm{~K} & \mathrm{~K} \\
1 / w_{1 n} & 1 / w_{2 n} & \mathrm{~K} & 1
\end{array}\right]
$$

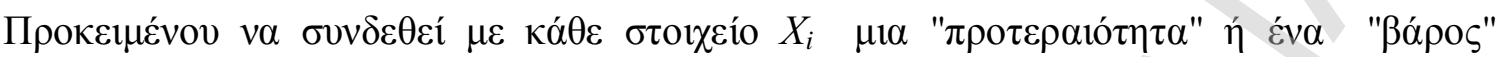

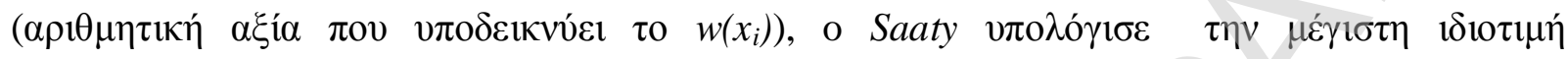

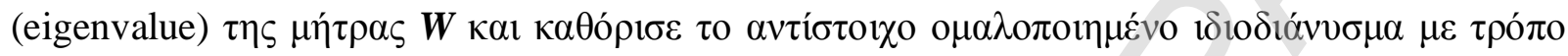

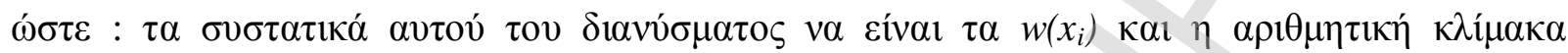

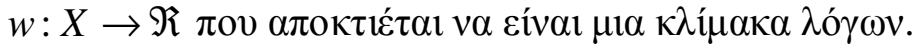

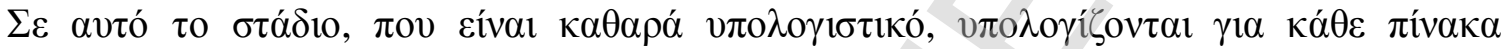

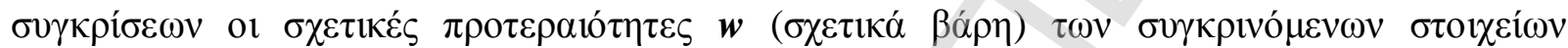

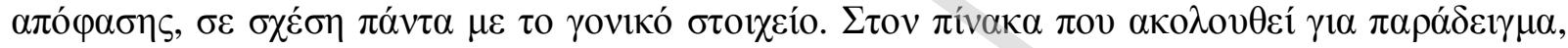

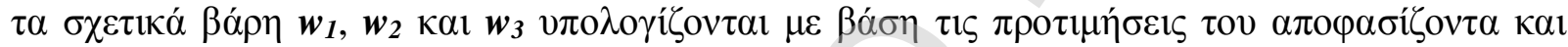

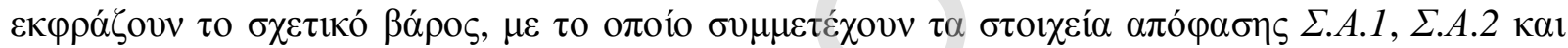

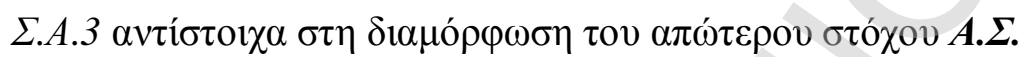

\begin{tabular}{|c|c|c|c|c|}
\hline.$\Sigma$. & .A.1 &.$A .2$ &.$A .3^{2}$ & $\alpha ́ \rho \eta$ \\
\hline .A.1 & 1 & & & 1 \\
\hline.$A .2$ & 12 & & 3 & 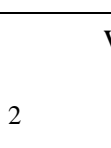 \\
\hline . A.3 & $/ 7$ & $1 / 3$ & & 3 \\
\hline
\end{tabular}

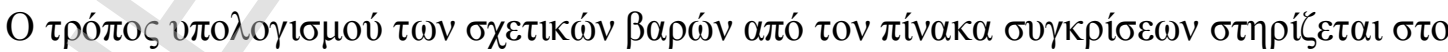

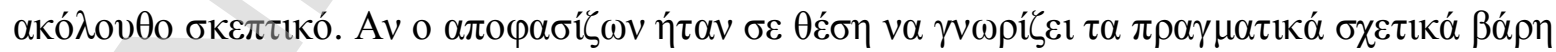

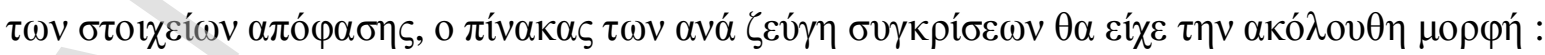




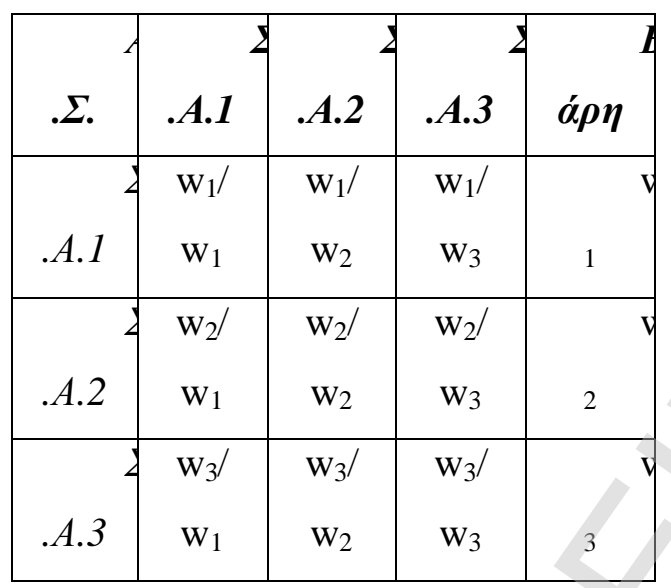

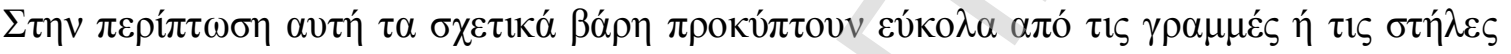

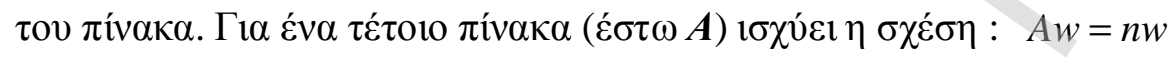

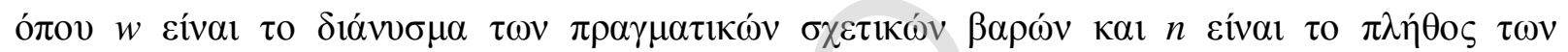

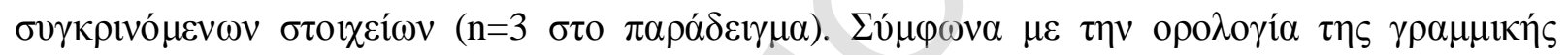

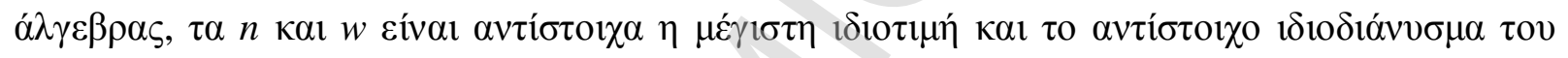

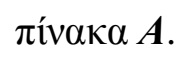

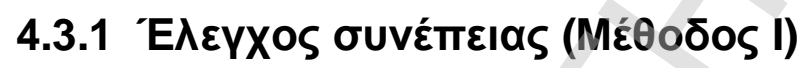

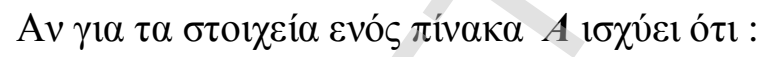

$$
a_{i j} \times a_{j k}=a_{i k} \gamma l \alpha \kappa \alpha \dot{\theta} \theta \varepsilon(i, j, k)
$$

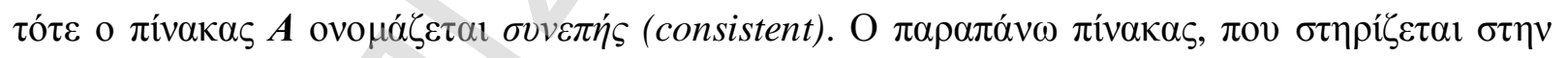

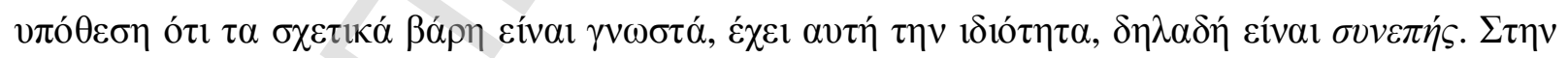

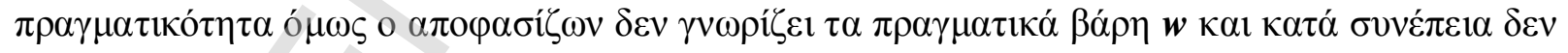

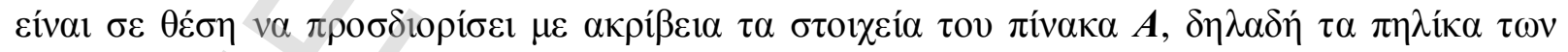

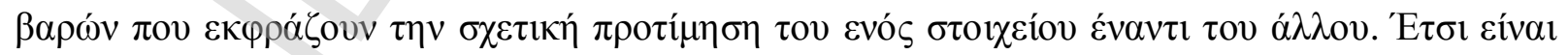

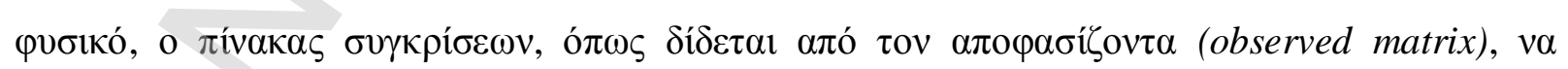

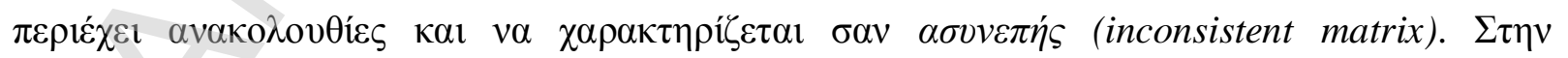

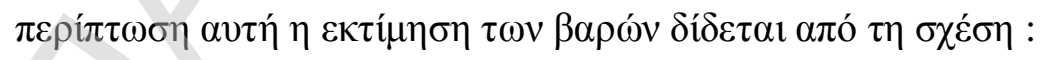

$$
\hat{A} \hat{w}=\lambda_{\max } \hat{w} \text { ó } \pi \text { ov } \hat{A}, \lambda_{\max }, \hat{w}
$$




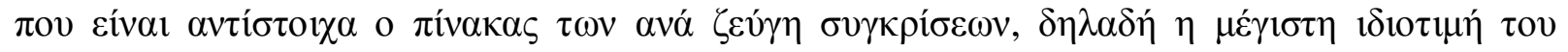

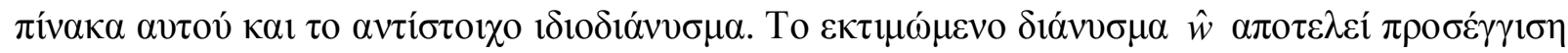

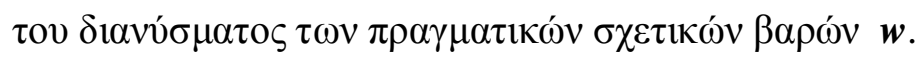

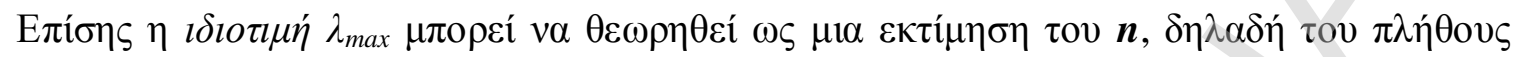

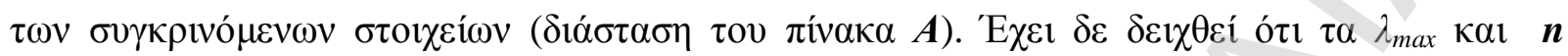

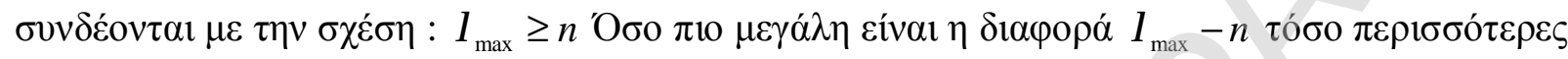

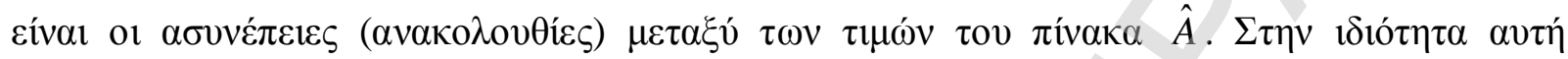

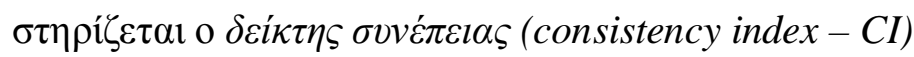

$$
\begin{gathered}
C I=\left(\lambda_{\max }-n\right) /(n-1) \quad \kappa \alpha 1 \text { o } \lambda o ́ \gamma o \varsigma ~ \sigma v v \varepsilon ́ \pi \varepsilon l \alpha \varsigma(\text { consistency ratio }-C R) \\
C R=\left(C I / R I_{n}\right) * 100
\end{gathered}
$$

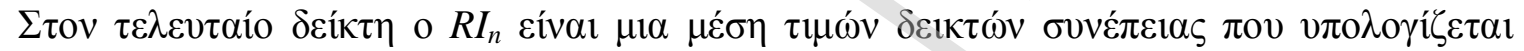

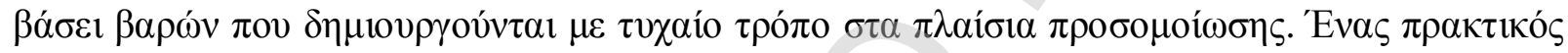

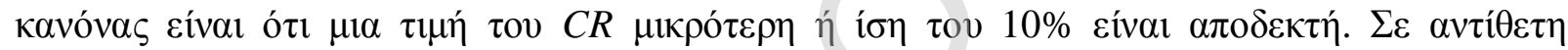

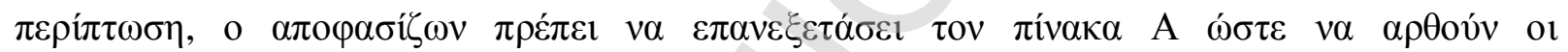

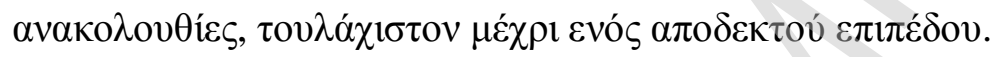

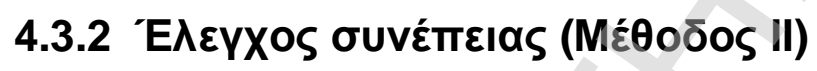

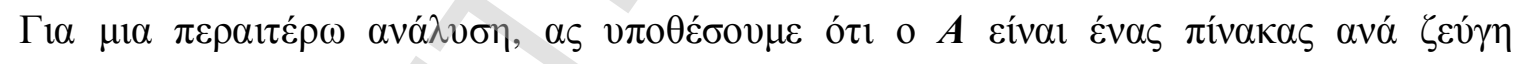

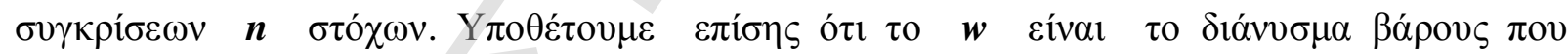

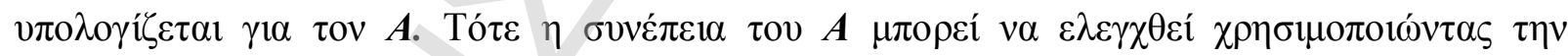

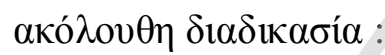

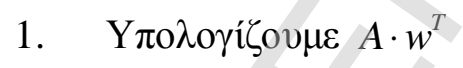

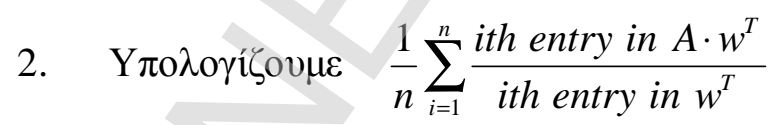

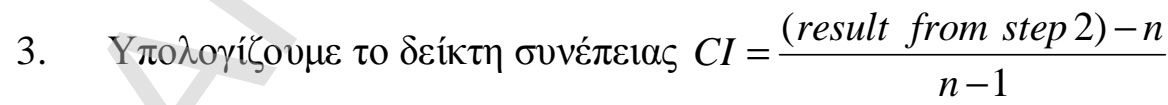

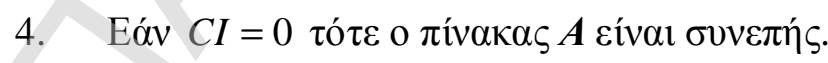

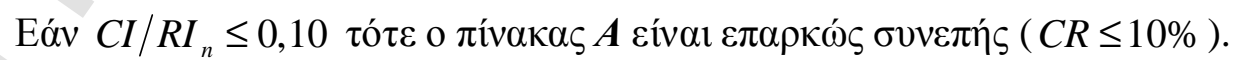

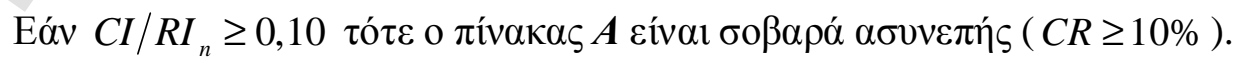




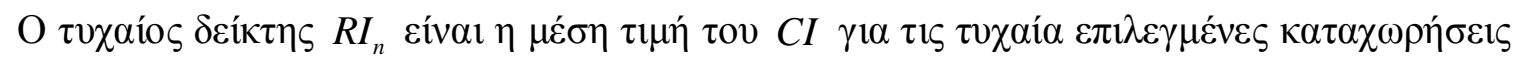

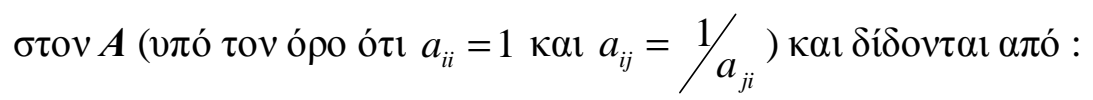

\begin{tabular}{|l|l|l|l|l|l|l|l|}
\hline $\boldsymbol{n}$ & $\mathbf{2}$ & $\mathbf{3}$ & $\mathbf{4}$ & $\mathbf{5}$ & $\mathbf{6}$ & $\mathbf{7}$ & $\boldsymbol{\cdots}$ \\
\hline $\boldsymbol{R I}_{\boldsymbol{n}}$ & 0 & 0,58 & 0,90 & 1,12 & 1,24 & 1,32 & $\cdots$ \\
\hline
\end{tabular}

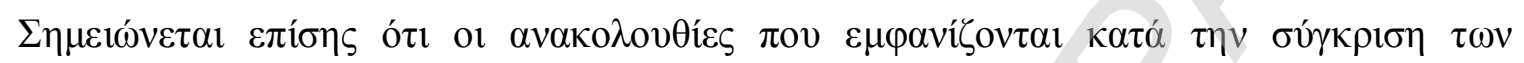

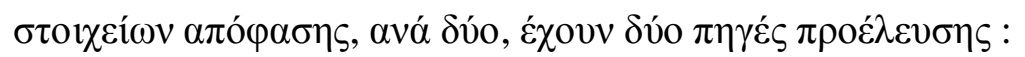

- $\quad \tau o v \alpha \pi \circ \varphi \alpha \sigma i \zeta o v \tau \alpha \kappa \alpha \imath$

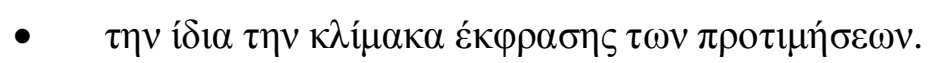

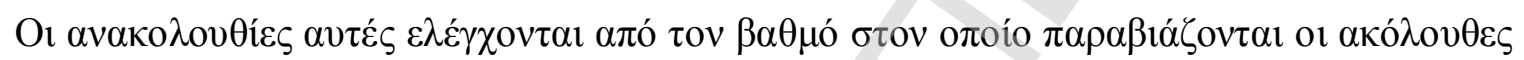

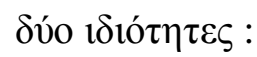

$$
\begin{aligned}
& \text { (a) Av } a_{i j}>1 \kappa \alpha l a_{j k}>1 \tau o ́ t \varepsilon \kappa \alpha l a_{i k}>1 \\
& \text { ( } \beta) a_{i j} \times a_{j k}=a_{i k} \gamma \alpha \kappa \alpha \dot{\theta} \varepsilon(i, j, k)
\end{aligned}
$$

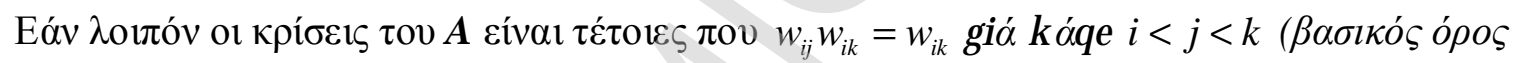

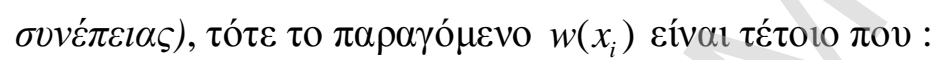

$$
w_{i j}=w\left(x_{i}\right) / w\left(x_{j}\right) \gamma l \alpha ́ \kappa \dot{\theta} \theta \varepsilon i<j .
$$

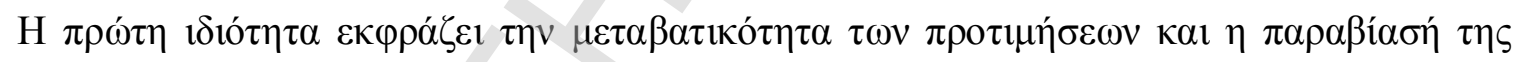

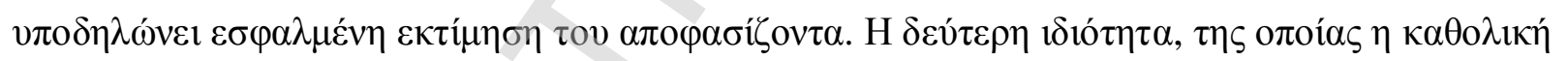

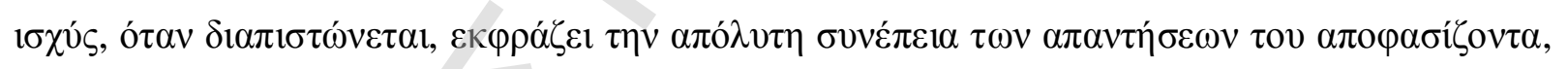

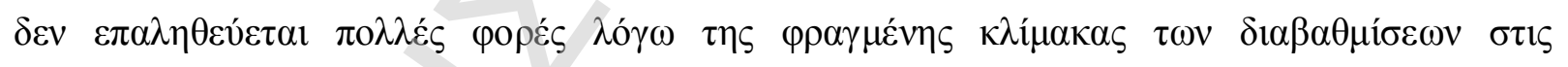

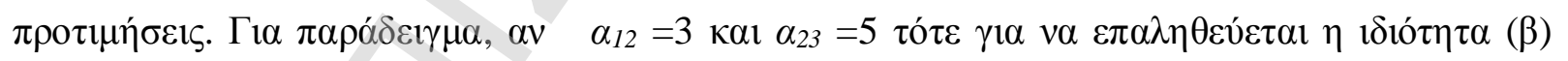

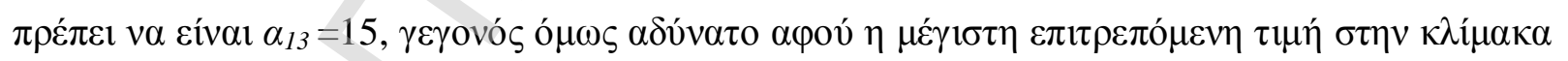

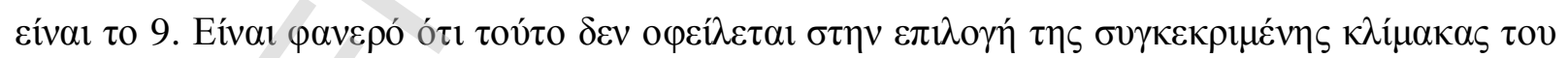

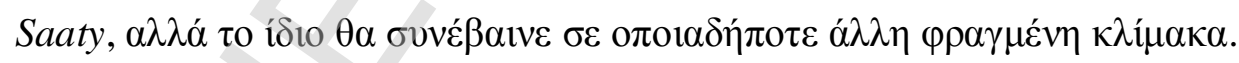

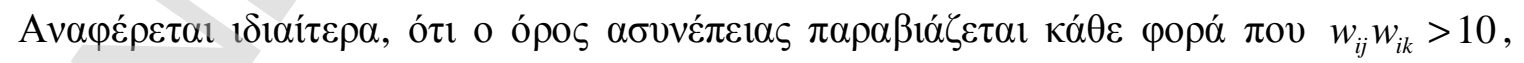

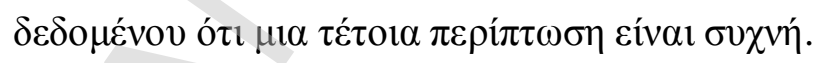

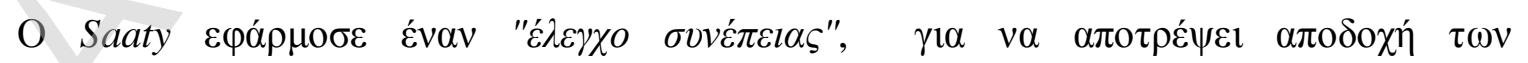

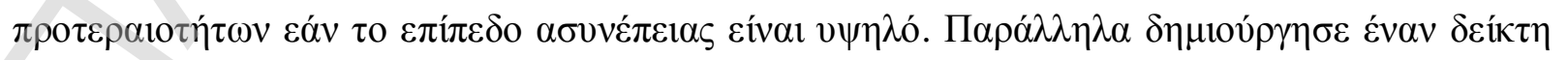

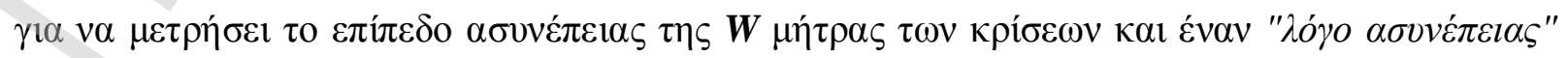

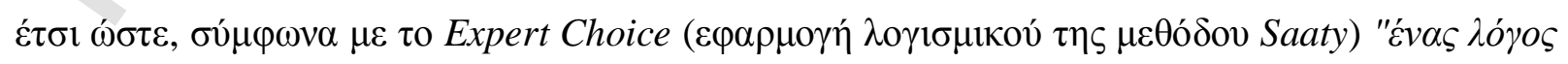

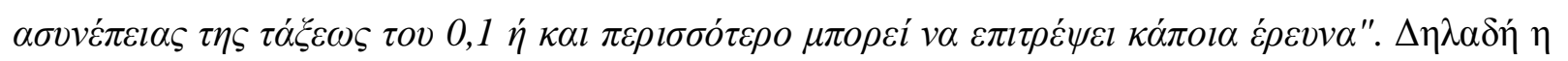




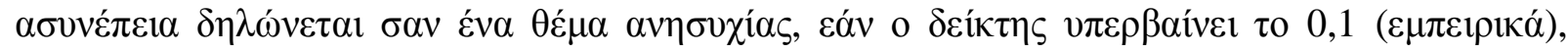

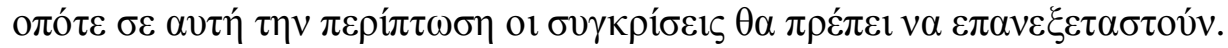

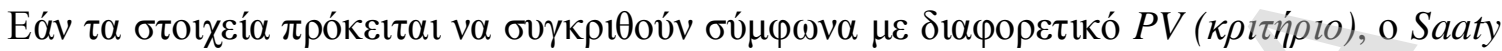

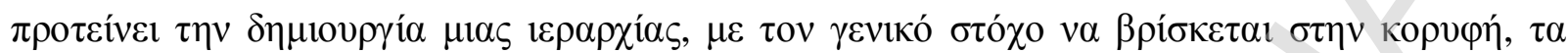

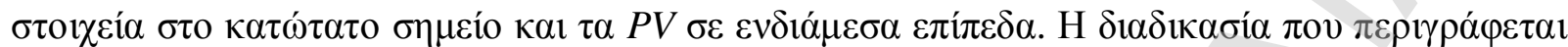

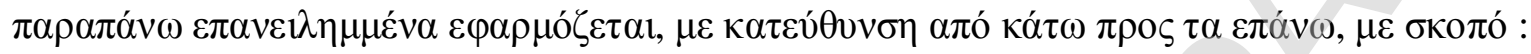

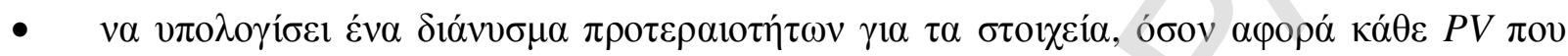

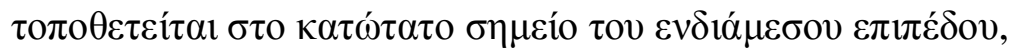

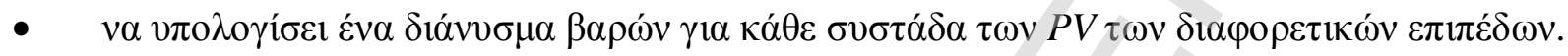

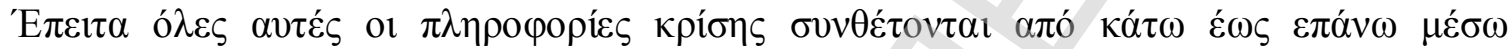

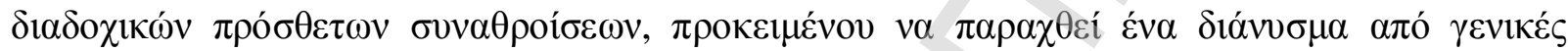

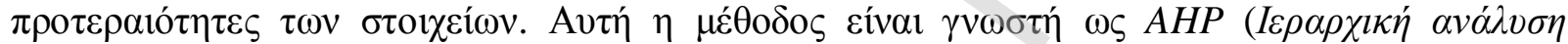
$\alpha \pi o \varphi \alpha ́ \sigma \varepsilon \omega v)$.

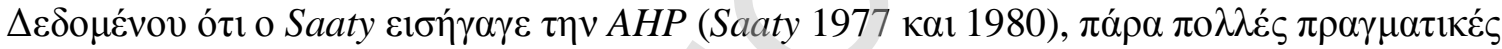

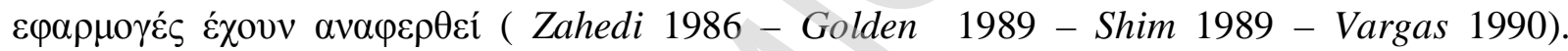

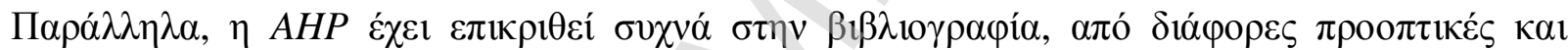

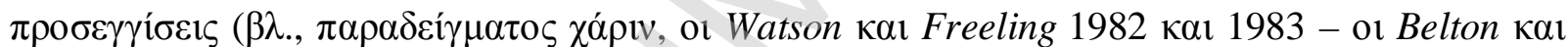
Gear 1985 - o French 1988 - Holder 1990 - o Dyer 1990 - oı Barlizai kaı Golany 1994 - Salo

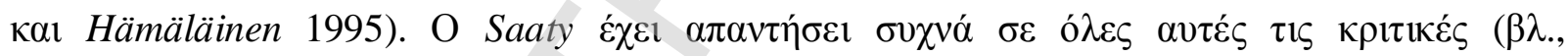

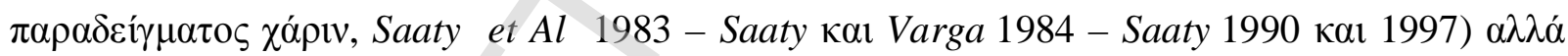

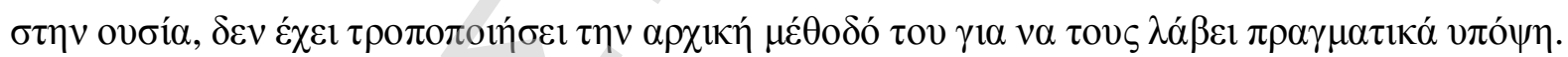

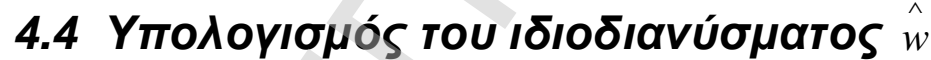

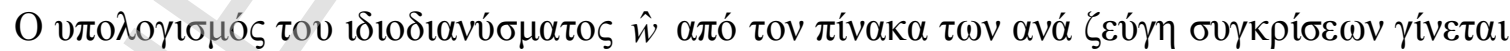
$\mu \varepsilon \tau \eta v \varepsilon \xi \eta \dot{\eta} \varsigma \varepsilon \pi \alpha v \alpha \lambda \eta \pi \tau 1 \kappa \eta ́ ~ \delta 1 \alpha \delta 1 \kappa \alpha \sigma i ́ \alpha$ :

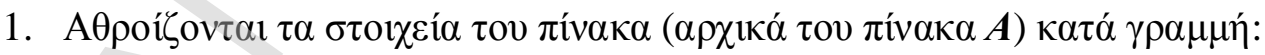

$s_{i}=\sum_{j} a_{i j} \gamma 1 \alpha \kappa \alpha \dot{\theta} \varepsilon \boldsymbol{i}$.

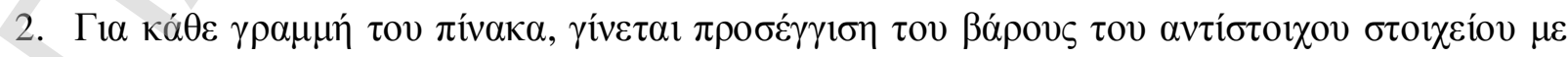

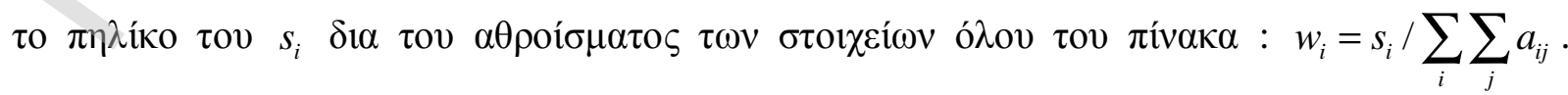




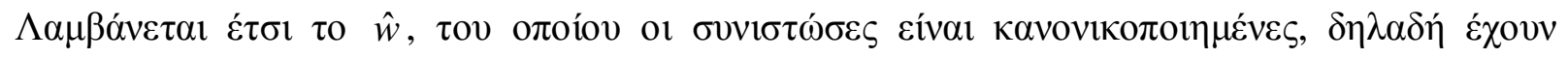

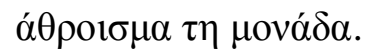

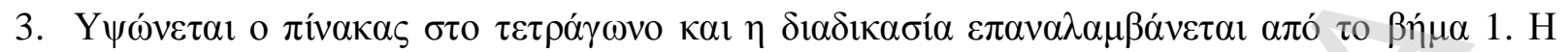

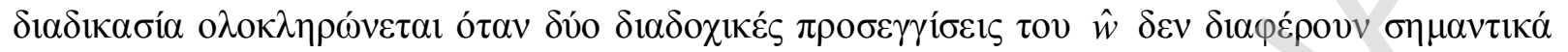

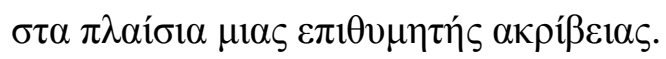

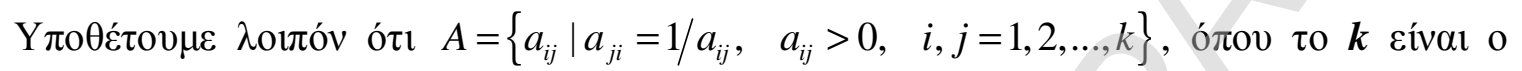

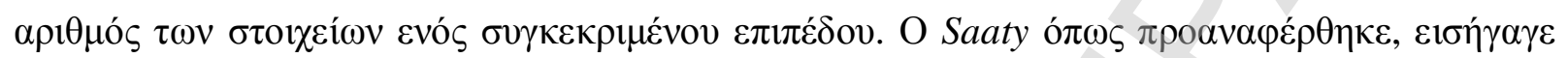

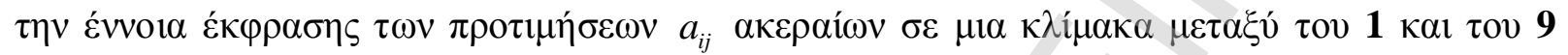

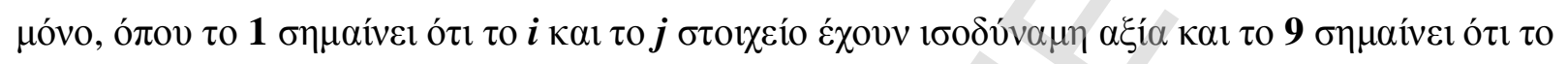

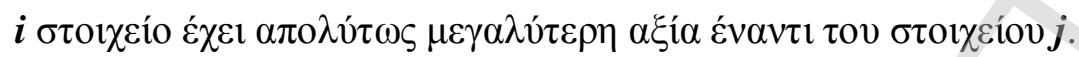

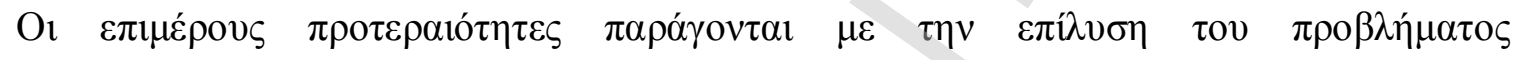

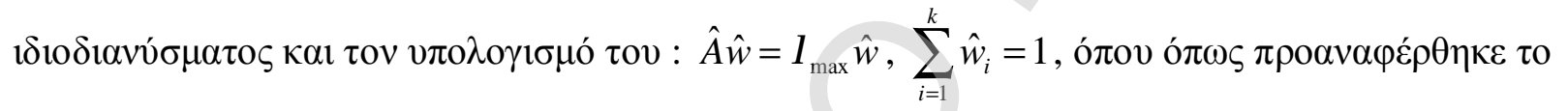

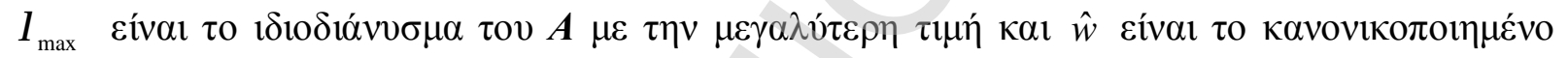

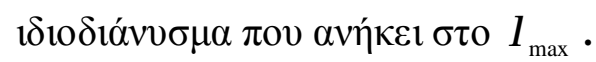

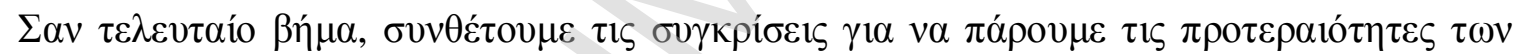

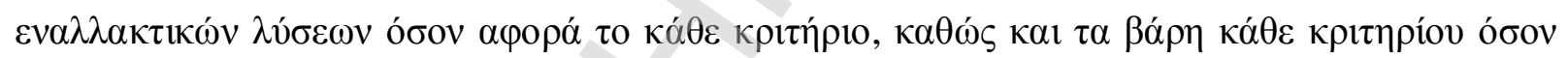

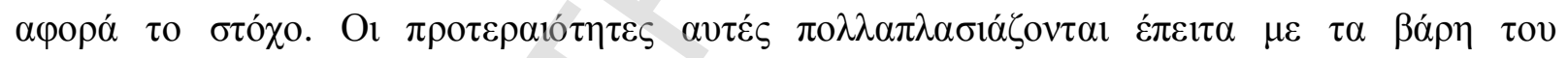

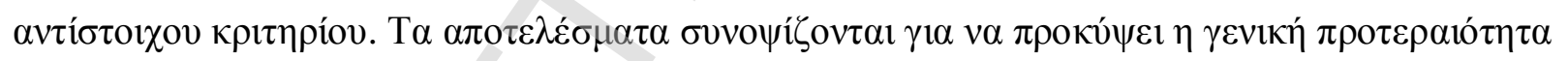

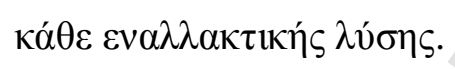

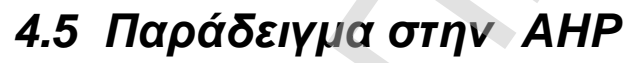

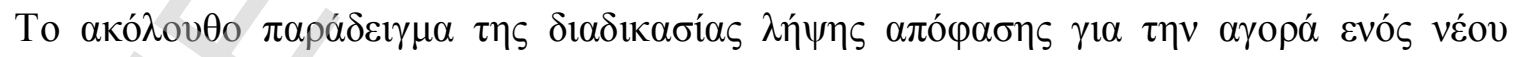

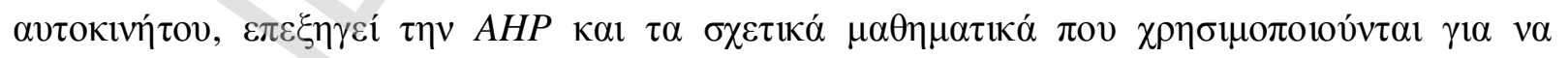

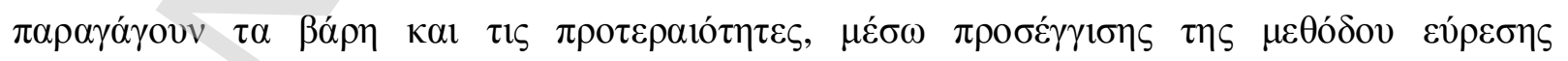

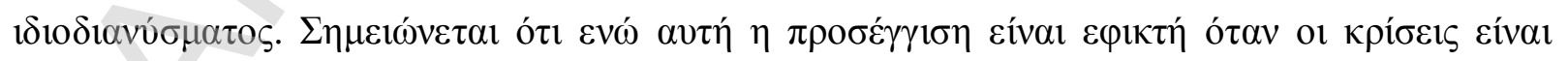

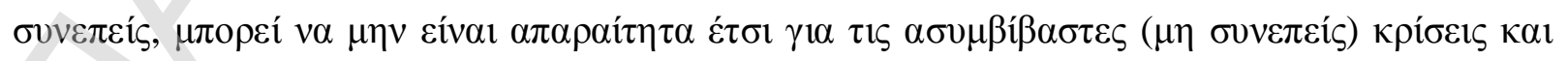

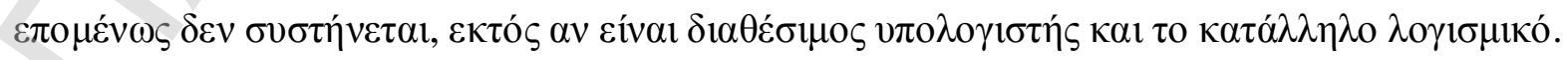

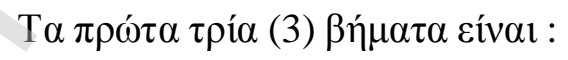

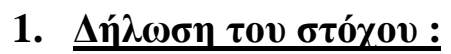




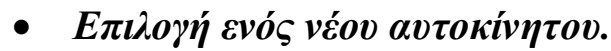

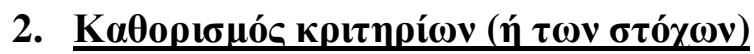

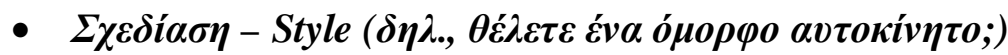

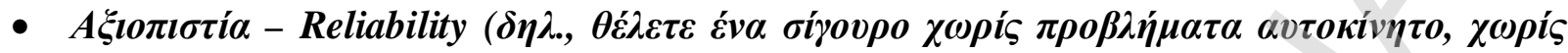

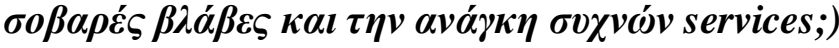

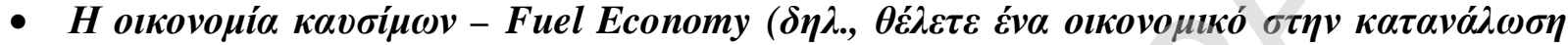

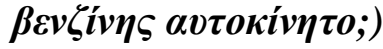

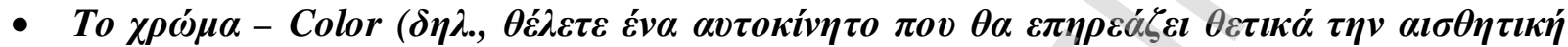

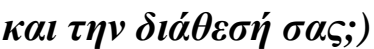

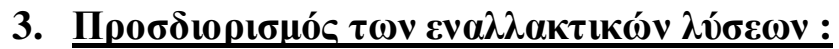

- Opel

- Fiat

- Ford

- Citroen

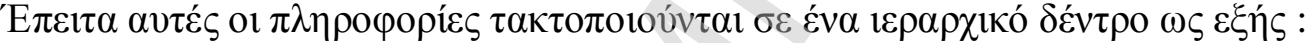




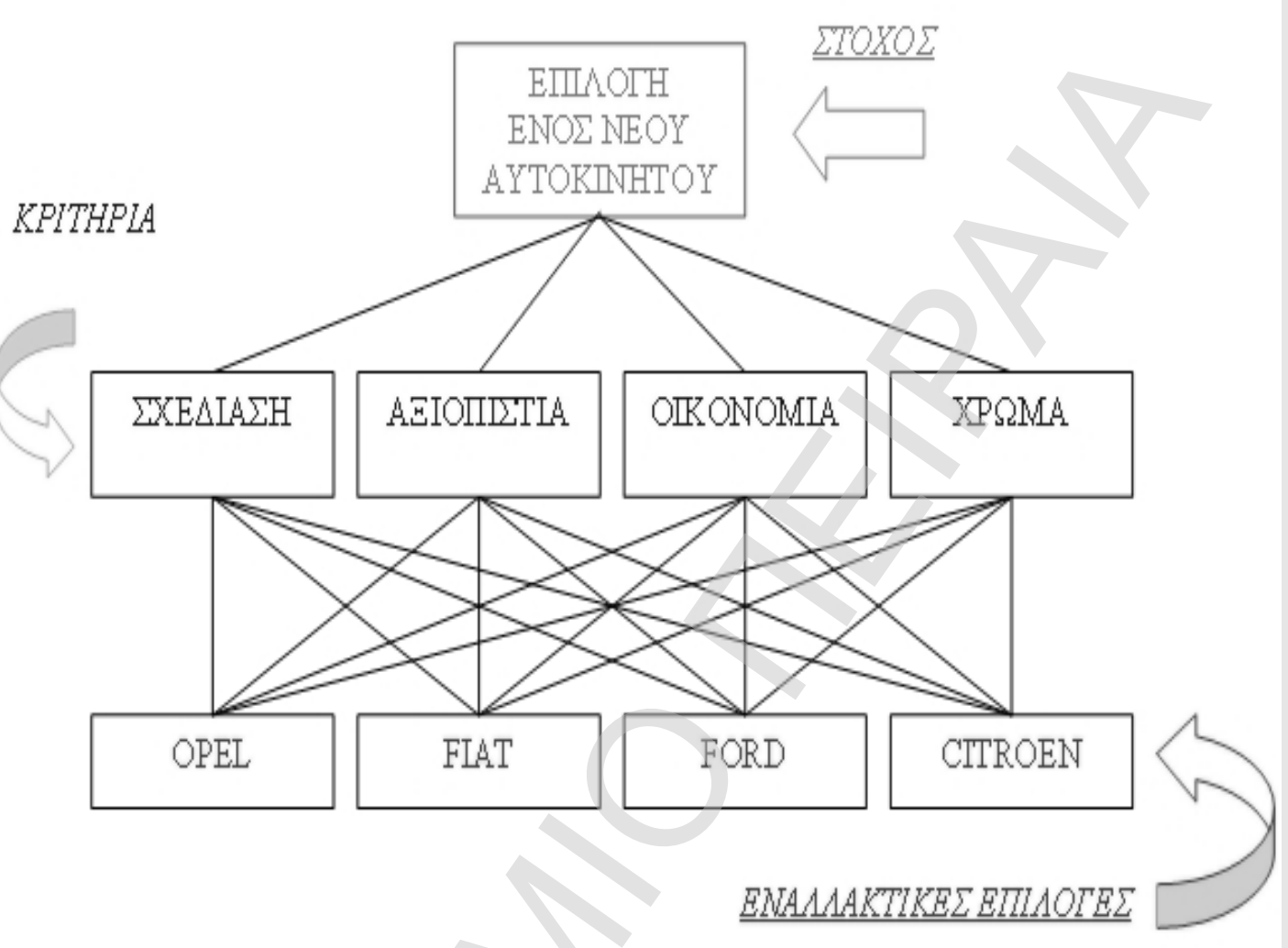

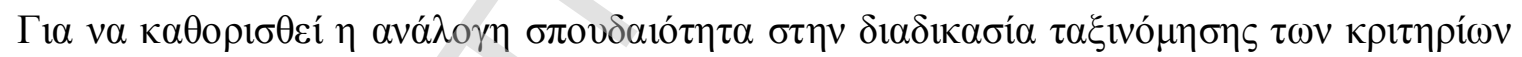

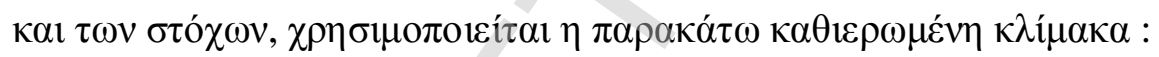

$$
\begin{aligned}
& 1 \Rightarrow 1 \sigma o \delta \dot{v} \alpha \mu
\end{aligned}
$$

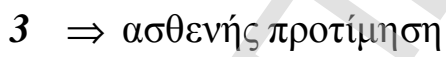

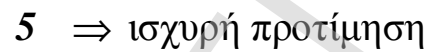

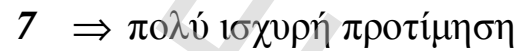

$$
\begin{aligned}
& \left.9 \Rightarrow \alpha \pi \delta ́ \lambda v \tau \eta \pi \rho \tau^{\prime} \mu \eta \eta\right\rceil
\end{aligned}
$$

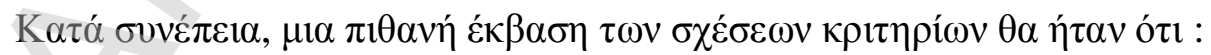

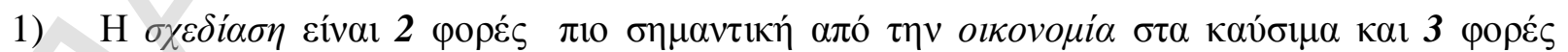

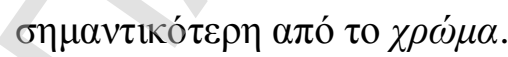

2) Н Н

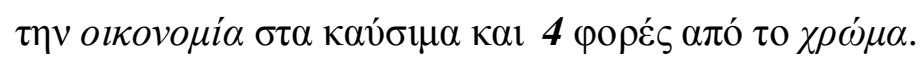




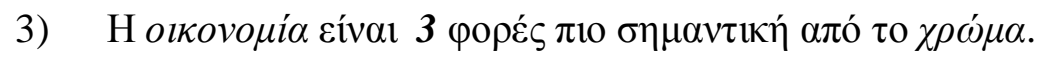

'О

\begin{tabular}{|c|c|c|c|c|}
\hline & $\Sigma X E \Delta I A \Sigma H$ & 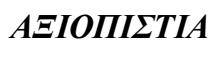 & OIKONOMIA & XPQMA \\
\hline$\Sigma X E \Delta I A \Sigma H$ & $1 / 1$ & $1 / 1,5$ & $2 / 1$ & $3 / 1$ \\
\hline 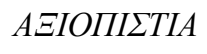 & $1,5 / 1$ & $1 / 1$ & & $4 / 1$ \\
\hline OIKONOMIA & $1 / 2$ & $1 / 3$ & $1 / 1$ & $3 / 1$ \\
\hline$X P \Omega M A$ & $1 / 3$ & $1 / 4$ & $1 / 3$ & $1 / 1$ \\
\hline
\end{tabular}

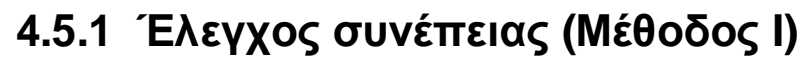

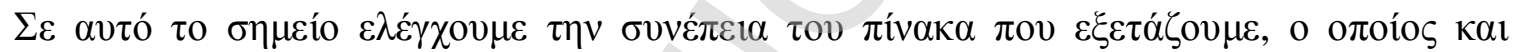

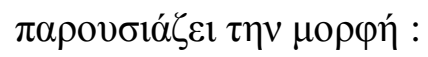

$$
A=\left[\begin{array}{cccc}
1 & \frac{2}{3} & 2 & 3 \\
\frac{3}{2} & 1 & 3 & 4 \\
\frac{1}{2} & \frac{1}{3} & 1 & 3 \\
\frac{1}{3} & \frac{1}{4} & \frac{1}{3} & 1
\end{array}\right]
$$

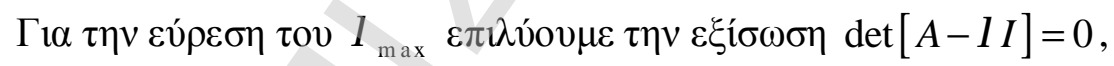

$$
\text { о́лоv: } \operatorname{det}\left[\begin{array}{cccc}
1-\lambda & \frac{2}{3} & 2 & 3 \\
\frac{3}{2} & 1-\lambda & 3 & 4 \\
\frac{1}{2} & \frac{1}{3} & 1-\lambda & 3 \\
\frac{1}{3} & \frac{1}{4} & \frac{1}{3} & 1-\lambda
\end{array}\right]=0
$$

$\eta \dot{n}$ 


$$
\begin{aligned}
& (1-\lambda)\left[\begin{array}{ccc}
1-\lambda & 3 & 4 \\
\frac{1}{3} & 1-\lambda & 3 \\
\frac{1}{4} & \frac{1}{3} & 1-\lambda
\end{array}\right]-\left(\frac{2}{3}\right)\left[\begin{array}{ccc}
\frac{3}{2} & 3 & 4 \\
\frac{1}{2} & 1-\lambda & 3 \\
\frac{1}{3} & \frac{1}{3} & 1-\lambda
\end{array}\right]+2\left[\begin{array}{ccc}
\frac{3}{2} & 1-\lambda & 4 \\
\frac{1}{2} & \frac{1}{3} & 3 \\
\frac{1}{3} & \frac{1}{4} & 1-\lambda
\end{array}\right]-3\left[\begin{array}{ccc}
\frac{3}{2} & 1-\lambda & 3 \\
\frac{1}{2} & \frac{1}{3} & 1-\lambda \\
\frac{1}{3} & \frac{1}{4} & \frac{1}{3}
\end{array}\right]= \\
& =(1-\lambda)\left[(1-\lambda)\left[\begin{array}{cc}
1-\lambda & 3 \\
\frac{1}{3} & 1-\lambda
\end{array}\right]-3\left[\begin{array}{cc}
\frac{1}{3} & 3 \\
\frac{1}{4} & 1-\lambda
\end{array}\right]+4\left[\begin{array}{cc}
\frac{1}{3} & 1-\lambda \\
\frac{1}{4} & \frac{1}{3}
\end{array}\right]\right]- \\
& -\left(\frac{2}{3}\right)\left[\left(\frac{3}{2}\right)\left[\begin{array}{cc}
1-\lambda & 3 \\
\frac{1}{3} & 1-\lambda
\end{array}\right]-3\left[\begin{array}{cc}
\frac{1}{2} & 3 \\
\frac{1}{3} & 1-\lambda
\end{array}\right]+4\left[\begin{array}{cc}
\frac{1}{2} & 1-\lambda \\
\frac{1}{3} & \frac{1}{3}
\end{array}\right]\right]+ \\
& +2\left[\left(\frac{3}{2}\right)\left[\begin{array}{cc}
\frac{1}{3} & 3 \\
\frac{1}{4} & 1-\lambda
\end{array}\right]-(1-\lambda)\left[\begin{array}{cc}
\frac{1}{2} & 3 \\
\frac{1}{3} & 1-\lambda
\end{array}\right]+4\left[\begin{array}{cc}
\frac{1}{2} & \frac{1}{3} \\
\frac{1}{3} & \frac{1}{4}
\end{array}\right]\right]- \\
& -3\left[\left(\frac{3}{2}\right)\left[\begin{array}{cc}
\frac{1}{3} & 1-\lambda \\
\frac{1}{4} & \frac{1}{3}
\end{array}\right]-(1-\lambda)\left[\begin{array}{cc}
\frac{1}{2} & 1-\lambda \\
\frac{1}{3} & \frac{1}{3}
\end{array}\right]+3\left[\begin{array}{cc}
\frac{1}{2} & \frac{1}{3} \\
\frac{1}{3} & \frac{1}{4}
\end{array}\right]\right]=0
\end{aligned}
$$

$\delta \eta \lambda \alpha \delta \eta \dot{0}$

$$
(1-\lambda)^{4}-6(1-\lambda)^{2}+\frac{221}{24}(1-\lambda)-\frac{101}{24}=0
$$

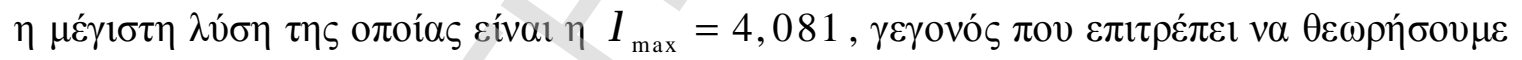

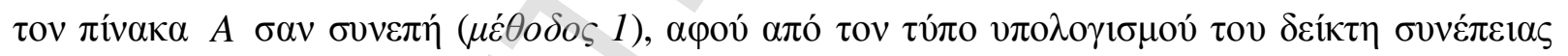
$C I=\frac{\lambda_{\max }-n}{n-1} \pi \rho$ оќ́ $\pi \tau \varepsilon \imath$ ó $\tau 1 ~ C I=0,027<0,10$.

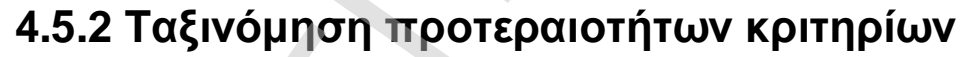

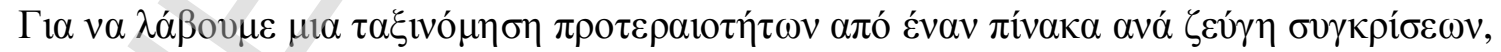

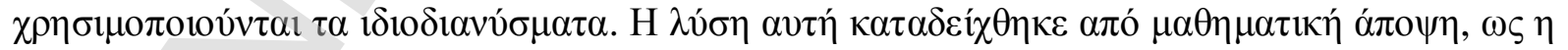

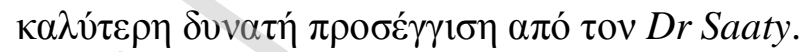

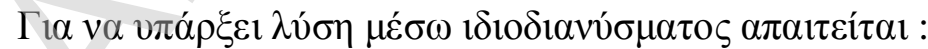

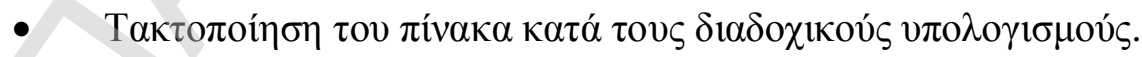

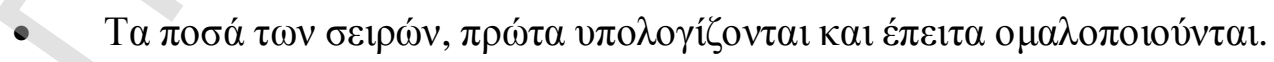

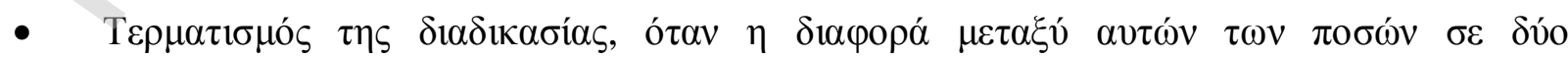

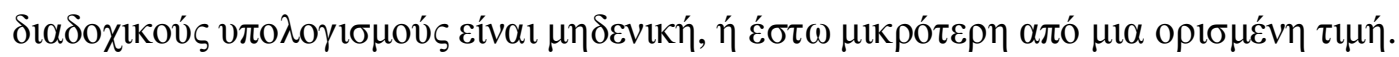




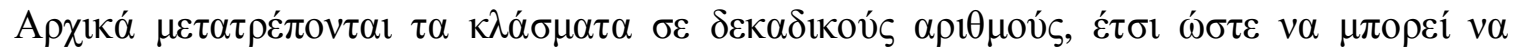

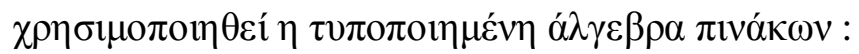

\begin{tabular}{|c|c|c|c|c|}
\hline & $\Sigma X E \Delta I A \Sigma H$ & $\begin{array}{l}A \Xi I O H I \\
\Sigma T I A \\
\end{array}$ & $\begin{array}{l}\text { OIKONO } \\
\text { MIA }\end{array}$ & $\begin{array}{l}\text { XPQM } \\
A \\
\end{array}$ \\
\hline$\Sigma X E \Delta I A \Sigma H$ & 1,0000 & 0,6666 & 2,0000 & 3,0000 \\
\hline 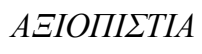 & 1,5000 & 1,0000 & 3,0000 & 4,0000 \\
\hline OIKONOMIA & 0,5000 & $\mathbf{0 , 3 3 3 3}$ & 1,0000 & 3,0000 \\
\hline$X P \Omega M A$ & $\mathbf{0 , 3 3 3 3}$ & 0,2500 & $\mathbf{0 , 3 3 3 3}$ & 1,0000 \\
\hline
\end{tabular}

\section{Bíua 1.}

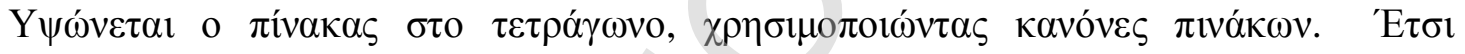

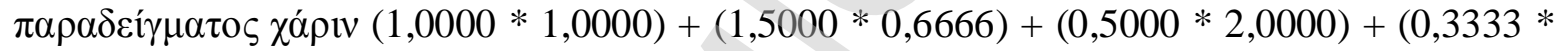

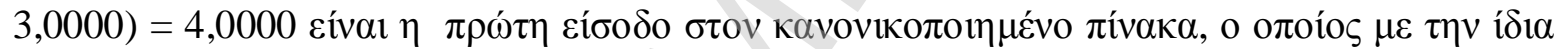

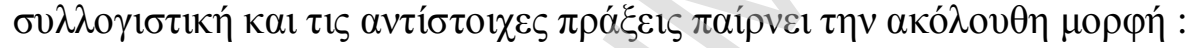

\begin{tabular}{|c|c|c|c|}
\hline 4,000 & 2,750 & 7,0000 & 14,6666 \\
\hline $\begin{array}{l}\mathbf{n} \\
5,833 \\
3\end{array}$ & $\begin{array}{l}0,000 \\
0\end{array}$ & 10,3333 & 21,5000 \\
\hline $\begin{array}{r}2,500 \\
\Omega\end{array}$ & $\begin{array}{l}1,750 \\
\Omega\end{array}$ & 4,0000 & 8,8333 \\
\hline $\begin{array}{r}1,208 \\
3\end{array}$ & $\begin{array}{l}0,833 \\
3\end{array}$ & 2,0833 & 4,0000 \\
\hline
\end{tabular}

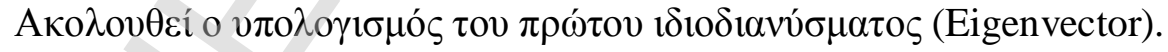

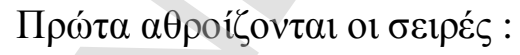

\begin{tabular}{|c|c|c|c|c|}
\hline $\mathbf{4 , 0 0 0 0} \mathbf{2 , 7 5 0 0}$ & $\mathbf{7 , 0 0 0 0}$ & $\mathbf{1 4 , 6 6 6 6}$ & $\mathbf{2 8 , 4 1 6 6}$ \\
\hline $\mathbf{5 , 8 3 3 3}$ & 4,0000 & 10,3333 & 21,5000 & $=41,6666$ \\
\hline $\mathbf{2 , 5 0 0 0}$ & 1,7500 & 4,0000 & 8,8333 & $=17,0833$ \\
\hline
\end{tabular}




\begin{tabular}{|l|l|l|l|l|}
\hline $\mathbf{1 , 2 0 8 3}$ & 0,8333 & 2,0833 & 4,0000 & $=8,1249$ \\
\hline
\end{tabular}

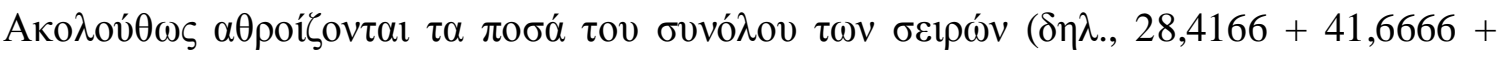

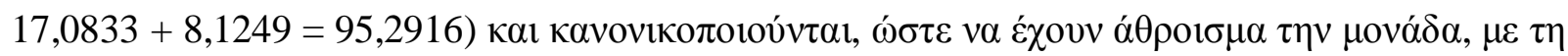

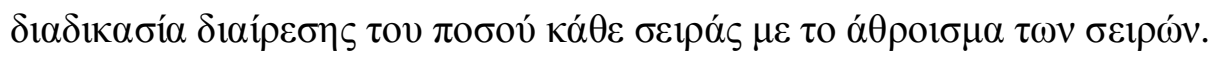

$$
\begin{aligned}
& 28,4166 / 95,2914= 0,2982 \\
& 41,6666 / 95,2914= 0,4372 \\
& 17,0833 / 95,2914= 0,1793 \\
& 8,1249 / 95,2914= \underline{0,0853} \\
& 1,0000
\end{aligned}
$$

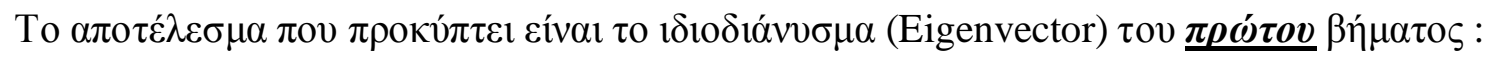

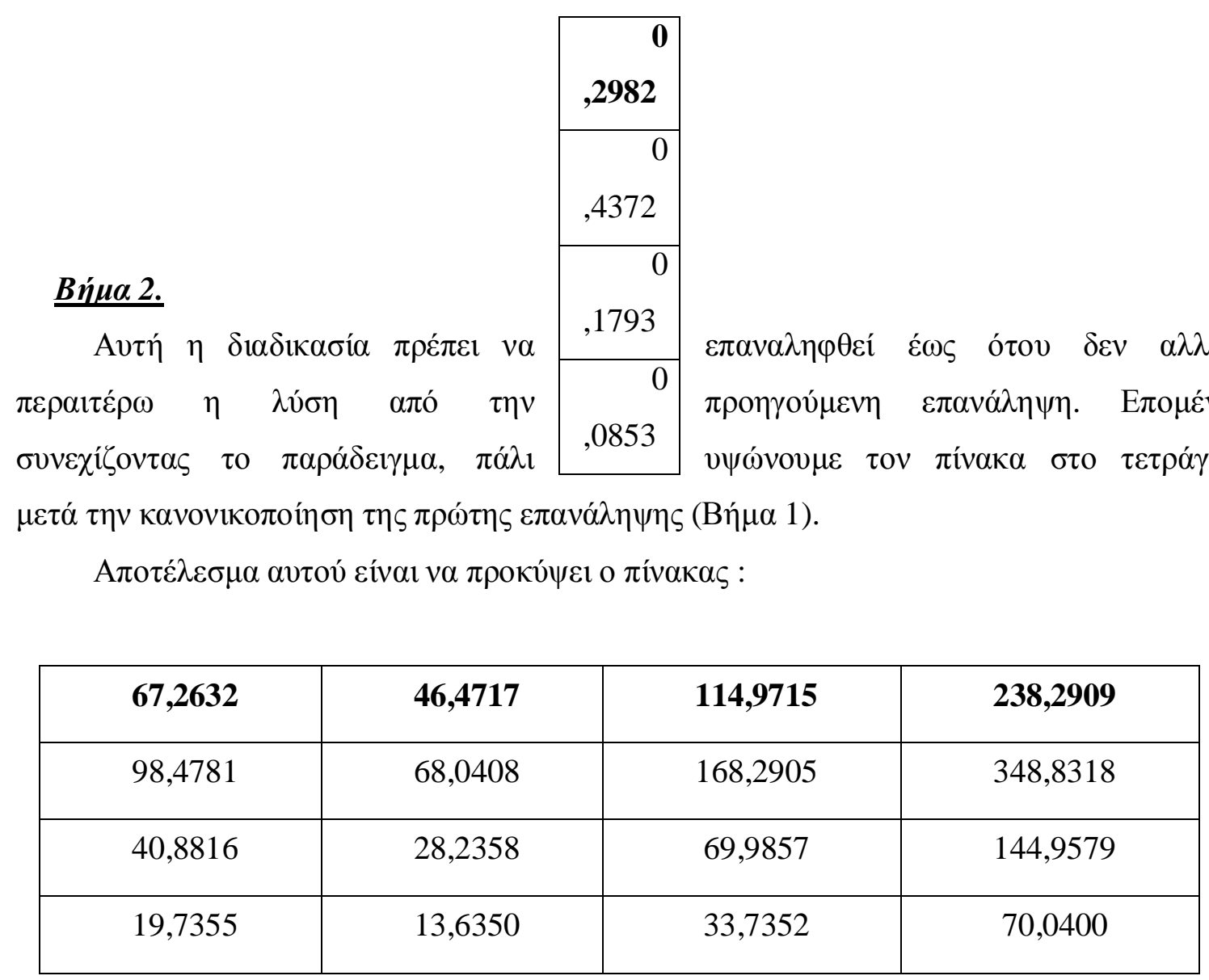

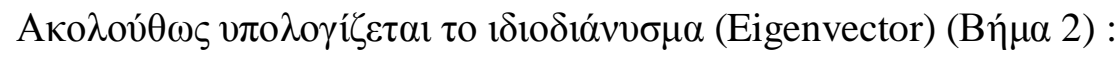




\begin{tabular}{|c|l|l|l|l|}
\hline $\mathbf{6 7 , 2 6 3 2}$ & $\mathbf{4 6 , 4 7 1 7}$ & $\mathbf{1 1 4 , 9 7 1 5}$ & $\mathbf{2 3 8 , 2 9 0 9}$ & $\mathbf{4 6 6 , 9 9 7 3}$ \\
\hline 98,4781 & 68,0408 & 168,2905 & 348,8318 & $=683,6412$ \\
\hline 40,8816 & 28,2358 & 69,9857 & 144,9579 & $=284,0609$ \\
\hline 19,7355 & 13,6350 & 33,7352 & 70,0400 & $=137,1458$ \\
\hline
\end{tabular}

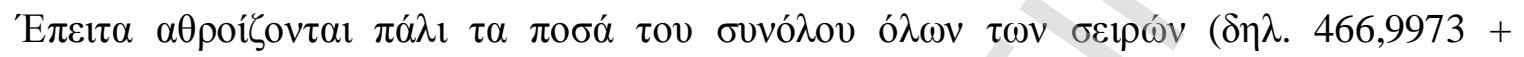

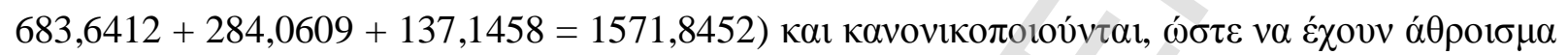

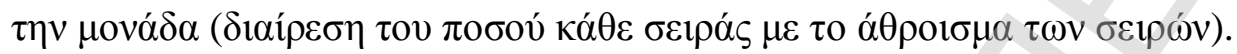

$$
\begin{aligned}
& 466,9973 / 1571,8452=0,2971 \\
& 683,6412 / 1571,8452=0,4349 \\
& 284,0609 / 1571,8452=0,1807 \\
& 137,1458 / 1571,8452= \underline{0,0873} \\
& 1,0000
\end{aligned}
$$

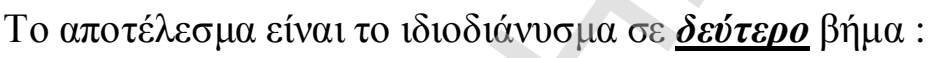

\section{Bíma 3.}

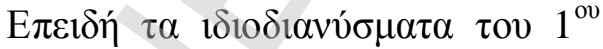
$\delta 1 \alpha \delta 1 \kappa \alpha \sigma i ́ \alpha \quad \pi \rho \varepsilon ́ \pi \varepsilon \imath \quad v \alpha \quad \varepsilon \pi \alpha v \alpha \lambda \eta \varphi \theta \varepsilon i ́$.

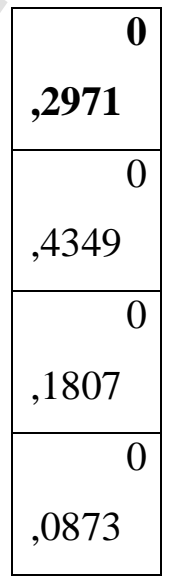

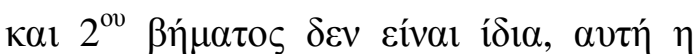

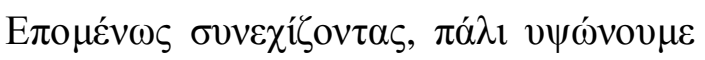

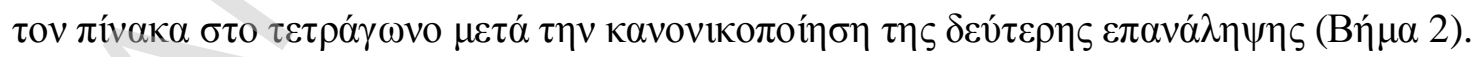

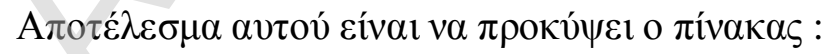

\begin{tabular}{|l|l|l|l|}
\hline 18503,79 & 12783,21 & 31639,25 & 65594,94 \\
71 & 27 & 42 & 11 \\
\hline
\end{tabular}




\begin{tabular}{|l|l|l|l|}
\hline $\mathbf{2 7 0 8 9 , 8 4}$ & 18714,12 & 46318,63 & 96028,44 \\
$\mathbf{2 1}$ & 84 & 97 & 74 \\
\hline $\mathbf{1 1 2 5 2 , 3 7}$ & 7773,622 & 19240,21 & 39889,07 \\
$\mathbf{7 8}$ & 1 & 31 & 54 \\
\hline $\mathbf{5 4 3 1 , 6 5 1}$ & 3752,416 & 9287,465 & 19254,91 \\
$\mathbf{3}$ & 6 & 5 & 45 \\
\hline
\end{tabular}

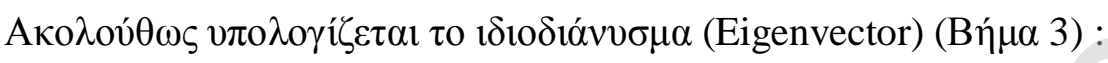

\begin{tabular}{|l|l|c|l|l|}
\hline $\mathbf{1 8 5 0 3}, 7971$ & $\mathbf{1 2 7 8 3 , 2 1 2 7}$ & $\mathbf{3 1 6 3 9 , 2 5 4 2}$ & $\mathbf{6 5 5 9 4 , 9 4 1 1}$ & $=\mathbf{1 2 8 5 2 1 , 2 0 5 0}$ \\
\hline 27089,8421 & 18714,1284 & $\begin{array}{c}463 \\
18,6397\end{array}$ & 96028,4474 & $=188150,0577$ \\
\hline 11252,3778 & 7773,6221 & $\begin{array}{c}192 \\
40,2131\end{array}$ & 39889,0754 & $=78155,2883$ \\
\hline 5431,6513 & 3752,4166 & 928 & 19254,9145 & $=37726,4479$ \\
\hline
\end{tabular}

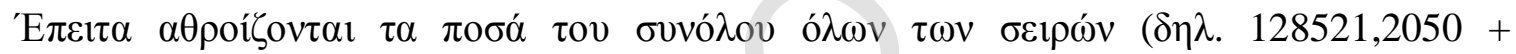

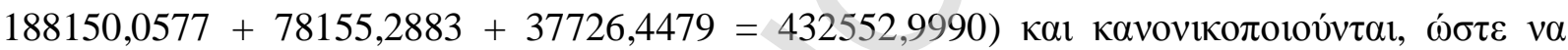

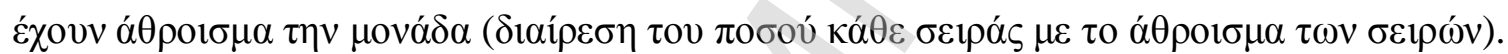




$$
\begin{array}{r}
128521,2050 / 432552,9990=0,2971 \\
188150,0577 / 432552,9990=0,4350 \\
78155,2883 / 432552,9990=0,1807 \\
37726,4479 / 432552,9990=\underline{0,0872} \\
1,0000
\end{array}
$$

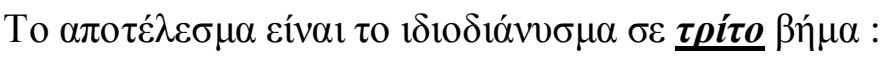

\section{Bíma 4.}

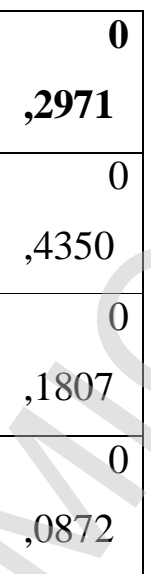

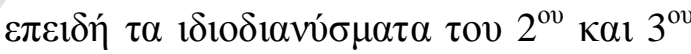

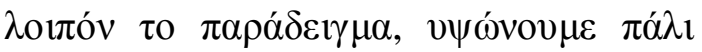

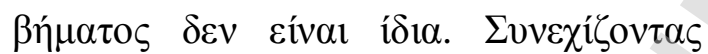

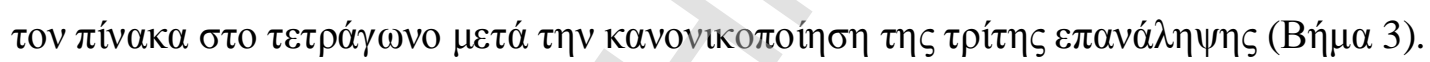

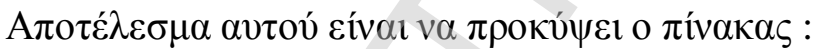

\begin{tabular}{|r|r|r|c|}
\hline $\mathbf{1 4 0 0 9 7 8 6 2 4 , 6 1 3 9}$ & $\mathbf{9 6 7 8 5 5 8 0 8 , 8 0 3 8}$ & $\mathbf{2 3 9 5 5 0 4 1 0 4 , 8 0 4 0}$ & $\mathbf{4 9 6 6 3 9 3 1 2 2 , 9 5 8 3}$ \\
\hline 2050978383,1231 & 1416903375,2273 & 3506925123,1364 & 7270606958,8218 \\
\hline 851951829,5413 & 588564673,6900 & 1456734648,8416 & 3020122957,6161 \\
\hline 411246846,6856 & 284106868,1733 & 703182398,3713 & 1457847732,5308 \\
\hline
\end{tabular}

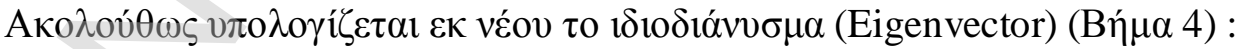

\begin{tabular}{|c|c|c|c|c|}
\hline $\mathbf{1 4 0 0 9 7 8 6 2 4 , 6 1 3 9}$ & $\mathbf{9 6 7 8 5 5 8 0 8 , 8 0 3 8}$ & $\mathbf{2 3 9 5 5 0 4 1 0 4 , 8 0 4 0}$ & $\mathbf{4 9 6 6 3 9 3 1 2 2 , 9 5 8 3}$ & $\mathbf{9 7 3 0 7 3 1 6 6 1 , 1 8 0 0}$ \\
\hline 2050978383,1231 & 1416903375,2273 & 3506925123,1364 & 7270606958,8218 & $=14245413840,3085$ \\
\hline 851951829,5413 & 588564673,6900 & 1456734648,8416 & 3020122957,6161 & $=5917374109,6890$ \\
\hline 411246846,6856 & 284106868,1733 & 703182398,3713 & 1457847732,5308 & $=2856383845,7610$ \\
\hline
\end{tabular}




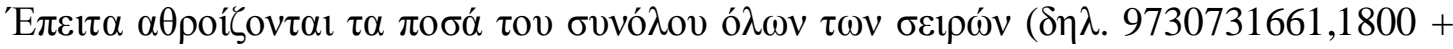
$14245413840,3085+5917374109,6890+2856383845,7610=32749903456,9385) \kappa \alpha 1$

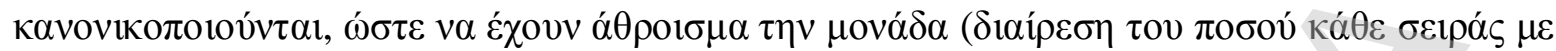

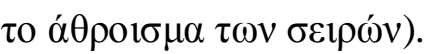

$$
\begin{array}{r}
9730731661,1800 / 32749903456,9385=0,2971 \\
14245413840,3085 / 32749903456,9385=0,4350 \\
5917374109,6890 / 32749903456,9385=0,1807 \\
2856383845,7610 / 32749903456,9385=\underline{0,0872} \\
1,0000
\end{array}
$$

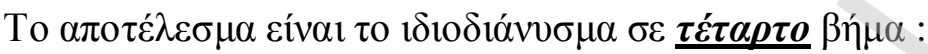

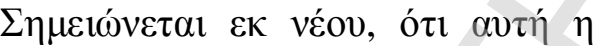

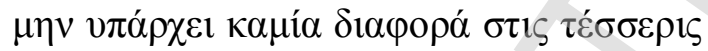

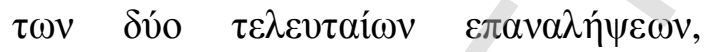

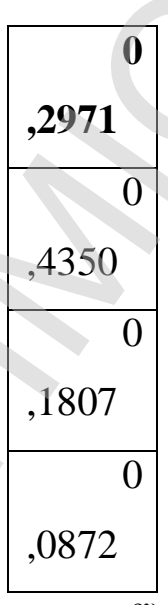

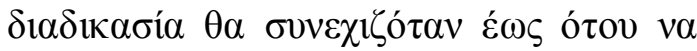

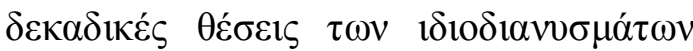

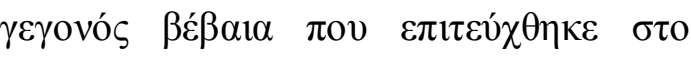

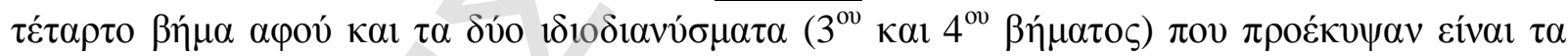

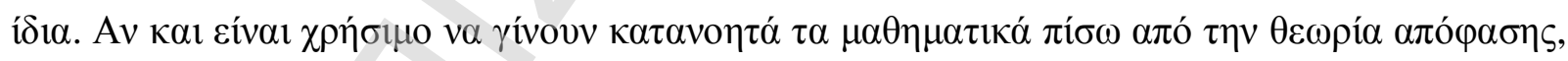

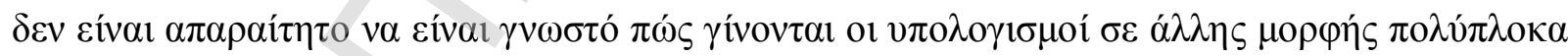

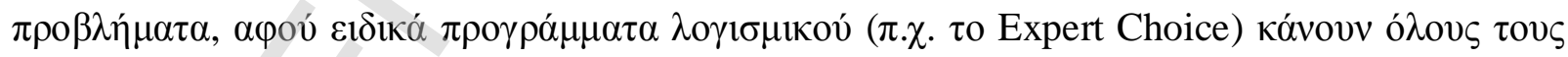

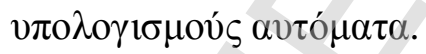

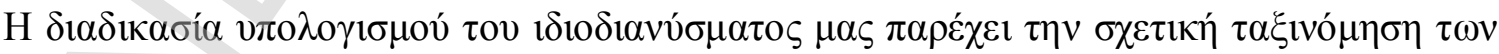

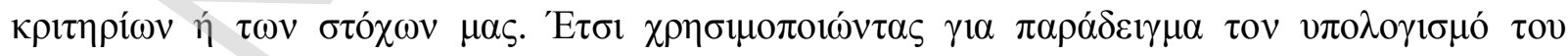

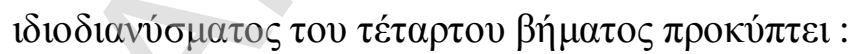




\subsection{3}

\begin{tabular}{|c|c|c|c|}
\hline $\begin{array}{l}\mathbf{\Sigma X E \Delta I} \\
\mathbf{A} \Sigma \mathbf{H}\end{array}$ & $\begin{array}{r}0 \\
, 2971\end{array}$ & 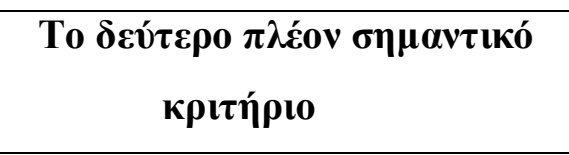 & 'EגعYXOS \\
\hline 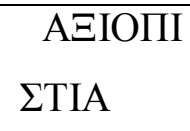 & $\begin{array}{r}0 \\
, 4350\end{array}$ & 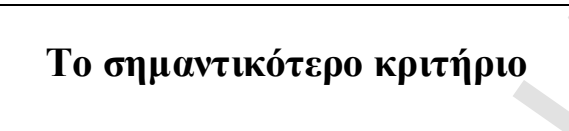 & \\
\hline $\begin{array}{l}\text { OIKON } \\
\text { OMIA }\end{array}$ & $\begin{array}{r}0 \\
, 1807\end{array}$ & 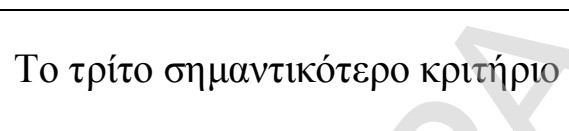 & \\
\hline $\begin{array}{l}\mathrm{XP \Omega M} \\
\mathrm{A}\end{array}$ & $\begin{array}{r}0 \\
0872\end{array}$ & 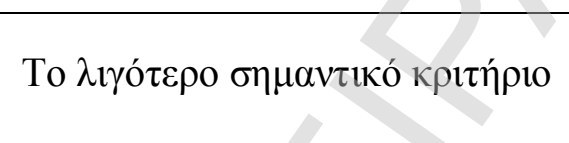 & \\
\hline
\end{tabular}

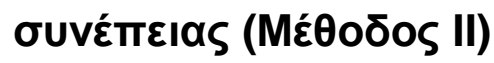

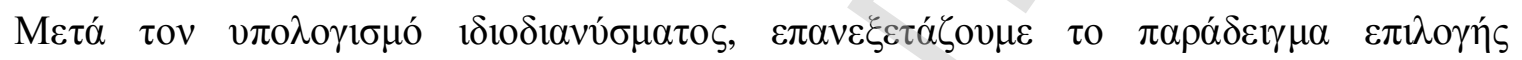

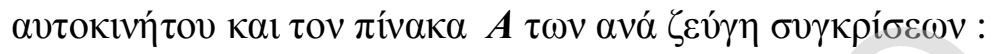

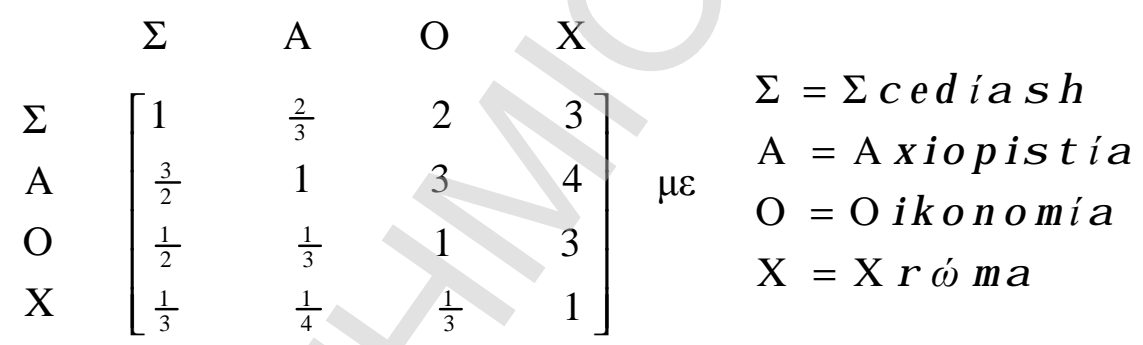

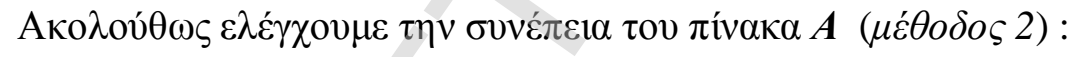

1. $A \cdot w^{T}=\left[\begin{array}{cccc}1 & \frac{2}{3} & 2 & 3 \\ \frac{3}{2} & 1 & 3 & 4 \\ \frac{1}{2} & \frac{1}{3} & 1 & 3 \\ \frac{1}{3} & \frac{1}{4} & \frac{1}{3} & 1\end{array}\right] * \frac{\left[\begin{array}{l}0,2971 \\ 0,4350 \\ 0,1807 \\ 0,0872\end{array}\right]}{1} \frac{\left[\begin{array}{l}1,2101 \\ 1,7716 \\ 0,7359 \\ 0,3552\end{array}\right]}{4,0727}$

2. $\quad \frac{1}{n} \sum_{i=1}^{n} \frac{\text { ith entry in } A \cdot w^{T}}{\text { ith entry in } w^{T}}=\frac{1}{4} \cdot\left(\frac{1,2101}{0,2971}+\frac{1,7716}{0,4350}+\frac{0,7359}{0,1807}+\frac{0,3552}{0,0872}\right)=4,0728$

3. $C I=\frac{\text { (result from step } 2)-n}{n-1}=\frac{4,0728-4}{3}=0,0243$

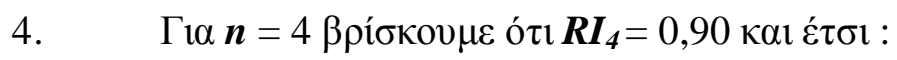




$$
C I / R I_{4}=\frac{0,02443}{0,90}=0,027<0,10
$$

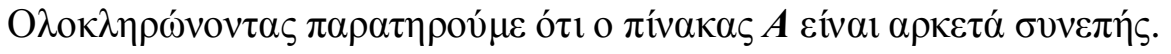

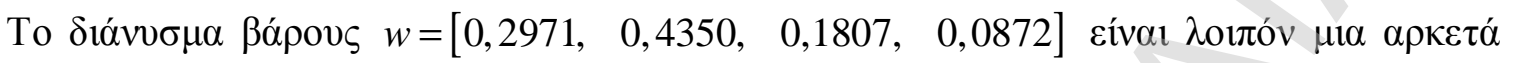
$\kappa \alpha \lambda \eta ́ ~ \pi \rho о \sigma \varepsilon ́ \gamma \gamma \imath \sigma \eta$.

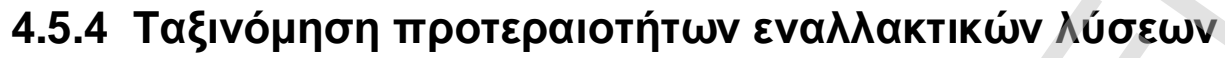

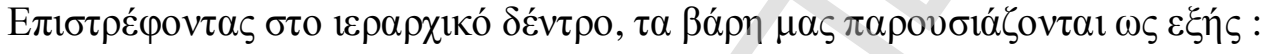

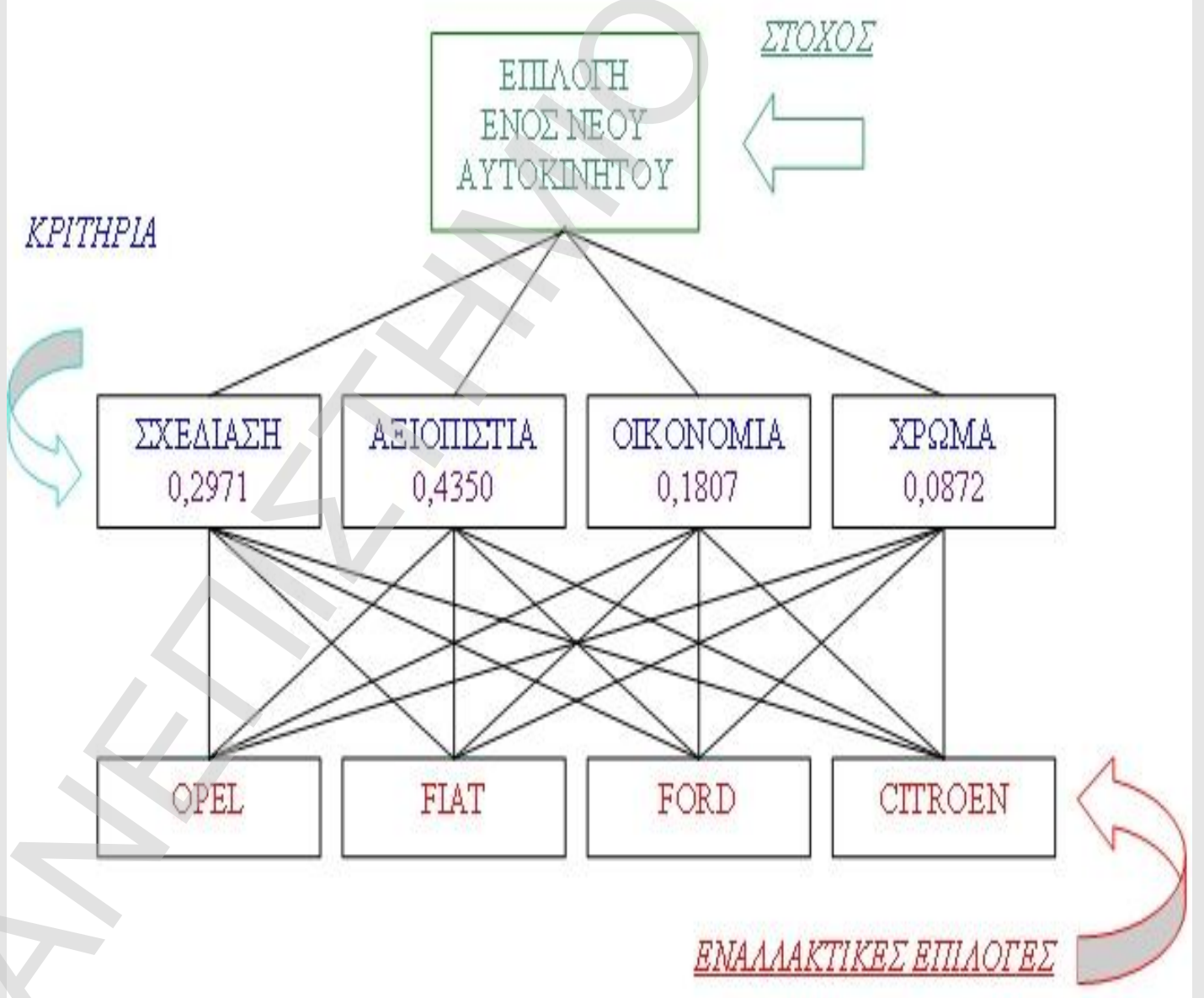

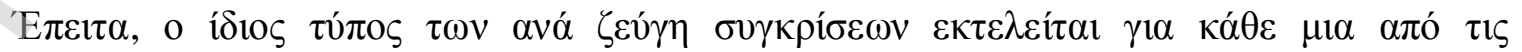

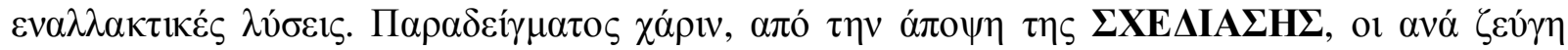




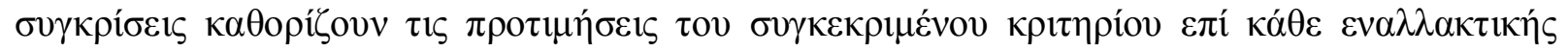

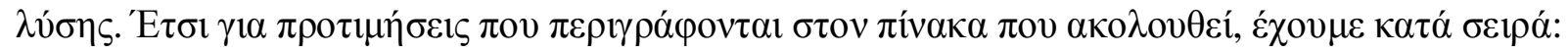

\begin{tabular}{|c|c|c|c|c|}
\hline $\begin{array}{ll}\underline{\Sigma \mathrm{\Sigma XE}} & \\
\underline{\underline{\Sigma}} & \end{array}$ & OPEL & FIAT & FORD & $\begin{array}{l}\text { CITROE } \\
\mathrm{N}\end{array}$ \\
\hline OPEL & $1 / 1$ & $2 / 1$ & $1 / 3$ & $1 / 2$ \\
\hline FIAT & $1 / 2$ & $1 / 1$ & $1 / 4$ & $1 / 3$ \\
\hline FORD & $3 / 1$ & $4 / 1$ & $1 / 1$ & $3 / 1$ \\
\hline CITROEN & $2 / 1$ & $3 / 1$ & $1 / 3$ & $1 / 1$ \\
\hline
\end{tabular}

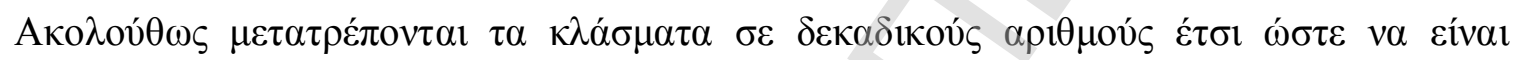

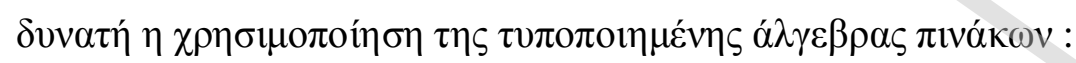

\begin{tabular}{|c|c|c|c|c|}
\hline & OPEL & FIAT & FORD & CITROEN \\
\hline OPEL & 1,0000 & 2,0000 & 0,3333 & 0,5000 \\
\hline FIAT & 0,5000 & 1,0000 & 0,2500 & 0,3333 \\
\hline FORD & 3,0000 & 4,0000 & 1,0000 & 3,0000 \\
\hline CITROEN & 2,0000 & 3,0000 & 0,3333 & 1,0000 \\
\hline
\end{tabular}

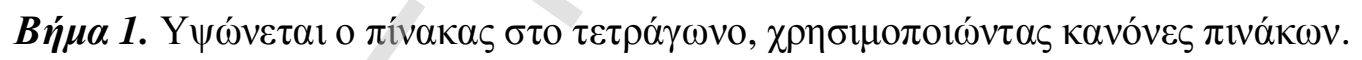

\begin{tabular}{|c|c|c|c|}
\hline $\mathbf{4 , 0 0 0 0}$ & $\mathbf{6 , 8 3 3 3}$ & $\mathbf{1 , 3 3 3 3}$ & $\mathbf{2 , 6 6 6 7}$ \\
\hline 2,4167 & 4,0000 & 0,7778 & 1,6667 \\
\hline 14,0000 & 23,0000 & 4,0000 & 8,8333 \\
\hline 6,5000 & 11,3333 & 2,0833 & 4,0000 \\
\hline
\end{tabular}




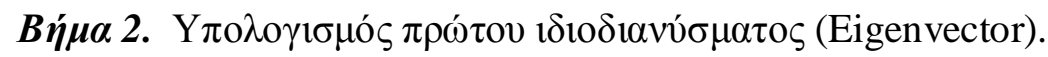

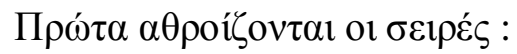

\begin{tabular}{|c|c|c|c|c|}
\hline $\mathbf{4 , 0 0 0 0}$ & $\mathbf{6 , 8 3 3 3}$ & $\mathbf{1 , 3 3 3 3}$ & $\mathbf{2 , 6 6 6 7}$ & $=$ \\
& & & & $\mathbf{1 4 , 8 3 3 3}$ \\
\hline 2,4167 & 4,0000 & 0,7778 & 1,6667 & $=$ \\
& & & & 8,8611 \\
\hline 14,0000 & 23,0000 & 4,0000 & 8,8333 & $=$ \\
& 11,3333 & 2,0833 & 4,0000 & $=$ \\
\hline 6,5000 & & & & 23,9163 \\
\hline
\end{tabular}

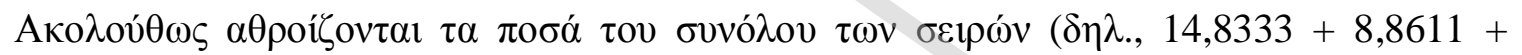

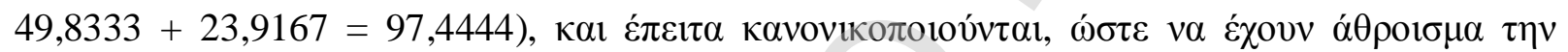

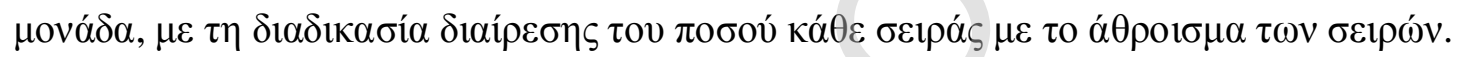

$$
\begin{aligned}
& 14,8333 / 97,4444= 0,1522 \\
& 8,8611 / 97,4444= 0,0909 \\
& 49,8333 / 97,4444= 0,5114 \\
& 23,9167 / 97,4444=\underline{0,2455} \\
& 1,0000
\end{aligned}
$$

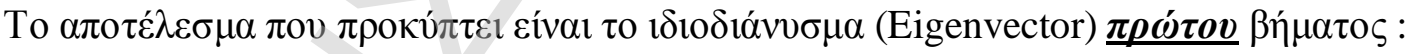

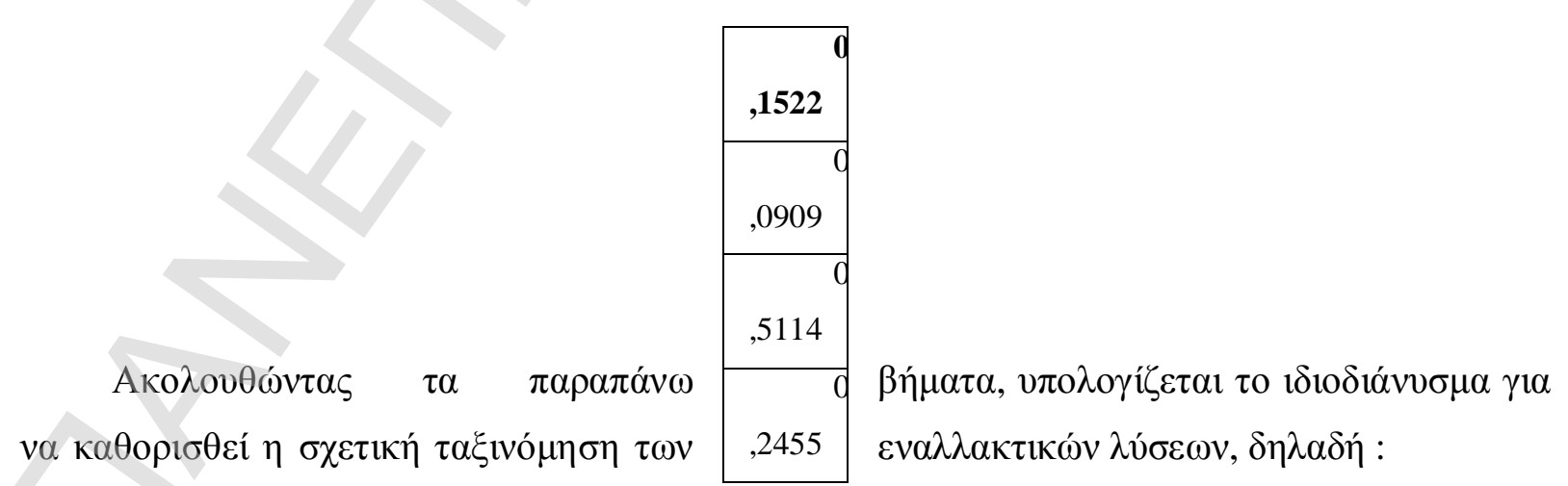




\begin{tabular}{|c|c|}
\hline OPEL & $\mathbf{0 , 1 5 2 2}$ \\
\hline FIAT & $\mathbf{0 , 0 9 0 9}$ \\
\hline FORD & $\mathbf{0 , 5 1 1 4}$ \\
\hline CITROEN & $\mathbf{0 , 2 4 5 5}$ \\
\hline
\end{tabular}

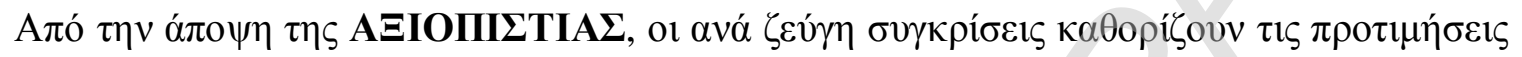

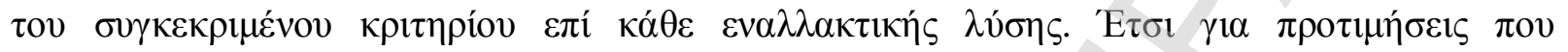

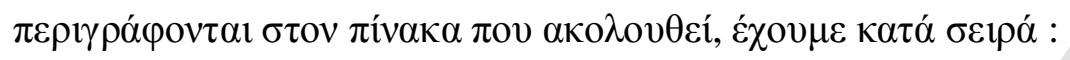

\begin{tabular}{|c|c|c|c|c|}
\hline 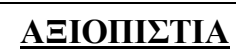 & OPEL & FIAT & FORD & CITROEN \\
\hline OPEL & $1 / 1$ & $3 / 1$ & $1 / 2$ & $2 / 1$ \\
\hline FIAT & $1 / 3$ & $1 / 1$ & $1 / 4$ & $1 / 3$ \\
\hline FORD & $2 / 1$ & $4 / 1$ & $1 / 1$ & $3 / 1$ \\
\hline $\begin{array}{ll} & \text { CITROE } \\
\mathrm{N} & \end{array}$ & $1 / 2$ & & $1 / 3$ & $1 / 1$ \\
\hline
\end{tabular}

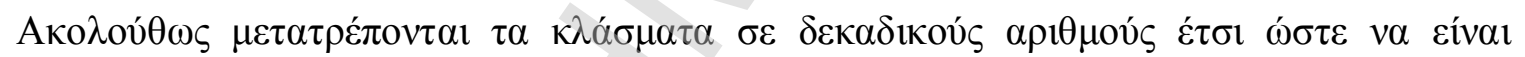

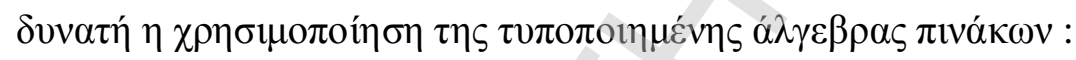

\begin{tabular}{|c|c|c|c|c|}
\hline & OPEL & FIAT & FORD & $\begin{array}{c}\text { CITRO } \\
\text { EN }\end{array}$ \\
\hline OPEL & 1,0000 & 3,0000 & 0,5000 & 2,0000 \\
\hline FIAT & 0,3333 & 1,0000 & 0,2500 & 0,3333 \\
\hline FORD & 2,0000 & 4,0000 & 1,0000 & 3,0000 \\
\hline $\begin{array}{c}\text { CITROE } \\
\text { N }\end{array}$ & 0,5000 & 3,0000 & 0,3333 & 1,0000 \\
\hline
\end{tabular}

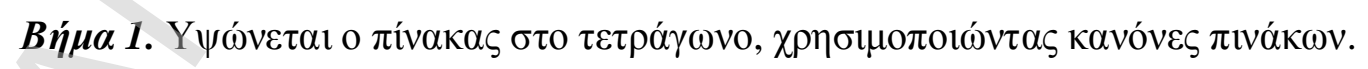

\begin{tabular}{|c|c|c|c|}
\hline $\mathbf{4 , 0 0 0 0}$ & $\mathbf{1 4 , 0 0 0 0}$ & $\mathbf{2 , 4 1 6 7}$ & $\mathbf{6 , 5 0 0 0}$ \\
\hline 1,3333 & 4,0000 & 0,7778 & 2,0833 \\
\hline 6,8333 & 23,0000 & 4,0000 & 11,3333 \\
\hline
\end{tabular}




\begin{tabular}{|l|l|l|l|}
\hline 2,6667 & 8,8333 & 1,6667 & 4,0000 \\
\hline
\end{tabular}

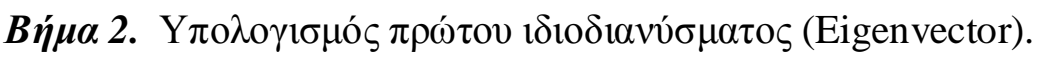

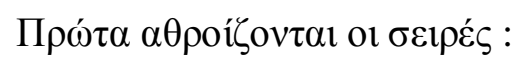

\begin{tabular}{|r|r|r|r|l|}
\hline $\mathbf{4 , 0 0 0 0}$ & $\mathbf{1 4 , 0 0 0 0}$ & $\mathbf{2 , 4 1 6 7}$ & $\mathbf{6 , 5 0 0 0}$ & $=\mathbf{2 6 , 9 1 6 7}$ \\
\hline 1,3333 & 4,0000 & 0,7778 & 2,0833 & $=8,1944$ \\
& & & & \\
\hline 6,8333 & 23,0000 & 4,0000 & 11,3333 & $=45,1667$ \\
\hline 2,6667 & 8,8333 & 1,6667 & 4,0000 & $=17,1667$ \\
\hline
\end{tabular}

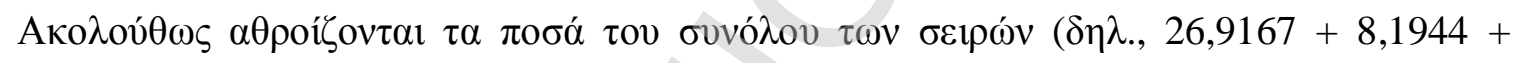

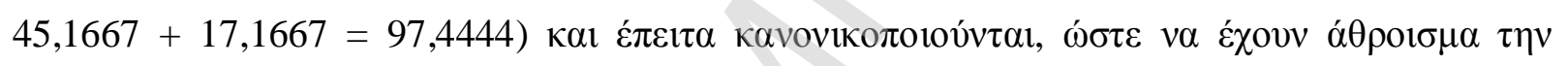

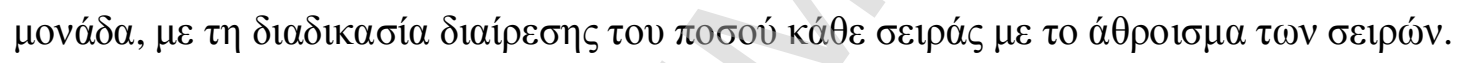

$$
\begin{aligned}
& 26,9167 / 97,4444=0,2762 \\
& 8,1944 / 97,4444=0,0841 \\
& 45,1667 / 97,4444=0,4635 \\
& 17,1667 / 97,4444= \underline{0,1762} \\
& 1,0000
\end{aligned}
$$

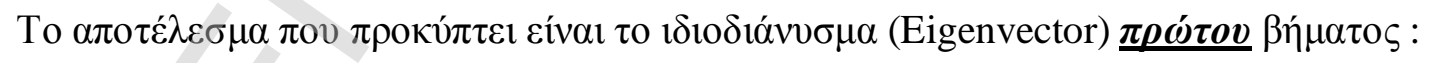




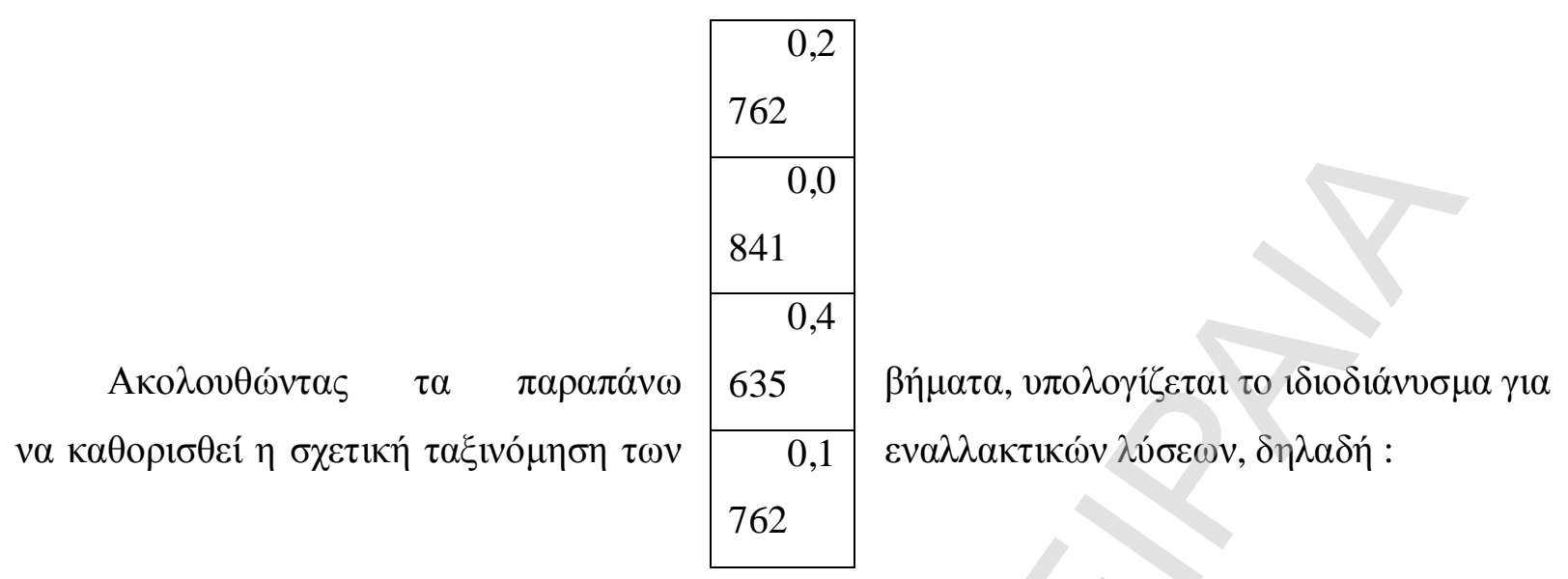




\begin{tabular}{|l|l|}
\hline OPEL & $\mathbf{0 , 2 7 6 2}$ \\
\hline FIAT & $\mathbf{0 , 0 8 4 1}$ \\
\hline FORD & $\mathbf{0 , 4 6 3 5}$ \\
\hline CITROEN & $\mathbf{0 , 1 7 6 2}$ \\
\hline
\end{tabular}

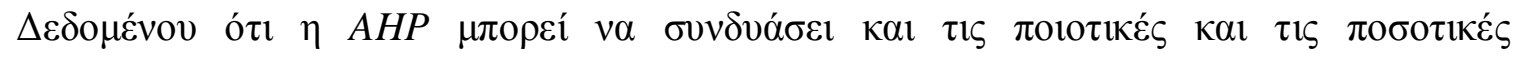

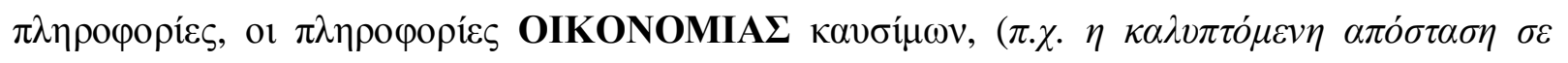

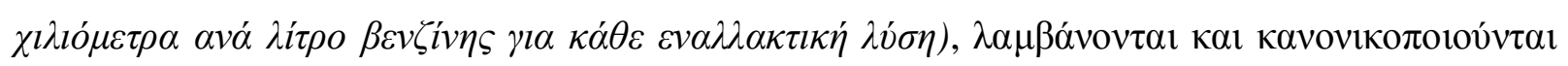

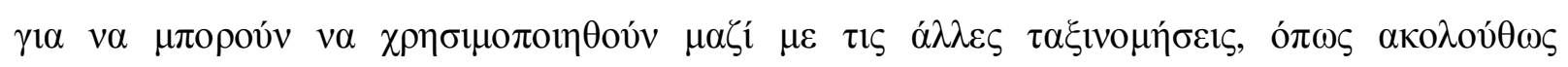
$\pi \alpha \rho o v \sigma 1 \alpha ́ \zeta \varepsilon \tau \alpha 1$ :

\begin{tabular}{|c|c|c|c|}
\hline OPEL & $12 \chi i \lambda . / \lambda i ́ \tau \rho o$ & $\Rightarrow \quad 12 / 42$ & $=0,2857$ \\
\hline FIAT & $9 \chi 1 \lambda . / \lambda i ́ \tau \rho o$ & $\Rightarrow \quad 09 / 42$ & $=0,2143$ \\
\hline FORD & $11 \chi i \lambda . / \lambda i ́ \tau \rho o$ & $\Rightarrow 11 / 42$ & $=0,2619$ \\
\hline CITROEN & $10 \chi 1 \lambda . / \lambda i ́ \tau \rho o$ & $\Rightarrow 10 / 42$ & $=0,2381$ \\
\hline$\Sigma \mathrm{YNO} \Lambda \mathrm{ON}$ & 42 & & 1,0000 \\
\hline
\end{tabular}

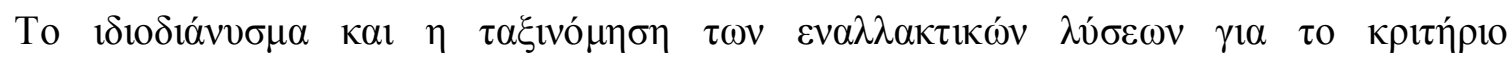

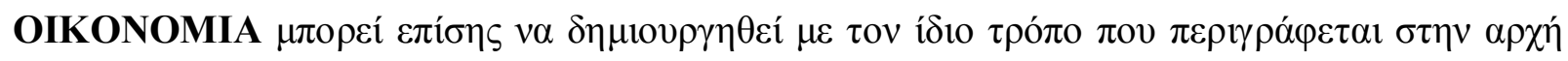

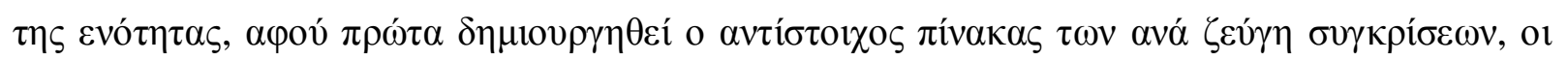

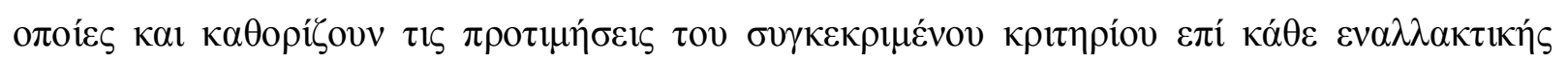

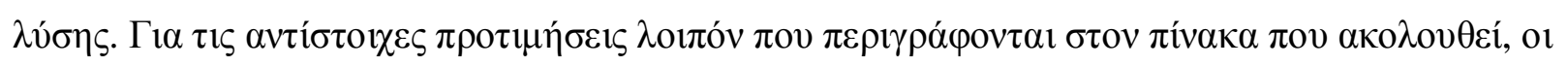

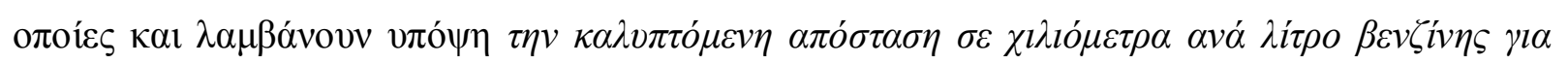

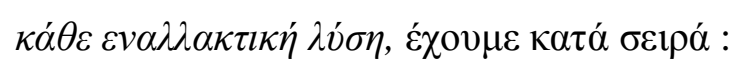

\begin{tabular}{|c|c|c|c|c|}
\hline $\begin{array}{c}\text { OIKON } \\
\text { OMIA }\end{array}$ & OPEL & FIAT & FORD & $\begin{array}{c}\text { CITRO } \\
\text { EN }\end{array}$ \\
\hline OPEL & $1 / 1$ & $12 / 9$ & $12 / 11$ & $12 / 10$ \\
\hline FIAT & $9 / 12$ & $1 / 1$ & $9 / 11$ & $9 / 10$ \\
\hline FORD & $11 / 12$ & $11 / 9$ & $1 / 1$ & $11 / 10$ \\
\hline CITROE & $10 / 12$ & $10 / 9$ & $10 / 11$ & $1 / 1$ \\
\hline
\end{tabular}




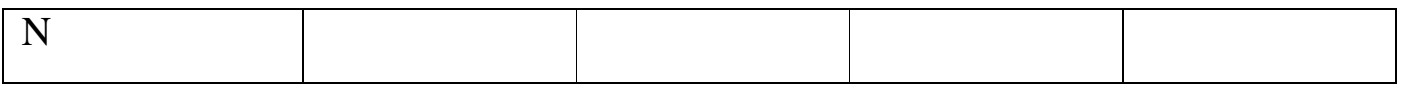

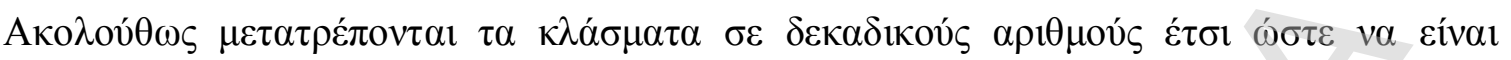

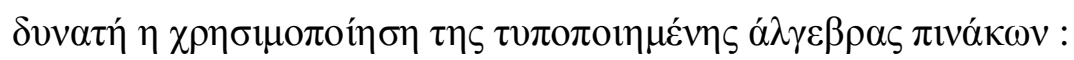

\begin{tabular}{|c|c|c|c|c|}
\hline & OPEL & FIAT & FORD & $\begin{array}{l}\text { CITRO } \\
\text { EN }\end{array}$ \\
\hline OPEL & 1,0000 & 1,3333 & 1,0909 & 1,2000 \\
\hline FIAT & 0,7500 & 1,0000 & 0,8182 & 0,9000 \\
\hline FORD & 0,9167 & 1,2222 & 1,0000 & 1,1000 \\
\hline $\begin{array}{ll} & \text { CITRO } \\
\text { EN } & \end{array}$ & 0,8333 & 1,1111 & 0,9091 & 1,0000 \\
\hline
\end{tabular}

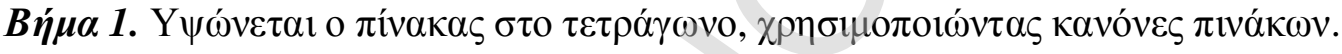

\begin{tabular}{|c|c|c|c|}
\hline $\mathbf{4 , 0 0 0 0}$ & $\mathbf{5 , 3 3 3 3}$ & $\mathbf{4 , 3 6 3 6}$ & $\mathbf{4 , 8 0 0 0}$ \\
\hline 3,0000 & 4,0000 & 3,2727 & 3,6000 \\
\hline 3,6667 & 4,8889 & 4,0000 & 4,4000 \\
\hline 3,3333 & 4,4444 & 3,6364 & 4,0000 \\
\hline
\end{tabular}

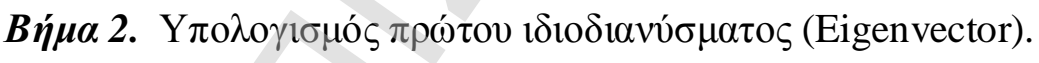

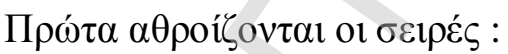

\begin{tabular}{|c|c|c|c|l|}
\hline $\mathbf{4 , 0 0 0 0}$ & $\mathbf{5 , 3 3 3 3}$ & $\mathbf{4 , 3 6 3 6}$ & $\mathbf{4 , 8 0 0 0}$ & $=\mathbf{1 8 , 4 9 7 0}$ \\
\hline 3,0000 & 4,0000 & 3,2727 & 3,6000 & $=13,8727$ \\
\hline 3,6667 & 4,8889 & 4,0000 & 4,4000 & $=16,9556$ \\
\hline 3,3333 & 4,4444 & 3,6364 & 4,0000 & $=15,4141$ \\
\hline
\end{tabular}




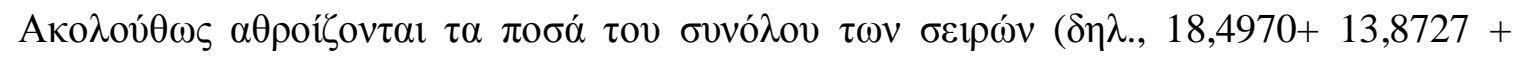

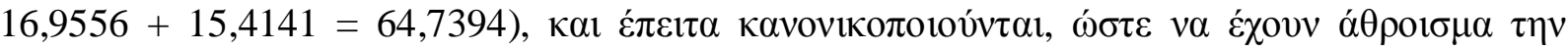

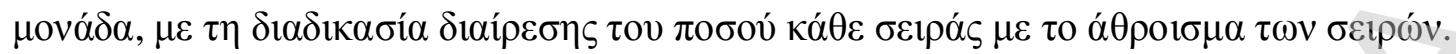

$$
\begin{aligned}
& 18,4970 / 64,7394=0,2857 \\
& 13,8727 / 64,7394=0,2143 \\
& 16,9556 / 64,7394=0,2619 \\
& 15,4141 / 64,7394=\underline{0,2381} \\
& 1,0000
\end{aligned}
$$

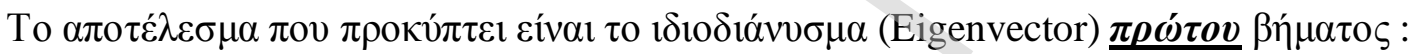

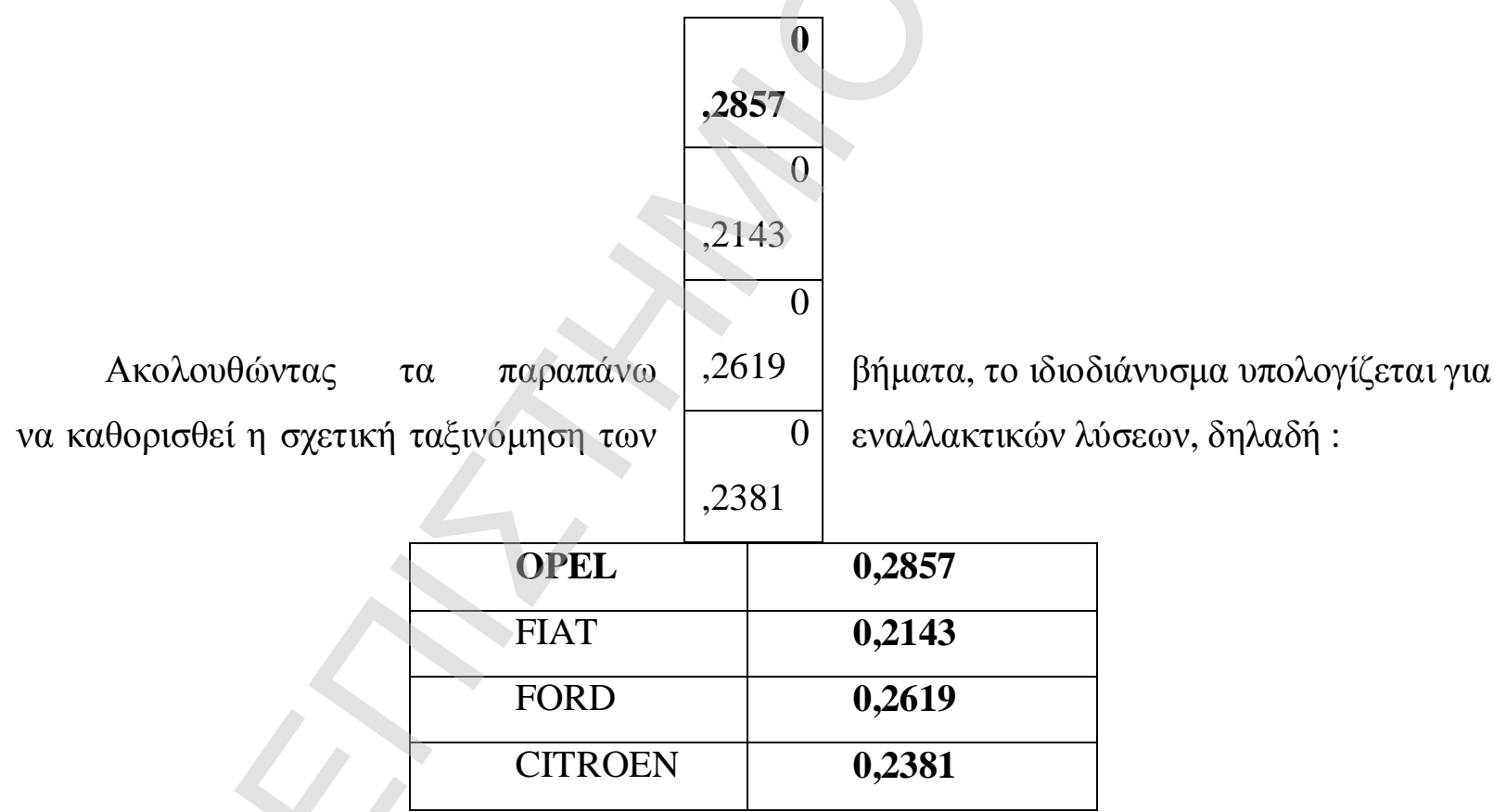

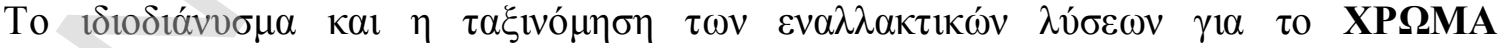

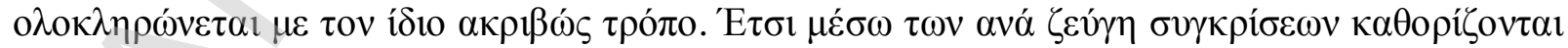

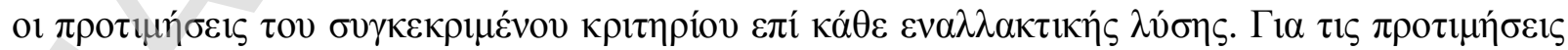

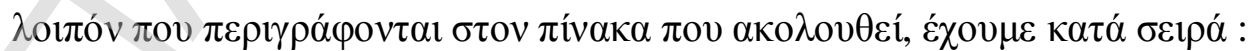




\begin{tabular}{|c|c|c|c|c|}
\hline$\underline{\mathbf{X P \Omega M}}$ & OPEL & FIAT & FORD & $\begin{array}{c}\text { CITRO } \\
\text { EN }\end{array}$ \\
\hline OPEL & $1 / 1$ & $1,5 / 1$ & $1 / 2$ & $1,5 / 1$ \\
\hline FIAT & $1 / 1,5$ & $1 / 1$ & $1 / 2,5$ & $1 / 3$ \\
\hline FORD & $2 / 1$ & $2,5 / 1$ & $1 / 1$ & $3 / 1$ \\
\hline CITRO & $1 / 1,5$ & $3 / 1$ & $1 / 3$ & $1 / 1$ \\
EN & & & & \\
\hline
\end{tabular}

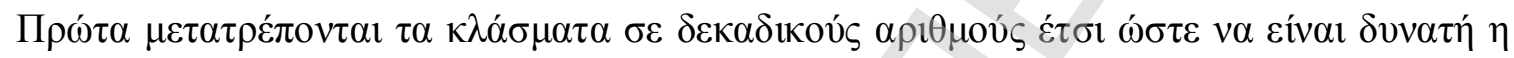

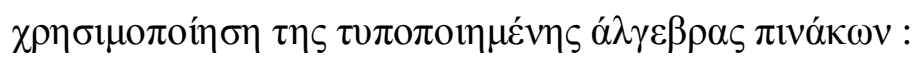

\begin{tabular}{|c|c|c|c|c|}
\hline & OPEL & FIAT & FORD & $\begin{array}{c}\text { CITRO } \\
\text { EN }\end{array}$ \\
\hline OPEL & 1,0000 & 1,5000 & 0,5000 & 1,5000 \\
\hline FIAT & 0,6667 & 1,0000 & 0,4000 & 0,3333 \\
\hline FORD & 2,0000 & 2,5000 & 1,0000 & 3,0000 \\
\hline CITROE & 0,6667 & 3,0000 & 0,3333 & 1,0000 \\
\hline
\end{tabular}

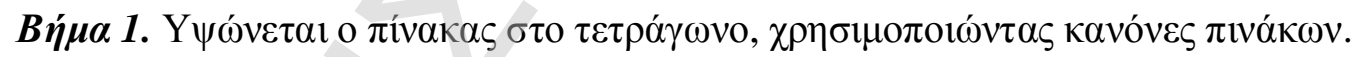

\begin{tabular}{|c|c|c|c|}
\hline $\mathbf{4 , 0 0 0 0}$ & $\mathbf{8 , 7 5 0 0}$ & $\mathbf{2 , 1 0 0 0}$ & $\mathbf{5 , 0 0 0 0}$ \\
\hline 2,3556 & 4,0000 & 1,2444 & 2,8667 \\
\hline 7,6667 & 17,0000 & 4,0000 & 9,8333 \\
\hline 4,0000 & 7,8333 & 2,2000 & 4,0000 \\
\hline
\end{tabular}

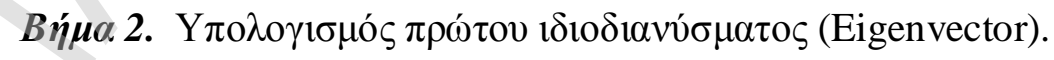

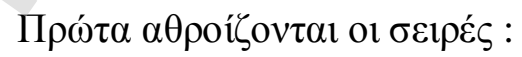




\begin{tabular}{|c|c|c|c|l|}
\hline $\mathbf{4 , 0 0 0 0}$ & $\mathbf{8 , 7 5 0 0}$ & $\mathbf{2 , 1 0 0 0}$ & $\mathbf{5 , 0 0 0 0}$ & $=\mathbf{1 9 , 8 5 0 0}$ \\
\hline 2,3556 & 4,0000 & 1,2444 & 2,8667 & $=10,4667$ \\
\hline 7,6667 & 17,0000 & 4,0000 & 9,8333 & $=38,5000$ \\
\hline 4,0000 & 7,8333 & 2,2000 & 4,0000 & $=18,0333$ \\
\hline
\end{tabular}

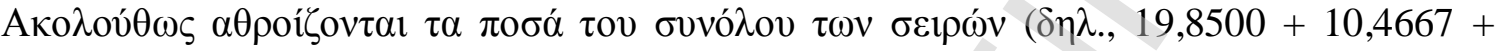

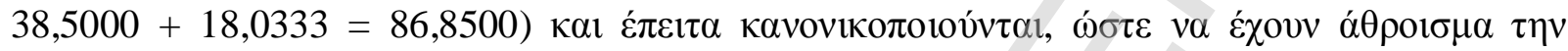

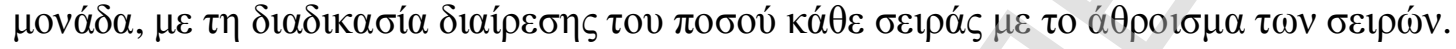

$$
\begin{aligned}
& 19,8500 / 86,8500=0,2286 \\
& 10,4667 / 86,8500=0,1205 \\
& 38,5000 / 86,8500=0,4433 \\
& 18,0333 / 86,8500=\underline{0,2076} \\
& 1,0000
\end{aligned}
$$

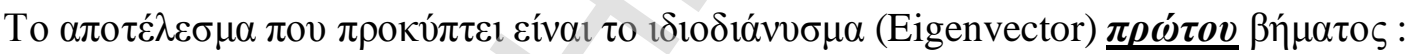

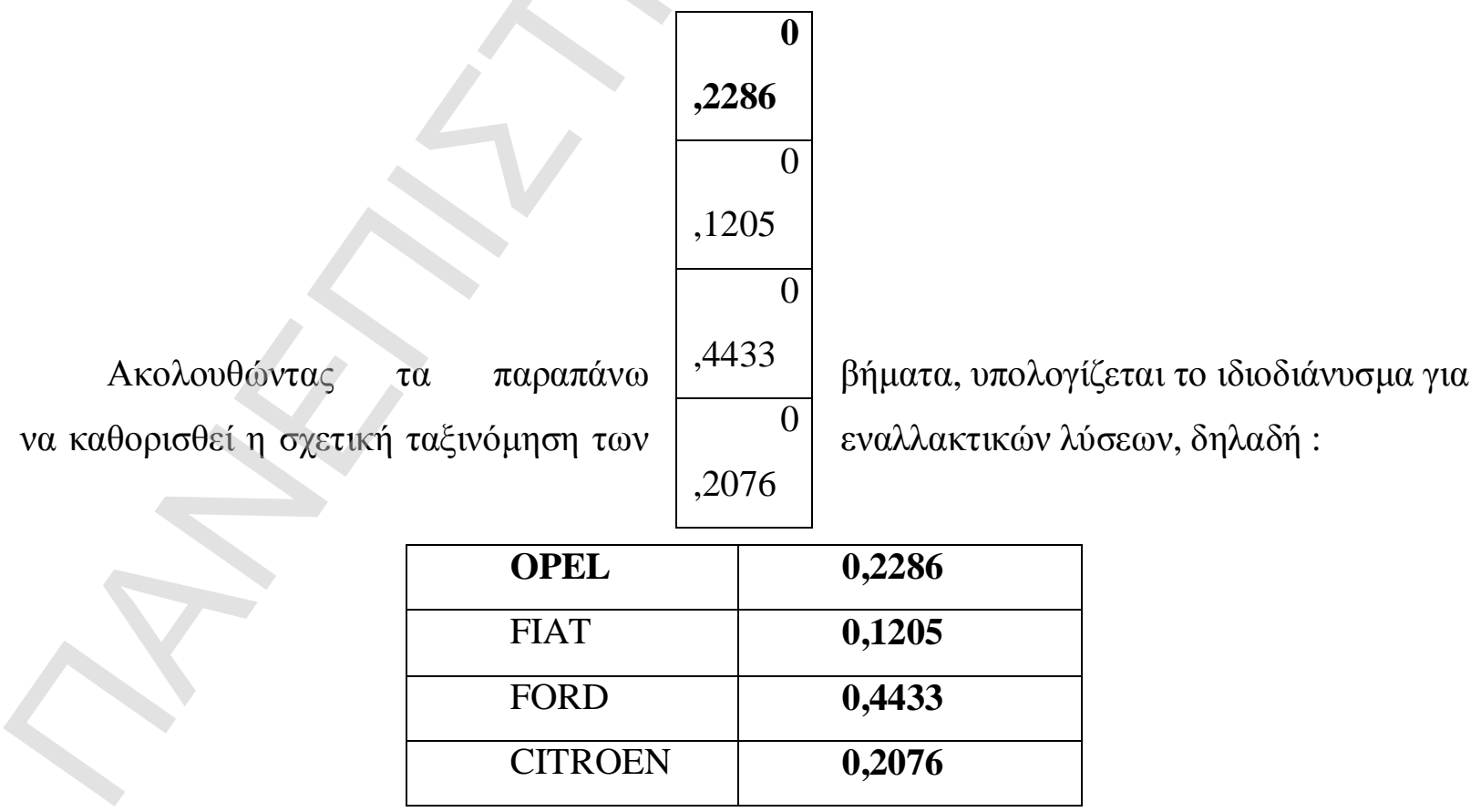




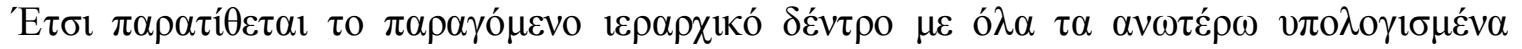

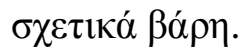




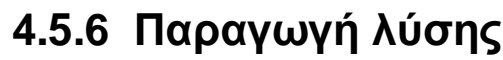

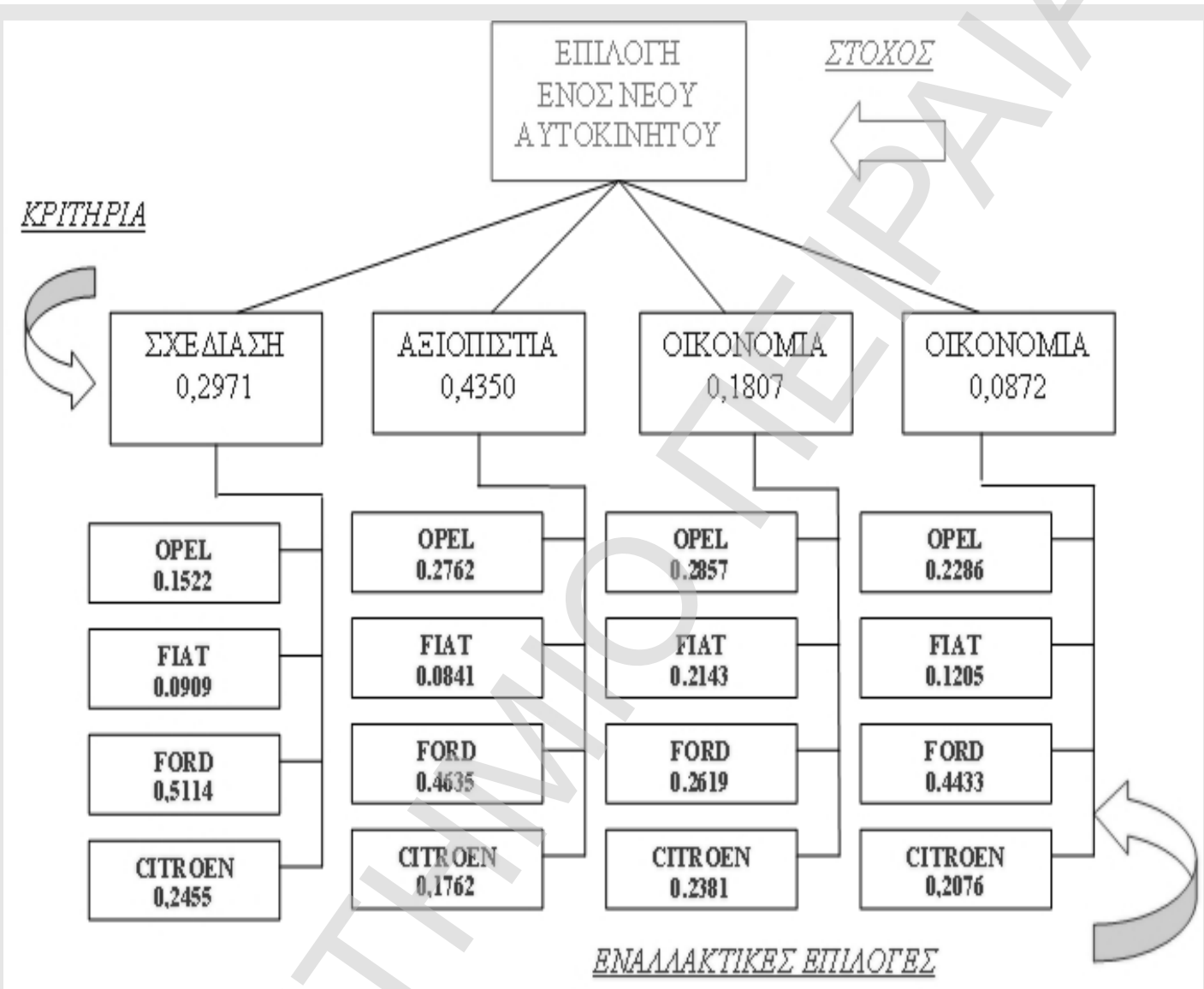

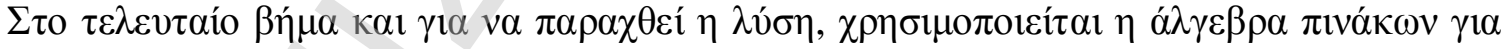

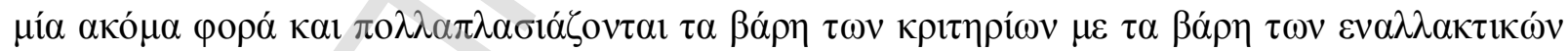

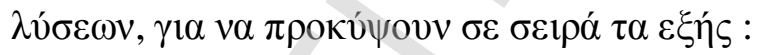




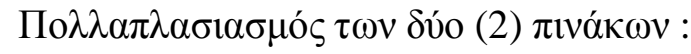

\begin{tabular}{|c|c|c|c|c|}
\hline & $\Sigma X E \Delta I A \Sigma H$ & 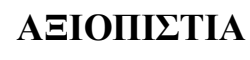 & OIKONOMIA & ХР囚МА \\
\hline OPEL & 0,1522 & 0,2762 & 0,2857 & 0,2286 \\
\hline FIAT & 0,0909 & 0,0841 & 0,2143 & 0,1205 \\
\hline FORD & 0,5114 & 0,4635 & 0,2619 & 0,4433 \\
\hline CITROEN & 0,2455 & 0,1762 & 0,2381 & 0,2076 \\
\hline
\end{tabular}

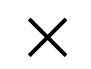

\begin{tabular}{|c|c|}
\hline $\begin{array}{l}\text { KPITH } \\
\text { PIO }\end{array}$ & $\begin{array}{r}\text { B } \\
\text { APOE }\end{array}$ \\
\hline$\Sigma \mathrm{XE} \Delta \mathrm{I}$ & 0 \\
\hline $\mathrm{A} \Sigma \mathrm{H}$ & 2971 \\
\hline АछІОПI & 0 \\
\hline$\Sigma \mathrm{TIA}$ & 4350 \\
\hline OIKON & 0 \\
\hline OMIA & 1807 \\
\hline $\mathrm{XP} \Omega \mathrm{M}$ & 0 \\
\hline A & 0872 \\
\hline
\end{tabular}

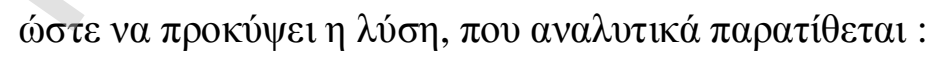

$$
0,2971 \times\left(\begin{array}{c}
0,1522 \\
0,0909 \\
0,5114 \\
0,2454
\end{array}\right)+0,4350 \times\left(\begin{array}{c}
0,2762 \\
0,0841 \\
0,4635 \\
0,1762
\end{array}\right)+0,1807 \times\left(\begin{array}{c}
0,2857 \\
0,2143 \\
0,2619 \\
0,2381
\end{array}\right)+0,08^{\prime}
$$

$$
=\left(\begin{array}{l}
0,0452 \\
0,0270 \\
0,1520 \\
0,0729
\end{array}\right)+\left(\begin{array}{l}
0,1201 \\
0,0366 \\
0,2016 \\
0,0766
\end{array}\right)+\left(\begin{array}{l}
0,0516 \\
0,0387 \\
0,0473 \\
0,0430
\end{array}\right)+\left(\begin{array}{l}
0,0199 \\
0,0105 \\
0,0387 \\
0,0181
\end{array}\right)=\left(\begin{array}{l}
0,2369 \\
0,1128 \\
0,4396 \\
0,2107
\end{array}\right)
$$




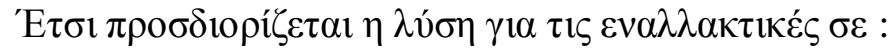

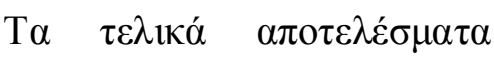

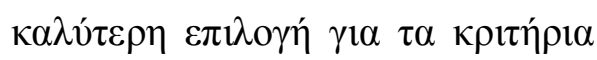

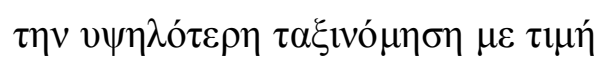

\begin{tabular}{|c|r|}
\hline OP & $\begin{array}{r}\text { O } \\
\text { EL }\end{array}$ \\
\hline FIA & 0 \\
T &, 1128 \\
\hline FOR & $\mathbf{0}$ \\
D &, $\mathbf{4 3 9 6}$ \\
\hline CIT & 0 \\
ROEN &, 2107 \\
\hline
\end{tabular}

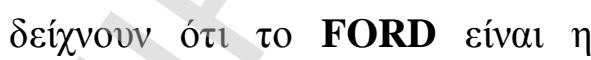

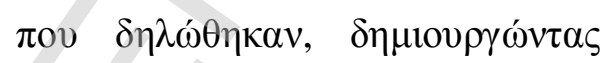
0,4396 .

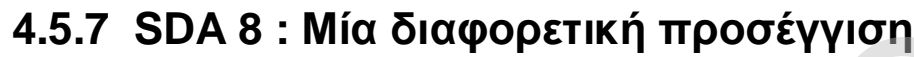

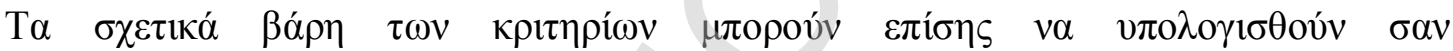

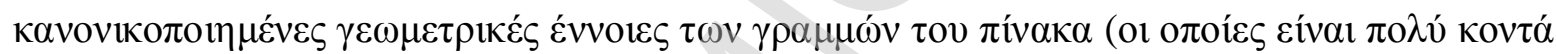

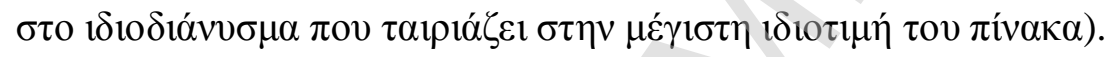

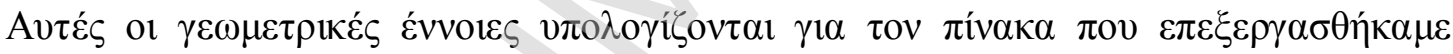

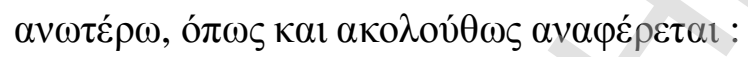

\begin{tabular}{|c|c|c|c|c|}
\hline & $\Sigma X E \Delta I A \Sigma H$ & 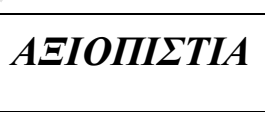 & OIKONOMIA & XPQM \\
\hline$\Sigma X E \triangle I A \Sigma H$ & $1 / 1$ & $1 / 1,5$ & $2 / 1$ & $3 / 1$ \\
\hline 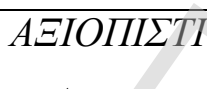 & $1,5 / 1$ & $1 / 1$ & $3 / 1$ & $4 / 1$ \\
\hline$\frac{4}{O I K O N O M}$ & + & $1 / 3$ & $1 / 1$ & $3 / 1$ \\
\hline$X P \Omega M A$ & $1 / 3$ & $1 / 4$ & $1 / 3$ & $1 / 1$ \\
\hline
\end{tabular}

$$
\begin{aligned}
& m_{1}=\sqrt[4]{1 \times \frac{2}{3} \times 2 \times 3} \\
& m_{2}=\sqrt[4]{1,5 \times 1 \times 3 \times 4} \\
& m_{3}=\sqrt[4]{\frac{1}{2} \times \frac{1}{3} \times 1 \times 3} \\
& m_{4}=\sqrt[4]{\frac{1}{3} \times \frac{1}{4} \times \frac{1}{3} \times 1}
\end{aligned}
$$


А $\pi^{\prime}$ ó $\pi$ оv $\pi$ роки́ $\pi \tau \varepsilon \imath$ ó $\tau 1: \mathrm{m}_{1}=1,9999, \mathrm{~m}_{2}=4,2426, \mathrm{~m}_{3}=0,7071 \& \mathrm{~m}_{4}=0,1667$.

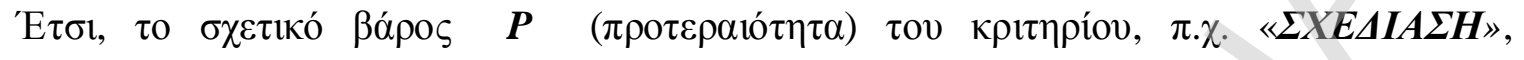

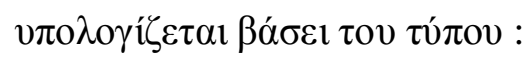

$$
p_{1}=\frac{m_{1}}{m_{1}+m_{2}+m_{3}+m_{4}} \kappa \alpha \imath \pi \rho \text { }
$$

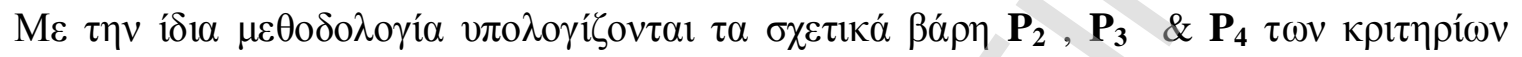

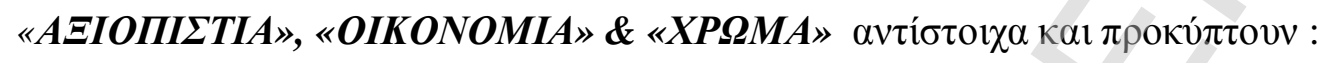

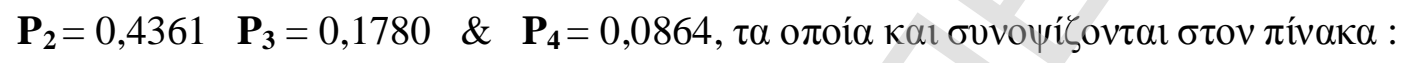

\begin{tabular}{|c|c|c|c|c|c|}
\hline & $\Sigma X E \Delta I A \Sigma H$ & 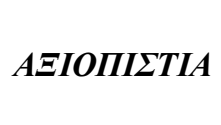 & OIKONOMIA & XPQMA & 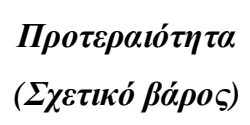 \\
\hline$\Sigma X E \triangle I A \Sigma H$ & $1 / 1$ & $1 / 1,5$ & $2 / 1$ & $3 / 1$ & 0,2994 \\
\hline АЕІОПIГТІА & $1,5 / 1$ & $1 / 1$ & $3 / 1$ & $4 / 1$ & 0,4361 \\
\hline OIKONOMIA & $1 / 2$ & $1 / 3$ & $1 / 1$ & $3 / 1$ & 0,1780 \\
\hline$X P \Omega M A$ & $1 / 3$ & $1 / 4$ & $1 / 3$ & $1 / 1$ & 0,0864 \\
\hline
\end{tabular}

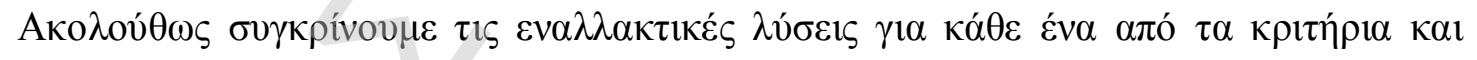

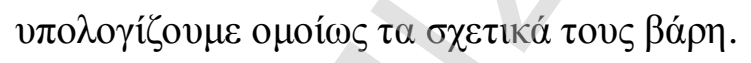

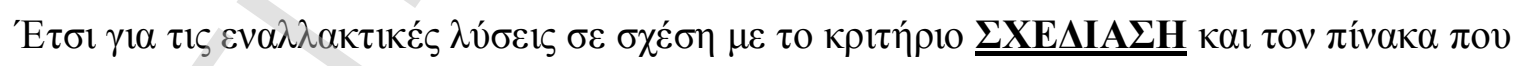

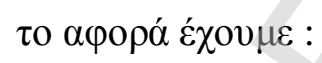

$$
\begin{aligned}
& m_{1}=\sqrt[4]{1 \times 2 \times \frac{1}{3} \times \frac{1}{2}} \\
& m_{2}=\sqrt[4]{\frac{1}{2} \times 1 \times \frac{1}{4} \times \frac{1}{3}} \\
& m_{3}=\sqrt[4]{3 \times 4 \times 1 \times 3} \\
& m_{4}=\sqrt[4]{2 \times 3 \times \frac{1}{3} \times 1}
\end{aligned}
$$




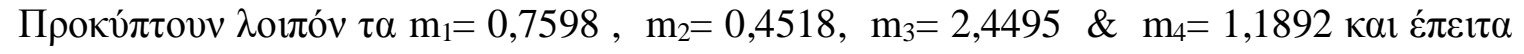

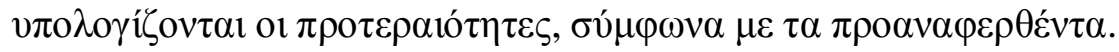

\begin{tabular}{|c|c|c|c|c|c|}
\hline$\underline{\Sigma X E \Delta I A \Sigma H}$ & OPEL & FIAT & FORD & CITROEN & 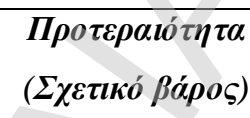 \\
\hline OPEL & $1 / 1$ & $2 / 1$ & $1 / 3$ & $1 / 2$ & 0,1566 \\
\hline FIAT & $1 / 2$ & $1 / 1$ & $1 / 4$ & $1 / 3$ & 0,0931 \\
\hline FORD & $3 / 1$ & $4 / 1$ & $1 / 1$ & $3 / 1$ & 0,5050 \\
\hline $\begin{array}{l}\text { CITROE } \\
\mathrm{N}\end{array}$ & $2 / 1$ & $3 / 1$ & $1 / 3$ & & 0,2452 \\
\hline
\end{tabular}

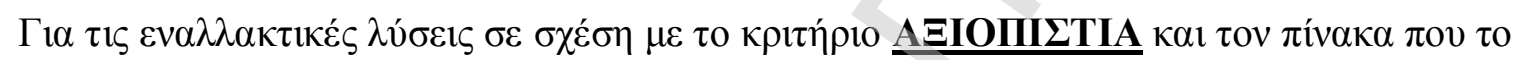

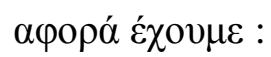

$$
\begin{aligned}
& m_{1}=\sqrt[4]{1 \times 3 \times \frac{1}{2} \times 2} \\
& m_{2}=\sqrt[4]{\frac{1}{3} \times 1 \times \frac{1}{4} \times \frac{1}{3}} \\
& m_{3}=\sqrt[4]{2 \times 4 \times 1 \times 3} \\
& m_{4}=\sqrt[4]{\frac{1}{2} \times 3 \times \frac{1}{3} \times 1}
\end{aligned}
$$

\begin{tabular}{|c|c|c|c|c|c|}
\hline 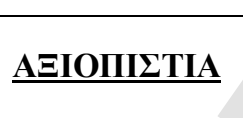 & OPEL & FIAT & FORD & CITROEN & 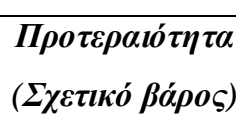 \\
\hline OPEL & $1 / 1$ & $3 / 1$ & $1 / 2$ & $2 / 1$ & 0,2754 \\
\hline FIAT & $1 / 3$ & $1 / 1$ & $1 / 4$ & $1 / 3$ & 0,0854 \\
\hline FORD & $2 / 1$ & $4 / 1$ & $1 / 1$ & $3 / 1$ & 0,4632 \\
\hline CITROEN & $1 / 2$ & $3 / 1$ & $1 / 3$ & $1 / 1$ & 0,1760 \\
\hline
\end{tabular}

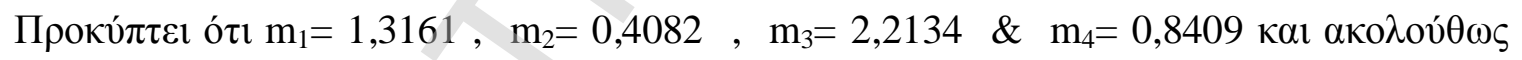

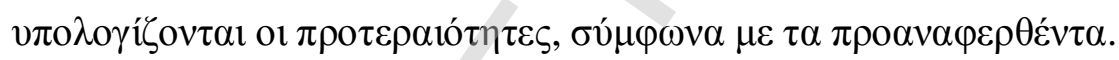

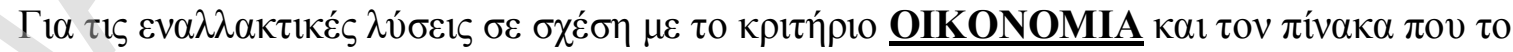

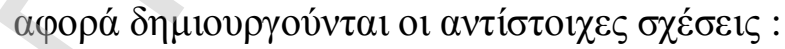




$$
\begin{aligned}
& m_{1}=\sqrt[4]{1 \times \frac{12}{9} \times \frac{12}{11} \times \frac{12}{10}} \\
& m_{2}=\sqrt[4]{\frac{9}{12} \times 1 \times \frac{9}{11} \times \frac{9}{10}} \\
& m_{3}=\sqrt[4]{\frac{11}{12} \times \frac{11}{9} \times 1 \times \frac{11}{10}} \\
& m_{4}=\sqrt[4]{\frac{10}{12} \times \frac{10}{9} \times \frac{10}{11} \times 1}
\end{aligned}
$$

\begin{tabular}{|c|c|c|c|c|c|}
\hline OIKONOMIA & OPEL & FIAT & FORD & CITROEN & 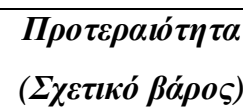 \\
\hline OPEL & $1 / 1$ & $9^{12 /}$ & $12 / 11$ & $12 / 10$ & 0,2857 \\
\hline FIAT & $9 / 12$ & $1 / 1$ & $9 / 11$ & $9 / 10$ & 0,2143 \\
\hline FORD & $11 / 12$ & $9^{11 /}$ & & $11 / 10$ & 0,2619 \\
\hline $\begin{array}{l}\text { CITROE } \\
\mathrm{N}\end{array}$ & $10 / 12$ & $9^{10 /}$ & $10 / 11$ & $1 / 1$ & 0,2381 \\
\hline
\end{tabular}

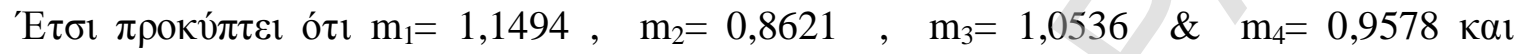

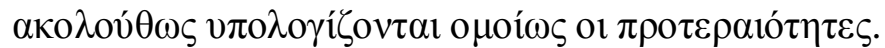

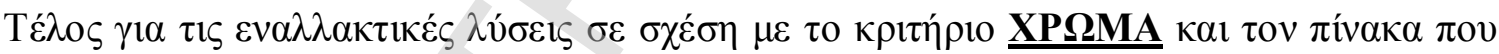

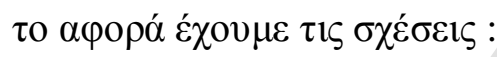

$$
\begin{aligned}
& m_{1}=\sqrt[4]{1 \times \frac{3}{2} \times \frac{1}{2} \times 1,5} \\
& m_{2}=\sqrt[4]{\frac{2}{3} \times 1 \times 0,4 \times \frac{1}{3}} \\
& m_{3}=\sqrt[4]{2 \times 2,5 \times 1 \times 3} \\
& m_{4}=\sqrt[4]{\frac{2}{3} \times 3 \times \frac{1}{3} \times 1}
\end{aligned}
$$

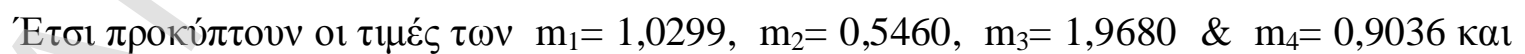

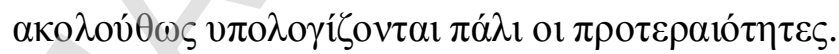




\begin{tabular}{|c|c|c|c|c|c|}
\hline$\underline{\text { XРММА }}$ & OPEL & FIAT & FORD & CITROEN & 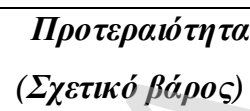 \\
\hline OPEL & $1 / 1$ & ${ }^{1,5}$ & $1 / 2$ & $1,5 / 1$ & 0,2316 \\
\hline FIAT & $1 / 1,5$ & $1 / 1$ & $1 / 2,5$ & $1 / 3$ & 0,1228 \\
\hline FORD & $2 / 1$ & $/ 1^{2,5}$ & $1 / 1$ & $3 / 1$ & 0,4425 \\
\hline $\begin{array}{l}\text { CITROE } \\
\mathrm{N}\end{array}$ & $1 / 1,5$ & $3 / 1$ & $1 / 3$ & & 0,2032 \\
\hline
\end{tabular}

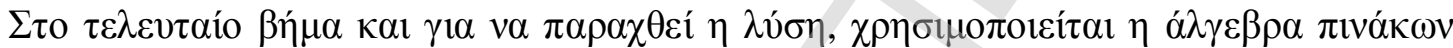

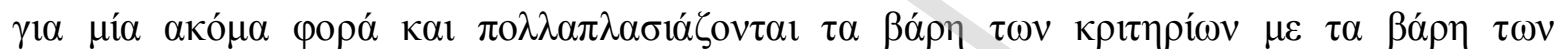

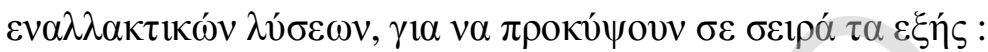

$$
\begin{aligned}
& 0,2994 \times\left(\begin{array}{l}
0,1566 \\
0,0931 \\
0,5050 \\
0,2452
\end{array}\right)+0,4361 \times\left(\begin{array}{l}
0,2754 \\
0,0854 \\
0,4632 \\
0,1760
\end{array}\right)+0,1780 \times\left(\begin{array}{l}
0,2857 \\
0,2143 \\
0,2619 \\
0,2381
\end{array}\right)+0,0864 \times\left(\begin{array}{l}
0,2316 \\
0,1228 \\
0,4425 \\
0,2032
\end{array}\right)= \\
& =\left(\begin{array}{l}
0,0469 \\
0,0279 \\
0,1512 \\
0,0734
\end{array}\right)+\left(\begin{array}{l}
0,1201 \\
0,0372 \\
0,2020 \\
0,0768
\end{array}\right)+\left(\begin{array}{l}
0,0509 \\
0,0381 \\
0,0466 \\
0,0424
\end{array}\right)+\left(\begin{array}{l}
0,0200 \\
0,0106 \\
0,0382 \\
0,0176
\end{array}\right)=\left(\begin{array}{l}
0,2379 \\
0,1139 \\
0,4380 \\
0,2101
\end{array}\right)
\end{aligned}
$$

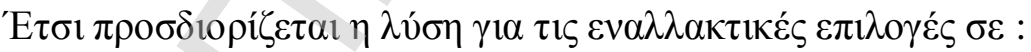

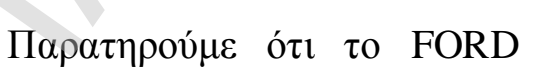

\begin{tabular}{|c|c|}
\hline $\begin{array}{ll} & \text { OP } \\
\text { EL }\end{array}$ & ,2379 \\
\hline $\begin{array}{ll} & \text { FI } \\
\text { AT } & \end{array}$ & 1139 \\
\hline FORD & ,4380 \\
\hline $\begin{array}{r}\text { CI } \\
\text { TROEN }\end{array}$ & ,2101 \\
\hline
\end{tabular}
$\kappa \rho \imath \eta ́ p l \alpha \quad \kappa \alpha \imath \quad \tau \alpha \quad \beta \alpha ́ \rho \eta \quad \pi 0 v$

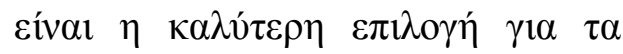

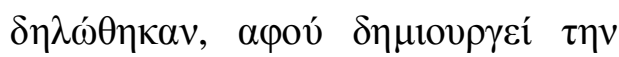

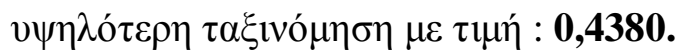




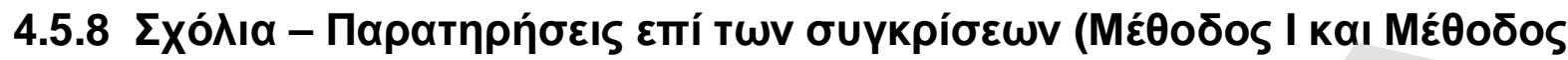 II)}

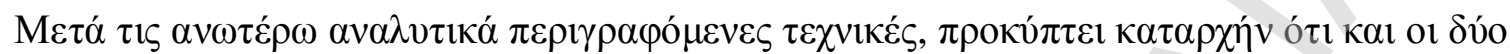

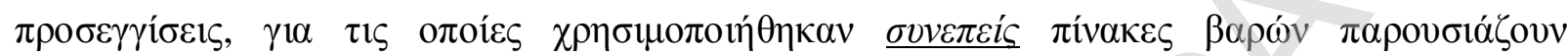

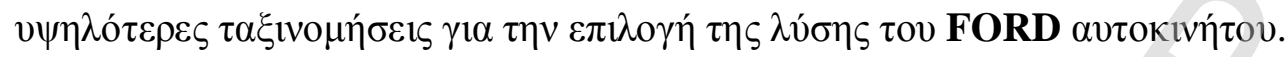

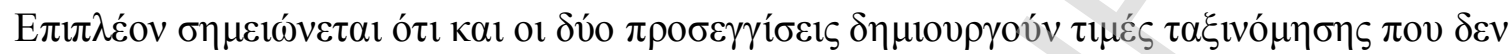

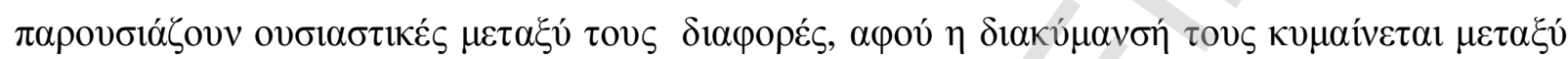
$0,2 \%$ ка $1 \%$.

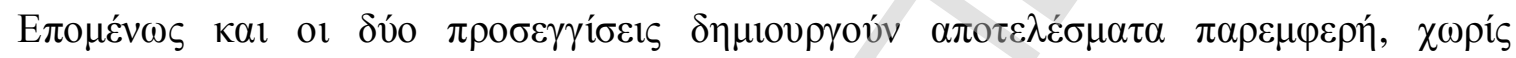

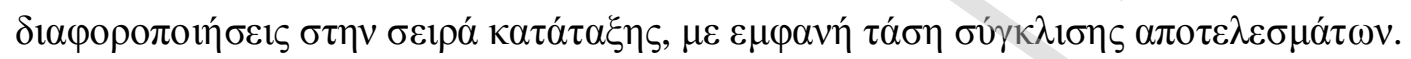

\begin{tabular}{|c|c|c|}
\hline 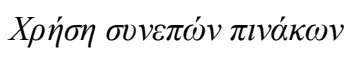 & $1^{\eta}$ ПРОГЕГГІऽН & $2^{\eta}$ ПРОГЕГГІऽН \\
\hline OPEL & 0,2369 & 0,2379 \\
\hline FIAT & 0,1128 & 0,1139 \\
\hline FORD & 0,4396 & 0,4380 \\
\hline CITROEN & 0,2107 & 0,2101 \\
\hline
\end{tabular}

\section{$4.6 \Sigma u \mu \pi \varepsilon \rho ı$ І}

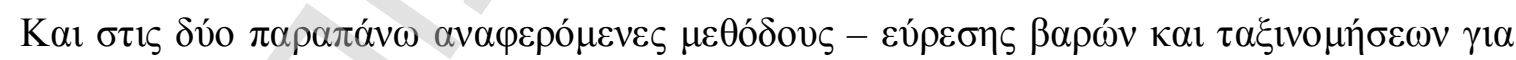

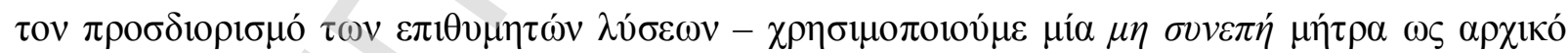

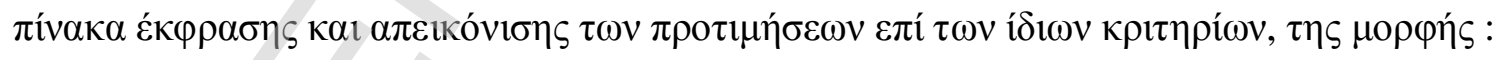

\begin{tabular}{|c|c|c|c|c|}
\hline$+\sqrt{2}$ & $\Sigma X E \Delta I A \Sigma H$ & АЕІОПІЕТІА & $\begin{array}{l}\text { OIKONO } \\
\text { MIA }\end{array}$ & $X P \Omega M A$ \\
\hline$\Sigma X E \Delta I A \Sigma H$ & $1 / 1$ & $2 / 1$ & $2 / 1$ & $2 / 1$ \\
\hline 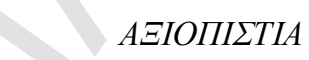 & $1 / 2$ & $1 / 1$ & $1 / 3$ & $5 / 1$ \\
\hline OIKONOMIA & $1 / 2$ & $3 / 1$ & $1 / 1$ & $1 / 8$ \\
\hline
\end{tabular}




\begin{tabular}{|l|l|l|l|l|}
\hline$X P \Omega M A$ & $1 / 2$ & $1 / 5$ & $8 / 1$ & $1 / 1$ \\
\hline
\end{tabular}

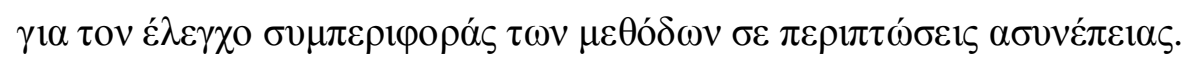

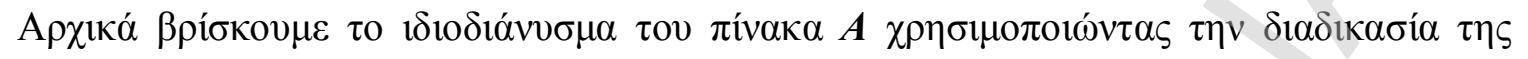

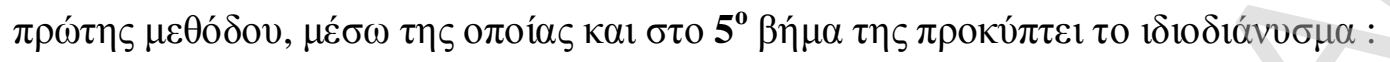

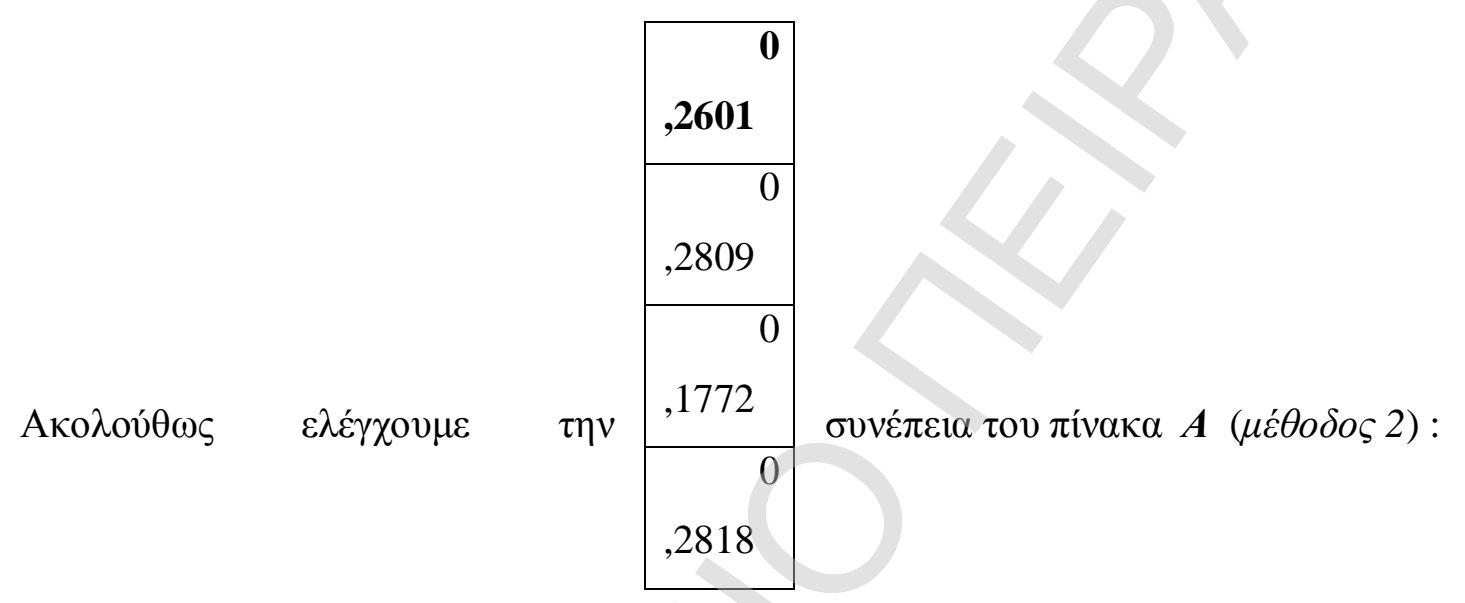

1. $A \cdot w^{T}=\left[\begin{array}{cccc}1 & 2 & 2 & 2 \\ \frac{1}{2} & 1 & \frac{1}{3} & 5 \\ \frac{1}{2} & 3 & 1 & \frac{1}{8} \\ \frac{1}{2} & \frac{1}{5} & 8 & 1\end{array}\right] * \frac{\left[\begin{array}{l}0,2601 \\ 0,2809 \\ 0,1772 \\ 0,2818\end{array}\right]}{1}=\frac{\left[\begin{array}{l}1,7399 \\ 1,8790 \\ 1,1852 \\ 1,8856\end{array}\right]}{6,6897}$

2. $\frac{1}{n} \sum_{i=1}^{n} \frac{\text { ith entry in } A \cdot w^{T}}{\text { ith entry in } w^{T}}=\frac{1}{4} \cdot\left(\frac{1,7399}{0,2601}+\frac{1,8790}{0,2809}+\frac{1,1852}{0,1772}+\frac{1,8856}{0,2818}\right)=6,6896$

3. $C I=\frac{\text { (result from step } 2)-n}{n-1}=\frac{6,6897-4}{3}=0,8965$

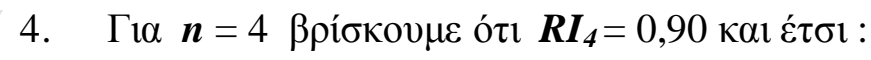

$$
C I / R I_{4}=\frac{0,8965}{0,90}=0,9961>>0,10
$$




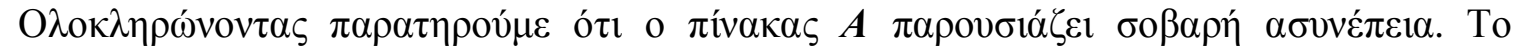

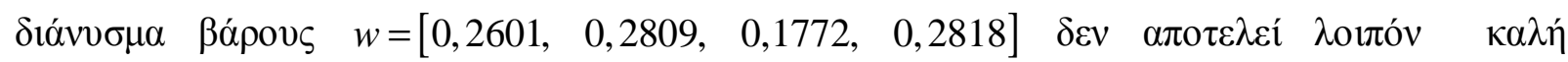
$\pi \rho 0 \sigma \varepsilon ́ \gamma \gamma 1 \sigma \eta$.

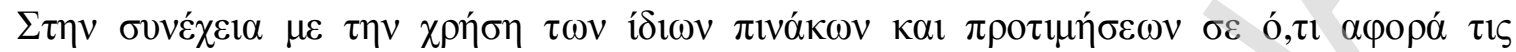

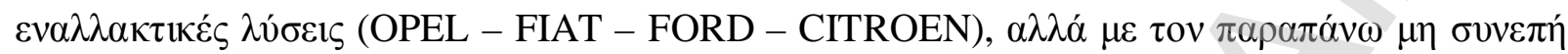

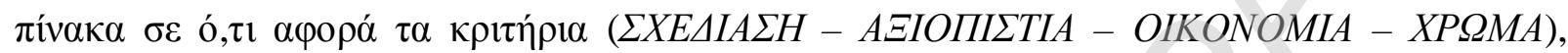

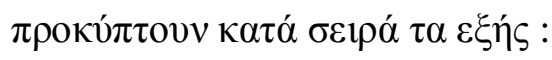

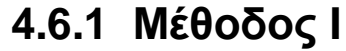

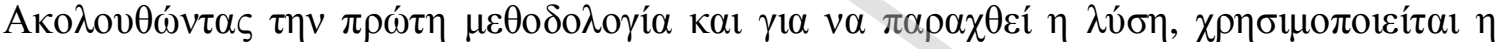

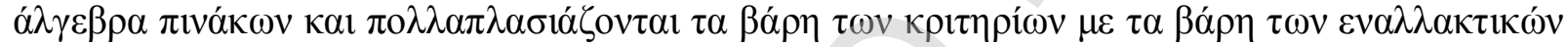

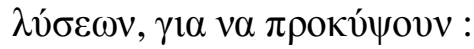

\begin{tabular}{|c|c|c|c|c|}
\hline & $\Sigma$ XEAIA $\Sigma H$ & 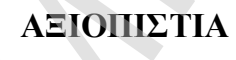 & OIKONOMIA & XPQMA \\
\hline OPEL & 0,1522 & 0,2762 & 0,2857 & 0,2286 \\
\hline FIAT & 0,0909 & 0,0841 & 0,2143 & 0,1205 \\
\hline FORD & 0,5114 & 0,4635 & 0,2619 & 0,4433 \\
\hline CITROEN & 0,2455 & 0,1762 & 0,2381 & 0,2076 \\
\hline
\end{tabular}




\begin{tabular}{|c|r|}
\hline $\begin{array}{c}\text { KPITH } \\
\text { PIO }\end{array}$ & $\begin{array}{r}\text { B } \\
\text { APO } \Sigma\end{array}$ \\
\hline$\Sigma X E \Delta I$ & 0, \\
$A \Sigma H$ & 2601 \\
\hline$A \Xi I O \Pi$ & 0, \\
$I \Sigma T I A$ & 2809 \\
\hline$O I K O N$ & 0, \\
$O M I A$ & 1772 \\
\hline$X P \Omega M$ & 0, \\
$A$ & 2818 \\
\hline
\end{tabular}

$$
=\ldots
$$

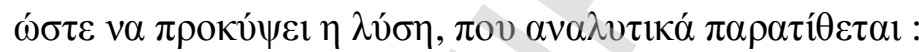

$$
0,2601 \times\left(\begin{array}{c}
0,1522 \\
0,0909 \\
0,5114 \\
0,2454
\end{array}\right)+0,2809 \times\left(\begin{array}{c}
0,2762 \\
0,0841 \\
0,4635 \\
0,1762
\end{array}\right)+0,1772 \times\left(\begin{array}{c}
0,2857 \\
0,2143 \\
0,2619 \\
0,2381
\end{array}\right)+0,281
$$

$$
=\left(\begin{array}{l}
0,0396 \\
0,0237 \\
0,1330 \\
0,0639
\end{array}\right)+\left(\begin{array}{l}
0,0776 \\
0,0236 \\
0,1302 \\
0,0495
\end{array}\right)+\left(\begin{array}{l}
0,0506 \\
0,0380 \\
0,0464 \\
0,0422
\end{array}\right)+\left(\begin{array}{l}
0,0644 \\
0,0340 \\
0,1249 \\
0,0585
\end{array}\right)=\left(\begin{array}{l}
0,2322 \\
0,1193 \\
0,4345 \\
0,2140
\end{array}\right)
$$

\begin{tabular}{|c|c|}
\hline OP & 0 \\
\hline EL & 2322 \\
\hline FI & 0 \\
\hline AT & ,1193 \\
\hline FO & 0 \\
\hline RD & 4345 \\
\hline CI & 0 \\
\hline TROEN & 2140 \\
\hline
\end{tabular}

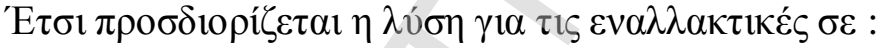

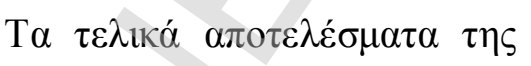

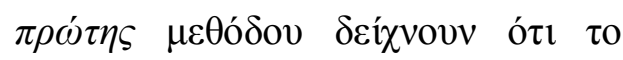

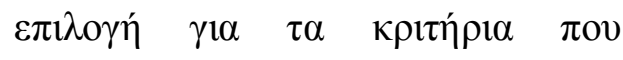

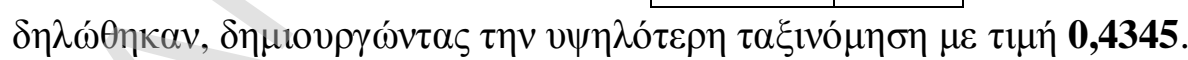

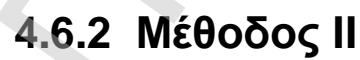




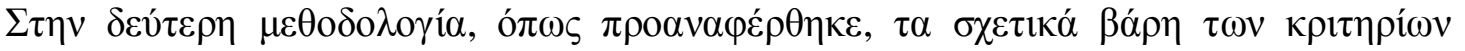

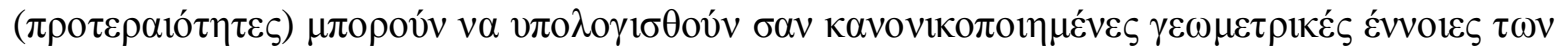

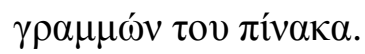

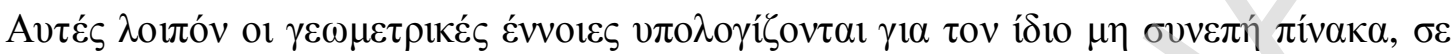

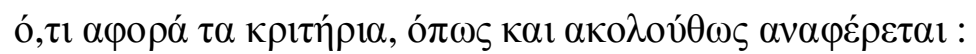

\begin{tabular}{|c|c|c|c|c|}
\hline & $\Sigma X E \Delta I A \Sigma H$ & АЕІОПІЕТІА & OIKONOMIA & $\begin{array}{l}X P \Omega M \\
A \\
\end{array}$ \\
\hline$\Sigma X E \Delta I A \Sigma H$ & $1 / 1$ & $2 / 1$ & $2 / 1$ & $2 / 1$ \\
\hline АछІОПІГТІА & $1 / 2$ & $1 / 1$ & $1 / 3$ & $5 / 1$ \\
\hline OIKONOMIA & $1 / 2$ & $3 / 1$ & $1 / 1$ & $1 / 8$ \\
\hline$X P \Omega M A$ & $1 / 2$ & $1 / 5$ & $8 / 1$ & $1 / 1$ \\
\hline
\end{tabular}




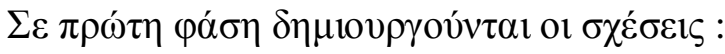

$$
\begin{aligned}
& m_{1}=\sqrt[4]{1 \times 2 \times 2 \times 2} \\
& m_{2}=\sqrt[4]{\frac{1}{2} \times 1 \times \frac{1}{3} \times 5} \\
& m_{3}=\sqrt[4]{\frac{1}{2} \times 3 \times 1 \times \frac{1}{8}} \\
& m_{4}=\sqrt[4]{\frac{1}{2} \times \frac{1}{5} \times 8 \times 1}
\end{aligned}
$$

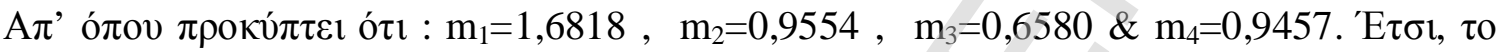

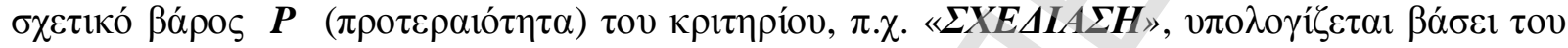

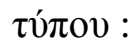

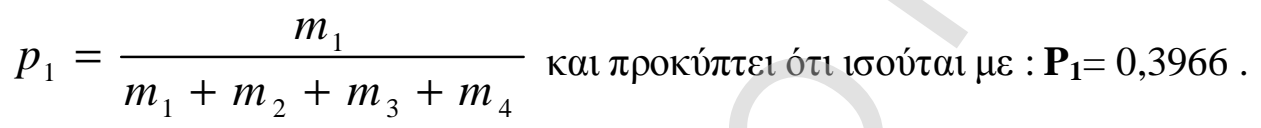

\begin{tabular}{|c|c|c|c|c|c|}
\hline & $\Sigma X E \Delta I A \Sigma H$ & AछIOПIITIA & ОІКОNОМІА & XPQMA & 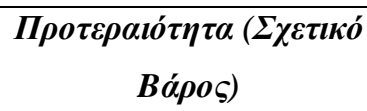 \\
\hline$\Sigma X E \Delta I A \Sigma H$ & $1 / 1$ & $2 / 1$ & $2 / 1$ & $2 / 1$ & 0,3966 \\
\hline 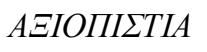 & $1 / 2$ & $1 / 1$ & $1 / 3$ & $5 / 1$ & 0,2253 \\
\hline OIKONOMIA & & $3 / 1$ & $1 / 1$ & $1 / 8$ & 0,1552 \\
\hline$X P \Omega M A$ & & $1 / 5$ & $8 / 1$ & $1 / 1$ & 0,2230 \\
\hline
\end{tabular}

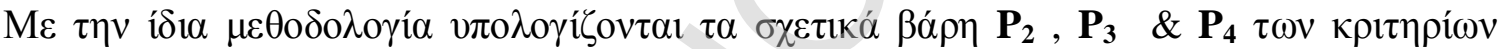

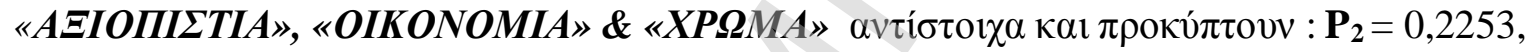

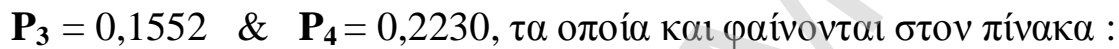

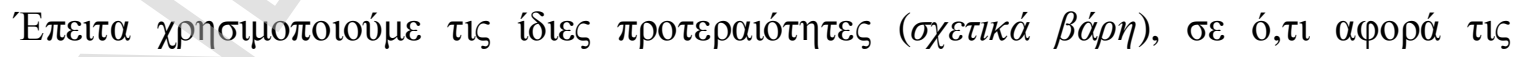

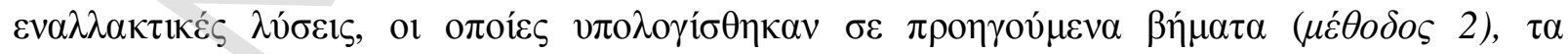

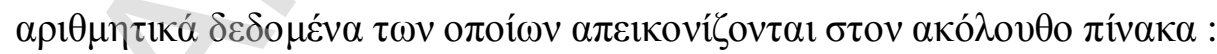




\begin{tabular}{|c|c|c|c|c|}
\hline 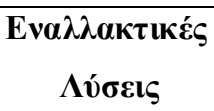 & $\Sigma \chi \varepsilon \delta i ́ \alpha \sigma \eta$ & 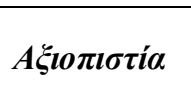 & $\begin{array}{l}\text { Oıкоvонí } \\
\alpha\end{array}$ & 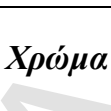 \\
\hline OPEL & 0,1566 & 0,2754 & 0,2857 & 0,2316 \\
\hline FIAT & 0,0931 & 0,0854 & 0,2143 & 0,1228 \\
\hline FORD & 0,5050 & 0,4632 & 0,2619 & 0,4425 \\
\hline CITROEN & 0,2452 & 0,1760 & 0,2381 & 0,2032 \\
\hline
\end{tabular}

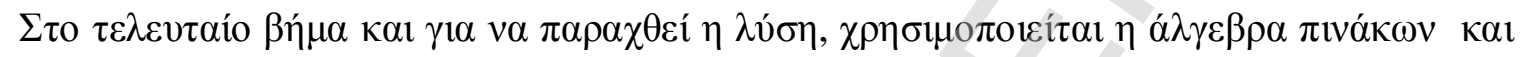

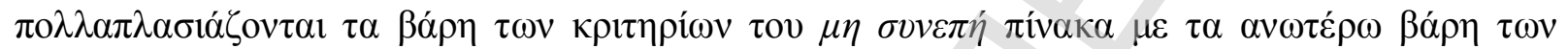

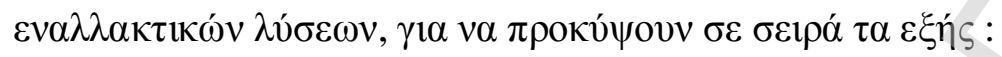

$$
\begin{aligned}
& 0,3966 \times\left(\begin{array}{l}
0,1566 \\
0,0931 \\
0,5050 \\
0,2452
\end{array}\right)+0,2253 \times\left(\begin{array}{l}
0,2754 \\
0,0854 \\
0,4632 \\
0,1760
\end{array}\right)+0,1552 \times\left(\begin{array}{l}
0,2857 \\
0,2143 \\
0,2619 \\
0,2381
\end{array}\right)+0,2230 \times\left(\begin{array}{l}
0,2316 \\
0,1228 \\
0,4425 \\
0,2032
\end{array}\right)= \\
& =\left(\begin{array}{l}
0,0604 \\
0,0361 \\
0,2028 \\
0,0974
\end{array}\right)+\left(\begin{array}{l}
0,0622 \\
0,0189 \\
0,1044 \\
0,0397
\end{array}\right)+\left(\begin{array}{l}
0,0443 \\
0,0333 \\
0,0406 \\
0,0370
\end{array}\right)+\left(\begin{array}{l}
0,0510 \\
0,0269 \\
0,0988 \\
0,0463
\end{array}\right)=\left(\begin{array}{l}
0,2179 \\
0,1151 \\
0,4467 \\
0,2203
\end{array}\right)
\end{aligned}
$$

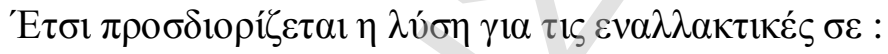

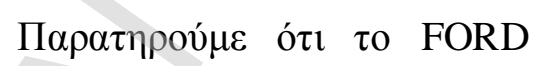

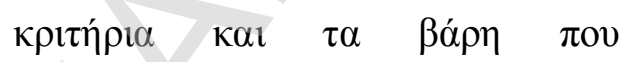

\begin{tabular}{|c|c|}
\hline $\begin{array}{ll} & \text { OP } \\
\text { EL } & \end{array}$ &, 2179 \\
\hline $\begin{array}{ll} & \text { FI } \\
\text { AT } & \end{array}$ &, 1151 \\
\hline $\begin{array}{ll} & \mathrm{FO} \\
\mathrm{RD} & \end{array}$ & $\begin{array}{r}0 \\
, 4467\end{array}$ \\
\hline $\begin{array}{r}\text { CI } \\
\text { TROEN }\end{array}$ & 2203, \\
\hline
\end{tabular}

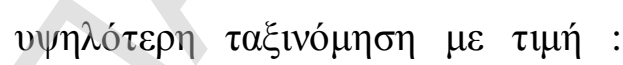

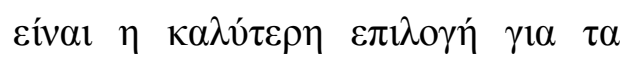

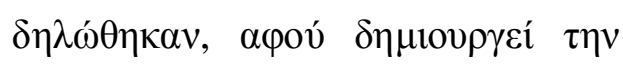
0,4467 . 


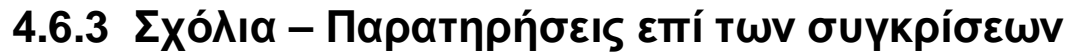

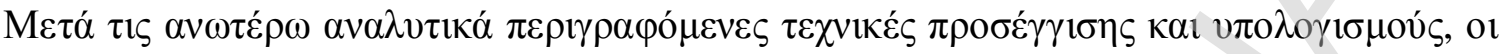

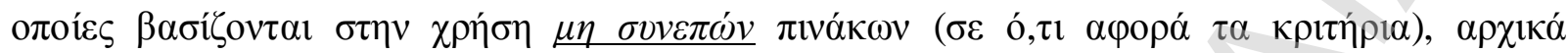

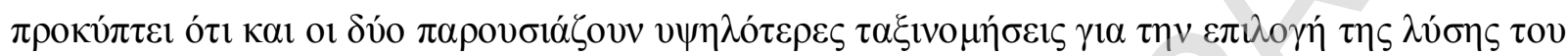

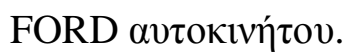

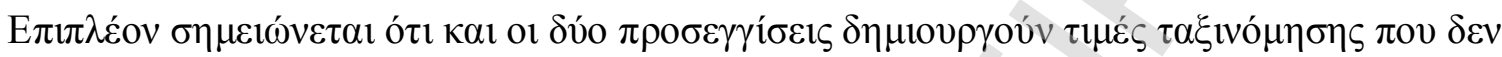

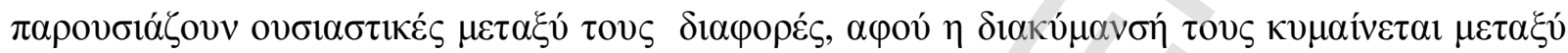
$0,42 \%$ ка $1,43 \%$.

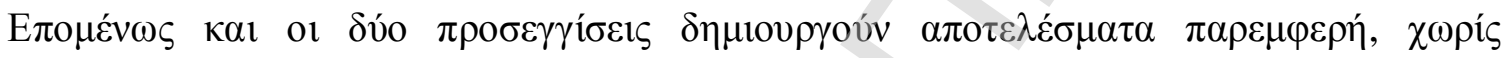

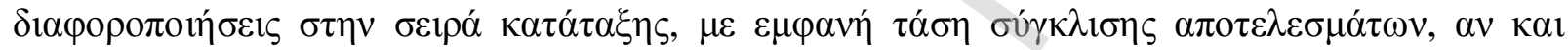

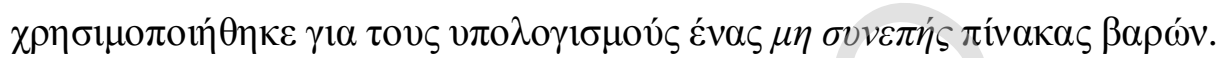

\begin{tabular}{|c|c|c|}
\hline$X \rho \dot{\sigma} \sigma \eta \mu \eta{ }^{\prime} \sigma v v \varepsilon \pi \dot{v} v \pi v a ́ \kappa \omega v$ & $I^{\prime \prime}$ ПРОГЕГГІГН & $2^{\eta}$ ПРОЕЕГГІऽН \\
\hline OPEL & 0,2322 & 0,2179 \\
\hline FIAT & 0,1193 & 0,1151 \\
\hline FORD & $\mathbf{0 , 4 3 4 5}$ & 0,4467 \\
\hline CITROEN & 0,2140 & 0,2203 \\
\hline
\end{tabular}




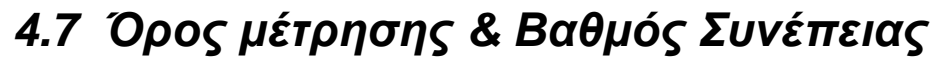

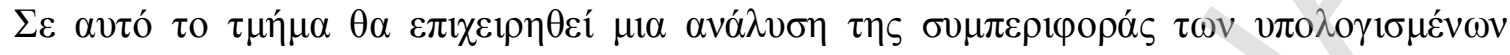

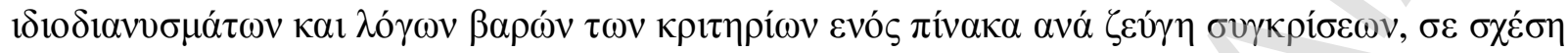

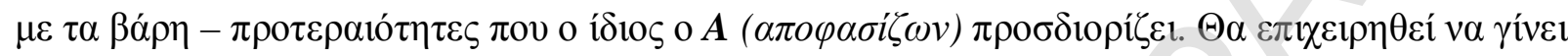

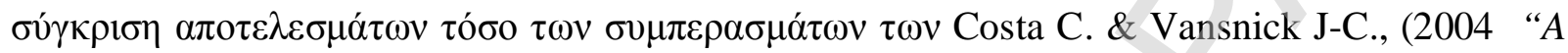
fundamental criticism to Saaty's use of the eigenvalue procedure to derive priorities" ) ófo $\mu \varepsilon \tau \alpha$

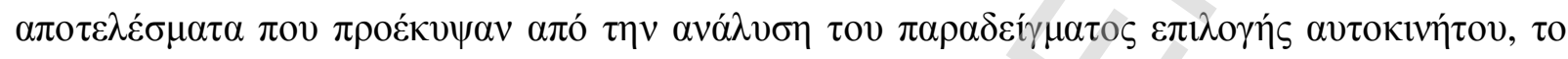

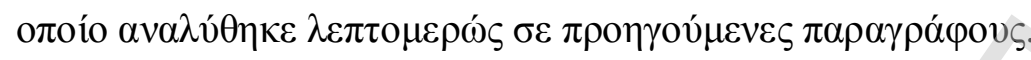

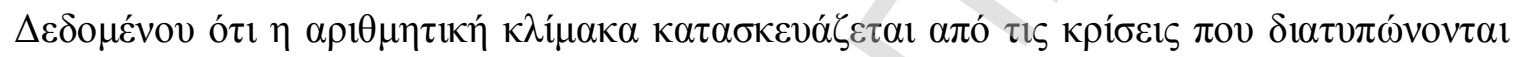

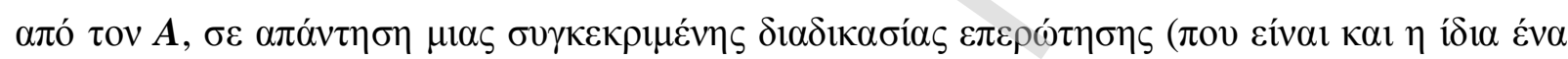

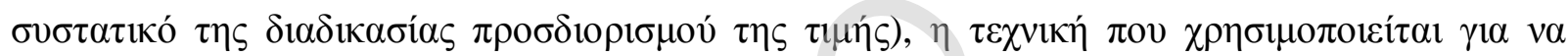

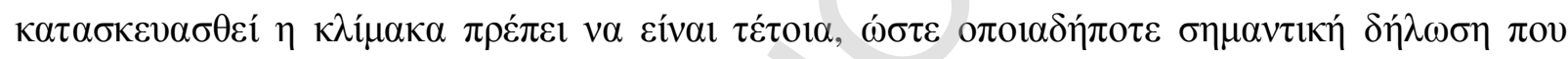

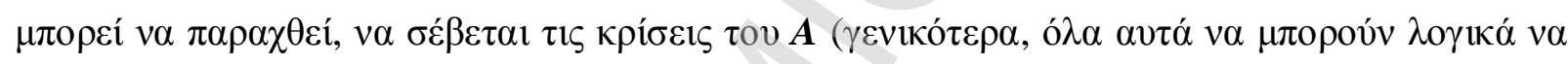

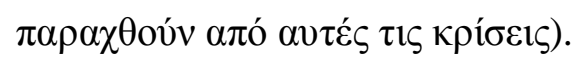

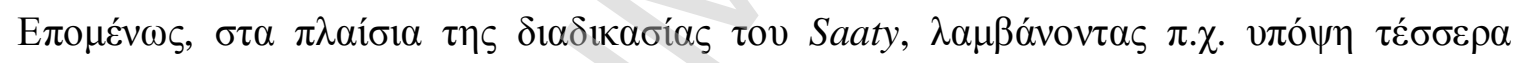

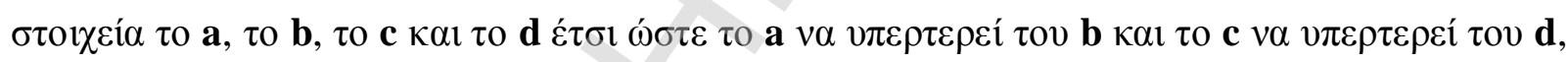

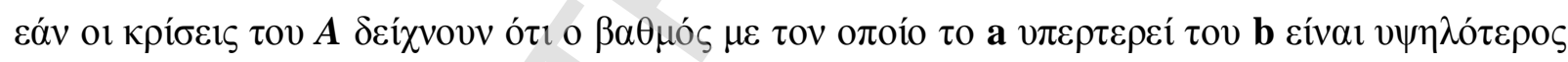

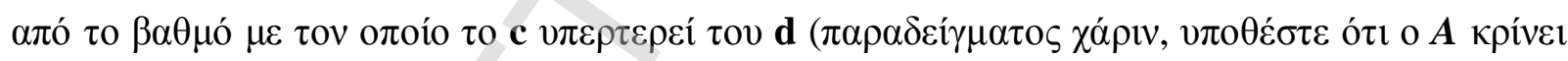

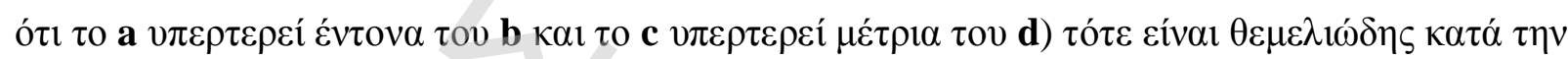

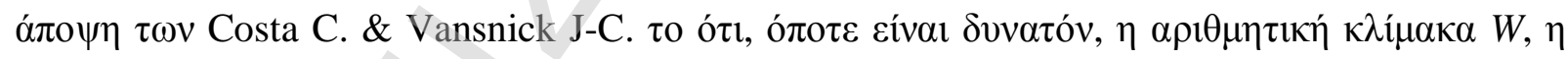

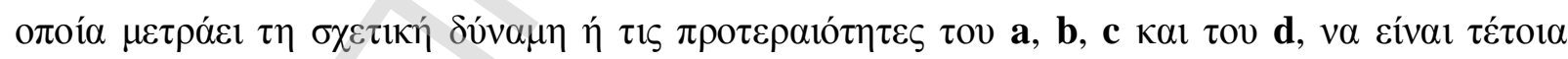

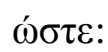

$$
\frac{w(a)}{w(b)}>\frac{w(c)}{w(d)}
$$

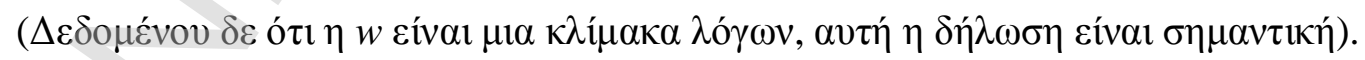

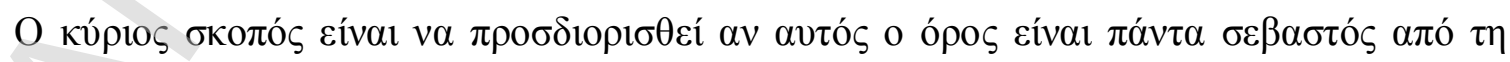

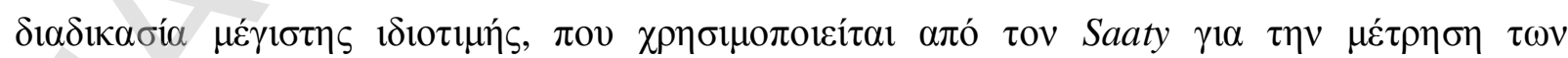

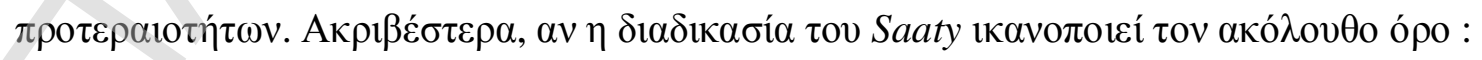

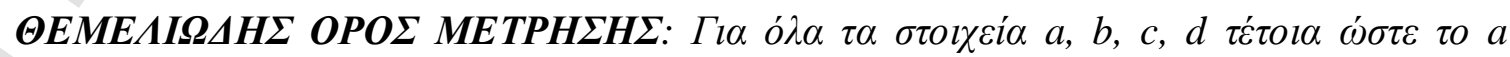

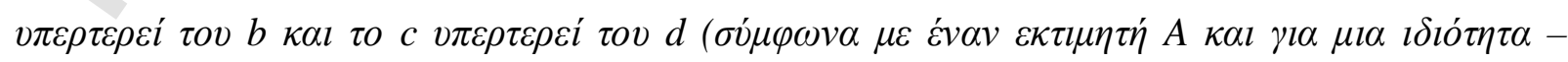

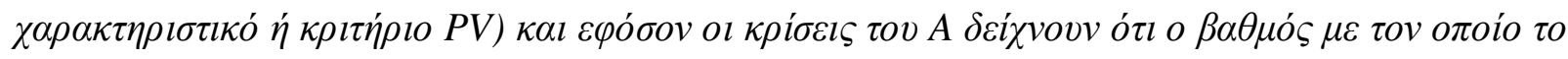




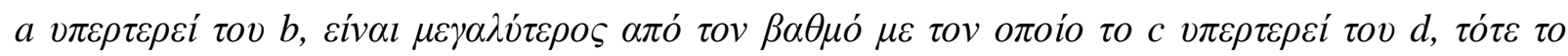

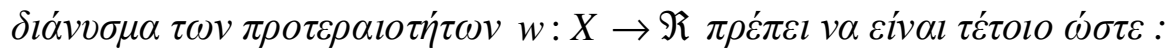

$$
w(a) / w(b)>w(c) / w(d) .
$$

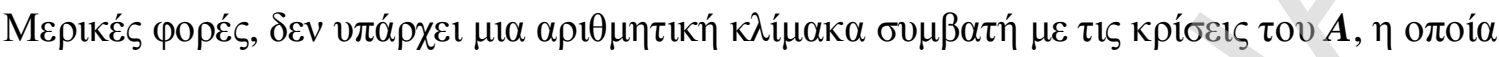

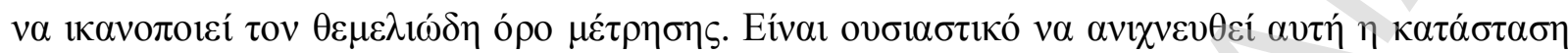

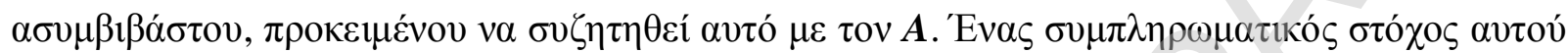

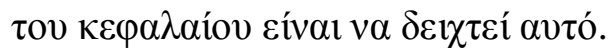

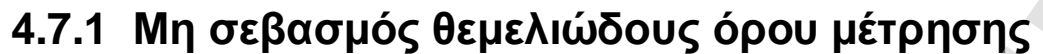

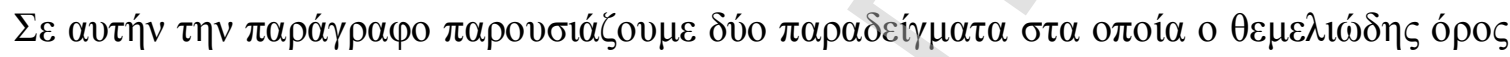

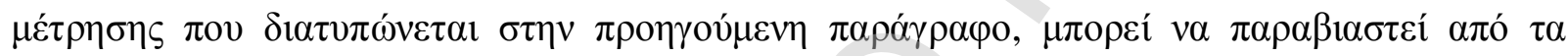

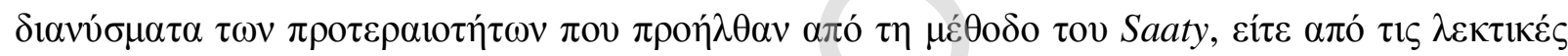

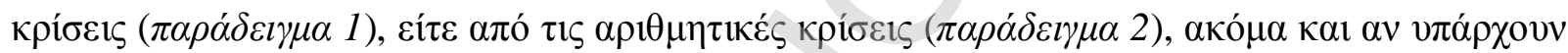

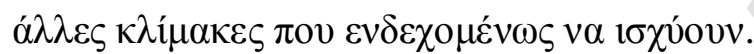

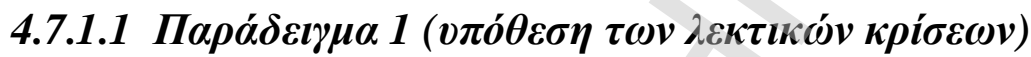

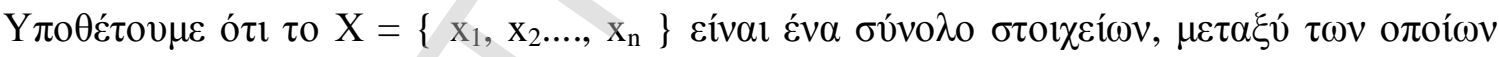

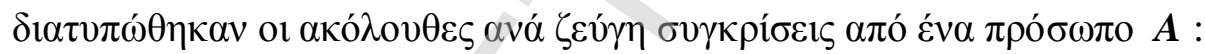

\begin{tabular}{|c|c|}
\hline$\left\{\mathrm{x}_{1}, \mathrm{x}_{2}\right\}$ & 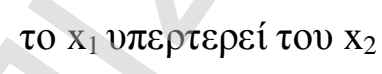 \\
\hline$\left\{\mathrm{x}_{1}, \mathrm{x}_{3}\right\}$ & 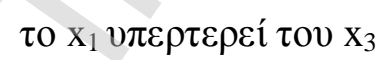 \\
\hline$\left\{\mathrm{x}_{1}, \mathrm{x}_{4}\right\}$ & 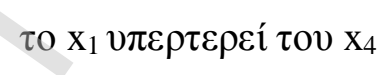 \\
\hline & 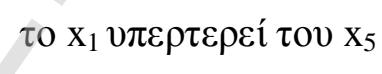 \\
\hline & 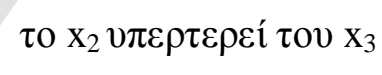 \\
\hline$\left\{\mathrm{x}_{2}, \mathrm{x}_{4}\right\}$ & 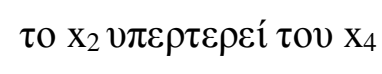 \\
\hline$\left\{\mathrm{x}_{2}, \mathrm{x}_{5}\right\}$ & 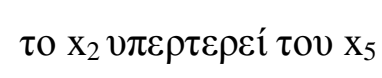 \\
\hline$\left\{x_{3}, x_{4}\right\}$ & 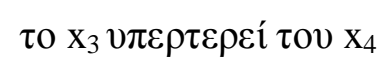 \\
\hline$\left\{\mathrm{x}_{3}, \mathrm{x}_{5}\right\}$ & 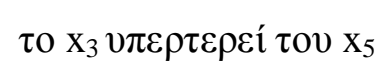 \\
\hline$\left\{\mathrm{x}_{4}, \mathrm{x}_{5}\right\}$ & 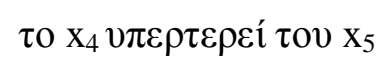 \\
\hline
\end{tabular}

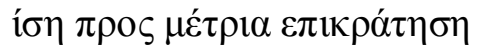

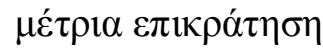

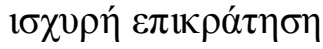

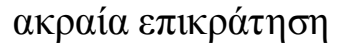

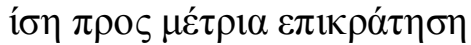

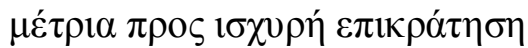

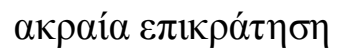

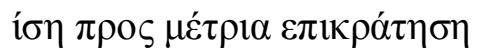

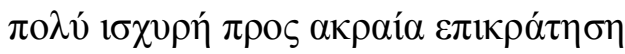

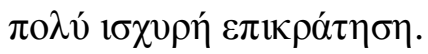




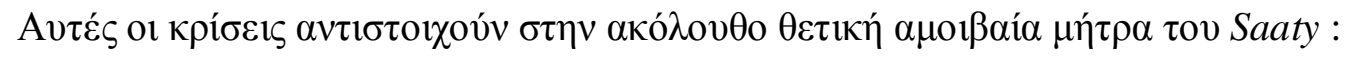

$$
\left[\begin{array}{lcccc}
1 & 2 & 3 & 5 & 9 \\
1 / 2 & 1 & 2 & 4 & 9 \\
1 / 3 & 1 / 2 & 1 & 2 & 8 \\
1 / 5 & 1 / 4 & 1 / 2 & 1 & 7 \\
1 / 9 & 1 / 9 & 1 / 8 & 1 / 7 & 1
\end{array}\right]
$$

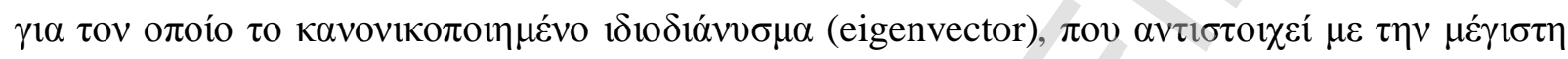

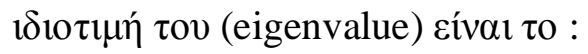

$$
\left(\begin{array}{l}
0,426 \\
0,281 \\
0,165 \\
0,101 \\
0,027
\end{array}\right)
$$

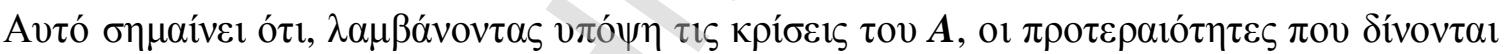

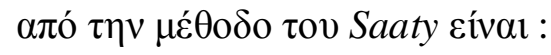

$$
\begin{aligned}
& w\left(x_{1}\right)=0,426 \\
& w\left(x_{2}\right)=0,281 \\
& w\left(x_{3}\right)=0,165 \\
& w\left(x_{4}\right)=0,101 \\
& w\left(x_{5}\right)=0,027
\end{aligned}
$$

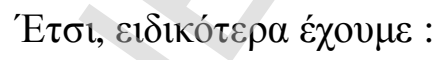

$$
\frac{w\left(x_{1}\right)}{w\left(x_{4}\right)} \approx 4,218 \kappa \alpha \mathrm{l} \frac{w\left(x_{4}\right)}{w\left(x_{5}\right)} \approx 3,741
$$

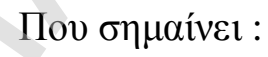

$$
\frac{w\left(x_{1}\right)}{w\left(x_{4}\right)}>\frac{w\left(x_{4}\right)}{w\left(x_{5}\right)}
$$




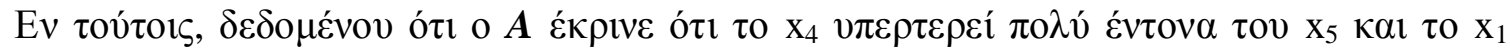

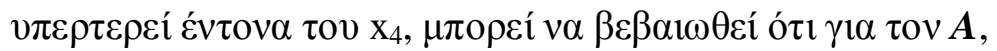

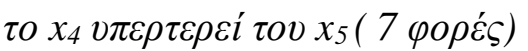

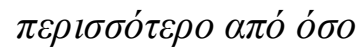

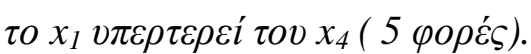

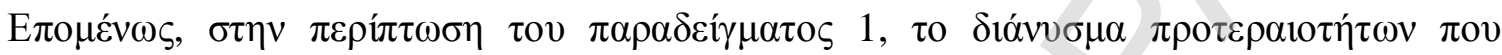

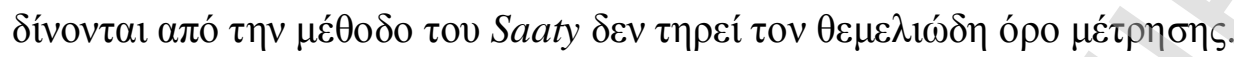

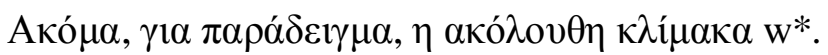

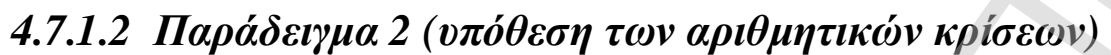

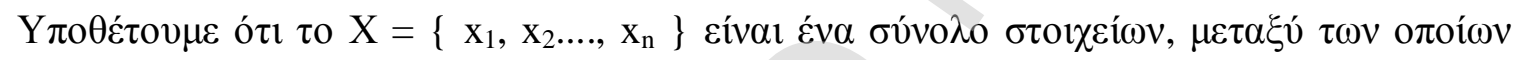

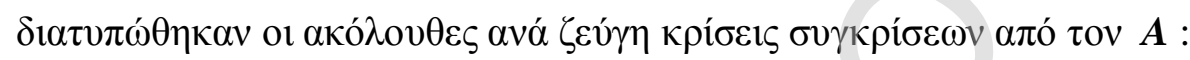

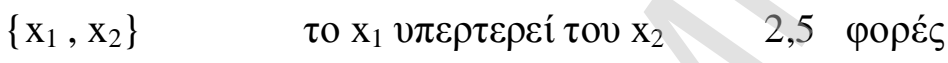

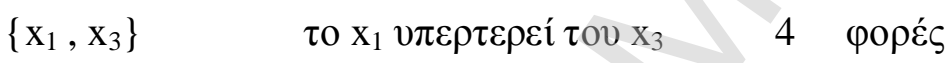

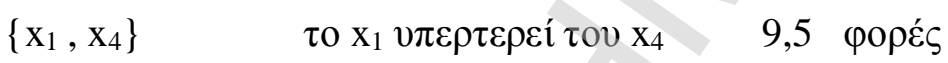

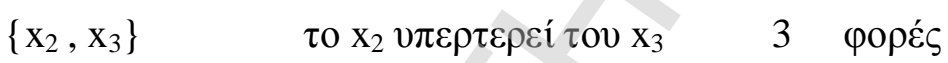

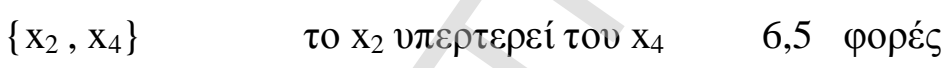

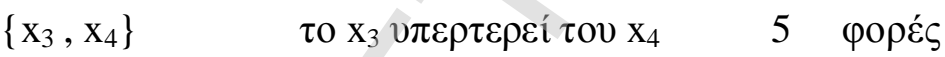

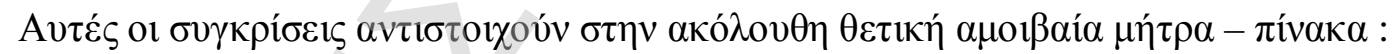

$$
\left[\begin{array}{lccc}
1 & 2,5 & 4 & 9,5 \\
1 / 2,5 & 1 & 3 & 6,5 \\
1 / 4 & 1 / 3 & 1 & 5 \\
1 / 9,5 & 1 / 6,5 & 1 / 5 & 1
\end{array}\right]
$$

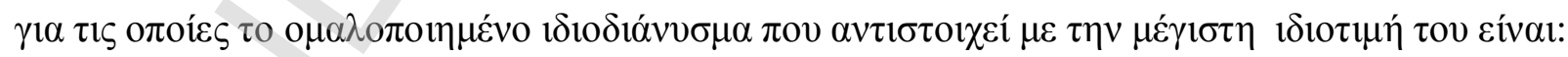

$$
\left[\begin{array}{l}
0,533 \\
0,287 \\
0,139 \\
0,041
\end{array}\right]
$$

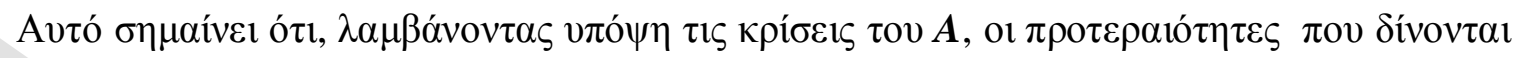

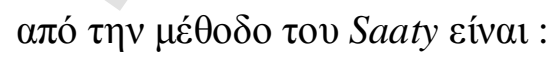




$$
\begin{aligned}
& w\left(x_{1}\right)=0,533 \\
& w\left(x_{2}\right)=0,287 \\
& w\left(x_{3}\right)=0,139 \\
& w\left(x_{4}\right)=0,041
\end{aligned}
$$

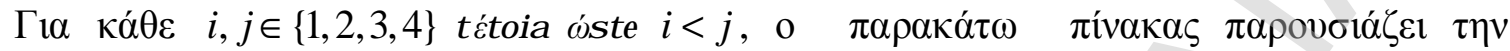

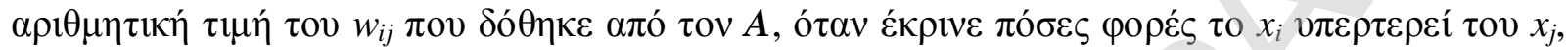

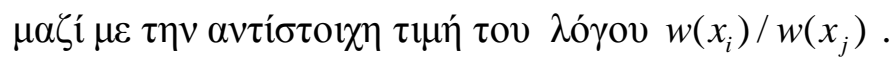

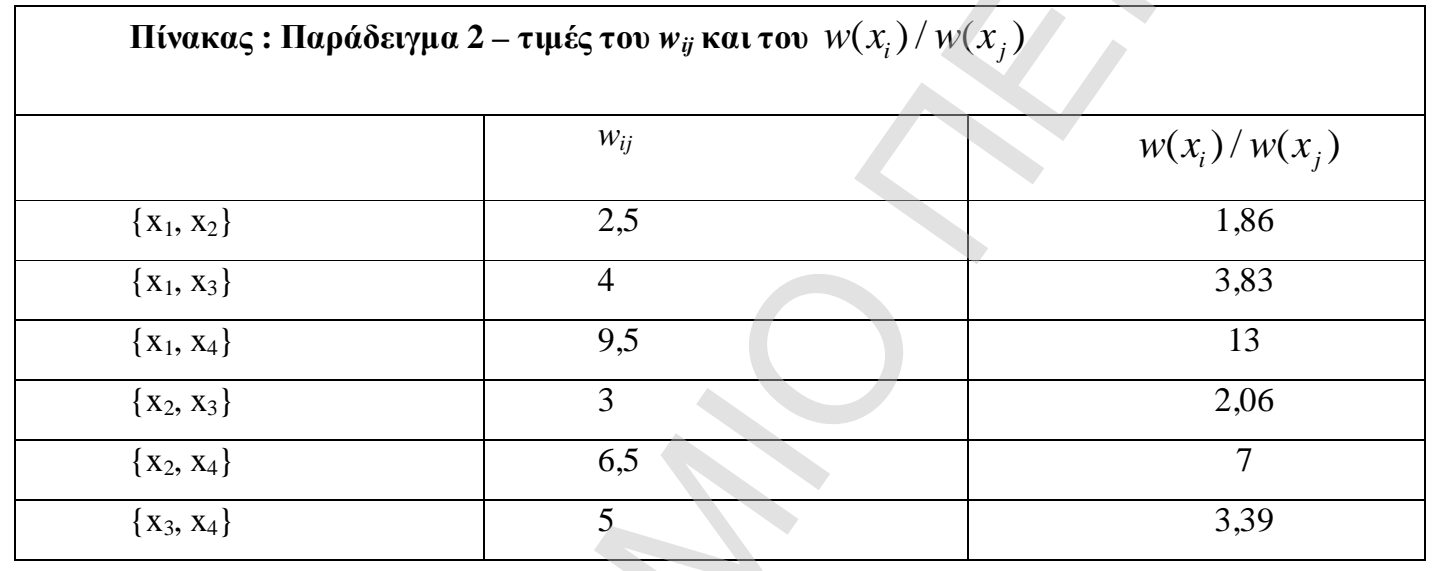

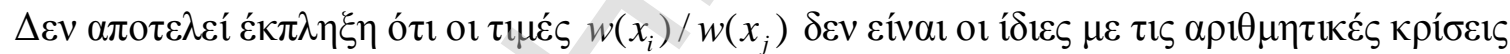

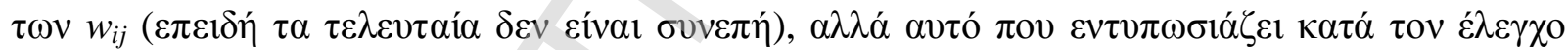

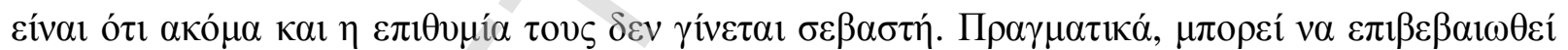

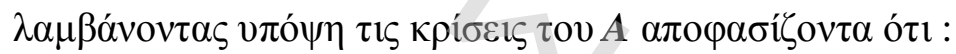

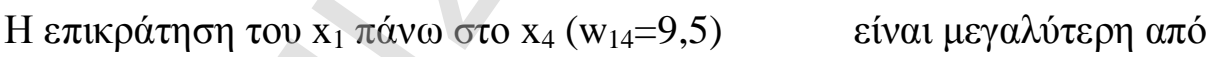

$\tau \eta v \varepsilon \pi$

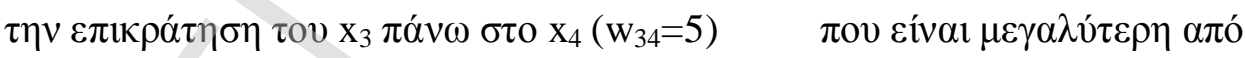

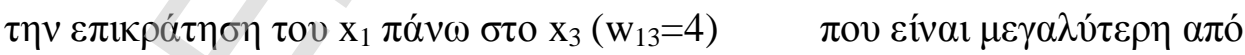

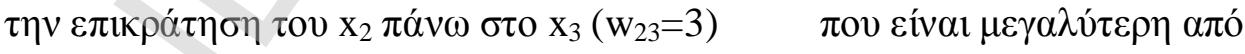

$\tau \eta v \varepsilon \pi \iota \kappa \rho \alpha ́ \tau \eta \sigma \eta ~ \tau o v ~ \mathrm{x}_{1} \pi \alpha ́ v \omega \sigma \tau \mathrm{O} \mathrm{x}_{2}\left(\mathrm{w}_{12}=2,5\right)$

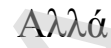

$$
3,39=\frac{w\left(x_{3}\right)}{w\left(x_{4}\right)}<\frac{w\left(x_{1}\right)}{w\left(x_{3}\right)}=3,83 .
$$

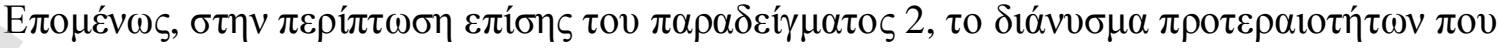

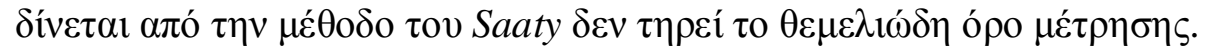




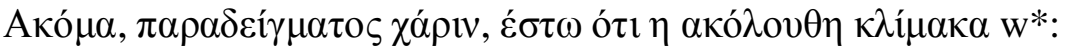

$$
\begin{aligned}
& w^{*}\left(x_{1}\right)=0,48 \\
& w^{*}\left(x_{2}\right)=0,32 \\
& w^{*}\left(x_{3}\right)=0,16 \\
& w^{*}\left(x_{4}\right)=0,04
\end{aligned}
$$

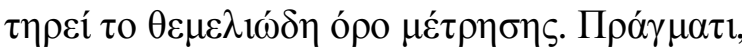

$$
\frac{w^{*}\left(x_{1}\right)}{w^{*}\left(x_{4}\right)}=12>\frac{w^{*}\left(x_{2}\right)}{w^{*}\left(x_{4}\right)}=8>\frac{w^{*}\left(x_{3}\right)}{w^{*}\left(x_{4}\right)}=4>\frac{w^{*}\left(x_{1}\right)}{w^{*}\left(x_{3}\right)}=3>\frac{w^{*}\left(x_{2}\right)}{w^{*}\left(x_{3}\right)}=2>\frac{w^{*}\left(x_{1}\right)}{w^{*}\left(x_{2}\right)}=1,5
$$

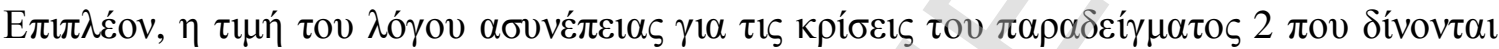

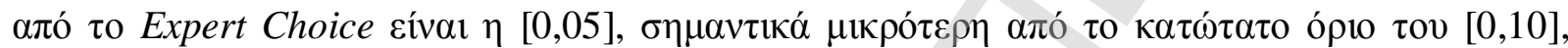

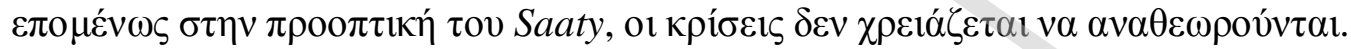

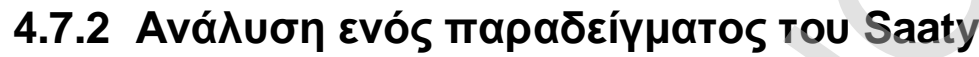

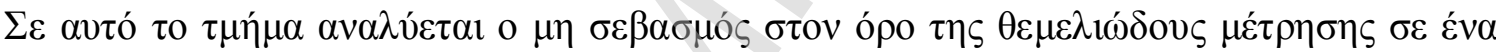

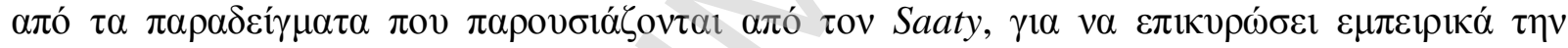

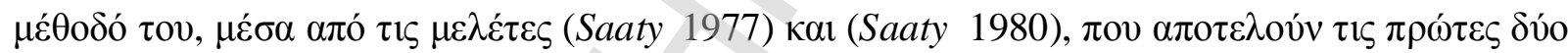

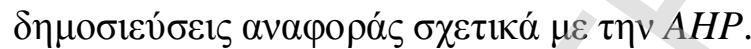

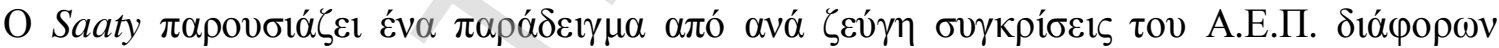

\begin{tabular}{|c|c|c|c|c|c|c|c|}
\hline & & $U, S . S . R$ & China & France & $U \cdot K$ & Japan & $w . C$ \\
\hline$U \cdot S$ & 1 & 4 & 9 & 6 & 6 & 5 & 5 \\
\hline$U . S . S . R$ & $\frac{1}{4}$ & 1 & 7 & 5 & 5 & 3 & 4 \\
\hline Chin $a$ & $\frac{1}{9}$ & $\frac{1}{7}$ & 1 & $\frac{1}{5}$ & $\frac{1}{5}$ & $\frac{1}{7}$ & $\frac{1}{5}$ \\
\hline France & $\frac{1}{6}$ & $\frac{1}{5}$ & 5 & 1 & 1 & $\frac{1}{3}$ & $\frac{1}{3}$ \\
\hline$U \cdot K$ & $\frac{1}{6}$ & $\frac{1}{5}$ & 5 & 1 & 1 & $\frac{1}{3}$ & $\frac{1}{3}$ \\
\hline Japan & $\frac{1}{5}$ & $\frac{1}{3}$ & 7 & 3 & 3 & 1 & 2 \\
\hline W. Germany & $\frac{1}{5}$ & $\frac{1}{4}$ & 5 & 3 & 3 & $\frac{1}{2}$ & 1 \\
\hline
\end{tabular}

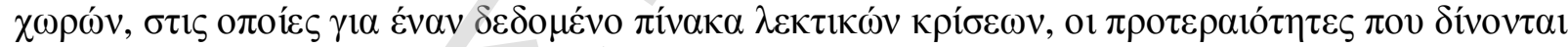

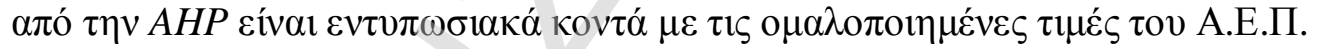

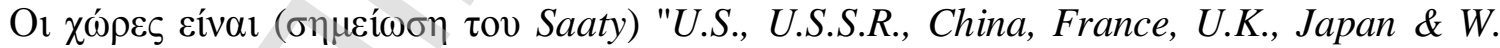

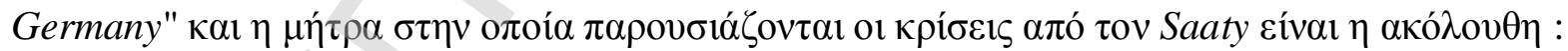




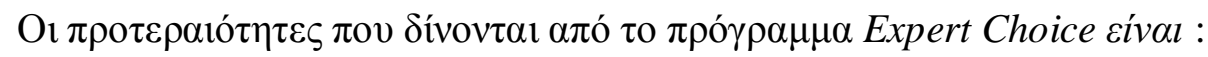

$\begin{array}{ll}\mathrm{w}(\text { U.S. }) & =0,427 \\ \mathrm{w}(\text { U.S.S.R. }) & =0,230 \\ \mathrm{w}(\text { China }) & =0,021 \\ \mathrm{w}(\text { France }) & =0,052 \\ \mathrm{w}(\text { U.K. }) & =0,052 \\ \mathrm{w}(\text { Japan }) & =0,123 \\ \mathrm{w}(\text { W. Germany }) & =0,094 .\end{array}$

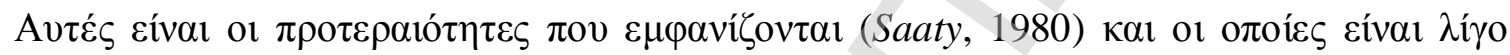

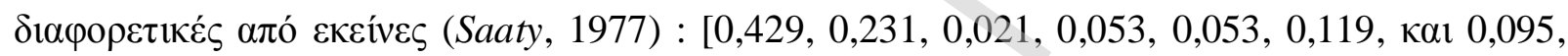

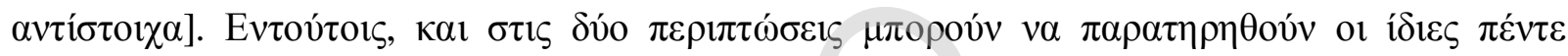

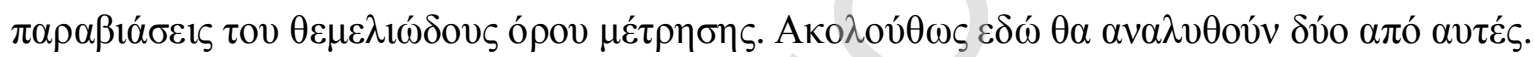

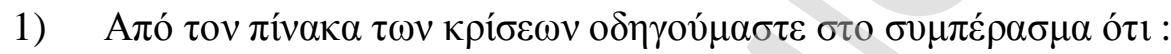

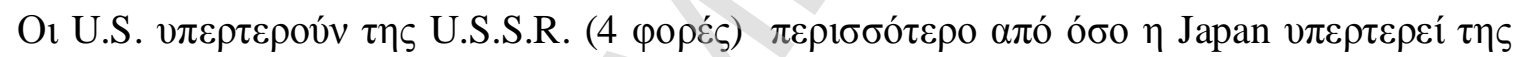
France $\left(3\right.$ $\left.\varphi \rho \varepsilon_{\varsigma}\right)$.

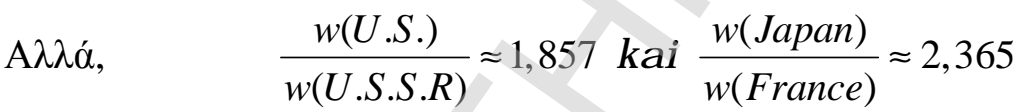

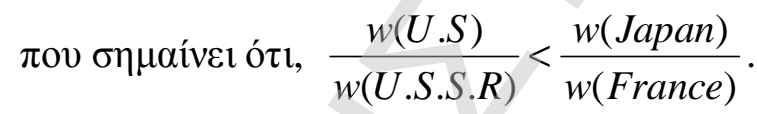

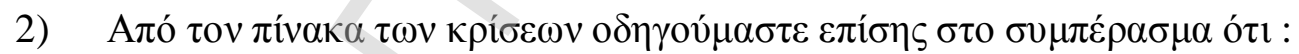

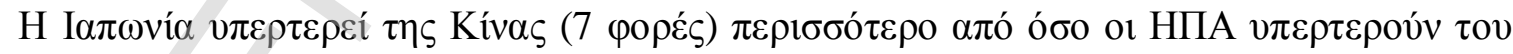

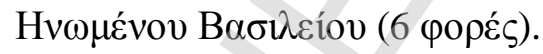

$\mathrm{A} \lambda \lambda \alpha \dot{\alpha}, \frac{w(\text { Japan })}{w(\text { China })} \approx 5,857 \kappa \alpha \imath \frac{w(\text { U.S. })}{w(\text { U.K. })} \approx 8,212$

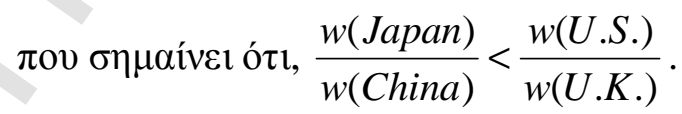




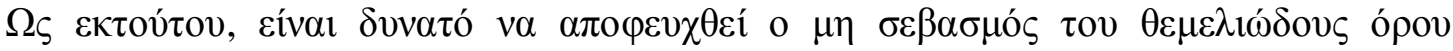

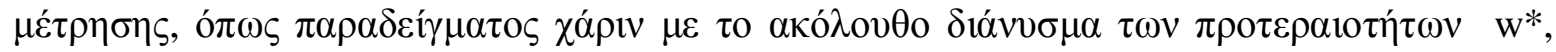

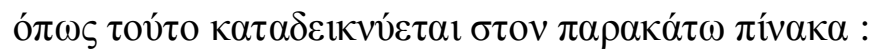

$\begin{array}{ll}\mathrm{w}^{*}(\text { U.S. }) & =0,414 \\ \mathrm{w}^{*}(\text { U.S.S.R. }) & =0,217 \\ \mathrm{w}^{*}(\text { China }) & =0,019 \\ \left.\mathrm{w}^{*} \text { (France }\right) & =0,069 \\ \mathrm{w}^{*}(\text { U.K. }) & =0,069 \\ \mathrm{w}^{*}(\text { Japan }) & =0,117 \\ \mathrm{w}^{*}(\text { W. Germany }) & =0,095 .\end{array}$

\begin{tabular}{|c|c|}
\hline \multicolumn{2}{|c|}{ 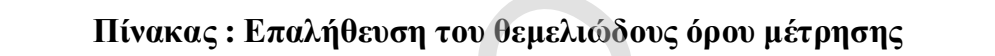 } \\
\hline 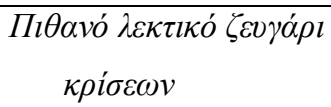 & 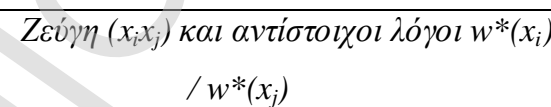 \\
\hline 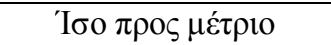 & (Japan, W. Germany): 1,23 \\
\hline & $\begin{array}{l}\text { (W. Germany, France): 1,38 } \\
\text { (W. Germany, U.K.): 1,38 } \\
\text { (Japan, France): 1,70 } \\
\text { (Japan, U.K.): } 1,70 \\
\text { (U.S.S.R., Japan): 1,85 }\end{array}$ \\
\hline 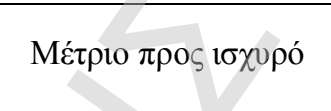 & $\begin{array}{l}\text { (U.S., U.S.S.R.): } 1,91 \\
\text { (U.S.S.R., W. Germany): } 2,28\end{array}$ \\
\hline 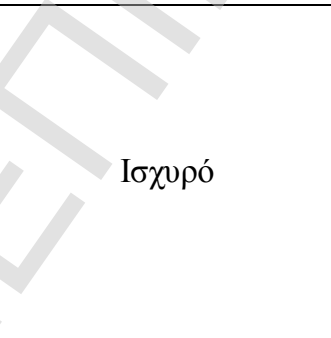 & $\begin{array}{l}\text { (U.S.S.R., France): } 3,14 \\
\text { (U.S.S.R., U.K.): 3,14 } \\
\text { (U.S., Japan): 3,54 } \\
\text { (U.K., China): 3,63 } \\
\text { (France, China): 3,63 } \\
\text { (U.S., W. Germany): 4,36 }\end{array}$ \\
\hline 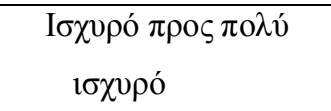 & $\begin{array}{l}\text { (U.S., France): 6,00 } \\
\text { (U.S., U.K.): } 6,00\end{array}$ \\
\hline 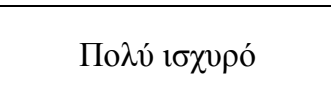 & $\begin{array}{l}\text { (Japan, China): 6,16 } \\
\text { (U.S.S.R., China): 11,42 }\end{array}$ \\
\hline 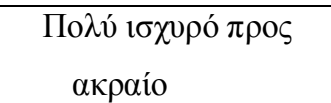 & $\varnothing$ \\
\hline
\end{tabular}




\begin{tabular}{|c|c|}
\hline Aкраío & (U.S., China): 21,79 \\
\hline
\end{tabular}

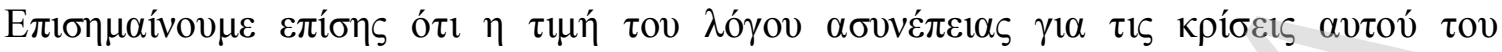

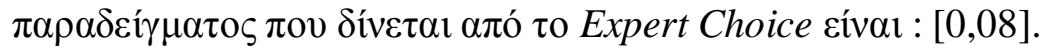

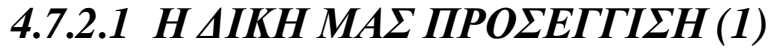

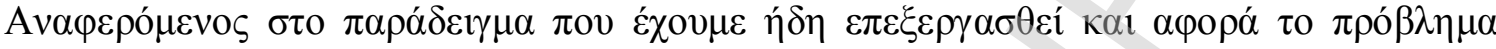

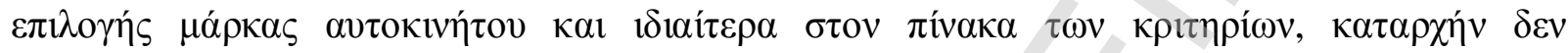

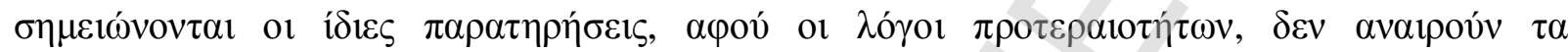

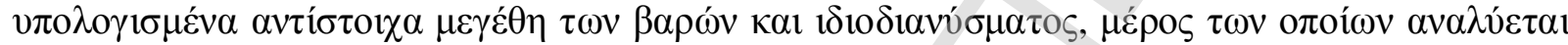
$\alpha \kappa \circ \lambda \circ \theta_{\theta \omega \varsigma}$.

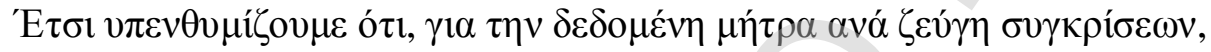

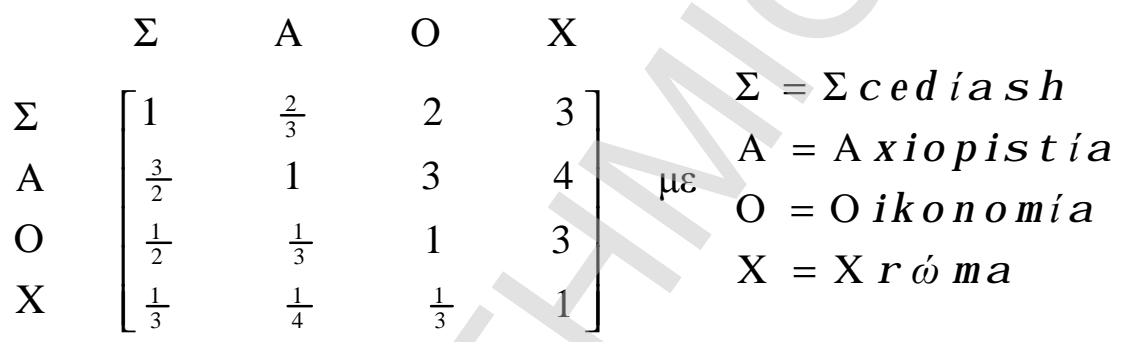

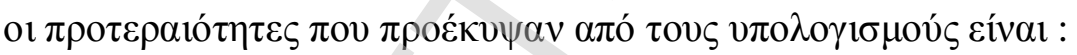




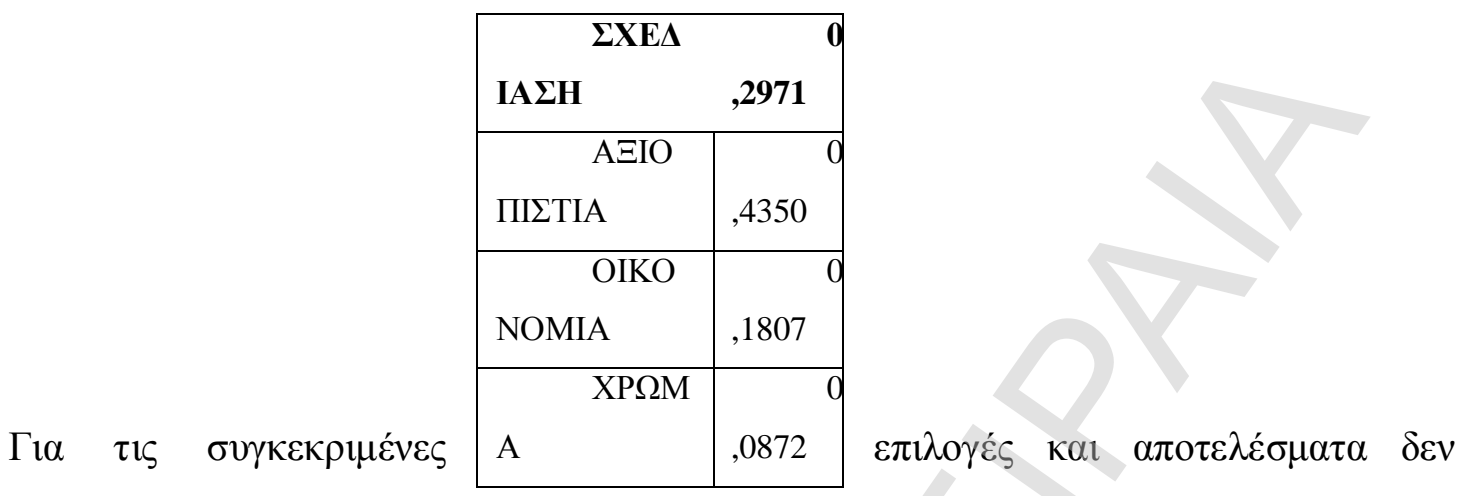

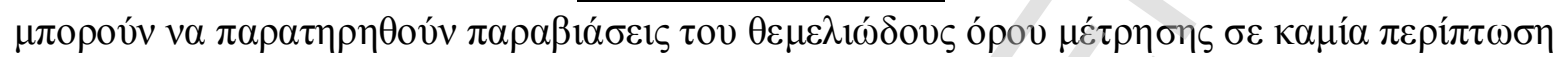

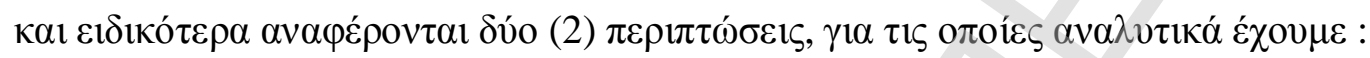

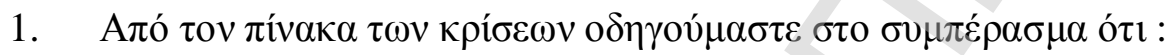

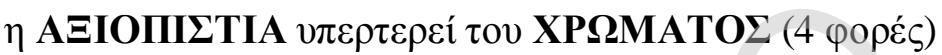

$\pi \varepsilon \rho 1 \sigma \sigma o ́ \tau \varepsilon \rho o ~ \alpha \pi$ ó $\sigma o$

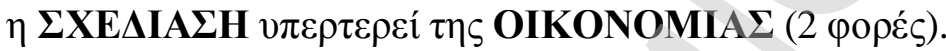

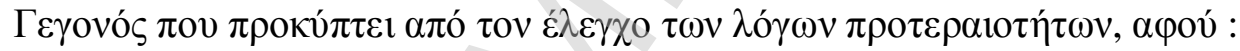

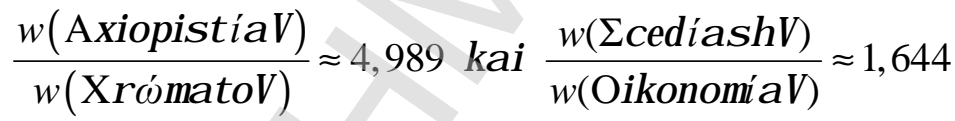

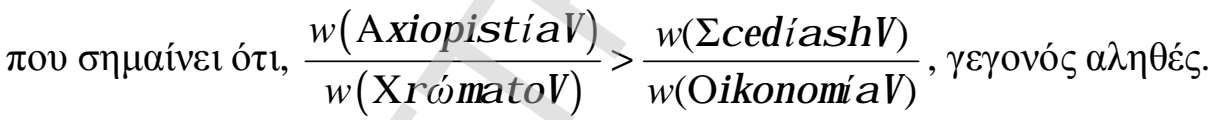

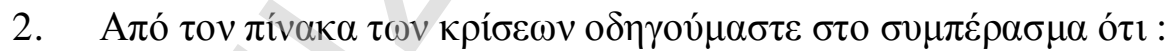

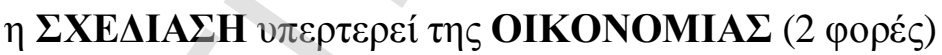

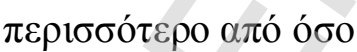

$\eta \mathbf{A \Xi I O \Pi I \Sigma T I A ~ v \pi \varepsilon \rho \tau \varepsilon \rho \varepsilon i ́ ~} \tau\rceil \varsigma \mathbf{\Sigma X E \Delta I A \Sigma H \Sigma ~ ( 3 / 2 ~ \varphi о \rho \varepsilon ́ \varsigma ) . ~}$

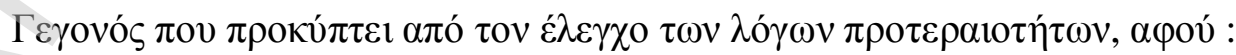

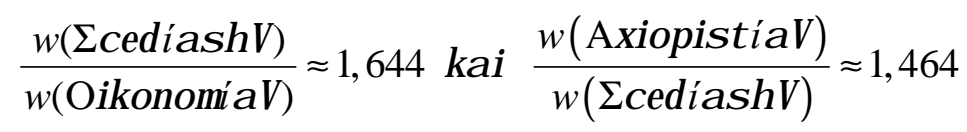

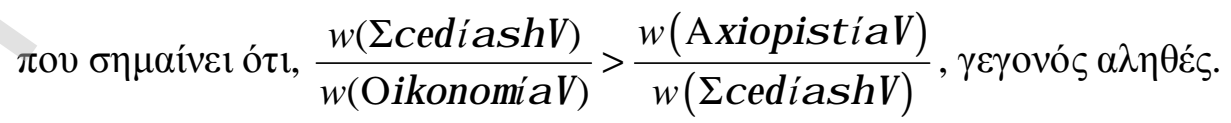




\begin{tabular}{|c|c|}
\hline \multicolumn{2}{|c|}{ 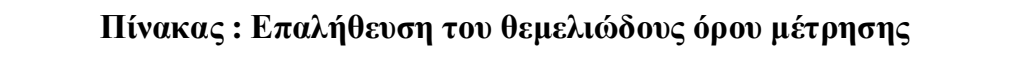 } \\
\hline 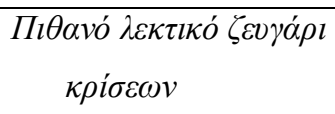 & 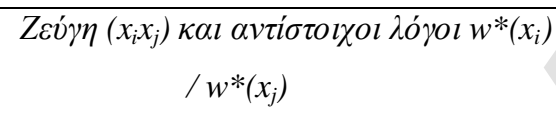 \\
\hline 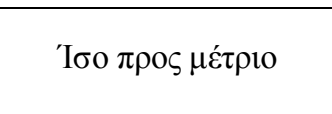 & 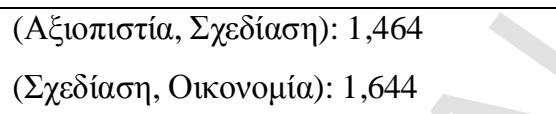 \\
\hline Mét $\rho ı$ & 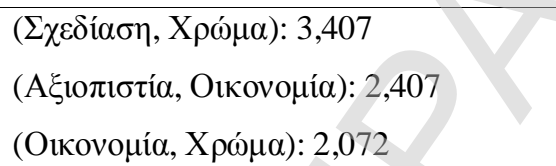 \\
\hline 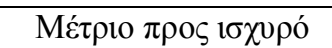 & 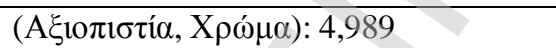 \\
\hline
\end{tabular}

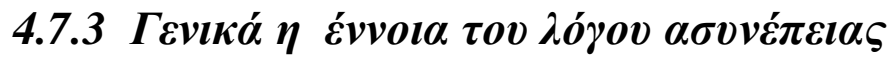

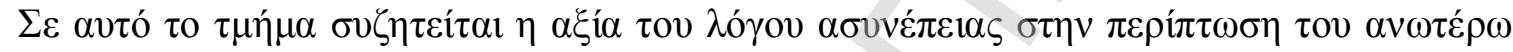

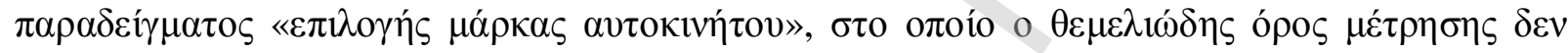

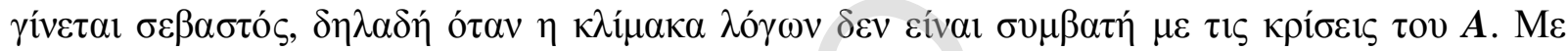

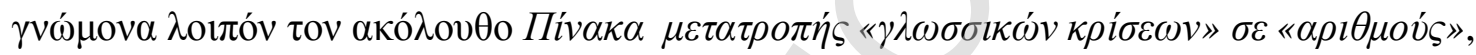

\begin{tabular}{|c|c|}
\hline \multicolumn{2}{|c|}{ 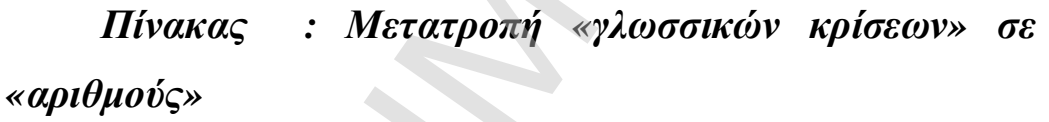 } \\
\hline 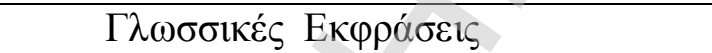 & $\Sigma \chi \varepsilon \tau \imath$ соі́ $\alpha \rho \imath \theta \mu$ оí \\
\hline Iøך $\alpha \xi i ́ \alpha$ & 1 \\
\hline 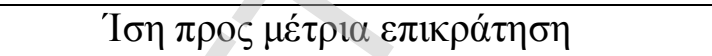 & 2 \\
\hline 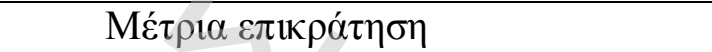 & 3 \\
\hline 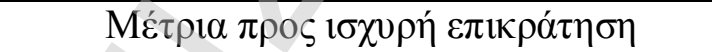 & 4 \\
\hline 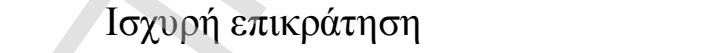 & 5 \\
\hline 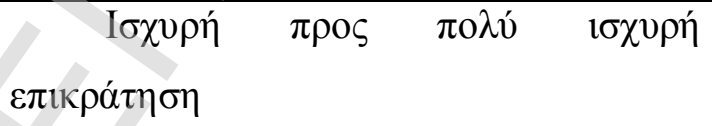 & 6 \\
\hline 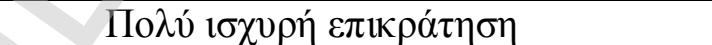 & 7 \\
\hline 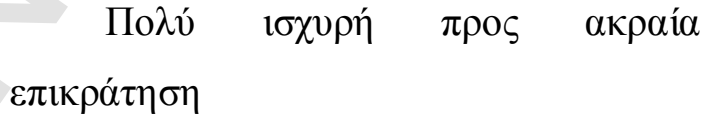 & 8 \\
\hline 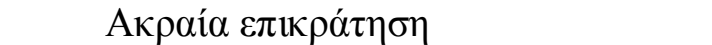 & 9 \\
\hline
\end{tabular}




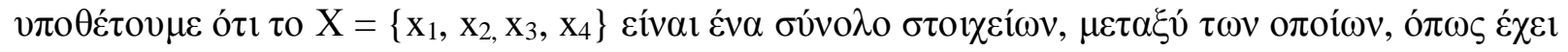

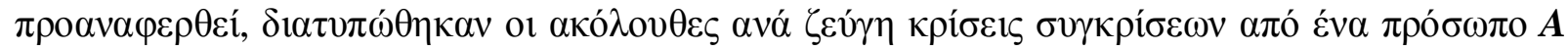
$(\alpha \pi \circ \varphi \alpha \sigma i \zeta \omega v)$.

\begin{tabular}{|c|c|c|c|c|c|c|}
\hline & $\Sigma$ & A & $\mathrm{O}$ & \multicolumn{2}{|l|}{$X$} & \\
\hline$\Sigma$ & {$[1$} & $\frac{2}{3}$ & 2 & 3 & & $\Sigma=\Sigma \chi \varepsilon \delta i \alpha \sigma \eta$ \\
\hline A & $\frac{3}{2}$ & 1 & 3 & 4 & $\mu \varepsilon$ & $\mathrm{A}=\mathrm{A} \xi i 0 \pi \imath \sigma \tau i \alpha$ \\
\hline $\mathrm{O}$ & $\frac{1}{2}$ & $\frac{1}{3}$ & 1 & 3 & & $\mathrm{X}=\mathrm{X}$ \\
\hline$X$ & $\frac{1}{3}$ & $\frac{1}{4}$ & $\frac{1}{3}$ & 1 & & $\Delta-\alpha \beta \cos$ \\
\hline
\end{tabular}

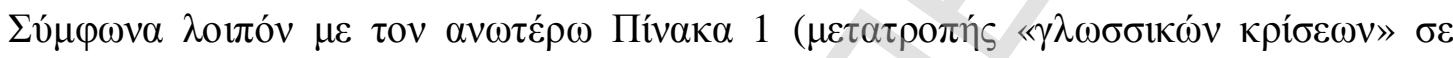

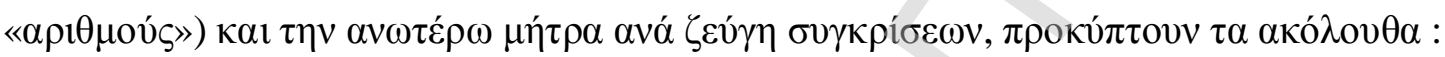

\begin{tabular}{|c|c|c|}
\hline$\left\{\mathrm{x}_{2}, \mathrm{x}_{1}\right\}$ & 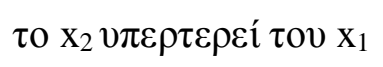 & 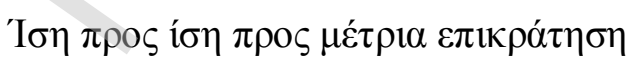 \\
\hline$\left\{\mathrm{x}_{1}, \mathrm{x}_{3}\right\}$ & 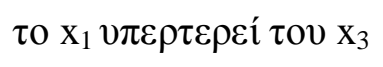 & 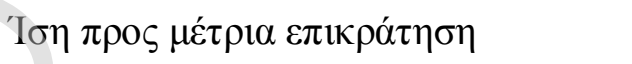 \\
\hline$\left\{\mathrm{x}_{1}, \mathrm{x}_{4}\right\}$ & 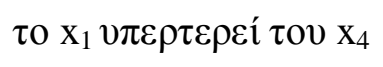 & 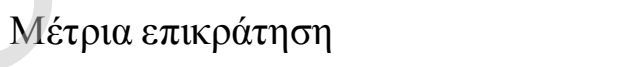 \\
\hline$\left\{\mathrm{x}_{2}, \mathrm{x}_{3}\right\}$ & 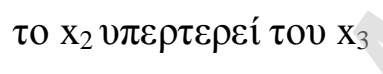 & 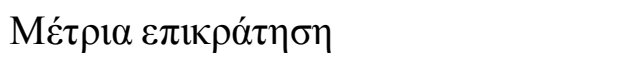 \\
\hline$\left\{x_{2}, x_{4}\right\}$ & 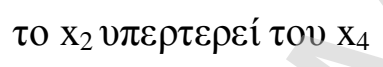 & 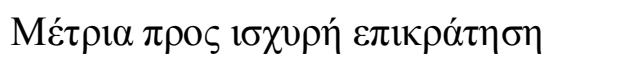 \\
\hline$\left\{x_{3}, x_{4}\right\}$ & $\tau \mathrm{O} \mathrm{x}_{3} v \pi \varepsilon \rho \tau \varepsilon \rho \varepsilon i ́$ $\tau \mathrm{ov} \mathrm{x}_{4}$ & 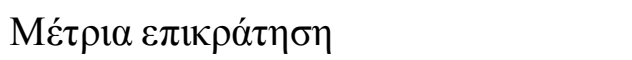 \\
\hline
\end{tabular}

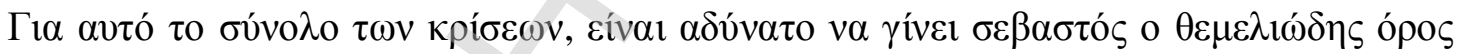

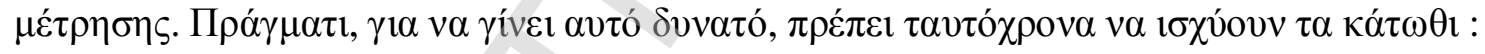

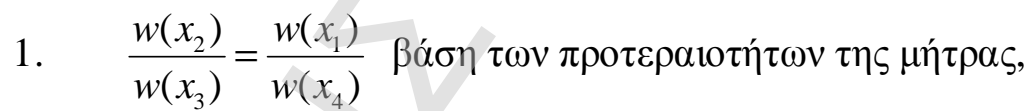

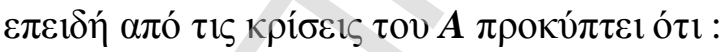

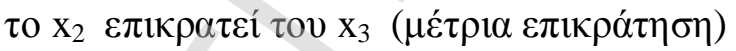

óбo

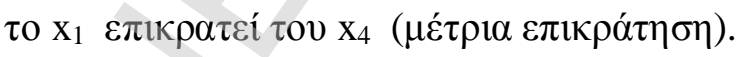

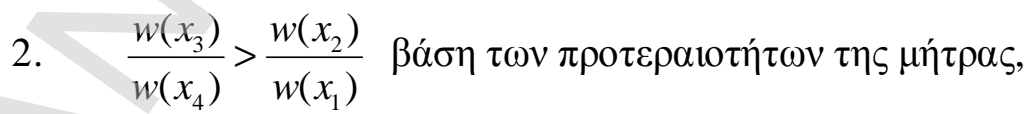

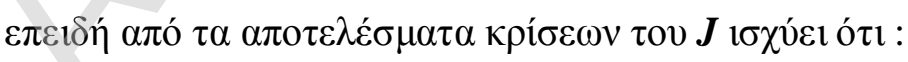

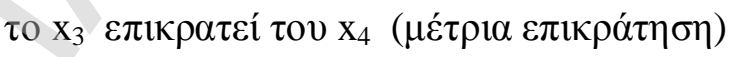

$\pi \varepsilon \rho 1 \sigma \sigma o ́ \tau \varepsilon \rho о \alpha \pi$ ó óбo

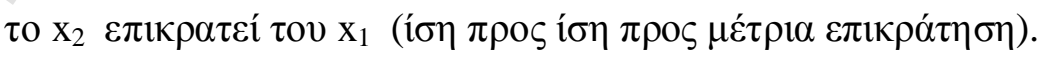




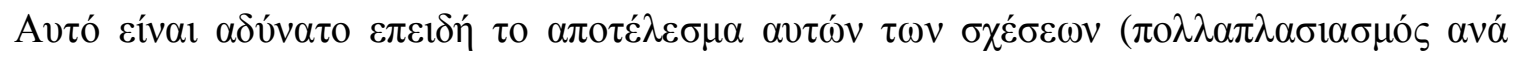

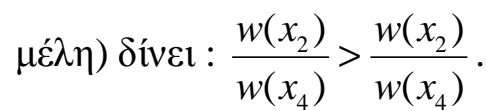

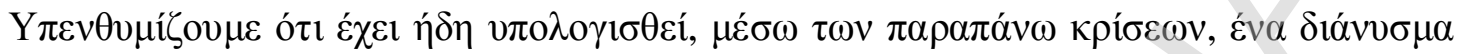

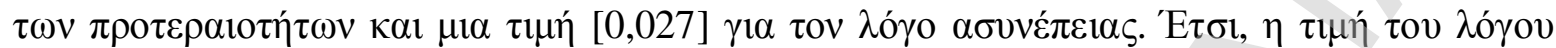

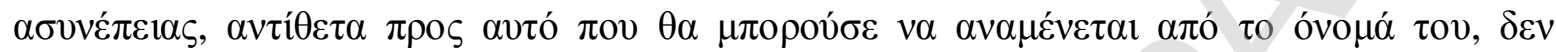

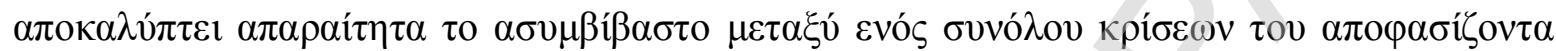

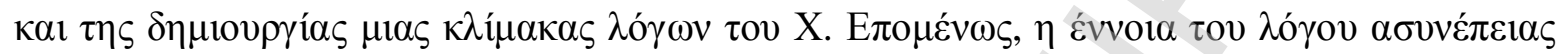

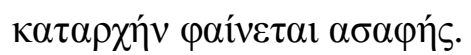

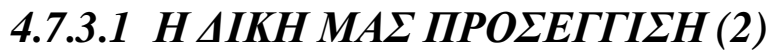

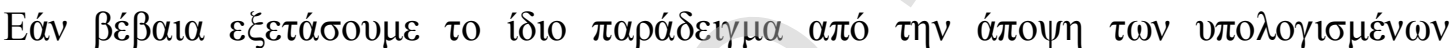

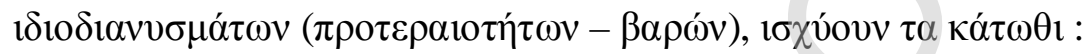

1. $\frac{w\left(x_{2}\right)}{w\left(x_{3}\right)}<\frac{w\left(x_{1}\right)}{w\left(x_{4}\right)}, \quad \alpha \varphi \circ v^{\prime}$

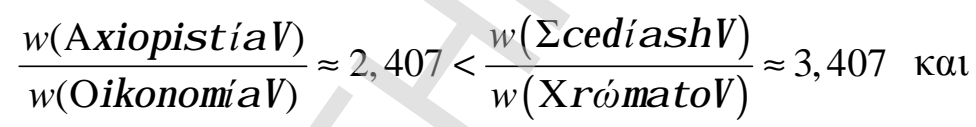

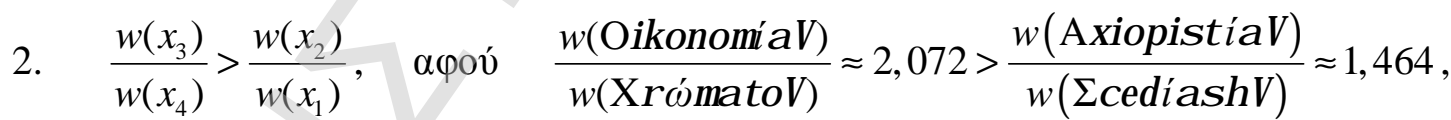

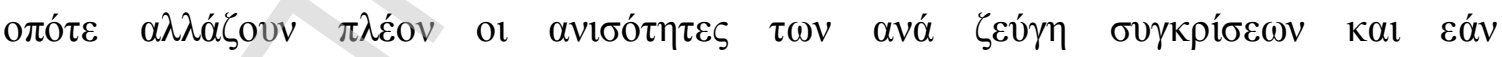

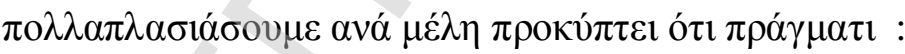

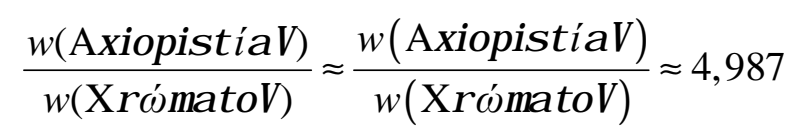

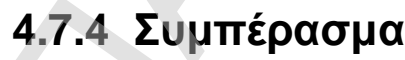

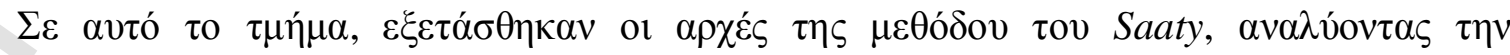

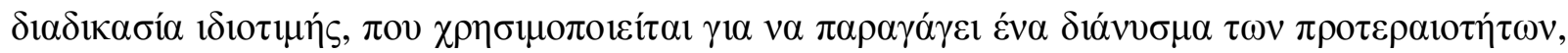




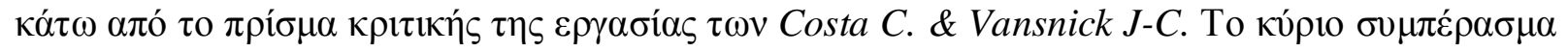

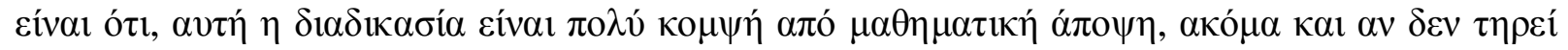

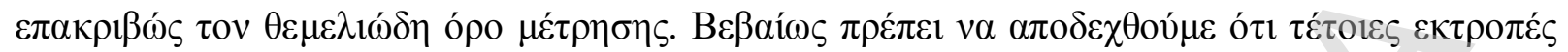

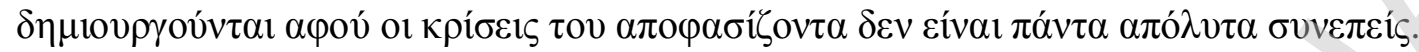

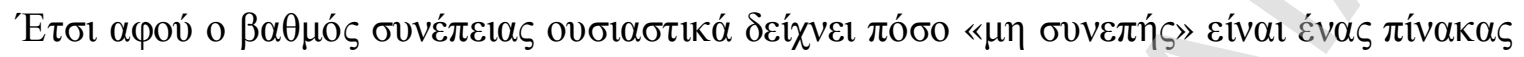

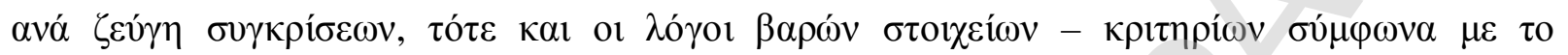

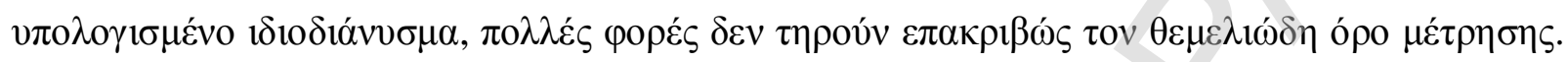

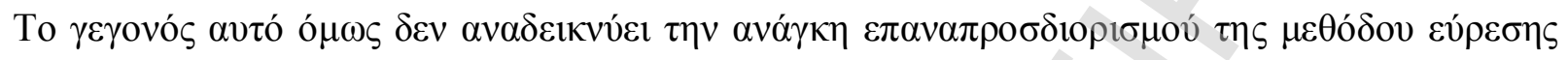

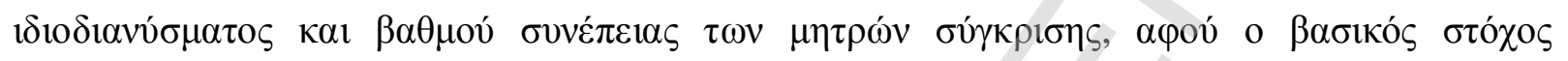

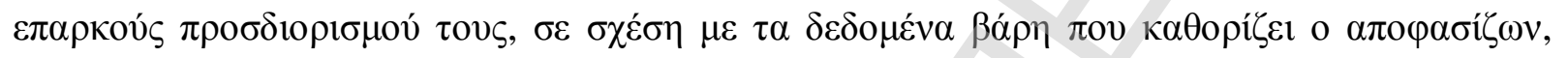

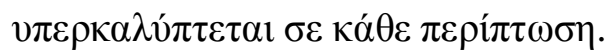

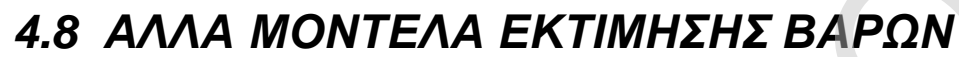

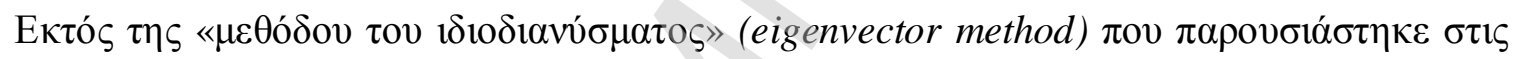

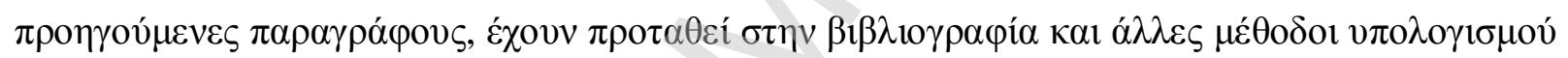

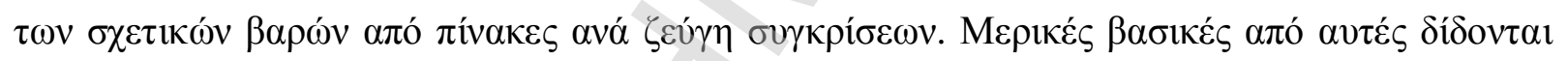

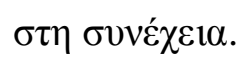

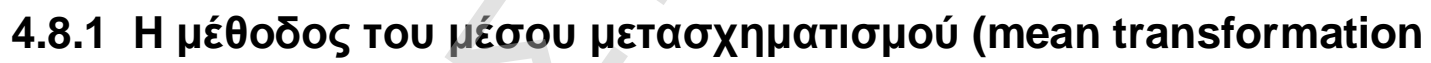 method)}

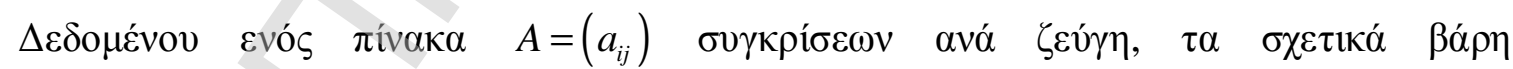

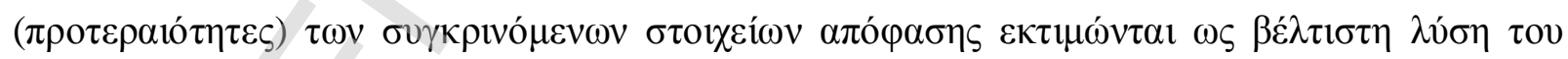

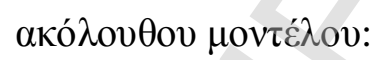

$$
\min _{w_{j}>0} \sum_{i=1}^{n} \sum_{j=1}^{n}\left(b_{i j}-w_{j}\right)^{2}
$$

Oлov:

$$
b_{i j}=a_{i j} / \sum_{i} a_{i j}
$$

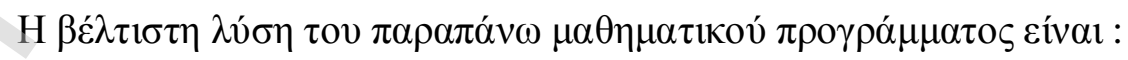




$$
w_{i}=\frac{1}{n} \sum_{j} b_{i j}
$$

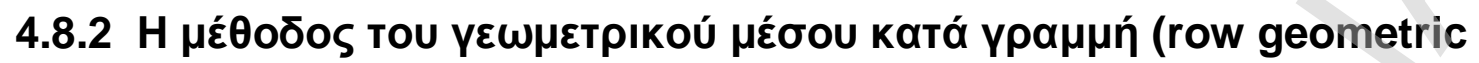 mean)}

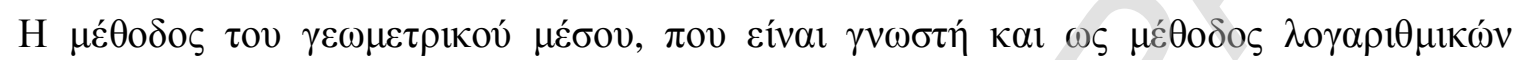

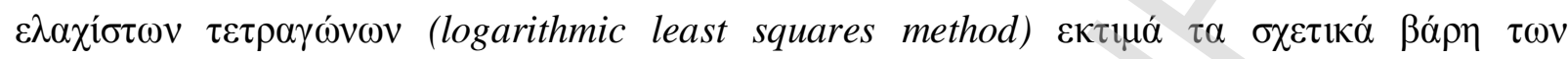

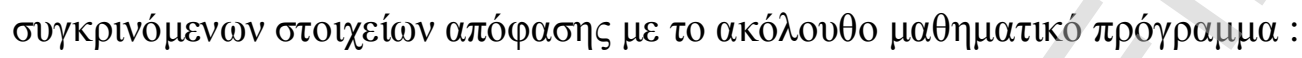

$$
\min _{w_{j}>0} \sum_{i \neq j}\left[\ln a_{i j}-\left(\ln w_{i}-\ln w_{j}\right)\right]^{2}
$$

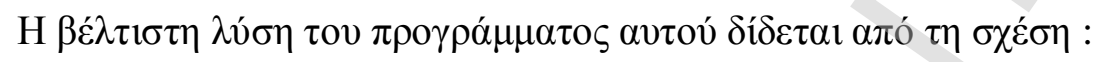

$$
w_{i}=\left(\prod_{j=1}^{n} a_{i j}\right)^{1 / n}
$$

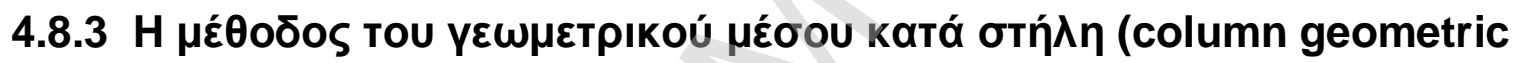 mean)}

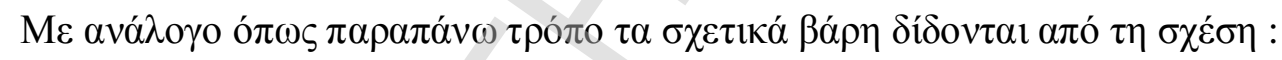

$$
w_{j}=\left(\prod_{i=1}^{n} a_{i j}\right)^{1 / n}
$$

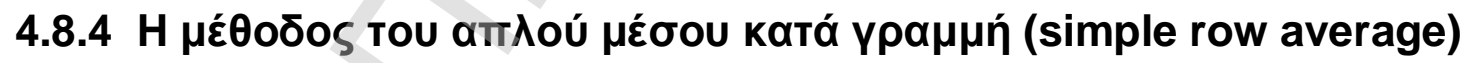

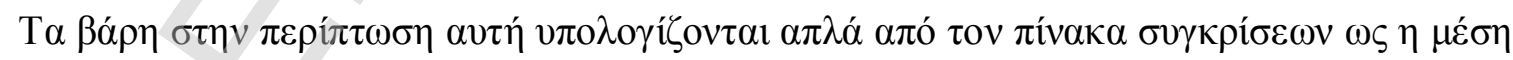

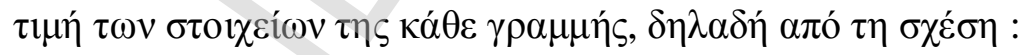

$$
w_{i}=\frac{1}{n} \sum_{j=1}^{n} a_{i j}
$$




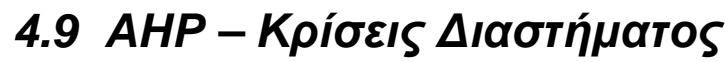

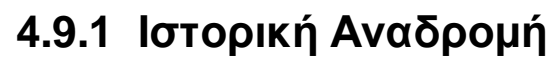

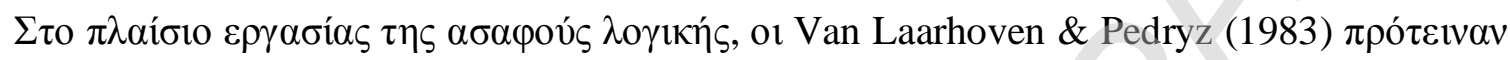

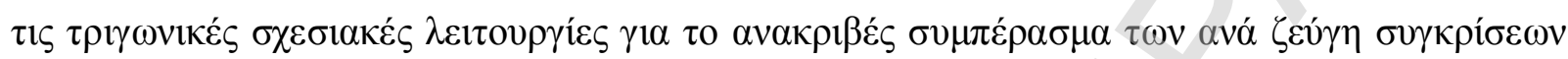

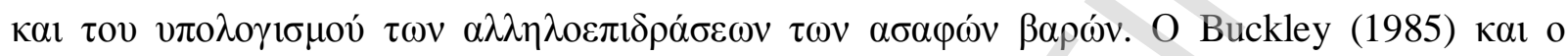

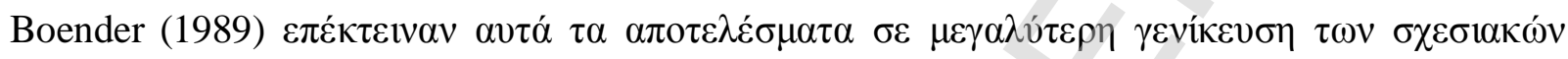

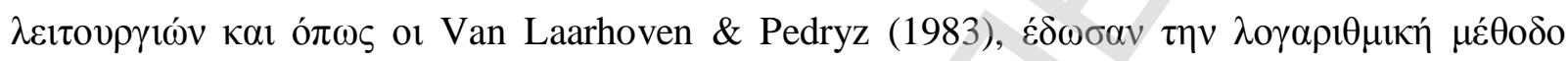

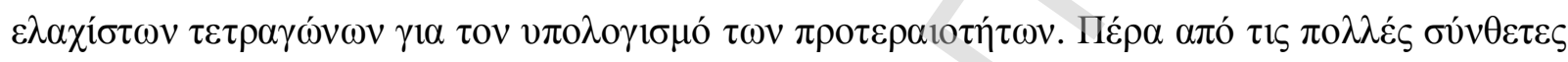

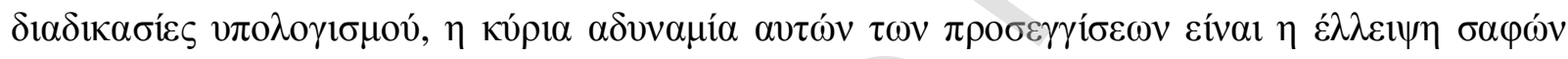

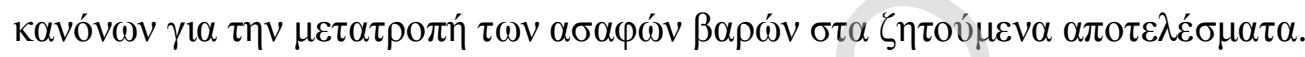

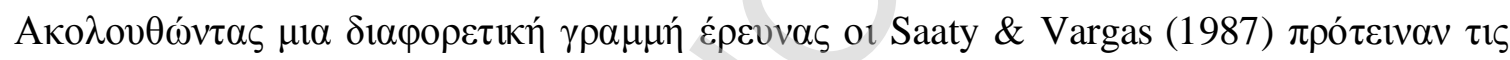

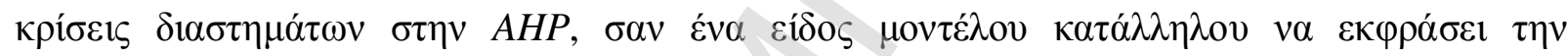

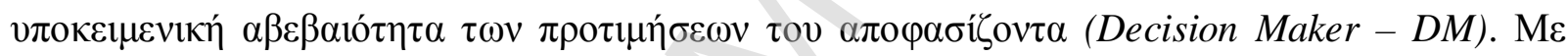

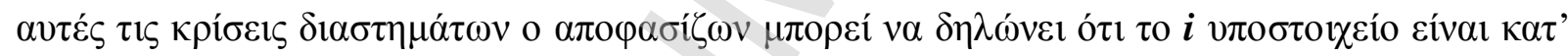

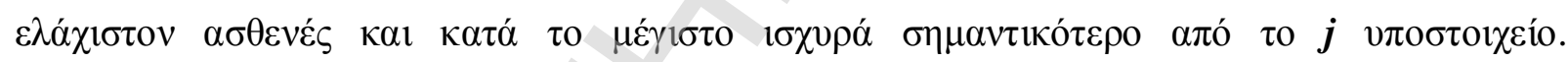

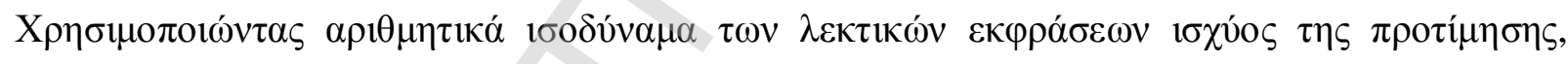

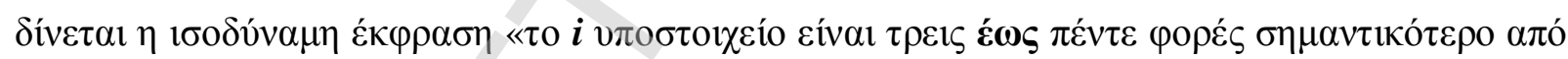

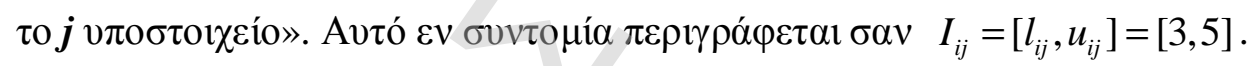

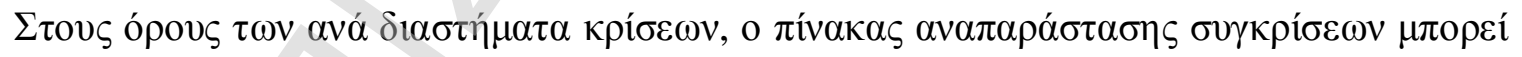
$v \alpha \gamma \rho \alpha \varphi \tau \varepsilon i ́$ :

$$
\left(\begin{array}{cccc}
1 & <l_{12}, u_{12}> & \mathrm{L} & <l_{1 n}, u_{1 n}> \\
<l_{21}, u_{21}> & 1 & \mathrm{~L} & <l_{2 n}, u_{2 n}> \\
\mathrm{M} & \mathrm{M} & \mathrm{M} \\
<l_{n 1}, u_{n 1}> & <l_{n 2}, u_{n 2}> & \mathrm{L} & 1
\end{array}\right)
$$

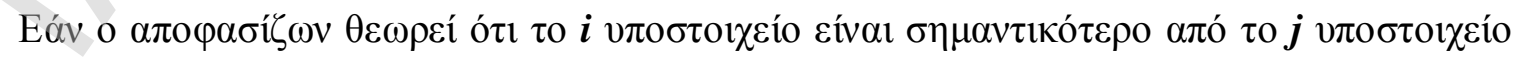

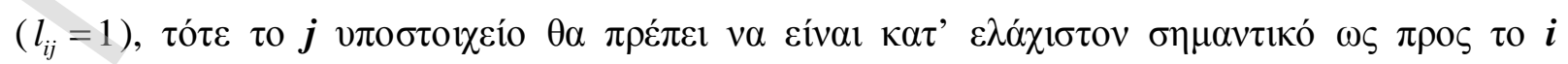




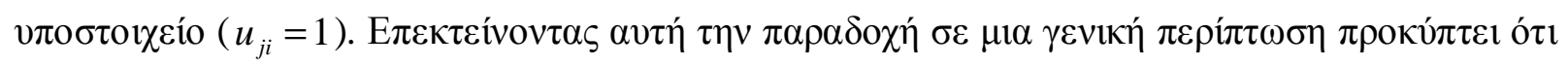
$l_{i j} u_{j i}=1 \gamma 1 \alpha i \neq j$.

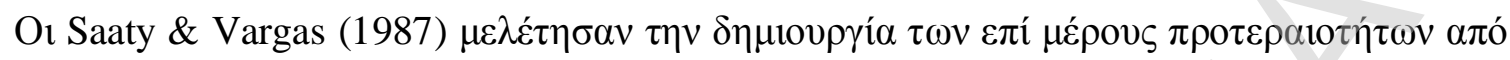

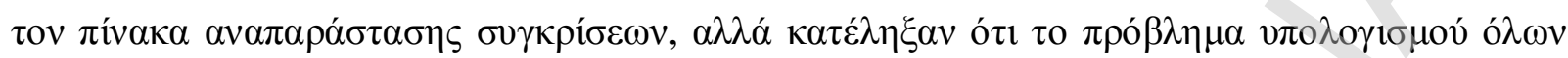

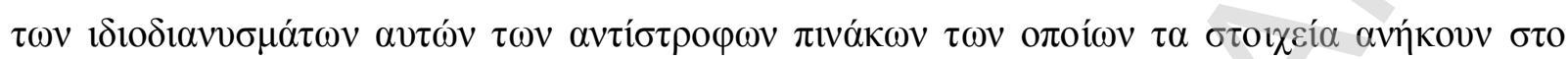

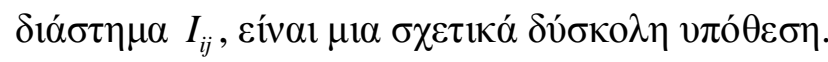

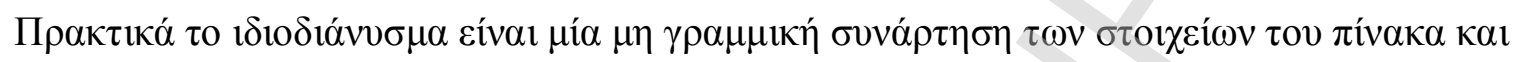

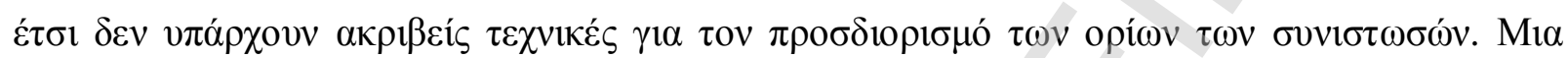

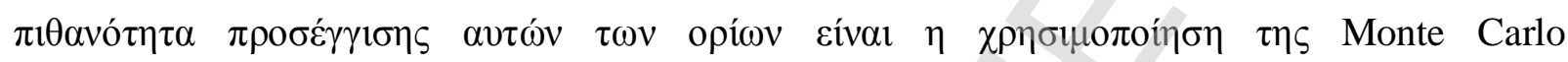

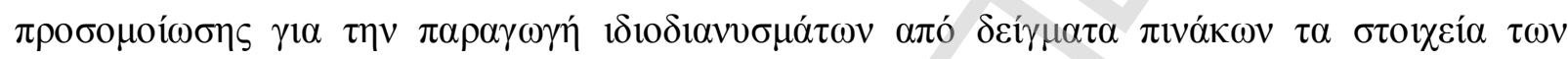

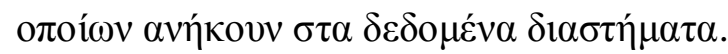

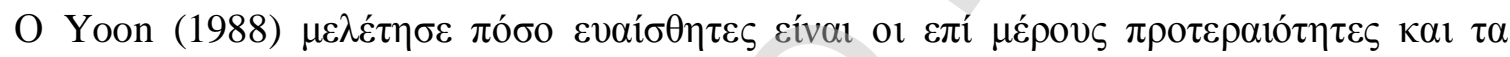

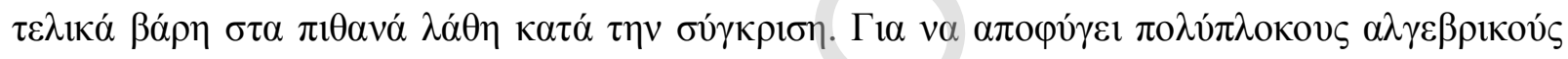

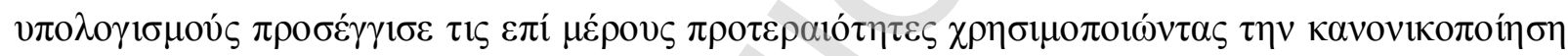

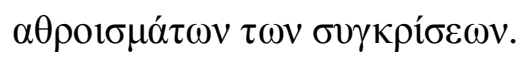

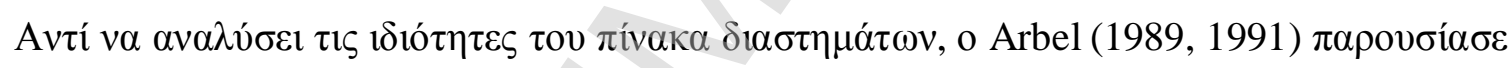

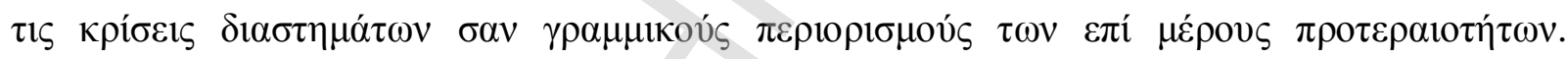

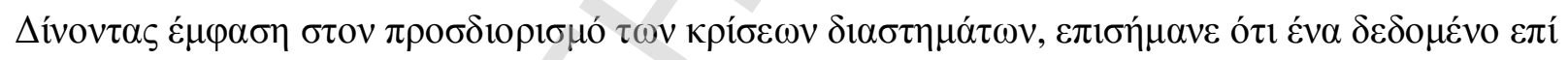

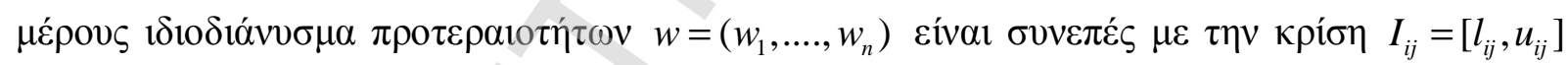

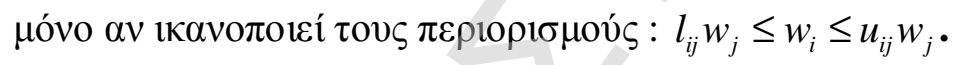

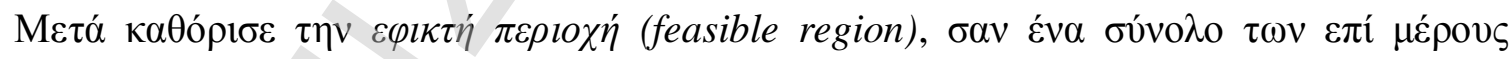

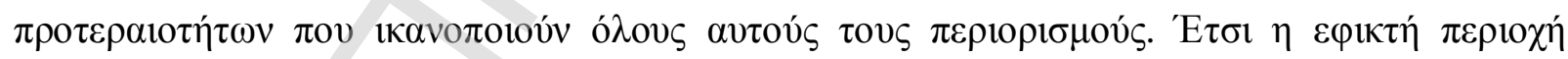

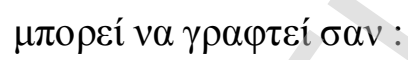

$$
S=Q^{n} \cap\left\{w \mid l_{i j} w_{j} \leq w_{i} \leq u_{i j} w_{j}\right\} \text { ó } \pi \text { ov } Q^{n}=\left\{\left(w_{1}, \ldots ., w_{n}\right) \mid w_{i} \geq 0, \sum_{i=1}^{n} w_{i}=1\right\}
$$

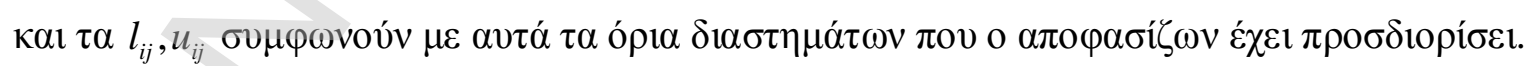

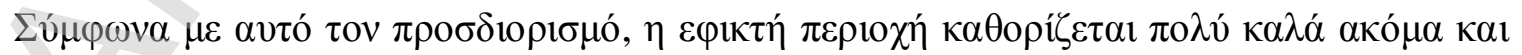

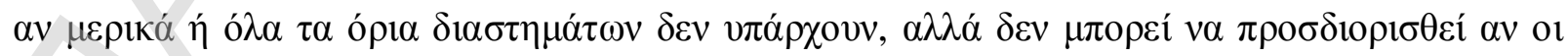

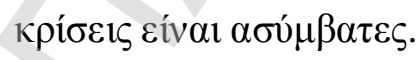

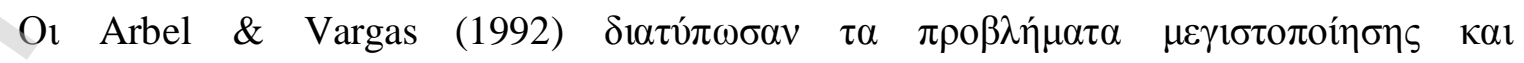

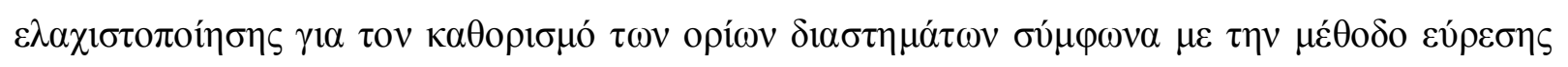




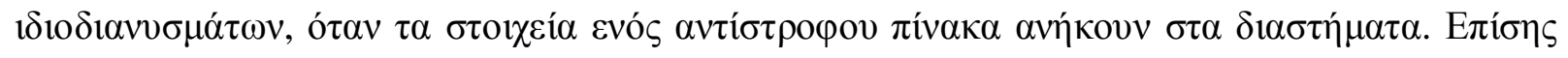

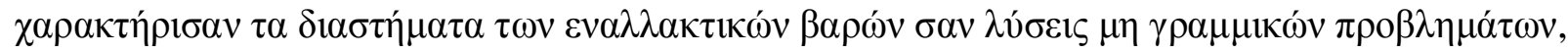

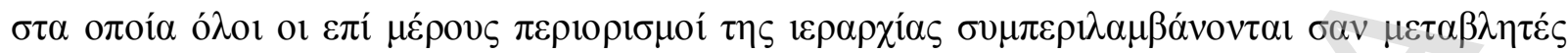
$\alpha \pi$ ó $\alpha \sigma\rceil \zeta$.

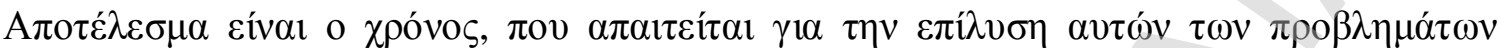

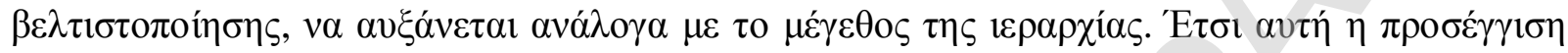

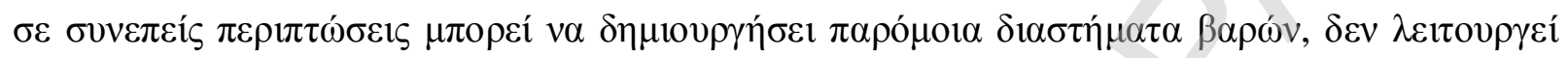

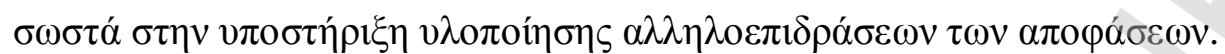

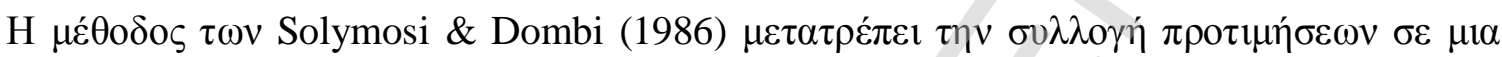

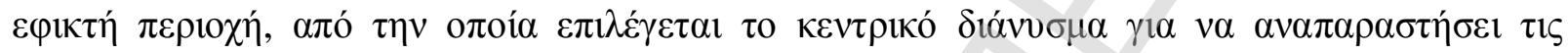

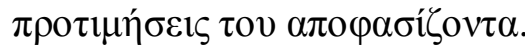

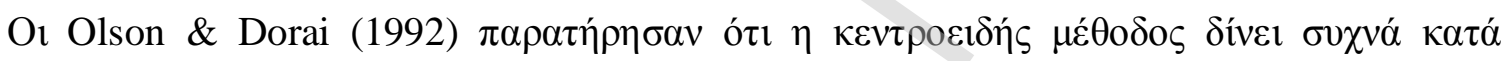

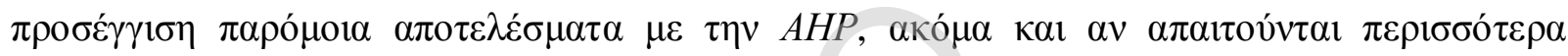

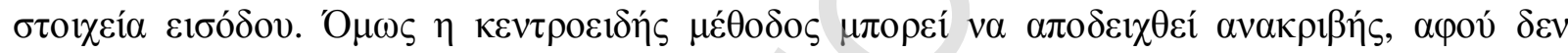

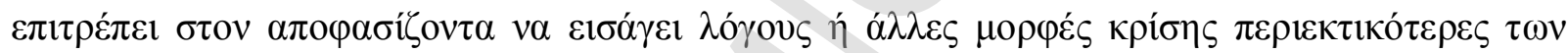

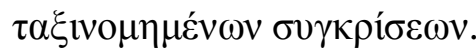

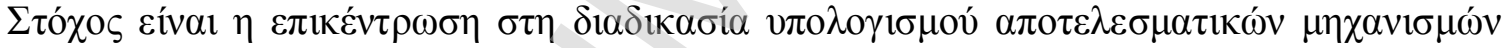

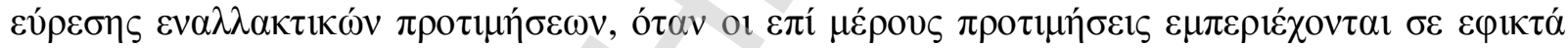

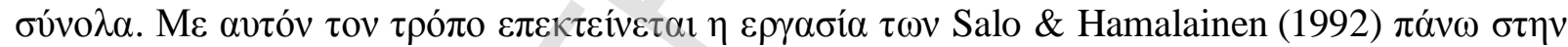

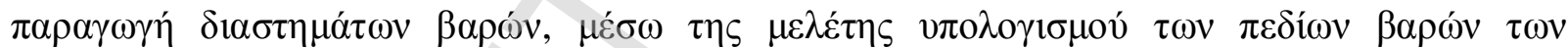

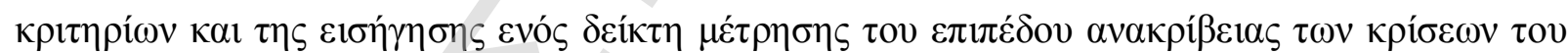
$\alpha \pi \circ \varphi \alpha \sigma i \zeta{ }_{0} \tau \tau \alpha$.

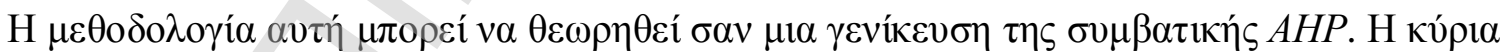

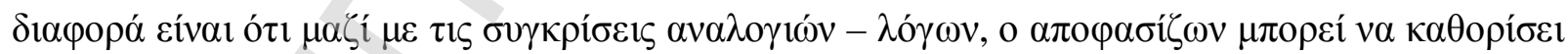

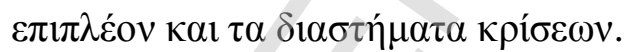

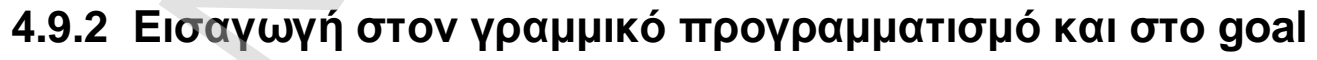 programming model}

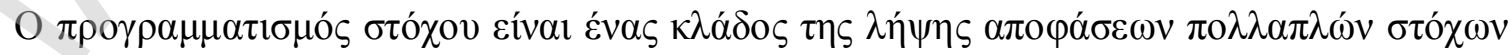

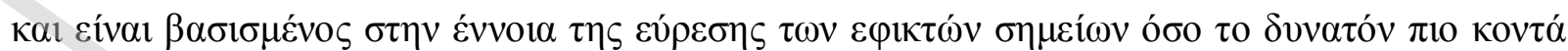

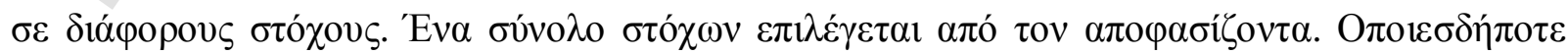




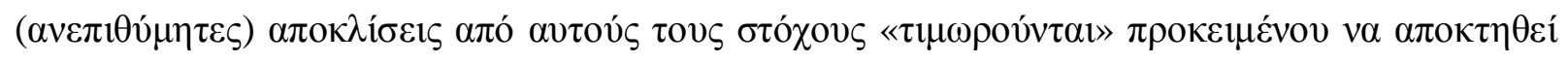

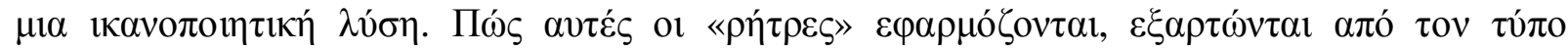

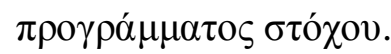

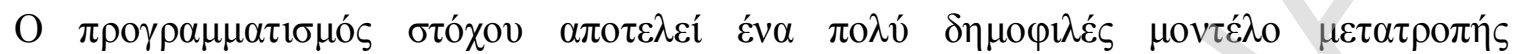

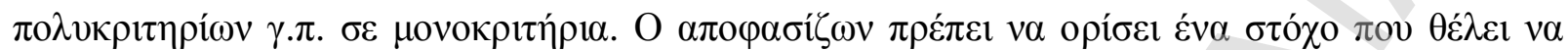

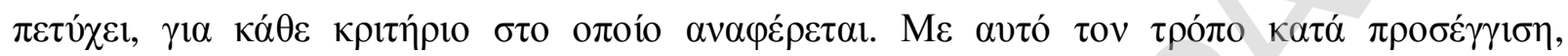

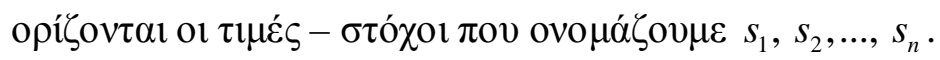

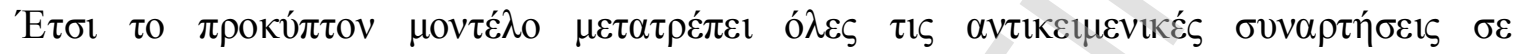

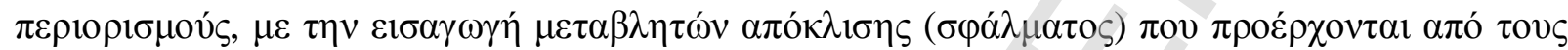

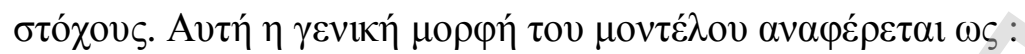

$$
[\min ] z=\sum_{i=1}^{n} p_{i} f_{i}\left(d_{1}^{-}, d_{1}^{+}, d_{2}^{-}, d_{2}^{+}, \ldots, d_{n}^{-}, d_{n}^{+}\right)
$$

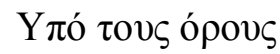

$$
\begin{gathered}
\sum_{j=1}^{l} c_{i j} x_{j}+d_{i}^{-}-d_{i}^{+}=s_{i}, \quad i=1,2, \ldots, n \\
x \in A \\
d_{i}^{-} \geq 0, d_{i}^{+} \geq 0, \quad i=1,2, \ldots, n
\end{gathered}
$$

'О

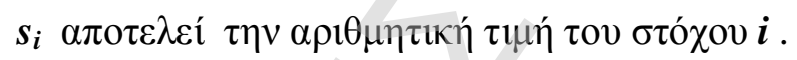

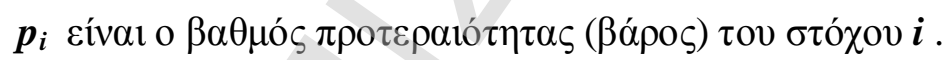

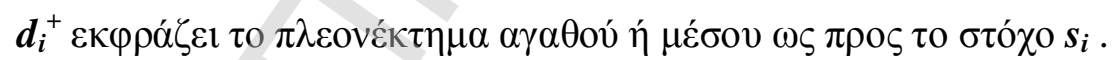

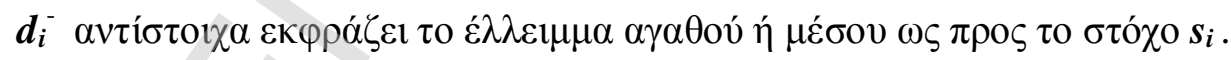

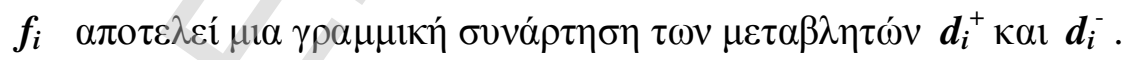

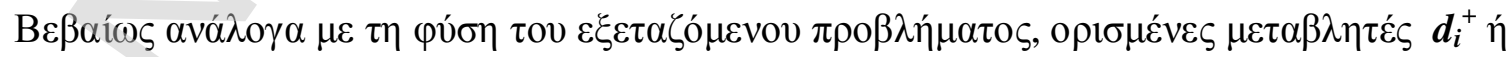

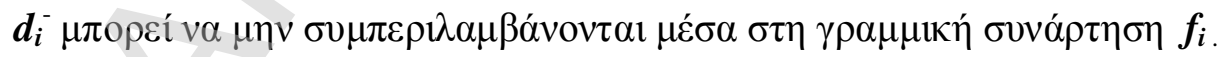

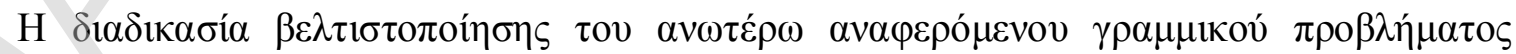

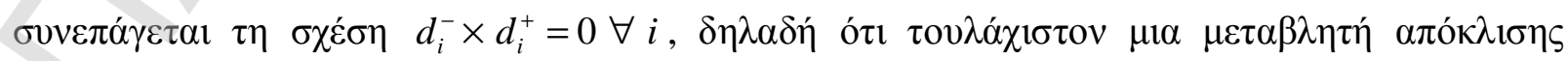

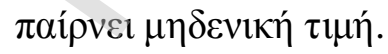




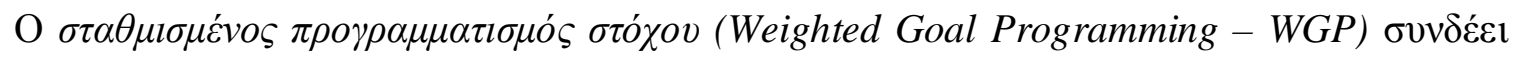

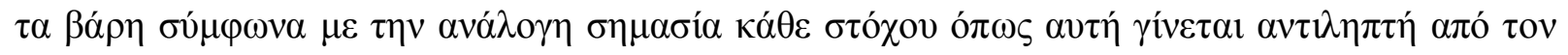

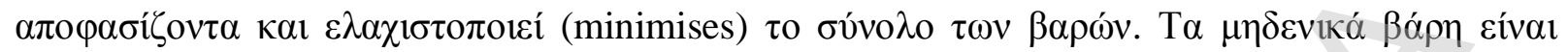

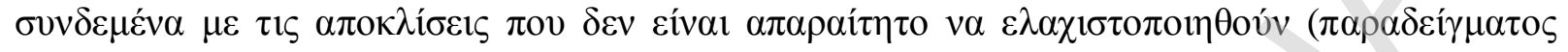

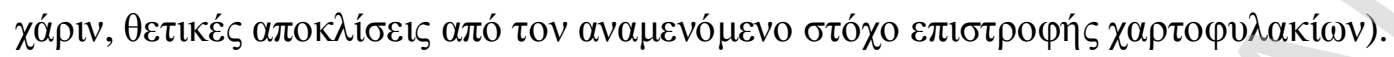

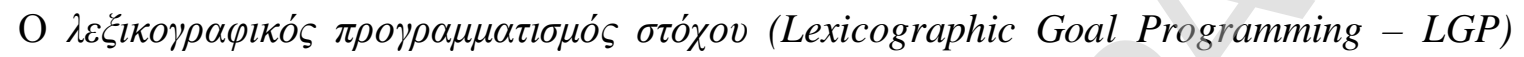

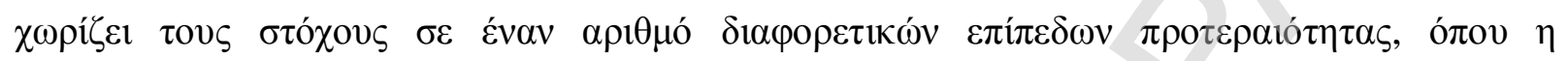

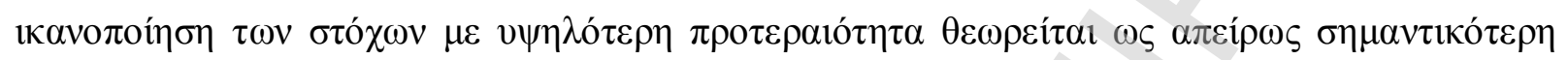

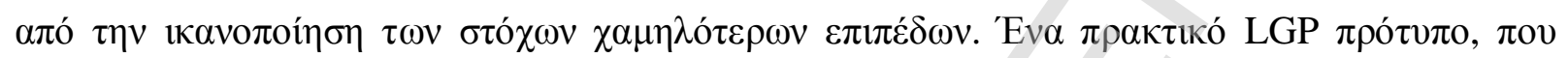

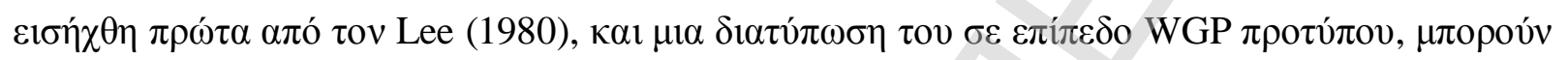

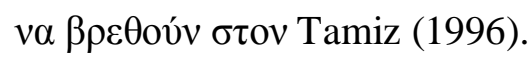

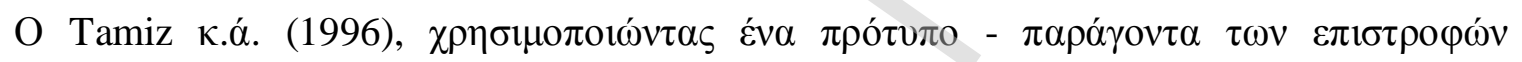

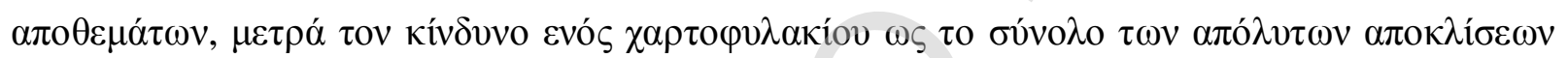

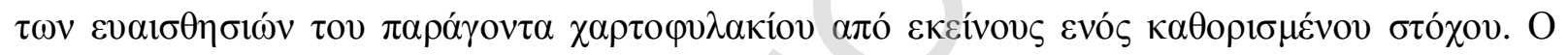

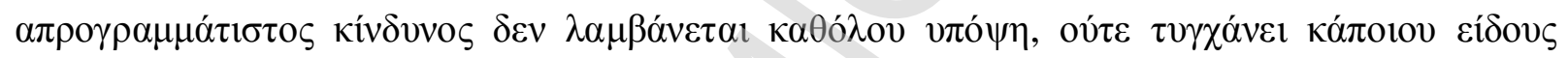

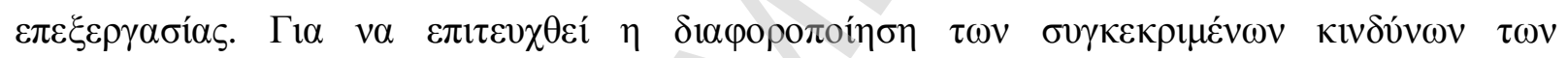

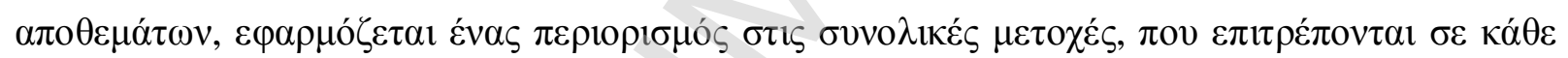

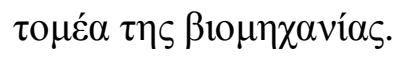

\subsubsection{Weighted Goal Programming - WGP}

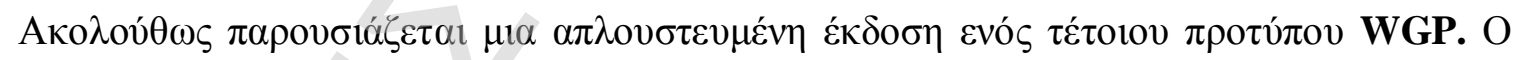

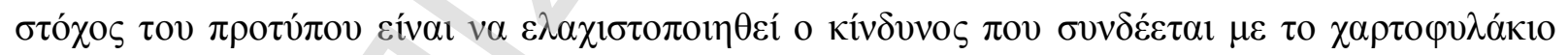

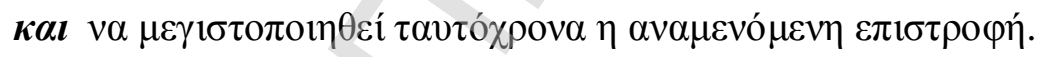

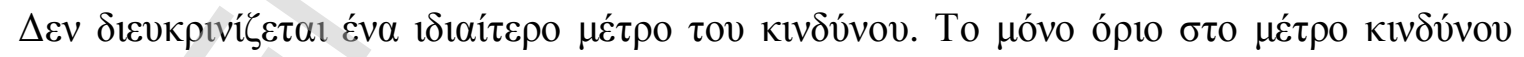

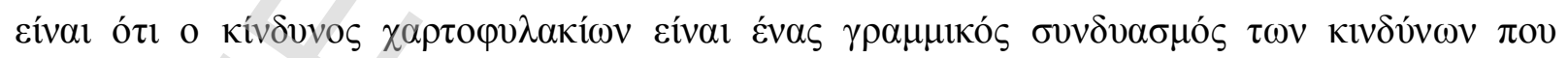

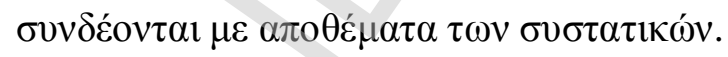

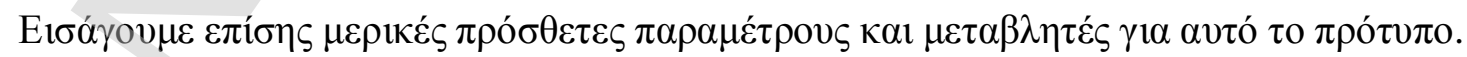

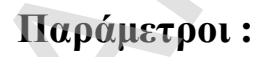

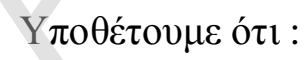

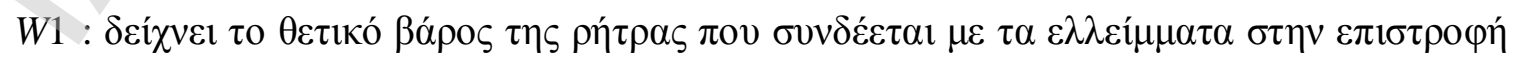

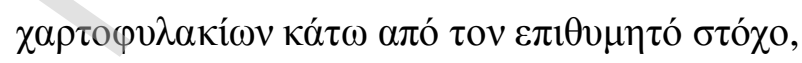




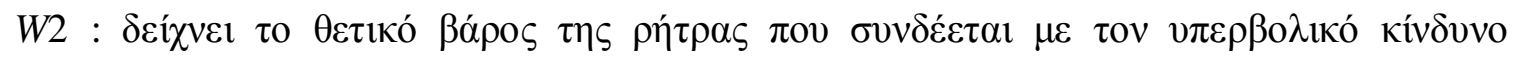

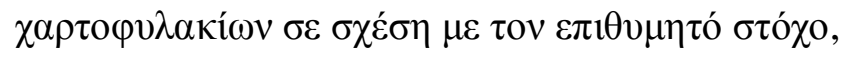

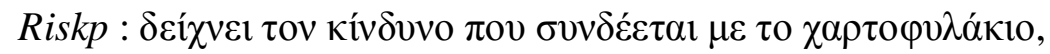

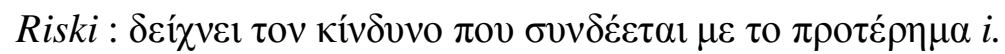

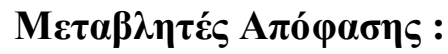

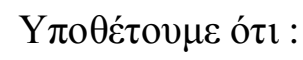

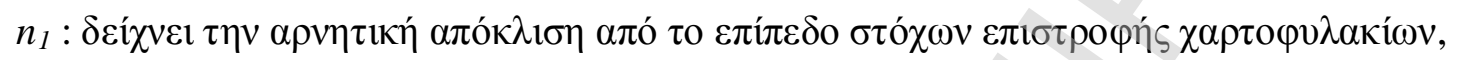

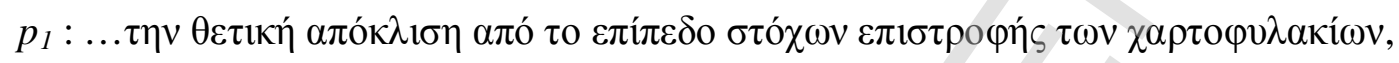

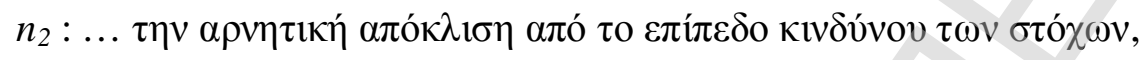

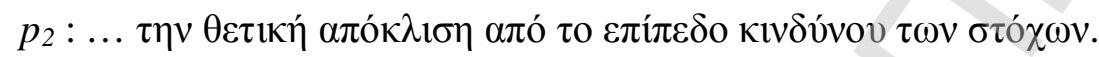

\section{WGP :}

Min $\mathrm{Z}_{\mathrm{WGP}}=W_{1} n_{1}+W_{2} p_{2}$

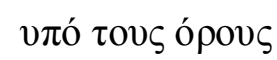

$\sum_{i=1}^{N} x_{i} \mu_{\mathrm{i}}+n_{1}-p_{1}=\rho$

$\sum_{i=1}^{N} \operatorname{Risk}_{i} x_{i}+n_{2}-p_{2}=\operatorname{Risk}_{p}$

$\sum_{i=1}^{N} x_{i}=1$

$n_{1}, n_{2}, p_{1}, p_{2} \geq 0$

$x_{i} \geq 0 \quad i=1, \ldots, N$

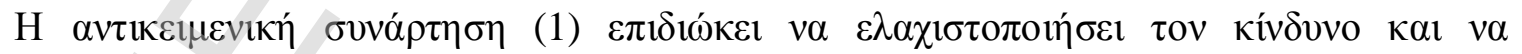

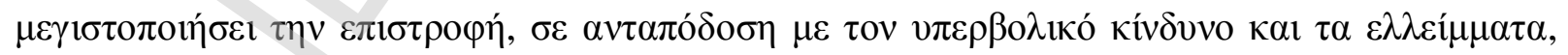

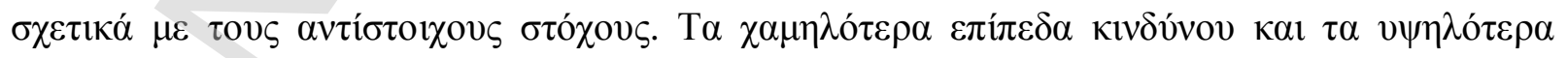

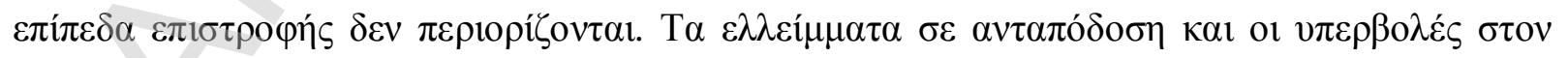

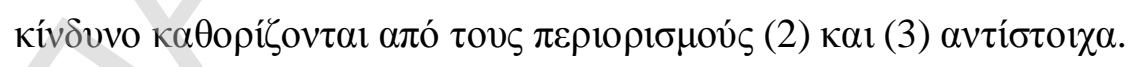




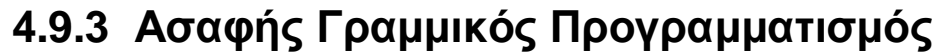

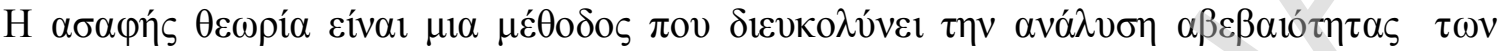

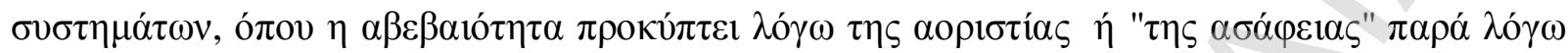

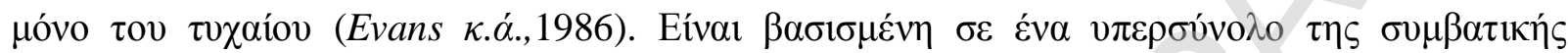

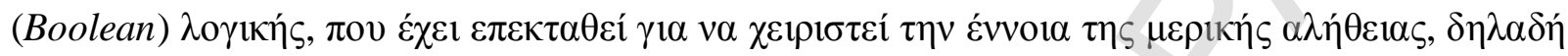

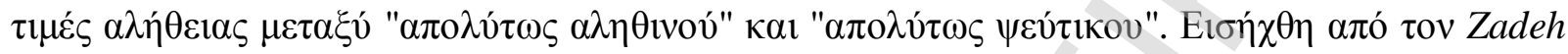

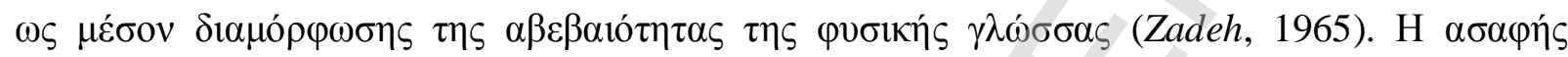

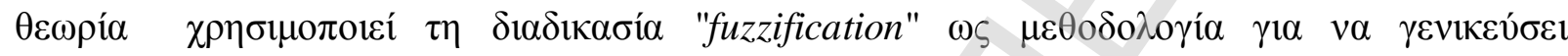

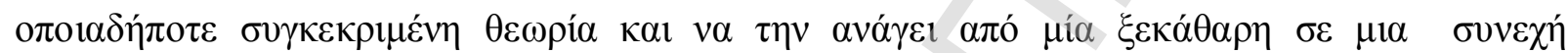

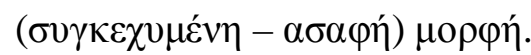

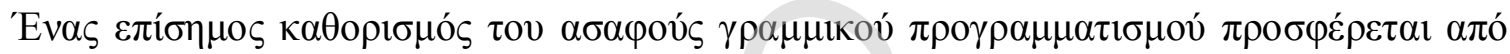

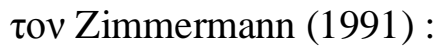

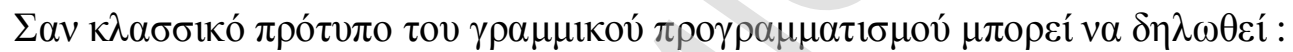

$$
\begin{aligned}
& \text { maximize } f(x)=c^{T} x
\end{aligned}
$$

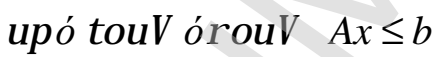

$$
\begin{aligned}
& x \geq 0 \\
& \mu \varepsilon \quad c, x \in \mathfrak{R}^{n}, b \in \mathfrak{R}^{m}, a \in \mathfrak{R}^{m \times n}
\end{aligned}
$$

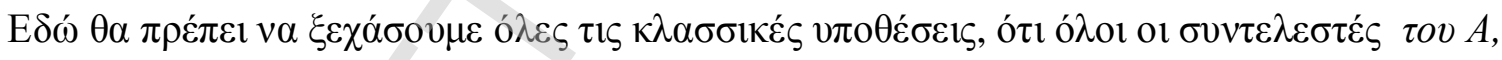

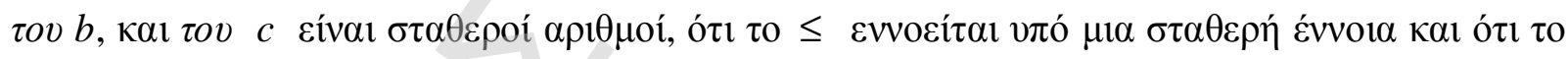

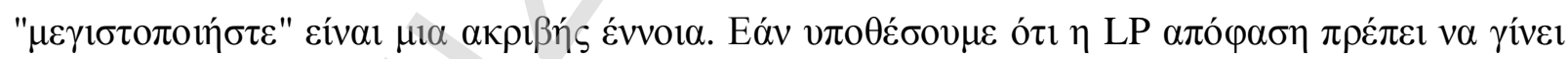

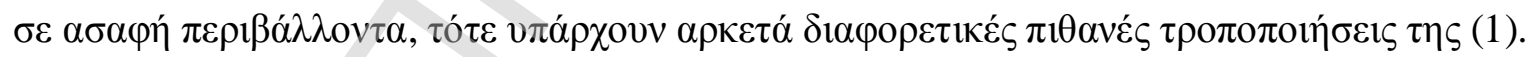

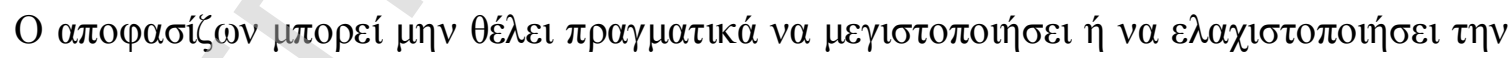

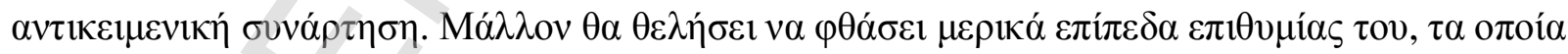

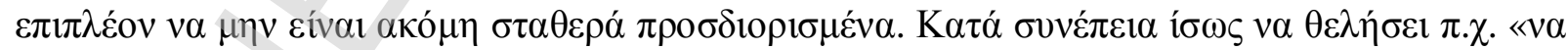

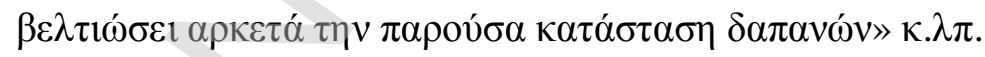

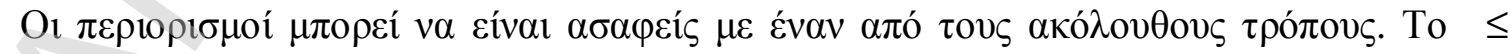

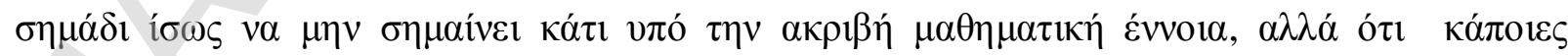

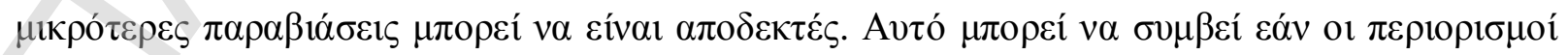

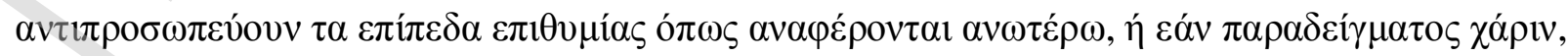

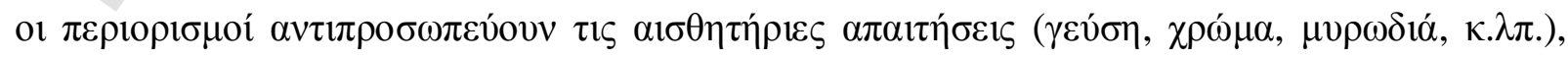

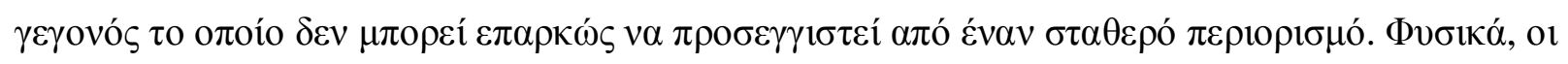




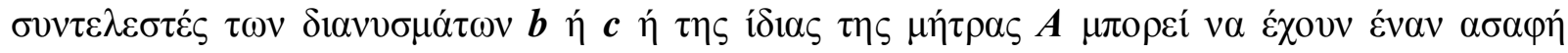

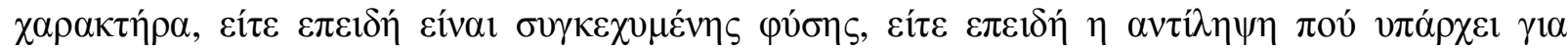

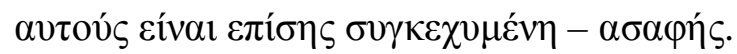

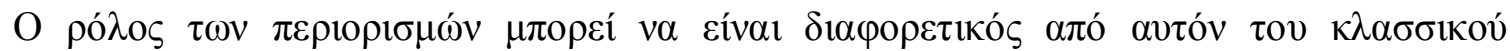

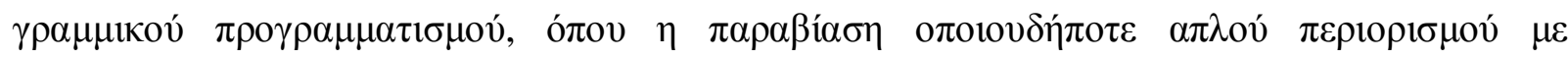

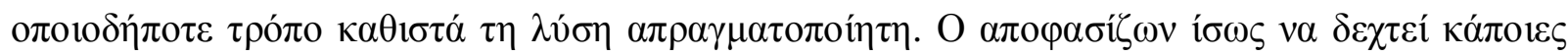

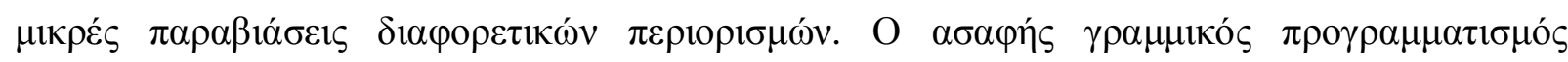

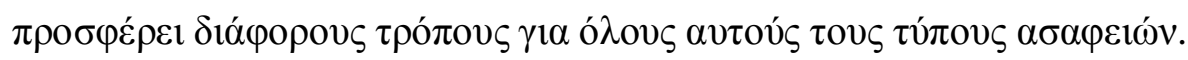

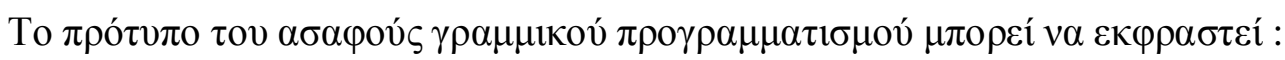

$$
\begin{array}{rlrl}
\max \text { imize } & c^{T} & \approx z \\
\text { vлó tovs ópovs } & A x & \approx b b \\
x & \geq 0
\end{array}
$$

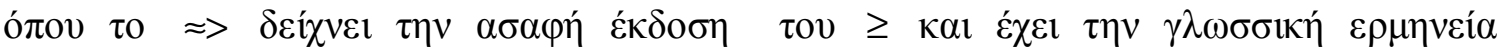

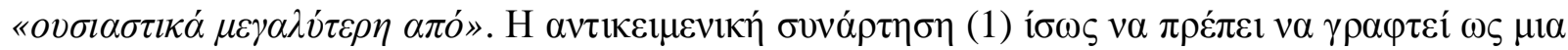

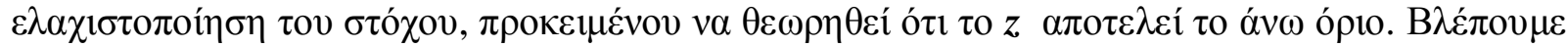

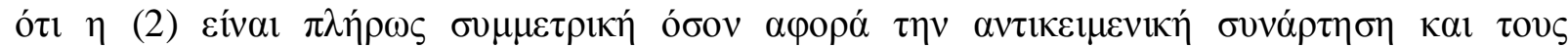
$\pi \varepsilon \rho 10 \rho i \sigma \mu o u ́ s$.

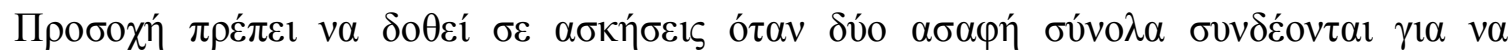

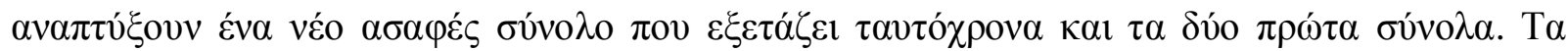

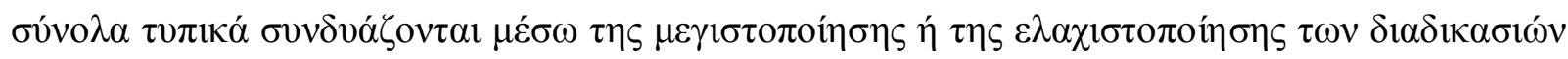

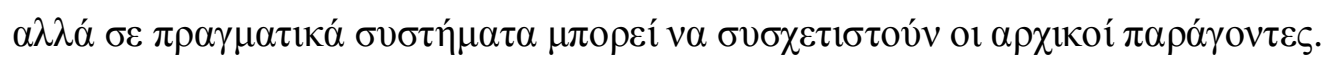

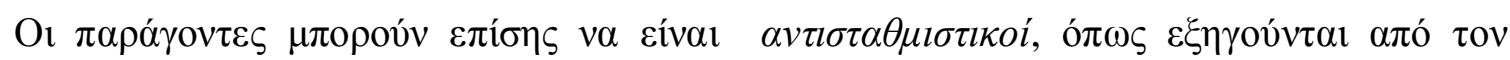
Zimmermann (1991) :

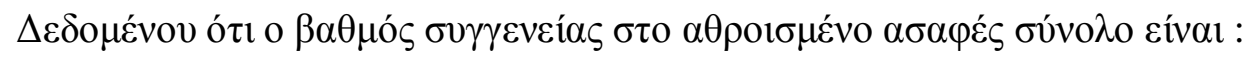

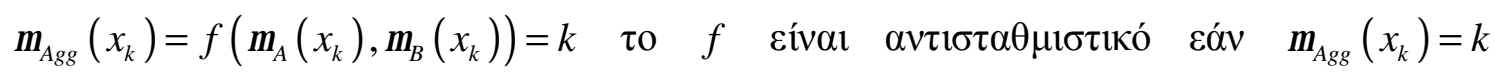

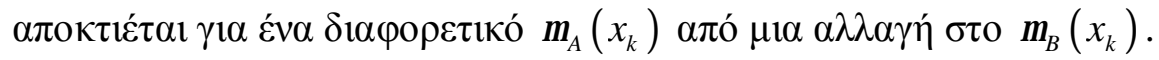

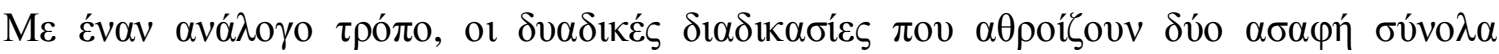

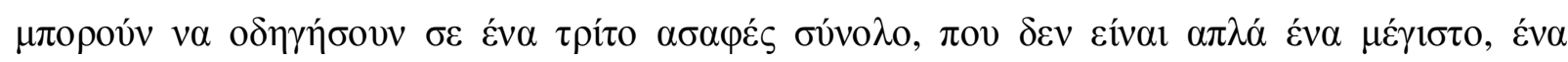

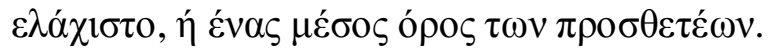




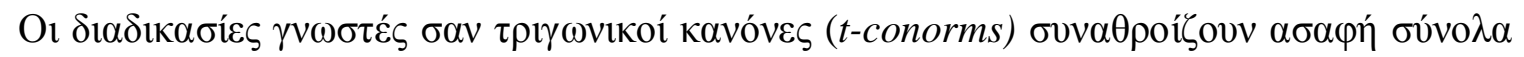

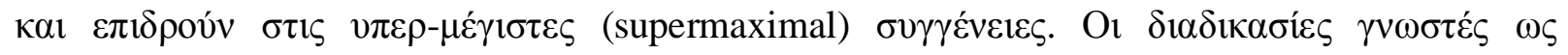

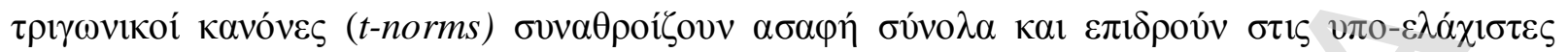

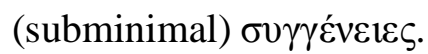

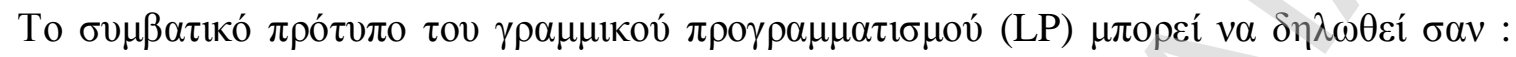
$\langle c, x\rangle \rightarrow \min v \pi o ́$ đov ópo $A x \leq b$,

о́лоv

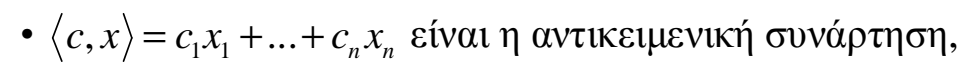

- $x=\left(x_{1}, \ldots, x_{n}\right)$ cív $\alpha \imath \eta \mu \varepsilon \tau \alpha \beta \lambda \eta \tau \eta ́ ~ \alpha \pi o ́ \varphi \alpha \sigma \eta \varsigma$,

$\bullet(A x)_{i}=\left\langle a_{i}, x\right\rangle=a_{i 1} x_{1}+\ldots+a_{i n} x_{n}$

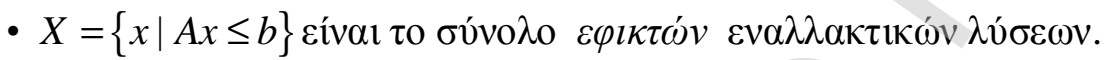

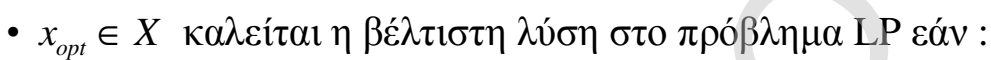

$\left\langle c, x_{\text {opt }}\right\rangle \leq\langle c, x\rangle \gamma \dot{\alpha} \kappa \alpha \dot{\theta} \theta \varepsilon \quad x \in X$.

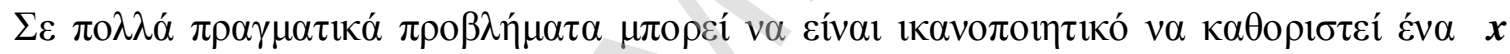

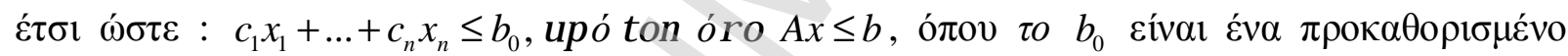

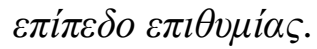

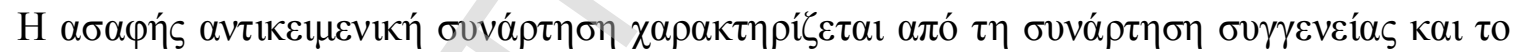

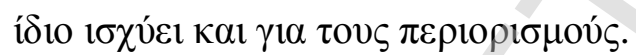

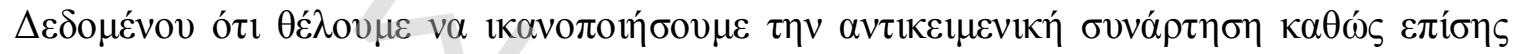

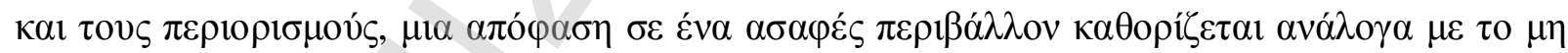

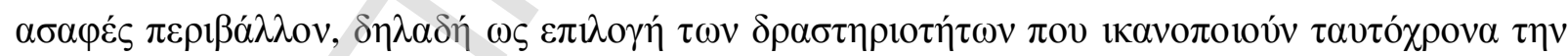

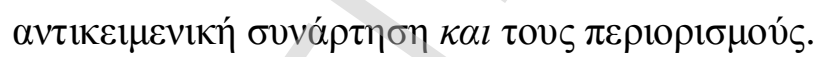

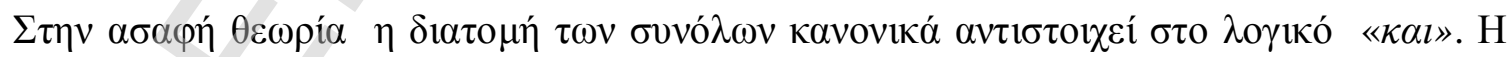

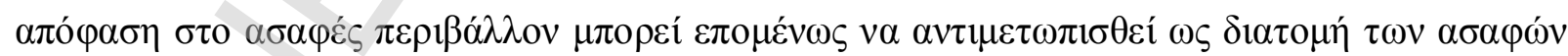

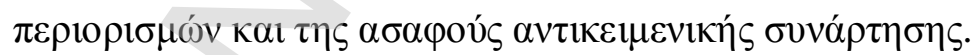

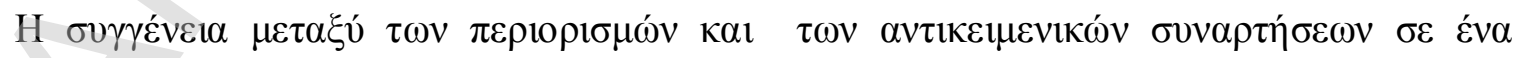

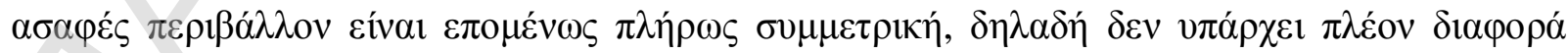

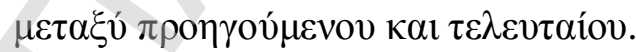

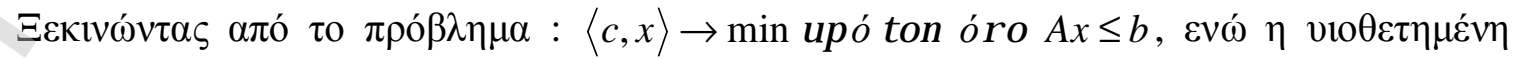

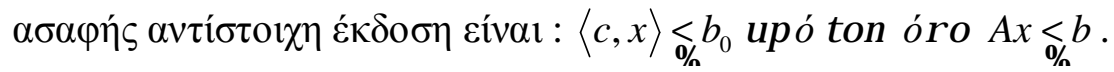


$\Delta \eta \lambda \alpha \delta \eta$

$$
\begin{gathered}
c_{1} x_{1}+\ldots+c_{n} x_{n} \delta b_{0} \\
a_{i 1} x_{1}+\ldots+a_{i n} x_{n} \delta_{\delta / n}, i=1, \ldots, m .
\end{gathered}
$$

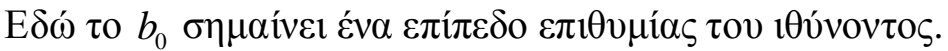

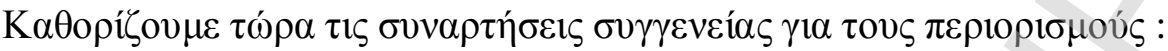

$$
\mu_{i}(x)=\left\{\begin{array}{lc}
1 & \varepsilon \dot{\alpha} \quad\left\langle a_{i}, x\right\rangle \leq b_{i} \\
1-\frac{\left\langle a_{i}, x\right\rangle-b_{i}}{d_{i}} \varepsilon \dot{\alpha}^{\prime} & b_{i}<\left\langle a_{i}, x\right\rangle \leq b_{i}+d_{i} \\
0 & \varepsilon \dot{\alpha} N\left\langle a_{i}, x\right\rangle>b_{i}+d_{i}
\end{array}\right\}
$$

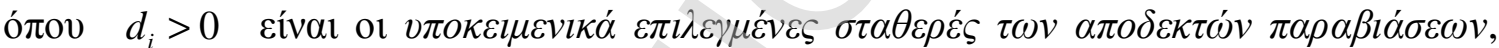
$i=1, \ldots, m$.

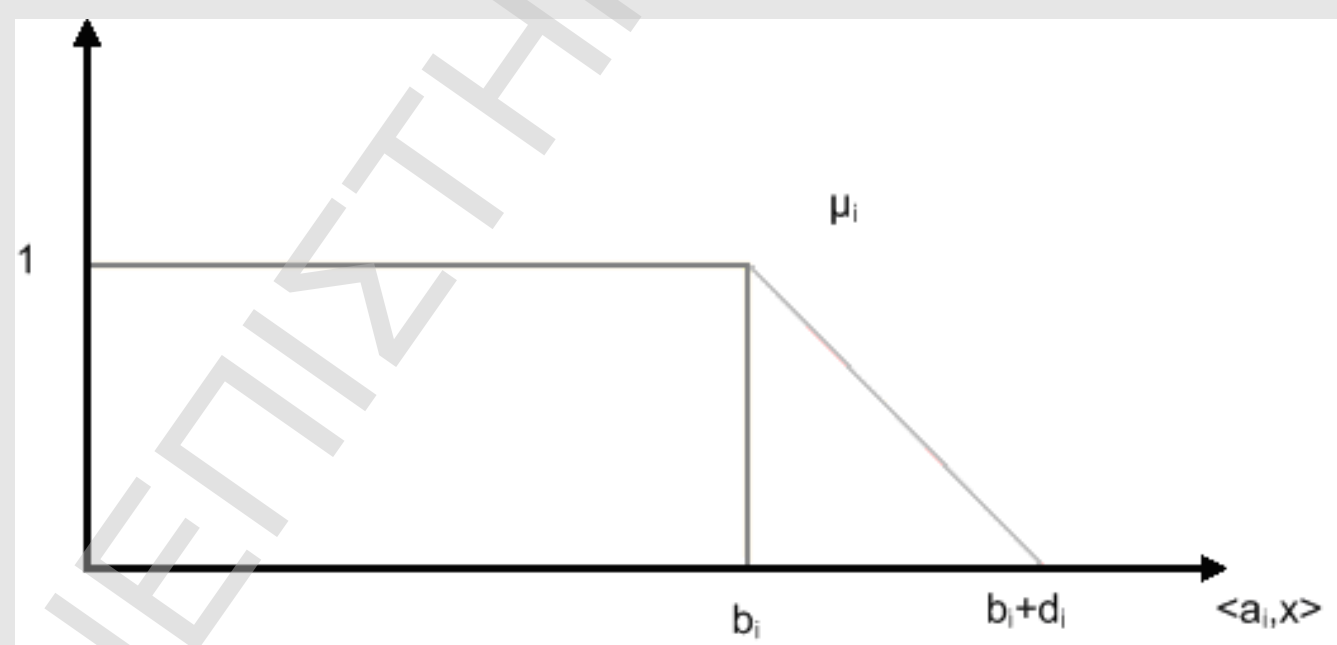

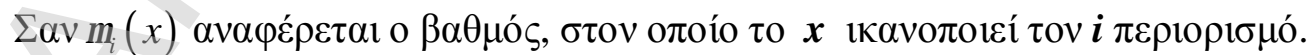

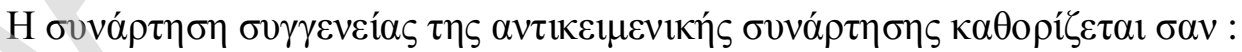




$$
\mu_{0}(x)=\left\{\begin{array}{ll}
1 & \varepsilon \dot{N}\langle c, x\rangle \leq b_{0} \\
1-\frac{\langle c, x\rangle-b_{0}}{d_{0}} & \varepsilon \dot{\alpha} \quad b_{0}<\langle c, x\rangle \leq b_{0}+d_{0} \\
0 & \varepsilon \dot{\alpha} \quad\langle c, x\rangle>b_{0}+d_{0}
\end{array}\right\}
$$

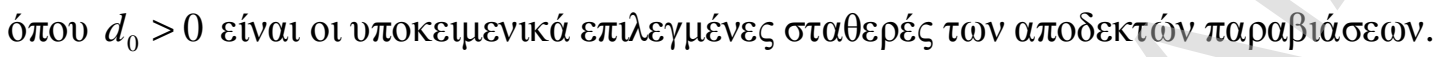




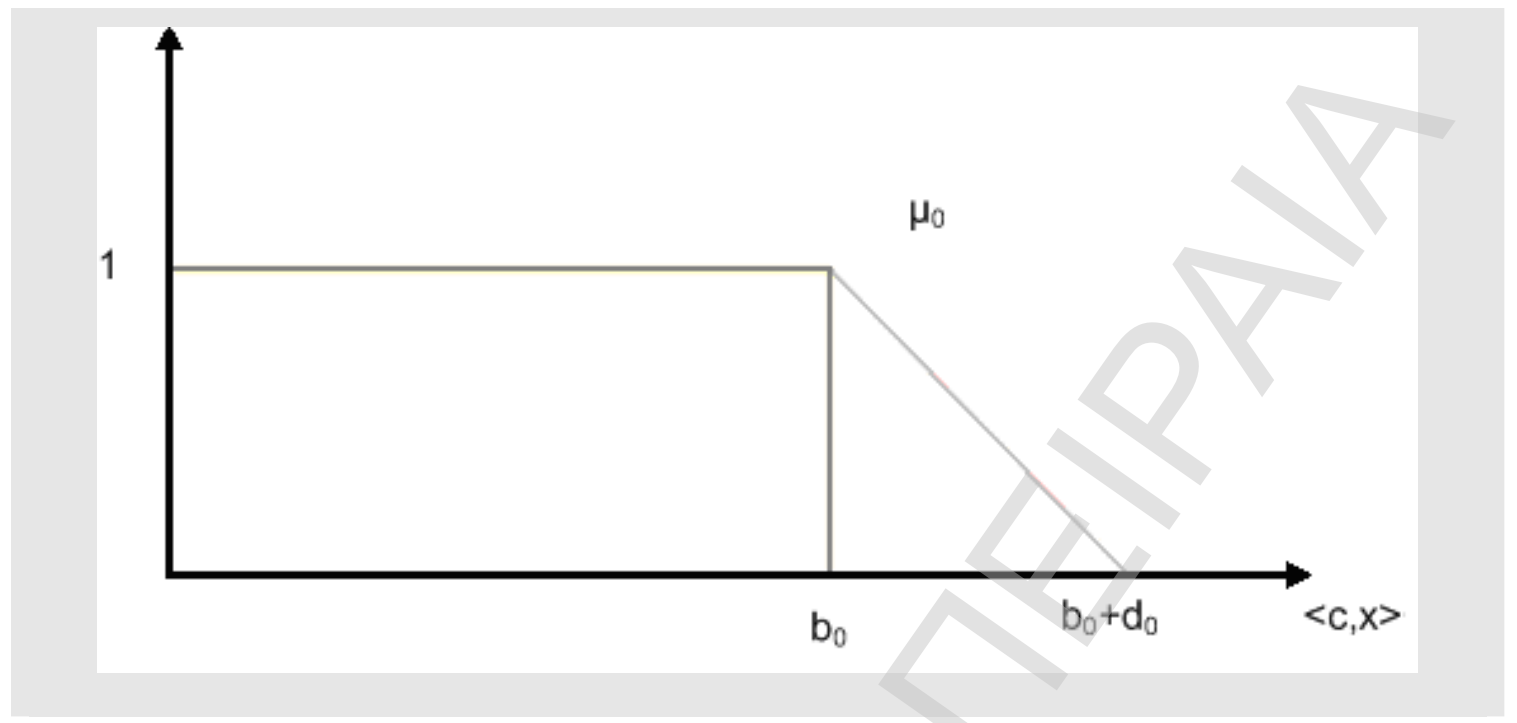

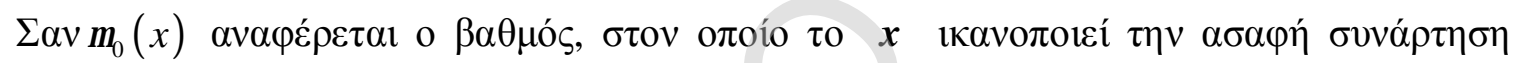

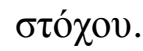

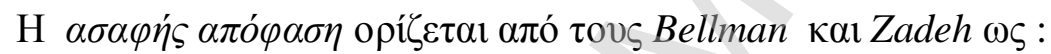

$$
D(x)=\min \left\{\mu_{0}(x), \mu_{1}(x), \ldots, \mu_{m}(x)\right\}
$$

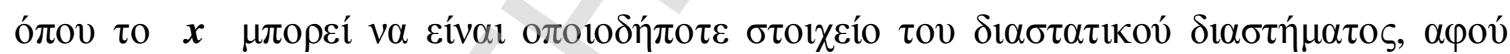

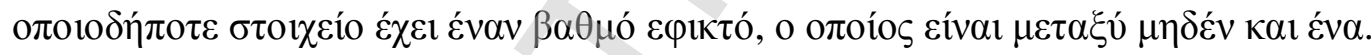

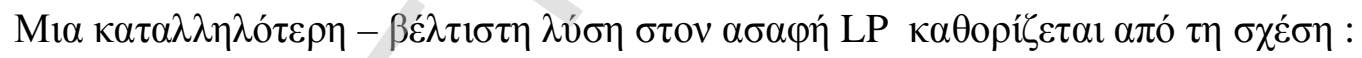

$$
D\left(x^{*}\right)=\max _{x \in \square^{n}} D(x)
$$

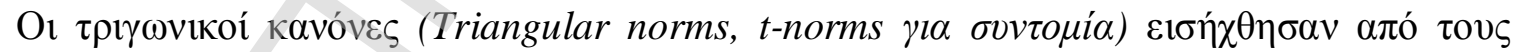

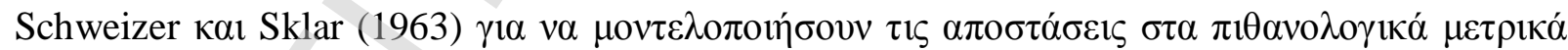

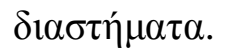

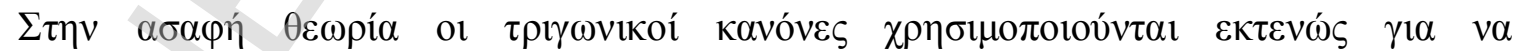

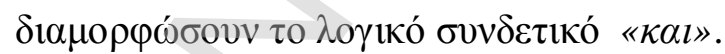

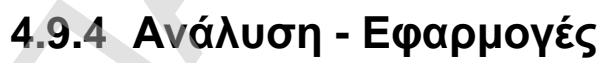

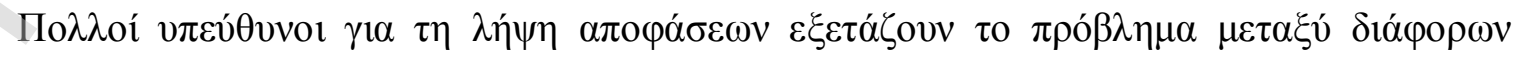

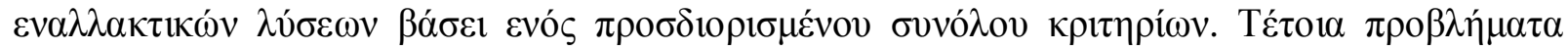




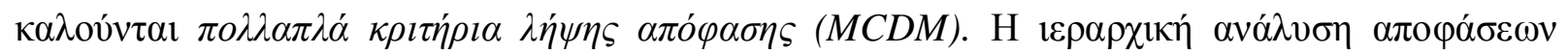

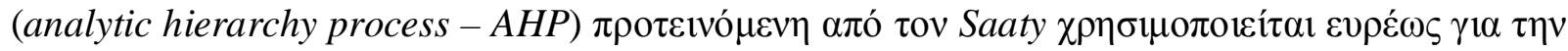

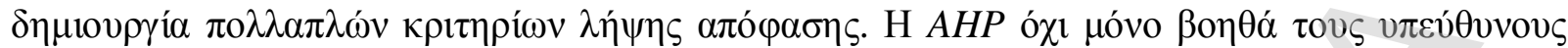

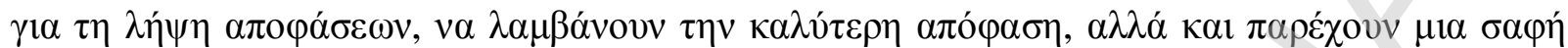

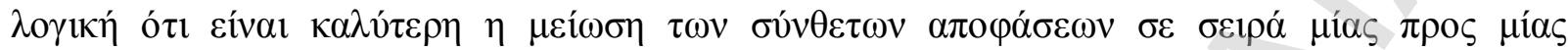

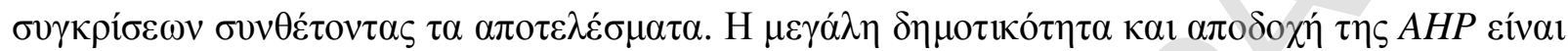

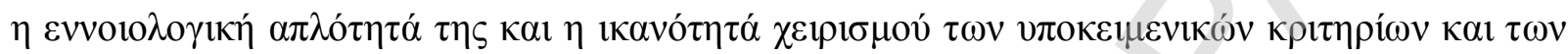

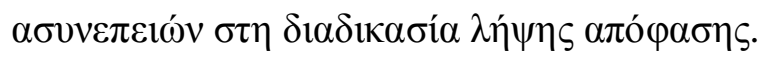

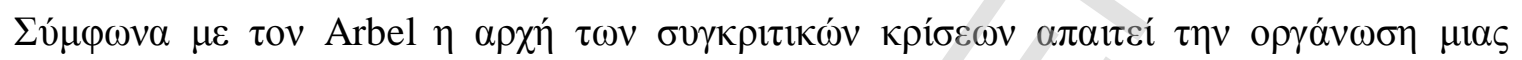

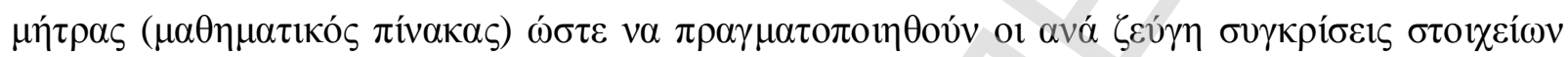

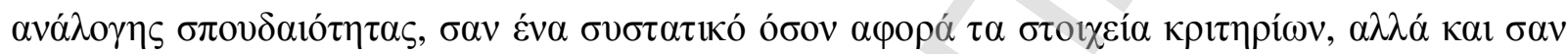

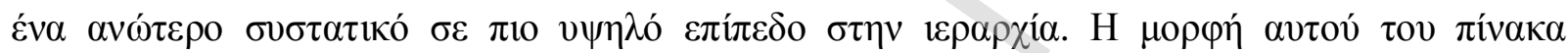

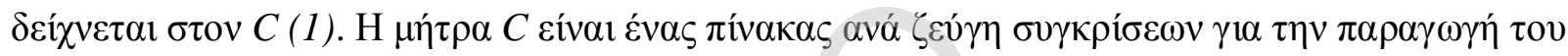

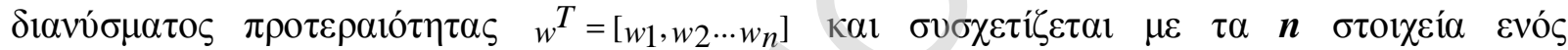

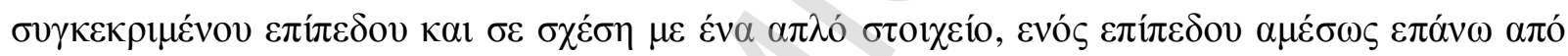

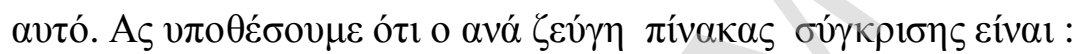

$$
C=\left[\begin{array}{ccccc}
\frac{w_{1}}{w_{1}} & \frac{w_{1}}{w_{2}} & \frac{w_{1}}{w_{3}} & \cdots & \frac{w_{1}}{w_{n}} \\
\frac{w_{2}}{w_{1}} & \frac{w_{2}}{w_{2}} & \frac{w_{2}}{w_{3}} & \cdots & \frac{w_{2}}{w_{n}} \\
\frac{w_{3}}{w_{1}} & \frac{w_{3}}{w_{2}} & \frac{w_{3}}{w_{3}} & \cdots & \frac{w_{3}}{w_{n}} \\
\cdots & \cdots & \cdots & \cdots \\
\frac{w_{n}}{w_{1}} & \frac{w_{n}}{w_{2}} & \frac{w_{n}}{w_{3}} & \cdots & \frac{w_{n}}{w_{n}}
\end{array}\right]
$$

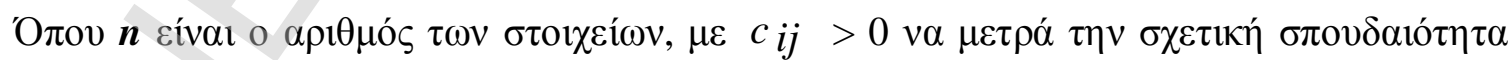

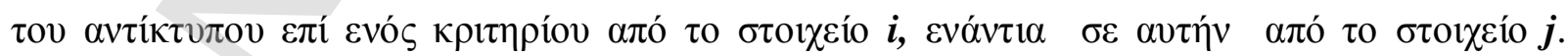

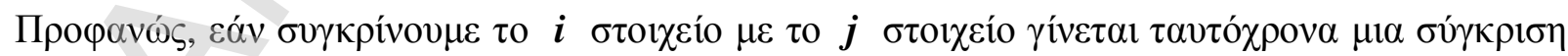

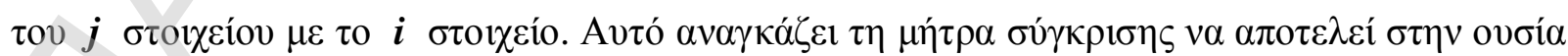

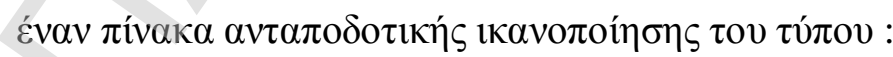

$$
c_{i j}=\frac{1}{c_{j i}}
$$




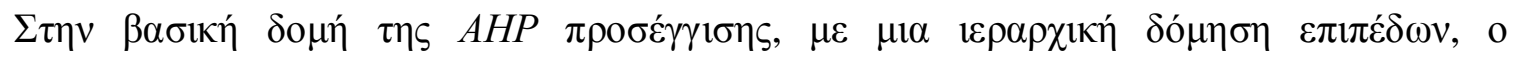

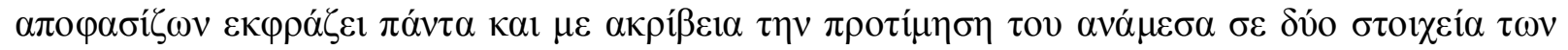

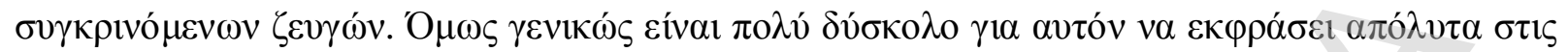

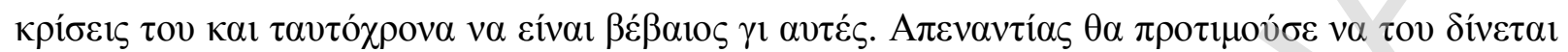

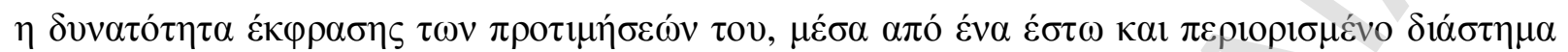
$\tau \varkappa \mu \omega ́ v$.

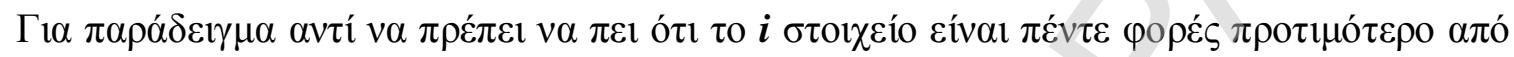

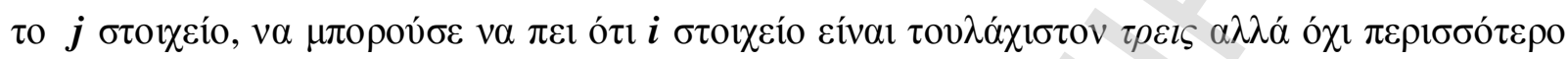

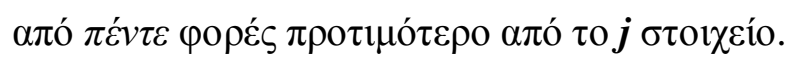

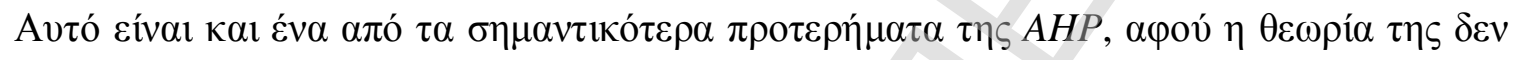

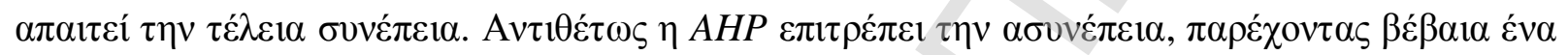

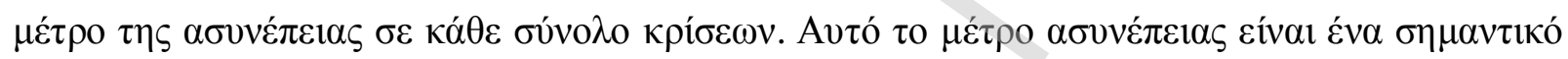

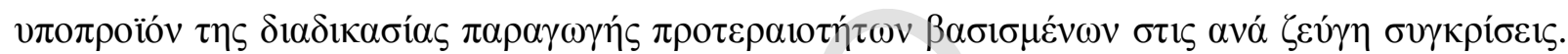

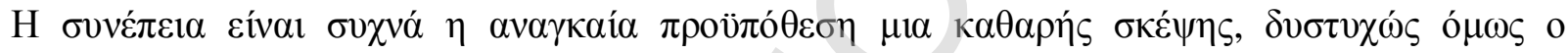

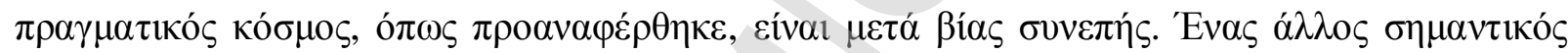

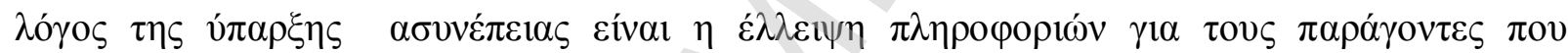

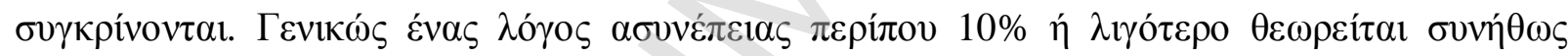
$\alpha \pi \circ \delta \varepsilon \kappa \tau$ ćs.

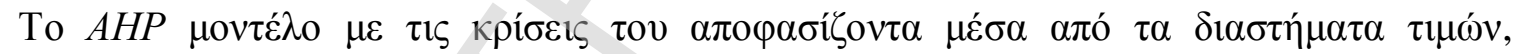

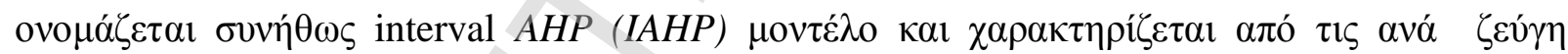

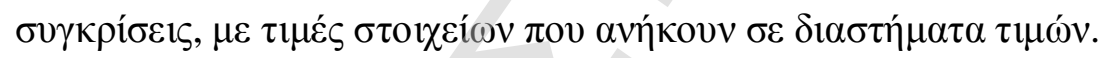

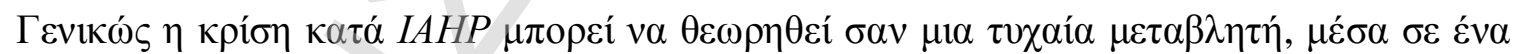

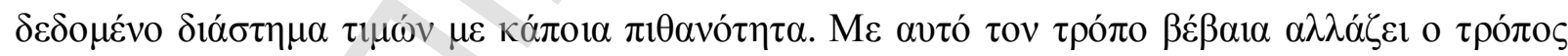

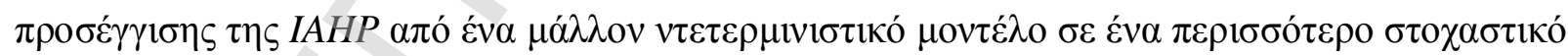

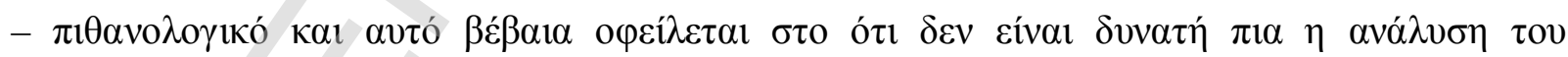

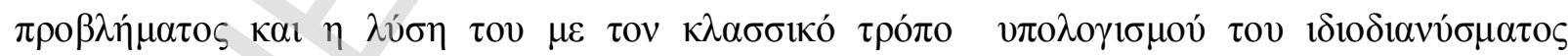
(eigenvector).

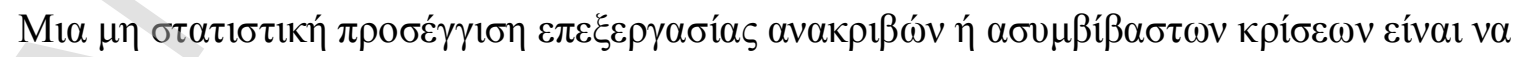

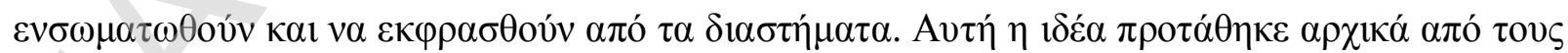

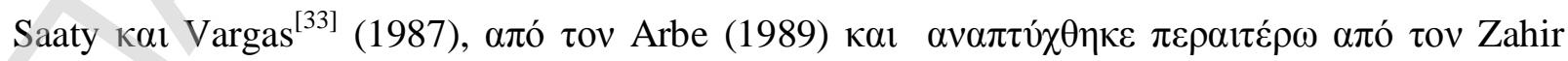

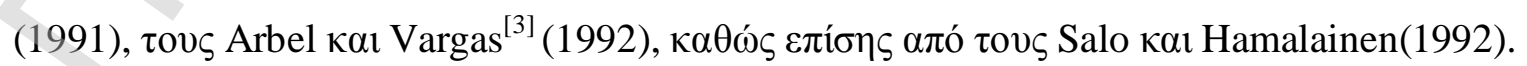

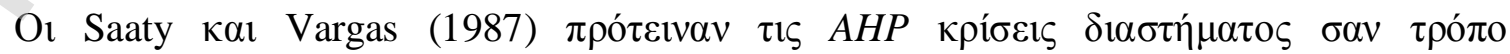

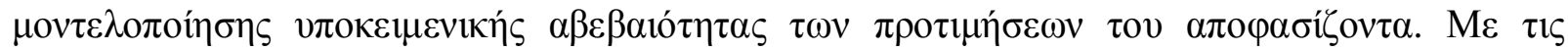




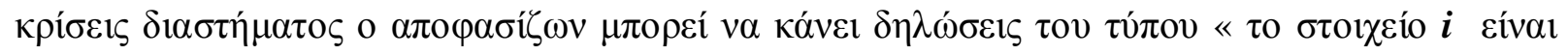

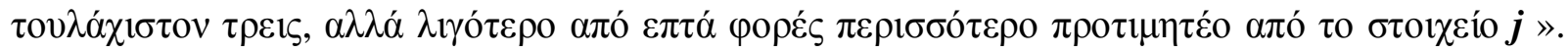

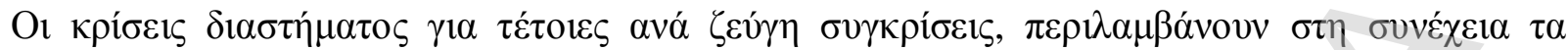

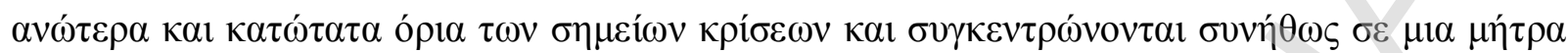

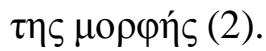

$$
C=\left[\begin{array}{ccccc}
1 & {\left[\begin{array}{ll}
l_{12} & u_{12}
\end{array}\right]} & {\left[\begin{array}{ll}
l_{13} & u_{13}
\end{array}\right]} & \ldots & {\left[\begin{array}{ll}
l_{1 n} & u_{1 n}
\end{array}\right]} \\
\left.\begin{array}{cc}
\frac{1}{u_{12}}, & \frac{1}{l_{12}}
\end{array}\right] & \left.\begin{array}{cc}
l_{23} & u_{23}
\end{array}\right] & \ldots & {\left[\begin{array}{ll}
l_{2 n} & u_{2 n}
\end{array}\right]} \\
{\left[\begin{array}{cc}
\frac{1}{u_{13}}, & \frac{1}{l_{13}}
\end{array}\right]\left[\begin{array}{ccc}
\frac{1}{u_{23}}, & \frac{1}{l_{23}}
\end{array}\right]} & 1 & \ldots & {\left[\begin{array}{ll}
l_{3 n} & u_{3 n}
\end{array}\right]} \\
\ldots & \ldots & \ldots & \ldots \\
{\left[\frac{1}{u_{1 n}},\right.} & \left.\frac{1}{l_{1 n}}\right]\left[\begin{array}{cc}
\frac{1}{u_{2 n}}, & \left.\frac{1}{l_{2 n}}\right]
\end{array}\right]\left[\begin{array}{cc}
\frac{1}{u_{3 n}}, & \frac{1}{l_{3 n}}
\end{array}\right] & \ldots & 1
\end{array}\right]
$$

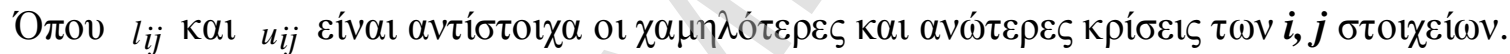

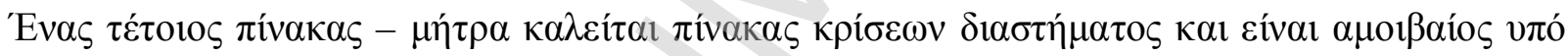

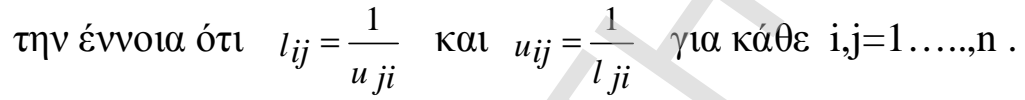

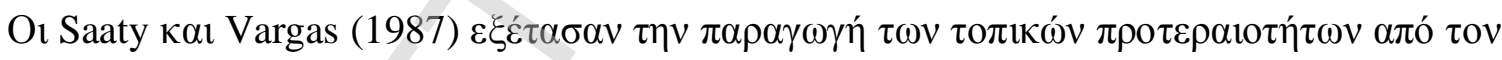

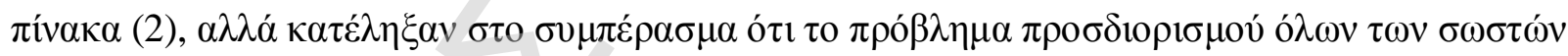

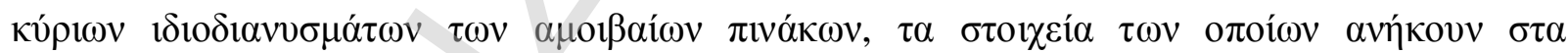

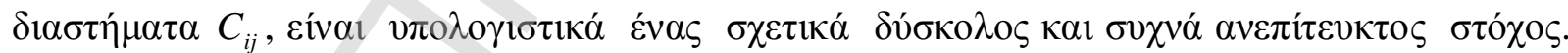

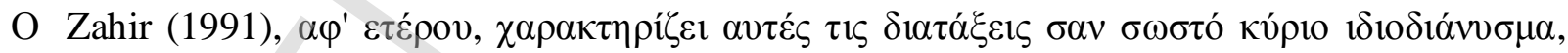

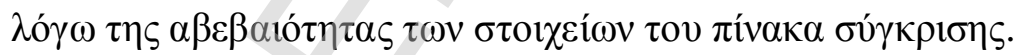

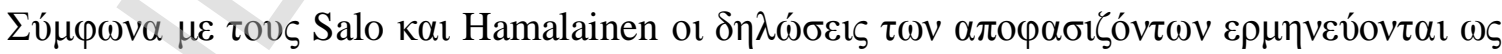

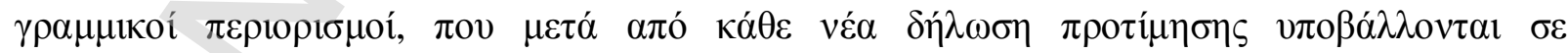

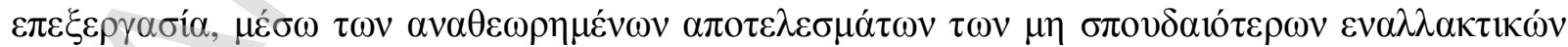

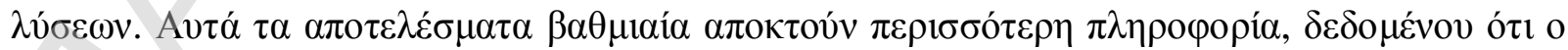

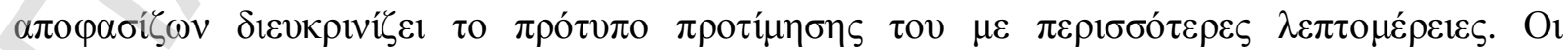

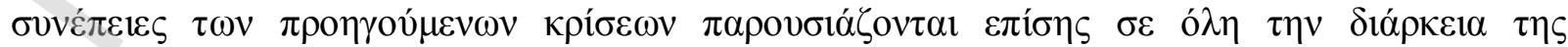




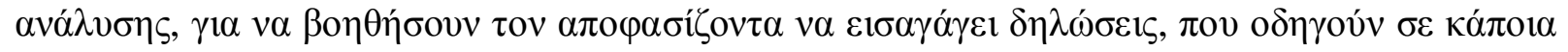
$\alpha \pi \circ \varphi \alpha \sigma \iota \tau \tau 1 \kappa o ́ \tau \varepsilon \rho \alpha \alpha \pi \mathrm{\alpha} \varepsilon \lambda \varepsilon \dot{\sigma} \sigma \mu \alpha \alpha$.

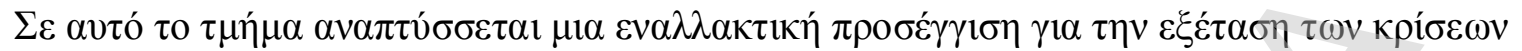

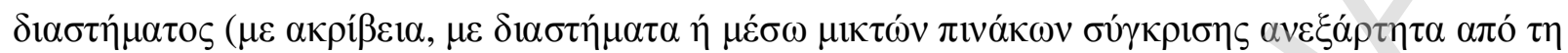

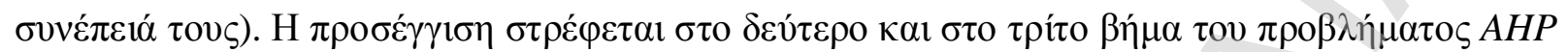

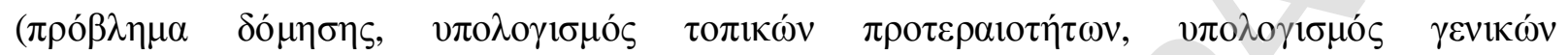

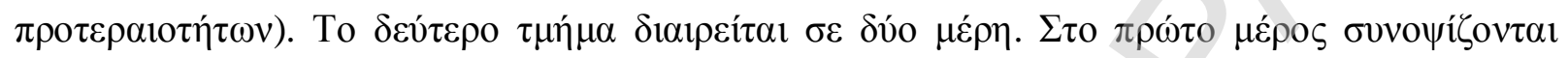

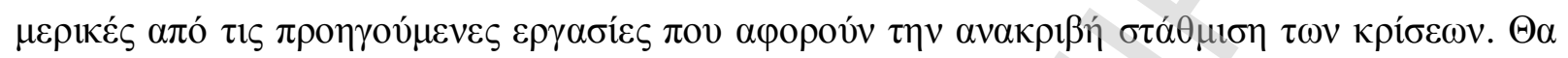

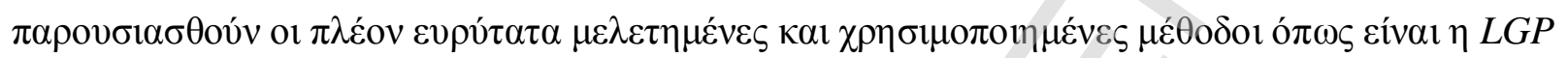
(Lexicographic Goal Programming) кaı $\eta$ FLP (Fuzzy Linear Programming).

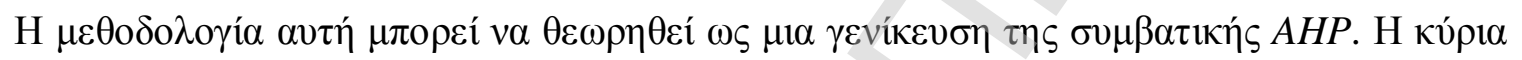

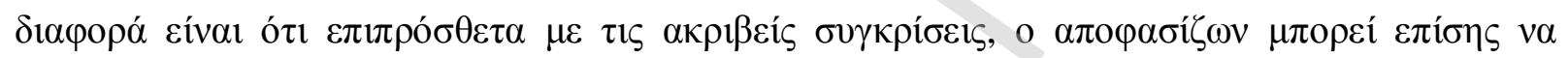

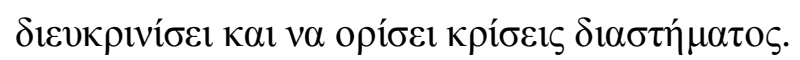

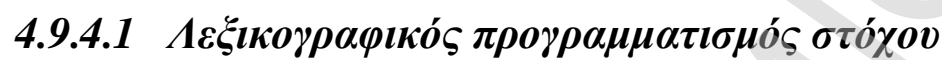

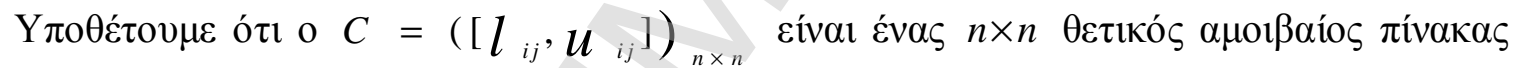

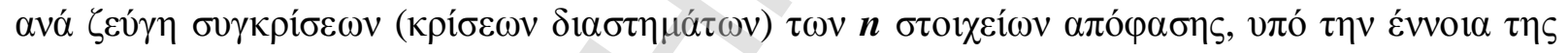

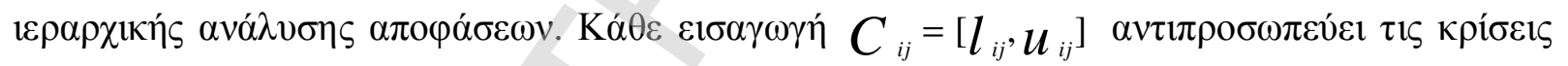

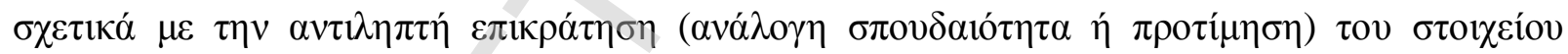

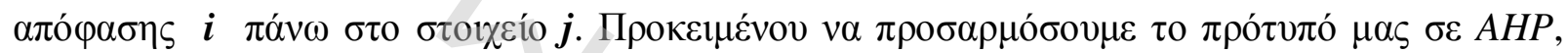

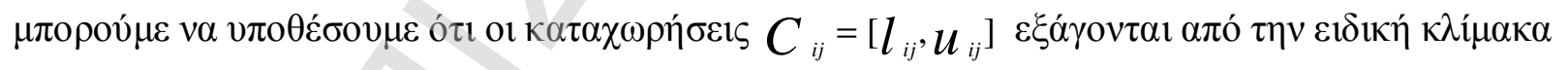

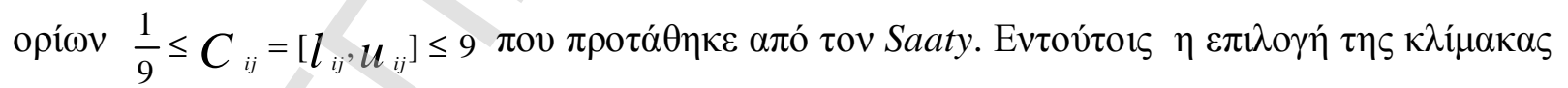

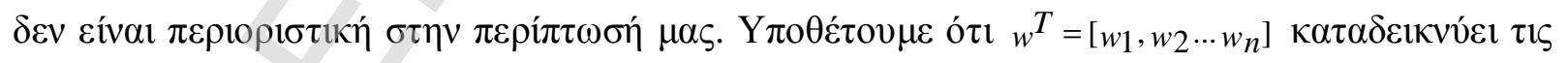

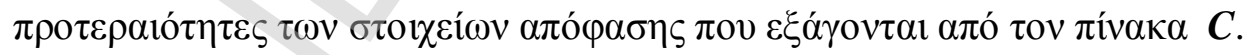

'E $\tau \sigma \imath \varepsilon \dot{\chi} \chi 00 \mu \varepsilon:$

$$
S=\left\{\left(w_{1}, \mathcal{W}_{2}, \cdots \mathcal{W}_{n}\right): l_{i j} \leq \frac{\mathcal{W}_{i}}{w_{j}} \leq u_{i j}, w_{i} \geq 0, \quad \sum_{i=1}^{n} \mathcal{W}_{i}=1, \gamma \dot{\alpha} \dot{\alpha}, j, \ldots, n\right\}
$$

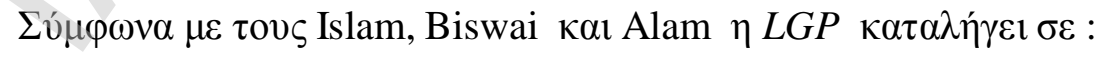




$$
\begin{aligned}
& \mathrm{m} \text { in } a=\left(p_{n n}+n_{n n}, \sum_{i=1}^{n-1} \sum_{j=i+1}^{n}\left(p_{i j}+p_{i j}^{\prime}\right)\right) \\
& v \pi \dot{o} \tau \mathrm{o} v \varsigma \quad \dot{\rho} \mathrm{\rho} v \varsigma: \\
& -w_{i}+l_{i j} w_{j}+n_{i j}-p_{i j}=0 \\
& w_{i}-u_{i j} w_{j}+n_{i j}^{\prime}-p_{i j}^{\prime}=0 \\
& i=1,2,3, \ldots n-1, \quad j=i+1, \ldots, n \\
& \sum_{i=1}^{n} w_{i}+n_{n n}-p_{n n}=1 \\
& \mu \varepsilon \quad w_{i}, n_{i j}, p_{i j}, p_{i j}^{\prime}, n_{i j}^{\prime} \geq 0 \quad \gamma l \dot{\alpha} \kappa \dot{\alpha} \theta \varepsilon \quad i, j
\end{aligned}
$$

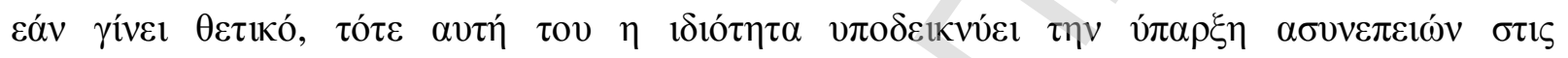

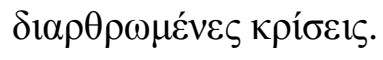

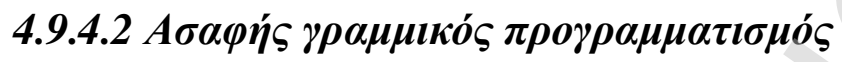

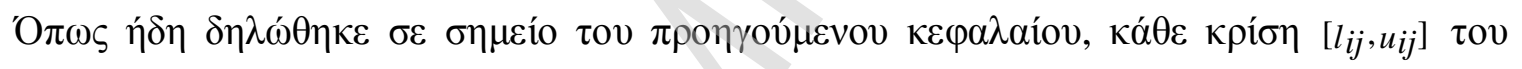

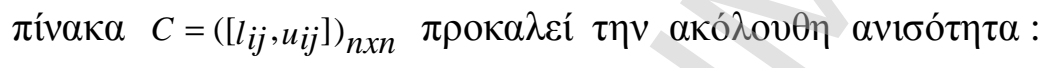

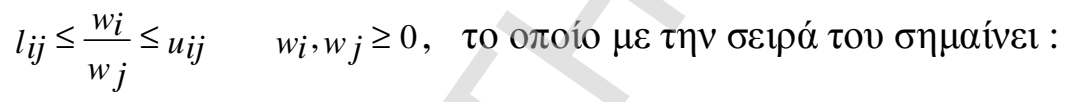

$$
\begin{aligned}
& -w_{i}+l_{i j} w_{j} \leq 0 \quad \kappa \alpha \imath \quad w_{i}-u_{i j} \leq 0
\end{aligned}
$$

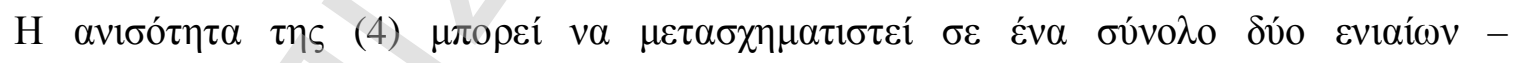

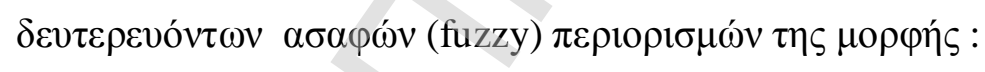

$$
-w_{i}+l_{i j} w_{j} \tilde{\leq} 0 \quad \kappa \alpha \imath \quad w_{i}-u_{i j} \tilde{\leq} 0
$$

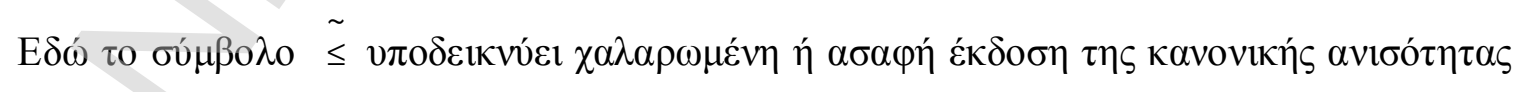

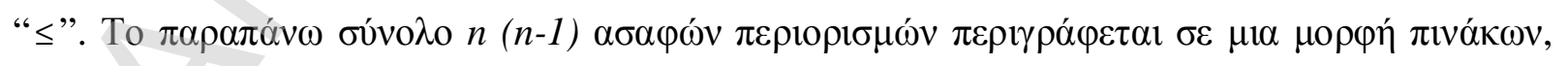
ó $\pi \omega \varsigma$ :

$$
C \mathrm{w} \leq 0
$$




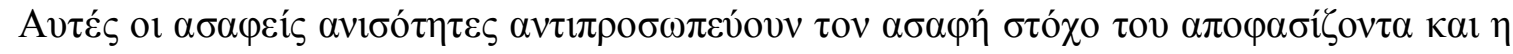

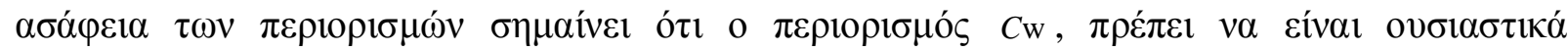

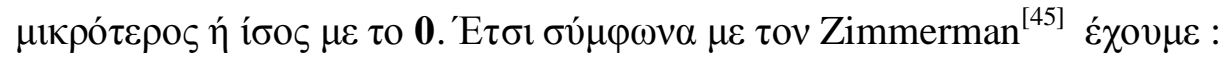

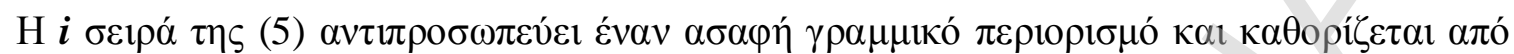

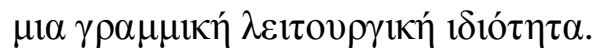

$$
\mu_{1}\left(C_{i^{w}}\right)=\left\{\begin{array}{cc}
1 & \left(C_{i^{w}}\right) \leq b_{i}^{\prime} \\
1-\frac{\left(C_{i^{w}}\right)-b_{i}^{\prime}}{d_{i}} & b_{i}^{\prime} \leq\left(C_{i}{ }_{i}\right) \leq b_{i}^{\prime}+d_{i} \\
0 & \left(C_{i}{ }^{w}\right) \geq b_{i}^{\prime}+d_{i}
\end{array}\right.
$$

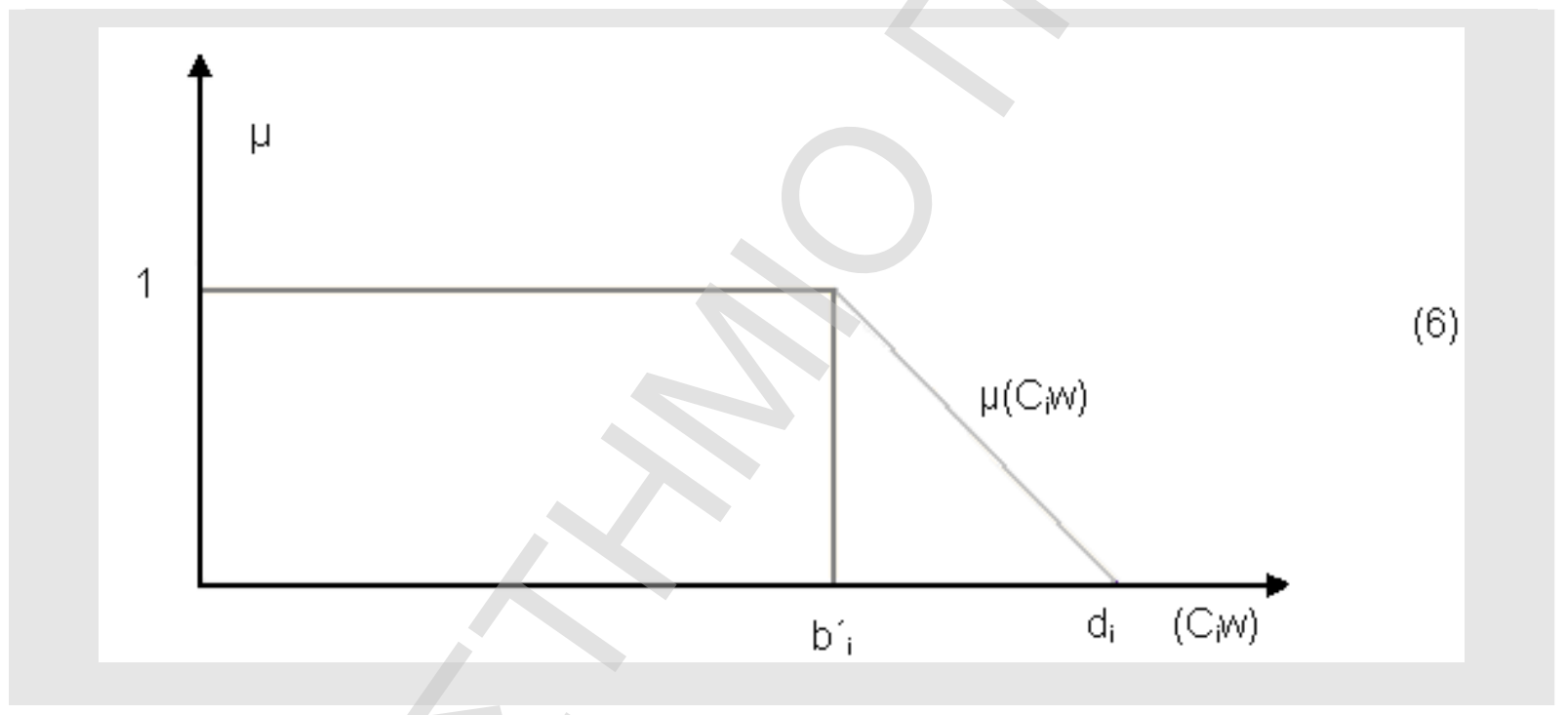

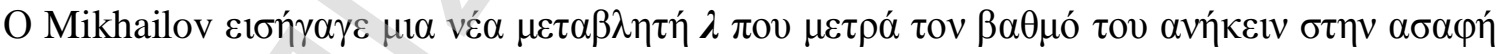

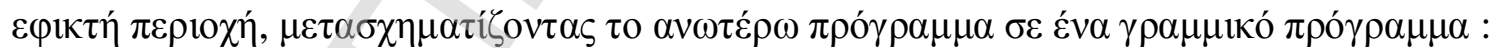

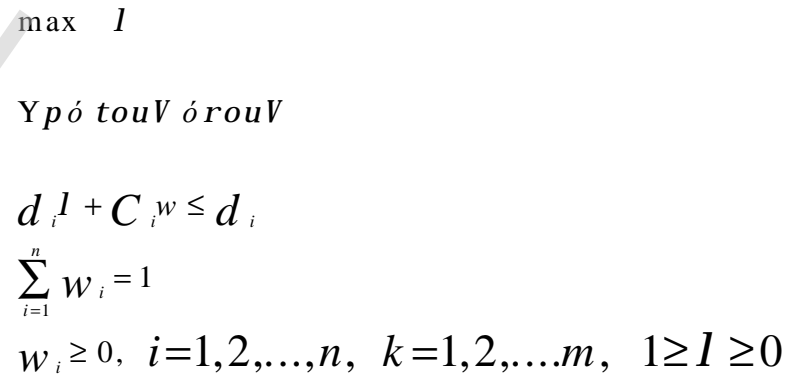




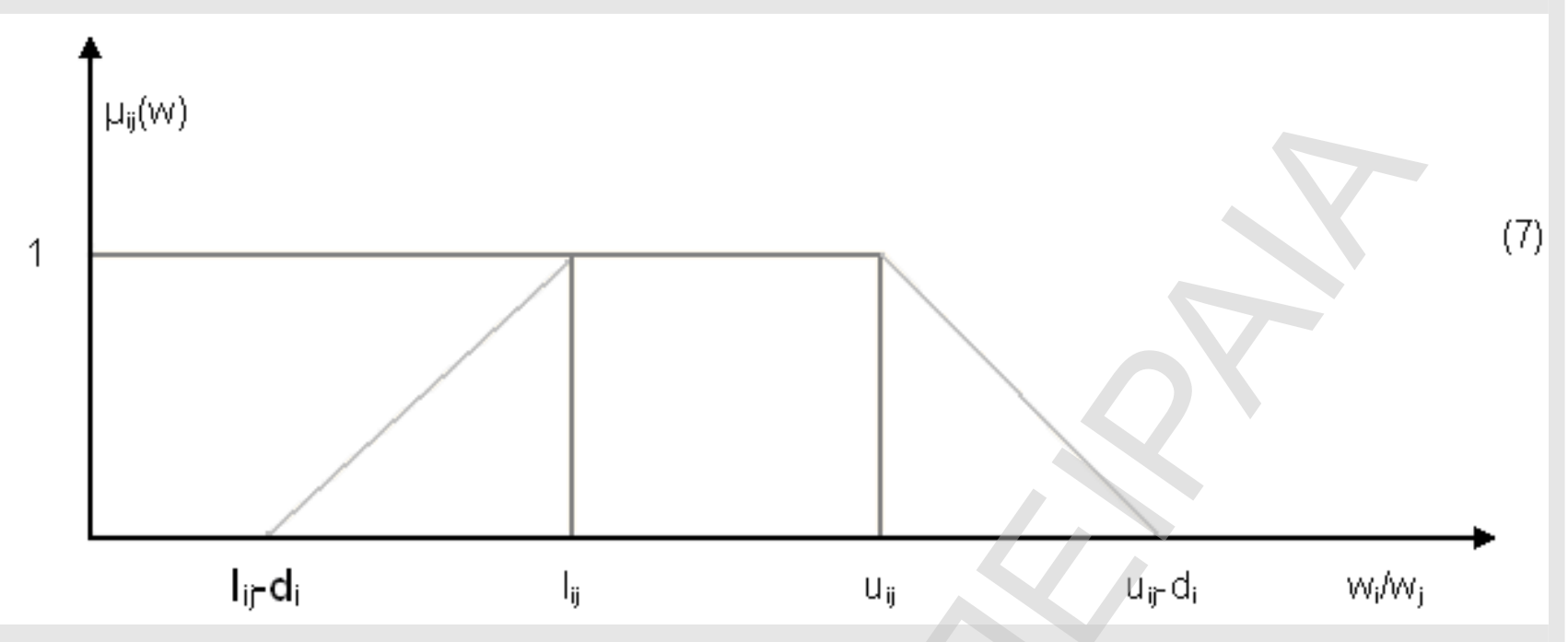

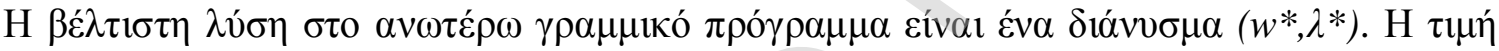

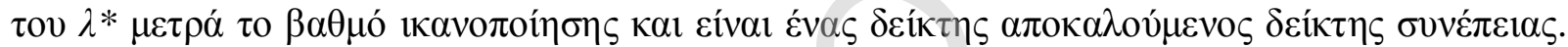

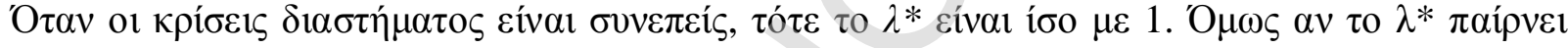

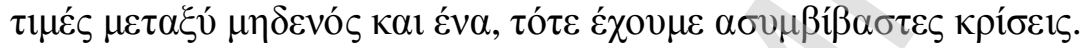

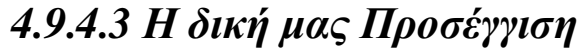

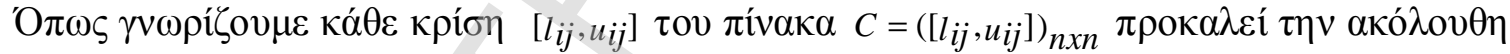
$\alpha v 1 \sigma o ́ \tau \eta \tau \alpha:$

$$
l_{i j} \leq \frac{w_{i}}{w_{j}} \leq u_{i j} \quad w_{i}, w_{j} \geq 0
$$

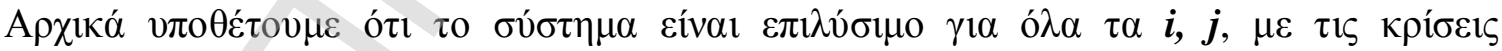

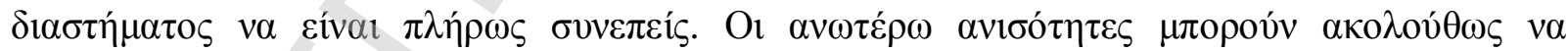
$\varepsilon \kappa \varphi \rho \alpha \sigma \tau o u ́ v \omega \varsigma \varepsilon \xi \dot{n} \varsigma$ :

$$
\frac{w_{i}}{w_{j}}=l_{i j}+s_{i j}\left(u_{i j}-l_{i j}\right) \Rightarrow w_{i}=w_{j} l_{i j}+w_{j} s_{i j}\left(u_{i j}-l_{i j}\right) \mu \varepsilon \quad s_{i j} \in[0,1]
$$

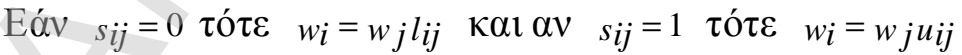

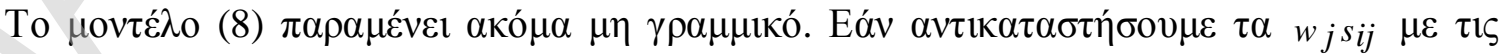

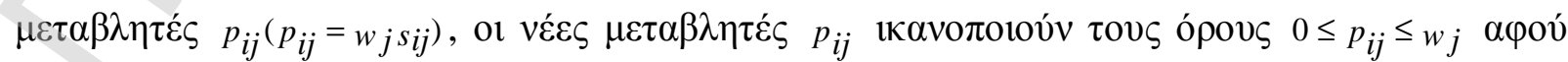




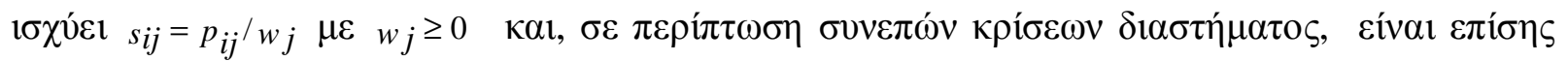

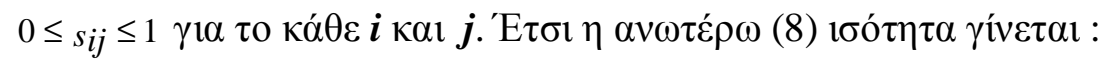

$$
w_{i}=w_{j} l_{i j}+p_{i j}\left(u_{i j}-l_{i j}\right)
$$

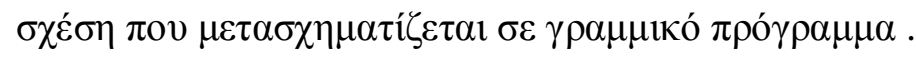

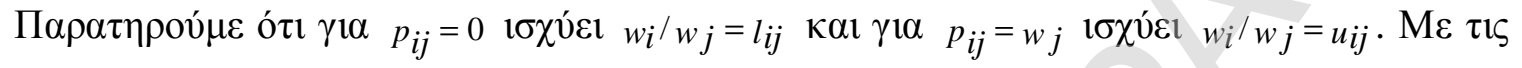

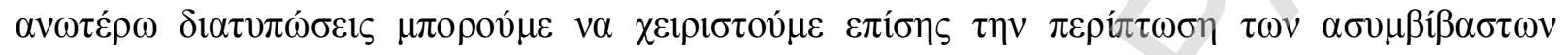

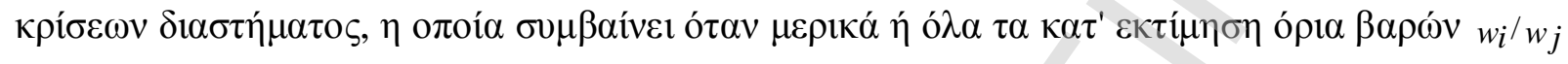

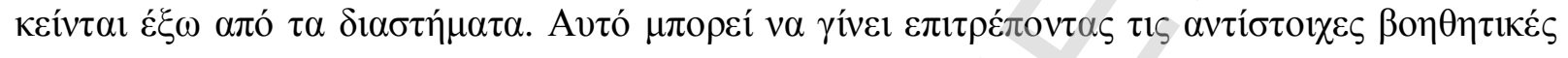

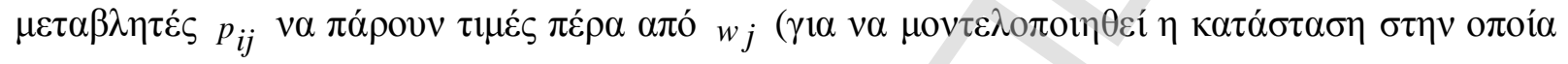

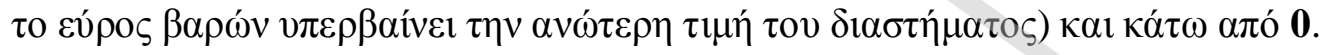

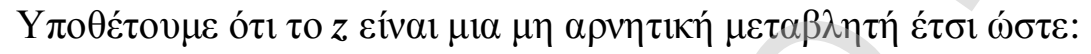

$$
-z \leq p_{i j \leq} w_{j}+z \text {. }
$$

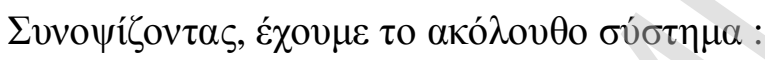

$$
\begin{aligned}
& w_{i}=w_{j} l_{i j}+w_{j} s_{i j}\left(u_{i j}-l_{i j}\right)=w_{j} l_{i j}+p_{i j}\left(u_{i j}-l_{i j}\right) \\
& -z \leq p_{i j \leq} w_{j}+z \\
& o r \\
& w_{i-} w_{j} l_{i j}-p_{i j}\left(u_{i j}-l_{i j}\right)=0 \\
& p_{i j-} w_{j}-z \leq 0 \\
& -z-p_{i j \leq} 0
\end{aligned}
$$

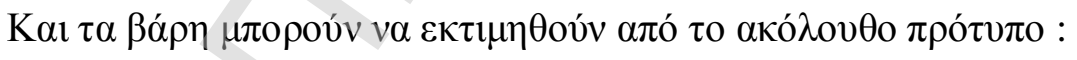




$$
\begin{aligned}
& \min \quad z \\
& w_{i-} w_{j} l_{i j}-p_{i j}\left(u_{i j}-l_{i j}\right)=0 \\
& p_{i j-} w_{j}-z \leq 0 \\
& -z-p_{i j \leq} 0 \\
& \sum_{j=1}^{n} w_{j}=1 \\
& w_{j} \geq 0, z \geq 0 \\
& p_{i j} \quad \text { free }
\end{aligned}
$$

\begin{tabular}{|c|c|c|c|c|}
\hline & $\mathbf{A}$ & B & $\bar{C}$ & D \\
\hline A & 1 & {$\left[\begin{array}{ll}1 & 2\end{array}\right]$} & {$\left[\begin{array}{ll}1 & 2\end{array}\right]$} & {$\left[\begin{array}{ll}2 & 5\end{array}\right]$} \\
\hline B & & 1 & {$\left[\begin{array}{ll}2 & 5\end{array}\right]$} & {$\left[\begin{array}{ll}4 & 5\end{array}\right]$} \\
\hline $\mathrm{C}$ & & & 1 & {$\left[\begin{array}{ll}2 & 3\end{array}\right]$} \\
\hline $\mathrm{D}$ & & & & 1 \\
\hline
\end{tabular}

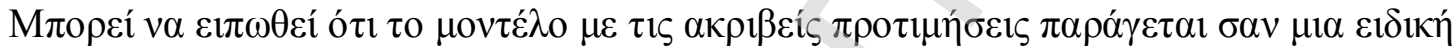

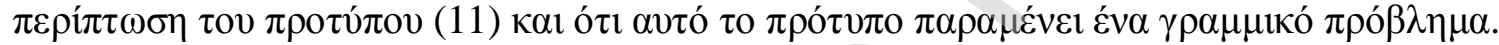

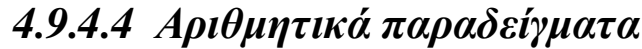

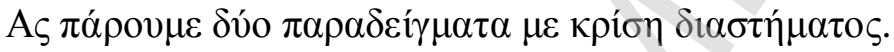

Пívakas (1)

\begin{tabular}{|l|l|l|l|l|}
\hline & \multicolumn{1}{|c|}{$\mathrm{A}$} & \multicolumn{1}{c|}{$\mathrm{C}$} & \multicolumn{1}{c|}{ C } & D \\
\hline $\mathrm{A}$ & 1 & {$\left[\begin{array}{ll}1 & 2\end{array}\right]$} & {$\left[\begin{array}{ll}1 & 2\end{array}\right]$} & {$\left[\begin{array}{ll}2 & 3\end{array}\right]$} \\
\hline $\mathrm{B}$ & & 1 & {$\left[\begin{array}{ll}3 & 5\end{array}\right]$} & {$\left[\begin{array}{ll}4 & 5\end{array}\right]$} \\
\hline $\mathrm{C}$ & & & 1 & {$\left[\begin{array}{ll}6 & 8\end{array}\right]$} \\
\hline $\mathrm{D}$ & & & & 1 \\
\hline
\end{tabular}

Пívakas (2)

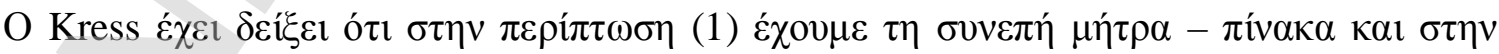

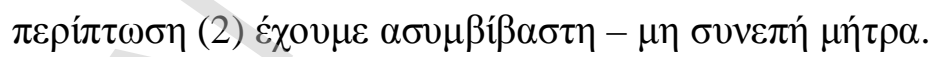

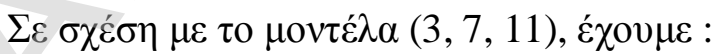




\begin{tabular}{|c|c|c|c|c|c|c|}
\hline & \multicolumn{3}{|c|}{ ПЕРІПТ ДЕН (1) } & \multicolumn{3}{|c|}{ ПЕРІПТ ДУН (2) } \\
\hline & LGP & $\begin{array}{l}\text { FLP } \\
\mathrm{di}=1\end{array}$ & D.D. & LGP & $\begin{array}{l}\text { FLP } \\
\mathrm{di}=1\end{array}$ & D.D. \\
\hline W1 & 0,3636 & 0,3636 & 0,3636 & 0,3030 & 0,31818 & 0,3103 \\
\hline $\mathrm{W} 2$ & 0,3636 & 0,3636 & 0,3636 & 0,4545 & 0,43181 & 0,4137 \\
\hline W3 & 0,1818 & 0,1818 & 0,1818 & 0,1515 & 0,19318 & 0,2068 \\
\hline W4 & 0,0909 & 0,0909 & 0,0909 & 0,0910 & 0,05681 & 0,0689 \\
\hline & $\alpha=0$ & $\lambda=1$ & $\mathrm{z}=0$ & $\alpha=0,5758$ & $\lambda=0,8522$ & $z=0,1034$ \\
\hline
\end{tabular}

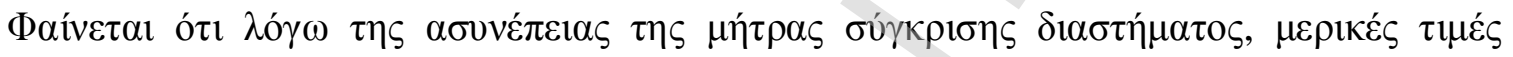

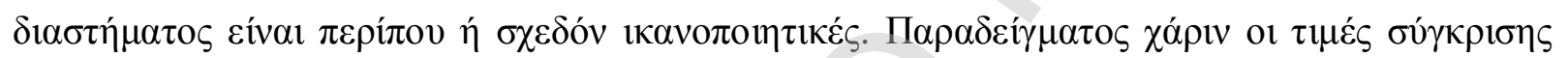

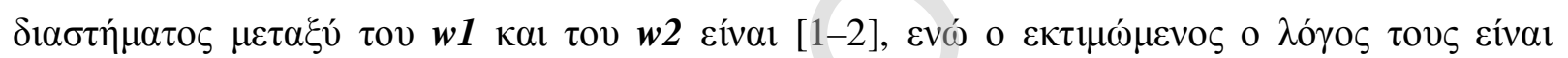
$\frac{w 1}{w 2}=\frac{0,3103}{0,4137}=0,75$.

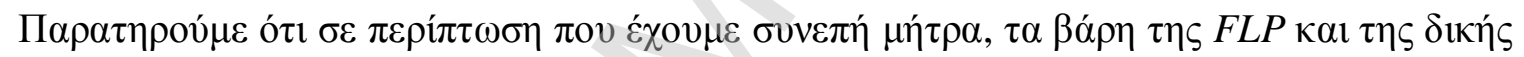
$\mu \alpha \varsigma \pi \rho \circ \sigma \dot{\gamma} \gamma \gamma 1 \sigma \eta \varsigma \sigma v \mu \pi i ́ \pi \tau 0 v v$.

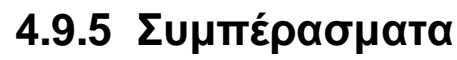

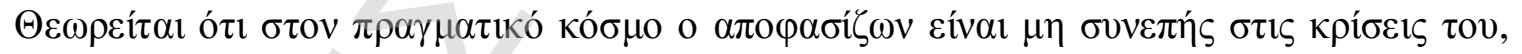

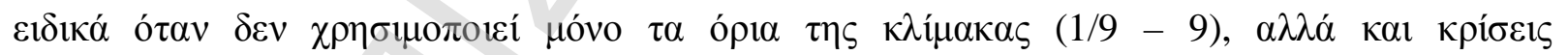

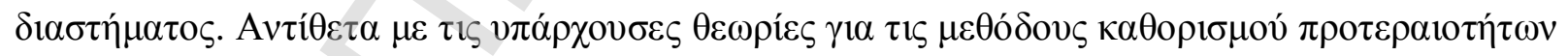

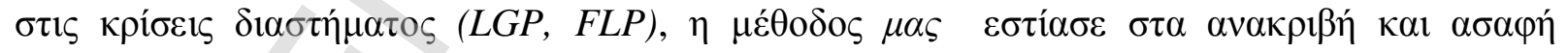

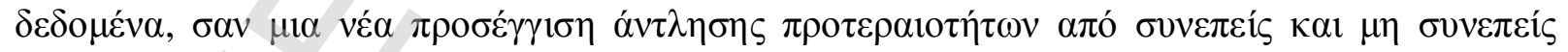

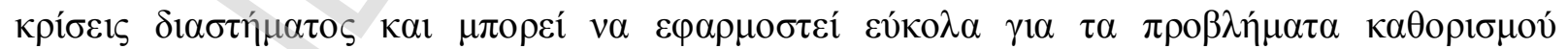

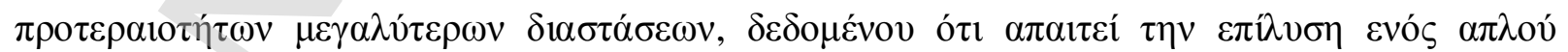

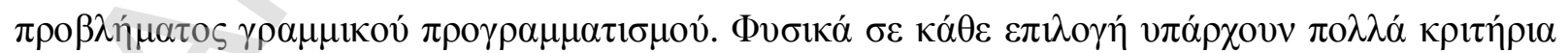

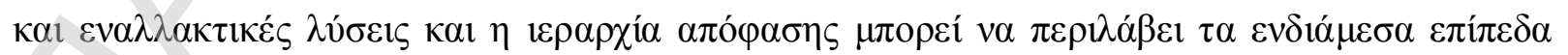

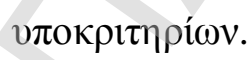




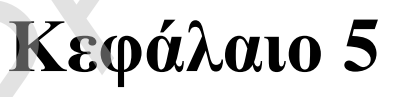

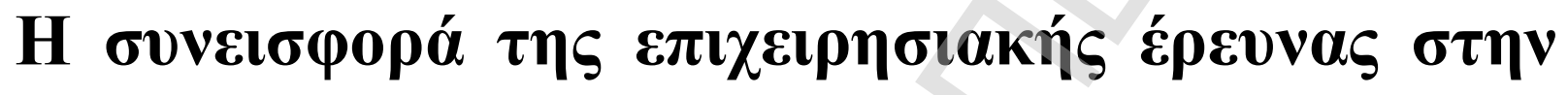

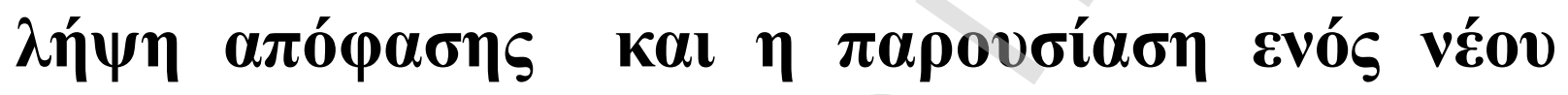

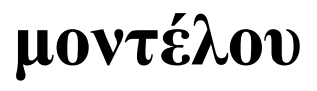

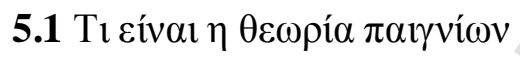

$5.2 \Sigma v v \delta v \alpha \sigma \tau 1 \kappa \eta ́ ~ A v \alpha ́ \lambda v \sigma \eta$

5.3 Avó $\lambda v \sigma \eta \gamma \nu \omega \mu \omega ́ v$

$5.4 \Sigma v \mu \pi \varepsilon \dot{\varepsilon} \rho \alpha \mu \alpha$ 


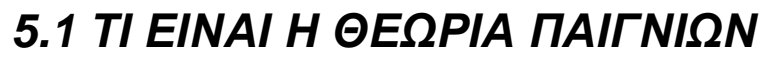

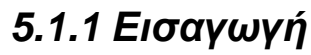

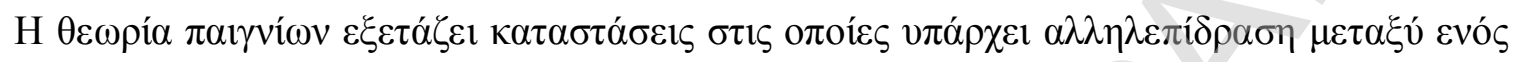

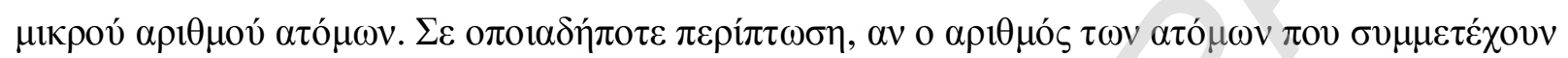

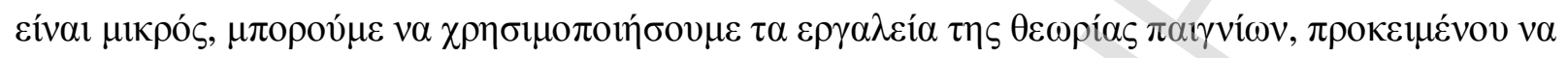

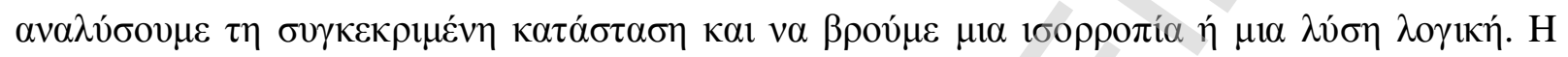

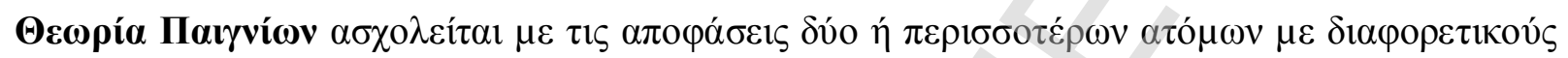

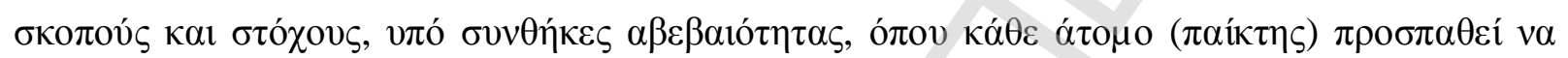

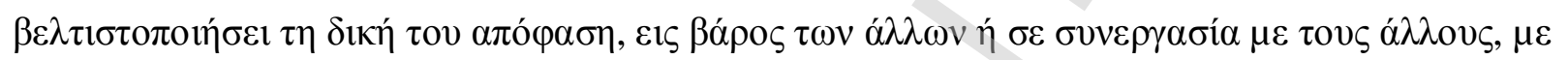

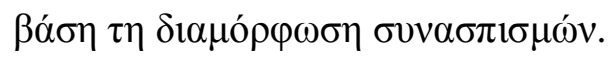

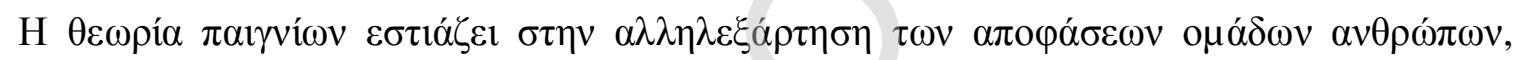

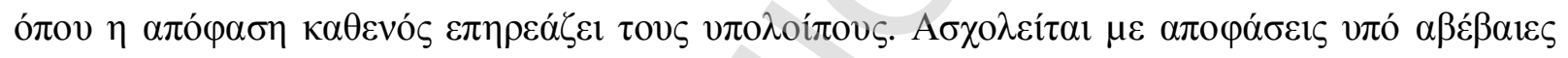

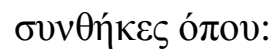

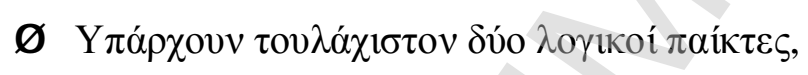

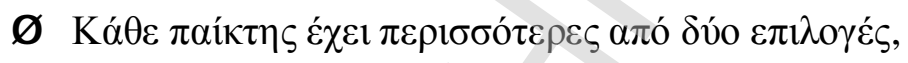

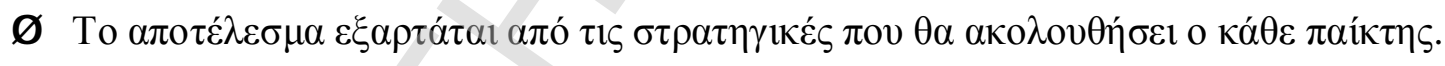

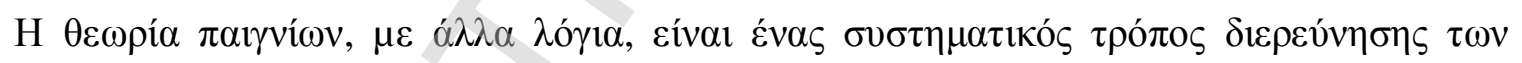

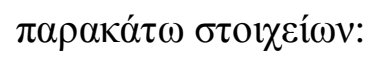

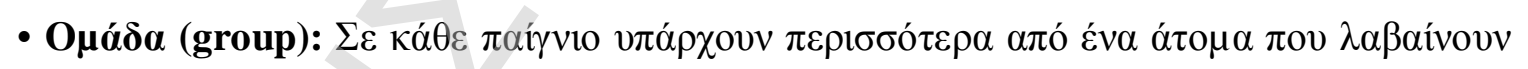

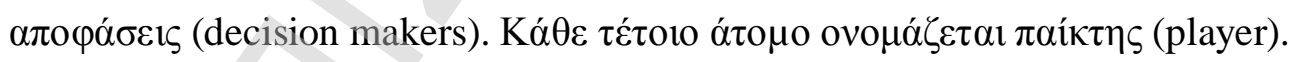

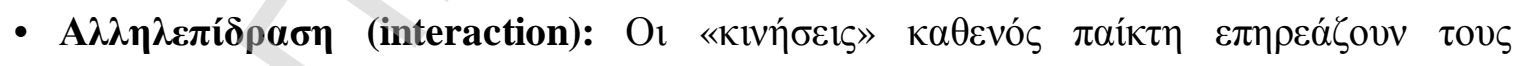

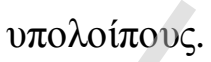

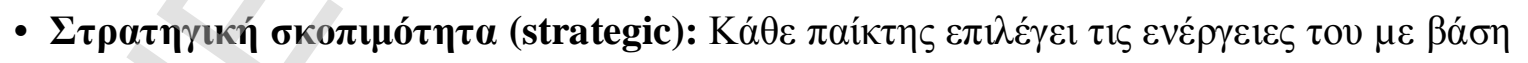
$\tau \eta v \varepsilon \rho \mu \eta v \varepsilon i ́ \alpha \alpha \omega v \alpha \lambda \lambda \eta \lambda \varepsilon \pi i \delta \rho \alpha ́ \sigma \varepsilon \omega v$.

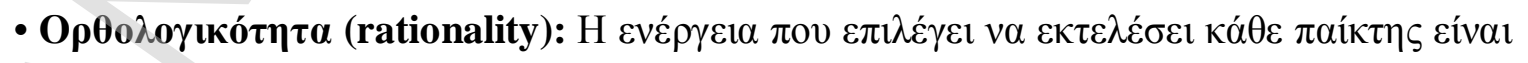

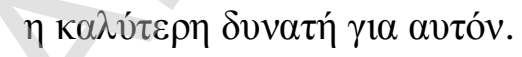

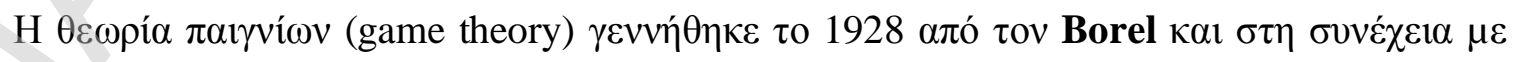

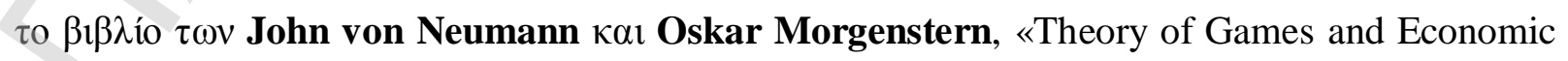

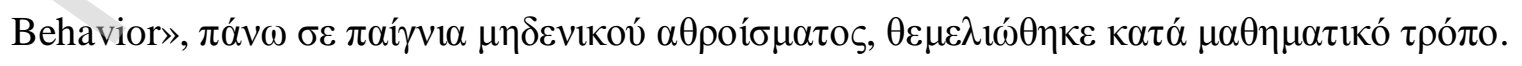

Oı J. von Neumann кaı O. Morgenstern : 
I. 'O

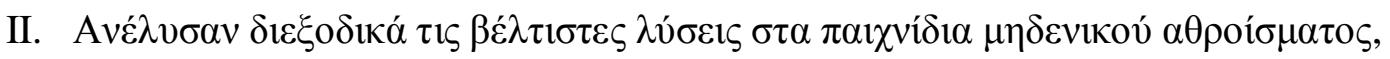

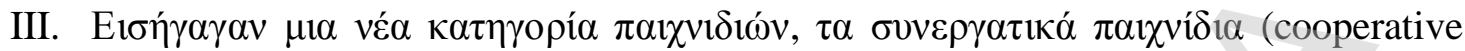
games).

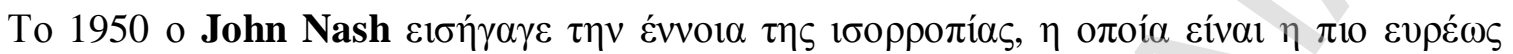

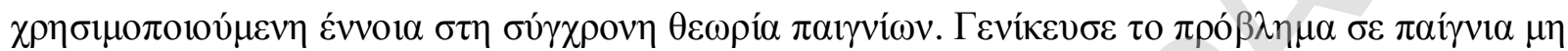

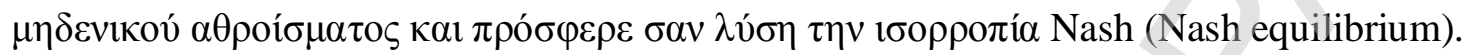

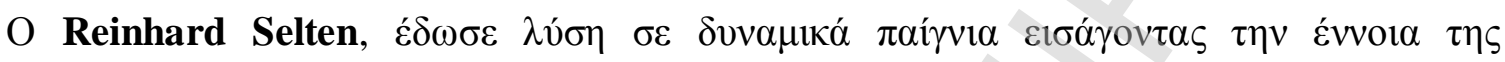

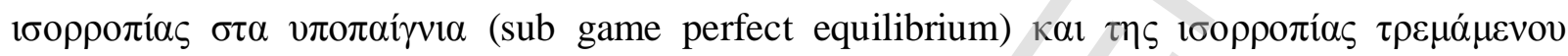
$\chi \varepsilon \rho ı v ́$ (trembling hand perfect equilibrium)

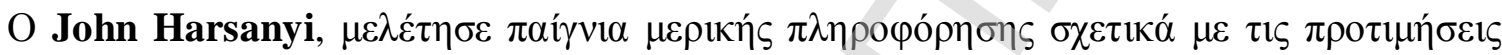

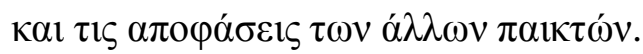

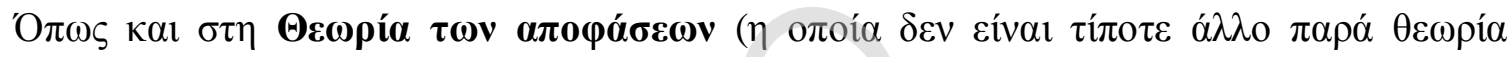

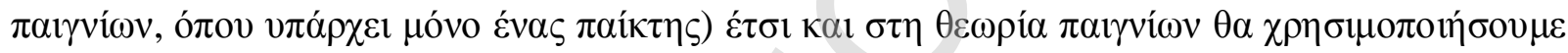

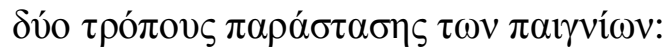

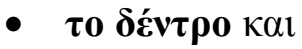

- $\tau \eta \mu \eta ́ \tau \rho \alpha$

Пıо $\alpha v \alpha \lambda v \tau \imath \kappa \alpha ́:$

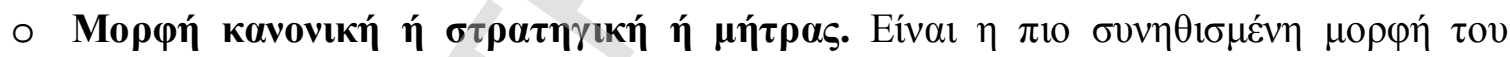

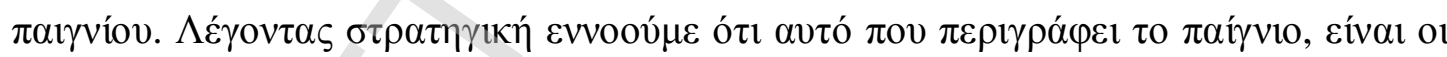

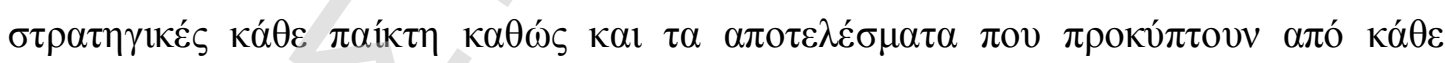

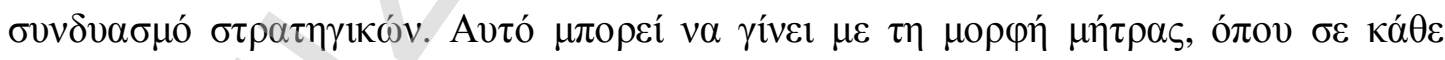

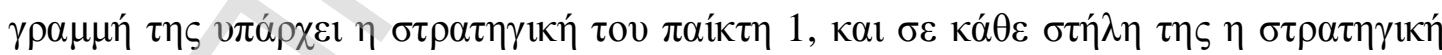

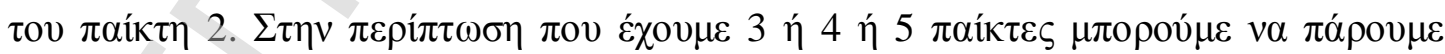
$\mu \eta ́ \tau \rho \alpha \pi \varepsilon \rho \iota \sigma \sigma o \tau \varepsilon ́ \rho \omega v \delta 1 \alpha \sigma \tau \alpha ́ \sigma \varepsilon \omega v$.

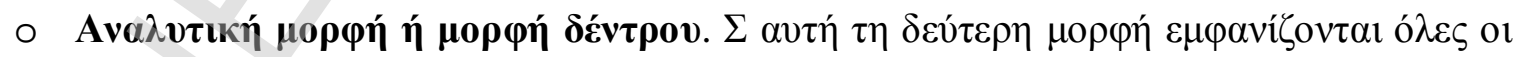

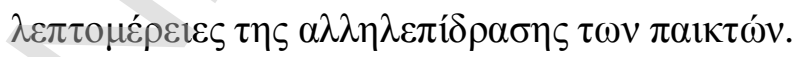

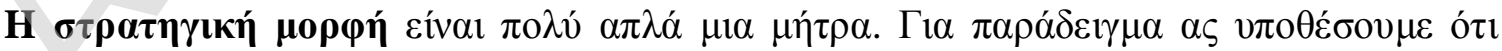

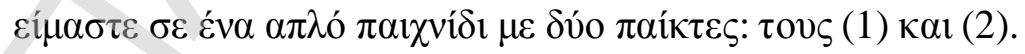

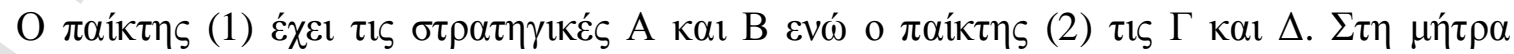

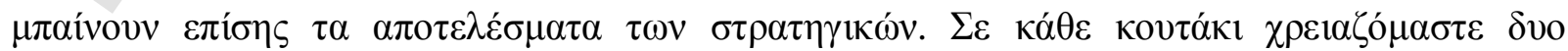

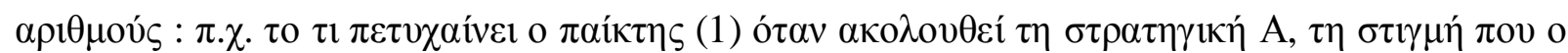




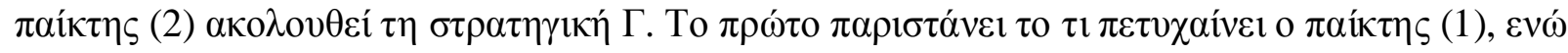

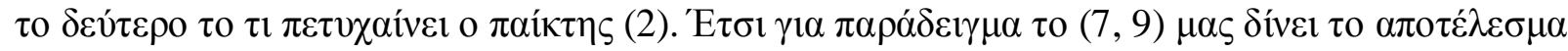

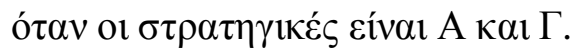

\begin{tabular}{|c|c|c|}
\hline & $\Gamma$ & $\Delta$ \\
\hline A & 7,9 & 3,10 \\
\hline B & 9,19 & 0,7 \\
\hline
\end{tabular}

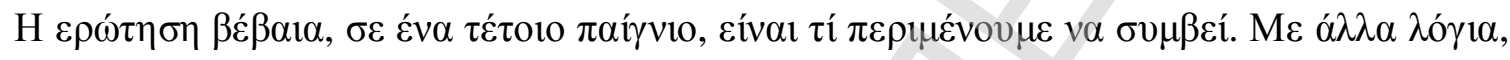

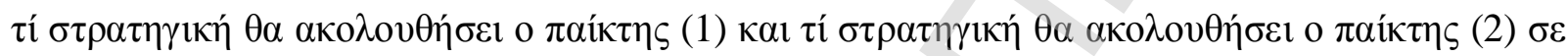

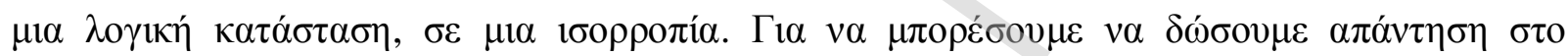

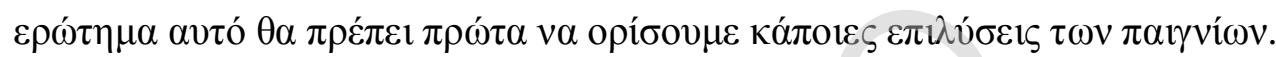

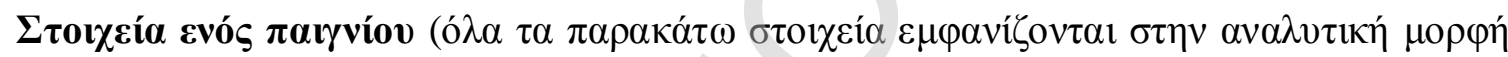

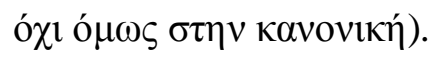

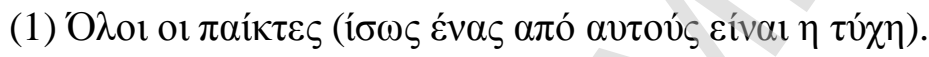

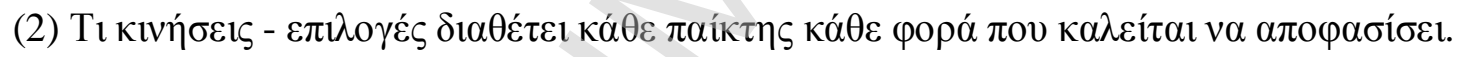

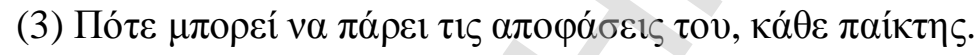

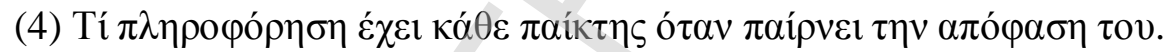

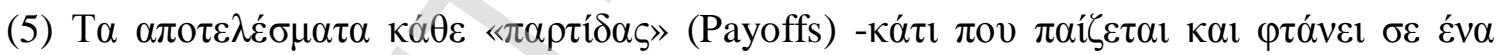

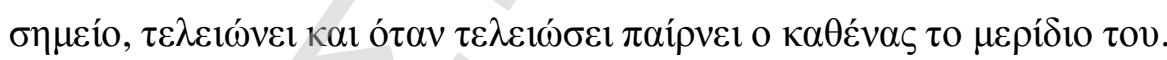

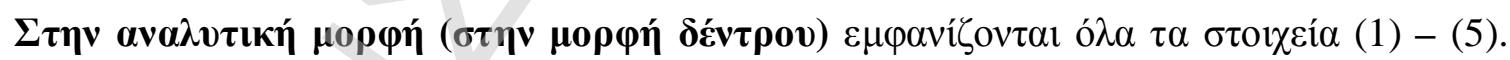

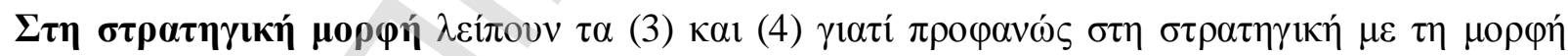

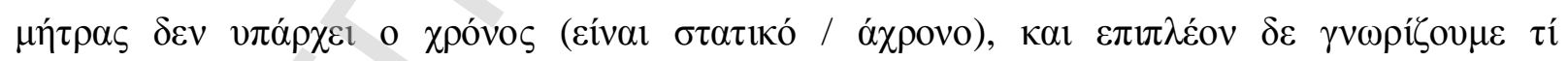

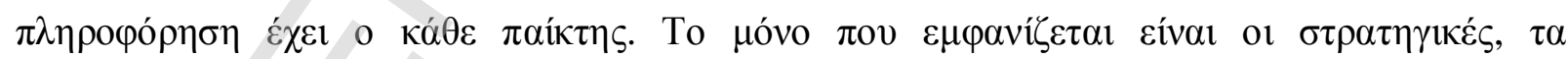
$\alpha \pi \mathrm{o} \varepsilon \lambda \varepsilon \dot{\sigma} \sigma \mu \alpha \tau \alpha$ (pay oft) $\kappa \alpha \imath$ ol $\pi \alpha i ́ \kappa \tau \varepsilon \varsigma$.

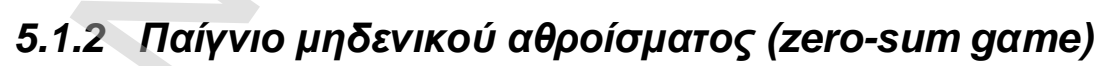

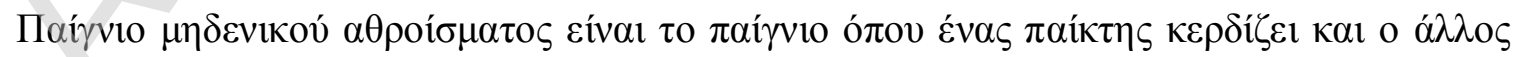

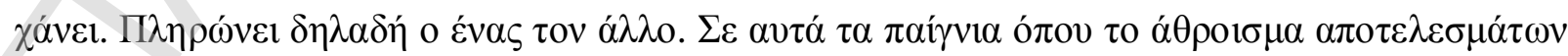

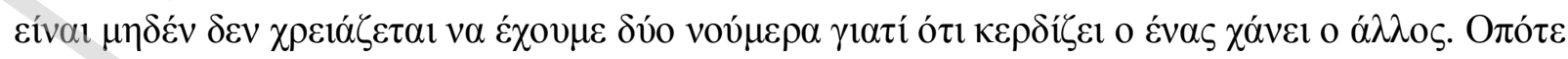

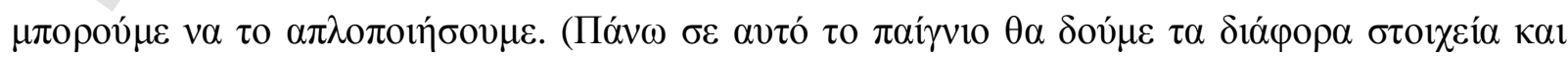




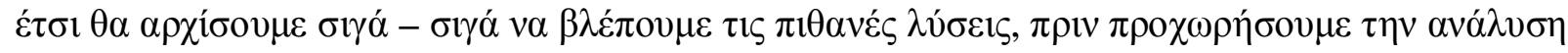
$\mu \alpha \varsigma \sigma \varepsilon \beta \alpha ́ \theta 0 \varsigma)$.

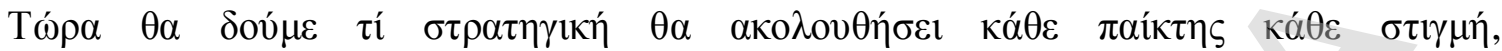

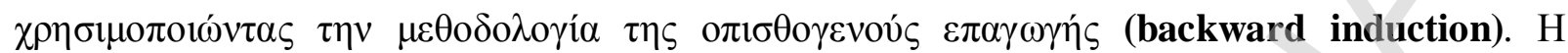

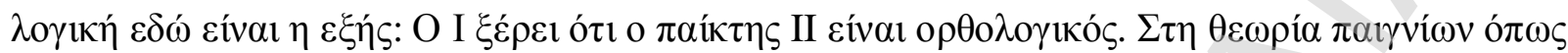

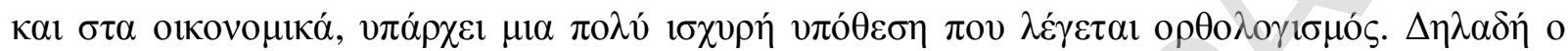

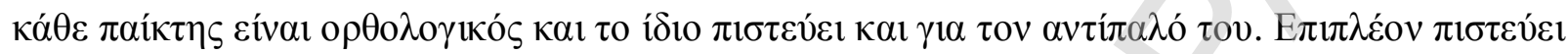

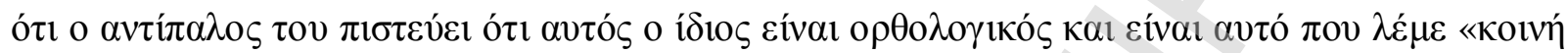

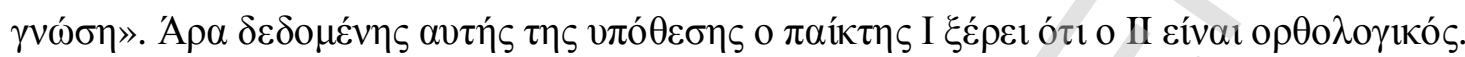

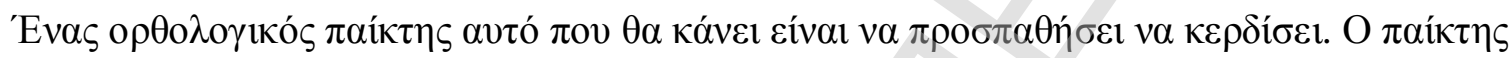

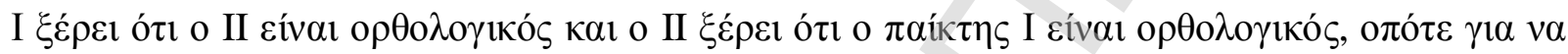

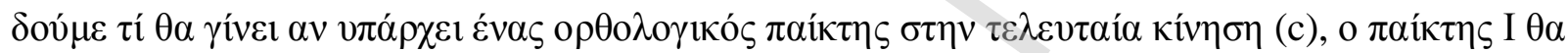

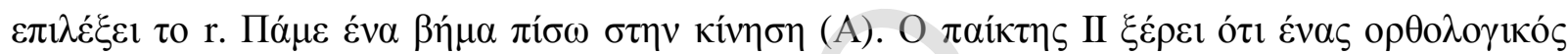

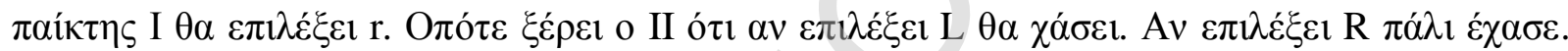

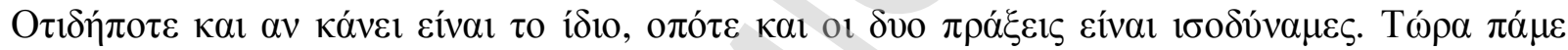

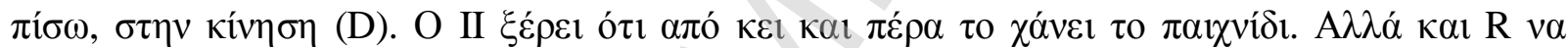

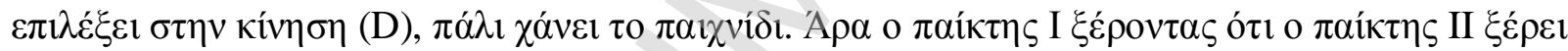

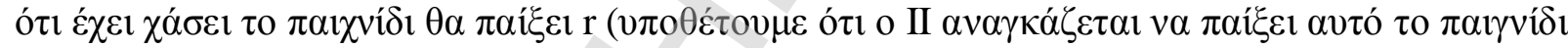

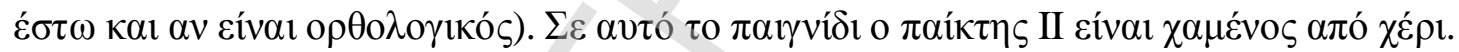

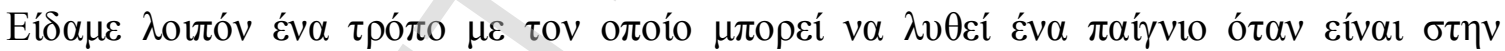

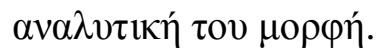

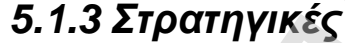

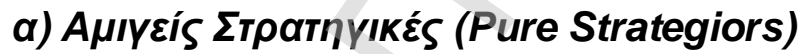

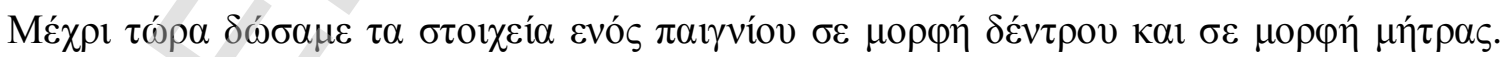

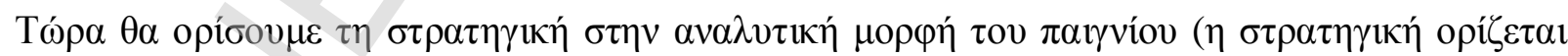

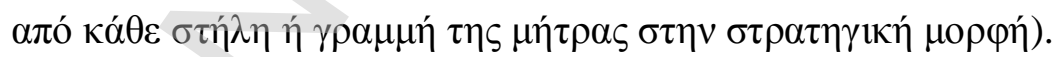

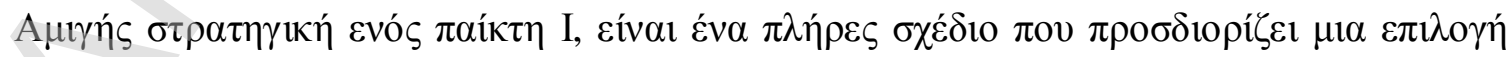

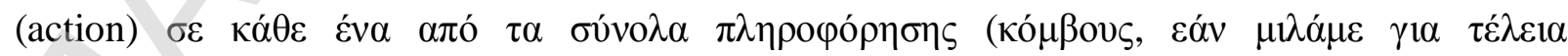

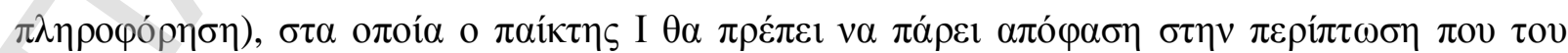

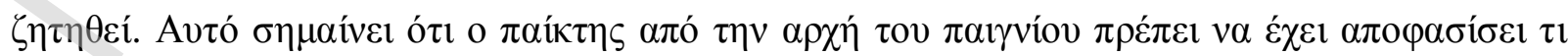

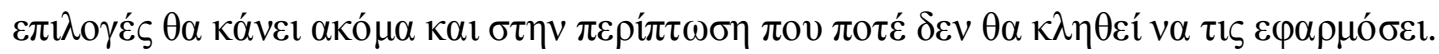




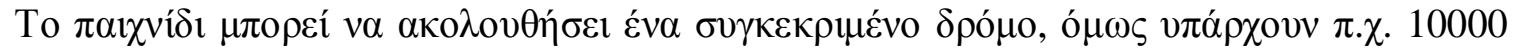

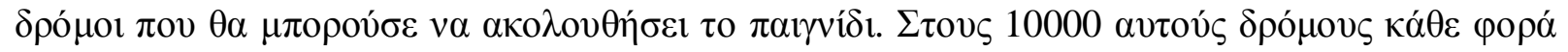

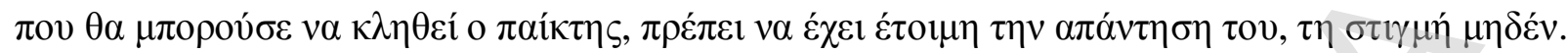

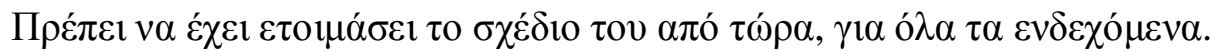

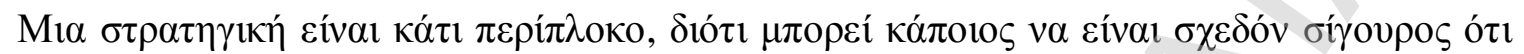

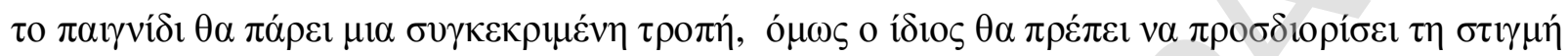

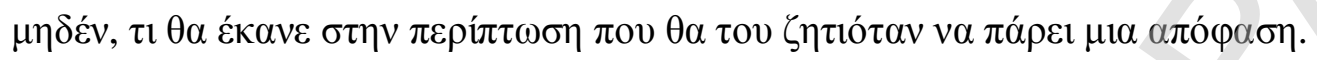

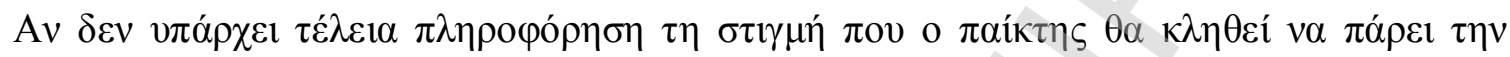

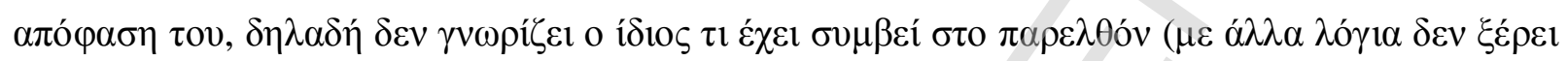

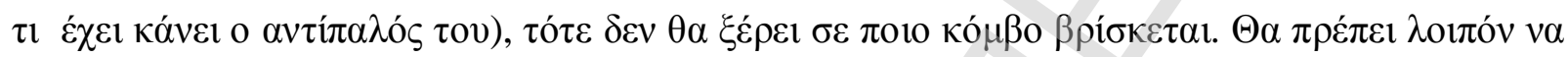

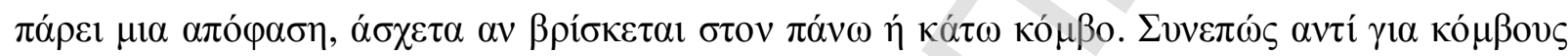

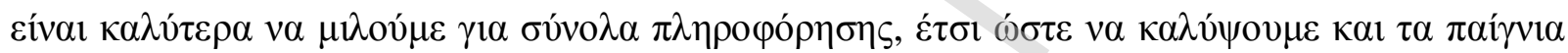

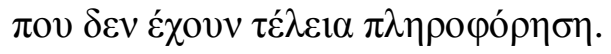

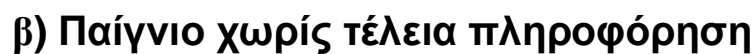

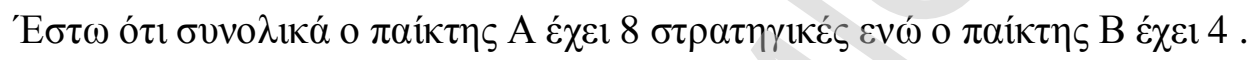

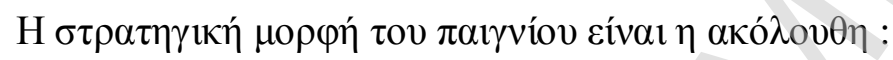

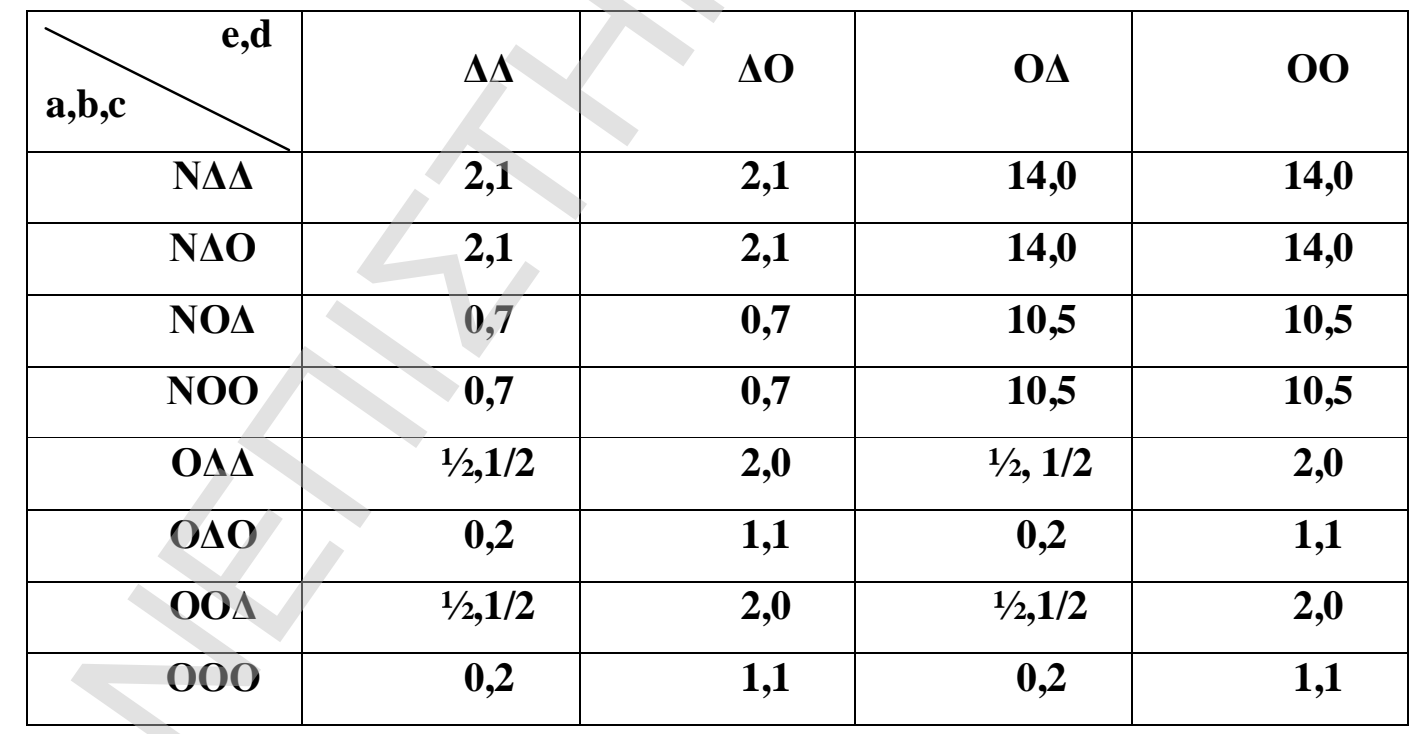

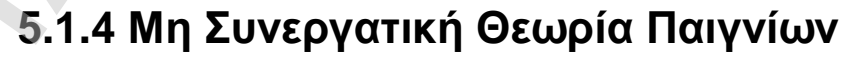

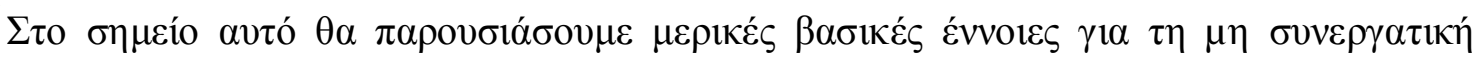

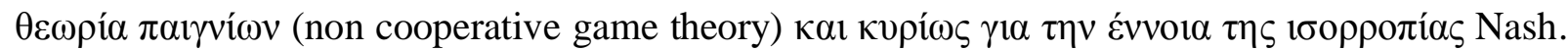




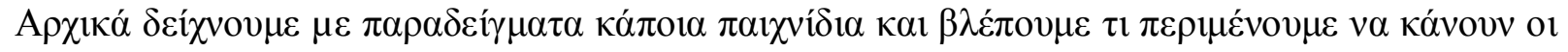

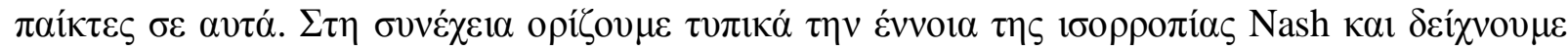

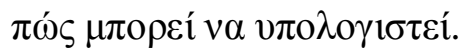

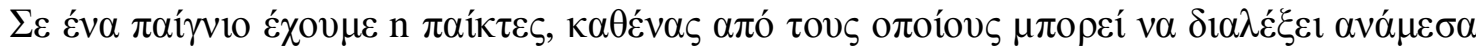

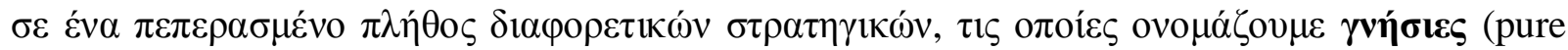

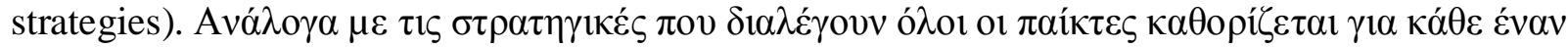

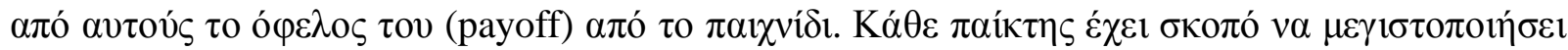

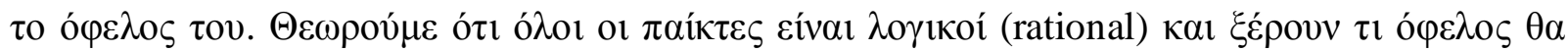

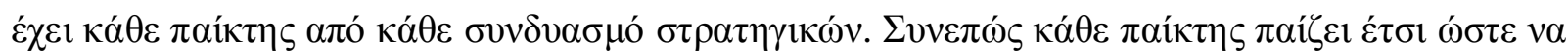

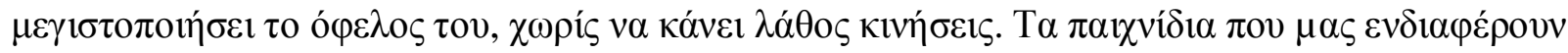

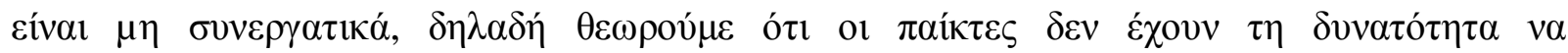

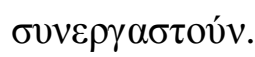

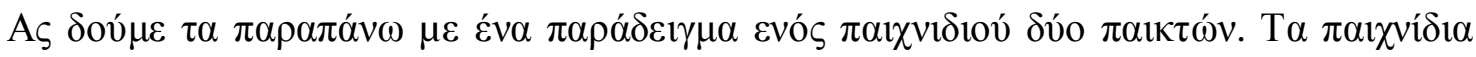

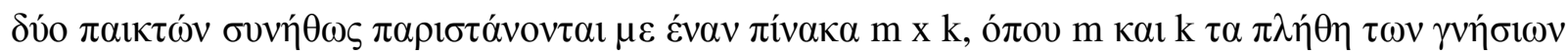

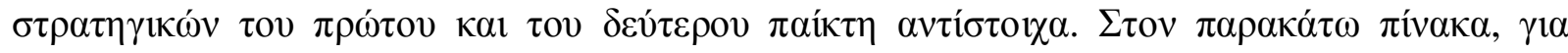

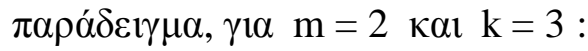

\begin{tabular}{|c|c|c|c|}
\hline $\mathrm{A}$ & $\mathrm{B} 1$ & $\mathrm{~B} 2$ & $\mathrm{~B} 3$ \\
\hline $\mathrm{A} 1$ & $(1,1)$ & $(2,-2)$ & $(2,-3)$ \\
\hline $\mathrm{A} 2$ & $(5,6)$ & $(1,1)$ & $(2,-2)$ \\
\hline
\end{tabular}

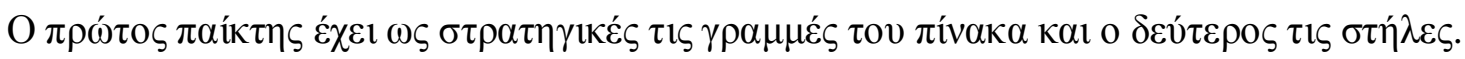

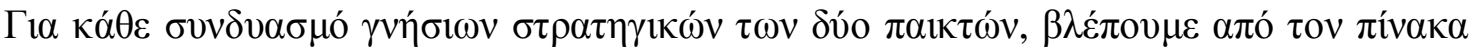

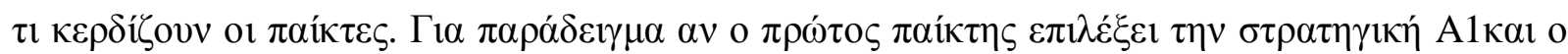

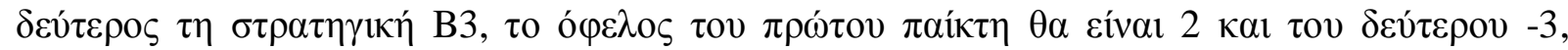

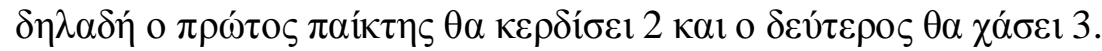

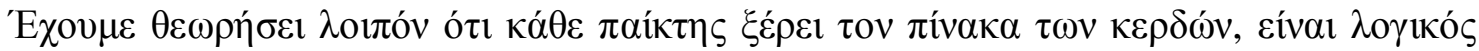

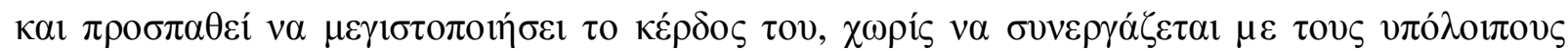

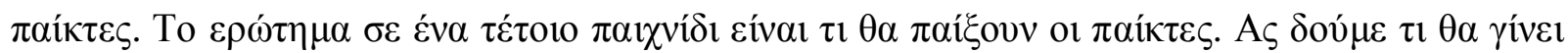




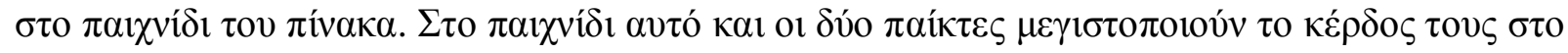

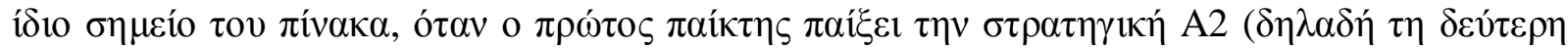

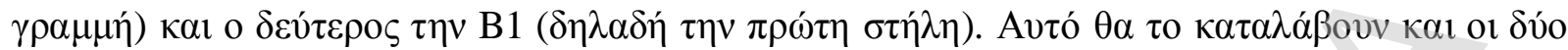
$\pi \alpha i \kappa \tau \varepsilon \varsigma \kappa \alpha 1 \theta \alpha \pi \alpha i \xi o v v \tau ı \varsigma \alpha v \tau i ́ \sigma \tau o \chi \chi \varepsilon \varsigma \sigma \tau \rho \alpha \tau \eta \gamma 1 \kappa \varepsilon ́ \varsigma$.

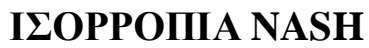

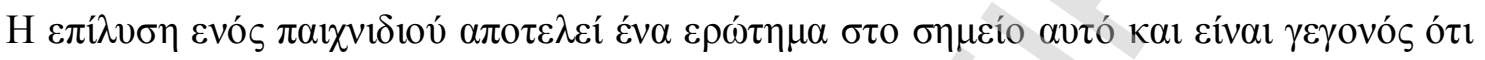

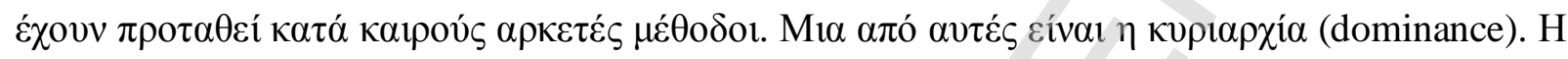

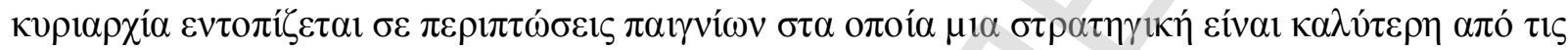

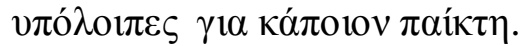

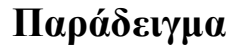

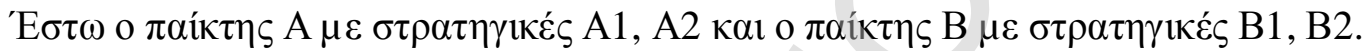

\section{ПаIKTnৎ B}

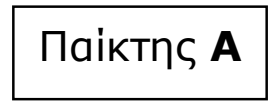

\begin{tabular}{|c|c|c|}
\hline & B1 & B2 \\
\hline $\mathbf{A 1}$ & $(9,6)$ & $(8,2)$ \\
\hline $\mathbf{A 2}$ & $(1,1)$ & $(3,3)$ \\
\hline
\end{tabular}

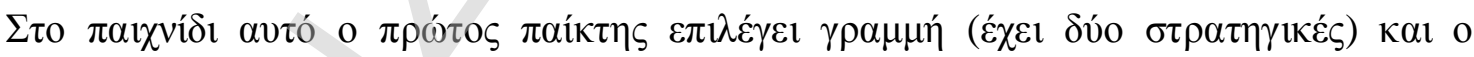

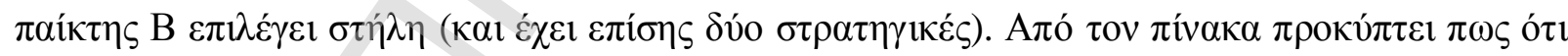

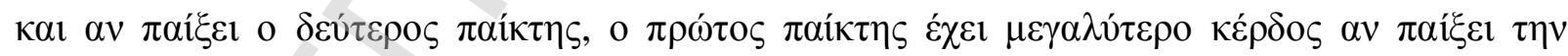

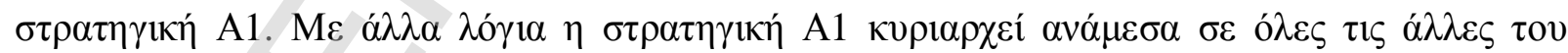

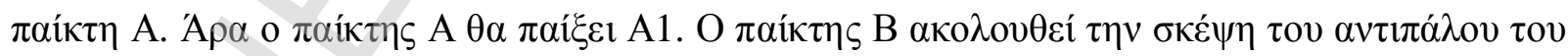

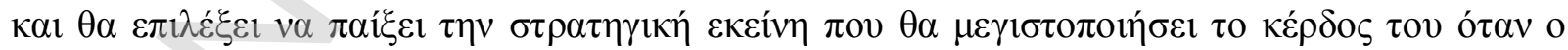

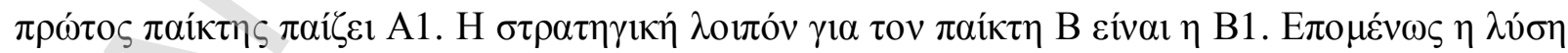

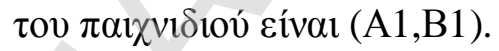

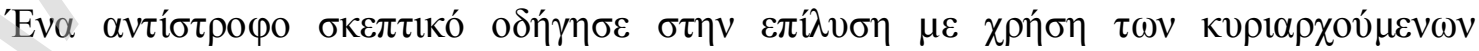

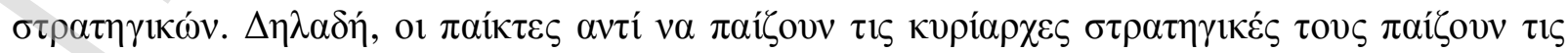

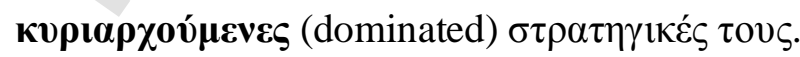




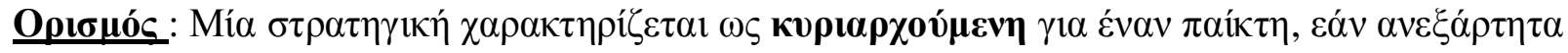

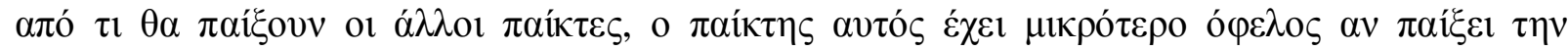

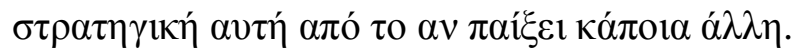

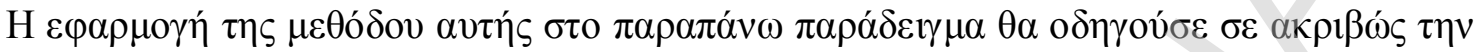

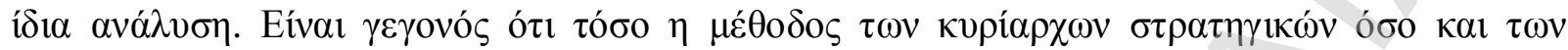

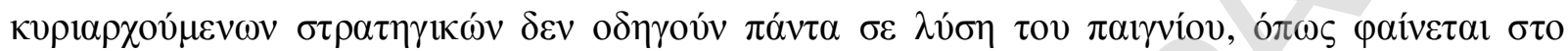

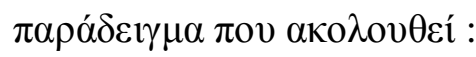

\begin{tabular}{|c|c|c|}
\hline $\mathbf{A}$ & $\mathbf{B}$ & $\mathbf{B 1}$ \\
\hline $\mathbf{A 1}$ & $(4,5)$ & $\mathbf{B 2}$ \\
\hline $\mathbf{A 2}$ & $(2,0)$ & $(0,1)$ \\
\hline
\end{tabular}

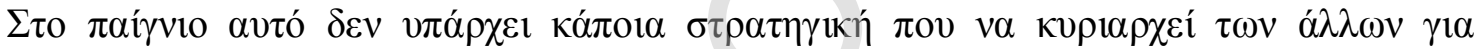

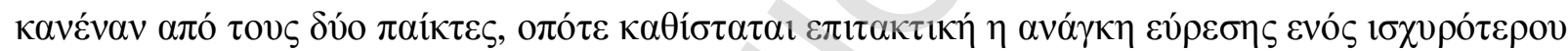

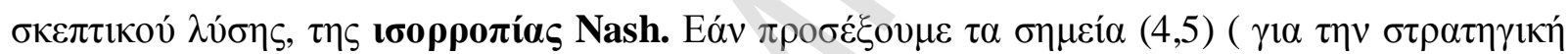

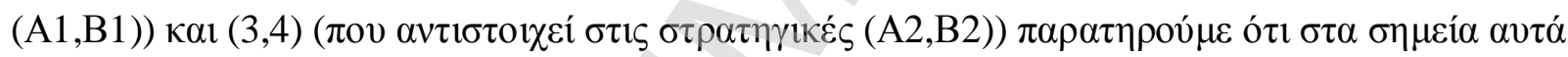

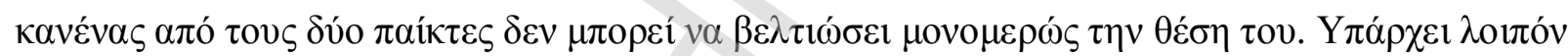

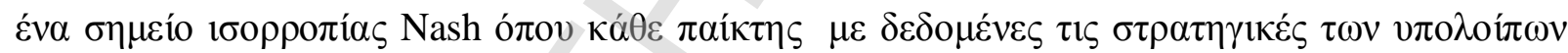

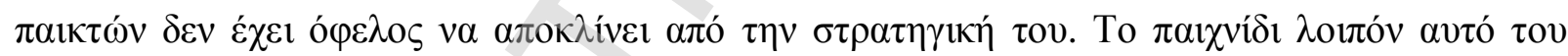

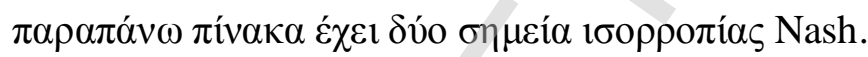

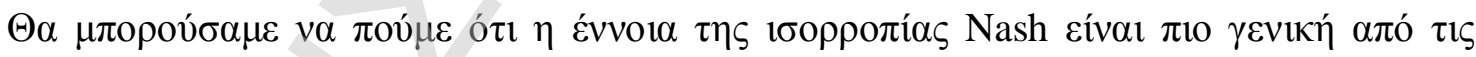

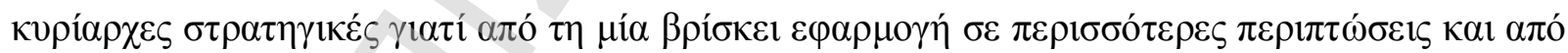

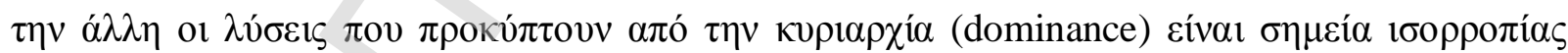
Nash.

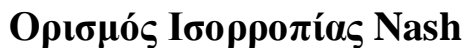

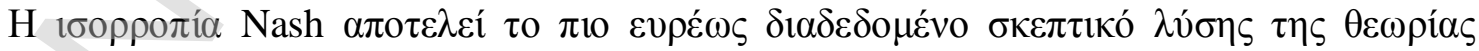

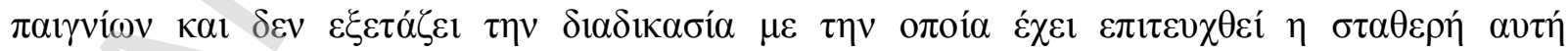
$\kappa \alpha \tau \alpha ́ \sigma \tau \alpha \sigma \eta$.

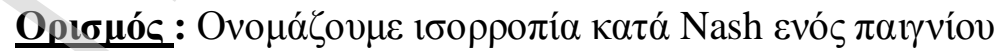

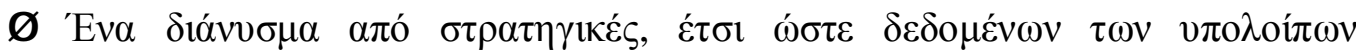

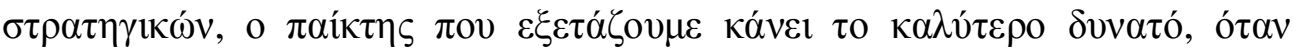




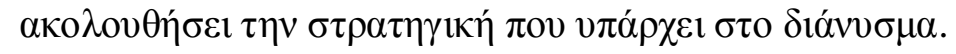

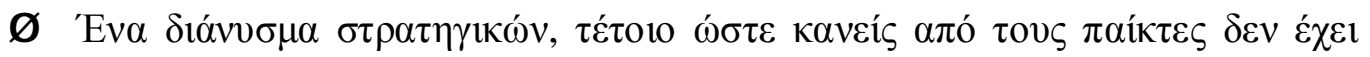

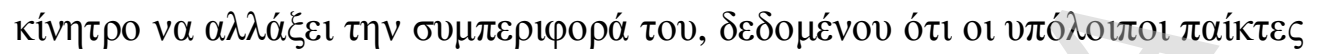

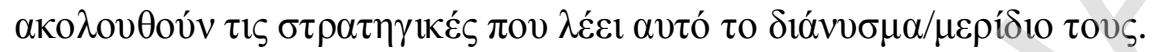

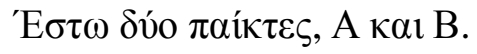

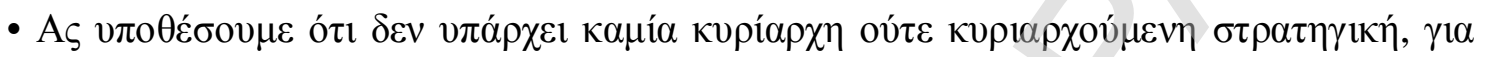

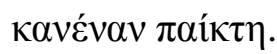

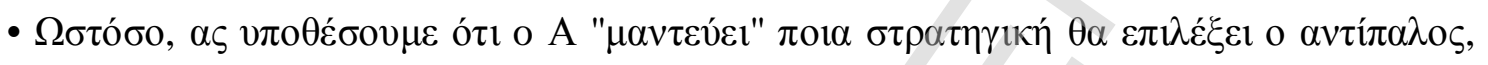
$\varepsilon \dot{\varepsilon} \sigma \omega \mathrm{s}_{\mathrm{B}}^{*}$.

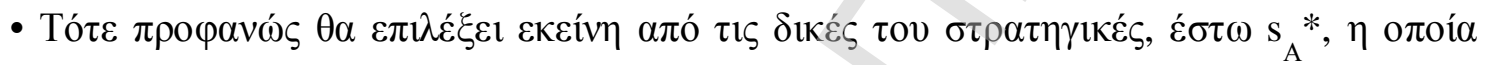

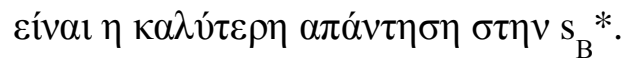

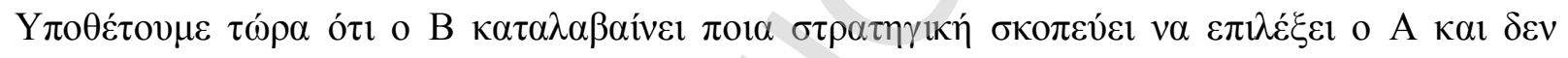
$\alpha \lambda \lambda \alpha ́ \zeta \varepsilon 1 \tau \eta \sigma \tau \rho \alpha \tau \eta \gamma 1 \kappa \eta ́ \tau o v \mathrm{~s}_{\mathrm{B}}^{*}$.

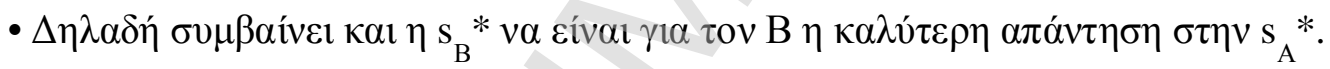

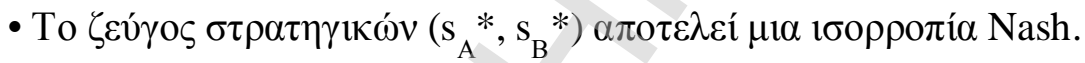

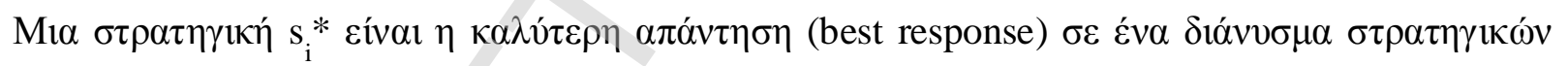

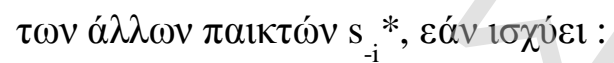

$$
\mathbf{u}_{i}\left(\mathbf{s}_{i}^{*}, \mathbf{s}_{-i}^{*}\right) \geq \mathbf{u}_{i}\left(\mathbf{s}_{i}, \mathbf{s}_{-i}^{*}\right)
$$

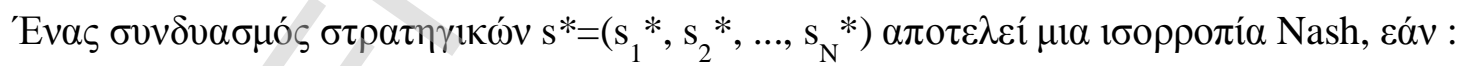

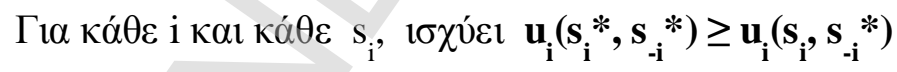

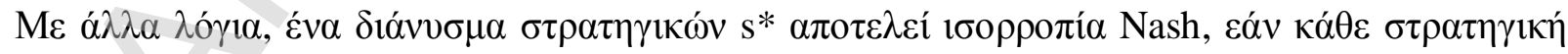

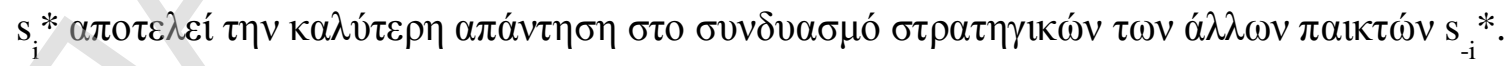

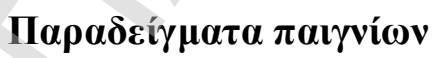

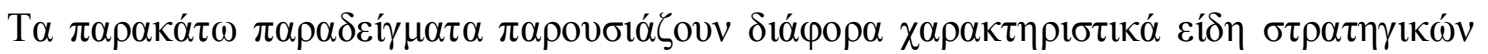

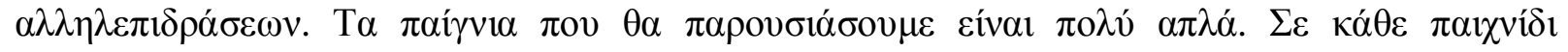




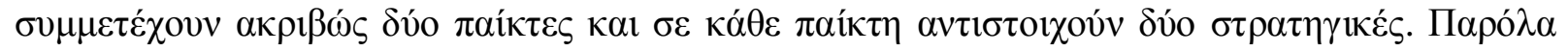

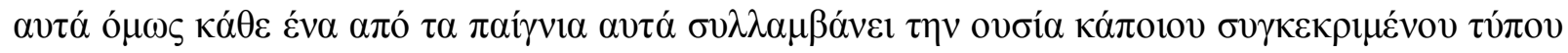

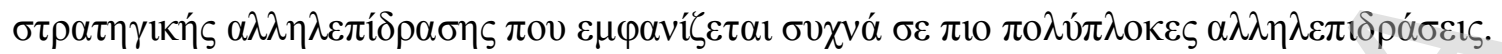

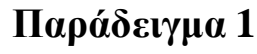

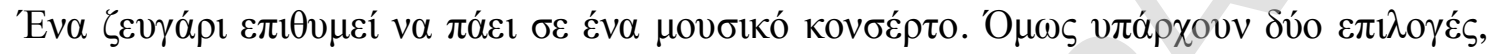

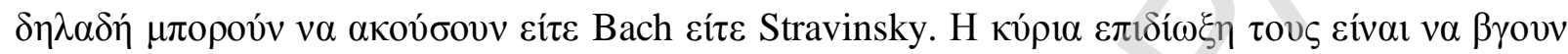

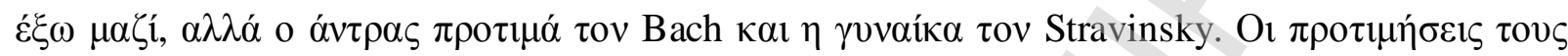

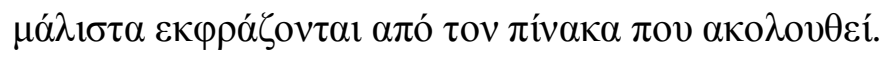

\begin{tabular}{|c|c|c|c|}
\hline & $\mathbf{A}$ & $\mathbf{B}$ & $\mathbf{S}$ \\
\hline $\mathbf{T}$ & $\mathbf{B}$ & $(2,1)$ & $(0,0)$ \\
\hline $\mathbf{S}$ & $(0,0)$ & $(1,2)$ \\
\hline
\end{tabular}

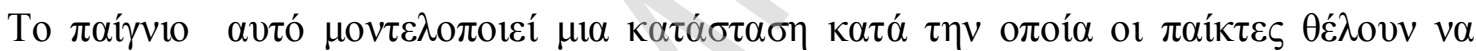

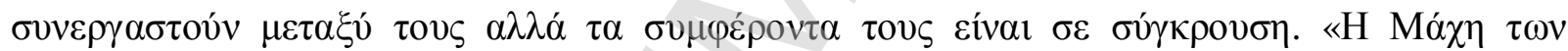

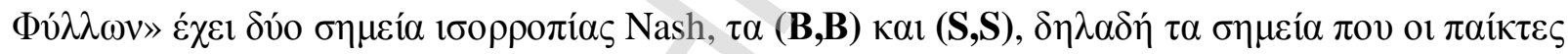

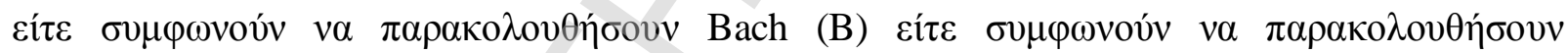
Stravinsky (S).

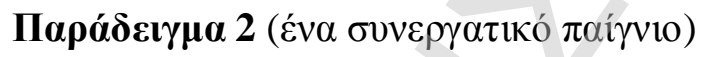

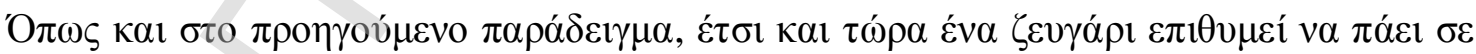

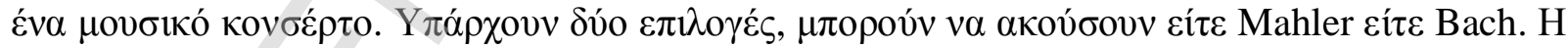

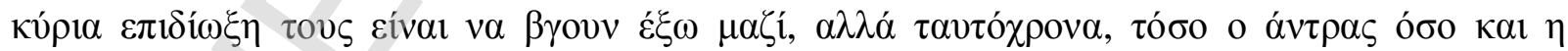

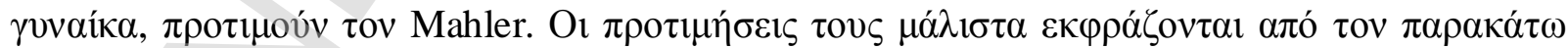

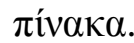




\section{AN $\triangle \mathrm{PA} \boldsymbol{\Sigma}$}

\begin{tabular}{|c|c|c|c|}
\hline & & $\mathbf{M}$ & B \\
\hline \multirow{2}{*}{ ГYNAIKA } & $\mathbf{M}$ & $(2,2)$ & $(0,0)$ \\
\hline & B & $(0,0)$ & $(1,1)$ \\
\hline
\end{tabular}

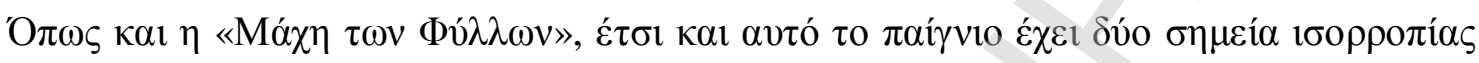

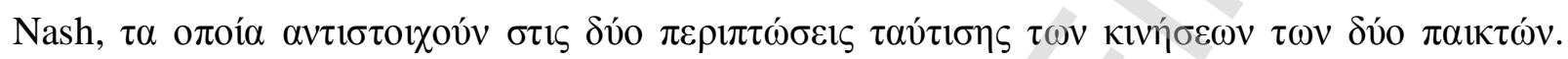

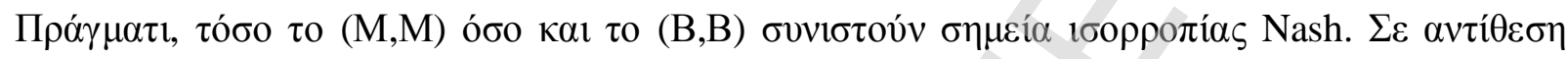

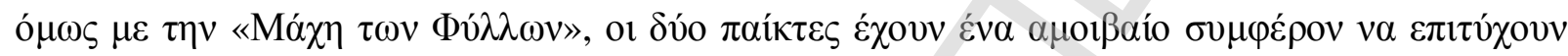

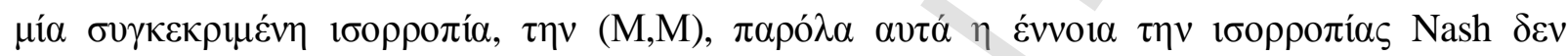

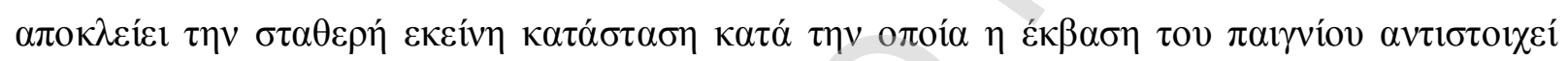
$\sigma \tau \eta v \ll \kappa \alpha \tau \omega ́ \tau \varepsilon \rho \eta » 1 \sigma o \rho \rho о \pi i ́ \alpha(B, B)$.

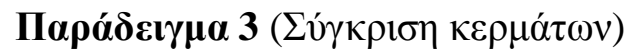

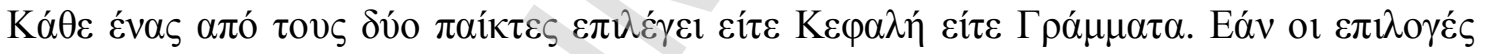

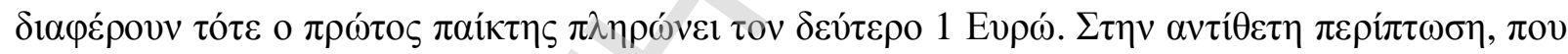

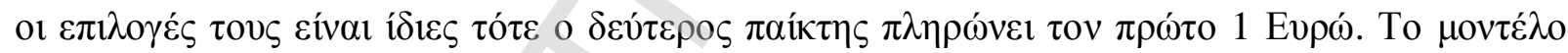

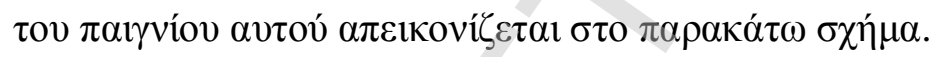

\begin{tabular}{|c|c|c|}
\hline 7 & M & B \\
\hline $\mathbf{M}$ & $(1,-1)$ & $(-1,1)$ \\
\hline B & $(-1,1)$ & $(1,-1)$ \\
\hline
\end{tabular}

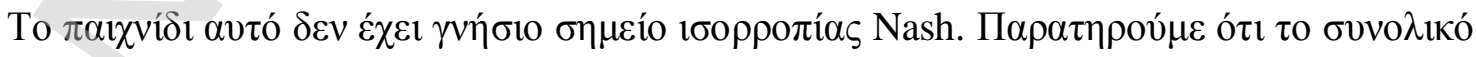

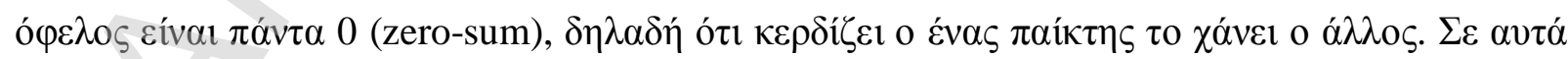

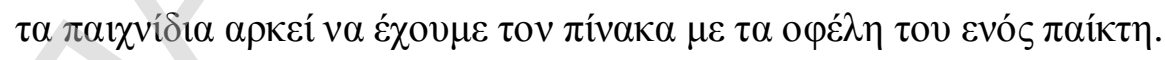




\section{ПАРА $\triangle O \mathbf{\Xi A}$}

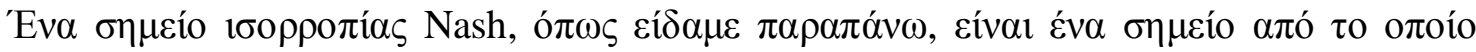

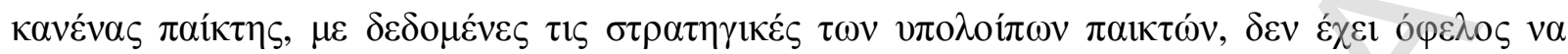

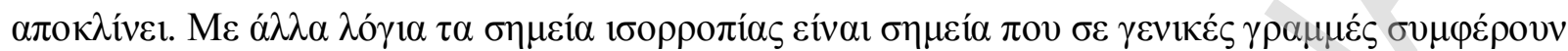

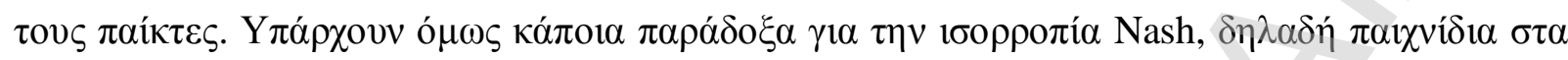

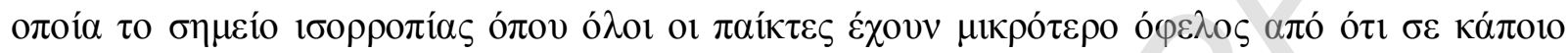

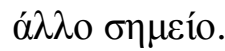

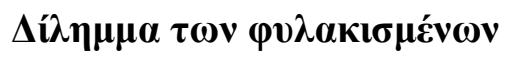

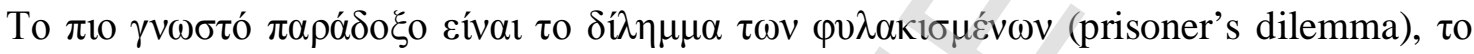

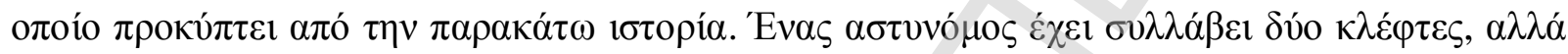

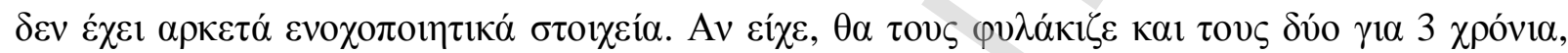

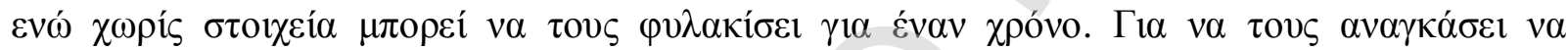

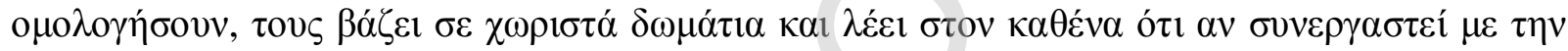

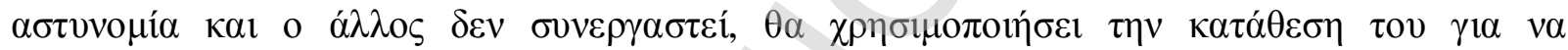

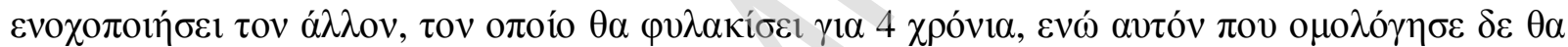

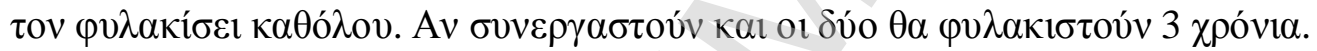

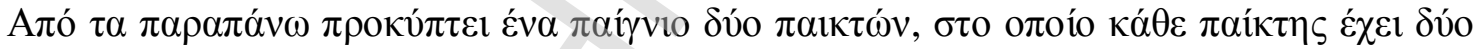

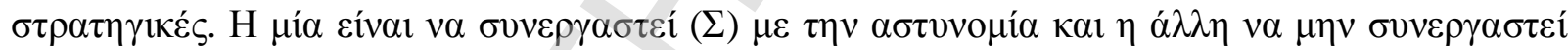

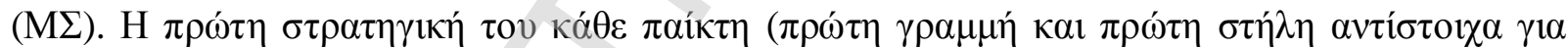

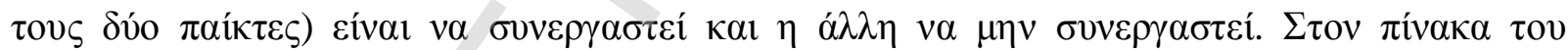

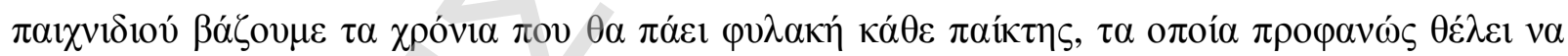
$\varepsilon \lambda \alpha \chi 1 \sigma \tau 0 \pi \circ \eta \dot{\sigma \varepsilon l . ~}$

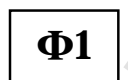

\begin{tabular}{|c|c|c|}
\hline & $\boldsymbol{\Sigma}$ & $\mathbf{M} \boldsymbol{\Sigma}$ \\
\hline $\boldsymbol{\Sigma}$ & $(3,3)$ & $(0,4)$ \\
\hline $\mathbf{M} \boldsymbol{\Sigma}$ & $(4,0)$ & $(1,1)$ \\
\hline
\end{tabular}

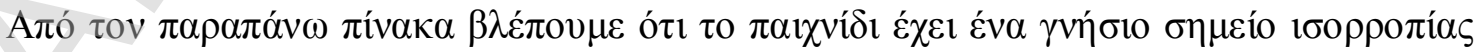

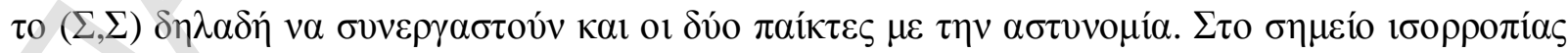

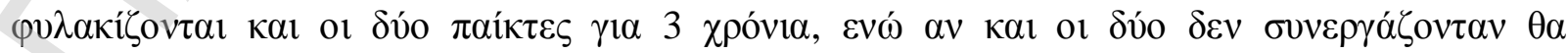

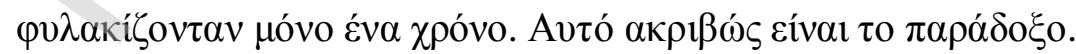




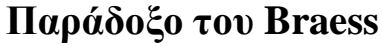

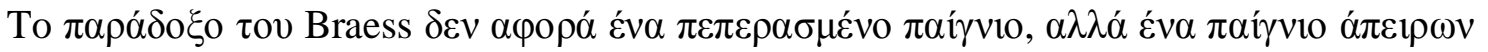

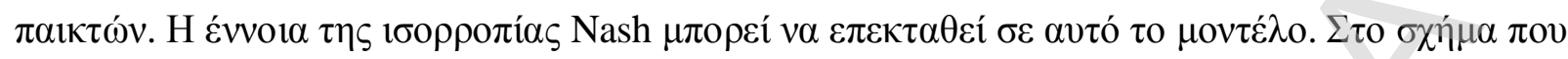

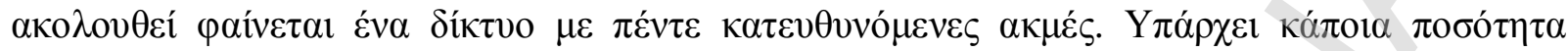

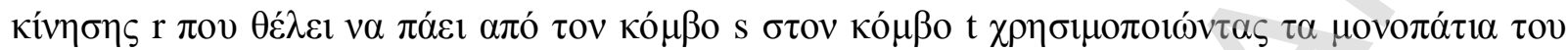

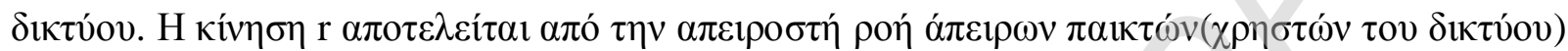

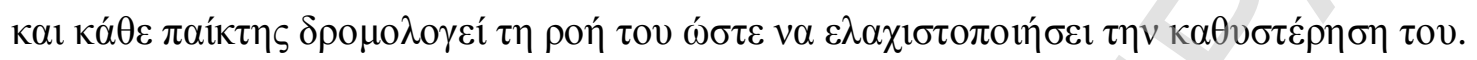

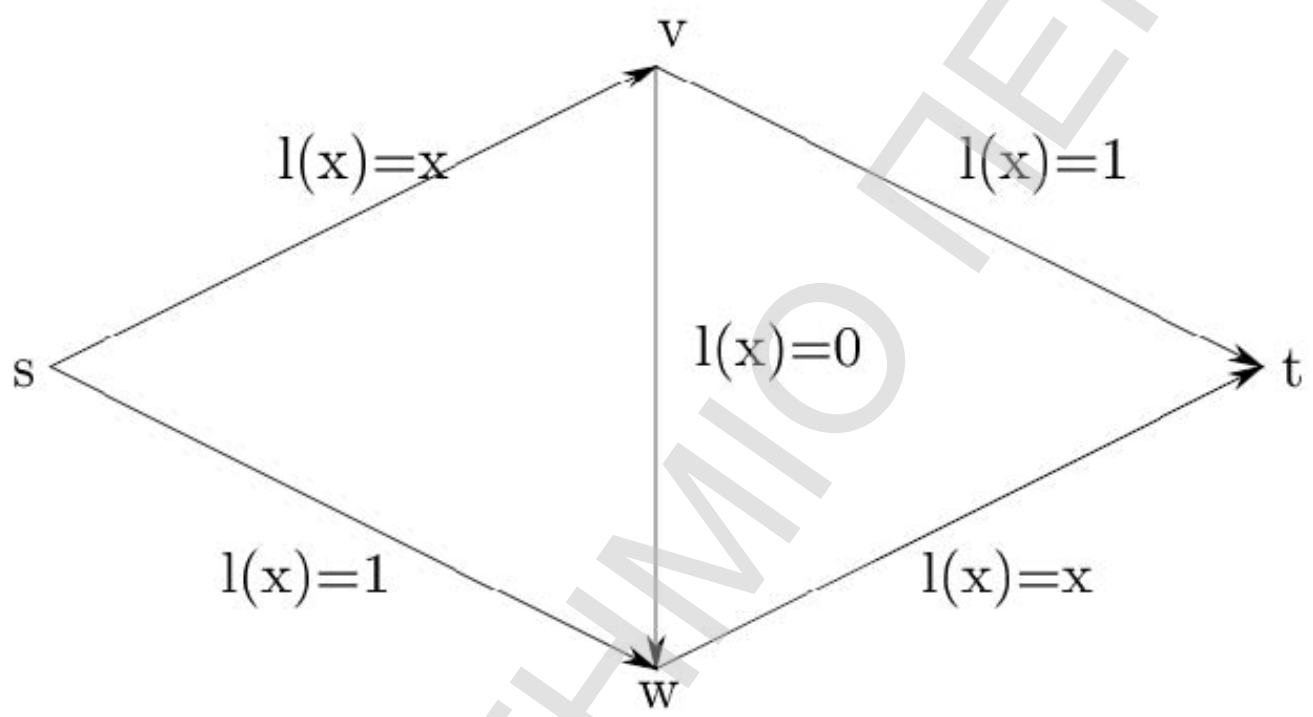

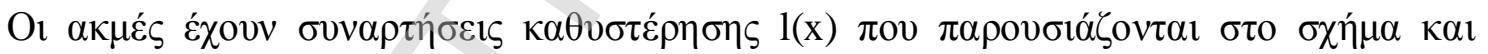

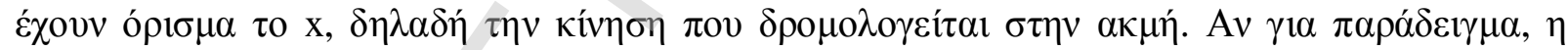

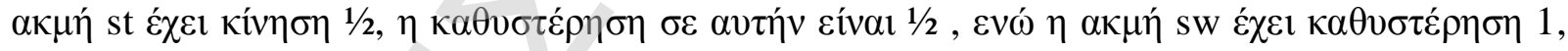

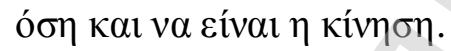

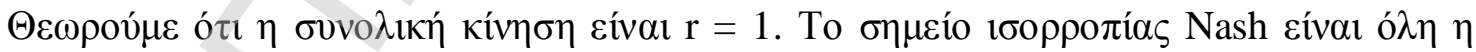

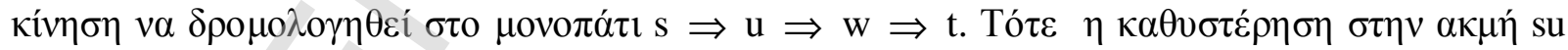

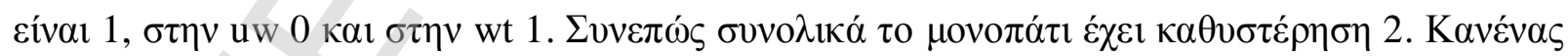

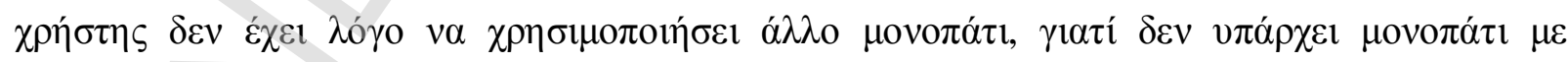

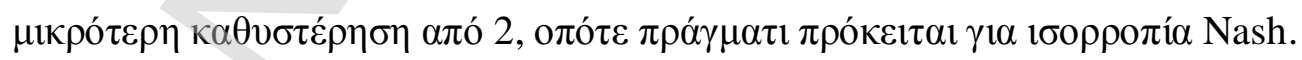

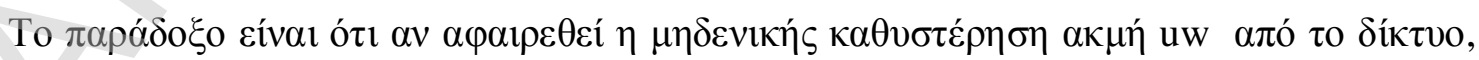

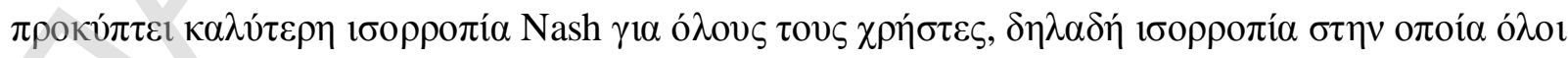

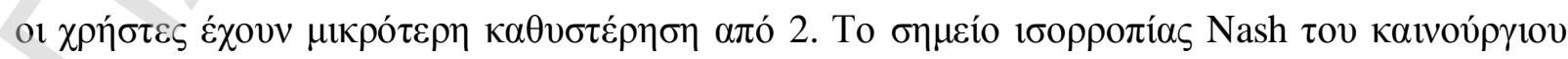

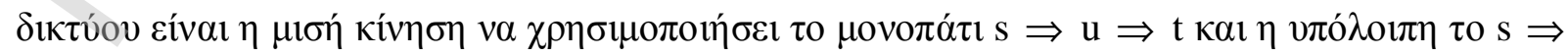

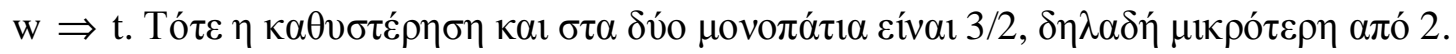




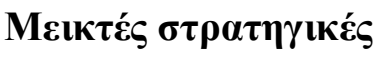

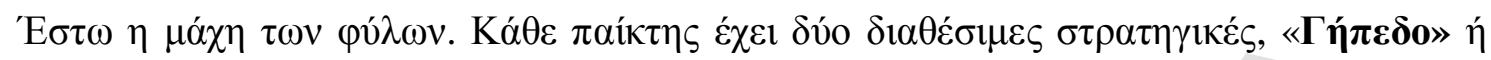

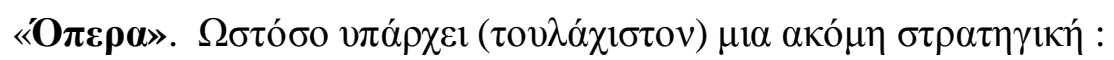

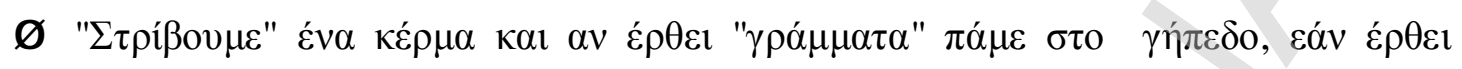

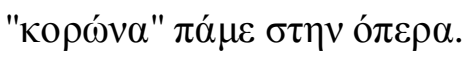

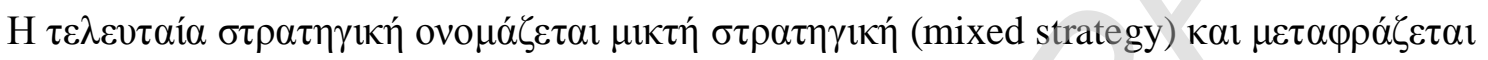

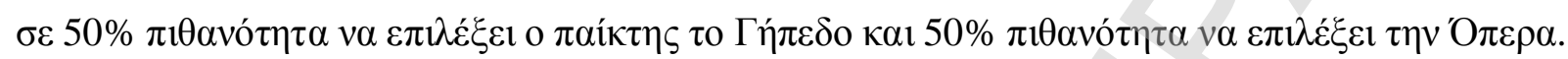

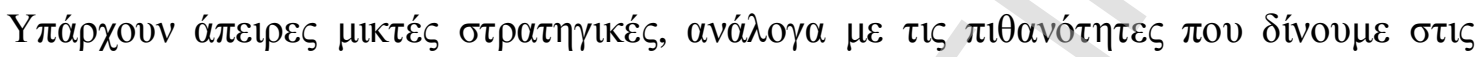
$\delta i \alpha ́ \varphi \circ \rho \varepsilon \varsigma \varepsilon \pi \imath \lambda \circ \gamma \varepsilon \dot{\varepsilon}$.

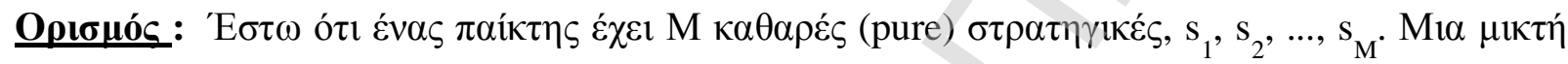

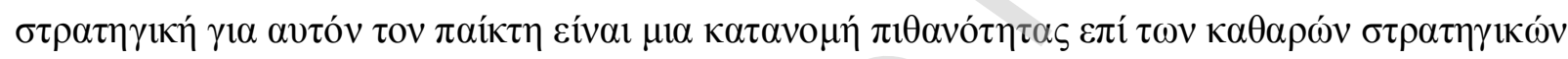

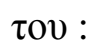

$$
\left(\mathrm{p}_{1}, \mathrm{p}_{2}, \ldots, \mathrm{p}_{\mathrm{M}}\right), \dot{\varepsilon} \tau \sigma \mathrm{\iota} \sigma \tau \tau \varepsilon \mathrm{p}_{1}+\mathrm{p}_{2}+\ldots+\mathrm{p}_{\mathrm{M}}=1
$$

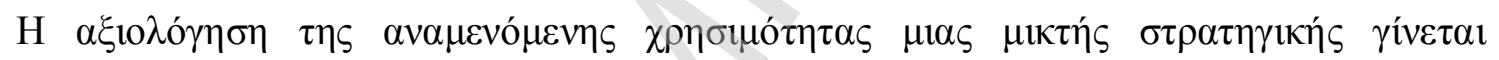

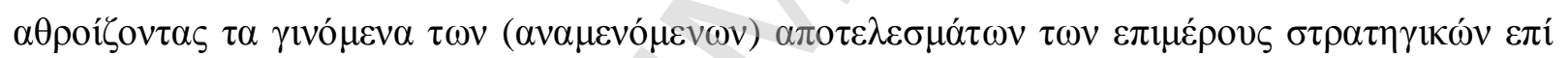
$\tau \imath \varsigma \alpha v \tau i ́ \sigma \tau 0 \imath \chi \varepsilon \varsigma \pi \imath \theta \alpha$ ó $\eta \tau \varepsilon \varsigma$.

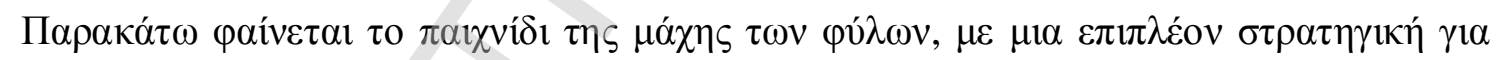

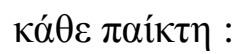

\begin{tabular}{|c|c|c|c|}
\hline $\mathbf{A}$ & Гท́л $\pi \varepsilon \delta 0$ & 'Олєра & $0.5-0.5$ \\
\hline Ги́л $\boldsymbol{\varepsilon} \delta 0$ & $(3,1)$ & $(0,0)$ & $(1.5,0.5)$ \\
\hline О $\pi \varepsilon \rho \alpha$ & $(0,0)$ & $(1,3)$ & $(0.5,1.5)$ \\
\hline $0.5-0.5$ & $(1.5,0.5)$ & $(0.5,1.5)$ & $(1,1)$ \\
\hline
\end{tabular}

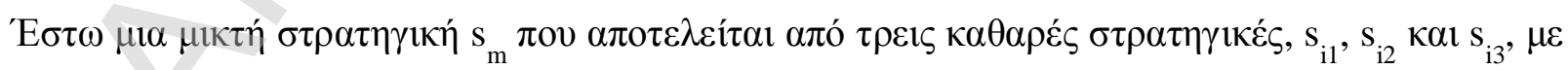

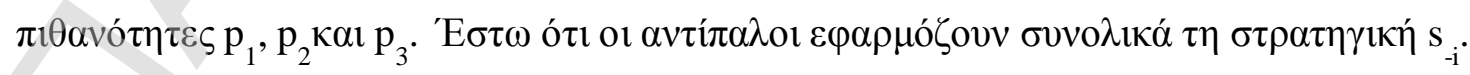




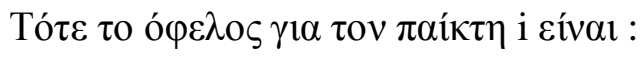

$$
\mathrm{u}\left(\mathrm{s}_{\mathrm{m}}, \mathrm{s}_{-\mathrm{i}}\right)=\mathrm{p}_{1} \cdot \mathrm{u}\left(\mathrm{s}_{\mathrm{i} 1}, \mathrm{~s}_{-\mathrm{i}}\right)+\mathrm{p}_{2} \cdot \mathrm{u}\left(\mathrm{s}_{\mathrm{i} 2}, \mathrm{~s}_{-\mathrm{i}}\right)+\mathrm{p}_{3} \cdot \mathrm{u}\left(\mathrm{s}_{\mathrm{i} 3} \mathrm{~s}_{-\mathrm{i}}\right)
$$

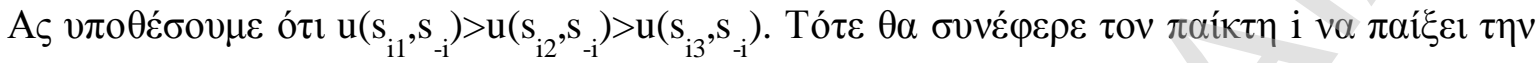

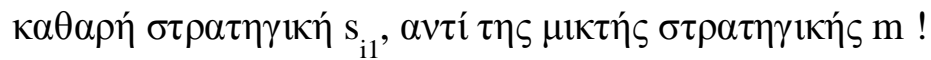

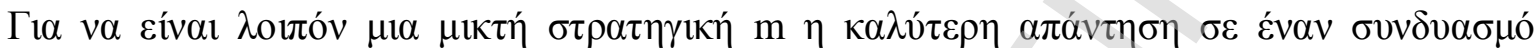

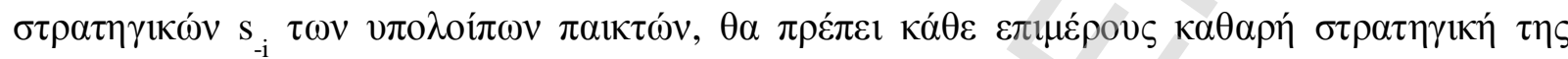

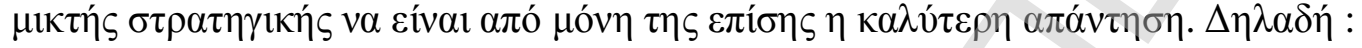

$$
\mathbf{u}\left(\mathbf{s}_{\mathbf{m}}, \mathbf{s}_{-i}\right)=\mathbf{u}\left(\mathbf{s}_{\mathrm{i} 1}, \mathbf{s}_{-\mathrm{i}}\right)=\mathbf{u}\left(\mathbf{s}_{\mathrm{i} 2}, \mathbf{s}_{-\mathrm{i}}\right)=\mathbf{u}\left(\mathbf{s}_{\mathrm{i} 3} \mathbf{s}_{-\mathrm{i}}\right)
$$

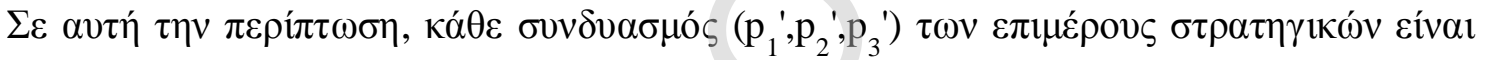

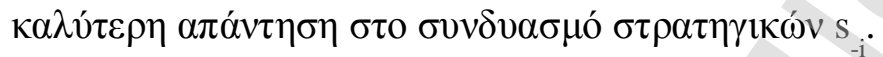

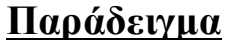

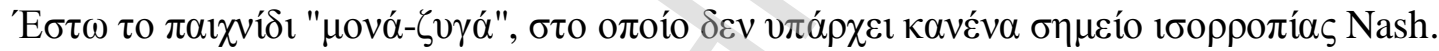

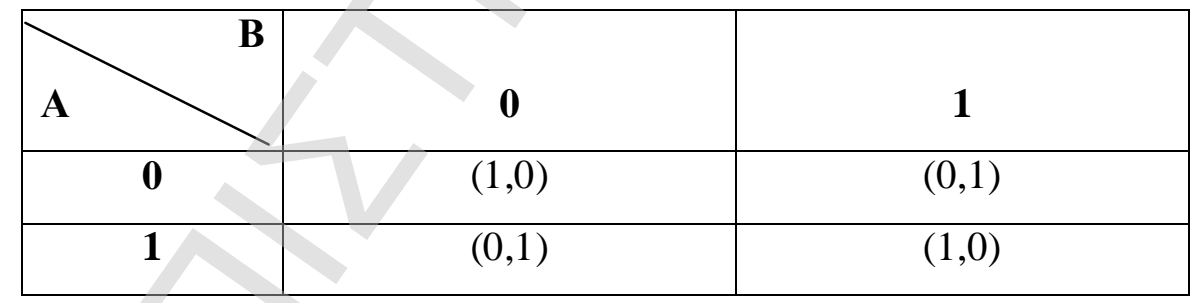

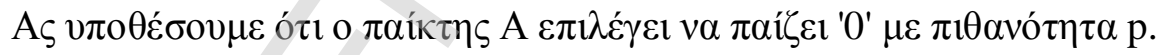

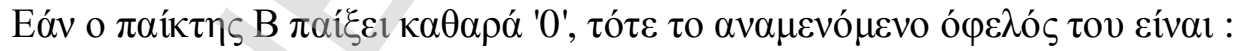

$$
\mathrm{Eu}_{\mathrm{B}}\left(\mathrm{O}^{\prime}\right)=\mathrm{p} \cdot 0+(1-\mathrm{p}) \cdot 1=1-\mathrm{p}
$$

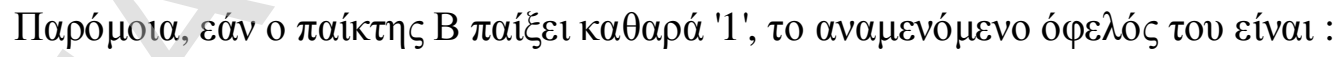

$$
\mathrm{Eu}_{B}\left({ }^{\prime} 1^{\prime}\right)=\mathrm{p} \cdot 1+(1-\mathrm{p}) \cdot 0=\mathrm{p}
$$

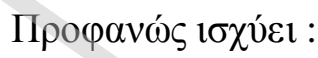

$$
\mathrm{Eu}_{B}\left({ }^{\prime} 0^{\prime}\right)>\mathrm{Eu}_{\mathrm{B}}\left({ }^{\prime} 1^{\prime}\right) \Leftrightarrow(1-\mathrm{p})>\mathrm{p} \Leftrightarrow \mathrm{p}<1 / 2
$$


Eóv $\mathrm{p}=1 / 2, \tau$,

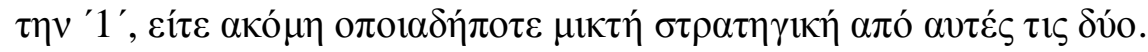

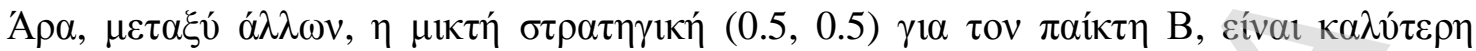

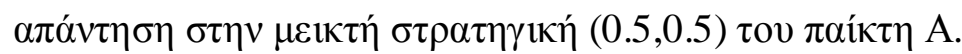

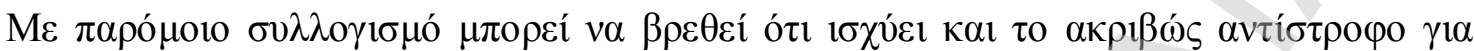

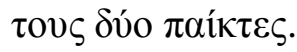

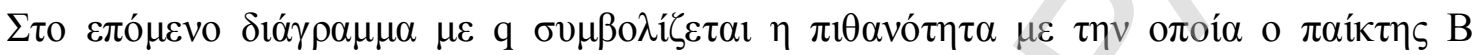
$\varepsilon \pi \imath \lambda \varepsilon \dot{\gamma \varepsilon \iota}$ ' 0 '.

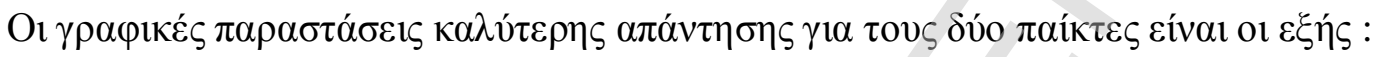

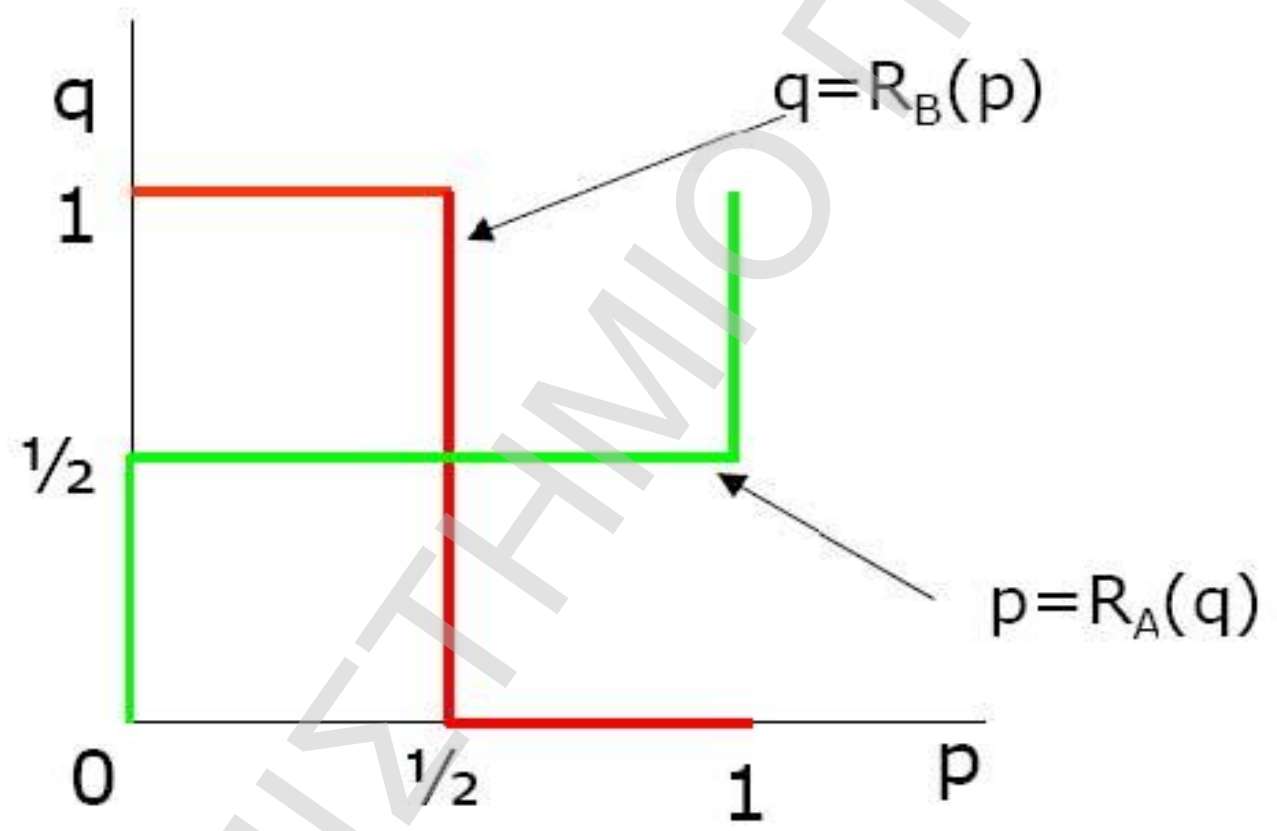

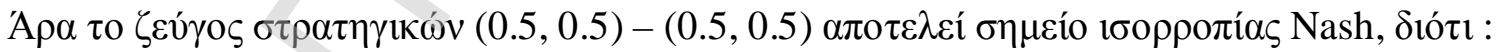

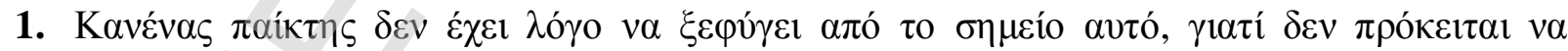
$\kappa \varepsilon \rho \delta i ́ \sigma \varepsilon l ~ \alpha ́ \mu \varepsilon \sigma \alpha$.

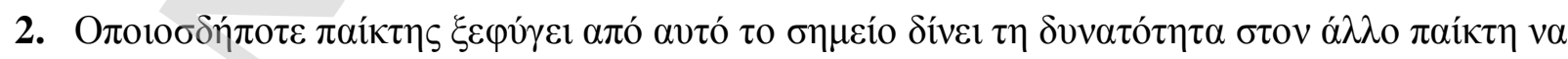

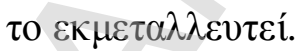

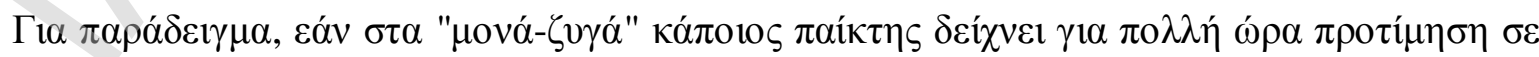

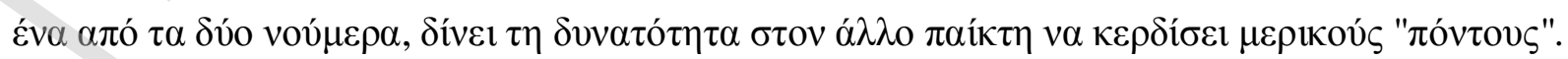




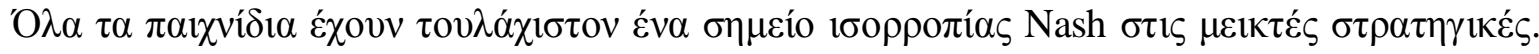

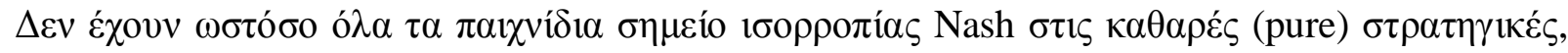

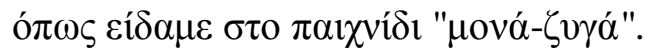

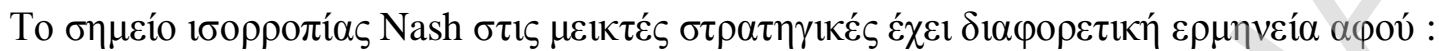

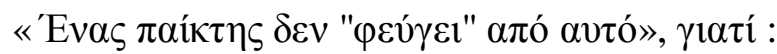

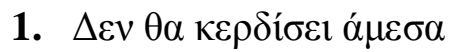

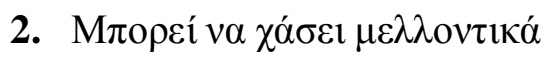

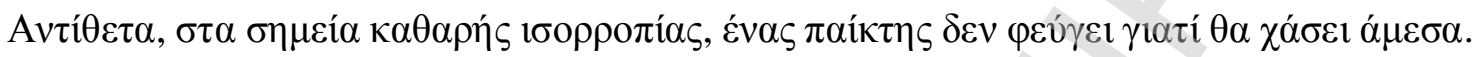

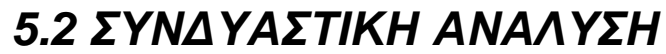

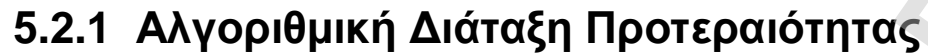

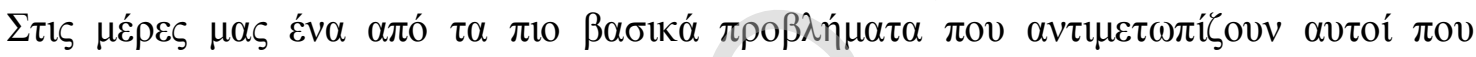

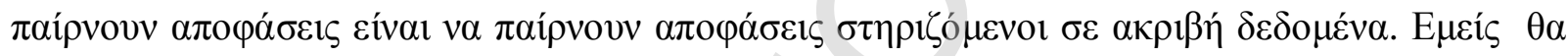

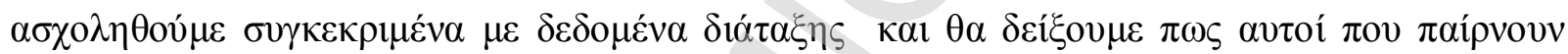

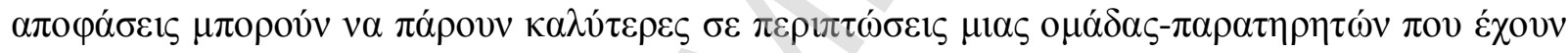

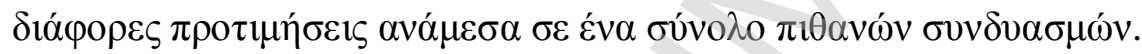

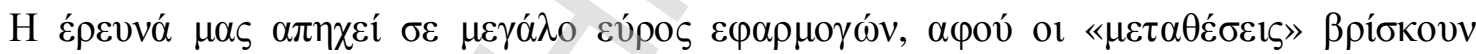

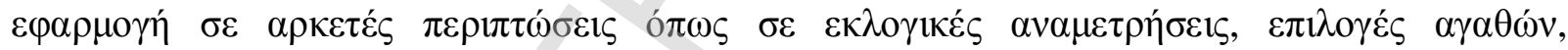
$\varepsilon \pi \varepsilon v \delta v ́ \sigma \varepsilon \omega v, \delta 1 \alpha ́ \tau \alpha \xi \eta \mu \eta \chi \alpha \vee \eta \mu \alpha ́ \tau \omega \nu \kappa . \alpha$.

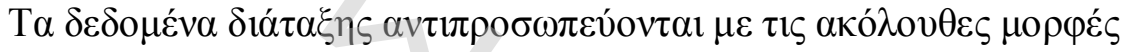

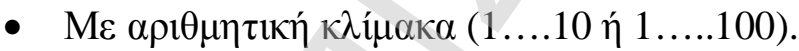

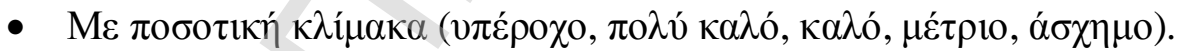

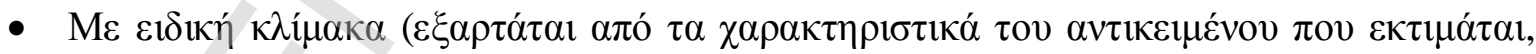
$\left.\pi \cdot \chi . \psi \eta \lambda o^{\prime}, \kappa o v \tau o ́, \pi \alpha \chi v ́, \lambda \varepsilon \pi \tau o ́\right)$.

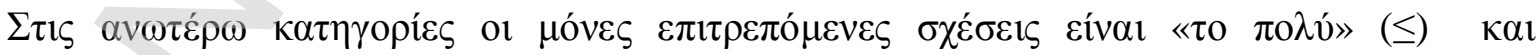

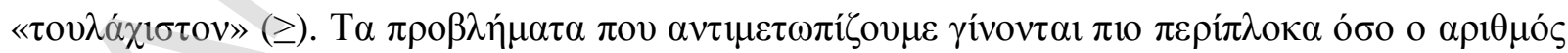

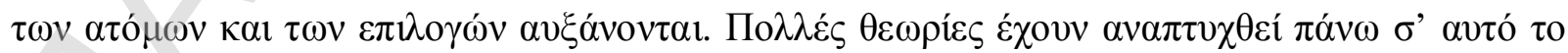

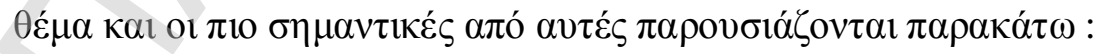

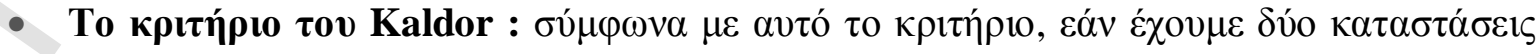

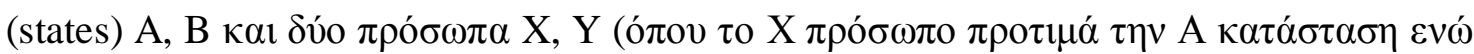




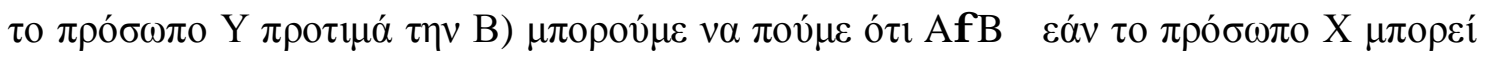

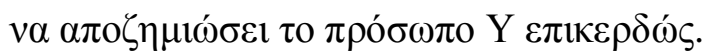

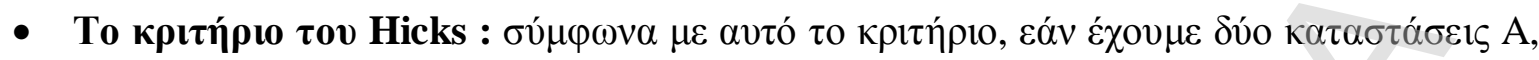

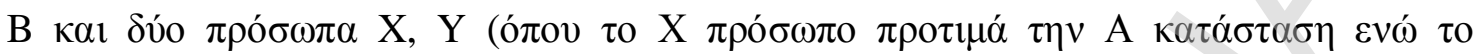

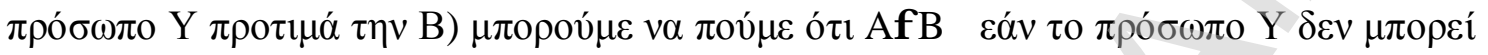

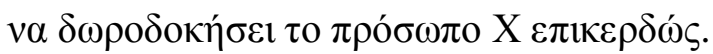

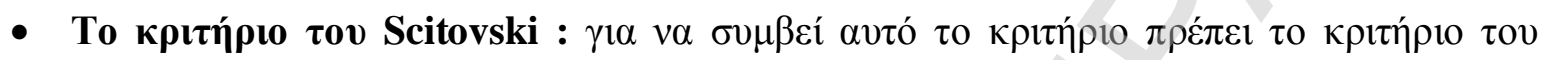

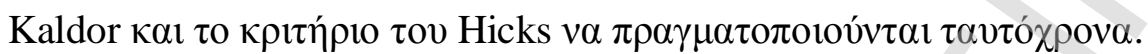

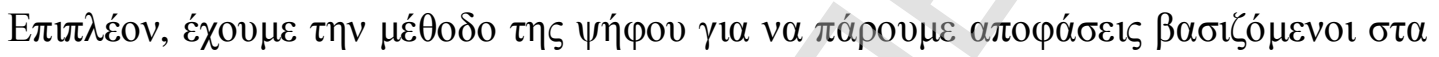

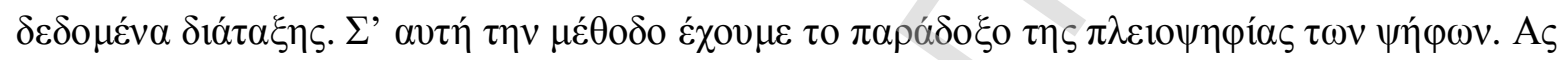

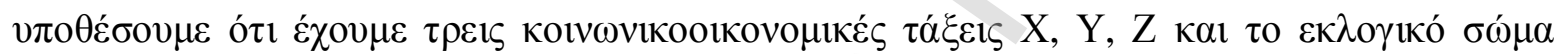

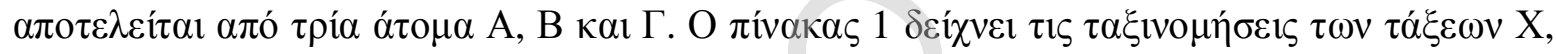

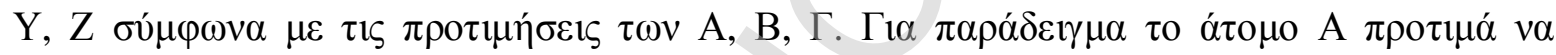

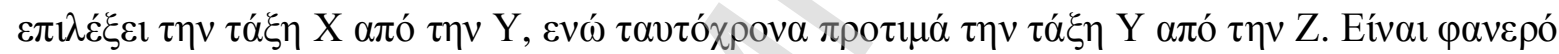

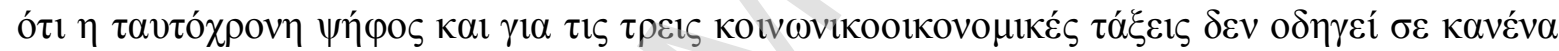

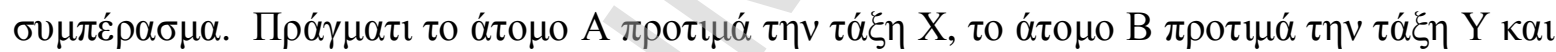

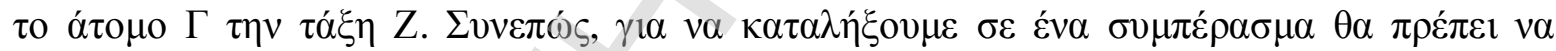

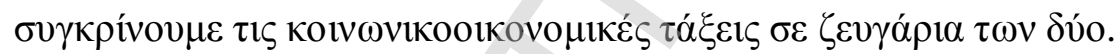

\begin{tabular}{|c|c|c|c|}
\hline ЕКАЕКТОРЕЕ & \multirow{2}{*}{ A } & \multirow{2}{*}{ B } & \multirow{2}{*}{$\Gamma$} \\
\hline KАTATAЕН & & & \\
\hline 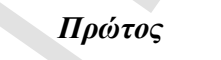 & $\mathbf{x}$ & $\mathbf{Y}$ & $\mathbf{Z}$ \\
\hline 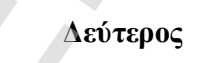 & $\mathbf{Y}$ & $\mathbf{Z}$ & $\mathbf{X}$ \\
\hline Tрі́тоs & $\mathbf{Z}$ & $\mathbf{X}$ & $\mathbf{Y}$ \\
\hline
\end{tabular}

Пívakas 1

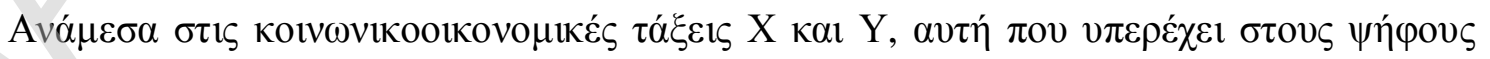

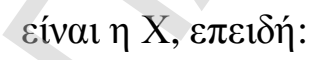

Xf Y $\gamma 1 \alpha \tau$ o A, XpY $\gamma 1 \alpha \tau$ o B, Xf Y $\gamma 1 \alpha \tau$, Г. 


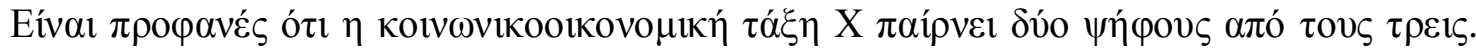

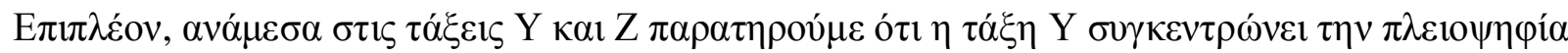
$\tau \omega \nu \psi \eta ́ \varphi \omega v \gamma 1 \alpha \tau i ́:$

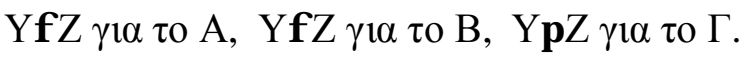

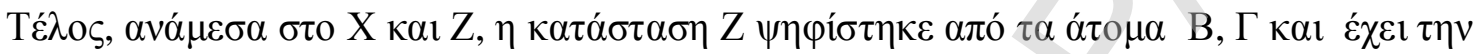

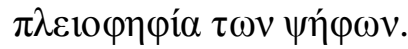

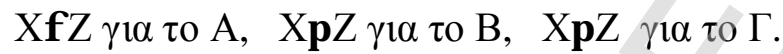

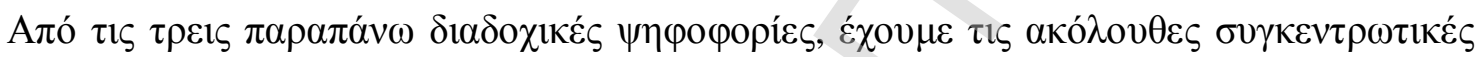
$\pi \rho о \tau \mu \eta \dot{\sigma \varepsilon \varepsilon \varsigma . ~}$

\section{Xf Y, YfZ, XpZ}

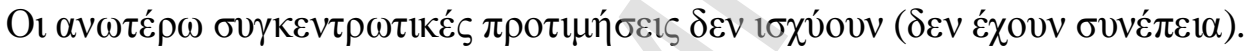

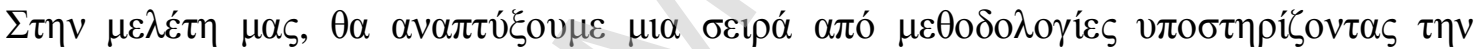

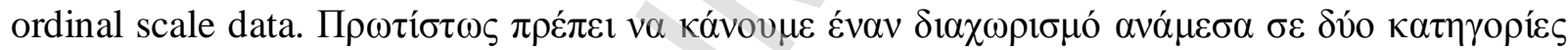

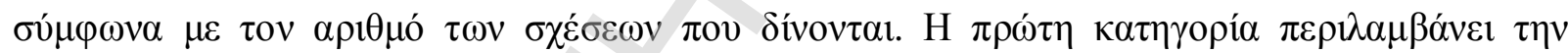

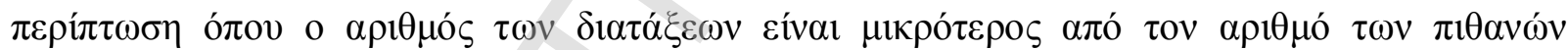

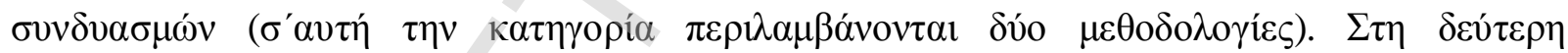

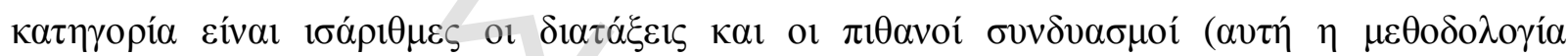

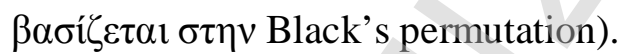

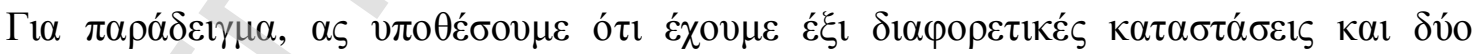

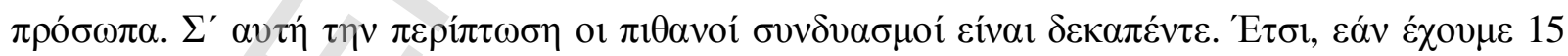

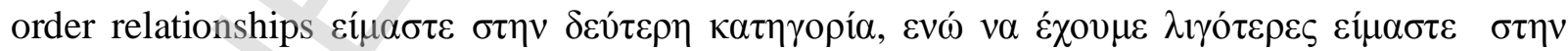

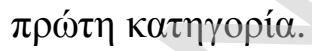

\subsection{2 При́тп Катпуорі́a}

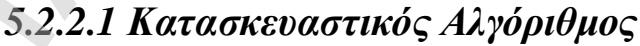

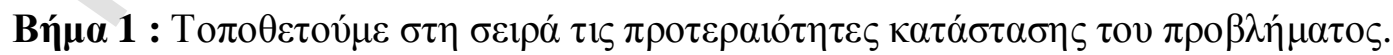




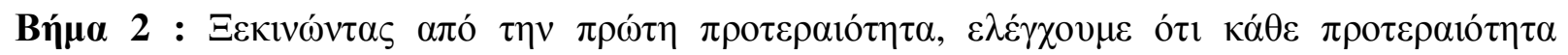

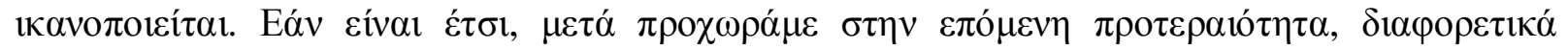

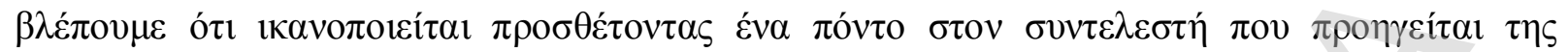

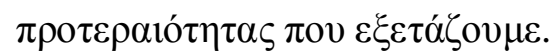

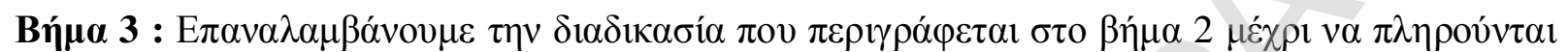

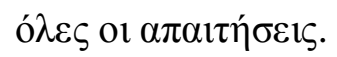

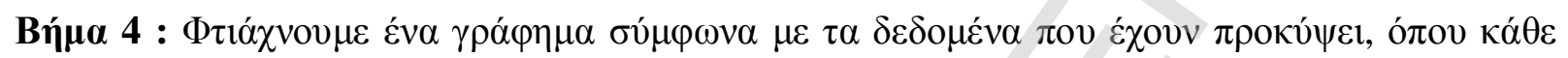

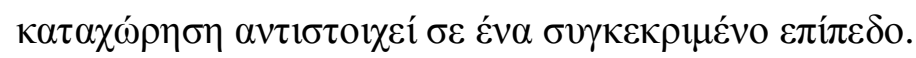

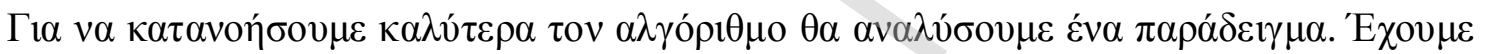

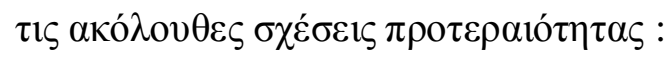

Af B, Af $\Gamma$, Af E, Bf E, Гf B, $\Delta f A, \Delta f E, \Delta f Z, Z f \Gamma, Z f E$.

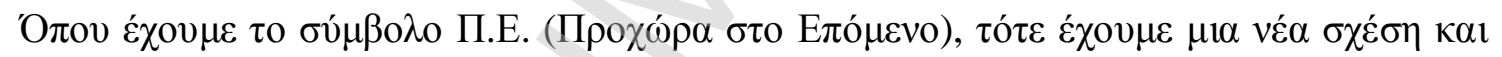

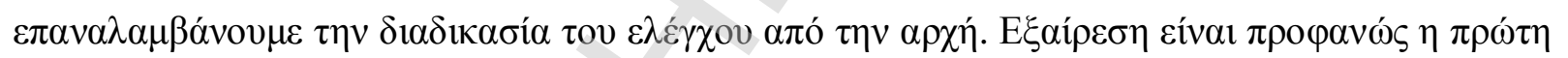

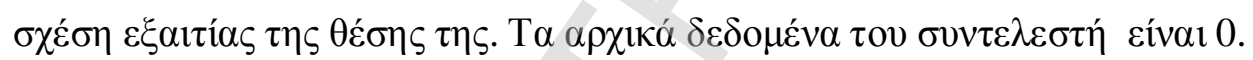

\begin{tabular}{|c|l|l|l|l|l|l|l|}
\hline 1 & Af $\mathrm{B}$ & $\begin{array}{l}\mathrm{A}=1, \\
\mathrm{~B}=0\end{array}$ & $\begin{array}{l}\mathrm{A}=2, \\
\mathrm{~B}=1\end{array}$ & $\begin{array}{l}\mathrm{A}=2, \\
\mathrm{~B}=1\end{array}$ & $\begin{array}{l}\mathrm{A}=3, \\
\mathrm{~B}=1\end{array}$ & $\begin{array}{l}\mathrm{A}=3, \\
\mathrm{~B}=1\end{array}$ & $\begin{array}{l}\mathrm{A}=3, \\
\mathrm{~B}=1\end{array}$ \\
\hline 2 & Af $\Gamma$ & $\begin{array}{l}\mathrm{A}=1, \\
\Gamma=0\end{array}$ & $\begin{array}{l}\mathrm{A}=2, \\
\Gamma=0\end{array}$ & $\begin{array}{l}\mathrm{A}=3, \\
\Gamma=2\end{array}$ & $\begin{array}{l}\mathrm{A}=3, \\
\Gamma=2\end{array}$ & $\begin{array}{l}\mathrm{A}=3, \\
\Gamma=2\end{array}$ & $\begin{array}{l}\mathrm{A}=3, \\
\Gamma=2\end{array}$ \\
\hline 3 & Af $\mathrm{E}$ & $\begin{array}{l}\mathrm{A}=1, \\
\mathrm{E}=0\end{array}$ & $\begin{array}{l}\mathrm{A}=2, \\
\mathrm{E}=0\end{array}$ & П.E. & $\begin{array}{l}\mathrm{A}=3, \\
\mathrm{E}=0\end{array}$ & $\begin{array}{l}\mathrm{A}=3, \\
\mathrm{E}=0\end{array}$ & $\begin{array}{l}\mathrm{A}=3, \\
\mathrm{E}=0\end{array}$ \\
\hline 4 & B $\mathrm{E}$ & $\begin{array}{l}\mathrm{B}=1, \\
\mathrm{E}=0\end{array}$ & $\begin{array}{l}\mathrm{B}=1, \\
\mathrm{E}=0\end{array}$ & & $\begin{array}{l}\mathrm{B}=1, \\
\mathrm{E}=0\end{array}$ & $\begin{array}{l}\mathrm{B}=1, \\
\mathrm{E}=0\end{array}$ & $\begin{array}{l}\mathrm{B}=1, \\
\mathrm{E}=0\end{array}$ \\
\hline 5 & Гf $\mathrm{B}$ & П.Е. & $\begin{array}{l}\Gamma=2, \\
\mathrm{~B}=1\end{array}$ & & $\begin{array}{l}\Gamma=2, \\
\mathrm{~B}=1\end{array}$ & $\begin{array}{l}\Gamma=2, \\
\mathrm{~B}=1\end{array}$ & $\begin{array}{l}\Gamma=2, \\
\mathrm{~B}=1\end{array}$ \\
\hline 6 & $\Delta^{\mathrm{f}} \mathrm{A}$ & & $\Pi . \mathrm{E}$. & & $\begin{array}{l}\Delta=4, \\
\mathrm{~A}=3\end{array}$ & $\begin{array}{l}\Delta=4, \\
\mathrm{~A}=3\end{array}$ & $\begin{array}{l}\Delta=4, \\
\mathrm{~A}=3\end{array}$ \\
\hline
\end{tabular}




\begin{tabular}{|c|l|l|l|l|l|l|l|}
\hline 7 & $\Delta \mathrm{f}^{\mathrm{E}}$ & & & & П.E. & $\begin{array}{l}\Delta=4, \\
\mathrm{E}=0\end{array}$ & $\begin{array}{l}\Delta=4, \mathrm{E} \\
=0\end{array}$ \\
\hline 8 & $\Delta \mathrm{f} \mathrm{Z}$ & & & & & $\begin{array}{l}\Delta=4, \\
\mathrm{Z}=0\end{array}$ & $\begin{array}{l}\Delta=4, \mathrm{Z} \\
=3\end{array}$ \\
\hline 9 & $\mathrm{Z} \mathrm{f}^{\mathrm{f}}$ & & & & & $\begin{array}{l}\mathrm{Z}=3, \\
\Gamma=2\end{array}$ & $\begin{array}{l}\mathrm{Z}=3, \\
\Gamma=2\end{array}$ \\
\hline 10 & $\mathrm{Zf} \mathrm{E}$ & & & & & П.E. & $\begin{array}{l}\mathrm{Z}=3, \\
\mathrm{E}=0\end{array}$ \\
\hline
\end{tabular}

Пívakas 2

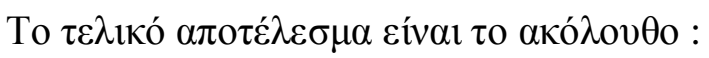

$$
\mathrm{A}=3, \mathrm{~B}=1, \Gamma=2, \Delta=4, \mathrm{E}=0, \mathrm{Z}=3 .
$$

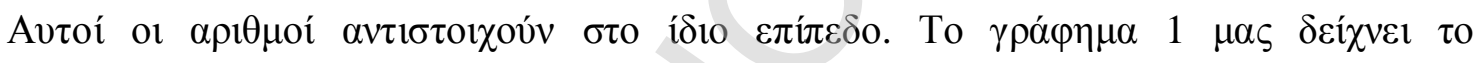

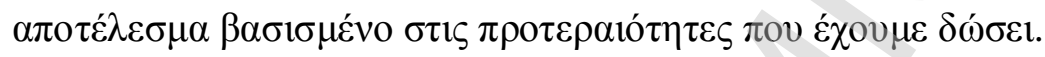

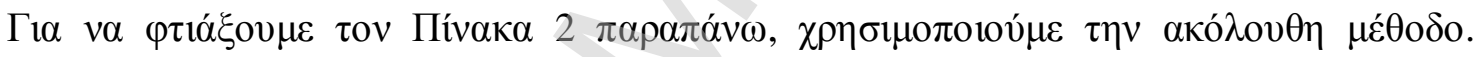

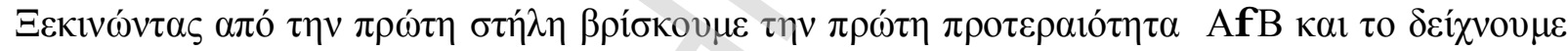

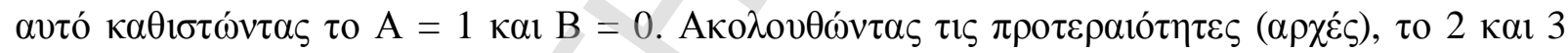

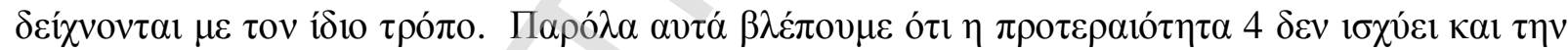

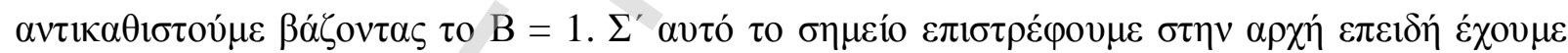

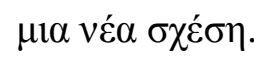

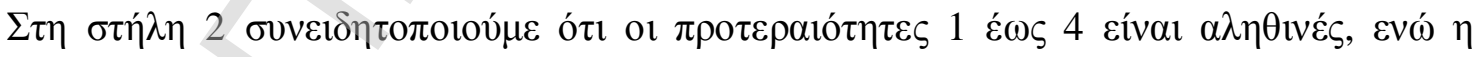

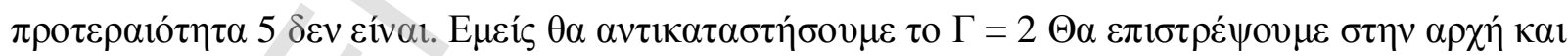

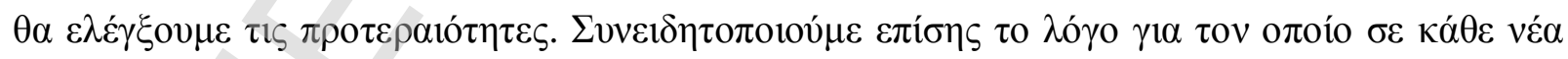

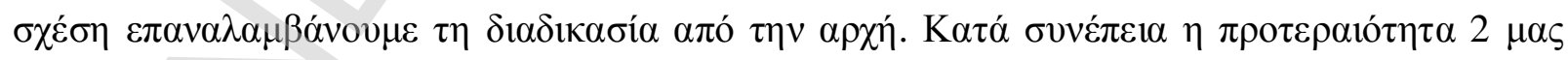

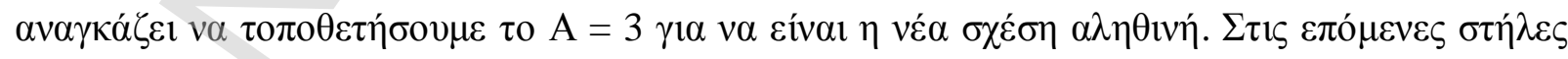

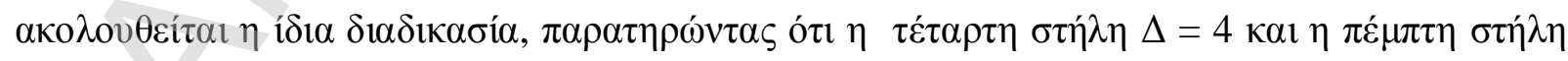

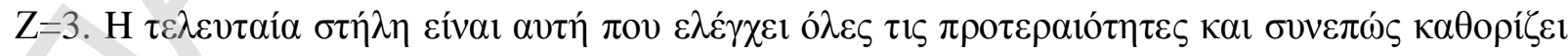

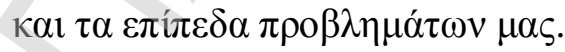




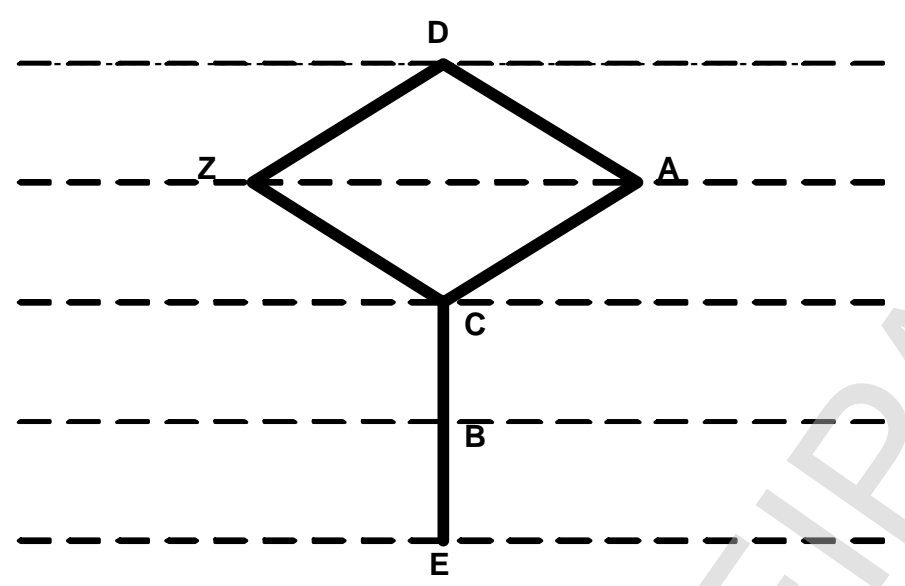

Гра́фү $\mu \alpha 1$

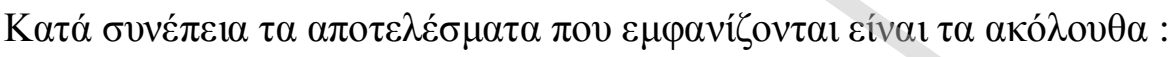

1. $\triangle \mathrm{A} Г \mathrm{BE}$

2. $\triangle \mathrm{ZГВЕ}$

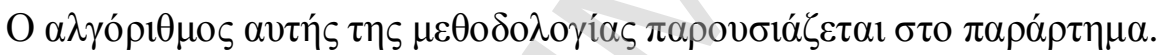

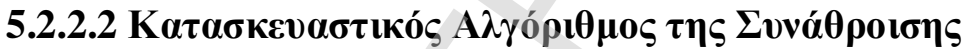

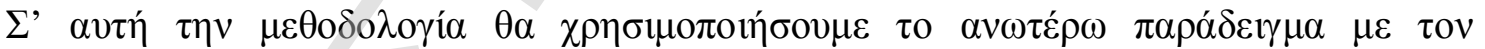

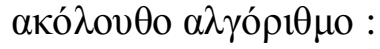

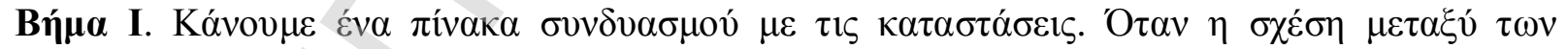

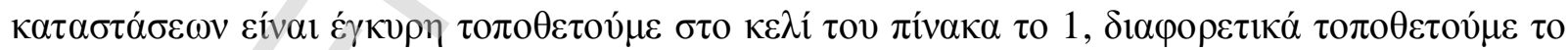
0.

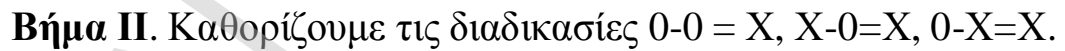

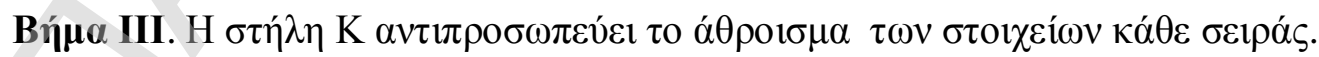

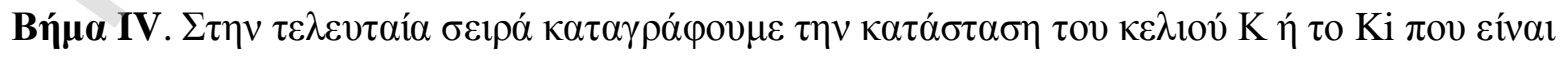
í 


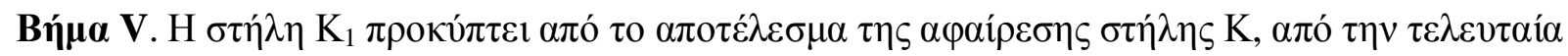

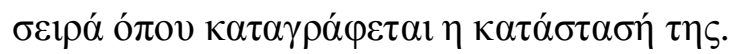

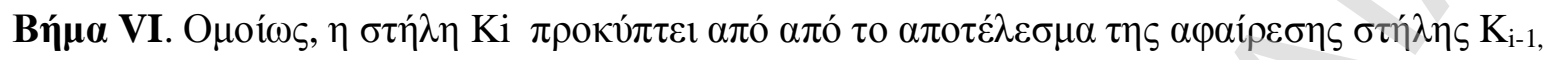

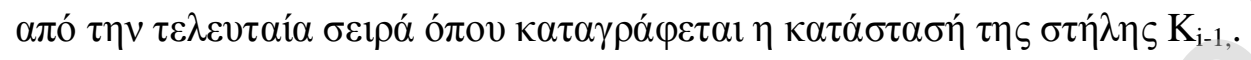

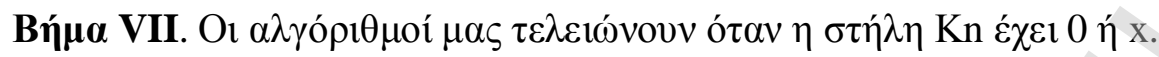

\begin{tabular}{|c|c|c|c|c|c|c|c|c|c|c|c|c|}
\hline $\boldsymbol{\sigma}(\mathbf{i})$ & & $\boldsymbol{\sigma}(\mathbf{1})$ & $\boldsymbol{\sigma}(\mathbf{2})$ & $\boldsymbol{\sigma}(\mathbf{3})$ & $\boldsymbol{\sigma}(\mathbf{4})$ & $\boldsymbol{\sigma}(\mathbf{5})$ & $\boldsymbol{\sigma}(\mathbf{6})$ & $\mathbf{K}$ & $\mathbf{K}_{\mathbf{1}}$ & $\mathbf{K}_{\mathbf{2}}$ & $\mathbf{K}_{\mathbf{3}}$ & $\mathbf{K}_{\mathbf{4}}$ \\
\hline $\mathbf{1}$ & $\mathbf{A}$ & 0 & 1 & 1 & 0 & 1 & 0 & 3 & 2 & 1 & 0 & $\mathrm{X}$ \\
\hline $\mathbf{2}$ & $\mathbf{B}$ & 0 & 0 & 0 & 0 & 1 & 0 & 1 & 0 & $\mathrm{X}$ & $\mathrm{X}$ & $\mathrm{X}$ \\
\hline $\mathbf{3}$ & $\boldsymbol{\Gamma}$ & 0 & 1 & 0 & 0 & 0 & 0 & 1 & 1 & 0 & $\mathrm{X}$ & $\mathrm{X}$ \\
\hline $\mathbf{4}$ & $\boldsymbol{\Delta}$ & 1 & 0 & 0 & 0 & 1 & 1 & 3 & 2 & 2 & 2 & 0 \\
\hline $\mathbf{5}$ & $\mathbf{E}$ & 0 & 0 & 0 & 0 & 0 & 0 & 0 & $\mathrm{X}$ & $\mathrm{X}$ & $\mathrm{X}$ & $\mathrm{X}$ \\
\hline $\mathbf{6}$ & $\mathbf{Z}$ & 0 & 0 & 1 & 0 & 1 & 0 & 2 & 1 & 1 & 0 & $\mathrm{X}$ \\
\hline $\begin{array}{c}\text { TE } \boldsymbol{M} \\
\text { EYT } \\
\text { AIA }\end{array}$ & & & & & & & & $\mathrm{E}$ & $\mathrm{B}$ & $\Gamma$ & $\mathrm{A}, \mathrm{Z}$ & $\Delta$ \\
\hline
\end{tabular}

Пívakas 3

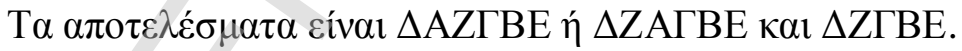




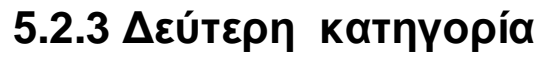

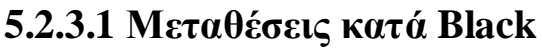

\section{Opıбноí :}

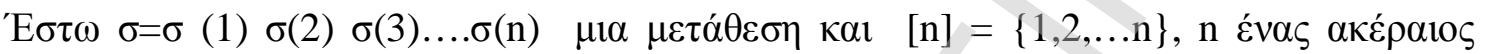

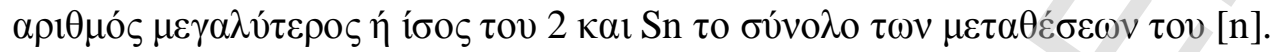

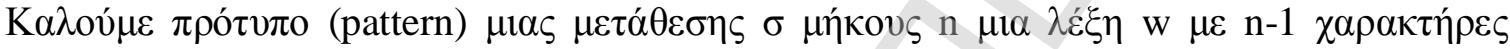

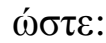

$$
\begin{gathered}
\mathrm{w}=\mathrm{Z}_{1} \mathrm{Z}_{2} \cdots \cdots \mathrm{zn}-1, \mathrm{Z}_{\mathrm{i}} \in\{-1,+1\}^{\mathrm{n}-1} \\
\mathrm{Z}_{\mathrm{i}}=\left\{\begin{array}{lll}
-1 & \alpha \nu & \sigma(\mathrm{i})>\sigma(\mathrm{i}+1) \\
+1 & \alpha \nu & \sigma(\mathrm{i})<\sigma(\mathrm{i}+1)
\end{array} \quad i \in[n-1]\right.
\end{gathered}
$$

$\Gamma 1 \alpha \kappa \alpha ́ \theta \varepsilon \sigma \in \mathrm{Sn} \sigma v \mu \beta \mathrm{o} \lambda i \zeta \zeta o v \mu \varepsilon \mu \varepsilon$

$$
\mathrm{A}^{+}(\sigma) \equiv\{\mathrm{i} \in[2, \mathrm{n}]: \sigma(\mathrm{i})>\sigma(1)\} \quad \text { Kal } \quad \mathrm{A}^{-}(\sigma) \equiv\{\mathrm{i} \in[2, \mathrm{n}]: \sigma(\mathrm{i})<\sigma(1)\}
$$

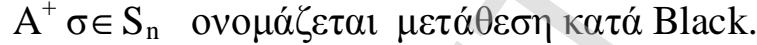

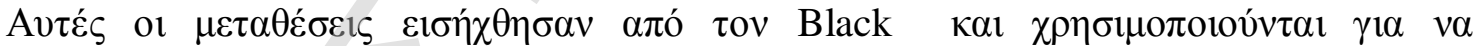

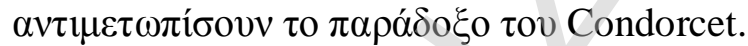

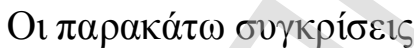

\begin{tabular}{|l|l|l|l|l|l|l|}
\hline$A^{f} B$ & $A^{f} \Gamma$ & $A^{f} \Delta$ & $A^{f} E$ & $A^{f} F$ & $A^{f} H$ & $B^{f} \Gamma$ \\
\hline$B^{f} \Delta$ & $B^{f} E$ & $B^{f} Z$ & $B^{f} H$ & $\Gamma^{f} \Delta$ & $\Gamma^{f} E$ & $\Gamma^{f} F$ \\
\hline$\Gamma^{f} Z$ & $\Delta^{f} E$ & $\Delta^{f} Z$ & $\Delta^{f} H$ & $E^{f} Z$ & $E^{f} H$ & $F^{f} H$ \\
\hline
\end{tabular}

Пívakas 4

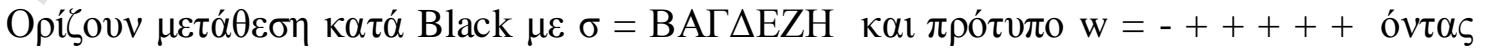

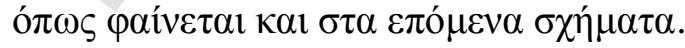




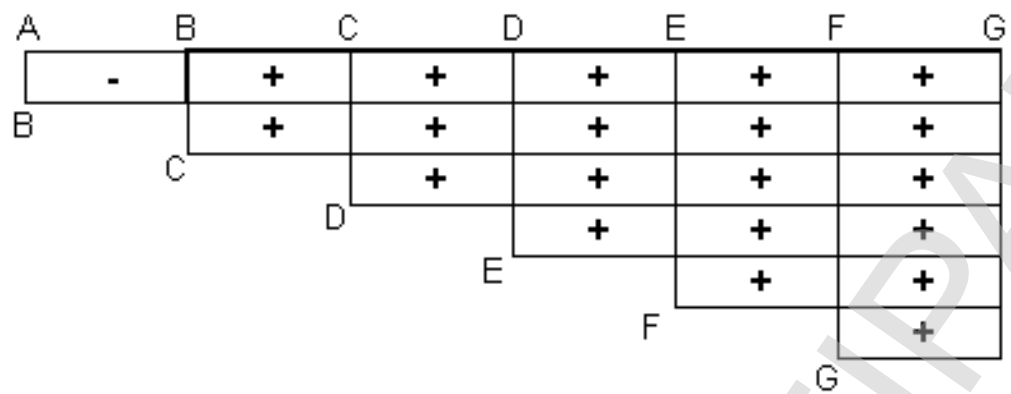

Пívaкas 1

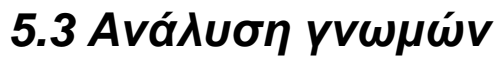

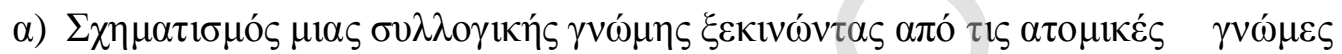

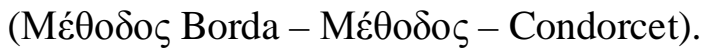

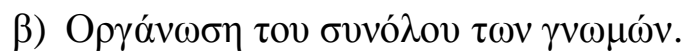

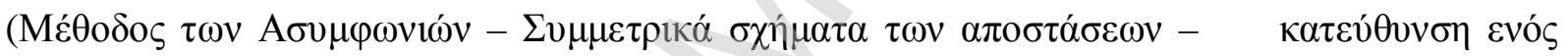

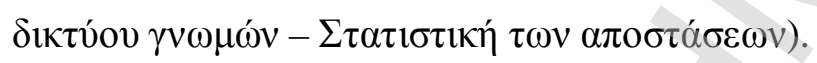

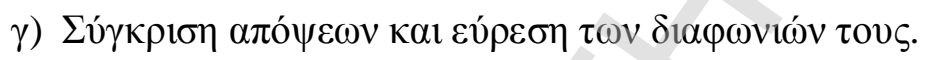

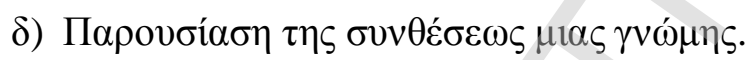

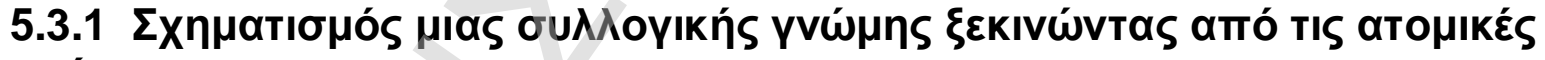

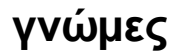

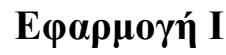

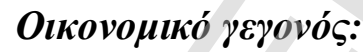

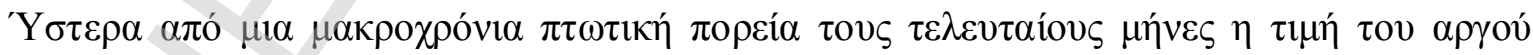

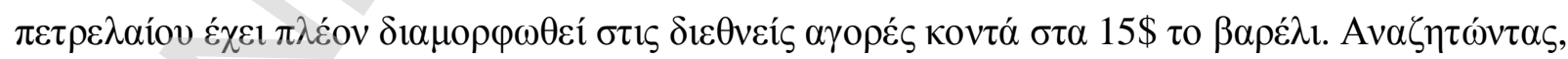

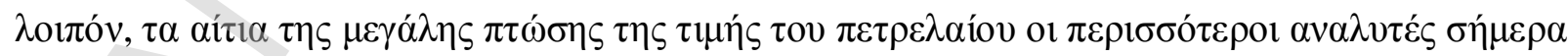
$\pi \imath \tau \varepsilon v ́ 0 v \nu \tau \alpha \varepsilon \xi \dot{\eta} \zeta$ :

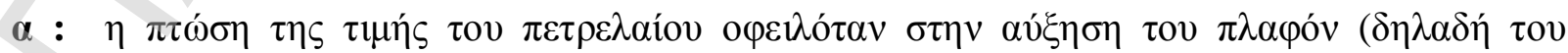

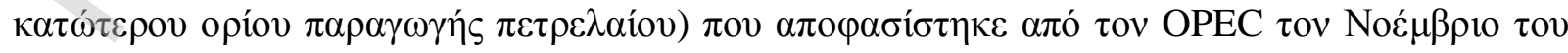

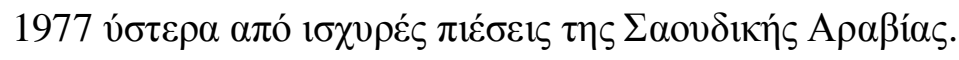




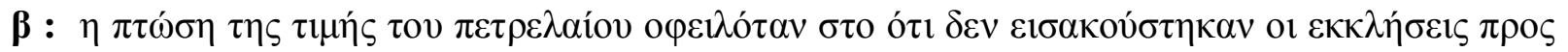

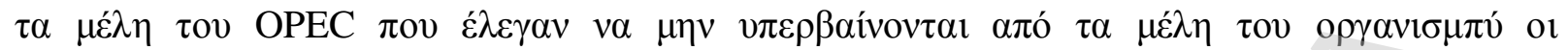

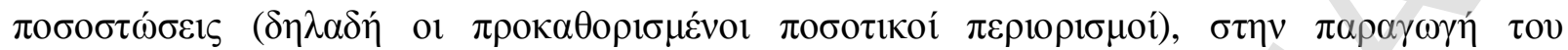

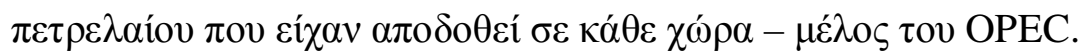

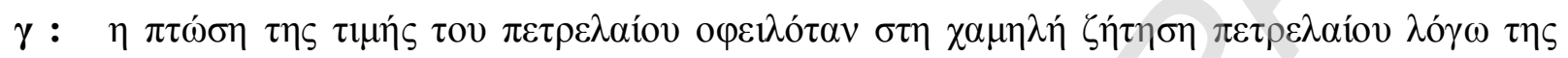

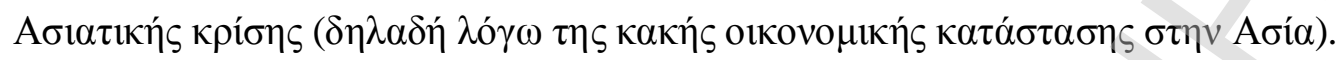

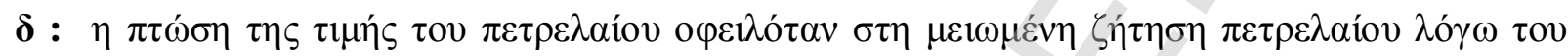

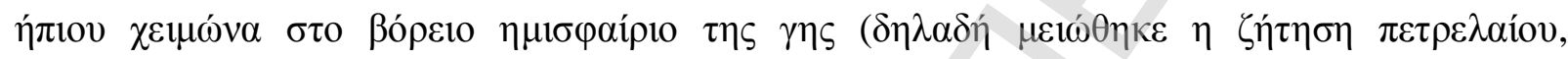

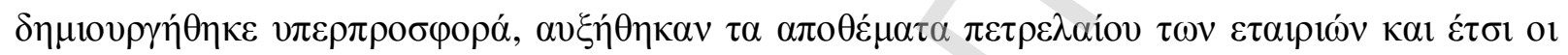

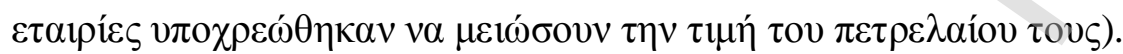

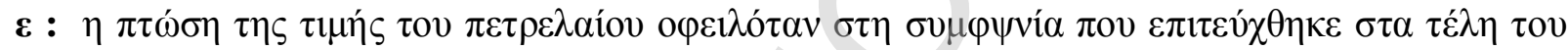

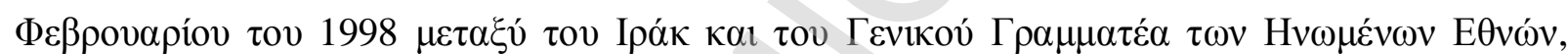

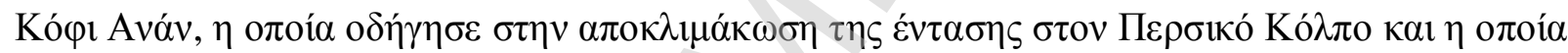

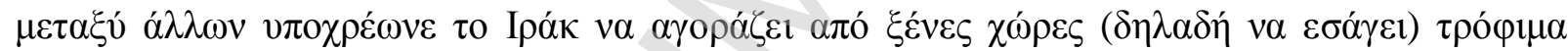

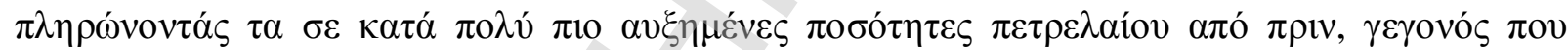

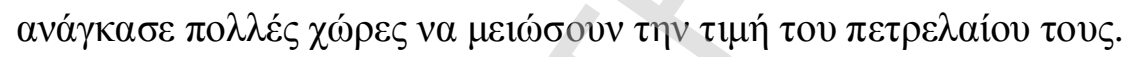

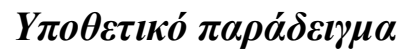

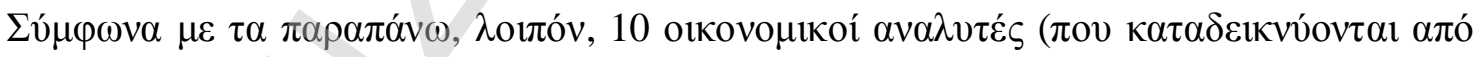

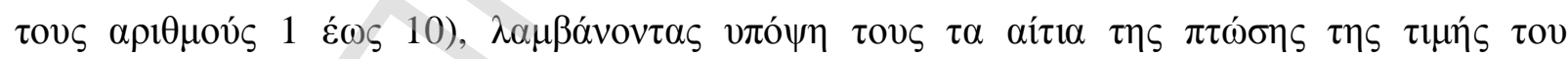

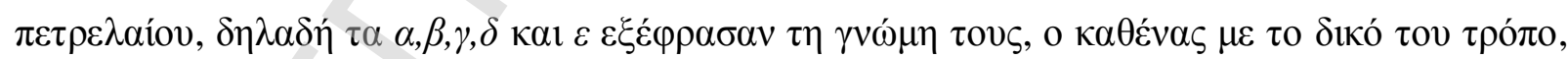

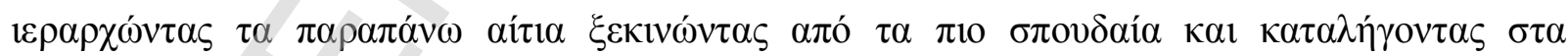

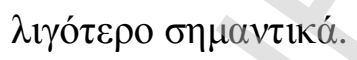

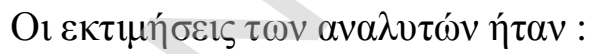

$\begin{array}{llllll}1: & \alpha & \beta & \gamma & \delta & \varepsilon \\ 2: & \beta & \gamma & \alpha & \varepsilon & \delta \\ 3: & \varepsilon & \alpha & \gamma & \delta & \beta \\ 4: & \gamma & \alpha & \beta & \delta & \varepsilon \\ 5: & \delta & \gamma & \alpha & \beta & \varepsilon \\ 6: & \alpha & \gamma & \delta & \beta & \varepsilon \\ 7: & \varepsilon & \delta & \gamma & \alpha & \beta \\ 8: & \gamma & \varepsilon & \alpha & \beta & \delta\end{array}$




$\begin{array}{rlllll}9: & \beta & \delta & \gamma & \varepsilon & \alpha \\ 10: & \delta & \alpha & \varepsilon & \gamma & \beta\end{array}$

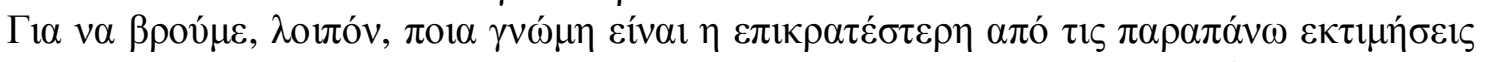

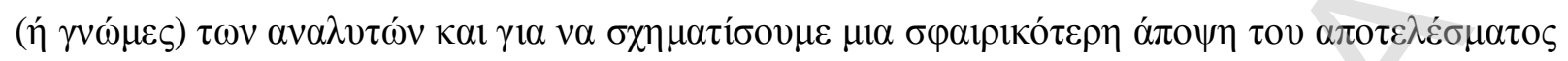

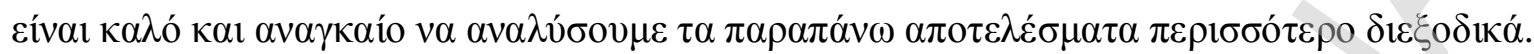

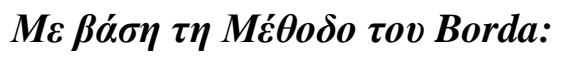

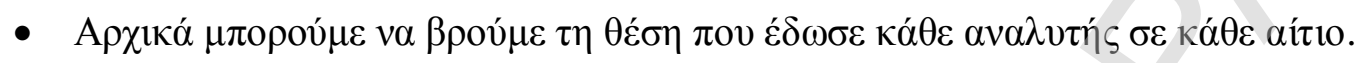

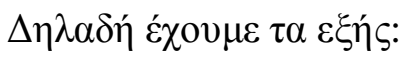

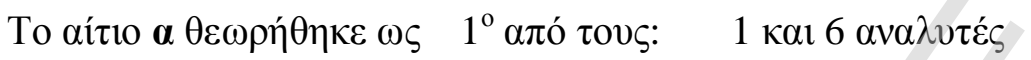

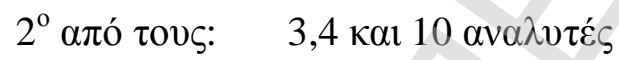

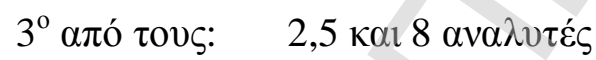

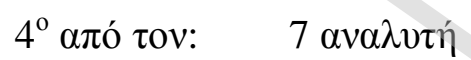

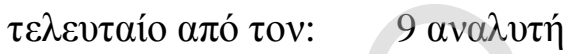

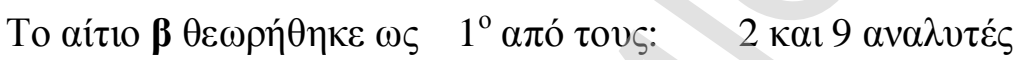

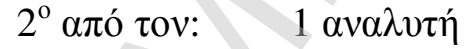

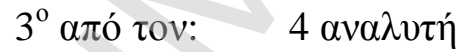

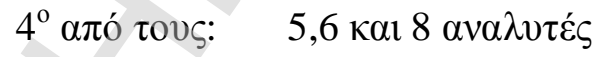

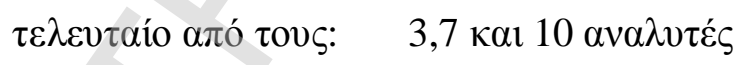

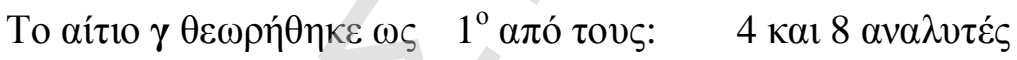

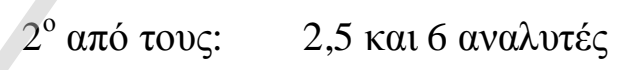

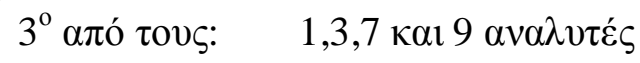

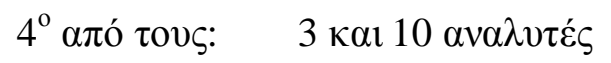

$\tau \varepsilon \lambda \varepsilon v \tau \alpha i ́ o ~ \alpha \pi o ́ ~ \kappa \alpha v \varepsilon ́ v \alpha v \alpha v \alpha \lambda v \tau \eta ́$

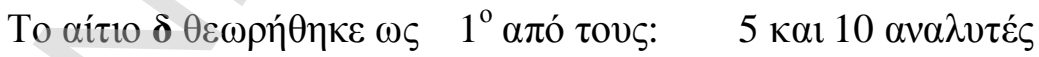

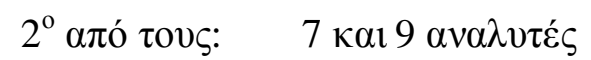

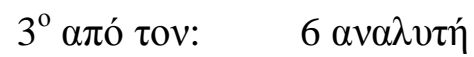

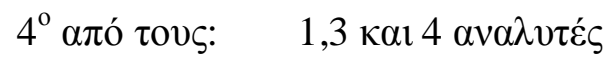

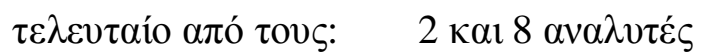




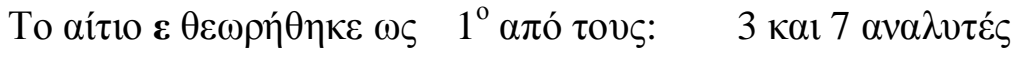

$2^{\circ}$ a

$3^{\circ} \alpha \pi$ cov: $\quad 10 \alpha v \alpha \lambda v \tau \eta ́$

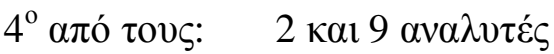

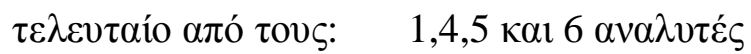

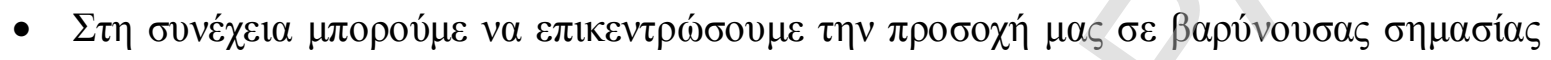

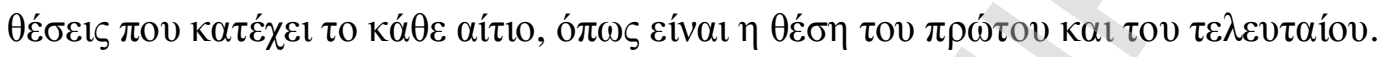

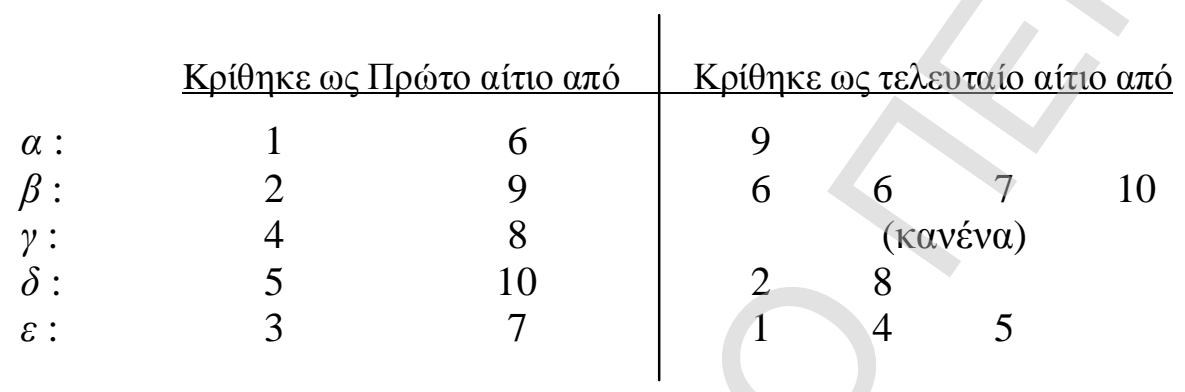

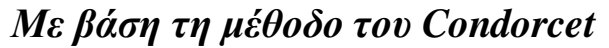

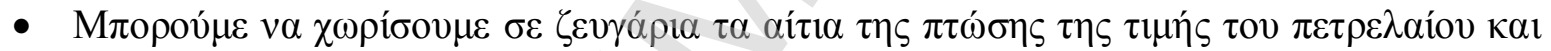

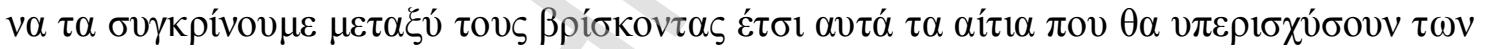
$\alpha \dot{\alpha} \lambda \omega \nu$ :

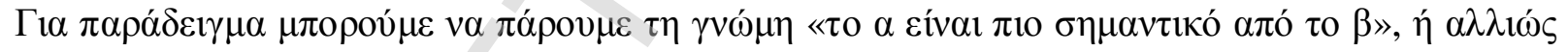

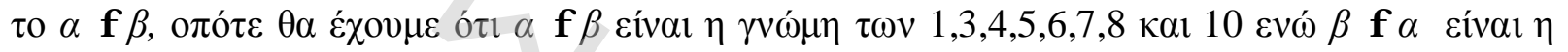

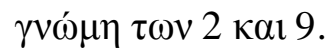

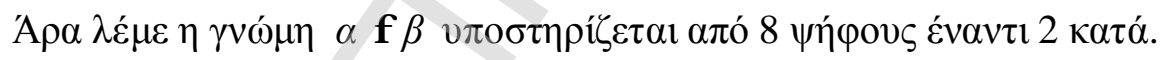

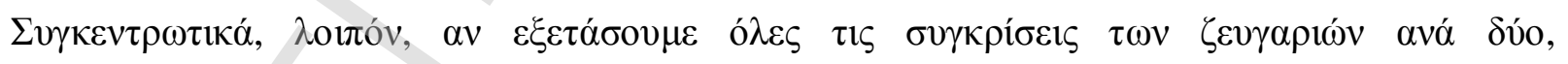

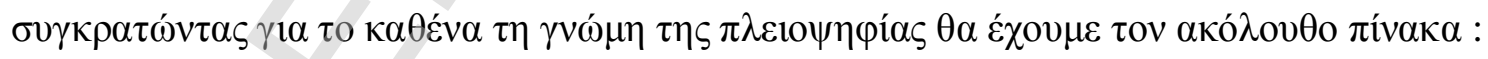

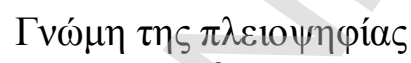

$$
\begin{aligned}
& \alpha \mathrm{f} \beta \\
& \gamma \mathrm{f} \beta \\
& \gamma \mathrm{f} \varepsilon \\
& \alpha \mathrm{f} \delta \\
& \alpha \mathrm{f} \varepsilon \\
& \gamma \mathrm{f} \alpha \\
& \delta \mathrm{f} \varepsilon \\
& \gamma \mathrm{f} \delta
\end{aligned}
$$

$$
\begin{aligned}
& \text { Apı } \theta \mu \text { ó } \varsigma \alpha v \alpha \lambda v \tau \omega ́ v
\end{aligned}
$$

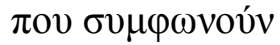

$$
8
$$$$
7
$$$$
7
$$

6

6

6

6
Apı $\theta \mu$ ó $\varsigma \alpha v \alpha \lambda v \tau \omega ́ v$

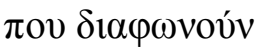




$\begin{array}{lll}\beta \mathrm{f} \varepsilon & 6 & 4 \\ \beta \mathrm{f} \delta & 5 & 5 \\ \delta \mathrm{f} \beta & 5 & 5 \\ \varepsilon \mathrm{f} \beta & 4 & 6 \\ \delta \mathrm{f} \gamma & 4 & 6\end{array}$

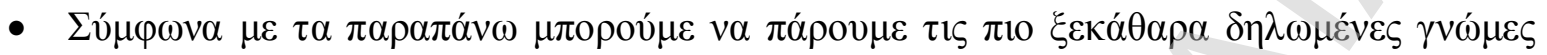

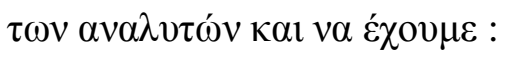

\begin{tabular}{|c|c|c|c|}
\hline 8 & 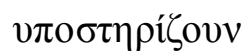 & ó $\tau \imath$ & $\alpha f \beta$ \\
\hline 7 & 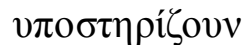 & ó $\tau \imath$ & $\gamma f \beta$ \\
\hline 7 & 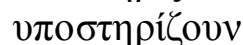 & ó $\tau \imath$ & $\gamma f \varepsilon$ \\
\hline & 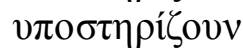 & ó $\tau \imath$ & $\gamma f \delta$ \\
\hline & 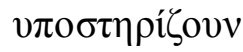 & ó $\tau \imath$ & $\alpha f \delta$ \\
\hline & 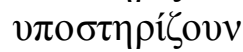 & ó $\tau 1$ & $\alpha f \varepsilon$ \\
\hline & 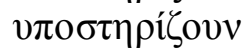 & ó $\tau \imath$ & $\gamma f \alpha$ \\
\hline & 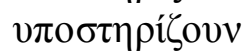 & ó $\tau \imath$ & $\delta f$ \\
\hline & 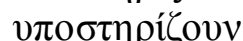 & ó $\tau 1$ & $\beta f \varepsilon$ \\
\hline
\end{tabular}

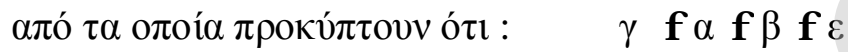

$\gamma f \alpha f \delta f \varepsilon$

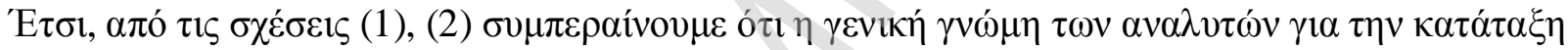

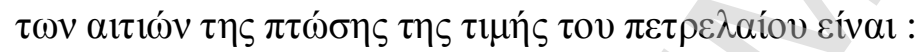

$\gamma f \alpha f \delta f \varepsilon$

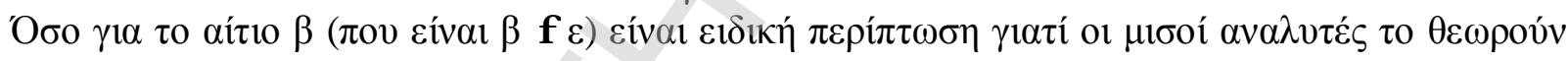

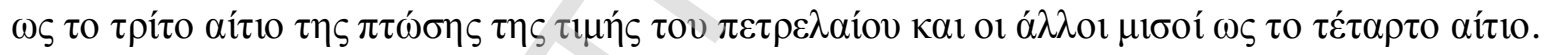

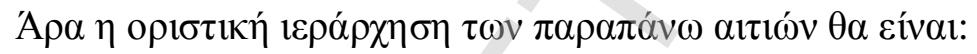

$$
\gamma \alpha \beta \delta \varepsilon \quad \eta \dot{\gamma} \quad \gamma \alpha \delta \beta \varepsilon
$$

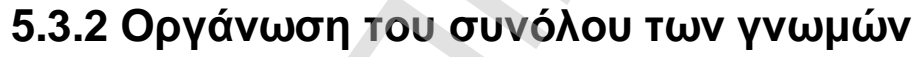

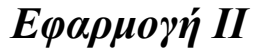

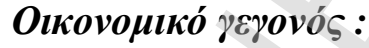

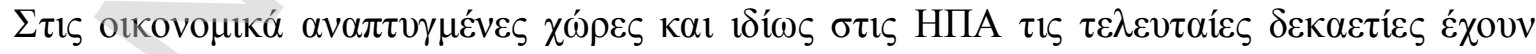

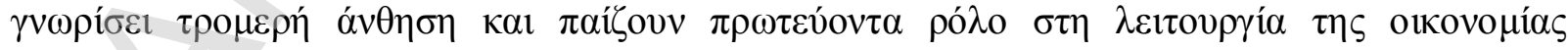

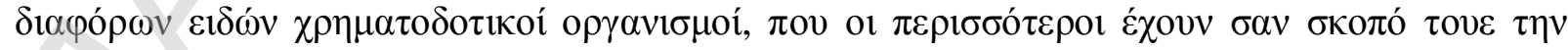

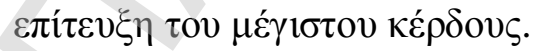

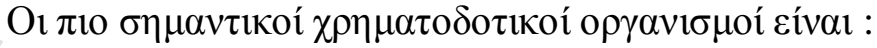




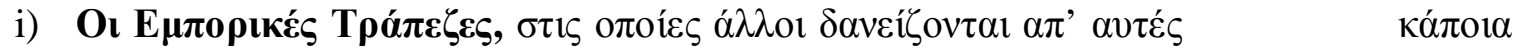

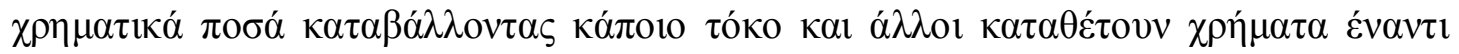

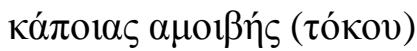

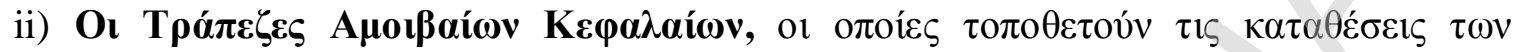

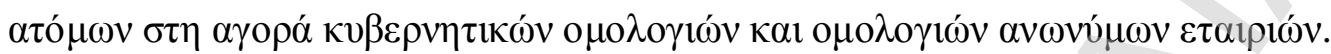

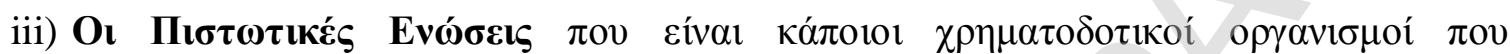
$\delta \eta \mu 10 v \rho \gamma o v ́ v \tau \alpha 1 \alpha \pi$ vं

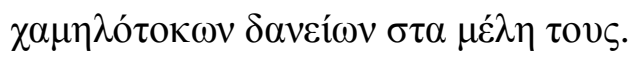

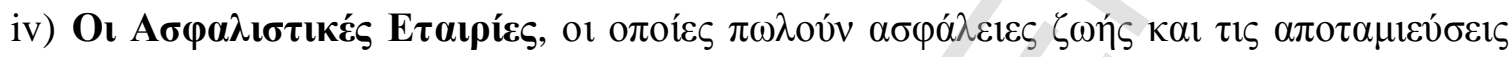

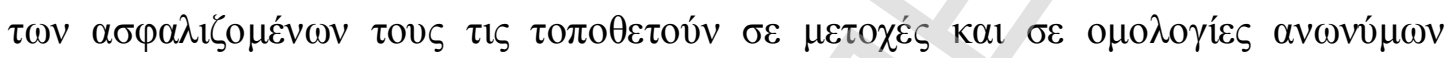

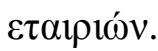

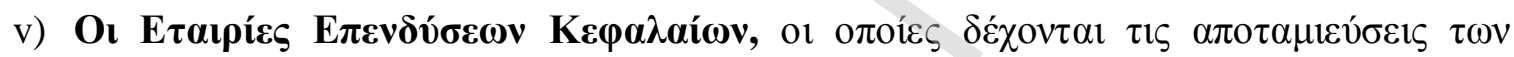

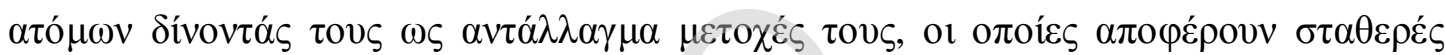

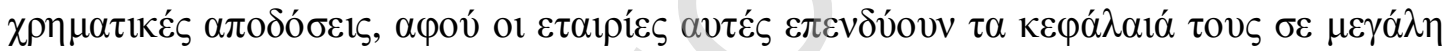

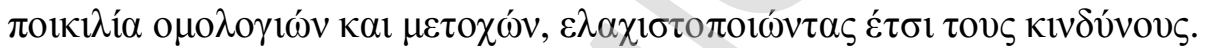

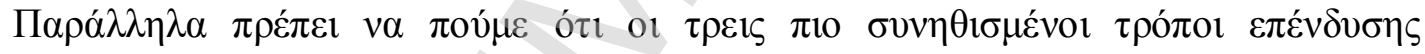

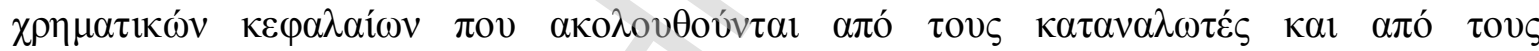

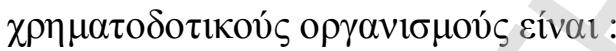

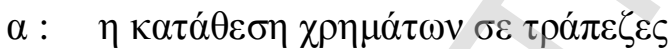

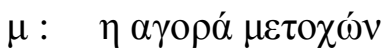

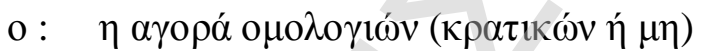

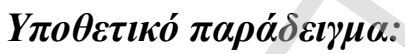

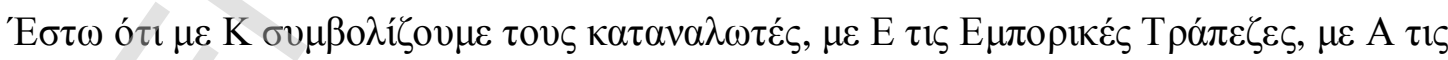

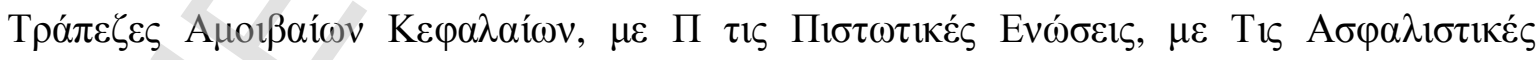

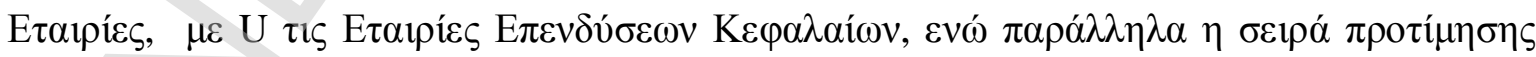

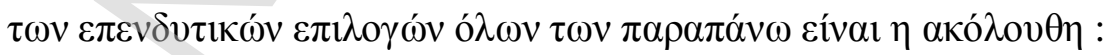

$$
\begin{aligned}
& \gamma \text { f } \alpha \mathrm{f} \beta=\mathrm{K} \\
& \alpha \text { f of } \mu=\mathrm{E} \\
& \mu \text { f } \alpha \mathrm{f} \text { o }=\mathrm{A} \\
& \mu \mathrm{f} \text { of } \alpha=\Pi \\
& \text { o f } \alpha \mathrm{f} \mu=\mathrm{T} \\
& \text { of } \mu \mathrm{f} \alpha=\mathrm{U}
\end{aligned}
$$




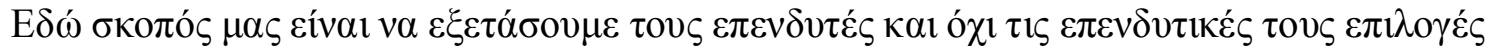

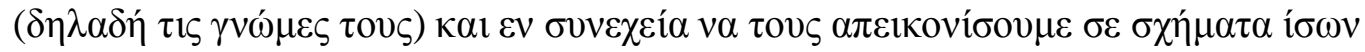
$\alpha \pi 0 \sigma \tau \alpha ́ \sigma \varepsilon \omega v$.

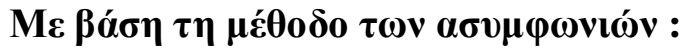

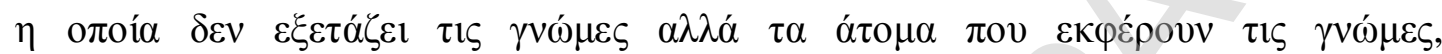

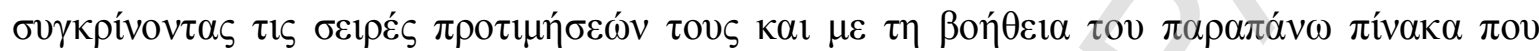

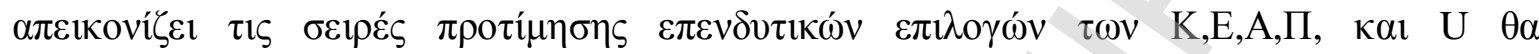

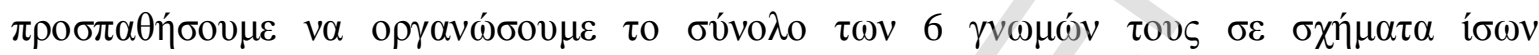

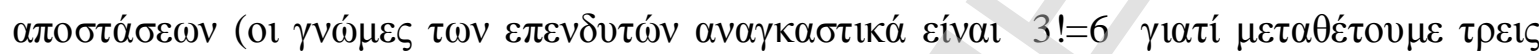

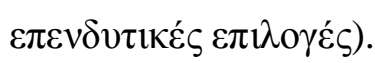

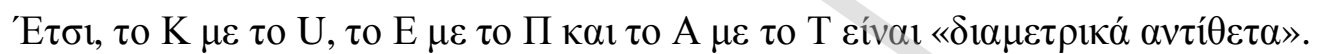

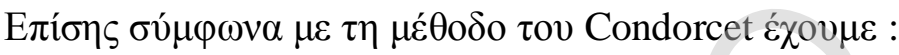

\begin{tabular}{|c|c|c|c|}
\hline $\mathrm{K}$ & $\alpha f \mu f o$ & $\delta \eta \lambda \alpha \delta \eta ́$ & $(\alpha f \mu) \kappa \alpha l(\alpha f o) \kappa \alpha l(\mu f \circ)$ \\
\hline $\mathrm{E}$ & $\alpha$ f of $\mu$ & $\delta \eta \lambda \alpha \delta \eta ́$ & $(\alpha f o) \kappa \alpha l(\alpha f \mu) \kappa \alpha l(o f \mu)$ \\
\hline A & $\alpha f \mu f o$ & $\delta \eta \lambda \alpha \delta \eta^{\prime}$ & $(\mu f \alpha) \kappa \alpha \iota(\mu f o) \kappa \alpha l(\alpha f$ \\
\hline$\Pi$ & $\alpha f \mu f o$ & $\delta \eta \lambda \alpha \delta \dot{~}$ & $(\alpha f \mu) \kappa \alpha l(\alpha f o) \kappa \alpha l(\mu f$ \\
\hline $\mathrm{T}$ & $o f \alpha f \mu$ & $\delta \eta \lambda \alpha \delta \eta^{\prime}$ & $(\mathrm{o} f \alpha) \kappa \alpha l(\alpha f \mu) \kappa \alpha l(o f$ \\
\hline $\mathrm{U}$ & $o f \mu f \alpha$ & $\delta \eta \lambda \alpha \delta \eta^{\prime}$ & $(o f \mu) \kappa \alpha l(\mu f \alpha) \kappa \alpha l$ (o $f$ \\
\hline
\end{tabular}

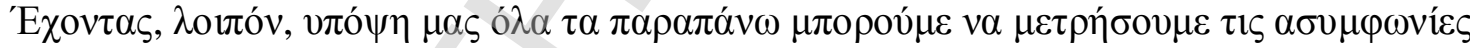

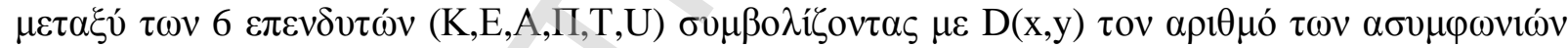
$\mu \varepsilon \tau \alpha \xi \dot{v} \delta$ v́o $\tau \chi \chi \alpha i ́ \omega v \varepsilon \pi \varepsilon v \delta v \tau \omega ́ v \mathrm{x}, \mathrm{y}$.

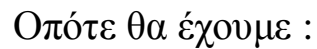

$\begin{array}{lll}\mathrm{D}(\mathrm{K}, \mathrm{E})=1 & \alpha \varphi \circ v^{\prime} & \alpha \mathrm{f} \mu \mathrm{fo}=\mathrm{K} \\ & & \alpha \mathrm{f} \text { of } \mu=\mathrm{E} \\ \mathrm{D}(\mathrm{K}, \mathrm{A})=1 & \alpha \varphi \circ v^{2} & \alpha \mathrm{f} \mu \mathrm{f}=\mathrm{K} \\ & & \mu \mathrm{f} \alpha \mathrm{fo}=\mathrm{A}\end{array}$

ouoíws

$\mathrm{D}(\mathrm{E}, \mathrm{T})=1$

$\mathrm{D}(\mathrm{A}, \Pi)=1$

$\mathrm{D}(\mathrm{T}, \mathrm{U})=1$

$\mathrm{D}(\Pi, \mathrm{U})=1$

$\mathrm{D}(\mathrm{K}, \mathrm{T})=2$

$\mathrm{D}(\mathrm{K}, \Pi)=1$

$\mathrm{D}(\mathrm{E}, \mathrm{U})=2$

$\mathrm{D}(\mathrm{A}, \mathrm{U})=2$

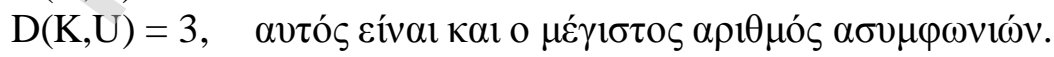




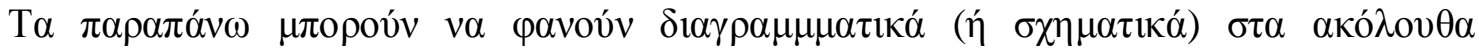

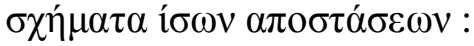

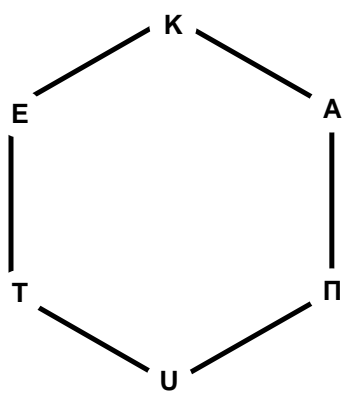

$\delta \eta \lambda \alpha \delta \eta \dot{~}$

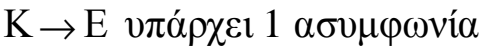

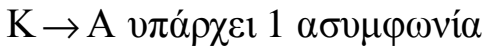

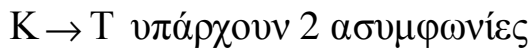

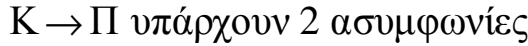

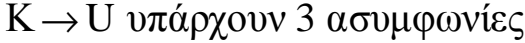

$\kappa . \lambda . \pi$.

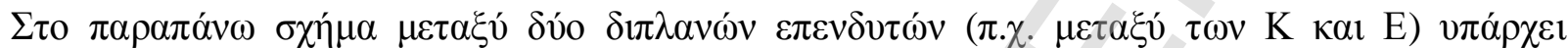

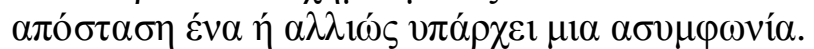

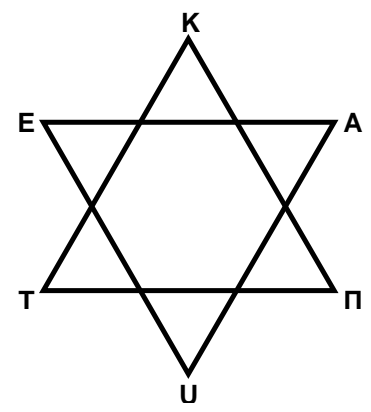

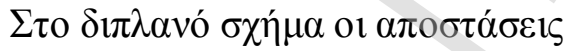

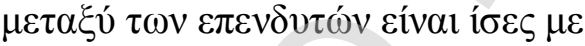

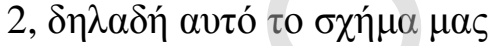

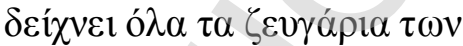

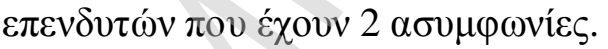

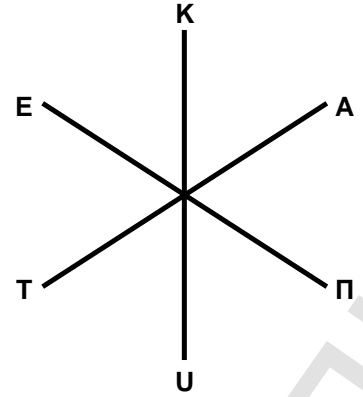

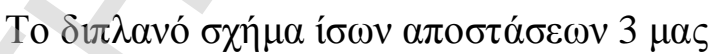

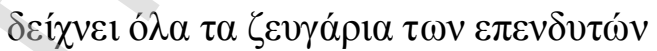

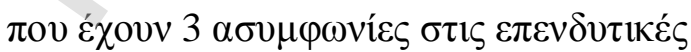

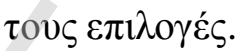

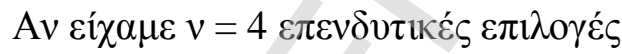

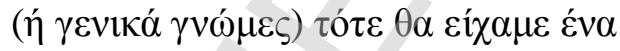

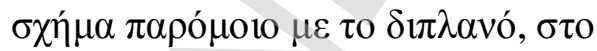

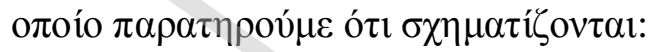

$6 \tau \varepsilon \tau \rho \alpha ́ \pi \lambda \varepsilon v \rho \alpha$

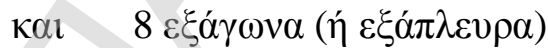

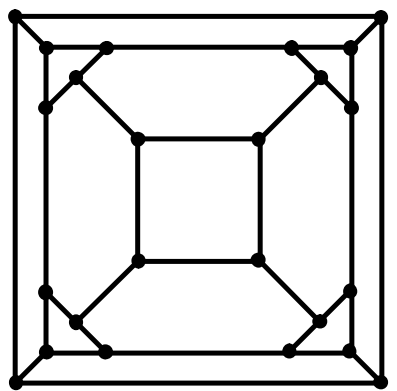

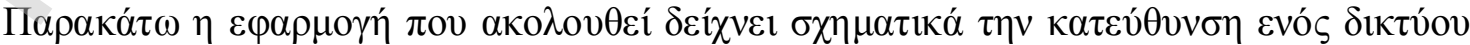

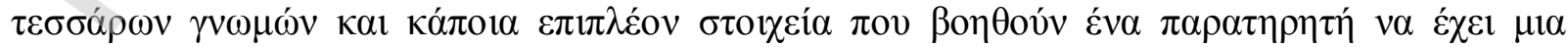

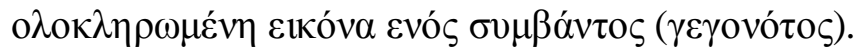




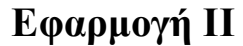

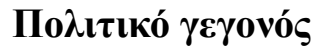

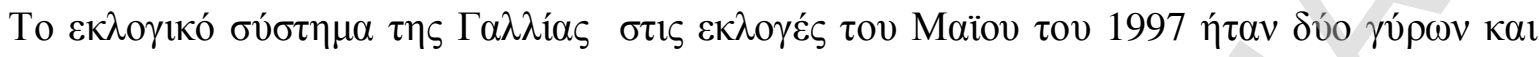

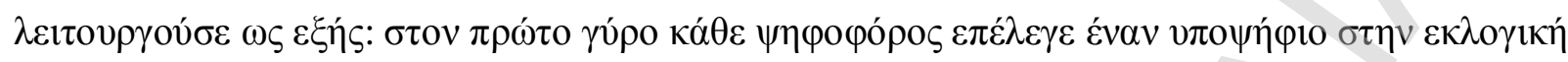

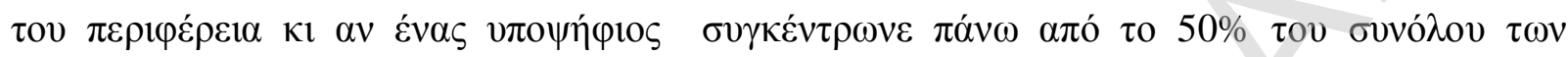

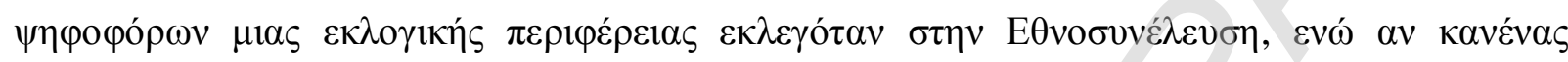

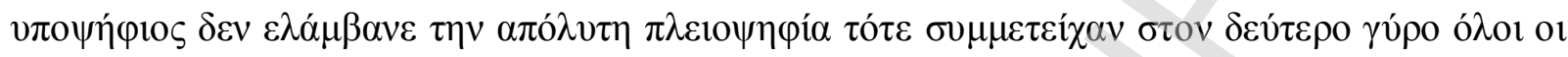

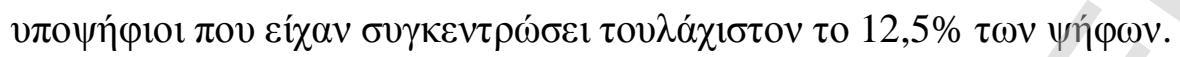

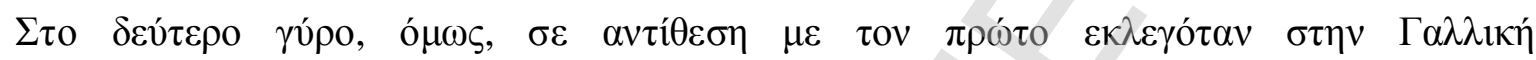

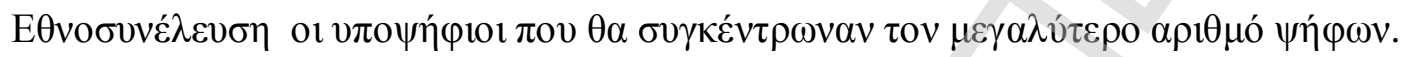

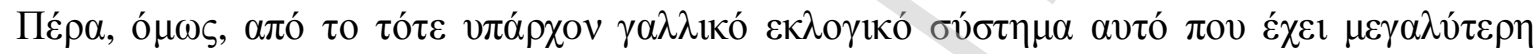

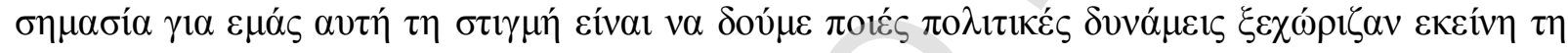

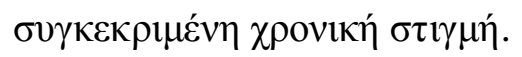

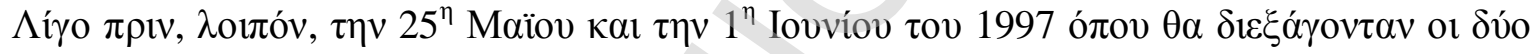

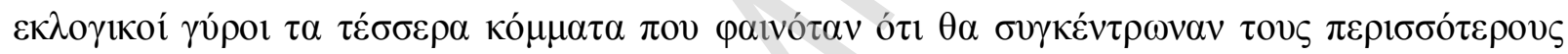

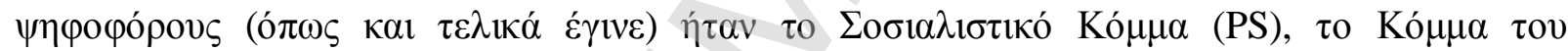

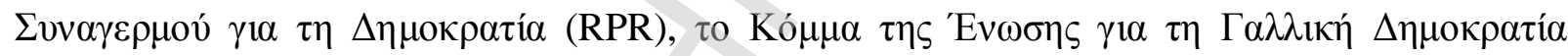

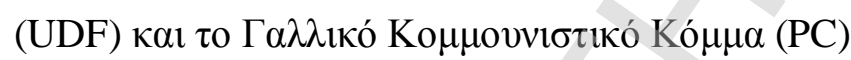

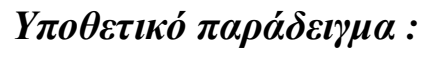

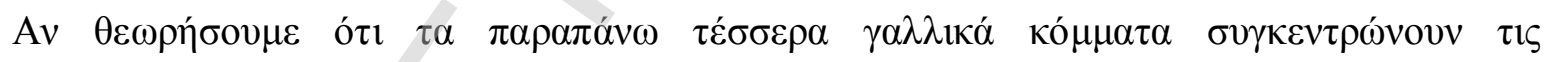

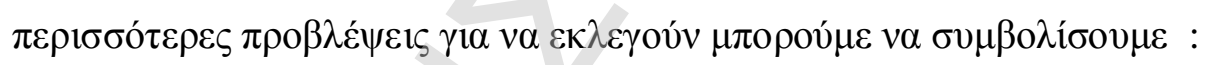

$$
\mathrm{P}_{1 \mathrm{~V} 2} \mu \varepsilon \mathrm{K}_{(1 v 2)}=K_{1}^{-} \cup K_{2}^{-}=\{\gamma \beta, \delta \alpha, \delta \beta, \delta \gamma, \varepsilon \alpha, \varepsilon \beta, \varepsilon \gamma, \varepsilon \delta\}
$$

\section{4 ГYMПEPAГMATA}

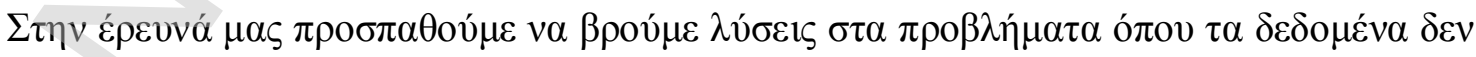

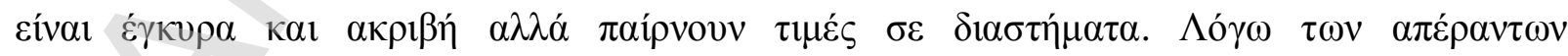

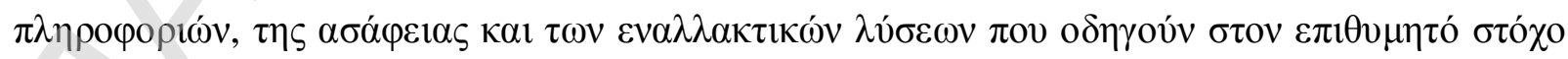

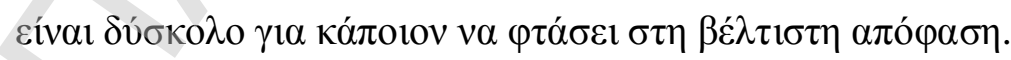

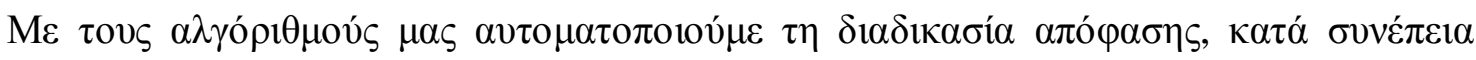

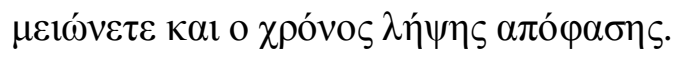




\section{The algorithm}

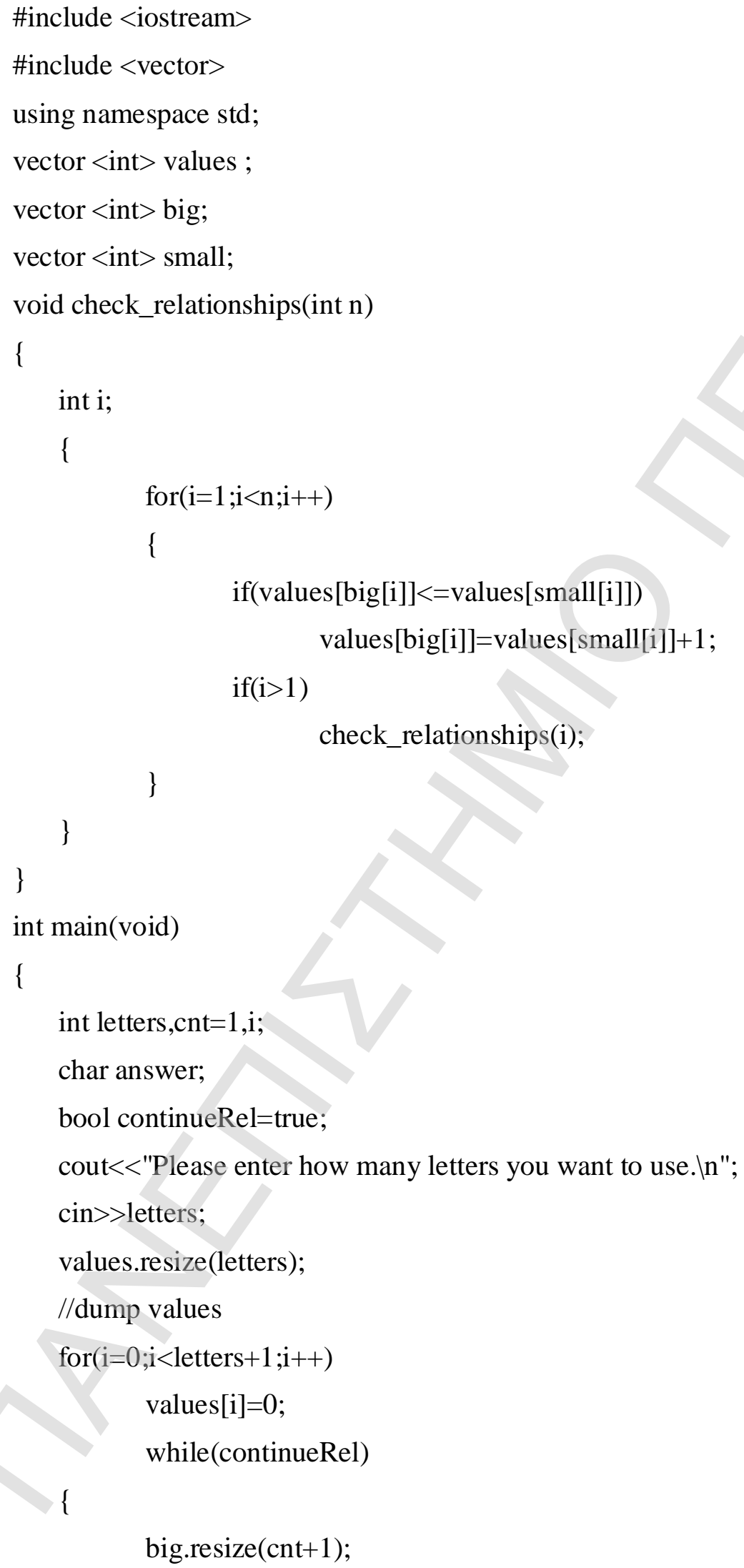


small.resize(cnt+1);

cout $<<$ "Enter the new relationshipln";

cout $<<$ "What letter is bigger? ";

cin >>big[cnt];

cout $<<$ "What letter is smaller? ";

cin >>small[cnt];

cout $<<$ "Continue? $\ln$ ";

cin >>answer;

continueRel $=\left(\right.$ answer $\left.=={ }^{\prime} y^{\prime}\right)$;

cnt++;

\}

cout $<<$ "The relations that you have entered are:"<<endl;

for $(\mathrm{i}=1 ; \mathrm{i}<\mathrm{cnt} ; \mathrm{i}++)$

cout $<<$ big $[\mathrm{i}]<<" \quad>\quad "<<$ small $[\mathrm{i}]<<$ endl;

check_relationships(cnt);

//output the found values

for $(\mathrm{i}=1 ; \mathrm{i}<$ letters $+1 ; \mathrm{i}++)$

cout $<<$ "letter $"<<\mathrm{i}<<"=$ "<<values $[\mathrm{i}]<<$ endl;

$1\}$ 


\section{Keфá̀aıo 6}

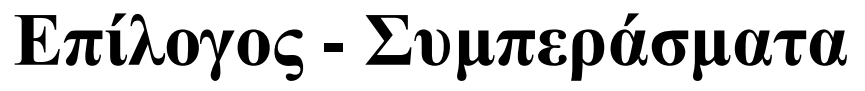

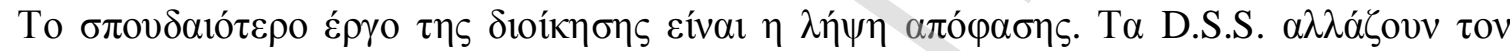

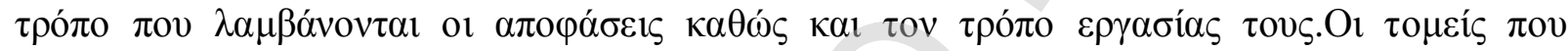
$\varepsilon \pi \eta \rho \varepsilon \alpha ́ \zeta o v \tau \alpha 1$ عíval :

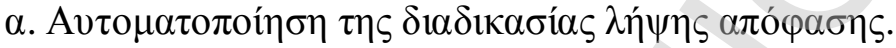

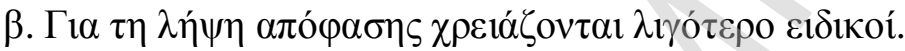

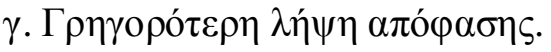

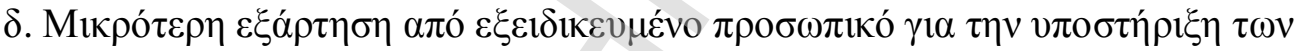
$\delta 1 \varepsilon v \theta u v \tau \omega ́ v$.

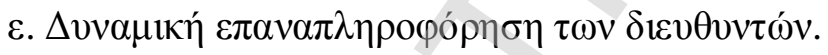

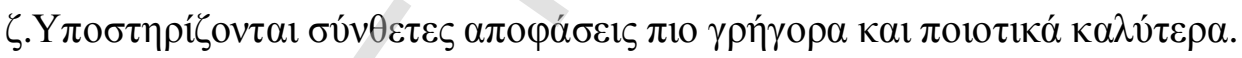

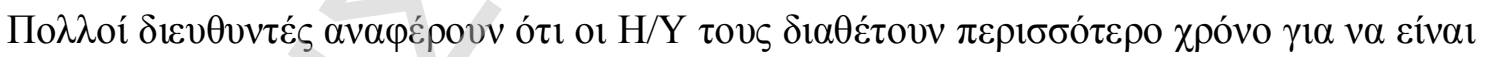

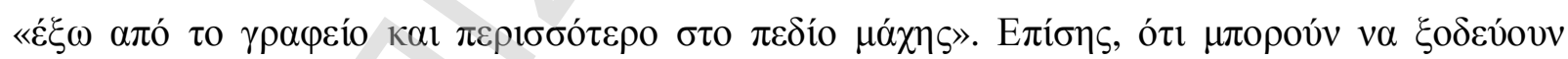

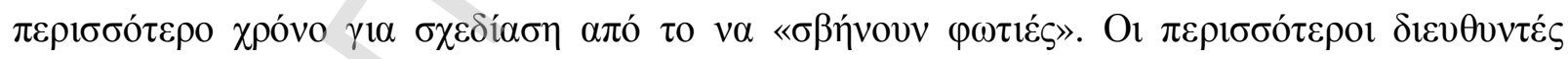

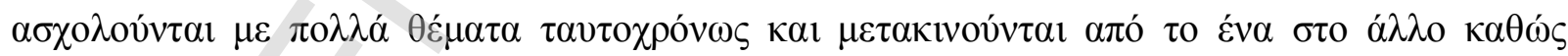

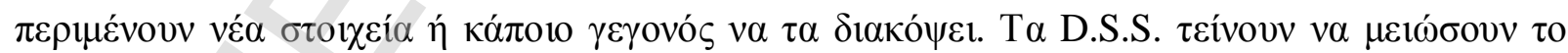

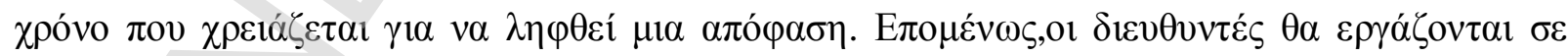

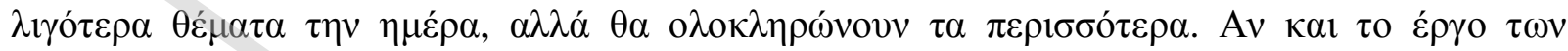

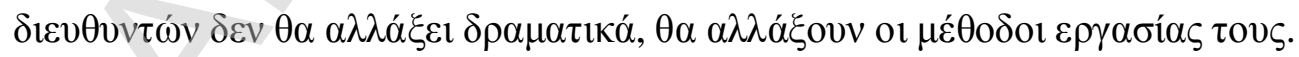




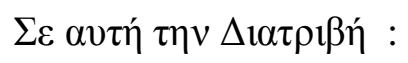

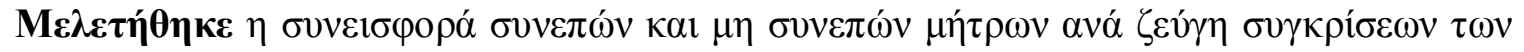

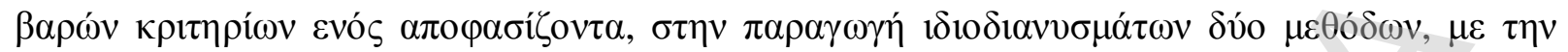

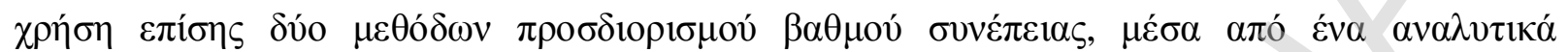

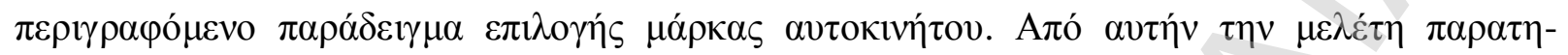
$\rho \eta ́ \theta \eta \kappa \alpha \nu \tau \alpha \varepsilon \xi \dot{\eta} \varsigma$ :

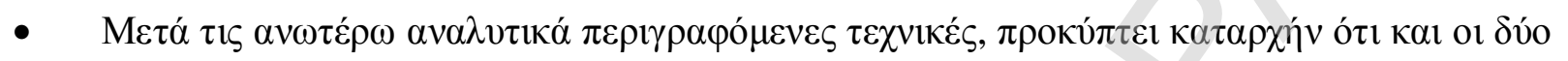

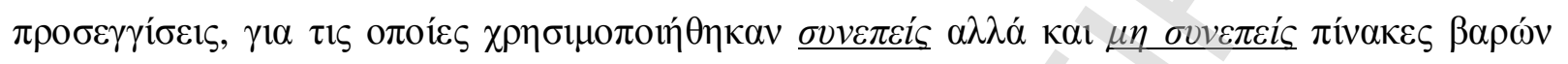

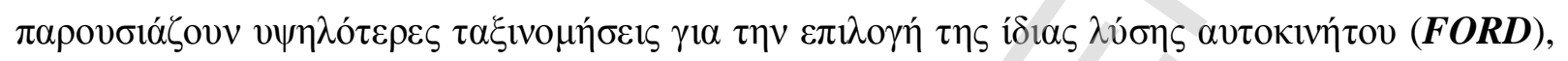

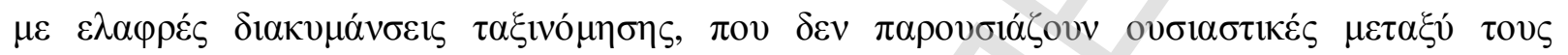

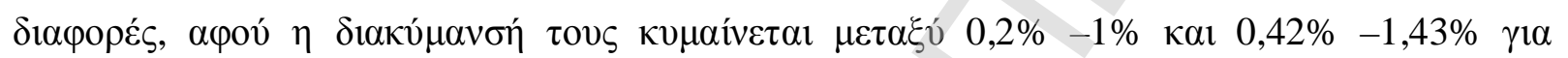

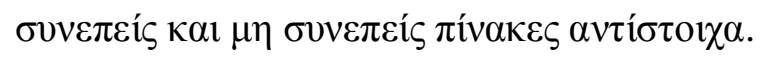

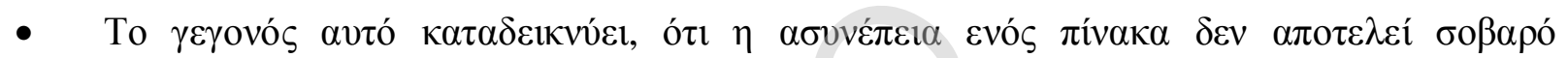

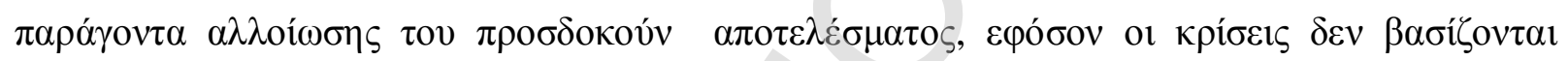

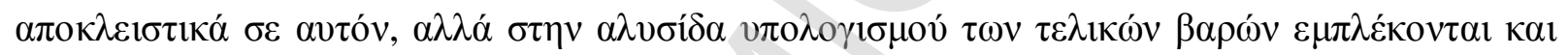

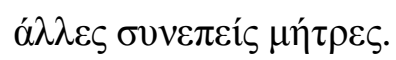

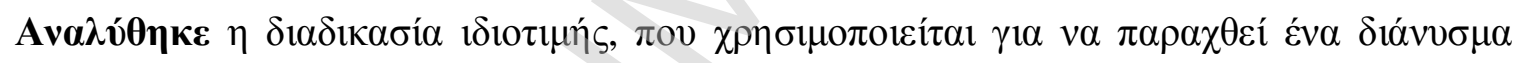

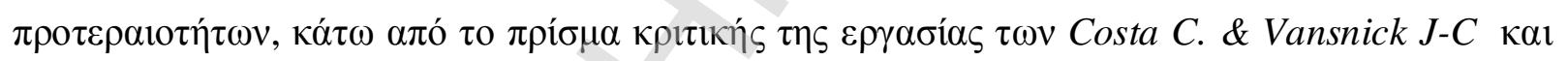
$\pi \alpha \rho \alpha \tau \eta \rho \dot{\theta \eta} \kappa \alpha \nu \tau \alpha \varepsilon \xi \dot{\eta} \varsigma$ :

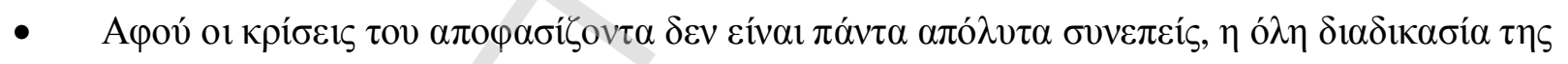

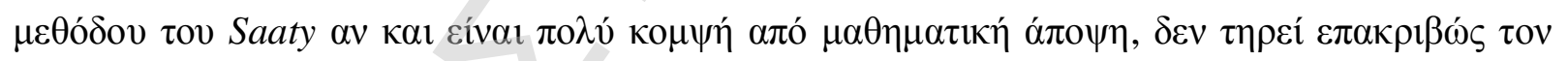

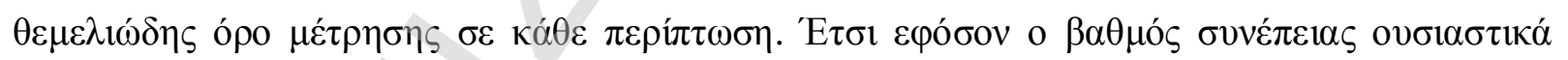

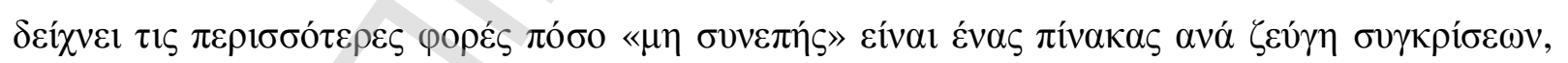

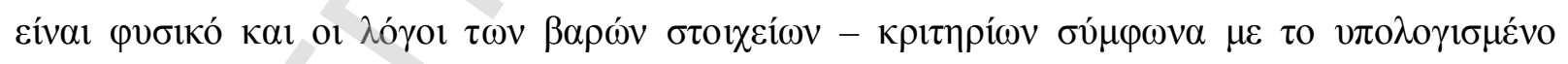

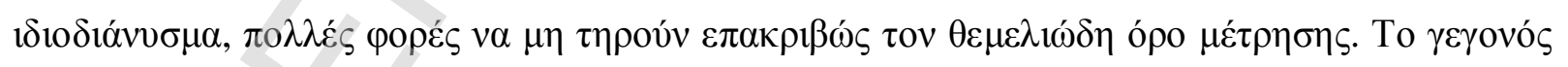

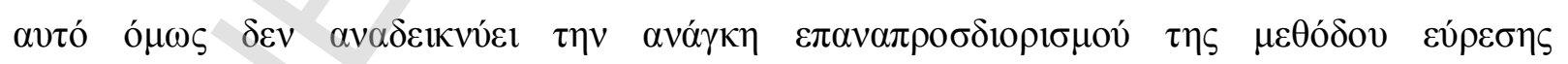

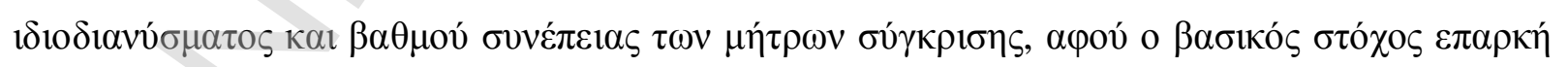

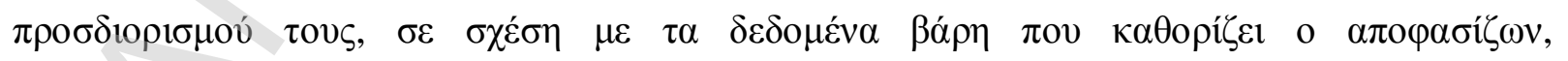

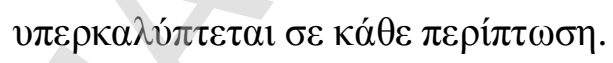

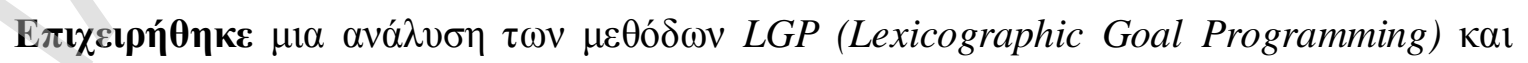

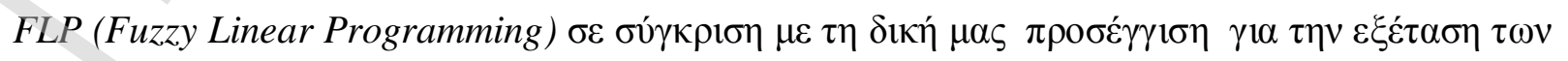

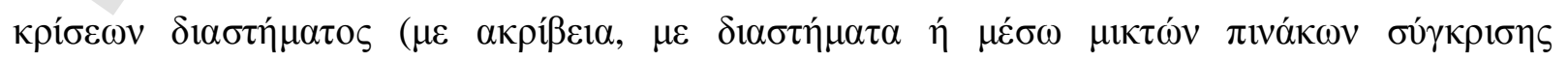




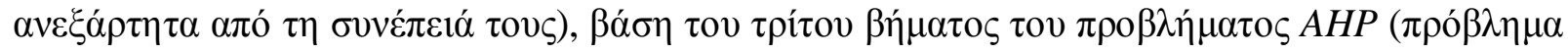

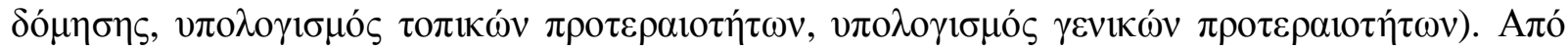
$\alpha v \tau \eta ́ v \tau \eta \nu \alpha v \alpha ́ \lambda v \sigma \eta \pi \alpha \rho \alpha \tau \eta \rho \dot{\theta} \theta \eta \kappa \alpha \nu \tau \alpha \varepsilon \xi \dot{\eta} \varsigma$ :

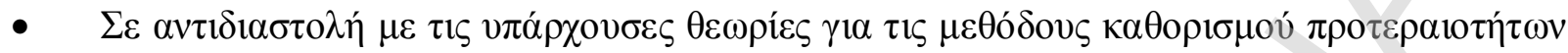

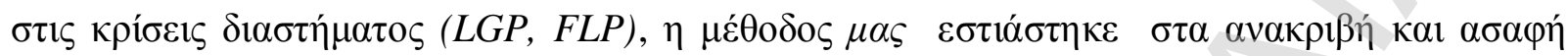

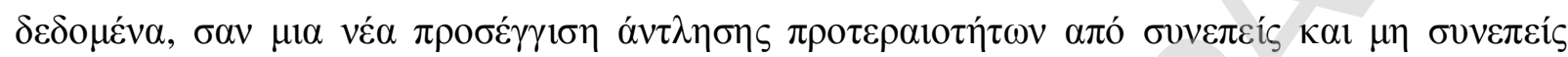

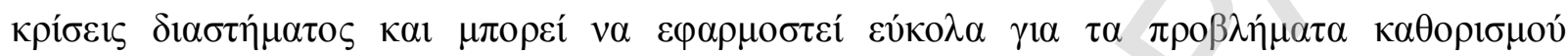

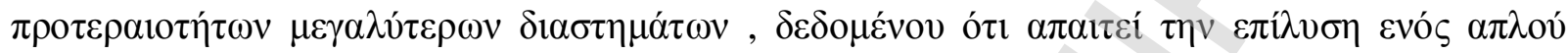

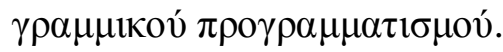

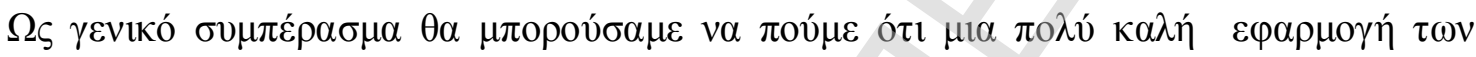

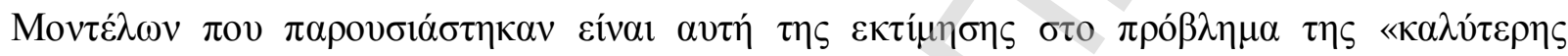

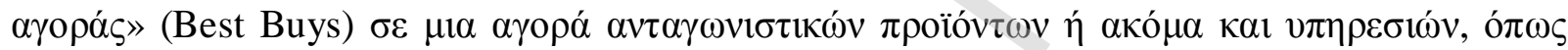

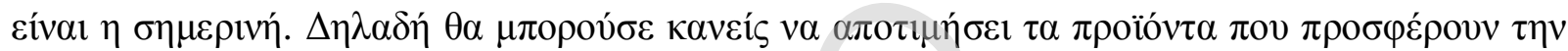

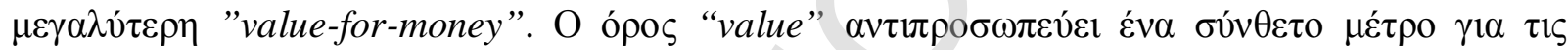

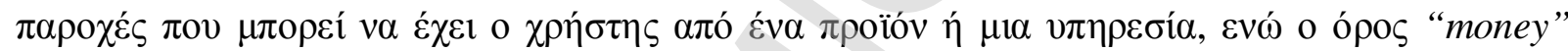

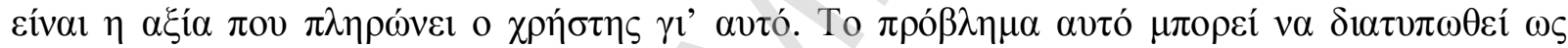

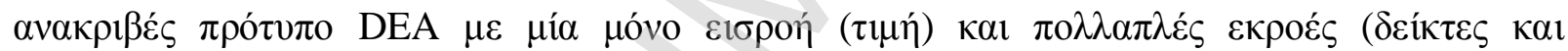

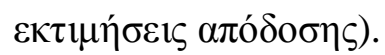

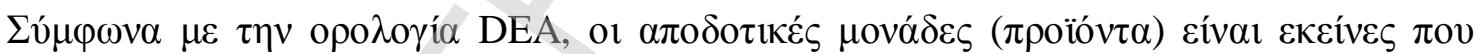

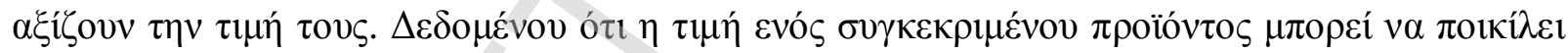

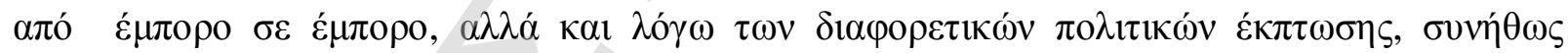

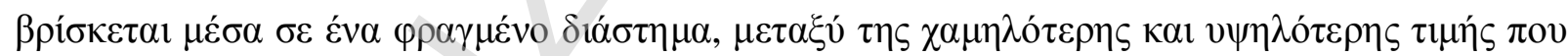

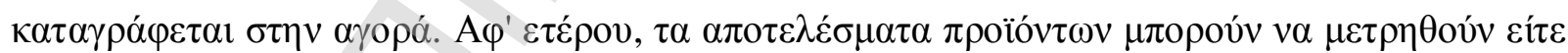

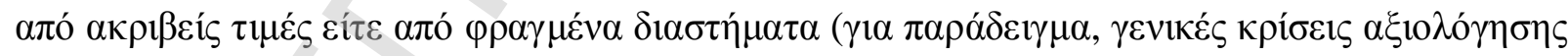

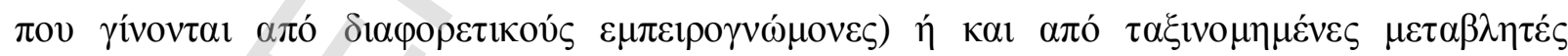

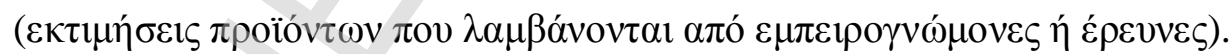

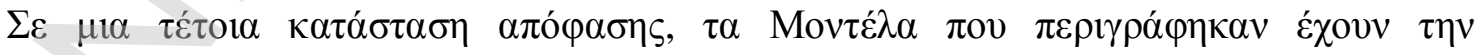

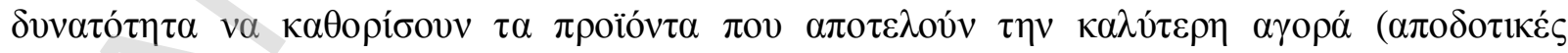

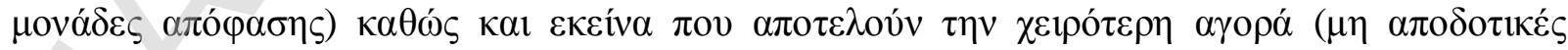

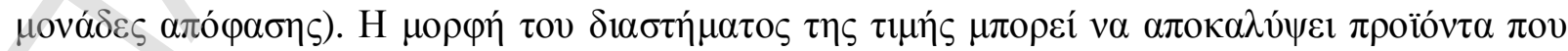

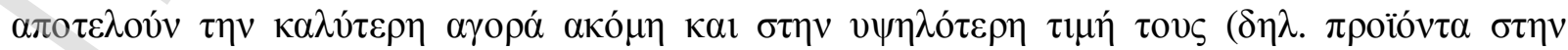
$\left.\kappa \alpha \tau \eta \gamma o \rho i ́ \alpha \mathrm{E}^{++}\right)$. 


\section{ПАРАPTHMATA}

\section{ПAPAPTHMA A'}

\section{1. ПЕРІИНЧН}

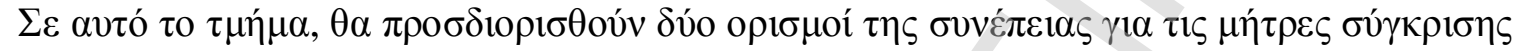

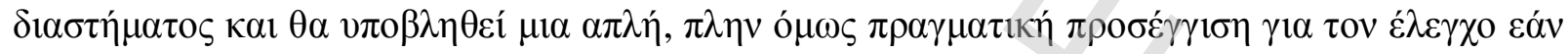

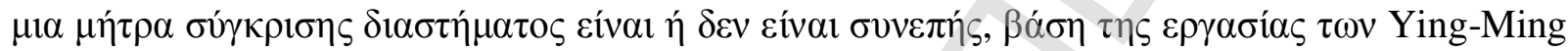
Wang, Jian-Bo Yang \& Dong-Ling Xu (Manchester School of Management), $\chi \omega \rho i ́ \varsigma ~ v \alpha \pi \rho \varepsilon ́ \pi \varepsilon ı ~ v \alpha$

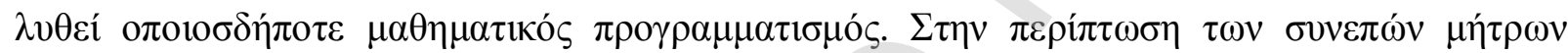

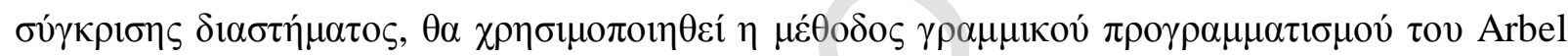

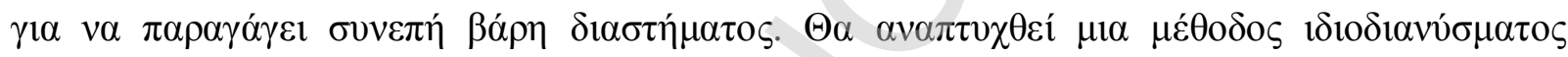

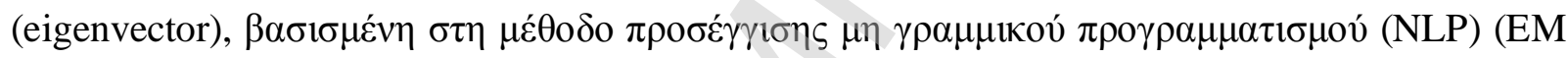

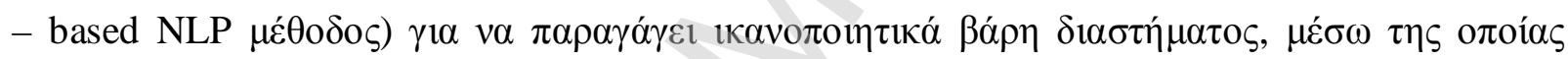

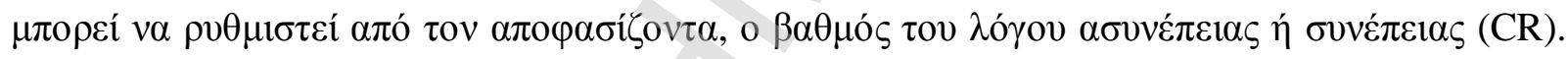

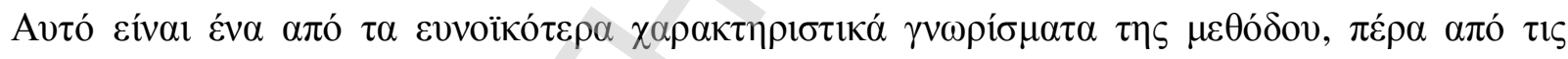

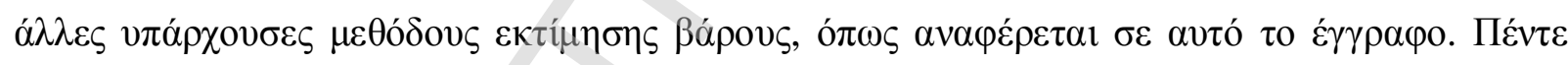

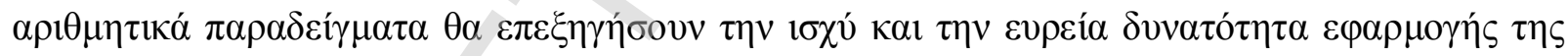

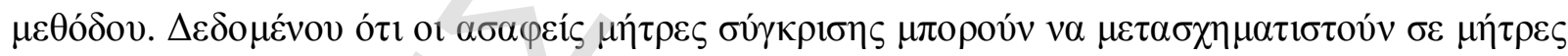

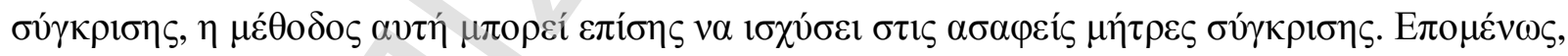

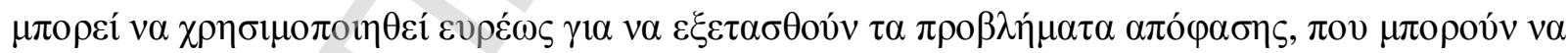

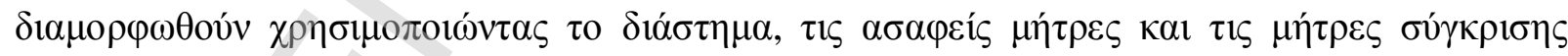

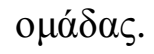




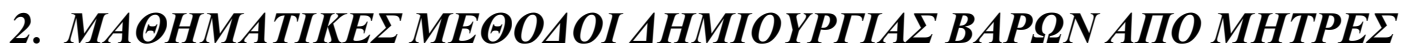

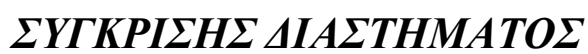

\section{$2.1 E \imath \sigma \alpha \gamma \omega \gamma \eta ́$}

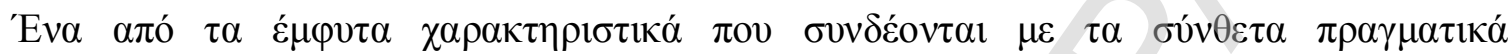

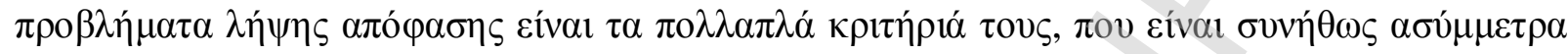

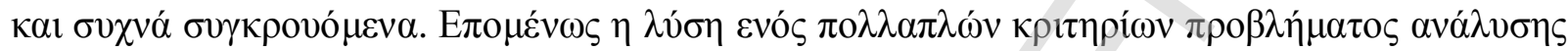

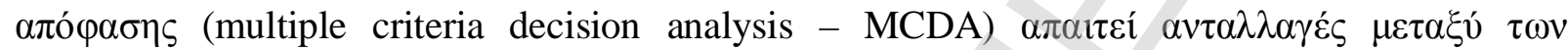

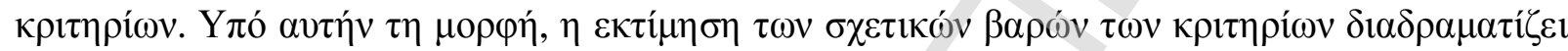

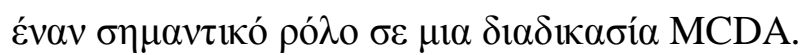

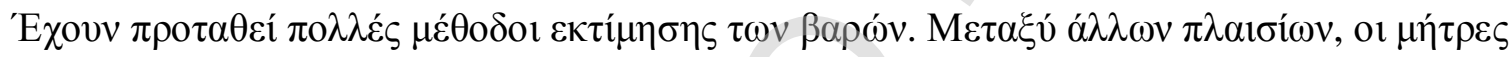

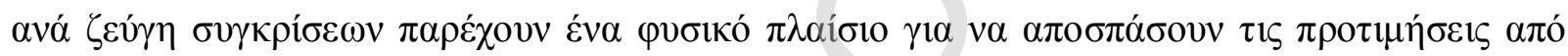

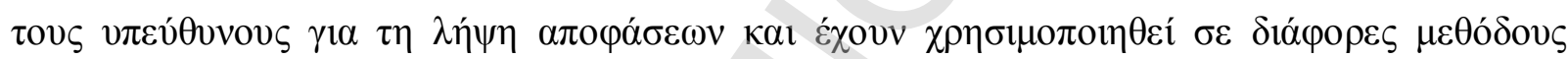

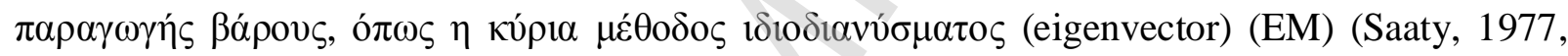

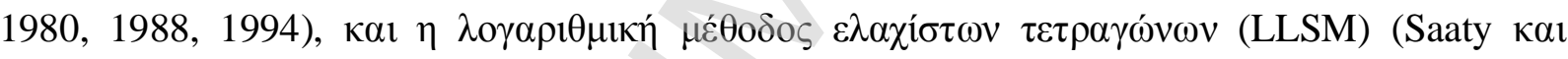

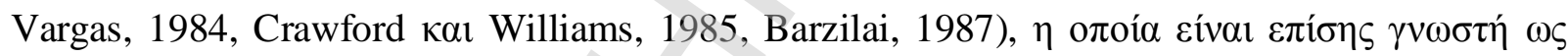

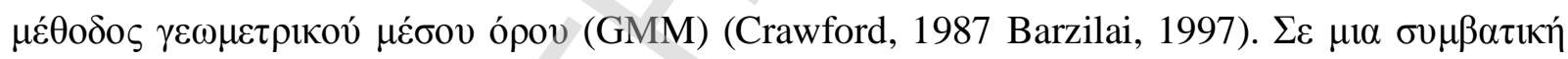

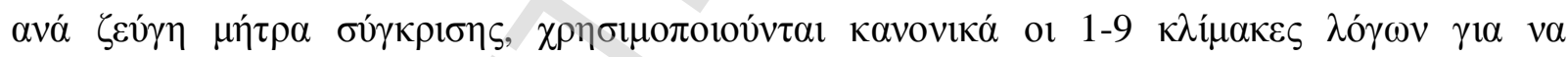

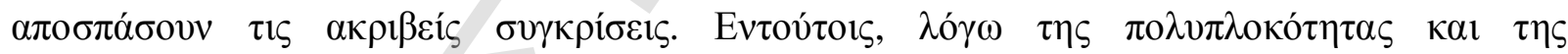

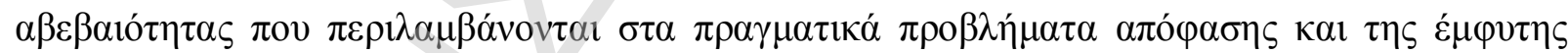

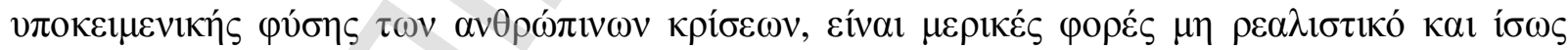

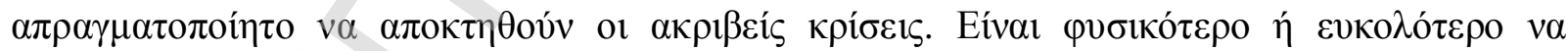

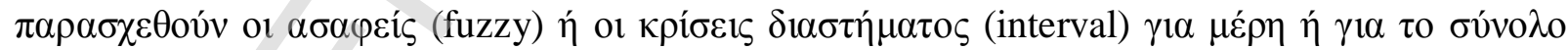

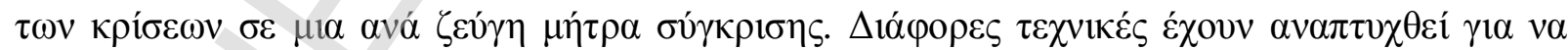

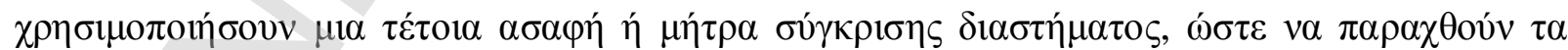

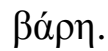

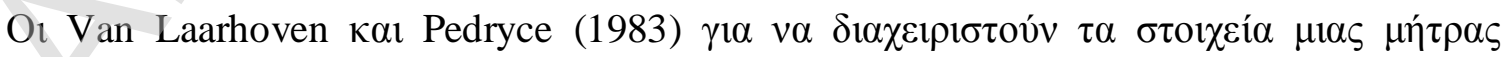

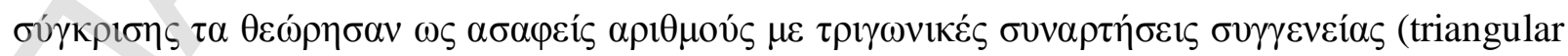

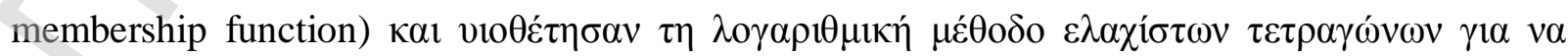

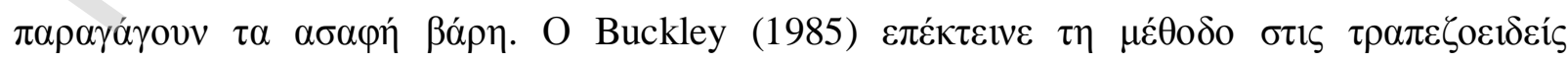

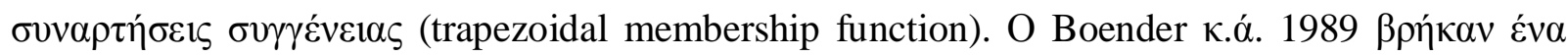




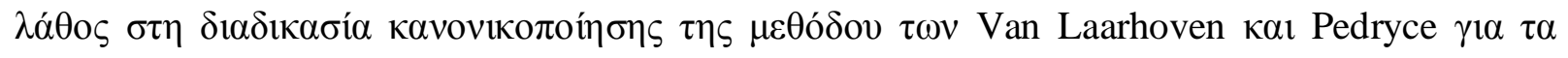

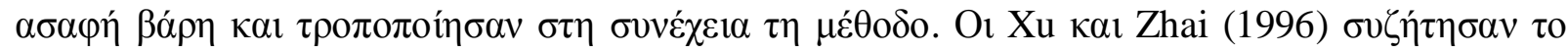

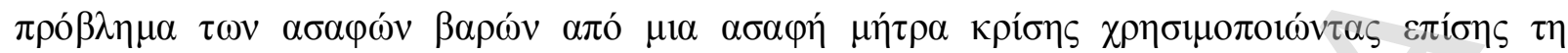

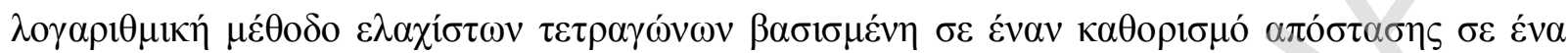

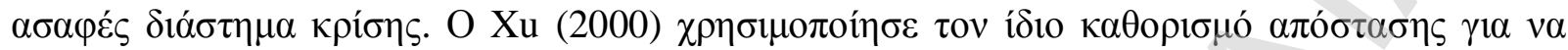

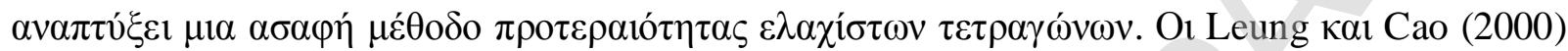

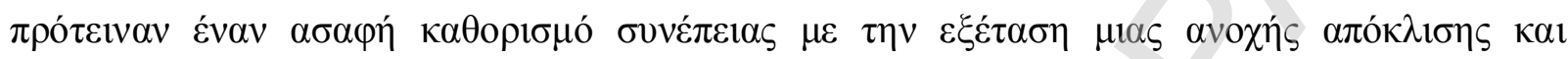

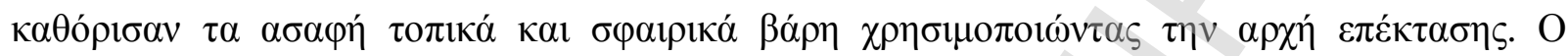

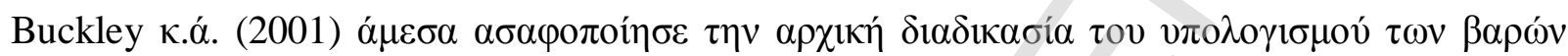

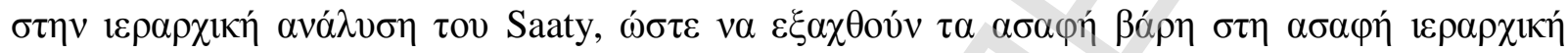

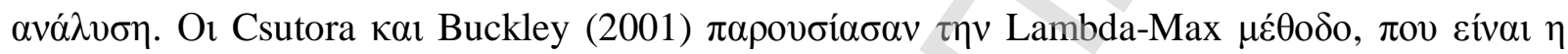

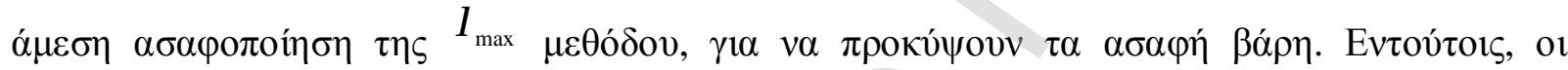

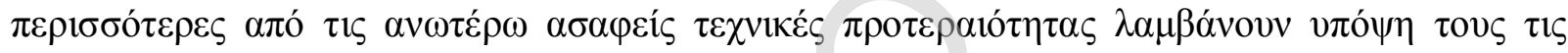

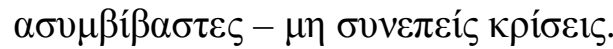

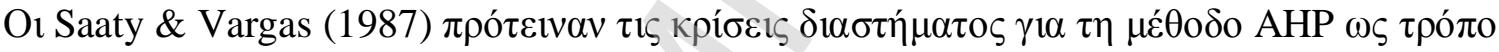

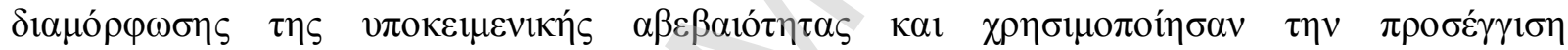

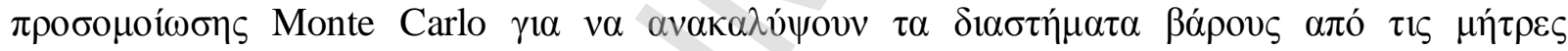

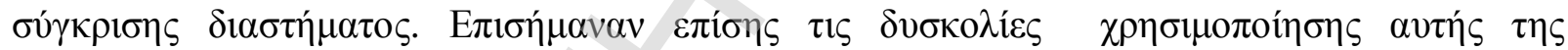

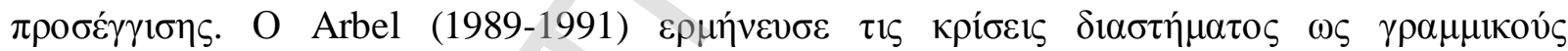

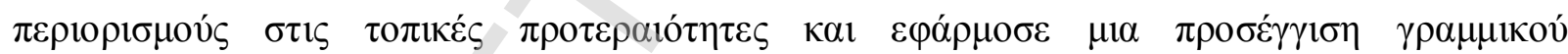

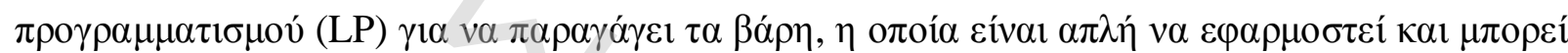

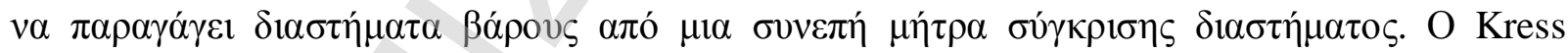

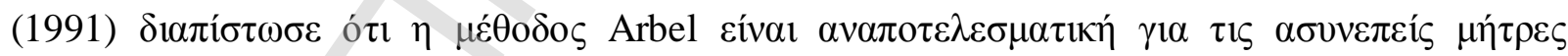

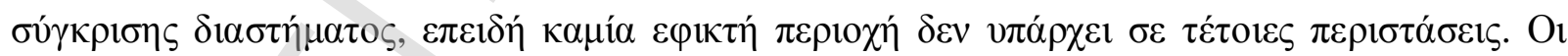

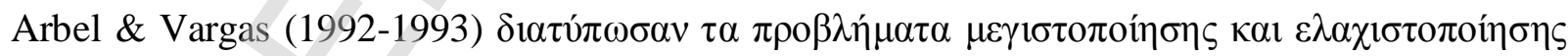

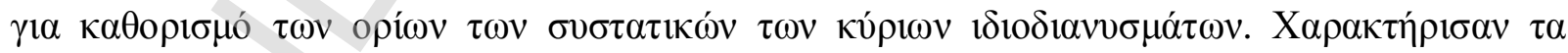

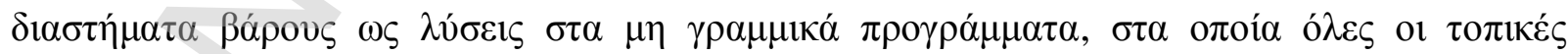

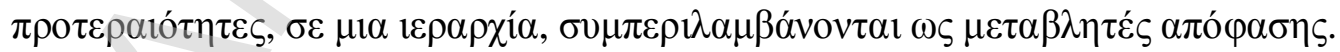

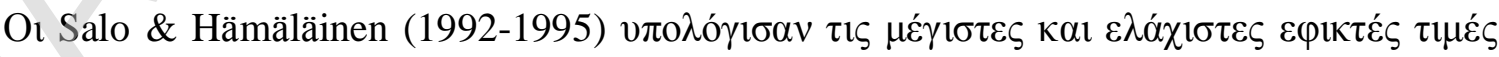

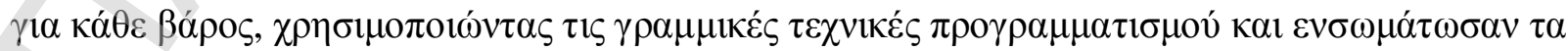

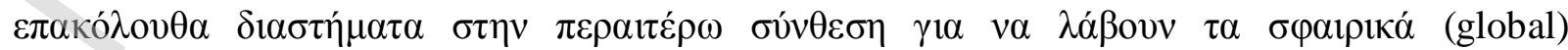

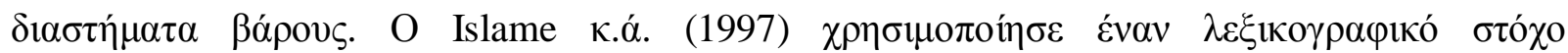




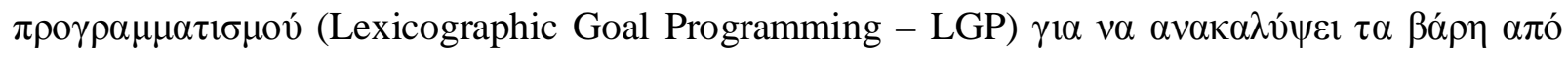

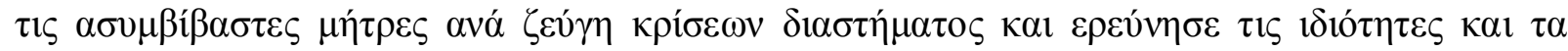

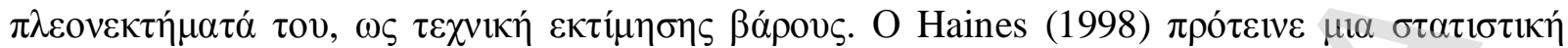

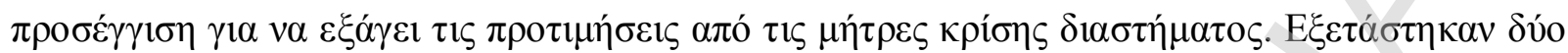

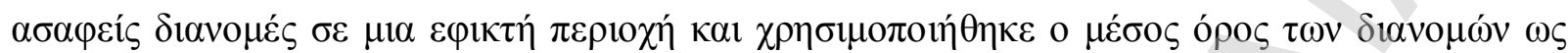

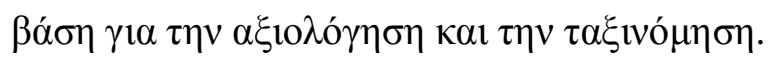

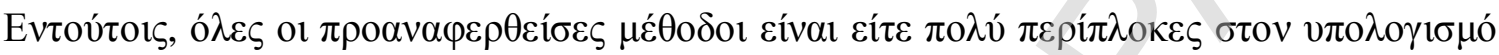

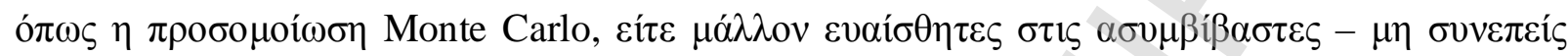

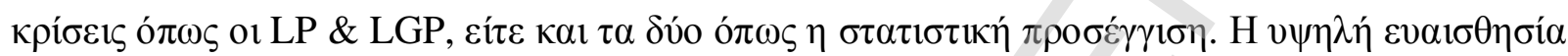

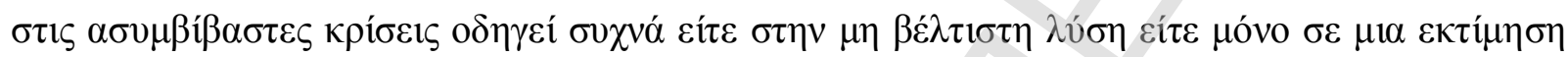

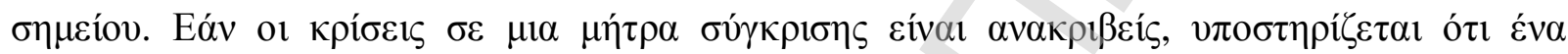

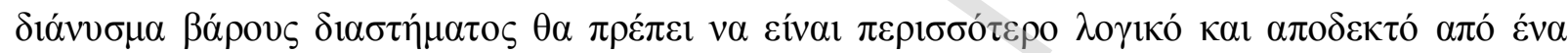

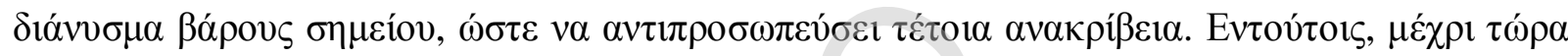

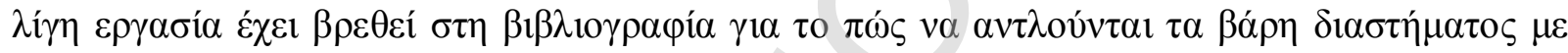

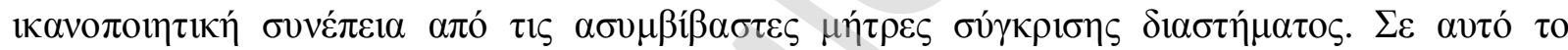

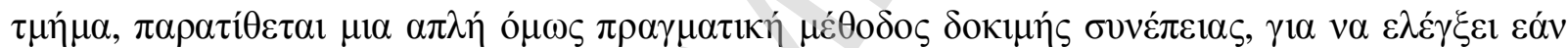

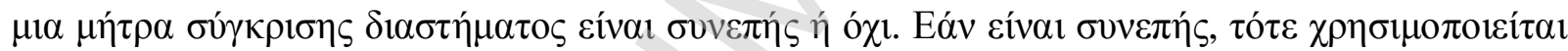

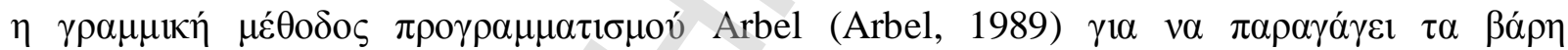

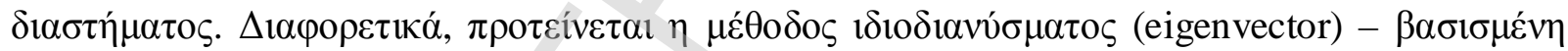

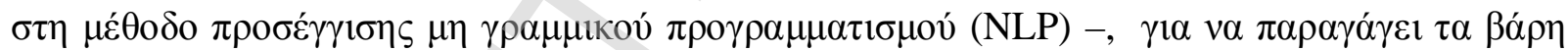

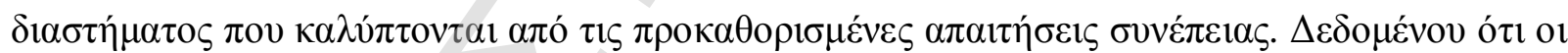

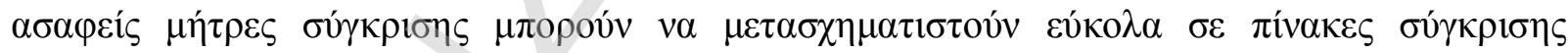

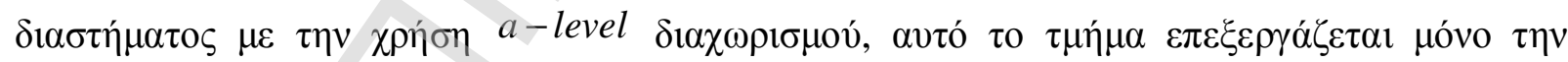

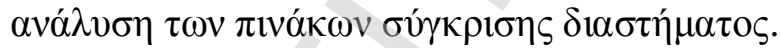

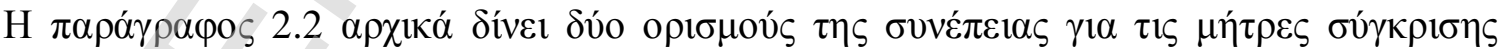

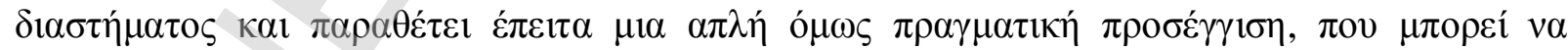

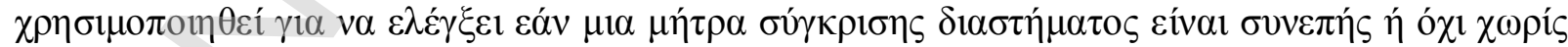

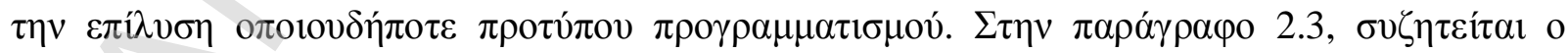

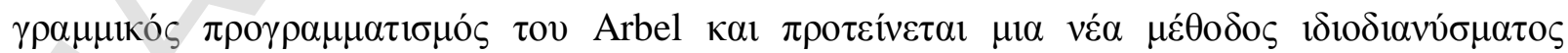

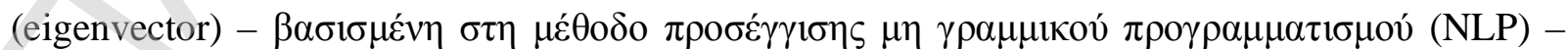

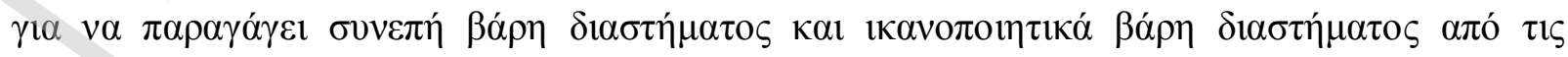

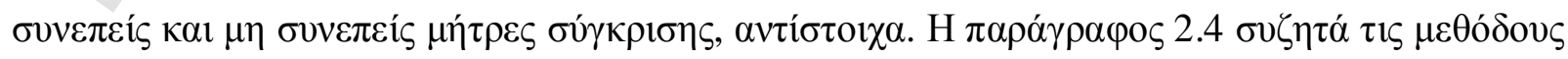




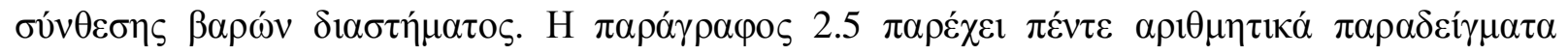

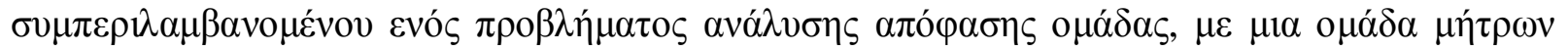

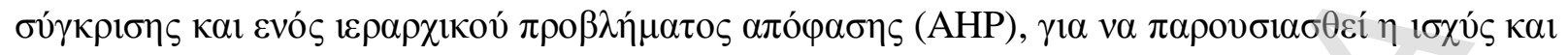

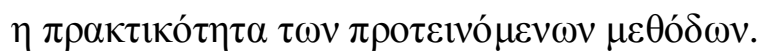

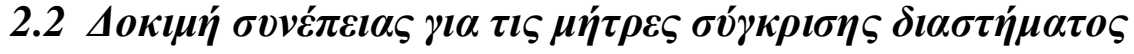

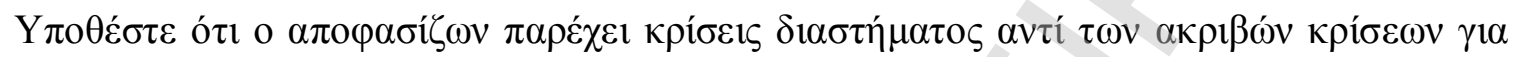

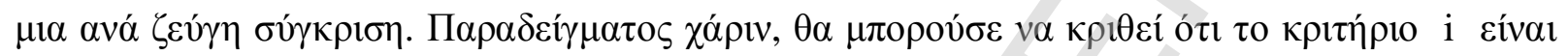

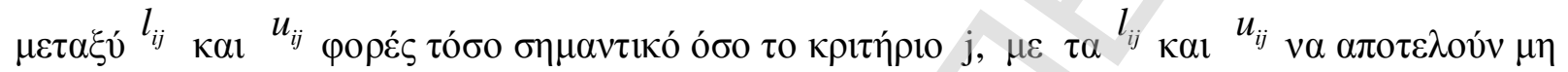

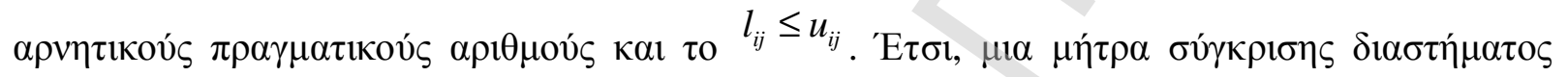

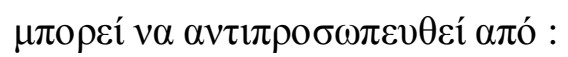

$$
A=\left[\begin{array}{cccc}
1 & {\left[l_{12}, u_{12}\right]} & \mathrm{L} & {\left[l_{1 n}, u_{1 n}\right]} \\
{\left[l_{21}, u_{21}\right]} & 1 & \mathrm{~L} & {\left[l_{2 n}, u_{2 n}\right]} \\
\mathrm{M} & \mathrm{M} & \mathrm{M} & \mathrm{M} \\
{\left[l_{n 1}, u_{n 1}\right]} & {\left[l_{n 2}, u_{n 2}\right]} & \mathrm{L} & 1
\end{array}\right]
$$

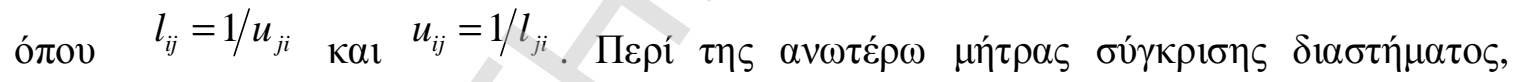

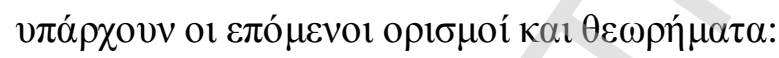

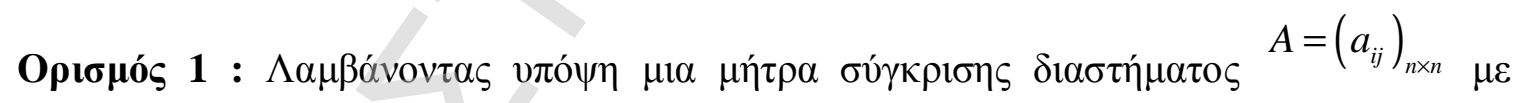

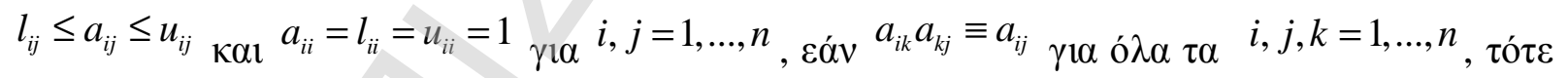

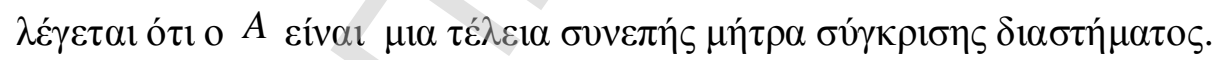

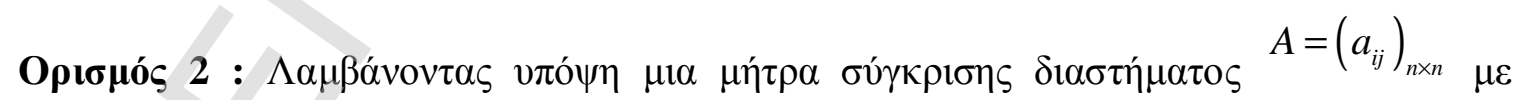

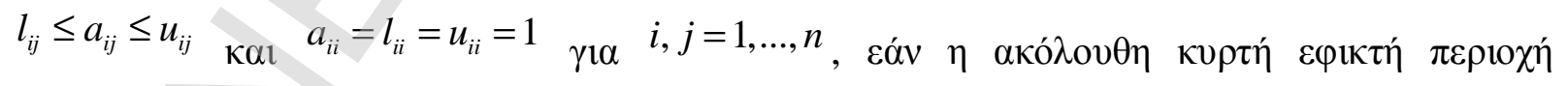
$S_{w}=\left\{w=\left(w_{1}, \ldots, w_{n}\right) \mid l_{i j} \leq w_{i} / w_{j} \leq u_{i j}, \sum_{i=1}^{n} w_{i}=1, w_{i} \geq 0\right\}$

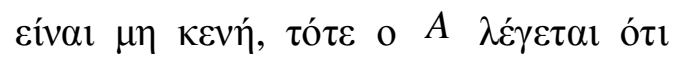

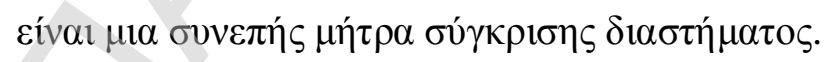

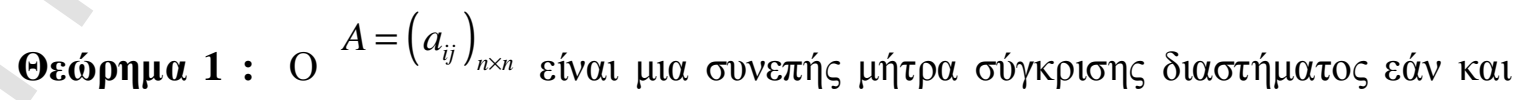

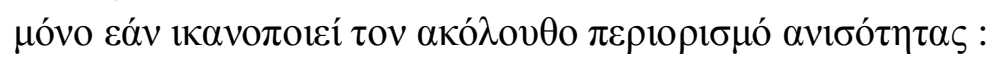




$$
\max _{k}\left(l_{i k} l_{k j}\right) \leq \min _{k}\left(u_{i k} u_{k j}\right), \gamma 1 \alpha \kappa \alpha \dot{\theta} \theta \varepsilon{ }^{i, j, k=1, \ldots, n}
$$

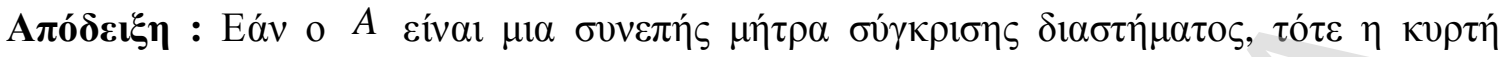

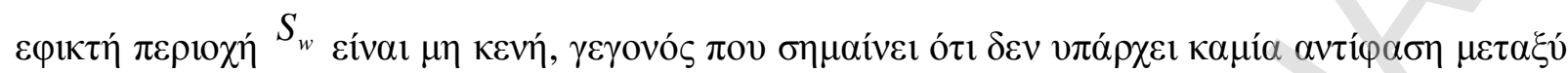

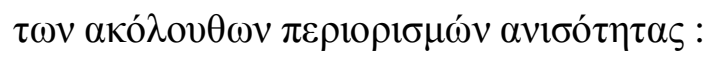

$$
\begin{aligned}
& l_{i k} \leq w_{i} / w_{k} \leq u_{i k}, \quad i, k=1, \ldots, n \\
& l_{k j} \leq w_{k} / w_{j} \leq u_{k j}, \quad k, j=1, \ldots, n \\
& l_{i j} \leq w_{i} / w_{j} \leq u_{i j}, \quad i, j=1, \ldots, n
\end{aligned}
$$

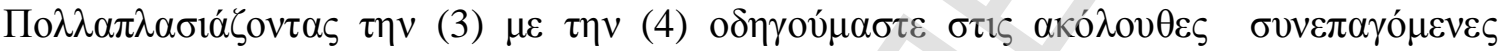
$\varepsilon \dot{\varepsilon} \mu \mu \varepsilon \sigma \varepsilon \varsigma \alpha \nu 1 \sigma o ́ \tau \eta \tau \varepsilon \varsigma:$

$$
l_{i k} l_{k j} \leq w_{i} / w_{j} \leq u_{i k} u_{k j}, \quad i, j, k=1, \ldots, n
$$

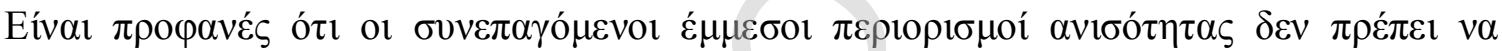

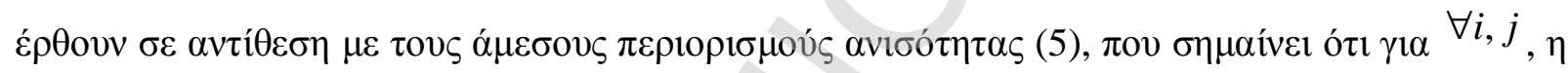
(2) $1 \sigma \chi v ́ \varepsilon 1 \pi \alpha ́ v \tau \alpha$.

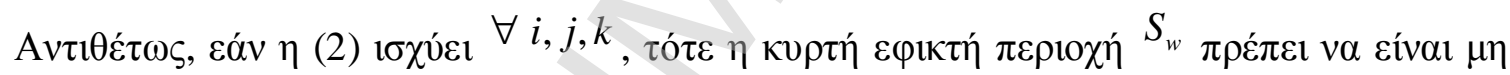

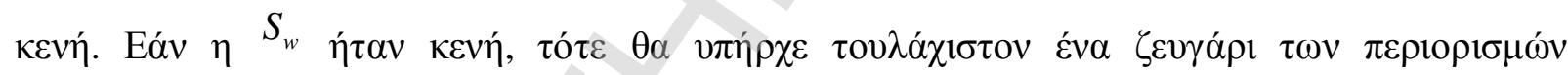

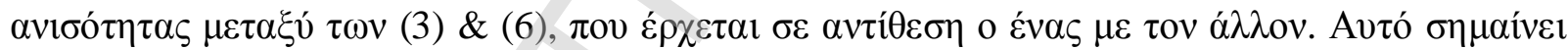

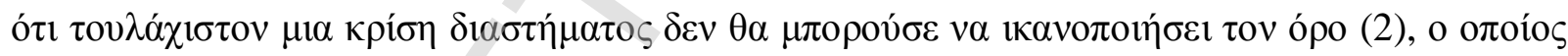

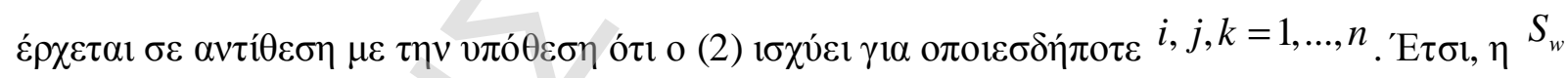

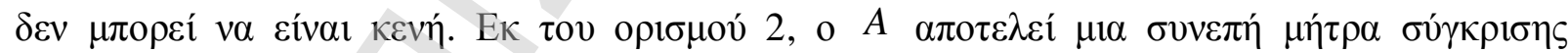
$\delta 1 \alpha \sigma \tau \eta ́ \mu \alpha \tau o \varsigma$.

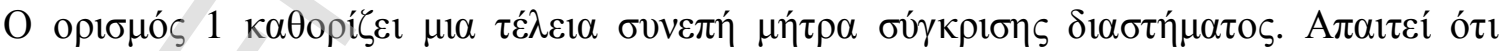

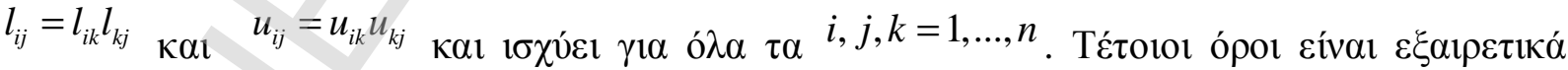

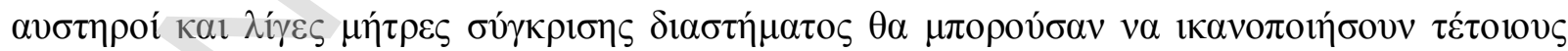

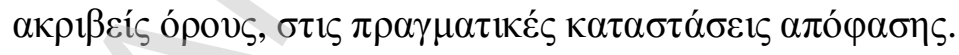

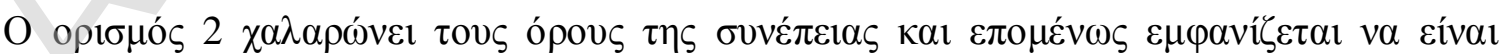

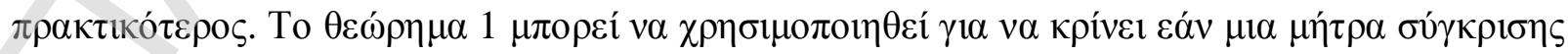

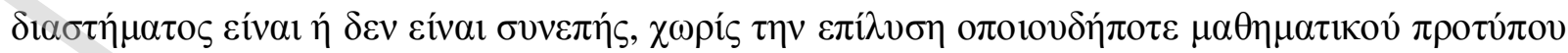

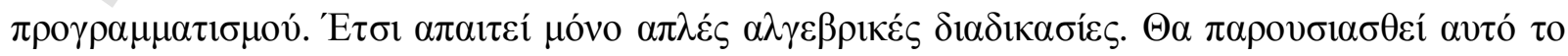




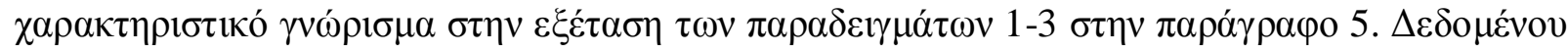

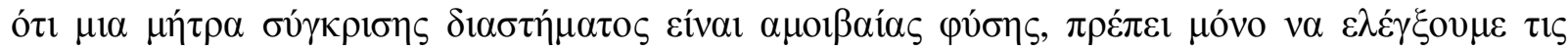

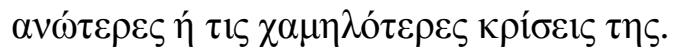

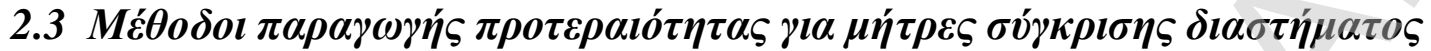

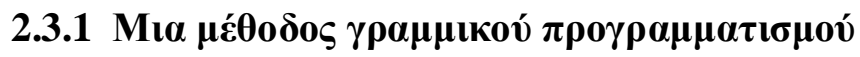

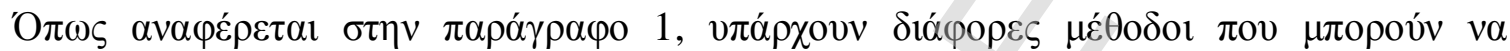

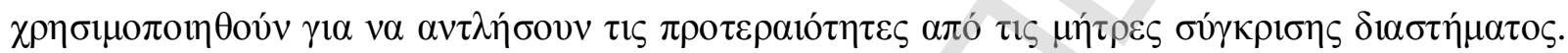

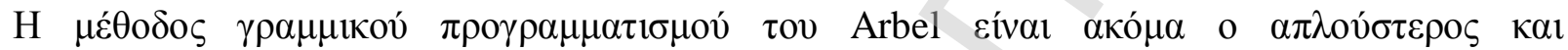

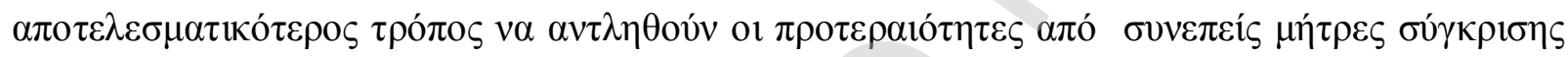

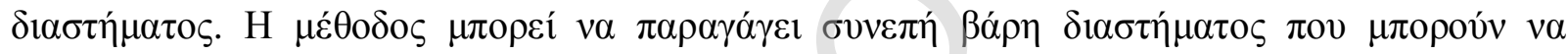

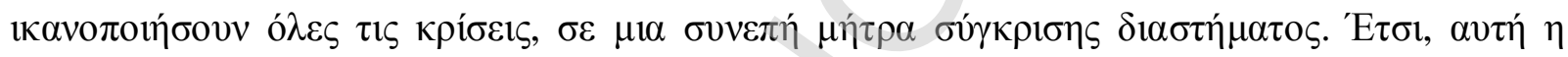

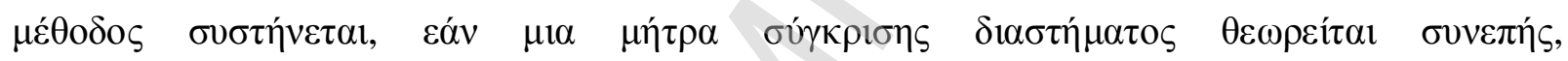

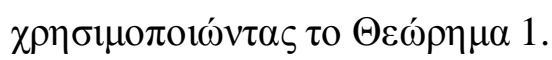

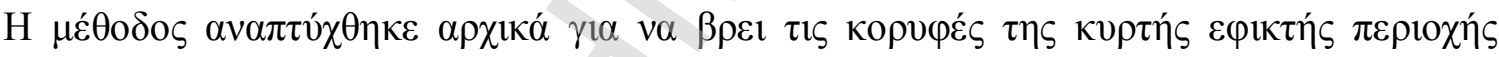

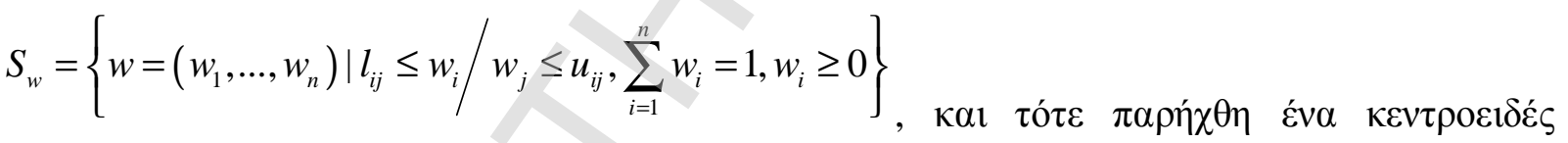

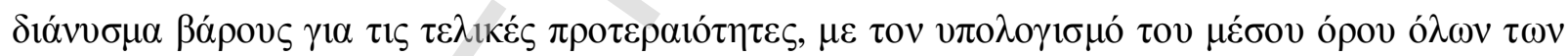

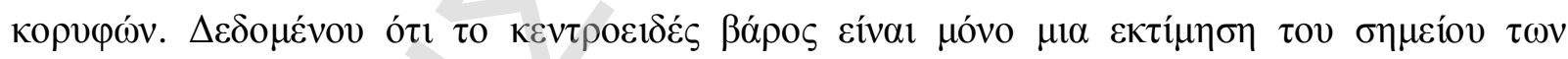

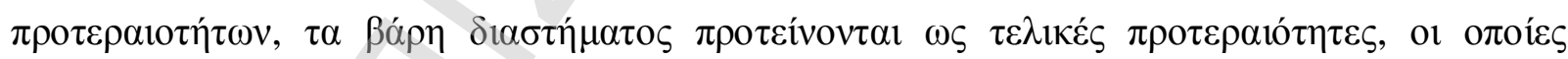

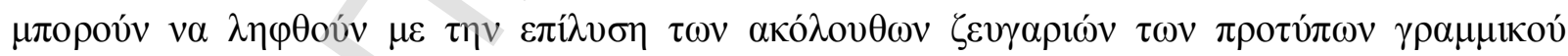
$\pi \rho о \gamma \rho \alpha \mu \mu \tau \tau \sigma \mu о \cup ́(\mathrm{LP})$ :

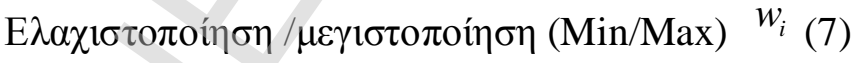

$$
\text { Yлó tov ópo } W \in S_{w}
$$

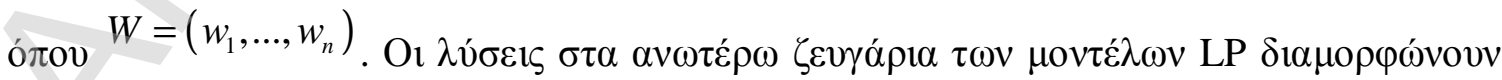

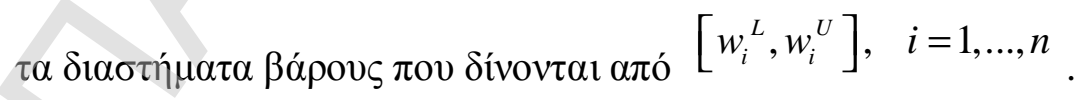




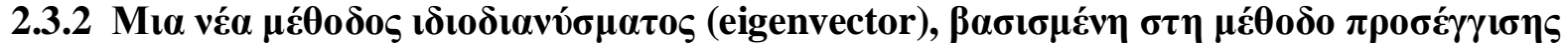

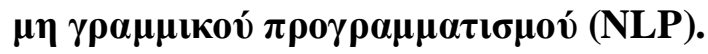

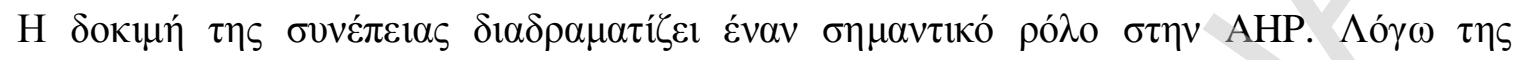

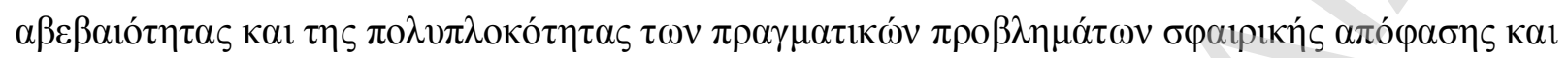

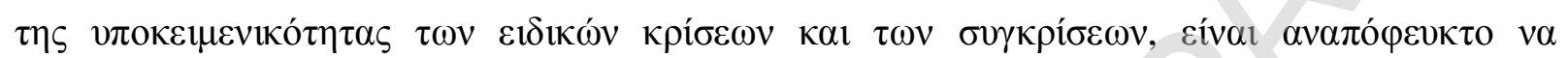

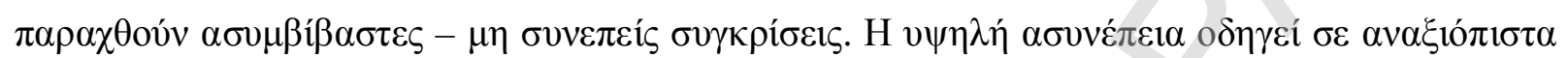

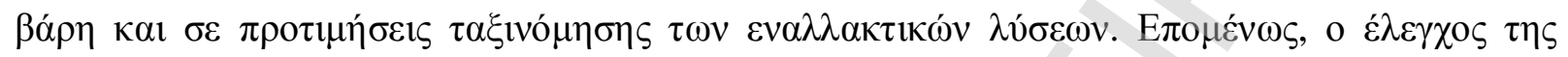

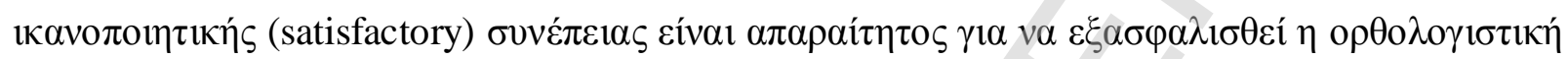

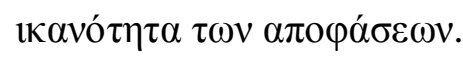

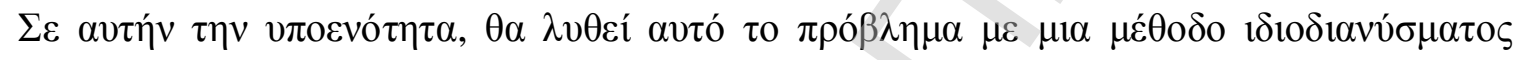

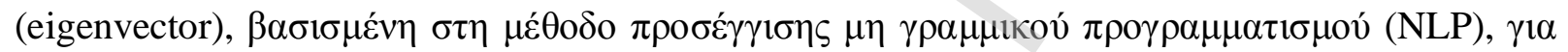

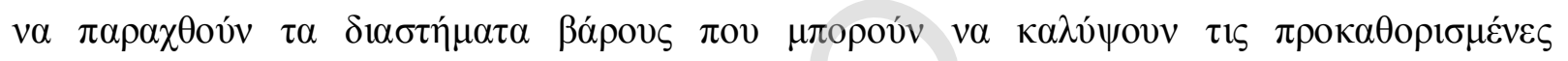

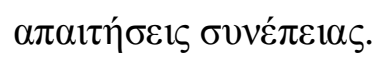

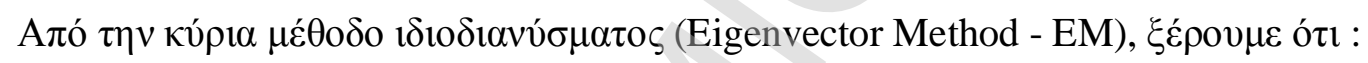

$$
\hat{A} W=\lambda_{\max } W
$$

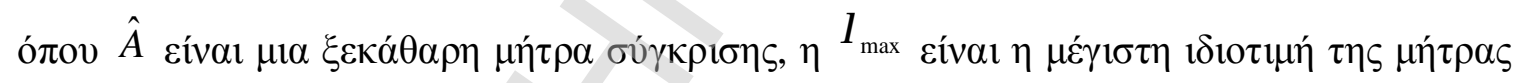

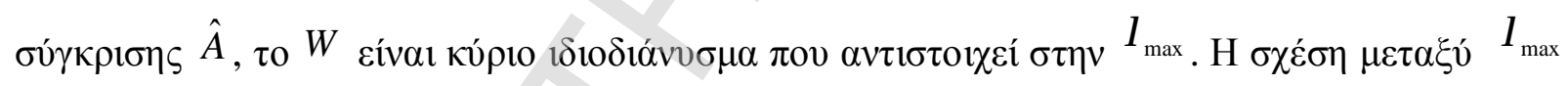

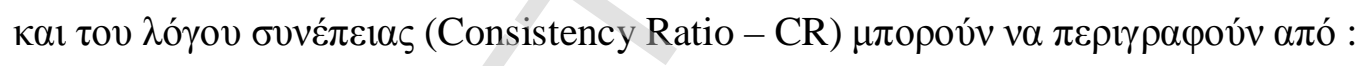

$$
C R=\frac{C I}{R I}=\frac{\lambda_{\max }-n}{n-1} / R I=\frac{\lambda_{\max }-n}{(n-1) R I}
$$

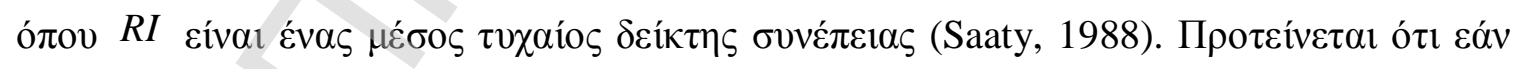

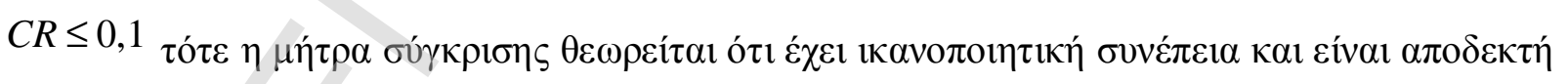

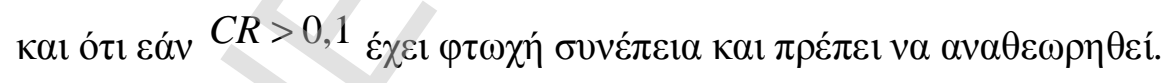

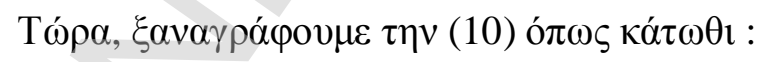

$$
\lambda_{\text {max }}=n+(n-1) R I \cdot C R
$$

H $\alpha \nu \tau \iota \kappa \alpha \tau \alpha ́ \sigma \tau \alpha \sigma \eta \tau \eta \varsigma(11) \sigma \varepsilon \tau \eta \nu(9) \pi \alpha \rho \alpha ́ \gamma \varepsilon \iota:$

$$
\hat{A} W=[n+(n-1) R I \cdot C R] W
$$

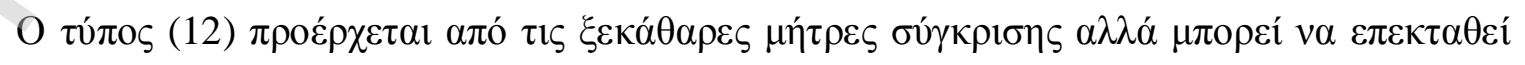

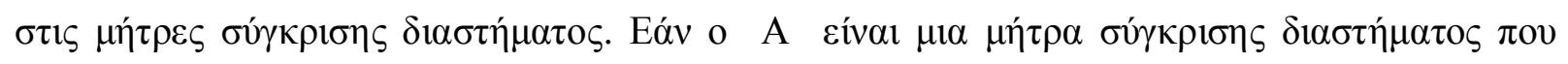




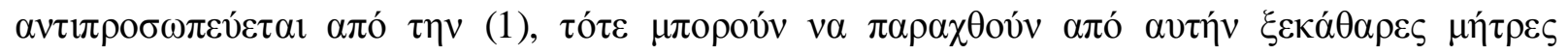

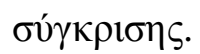

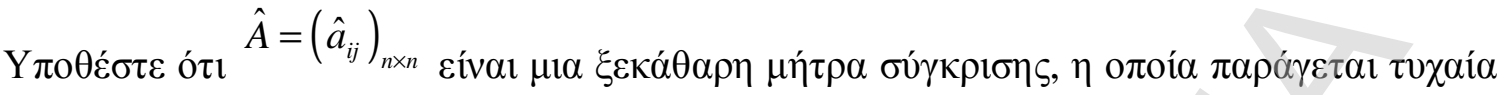

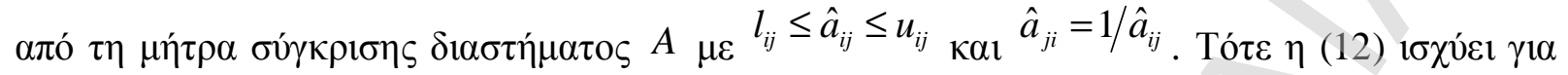

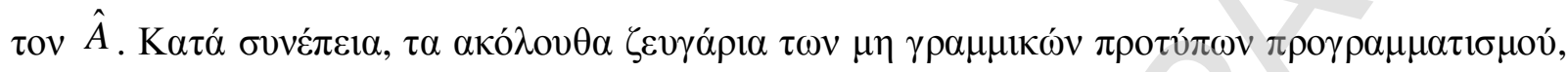

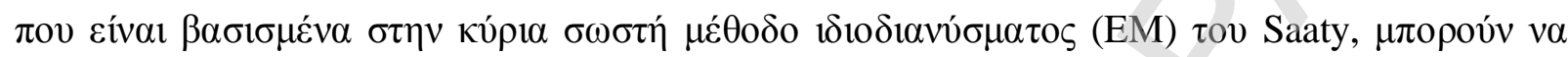

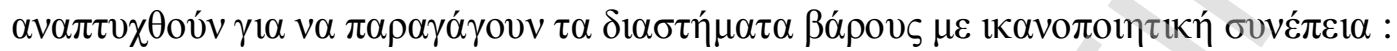

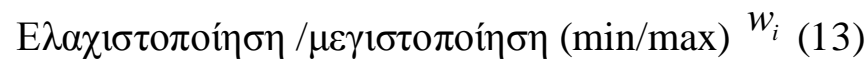

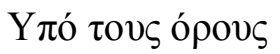

$$
\begin{aligned}
& \sum_{j=1}^{i-1} \frac{w_{j}}{\hat{a}_{i j}}-(n-1)(1+R I \cdot C R) w_{i}+\sum_{j=i+1}^{n} \hat{a}_{i j} w_{i}=0, \quad i=1, \ldots, n \\
& \sum_{i=1}^{n} w_{i}=1 \\
& l_{i j} \leq \hat{a}_{i j} \leq u_{i j}, \quad i=1, \ldots, n, \quad j=i+1, \ldots, n \\
& C R \leq 0,1
\end{aligned}
$$

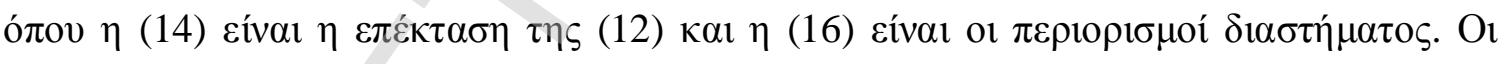

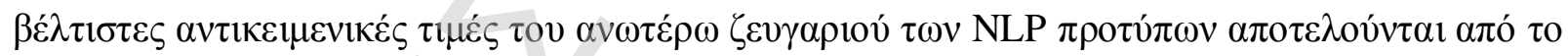

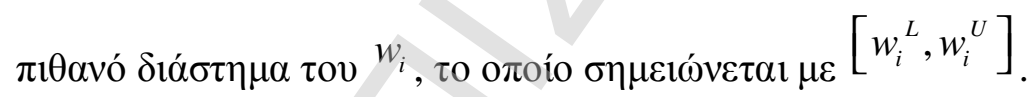

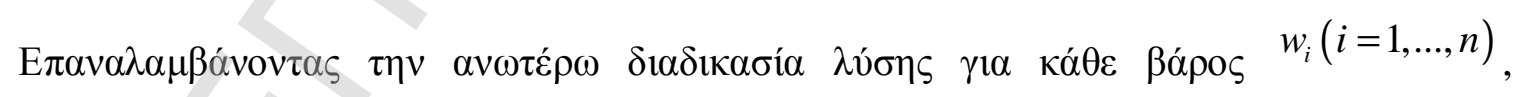

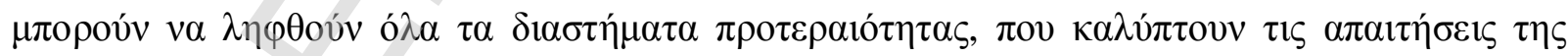

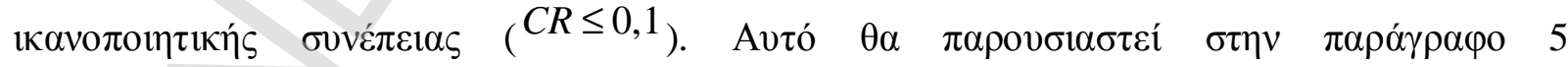

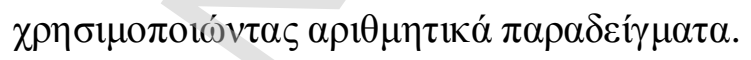

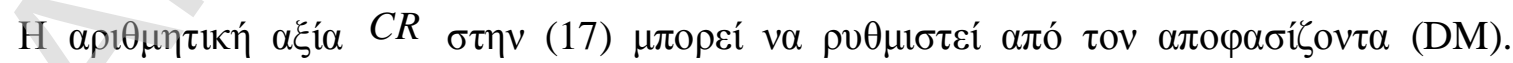

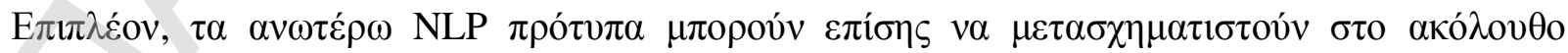

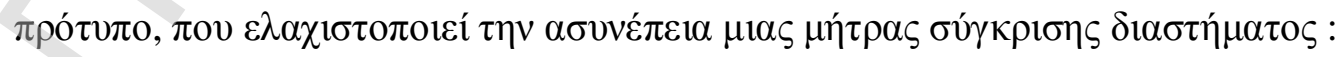

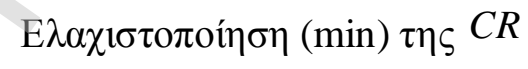

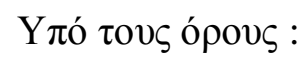




$$
\begin{aligned}
& \sum_{j=1}^{i-1} \frac{w_{j}}{\hat{a}_{i j}}-(n-1)(1+R I \cdot C R) w_{i}+\sum_{j=i+1}^{n} \hat{a}_{i j} w_{i}=0, \quad i=1, \ldots, n \\
& \sum_{i=1}^{n} w_{i}=1 \\
& l_{i j} \leq \hat{a}_{i j} \leq u_{i j}, \quad i=1, \ldots, n, \quad j=i+1, \ldots, n
\end{aligned}
$$

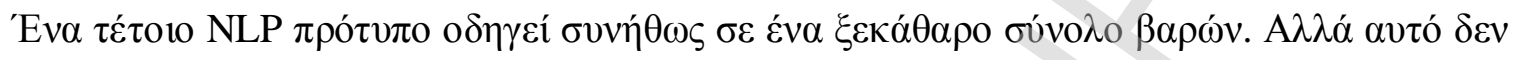

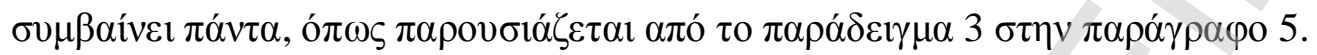

\section{$2.4 \Sigma v ́ v \theta \varepsilon \sigma \eta \tau \omega v \beta \alpha \rho \omega ́ v \delta ı \alpha \sigma \tau \eta ́ \mu \alpha \tau o \varsigma$}

\begin{tabular}{|c|c|c|c|c|c|}
\hline 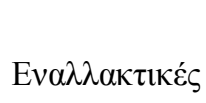 & 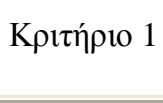 & Kpıtŕ́pıo 2 & $\ldots$ & Крıт́́рı $m$ & \multirow{2}{*}{ 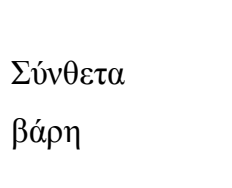 } \\
\hline$\lambda v ́ \sigma \varepsilon 1 \varsigma$ & & {$\left[w_{2}{ }^{L}, w_{2}^{U}\right]$} & $\ldots$ & {$\left[w_{m}{ }^{L}, w_{m}{ }^{U}\right]$} & \\
\hline$A_{1}$ & {$\left[w_{11}{ }^{L}, w_{11}\right.$} & {$\left[w_{12}{ }^{L}, w_{12}{ }^{U}\right.$} & $\cdots$ & {$\left[w_{1 m}{ }^{L}, w_{1 m}{ }^{U}\right.$} & {$\left[w_{A_{1}}^{L}, w_{A_{1}}^{U}\right]$} \\
\hline$A_{2}$ & {$\left[w_{21}{ }^{L}, w_{21}\right.$} & {$\left[w_{22}{ }^{L}, w_{22}{ }^{L}\right.$} & $\cdots$ & {$\left[w_{2 m}{ }^{L}, w_{2 m}{ }^{U}\right.$} & {$\left[w_{A_{2}}{ }^{L}, w_{A_{2}}^{U}\right]$} \\
\hline$N$ & M & $\Lambda$ & $\ldots$ & $\Lambda$ & $\vdots$ \\
\hline & {$\left[w_{n 1}{ }^{L}, w_{n}\right.$} & {$\left[w_{n 2}{ }^{L}, w_{n 2}{ }^{l}\right.$} & $\cdots$ & {$\left[w_{n m}{ }^{L}, w_{n m}{ }^{U}\right.$} & {$\left[w_{A_{K}}^{L}, w_{A_{K}}^{U}\right]$} \\
\hline
\end{tabular}

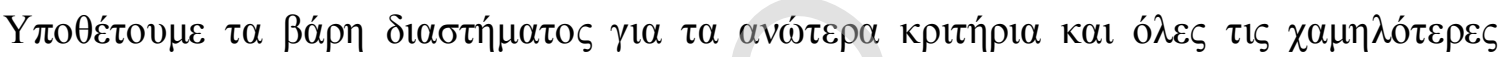

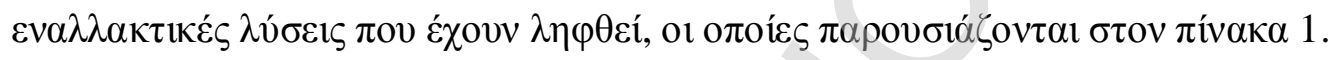

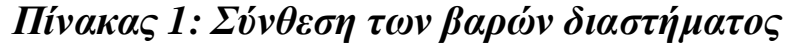

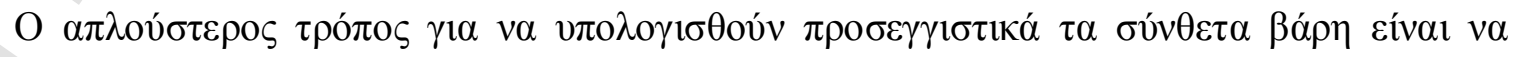

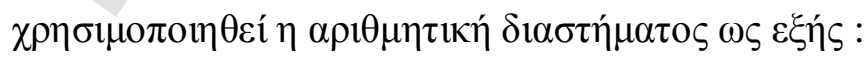




$$
\begin{aligned}
& w_{A_{1}}{ }^{L}=\sum_{j=1}^{m} w_{i j}^{L} w_{j}^{L} \quad i=1, \ldots, n \\
& w_{A_{1}}{ }^{U}=\sum_{j=1}^{m} w_{i j}^{U} w_{j}^{U} \quad i=1, \ldots, n
\end{aligned}
$$

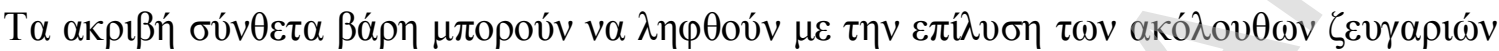
$\tau \omega v \pi \rho \circ \tau v \pi \omega v$ LP :

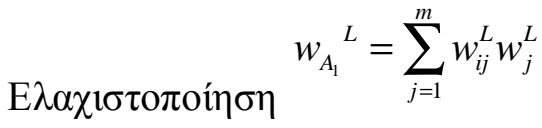

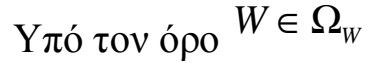

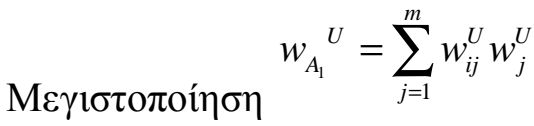

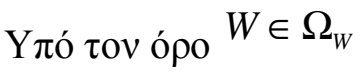

о́лоv $W=\left(w_{1}, \ldots, w_{m}\right)$ $\alpha \alpha 1$

$$
\Omega_{w}=\left\{w=\left(w_{1}, \ldots, w_{m}\right) \mid w_{j}{ }^{L} \leq w_{j} \leq w_{j}^{U}, \sum_{j=1}^{m} w_{j}=1, w_{j} \geq 0\right\}
$$

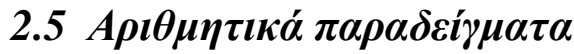

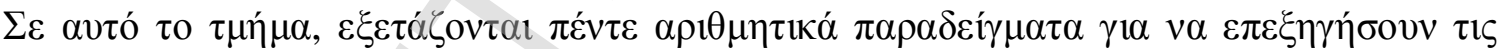

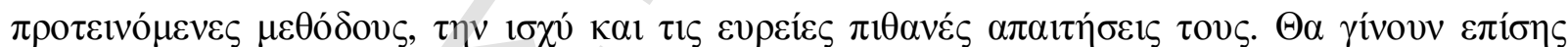

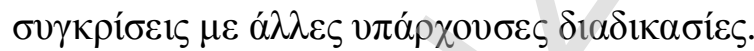

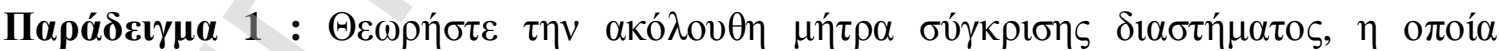

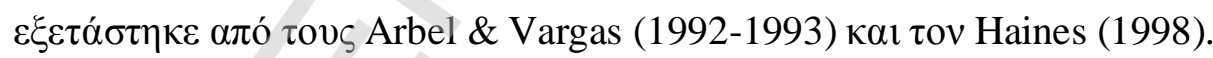

$$
A=\left[\begin{array}{cccc}
1 & {[2,5]} & {[2,4]} & {[1,3]} \\
{\left[\frac{1}{5}, \frac{1}{2}\right]} & 1 & {[1,3]} & {[1,2]} \\
{\left[\frac{1}{4}, \frac{1}{2}\right]} & {\left[\frac{1}{3}, 1\right]} & 1 & {\left[\frac{1}{2}, 1\right]} \\
{\left[\frac{1}{3}, 1\right]} & {\left[\frac{1}{2}, 1\right]} & {[1,2]} & 1
\end{array}\right]
$$




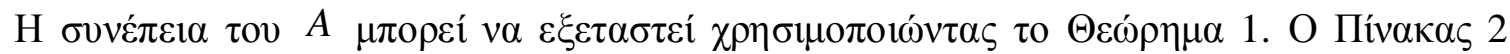

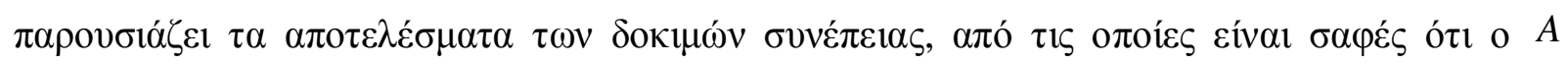

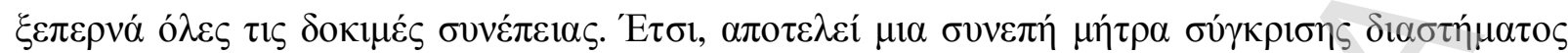

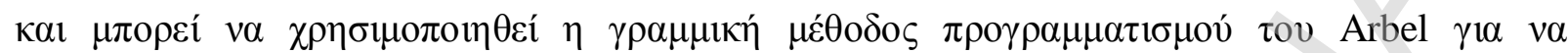

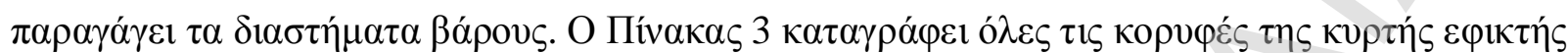

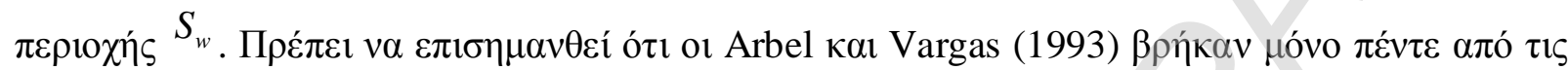

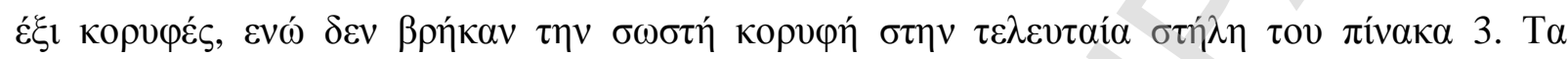

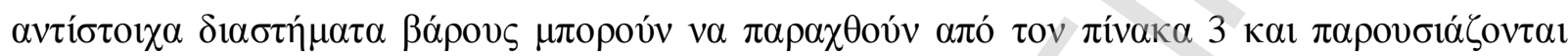

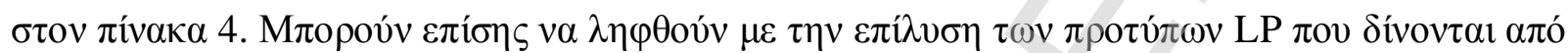

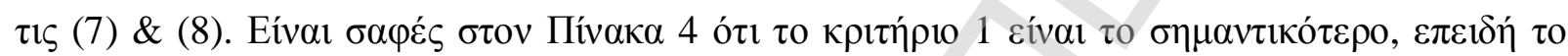

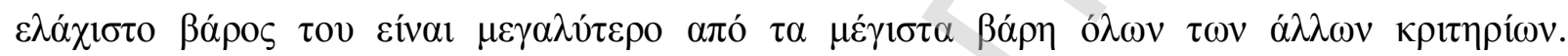

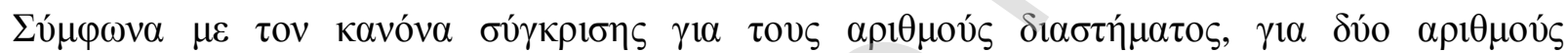

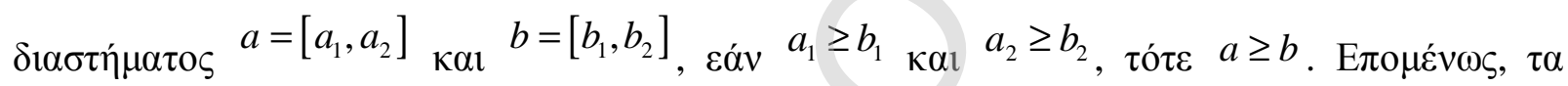

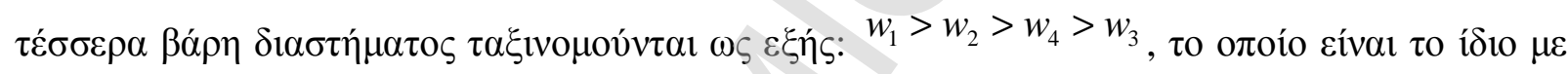

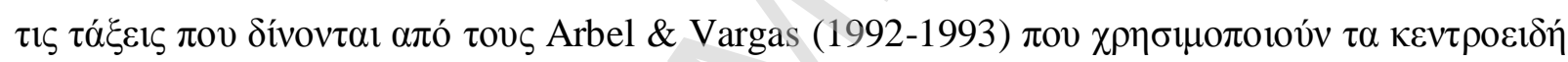

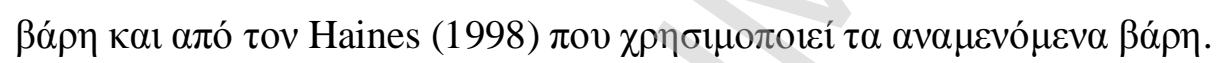

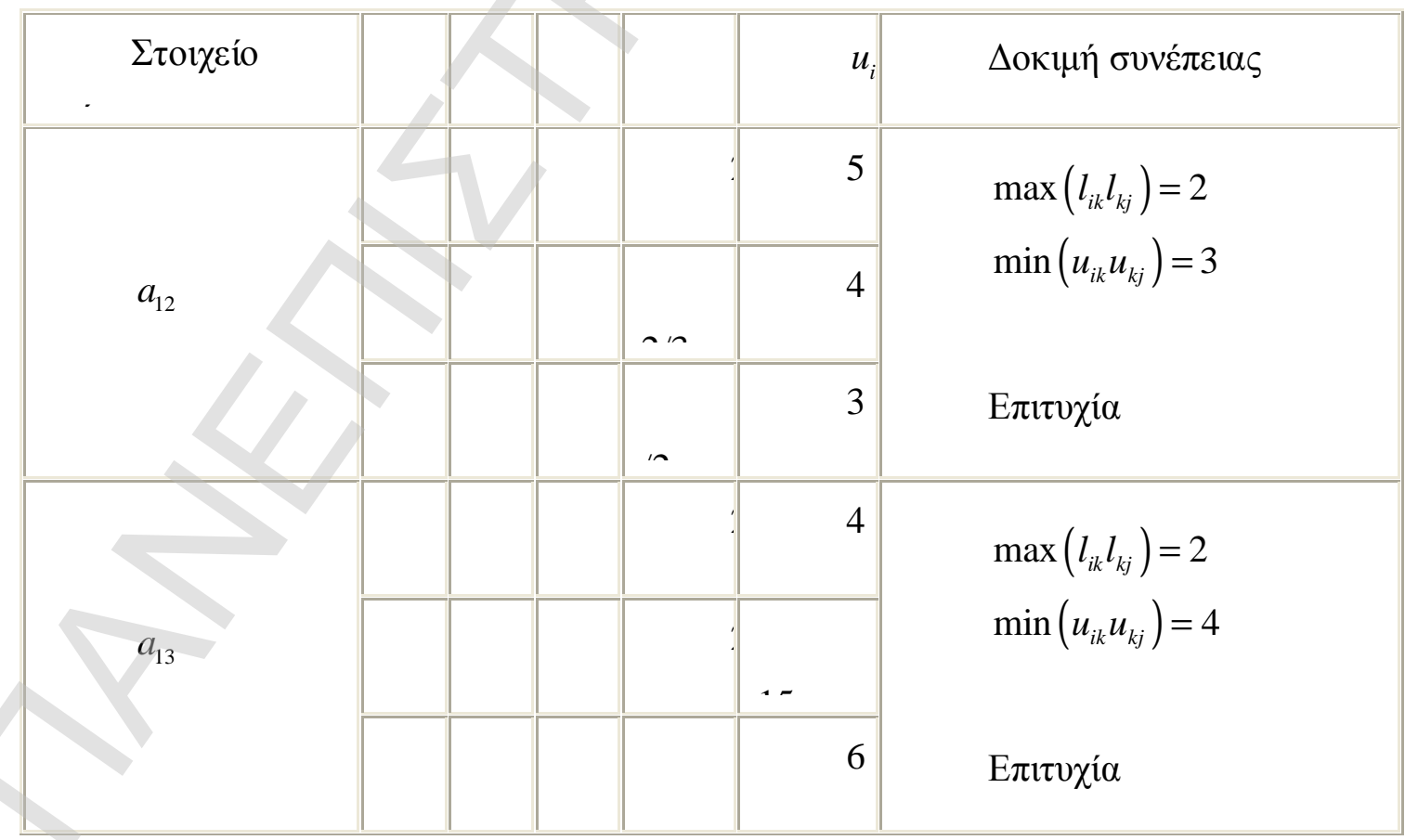




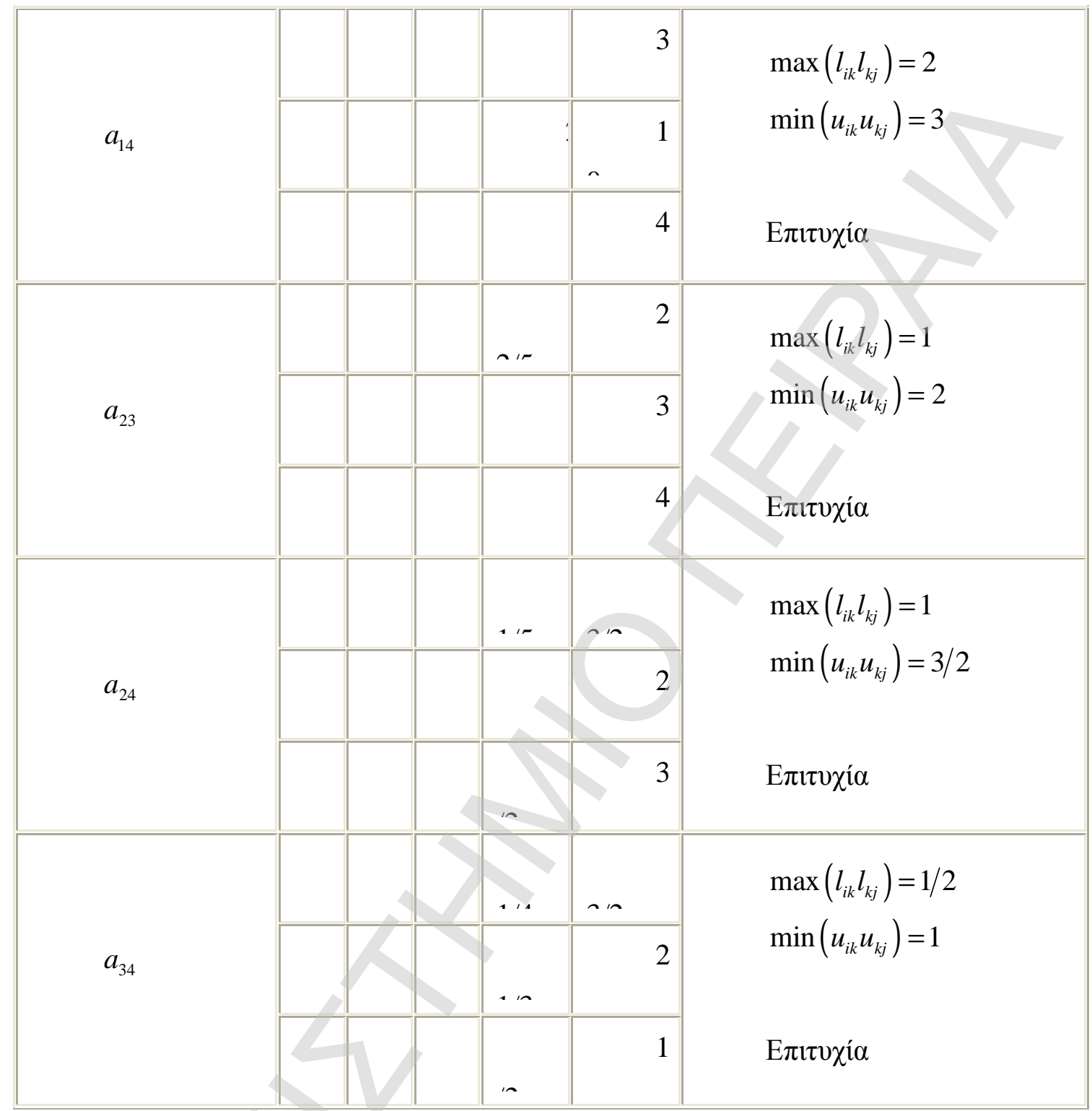

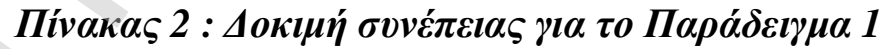




\begin{tabular}{|c|c|c|c|c|c|}
\hline 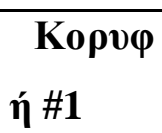 & $\begin{array}{l}\text { Kopv } \\
\varphi \eta ́ ~ \# 2\end{array}$ & $\begin{array}{l}\text { Kopv } \\
\varphi \eta ́ ~ \# 3\end{array}$ & $\begin{array}{l}\text { Kopv } \\
\varphi \eta ́ \text { \#4 }\end{array}$ & $\begin{array}{l}\text { Kopv } \\
\varphi \eta ́ \text { \#5 }\end{array}$ & $\begin{array}{l}\text { Kopv } \\
\varphi \eta ́ \text { \#6 }\end{array}$ \\
\hline 0,522 & 0,500 & 0,400 & 0,444 & 0,480 & 0,462 \\
\hline 0,174 & 0,167 & 0,200 & 0,222 & 0,240 & 0,231 \\
\hline 0,130 & 0,167 & 0,200 & 0,111 & 0,120 & 0,154 \\
\hline 0,174 & $\begin{array}{l}0,167 \\
(0,174 *)\end{array}$ & 0,200 & 0,222 & 0,160 & 0,154 \\
\hline
\end{tabular}

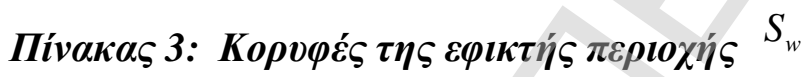

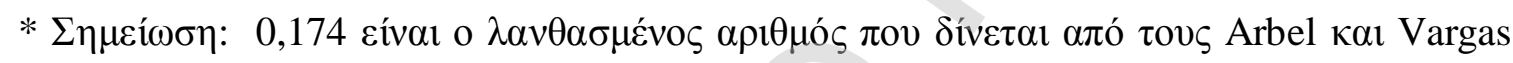
(1993).

\begin{tabular}{|r|r|r|r|l|}
\hline$W$ & $w_{1}$ & $w_{2}$ & $w_{3}$ & $w_{4}$ \\
\hline$w_{i}^{L}=\min w_{i}$ & 0,400 & 0,167 & 0,111 & 0,154 \\
\hline$w_{i}^{U}=\min w_{i}$ & 0,522 & 0,240 & 0,200 & 0,222 \\
\hline
\end{tabular}

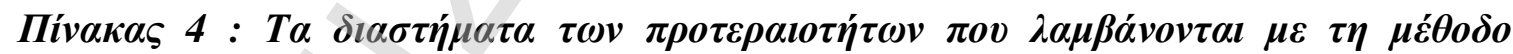

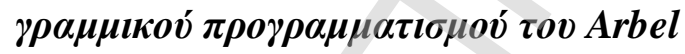




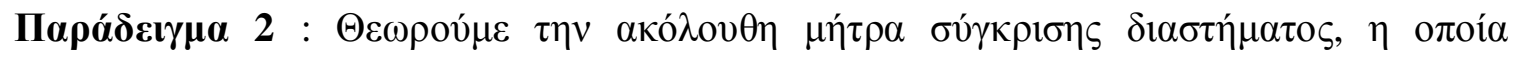

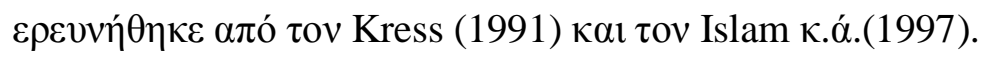

$$
A=\left[\begin{array}{cccc}
1 & {[1,2]} & {[1,2]} & {[2,3]} \\
{\left[\frac{1}{2}, 1\right]} & 1 & {[3,5]} & {[4,5]} \\
{\left[\frac{1}{2}, 1\right]} & {\left[\frac{1}{5}, \frac{1}{3}\right]} & 1 & {[6,8]} \\
{\left[\frac{1}{3}, \frac{1}{2}\right]} & {\left[\frac{1}{5}, \frac{1}{4}\right]} & {\left[\frac{1}{8}, \frac{1}{6}\right]} & 1
\end{array}\right]
$$

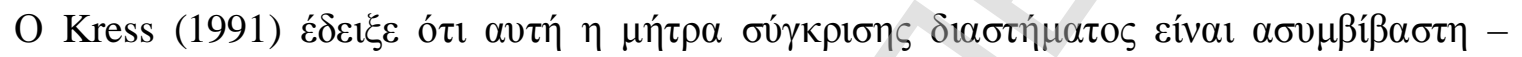

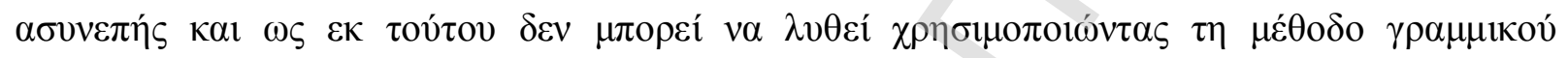

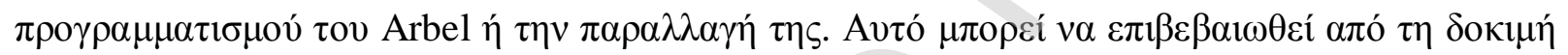

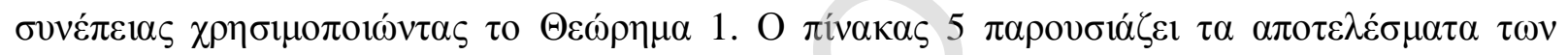

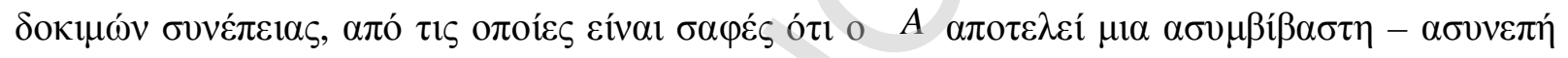

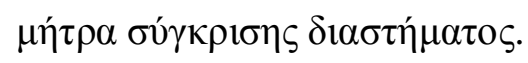

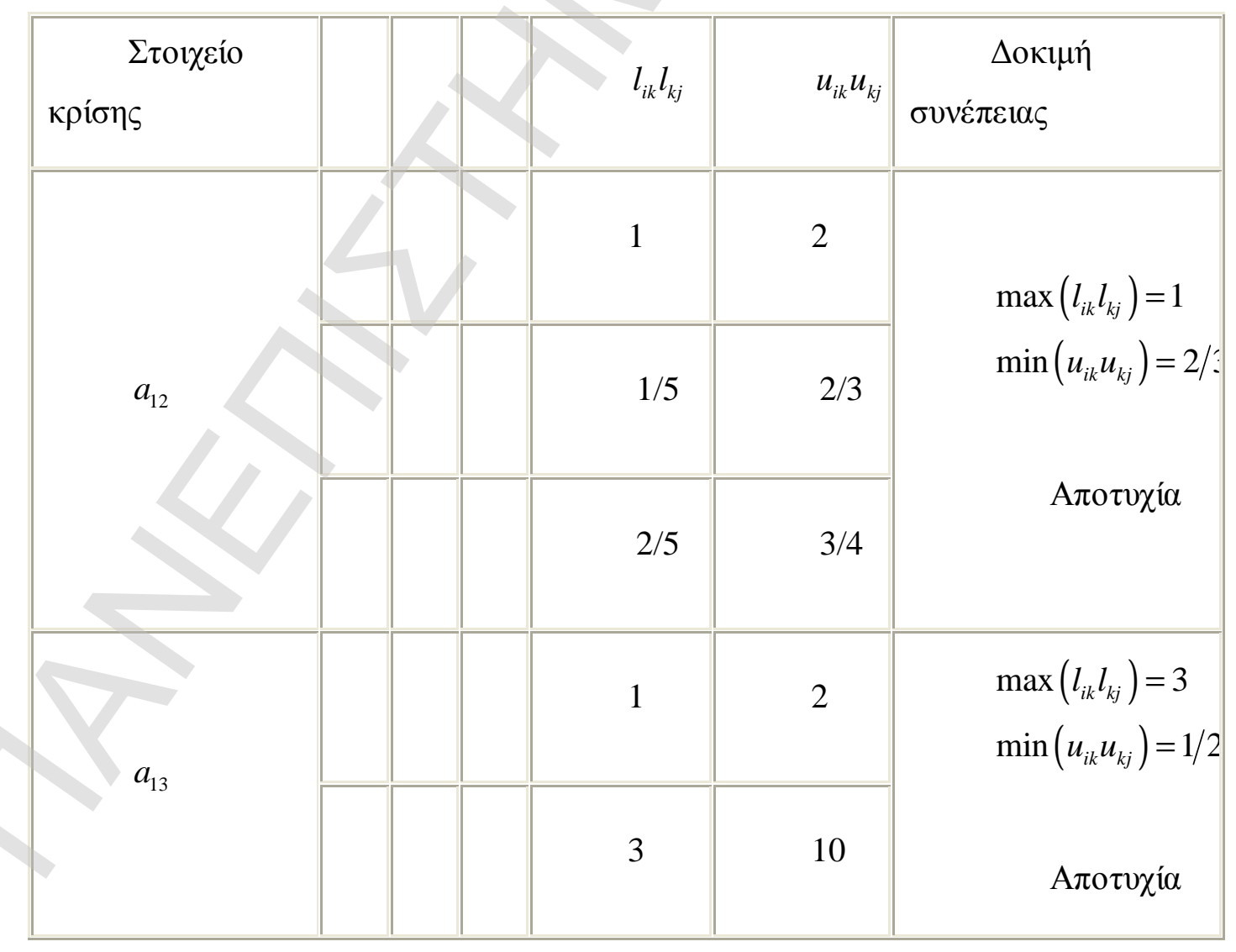




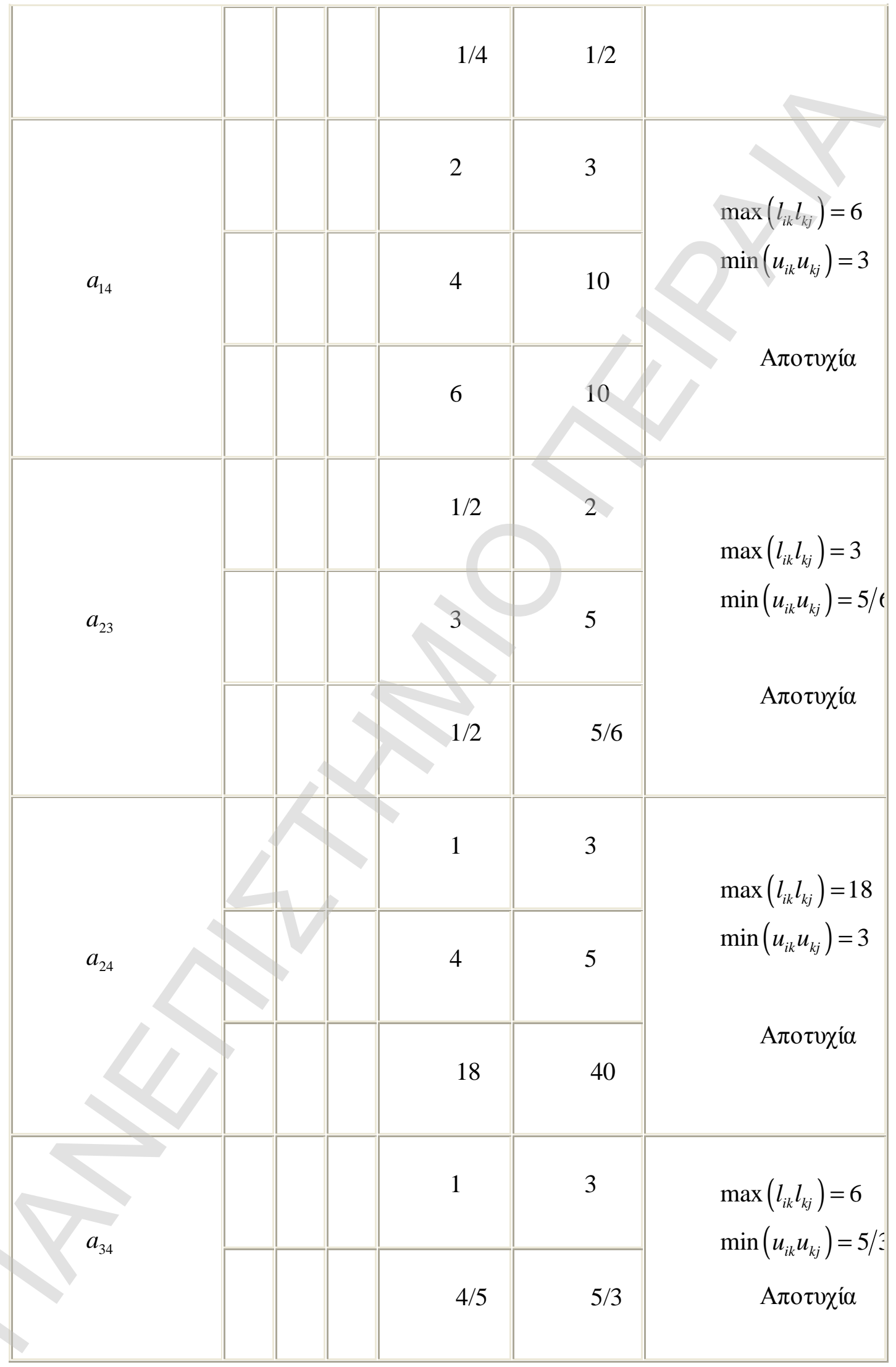




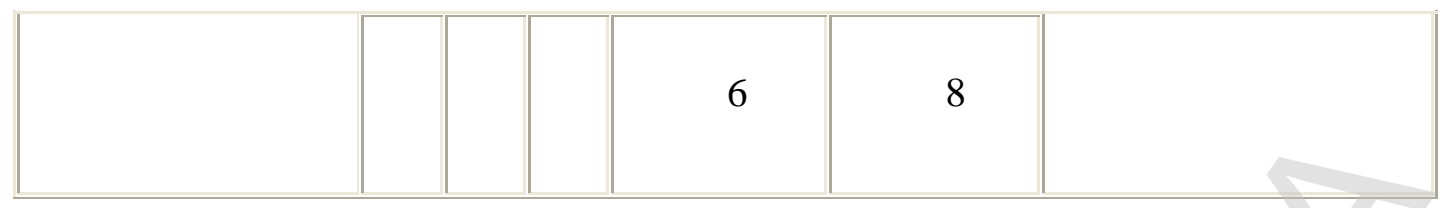

\section{Пívakas 5}

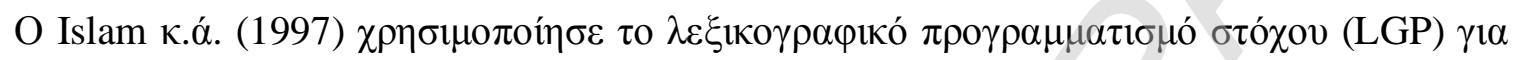

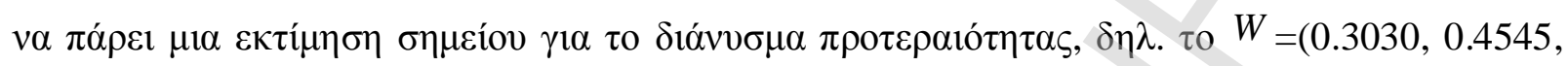

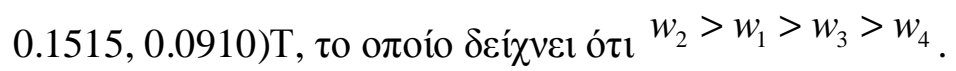

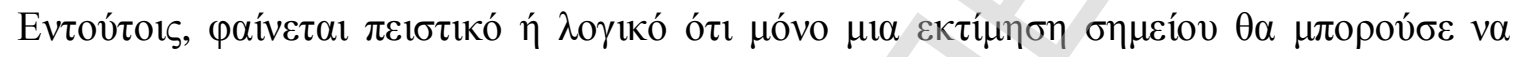

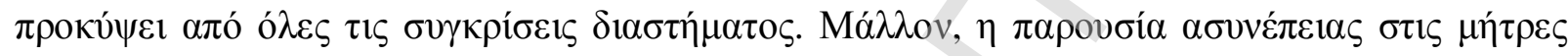

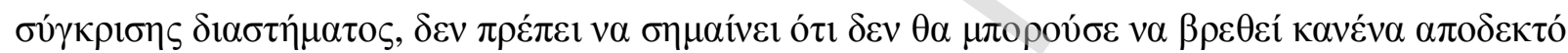

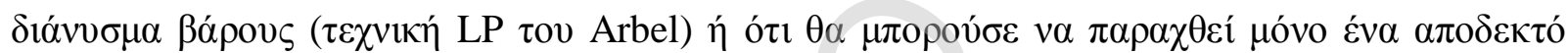

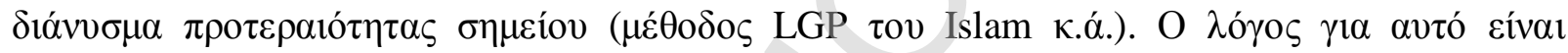

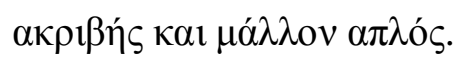

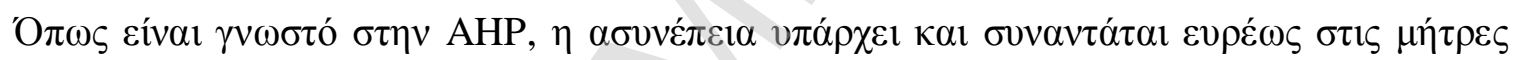

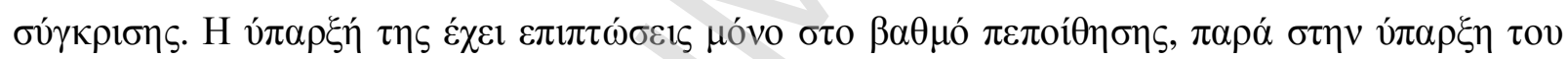

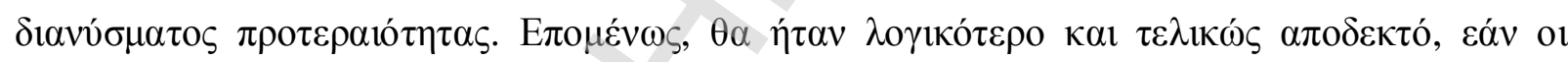

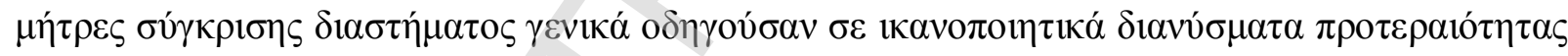
$\delta i \alpha \sigma \tau \eta ́ \mu \alpha \tau o \varsigma$.

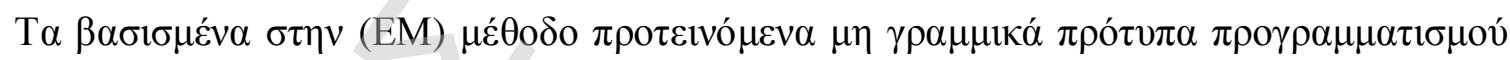

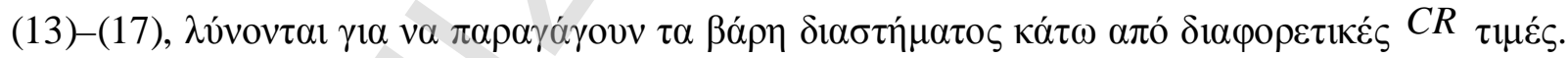

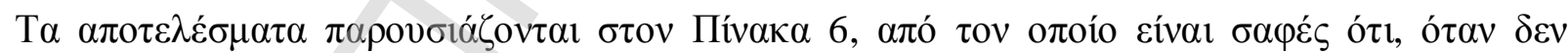

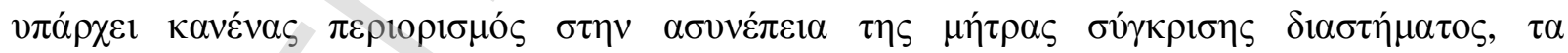

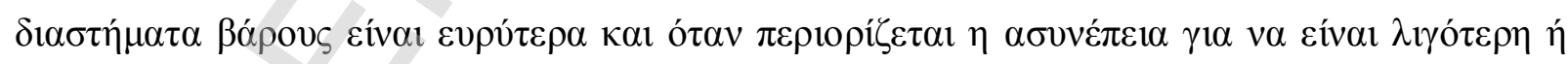

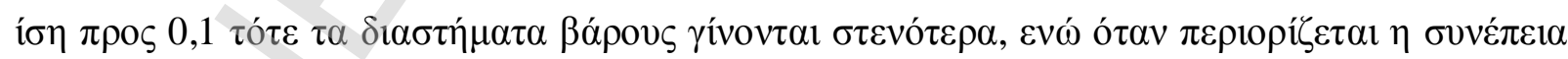

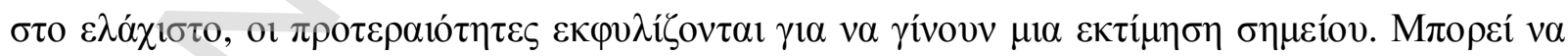

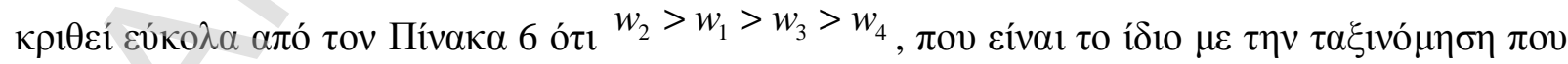

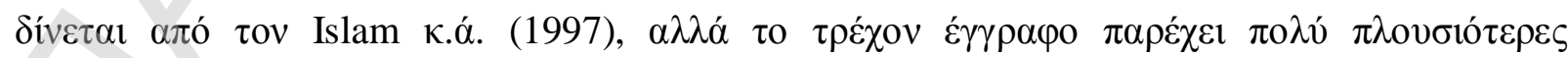

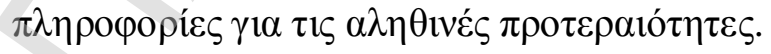




\begin{tabular}{|c|c|c|c|c|c|c|c|c|c|}
\hline \multirow{2}{*}{ 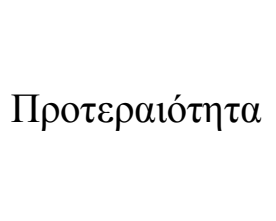 } & \multicolumn{3}{|c|}{$\min C R=0,0831$} & \multicolumn{3}{|c|}{ 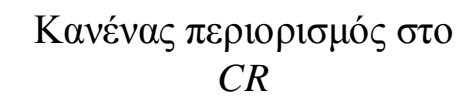 } & \multicolumn{3}{|c|}{ 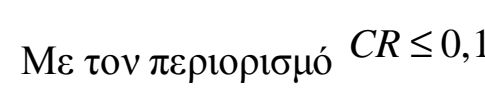 } \\
\hline & $w_{i}^{L}$ & $w_{i}^{U}$ & 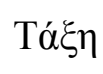 & $w_{i}^{L}$ & $w_{i}^{U}$ & 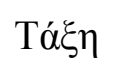 & $w_{i}^{L}$ & $w_{i}^{U}$ & 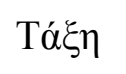 \\
\hline$w_{1}$ & 0,2812 & 0,2812 & 2 & 0,2282 & 0,3830 & 2 & 0,2577 & 0,3259 & 2 \\
\hline$w_{2}$ & 0,4132 & 0,4132 & 1 & 0,3264 & 0,4758 & 1 & 0,3878 & 0,4297 & 1 \\
\hline$w_{3}$ & 0,2386 & 0,2386 & 3 & 0,1792 & 0,2819 & 3 & 0,2116 & 0,2579 & 3 \\
\hline$w_{4}$ & 0,0670 & 0,0670 & 4 & 0,0562 & 0,0843 & & 0,0637 & 0,0743 & 4 \\
\hline
\end{tabular}

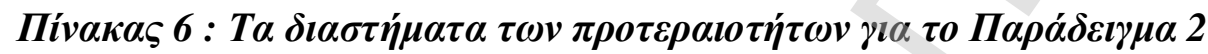

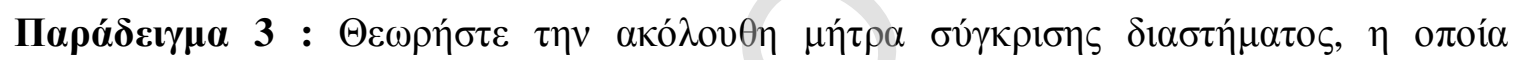

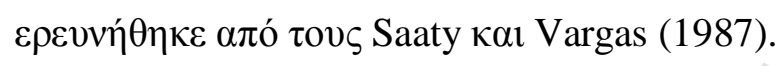

$$
A=\left[\begin{array}{cccc}
1 & {[2,4]} & {[3,5]} & {[3,5]} \\
{\left[\frac{1}{4}, \frac{1}{2}\right]} & 1 & {\left[\frac{1}{2}, 1\right]} & {[2,5]} \\
{\left[\frac{1}{5}, \frac{1}{3}\right]} & {[1,2]} & 1 & {\left[\frac{1}{3}, 1\right]} \\
{\left[\frac{1}{5}, \frac{1}{3}\right]} & {\left[\frac{1}{5}, \frac{1}{2}\right]} & {[1,3]} & 1
\end{array}\right]
$$

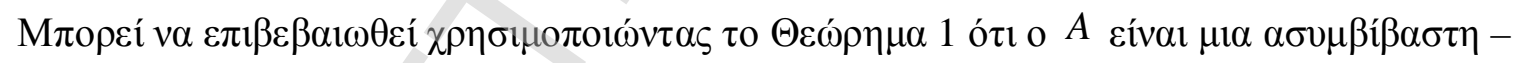

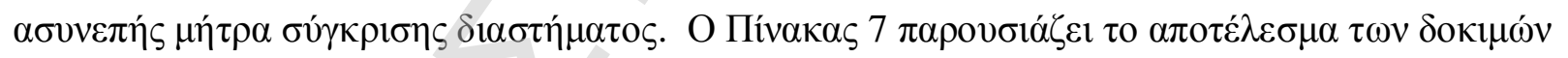

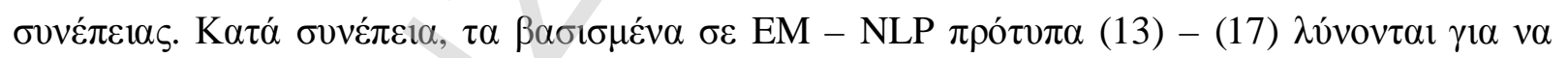

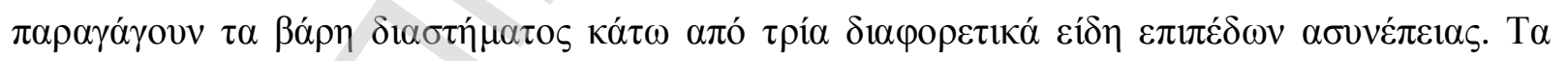

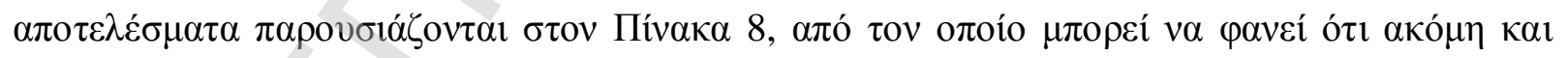

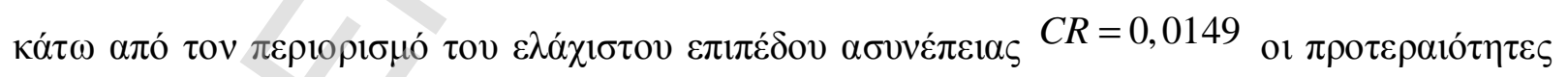

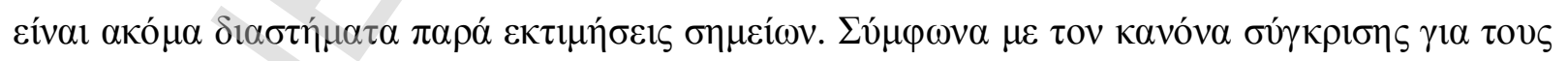

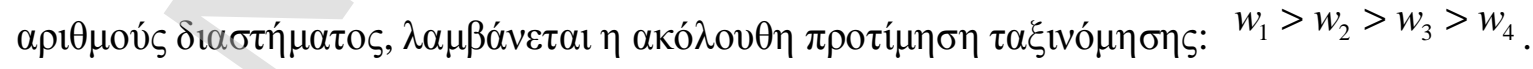

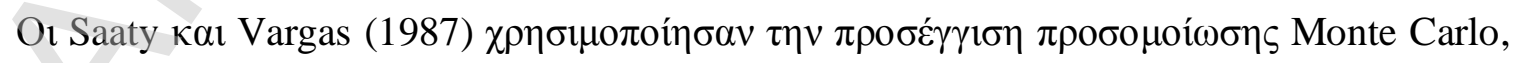

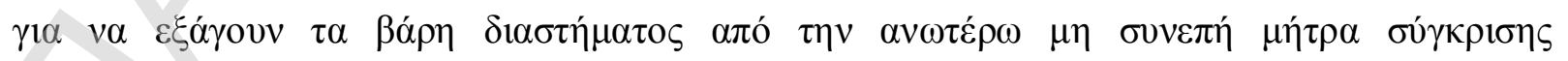

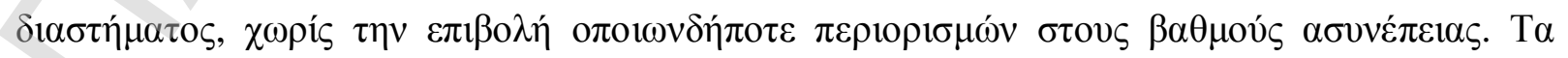

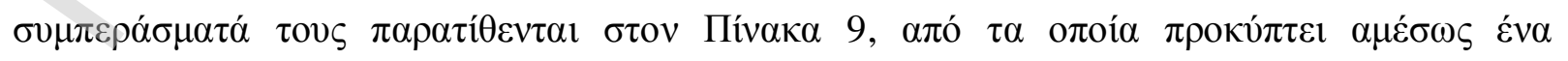

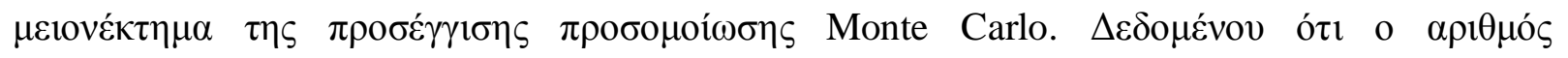




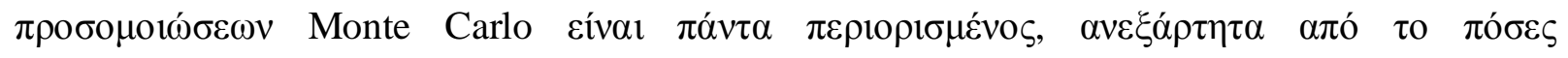

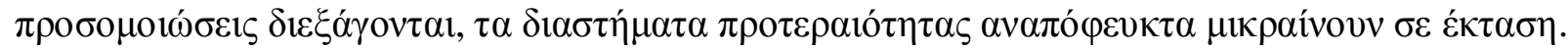

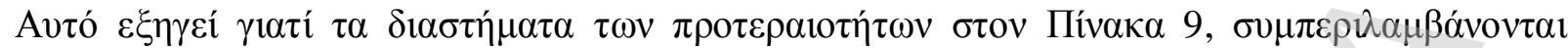

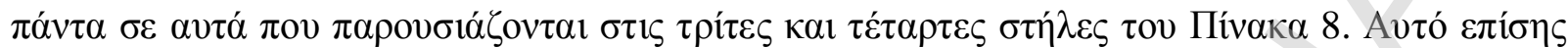

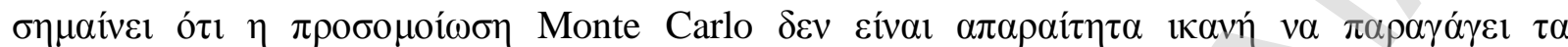

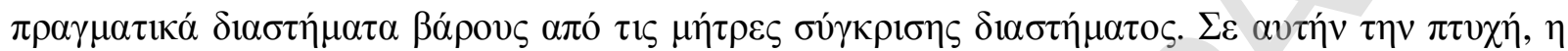

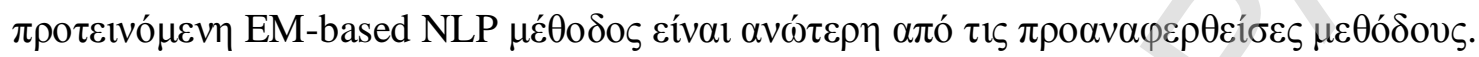

\begin{tabular}{|c|c|c|c|c|c|c|}
\hline 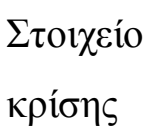 & $i$ & $j$ & $k$ & $l_{i k} l_{k j}$ & & 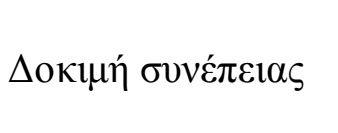 \\
\hline \multirow{3}{*}{$a_{12}$} & 1 & 2 & 1 & 2 & 4 & \multirow{2}{*}{$\begin{array}{l}\max \left(l_{i k} l_{k j}\right)=3 \\
\min \left(u_{i k} u_{k j}\right)=5 / 2\end{array}$} \\
\hline & 1 & 2 & 3 & 3 & 10 & \\
\hline & 1 & 2 & 4 & $3 / 5$ & $5 / 2$ & 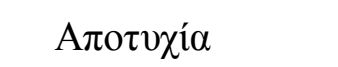 \\
\hline \multirow{3}{*}{$a_{13}$} & 1 & 3 & 1 & 3 & 5 & \multirow{2}{*}{$\begin{array}{l}\max \left(l_{i k} l_{k j}\right)=3 \\
\min \left(u_{i k} u_{k j}\right)=4\end{array}$} \\
\hline & 1 & 3 & 2 & 1 & 4 & \\
\hline & 1 & 3 & 4 & 3 & 15 & 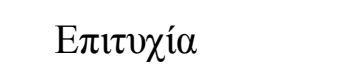 \\
\hline \multirow{6}{*}{$a_{14}$} & 1 & 4 & 1 & 3 & 5 & \multirow{2}{*}{$\begin{array}{l}\max \left(l_{i k} l_{k j}\right)=4 \\
\min \left(u_{i k} u_{k j}\right)=5\end{array}$} \\
\hline & 1 & 4 & 2 & 4 & 20 & \\
\hline & 1 & 4 & 3 & 1 & 5 & 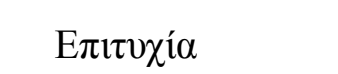 \\
\hline & 2 & 3 & 1 & $3 / 4$ & $5 / 2$ & \multirow{2}{*}{$\begin{array}{l}\max \left(l_{i k} l_{k j}\right)=2 \\
\min \left(u_{i k} u_{k j}\right)=1\end{array}$} \\
\hline & 2 & 3 & 2 & $1 / 2$ & 1 & \\
\hline & 2 & 3 & 4 & 2 & 15 & 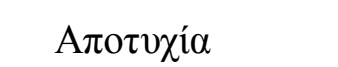 \\
\hline
\end{tabular}




\begin{tabular}{|c|c|c|c|c|c|c|}
\hline \multirow{3}{*}{$a_{24}$} & 2 & 4 & 1 & $3 / 4$ & $5 / 2$ & \multirow{3}{*}{ 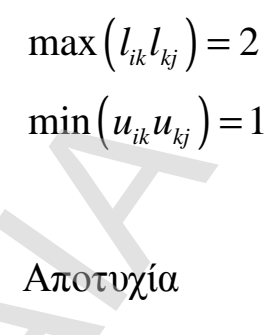 } \\
\hline & 2 & 4 & 2 & 2 & 5 & \\
\hline & 2 & 4 & 3 & $1 / 6$ & 1 & \\
\hline \multirow{3}{*}{$a_{34}$} & 3 & 4 & 1 & $3 / 5$ & & \multirow{2}{*}{$\begin{array}{l}\max \left(l_{i k} l_{k j}\right)=2 \\
\min \left(u_{i k} u_{k j}\right)=1\end{array}$} \\
\hline & 3 & 4 & 2 & 2 & & \\
\hline & 3 & 4 & 3 & $1 / 3$ & & 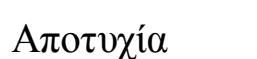 \\
\hline
\end{tabular}

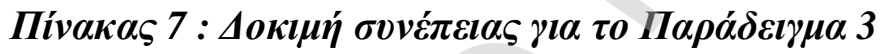




\begin{tabular}{|c|c|c|c|c|c|c|c|c|c|}
\hline \multirow{2}{*}{ 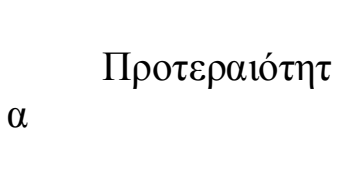 } & \multicolumn{3}{|c|}{$\min C R=0,0149$} & \multicolumn{3}{|c|}{ 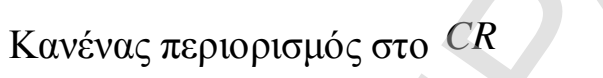 } & \multicolumn{3}{|c|}{ 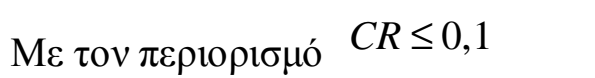 } \\
\hline & $w_{i}^{L}$ & $w_{i}^{U}$ & 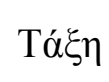 & $w_{i}^{L}$ & $w_{i}^{U}$ & 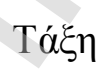 & $w_{i}^{L}$ & $w_{i}^{U}$ & 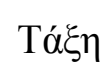 \\
\hline$w_{1}$ & 0,492 & 0,562 & 1 & 0,381 & 0,601 & 1 & 0,432 & 0,601 & 1 \\
\hline$w_{\curvearrowright}$ & 0,180 & 0,209 & 2 & 0,139 & 0,305 & 2 & 0,139 & 0,288 & 2 \\
\hline$w_{2}$ & 0,143 & 0,166 & 3 & 0,101 & 0,214 & 3 & 0,103 & 0,212 & 3 \\
\hline$w_{1}$ & 0,113 & 0,132 & 4 & 0,085 & 0,208 & 4 & 0,087 & 0,193 & 4 \\
\hline
\end{tabular}

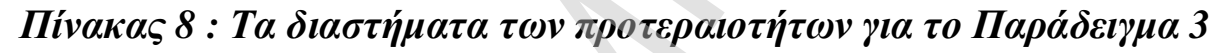

\begin{tabular}{|c|c|c|c|c|}
\hline$W$ & $w_{1}$ & $w_{2}$ & $w_{3}$ & $w_{4}$ \\
\hline$w_{i}^{\min }$ & 0,4374 & 0,1654 & 0,1111 & 0,1011 \\
\hline$w_{i}^{\max }$ & 0,5696 & 0,2708 & 0,1971 & 0,1633 \\
\hline 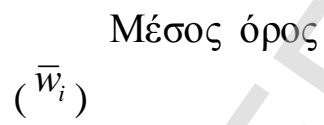 & 0,5093 & 0,2131 & 0,1496 & 0,1280 \\
\hline
\end{tabular}

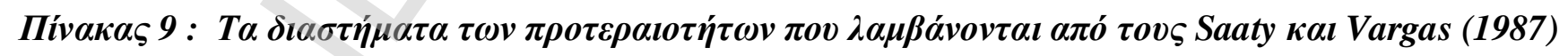




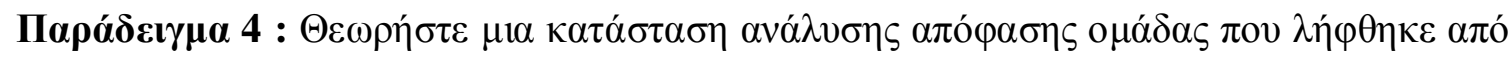

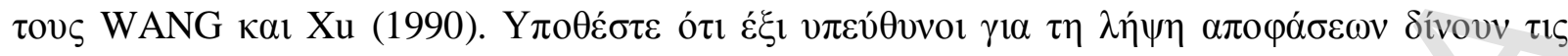

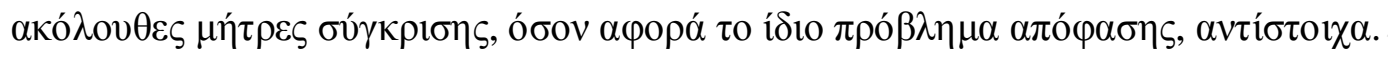

$$
\begin{aligned}
& A_{1}=\left[\begin{array}{ccccc}
1 & 3 & 5 & 4 & 7 \\
\frac{1}{3} & 1 & 3 & 2 & 5 \\
\frac{1}{5} & \frac{1}{3} & 1 & \frac{1}{2} & 3 \\
\frac{1}{4} & \frac{1}{2} & 2 & 1 & 3 \\
\frac{1}{7} & \frac{1}{5} & \frac{1}{3} & \frac{1}{3} & 1
\end{array}\right], \quad A_{2}=\left[\begin{array}{ccccc}
1 & 4 & 3 & 5 & 8 \\
\frac{1}{4} & 1 & 4 & 3 & 6 \\
\frac{1}{3} & \frac{1}{4} & 1 & 1 & 5 \\
\frac{1}{5} & \frac{1}{3} & 1 & 1 & 7 \\
\frac{1}{8} & \frac{1}{6} & \frac{1}{5} & \frac{1}{7} & 1
\end{array}\right], \quad A_{3}=\left[\begin{array}{ccccc}
1 & \frac{1}{2} & 3 & 2 & 5 \\
2 & 1 & 5 & 1 & 2 \\
\frac{1}{3} & \frac{1}{5} & 1 & 2 & \frac{1}{2} \\
\frac{1}{2} & 1 & \frac{1}{2} & 1 & 5 \\
\frac{1}{5} & \frac{1}{2} & 2 & \frac{1}{5} & 1
\end{array}\right] \\
& A_{5}=\left[\begin{array}{ccccc}
1 & 2 & 6 & 3 & 3 \\
\frac{1}{2} & 1 & 2 & 5 & 4 \\
\frac{1}{6} & \frac{1}{2} & 1 & \frac{1}{2} & 1 \\
\frac{1}{3} & \frac{1}{5} & 2 & 1 & 5 \\
\frac{1}{3} & \frac{1}{4} & 1 & \frac{1}{5} & 1
\end{array}\right], A_{4}=\left[\begin{array}{ccccc}
1 & 3 & 5 & 2 & 6 \\
\frac{1}{3} & 1 & 1 & 3 & 2 \\
\frac{1}{5} & 1 & 1 & 4 & 5 \\
\frac{1}{2} & \frac{1}{3} & \frac{1}{4} & 1 & \frac{1}{2} \\
\frac{1}{6} & \frac{1}{2} & \frac{1}{5} & 2 & 1
\end{array}\right] \quad A_{6}=\left[\begin{array}{ccccc}
1 & 2 & 5 & 4 & 9 \\
\frac{1}{2} & 1 & 3 & 2 & 6 \\
\frac{1}{5} & \frac{1}{3} & 1 & 1 & 2 \\
\frac{1}{4} & \frac{1}{2} & 1 & 1 & 3 \\
\frac{1}{9} & \frac{1}{6} & \frac{1}{2} & \frac{1}{3} & 1
\end{array}\right]
\end{aligned}
$$

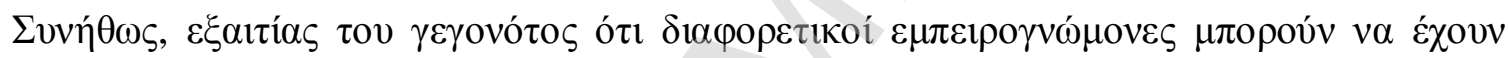

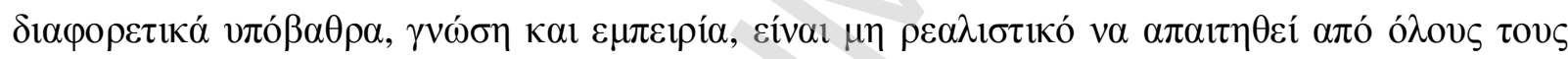

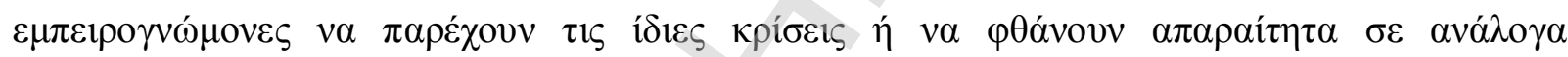

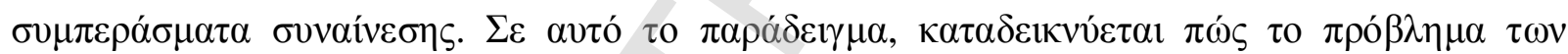

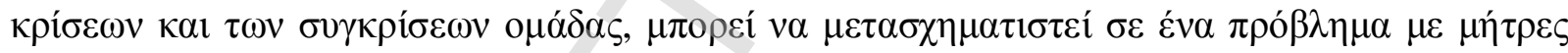

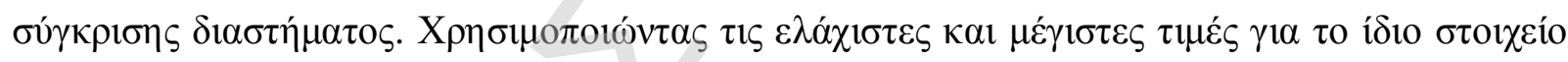

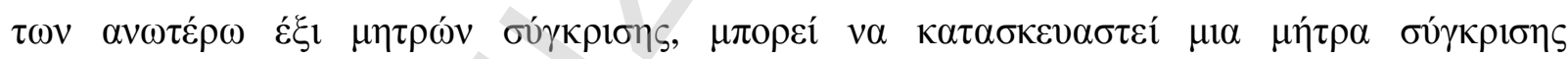

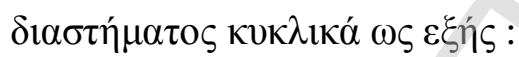

$$
A=\left[\begin{array}{ccccc}
1 & {\left[\frac{1}{2}, 4\right]} & {[3,6]} & {[2,5]} & {[3,9]} \\
{\left[\frac{1}{4}, 2\right]} & 1 & {[1,5]} & {[1,5]} & {[2,6]} \\
{\left[\frac{1}{6}, \frac{1}{3}\right]} & {\left[\frac{1}{5}, 1\right]} & 1 & {\left[\frac{1}{2}, 4\right]} & {\left[\frac{1}{2}, 5\right]} \\
{\left[\frac{1}{5}, \frac{1}{2}\right]} & {\left[\frac{1}{5}, 1\right]} & {\left[\frac{1}{4}, 2\right]} & 1 & {\left[\frac{1}{2}, 7\right]} \\
{\left[\frac{1}{9}, \frac{1}{3}\right]} & {\left[\frac{1}{6}, \frac{1}{2}\right]} & {\left[\frac{1}{5}, 2\right]} & {\left[\frac{1}{7}, 2\right]} & 1
\end{array}\right]
$$

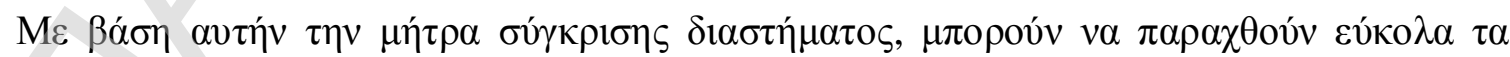

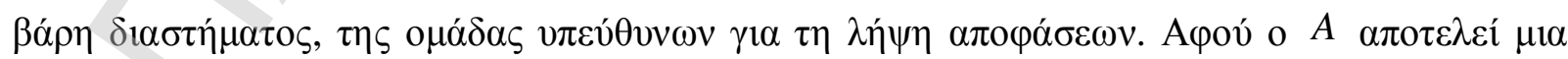

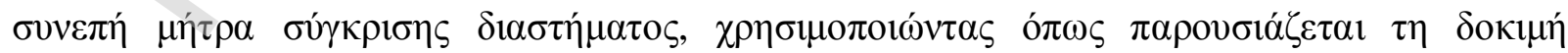

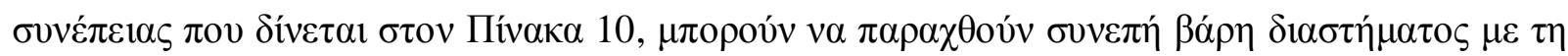




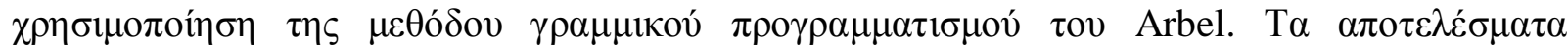

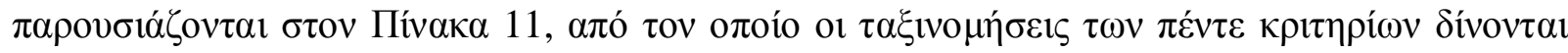
$\alpha \pi \mathrm{ó}^{w_{1}}>w_{2}>w_{4}>w_{3}>w_{5}$.

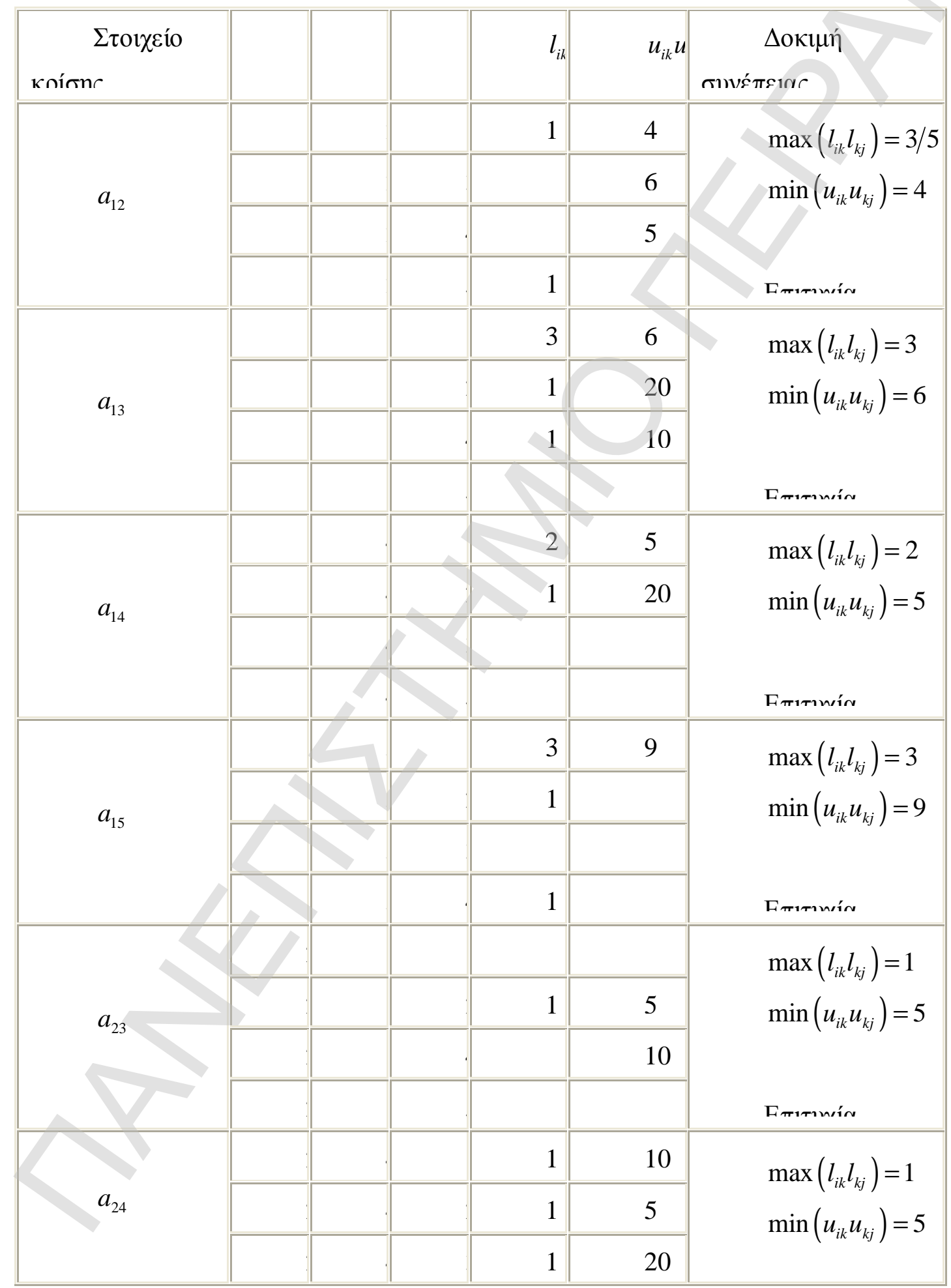




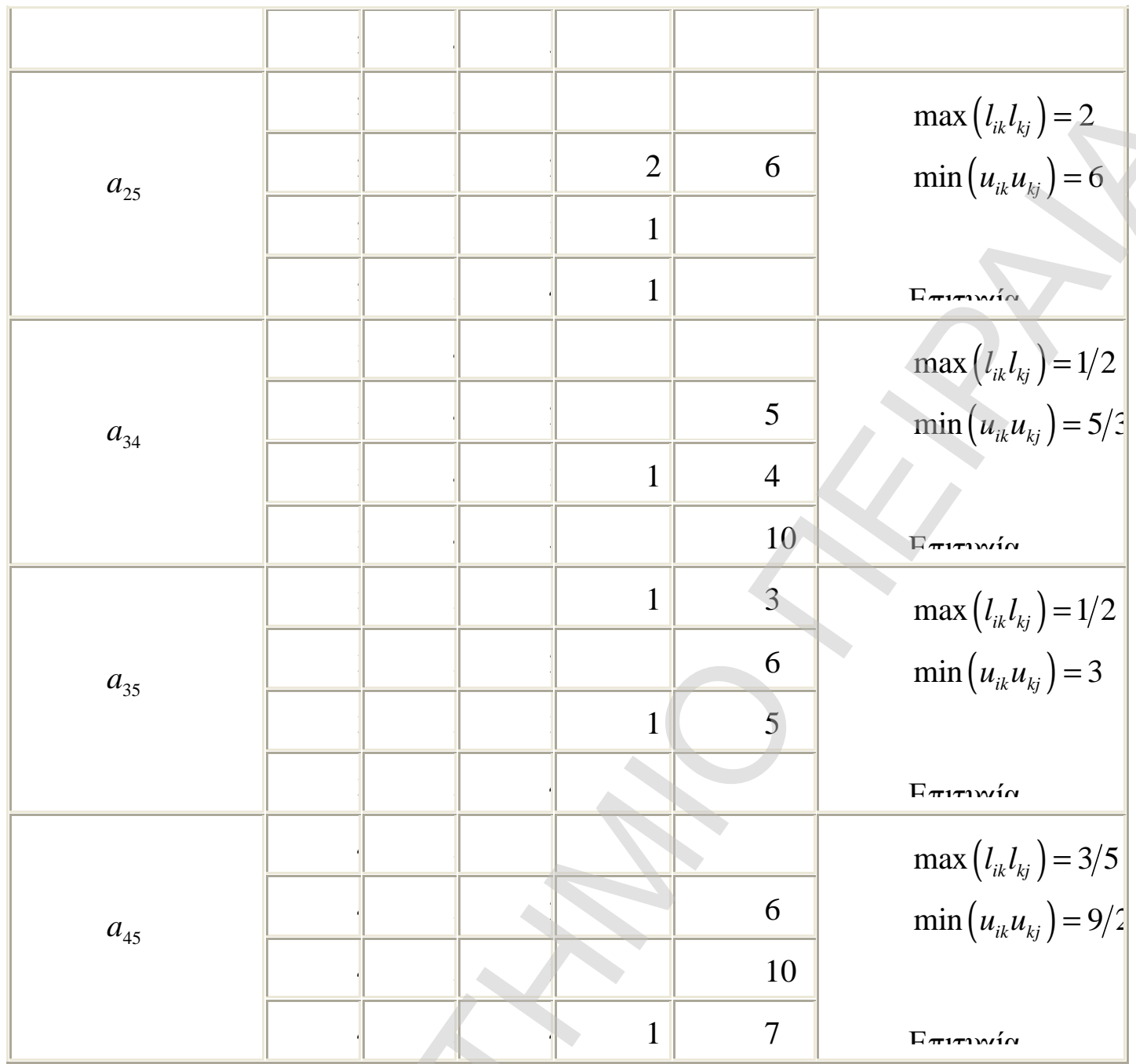

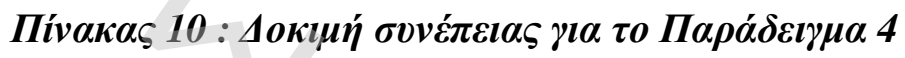

\begin{tabular}{|r|r|r|r|r|r|}
\hline$W$ & $w_{1}$ & $w_{2}$ & $w_{4}$ & $w_{3}$ & $w_{5}$ \\
\hline$w_{i}^{L}=\min w_{i}$ & 0,2609 & 0,1333 & 0,0625 & 0,0698 & 0,0426 \\
\hline$w_{i}^{U}=\min w_{i}$ & 0,5788 & 0,4615 & 0,1685 & 0,2118 & 0,1408 \\
\hline
\end{tabular}

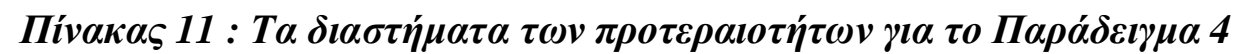




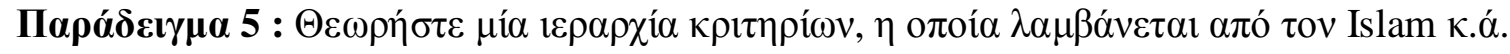

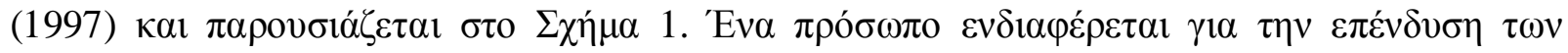

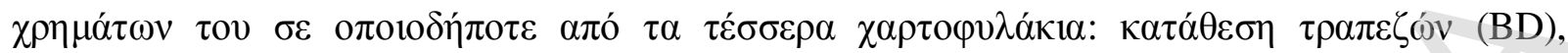

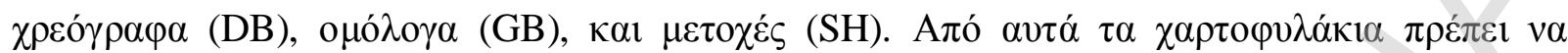

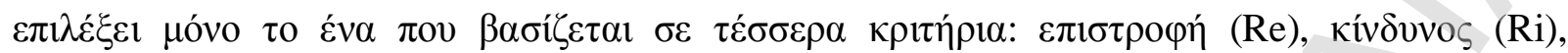

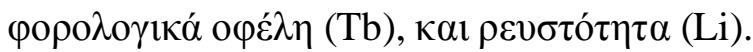

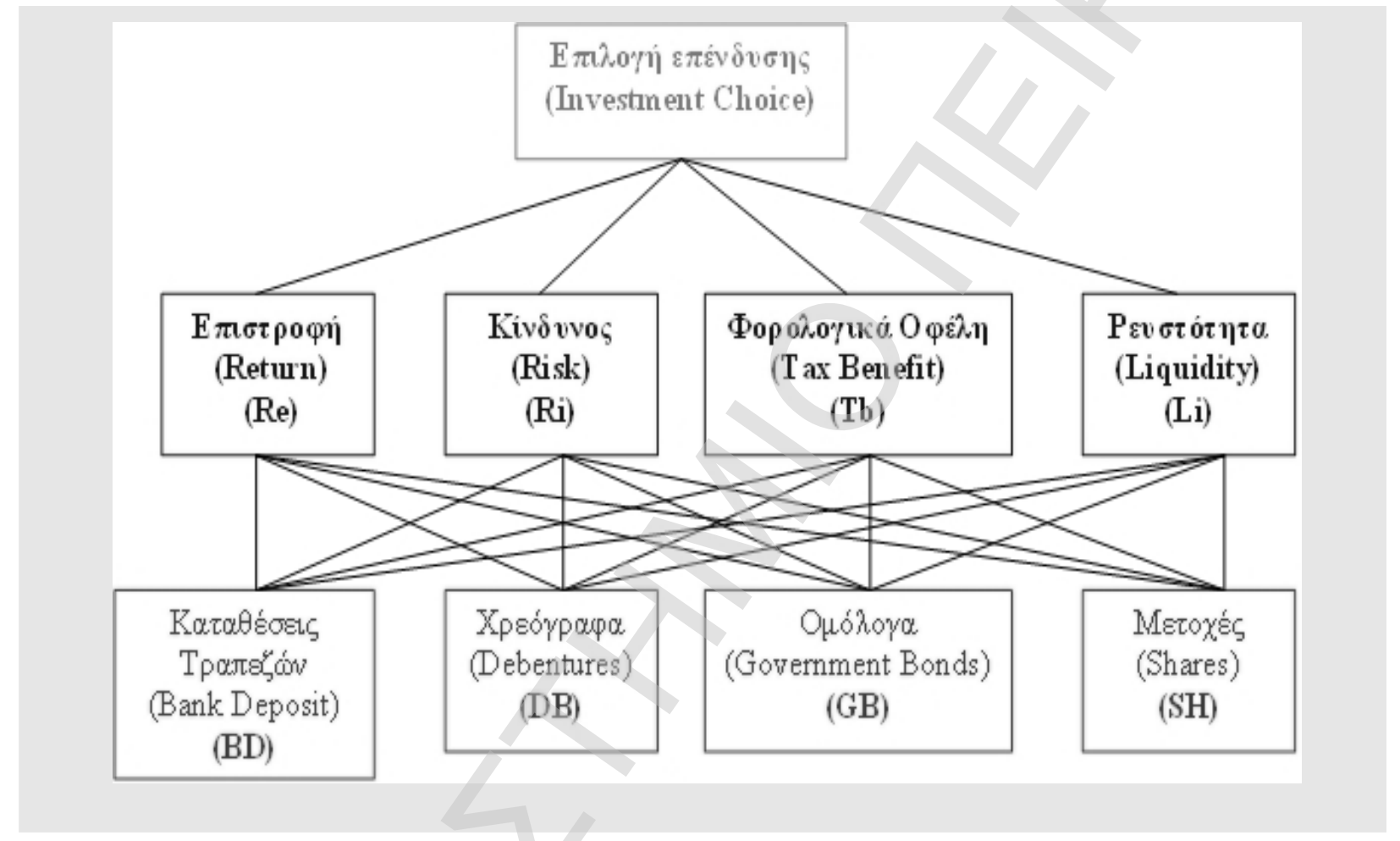

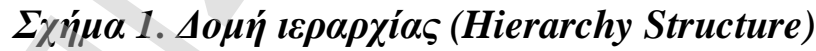

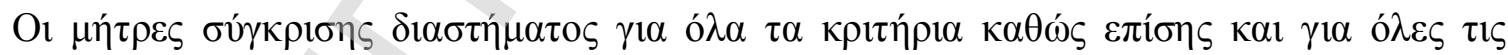

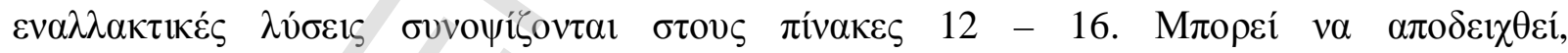

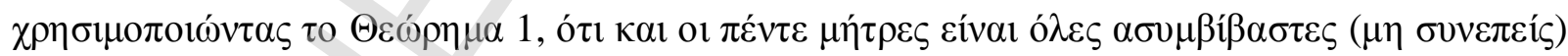

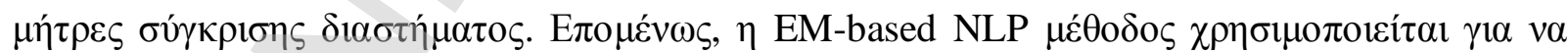

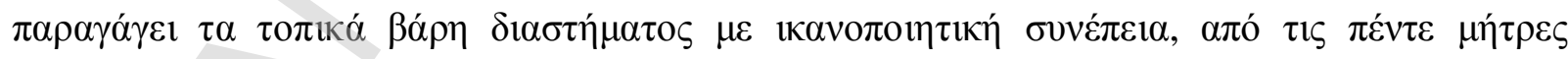

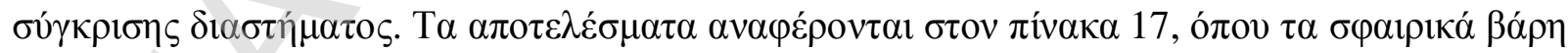

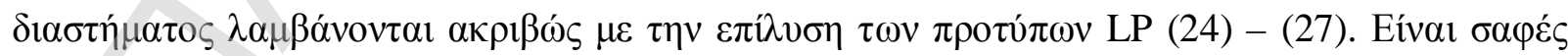

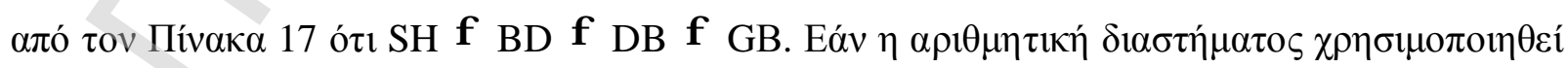

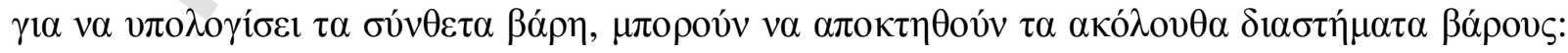
$\mathrm{BD}=[0,21320,3012], \mathrm{DB}=[0,1938$ 0,2808], $\mathrm{GB}=[0,1104 \quad 0,1624], \mathrm{SH}=[0,34290,4300]$. 


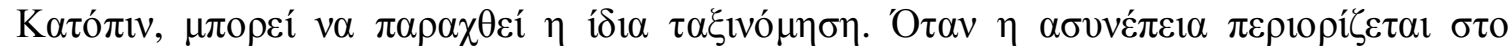

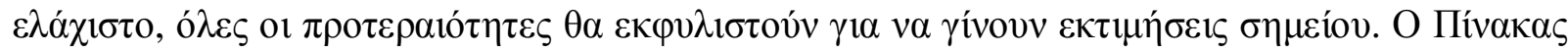

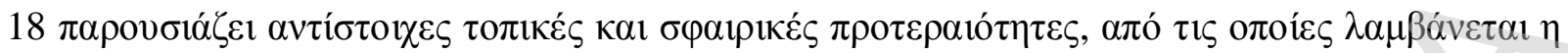

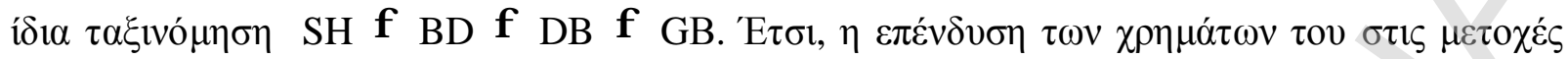

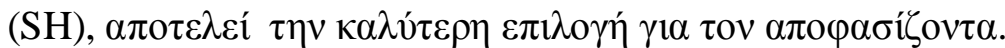

\begin{tabular}{|c|c|c|c|c|}
\hline & $\operatorname{Re}$ & $\mathrm{Ri}$ & $\mathrm{Tb}$ & $\mathrm{Li}$ \\
\hline $\operatorname{Re}$ & 1 & {$\left[\begin{array}{ll}3 & 4\end{array}\right]$} & {$\left[\begin{array}{ll}5 & 6\end{array}\right]$} & {$\left[\begin{array}{ll}6 & 7\end{array}\right.$} \\
\hline $\mathrm{Ri}$ & & 1 & [4 51 & {$\left[\begin{array}{ll}5 & 6\end{array}\right]$} \\
\hline $\mathrm{Tb}$ & & & + & [3 4 4 \\
\hline $\mathrm{Li}$ & & & & 1 \\
\hline
\end{tabular}

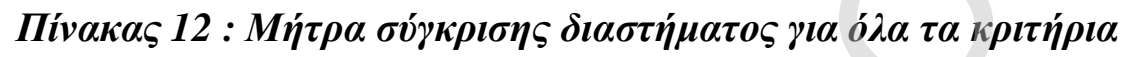

\begin{tabular}{|c|c|c|c|c|}
\hline $\mathrm{Re}$ & $\mathrm{BD}$ & $\mathrm{DB}$ & GB & $\mathrm{SH}$ \\
\hline $\mathrm{BD}$ & 1 & {$[1 / 4]$} & {$\left[\begin{array}{ll}3 & 4\end{array}\right]$} & {$[1 / 6$} \\
\hline DB & & 1 & {$\left[\begin{array}{ll}6 & 7\end{array}\right]$} & {$[1 / 5$} \\
\hline GB & & & 1 & {$[1 / 7$} \\
\hline SH & & & & 1 \\
\hline
\end{tabular}

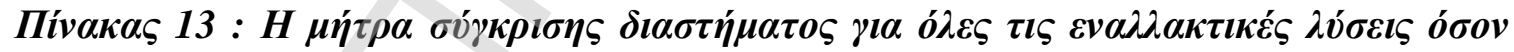

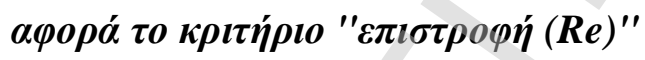




\begin{tabular}{|c|c|c|c|c|}
\hline Ri & BD & DB & GB & SH \\
\hline BD & 1 & {$\left[\begin{array}{ll}3 & 4\end{array}\right]$} & {$\left[\begin{array}{ll}4 & 5\end{array}\right]$} & {$\left[\begin{array}{ll}6 & 7\end{array}\right]$} \\
\hline DB & & 1 & {$\left[\begin{array}{ll}3 & 4\end{array}\right]$} & {$\left[\begin{array}{ll}5 & 6\end{array}\right]$} \\
\hline GB & & & 1 & {$\left[\begin{array}{ll}4 & 5\end{array}\right]$} \\
\hline SH & & & & 1 \\
\hline
\end{tabular}

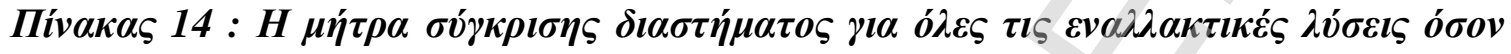

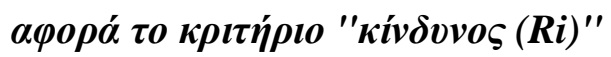

\begin{tabular}{|c|c|c|c|c|}
\hline Tb & BD & DB & GB & SH \\
\hline BD & 1 & 1 & {$[1 / 6$} & {$[1 / 4$} \\
\hline DB & & 1 & {$[1 / 6$} & {$[1 / 4$} \\
\hline GB & & & 1 & {$\left[\begin{array}{ll}5 \\
\hline\end{array}\right.$} \\
\hline SH & & & & 1 \\
\hline
\end{tabular}

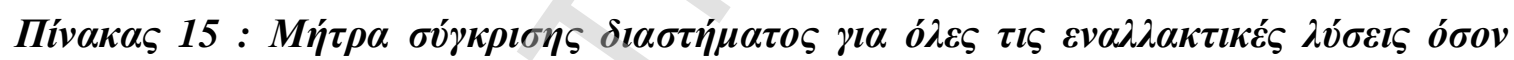

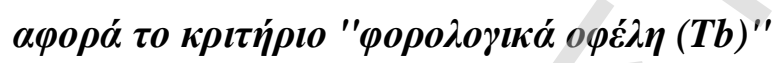

\begin{tabular}{|c|c|c|c|c|}
\hline Li & BD & DB & GB & SH \\
\hline BD & 1 & {$\left[\begin{array}{ll}3 & 4\end{array}\right]$} & 6 & {$\left[\begin{array}{ll}6 & 7\end{array}\right]$} \\
\hline DB & 1 & {$\left[\begin{array}{ll}3 & 4\end{array}\right]$} & {$\left[\begin{array}{ll}3 & 4\end{array}\right]$} \\
\hline GB & & 1 & {$\left[\begin{array}{ll}3 & 4\end{array}\right]$} \\
\hline SH & & & & 1 \\
\hline
\end{tabular}




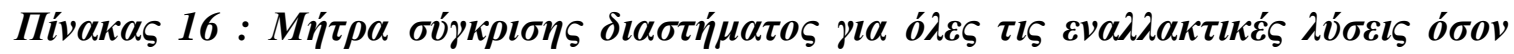

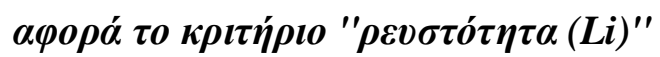

\begin{tabular}{|c|c|c|c|c|c|}
\hline & $\mathrm{Re}$ & $\mathrm{Ri}$ & $\mathrm{Tb}$ & $\mathrm{Li}$ & 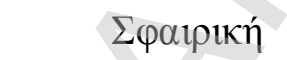 \\
\hline & {$[0,5365-$} & & & & $\pi \rho \circ \tau \varepsilon \rho \alpha$ ló $\tau \eta \tau \alpha$ \\
\hline $\mathrm{BD}$ & {$[0,0936-$} & & & & {$[0,2198-$} \\
\hline DB & {$[0,2209-$} & & & & {$[0,2065-$} \\
\hline GB & {$[0,0454-$} & & & & {$[0,1146-$} \\
\hline $\mathrm{SH}$ & {$[0,5794-$} & & & & {$[0,3474-$} \\
\hline
\end{tabular}

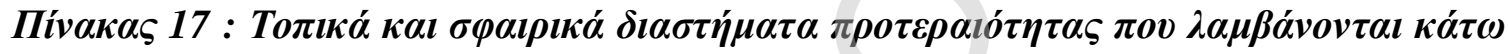

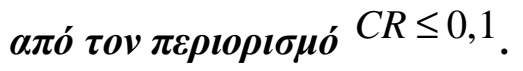

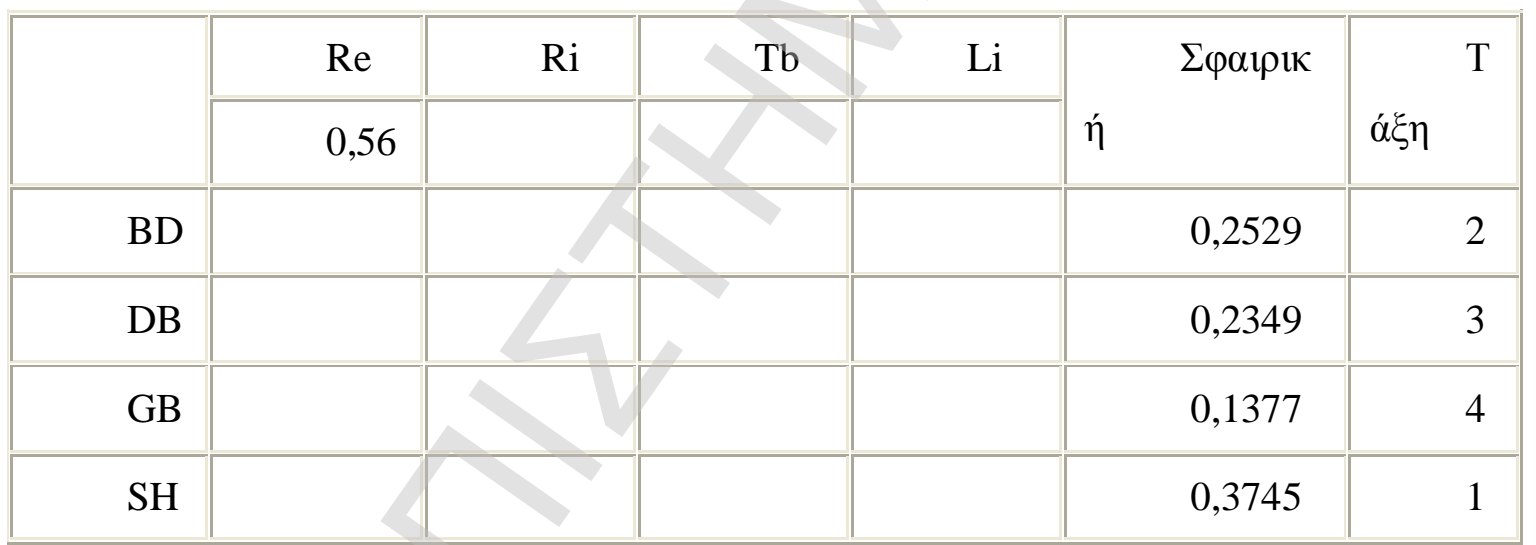

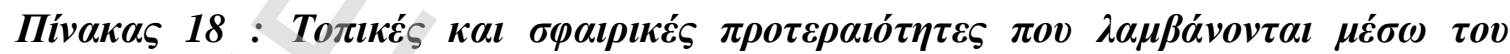
$\varepsilon \lambda \alpha ́ \chi l \sigma \tau o v$ CR . 


\section{APOPA}

[1] Arbel A., 1989 : “Approximate articulation of preference and priority derivation”, European Journal of Operational Research 43, 317-326.

[2] Arbel A., 1991 : "A linear programming approach for processing approximate articulation of preference”, In: Korhonen, P., Lewandowski, A. and Wallenius, J. (Eds.), Multiple Criteria Decision Support. Lecture Notes in Economics and Mathematical Systems 356, SpringVerlag, Berlin, 79-86.

[3] Arbel A., Vargas, L.G., 1992 : "The analytic hierarchy process with interval judgments", In: Goicoechea, A., Duckstein, L., Zoints, S. (Eds.), Multiple Criteria Decision Making. Proceedings of the Ninth International Conference held in Fairfax, Virginia, 1990, Springer, New York, 61-70.

[4] Arbel A., Vargas, L.G., 1993 : "Preference simulation and preference programming: robustness issues in priority deviation”, European Journal of Operational Research 69, 200-209.

[5] Barzilai J., 1997 : "Deriving weights from pairwise comparison matrices”, Journal of Operational Research Society, 48, 1226-1232.

[6] Barzilai, J., Cook, W.D., Golany, B., 1987 : “Consistent weights for judgments matrices of the relative importance of alternatives", Operations Research 6(3), 131-134.

[7] Belton, V., Gear, A.E., 1985 : “The legitimacy of rank reversal - a comment", Omega, 13, $3(143-144)$.

[8] Bonder, C.G.E., de Graan, J.G., Lootsma, F.A., 1989 : "Multicretia decision analysis with fuzzy pairwise comparisons", Fuzzy Sets and Systems 29, 133-143. 
[9] Buckley, J.J., Feuring, T., Hayashi, Y., 2001 : "Fuzzy hierarchical analysis revisited", European Journal of Operational Research 129,48-64.

[10] Chu, A., Kalaba, R., Springarn, K., 1979 : “A comparison of two methods for determing the weights of belonging to fuzzy sets", Journal of Optimization Theory and Applications 27, 531-541.

[11] Cogger, K.O., Yu, P.L., 1985 : “Eigenweight vectors and least-distance approximation for revealed preference in pairwise weight ratios", Journal of Optimization Theory and Applications 46(4), 483-491.

[12] Crawford, G., Williams, C.A., 1985 : "A note on the analysis of subjective judgment matrices", Journal of Mathematical Psychology 29, 387-405.

[13] Crawford, G.B., 1987 : "The geometric mean procedure for estimating the scale of a judgment matrix", Mathematical Modelling 9(3-5), 327-334.

[14] Csutora, R., Buckley, J.J., 2001 : "Fuzzy hierarchical analysis: the Lamda-Max method", Fuzzy Sets and Systems 120, 181-195.

[15] Dyer, J.S., 1990(a) : "Remarks on the Analytic Hierarchy Process", Management Science, 36, 3 (249-258).

[16] Dyer, J.S., 1990(b) : “A clarification of 'Remarks on the Analytic Hierarchy Process"”, Management Science, 36, 3 (274-275).

[17] Rench, S., 1988 : "Decision Theory : An Introduction to the Mathematics of Rationality", Ellis Horwood Limited, Chichester.

[18] Golden, B.L., Wasil, E.A., Harker, P.T. (eds.), 1989 : "The Analytic Hierarchy Process: Applications and Studies", Springer-Verlag, New York. 
[19] Haines, L.M., 1998 : "A statistical approach to the analytic hierarchy process with interval judgments", (I). Distributions on feasible regions. European Journal of Operational Research 110,112-125.

[20] Holder, R. D., 1990 : "Some comments on the Analytic Hierarchy Process", Journal of the Operational Research Society, 41, 11 (1073-1076).

[21] Islam,R., Biswal, M.P., Alam,S.S., 1997 : "Preference programming and inconsistent interval judgments”, European Journal of Operational Research 97,53-62.

[22] Leung, L.C., Cao, D., 2000 : "On consistency and ranking of alternatives in fuzzy AHP”, European Journal of Operational Research 124, 102-113.

[23] Mikhailov L. : "A Fuzzy approach to deriving priorities from interval pairwise comparison judgements", European Journal of Operational Research (2003- in press).

[24] Saaty, T.L., 1977 : “A scaling method for priorities in hierarchical structures”, Journal of Mathematical Psychology, 15 (234-281).

[25] Saaty, T.L., 1980 : “The Analytic Hierarchy Process”, McGraw-Hill, New York.

[26] Saaty, T.L., 1989 : "Decision making, scaling, and number crunching”, Decision Sciences, 20 (404-409).

[27] Saaty, T.L., 1990 : "An exposition of the AHP in reply to the paper 'Remarks on the Analytic Hierarchy Process", Management Science, 36, 3 (259-268).

[28] Saaty, T.L., 1988 : "Multicriteria Decision Making: The Analytic Hierarchy Process", RWS Publications, Pittsburgh, PA.

[29] Saaty, T.L., 1994 : "Fundamentals of Decision Making and Priority Theory with the Analytic Hierarchy Process", RWS Publications, Pittsburgh, PA. 
[30] Saaty, T.L., Vargas, L.G., 1984 : "The legitimacy of rank reversal”, Omega, 12, 5 (513-516).

[31] Saaty, T.L., Vargas, L.G., 1984, “Comparison of eigenvalue, logarithmic least squares and least squares methods in estimating ratios", Mathematical Modelling 5, 309-324.

[32] Saaty, T.L., Vargas, L.G., Wendell, R.E., 1983 : “Assessing attribute weights by ratios", Omega, 11, 1 (9-12).

[33] Saaty T.L and Vargas L.G. : "Uncertainly and rank order in the analytic hierarchy process", European Journal of Operational Research 32 (1987) 107-117.

[34] Salo, A., Hämäläinen, R.P., 1992 : "Processing interval judgments in the analytic hierarchy process", In: Goicoechea, A., Duckstein, L., Zoints, S. (Eds.), Multiple Criteria Decision Making. Proceedings of the Ninth International Conference held in Fairfax, Virginia, 1990, Springer, New York, 359-372.

[35] Salo, A., Hämäläinen, R.P., 1995 : "Preference programming through approximate ratio comparisons", European Journal of Operational Research 82,458-475.

[36] Shim, J.P., 1989 : "Bibliography research on the Analytic Hierarchy Process (AHP)", Socio-Economic Planning Sciences, 23 (161-167).

[37] Takeda, E., Cooger, K.O., Yu, P.L., 1987 : "Estimating criterion weights using eigenvectors: A comparative study”, European Journal of Operational Research 29, 360-369.

[38] Vargas, L.G., 1990 : An overview of the Analytic Hierarchy Process and its applications", European Journal of Operational Research, 48 (2-8).

[39] Wang,Y.M., Xu, N.R., 1990 : "Linear programming method for solving the groupAHP”, J ournal of Southeast University 20(6), 58-63 (in Chinese). 
[40] Watson, S.R., Freeling, A.N.S., 1982 : “Assessing attribute weights", Omega, 10, 6 (582-583).

[41] Watson, S.R., Freeling, A.N.S., 1983 : “Comment on: Assessing attribute weights by ratios", Omega, 11, 1 (13).

[42] Xu, R., 2000 : "Fuzzy least-squares priority method in the analytic hierarchy process", Fuzzy Sets and Systems 112, 359-404.

[43] Xu, R., Zhai, X., 1996 : "Fuzzy logarithmic least squares ranking method in analytic hierarchy process", Fuzzy Sets and Systems 77, 175-190.

[44] Zahedi, F., 1986 : "The Analytic Hierarchy Process - A survey of the method and its applications", Interfaces, 16, 4 (96-108).

[45] Zimmerman H-J., 1985 : "Fuzzy Set Theory and Its Application ”, Second Edition (1991), Kluwer Academic Publishers, Dordrecht.

[46] Despotis D.K. : "Data envelopment analysis with imprecise data”, European Journal of Operational Research 140 (2002) 24-36.

[47] Kress M. : "Approximate articulation of preference and priority derivation - A comment”, European Journal of Operational Research 52 (1991) 382-383.

[48] Gorry G. M., and M. S. Scott-Morton : “ A framework for management Information Systems.” Sloan Management Review, Fall 1971.

[49] Simon H. : “The new Science of management decision”. Englewood Cliffs, NJ: Prentice Hall, 197.

[50] Anthony, R. N. : "Planning and Control Systems: A framework for analysis." Cambridge MA : Harvard University Graduate school of business, 1965. 
[51] Scott-Morton, M. S. : "Management Decision Systems: computer based support for Decision Making." Cambridge, MA : division of research, Harvard University, 1971.

[52] Finholt, T. and Sproull : "Electronic Groups at work.". Organization science, vol 1, No1, 1990.

[53] Rockart ,J. F. and Delong D. : "Executive Support Systems.” Homewood, IL :Dow Jones -Irwin, 1988.

[54] Rich E. and Knight K. : “Artificial Intelligence”. 2nd edition, New York, Mc Graw Hill 1991.

[55] Negoita,C.F. : “Expert systems and fuzzy systems”. Menlo Park, California : Benjamin-Cummings 1985.

[56] Zadeh,L.A. : "Coping with the imprecision of the real world" Communications of ACM-April 1984.

[57] Meredith, J.R. : “The Implementation of Computer Based Systems." Journal of Operational Management (October 1981).

[58] Liebowitz, J. : "Possible Societal Impacts of Artificial Intelligence.” Information Age (July 1989).

[59] Sviolka, J.J. : "An Examination of the Impact of Expert Systems on the firm : the Case XCON.” MIS Quarterly (June 1990).

[60] Senker P. : "Implications of Expert Systems for Skill Requirements and working Life." AI and Society 3 (1989). 


\section{BIBАIOГРАФIA}

[1] Steven Alter, "Information Systems-A Management respective", 3rd Edition, Addison-Wesley, Longman.

[2] Joseph P.Bigus, 'Data Mining With Neural Networks', Mc Graw-Hill, 1996.

[3] Vasant Dhar-Roger Stein, 'Intelligent Decision Support Methods - The Science of Knowledge Work', Prentice-Hall Inc., 1997.

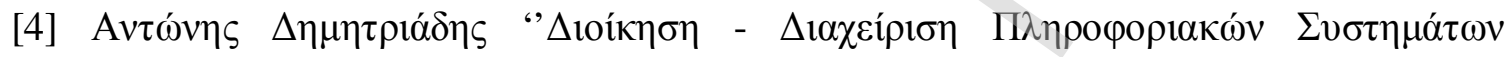

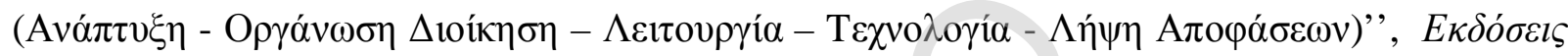

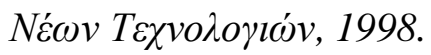

[5] Efraim Turban,'Decision Support and Expert Systems-Management Support Systems", 3rd Edition, Macmillan Publ.Co,1993.

[6] Michel R.Klein- Leif B.Methlie, 'Knowledge - based Decision Support Systems- With Applications in Business', 2rd Edition, Wiley \& Sons Ltd, 1995.

[7] M.G.Singh - J.C.Bennavail, "Knowledge Support Systems For Improving Managerial Decisions",

[8] Efraim Turban, Jay E. Aronson, "Decision Support Systems and Intelligence Systems", Prentice Hall, 1998.

[9] Kenneth C. Laudon, Jane Price Laudon, "Information Systems. A problem-solving approach", Third edition, The Dryden Press, 1995.

[10] Turban, McLean, Wetherbe, "Information Technology for Management. Making Connections for Strategic Advantage”, Second edition, John Wiley \& sons Inc., 1999. 
[11] Decision Support Systems : "An Organizational Perspective”, Keen, Peter,GW and Scott Morton, Michael S., Addison-Wesley, 1978.

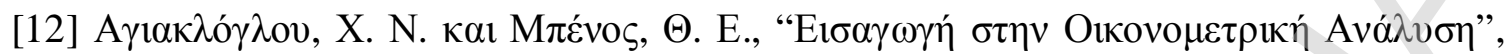

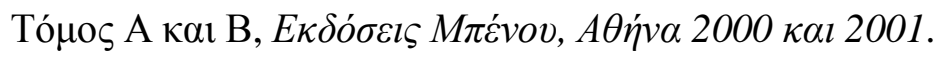

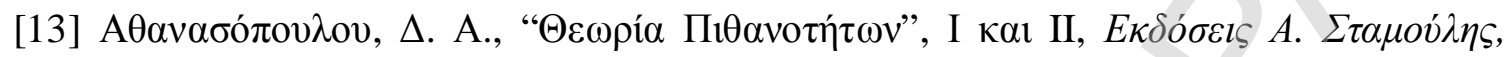

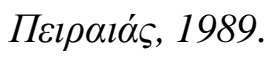

[14] АӨ

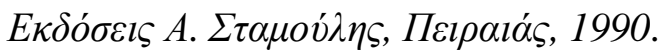

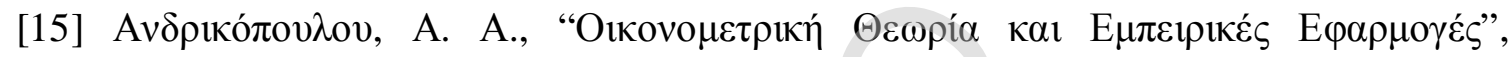

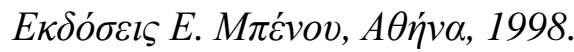

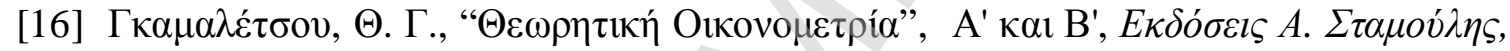

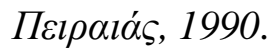

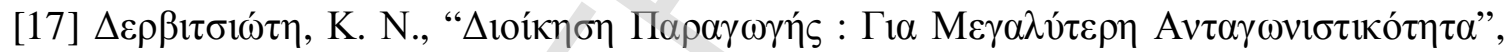

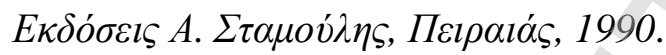

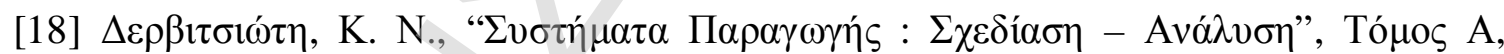

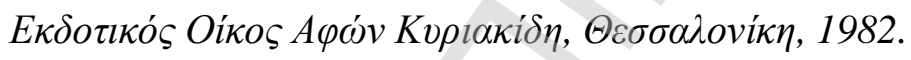

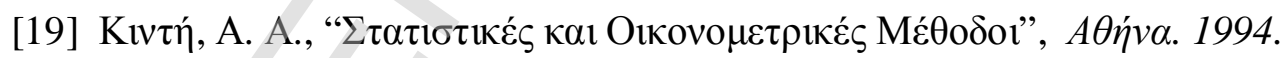

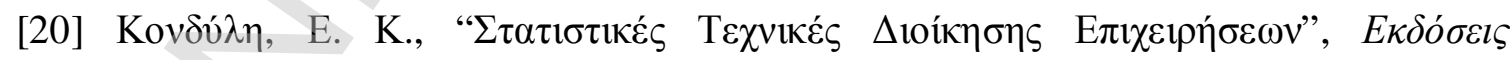

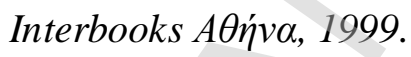

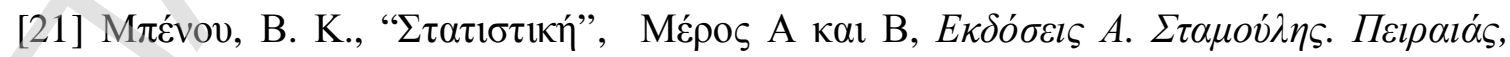
1999. 


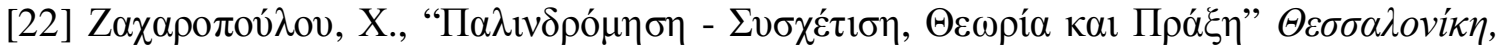
1994.

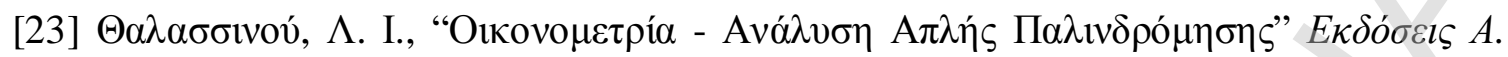

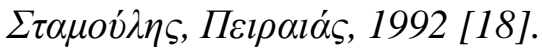

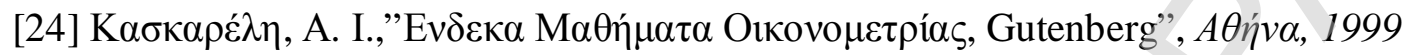

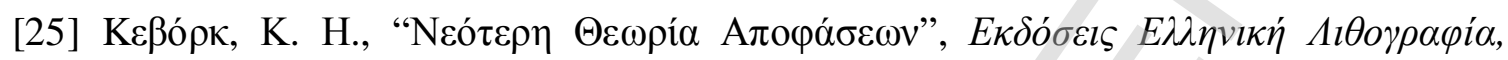

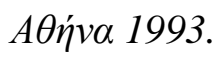

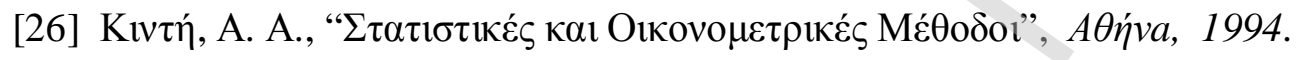

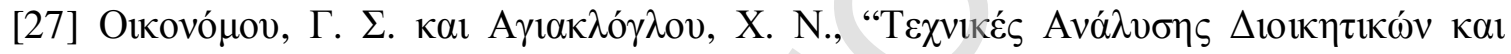

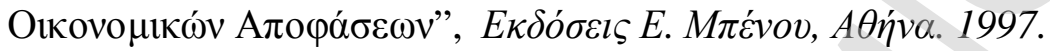

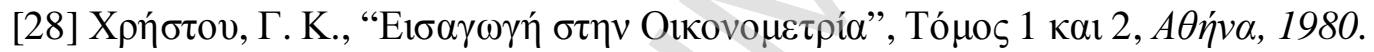

